\title{
Pests and
}

vector-borne diseases

\section{in the livestock industry}


Pests and vector-borne diseases in the livestock industry 


\title{
Pests and vector-borne diseases in the livestock industry
}

Ecology and control of vector-borne diseases

\section{Volume 5}

\author{
edited by: \\ Claire Garros, \\ Jérémy Bouyer, \\ Willem Takken \\ and \\ Renate C. Smallegange
}

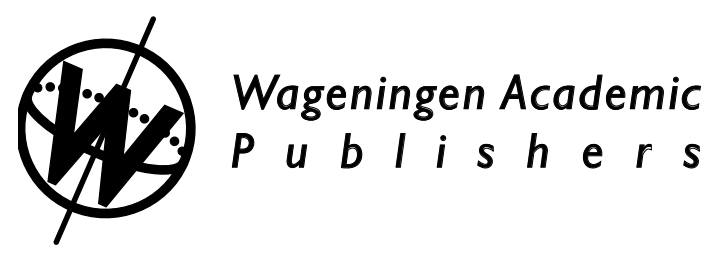


EAN: 9789086863150

e-EAN: 9789086868636

ISBN: 978-90-8686-315-0

e-ISBN: 978-90-8686-863-6

DOI: 10.3920/978-90-8686-863-6

ISSN: 1875-0699

Culicoides on front cover by: Jean-Baptiste Ferré (EID-Méd)

Cow on front cover by: Renaud Lancelot (Cirad) Trap on front cover by: Claire Garros (Cirad) Culicoides on back cover by: Hans M. Smid (bugsinthepicture.com)

First published, 2018

(c) Wageningen Academic Publishers

The Netherlands, 2018
This work is subject to copyright. All rights are reserved, whether the whole or part of the material is concerned. Nothing from this publication may be translated, reproduced, stored in a computerised system or published in any form or in any manner, including electronic, mechanical, reprographic or photographic, without prior written permission from the publisher:

Wageningen Academic Publishers, P.O. Box 220, 6700 AE Wageningen, the Netherlands,

Copyright@WageningenAcademic.com, www.WageningenAcademic.com

The individual contributions in this publication and any liabilities arising from them remain the responsibility of the authors.

The publisher is not responsible for possible damages, which could be a result of content derived from this publication. 


\section{Ecology and control of vector-borne diseases}

In the past century, many advances were made in the control of vector-borne diseases. Malaria disappeared from the northern hemisphere, diseases such as typhus, Bartonella and yellow fever were seriously reduced in prevalence and in many countries effective methods of disease control contributed to a greatly reduced incidence of such diseases. Most of these advances were beneficial to the industrialised world, whereas underdeveloped countries continued to suffer much as before. Indeed, several diseases such as malaria, Rift Valley fever and African sleeping sickness are still highly prevalent in parts of the tropics. 'New' vector-borne diseases such as dengue, chikungunya fever and West Nile fever, have emerged and are invading previously disease-free regions. The discovery of new drugs and vaccines has made great advances and allows for the effective treatment and control of many diseases. In contrast, vector control has lagged behind in development, even though it is realised that effective vector control would allow for an immediate interruption of the transmission of disease, and aid in disease control and eradication. In the last decade new initiatives on vector control have been undertaken, leading to a rapid development of effective and lasting methods of vector control. For example, the Roll Back Malaria control programme of the World Health Organization has led to significant reductions in malaria in many countries. In order to achieve further advances, however, additional tools are required. The development of molecular genetics has provided new insight in vector biology and behaviour, which is being used for developing new strategies of vector control. Advances in geographic information systems allow for precision targeting of interventions. The collective information on new developments in vector ecology and control for vector-borne diseases is scattered over numerous periodicals and electronic databases. This book series intends to bring together this information in sequential volumes arranged around selected themes that are currently of interest.

Willem Takken is the senior editor of the series. Each volume is co-edited by one or more guest editors, which in Volume 5 are Claire Garros, Renate C. Smallegange and Jérémy Bouyer. The editors of the current volume are well-known experts in the field of livestock pests. 


\section{Table of contents}

1. Livestock pests and vector-borne diseases - a much neglected subject

Willem Takken, Jérémy Bouyer, Renate C. Smallegange and Claire Garros

Case studies of livestock pests

2. Arthropod pests in the poultry industry

Olivier Sparagano, Davide Di Domenico, Claudio Venturelli, Elias Papadopoulos,

Renate C. Smallegange and Annunziata Giangaspero

3. Veterinary importance and integrated management of Brachycera flies in dairy farms Frédéric Baldacchino, Marc Desquesnes, Gérard Duvallet, Timothy Lysyk and Steve Mihok

4. Acaricides: current status and sustainable alternatives for controlling the cattle tick, Rhipicephalus microplus, based on its ecology Isabel K.F. de Miranda Santos, Gustavo R. Garcia, Priscila Silva Oliveira,

Cecilia J. Veríssimo, Luciana M. Katiki, Leandro Rodrigues, Matias P.J. Szabó and

Christine Maritz-Olivier

5. Sheep myiasis: a one health perspective

Douglas D. Colwell and Richard Wall

Case studies of vector borne diseases in livestock

6. Integrated control of trypanosomosis

Geoffrey Gimonneau, Jean-Baptiste Rayaisse and Jérémy Bouyer

7. Prevention and control of tick-borne anaplasmosis, cowdriosis and babesiosis in the cattle industry

Martin Pfeffer, Nina Król and Anna Obiegala

8. Mosquito-borne diseases in the livestock industry Nonito Pagès and Lee W. Cohnstaedt

9. Case studies of vector-borne diseases in livestock: bluetongue virus Miguel Á. Miranda 
10. Public-private partnership enabled use of anti-tick vaccine for integrated cattle fever tick eradication in the USA

Adalberto A. Pérez de León, Suman Mahan, Matthew Messenger, Dee Ellis, Kevin

Varner, Andy Schwartz, Dan Baca, Renato Andreotti, Manuel Rodríguez Valle,

Rodrigo Rosario Cruz, Delia Inés Domínguez García, Myrna Comas Pagan, Carmen Oliver Canabal, Jose Urdaz, Francisco Collazo Mattei, Fred Soltero, Felix Guerrero and Robert J. Miller

11. Biological control with parasitoids

Erika T. Machtinger and Christopher J. Geden

12. Biological control of livestock pests: entomopathogens

Emma N.I. Weeks, Erika T. Machtinger, Diana Leemon and Christopher J. Geden

13. Semiochemical tools for a new generation of livestock pest control Victor A. Brugman, Renate C. Smallegange and James G. Logan

14. Genetic control of vectors Jérémy Bouyer and Eric Marois

15. Biosecurity: methods to reduce contact risks between vectors and livestock Bastiaan G. Meerburg and Bruce Schoelitsz

16. The Fly Simulator: a simulation model of stable flies and their control Gösta Nachman and Henrik Skovgård

\section{Impact of vector control}

17. Case study: costs of Culicoides-borne arboviral diseases

Helen Roberts, Emily Nixon and Clemens Matt

18. Controlling tsetse - what does it cost? Alexandra P.M. Shaw

19. Acceptability of vector control actions or co-production of innovations? Fanny Bouyer and Eduardo Chia 
20. Control of vector-borne diseases in the livestock industry: new opportunities and challenges

Claire Garros, Jérémy Bouyer, Willem Takken and Renate C. Smallegange

About the editors

Contributors

583

Reviewers

589

Keyword index

591 


\title{
1. Livestock pests and vector-borne diseases - a much neglected subject
}

\begin{abstract}
Willem Takken ${ }^{1 *}$, Jérémy Bouyer ${ }^{2,3,4}$, Renate C. Smallegange ${ }^{1}$ and Claire Garros ${ }^{4,5}$
'Laboratory of Entomology, Wageningen University \& Research, P.O. Box 16, 6700 AA Wageningen, the Netherlands; ' Unité Mixte de Recherche ASTRE 'Animal, Santé, Territoires, Risques et Ecosystèmes', Campus international de Baillarguet, Centre de Coopération Internationale en Recherche Agronomique pour le Développement (CIRAD), 34398 Montpellier, France; ${ }^{3}$ Insect Pest Control Laboratory, Joint FAO/IAEA Programme of Nuclear Techniques in Food and Agriculture, 1400 Vienna, Austria; ${ }^{4}$ ASTRE, University of Montpellier, CIRAD, INRA, Montpellier, France; ${ }^{5}$ CIRAD, UMR ASTRE, 97491 Sainte-Clotilde, La Réunion, France; willem.takken@wur.nl
\end{abstract}

\begin{abstract}
Worldwide the livestock industry, from small farmers to large industrialized farms, is affected by arthropods and arthropod-borne diseases at various scales, which cause huge losses and are a constraint to socio-economic development. Farmers make considerable efforts to prevent and control pest and disease incidence, often requiring the use of vaccines, if available, drugs and pesticides. Examples of current problems are presented, to set the stage for the detailed and state-of-the-art presentations of specific cases of livestock pests and their associated diseases and modern methods of prevention and control.
\end{abstract}

Keywords: livestock, pests, arthropods, vector-borne disease, prevention, control

\section{Introduction}

Livestock forms one of the important economic pillars of human societies as the principal source of animal protein in nutrition. The animals are commonly held in small or large flocks and herds, and depending on the state of the economy as well as cultural practice, in purposefully-designed buildings, outdoor fields or allowed to range free, where they may even cross the border between countries. Many species of arthropods have formed associations with animals, feeding on animal waste, carcasses as well as on skin, live tissue and body fluids (Williams 2009). Notably among the latter group, several arthropods transmit infectious pathogens (viruses, bacteria, protozoa, nematodes) that can be fatal to the animals, and cause huge economic loss to livestock holders (FAO 2016). Methods of animal husbandry include measures for the prevention of arthropod pests and vector-borne diseases. These require often large investments and are mostly recurrent, creating a constant expense for the farmer and livestock industry. Although arthropods that act as ectoparasites or cause myiasis can inflict considerable damage, the vector-borne diseases deserve specific attention, as these can cause rapid and dramatic losses due to illness and death.

Global industrialization, followed by economic development and rapid growth of the human population, led to livestock production systems that can meet the demand for food from the increasingly-urbanized societies. These developments required a drastic change in methods of animal husbandry, allowing for significant increases in the numbers of livestock held by one farmer. This, in turn, required changes in the way animals are being housed, looked after, fed and milked (cattle, sheep and goats). Similarly, poultry and pigs are kept at increasingly larger scales. The significant increases in the size of livestock production systems, often housing or herding thousands of animals at close range of each other, however, has provided arthropods and their 
associated pathogens unprecedented opportunities for growth and disease infestation. Small and low-tech farming systems, as are still widely found in many lower income countries, experience different effects of arthropods and their associated pathogens than large, highly-intensified farming systems as are now often found in the industrialized world. In the former systems, lack of resources for pest and disease control as well as insufficient or low quality animal food are often bottlenecks for healthy animal production. By contrast, in farming systems of the industrialized countries, resources for pest and disease prevention and control are widely available, and there is no shortage of animal food. Here, the risk is created by housing huge numbers of animals, notably pigs and poultry, in a single facility where the animals are in close contact, with little room for movement. In such a situation, livestock pests have an opportunity to thrive, developing rapidly and causing loss in animal production. Examples are outbreaks of the red poultry mite (George et al. 2015), fleas and mite infestations in swine (Williams 2009), and hornflies in cattle (Swiger and Payne 2017).

More serious are the infectious pathogens transmitted by a wide range of arthropods such as animal trypanosomosis, bluetongue, Rift Valley fever, babesiosis and African swine fever. These pathogens may cause huge losses in a very short time, and are often not easy to control, depending on the availability of vaccines and drugs, as well as arthropod control (FAO 2016; Giordani et al. 2016; Maclachlan and Mayo 2013; Paul-Pierre 2009).

Many arthropod pests of livestock as well as the vectors of pathogenic agents affecting livestock are managed by classical control with insecticides. For the control of the red poultry mite (Dermanyssus gallinae (De Geer)), for example, the chemicals are sprayed on the inner walls, floor and ceilings of poultry stables in order to kill any life stage of the parasite. This has led to the widespread development of insecticide resistance in the mites, rendering such chemicals ineffective. Inevitably, producers began looking for alternative methods of mite control, including heat treatment, steam treatment and silica-based acaricides (poultry mite dust). Sometimes novel combinations of pesticides are being used, leading to environmental and human health disasters as recently occurred in the Netherlands, where the phenyl-pyrazole fipronil was illegally mixed with the natural (and approved) products menthol and eugenol. Fipronil is considered a systemic insecticide, and was subsequently detected in chicken eggs (MacLachlan 2008). These were considered unfit for human consumption and destroyed. As fipronil was not registered for use in poultry houses, this caused a huge scandal with high losses to the economies of the Netherlands and other European countries (Gary 2017; https://english.nvwa.nl/topics/fipronil-in-eggs). House flies (Musca domestica L.) can be a huge problem in pig and poultry farms, where the insects thrive in the animal dung, especially if this is not removed at frequent intervals. The flies are globally resistant to a wide range of insecticides, requiring alternative strategies such as the use of biopesticides like entomopathogenic fungi, pathogenic bacteria and viruses (Acharya et al. 2015; Geden 2012; Vallejo et al. 2013). These biopesticides are promising tools for inclusion in integrated pest management strategies.

These examples of the control of the red poultry mite and of house flies underscore the fragility of the livestock industry's dependence on synthetic insecticides, and lead to high risks for animal health and animal production systems alike. Alternative strategies, other than insecticides, are needed to develop a sustainable and environmentally-acceptable pest control for the livestock industry. In the low-income countries insecticide resistance is usually less a problem than in the industrialized countries, where long-term and extensive use of pesticides has led to the resistance of a large number of pest species, including disease vectors. However, the development plans and associated economic growth is likely to lead to resistance similar to currently in the industrialized 
countries. To avoid a crisis in the livestock industry, it is realized that different strategies for arthropod pest control are required that depend significantly less on synthetic insecticides than is currently the case. On the way to sustainable pest control, strategies using biological control, biorational and mechanical control and genetic tools are being developed, many of which are already being practiced at relatively large scale.

One of the big challenges to integrate the innovative tools to conventional control based on biocides is a full understanding and application of area-wide integrated pest management (AWIPM) concepts (Vreysen et al. 2007) that is still lacking in the field of vector control. 'Area-wide' supposes a centralized approach of insect control with a preventive approach, addressing a full population of vectors in a given area, and accounting for dispersal and migration from surrounding areas. This approach is essential for sustainable management of resistances whereas vector control is presently mostly achieved on a 'field-by field' basis, each farmer acting almost independently from his neighbours. The examples of screwworms and tick eradication projects presented in this volume are nice illustrations on how an Area Wide approach can drive huge benefits at the regional scale. On the other hand, 'IPM' supposes a full understanding of the ecology of the target population to be controlled and of environmental and socio-economic impacts of vector control on the medium and long term that is essential to make it sustainable. The examples of stable fly (Stomoxys calcitrans (L.)) and tsetse fly (Glossina spp.) control presented in this volume are good illustrations of this approach but need to become the rule whereas against some vectors like biting midges, larval ecology is still not yet fully elucidated.

The current volume of the Ecology and Control of Vector-borne Diseases series focuses on common arthropod pests in the livestock industry, the main arthropod-borne diseases affecting livestock and state-of-the-art methods of control. We aim to provide a reference for professionals, researchers and students, so that the reader can become familiarized with the topic and understand if and how prevention and control can be executed in order to minimize damage and allow for a healthy and productive livestock industry. Main pests and diseases are discussed, although due to a lack of experts available for the review, some topics could not be included (e.g. arthropod pests of swine).

A large number of experts has contributed to the book, so that a truly global coverage of the problem and solutions has been achieved.

\section{References}

Acharya N, Rajotte EG, Jenkins NE and Thomas MB (2015) Potential for biocontrol of house flies, Musca domestica, using fungal biopesticides. Biocontrol Sci Technol 25: 513-524.

Food and Agriculture Organisation (FAO) (2016) Economic analysis of animal diseases. FAO, Rome, Italy.

Gary F (2017) The first lessons of the egg crisis involving fipronil. Le Nouveau Praticien Veterinaire Elevages et Santé 37: 8.

Geden CJ (2012) Status of biopesticides for control of house flies. J Biopest 5: 1-11.

George DR, Finn RD, Graham KM, Mul MF, Maurer V, Moro CV and Sparagano OAE (2015) Should the poultry red mite Dermanyssus gallinae be of wider concern for veterinary and medical science? Parasit Vectors 8: 178.

Giordani F, Morrison LJ, Rowan TG, De Koning HP and Barrett MP (2016) The animal trypanosomiases and their chemotherapy: a review. Parasitology 143: 1862-1889.

MacLachlan DJ (2008) Transfer of fat-soluble pesticides from contaminated feed to poultry tissues and eggs. Br Poult Sci 49: 290-298. 
Maclachlan NJ and Mayo CE (2013) Potential strategies for control of bluetongue, a globally emerging, Culicoidestransmitted viral disease of ruminant livestock and wildlife. Antivir Res 99: 79-90.

Paul-Pierre P (2009) Emerging diseases, zoonoses and vaccines to control them. Vaccine 27: 6435-6438.

Swiger SL and Payne RD (2017) Selected insecticide delivery devices for management of horn flies (Haematobia irritans) (Diptera: Muscidae) on beef cattle. J Med Entomol 54: 173-177.

Vallejo CR, Lee JA, Keesling JE, Geden CJ, Lietze VU and Boucias DG (2013) A mathematic model that describes modes of MdSGHV transmission within house fly populations. Insects 4: 683-693.

Vreysen M, Robinson AS and Hendrichs J (2007) Area-wide control of insect pests, from research to field implementation. Springer, Dordrecht, the Netherlands, $789 \mathrm{pp}$.

Williams RE (2009) Veterinary entomology: livestock and companion animals. CRC Press, Boca Raton, FL, USA, 376 pp. 
Case studies of livestock pests 


\title{
2. Arthropod pests in the poultry industry
}

\begin{abstract}
Olivier Sparagano ${ }^{1 *}$, Davide Di Domenico ${ }^{2}$, Claudio Venturelli ${ }^{3}$, Elias Papadopoulos ${ }^{4}$, Renate C. Smallegange 5 and Annunziata Giangaspero ${ }^{6}$

${ }^{1}$ Centre for Agroecology, Water and Resilience (CAWR), Coventry University, Coventry, CV1 5FB, United Kingdom; ${ }^{2}$ Mellivora Pest Management and Consulting, Via F. Coppi 20, 40033 Casalecchio di Reno (BO), Italy; ${ }^{3}$ AUSL of Romagna, U.O. Igiene e Sanità Pubblica - Cesena, Via M. Moretti, 99, 47521 Cesena (FC), Italy; ${ }^{4}$ Laboratory of Parasitology and Parasitic Diseases, School of Veterinary Medicine, Faculty of Health Sciences, Aristotle University of Thessaloniki, Thessaloniki, Greece; ${ }^{5}$ Laboratory of Entomology, Wageningen University \& Research, P.O. Box 16, 6700 AA Wageningen, the Netherlands; ${ }^{6}$ Department of Science of Agriculture, Food and Environment, University of Foggia, Via Napoli 25, 71121 Foggia, Italy; olivier.sparagano@coventry.ac.uk
\end{abstract}

\section{Abstract}

This chapter is presenting the main arthropod pests encountered in the poultry sector, including flies, cockroaches, lice, mites and the lesser mealworm. Each subsection highlights the biological characteristics, life cycle, prevalence, risks to animals and humans, and control methods used on farms with or without the presence of the birds, of each pest group. Some legal directives banning chemicals or imposing new welfare issues can also support these pest proliferations alongside resistance observed in many of these pest species, making control methods more difficult and relying on a combination of approaches to prevent control failures and income reduction. An integrated pest management programme for poultry arthropod pests would have three main components with the goal of keeping these pests below the density where their damage involves costs, welfare and production issues. These components are: (1) biological control methods; (2) chemical control methods; and (3) an educational approach for professionals involved in the poultry sector (farm staff, veterinarians, technologists and industrialists).

Keywords: cockroaches, flies, integrated pest management, lesser mealworm, lice, mites, poultry

\section{General introduction}

Livestock production contributes to $40 \%$ of the total global agricultural production, and supports the livelihood and food security of almost 1.3 billion people. The livestock sector is one of the fastest growing parts of the agricultural economy, with China representing $39 \%$ of this global production, with an increase worldwide of 153\% between 1983 and 2013 (increase of 388\% for Asia during the same period; http://www.fao.org/animal-production/en).

Currently, $92 \%$ of the world's egg production is related to laying hens (http://www.fao.org/ resources/infographics/infographics-details/en/c/284410). The United States Department of Agriculture had forecasted a 1\% increase in broiler production in 2017 up to 89.5 million tonnes with increases in Brazil, USA, the European Union, and India that will compensate for the predicted decline in China. However, China is still the top egg producer with around 25 million tonnes per year. The global egg production is expected to reach 100 million tonnes by 2035. In 2014 it was estimated that 7.2 billion hens produce 1,320 billion eggs (http://freerangereggs.blogspot. co.uk/2017/05/world-egg-production-set-to-hit-100.html). One large egg (50 g) provides 27\% selenium, $25 \%$ vitamin B12, $23 \%$ choline, $15 \%$ riboflavin, $13 \%$ protein, $11 \%$ phosphorus, and $9 \%$ vitamin $D$ of the daily requirements for humans (http://www.fao.org/resources/infographics/ infographics-details/en/c/284410). 
The egg and poultry meat markets are growing globally and poultry diseases would have a tremendous impact on animal welfare, farmers' livelihood, food safety and consumer trust. This chapter covers, in a non-exhaustive way, the major arthropod pests affecting the poultry industry.

\section{Flies}

\section{Introduction}

Flies affecting poultry production can be categorised as 'premise arthropod pests' because their presence is mainly related to poultry manure and other ideal media/substrates that favours the development of large fly populations. The general term 'fly' indicates a group of small and mediumsized insects belonging to the order Diptera. In the poultry industry, the most important flies of veterinary and/or human interest belong to the infraorders Tabanomorpha (family Stratiomyidae), and Muscomorpha (families Muscidae, Fanniidae, Calliphoridae and Drosophilidae) (Kettle 1995).

In poultry environments, fly species are distinctly synanthropic (Kettle 2000; Nichols 2005), i.e. related to humans and their environment (houses, farms, animals, sheds, etc.). The most common species are, in order of importance, Musca domestica L., Fannia canicularis (L.), Ophyra aenescens (Wiedemann), Hermetia illucens (L.), Muscina stabulans (Fallen), Phormia spp., Lucilia spp., Calliphora spp., Drosophila repleta Wollaston (non-biting flies), and Stomoxys calcitrans (L.) (biting fly).

These species can cause serious damage to poultry production; their presence depending on the breeding systems, i.e. on environmental factors and management practices. Flies cause significant health problems on farms, as demonstrated by the vast bibliography concerning the vector capacity of flies, and control measures to be taken on farms, together with the high annual cost for farmers associated with the prevention and control of fly populations.

Fly populations are largest in the various types of caged-layer production systems, and thrive under the slats in broiler breeder houses. Breeding habitats for flies are provided by accumulations of manure, high humidity levels (up to $75 \%$ ), temperatures (above $20^{\circ} \mathrm{C}$ ), the season (springautumn), the presence of other breeding media (e.g. broken eggs, dead birds, feed spills), the duration of the poultry production cycle, and are also affected by the presence/absence of natural fly predators and parasites (James et al. 2004).

Besides a general description of the morphology and biology of flies, this chapter deals with the most economically important fly species found in European poultry farms and the management practices for their control, providing only the most relevant and updated references.

\section{Morphology and biology}

At the adult stage, flies are small to medium-sized $(5-12 \mathrm{~mm})$; size can vary within the same species because it is not genetically based but depends on the food availability during the larval stages.

In the majority of species, the body is covered by pollinosities forming striae, macules, spots, and patterns, and also cuticular appendages, such as bristles, hairs and setae. The head bears two large compound eyes consisting of numerous hexagonal elements $(4,000)$ or ommatidia. Non-biting flies have lapping-sponging type retractile mouthparts for feeding on secretions and animal or human excretions, whereas biting flies have piercing non-retractile mouthparts for blood-feeding. In non-biting flies (e.g. Musca spp.), the proboscis is flexible and ends in two bilobate structures 
(labella), crossed by a series of small grooves or pseudotracheae and prestomal teeth (Figure 1 and 2). Biting flies (e.g. Stomoxys) have a non-retractile proboscis (terminating in little-developed labella, with strong prestomal teeth; Figure 3 and 4) for skin penetration.

The lifecycle displays a holometabolous metamorphosis with four morphologically distinct stages: egg, larva (I, II and III instars), pupa and adult. The eggs are oval and slightly concave, no longer than $1 \mathrm{~mm}$, and range in colour from white to creamish-brown. The whitish cylindrical larva consists of thirteen segments, twelve of which are visible. The anterior region is pointed and bears the mouthparts: a pair of hooks protruding from the oral opening. The anterior spiracles are fanlike structures consisting of a number of finger-like elements, which are clearly visible in mature larvae. The larvae have truncated or rounded ends, depending on the species. The posterior spiracles on the last segment have two respiratory openings with a chitinous ring (peritrema).

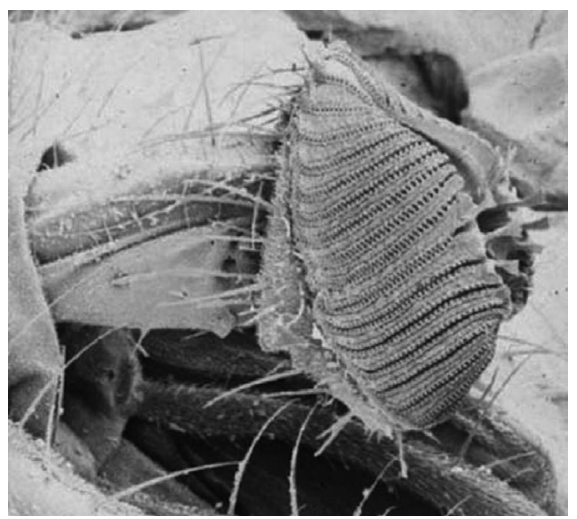

Figure 1. Mouthparts of a Musca spp. showing the labella with prestomal teeth (photo by A. Giangaspero).

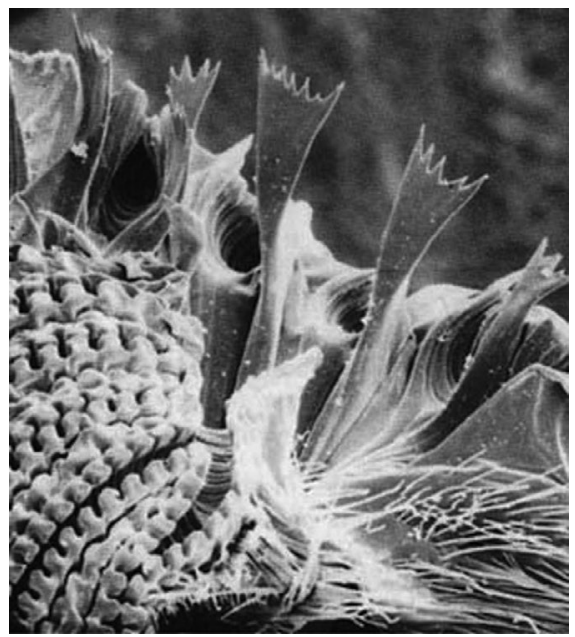

Figure 2. Detailed view of the prestomal teeth of a Musca spp. (photo by A. Giangaspero). 


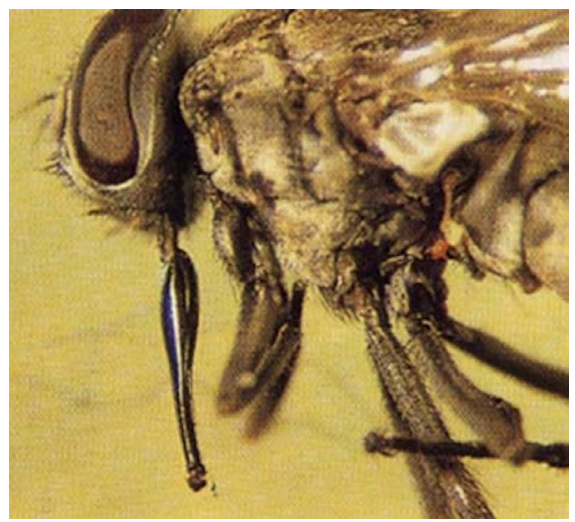

Figure 3. Proboscis of Stomoxys calcitrans (photo by A. Giangaspero).

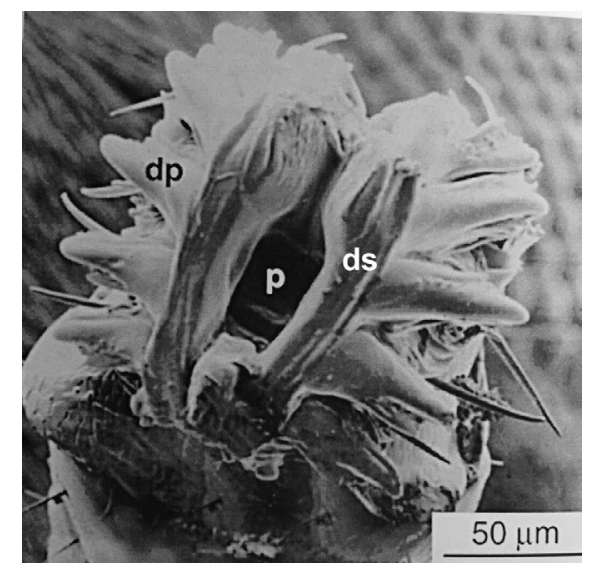

Figure 4. Reduced labella and prestomal teeth of Stomoxys calcitrans ( $p=$ prestomum; $d s=$ discal sclerites; $d p=$ prestomal teeth) (photo by A. Giangaspero).

The pupa is a barrel-like structure formed by the thickening and contraction of the outer cuticle of the mature larva (Giangaspero 1997; Greenberg 1973).

Fly larvae live and feed on soft wet organic materials (manure, decomposing animals, wet food). Below $10^{\circ} \mathrm{C}$ and above $45^{\circ} \mathrm{C}$ their vital functions stop. The ideal temperature range for young larvae is $30-37^{\circ} \mathrm{C}$, but later stages require lower temperatures; stage III larvae require temperatures of approximately $15-20^{\circ} \mathrm{C}$. At these temperatures, larval development from stage I to stage III occurs in 8-12 days. During their development and moults larvae change in colour from milky white to brownish-red. At the end of stage III, the larvae stop feeding and pupate in the same medium in which they have developed as larvae or in soil. Pupation lasts 3-4 days at a temperature of $30{ }^{\circ} \mathrm{C}$. The last segment of the pupa splits when the adult has formed (Figure 5). Using its ptilinus (a haemolymph-filled structure situated between the ocular region and the antenna base) (Figure 6), 


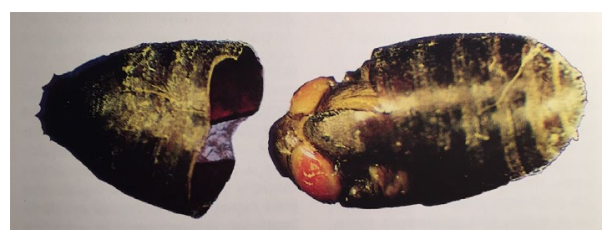

Figure 5. Muscomorphid fly emerging from the puparium (photo by A. Giangaspero).

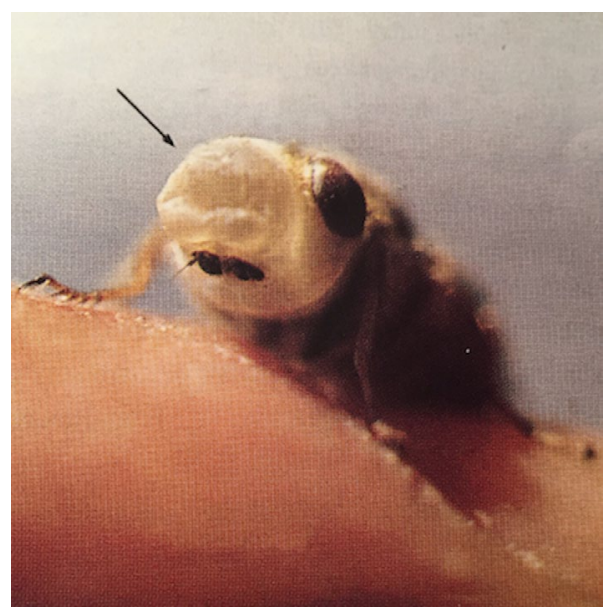

Figure 6. Muscomorphid fly immediately after emergence from the puparium showing its ptilinus (arrow) (photo by A. Giangaspero).

the fly pushes against the cap of the puparium. The fly moves on the substrate as its wings dry and the exoskeleton begins to harden and darken. Shortly afterwards, the ptilinus loses its function and retracts. The complete development cycle from egg to adult fly takes from 7 to 21 days, depending on the temperature (Giangaspero 1997; Greenberg 1973).

\section{Health risks for animals}

The pathogenic role of some fly species has been proven, whereas for other species it can only be hypothesized. Flies have a pathogenic role during the larval and adult stages, which can be direct or indirect. This will be discussed in more detail below.

Flies responsible for damage during the larval stage cause a disease called myiasis, i.e. 'infestations of humans and vertebrate animals with dipterous larvae that, at least for a certain period, feed on living or dead tissue and complete their development on or within the body of the vertebrate host' (Zumpt 1965). Larvae of the flies associated with poultry production mainly infest existing wounds, and most frequently cause cutaneous myiasis. Most species responsible for wound myiasis belong to the Calliphoridae. 
In the adult stage, non-biting-flies (genera Musca, Ophyra) irritate animals with their presence and are therefore known as nuisance flies; these species feed on body secretions and wounds, thereby annoying and disturbing the animals. Biting flies (genus Stomoxys) not only irritate animals with their bite but also suck their blood, thus causing various degrees of anaemia.

Flies are responsible for transmitting several major pathogens. Biting and non-biting flies can be passive or active vectors of pathogenic microorganisms, such as viruses, rickettsiae, bacteria, protozoa, and helminths (Föster et al. 2010; Greenberg 1973; Kettle 1995).

Since flies feed or rest on decaying material, they can easily be responsible for the mechanical transmission of pathogens. The fly's morphology and structure make it particularly suitable for transporting pathogens acquired from the environment (faeces, garbage) or from any animals with wounds, secretions, etc., during feeding. The fly's body is covered with abundant hairs, and its claws and pads have fine hairy structures and sticky elements that trap microorganisms (Sukontason et al. 2006). The mechanical action of the prestomal teeth (highly or little developed) can damage the skin, eyes or any delicate epithelia, thus creating the ideal conditions for bacterial contamination (Giangaspero 1997).

When muscoid flies complete their meal, they partially regurgitate the food and defecate, producing in non-biting flies transparent and thick spots, respectively, which contain pathogens and can be found on all farm surfaces (equipment, windows, buildings, eggs, etc.) or even transferred to animal mucosa or wounds (Bahrndorff et al. 2013); in biting-flies, particularly in $S$. calcitrans, pathogens are transferred prior to blood-sucking (Butler et al. 1977); however, digestive secretions of biting-flies may inhibit the pathogen survival (Baldacchino et al. 2013).

The efficiency of the fly's pathogenic role is closely related to the structure of its mouthparts and its feeding behaviour. When non-biting flies (e.g. Musca spp.) feed, the labella or the labellar disks press on the surface, and the fly sucks liquid or fine particles through its pseudotracheae (Figure 1). If the food is solid, the fly turns back its labella to expose its prestomal teeth (Figure 2); these scratch the surface, and strong muscles force the food into the oral aperture (prestomum), injuring the animal mucosae or skin and allowing pathogens to enter. Hematophagous species (e.g. Stomoxys) press their little-developed labella on the host skin and their sharp teeth scratch the surface (Figure 3 and 4). This allows the proboscis to pierce the skin with a drilling action until it locates blood vessels (Giangaspero 1997).

Mechanical transmission is the easiest way for pathogens to spread, and this involves no developmental stage of the pathogens within the vector itself, whereas transmission depends on whether the pathogen is persistent (i.e. it remains viable for a long period, perhaps months, outside the host), or non-persistent (i.e. remains viable for only a limited period; hours or days). Cyclical transmission is a more complex type of transmission, since it involves a period in which the pathogen develops within the insect vector.

\section{Health risks for humans}

Excessive numbers of house flies and other filth flies in poultry farms become intolerable because they irritate workers. Flies are an additional concern for people living near poultry farms; because they disperse to nearby homes, the infestation can often lead to legal disputes and the violation of local public health laws and regulations. As reservoirs and vectors of a wide variety of pathogens, including Campylobacter spp., Salmonella spp., Escherichia coli, Staphylococcus spp., etc. (see 
Bahrndorff et al. 2013), flies can also affect human health. In poultry houses, the level of flies contaminated by Enterobacteriaceae, E. coli, and Staphylococcus spp. coagulase positive ranges between $10^{1}$ and $10^{2} \mathrm{cfu} / \mathrm{fly}$ (Barreiro et al. 2013). The most impressive case is campylobacteriosis, whose incidence cases among humans has been shown to correlate with the prevalence of Campylobacter spp. among broiler chickens (Bahrndorff et al. 2013; Patrick et al. 2004).

House flies are usually found within $500 \mathrm{~m}$ from a breeding source, but may disperse over distances of up to $30 \mathrm{~km}$. If manure that was spread on fields contains pupae and third-instar larvae, adult flies may continue to develop, and emerge and disperse, causing distress to those living and working in the vicinity. Homes relatively close to poultry facilities (within $800 \mathrm{~m}$ ) are 83 times more at risk for flies than homes far from poultry farms. Another consequence of poultry presence for humans is related to the use of pesticides: their use for fly and other pest control can pollute groundwater and surface water (Maheshwari 2013).

\section{The most important fly species: a brief description}

\section{House fly}

\section{General morphology}

The house fly (M. domestica) is 5-8 $\mathrm{mm}$ long. The thorax is grey, with four large dark longitudinal dorsal stripes. The abdomen has yellowish sides on the basal half, while the posterior portion is blackish-brown (Figure 7). Sexual dimorphism is marked (Greenberg 1973).

\section{Biology}

The house fly is an endophilic and eusynanthropic species, i.e. it lives closely with humans and can complete its entire life cycle in the homes of humans, or in dairies, poultry houses, stables, food processing factories and other domestic areas and buildings. This extreme adaptability to different substrates is connected with its poorly developed olfactory sense; in fact, unlike other species, $M$.

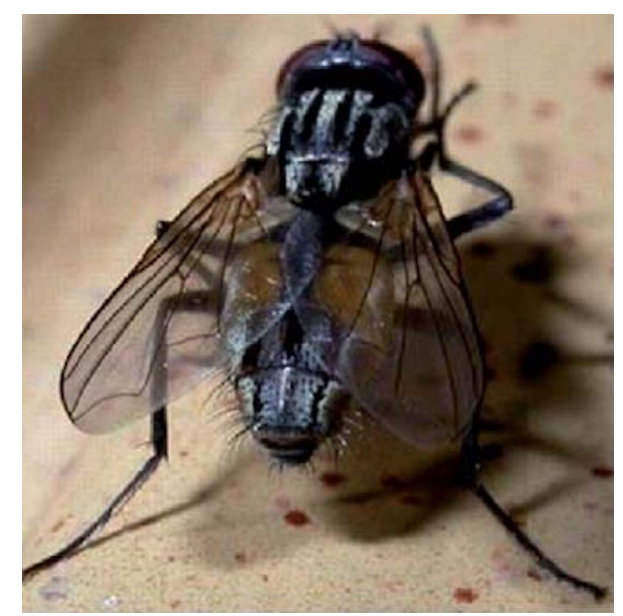

Figure 7. Musca domestica (male). 
domestica has only 550-600 sensory receptors, i.e. only 1/7 of those possessed by other Muscidae (Bahrndorff et al. 2013). Its rate of development depends on food availability and temperature. A female house fly may lay four to six batches of eggs consisting of 75-150 eggs. Adult house flies may live 15-30 days. Manure and spilled feed are the principal breeding media for house flies in poultry houses, and fly survival, growth; reproduction is greatly influenced by manure quality, moisture, temperature and other conditions (Barnard et al. 1995; Shah et al. 2016). This rapid development and the large numbers of eggs produced mean that large populations can build up in a short time. In temperate regions, ten to twelve generations can occur per year. In colder regions, where breeding is restricted to the warmer months, there are four to six generations per year. Overwintering takes place in the larval or pupal stage (reviewed by Kettle 1995; West 1951).

\section{Pathogenic role}

House flies are usually the most abundant and irritating fly species, and cause great economic and sanitary problems. In modern poultry production, flies can cause immense damage to the birds because the flies transmit diseases. The house fly is the primary pest of most fly management and control programmes (Wilhoit et al. 1991a, 1991b). Evidence that muscoid flies act as mechanical vectors of gastrointestinal (Gl) pathogens has been documented widely (Ugbogu et al. 2006). Their continuous movement between human and animal food, faeces, organic waste, refuse, manure and carcasses make them ideal vectors of human and animal pathogens, since they connect clean and contaminated areas.

Pathogens that can be transmitted by house flies include Salmonella spp., E. coli, streptococci and at least 100 other pathogen species have been detected inside or on the house fly body (reviewed by Greenberg 1973; reviewed by Kettle 1995). A relationship has been reported between the necrotic enteritis caused by Clostridium perfringens isolated from house fly and increased chicken mortality (Dhillon et al. 2004). M. domestica plays an important role as a vector of Campylobacter spp. in broiler flocks (Hald et al. 2004; Mull et al. 2015a), and fly prevention in broiler facilities succeeded in reducing Campylobacter positive flocks from $43.3 \%$ (in three years without fly nets) to $9.9 \%$ (in four years with fly nets) (Hald et al. 2008). It has also been shown that the entrance of house flies into poultry houses constitutes a considerable risk for colonization of broilers with multidrug-resistant E. coli (Solà-Ginés et al. 2015).

House flies are also known to carry viruses responsible of diseases in poultry, such as the exotic Newcastle Disease (Barin et al. 2010) or Rotavirus (Tan et al. 1997), but one of the most threatening diseases for birds and humans is avian influenza (Al). The strain (H5N1) currently spreading from Asia to Europe is not only highly pathogenic for poultry, but has the potential to infect humans, and a number of fatal cases linked to domestic poultry have been reported. The responsibility of house flies in serving as vectors in HPAI-H5N1 virus transmission in chickens has been demonstrated under experimental conditions (Wanaratana et al. 2013). House flies may also be carriers of protozoan oocysts, nematode eggs and cestodes of poultry (Barnard 2003; Graczyk et al. 2005).

\section{Lesser house fly}

\section{General morphology}

The lesser house fly (Fannia canicularis) is a slender insect $(5-6 \mathrm{~mm})$, smaller than M. domestica. The thorax is crossed by three longitudinal indistinct stripes, and the narrow elongated abdomen is 
yellowish-white in males. F. canicularis larvae have peculiar features, they are flattened and brown with numerous spiny projections and the pupae have a similar appearance.

When at rest, F. canicularis overlaps its wings, and therefore has a more ' $V$ ' shaped wing outline than house fly. F. canicularis larvae have spiny processes, as well as the pupa but are darker in colour and have a toughened outer skin (Greenberg 1973).

\section{Biology}

This is a ubiquitous, polyphagous, endophilic and synanthropic species like $M$. domestica, with which it also shares the substrates of larval development. F. canicularis is more common at lower temperatures than the house fly and often reaches high numbers in spring and early summer. However, F. canicularis breeds throughout the winter in many regions, and the winter breeding causes the population to rise in the spring.

F. canicularis develops from egg to adult more slowly than the house fly; it usually takes 18-22 days at $22-24^{\circ} \mathrm{C}$, but may last $30-40$ days under cooler conditions (below $20^{\circ} \mathrm{C}$ ). Larvae are often found in manure together with house fly larvae, but F. canicularis can breed in drier manure $(55-60 \% \mathrm{RH})$ than house flies (60-75\%).

A characteristic of $F$. canicularis is the male's tendency to hover or fly slowly in small circles in poultry sheds or in shaded and protected areas outside of the sheds. Females are less active and mostly found near manure or other breeding sites (Greenberg 1973).

\section{Pathogenic role}

F. canicularis is a mechanical vector of Giardia, Entamoeba, Escherichia, Shigella and staphylococci, and is also known to be associated with outbreaks of the exotic Newcastle Disease (Axtell 1999; Greenberg 1973).

\section{Dump flies or black garbage flies}

\section{General morphology}

Adults of dump flies or black garbage flies (Ophyra aenescens) are black, shiny and slender, about half the size of house flies (6-6.5 mm long). The head has a bright clear triangle between the eyes, and the chest and abdomen are black. O. aenescens is easily distinguished from other species of Ophyra (Ophyra capensis (Wiedemann) and Ophyra leucostoma (Wiedemann), since both sexes have yellow palps.

\section{Biology}

This polyphagous and exophilic species uses the same development sites as M. domestica. It is mainly found on refuse and is common in caged-layer and broiler breeder houses. The black dump fly's life cycle is similar to the house fly and takes 14 to 45 days to complete.

This species almost never enters homes. Although presenting similar behaviour to $F$. canicularis, $O$. aenescens is less commonly seen flying slowly in circles in a poultry house. Dump fly larvae prey on small arthropods in the manure, including house fly larvae, and 0 . aenescens is sometimes 
promoted as a biological control agent against the house fly. Unlike the house fly and lesser house fly, black dump flies characteristically remain on their food source at night rather than resting on the ceiling or on outdoor vegetation. These flies have a more limited range $(6-8 \mathrm{~km})$ than the house fly and lesser house fly (Greenberg 1973).

\section{Pathogenic role}

The black dump fly is known to carry pathogens (Salmonella, Campylobacter, E. coli) on its body surface or in its digestive system, in much the same way as the house fly (Olsen and Hammack 2000; Szalanski et al. 2004). Given the frequency with which O. aenescens is found in poultry farms, the detection of avian influenza virus in this fly species is extremely important (Sievert et al. 2008). Very large populations of dump flies will sometimes spread and may cause annoyance to the neighbourhood, although dump flies tend to disperse less readily than the house fly. Dump flies leave faecal and regurgitation spots, for example on light fixtures, equipment and eggs, in a similar way as house flies (Adams 1984; Skidmore 1985).

\section{Soldier fly}

\section{General morphology}

Adult black soldier flies (Hermetia illucens) are large, slender and slow flying.

\section{Biology}

This species is common in poultry manure worldwide and may be especially abundant in high-rise deep pit caged layer houses and under the slats in breeder houses (Shepard et al. 1994). The soldier fly oviposits egg masses on drier (i.e. external or marginal) areas of manure. Larval development (5 instars) is slow (2-4 weeks) and, depending on temperature and other environmental conditions, pupation occurs in spring and lasts from 9 days to 5 months (Oliveira et al. 2014). A complete life cycle from egg to adult in poultry houses takes 40-60 days, and larvae can thus become extremely abundant. This species may be found resting on vegetation around a poultry house, as well as on the manure and surfaces inside the house (Axtell 1999).

The robust larvae churn up the manure and physically render the habitat less suitable for the house fly and other muscoid flies and in this way may prevent the development of large house fly populations.

\section{Pathogenic role}

They do not readily disperse in sufficient numbers to cause problems in nearby residences or businesses. Large numbers of soldier fly larvae discourage house fly development, and the larvae have been observed actually preying on other fly larvae in rare circumstances. The larvae cause the manure to liquefy, which makes it difficult to remove, and it may flow onto walkways and undermine the foundations of poultry houses. In breeder houses, liquefied manure may flow from under the slats and attach to birds' feet. It can then adhere to eggs as they are laid in nest boxes, and pathogens may enter the egg through its moist shell. Some pathogens transmitted in this way can reduce hatching rates and chick growth (Axtell 1999; Zheng et al. 2013). 


\section{False stable fly}

\section{General morphology}

Muscina stabulans (the false stable fly) is larger and more robust than the common house fly (8-12 $\mathrm{mm}$ long), but is also dark grey in colour with four dark stripes on the thorax. However, the rear lobe of the thorax is tan-coloured and the legs are orange or cinnamon (Figure 8) (Greenberg 1973).

\section{Biology}

This polyphagous exophilic species prefers bird droppings, especially of chickens, but also breeds on human and sheep faeces.

It is frequently found on the external walls of houses, but rarely enters. M. stabulans breeds in cooler conditions than house flies and takes longer to complete its life cycle (Greenberg 1973).

\section{Pathogenic role}

It is a vector of numerous pathogens: enterovirus, foot-and-mouth disease virus, E. coli, Salmonella, Proteus, Brucella, and staphylococci (reviewed by Bidawid et al. 1978; Greenberg 1973).

\section{Blowflies}

\section{General morphology}

Blowflies (Calliphora, Lucilia, Phormia spp.) are medium to large $(6-12 \mathrm{~mm})$ flies. The thorax and abdomen are coloured with bright metallic colours: blue, green or copper. The thorax has no longitudinal stripes but has strong bristles.

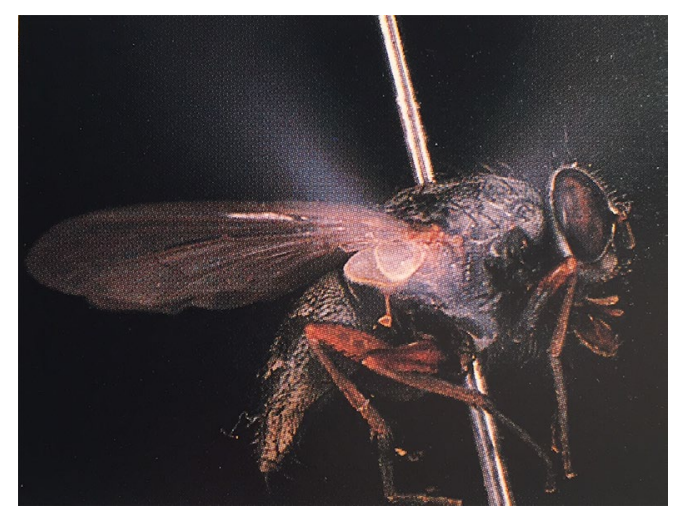

Figure 8. Muscina stabulans (photo by A. Giangaspero). 


\section{Biology}

These eusynanthropic exophilic species prefer poultry manure, faeces and rotting food, decaying animal carcasses (rodents and birds) and sites contaminated by broken eggs, where the larvae develop into robust adults in less than a week (Axtell 1999). Most blowflies develop when there is incorrect disposal of dead birds in a poultry farm. They enter homes or other indoor environments through open windows. Their remarkable sense of smell means that they are attracted to decaying meat, fish or cheese even from a considerable distance. They prefer not to stay indoors, and will fly into windows in an attempt to get outside.

\section{Pathogenic role}

Adults can lay eggs on exposed wounds in which the larvae develop, causing facultative myiasis (Al-Khalidi and Shareef 1985; Zumpt 1965). They are also mechanical vectors of several viruses (enteroviruses), bacteria (Salmonella, Escherichia, Aereobacter, Proteus, staphylococci, Shigella, Clostridium, etc.), protozoans (Giardia, Toxoplasma) and worms (Trichuris, Ascaris) (reviewed by Förster et al. 2007; Greenberg 1973).

\section{Black fruit fly}

\section{General morphology}

While there is no common name, Drosophila repleta has been referred to as the black fruit fly. The black fruit fly is a very small (3-4 mm long) dull-brown non-biting species that breeds in poultry manure and may become a nuisance due to its large numbers (Greenberg 1973).

\section{Biology}

Common worldwide, D. repleta prefers to rest in the cooler areas of poultry houses (Harrington and Axtell 1994). It is most abundant in caged-layer houses, even when there is a flush or scraper system for manure removal. Small accumulations of manure are sufficient to allow the development of large fly populations. Adults often congregate around feed troughs and along the manure-crusted edges of walkways. Invasion of the adjacent egg sorting and packing facilities causes annoyance to workers.

\section{Pathogenic role}

Staphylococci and Salmonella have been isolated from Drosophila spp. (Greenberg 1973) but its pathogenic role on poultry farms is unclear.

\section{Stable fly}

\section{General morphology}

Similar in appearance to M. domestica, S. calcitrans is $6-8 \mathrm{~mm}$ long, but its long rigid proboscis makes it easily recognisable (Figure 3 ). The grey thorax has four longitudinal stripes, and there are three dark circular spots on abdominal segments II and III (Figure 9). Sexual dimorphism is not marked (Greenberg 1973). 


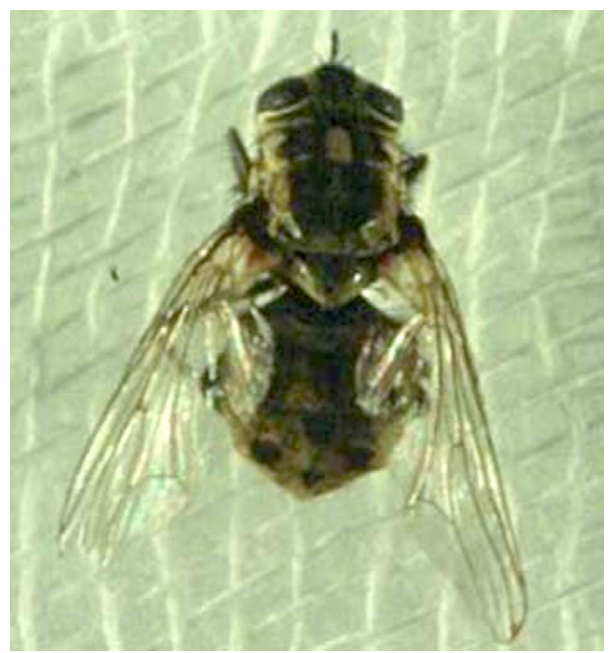

Figure 9. Stomoxys calcitrans, female (photo by A. Giangaspero).

\section{Biology}

This haematophagous fly's larval habitat consists of all substrates where there is intense bacterial activity. The stable fly may breed in poultry shed litter and poultry manure used as fertiliser for horticultural crops and for turf production (James et al. 2004), but seldom in significant numbers. Both sexes are bloodsucking. S. calcitrans can attack humans and cause great nuisance, mostly biting around the ankles. Median immature developmental times range from 60 days at $15^{\circ} \mathrm{C}$ to 12 days at $30^{\circ} \mathrm{C}$ (Lysyk 1998).

\section{Pathogenic role}

S. calcitrans can suck 11-15 $\mu \mathrm{l}$ of blood per meal, and females take $50 \%$ more blood than males (Schowalter and Klowden 1979). In addition to the mechanical irritation caused by the proboscis, the feeding behaviour of this species makes it particularly suited to the active and passive transmission of pathogenic microorganisms of livestock (reviewed by Baldacchino et al. 2013). This biting species is capable of carrying Campylobacter in North American poultry facilities (Szalanski et al. 2004) and the fowl poxvirus (Greenberg 1973).

\section{Control}

To achieve satisfactory control of flies in poultry facilities, several methods should be used following integrated pest management (IPM) principles, a framework to reduce the risks and impacts of pesticide use on human health and the environment (Axtell 1999; Barzman et al. 2015; Durel et al. 2015; EC 2009).

With respect to Directive 2009/128/EC (EC 2009), which focuses on crop protection, Durel et al. (2015) identified the following eight lines of action for the implementation of IPM in farm animal operations: (1) prevention and/or suppression of harmful organisms; (2) monitoring of harmful 
organisms; (3) application of animal protection measures; (4) sustainable biological, physical, and other non-chemical methods; (5) the animal protection product applied has to be as specific as possible; (6) use of pesticides at necessary levels; (7) application of anti-resistance strategies to maintain the effectiveness of the products; and (8) evaluation of the success of the applied protection measures. Methods that could be applied within this framework are briefly discussed below.

Avoiding the introduction of flies into a poultry facility is an important first step. This can be achieved by making sure doors close quickly and properly, as well as screening of doors, windows, and ventilation openings (Hald et al. 2008).

Poultry manure is known to be attractive to and a good breeding medium for $M$. domestica and other 'filth' flies (Axtell 1999; Merdan 2012; Shah et al. 2016; Smallegange 2003). The same applies for rodent carcasses, which volatile odorous components are attractive to Calliphora vicina Robineau-Desvoidy (Johansen et al. 2014). Therefore, proper rodent and waste management around and in poultry facilities is crucial (Axtell 1999).

Monitoring is an important aspect of IPM, serving as an early warning system, providing the opportunity to estimate fly population sizes, and to identify the insect species that are present correctly (Durel et al. 2015). The latter is key to a successful fly control because differences in physiological and ecological needs of different fly species indicate where and which control measures should be applied (see above). Several methods to monitor flies are available, among which spot and sticky cards (Qiu et al. 2007; Smallegange and Den Otter 2007). These monitoring techniques are also applicable for evaluation of the impact of the applied control measures.

In addition to sticky traps, visual targets, ultraviolet light, odour baited, and electrocuting traps (e.g. Baldacchino et al. 2018; Geden 2006; Geden et al. 2009; Qiu et al. 2007; Smallegange and Den Otter 2007; Welch 2006) are non-chemical control methods that can be applied. Although trapping techniques hold considerable promise for future development, more research is needed to improve the efficacy of the traps and to assess their effect and correct placement in poultry facilities; the light conditions and competing odour sources present may hamper the succes of lure-and-kill traps (Hanley et al. 2009; Smallegange 2003).

Moreover, biological control agents (natural enemies) can be introduced for control purposes. Chicken faeces inoculated with the bacterium Bacillus thuringiensis (Bti) or oral administration of Bti to chicken reduced pupal and adult emergence of $M$. domestica (Geden 2012; Merdan 2012). Also the application of oil-formulated spores of the fungal pathogens Beauveria bassiana and Metarhizium anisopliae shows promise in suppressing fly populations in poultry facilities, although biotic and abiotic factors such as ammonia levels have an effect on its effectiveness (Acharya 2015; Acharya et al. 2015a, 2015b; Geden 2012; Kaufman et al. 2005; Oliveira et al. 2014; Weeks et al. 2018). Studies examining the effect of nematodes on flies in poultry houses had opposing effects and therefore render for re-examination (Geden 2012). Various parasitoid species can be released in poultry facilities to control flies; this approach is described in detail by Machtinger and Geden (2018). Nachman and Skovgård (2018) have developed a simulation model to show how control of S. calcitrans by the pteromalid parasitoid Spalangia cameroni Perkins can be conducted efficiently.

To avoid damage to the environment, animal and human health, chemical control methods (insecticides) should be restricted to a minimum. The high developmental rate of flies, frequent 
and improper insecticide use can easily result in resistance due to a continued selection pressure (e.g. Abbas et al. 2016).

The aforementioned measures can be included in biosecurity protocols used to prevent the introduction and spread of vector-borne diseases in poultry flocks (Axtell 1999). However, application of control methods depends on the local circumstances, finances, fly species present, fly population size and age structure, the source and degree of the fly nuisance, and the presence of natural enemies, and can, therefore, differ per location and over time. For example, ventilation systems of poultry houses in the USA are different compared to those used in Europe, possibly making fly screens less effective in the USA (Sahin et al. 2015). Differences in production practices between countries will affect the success of biosecurity and hygiene approaches, posing a challenge for development of control protocols that can be used globally (Sahin et al. 2015).

Currently, Durel et al. (2015) consider prevention and suppression (i.e. removal of larval development and adult feeding and resting sites by sanitation and mechanical control measures), and monitoring the only realistic options within the implementation of IPM strategies in farm animal facilities. Recent sequencing of the genome of $M$. domestica will give insights in its biology, its adaptation to high pathogen loads, the mechanisms of insecticide resistance, and may result in the development of innovative control methods for this important disease vector (Scott et al. 2014).

\section{Alphitobius diaperinus (lesser mealworm)}

\section{Introduction}

A. diaperinus (Panzer), also known as 'lesser mealworm' and 'litter beetle', is one of the most widespread pests of general stored food products and a vector-competent reservoir of several zootechnical pathogens and parasites (Francisco and Prado 2001). Except for the house fly, it can be considered the most important pest of the poultry industry as its massive development can cause a range of problems, such as damage to the housing facilities, for example to the insulating material of walls when they search for suitable pupation sites, and a more proper biological damage as said before (Axtell 1999). On the other hand, the breeding of A. diaperinus has an economic interest as this tenebrionid in its larval stage, the so called 'buffalo worm', can be used as food supplying a good nourishment for reptiles, sauria and birds. They are also suitable for human consumption being characterized by a sweet taste similar to the honeycomb moth (Galleria mellonella (L.)) with an aftertaste of hazelnut. They are generally served steamed, roasted, fried, pan-roasted or in powder as flour (e.g. processing of cakes). They are even suitable for other general purposes such as the cleaning of carcasses for museum displays and for the decomposition of animal remains (Dunford and Kaufman 2006).

\section{Morphology and biology}

Lesser mealworm adults are black or brownish-black. The young adult is milky coloured but it rapidly darkens to brown or black-brownish which is its definitive colour. They are approximately $6.3 \mathrm{~mm}$ long and the integument is coarsely punctured. Male and female adults are very similar except for some minimal differences that can be observed with a microscope (Bousquet 1990).

Within 6-10 days after eclosion, female beetles oviposit in sheltered areas of the house for example in the moist litter and along the building walls. Eggs are approximately $0.8 \mathrm{~mm}$ in length, creamy 
white coloured. Adults can live for several months, with females continuing to produce eggs most of their life at one to five day intervals. Larvae hatch in a few days depending on the temperature ( 3 days at $35^{\circ} \mathrm{C}$ and 10 days at $21^{\circ} \mathrm{C}$ ). Egg hatching is minimal under $15^{\circ} \mathrm{C}$ and over $38^{\circ} \mathrm{C}$. There are approximately six to ten larval instars depending on environmental conditions, and larvae progressively darken in colour. The last larval stage is creamy brown with a yellowish area on the ventral surface, varying in length from 1.2 to $1.9 \mathrm{~cm}$. They are shiny in appearance with no evident hairs or stings. Larvae have a segmented body with abdominal tip tapering posteriorly, three pairs of short legs. The duration of the larval instars may vary with environmental conditions ranging from 30 to 200 days. The last larval stages disperse to find isolated pupation sites, where they construct pupal cells. This may occur into litter, manure, soil and even into thermal insulation materials in buildings. Pupae are approximately creamy white to tan coloured, they are able to more around even with no mobile legs. Pupal stage may persist for 4 to 14 days depending on temperature. The entire life cycle (egg - six or more larval instars - pupa - adult) requires from 40 to 80 days in litter and manure into a building, where the temperature ranges from 21 to $35^{\circ} \mathrm{C}$. A. diaperinus has a good tolerance for humidity variations in every life stage and it has a good development even in dry habitats (Axtell 1994; Bousquet 1990; Dass et al. 1984; Rueda and Axtell 1996).

\section{Habitat}

A. diaperinus, because of its tropical origin, is well suited for warm, humid conditions and it has been observed in a wide variety of habitats such as bird nests, bat colonies/caves, and in rodent living areas (Dunford and Kaufman 2006). Both adults and larvae are abundant in both manure and litter from henneries and poultry farms. The term litter means an ensemble of manure and material from floor (wood chippings, rice hulls and other vegetal materials); manure consists only of poultry excreta with no litter. Both manure and litter contain feed remains. Litter is suitable for free range poultry farms (inside the building) such as chicks, broilers and turkey farm, where manure is accumulated under the cages and perches (Axtell and Arends 1990; Francisco and Prado 2001; Geden and Axtell 1978). These situations favour a massive presence of A. diaperinus, especially when old litter is being reused for more breeding cycles.

The floor of breeding houses may vary from soil to hard surfaces. In the case of soil, mature larvae may disperse finding pupation sites directly under the litter, building pupal cells into soil. In both cases pupation sites may occur in thicker portion of the litter where it could be easier to dig and build a pupal cell (Pfeiffer and Axtell 1980; Rueda and Axtell 1996). Populations of A. diaperinus in a manure habitats represent a worse problem particularly in battery cages, where manure accumulates in the building for several months. In this case, the drier portions of manure are very suitable for the development of 'litter beetle'. Food and water dispenser in broilers farming houses are collocated at $90 \mathrm{~cm}$ above the ground, and the dry manure beneath many lead to massive infestation. A complete cleaning and sanitation is normally carried only after 15 months when a breeding cycle is completed and a new one is about to start (Axtell and Arends 1990; ChernakiLeffer et al. 2007; Pfeiffer and Axtell 1980; Rueda and Axtell 1997).

\section{Behaviour}

Both larvae and adults of $A$. diaperinus are able to move rapidly. When disturbed they can quickly hide into the litter media or the manure. When captured and brought out of their habitat, they tend to stay still simulating death for minutes, then suddenly they may move rapidly again (Pfeiffer and Axtell 1980). 
Adults and larvae usually form huge groups in several areas of manure and litter. It is usual to find clusters beneath shelves, feed and water disperse laying on the manure or litter. In case of overpopulation, cannibalism appears as a common behaviour, in particular from adults and mature larvae towards egg and young larvae. As carrion insects they can cluster even beneath the carcass of dead animals. By contrast, they do not attack healthy birds (Chernaki-Leffer et al. 2007; Rueda and Axtell 1997).

Frequently a migration of larvae and adults (mostly during night-time, even though adults can move also during daytime) from the bottom to higher parts of walls and columns has been observed in poultry farms. An explanation of this phenomena relies in the fact that larvae move, in search of pupation sites and the relative frequency of their migration depends on the population density; the higher the density of population and more frequent will be the migration. This migration is also connected with a low availability of pupation sites in manure, litter and ground floor. Larvae are able to damage almost any kind of insulating material, digging galleries into it. They evolve in pupae within and when adults, they return to manure or litter to feed and oviposit (Lambkin et al. 2008; Salin et al. 2000).

Evidence has been collected also of clusters of $A$. diaperinus close to dust aspiration systems in the walls of poultry houses, probably aiming at moving from the house to new habitats. These phenomena are difficult to observe since these migrations occur during the night (Chernaki-Leffer et al. 2007; Salin et al. 2000).

\section{Economic relevance}

\section{Damage to poultry houses}

Modern poultry houses are designed to reduce the range of variation of inner temperatures during the year. Given the local meteorological conditions, insulating materials generally made of polyurethane are fitted under the roof to simulate the thickness of the walls of the building (Geden and Axtell 1978; Salin et al. 2000). The galleries of A. diaperinus are responsible of as much as $30 \%$ depreciation of insulating materials in few years. This aspect not only impacts the building value shortening its lifetime, but also affects the inner microclimate since the damages can lead to an insufficient insulation causing loss of production. The substitution of insulation is estimated in hundred thousand euros in expenditure as well as a fall in production during the repair work. Wooden insulation may lead to less damage even though this may be damaged as well (Geden and Axtell 1978; Lambkin et al. 2008; Salin et al. 2000).

\section{Hygienic aspects}

A. diaperinus is a well-known vector of a number of poultry pathogens and parasites, such as fungi (Aspergillus), bacteria (E. coli, Salmonella, Bacillus, Streptococcus), viruses of leucosis (Marek's disease and Gumboro disease). Salmonella typhimurium may lead to gastroenteritis and poultry products represent a source of infection. A. diaperinus represents a reservoir for Salmonella, and the presence of Salmonella has been demonstrated in its faecal production even 28 days after ingestion of contaminated material. Infected larvae lead to infection of the successive development stages. There is evidence of Salmonella infection in broilers after ingestion of adults of $A$. diaperinus. Such infections occur quite often since young broilers actively feed on $A$. diaperinus in the litter. In its first ten days of life broilers eat some 450 larvae per day of $A$. diaperinus, and a 
young broiler may capture some 200 larvae per day even in the presence of other feed sources (Crippen and Poole 2012; Hazeleger et al. 2008; Leffer et al. 2010; Roche et al. 2009).

\section{Control}

Control of A. diaperinus in the poultry farm may be challenging and it requires the implementation of a management effort well integrated with the poultry raising techniques. How and when to implement control techniques may vary case by case, ranging on the type of poultry farm, even though some general aspects may be underlined as described below.

\section{Biological control}

The artificial environment of a poultry house is an ideal place for the development of huge populations of $A$. diaperinus because the lack of competitors that can be present in a natural environment. Only few pathogens of $A$. diaperinus have been studied and none for practical usage. In a few cases, under particular conditions, a significant mortality of a relevant number of individuals in a population $A$. diaperinus may occur by the action of some natural pathogens including nematodes (Steinernema feltia), fungi (B. bassiana) and protozoa (Farinocystis tribolii) (Chernaki-Leffer et al. 2007; Rezende et al. 2009).

\section{Hygienic measures}

The frequent and complete cleanings of poultry facilities, the removal and change of litter at every production cycle leads to control of the population size of $A$. diaperinus. This practice, known for its high costs (in terms of days off in production, cost of litter), is often partially or not implemented. This technique remains the best way to control an A. diaperinus population, together with, under cold climate conditions, the aeration of holding facilities to let the inner temperature get around $0{ }^{\circ} \mathrm{C}$.

\section{Chemical control}

Insecticides in granules, spray or powder may be used directly on litter, and as spray and powder on the floor under the litter between production cycles. Insecticides may also be applied on inner surfaces, such as walls and columns, to reduce the migration or larvae and adults from the ground floor to the external environment. The use of insecticides must be guided by the instructions on the label and MSDS, and great attention must be paid to the presence of animals in the sprayed area. Pyrethoids may grant a longer half-life and a combined efficacy towards other pests such as house fly and other ectoparasites. A longterm control of $A$. diaperinus is almost impossible to achieve since in a suitable environment the population can rapidly reach a huge dimension. Even the most accurate disposal of insecticides alongside the walls and insulating materials cannot reach every pupa and adults hidden in cracks in the wall and ground floor; these survivors will be the pioneers of new colonies.

The objective of chemical control is the sudden reduction of the dimension of the population of A. diaperinus and its restraint in time with the reiteration of controlled application of pesticides. In normal conditions an active control can be achieved with one treatment per year during the cleaning period between two production cycles. Pest control, in this way, makes sense only when fully integrated in the schedule of poultry activities, harmonizing with the needs for production 
and the monitoring of the levels of infestation in poultry houses (Hinton and Moon 2003; Tomberlin et al. 2008).

\section{Monitoring}

Normally the monitoring of the population size on a weekly base allows for an active assessment of the efficacy of the control programme as well as the need for implementation of new strategies. A direct approach consists of a person walking in the poultry facility, searching for the most probable hot spots, e.g. alongside the walls, inspecting the litter especially under the food and water dispenser. In the search for $A$. diaperinus other places must be inspected like under the carcass of dead animals and in the areas where litter is compact and dry. (Hinton and Moon 2003).

\section{Cockroaches}

\section{Introduction}

Cockroaches belong to the order Blattodea (Greek: blatta $=$ an insect that shuns light). Due to their excellent ability of environmental adaptation, cockroaches are considered as one of the most successful group of animals living on earth for over 300 million years. Although they mostly prefer sub-tropical and tropical areas, they have followed humans and commercial movements around the world so now they have colonized all continents. In fact, even in the late Paleozoic era (Carboniferous), cockroaches were considered to be one of the largest orders of insects (Grandi 1996). Currently, 4,000 species and 460 genera belong to this order and three of them represent a problem for the poultry houses, especially caged-layers and broiler breeders. The most common cockroach species that are likely to occur in poultry houses are the German cockroach, Blattella germanica (Linnaeus), the American cockroach, Periplaneta americana (Linnaeus), and the oriental cockroach, Blatta orientalis (Linnaeus).

\section{Morphology and biology}

The order Blattodea consists of terrestrial species of various dimensions, with flat, almost darkbrown coloured bodies although in some cases the body colour might seem to be brick-red (Tremblay 2005). The mouthparts of these animals are adapted for chewing so they have strong and long mandibles. They have a well-developed and protective pronotum, which covers the head and thread-like antennas composed of many segments.

When disturbed, cockroaches can run fast. Some cockroach species have wings but they rarely use them for flying. In some cases the wings might be regularly developed while in others, they might be reduced, completely absent or atrophic. The anterior wings are slightly sclerotized and their name derives from tegmen. The last segment of the cockroach's abdomen has openings for the glands, which release a repugnant odour and another one for the attractive glands. It should be noted that cockroaches move freely inside buildings through electrical conduits, water conductors like air conditioners or heaters, which act as a protective refuge.

A cockroach passes through three stages during its life cycle: egg, nymph and adult. The Blattodea reproduction is amphigonic and they have a heterometabolous development: based on the type of species, they may have an oviparous, ovoviviparous or viviparous reproduction. The species explored in this chapter have an ootheca divided in 10 to 30 vertical cells and each cell contains an egg. However, the number of eggs may vary based on each cockroach species. The ootheca 
is formed by the segregation of colleterial glands and can be morphologically different in each species (Grandi 1996). Recently hatched nymphs have a shape resembling the adults. Obviously, a nymph has a much smaller body size and does not have any wings. In the ovoviviparous species, the ootheca is held by the mother near the genital opening, until a little before the eggs are ready to hatch. Nymphs can grow over a period of weeks and they are always more numerous than adults.

Cockroaches are phytophagous but animal proteins can become part of their diet: if the population becomes too numerous, cockroaches can become cannibalistic out of necessity (Trematerra and Gentile 2008).

B. germanica is usually brown coloured and it can grow up to $1 \mathrm{~cm}$. It is identified by the two dark stripes near its head. A female German cockroach can deposit from 4 to 8 ootheca during its life time. Each ootheca can contain around 20-30 eggs. The ootheca is carried by the female, attached to its abdomen until a little before than the hatching. It takes a German cockroach around 3 to 4 months to complete its life cycle. Although equipped with wings, they rarely use them for flight.

The German cockroach can be considered as one of the most problematic pest species because of its rapid multiplication skills and its ability to colonize climate controlled warm and humid environments. The species can mechanically transmit various pathogenic agents. These species is only occasionally seen in poultry houses thus it is not considered as an actual health problem.

P. americana can be recognized by its dark-brown colour while the head can have a slightly lighter colour. It lives in moist places like basements, under-sinks or sewers. The adults have wings although they use them for gliding instead of flying. They are good climbers and they can reach very high points on smooth, vertical surfaces. Male American cockroaches are thinner, longer and their wings are pointier while the females have a stockier, round body with round wings.

During their lives, the females deposit around 50 ootheca and each of them contains around 8-16 eggs. The time from egg to adult is about one year and the adults can grow up to $4 \mathrm{~cm}$. Adult $P$. americana can live up to two years in warm and humid environments; they can also resist hunger for a long time.

B. orientalis usually prefers warm, humid and dark environments like wood piles, leaf litters, animal waste, sewers or animal farms, food industries and bakeries. However, in some cases it might also be found in houses. This species is considered voracious and omnivorous. The sexual dimorphism of this species becomes clear by observing the male regularly developed wings and the female short anterior and atrophic posterior wings, which are not designed for flying (Masutti and Zangheri 2001).

This species can frequently be found in organic material of poultry houses. It moves easily on the ground where it creates a numerous colony. Adult males have wings, although they cannot fly. On the other hand, the females, which are larger and heavier, have a sketchy set of wings which cannot be used for flying. During its life cycle, females lay around 5 to 10 ootheca, each containing 16 to 20 eggs; only about fifteen of them are fertile. This makes their removal for disinfestation more difficult. The female oriental cockroach deposits its ootheca in crevices and narrow spaces in the wall where the eggs develop and hatch into nymphs. Under warm conditions, within a humidity range of $30-70 \%$, the eggs can grow into adults over a year. They are almost black and 2-2.4 cm long (Sùss and Locatelli 2001). 
This species causes remarkable damages in food commodities because next to consuming the food, they also contaminate it with their repugnatory gland segregations, causing the food supply to become non-edible. They might also damage paper and fabric products releasing a substance which might stain some surfaces and leave an unpleasant odour. The infested materials contain the cockroach's dead skin, saliva and faeces.

\section{Sanitary importance}

Cockroaches are described as the most well-known insect pest. They are not easy to find during the day as they hide in cracks and crevices of poultry houses during the day and they come out at night to search for food and water. However, they might be traced also during the day if specific and adequate instruments are used. Since they live inside drainage and toilet systems, they can carry various human and animal pathogens like bacteria, viruses and intestinal parasites although there is no scientific evidence that they can transmit disease agents (Brenner and Kramer 2002). The most precarious aspect about the Blattodea is the disgorgement of the swollen food and its defecation during feeding because during this process, this contaminates human and animal food. In addition to these harmful effects, the decomposed body of a cockroach can cause respiratory allergies (Kang et al. 1979). Therefore, cockroaches are considered as a risk for the public health and an large cockroach population is a sign of inadequate hygienic conditions.

In poultry sites, cockroaches invade the egg handling and storage facilities, where their large numbers are a nuisance and the odour is unpleasant. The roaches stain the surfaces and leave fecal deposits. Roaches are easily transported from one facility to another in contaminated egg cases.

\section{Control}

The battle against cockroaches should be carefully planned and performed with adequate instruments. Regular hygiene rules for keeping living and working places clean should be adhered to in order to avoid the presence and settlement of cockroaches (Di Domenico et al. 2009). Their preference of living in places like animal farms is due to the maintenance of large food supplies and to the humid conditions which characterize these spaces and help the expansion of their population. In such settings, livestock waste, food waste and food storage should be adequately managed. It should be noted that the scrupulous management of any kind of material has an important role in prevention activities. These materials should be kept in order and they should remain undamaged; they should be placed above from the ground and distant from the walls. In this way, noticing eventual traces of these infestants and controlling them would be easier.

The monitoring plan with attractive sticky traps (activated with food lures or sexual pheromones) is the first step of an adequate prevention programme. In addition to this, the sewage discharges should be controlled in order to detect eventual infestations. If necessary, cockroaches can be attacked using more responsive instruments for the environment like gel-material as these are easier to manage. However, they should be applied with attention, avoiding dusty spaces in order to make sure they function well. In these cases, liquid and dislodging substances should be applied: obviously, the ideal molecules available in commerce should be chosen. New biocide laws have reduced the amount of products that can be used but pyrethroids are still a valid solution, as long as indications and dosages indicated on the label are respected. Insect growth regulators (IGRs), such as methoprene, and pyriproxifen, which interfere with the development of the insect, can be used as alternative to insecticides. Once the IGR is taken in by the cockroach, it starts being effective during the transition from nymph stage to adulthood. As an IGR does not let the 
cockroach reach adulthood so it also stops their reproduction; this is why it is considered to be one of the most effective prevention methods.

It should be noted that since the cockroach eggs are protected by the ootheca, they are basically resistant to treatments with insecticides. So, spaces where the Blattodea may hide should be monitored in order to remove and control them: a three-week long persistent product should be applied so that it can be effective on new-born nymphs.

\section{Chicken lice}

\section{Introduction}

Lice are permanent obligate ectoparasites, characterised by high host specificity and worldwide distribution. In poultry, lice infestation is particularly common and may cause an important disease named pediculosis. This insect belongs to the order Phthiraptera, which is divided into different sub-orders Mallophaga and Anoplura. In poultry the sub-order Mallophaga (chewing lice) practically attracts all scientific interest. This sub-order is further divided Amblycera and Ichnocera.

More than 40 species of Mallophaga have been reported from domestic chicken. Birds frequently harbour several species on the same time. Several species are found on chicken, most important being Menacanthus stramineus (Nitzsch) (chicken body louse), Cuclotogaster heterographus (Nitzsch in Giebel) (head louse), Menopon gallinae (L.) (shaft louse), Lipeurus caponis (L.) (wing louse), Goniocotes gallinae De Geer (fluff louse), Goniodes dissimilis Denny (brown chicken louse) and Goniodes gigas (Taschenberg) (large chicken louse) (Sychra et al. 2008; Taylor et al. 2007).

\section{Morphology and biology}

Lice, like all insects, have a 3-part segmented body consisting of the head, the thorax and the abdomen. They have short antennae with 3-5 segments, no wings and their body is characteristically dorso-ventrally flattened. Their size may reach up to $3 \mathrm{~mm}$, depending on the species. Chewing lice are characterised by possession of broad, chewing-type, mandibles located ventrally on the head and a pair of segmented maxillary palps (Figure 10 and 11).

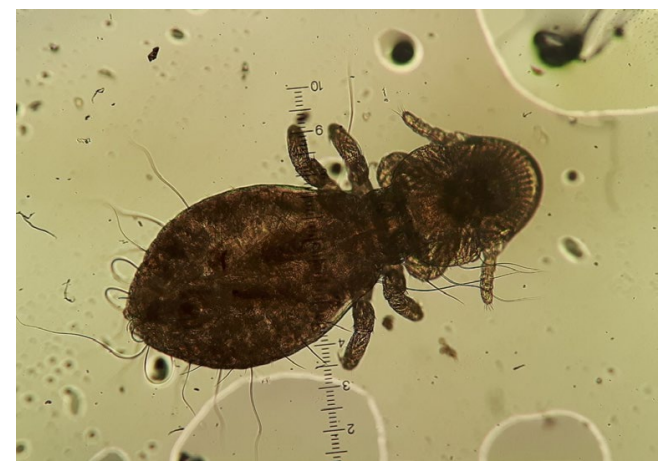

Figure 10. Mallophaga bird louse. 


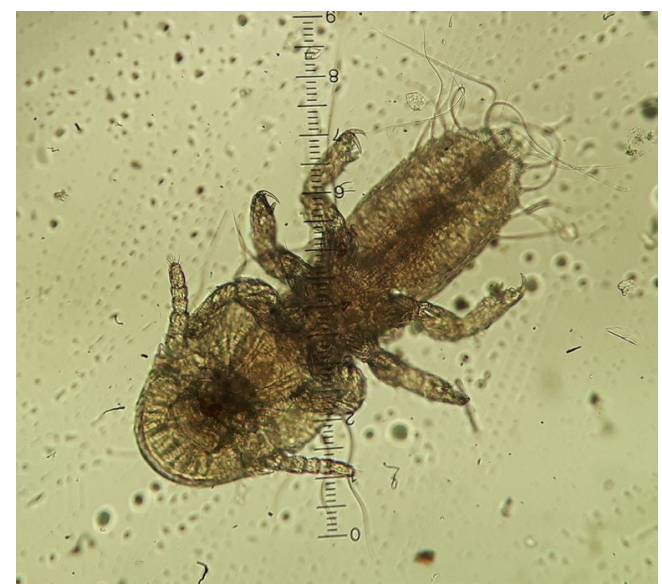

Figure 11. Mallophaga bird louse (note the pair of claws at the end of the tarsus).

Chewing lice are obligatory parasites and spend their entire life cycle on the birds. They live approximately one month and lay eggs ('nits'), which are attached (glued) to the base of feathers, often in clusters (masses), and require 4-7 days to hatch. Eggs are whitish and they may be seen with the naked eye (Figure 12). There is no true metamorphosis and from the eggs hatches a nymph which appears similar to the adult form but is smaller in size. There are three nymphal moults in order to produce the adult and this lasts approximately 2-3 weeks.

Chewing lice can digest keratin and therefore are able to eat feather products, skin scales and scabs, but M. stramineus may also consume blood. Transfer of lice is usually made by physical

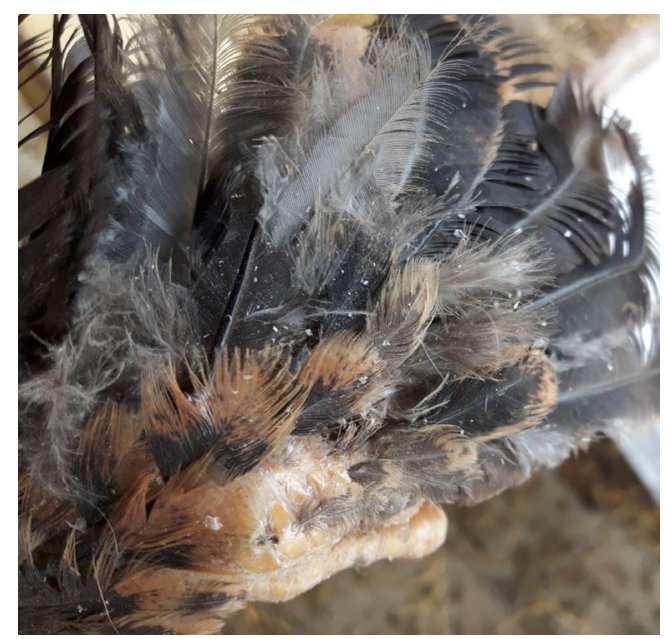

Figure 12. Louse eggs (nits) on bird feathers and scales. 
direct contact and rarely by the beddings, as lice cannot survive long off their hosts. In hobby farms, a seasonal fluctuation may be seen with higher parasite burdens during winter.

\section{Risks to animals}

Lice are not very pathogenic to mature birds, but lice-infested chicks may die. Their pathogenic effect is usually a function of their density. Lice infestation frequently accompanies manifestations of poor health, such as internal parasitism, infectious disease, malnutrition as well as poor sanitation and unhygienic conditions. Infested chicken are restless, self-wounded due to scratching and feather plucking, emaciated and anaemic, with damaged feathers and reduced performance. Some lice species may pierce the pulp of feathers or the skin by their biting or scratching mandibles and thus lick the excreted blood; they may be extremely dangerous especially to young birds, even if they only feed by nibbling along the feather surface and/or eat epidermal debris. Lice may cause irritation of the skin, restlessness, overall weakening, cessation of feeding, loss of weight, inferior laying capacity, and skin lesions that may become sites of secondary infection. In cases of heavy infestation, birds may present lethargy and eventually die (Taylor et al. 2007).

\section{Risks to humans}

Bird lice are host-specific and do not infest humans. However, insecticide residues in eggs or meat can be harmful to consumers, if withdrawal periods are not respected.

\section{Impact on poultry production}

Lice infestation of chicken is a very important cause of production losses in both traditional and intensive rearing systems. They have a very wide geographical range, being present in all continents, which is closely related to, among other human activities, transportation of poultry that leads to long-distance dispersal of these parasites.

In trials with experimental infestation of laying hens with the body louse $M$. stramineus, it was found that egg production was decreased up to $46 \%$, depending on the age of chicken. In the same experiments, it was also shown that the average hen weight decreased up to $450 \mathrm{~g}$ compared with non-infested control hens of the same age. In both cases the decrease rate was correlated to the lice burdens (DeVaney, 1976). On the contrary, Khan et al. (2011) demonstrated only a nonsignificant reduction in the egg production of hens parasitized with Menopon gallinae (L.) and Lipeurus iawrensis tropicalis Peters, i.e. 3.4 and 13.3\% reduction, respectively. Additionally to the drop of egg production and weight gain, male reproduction may also be affected.

\section{Welfare}

Beak trimming, an important welfare issue of chicken, seems to have a direct impact on the high louse populations on most commercial caged laying hens. The effects of beak condition on lice populations and preening in laying hens were investigated by Vezzoli et al. (2015) by using beaktrimmed and beak-intact caged hens infested with chicken body lice. This study confirmed previous studies showing that an intact beak is important for reducing ectoparasite infestations. Preening behaviour increased in response to lice infestation, but only in beak-trimmed hens; preening behaviour and louse load were correlated at peak infestation. However, lice-infested hens directed preening behaviour predominantly towards the vent where these parasites are typically found. In agreement to the above study, are the findings of Mullens et al. (2010) who reported that louse 
distribution on the body of hens and louse population age structure were also influenced by host beak condition. Beak-intact hens had a higher proportion of lice under the wings, whereas beaktrimmed hens had the majority of lice on the lower abdomen. Louse populations on beak-trimmed hens also comprised relatively more immature stages than populations found on beak-intact hens. The effects are likely related to decreased grooming efficiency by beak-trimmed hens and the higher host density.

\section{Control}

All the various lice species are controlled by the same methods, i.e. spraying with the pyrethroids, carbaryl, coumaphos, dichlorvos, malathion and other insecticides. Treatment is required to be repeated at a 10-day interval, because only the mature forms will be controlled as most of the available insecticides do not kill the eggs. Furthermore, lice must be controlled on all the birds in the environment at the same time in order to avoid any cross-contamination.

Al-Quraishy et al. (2012) reported the successful treatment of severely infested birds by either a short dipping of the whole fowl into the 1:33 dilution (with tap water) of a neem seed extract (MiteStop $^{\oplus}$; Alpha-Biocare, Boca Raton, FL, USA) or by spraying them with the freshly diluted product. It was seen that the dead lice dropped down from the feathers as soon as they were dry again. As a precaution, a second treatment was done by some owners one week after the first one in order to eliminate all stages, which eventually might have hatched from untouched nits during the time interval between the two treatments. When treating in vitro cut-off feathers contaminated with L. caponis, it was seen under the stereomicroscope, that the lice moved away from the 1:33 water-diluted active compound, indicating that there is also a repellent effect. Treated $L$. caponis stopped leg movements within $3 \mathrm{~min}$. and died on their feathers within 1-20 min. By then, the visible effects of treatment, trembling movements of their legs and convulsions of their intestine, finally stopped.

In the experiments of Martin and Mullens (2012) cage-free hens received a dust box with sand plus diatomaceous earth, kaolin clay or sulphur. Ectoparasite populations on dust bathing hens (users) were compared with those on non-user cage-free and caged hens. All materials reduced lice on user hens by $80-100 \%$ after one week of dust box use. However, diatomaceous earth and kaolin failed to reduce ectoparasites on non-user hens, and ectoparasites on user hens recovered after dust box removal. This is the first experimental study to show that bird dust bathing in naturally and widely available dust materials (particularly kaolin) can suppress ectoparasites and this effect is probably adaptive.

\section{Poultry mites}

\section{Introduction}

Mites are with the ticks in the sub-class Acari. The poultry red mite, Dermanyssus gallinae (De Geer 1778) and the northern fowl mite, Ornithonyssus sylviarum (Canestrini and Fanzago 1877) are the most important mites for the poultry industry. The former is more important in Europe while the latter is a serious problem in North America. However, reports on both mites can now be found on both sides of the Atlantic. In Europe prevalence could reach easily 80-90\% (Sparagano et al. 2009) and new control methods are now explored at laboratory level with some reaching farm applications (Sparagano et al. 2014). 


\section{Morphology and biology}

D. gallinae is a relatively small ectoparasitic mite approximately 0.5 to $1.5 \mathrm{~mm}$ in length and varies in colour from grey to brown/red depending on feeding status. See Di Palma et al. (2012) for some identification keys.

This mite displays relative plasticity in terms of host specificity, although it remains associated primarily with birds in general and laying hens in particular (Roy and Chauve 2007). In contrast to O. sylviarum, the majority of the $D$. gallinae life cycle is spent off the host where mites seek refuge in secluded areas, such as cracks formed by timber joints, aggregating together in response to both thigmokinesis and pheromone cues. For more information on mite movement see Pritchard et al. (2015). Dermanyssus gallinae locates its hosts using a combination of temperature stimuli, chemical signals, and responses to vibration and carbon dioxide (Kilpinen, 2005). Once on a host, mites feed for short periods of up to an hour, doing so every 2-4 days and typically (although not exclusively) during periods of darkness. Larvae do not feed, and though adult males may feed, they are thought to do so only intermittently.

Conditions within poultry houses are well suited to $D$. gallinae population growth, where temperatures between 10 and $35{ }^{\circ} \mathrm{C}$ and high relative humidity $(>70 \%)$ facilitate $D$. gallinae reproduction and development. Consequently, weekly doubling of populations is possible in egg-laying facilities. D. gallinae densities commonly reach up to 50,000 mites per bird in caged systems, although densities can reach 500,000 mites per bird in severe cases (Kilpinen et al. 2005). D. gallinae may be present year-round, but highest densities occur during hot and humid seasons (Nordenfors and Hoglund 2000).

The life cycle has two nymphal stages, deutonymph and protonymph. The eggs and larvae do not need to feed on blood and therefore would stay away from birds in poultry farms while the two nymphal stages and the adults (sexualised only at that stage) would need blood to continue their life cycle or for the adult female to lay eggs. The morphology of these stages is described in Di Palma et al. 2012.

\section{Prevalence}

The poultry red-mite, Dermanyssus gallinae is well known worldwide and a prevalence around $80 \%$ of the surveyed laying farms is an average situation in Europe (George et al. 2015). In the UK, between 60 and $85 \%$ of commercial egg-laying facilities may be infested with D. gallinae. More recent figures suggest a greater variation in prevalence both in the UK and throughout Europe, with all sampled facilities and poultry production systems (barns, cages, aviaries) infested in certain countries (George et al. 2015; Sparagano et al. 2009). In Europe, the introduction of $D$. gallinae into poultry houses is considered to occur almost exclusively via the trade route (i.e. the movement of birds, egg crates, etc., between premises), although elsewhere (e.g. in Brazil) infestation of flocks occurs via both trade and wild birds, many species of which may serve as hosts.

\section{Vector-borne diseases and potential vaccine development}

Even relatively small mite populations that may not affect health through feeding per se may have significant impact, as D. gallinae may serve as a disease vector (De Luna et al. 2009; Huong et al. 2014; Valiente-Moro et al. 2007). Recently avian influenza was shown to be transmitted 
by D. gallinae (Sommer et al. 2016). Although the absolute vector competence of D. gallinae is unconfirmed, its potential to spread disease, including to humans, should not be underestimated. In addition to spreading disease, infestation may limit hen immunological responses to pathogens. Heavy infestations are reported to reduce antibody titres to some viral vaccines or suppress host antibody production. Some authors postulate that mites adopt a feeding strategy that involves minimal interference or modulation of host immunity, which could support these findings (Harrington et al. 2010). This same work suggests that D. gallinae might determine host immunecompetence via progeny survival rates. Although the reason is unknown, hens appear unable to mount a sufficiently effective immune response to $D$. gallinae, as supported by a lack of correlation between anti-D. gallinae immunoglobulin $Y$ levels and mite infestation levels.

The development of a vaccine against $D$. gallinae has made real progress in the last decade; from whole mite antigens (Arkle et al. 2008), to recombinant antigen (Harrington et al. 2009) to several vaccine candidates (Wright et al. 2016) and a dual approach. This has progressed faster since the publication of the mite transcriptomes (Schicht et al. 2013, 2014), allowing research groups working on vaccines to identify new potential vaccine candidates.

\section{Control}

\section{Chemicals}

There are only two main compounds that are approved for use in European countries - spinosad (under the trade name Elector; Elanco, Greenfield, IN, USA) and phoxim (under the trade name ByeMite; Bayer Healthcare, Leverkusen, Germany) are still the leading molecules being used officially. However, they are no longer fully effective and not available in all countries, and farmers have used many products which have not been approved for use on layers. For the past few years some products have been banned in the EU for safety reasons, which reduces the treatment option even more (Flochlay Sigognault et al. 2017). Sadly, in 2017 it was discovered that the acaricide fipronil, banned for use on poultry, had been illegally applied to anti-poultry mite products in the Netherlands, affecting the eggs and chicken meat products. By the time of the discovery several other European countries had been similarly affected causing the complete ban of chicken eggs and a huge loss to the producers (Gary 2017).

Resistance against carbaryl and permethrin has already been observed in several European countries (Marangi et al. 2009). However, there are ways to work against this increasing acaricide resistance by interfering with their resistance pathways (Graham et al. 2016) or adapting management processes (Abbas et al. 2014).

Non chemical products such as matrix-based products (Dergal or also known as Freeze from ICP Pharma, Saint Petersburg, FL, USA) target the immobilisation of $D$. gallinae and other poultry pests. Recently a new product, EXZOLT ${ }^{\oplus}$, has been put on the European market by MSD Animal Health (Boxmeer, the Netherlands) using the fluralaner molecule. This product which can be used in the presence of the birds and kills the mites very quickly to prevent female mites to have time to lay the eggs.

\section{Biological treatments}

At farm level it is now seen that farmers can use, as an alternative control method but within an integrated pest management complementary approach, predators against D. gallinae (Ali et al. 
2012; Lesna et al. 2009, 2012) with Androlis ${ }^{\circledR}$ and Taurrus ${ }^{\circledR}$ (APPI, Paris, France) predators being used predominantly. The efficacy of such approach depended of the predation and hunting skills of the predators and was highly influenced by the poultry system targeted with higher performances with floor-reared birds and less impact on cages as usually those predators are heavier and struggle to climb on walls and cages.

Entomopathogenic fungi have been used in pilot trials but struggled to be performing well under farm environmental conditions not always favourable to the fungi (Karsburg et al. 2016). Plantderived products (PDPs) have also been used as some products are showing repellent, toxicity or attractant properties to be used to divert and/or kill these mites.

One field expanding rapidly is the use of essential oils (EOs) or PDPs or plant extracts. Some EOs or PDPs can exhibit repellent or toxic effects or both. Several studies have shown the high mortality with such products (George et al. 2008, 2010a; Immediato et al. 2016; Nechita et al. 2015) but with environmental conditions such as temperature, dust and humidity affecting their efficacy (George et al. 2010b). Some have shown toxic effects beyond the D. gallinae group which can have negative impact by also affecting non-targeted insects, and vertebrates including chickens and humans. As some modes of interactions showed an effect on the acetylcholinesterase it is paramount to check the action spectrum of new EOs/PDPs (George et al. 2010c). Furthermore the volatility of such compounds makes their efficacy lasting only for a few hours/days and a need a possible improvement of their performance would be to encapsulate or trap such compound with a constant, slow release on a longer term to develop long-lasting effects.

Finally, some new biological potentials have not been fully explored, such as the symbionts found inside $D$. gallinae. These could be targeted to destabilise the arthropod-symbiont relationships (De Luna et al. 2009).

\section{Physical treatment}

Heat and steam have been used in some countries to treat the buildings and cages between flocks (Kilpinen 2001; Kilpinen and Mullens 2004), but this is seen as expensive and can affect the structural integrity of cages, doors and other equipment (Mul 2009b). However, it is important to highlight that such an approach can be counter-productive if the mite colonies are not killed directly by such treatment as it would bring the humidity and warmth they need to thrive.

A Dutch company has put the Q-Perch on the market (www.stikdesign.nl) based on electric wires under the perches were the birds can rest, while ICP Pharma has put an immobilising spray (DerGal, also sold as Freeze) on the market. When mites cross the wires they would be electrocuted. However, such setting can only work in this specific environmental conditions and not when birds are in cages or on the floor.

Organic system and farms not wishing to use chemicals are using in increasing numbers the diatomaceous earth or other silica-based products or even dust (Kilpinen and Steenberg 2009). They dessicate the mites and rupture their cuticle. However, small silica particles can have a negative effect on humans due to aerosol formation and lung deterioration and possible respiratory problems (Flochlay-Sigognault et al. 2017). 


\section{Integrated pest management}

IPM is well established in other production sectors, and recommended. combined treatment regimens for poultry pests, including D. gallinae, and date back many years (Axtell 1999). Today, poultry producers often implement some form of biosecurity in conjunction with multifaceted treatment programs involving cleaning/disinfecting and the use of physical agents and synthetic acaricides. Compared with other sectors, however, comprehensive IPM remains relatively rare in the poultry industry; the full potential of this technique for D. gallinae control is perhaps not achieved yet.

Of the various $D$. gallinae management approaches that have been reviewed here, many would be amenable to integration. Should a vaccine against $D$. gallinae become available, it likely could be used in conjunction with any other control method, similar to the hazard analysis and critical control point method (Mul and Koenraadt 2009). Similarly, the efficacy of novel pesticides, inert products, semiochemicals, and IGRs would be unaffected (and perhaps even improved) by advances in animal/premise husbandry techniques, as all of these would be compatible with one another as well as with conventional $D$. gallinae control. Such compatibility would not necessarily imply benefit, however, and would not be universal for all the management options considered. Broad-spectrum approaches, such as the use of some novel pesticides, inert dusts, IGRs, and heat treatment, would likely have an adverse effect on natural enemies and biological control. Although this effect may limit their use in IPM, they could be employed to target multiple invertebrate pests with a single application, as shown by MiteStop ${ }^{\circledR}$ (Walldorf et al. 2012) and some EOs (George et al. 2010a, 2010b, 2010c). Much remains to be done to promote comprehensive IPM for D. gallinae and to devise optimal treatment regimens, of which the latter has seen the least progress in the past 10-15 years. Recent advances in D. gallinae population modelling, coupled with empirical research, should facilitate work in this area, revealing optimal IPM strategies for immediate and future use (Huber et al. 2011).

Interestingly, the biosecurity levels can have secondary effects on Salmonella levels as D. gallinae has been proven as a potential vector for bacteria and could explain why there are still Salmonella foci in some countries still applying vaccination against this pathogen (Sylejmani et al. 2016).

One of the key issues is to evaluate the mite populations in a shed/house and decide when to treat. A recent automated method could help farmers and veterinarians to assess more accurately the mite population dynamic and develop a more adapted, time sensitive treatment strategy (Mul et al. 2015b). Finally, Mul (2017) has been focussing on these issues and brought new understanding of the key surveillance parameters to take into consideration.

\section{General conclusion}

This chapter has shown the extensive number of arthropod pests that can damage the poultry industry and their effect on birds, farm workers and production parameters. Some countries rely on the products available on their national market and what their national legislation allows them to use. It has been shown that an IPM approach would reduce possible acaricide resistance and improve production performance. Mixed control methods seem to bring such higher performance by reducing the potential for resistance and further limiting mite populations from re-infestation (Kilpinen and Steenberg 2016; Steenberg and Kilpinen 2014). 
The urgent need for innovative treatment approaches is now expected by many poultry production stakeholders. The absence of an effective treatment is stated in most of the scientific papers and has been recognized by the EU Commission, which created and funds the COST Action FA 1404 Research Platform (COREMI, Control of Red MItes) to'rid laying hens of a common diseasespreading pest' (www.coremi.eu). This four-year project started in December 2014 and involves representatives from almost all EU countries, Turkey, Israel, Albania and Canada. One of the major conclusions from recent COST conferences was that no single treatment method is sufficient to control D. gallinae. Increased use of IPM, improved biosecurity measures to prevent transmission of mites, and mite infestation monitoring are considered the best current strategies to control $D$. gallinae infestation.

IPM could bring long-lasting prevention strategies, which could reduce the negative impact of all the ectoparasites presented in this chapter. A gold standard approach could be to find a method which would affect all of them without targeting other beneficial insects, but such mode of action with such a specific spectrum would be unlikely and multiple treatments would be needed for the eradication of all these pests at once.

Interestingly, several sections in this chapter showed that B. bassiana or arthropod predators could be a solution for more than one arthropod pest species, highlighting the possibility for one biological control method to work effectively for more than one pest.

These arthropod pests, however, have developed counter-reaction mechanisms leading to an on-going race to identify new products, control methods and understanding better their biology, physiology and genetic make-up is paramount to identify new control approaches. More research and information is needed to identify new control targets for the above pests and identify future control methods giving sustainable and long-lasting protection to the poultry industry.

\section{Acknowledgements}

This article is based upon work from COST Action (FA1404-COREMI), supported by COST (European Cooperation in Science and Technology).

\section{References}

Abbas N, ljaz M, Shad SA and Binyameen M (2016) Assessment of resistance risk to fipronil and cross resistance to other insecticides in the Musca domestica L. (Diptera: Muscidae). Vet Parasit 223: 71-76.

Abbas RZ, Colwell DD, lqbal Z and Khan A (2014) Acaricidal drug resistance in poultry red mite (Dermanyssus gallinae) and approaches to its management. World's Poult Sci J 70: 113-124.

Acharya N (2015) House fly (Musca domestica L.) management in poultry production using fungal biopesticides. PhDthesis, Pennsylvania State University, State College, PA, USA.

Acharya N, Rajotte EG, Jenkins NE and Thomas MB (2015a) Influence of biotic and abiotic factors on the persistence of a Beauveria bassiana biopesticide in laboratory and high-rise poultry house settings. Biocontrol Sci Technol 25: 1317-1332.

Acharya N, Rajotte EG, Jenkins NE and Thomas MB (2015b) Potential for biocontrol of house flies, Musca domestica, using fungal biopesticides. Biocontrol Sci Technol 25: 513-524.

Adams RG (1984) Ophyra species as predators in animal houses, with a key to species occurring in Europe (Diptera: Muscidae). Entomol Gaz 35: 243-246.

Al-Khalidi NW and Shareef AM (1985) Myiasis in a turkey. Avian Dis 29: 1235-1238. 
Al-Quraishy S, Abdel-Ghaffar F, Al-Rasheid KA, Mehlhorn J and Mehlhorn H (2012) Effects of a neem seed extract (MiteStop ${ }^{\oplus}$ ) on mallophages (featherlings) of chicken: in vivo and in vitro studies. Parasitol Res 110: 617-622.

Ali W, George DW, Shiel RS, Sparagano OAE and Guy JH (2012) Laboratory screening of potential predators of the poultry red mite (Dermanyssus gallinae) and assessment Hypoaspis miles performance under varying biotic and abiotic conditions. Vet Parasit 187: 341-344.

Arkle A, Harrington D, De Luna C, George D, Guy J and Sparagano OA (2008) Immunological control of poultry red mite: the use of whole mite antigens as a candidate vaccine. Ann N Y Acad Sci 1149: 36-40.

Axtell RC and Arends JJ (1990) Ecology and management of arthropod pests of poultry. Ann Rev Entomol 35: 101-126.

Axtell RC (1994) Biology and economic importance of the darkling beetle in poultry houses. Poultry Supervisors' Short Course, North Carolina State University, Raleigh, NC, USA.

Axtell RC (1999) Poultry integrated pest management: status and future. J Integr Pest Manag 4: 53-73.

Bahrndorff S, Rangstrup-Christensen L, Nordentoft S and Hald B (2013) Foodborne disease prevention and broiler chickens with reduced Campylobacter infection. Emerg Infect Dis 19: 425-430.

Baldacchino F, Muenworn V, Desquesnes M, Desoli F, Charoenviriyaphap T and Duvallet G (2013) Transmission of pathogens by Stomoxys flies (Diptera, Muscidae): a review. Parasite 20: 26.

Baldacchino F, Desquesnes M, Duvallet G, LysykT and MihokS (2018) Veterinary importance and integrated management of Brachycera flies in dairy farms. In: Garros C, Bouyer J, Takken W and Smallegange RC (eds.) Pests and vector-borne diseases in the livestock industry. Ecology and Control of Vector-borne diseases. Vol. 5. Wageningen Academic Publishers, Wageningen, the Netherlands, pp. 55-90.

Barin A, Arabkhazaeli F, Rahbari S and Madani SA (2010) The housefly, Musca domestica, as a possible mechanical vector of Newcastle disease virus in the laboratory and field. Med Vet Entomol 24: 88-90.

Barnard DR (2003) Control of fly borne diseases. Pesticid Outl 14: 222-228.

Barnard DR, Harms RH and Sloan DR (1995) Influence of nitrogen, phosphorus, and calcium in poultry manure on survival, growth, and reproduction in the house fly (Diptera: Muscidae). Environ Entomol 24: 1297-1301.

Barreiro C, Albano H, Silva J and Teixeira P (2013) Role of flies as vectors of foodborne pathogens in rural areas. ISRN Microbiol 2013: 718780.

Barzman M, Bàrberi P, Birch ANE, Boonekamp P, Dachbrodt-Saaydeh S, Graf B, Hommel B, Jensen JE, Kiss J, Kudsk P, Lamichhane JR, Messéan A, Moonen A-C, Ratnadass A, Ricci P, Sarah JL and Sattin M (2015) Eight principles of integrated pest management. Agron Sustain Dev 35: 1199-1215.

Brenner RJ and Kramer RD (2002) Cockroaches (Blattaria). In: Mullen GR and Durden LA (eds.) Medical and veterinary entomology. Academic Press, London, UK.

Bidawid SP, Edeson JF, Ibrahim J and Matossian RM (1978) The role of non-biting flies in the transmission of enteric pathogens (Salmonella species and Shigella species) in Beirut, Lebanon. Ann Trop Med Parasitol 72: 117-121.

Bousquet $Y$ (1990) Beetles associated with stored products in Canada: an identification guide. Research branch agriculture Canada publication 1837. Canadian Government Publishing Centre Supply and Services Ottawa, Canada, $214 \mathrm{pp}$.

Butler JF, Kloft WJ, DuBose LA and Kloft ES (1977) Recontamination of food after feeding a ${ }^{32} \mathrm{P}$ food source to biting Muscidae. J Med Entomol 13:567-571

Chernaki-Leffer AM, Almeida LM, Sosa-Gómez DR, Anjos A and Vogado KM (2007) Populational fluctuation and spatial distribution of Alphitobius diaperinus (Panzer) (Coleoptera: Tenebrionidae) in a poultry house. Cascavel, Parana state, Brazil. Braz J Biol 67: 209-213.

Crippen TL and Poole TL (2012) Lesser mealworm on poultry farms: a potential arena for the dissemination of pathogens and antimicrobial resistance. In: Callaway TR and Edrington TS (eds.) On-farm strategies to control foodborne pathogens. NOVA Science Publishers, New York, NY, USA.

Dass R, Paul AVN and Agarwal RA (1984) Feeding potential and biology of lesser mealworm, Alphitobius diaperinus (Panz.) (Col., Tenebrionidae), preying on Corcyra cephalonica St. (Lep., Pyralidae). Z Angew Entomol 98(1-5): 444-447.

De Luna CJ, Moro CV, Guy JH, Zenner L and Sparagano OA (2009) Endosymbiotic bacteria living inside the poultry red mite (Dermanyssus gallinae). Exp Appl Acarol 48: 105-113.

DeVaney JA (1976) Effects of the chicken body louse, Menacanthus stramineus, on caged layers. Poult Sci 55: 430-435.

Pests and vector-borne diseases in the livestock industry 
Dhillon AS, Roy P, Lauerman L, Schaberg D, Weber S, Bandli D and Wier F (2004) High mortality in egg layers as a result of necrotic enteritis. Avian Dis 48: 675-680.

Di Domenico D, Venturelli C and Piumi M (2009) Studi Pratici per il controllo degli infestanti nelle industrie alimentari. ECOD, Milano, Italy.

Di Palma A, Giangaspero A, Cafiero MA and Germinara GS (2012) A gallery of the key characters to ease identification of Dermanyssus gallinae (Acari: Gamasida: Dermanyssidae) and allow differentiation from Ornithonyssus sylviarum (Acari: Gamasida: Macronyssidae). Parasit Vectors 5: 104.

Dunford JC and Kaufman PE (2006) Lesser mealworm, Alphitobius diaperinus. University of Florida, Gainesville, FL, USA. Available at: https://tinyurl.com/y8tw4qb9.

Durel L, Estrada-Peña A, Franc M, Mehlhorn H and Bouyer J (2015) Integrated fly management in European ruminant operations from the perspective of directive 2009/128/EC on sustainable use of pesticides. Parasitol Res 114: 379-389.

European Commission (EC) (2009) Directive 2009/128/EC of the European Parliament and of the Council of 21 October 2009 establishing a framework for Community action to achieve the sustainable use of pesticides. O J EU L309: 1-26.

Flochlay Sigognault A, Thomas E and Olivier Sparagano O (2017) Poultry red mite (Dermanyssus gallinae) infestation: a broad impact parasitological disease that still remains a significant challenge for the egg-laying industry in Europe. Parasit Vectors 10: 357.

Förster M, Klimpel S, Mehlhorn H, Sievert K, Messler S and Pfeffer K (2007) Pilot study on synanthropic flies (e.g. Musca, Sarcophaga, Calliphora, Fannia, Lucilia, Stomoxys) as vectors of pathogenic microorganisms. Parasitol Res 101: 243-246.

Francisco O and Prado AP (2001) Characterization of the larval stages of Alphitobius diaperinus (Panzer) (Coleoptera: Tenebrionidae) using head capsule width. Revista Brasileira de Biologia 61: 125-131.

Gary F (2017) The first lessons of the egg crisis involving fipronil. Le Nouveau Praticien Veterinaire Elevages et Sante $37: 8$.

Geden CJ (2006) Visual targets for capture and management of house flies, Musca domestica L. JVector Ecol 31: 152-157.

Geden CJ (2012) Status of biopesticides for control of house flies. J Biopest 5: 1-11.

Geden CJ and Axtell RC (1978) Factors affecting climbing and tunneling behavior of the lesser mealworm, Alphitobius diaperinus (Coleoptera: Tenebrionidae). J Econ Entomol 80: 1197-1204.

Geden CJ, Szumlas DE and Walker TW (2009) Evaluation of commercial and field-expedient baited traps for house flies, Musca domestica L. (Diptera: Muscidae). J Vector Ecol 34: 99-103.

George DR, Guy JH, Arkle S, Harrington D, De Luna CJ, Okello E, Shiel RS, Port G and Sparagano OA (2008) Use of plantderived products to control arthropods of veterinary importance; a mini-review. Ann N-Y Acad Sci 1149: 23-26.

George DR, Sparagano OAE, Port G, Okello E, Shiel RS and Guy JH (2010a) Toxicity of plant essential oils to different life stages of the poultry red mite, Dermanyssus gallinae, and non-target invertebrates. Med Vet Entomol 24: 9-15.

George DR, Sparagano OAE, Port G, Okello E, Shiel RS and Guy JH (2010b) Environmental interactions with the toxicity of plant essential oils to the poultry red mite Dermanyssus gallinae. Med Vet Entomol 24: 1-8.

George DR, Sparagano OAE, Port G, Okello E, Shiel RS and Guy JH (2010c) The effect of essential oils on laying hen welfare when deployed as acaricides for the poultry red mite, Dermanyssus gallinae. Anim Welf 19: 265-273.

George D, Finn RD, Graham KM, Mul MF, Maurer V, Valiente Moro C and Sparagano OAE (2015) Should the poultry red mite Dermanyssus gallinae be of wider concern for veterinary and medical science? Parasit Vectors 8: 178.

Giangaspero A (1997) Le mosche di interesse veterinario. I Muscidae (Flies of veterinary interest. Muscidae). Edagricole, Calderini, Bologna, Italy.

Graczyk TK, Knight R and Tamang L (2005) Mechanical transmission of human protozoan parasites by insects. Clin Microbiol Rev 18: 128-132.

Graham KM, Sparagano OAE and Finn RD (2016) Isolation of the monooxygenase complex from Rhipicephalus (Boophilus) microplus - clues to understanding acaricide resistance. Ticks Tick Borne Dis 7: 614-623.

Grandi G (1996) Istituzioni di entomologia generale. Calderini, Bologna, IT.

Greenberg B (1973) Flies and diseases. Princeton University Press, Princeton, NJ, USA. 
Hald B, Skovgard H, Bang DD, Pedersen K, Dybdahl J, Jespersen JB and Madsen M (2004) Flies and Campylobacter infection of broiler flocks. Emerg Infect Dis 10: 1490-1492.

Hald B, Skovgård H, Pedersen K and Bunkenborg H (2008) Influxed insects as vectors for Campylobacter jejuni and Campylobacter coli in Danish broiler houses. Poult Sci 87: 1428-1434.

Hanley ME, Cruickshanks KL, Dunn D, Stewart-Jones A and Goulson D (2009) Luring houseflies (Musca domestica) to traps: do cuticular hydrocarbons and visual cues increase catch? Med Vet Entomol 23: 26-33.

Harrington LC and Axtell RC (1994) Comparisons of sampling methods and seasonal abundance of Drosophila replete in caged-layer poultry houses. Med Vet Entomol 8: 331-339.

Harrington D, Canales M, de la Fuente J, Robinson K, Guy J and Sparagano O (2009). Immunisation with recombinant proteins subolesin and Bm86 for the control of Dermanyssus gallinae in poultry. Vaccine 27: 4056-4063.

Harrington DW, Robinson K and Sparagano OA (2010) Immune responses of the domestic fowl to Dermanyssus gallinae under laboratory conditions. Parasitol Res 106: 1425-1434.

Hazeleger WC, Bolder MN, Beumer RR and Jacobs-Reitsma WF (2008) Darkling beetles (Alphitobius diaperinus) and their larvae as potential vectors for the transfer of Campylobacter jejuni and Salmonella enterica serovar paratyphi B variant Java between successive broiler flocks. Appl Environ Microbiol 74: 6887-6891.

Hinton JL and Moon RD (2003) Arthropod populations in high-rise, caged layer houses after three manure cleanout treatments. J Econ Entomol 96: 1352-1361.

Huber K, Zenner L and Bicout DJ (2011) Modelling population dynamics and response to management options in the poultry red mite Dermanyssus gallinae (Acari: Dermanyssidae). Vet Parasitol 176: 65-73.

Huong CT, Murano T, Uno Y, Usui T and Yamaguchi T (2014) Molecular detection of avian pathogens in poultry red mite (Dermanyssus gallinae) collected in chicken farms. J Vet Med Sci 76: 1583-1587.

Immediato D, Figueredo LA, latta R, Otranto D and Cafarchia C (2016) Essential oils and Beauveria bassiana against Dermanyssus gallinae (Acari: Dermanyssidae): towards new natural acaricides. Vet Parasitol 229: 159-165.

James P, Krawec C, Glatz P and Critchley K (2004) Fly control in layer sheds - an integrated approach. Pig and Poultry Production Institute, South Australian Research and Development Institute, Roseworthy Campus, Roseworthy, Australia.

Johansen H, Solum M, Knudsen GK, Hågvar EB, Norli HR and Aak A (2014) Blow fly responses to semiochemicals produced by decaying carcasses. Med Vet Entomol 28: 26-34.

Kang B, Vellody D, Homburger H and Yunginger JW (1979) Cockroach cause of allergic asthma. Its specificity and immunologic profile. J Allergy Clin Immunol 63: 80-86.

Karsburg CR, Alves LFA, Oliveira DGP and Rohde C (2016) Activity of some Brazilian isolates of entomopathogenic fungi against the poultry red mite Dermanyssus gallinae De Geer (Acari: Dermanyssidae). Rev Bras Cienc Avic 18: 457-460.

Kaufman PE, Reasor C, Rutz DA, Ketzis JK and Arends JJ (2005) Evaluation of Beauveria bassiana applications against adult house fly, Musca domestica, in commercial caged-layer poultry facilities in New York state. Bio Control 33: 360-367.

Kettle DS (ed) (1995) Medical and veterinary entomology (2 ${ }^{\text {nd }}$ Ed.). CAB International, Wallingford, UK, 725 pp.

Khan V, Arya G, Ahmad A and Kumar Saxena A (2011) Poultry lice (Phthiraptera: Insecta) parasitism on caged layers. Indian Vet J 88: 68-70.

Kilpinen $O$ (2001) Activation of the poultry red mite, Dermanyssus gallinae (Acari: Dermanyssidae), by increasing temperatures. Exp Appl Acarol 25(10-11): 859-867.

Kilpinen $\mathrm{O}$ (2005) How to obtain a bloodmeal without being eaten by a host: the case of poultry red mite, Dermanyssus gallinae. Physiol Entomol 30: 232-240.

Kilpinen $O$ and Mullens BA (2004) Effect of food deprivation on response of the mite, Dermanyssus gallinae, on heat. Med Vet Entomol 18: 368-371.

Kilpinen O, Roepstorff A, Permin A, Norgaard-Nielsen G, Lawson LG and Simonsen HB (2005) Influence of Dermanyssus gallinae and Ascaridia galli infections on behaviour and health of laying hens (Gallus gallus domesticus). Br Poult Sci 46: 26-34.

Kilpinen $O$ and Steenberg T (2009) Inert dusts and their effects on the poultry red mite (Dermanyssus gallinae). Exp Appl Acarol 48(1-2): 51-62. 
Kilpinen $\mathrm{O}$ and Steenberg T (2016) Repellants activity of dessicant dusts and conidia of the entomopathogenic fungus Beauveria bassiana when tested against the poultry red mites (Dermanyssus gallinae) in laboratory experiments. Exp Appl Acarol 70: 329-341.

Lambkin TA, Kopittke RA, Rice SJ, Bartlett JS and Zalucki MP (2008) Factors affecting localized abundance and distribution of lesser mealworm in earth-floor broiler houses in subtropical Australia. J Econ Entomol 101: 61-67.

Leffer AM, Kuttel J, Martins LM, Pedroso AC, Astolfi- Ferreira CS, Ferreira F and Ferreira AJP (2010) Vectorial competence of larvae and adults of Alphitobius diaperinus in the transmission of Salmonella enteritidis in poultry. Vector-Borne Zoonotic Dis 10: 481-487.

Lesna I, Wolfs P, Faraji F, Komdeur J and Sabelis MW (2009) Candidate predators for biological control of the poultry red mite Dermanyssus gallinae. Exp Appl Acarol 48(1-2): 63-80.

Lesna I, Sabelis MW, Van Niekerk TGCM and Komdeur J (2012) Laboratory tests for controlling poultry red mites (Dermanyssus gallinae) with predatory mites in small 'laying hens' cages. Exp Appl Acarol 58: 371-383.

Lysyk TJ (1998) Relationships between temperature and life-history parameters of Stomoxys calcitrans (Diptera: Muscidae). J Med Entomol 35: 107-119.

Machtinger ET and Geden CJ (2018) Biological control with parasitoids. In: Garros C, Bouyer J, Takken W and Smallegange $\mathrm{RC}$ (eds.) Pests and vector-borne diseases in the livestock industry. Ecology and Control of Vector-borne diseases. Vol. 5. Wageningen Academic Publishers, Wageningen, the Netherlands, pp. 299-335.

Maheshwari S (2013) Environmental impacts of poultry production. Poult Fish Wildl Sci 1: 101.

Marangi M, De Luna CJ, Cafiero MA, Camarda A, Le Bouquin S, Huonnic D, Giangaspero A and Sparagano OAE (2009) Phylogenetic relationship between Dermanyssus gallinae populations in European countries based on mitochondrial CO1 gene sequences. Exp Appl Acarol 48(1-2): 143-155.

Martin CD and Mullens BA (2012) Housing and dustbathing effects on northern fowl mites (Ornithonyssus sylviarum) and chicken body lice (Menacanthus stramineus) on hens. Med Vet Entomol 26: 323-333.

Masutti L and Zangheri S (2001) Entomologia generale e applicata. CEDAM, Padova, Italy.

Merdan BA (2012) Bacillus thuringiensis as a feed additive to control Musca domestica associated with poultry houses. J Basic Appl Zool 65: 83-87.

Mul MF and Koenraadt CJM (2009) Preventing introduction and spread of Dermanyssus gallinae in poultry facilities using the HACCP method. Exp Appl Acarol 48: 167-181.

Mul MF, Van Niekerk T, Chirico J, Maurer V, Kilpinen O, Sparagano O, Thind B, Zoons J, Moore D, Bell B, Gjevre A-G and Chauve $C$ (2009) Control methods for Dermanyssus gallinae in systems for laying hens: results of an international seminar. Worlds Poult Sci J 65: 589-599.

Mul MF, Smallegange RC and Brooks M (2015a) Preventieve maatregelen tegen huisvliegen in vleeskuikenstallen; voorkomen van introductie van Campylobacter door vliegen op vleeskuikenbedrijven. Livestock Research Rapport 836, Wageningen University \& Research, Wageningen Livestock Research, Wageningen, the Netherlands, 28 pp.

Mul MF, Van Riel JW, Meerburg BG, George DR, Groot Koerkamp PWG (2015b) Validation of an automated mite counter for Dermanyssus gallinae in experimental laying hen cages. Exp Appl Acarol 66: 589-604.

Mul MF (2017) Advancing integrated pest management for Dermanyssus gallinae in laying hen facilities. PhD-thesis, Wageningen University, Wageningen, the Netherlands, $194 \mathrm{pp}$.

Mullens BA, Chen BL and Owen JP (2010) Beak condition and cage density determine abundance and spatial distribution of northern fowl mites, Ornithonyssus sylviarum, and chicken body lice, Menacanthus stramineus, on caged laying hens. Poult Sci 89: 2565-2572.

Nachman G and Skovgård H (2018) The Fly Simulator: a simulation model of stable flies and their control. In: Garros C, Bouyer J, Takken W and Smallegange RC (eds.) Pests and vector-borne diseases in the livestock industry. Ecology and Control of Vector-borne diseases. Vol. 5. Wageningen Academic Publishers, Wageningen, the Netherlands, pp. 465-494.

Nechita IS, Poirel MT, Cozma V and Zenner L (2015) The repellent and persistent toxic effects of essential oils against the poultry red mite, Dermanyssus galliane. Vet Parasitol 214(3-4): 348-352.

Nichols GL (2005) Fly transmission of Campylobacter. Em Inf Dis 11: 361-364. 
Nordenfors $\mathrm{H}$ and Hoglund J (2000) Long term dynamics of Dermanyssus gallinae in relation to mite control measures in aviary systems for layers. Br Poult Sci 41:533-540.

Oliveira D, Alves L and Sosa-Gómez D (2014) Advances and perspectives of the use of the entomopathogenic fungi Beauveria bassiana and Metarhizium anisopliae for the control of arthropod pests in poultry production. Rev Bras Ciênc Avíc 16: 1-12.

Olsen AR and Hammack TS (2000) Isolation of Salmonella spp. from the housefly, Musca domestica L., and the dump fly, Hydrotaea aenescens (Wiedemann) (Diptera: Muscidae), at caged-layer houses. J Food Prot 63: 958-960.

Patrick ME, Christiansen LE, Waino M, Ethelberg S, Madsen H and Wegener HC (2004) Effects of climate on incidence of Campylobacter spp. in humans and prevalence in broiler flocks in Denmark. Appl Environ Microbiol 70: 7474-7480.

Pfeiffer DG and Axtell RC (1980) Coleoptera of poultry manure in caged layer houses in North Carolina. Environ Entomol 9:21-28.

Pritchard J, Kuster T, Sparagano $O$ and Tomley $F$ (2015) Undertsanding the biology and control of the poultry red mite Dermanyssus gallinae: a review. Avian Pathol 44: 143-153.

Qiu YT, Spitzen J, Smallegange RC and Knols BGJ (2007) Monitoring systems for adult insect pests and disease vectors. In: Takken T and Knols BGJ (eds.) Emerging pests and vector-borne diseases in Europe. Ecology and Control of Vector-borne diseases. Vol. 1. Wageningen Academic Publishers, Wageningen, the Netherlands.

Rezende SRF, Curvello FA, Fraga ME, Reis RCS, Castilho AMC and Agostinho TSPV (2009) Control of the Alphitobius diaperinus (Panzer) (Coleoptera: Tenebrionidae) with entomopathogenic fungi. Braz J Poult Sci 11: 121-127.

Roche AJ, Cox NA, Richardson LJ, Buhr RJ, Cason JA, Fairchild BD and Hinkle NC (2009) Transmission of Salmonella to broilers by contaminated larval and adult lesser mealworms, Alphitobius diaperinus (Coleoptera: Tenebrionidae). Poult Sci 88: 44-48.

Roy L and Chauve CM (2007) Historical review of the genus Dermanyssus Duges, 1834 (Acari: Mesostigmata: Dermanyssidae). Parasite 14: 87-100.

Rueda LM and Axtell RC (1996) Temperature-dependent development and survival of the lesser mealworm, Alphitobius diaperinus. Med Vet Entomol 10: 80-86.

Rueda LM and Axtell RC (1997) Arthropods in litter of poultry (broiler chicken and turkey) houses. J Agri Urban Entomol 14: 81-91.

Sahin O, Kassem II, Shen Z, Lin J, Rajashekara G and Zhang Q (2015) Campylobacter in poultry: ecology and potential interventions. Avian Dis 59: 185-200.

Salin C, Delettre YR, Cannavacciuolo M and Vernon P (2000) Spatial distribution of Alphitobius diaperinus (Panzer) (Coleoptera: Tenebrionidae) in the soil of a poultry house along a breeding cycle. Eur J Soil Biol 36: 107-115.

Schicht S, Qi W, Poveda L and Strube C (2013) The predicted secretome and transmembranome of the poultry red mite Dermanyssus gallinae. Parasite Vectors 6: 259.

Schicht S, Qi W, Poveda L and Strube C (2014) Whole transcriptome analysis of the poultry red mite Dermanyssus gallinae (De Geer, 1778). Parasitology 141: 336-346.

Schowalter TD and Klowden MJ (1979) Blood meal size of the stable fly, Stomoxys calcitrans, measured by the HiCN method. Mosq News 39: 110-112.

Scott JG, Warren WC, Beukeboom LW, Bopp D, Clark AG, Giers SD, Hediger M, Jones AK, Kasai S, Leichter CA, Li M, Meisel RP, Minx P, Murphy TD, Nelson DR, Reid WR, Rinkevich FD, Robertson HM, Sackton TB, Sattelle DB, Thibaud-Nissen F, Tomlinson C, Van de Zande L, Walden KK, Wilson RK and Liu N (2014) Genome of the house fly, Musca domestica L., a global vector of diseases with adaptations to a septic environment. Genome Biol 15: 466.

Shah RM, Azhar F, Shad SA, Walker WB, Azeem M and Binyameen M (2016) Effects of different animal manures on attraction and reproductive behaviors of common house fly, Musca domestica L. Parasitol Res 115: 3585-3598.

Shepard DC, Newton GL, Thompson SA and Savage S (1994) A value added manure management system using the black soldier fly. Biores Tech 50: 275-279.

Sievert K, Alvarez R, Cortada R and Valks M (2008) House flies and the avian influenza threat. Int Poult Prod 14: 7-9.

Skidmore P (1985) The biology of the Muscidae of the world. W. Junk Publishers, Dordrecht, the Netherlands.

Smallegange RC (2003) Attractiveness of different light wavelengths, flicker frequencies and odours to the housefly (Musca domestica L.). PhD thesis, Groningen University, Groningen, the Netherlands. 
Smallegange RC and Den Otter CJ (2007) Houseflies, annoying and dangerous. In: Takken T and Knols BGJ (eds.) Emerging pests and vector-borne diseases in Europe. Ecology and Control of Vector-borne diseases. Vol. 1. Wageningen Academic Publishers, Wageningen, the Netherlands.

Solà-Ginés M, González-López JJ, Cameron-Veas K, Piedra-Carrasco N, Cerdà-Cuéllar M and Migura-Garcia L (2015) Houseflies (Musca domestica) as vectors for extended-spectrum $\beta$-lactamase-producing Escherichia coli on Spanish broiler farms. Appl Environ Microbiol 81: 3604-3611.

Sommer D, Heffels-Redmann U, Kohler K, Lierz M and Kaleta EF (2016). Role of the poultry red mite (Dermanyssus gallinae) in the transmission of Avian Influenza A virus. Tierarztl Prax Ausg G Grosstiere Nutztiere 44: 26-33.

Sparagano O, Pavlicevic A, Murano T, Camarda A, Sahibi H, Kilpinen O, Mul M, Van Emous R, Le Bouquin S, Hoele K and Assunta Cafiero M (2009) Prevalence and key figures for the poultry red mite Dermanyssus gallinae infections in poultry farm systems. Exp Appl Acarol 48: 3-10.

Sparagano OAE, George DR, Harrington DWJ and Giangaspero A (2014) Significance and control of the poultry red mite, Dermanyssus gallinae. Ann Rev Entomol 59: 447-466.

Steenberg T and Kilpinen $O$ (2014). Synergistic interaction between the fungus Beauveria bassiana and dessicant dusts applied against the poultry red mites (Dermanyssus gallinae). Exp Appl Acarol 62: 511-524.

Sukontason KL, Bunchu N, Methanitikorn R, Chaiwong T, Kuntalue B and Sukontason K (2006) Ultrastructure of adhesive device in fly in families Calliphoridae, Muscidae and Sarcophagidae, and their implication as mechanical carriers of pathogens. Parasitol Res 98: 477-481.

Sùss L and Locatelli DP (2001) Gli Artropodi. I parassiti delle derrate. Calderini, Bologna, Italy.

Sychra O, Harmat P and Literak I (2008) Chewing lice (Phthiraptera) on chickens (Gallus gallus) from small backyard flocks in the eastern part of the Czech Republic. Vet Parasitol 152: 344-348.

Sylejmani D, Musliu A, Ramadani N, Sparagano O and Hamidi A (2016) Association between the Level of biosecurity and occurrence of Dermanyssus gallinae and Salmonella spp. in layer farms. Avian Dis 60: 454-459.

Szalanski AL, Owens CB, McKay T and Steelman CD (2004). Detection of Campylobacter and Escherichia coli 0157:H7 from filth flies by polymerase chain reaction. Med Vet Entomol 18: 241-246.

Tan SW, Yap KL and Lee HL (1997) Mechanical transport of rotavirus by the legs and wings of Musca domestica (Diptera: Muscidae). J Med Entomol 34: 527-531.

Taylor MA, Coop RL and Wall RL (2007) Veterinary parasitology ( ${ }^{\text {rd }}$ Ed.). Blackwell Publishing, Oxford, UK, 874 pp.

Tomberlin JK, Richman D and Myers HM (2008) Susceptibility of Alphitobius diaperinus (Coleoptera: Tenebrionidae) from broiler facilities in Texas to four insecticides. J Econ Entomol 101: 480-483.

Trematerra P and Gentile P (2008) Gli animali infestanti in molini e pastifici. Chiriotti, Pinerolo, Italy.

Tremblay E (2005) Entomologia applicata. Vol. 2. Liguori, Napoli, Italy.

Ugbogu OC, Nwachukwu NC and Ogbuagu UN (2006) Isolation of Salmonella and Shigella species from house flies (Musca domestica L.) in Uturu, Nigeria. Afr J Biotechnol 5: 1090-1091.

Valiente-Moro C, Desloire C, Chauve C and Zenner L (2007) Detection of Salmonella spp.in D. gallinae using FTA ${ }^{\oplus}$ filterbased polymerase chain reaction. Med Vet Entomol 21: 148-152.

Vezzoli G, Mullens BA and Mench JA (2015) Relationships between beak condition, preening behaviour and ectoparasite infestation levels in laying hens. Poult Sci 94: 1997-2007.

Walldorf V, Mehlhorn H, Al-Quraishy S, Al-Rasheid KA, Abdel-Ghaffar F and Mehlhorn J (2012) Treatment with a neem seed extract (MiteStop ${ }^{\oplus}$ ) of beetle larvae parasitizing the plumage of poultry. Parasitol Res 110: 623-627.

Wanaratana S, Amonsin A, Chaisingh A, Panyim S, Sasipreeyajan J and Pakpinyo S (2013) Experimental assessment of houseflies as vectors in avian influenza subtype H5N1 transmission in chickens. Avian Dis 57: 266-272.

Weeks ENI, Machtinger ET, Leemon D and Geden CJ (2018) Biological control of livestock pests: entomopathogens. In: Garros C, Bouyer J, Takken W and Smallegange RC (eds.) Pests and vector-borne diseases in the livestock industry. Ecology and Control of Vector-borne diseases. Vol. 5. Wageningen Academic Publishers, Wageningen, the Netherlands, pp. 337-387.

Welch RM (2006) Relative effectiveness of commercial fly traps and ammonium carbonate/yeast in catching house flies (Diptera: Muscidae). PhD thesis, University of Florida, Gainesville, FL, USA. 
West LS (1951) The housefly. Its natural history, medical importance, and control. Comstock Publishing Company, Cornell University Press, Ithaca, NY, USA.

Wilhoit LR, Axtell RC and Stinner RE (1991a) Estimating manure temperatures from air temperatures and results of its use in models of filth fly (Diptera: Muscidae) development. Environmental Entomology 20: 635-43.

Wilhoit LR, Stinner RE and Axtell RC (1991b) Computer simulation model of house fly management in confined-animal production systems. North Carolina Agricultural Research Service Technical Bulletin 296: 1-81.

Wright HW, Bartley K, Huntley JF and Nisbet AJ (2016) Characterisation of tropomyosin and paramyosin as vaccine candidate molecules for the poultry red mite, Dermanyssus gallinae. Parasit Vectors 9: 544.

Zheng L, Crippen TL, Singh B, Tarone AM, Dowd S, Yu Z, Wood TK and Tomberlin JK (2013) A survey of bacterial diversity from successive life stages of black soldier fly (Diptera: Stratiomyidae) by using 16S rDNA pyrosequencing. J Med Entomol 50: 647-658.

Zumpt $F$ (1965) Myiasis in man and animals in the old world. A text book for physicians, veterinarians and zoologists. Butterworth and Co Ltd., London, UK, pp. 109. 


\title{
3. Veterinary importance and integrated management of Brachycera flies in dairy farms
}

\begin{abstract}
Frédéric Baldacchino ${ }^{1,2^{*}}$, Marc Desquesnes ${ }^{3,4}$, Gérard Duvallet ${ }^{5}$, Timothy Lysyk ${ }^{6}$ and Steve Mihok ${ }^{7}$
${ }^{1}$ Research and Innovation Centre, Fondazione Edmund Mach, Via Mach 1, 38010 San Michele all'Adige, Italy; ${ }^{2}$ Direction départementale de la protection des populations Nord, 93-95 boulevard Carnot, 59046 Lille cedex, France; ${ }^{3}$ Cirad, Avenue Agropolis, 34398 Montpellier Cedex 5, France; ${ }^{4}$ Kasetsart University, 50 Ngam Wong Wan Rd, Ladyaow Chatuchak, Bangkok 10900, Thailand; ${ }^{5}$ Centre d'Écologie fonctionnelle et évolutive, UMR5175, Université Paul-Valéry, Route de Mende, 34199 Montpellier Cedex 5, France; ${ }^{6}$ Agriculture and Agri-Food Canada, $540311^{\text {st }}$ Avenue South Lethbridge, AB, T1J 4B1, Canada; 7388 Church Street, Russell, ON, K4R 1A8, Canada; fredericbaldacchino@yahoo.fr
\end{abstract}

\begin{abstract}
Adult Brachycera can affect dairy cattle through their feeding behaviour. The main families of veterinary importance are Tabanidae, Athericidae, Rhagionidae, Muscidae, Glossinidae and Hippoboscidae. Non-biting flies such as house flies and face flies ingest liquids from animal tissues using sponging mouthparts, whereas biting flies such as tabanids, stable flies and tsetse flies take blood from hosts using piercing-sucking mouthparts. Thus, Brachycera flies may impact livestock through both direct effects (nuisance, skin injuries, blood losses) and indirect effects (pathogen transport or transmission), contributing to economic losses for dairy production. Nonbiting flies are mainly mechanical carriers of pathogens, especially bacteria (e.g. Escherichia coli, Moraxella bovis, Staphylococcus aureus). Tabanids and biting muscid flies are mainly mechanical vectors of pathogens including bacteria (e.g. Bacillus anthracis, Anaplasma marginale), protozoa (e.g. Besnoitia besnoiti, Trypanosoma spp.) and viruses (e.g. lumpy skin disease virus), whereas tsetse flies are biological vectors of trypanosomes causing African Animal Trypanosomosis. Brachycera flies are also developmental vectors of several nematodes (Thelazia spp., Parafilaria bovicola, Stephanofilaria stilesi). Today, several control methods are available for the integrated management of flies inside livestock facilities and in pastures, including environmental methods (sanitation), biological methods (parasitoids, entomopathogenic fungi), chemical methods (insect growth regulators, insecticides) and mechanical methods (traps, targets). Facing the growing concern of increasing fly populations related to changing climate and land use, there is a crucial need to better assess their direct impact and their role in the epidemiology of (re)emerging flyborne pathogens and to improve the effectiveness and sustainability of control methods.
\end{abstract}

Keywords: house fly, horse fly, stable fly, tsetse fly, mechanical transmission, vector-borne disease, vector control

\section{Introduction}

The Diptera is a large and diverse order of insects and is divided into two suborders: the more primitive Nematocera and the Brachycera (McAlpine and Wood 1989). Nematocera flies have long antennae (e.g. mosquitoes) whereas Brachycera flies have short antennae (e.g. horse flies and house flies). The suborder Brachycera includes three infraorders: Tabanomorpha (e.g. horse flies), Asilomorpha (no species of veterinary importance) and Muscomorpha (e.g. house flies).

In this chapter, we consider only Brachycera that affect cattle as adults through their feeding behaviour (Figure 1). We exclude myiasis-causing flies (e.g. warble flies) whose larvae develop 
A

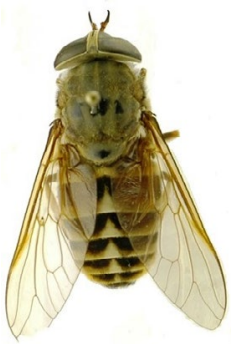

D

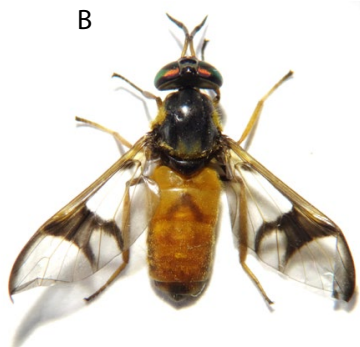

$\mathrm{E}$

E
C

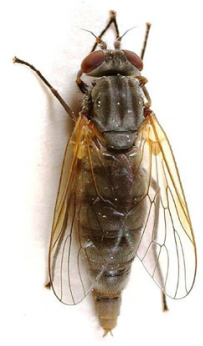

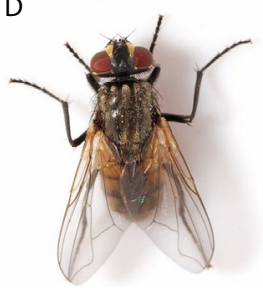

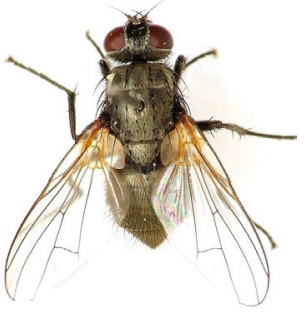

$\mathrm{F}$

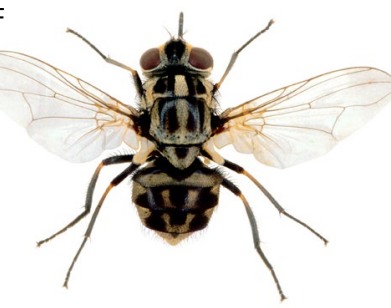

G

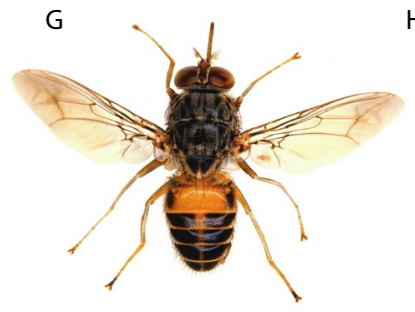

$\mathrm{H}$

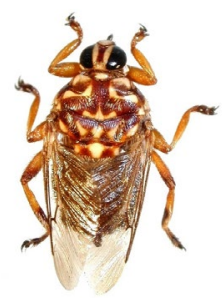

Figure 1. Examples of Brachycera species (A) Tabanus sudeticus (photo by F. Baldacchino), (B) Chrysops flaviventris (photo by M. Desquesnes), (C) Symphoromyia hirta (photo by T. Murray), (D) Musca domestica (photo by P. Bolson), (E) Hydrotaea irritans (photo by L. Karlsson), (F) Stomoxys calcitrans (photo by S. Mihok), (G) Glossina pallidipes (photo by S. Mihok), and (H) Hippobosca variegata (photo by M. Desquesnes).

and migrate in mammalian hosts (Hall et al. 2016). Flies can be grouped by feeding behaviour; nonbiting flies ingest liquids from surfaces and animal tissues using sponging mouthparts, whereas biting flies take blood from hosts using piercing-sucking mouthparts (Lehane 2005). Brachycera may have an impact on livestock through both direct effects (nuisance, skin injuries, blood loss) and indirect effects (pathogen transmission) (Table 1), contributing to economic losses for dairy production (Baldacchino et al. 2014a; Foil and Hogsette 1994; Thomas and Jespersen 1994). Their economic impact can be highly significant in dairy cattle; as an illustration of this impact, the median annual milk loss due to stable flies in the USA was estimated around $140 \mathrm{~kg}$ per year (Taylor et al. 2012).

More specifically, here we discuss the different transmission routes of pathogens by describing: (1) mechanical transport as the transmission of a pathogen via mechanical dislodgment from the exoskeleton, faecal deposition, and regurgitation, usually by a non-biting fly; (2) mechanical transmission as the transmission of a pathogen by a biting fly via contaminated blood on 


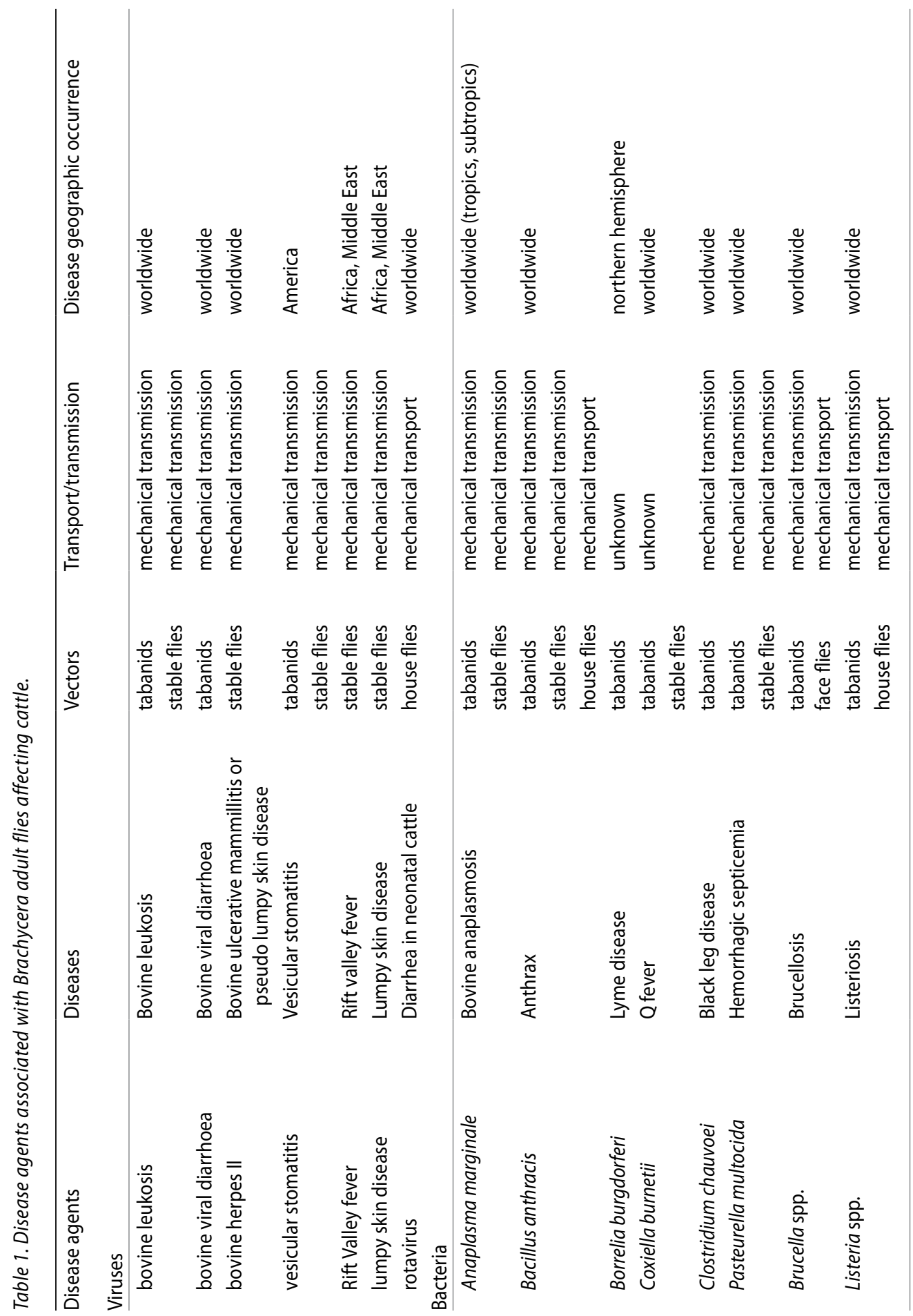




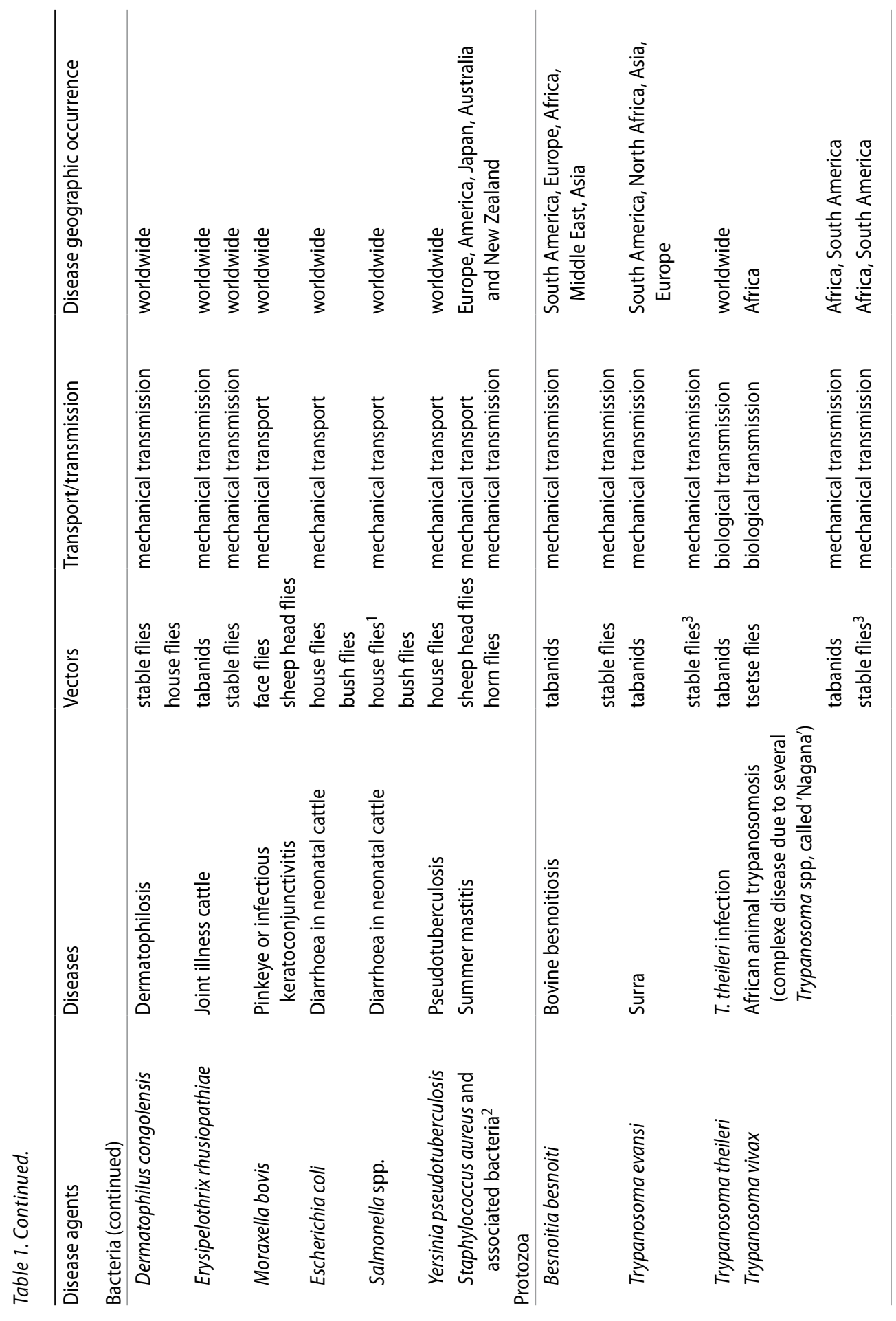




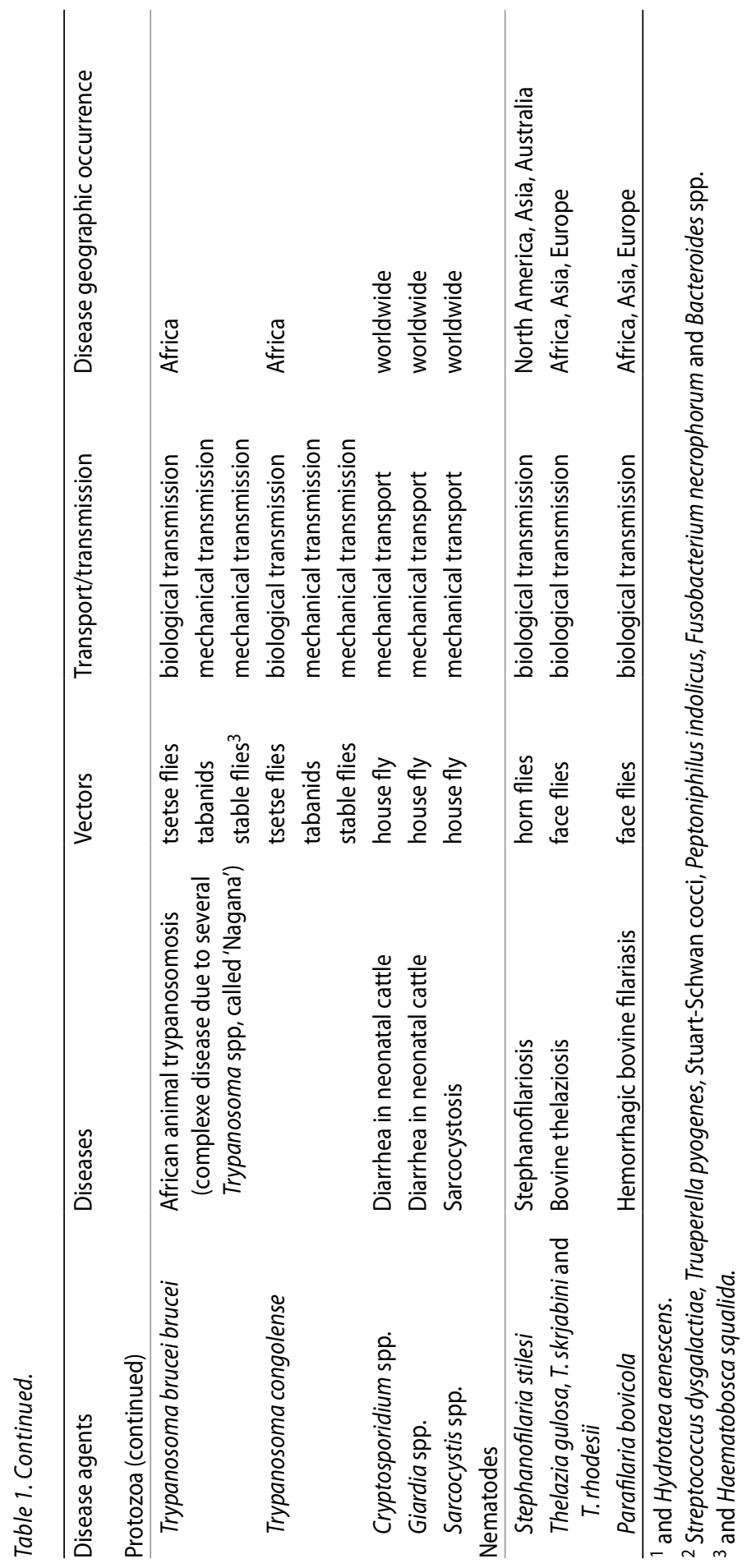


mouthparts without amplification or development within the fly; and (3) biological transmission as the transmission of a pathogen by a biting fly generally via saliva after amplification and/or development within the fly (Baldacchino et al. 2014a; Graczyk et al. 2005).

Facing the growing concern of flies as nuisance and vectors of pathogenic agents as well as the threat to pollinators by insecticides used for fly control, requires improvement of the effectiveness and sustainability of integrated pest management favouring ecosystem services (Baldacchino et al. 2014a; Floate et al. 2013). This chapter presents the different fly control methods that can be implemented in cattle facilities and pastures, focusing on alternatives to the traditional chemical methods. Thus, we will also describe environmental methods including sanitation, dung degradation by beetles, patch-burn grazing and animal grazing management, biological methods such as the use of parasitoids, entomopathogenic fungi, plant essential oils, bacterial toxins and natural predators, and behavioural and mechanical methods mainly based on attractive traps and targets (Figure 2).
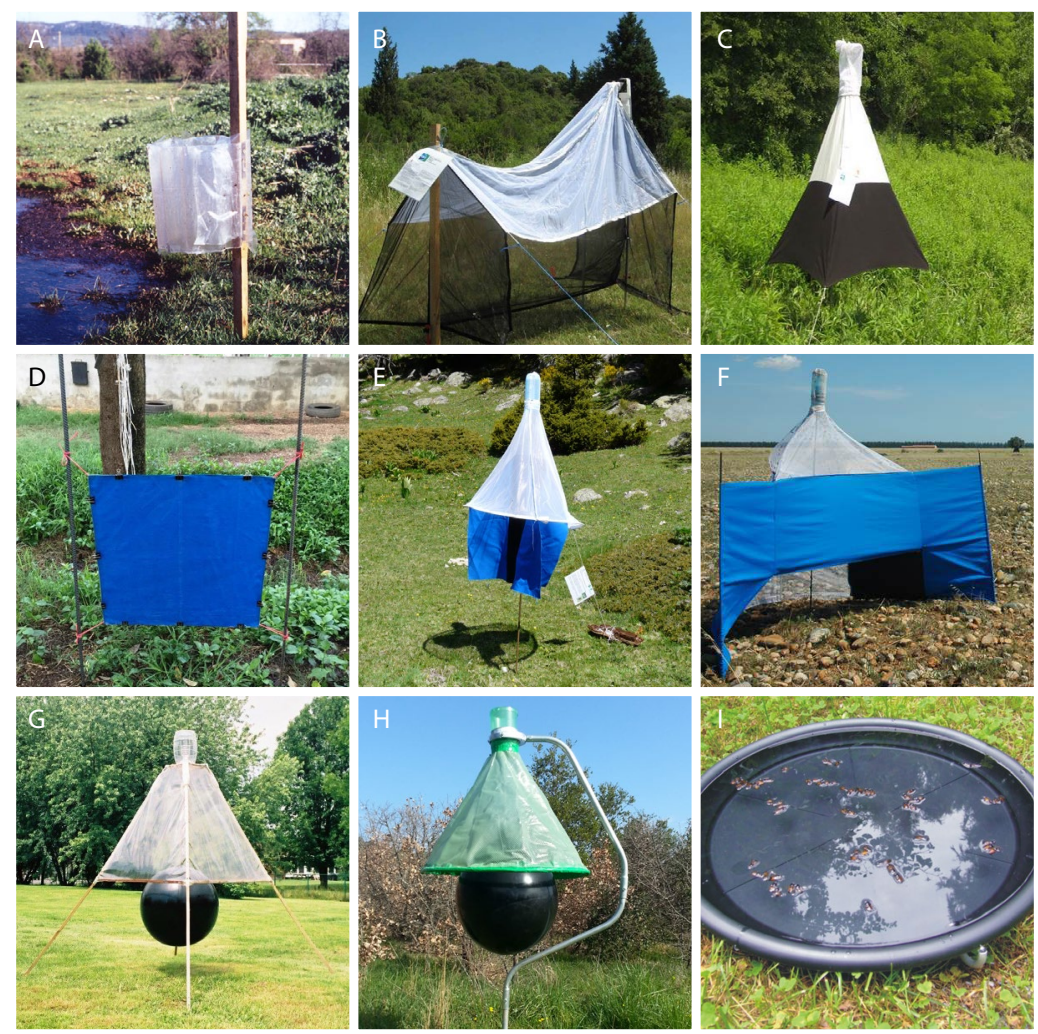

Figure 2. Examples of trapping devices for tabanids, stable flies and tsetse flies: (A) Broce trap (photo by G. Duvallet), (B) malaise trap (photo by S. Krčmar), (C) canopy trap (photo by S. Krčmar), (D) blue target (photo by M. Desquesnes), (E) Vavoua trap (photo by F. Baldacchino), (F) Nzi trap (photo by F. Baldacchino), (G) Manitoba trap (photo by S. Mihok), (H) H trap (photo by G. Duvallet) and (I) horizontally polarizing liquid trap (photo by G. Horváth). 


\title{
Biology and ecology of Brachycera flies affecting dairy cattle
}

\author{
Tabanidae and Athericidae (superfamily Tabanoidea), and Rhagionidae (superfamily \\ Rhagionoidea)
}

\section{Tabanidae}

Tabanid flies have stout bodies and large brilliant-coloured eyes. The family Tabanidae includes around 4,400 species in 144 genera. Tabanids are commonly referred to as horse flies (subfamily Tabaninae) and deer flies (subfamily Chrysopsinae) (Baldacchino et al. 2014a). Other names such as clegs (i.e. Haematopota genus) and March flies are also used. Tabanids attack mainly large mammals such as cattle, horses and deer, and preferentially feed on the belly and legs.

Females usually lay 100-800 eggs in a single mass and embryogenesis requires 2-21 days depending on species and climatic conditions. Tabanid larvae are found in mostly moist biotopes with diverse soil moisture and structure, plant composition, and other physical parameters (Andreeva et al. 2009). Most larvae are found in stream borders, littoral areas or forest litter, but they can also develop in other habitats due to their adaptability and hardiness. Tabanid larvae are predators of worms, larvae of other Diptera, and are occasionally cannibalistic. They can survive a long time without feeding and can hibernate over 2-3 winters in northern regions. Most temperate species are univoltine, whereas some tropical species can be bi- or tri-voltine. Pupation occurs in dry places and lasts 1-3 weeks. After emergence, adults of both sexes feed on nectar and other natural plant sugar deposits for body maintenance, flight and mating. After mating, haematophagous females seek a blood meal using their large mouthparts to cut the skin and create a micro-hematoma. Oviposition occurs 3-11 days after feeding.

In temperate climates, tabanids are highly active during the warmer months, i.e. summer, whereas in the tropics, tabanid activity usually peaks during the rainy season (Desquesnes 2004). The activity season lasts 2-3 months with a succession of species. Female tabanids are generally active during the day with a unimodal or a bimodal pattern. Daily activity patterns are related to meteorological variables such as temperature, relative humidity, wind speed or atmospheric pressure, and each species responds differently (Van Hennekeler et al. 2011). In temperate climates, tabanid activity peaks typically at noon or in the early afternoon during the hottest hours of the day (Baldacchino et al. 2014b). In tropical areas with high rainfall, most tabanid species are active early in the morning and late in the afternoon during the coolest hours of the day (Raymond 1989).

\section{Athericidae and Rhagionidae}

Athericid flies, also named water snipe or ibis flies, and rhagionid flies, also named snipe flies, are medium-sized and sombrely coloured flies with slender bodies, long legs and often spotted wings. They are mostly predators of other insects, except some genera that are blood feeders, Suragina (Athericidae) in the Nearctic region, Symphoromyia (Rhagionidae) in western North America and Spaniopsis (Rhagionidae) in Australia. These flies have only one generation per year. Larvae prey generally on other insect larvae. Suragina larvae inhabit flowing water, whereas Symphoromyia larvae are usually found in wet soil, leaf mould and rotting wood. They prey on other insect larvae, earthworms and other soil fauna (Hall and Gerhardt 2002; Lehane 2005). Symphoromyia bites are very painful, especially on the head, causing a high degree of public nuisance (Hall and Gerhardt 2002; Lehane 2005). When abundant, snipe flies may cause large animals to seek shelter or to move to higher elevation where fly activity is reduced (Burger 1995). However, they have not been 
implicated in the transmission of any pathogens. In California, snipe flies are active from April to mid-July; in Yellowstone, their biting activity peaks in July (Burger 1995).

\section{Muscidae (superfamily: Muscoidea)}

Muscid flies are brownish grey to black coloured, often with dark longitudinal stripes on the thorax and dark spots on the abdomen (Moon 2002). They possess wings that are longer than the abdomen. The family Muscidae includes approximately 9,000 species in 190 genera (Moon 2002). Muscid flies that exploit foods and habitats created by agriculture and human activities are often called synanthropic (e.g. Musca domestica L.), whereas muscid flies associated with cattle are called symbovine (e.g. Musca autumnalis De Geer and Haematobia irritans (L.)) (Thomas and Jespersen 1994). Muscid flies are almost all oviparous. Females lay eggs singly or in groups in various habitats. Filth flies oviposit on manure, soiled bedding and rotting feeds (e.g. hay and silage); dung flies oviposit on or into fresh cattle dung pats; sweat flies oviposit in plant litter or decomposing dung in grasslands and forests.

The larvae of muscid flies are known as maggots and have three instars in all species (Moon 2002). Most muscid larvae are saprophagous, filtering bacteria, yeasts and small organic particles in wet habitats. Third instars of the sweat flies are facultative predators and they can contribute to the natural control of other flies. Development time from egg to adult ranges from one to six weeks depending on the species, temperature (optimal: $27-32^{\circ} \mathrm{C}$ ) and food supply (Moon 2002). In pastures, horn flies and face flies both develop in mainly intermediate-sized dung pats, where they may co-exist (Fowler and Mullens 2016). Larval survival is affected by moisture, temperature and dung microbiota.

Muscid flies are usually active during the day for flying, host location, feeding, mating and oviposition. Most species are exophilic. Females feed only for a few minutes on their hosts to complete a meal and then rest in the nearby environment. An exception is the horn fly which remains on its host almost all the time, leaving the host just for laying eggs on fresh dung pats or after disturbances (Kuramochi 2000). Most muscid flies are multivoltine, having two or more generations per breeding season. In temperate areas, population growth outside buildings is restricted to spring, summer and autumn. In winter, house fly and stable fly adults can survive in protected and warmed places, whereas horn flies and sweat flies overwinter as pupae and larvae, respectively. In warm climates, adult flies are present throughout the year.

Non-biting muscid flies

\section{Musca domestica (house fly)}

The house fly occurs worldwide. It is often the most abundant insect in livestock buildings. Adults feed on available blood, sweat, tears, saliva and other body fluids from cattle as well as liquids in the environment (Graczyk et al. 2001). In dairy farms, major breeding sites are livestock manure and soiled bedding. Population growth is continuous in tropical and subtropical regions, but interrupted by winter in temperate regions as the house flies do not have a freeze-tolerant life stage (Moon 2002). However, house flies can overwinter within heated animal facilities in colder climates (Black and Krafsur 1986). Adults are diurnal with a bimodal activity rhythm peaking at the crepuscular hours of the day (Thomas and Jespersen 1994). Their flight dispersal range is about three km, but some individuals can fly up to $30 \mathrm{~km}$ (Graczyk et al. 2001; Nazni et al. 2005). 


\section{Musca autumnalis (face fly)}

The face fly occurs in the temperate region of the northern hemisphere (Krafsur and Moon 1997). It is endemic to Europe and western Asia and was introduced in North America (Vockeroth 1953). Face flies inhabit moist pastures with trees near ponds and streams (Depner 1969). They rest on vertical surfaces such as fence posts and trees, and visit cattle to feed on various facial secretions, wounds and blood. Females oviposit in fresh cattle dung (Hammer 1941). Face flies overwinter as diapausing unmated adults in buildings or hollow trees. This species has a high dispersal capacity of up to $12 \mathrm{~km}$ in five days (Thomas and Jespersen 1994).

\section{Musca vetustissima (bush fly)}

The bush fly occurs in the Australasian region and is found in open country avoiding heavily forested areas. It is a highly irritating fly which feeds on secretions and wounds and prevents skin sores from healing (Thomas and Jespersen 1994). Bush flies are active during the day, avoiding shade unless temperatures rise over $35^{\circ} \mathrm{C}$, and rest in vegetation during the night. Females lay their eggs on fresh dung since eggs are very susceptible to desiccation, and larvae prefer moist dung. There is no diapausing stage; hence populations die out in winter in the southern part of Australia. In the north, the population is highest in autumn, and flies move south using warm winds. The population of Musca vetustissima Walker is generally highest after the wet season, when cattle feed on rapidly-growing grass (Thomas and Jespersen 1994).

\section{Hydrotaea irritans (sheep head fly)}

The sheep head fly is confined to the Palearctic region and can be found in large numbers in northern European countries, especially Denmark and Great Britain (Ball 1984; Jensen et al. 1993). Sheep head flies feed on secretions around the face and udder, but also on wounds caused by biting flies (Thomas and Jespersen 1994). Hydrotaea irritans (Fallen) produces one generation per year and is active from June to September. In late summer, females lay eggs around the edges of grass-covered fields with moist soil. The second instar larvae are saprophagous whereas the third instar larvae are predators on other insect larvae as well as cannibalistic. Hy. irritans overwinters as larvae and pupation occurs usually in early May (Thomas and Jespersen 1994).

\section{Musca crassirostris (cattle fly)}

Musca crassirostris Stein is a true haematophagous fly whose mouthparts are adapted through the development of strong prestomal teeth. These are used to abrade or rasp away the skin of hosts to feed on blood. This species is present in the Palaearctic, Afrotropical and Oriental Regions (Walker 1994), hence found in parts of Europe, through Africa to the Middle East to South-East Asia. It is also called the 'Indian fly' or 'cattle fly' since it is abundant around cattle (Russel 2013). Due to the fact that it is not a true biting fly, $M$. crassirostris, as well as some other true haematophagous but non-biting flies such as Musca inferior and Musca planiceps are probably the most neglected haematophagous insects (Patton and Senior-White 1924).

\section{Other flies}

Hydrotaea spp., named sweat flies, and Morellia spp. are cattle-visiting flies in pastures and are considered less important in veterinary health (Jensen et al. 1993). Morellia spp. (e.g. Morellia hortorum (Fallen) and Morellia simplex (Loew)) and Hydrotaea spp. (e.g. Hydrotaea meteorica L. 
and Hydrotaea albipuncta (Zetterstedt)) feed mainly on lachrymal discharges (Madsen et al. 1991). Morellia flies and sweat flies develop in dung and overwinter as larvae (Ball 1984). In north-east England, M. hortorum was found active from May to September with two well-defined peaks in early June and July, whereas sweat flies occurred throughout the season with no obvious pattern (Ball 1984).

\section{Biting muscid flies}

Biting muscid flies of veterinary importance belong to the genera Stomoxys, Haematobia and Haematobosca (Zumpt 1973). Both sexes of these flies are haematophagous.

\section{Stomoxys spp.}

Stomoxys flies include 18 described species. Stomoxys calcitrans L. (stable fly) has a worldwide distribution; other species such as Stomoxys niger L., Stomoxys sitiens Rondani and Stomoxys indicus Picard are exclusively present in tropical areas of the African and/or Asian continents (Dsouli-Aymes et al. 2011; Zumpt 1973). Like M. domestica, S. calcitrans is primarily associated with livestock confinements, e.g. dairies and feedlots, but in the last few decades, it has also become a major pest of cattle on pastures (Broce et al. 2005). Stable flies usually feed on the legs, especially the front legs attracting $45 \%$ of the total number of stable flies on an animal (Lysyk 1995). After feeding, adult flies rest in protected areas on vertical surfaces (e.g. vegetation, fence, building sides).

Stable fly larvae develop in manure, litter, spilled feed (e.g. hay and green chop), silage and decaying vegetation (e.g. sugar cane) (Foil and Hogsette 1994). In pastures, winter feeding sites of hay are a main source of stable flies (Broce et al. 2005). No true diapause stage has been identified. They may overwinter as larvae in sheltered areas indoors (Beresford and Sutcliffe 2009) or in mounds of silage, manure or vegetation (Berkebile et al. 1994). Local populations can also persist in livestock facilities (Moon 2002). Stable flies may disperse $5 \mathrm{~km}$ or more for host location (Foil and Hogsette 1994).

\section{Haematobia irritans}

The two common Haematobia pest species of livestock are the horn fly Haematobia irritans irritans, and the buffalo fly Haematobia irritans exigua. The horn fly is found in Europe, North Africa, Asia Minor and the Americas, whereas the buffalo fly is found in China, India, South East Asia, down to Indonesia, Malaysia, Papua New Guinea and northern Australia (Foil and Hogsette 1994). Horn flies are pests of cattle on pastures. Adult flies live in intimate association with cattle hosts. They feed repeatedly on the back and sides of the cattle and leave only to oviposit or disperse among hosts (Kuramochi 2000). Gravid females move to the hind legs and fly to the dung pat immediately for egg deposition. Diapausing pupae overwinter beneath the pats (McLintock and Depner 1954). The flight dispersal range can exceed $5 \mathrm{~km}$.

\section{Haematobosca spp.}

Haematobosca species are pests of pastured cattle as well as wild ungulates. Haematobosca stimulans (Meigen) is commonly observed on cattle in north Europe (Ball 1984; Jensen et al. 1993), while Haematobosca squalida (Grunberg) and Haematobosca latifrons (Malloch) are African species found on bullocks and buffalos (Kangwagye 1973; Mihok et al. 1996). H. stimulans is found on 
the back or flanks of cattle (Hillerton et al. 1984). Females oviposit only in fresh dung pats like Ha. irritans, but unlike this species, Haematobosca adults do not remain on host animals and are found mostly on pasture vegetation (Burger and Anderson 1974). Haematobosca overwinters at the pupal stage in dung. In Europe, $\mathrm{H}$. stimulans is present throughout the grazing period, generally in lower number compared to other biting flies, with a peak in late summer (Ball 1984; Jensen et al. 1993).

\section{Glossinidae and Hippoboscidae (superfamily Hippoboscoidea)}

\section{Glossinidae}

The Glossinidae is represented by one genus Glossina that includes 31 species, namely tsetse flies, often simply referred to as tsetse. Eight to ten species are considered to be of health importance (Vreysen et al. 2013). Glossina spp. are tan or brown flies with the base of the proboscis typically swollen, whereas the biting muscid flies have a thinner base of the proboscis (Krinsky 2002). Tsetse flies occur throughout sub-Saharan Africa, with a few extending into the Arabian Peninsula (Glossina tachinoides Westwood, Glossina morsitans submorsitans Newstead and Glossina fuscipes fuscipes Newstead) (Vreysen et al. 2013). The genus Glossina is divided into three functional taxonomic groups (fusca, palpalis and morsitans) based on morphological characters, habitat requirements and preferred hosts (Lane and Crosskey 1993). The fusca-group is mainly found in lowland rain forests and isolated relic forests with the exception of Glossina longipennis Corti common in open savannah. The palpalis-group inhabits lowland rain forests and humid savannah along river systems; however some species can live in drier savannah areas and have also adapted to peridomestic habitats. The morsitans-group is common in savannah woodlands (Vreysen et al. 2013). Tsetse flies feed on a wide variety of hosts and host preference varies greatly among species (Krinsky 2002). A few examples of species commonly feeding on cattle in savannah woodlands are G. morsitans, Glossina fusca Walker, Glossina pallidipes Austen and Glossina longipalpis Wiedemann (Lehane 2005). Tsetse flies are more likely to feed on calm individuals instead of individuals irritated by other biting flies (Krinsky 2002). They usually feed on the belly and legs (Torr et al. 2007).

Tsetse flies reproduce by adenotrophic viviparity, i.e. the egg hatches in the female's reproductive tract and the three larval stages feed on 'milk' produced by the female's reproductive accessory glands until development is completed (Gooding and Krafsur 2005). After parturition, mature larvae burrow into the ground and quickly pupariate; adults emerge about 30 days later. Female tsetse flies most likely mate once, but polyandry has been detected in $G$. f. fuscipes wild populations (Bonomi et al. 2011). In nature, they probably have two reproducing offspring during their lifespan. Tsetse flies are fast flyers and have great dispersal capacity depending on species and landscape. Savannah species such as G. pallidipes may disperse up to $2 \mathrm{~km}$, whereas riverine species such as G. palpalis have greater dispersal capacity, up to $12 \mathrm{~km}$. (G. p. gambiensis - $25 \mathrm{~km}$ ). In addition, the dispersal capacity of riverine species such as G. p. gambiensis and G. tachinoides is higher in fragmented habitats (Vreysen et al. 2013).

\section{Hippoboscidae}

Hippoboscid flies are tough, leathery and dorso-ventrally flattened flies with a prognathous head broadly confluent with the thorax. The Hippoboscidae contains approximately 200 species subdivided into three subfamilies, Lipopteninae, Ornithomyiinae and Hippoboscinae, parasitizing birds and/or mammals (Hutson 1984). The species occurring on mammals are often called keds (Paakkonen et al. 2010). Two Hippoboscinae species are parasites of cattle, Hippobosca equina $\mathrm{L}$. 
and Hippobosca variegata Megerle. H. equina is a common parasite of Equidae but also a facultative cattle pest. It occurs in Europe, northern Africa and western Asia (Lloyd 2002). H. variegata is also a parasite of horses and cattle, present in Africa, Asia and southern Europe.

Both sexes are haematophagous, and Hippobosca spp. commonly feed several times a day on a host (Lehane 2005). Like tabanids, keds are pool feeders cutting the skin of their hosts and consuming the blood that accumulates in the wound (Paakkonen et al. 2010). The Hippoboscidae are larviparous, and the larvae remain in the uterus where they are nourished by a pair of accessory glands or milk glands (Lloyd 2002). H. equina females deposit their larvae in humus under bracken (Lehane 2005). Shortly after larviposition, the larval integument hardens to form a puparium. In temperate areas, hippoboscid flies overwinter in the pupal stage (Lehane 2005). Pupae produced in summer take about one month to develop, and peak numbers of flies occur during the warmest months.

\section{Direct and indirect effects, i.e. transport, mechanical and biological transmission of pathogens}

\section{Direct effects}

Non-biting flies such as face flies cause discomfort in cattle especially by feeding on facial secretions (Moon 2002). However, these direct effects on cattle growth and dairy cow lactation seem negligible. For example, 2 to 18 face flies per face did not appear to affect cattle production (Krafsur and Moon 1997). Biting flies cause more annoyance, skin injuries and blood loss. The annoyance level is related to fly abundance and to the pain of the bites. For instance, tabanid and hippoboscid bites are particularly painful as they are using their mouthparts to cut the skin in a scissor-like fashion creating a micro-hematoma (Lehane 2005); this mode of biting is referred to as 'telmophagia'. Fly bites may also cause local reactions (e.g. dermal nodules) due to the physical action of the mouthparts and the chemical action of the saliva (Baldacchino et al. 2013). In addition, fly bites result in blood loss due to two actions, feeding and oozing. The blood loss is related to the blood meal size of the biting flies. The blood meal size can range from 10-15 $\mu$ for stable flies to $600 \mu \mathrm{l}$ for the largest tabanid species (Baldacchino et al. 2013, 2014a). Natural oozing from feeding punctures may also attract non-biting flies for feeding or myiasis-causing flies for oviposition. Non-biting flies can extend the duration of the bleeding. Finally, feeding punctures may facilitate secondary infections (Baldacchino et al. 2013).

Flies induce host-defensive reactions in cattle such as eye blinks, head throws, ear flaps, leg stamps, tail flicks and skin twitches (Baldacchino et al. 2014c; Moon 2002; Mullens et al. 2006). In pastures, cattle may also use escaping or hiding behaviours, e.g. going into a forest or a water body, or defensive herding. These host reactions related to the fly annoyance induce stress, energy loss, reduced feeding time, and a decreased food intake. Vitela-Mendoza et al. (2016) demonstrated that plasma cortisol concentration as stress indicator in dairy cows was linearly related to the number of stable flies on a cow and the frequency of fly-dislodging behaviours. Thus, biting flies can affect animal growth and milk production with a significant economic impact, whereas little or no loss of productivity is typically observed in the presence of non-biting flies (Moon 2002). Horse flies, stable flies, horn flies and buffalo flies are probably the most annoying flies because of their painful biting behaviour and their abundance. For instance, economic thresholds were estimated at about 25-50 flies per animal per day for S. calcitrans and 200 flies per animal per day for $\mathrm{Ha}$. irritans irritans. Studies on the economic impact of biting flies showed contrasting results depending on fly abundance, climate, landscape, management techniques, breed type 
and calving period (Foil and Hogsette 1994). For example, an average of 90 horse flies on heifers resulted in a weight loss of $100 \mathrm{~g}$ per animal per day, and an average of 90 horn flies on noncastrated Nelore calves resulted in weight loss of $130 \mathrm{~g}$ per animal per day (Foil and Hogsette 1994; Maciel et al. 2015). By contrast, Hogsette et al. (1991) observed that cattle could tolerate an average of 200 horn flies for 70 days without any adverse impacts. Biting flies have been also shown to reduce milk production of dairy cows. An average milk production loss was estimated at $0.7 \%$ per stable fly per cow (Bruce and Decker 1958). By modelling the effects of buffalo fly on milk production, Jonsson and Mayer (1999) predicted a no adverse effect of below 30 flies per cow, but a loss in milk production of $2.6 \mathrm{ml}$ per fly per day at an infestation level of 200 flies per cow. Biting flies may also affect milk quality, i.e. the percent milk fat, solids-no-fat, urea nitrogen and/or protein (Mays et al. 2014).

\section{Indirect effects}

\section{Mechanical transport}

\section{Non-biting flies as mechanical carriers of pathogens}

Non-biting flies can carry a wide range of pathogens on the mouthparts, body, and leg hairs (i.e. setae) or foot pads (i.e. pulvilli) (Graczyk et al. 2005). In particular, pathogens can attach on the numerous setae present on the tarsomeres, and the sticky substance secreted through the tip of these setae enhances the adhesion of pathogens (Sukontason et al. 2006). Furthermore, fly tarsi efficiently trap the pathogens present on cattle faeces because of the faecal viscosity. Pathogens can also adhere to the fly body because the exoskeleton has certain electrostatic charges (Graczyk et al. 2005). Finally, protozoan parasites ingested by the fly can either be regurgitated, or deposited in faecal spots after transit through the gastrointestinal tract (Graczyk et al. 2005).

\section{Main pathogens mechanically transported by non-biting flies}

\section{- Bacteria}

Numerous bacteria have been associated with non-biting flies, especially the house fly. $M$. domestica is a mechanical carrier of Escherichia coli, Staphylococcus aureus, Campylobacter spp., Yersinia enterocolitica, Pseudomonas spp., Chlamydia spp., Klebsiella spp., Enterobacter spp., Enterococcus spp., Proteus spp., Acinetobacter spp., Viridans streptococci, Shigella spp., Vibrio spp., Morganella morganii, Providencia stuartii, Aeromonas spp., etc. (Graczyk et al. 2001). In Australia, E. coli, Enterococcus faecalis, Salmonella spp., and Shigella spp. have also been isolated from the bush fly $M$. vetustissima (Vriesekoop and Shaw 2010). Animal manure is a recognized source of zoonotic enteropathogens. Thus, bacteria are more frequently isolated from flies collected in animal facilities with the highest bacterial loading. Vriesekoop and Shaw (2010) found that bacterial populations per bush fly were higher in a farm environment $\left(9.1 \times 10^{4} \mathrm{cfu}\right.$ per fly) compared to an urban environment; $E$. coli was the most common pathogen isolated.

E. coli is the most important bacterial cause of diarrhoea in calves during the first week of life, but other bacteria are also implicated in diarrhoea in neonatal calves: Salmonella typhimurium, Salmonella dublin, Campylobacter jejuni, Y. enterocolitica (Aiello 2012). Enteropathogens are generally excreted by healthy adult cattle and transmitted to calves by faecal-oral contact. However, flies may act as an epidemiological link between animals and humans, especially in the vicinity of animal facilities. In Thailand, the increase of house fly populations in animal pens was 
associated with an increase of diarrhoea incidence in humans, and enterotoxigenic $E$. coli, Shigella spp. and Vibrio spp. were isolated from fly pools (Echeverria et al. 1983).

M. autumnalis can transmit the bacterium Moraxella bovis, the agent of infectious bovine keratoconjunctivitis or pinkeye (Krafsur and Moon 1997). Pinkeye occurs most frequently among calves and yearling cattle, and it can progress to blindness. Under laboratory conditions, contaminated face flies have been demonstrated to transport on the tarsi and mouthparts, and/ or regurgitate viable bacteria even several hours after exposure (Gerhardt et al. 1982). In the field, face fly abundance has been positively correlated with pinkeye incidence, and $M$. bovis has been isolated in natural fly populations (Hall 1984; Krafsur and Moon 1997). Face flies may also facilitate the infection by scarifying the host conjunctiva during feeding (Moon 2002). Although pinkeye may occur without the presence of face flies (it was enzootic in the United-States before face fly introduction), they are important in the spread of M. bovis.

Hy. irritans is a mechanical carrier of summer mastitis pathogens, and lesions on the teat orifice appear to be a predisposing factor in the development of the disease (Chirico et al. 1997). Summer mastitis is an aetiologically complex disease, and mixed cultures of various bacteria are commonly isolated from udder secretions of affected cows: S. aureus, Streptococcus dysgalactiae, Trueperella pyogenes (formerly named Actinomyces pyogenes), Stuart-Schwan cocci, Peptoniphilus indolicus, Fusobacterium necrophorum and Bacteroides spp. (Chirico et al. 1997). Summer mastitis is also called heifer mastitis as it mainly affects pastured heifers, but dry cows can also be affected. In heifers, it may be defined as a subclinical mastitis that threatens production and udder health in the first lactation (De Vliegher et al. 2012). This disease may be costly because of milk production losses, premature culling, additional labour, and veterinary treatments.

Biting flies may also act as mechanical carriers of pathogens. Castro et al. (2010) isolated 20 microbial agents on the external surface of stable flies collected from dairy farms in Brazil. E. coli, Enterobacter spp. and Salmonella spp. were the most frequently identified. Moreover, stable flies are presumed to transmit Dermatophilus congolensis, a Gram-positive coccobacillary actinomycete causing a dermatitis disease in ruminants, named dermatophilosis or cutaneous streptothricosis. Under laboratory conditions, D. congolensis was observed on S. calcitrans legs, and rabbits have been successfully infected through contaminated stables flies as well as house flies (Richard and Pier 1966). Thus, the disruption of the skin was not necessary for the transmission of $D$. congolensis.

\section{- Protozoa}

M. domestica is a mechanical carrier of various protozoan parasites: Sarcocystis spp., Giardia spp., Toxoplasma gondii, Cryptosporidium parvum, Isospora spp., Entamoeba spp., Endolimax nana, Trichomonas spp., Hammondia spp. (Graczyk et al. 1999, 2001). C. parvum is the causative agent of cryptosporidiosis, a zoonotic diarrheal disease affecting neonatal calves. Under laboratory conditions, C. parvum oocysts were detected by immunofluorescent antibodies on the exoskeleton of adult house flies and in their digestive tracts after exposure of flies to bovine diarrheal faeces containing oocysts (Graczyk et al. 1999). In bioassays, C. parvum oocysts transported on the exoskeletons of house flies and eluted from their faecal and vomit droplets were infectious to neonatal mice. The epidemiologic involvement of house flies in the transmission of C. parvum is difficult to assess as transmission may occur directly from calf to calf or indirectly by faecal contamination of food or water (Graczyk et al. 2005). This same aspect (difficulty in establishing what proportion of infection is due to insect contamination) holds for many pathogens that are transmitted mechanically by insects. 
Viruses transmitted by M. domestica include poliovirus, coxsackievirus, and enteroviruses such as rotavirus (Graczyk et al. 2001; Tan et al. 1997). The walking behaviour of the house fly has been shown to be an important mode of fly contamination by rotavirus particles. An increase in the viscosity of the contact surface enhanced the proportion of flies contaminated; the majority of the particles were deposited on the first contact surface (Tan et al. 1997). In contrast, M. autumnalis was found to retain the bovine herpes virus-1 (BHV1) for only a short period of time, and experimental transmission to Hereford calves was unsuccessful (Johnson et al. 1991).

\section{Mechanical transmission}

\section{Biting flies as mechanical vectors of pathogens}

Mechanical transmission appears to occur through either contamination of mouthparts or regurgitation of digestive tract contents (Baldacchino et al. 2013). Tabanids and biting muscid flies (Stomoxys, Haematobia and Haematobosca) are the most important mechanical vectors since they transmit a wide range of pathogens. Several factors can affect the probability of mechanical transmission such as the biting behaviour of flies, interruption of blood-feeding, quantity of blood on mouthparts, sensitivity to host defensive reactions, tendency to switch individuals, biting intensity and fly abundance (Baldacchino et al. 2014a). Interrupted feeding is essential in mechanical transmission. After interruption, the fly may pursue a single host to complete its blood meal. This behaviour is called feeding persistence and depends on the species. In Australia, Tabanus pallipennis Macquart was observed to be very sensitive to host defensive reactions; it required as many as nine partial meals before reaching engorgement (Muzari et al. 2010). Blood meal residues on mouthparts influence the amount of infectious material transmitted between hosts. The amount of blood remaining on mouthparts after feeding varies among biting flies; it was estimated at $0.4 \mu \mathrm{l}$ for S. calcitrans and $25.6 \mu \mathrm{l}$ for Tabanus fuscicostatus Hine (Scoles et al. 2005, 2008). Because of the short survival times of pathogens on mouthparts, mechanical transmission is most effective immediately or few minutes after feeding on an infected host. However, delayed transmission up to 72 hours has been observed experimentally with Stomoxys flies, which is thought to be related to a regurgitation of parasites from the crop of the flies (Bouet and Roubaud 1912). Finally, the success of mechanical transmission is related to the density of biting flies, and the peak of infection by a mechanically-transmitted pathogen is generally synchronized with the peak of fly populations (Baldacchino et al. 2014a).

\section{Main pathogens mechanically transmitted by biting flies}

\section{- Bacteria}

Tabanids and biting muscid flies have been associated with several bacteria including Bacillus anthracis, Anaplasma marginale, Brucella spp., Pasteurella multocida, Erysipelothrix rhusiopathiae, Coxiella burnetii through isolation from wild specimens, experimental and/or natural transmission (Baldacchino et al. 2013, 2014a; Krinsky 1976); horn flies have been associated only with S. aureus causing mastitis in dairy heifers during a field control trial (Ryman et al. 2013).

B. anthracis is responsible for anthrax, a non-contagious zoonotic disease inducing pulmonary, gastro-intestinal or cutaneous symptoms (e.g. a boil-like skin lesion). Herbivores usually become infected by ingesting spores dispersed in pastures. However, the bacterium B. anthracis can be 
mechanically transmitted by tabanids and biting muscid flies, potentially spreading the disease. Turell and Knudson (1987) were able to transmit B. anthracis to a susceptible guinea pig with $S$. calcitrans immediately or four hours after exposure to a bacteremic guinea pig. Kraneveld and Djaenoedin (1940) infected horses and water buffaloes by immediate transfer feeding or 48-hours delayed feeding with Tabanus rubidus Wiedemann. Interestingly, non-biting flies such as house flies can act as carriers of $B$. anthracis spores (Fasanella et al. 2010). Indeed, Fasanella et al. (2010) detected anthrax spores in faeces and vomitus of $M$. domestica previously infected by feeding on anthrax-infected rabbit carcass or anthrax contaminated blood.

A. marginale is the etiological agent of bovine anaplasmosis. The rickettsiae $A$. marginale parasitize erythrocytes resulting in development of mild to severe anaemia and icterus with general clinical signs such as fever, weight loss, abortion and lethargy (Kocan et al. 2010). A. marginale can be transmitted mechanically by biting flies and biologically by ticks. Natural experiments have shown the transmission of $A$. marginale between splenectomised cattle by tabanids and stable flies (Hawkins et al. 1982; Potgieter et al. 1981). Scoles et al. (2005) demonstrated that mechanical transmission by S. calcitrans was at least two orders of magnitude less efficient than biological transmission by the tick Dermacentor andersoni Stiles; however, one-host ticks such as Rhipicephalus microplus (Canestrini) would act as amplificator only, rather than vectors, since they very rarely move from a host to another (Mason and Norval 1981). On the other hand, attempts to transmit $A$. marginale by buffalo flies were unsuccessful (Allingham et al. 1994). In nature, ticks likely contribute to the amplification of the parasite, whereas biting flies may be responsible for transmission and spreading, as suggested by field surveys. In Costa Rica, Oliveira et al. (2011) found that the seroprevalence of $A$. marginale in dairy herds was related to the presence of tabanids and stable flies. In Hungary, Hornok et al. (2008) detected four specimens of $T$. bovinus positive for $A$. marginale by PCR providing evidence for the role of tabanids as mechanical vector of $A$. marginale.

\section{- Protozoa}

Tabanids and biting muscid flies can act as mechanical vectors of Besnoitia besnoiti, Trypanosoma brucei, Trypanosoma congolense, Trypanosoma vivax and Trypanosoma evansi. B. besnoiti is responsible for bovine besnoitiosis, a widely distributed disease currently re-emerging in Europe (Alvarez-Garcia et al. 2013). T. congolense, T. vivax and to a lesser extent T. brucei, are the main causative agents of African animal trypanosomosis (AAT) or nagana in cattle, considered the greatest health constraint for livestock production in sub-Saharan Africa (Auty et al. 2015; Vreysen et al. 2013). T. evansi is the causative agent of surra, a disease affecting mainly camels, equines and dogs; T. evansi is considered as a mild pathogen in cattle in Africa and the Americas, however its clinical and economic impacts are strong in cattle and buffaloes in Asia where bovines may serve as reservoirs of infection (Desquesnes et al. 2013). Recently two outbreaks of surra were observed in Spain and the French mainland; this stresses the risk of spreading exotic pathogens through the non-specific mechanical transmission by cosmopolitan Brachycera flies in Europe (Gutierrez et al. 2010).

Bovine besnoitiosis is clinically characterized by three successive stages: an acute stage with pyrexia and anorexia, subacute and chronic stages with generalized oedema, skin lesions and weight loss (Gollnick et al. 2015). Death can occur in about $10 \%$ of the cases (Jacquiet et al. 2010). The causative agent is a cyst-forming apicomplexan parasite, $B$. besnoiti. Mechanical transmission of $B$. besnoiti has been demonstrated between cattle with $S$. calcitrans and African tabanid species (e.g. Atylotus nigromaculatus Ricardo and Tabanocella denticornis Wiedemann) (Bigalke 1968). Bigalke (1968) estimated that numerous stable fly bites were required for transmission $(>50.000)$, 
whereas only three horse fly bites were sufficient. This difference might be explained by the low volume of blood residues, and consequently the low parasite burden, in the mouthparts of S. calcitrans (Lienard et al. 2013). However, there are several field observations implicating S. calcitrans as a vector of $B$. besnoiti. In a dairy farm in southwestern France, Lienard et al. (2011) found that the seroprevalence of $B$. besnoiti increased in the two-months before turn-out in association with high stable fly activity inside the facilities.

AAT clinical signs are primarily intermittent fever, anaemia and weight loss; then cattle usually have a chronic course of the disease with loss of milk and meat production, abortion and death. Trypanosoma spp., responsible for nagana, are predominantly transmitted by tsetse flies, their biological vectors (Holmes 2013) (see below). Mechanical transmission of Trypanosoma spp. by tabanids and biting muscid flies may augment natural transmission, as demonstrated in semifield conditions, notably for T. vivax (Desquesnes and Dia 2003a, 2004), and also possibly for $T$. congolense (Desquesnes and Dia 2003b). In South America where tsetse flies do not occur, $T$. vivax is only transmitted mechanically by biting flies. Under experimental conditions, $T$. vivax has been mechanically transmitted with neotropical tabanid species (e.g. Tabanus nebulosus De Geer, Tabanus importunus Wiedemann and Cryptotylus unicolor (Wiedemann), Afrotropical tabanid species (e.g. Atylotus agrestis (Wiedemann) and Atylotus fuscipes Ricardo), African biting muscid fly species (e.g. S. niger and H. squalida) (Mihok et al. 1995). The importance of mechanical transmission of $T$. vivax is also supported by several epidemiological studies with tabanids or biting muscid flies as vectors. The prevalence of $T$. vivax infections appears to be correlated with the density of biting flies in several tsetse free areas of Africa and in South America (Birhanu et al. 2015; Cherenet et al. 2004; Cuglovici et al. 2010; Rahman 2005). A mathematical model of mechanical transmission by tabanids has been developed to predict the incidence of the infection based on the initial prevalence, the parasitaemia of infected animals and the density of biting insects (Desquesnes et al. 2009).

\section{- Viruses}

Several viruses can be transmitted mechanically by tabanids and/or biting muscid flies including the causative agents of bovine leucosis, bovine viral diarrhoea, vesicular stomatitis, Rift Valley fever, lumpy skin disease and bovine ulcerative mammillitis, also named pseudo lumpy skin disease. However, the mechanical transmission of these pathogens is not the major route of transmission. In most cases, viruses have been isolated in biting fly specimens and/or mechanically transmitted under experimental conditions. Natural transmission was conducted successfully between cattle for the viruses of bovine leukosis and bovine viral diarrhoea by the tabanid species, T. fuscicostatus and Haematopota pluvialis (L.), respectively (Baldacchino et al. 2014a). To date, few epidemiological observations have supported the role of biting flies in the transmission of viruses in cattle. In Israeli dairy farms, lumpy skin disease outbreaks were associated with a high relative abundance of stable flies, and stable flies carried by wind were suspected to spread the virus (Kahana-Sutin et al. 2016; Yeruham et al. 1995). For bovine ulcerative mammillitis, Gibbs et al. (1972) also observed a seasonal prevalence which could be related to stable fly activity.

\section{Biological (cyclical) transmission}

\section{Tsetse flies as biological vectors of trypanosomes}

Tsetse flies are responsible for the cyclical transmission of trypanosomes causing AAT, i.e. T. congolense, T. vivax and T. brucei (Auty et al. 2015). After ingestion by the tsetse fly from a 
trypanosome-infected animal, trypanosomes multiply exclusively in the proboscis (T. vivax), or in the midgut and then migrate to mature in the salivary glands ( $T$. brucei) or the proboscis ( $T$. congolense) (Holmes 2013). There, they are transformed into epimastigotes (uninfective stage) and after further multiplication they are transformed into trypomastigotes (infective stage). The life cycle lasts from one week for T. vivax to a few weeks for T. brucei. Trypanosome infection levels in tsetse flies are generally low due to endogenous factors (tsetse species, sex, age, host preference and concurrent infections such as bacterial flora), ecological factors (climatic conditions and host availability) and parasite/host factors (number of trypanosomes, Trypanosoma spp., host breed, host behaviour and attractiveness) (Holmes 2013; Soumana et al. 2013). Wildlife maintain a sylvatic cycle of the disease, whereas the peridomestic cycle involves mainly livestock as hosts for tsetse flies and as a reservoir of trypanosomes (Van den Bossche et al. 2010). The epidemiology, dynamics and impact of AAT are affected by anthropogenic environmental changes, including the encroachment of livestock into protected areas with many tsetse flies and wildlife (Ogutu et al. 2011). Despite the recent decrease in tsetse fly habitats due to population growth, some species have adapted and persist in microclimatic niches. In West Africa, riverine tsetse fly species in particular are more adaptable than savannah species and are responsible for much trypanosome transmission (Van den Bossche et al. 2010).

\section{Tabanids as biological vectors of Trypanosoma theileri}

Trypanosoma theileri is a stercorarian parasite multiplying in the midgut of numerous tabanids and found as infective metacyclic stages in the terminal part of the insect gut (Hoare 1972); it is widely distributed in cattle, but few clinical cases have been reported (Villa et al. 2008). T. theileri is considered a non-pathogenic and cosmopolitan trypanosome in bovines.

\section{Flies as developmental vectors of nematodes}

Flies can be developmental vectors or intermediate hosts of parasites, i.e. the parasite undergoes a developmental transformation in the vector without any multiplication (Lehane 2005). Face flies are exclusive developmental vectors of Thelazia gulosa, Thelazia skrjabini and Thelazia rhodesii (Nematoda: Thelaziidae) responsible for bovine thelaziosis (Krafsur and Moon 1997). Females of Thelazia spp. reside in the lachrymal ducts of cattle. The microfilariae infect eye-feeding flies, and larvae develop in the fly's haemocoel. Extrinsic development by Thelazia spp. required a minimum of nine days in experimentally infected flies at $27^{\circ} \mathrm{C}$. Thelazia spp. are widespread and abundant, but cause asymptomatic infection in cattle. In addition, face flies can transmit Parafilaria bovicola (Nematoda: Filarioidea) causing haemorrhagic bovine filariasis, also known as 'green muscle disease' which may cause notable carcass losses (Krafsur and Moon 1997). Female P. bovicola pierce their host's skin and lay eggs in the exudate that oozes from external 'bleeding points'. Face flies acquire microfilariae when feeding on the exudate, and $P$. bovicola larvae subsequently develop in the fly's haemocoel. In South Africa, P. bovicola is transmitted by other Musca spp. (Nevill 1985). Finally, horn flies are developmental vectors of Stephanofilaria stilesi (Nematoda: Filarioidea), causing stephanofilariosis, a circumscribed dermatitis along the ventral midline of cattle (Moon 2002). In Indonesia and Australia the most common lesion in cattle is found at the medial canthus of the eye, followed by the neck and the sternum, with rare lesions on the head (Johnson 1989). Female horn flies ingest microfilaria by feeding on the lesion. The development of microfilariae to third-stage infective larvae in the fly's haemocoel lasts 2-3 weeks. Active lesions are covered with blood or serous exudate. Other Stephanofilaria spp. parasitize livestock and wildlife in various parts of the world and are transmitted by a variety of muscid flies, including 
Stomoxys spp. (Johnson 1987). The vectors of several other nematodes transmitted by Musca spp. have not been extensively studied in modern times (Stoffolano 1970).

\section{Control methods available for an integrated management of flies}

Given the potentially large economic impact of flies on livestock production, livestock holders will aim to keep this impact as small as possible, by using preventive as well as curative fly control methods. Ideally, these should be based on the principle of integrated pest management, which is defined as an ecosystem approach to crop and livestock production and protection that combines different management strategies and practices to allow production of healthy animals and minimize the use of pesticides (FAO, Rome).

\section{Environmental control}

\section{Sanitation}

Sanitation is the gold standard control method for flies such as house flies, stable flies or horn flies when they develop on fresh dung or in manure near livestock confinements. However, this sanitation is impractical for horn flies or horse flies breeding in fresh dung pats or natural habitats, respectively, scattered on pastures and other areas (e.g. forests, inland waters) (Baldacchino et al. 2014a; Floate et al. 2013). The elimination of manure, litter and spilled feed should be the highest priority to control stable fly populations in confined animal facilities (Baldacchino et al. 2013).

\section{Dung degradation by beetles}

Dung beetles (Coleoptera: Scarabaeidae) can help in controlling flies breeding in dung pats such as horn flies and face flies by accelerating the degradation of cattle dung on pastures. Accelerated degradation by dung beetles provides an ecosystem service as it prevents the development of flies (e.g. horn flies), it increases the surface of pastures for grazing and it enriches the soil with nitrogen and minerals (Floate et al. 2013). In Australia, many dung beetle species such as Onthophagus spp. have been introduced and have demonstrated to compete successfully with bush flies (Thomas and Jespersen 1994). Dung beetle development should then always be preserved.

\section{Patch-burn grazing}

Patch-burn grazing (PBG) is applied by burning spatially discrete patches of a pasture and allowing cattle to graze where they want to (Scasta et al. 2015). It results in a mosaic of patches with a structural heterogeneity of the vegetation due to the interaction of fire and grazing. PBG benefits biodiversity, but it can also limit fly populations. In lowa and Oklahoma (USA), horn flies were reduced by $41 \%$ with PBG management compared to traditional management without fire (Scasta et al. 2012). PBG may also affect other flies such as stable flies and face flies. The efficacy of PBG in controlling flies might be explained by the combustion of dung containing horn fly pupae or the reduction of shrubs and trees used as resting sites for the face fly (Scasta et al. 2015).

\section{Animal grazing management}

Biting flies can be highly abundant in areas offering favourable ecological conditions for larval development and adult activity (host seeking, resting and oviposition). Thus, landscape and altitude can be determining factors for their distribution. For instance, many tabanid species are 
active at the pasture-forest ecotone; some snipe fly species are more active in open areas, whereas others are more active in forested areas (Baldacchino et al. 2014b; Burger 1995). Moreover, biting fly populations generally decrease at high elevations in a mountainous environment. When animals are free-roaming, they naturally select areas with low biting fly activity such as dense thickets, windy hilltops, deep forests or water ponds (Baldacchino et al. 2014a). Grazing management might be used to avoid pastures with high fly populations and spatial analysis can be helpful for identifying and delimiting these hot spots (Sciarretta et al. 2010). However, practically, it is rare that zootechnical optimization of the pasture and fly control are in agreement.

\section{Biological control}

\section{Parasitoids}

Pupal parasitoids (Hymenoptera: Pteromalidae) such as Spalangia spp. (e.g. Spalangia cameroni Perkins) and Muscidifurax spp. (e.g. Muscidifurax raptor Girault \& Sanders) have a high potential for the biological control of flies, especially M. domestica and S. calcitrans (Machtinger and Geden 2018; Nachman and Skovgård 2018; Petersen et al. 1995, Skovgård and Steenberg 2002). The parasitoid wasps lay their eggs in immature flies and, the resulting wasp offspring feed on the fly pupa and eventually kill it. The immature parasitoid wasp will then develop into an adult, emerge from the fly pupa, and repeat the life cycle (Baldacchino et al. 2013). Pupal parasitoids have been successfully used in dairy farms (Geden et al. 1992; Skovgård and Nachman 2004). However, this approach may not have a significant impact due to the number and/or the distance between the release stations of parasitoids (Machtinger et al. 2015; McKay and Galloway 1999). The highest rates of total parasitism of $M$. domestica by S. cameroni were recorded at less than $5 \mathrm{~m}$ from the release station suggesting that pupal parasitoids should be released in close proximity to host development areas (Machtinger et al. 2015).

Although pupal parasitoids offer some measures of control they do not produce immediate results. Therefore, biological control should not be used alone, but in concert with other methods, such as sanitation (Baldacchino et al. 2013). Some studies suggested that insecticide use can sometimes be compatible with parasitoid populations. Compatibility between a parasitoid wasp Spalangia endius Walker and insecticides (see below) against $M$. domestica has been evaluated by Burgess and King (2015). Spinosad appeared to be the most suitable compound both for parasitoid survival and fly mortality. However, the authors recommend minimizing the overlap between mass release of parasitoids and insecticide applications and keeping insecticides away from areas with parasitoids.

\section{Entomopathogenic fungi}

Entomopathogenic fungi naturally present in farms can be used for controlling flies (Weeks et al. 2018). The pathogenicity of different isolates of entomopathogenic fungi has been determined under laboratory conditions against $\mathrm{M}$. domestica, Ha. irritans, S. calcitrans and Glossina spp. (Lohmeyer and Miller 2006; Lopez-Sanchez et al. 2012; Maniania and Ekesi 2013; Mishra et al. 2011; Mochi et al. 2010; Watson et al. 1995); and several commercial formulations are already available (Weeks et al. 2016). The pathogenic effect of fungi on flies may vary widely depending on the fly stage, the fungal species and isolates, the conidia concentration, and the fungal formulation (e.g. powder or liquid) (Mochi et al. 2010; Watson et al. 1995). Entomopathogenic fungi are most effective against adult flies, but they can also affect eggs, larvae and/or pupae. They also possess an oviposition deterrent effect against house flies and stable flies (Machtinger et al. 
2016). Among the different species tested, Metarhizium anisopliae and Beauveria bassiana appear to be the most pathogenic. Several field studies have shown the potential of entomopathogenic fungi as biocontrol agents for adult flies. Galindo-Velasco et al. (2015) observed that $M$. anisopliae caused a $94-100 \%$ reduction in horn fly infestation after spraying stabled cattle with an aqueous conidial suspension $\left(1 \times 10^{8}\right.$ conidia/ml). Mochi et al. (2009) observed a decrease by $50 \%$ in the horn fly number on heifers sprayed by a $M$. anisopliae suspension $\left(3 \times 10^{10}\right.$ conidia/l) compared to untreated heifers. Cruz-Vazquez et al. (2015) demonstrated the effectiveness of a suspension of $M$. anisopliae $\left(1 \times 10^{8}\right.$ conidia/ml) applied to Holstein cows using a backpack sprayer to control stable fly infestations and reduce cow defensive behaviours. Finally, Maniania and Ekesi (2013) achieved a reduction in tsetse fly populations by the large deployment of pyramidal traps mounted with contamination devices charged with M. anisopliae conidia.

\section{Plant essential oils}

Several plant essential oils repel various fly species; their effectiveness depends on their volatile bioactive constituents, the formulation and the application method (Lachance and Grange 2014). Most studies have been conducted under laboratory conditions, but some successful experiments have also been achieved in the field. In India, a 3.5\% emulsifiable formulation of peppermint oil (Mentha piperita) showed reductions in house fly density of $96 \%$ on treated cattle over four hours (Kumar et al. 2011). In the United-States, a 15\% oil-based formulation of catnip oil (Nepeta cataria) provided more than $95 \%$ protection for up to six hours on cattle against stable flies (Zhu et al. 2012). In Canada, Lachance et al. (2014) tested six essential oils diluted in a 5\% sunflower oil-based formulation against horn flies and house flies on pastured cows and indoor heifers: basil (Ocimum basilicum), geranium (Pelargonium graveolens), lavender (Lavandula angustifolia), lemongrass (Cymbopogon citratus), peppermint and pine (Pinus sylvestris). All essential oils repelled more than $75 \%$ of the flies on the treated body parts for up to 6-8 hours. The carrier oil alone also appeared to repel flies acting probably as a mechanical barrier to biting. In Brazil, a $5 \%$ formulation of essential oils of tea tree (Melaleuca alternifolia) and andiroba (Carapa guianensis) reduced by 61.6 and $57.7 \%$, respectively, the number of horn flies on Holstein cows at 24 hours (Klauck et al. 2014).

Essential oils also possess insecticidal properties or may act as antifeedants, oviposition deterrents or insect growth regulators (Lachance and Grange 2014). Klauck et al. (2014) found that tea tree and andiroba oils have an adulticidal activity against house flies and horn flies, the latter being the most susceptible with $100 \%$ mortality after four hours. Zhu et al. (2014) observed more than $98 \%$ inhibition of stable fly larval growth and female oviposition in larval and oviposition substrate treated with encapsulated catnip oil. The encapsulation process ensures an increased longevity and stability of the product because essential oils are volatiles and often short-lived. However, the very short effect of all these plant-based products remains so far a strong obstacle to their use and adoption in the livestock breeding.

\section{Bacterial toxins}

Bacillus thuringiensis is a naturally occurring bacterium that produces insecticidal proteins potentially active against various insects (Ammons et al. 2009). As an example, B. thuringiensis israelensis is widely used as larvicide against mosquitoes. Several isolates of $B$. thuringiensis have been identified as active against larvae of house fly, stable fly and horn fly under laboratory conditions (Hodgman et al. 1993, Lysyk et al. 2010). Furthermore, B. thuringiensis thompsoni was proven to cause adult mortality of stable flies when fed in blood or applied topically (Lysyk et al. 
2012). Direct spray applications in defined development sites or oral formulations for cows might be investigated under field conditions in cattle farms (Lysyk et al. 2010, 2012).

B. thuringiensis may also be a useful mortality agent for tsetse flies, based on the isolation of unique strain(s) from animal facilities housing waterbuck in Kenya the 1990's (Mihok et al. 1991; Osir and Vundla 1999).

\section{Natural predators}

Natural predators of flies such as mites, insects and birds are commonly present in livestock barns and in pastures. Mites of the family Macrochelidae are naturally present in cow manure (Cicolani 1992). They prey on the eggs and larvae of dung-breeding flies (Krantz 1983). The use of Macrocheles muscaedomesticae Scopoli in cow manure was proved effective in controlling the house fly, but not the face fly (Singh et al. 1966). In Australia, Macrocheles spp. have shown to cause considerable mortality of the bush fly and the buffalo fly (Halliday and Holm 1987). Insects can be predators of flies at the immature stages or adult stage. Beetles such as histerids and staphylinids prey on eggs and larvae (Hu and Frank 1997; Summerlin et al. 1991); Philonthus spp. are frequent inhabitants of cattle dung and effective predators of the horn fly. Several species of the family Asilidae (Diptera), Sphecidae (Hymenoptera) and Crabronidae (Hymenoptera) prey on adult flies. For instance, predation by bembicine wasps (Hymenoptera: Crabronidae) has shown some efficacy in the reduction of tabanid populations around cattle (Roberts and Wilson 1967). Finally, domestic and wild birds can act as insect predators. The guinea fowl is an excellent horsefly and tick predator, and livestock quickly becomes accustomed to its presence; it is therefore doubly beneficial to associate this species with livestock when tabanids are active (Desquesnes 2004). The egret (Bubulcus ibis) catches horseflies when they land on livestock to bite; very often, livestock herds are escorted by these white birds which can be three or four per head of cattle. Natural predators may contribute to the reduction of the seasonal parasitic load on cattle; however, they may have limited impacts on persistence of fly populations. For instance, there is evidence that to maintain a tabanid population, only $2 \%$ of the females need to fulfil the normal cycle (Foil and Hogsette 1994).

\section{Chemical controls}

Insecticides are routinely used for fly control as larvicides or adulticides. Their efficacy depends on the compound and its formulation, the application method and setting, the fly species and the susceptibility/resistance level to a particular insecticide (Baldacchino et al. 2014a; Foil and Hogsette 1994). Larvicides can be applied directly to cow manure to control fly immature stages, and several insect growth regulators (IGRs) such as pyriproxyfen, cyromazine, diflubenzuron, novaluron and methoprene are effective against the house fly, stable fly and/or horn fly (Bell et al. 2010; Liu et al. 2012; Lohmeyer and Pound 2012; Oyarzun et al. 2008). Adulticides can be applied directly on cattle using ear tags, sprays, pour-on, footbaths and self-application devices, or in the close environment of cattle as space sprays, residual premise sprays, insecticide-treated screens, and toxic sugar baits (Baldacchino et al. 2014a; Foil and Hogsette 1994). Different compounds are effective adulticides against flies: synthetic pyrethroids (e.g. permethrin, cypermethrin, deltamethrin and fenvalerate), organophosphate (e.g. diazinon), phenylpyrazole (e.g. fipronil), macrocyclic lactones (e.g. ivermectin), pyrrole derivatives (e.g. chlorfenapyr), neonicotinoids (e.g. imidacloprid), carbamate (e.g. methomyl) and spinosad (Bauer and Baumann 2015; Deacutis et al. 2006; Foil and Hogsette 1994; Oyarzun et al. 2008). However, the very short insecticidal effect of ivermectin and its very high toxicity for dung beetles limits its use for such a purpose (Lumaret 
and Errouissi 2002). Very high persistence in the environment and the very high insecticidal power of phenylpyrazole and neonicotinoids make them highly toxic for the pollinator insect fauna (Bonmatin et al. 2015). This strong environmental toxicity should preclude their use in agriculture. Today, synthetic pyrethroids are the most commonly used adulticides.

Next to the potential side effects of insecticidal use, another main issue is the rapid development of insecticide resistance in fly populations after intensive insecticide treatment in space and time, as illustrated by the popular use of insecticide ear tags in the 70s-80s for horn fly control (Foil and Hogsette 1994; Oyarzun et al. 2008). Nowadays, organophosphate and/or pyrethroid resistance are reported worldwide in different fly populations, especially horn flies, stable flies and house flies (Keiding 1999; Oyarzun et al. 2008; Pitzer et al. 2010; Salem et al. 2012). For instance, in Florida dairy farms, house fly resistance to pyrethroids is widespread, while imidacloprid resistance is emerging (Kaufman et al. 2010). Larvicide resistance toward IGRs have also been detected in house fly populations of Europe (Bell et al. 2010; Kristensen and Jespersen 2003).

Furthermore, insecticide residues in cattle dung may have lethal and sub-lethal effects on dungfeeding insects. Mann et al. (2015) found less adult Diptera emergence and a lower number of adult Coleoptera in freshly collected dung or dung collected up to one week from cattle treated with a deltamethrin pour-on formulation. Insecticides applied as pour-on or sprays on cattle contaminate the dung, by direct transfer in the anal area or by indirect transfer from the cattle licking themselves, affecting the dung fauna (Vale et al. 2015). Restricted applications of insecticides by footbaths or spraying on legs and/or belly allow for a reduction in the amount of insecticide used and hence minimize impacts on dung fauna (Bouyer et al. 2009; Torr et al. 2007). Moreover, they target biting flies that feed preferentially on the lower parts of cattle such as tsetse flies, horse flies and stable flies (Baldacchino et al. 2014c; Bouyer et al. 2009; Mullens et al. 2006). In Zimbabwe, deltamethrin spraying restricted to the belly and legs was effective in controlling G. pallidipes and G. m. morsitans (Torr et al. 2007). However, this restricted application required increasing the frequency of the treatments to 2-week intervals instead of monthly intervals (for whole body spraying). In Burkina Faso, footbath treatments using a formulation of deltamethrin 0.005\% applied every 2-3 days decreased the incidence of cattle nagana (Bouyer et al. 2009). In Chad, footbath treatments of a herd impacted significantly tsetse fly populations with a reduction of $80 \%$ in total tsetse fly catches after 6 months (Ndeledje et al. 2013). Thus, restricted application of insecticide should be recommended as a cost-effective and environmentally-friendly fly control method.

Recently, insecticide-treated nets (ITN) have been proposed as a novel tool for pest management in livestock providing a long lasting protection against flies (Bauer et al. 2012; Peck et al. 2014). In Ghana, deltamethrin-treated nets used as fences were effective in preventing fly attacks on cattle and reducing fly densities (Maia et al. 2010). Moreover, the insecticidal activity of ITN lasted up to nine months despite exposure to high rainfall and intensive sunlight, even if the time necessary to paralyze the insects increases (Maia et al. 2010).

Chemical compounds can also be used as insect repellents such as N,N-diethyl-3-methylbenzamide (DEET) or pyrethroids (notably metofluthrin and transfluthrin). DEET is the most widely used synthetic repellent, especially against mosquitoes. In Switzerland, DEET sprayed as an emulsion was effective against tabanids up to $4 \mathrm{~h}$ after repellent application (Herholz et al. 2016), which is far too short for a proper use in livestock. However, Zhu et al. (2012) observed a low repellent effect of DEET against stable flies compared to catnip oil. In French Guyana, spraying applications of deltamethrin and piperonyl butoxide (50 ppm of active ingredient; 2 l/animal) 
were effective in protecting cattle from tabanids for about 10 days (Raymond and Favre 1991, quoted by Desquesnes 2004).

\section{Mechanical control}

Physical tools such as screen, fan, smoke

The use of door and window screens prevents flies to enter the livestock production facilities, especially sensitive area such as the milk room (Thomas and Jespersen 1994). High powered fans are also effective in reducing fly nuisance during milking since high wind velocity inhibits flight activity and decrease the number of flies disturbing cattle.

Smoke generated by burning wood or plants can reduce the number of tsetse and other flies by more than 50\%, particularly when animals are close to the fire (Torr et al. 2011b). However, animals can prefer to rest for long periods under the smoke protection instead of grazing resulting in food intake decrease and economic losses. Some paradoxical effects may also be observed. Some flies such as horn flies and stable flies can even stick more to the cattle under the effect of the smoke, staying in close contact with the animal skin to avoid the smoke as much as possible (M. Desquesnes, personal observation).

\section{Traps and targets}

Many traps have been developed to attract biting flies using sensory cues such as colour, movement, heat and light intensity or polarization; there are many idiosyncrasies in terms of their relative efficiencies for different groups (Baldacchino et al. 2014a). Coloured traps made of blue and black fabrics are the ones most commonly used for tsetse fly sampling and control; some examples are the biconical, Vavoua, pyramidal, F3, epsilon, NGU, and Nzi traps (WHO 2013). Only a few of these 'tsetse' traps (e.g. the Nzi) simultaneously catch large numbers of stable flies and/ or horse flies (Gilles et al. 2007; Mihok et al. 2006). For horse flies, large open-style traps equipped with shiny black spherical targets are still in common use, especially in North America: some examples are the malaise, canopy, box, greenhead, Manitoba, $\mathrm{H}$ and Epps traps. Several years ago, Horváth et al. (2014) elegantly demonstrated that tabanids are strongly attracted to horizontally polarized light (e.g. as reflected from surface water), explaining the particular efficacy of traps that include certain types of shiny black materials for tabanids. This finding has now been studied in exquisite detail; resulting in the development of new innovative devices for catching and possibly controlling tabanids (Egri et al. 2013; Horváth et al. 2014).

Adhesive-coated traps have also been designed to control house flies and stable flies such as the Alsynite trap, the Williams trap, the Broce trap and the EZ trap (Broce 1988; Taylor and Berkebile 2006). As an example, large sticky traps were effective in controlling house flies and stable flies in dairy calf greenhouse facilities in New York, USA (Kaufman et al. 2005). Alsynite panels (fiberglass) are costly and have largely been superseded by other plastics on a retail basis; hence researchers have been developing new sticky traps based on more readily-available plastics such as Coroplast ${ }^{\circledR}$ (Great Pacific Enterprises Inc., Granby, Québec, Canada) white corrugated polypropylene (Beresford and Sutcliffe 2006). Zhu et al. (2015) also tested the attractiveness of white panels for stable flies and found that they were 2.5 times more effective than Alsynite traps. Interestingly, they also reported that, under laboratory conditions, young stable flies prefer white, whereas gravid females prefer blue. 
A practical walk-through trap has been developed to control horn flies; use of this system reduced horn fly numbers on pastured cattle from 54 to 73\% (Hall and Doisy 1989). This trap can be equipped with a vacuum system to increase fly catches and hence it can collect large numbers of horn flies, while still catching stable flies, house flies and face flies (Denning et al. 2014).

Insecticide-treated targets made of blue or black cloth with/without netting have been successfully used for tsetse fly control throughout Africa for many years (Lehane et al. 2016; Lindh et al. 2012; Torr et al. 2011a; Vale et al. 1988), but similar devices have yet to be adapted for the specific control of other biting flies (Hogsette et al. 2008). For tabanids, control on a large scale may not be practical given results from a trial where tabanids were monitored in Zimbabwe during a largescale tsetse control project (Vale et al. 1988), but targeted applications for livestock facilities/farms with smaller source populations should be possible. For instance, Beresford and Sutcliffe (2010) observed a decrease in stable fly populations by using permethrin-treated targets made of white Coroplast panels in Canadian dairy farms.

\section{Attractant and toxic baits}

The attractiveness of traps or toxic baits can be enhanced considerably by using odour attractants such as pheromones (e.g. (Z)-9-tricosene for M. domestica) or kairomones (e.g. carbon dioxide, animal urines, octenol, phenols) (Brugman et al. 2018).

The house fly pheromone, (Z)-9-tricosene, is added as attractant alone or in combination with sugar to many commercial house fly insecticide baits, widely used in indoor livestock units (Butler et al. 2007). However, odours associated with the baits are likely to increase or decrease fly activity around the baits. The efficacy of these baits depends on the target size and pheromone concentration (Hanley et al. 2004). This bait is ineffective in outdoor situations because of the short-range attractiveness of (Z)-9-tricosene and the weathering of active ingredients (Hanley et al. 2004).

Baits containing sugar were previously mainly used against non-biting flies like the house fly. However, attractive toxic sugar baits have been also tested successfully against biting Diptera (e.g. mosquitoes) as this method exploits the diet used by insects to sustain their daily activities (Müller et al. 2010); and it may become another useful tool for controlling biting flies.

Several chemical compounds in excretory products, glandular secretions and exudations have been identified as attractants for biting flies such as octenol (1-octen-3-ol) and several phenolic compounds (Baldacchino et al. 2014a; Gibson and Torr 1999). The attractiveness of a compound depends on the dose, the combination with other compounds and the species. Horn flies and stable flies are attracted by octenol, $m$ - and $p$-cresol in olfactometer assays in the laboratory, as are many other biting insects (Oyarzún et al. 2009; Tangtrakulwanich et al. 2015). In the field, a recent study found that 2-3 times more stable flies were caught by sticky white panels baited with phenol, $m$ - or $p$-cresol than non-baited panels (Zhu et al. 2015). Similarly, binary mixtures of phenol $+m$-cresol or phenol $+p$-cresol were effective in collecting stable flies using Alsynite traps (Tangtrakulwanich et al. 2015). Recent results for phenols as baits for targets are clearly worth exploring further, despite many negative results with traps (Mihok et al. 2007). Previous studies with phenols/urine as baits for stable flies with traps have not revealed any practical applications of baits other than octenol. Phenols are more consistently attractive for tabanids, but again with variation among species. Interestingly, synergism between different phenolic compounds and 
ammonia also exists, and may account for the high attractiveness of aged animal urines (Mihok and Lange 2012).

\section{Conclusions}

This chapter provides an overview of Brachycera adult flies as pests of dairy cattle causing: (1) direct effects related to annoyance/stress, skin injuries and blood losses with negative impacts on animal growth and milk production; and (2) indirect effects via the transport or the transmission of a wide range of pathogens, mechanically or biologically. Although limited in number, all studies agree on the strong direct impact of Brachycera flies in livestock, especially dairy cattle, which would justify the development of better and more effective integrated control. During the last decade, some of the pathogens transmitted by Brachycera flies and affecting livestock have (re) emerged as illustrated by the increase of lumpy skin disease or bovine besnoitiosis outbreaks in Europe (Alvarez-Garcia et al. 2013; Beard 2016). Some outbreaks also stress that newly introduced pathogens such as T. evansi in Europe can be spread easily through these mechanical vectors. In addition, the globalization of trade and the expansion of residential areas into rural landscapes with livestock facilities have increased the transmission risk of zoonotic diseases by flies (Förster et al. 2012). In the future, Brachycera flies may become a concern in animal health because of changes in climate (e.g. increased temperatures) and land use (e.g. intensive farming) affecting fly populations and, consequently, the epidemiology of fly-borne pathogens (Baldacchino et al. 2017; Smallegange and Den Otter 2007; Van den Bossche et al. 2010).

Therefore, there is a crucial need to better assess the role of flies as a direct burden and mechanical carrier or vector of pathogens through epidemiological and entomological studies and to develop innovative control methods. The intensive use of insecticides for fly control has shown two main issues, the rapid development of insecticide resistance in fly populations and the negative impact on non-target insects such as dung beetles or pollinators. This encourages the promotion of less polluting chemical control methods, or even better, biological control methods such as trapping or the use of parasitoids, entomopathogenic fungi or bacterial toxins. One promising prospect is the improvement of attractive traps or targets combined with repellent formulations for animals in a push-pull strategy.

\section{References}

Aiello SE (2012) Merck veterinary manual. Merck \& Co. Inc., Kenilworth, NJ, USA. Available at: https://tinyurl.com/ y9vm8lws.

Allingham PG, Leatch G and Kemp DH (1994) An attempt to transmit Anaplasma marginale by buffalo flies (Haematobia irritans exigua). Aust Vet J 71: 122-123.

Alvarez-Garcia G, Frey CF, Ortega Mora LM and Schares G (2013) A century of bovine besnoitiosis: an unknown disease re-emerging in Europe. Trends Parasitol 29: 407-415.

Ammons DR, Reyna A, Granados JC, Samlal MS and Rampersad JN (2009) An investigation of Bacillus thuringiensis in rectal-collected fecal samples of cows. Curr Microbiol 59: 532-536.

Andreeva VR, Kilic AY and Altunsoy F (2009) New contribution to information about Tabanidae (Diptera) adult and larvae from West Anatolia. J Entomol Res Soc 11: 19-30.

Auty H, Torr SJ, Michoel T, Jayaraman S and Morrison LJ (2015) Cattle trypanosomosis: the diversity of trypanosomes and implications for disease epidemiology and control. Rev Sci Tech OIE 34: 587-598.

Baldacchino F, Desquesnes M, Mihok S, Foil LD, Duvallet G and Jittapalapong S (2014a) Tabanids: neglected subjects of research, but important vectors of disease agents! Infect Genet Evol 28: 596-615. 
Baldacchino F, Krčmar S, Bernard C, Manon S and Jay-Robert P (2017) The impact of land use and climate on tabanid assemblages in Europe. Agr Ecosyst Environ 239: 112-118.

Baldacchino F, Muenworn V, Desquesnes M, Desoli F, Charoenviriyaphap T and Duvallet G (2013) Transmission of pathogens by Stomoxys flies (Diptera, Muscidae): a review. Parasite 20: 1-13.

Baldacchino F, Porciani A, Bernard C and Jay-Robert $P$ (2014b) Spatial and temporal distribution of Tabanidae in the Pyrenees mountains: the influence of altitude and landscape structure. B Entomol Res 104: 1-11.

Baldacchino F, Puech L, Manon S, Hertzog LR and Jay-Robert P (2014c) Biting behaviour of Tabanidae on cattle in mountainous summer pastures, Pyrenees, France, and effects of weather variables. B Entomol Res 104: 471-479.

Ball SG (1984) Seasonal abundance during the summer months of some cattle-visiting Muscidae (Diptera) in northeast England. Ecol Entomol 9: 1-10.

Bauer B and Baumann MPO (2015) Laboratory evaluation of efficacy and persistence of a 1\% w/w fipronil pour-on formulation (Topline ${ }^{\oplus}$ ) against Glossina palpalis gambiensis, Diptera: Glossinidae. Parasitol Res 114: 2919-2923.

Bauer B, Mehlitz D and Clausen PH (2012) Impact of insecticide-treated nets on insects of medical and veterinary relevance. In: Mehlhorn $\mathrm{H}$ (ed.) Arthropods as vectors of emerging diseases. Parasitology Research Monographs. Vol. 3. Springer-Verlag, Berlin Heidelberg, Germany, pp 137-150.

Beard PM (2016) Lumpy skin disease: a direct threat to Europe. Vet Rec 178: 557-558.

Bell HA, Robinson KA and Weaver RJ (2010) First report of cyromazine resistance in a population of UK house fly (Musca domestica) associated with intensive livestock production. Pest Manag Sci 66: 693-695.

Beresford DV and Sutcliffe JF (2006) Studies on the effectiveness of Coroplast sticky traps for sampling stable flies (Diptera: Muscidae), including a comparison to Alsynite. J Econ Entomol 99: 1025-1035.

Beresford DV and Sutcliffe JF (2009) Local infestation or long-distance migration? The seasonal recolonization of dairy farms by Stomoxys calcitrans (Diptera: Muscidae) in South Central Ontario, Canada. J Econ Entomol 102: 788-798.

Beresford DV and Sutcliffe JF (2010) Assessing pest control using changes in instantaneous rate of population increase: treated targets and stable fly populations case study. J Dairy Sci 93: 2517-2524.

Berkebile DR, Thomas GD and Campbell JB (1994) Overwintering of the stable fly (Diptera, Muscidae) in southeastern Nebraska. J Econ Entomol 87: 1555-1563.

Bigalke RD (1968) New concepts on the epidemiological features of bovine besnoitiosis as determined by laboratory and field investigations. Onderstepoort J Vet 35: 3-137.

Birhanu H, Fikru R, Said M, Kidane W, Gebrehiwot T, Hagos A, Alemu T, Dawit T, Berkvens D, Goddeeris BM and Buscher P (2015) Epidemiology of Trypanosoma evansi and Trypanosoma vivax in domestic animals from selected districts of Tigray and Afar regions, Northern Ethiopia. Parasit Vectors 8: 212.

Black WC and Krafsur ES (1986) Population biology and genetics of winter house fly (Diptera: Muscidae) populations. Ann Entomol Soc Am 79: 636-644.

Bonmatin JM, Giorio C, Girolami V, Goulson D, Kreutzweiser DP, Krupke C, Liess M, Long E, Marzaro M, Mitchell EAD, Noome DA, Simon-Delso N and Tapparo A (2015) Environmental fate and exposure; neonicotinoids and fipronil. Environ Sci Pollut R 22: 35-67.

Bonomi A, Bassetti F, Gabrieli P, Beadell J, Falchetto M, Scolari F, Gomulski LM, Regazzini E, Ouma JO, Caccone A, Okedi LM, Attardo GM, Guglielmino CR, Aksoy S and Malacrida AR (2011) Polyandry is a common event in wild populations of the tsetse fly Glossina fuscipes fuscipes and may impact population reduction measures. PLoS Neglect Trop Dis 5: e1190.

Bouet $\mathrm{G}$ and Roubaud E (1912) Expériences de transmission des trypanosomiases animales d'Afrique occidentale française par les stomoxes. B Soc Pathol Exot 5: 544-550.

Bouyer J, Stachurski F, Gouro AS and Lancelot R (2009) Control of bovine trypanosomosis by restricted application of insecticides to cattle using footbaths. Vet Parasitol 161: 187-193.

Broce AB (1988) An improved alsynite trap for stable flies, Stomoxys calcitrans (Diptera, Muscidae). J Med Entomol 25: 406-409.

Broce AB, Hogsette J and Paisley S (2005) Winter feeding sites of hay in round bales as major developmental sites of Stomoxys calcitrans (Diptera: Muscidae) in pastures in spring and summer. J Econ Entomol 98: 2307-2312. 
Bruce WN and Decker GC (1958) The relationship of stable fly abundance to milk production in dairy cattle. J Econ Entomol 51: 269-274.

Brugman VA, Smallegange RC and Logan JG (2018) Semiochemical tools for a new generation of livestock pest control. In: Garros C, Bouyer J, Takken W and Smallegange RC (eds.) Pests and vector-borne diseases in the livestock industry. Ecology and Control of Vector-borne diseases. Vol. 5. Wageningen Academic Publishers, Wageningen, the Netherlands, pp. 389-434.

Burger J (1995) Yellowstone's snipe fly summer. Yellowstone Science 3: 2-5.

Burger JF and Anderson JR (1974) Taxonomy and life history of the moose fly, Haematobosca alcis, and its association with the moose, Alces alces shirasi in Yellowstone National Park. Ann Entomol Soc Am 67: 204-214.

Burgess ERI and King BH (2015) Compatibility of the parasitoid wasp Spalangia endius (Hymenoptera: Pteromalidae) and insecticides against Musca domestica (Diptera: Muscidae) as evaluated by a new index. J Econ Entomol 108: 986-992.

Butler SM, Gerry AC and Mullens BA (2007) House fly (Diptera: Muscidae) activity near baits containing (Z)-9-tricosene and efficacy of commercial toxic fly baits on a Southern California dairy. J Econ Entomol 100: 1489-1495.

Castro BG, Souza MMS, Regua-Mangia AH and Bittencourt AJ (2010) Enterobacterial microbiota on Stomoxys calcitrans external surface. Transbound Emerg Dis 57: 22-24.

Cherenet T, Sani RA, Panandam JM, Nadzr S, Speybroeck N and Van Den Bossche P (2004) Seasonal prevalence of bovine trypanosomosis in a tsetse-infested zone and a tsetse-free zone of the Amhara Region, north-west Ethiopia. Onderstepoort J Vet Res 71: 307-312.

Chirico J, Jonsson P, Kjellberg S and Thomas G (1997) Summer mastitis experimentally induced by Hydrotaea irritans exposed to bacteria. Med Vet Entomol 11: 187-192.

Cicolani B (1992) Macrochelids mites (Acari, Mesostigmata) occuring in animal droppings in the pasture ecosystem in central Italy. Agr Ecosyst Environ 40: 47-60.

Cruz-Vazquez C, Carvajal Marquez J, Lezama-Gutierrez R, Vitela-Mendoza I and Ramos-Parra M (2015) Efficacy of the entomopathogenic fungi Metarhizium anisopliae in the control of infestation by stable flies Stomoxys calcitrans (L.), under natural infestation conditions. Vet Parasitol 212: 350-355.

Cuglovici DA, Bartholomeu DC, Reis-Cunha JL, Carvalho AU and Ribeiro MFB (2010) Epidemiologic aspects of an outbreak of Trypanosoma vivax in a dairy cattle herd in Minas Gerais state, Brazil. Vet Parasitol 169: 320-326.

De Vliegher S, Fox LK, Piepers S, McDougall S and Barkema HW (2012) Invited review: mastitis in dairy heifers: nature of the disease, potential impact, prevention, and control. J Dairy Sci 95: 1025-1040.

Deacutis JM, Leichter CA, Gerry AC, Rutz DA, Watson WD, Geden CJ and Scott JG (2006) Susceptibility of field collected house flies to spinosad before and after a season of use. J Agr Urban Entomol 23: 105-110.

Denning SS, Washburn SP and Watson DW (2014) Development of a novel walk-through fly trap for the control of horn flies and other pests on pastured dairy cows. J Dairy Sci 97: 4624-4631.

Depner KR (1969) Distribution of the face fly, Musca autumnalis (Diptera: Muscidae), in western Canada and the relationship between its environment and population density. Can Entomol 101: 97-100.

Desquesnes M (2004) Livestock trypanosomoses and their vectors in Latin America. OIE, Paris, France.

Desquesnes M, Biteau-Coroller F, Bouyer J, Dia ML and Foil L (2009) Development of a mathematical model for mechanical transmission of trypanosomes and other pathogens of cattle transmitted by tabanids. Int J Parasitol 39: 333-346.

Desquesnes M and Dia ML (2003a) Trypanosoma vivax: mechanical transmission in cattle by one of the most common African tabanids, Atylotus agrestis. Exp Parasitol 103: 35-43.

Desquesnes M and Dia ML (2003b) Mechanical transmission of Trypanosoma congolense in cattle by the African tabanid Atylotus agrestis. Exp Parasitol 105: 226-231.

Desquesnes M and Dia ML (2004) Mechanical transmission of Trypanosoma vivax in cattle by the African tabanid Atylotus fuscipes. Vet Parasitol 119: 9-19.

Desquesnes M, Holzmuller P, Lai D-H, Dargantes A, Lun Z-R and Jittaplapong S (2013) Trypanosoma evansi and surra: a review and perspectives on origin, history, distribution, taxonomy, morphology, hosts, and pathogenic effects. Biomed Res Int 2013: 194176. 
Dsouli-Aymes N, Michaux J, De Stordeur E, Couloux A, Veuille M and Duvallet G (2011) Global population structure of the stable fly (Stomoxys calcitrans) inferred by mitochondrial and nuclear sequence data. Infect Genet Evol 11:334-342.

Echeverria P, Harrison BA, Tirapat C and McFarland A (1983) Flies as source of enteric pathogens in a rural village in Thailand. Appl Environ Microb 46: 32-36.

Egri A, Blaho M, Szaz D, Kriska G, Majer J, Herczeg T, Gyurkovszky M, Farkas R and Horvath G (2013) A horizontally polarizing liquid trap enhances the tabanid-capturing efficiency of the classic canopy trap. B Entomol Res 103: 665-674.

Fasanella A, Scasciamacchia S, Garofolo G, Giangaspero A, Tarsitano E and Adone R (2010) Evaluation of the house fly Musca domestica as a mechanical vector for an anthrax. PLoS ONE 5: e12219.

Floate KD, Lysyk TJ and Gibson GAP (2013) Haematobia irritans L., horn fly, Musca domestica L., house fly, and Stomoxys calcitrans (L.), stable fly (Diptera: Muscidae). In: Mason PG and Gillespie DR (eds.) Biological control programmes in Canada 2001-2012. CABI, Wallingford, UK.

Förster M, Gestmann F, Mehlhorn H, Sievert K, Messler S, Neuhausen N, Petersdorf S and Pfeffer K (2012) Flies as vectors of parasites potentially inducing severe diseases in humans and animals. In: Mehlhorn $\mathrm{H}$ (ed.) Arthropods as vectors of emerging diseases. Parasitology Research Monographs. Vol. 3. Springer-Verlag, Berlin Heidelberg, Germany, pp. 227-253.

Foil LD and Hogsette JA (1994) Biology and control of tabanids, stables flies and horn flies. Rev Sci Tech OIE 13: 11251158.

Fowler FE and Mullens BA (2016) Dividing the pie: differential dung pat size utilization by sympatric Haematobia irritans and Musca autumnalis. Med Vet Entomol 30: 185-192.

Galindo-Velasco E, Lezama-Gutierrez R, Cruz-Vazquez C, Pescador-Rubio A, Angel-Sahagun CA, Ojeda-Chi MM, Rodriguez-Vivas RI and Contreras-Lara D (2015) Efficacy of entomopathogenic fungi (Ascomycetes: Hypocreales) against adult Haematobia irritans (Diptera: Muscidae) under stable conditions in the Mexican dry tropics. Vet Parasitol 209: 173-178.

Geden CJ, Rutz DA, Miller RW and Steinkraus DC (1992) Suppression of house flies (Diptera, Muscidae) on New-York and Maryland dairies using release of Muscidifurax raptor (Hymenoptera, Pteromalidae) in an integrated management program. Environ Entomol 21: 1419-1426.

Gerhardt RR, Allen JW, Greene WH and Smith PC (1982) The role of face flies in an episode of infectious bovine keratoconjunctivitis. J Am Vet Med Assoc 180: 156-159.

Gibbs EP, Johnson RH and Osborne AD (1972) Field observations on the epidemiology of bovine herpes mammillitis. Vet Rec 91: 395-401.

Gibson G and Torr SJ (1999) Visual and olfactory responses of haematophagous Diptera to host stimuli. Med Vet Entomol 13:2-23.

Gilles J, David JF, Duvallet G, De la Rocque S and Tillard E (2007) Efficiency of traps for Stomoxys calcitrans and Stomoxys niger niger on Reunion Island. Med Vet Entomol 21: 65-69.

Gollnick NS, Scharr JC, Schares G and Langenmayer MC (2015) Natural Besnoitia besnoiti infections in cattle: chronology of disease progression. BMC Vet Res 11: 35.

Gooding RH and Krafsur ES (2005) Tsetse genetics: contributions to biology, systematics, and control of tsetse flies. Annu Rev Entomol 50: 101-123.

Graczyk TK, Cranfield MR, Fayer R and Bixler H (1999) House flies (Musca domestica) as transport hosts of Cryptosporidium parvum. Am J Trop Med Hyg 61: 500-504.

Graczyk TK, Knight R, Gilman RH and Cranfield MR (2001) The role of non-biting flies in the epidemiology of human infectious diseases. Microb Infect 3: 231-235.

Graczyk TK, Knight R and Tamang L (2005) Mechanical transmission of human protozoan parasites by insects. Clin Microbiol Rev 18: 128-132.

Gutierrez C, Desquesnes M, Touratier L and Büscher P (2010) Trypanosoma evansi: recent outbreaks in Europe. Vet Parasitol 174: 26-29.

Hall MJR, Wall RL and Stevens JR (2016) Traumatic myiasis: a neglected disease in a changing world. Annu Rev Entomol 61: 159-176. 
Hall RD (1984) Relationship of the face fly (Diptera, Muscidae) to pinkeye in cattle - a review and synthesis of the relevant literature. J Med Entomol 21:361-365.

Hall RD and Doisy KE (1989) Walk-through trap for control of horn flies (Diptera, Muscidae) on pastured cattles. J Econ Entomol 82: 530-534.

Hall RD and Gerhardt RR (2002) Flies (Diptera). In: Mullen G and Durden L (eds.) Medical and veterinary entomology. Academic Press, San Diego, CA, USA., pp. 127-145.

Halliday RB and Holm E (1987) Mites of the family Macrochelidae as predators of 2 species of dung-breeding pest flies. Entomophaga 32: 333-338.

Hammer O (1941) Biological and ecological investigations on flies associated with pasturing cattle and their excrement. Vidensk Medd Dansk Naturh Foren 105: 191-393.

Hanley ME, Dunn DW, Abolins SR and Goulson D (2004) Evaluation of (Z)-9-tricosene baited targets for control of the housefly (Musca domestica) in outdoor situations. J Appl Entomol 128: 478-482.

Hawkins JA, Love JN and Hidalgo RJ (1982) Mechanical transmission of anaplasmosis by tabanids (Diptera, Tabanidae). Am J Vet Res 43: 732-734.

Herholz C, Kopp C, Wenger M, Mathis A, Wageli S and Roth N (2016) Efficacy of the repellent N,N-diethyl-3-methylbenzamide (DEET) against tabanid flies on horses evaluated in a field test in Switzerland. Vet Parasitol 221: 64-67.

Hillerton JE, Bramley AJ and Broom DM (1984) The distribution of 5 species of flies (Diptera, Muscidae) over the bodies of dairy heifers in England. B Entomol Res 74: 113-119.

Hoare CA (1972) The trypanosomes of mammals. A zoological monograph. Blackwell Scientific Publications, Oxford, UK.

Hodgman TC, Ziniu Y, Ming S, Sawyer T, Nicholls CM and Ellar DJ (1993) Characterization of Bacillus thuringiensis strain which is toxic to the house fly Musca domestica. FEMS Microbiol Lett 114: 17-22.

Hogsette JA, Nalli A and Foil LD (2008) Evaluation of different insecticides and fabric types for development of treated targets for stable fly (Diptera: Muscidae) control. J Econ Entomol 101: 1034-1038.

Hogsette JA, Prichard DL and Ruff JP (1991) Economic-effects of horn fly (Diptera, Muscidae) populations on beef-cattle exposed to 3 pesticide treatment regimes. J Econ Entomol 84: 1270-1274.

Holmes P (2013) Tsetse-transmitted trypanosomes - their biology, disease impact and control. J Invertebr Pathol 112: S11-S14.

Hornok S, Foeldvari G, Elek V, Naranjo V, Farkas R and De la Fuente J (2008) Molecular identification of Anaplasma marginale and rickettsial endosymbionts in blood-sucking flies (Diptera: Tabanidae, Muscidae) and hard ticks (Acari: Ixodidae). Vet Parasitol 154: 354-359.

Horváth G, Blahó M, Egri Á and Lerner A (2014) Applying polarization-based traps to insect control. In: Horváth G (ed.) Polarized light and polarization vision in animal sciences. Springer, Berlin Heidelberg, Germany.

Hu GY and Frank JH (1997) Predation on the horn fly (Diptera: Muscidae) by five species of Philonthus (Coleoptera: Staphylinidae). Environ Entomol 26: 1240-1246.

Hutson AM (1984) Keds, flat-flies and bat-flies. Diptera, Hippoboscidae and Nycteribiidae. Handbooks for the identification of British insects. Royal Entomological Society, St Albans, UK.

Jacquiet P, Lienard E and Franc M (2010) Bovine besnoitiosis: epidemiological and clinical aspects. Vet Parasitol 174: 30-36.

Jensen KMV, Jespersen JB and Nielsen BO (1993) Variation in density of cattle-visiting muscid flies between Danish inland pastures. Med Vet Entomol 7: 17-22.

Johnson GD, Campbell JB, Minocha HC and Broce AB (1991) Ability of Musca autumnalis (Diptera, Muscidae) to acquire and transmit bovine herpesvirus-1. J Med Entomol 28: 841-846.

Johnson SJ (1987) Stephanofilariasis - a review. Helminthological abstracts 56: 287-299.

Johnson SJ (1989) Studies on stephanofilariasis in Queensland. James Cook University, Douglas, Australia.

Jonsson NN and Mayer DG (1999) Estimation of the effects of buffalo fly (Haematobia irritans exigua) on the milk production of dairy cattle based on a meta-analysis of literature data. Med Vet Entomol 13: 372-376.

Kahana-Sutin E, Klement E, Lensky I and Gottlieb Y (2016) High relative abundance of the stable fly Stomoxys calcitrans is associated with lumpy skin disease outbreaks in Israeli dairy farms. Med Vet Entomol 2: 150-160.

Kangwagye TN (1973) Diurnal and nocturnal biting activity of flies (Diptera) in western Uganda. B Entomol Res 63: 17-29. 
Kaufman PE, Nunez SC, Mann RS, Geden CJ and Scharf ME (2010) Nicotinoid and pyrethroid insecticide resistance in houseflies (Diptera: Muscidae) collected from Florida dairies. Pest Manag Sci 66: 290-294.

Kaufman PE, Rutz DA, Frisch S (2005) Large sticky traps for capturing house flies and stable flies in dairy calf greenhouse facilities. J Dairy Sci 88: 176-181.

Keiding $J(1999)$ Review of the global status and recent development of insecticide resistance in field populations of the housefly, Musca domestica (Diptera: Muscidae). B Entomol Res 89: S7-S67.

Klauck V, Pazinato R, Stefani LM, Santos RC, Vaucher RA, Baldissera MD, Raffin R, Boligon A, Athayde M, Baretta D, Machado $G$ and Da Silva AS (2014) Insecticidal and repellent effects of tea tree and andiroba oils on flies associated with livestock. Med Vet Entomol 28: 33-39.

Kocan KM, De la Fuente J, Blouin EF, Coetzee JF and Ewing SA (2010) The natural history of Anaplasma marginale. Vet Parasitol 167: 95-107.

Krafsur ES and Moon RD (1997) Bionomics of the face fly, Musca autumnalis. Annu Rev Entomol 42: 503-523.

Kraneveld FC and Djaenoedin R (1940) Proeven over de overbrenging van miltvuur door den Tabanus rubidus Wied. op paard en buffet. Ned-Ind Blad Diergeneesk 52: 339-380.

Krantz GW (1983) Mites as biological control agents for dung-breeding flies, with special reference to the Macrochelidae. In: Hoy MA, Cunningham GL and Knutson L (eds.) Biological control of pests by mites. University of California, Berkeley, CA, USA.

Krinsky WL (1976) Animal disease agents transmitted by horse flies and deer flies (Diptera: Tabanidae). J Med Entomol 13: 224-275.

Krinsky WL (2002) Tsetse flies (Glossinidae). In: Mullen G and Durden L (eds.) Medical and veterinary entomolology. Academic Press, San Diego, CA, USA, pp. 303-316.

Kristensen M and Jespersen JB (2003) Larvicide resistance in Musca domestica (Diptera: Muscidae) populations in Denmark and establishment of resistant laboratory strains. J Econ Entomol 96: 1300-1306.

Kumar P, Mishra S, Malik A and Satya S (2011) Repellent, larvicidal and pupicidal properties of essential oils and their formulations against the housefly, Musca domestica. Med Vet Entomol 25: 302-310.

Kuramochi K (2000) Ovipositional behavior of the horn fly (Diptera: Muscidae) in the field. J Med Entomol 37: 461-466.

Lachance S and Grange G (2014) Repellent effectiveness of seven plant essential oils, sunflower oil and natural insecticides against horn flies on pastured dairy cows and heifers. Med Vet Entomol 28: 193-200.

Lane RP and Crosskey RW (1993) Medical insects and arachnids. Chapman and Hall, London, UK.

Lehane M (2005) The biology of blood-sucking in insects. Cambridge University Press, Cambridge, UK.

Lehane M, Alfaroukh I, Bucheton B, Camara M, Harris A, Kaba D, Lumbala C, Peka M, Rayaisse JB, Waiswa C, Solano P and Torr S (2016) Tsetse control and the elimination of Gambian sleeping sickness. PLoS Neglect Trop Dis 10: e0004437.

Lienard E, Salem A, Grisez C, Prevot F, Bergeaud JP, Franc M, Gottstein B, Alzieu JP, Lagalisse Y and Jacquiet P (2011) A longitudinal study of Besnoitia besnoiti infections and seasonal abundance of Stomoxys calcitrans in a dairy cattle farm of southwest France. Vet Parasitol 177: 20-27.

Lienard E, Salem A, Jacquiet P, Grisez C, Prevot F, Blanchard B, Bouhsira E and Franc M (2013) Development of a protocol testing the ability of Stomoxys calcitrans (Linnaeus, 1758) (Diptera: Muscidae) to transmit Besnoitia besnoiti (Henry, 1913) (Apicomplexa: Sarcocystidae). Parasitol Res 112: 479-486.

Lindh JM, Goswami P, Blackburn RS, Arnold SEJ, Vale GA, Lehane MJ and Torr SJ (2012) Optimizing the colour and fabric of targets for the control of the tsetse fly Glossina fuscipes fuscipes. Plos Neglect Trop Dis 6: e1661.

Liu SS, Li AY, Lohmeyer KH and De Leon AAP (2012) Effects of pyriproxyfen and buprofezin on immature development and reproduction in the stable fly. Med Vet Entomol 26: 379-385.

Lloyd JE (2002) Louse flies, keds, and related flies (Hippoboscoidea). In: Mullen G and Durden L (eds.) Medical and veterinary entomology. Academic Press, San Diego, CA, USA.

Lohmeyer KH and Miller JA (2006) Pathogenicity of three formulations of entomopathogenic fungi for control of adult Haematobia irritans (Diptera: Muscidae). J Econ Entomol 99: 1943-1947.

Lohmeyer KH and Pound JM (2012) Laboratory evaluation of novaluron as a development site treatment for controlling larval horn flies, house flies, and stable flies (Diptera: Muscidae). J Med Entomol 49: 647-651. 
Lopez-Sanchez J, Cruz-Vazquez C, Lezama-Gutierrez R and Ramos-Parra M (2012) Effect of entomopathogenic fungi upon adults of Stomoxys calcitrans and Musca domestica (Diptera: Muscidae). Biocontrol Sci Techn 22: 969-973.

Lumaret JP and Errouissi F (2002) Use of anthelmintics in herbivores and evaluation of risks for the non target fauna of pastures. Vet Res 33: 547-562.

Lysyk TJ (1995) Temperature and population-density effects on feeding activity of Stomoxys calcitrans (Diptera, Muscidae) on cattle. J Med Entomol 32: 508-514.

Lysyk TJ, Kalischuk-Tymensen LD, Rochon K and Selinger LB (2010) Activity of Bacillus thuringiensis isolates against immature horn fly and stable fly (Diptera: Muscidae). J Econ Entomol 103: 1019-1029.

Lysyk TJ, Kalischuk-Tymensen LD and Selinger LB (2012) Mortality of adult Stomoxys calcitrans fed isolates of Bacillus thuringiensis. J Econ Entomol 105: 1863-1870.

Machtinger ET and Geden CJ (2018) Biological control with parasitoids. In: Garros C, Bouyer J, Takken W and Smallegange $\mathrm{RC}$ (eds.) Pests and vector-borne diseases in the livestock industry. Ecology and Control of Vector-borne diseases. Vol. 5. Wageningen Academic Publishers, Wageningen, the Netherlands, pp. 299-335.

Machtinger ET, Geden CJ and Leppla NC (2015) Linear dispersal of the filth fly parasitoid Spalangia cameroni (Hymenoptera: Pteromalidae) and parasitism of hosts at increasing distances. PLoS ONE 10: e0129105.

Machtinger ET, Weeks ENI and Geden CJ (2016) Oviposition deterrence and immature survival of filth flies (Diptera: Muscidae) when exposed to commercial fungal products. J Insect Sci 16: 54.

Maciel W, Zanetti Lopes WD, Cruz B, Teixeira W, Felippelli G, Sakamoto CA, Favero FC, Buzzulini C, Soares V, Gomes LV, Bichuette M and Da Costa AJ (2015) Effects of Haematobia irritans infestation on weight gain of Nelore calves assessed with different antiparasitic treatment schemes. Prev Vet Med 118: 182-186.

Madsen M, Sorensen GH and Nielsen SA (1991) Studies on the possible role of cattle nuisance flies, especially Hydrotaea irritans in the transmission of summer mastitisin Denmark. Med Vet Entomol 5: 421-429.

Maia M, Clausen P-H, Mehlitz D, Garms R and Bauer B (2010) Protection of confined cattle against biting and nuisance flies (Muscidae: Diptera) with insecticide-treated nets in the Ghanaian forest zone at Kumasi. Parasitol Res 106: 1307-1313.

Maniania NK and Ekesi S (2013) The use of entomopathogenic fungi in the control of tsetse flies. J Invertebr Pathol 112: S83-S88.

Mann CM, Barnes S, Offer B and Wall R (2015) Lethal and sub-lethal effects of faecal deltamethrin residues on dungfeeding insects. Med Vet Entomol 29: 189-195.

Mason CA and Norval RAI (1981) The transfer of Boophilus microplus (Acarina: Ixodidae) from infested to uninfested cattle under field conditions. Vet Parasitol 8: 185-188.

Mays AR, Brown MA, Von Tunglen DL and Rosenkrans CF (2014) Milk production traits of beef cows as affected by horn fly count and sire breed type. J Anim Sci 92: 1208-1212.

McAlpine JE and Wood D (1989) Manual of neartic diptera. Vol. 3. Canadian Government Publishing Centre, Ottawa, Canada.

McKay T and Galloway TD (1999) Survey and release of parasitoids (Hymenoptera) attacking house and stable flies (Diptera: Muscidae) in dairy. Can Entomol 131: 743-756.

McLintock J and Depner KR (1954) A review of the life-history and habits of the hornfly, Siphona irritans (L.) (Diptera: Muscidae). Can Entomol 84: 20-33.

Mihok S, Carlson DA, Krafsur ES and Foil LD (2006) Performance of the Nzi and other traps for biting flies in North America. B Entomol Res 96: 387-397.

Mihok S, Carlson DA and Ndegwa PN (2007) Tsetse and other biting fly responses to Nzi traps baited with octenol, phenols and acetone. Med Vet Entomol 21:70-84.

Mihok S and Lange K (2012) Synergism between ammonia and phenols for Hybomitra tabanids in northern and temperate Canada. Med Vet Entomol 26: 282-290.

Mihok S, Maramba O, Munyoki E and Kagoiya J (1995) Mechanical transmission of Trypanosoma spp. by African stomoxyinae (Diptera, Muscidae). Trop Med Parasitol 46: 103-105.

Mihok S, Maramba O, Munyoki E and Saleh K (1996) Phenology of Stomoxyinae in a Kenyan forest. Med Vet Entomol 10: $305-316$. 
Mihok S, Olubayo RO and Wesonga DF (1991) Infection rates in Glossina morsitans morsitans fed on waterbuck and Boran cattle infected with Trypanosoma congolense. Acta Trop 49: 185-191.

Mishra S, Kumar P, Malik A and Satya S (2011) Adulticidal and larvicidal activity of Beauveria bassiana and Metarhizium anisopliae against housefly, Musca domestica (Diptera: Muscidae), in laboratory and simulated field bioassays. Parasitol Res 108: 1483-1492.

Mochi DA, Monteiro AC, Ribeiro Machado AC and Yoshida L (2010) Entomopathogenic fungal activity against pupae and adult Haematobia irritans (Diptera: Muscidae). Vet Parasitol 168: 105-110.

Mochi DA, Monteiro AC, Simi LD and Moraes Sampaio AA (2009) Susceptibility of adult and larval stages of the horn fly, Haematobia irritans, to the entomopathogenic fungus Metarhizium anisopliae under field conditions. Vet Parasitol 166: 136-143.

Moon RD (2002) Muscid Flies (Muscidae). In: Mullen G and Durden L (eds.) Medical and veterinary entomology. Academic Press, San Diego, CA, USA, pp. 279-301.

Mullens BA, Lii KS, Mao Y, Meyer JA, Peterson NG and Szijj CE (2006) Behavioural responses of dairy cattle to the stable fly, Stomoxys calcitrans, in an open field environment. Med Vet Entomol 20: 122-137.

Muzari MO, Skerratt LF, Jones RE and Duran TL (2010) Alighting and feeding behaviour of tabanid flies on horses, kangaroos and pigs. Vet Parasitol 170: 104-111.

Müller GC, Junnila A, Qualls W, Revay EE, Kline DL, Allan S, Schlein Y and Xue RD (2010) Control of Culex quinquefasciatus in a storm drain system in Florida using attractive toxic sugar baits. Med Vet Entomol 24: 346-351.

Nachman G and Skovgård H (2018) The Fly Simulator: a simulation model of stable flies and their control. In: Garros C, Bouyer J, Takken W and Smallegange RC (eds.) Pests and vector-borne diseases in the livestock industry. Ecology and Control of Vector-borne diseases. Vol. 5. Wageningen Academic Publishers, Wageningen, the Netherlands, pp. 465-494.

Nazni WA, Luke H, Wan Rozita WM, Abdullah AG, Sa'diyah I, Azahari AH, Zamree I, Tan SB, Lee HL and Sofian MA (2005) Determination of the flight range and dispersal of the house fly, Musca domestica (L.) using mark release recapture technique. Trop Biomed 22: 53-61.

Ndeledje N, Bouyer J, Stachurski F, Grimaud P, Belem AMG, Mbaindingatoloum FM, Bengaly Z, Alfaroukh IO, Cecchi G and Lancelot R (2013) Treating cattle to protect people? Impact of footbath insecticide treatment on tsetse density in chad. PLoS ONE 8: e67580.

Nevill EM (1985) The epidemiology of Parafilaria bovicola in the transvaal bushveld of South Africa. Onderstepoort J Vet Res 52: 261-267.

Ogutu JO, Owen-Smith N, Piepho HP and Said MY (2011) Continuing wildlife population declines and range contraction in the Mara region of Kenya during 1977-2009. J Zool 285: 99-109.

Oliveira JB, Montoya J, Romero JJ, Urbina A, Soto-Barrientos N, Melo ESP, Ramos CAN and Araujo FR (2011) Epidemiology of bovine anaplasmosis in dairy herds from Costa Rica. Vet Parasitol 177: 359-365.

Osir EO and Vundla WRM (1999) Characterization of the delta-endotoxin of a Bacillus thuringiensis isolate active against tsetse, Glossina morsitans, and a Stem Borer, Chilo partellus. Biocontrol Sci Techn 9: 247-258.

Oyarzún MP, Quiroz A and Birkett MA (2008) Insecticide resistance in the horn fly: alternative control strategies. Med Vet Entomol 22: 188-202.

Oyarzún MP, Palma R, Alberti E, Hormazabal E, Pardo F, Birkett MA and Quiroz A (2009) Olfactory response of Haematobia irritans (Diptera: Muscidae) to cattle-derived volatile compounds. J Med Entomol 46: 1320-1326.

Paakkonen T, Mustonen AM, Roininen H, Niemela P, Ruusila V and Nieminen P (2010) Parasitism of the deer ked, Lipoptena cervi, on the moose, Alces alces, in eastern Finland. Med Vet Entomol 24: 411-417.

Patton WS and Senior-White R (1924) The oriental species of the genus Musca Linnaeus. Rec Indian Museum 26: 553-577.

Peck GW, Ferguson HJ, LePage JT, Hebert VR, O'Neal SD and Walsh DB (2014) Evaluation of sunlight-exposed pyrethroidtreated netting for the control of face fly and housefly (Diptera: Muscidae). Pest Manag Sci 70: 123-129.

Petersen JJ, Watson DW and Cawthra JK (1995) Comparative effectiveness of 3 release rates for a pteromalid parasitoid (Hymenoptera) of house flies (Diptera) in beef cattle feedlots. Biol Control 5: 561-565.

Pitzer JB, Kaufman PE and Tenbroeck SH (2010) Assessing permethrin resistance in the stable fly (Diptera: Muscidae) in Florida by using laboratory selections and field evaluations. J Econ Entomol 103: 2258-2263. 
Potgieter FT, Sutherland B and Biggs HC (1981) Attempts to transmit Anaplasma marginale with Hippobosca rufipes and Stomoxys calcitrans. Onderstepoort J Vet Res 48: 119-122.

Rahman AHA (2005) Observations on the trypanosomosis problem outside the tsetse belts of Sudan. Rev Sci Tech OIE 24: $965-972$.

Raymond HL (1989) Temporal distribution of the main horse fly species (Diptera, Tabanidae) harmful to cattle in French Guyana. Ann Soc Entomol Fr 25: 289-294.

Richard JL and Pier AC (1966) Transmission of Dermatophilus congolensis by Stomoxys calcitrans and Musca domestica. Am J Vet Res 27: 419-423.

Roberts LW and Wilson BH (1967) Predation on horse flies by two bembicine wasp species in certain areas of southern Louisiana. J Econ Entomol 60: 412-415.

Russel RC, Otranto DP and Wall RL (2013) The encyclopedia of medical and veterinary entomology. CAB International, Wallingford, UK.

Ryman VE, Nickerson SC, Hurley DJ, Berghaus RD and Kautz FM (2013) Influence of horn flies (Haematobia irritans) on teat skin condition, intramammary infection, and serum anti-S. aureus antibody titres in Holstein heifers. Res Vet Sci 95: 343-346.

Salem A, Bouhsira E, Lienard E, Melou AB, Jacquiet P and Franc M (2012) Susceptibility of two European strains of Stomoxys calcitrans (L.) to cypermethrin, deltamethrin, fenvalerate, lambda-cyhalothrin, permethrin and phoxim. Int J Appl Res Vet M 10: 249-257.

Scasta JD, Engle DM, Talley JL, Weir JR, Fuhlendorf SD and Debinski DM (2015) Drought influences control of parasitic flies of cattle on pastures managed with patch-burn grazing. Rangeland Ecol Manag 68: 290-297.

Scasta JD, Engle DM, Talley JL, Weir JR, Stansberry JC, Fuhlendorf SD and Harr RN (2012) Pyric-herbivory to manage horn flies (Diptera: Muscidae) on cattle. Southwest Entomol 37: 325-334.

Sciarretta A, Tikubet G, Baumgartner J, Girma M and Trematerra P (2010) Spatial clustering and associations of two savannah tsetse species, Glossina morsitans submorsitans and Glossina pallidipes (Diptera: Glossinidae), for guiding interventions in an adaptive cattle health management framework. B Entomol Res 100:661-670.

Scoles GA, Broce AB, Lysyk TJ and Palmer GH (2005) Relative efficiency of biological transmission of Anaplasma marginale (Rickettsiales: Anaplasmataceae) by Dermacentor andersoni (Acari: Ixodidae) compared with mechanical transmission by Stomoxys calcitrans (Diptera: Muscidae). J Med Entomol 42: 668-675.

Scoles GA, Miller JA and Foil LD (2008) Comparison of the efficiency of biological transmission of Anaplasma marginale (Rickettsiales: Anaplasmataceae) by Dermacentor andersoni stiles (Acari: Ixodidae) with mechanical transmission by the horse fly, Tabanus fuscicostatus hine (Diptera: Muscidae). J Med Entomol 45: 109-114.

Singh P, King WE and Rodriguez JG (1966) Biological control of muscids as influenced by host preference of Macrocheles muscaedomesticae (Acarina: Macrochelidae). J Med Entomol 3: 78-81.

Skovgård H and Nachman G (2004) Biological control of house flies Musca domestica and stable flies Stomoxys calcitrans (Diptera: Muscidae) by means of inundative releases of Spalangia cameroni (Hymenoptera: Pteromalidae). B Entomol Res 94: 555-567.

Skovgård H and Steenberg T (2002) Activity of pupal parasitoids of the stable fly Stomoxys calcitrans and prevalence of entomopathogenic fungi in the stable fly and the house fly Musca domestica in Denmark. Biocontrol 47: 45-60.

Smallegange RC and Den Otter CJ (2007) Houseflies, annoying and dangerous. In: Takken W and Knols BGJ (eds.) Emerging pests and vector-borne diseases in Europe. Ecology and Control of Vector-borne diseases. Vol 1. Wageningen Academic Publishers, Wageningen, the Netherlands, pp. 281-292.

Soumana IH, Simo G, Njiokou F, Tchicaya B, Abd-Alla AMM, Cuny G and Geiger A (2013) The bacterial flora of tsetse fly midgut and its effect on trypanosome transmission. J Invertebr Pathol 112: S89-S93.

Stoffolano JG (1970) Nematodes associated with the genus Musca (Diptera: Muscidae). B Entomol Soc Am 16: 194-203.

Sukontason KL, Bunchu N, Methanitikorn R, Chaiwong T, Kuntalue B and Sukontason K (2006) Ultrastructure of adhesive device in fly in families calliphoridae, muscidae and sarcophagidae, and their implication as mechanical carriers of pathogens. Parasitol Res 98: 477-481.

Summerlin JW, Roth JP and Fincher GT (1991) Predation by 2 species of histerid beetles on the horn fly. Southwest Entomol 16: 45-49. 
Tan SW, Yap KL and Lee HL (1997) Mechanical transport of rotavirus by the legs and wings of Musca domestica (Diptera: Muscidae). J Med Entomol 34: 527-531.

Tangtrakulwanich K, Albuquerque TA, Brewer GJ, Baxendale FP, Zurek L, Miller DN, Taylor DB, Friesen KA and Zhu JJ (2015) Behavioural responses of stable flies to cattle manure slurry associated odourants. Med Vet Entomol 29: 82-87.

Taylor DB and Berkebile D (2006) Comparative efficiency of six stable fly (Diptera: Muscidae) traps. J Econ Entomol 99: 1415-1419.

Taylor DB, Moon RD and Mark DR (2012) Economic impact of stable flies (Diptera: Muscidae) on dairy and beef cattle production. J Med Entomol 49: 198-209.

Thomas $G$ and Jespersen JB (1994) Non-biting Muscidae and control methods. Rev Sci Tech OIE 13: 1159-1173.

Torr SJ, Chamisa A, Vale GA, Lehane MJ and Lindh JM (2011a) Responses of tsetse flies, Glossina morsitans morsitans and Glossina pallidipes, to baits of various size. Med Vet Entomol 25: 365-369.

Torr SJ, Mangwiro TNC and Hall DR (2011b) Shoo fly, don't bother me! Efficacy of traditional methods of protecting cattle from tsetse. Med Vet Entomol 25: 192-201.

Torr SJ, Maudlin I and Vale GA (2007) Less is more: restricted application of insecticide to cattle to improve the cost and efficacy of tsetse control. Med Vet Entomol 21: 53-64.

Turell MJ and Knudson GB (1987) Mechanical transmission of Bacillus anthracis by stable flies (Stomoxys calcitrans) and mosquitoes (Aedes aegypti and Aedes taeniorhynchus). Infect Immun 55: 1859-1861.

Vale GA, Hargrove JW, Chamisa A, Grant IF and Torr SJ (2015) Pyrethroid treatment of cattle for tsetse control: reducing its impact on dung fauna. PLoS Neglect Trop Dis 9: e0003560.

Vale GA, Lovemore DF, Flint S and Cockbill GF (1988) Odor-baited targets to control tsetse flies, Glossina spp. (Diptera, Glossinidae) in Zimbabwe. B Entomol Res 78: 31-49.

Van den Bossche P, De la Rocque S, Hendrickx G and Bouyer J (2010) A changing environment and the epidemiology of tsetse-transmitted livestock trypanosomiasis. Trends Parasitol 26: 236-243.

Van Hennekeler K, Jones RE, Skerratt LF, Muzari MO and Fitzpatrick LA (2011) Meteorological effects on the daily activity patterns of tabanid biting flies in northern Queensland, Australia. Med Vet Entomol 25: 17-24.

Villa A, Gutierrez C, Gracia E, Moreno B, Chacon G, Sanz PV, Buescher P and Touratier L (2008) Presence of Trypanosoma theileri in Spanish cattle. Animal biodiversity and emerging diseases: prediction and prevention. Ann NY Acad Sci 1149: 352-354.

Vitela-Mendoza I, Cruz-Vazquez C, Solano-Vergara J and Orihuela-Trujillo A (2016) Relationship between serum cortisol concentration and defensive behavioral responses of dairy cows exposed to natural infestation by stable fly, Stomoxys calcitrans. J Dairy Sci 99: 9912-9916.

Vockeroth JR (1953) Musca autumnalis Deg. in North America (Diptera: Muscidae). Can Entomol 85: 422-433.

Vreysen MJB, Seck MT, Sall B and Bouyer J (2013) Tsetse flies: their biology and control using area-wide integrated pest management approaches. J Invertebr Pathol 112: 15-25.

Vriesekoop F and Shaw R (2010) The Australian bush fly (Musca vetustissima) as a potential vector in the transmission of foodborne pathogens at outdoor eateries. Foodborne Pathog Dis 7: 275-279.

Walker A (1994) Arthropods of humans and domestic animals: a guide to preliminary identification. Chapman and Hall, London, UK.

Watson DW, Geden CJ, Long SJ and Rutz DA (1995) Efficacy of Beauveria bassiana for controlling the house fly and stable fly (Diptera, Muscidae). Biol Control 5: 405-411.

Weeks ENI, Machtinger ET, Leemon D and Geden CJ (2018) Biological control of livestock pests: entomopathogens. In: Garros C, Bouyer J, Takken W and Smallegange RC (eds.) Pests and vector-borne diseases in the livestock industry. Ecology and Control of Vector-borne diseases. Vol. 5. Wageningen Academic Publishers, Wageningen, the Netherlands, pp. 337-387.

Weeks ENI, Machtinger ET, Gezan SA, Kaufman PE and Geden CJ (2016) Effects of four commercial fungal formulations on mortality and sporulation in house flies (Musca domestica) and stable flies (Stomoxys calcitrans). Med Vet Entomol 31(1): 15-22.

World Health Organisation (WHO) (2013) Control and surveillance of human African trypanosomiasis: report of a WHO expert committee. WHO, Geneva, Switzerland. 
Frédéric Baldacchino et al.

Yeruham I, Nir O, Braverman Y, Davidson M, Grinstein H, Haymovitch M and Zamir O (1995) Spread of lumpy skin disease in Israeli dairy herds. Vet Rec 137: 91-93.

Zhu JJ, Berkebile DR, Dunlap CA, Zhang A, Boxler D, Tangtrakulwanich K, Behle RW, Baxendale F and Brewer G (2012) Nepetalactones from essential oil of Nepeta cataria represent a stable fly feeding and oviposition repellent. Med Vet Entomol 26: 131-138.

Zhu JJ, Wienhold BJ, Wehrle J, Davis D, Chen H, Taylor D, Friesen K and Zurek L (2014) Efficacy and longevity of newly developed catnip oil microcapsules against stable fly oviposition and larval growth. Med Vet Entomol 28: 222-227.

Zhu JJ, Zhang QH, Taylor DB and Friesen KA (2015) Visual and olfactory enhancement of stable fly trapping. Pest Manag Sci 72(9): 1765-1771.

Zumpt F (1973) The Stomoxyine biting flies of the world. Diptera: Muscidae. Taxonomy, biology economic importance and control measures. Gustav Fischer Verlag, Stuttgart, Germany. 


\title{
4. Acaricides: current status and sustainable alternatives for controlling the cattle tick, Rhipicephalus microplus, based on its ecology
}

Isabel K.F. de Miranda Santos ${ }^{1 *}$, Gustavo R. Garcia' ${ }^{1}$ Priscila Silva Oliveira ${ }^{1}$, Cecilia J. Veríssimo², Luciana M. Katiki ${ }^{2}$, Leandro Rodrigues ${ }^{2}$, Matias P.J. Szabó ${ }^{3}$ and Christine Maritz-Olivier ${ }^{4}$

${ }^{1}$ Department of Biochemistry and Immunology, Ribeirão Preto School of Medicine, University of São Paulo, Avenida Bandeirantes 3900, Monte Alegre, Ribeirão Preto, SP 14049-900, Brazil; ${ }^{2}$ São Paulo State Animal Science Institute, Rua Heitor Penteado, 56, Nova Odessa, SP 13460-000, Brazil; ${ }^{3}$ School of Veterinary Medicine, Federal University of Uberlândia, Av. Pará, 1720, Campus Umuarama-Bloco 2T, 38400-902 Uberlândia, MG, Brazil; ${ }^{4}$ Faculty of Agricultural and Natural Sciences, University of Pretoria, Department of Genetics, Private bag X20, Hatfield 0028 Pretoria, South Africa; imsantos@fmrp.usp.br

\begin{abstract}
Rhipicephalus microplus, the cattle tick, causes enormous losses for producers of beef, dairy and leather. Currently acaricides are the only method of treatment available to producers, but they are losing their efficacy due to alarming rates of resistance in tick populations, with new mechanisms of resistance being described regularly. Producers must invest in testing the level and type of resistance in field populations in order to select an effective treatment for subsequent seasons, to hinder spread of resistant ticks and achieve the most cost-effective strategy. Besides the levels of resistance to acaricides currently seen, these chemicals also impact greatly on the environment and on the quality of animal products consumed downstream in the food chain. In view of these problems, new and sustainable technologies to control infestations of ticks in cattle (and other commonly used meat-providing hosts) are urgently needed. Feasible alternatives consist of natural products from botanical extracts that kill ticks and/or have repellent properties, capable of modify the off- and on-host ecosystems of the tick. Modifications of the off-host ecosystem of the $R$. microplus tick consists of increasing its chances for desiccation, predation and/or infection of adults, eggs and larvae to reduce fecundity and hampering the host-seeking capacities of larvae and their vectorial capacity. The choice of a production system, including choice of pasture, and biological control agents can assist in achieving this goal. Modifications of the on-host ecology require that the bovine host mounts efficacious reactions to expel or repel ticks and/or hamper their blood-feeding and vectorial capacity; these responses can be induced by genetic selection, vaccination and/or interventions in the host's and in the tick's microbiota. Control strategies must consider endemic stability for maintaining adequate levels of immunity against tick-borne pathogens; they also require that producers understand their mechanisms of action in affecting tick biology in order to maintain adequate compliance and thus success.
\end{abstract}

Keywords: Rhipicephalus microplus, acaricides, on-host ecology, off-host ecology, microbiota, sustainable technologies

\section{Introduction}

This chapter focusses on information pertaining to Rhipicephalus microplus Canestrini, but much of it is applicable to the other important parasites for cattle, namely Rhipicephalus appendiculatus Neumann, Rhipicephalus decoloratus Koch and Rhipicephalus australis Fuller, mostly where novel strategies for control are concerned. It updates advances in ongoing studies on established or developing technologies as well as resistance to acaricides, tests for resistance and natural 
products and calls attention to novel opportunities where research could be warranted and efforts invested. The recent assembly and annotation of the $7.1 \mathrm{Gbp}$ genome of $R$. microplus (Barrero et al. 2017) revealed 38,827 putative gene loci, with 24,758 protein coding genes, of which 3,423 are unique to $R$. microplus or common to both $R$. microplus and Ixodes scapularis Say. This new and much-needed data provides a valuable opportunity for the scientific community to explore this parasite from a new angle in order to better understand its biology and develop sustainable interventions. It is already shedding light on mechanisms of resistance to acaricides. Gene editing in other haematophagous vectors, namely the anopheline vectors of malaria (Gantz et al. 2015; Hammond et al. 2016), is also proving to be another advance and is potentially applicable to the cattle tick, with multiplex strategies of gene editing being proposed in order to avoid evolution of resistance (Marshall et al. 2017).

\section{The impacts of infestations with the cattle tick, R. microplus}

In tropical and subtropical areas of the globe, the direct and indirect effects of infestations with $R$. microplus cause huge losses to livestock producers (Jongejan and Uilenberg 2004; Jonsson 2006; Jonsson et al. 2001). The magnitude of these losses can be assessed by the situation of producers in Brazil, home to the largest commercial herd of cattle in the world: the most recent estimate for direct losses was US\$ 3.4 billion in 2012 (Grisi et al. 2014). Computation of losses covers reduction of weight gain and productivity due to blood feeding, stress inflicted on the hosts by the ticks' bites, the toxic activity of a myriad of proteins that ticks salivate into their hosts' skin, the diseases that the ticks transmit, damage to leather and costs with applications of the acaricides currently employed to control infestations (Jonsson 2006; Seifert 1971). Dairy producers in Brazil estimate that controlling ticks and tick-borne diseases represents up to $60 \%$ of all costs to maintain the health of their animals, including vaccinations and controlling endoparasites and mastitis, also significant health problems in dairy farms. Not computed in these costs are the impacts of acaricides on the environment and on human and animal health. Since rainfall and temperature have important effects on the life cycle and survival of $R$. microplus, researchers expect the ongoing climate change to affect the problems ticks cause, although many feel that this specific issue warrants more knowledge about the ecology of this parasite in order to make precise predictions (reviewed by Estrada-Peña and Salman 2013; Estrada-Peña et al. 2017).

\section{The impacts of acaricides}

Acaricides are currently the main option employed by producers to control infestations with $R$. microplus. Indiscriminate practices in their handling have resulted in the appearance, worldwide, of large numbers of populations of $R$. microplus ticks resistant to many if not all commercially available acaricides and this phenomenon is further increasing worldwide. In addition to the problem of increasing resistance, the use of acaricides is not sustainable because they affect many types of fauna (Bai and Ogbourne 2016; Groh et al. 2015; Korta et al. 2003; Li et al. 2017; Werner and Moran 2008). There have been many studies on this issue and this chapter cites only the most recent ones in order to demonstrate that the environmental impact of acaricides is a persisting, if not increasing problem. While acaricides can act in synergy with entomopathogens, as shown by several studies (Bahiense and Bittencourt 2004; Farenhorst et al. 2010), they may also negatively affect the very entomopathogens that are desirable to control the cattle tick (Ferreira et al. 2016; Singh et al. 2010; Vieira et al. 2016). In addition, acaricides have to the potential to induce dysbioses in hosts (Nasuti et al. 2016) and possibly modify the microbial composition of soil (Qi and Wei 2017), with other negative consequences for many aspects of cattle and/or environmental health besides the latter's entomofauna. Finally, mainly due to mismanagement, among other factors, 
acaricides leave residues in beef and dairy products as attest recent publications (Macedo et al. 2015; Picinin et al. 2016; Qin et al. 2017), thus threatening consumers' health and food safety, as well as food security. Residues in animal products also create trade barriers (Lively 2013; Schröder et al. 2016) that can negatively affect national economies. The economic losses caused by these factors have not been computed in any study to date.

\section{Acaricides}

\section{Current status of resistance to acaricides}

One of the first reports of resistance to an acaricide probably is that describing resistance to organophosphorus insecticides in Australia (Shaw and Malcom 1964). Since then there have been reports describing resistance to all classes of acaricides and the number of reports has only increased. In the last five years there have been many further reports on different levels of resistance to most if not all of the acaricides most widely employed for control of $R$. microplus ticks (synthetic pyrethroids, organophosphates, cyclodienes, macrocyclic lactones, and the chitin synthesis inhibitor fluazuron) in all countries that are home to significant populations of cattle in tropical and subtropical climates: Argentina, Australia, Brazil, Mexico, India and South Africa, in addition to many other countries (Abbas et al. 2014; Guerrero et al. 2012a; Heath and Levot 2015; Lovis et al. 2013a; Rosario-Cruz et al. 2009; Rodríguez-Hidalgo et al. 2017; Rodríguez-Vivas et al. 2014). The most recent studies (Gaur et al. 2016; Heath and Levot 2015; Jyoti et al. 2016b; Klafke et al. 2017; Maciel et al. 2016; Robbertse et al. 2016; Rodríguez-Hidalgo et al. 2017; Van Wyk et al. 2016) reporting the frequency of resistance to acaricides (from 2015 to 2017 at the time of writing of this chapter,) were for farms in Australia, Brazil, Ecuador, India and South Africa. In Australia, so far formamidine, a benzoylphenyl urea and macrocyclic lactones are efficacious in recommended concentrations, but resistance has been detected to macrocyclic lactones in some strains. In the South of Brazil, the frequency of resistance in 104 field samples to amitraz, chlorpyriphos, ivermectin and fipronil was recently found to be $76.92,60.58,60.58$ and $53.85 \%$, respectively (Klafke et al. 2017). Multiple resistances to three or more compounds were found in $78.85 \%$ of the samples. As stated by the authors of that study, these are alarming figures. In the south of Brazil, a field tick population was recently found to be resistant to six classes of acaricides, including fluazuron (Reck et al. 2014). In the southeast and midwest regions of Brazil, four populations of $R$. microplus were diagnosed as resistant to fluazuron and efficacy of the association of a macrocyclic lactone, eprinomectin, with a new compound similar to fluazuron, novaluron, was inferior to $49 \%$. In a nation-wide study that employed one of two assays to evaluate the effect of pyrethroids, organophosphates, aminidines, and pyrethroid/organophosphate combinations on ticks, overall resistance was found to be $47.64 \%$ and efficacy of less than $90 \%$ (i.e. the percentage of ticks killed by the compound) was found in various locations throughout Brazil, including in states where resistance had never been found (Higa et al. 2016). In Ecuador, a resistance of $67 \%$ was reported for amitraz, ibid 50\% for alpha-cypermethrin and from 25 to $42 \%$ for ivermectin in twelve field populations analysed; the effective estimated dose needed to eliminate $99 \%$ of ticks (which is the minimum percentage of ticks that must killed in order for an acaricide to be considered effective) was higher than the dose recommended by the manufacturer (Rodríguez-Hidalgo et al. 2017). In India, all field isolates from the Punjab that were tested were found to be resistant against fenvalerate (Jyoti et al. 2016a); ticks collected from the Tohana region of Haryana state were found to be resistant to both deltamethrin and diazinon, while the ticks of the Agroha region of Haryana state were found to be susceptible (Gaur et al. 2016); resistance to amitraz and malathion was found in the field in the Jammu region (Dutta et al. 2017). In South Africa, a survey from 108 farms was analysed using molecular diagnostic tools and indicated for pyrethroid resistance a 
homozygous resistant $R$. microplus population of $58.8 \%$ and a heterozygous-resistant genotype of 8.8\% (Van Wyk et al. 2016). With regards to amitraz resistance, the frequencies of susceptible (AA/ $\mathrm{TT})$ and resistant $(\mathrm{CC} / \mathrm{CC})$ genotypes were calculated for the resistance linked single nucleotide polymorphisms (SNPs) occurring in the OCT/Tyr receptor gene with the majority of the population exhibiting the heterozygous (AC/TC) genotype at these nucleotide positions, with half of the population displaying the resistance $C$ allele for amitraz (Baron et al. 2015). In the Mnisi-area adjacent to the Kruger National Park, a high prevalence of alleles attributed to resistance against formamidines (amitraz) in the octopamine/tyramine (OCT/Tyr) receptor (55\%) and pyrethroids in the carboxylesterase (8\%) genes were observed. Overall, the sampled tick population was homozygous resistant to pyrethroid-based acaricides in the voltage-gated sodium channel gene (Robbertse et al. 2016). These results are from matter of course studies, relatively randomly focused surveys on resistance to specific acaricides undertaken with the resources available, but due to their sheer numbers, at this stage they reflect the dramatic situation of resistance to acaricides that more systematic studies would magnify.

\section{Molecular mechanisms of resistance to acaricides}

The mechanisms of resistance presented by $R$. microplus to pyrethroid, organophosphate, amitraz, fipronil and macrocyclic lactone acaricides have been well reviewed by Guerrero et al. (2012a). The following sections provide insight into mechanisms and how they can be exploited for the development of diagnostic assays to allow rapid screening of tick populations.

Our understanding of macrocyclic lactone resistance (based on the widely used ivermectin) was generated with a line of embryonic cells from $R$. microplus that is resistant to ivermectin (Pohl et al. 2014); this line was obtained by treating the ivermectin-sensitive cell line with increasing doses of the acaricide over a 46 week period. Generation of resistance depended on an increased expression of a gene encoding RmABCB10, an $A B C$ transporter of the B10 subfamily. Resistance diminished when the cell line was treated with cyclosporine, an inhibitor of $A B C$ transporters, supporting this mechanism. This cell line may assist in further understanding of resistance to acaricides and pave the way to studying resistance to other classes of acaricides in vitro. In addition, the same group also determined that this $A B C$ transporter also detoxifies heme from host blood by accumulating this compound in the hemosomes of tick gut cells (Lara et al. 2015).

The most common formamidine used for tick control is amitraz, for which the target site for in $R$. microplus has yet to be defined, which ultimately delays any further development with regard to screening assays for diagnostics. It was proposed that monoamine oxidase, alpha-2-adrenoceptors and the octopamine receptor could be candidates for the target site of amitraz, with the latter being the most probable in ticks (Jonsson and Hope 2007). It is thought that amitraz is a potential agonist of the octopaminergic system located in the tick synganglion. In the presence of amitraz, the octopamine receptor is activated and this overstimulation of synapses has lethal effects on the tick (Booth 1989; Lees and Bowman 2007). The octopamine receptor is a G-protein coupled receptor (GPCR) and in 1999 Baxter and Barker discovered that the $R$. microplus octopamine receptor sequence contained eight hydrophobic domains in contrast to the seven found in most GPCRs (the role of this additional transmembrane loop still needs to be elucidated). Previous studies have also shown that this receptor also regulates egg production in female ticks as well as the production of a wax-like layer along their oral cavities (Lees and Bowman 2007).

Again, studies by Pohl et al. (2014) contributed to our understanding using their in vitro system. Importantly, they showed that amitraz accumulated into the hemosomes by using the same 
transporter (see next section on formamidine resistance); in addition, an amitraz-resistant strain of $R$. microplus detoxified both amitraz and an inhibitor of heme-oxygenase more efficiently than the susceptible strain. This study did not compare the rate of accumulation of heme in the hemosomes of the amitraz-resistant and -sensitive strains of $R$. microplus, but this aspect merits examination since the hematocrits of tick-resistant indicine cattle and tick-susceptible taurine cattle differ consistently and significantly, being higher in indicine cattle (I.K.F. De Miranda Santos, unpublished observations; Hernandez et al. 2002); hosts with higher hematocrits may possibly interfere in the detoxification mechanisms of acaricide-resistant ticks.

Resistance to amitraz is complex and multigenic in nature, and involves recessive inheritance of resistant alleles (Fragoso-Sanchez et al. 2011; Li et al. 2004, 2005). To date no resistance mechanisms against amitraz have been shown inequivocally for $R$. microplus, however, several have been suggested. Li et al. (2004) illustrated that metabolic detoxification plays a role in amitraz resistance in Mexican tick strains by means of synergistic studies with enzyme inhibitors. These results were, however, variable across different $R$. microplus strains with the overall contribution of detoxifying enzymes being difficult to evaluate (Li et al. 2005). The Mexican Pesqueria strain was confirmed to convey metabolic resistance to amitraz by up-regulation of glutathione-Stransferase that was established by synergistic assays using diethylmaleate (Saldivar et al. 2008). It was then suggested that perhaps target site insensitivity could be the main mechanism of amitraz resistance, however, studies to illustrate this have not been conclusive (Fragoso-Sanchez et al. 2011; Guerrero et al. 2012a).

Based on sequence alignments between a susceptible Australian strain, the susceptible American Gonzalez strain, and the resistant Brazilian Santa Luiza strain two SNPs occurring in genes encoding the octopamine receptor maybe linked to amitraz resistance (Chen et al. 2007). Apart from this, there has yet been no direct evidence of target site resistance in the octopamine receptor.

Pyrethroids are synthetic composites encompassing similar attributes to extracts from the Chrysanthemum cinerariaefolium flowers. Pyrethrins represent the easily oxidized building blocks of pyrethroids (Anadon et al. 2009). There are two classes of pyrethroids, with a cyano-group present in class II but not class I. Additional substitutions such as chlorine or bromine ions can also be found within the different classes. The voltage-gated sodium channel is the well-established target of synthetic pyrethroids, which in arthropods occurs in nerve chemical synapses, allowing sodium ions to flow into the cell (Dong 2007). The inactivation gate of the sodium channel contains a motif of isoleucine-phenylalanine-methionine-threonine (IFMT), which plays an essential role in the activation of the voltage-gated sodium channel (Yu et al. 2005). Upon exposure to pyrethroids, the sodium channel undergoes hyperpolarization, causing neurons to swiftly liberate resulting in convulsions and eventual death of the arthropod. This effect is brought about by the prevention of the IFMT deactivation mechanism (Lees and Bowman 2007; Yu et al. 2005).

Metabolic resistance towards pyrethroids has been documented in the Coatzacoalcos Mexican $R$. microplus tick strain based on synergistic assays with piperonyl butoxide and triphenyl phosphate (Miller et al. 1999). It was also found within this particular strain that there was an up-regulation in the expression of an esterase (CzEst9) which seemed to be involved in the rapid hydrolysis of permethrin (Pruett et al. 2002). Further studies then corroborated that this carboxylesterase enzyme played a major role in pyrethroid resistance in the Mato Grosso Brazilian strain (Baffi et al. 2007). It appears that target site resistance is the major resistance mechanism to pyrethroids in R. microplus ticks. Three SNPs in the voltage-gated sodium channel have been reported to be associated with pyrethroid resistance in ticks. He et al. (1999) discovered the first SNP in domain III 
of the sodium channel in tick populations from Mexico, resulting in a phenylalanine to isoleucine mutation. This particular mutation seems to be confined to North America and has not been found in other regions of the world.

Target site mutations resulting in pyrethroid resistance were predominantly found to occur in domain II in other arthropods that were investigated (Soderlund and Knipple 2003). This subsequently led to the discovery of two SNP's in domain II S4-5 of the linker region of the sodium channel. Morgan et al. (2009) reported an amino acid change from leucine to isoleucine, while Jonsson et al. (2010) showed a glycine to valine substitution. Of the three mutations that have been reported, it has been suggested that those in domain III phenotypically convey a higher level of resistance while those reported by Morgan et al. (2009) confer moderate resistance (100400 fold). The domain II mutation reported by Jonsson et al. (2010) only conferred resistance to flumethrin (Guerrero et al. 2012a). Upon comparing all of the latter, it is clear that the most common mutation encountered is that published by Morgan et al. (2009). The study by Guerrero et al. (2001) represents the mutation published by He et al. (1999) in domain III, which appears to be persistent throughout Mexico. The mutation published by Jonsson et al. (2010) seems to have only been reported in Australia. In the presence of both metabolic and target site resistance within a tick, target site resistance has shown to be the major contributor to pyrethroid resistance. This is supported by the observation that apart from the mutations occurring in the voltagegated sodium channel, an additional mutation has been reported in the carboxylesterase gene that has been associated with pyrethroid resistance in Mexican tick populations (Hernandez et al. 2000, 2002). Baffi et al. (2007) suggested that this mutation was also involved in organophosphate resistance; however, others have not recognized this conception in the field.

Organophosphates and carbamates have been shown to target acetylcholinesterase (AChE, E 3.1.1.7) as a quasi-irreversible inhibitor in R. microplus ticks (Pruett, 2002; Pruett et al. 2002). $\mathrm{AChE}$, a serine hydrolase, is responsible for the degradation of the neurotransmitter acetylcholine (ACh) into acetate and choline at post-synaptic membranes. In the presence of an OP, the serine residue is phosphorylated in the enzyme active site, thereby disabling catalytic function (Pang et al. 2012). This prevents the breakdown of ACh into acetate and choline, leading to the buildup of ACh at the postsynaptic membrane and resulting in neuromuscular paralysis (Lees and Bowman 2007). Regarding resistance to products that target acetylcholinesterases, Bendele et al. (2015) determined that, among the three variants of the genes encoding these enzymes described in this species of tick, acetylcholinesterase 1 (AChE1) presented with 72 sequence variants in larvae from a mere seven strains of $R$. microplus, two of which were strongly associated with resistance to the organophosphate coumaphos. Jyoti et al. (2016b), in turn, examined sequences of the AChE3 gene in field isolates in which resistance to Malathion was detected and found evidence of non-synonymous mutations in the resistant isolates. Jamroz et al. (2000) showed that a carboxylesterase (Est10) was significantly abundant in organophosphate resistant Mexican strains, perhaps suggesting an involvement in the metabolic detoxification of the acaricide. Four coumaphos resistant Mexican strains were shown to confer resistance via an elevated cytochrome P450 mediated response, but the same was not obtained for diazinon resistant strains (Li et al. 2003). Glutathione S-transferase has also been associated with coumaphos resistance in the San Alfonso Mexican tick strain (Saldivar et al. 2008).

Target site resistant mechanisms remain unclear within AChEs largely due to the uncertainty surrounding the true identity of the transcript that is functionally pertinent to organophosphate resistance. As previously mentioned there are currently three BmAChEs that have been proposed in R. microplus. Researchers have extensively investigated these BmAChEs in the hope of discovering 
a mutation that confers resistance to organophosphates with unsuccessful results thus far (Baxter and Barker 1998; Hernandez et al. 1999; Temeyer et al. 2004). Temeyer et al. (2010) showed target site resistance in two malaoxon and paraoxon resistant $R$. microplus tick strains. The susceptible Deutch strain comprised of 13 amino acid substitutions, which seemed to be prevalent among susceptible strains. Two San Roman strains (SR4 and SR11) revealed 13 amino acid substitutions with nine being common between the two constructs. The resistant Tuxpan (Tx11) strain displayed four amino acid substitutions, which were unique to this particular construct and generated an expressed product which was insensitive to paraoxon. These results strongly suggest that organophosphate resistance is largely complex and perhaps multigenic in nature with the involvement of more than one AChE in the resistance phenotypic profile.

Cyclodienes, also known as organochlorides, are known to target the gamma-aminobutyric acid (GABA) gated chloride channel (Lees and Bowman, 2007). Dieldrin is a common cyclodiene acaricide however; its use has been prohibited in the vast majority of developing countries due to its excessive toxicity. The GABA-gated chloride channel plays an essential role at the neuromuscular junction and synapses in the central nervous system of the tick. The arrangement of the channel includes five protein chains that assemble to exhibit a pentameric like structure with receptor and membrane channel domains. Upon exposure to an acaricide such as dieldrin, the GABA neurotransmitter cannot bind to the receptor, thus preventing chloride ions from passing through the channel. This results in hyperexcitation at the nerve synapses consequentially leading to convulsion within the arthropod (Bloomquist 2003). Target site resistance has been reported against dieldrin in the GABA-gated chloride channel for $R$. microplus tick populations from Australia. A two base pair mutation results in an amino acid change from threonine to leucine. The mutation was present in $20 \%$ of tested samples, even though the chemical dieldrin was withdrawn from use in Australia approximately 20 years ago. This suggests that the mutation caused by exposure to dieldrin could be irreversible, or in the absence of selection pressure take prolonged periods of time to disappear from the gene pool. It has been suggested that the development of cross resistance may occur due to the presence of this mutation with other acaricides, which share the same target site (i.e. fipronil and macrocyclic lactones). Whether this actually takes place still has to be clarified. This particular mutation has the potential to shed some light onto the exposure of resistance mechanisms for other acaricides such as fipronil and macrocyclic lactones (Guerrero et al. 2012a).

It is seemingly clear that further investigation is required in the clarification and characterization of resistance mechanisms in $R$. microplus ticks both with regards to metabolic and target site resistance. A comprehensible explanation of these mechanisms will provide much needed insight for the future development of improved acaricides and their overall longevity in the field. Without this much-needed information, adequate tick control programs will remain elusive. With the assembly and annotation of the genome of $R$. microplus (Barrero et al. 2017) it was possible to observe significant diversity of several esterases similar to acetylcholinesterases; it was also possible to observe a lack of domains in certain cytochrome CYP proteins, including a novel CYP41, that could impact their interaction with exogenous compounds such as acaricides. This new resource can provide new insights on mechanisms of resistance to acaricides and assist in the development of new products.

\section{Strategies to evaluate resistance to acaricides}

There are several assays available that can be used to detect acaricide resistance in ticks. These include the larval packet test (Stone and Haydock 1962), the shaw larval immersion test (Shaw 
1966), adult immersion tests (Drummond et al. 1973) and larval tarsal tests (Lovis et al. 2011). These assays are time-consuming with most only providing results after 6 weeks. Regarding the design of tests to evaluate performance of acaricides in field samples of ticks, the larval package test has been reported to be the most reliable compared to the other options employed by investigators (larval immersion test and syringe immersion test) (Santos et al. 2013), but the recent study from Ecuador demonstrated that there were no statistical differences between the performances of the adult immersion, larval package and larval immersion tests (Rodríguez-Hidalgo et al. 2017). A simplified larval tarsal test was recently evaluated (Lovis et al. 2013b) While relying on equipment, which constrains its widespread application, it was comparatively superior to the larval package test because it detected higher resistance ratios to all compounds and allowed testing a larger number of compounds simultaneously. Synergistic assays have also been employed to identify the involvement of metabolic enzymes in acaricide-resistant tick populations. The synergists include triphenyl phosphate, piperonyl butoxide and diethylmaleate, which inhibit esterase activity, cytochrome P450 oxidase and glutathione S-transferase, respectively (Guerrero et al. 2012a; Li et al. 2007). As these synergistic assays are coupled to larval packet test assays, results are only obtained after 6 weeks.

As all of the latter assays do not have the ability to identify target site resistance mechanisms in tick populations, a more rapid approach to acaricide resistance diagnostics is required. Rapid screening for SNPs associated with acaricide resistance has gained much attention. An allelespecific assay has been developed for detection of SNPs in the voltage-gated sodium channel linked to pyrethroid resistance (Guerrero et al. 2001). A restriction fragment length polymorphism assay has been developed to detect resistance against pyrethroids in the carboxylesterase gene (Baffi et al. 2007) as well as amitraz resistance in the octopamine/tyramine receptor (Baron et al. 2015) and against organophospahtes and pyretrhoids (Faza et al. 2013). Finally, Brito et al. (2017) recently described a quantitative molecular assay to detect increases in synthesis of acetylcholinesterase 2 and esterase 1 in organophosphate-resistant strains of ticks in comparison to susceptible strains. In future, routine diagnositic analysis should be compulsory in areas of acaricide resistance to lessen the selective pressure and prevent spreading of resistant ticks to non-resistant areas.

\section{Strategies for using acaricides on cattle that alleviate emergence of resistance and contamination of products with residues}

Rural extension services have begun indicating two strategies for employing acaricides. Strategic or prophylactic control involves applying acaricides in anticipation to increases in infestations due to changes in seasons, rainfall, and pasture management, among other features (Jonsson and Matschoss 1998; Nari 1995). It is deemed to be especially effective in areas were seasons are well defined. As a result of this practice farmers observe cleaner pastures and thus lower tick loads, but enough ticks to ensure enzootic stability for effective immunity against tickborne diseases. Because it exposes reduced numbers of ticks to acaricides, this practice may also reduce selective pressure on ticks upon applying acaricides and thus delay emergence of resistant strains. It also reduces farmers' productions costs. However, formal studies have yet to be undertaken to comparatively examine the genetics and phenotypes of tick populations from properties employing conventional application of acaricides (i.e. according to manufacturers' recommendations) and properties employing strategic treatments. A limitation of strategic applications of acaricides is that producers must have, but rarely do have sufficient knowledge about tick biology, which is essential for commitment and adherence to this practice (Chevillon et al. 2007; Rocha et al. 2006). 
Another option is so-called targeted selective treatment, adopted from practices for treatment of endoparasites. It involves treating only the animals that maintain high tick loads (Martins et al. 2002; Molento et al. 2013) and considers that hosts are differentially infested and thus ticks are aggregated on hosts so that only a few individual bovines host the majority of parasites due to their particular sensitivities. These animals must be identified by the farmer, something that is very feasible on dairy farms with observations from day-to-day management and where animals are in service for long periods and exposed to several cycles of infestations. On the other hand, frequent observations are not feasible for most meat and rural farmers, especially in developing countries that are making use of communal grazing. In any case, this strategy, besides reducing costs, avoids or delays emergence of resistant parasites. Resistance is avoided because the parasites find a refuge in the non-treated animals and thus maintain high frequency of genotypes that render parasites amenable to acaricides (see, for example, Berk et al. 2016). However, its true effect in controlling infestations with R. microplus is still controversial: Morel et al. (2017) did not find evidence that selective treatment of animals carrying higher tick loads was effective for controlling infestations. Conversely, Martins et al. (2002) did verify that selective treatment reduced the number of acaricide applications and of costs by $10 \%$, but concluded that these numbers did not represent a sufficient gain for producers to justify the effort in identifying the animals to target. Futhermore, aggregation of ticks on certain hosts has been found by Calabrese et al. (2011) to be due to the 'simple bad luck' of a given host being in a more infested patch of space or, in the case of cattle and R. microplus, pasture. This seems to be corroborated by the study of Morel et al. (2017) because they also found that aggregation was related to the levels of ticks in pastures: the higher the abundance of $R$. microplus, the less the parasites aggregated on certain animals. In all, this suggests that there are no intrinsic differences in susceptibility. However, as will also be informed below in the topic on breeding for resistance, in the experience of some of the authors of this chapter, certain bovines do maintain consistently high tick loads in relation to other animals contemporary in the same pasture and management. In addition, since the reproductive efficiency is higher in ticks feeding on bovines carrying higher tick loads (Biegelmeyer et al. 2015), possibly due to less efficient immunity in these hosts, these animals may consequently produce more larvae and, thus, more heavily infested pastures. In all, to the best of the authors' knowledge, no study has been designed to identify bovines that consistently present high tick loads and that then treats these animals exclusively and compares the results with those found in a similar, but untreated group undergoing the same level of infestation; this design would assess the impact of targeted treatment because it would remove ticks from the more susceptible animals, while ticks feeding on more resistant animals would reproduce less efficiently.

Since hosts carrying lower tick loads may have more efficient anti-tick immunity, the effect of selective treatment on selective pressure must also be confirmed with genetic studies of tick populations (for which rapid genetic diagnostics will be invaluable; see previous section). While the genotype of the more resistant hosts may have an effect upon the genotypes of ticks feeding on them, a hypothesis that still needs to be tested, it probably does not select for acaricide resistance since the mechanisms involved in expelling skin parasites are different from those that poison them.

In regard to the link between genetic diversity (using conventional ITS2 sequencing), acaricide resistance status and evolutionary potential of a $R$. microplus population, when evaluating a population of ticks in South Africa, Robberste et al. (2016) found an abundance of heterozygosity in the population within the Oct/Tyr gene (associated with amitraz resistance) and homozygous resistant for the SNP in the voltage-gated sodium channel and carboxylesterase gene (both associated with pyrethroid resistance), combined with high haplotype diversity contrasted to low 
nucleotide diversity. Their evidence supports that the $R$. microplus population underwent rapid recent expansion with an excess of rare haplotypes. Most noteworthy is that that the majority of the rare, expanding haplotypes could be linked to multiple acaricide resistance. This to date is the first study to suggest a link between tick genotype, heritability of resistant markers and rapid expansion of multi-acacricide resistant tick populations, opening a window into understanding the rapid development of multi-resistant haplotypes. Lastly, a paper by Baron et al. (2015) indicated that the two SNPs associated with amitraz resistance are in gametic disequilibrium and that recombination was detected in the $\mathrm{OCT} / \mathrm{Tyr}$ receptor generating two recombinant haplotypes. They were also able to show that at least a minimum of two recombination events needed to occur in order to generate the resistant haplotypes observed in the field. These studies are paving the way to to enable future 'timing' of the development of resistance and early intervention in heterozygous populations.

\section{Alternatives to acaricides}

\section{Natural products for treating cattle}

Efforts to study phytotherapeutic products with acaricide action have increased in the last two decades, due to the serious problem of tick resistance to acaricides, to consumers' concerns about animal products with chemical residues, besides a general lack of perspective for releasing new chemicals in the market. Several recent reviews (Benelli et al. 2016; Borges et al. 2011; Campos et al. 2012; Ellse and Wall 2014; Zoubiri and Baaliouamer 2014) give us much of what has been published in recent years on this subject. Among plants with acaricidal and repellence potential for various species of ticks, the most studied were found within the following families: Asteraceae, Fabaceae, Solanaceae, Meliaceae and Verbenaceae. From a total of 83 plants species, three showed acaricidal effect with low lethal concentration (Euphorbia prostrata, Manilkara zapota and Piper tuberculatum var. Allenii) (Benelli et al. 2016).

The difficulty in transposing the efficiency obtained in the laboratory to the field is one of the main obstacles in the use of plants with acaricidal potential (Borges et al. 2011). The acaricide effect is dependent on the genotype of a plant (different genotypes having different percentages of active principles), parts of the plant, soil, environment, time of day the plant is harvested, etc., which results in the same species of plant having different acaricidal efficacies (Cruz et al. 2013; Kiss et al. 2012; Peixoto et al. 2015; Soares et al. 2016). In addition, there is an interaction between the plant and the tick genotypes, the acaricide effect of the plant or essential oil depending on the tick strain (Costa-Júnior et al. 2016; Melo et al. 2014). Toxicological studies of plant-based products should not be neglected in order to identify risks to human and cattle health, as well as risks to the environment. Some plants, like those from the Annonaceae family, for example, kill not only the pest that one wants to control, but also beneficial insects useful to plants and humans such as pollinators and natural enemies (Kiss et al. 2012; Roel 2001). In their review about essential oils and ectoparasite control, Ellse and Wall (2014) conclude that the use of essential oils in veterinary medicine to control ectoparasites has great potential, however, many studies are still needed for obtaining good standardization of active components, understanding of their action on the ectoparasites, and the best way to extract the active principles; experiments should also evaluate toxic effects on the mammalian hosts, in addition to the study of adjuvants and investigations on residues and shelf life, that allow their use. Kumar et al. (2016) foresee that the major problem of formulations with plant products is their standardization. According to these authors, the acaricide activity of 64 accessions of the same plants that were included in the best formulation, collected from 15 Indian states, ranged from 10 to $90 \%$, hence the importance of 
standardization. The solvent used for removing the plant active principle, especially in relation to essential oils, is another very important issue to consider when evaluating efficacy of plant extracts (Kiss et al. 2012).

In their review Campos et al. (Campos et al. 2012) cite various species of medicinal plants, such as citronella, eucalyptus, copaiba, cumin, and neem, from which essential oils are being studied and among which some already have proven to have acaricidal effects on cattle ticks. Some plant extracts are popular among producers due to empirical evidence and, therefore, have been evaluated in scientifically designed studies. Neem, in the form of a pie resulting from oil extraction or as oil extracted from the seed of Azadirachta indica, is commercially available in Brazil. It is known for its insecticidal action and is used by farmers worldwide (Roel 2001; Srivastava et al. 2008). However, scientific results on the efficacy of neem consumed as pie or oil to control the cattle tick in Brazil are controversial (Chagas et al. 2010; Furlong et al. 2002b; Giglioti et al. 2011; Lopes et al. 2004; Silva et al. 2002; Terassani et al. 2012; Valente et al. 2007). There are some commercial products available in Brazil based on garlic that are purported to control ectoparasites. An extract of Allium sativum supplied daily to animals in their concentrate supplement presented an overall efficiency of $64 \%$ compared to animals that did not receive the extract (Costa-Júnior and Furlong 2011). A similar study tested three different concentrations of residue from garlic processing and the animals with the highest ingestion of the residue had correspondingly less ticks and were not affected in weight gain (Alvarenga et al. 2004).

Extracts from plants rich in rotenoids have been tested in vitro and in vivo. Plants from the genus Derris, rich in rotenone, have been used in pest control. Prior to the Second World War, these plants were cultivated on an industrial scale in the Amazon in order to supply the foreign market, because chemical insecticides did not exist yet. In vitro tests showed an efficacy of $92.4 \%$ with $7.5 \mathrm{mg} / \mathrm{ml}$ and $97.8 \%$ with $10 \mathrm{mg} / \mathrm{ml}$ of rotenoids extracted from $D$. urucu on female ticks. A commercial product based on rotenone extracted from Lonchocarpus nicou roots was tested on cattle artificially and naturally infested with $R$. microplus larvae (Catto et al. 2009). The extract killed larvae and engorged females in vitro, but it was not efficient in vivo. Good results in in vitro tests on larvae and females of $R$. microplus were reported for extracts of Lonchocarpus floribundus roots (Machado et al. 2013).

Regarding tannin-rich plants, several have been tested on different developmental stages of $R$. microplus. Quassia amara, found in the Amazon, has been evaluated in different associations, either with citronella (Cymbopogon nardus) or Santa Maria herb (Chenopodium ambrosioides), in in vitro assays (Santos et al. 2013), providing efficacies of the order of $100 \%$. Black angico (Anadenanthera macrocarpa) was tested in vitro on larvae (Silva et al. 2013). The Andiroba tree (Carapa guianensis) contains another potential insecticide. The acaricidal effect of Andiroba seed oil on Anocentor nitens, R. microplus and Rhipicephalus sanguineus was studied in vitro: it presented $100 \%$ efficacy at all dilutions tested for all species (Farias et al. 2009, 2012). Other plants tested in vitro on $R$. microplus with good effects are Araucaria angustifolia (Castro et al. 2009), Sapindus saponaria (Fernandes et al. 2005), Simarouba versicolor (Pires 2006), and Pilocarpus microphyllus (Castro et al. 2016). The susceptibility of $R$. microplus larvae to different dissolution methods of thymol has also been studied (Matos et al. 2014).

One of the most promising herbal products studied for tick control due to its availability and low cost throughout many countries is eucalyptus oil. Studies indicate that the compound citronellal is the main component responsible for the acaricidal effect of oils extracted from several species of eucalyptus (Chagas et al. 2002). Holstein cows bathed with 4.0 I of a solution of $3.5 \%$ eucalyptus 
essential oil (Corymba citriodora) presented an average efficacy of $69.2 \%$, but it was superior than $90 \%$ on the $14^{\text {th }}$ and $21^{\text {th }}$ days after the bath, which means greater efficacy on younger tick instars, such as larvae and nymphs. A sensory test was performed to evaluate if the applied solution transferred taste of eucalyptus to milk and derivatives (yoghurt): consumers of milk and yoghurt from treated animals did not notice altered taste or odour in the milk, nor was there an adverse effect to the animals (Olivo et al. 2013). Interestingly, modifications in the citronellal molecule did not produce positive results in vivo (Chagas et al. 2014).

A high-throughput screening system based on a chimeric protein of the $R$. microplus tyramine receptor, the probable target of formamidine acaricides (Gross et al. 2015), with a G-protein for signalling was developed for screening new compounds in a terpenoid library with activity upon the parasite. Piperonyl alcohol, 1,4-cineole, carvacrol and isoeugenol were shown to activate the tyramine receptor (Gross et al. 2015). This promising technology can be expanded to other components of tick physiology in order to mine for new acaricides.

The essential oils that are found in plants generally also have a repellent effect, such as thymol (which, in addition to the repellent effect, killed R. microplus larvae) (Novelino et al. 2007), eugenol (Zeringóta et al. 2013) and an extract from Lippia alba (Lima et al. 2016). In addition, methods for detecting natural compounds with repellent activity on $R$. microplus larvae have been developed and standardized (Chagas and Rabelo 2012; Ferreira et al. 2017; Wanzala et al. 2014).

A summary on current researches about the effect of plants and essential oils upon R. microplus is presented in Table 1. This table shows the great efforts to investigate on products from the plant kingdom to control the cattle tick.

\section{The ecology of R. microplus: generating knowledge for windows to novel opportunities for tick control}

Given the current level of resistance to acaricides and the lack of new products in the pipeline, it is essential to acquire deeper understanding of the ecology of R. microplus in order to identify new windows of opportunity to develop technologies to control this parasite. According to Estrada-Pena et al. (2006), the cattle tick, R. microplus, was introduced from the bovid- and cervid inhabited forests of the Indian region to many tropical and subtropical areas of the world. With the recent reinstatement of $R$. australis as a separate species, Estrada-Pena et al. (2012) affirm that it is important to assess if both species are native to Asia, or if $R$. microplus is a tropical representative with a long history of successful colonization. According to these authors the current distribution of $R$. microplus comprises America, Africa, and south-eastern Asia. It must be emphasized that much of our information on the ecology and behaviour of the cattle tick was generated in the $20^{\text {th }}$ century in Australia and thus refers to R. australis. Even so, Australian knowledge was used successfully for several decades to understand $R$. microplus infestations and subtle differences in ecological requirements between $R$. microplus and $R$. australis are still unknown. Whatever the origin of $R$. microplus, it now thrives on grasslands intended for cattle raised throughout tropical and subtropical regions of the world. It is capable of infesting cattle in dry, anthropized-forested areas partially occupied by shrubs (Nava et al. 2013) and dessert-like areas such as those found in Namibia (Nyangiwe et al. 2013). These latter introductions are unusual, because previous studies have shown that temperature and humidity severely influence tick population dynamics (Corson et al. 2001). Due to tick migration being mainly dependant on host movement, and climate to a certain degree, these factors become essential in the future prediction of tick spread as well as the spread of acaricide resistance (Chevillon et al. 2013). 
Table 1. Research on plants and essential oils and their efficacy on Rhipicephalus microplus and other species of ticks.

\begin{tabular}{|c|c|c|}
\hline Plant or essential oil & Results & Reference \\
\hline Araucaria angustifolia & Partial effect of ethanolic extract at 30\% & Castro et al. (2009) \\
\hline Sapindus saponaria & $\begin{array}{l}\text { Good larvicidal power in low } \\
\text { concentrations }\end{array}$ & Fernandes et al. (2005) \\
\hline Simarouba versicolor & $\begin{array}{l}\text { Inhibition of } 100 \% \text { of the } R \text {. microplus } \\
\text { posture in aqueous extract (10\%) and } \\
\text { ethanolic extract }(12.5 \%)\end{array}$ & Pires (2006) \\
\hline Ruta graveolens & $\begin{array}{l}\text { Good in the form of homeopathy; no } \\
\text { effect in herbal form }\end{array}$ & Aurnheimer et al. (2012) \\
\hline Pilocarpus microphyllus & $\begin{array}{l}\text { Ethanolic extract and pilocarpine } \\
\text { hydrochloride had effect on larvae and } \\
\text { on the reproductive efficiency of in vitro } \\
\text { engorged females }\end{array}$ & Castro et al. (2016) \\
\hline $\begin{array}{l}\text { Extracts of wounded leaves from } \\
\text { Leucaena leucocephala }\end{array}$ & Reduced egg hatching by $47.7 \%$ & Wanderley et al. (2017) \\
\hline $\begin{array}{l}\text { Momordica charantia leaf extracts in } \\
\mathrm{ZnO} \text { nanoparticles }\end{array}$ & $\begin{array}{l}\text { Higher toxicity than acqueous extracts } \\
\text { against larvae }\end{array}$ & Gandhi et al. (2017) \\
\hline $\begin{array}{l}\text { Havardia albicans and Caesalpinia } \\
\text { gaumeri methanolic leaf extracts }\end{array}$ & $\begin{array}{l}\text { Modest acaricidal activity in adult and } \\
\text { larvae immersion tests and inhibition of } \\
\text { egg laying; atoxic administered in vivo }\end{array}$ & Rosado-Aguilar et al. (2017) \\
\hline $\begin{array}{l}\text { Various organic extracts of Crescentia } \\
\text { cujete L. fruit pulp }\end{array}$ & $\begin{array}{l}100 \% \text { of mortality of larvae in larval } \\
\text { packet test with } 10 \% \text { solution of ethyl } \\
\text { acetate phase }\end{array}$ & Pereira et al. (2017) \\
\hline $\begin{array}{l}\text { Comparison of essential oil from a } \\
\text { rare chemotype of Cinnamomum } \\
\text { verum Presl with benzyl benzoate }\end{array}$ & $\begin{array}{l}\text { Essential oil containing } 65 \% \text { benzyl } \\
\text { benzoate was } 3.3 \text { times more efficient } \\
\text { on larvae than benzyl benzoate alone; } \\
\text { no differences were found on engorged } \\
\text { females }\end{array}$ & Monteiro et al. (2017) \\
\hline $\begin{array}{l}\text { Prospection of } 13 \text { essential oils } \\
\text { extracted from many plants }\end{array}$ & In vitro significant effects of some oils & Domingues et al. (2014) \\
\hline Oil extract from Tagetes minuta plant & Almost 100\% efficacy in vivo test & Andreotti et al. (2013) \\
\hline $\begin{array}{l}\text { Oil extract from Allium sativum and } \\
\text { Allium cepa }\end{array}$ & $\begin{array}{l}100 \% \text { efficacy in vitro test on Boophilus } \\
\text { annulatus }\end{array}$ & Aboelhadid et al. (2013) \\
\hline $\begin{array}{l}\text { Limonene, limonene oxide, alcohol } \\
\beta \text {-amino derivatives }\end{array}$ & Efficacy in vitro for $R$. microplus larvae & Ferrarini et al. (2008) \\
\hline Oil of Calea serrata & Acaricidal properties on $R$. microplus & Ribeiro et al. (2011) \\
\hline $\begin{array}{l}\text { Oils extracted from Lippia graveolens, } \\
\text { Rosmarinus officinalis and A. sativum }\end{array}$ & $\begin{array}{l}\text { High larvae mortality in vitro tests (Lippia } \\
\text { and Allium oils) }\end{array}$ & Martinez-Velazquez et al. (2011) \\
\hline $\begin{array}{l}\text { Oil extracted from leaves of Lippia } \\
\text { graveolens }\end{array}$ & $\begin{array}{l}100 \% \text { mortality of female adults in vitro } \\
\text { tests at } 10 \% \text { concentration }\end{array}$ & Flores-Fernandez et al. (2016) \\
\hline $\begin{array}{l}\text { Carvacrol, (E)-cinnamaldehyde, trans- } \\
\text { anethole and linalool }\end{array}$ & $\begin{array}{l}\text { In vitro test on } R \text {. microplus and } \\
\text { Dermacentor nitens larvae. Linalool did } \\
\text { not have acaricidal effect }\end{array}$ & Senra et al. (2013) \\
\hline Eugenol & $\begin{array}{l}\text { In vitro repellent test on } R \text {. microplus and } \\
\text { D. nitens larvae }\end{array}$ & Zeringóta et al. (2013) \\
\hline
\end{tabular}


Table 1. Continued.

\begin{tabular}{|c|c|c|}
\hline Plant or essential oil & Results & Reference \\
\hline Eugenol & $\begin{array}{l}100 \% \text { mortality in vitro test on } \\
\text { R. microplus larvae }\end{array}$ & Valente et al. (2014) \\
\hline Eugenol & $\begin{array}{l}\text { In vivo Pour-on formulation not effective } \\
(5 \%)\end{array}$ & Valente et al. (2017) \\
\hline $\begin{array}{l}\text { Oil extract from Cymbopogon } \\
\text { wynterianus }\end{array}$ & $\begin{array}{l}\text { Efficacy in vitro (female adults and } \\
\text { larvae) } R \text {. microplus }\end{array}$ & Santos et al. (2012) \\
\hline $\begin{array}{l}\text { oil extracted from Cymbopogon } \\
\text { winterianus }\end{array}$ & $\begin{array}{l}\text { Efficacy in vitro (female adults and } \\
\text { larvae) R. microplus }\end{array}$ & Martins (2006) \\
\hline $\begin{array}{l}\text { oil extracted from Cymbopogon } \\
\text { winterianus }\end{array}$ & $\begin{array}{l}\text { In vivo applied Pour-on (pure) and spray } \\
\text { (diluted } 10 \% \text { in alcohol). Both forms } \\
\text { were different from control }(P<0.0001)\end{array}$ & Martins and Gonzalez (2007) \\
\hline $\begin{array}{l}\text { oil extracted from Cymbopogon } \\
\text { winterianus }\end{array}$ & $\begin{array}{l}\text { Spray }(4 \%) \text { applied in vivo. Necessary to } \\
\text { reapply the citronella oil after } 28 \text { days of } \\
\text { observation }\end{array}$ & Agnolin et al. (2010) \\
\hline $\begin{array}{l}\text { Five essential oils extracted from } \\
\text { three species of Ocimum }(0 . \\
\text { gratissimum, } 0 \text {. urticaefolium } 0 \text {. } \\
\text { canum) }\end{array}$ & $\begin{array}{l}\text { Acaricidal effect in vitro R. microplus } \\
\text { larvae of eugenol, thymol and, mainly, } \\
\text { elemycin }\end{array}$ & Hüe et al. (2015) \\
\hline $\begin{array}{l}\text { Eleven essential oils extracted from } \\
11 \text { plants }\end{array}$ & $\begin{array}{l}\text { Efficacy in vitro (female adults and } \\
\text { larvae) } R \text {. microplus. The essential oil } \\
\text { extracted from Curcuma longa rhizomes } \\
\text { had the best result }\end{array}$ & Chagas et al. (2016) \\
\hline
\end{tabular}

Awareness of the importance of tick ecology was boosted when Klompen et al. (1996) affirmed that tick-host association patterns may be explained as artefacts of biogeography and ecological specificity rather than by host specificity. This matter is still under debate, yet it is undisputable that the off-host environment is of major importance for survival of tick populations (and, by extention, that of any biological control agent to be used). The ecological and climate specificity is highlighted by the simple fact that, even though ubiquitous throughout several parts of the globe, $R$. microplus is not present in all areas of cattle raising, but rather at specific environments under certain climates. Notwithstanding the role of biogeography in off-host tick ecology, the hosts' physiology is also affected by the environment, as well as by its genetic composition, therefore, the on-host ecosystem also varies and strongly affects this species' biology. This fact can be gauged by the reproductive success of the female tick after feeding on hosts of different genetic compositions (Biegelmeyer et al. 2015), reproductive success being a driving force in evolution and natural selection. It also contributes to the displacement of other species of ticks, such as $R$. decoloratus in areas where they co-occur. This has serious consequences as $R$. decoloratus is the vector of Babesia bigeminia and is thus regarded as essential for upholding endemic stability to Babesia infection in Africa, in contrast to the lethal form of disease caused by Babesia bovis that is transmitted by R. microplus (Tønnensen et al. 2004).

Notable among several features of the ecology of $R$. microplus is that, probably driven by environmental selective pressure, they evolved a one-host or monoxen life cycle supposedly 
to allow parasitism of larger-sized, but highly mobile herbivorous mammals (Perissodactyla and Artiodactyla) with extensive home ranges (Apanaskevich and Oliver, 2014; Hoogstraal and Aeschlimann 1982). Balashov (1972) and Hoogstraal and Kim (1985) speculated that having a single host is an adaptation of this ectoparasite to large, migratory, grazing ungulates because the ability to moult to all developmental stages on the same vertebrate host reduces the number of necessary encounters and thus increases chances for maintenance of this parasite's life cycle. The off-host, non-parasitic life cycle of $R$. microplus was constrained to drop-off of engorged females followed by the search for an adequate microenvironment for oviposition, pre-ovipostion period, oviposition, egg incubation, hatching and host-seeking of larvae, while engorged larva and nymph moulting occurs on the host, dismissing the necessity of these stages from host seeking. Work that examined the large family of glycine-rich proteins found in saliva of different species of ticks and employed in its attachment to their hosts suggests that this monoxen tick acquired a comparatively larger diversity of genes encoding these proteins to sustain its ability to remain on a single host for approximately 21 days while relying on a comparatively short hypostome for puncturing and grabbing its host's skin (Maruyama et al. 2010). This is one aspect of tick biology to target, namely, interfering with attachment. This requires further understanding about the true functions of this large family of proteins.

Another notable feature of the ecology of R. microplus is the fact that the genetic composition of the bovine host affects its biology and reproductive success. Breeds of cattle present contrasting and heritable phenotypes for loads of the parasitic, blood feeding stage. When they are managed on pastures equally infested with $R$. microplus, breeds of indicine (Bos indicus) cattle carry significantly lower loads of ticks than most taurine (Bos taurus) breeds. Furthermore, when compared with $R$. microplus females feeding on taurines, those feeding on indicine cattle ingest significantly less blood, thus rendering significantly smaller egg masses. These differences in the reproductive success of female ticks are also seen in crosses of indicine and taurine cattle (Biegelmeyer et al. 2015). In contrast to indicine cattle, most taurine breeds, especially European breeds, even after undergoing repeated infestations, still carry levels of tick loads that are unacceptable in terms of animal productivity and health (Mattioli and Cassama 1995; Mattioli et al. 2000; Utech and Wharton 1982). These breed-dependent differences in the success of the tick's life cycle possibly occur because indicine breeds were domesticated in Asia (Bradley et al. 1998; MacHugh et al. 1997), which is also believed to be the place of origin of the parasite (Estrada-Peña et al. 2006), and a mutual agreement of acceptable boundaries and costs evolved from this particular parasitehost interface was reached. Interestingly, when deer and taurine cattle share grazing areas, the reproductive success is much lower in ticks feeding on deer. Furthermore, the effective population size in the group of parasites feeding on deer is approximately 75 times lower than that feeding on cattle (De Meeüs et al. 2010). This fact may have important consequences for control measures that exploit genetic resistance of the host and also for development of resistance to acaricides because it raises the following questions: what is the effective population size of ticks feeding on genetically resistant bovines compared to that of ticks feeding on susceptible hosts? What effects would bottlenecks have in selection of resistant SNPs should the effective population sizes differ?

Breeding cattle for resistance to infestations and also targeting tick proteins through vaccination can alter the ecosystem provided by the host (i.e. on-host ecology) by changing local and systemic reactions to tick bites, which have the potential to expel ticks attempting to feed or to reduce blood feeding, as demonstrated by the outcome of infestations in resistant hosts. The consequence is a reduction in the reproductive efficiency of ticks (Biegelmeyer et al. 2015) and their populations, if not their effective population size. Since cattle will spend at least two years in any given production system and $R$. microplus can complete up to four or even five life cycles 
a year, individual cattle are thus subjected to many infestations during their lifetime. This means that by the end of the parasitic cycle, $R$. microplus will potentially be confronted with all aspects of its host's innate and acquired immune responses and different levels of efficacy, depending on the breed of the bovine host.

Some level of interference in the off-host ecosystem is also possible, with positive and negative consequences for tick populations. The off-host ecosystem can be intentionally affected by producers by means of choices of the species of grass and other pastures employed for grazing, the intensity of grazing, the production system, whether confined or extensive, whether solely grazing or an agro-forestry consortium, with or without irrigation and extent of deforestation. These choices result in increased exposure of eggs and of free-living larvae (and, in certain instances, also of feeding ticks) to unfavourable physical conditions, to the chemicals and morphology of certain grasses on which cattle graze and/or to predators and infections with entomopathogens naturally present in the environment or delivered to it artificially; substances generated by host microbiota or provided by natural products can repel ticks. The optimal strategy will likely be an integrated approach that employs several or all of these basic strategies. It is also possible that one approach can synergize with another, resulting in an overall larger impact on tick populations in pastures.

\section{Off-host ecology of R. microplus}

\section{Season, climate and geography}

The drop-off rhythm of $R$. microplus is poorly understood but it seems to be influenced by season, attachment site, period of the day, host movement and location. In Brazil, De Paula and Furlong (2002) observed that from bovines taken to stalls most female $R$. microplus detached around 09:00 in summer and autumn, whereas a dual drop-off peak was observed in winter, the first at around 06:00 and the second at around 15:00. In the case of $R$. australis, females were observed to undergo rapid final engorgement during the night and detach early the next morning. But on cattle that were held in covered stalls, final engorgement commenced in the afternoon and peak numbers of engorged females were present at about midnight (Wharton and Utech 1970). Bianchi et al. (2003) observed in New Caledonia that morning detachment is faster for engorged females of $R$. australis attached on light/sun-exposed sites than for females attached on protected sites. Moreover, movement induced a more rapid drop-off of females attached on exposed sites than on those attached on protected sites.

After drop-off, engorged females are incapable of moving long distances on the ground, the overall theoretical picture being that they detach most when a favourable environment is to be met when off-host. One study performed in southeast Brazil (Brovini et al. 2003) showed that after engorged $R$. microplus females dropped from their hosts, they crawled for a sheltered place in the soil and buried themselves in crevices, under clumps of grass or under leaves. In that study, the search for shelter on the soil was distance-restricted; a mean of 7.2 and 12.2 centimetres in, respectively, summer and winter. Once in the shelter, oviposition and egg incubation will occur in the soil or similar hiding place. Shelters provide a relative comfort zone against more extreme climate variations above the soil, nonetheless climate variations will still affect engorged females. These issues are highlighted by a study in the tropics at low altitudes with hot and humid summers and dry winters in southeast Brazil: Oliveira et al. (1974) compared engorged female R. microplus kept on the ground surface to those kept $2 \mathrm{~cm}$ deep in the soil. Mortality of female ticks was higher in summer for those kept on the surface, whereas it was higher in winter for those kept 
underneath it. In the same study, mean daily egg output of engorged female was similar in the winter for females kept in both microenvironments, however, in the hot summer females kept on the surface laid significantly less eggs per day.

Laid eggs, the next phase of the off-host biology of $R$. microplus, are also prone to infections and predation and are unable to move to favourable microenvironments. The hatching rate of eggs is reduced in dry environment and at high ground temperatures $\left(>30^{\circ} \mathrm{C}\right)$ (Garris et al. 1990) and, on the other side, mean monthly temperatures below $20^{\circ} \mathrm{C}$ also affect negatively egg hatching (Canevari et al. 2017). While eggs remain in their shelter under a relatively stable environment, host-seeking larvae must climb on vegetation and wait for encounters with hosts. This is a particularly delicate part of the life cycle because ticks are exposed to open spaces and more extreme environmental variations. Larvae behavioural responses cope with many of these; they clump together in clusters to retain humidity, hide from the sun underneath leaves when necessary, or move to the ground to recover water content. These responses are, however, energy consuming and abbreviate the life span of larvae. For this reason, shade is a crucial factor for longer survival of $R$. microplus larvae and is strongly affected by the type of pasture. Short et al. (1989) for example, observed that survival of $50 \%$ of $R$. microplus larvae in Zimbabwe ranged from 40 to 58 days in long grass and 14 to 36 days in short grass, while Garris et al. (1990) reported that larvae in Puerto Rico can survive for up to 20 days longer in shaded or wooded areas than open grass areas. On the other hand, heavy rain showers also have a negative impact on $R$. microplus larvae (Cerny and De la Cruz 1971; Urdaz-Rodríguez et al. 2012). Cattle raising with highest stocking rates reduces home range and mobility of bovines and thus increases frequency of larvae-host encounter. Such conditions enormously favour tick survival and the parasite's population increases, many times to levels that are incompatible with the hosts' health and survival.

It is clear from the examples above that both temperature and humidity are driving factors for oviposition, egg incubation and for larvae longevity. Campos Pereira and Labruna (2008) grossly summarized $R$. microplus environmental survival limits during non-parasitic cycle; temperatures over $25^{\circ} \mathrm{C}$ reduces periods of oviposition and egg incubation but over $30^{\circ} \mathrm{C}$ mortality of females, eggs and larvae occur. Temperatures below $25^{\circ} \mathrm{C}$ extend periods of oviposition and egg incubation and below $15^{\circ} \mathrm{C}$ there is mortality of females and eggs in the environment. Relative humidity over $80 \%$ favours tick survival without altering length of life cycle, however below $80 \%$ death of ticks may occur by desiccation.

Duration of tick life cycle is also a major issue for $R$. microplus tick infestations and depends on environment and particularly climate. While the parasitic, on-host phase duration is relatively constant (21-23 days), the non-parasitic phase varies greatly. In Brazil it lasts generally 40-50 days in spring-summer and 70-120 days in autumn-winter according to Labruna (2008). Thus, off-host conditions will determine ticks' survival or death, but also the length of the life cycle and therefore the number of generations per year as well as tick abundance. In the southernmost range of its distribution in South America R. microplus undergoes three generations per year, the first in midlate spring, the second in summer and a third and largest in abundance in autumn (Canevari et al. 2017). Under favourable and at higher mean temperatures it may achieve as much as four and even five generations per year (Cerny and De la Cruz, 1971).

Under field settings all factors mentioned above interplay combined with daily and seasonal features. Various vegetation types (usually diverse grass species), latitude, altitude, temperature, humidity, wind, rain, host stocking rate and immune status, predators, pathogens and others, all interact in their effect upon the tick. Generic information in two countries provides a broad picture 
of these effects. Estrada-Peña et al. (2006) observed that R. microplus in Mexico is most common under warm and humid conditions. The tick avoids the colder central mountainous regions and Mexican plateau as in this zone the low temperature seems to be the major limiting factor. On the other hand, at warmer lower locations, humidity is a decisive factor for tick survival.

Lysyk (2008) showed that temperatures of the environment and of cattle skin can affect the engorgement rate of the tick Dermacentor andersoni Stiles: decreases in temperatures correlated with reduction in attachment and longer engorgement rates. This effect must also be assessed for $R$. microplus because many dairy producers already provide shade and mist for their milking animals in order to reduce thermal stress, which might also alleviate the damage done by tick infestations and could be a further incentive for more farmers to adopt this practice.

Hence, the daily and seasonal dynamism of environmental and micro environmental conditions is a crucial factor to determine the suitability of a given area for $R$. microplus populations. However, observations on $R$. microplus's ecological requirements and limits are still fragmented and scattered. They should ideally be determined for representative locations throughout the tick's range to be used in programs for tick overpopulation control.

Use of pastureland: grasses, rotations, fertilization, management systems and mixing tick-resistant and tick-susceptible breeds of cattle

Pasture for grazing animals can affect several aspects of the biology of $R$. microplus: among these are survival of females, eggs and larvae by providing more or less shade, and host-seeking behaviour by providing repellents or barriers to reach tips of leaves or reach the host. It has been estimated that the majority of a population of $R$. microplus ticks are in the pasture and only $5 \%$ are feeding upon their hosts (Campos Pereira and Labruna 2008). Thus, a great proportion of a tick population can be affected by the choice of pasture. For example, Pennisetum purpureum, or napier grass, benefited ticks because its morphology provides a microenvironment that protects ticks, including in the winter when ticks climb to the tips of leaves, increasing their chances for encounters with their hosts (Furlong et al. 2002a). Conversely, pastures composed of the genus Stylosanthes (Sutherst et al. 1982), of Melinis minutiflora (molasses grass) (Fernandez-Ruvalcaba et al. 2004; Prates et al. 1998), and of Andropogon gayanus (gamba grass) (Fernandez-Ruvalcaba et al. 2004) are known to immobilize, kill and/or repel ticks. The use of anti-tick grasses as part of an integrated management system for controlling cattle ticks was proposed many years ago by Thompson et al. (1978) While individual species of grass have been studied to answer various questions regarding animal nutrition, agronomical issues and effects on ticks, there has been no systematic evaluation of the most popular species of pastures in terms of all aspects. At the very least, a meta-analysis of previous studies is warranted to conclude which grasses furnish all desirable features or at least offer the best exchange in terms of both providing animal nutrition, agronomic suitability and controlling ticks; generally, only the first two features are considered among animal and crop scientists.

Pasture rotation has been adopted in the management of dairy cattle since it affords high quality feed for livestock. It also can assist in controlling tick infestations. However, in rotational grazing, the pasture must be left with lesser height: this contributes to control larvae, especially if the forage is a caespitose grass (i.e. erect growth, for example, those of the genus Panicum) because the solar rays are abler to penetrate to the ground, contributing to dehydrate eggs and larvae and kill engorged ticks (Veríssimo 2015). After cattle have finished grazing a pasture plot, it is a common practice in intensive production systems to fertilize the plot with urea in order to 
maintain the vitality of the pasture. Urea can kill the tick female preventing it from laying eggs (Cunha et al. 2008, 2010). However, recently Leal et al. (2017) found that urea had absolutely no effect on ticks in immersion assays of adults and larvae and in fertilized pastures. Since ammonia degraded from the urea is deemed to be the cause of mortality for ticks these authors speculate that additional factors in the studies by Cunha et al. $(2008,2010)$ may have led to ammonification of the fertilizer.

Crop-livestock integration practices, where crops and pastures are rotated on the same plot, also help to control ticks and other parasites, since all of them (endo- and ectoparasites) have a nonparasitic phase in the soil. If there is no grazing (even when waiting for a small period of the crop cycle), there will be no larvae to re-infest the animals. Cattle in newly formed pastures, grown previously for two consecutive years with maize crop, had significantly fewer tick larvae than those that occupied permanent pastures (Veríssimo et al. 1997). On the other hand, in agro-forestry systems, where distribution of pasture plots alternates with plots of trees planted for wood and generates more diversity per area, it is possible that its advantages could be offset by increasing the amount of shade, leaves covering the ground, etc., hence protecting engorged females, eggs and larvae if pasture rotation is not undertaken. This aspect of these systems merits evaluation since, according to Estrada-Peña and Salman (2013), changes in plant communities can benefit ticks.

Co-management of susceptible and resistant cattle has been recommended as a practice for tick control (Wharton et al. 1969) because larvae of $R$. microplus have difficulty fixing and completing the cycle on the tick resistant animals, which would act as 'aspirators' of larvae in the pasture, drastically reducing the infestation of the grass. However, in the experience of some of the authors of this chapter, this might not be the case: 300 heifers of a cross between a tick-susceptible and a tick-resistant breed that participated in a genome-wide association study, were managed for the entire period of the study on the same pasture and were visited and examined over a period 15 months; the tick loads remained unacceptably high in those initially identified as the most susceptible animals. This was observed regardless of the season and date of evaluation, therefore they did not seem to benefit from co-existing with the more resistant animals of the cross, many of which maintained negligible tick loads or no ticks throughout the study. However, it is possible that a ratio of resistant and susceptible animals must be maintained if tick populations are to be affected, but, to the best of our knowledge, the research that establishes this ratio has not been undertaken.

\section{The biota of the off-host and on-host ecosystems of R. microplus, of R. microplus itself and their effects on tick biology and transmission of pathogens}

Among the variables of the off-host and on-host ecosystems of $R$. microplus, mainly grasses and host skin, respectively, is the composition of the biotas that these ecosystems harbour. The biota of grasses that can affect the tick life cycle comprises the grasses themselves, already discussed above, as well as pathogens and predators of ticks; the skin biota comprises organisms that modify host products such as sweat and sebum and produce semiochemicals that affect tick behaviour.

\section{Biological control of R. microplus in pastures}

Biological control aims to diminish the effects of any potentially harsh chemicals on animals, humans and the environment. However, biological pesticides are generally not used due to their dependency on environmental factors, as well as the potential of harming non-target invertebrates 
despite their beneficial attributes (Domingos et al. 2013). Examples of different biological control agents that can be implemented for tick control are discussed below.

There are many natural enemies of $R$. microplus ticks among invertebrates (ants, diptera, forficulidae insects, beetles, spiders, etc.) and vertebrates (rats, mice, frogs, ants and birds, mainly chickens, ox peckers and egrets) (Veríssimo 2013a, 2015). Small lizards (Tropidurus torquatus), very common in South America, were observed eating engorged and semi-engorged females of $R$. microplus (Veríssimo and D'Agostino 2014). Avian tick predators include some fifty bird species that are known to prey on ticks. One such example is the yellow-billed oxpecker (Buphagus africanus) and the red-billed oxpecker (Buphagus erythrorhynchus). These birds are known for their preference for feeding on ectoparasites (Samish et al. 2004). However, like all predators of $R$. microplus, their usefulness is limited because they are generalist predators or are found only in specific geographical areas. This has turned attention to fungi and nematodes as entomopathogens and more effective enemies of $R$. microplus.

The fungi of the genera Metarhizium and Beauveria have been extensively studied to be used for the biological control of ticks (Kaaya et al. 2011; Monteiro et al. 2013). The fungus Metarhizium anisopliae controls ticks of the genus Rhipicephalus when sprayed on cattle (Kaaya et al. 2011), and when sprayed on pasture (Kaaya and Hedimbi 2012); when associated with the plant Solanum verbascifolium it increased the mortality of $R$. microplus ticks (Romo et al. 2013). A study carried out in Brazil revealed that a commercial formulation of M. anisopliae, Metarril SP Organico ${ }^{\circledR}$ (Koppert Biological Systems, Piracicaba, Brazil), added to $10 \%$ mineral oil, provided an average efficiency of $47.74 \%$ (Camargo et al. 2014). The product was also applied to infested animals in the field (two applications with a 3-day interval), again with $10 \%$ mineral oil. The product had an average efficacy of 75\% (Camargo et al. 2016). In spite of these reported effects, producers are not adopting these products. Possible reasons for concern about the efficacy of entomopathogenic fungi are their dependency on their host's behaviour, the reliance on their hosts preferences for the tick, as well as the overall susceptibility of the tick to the fungal infection. Additionally, they also display slow killing rates and require high humidity for germination and sporulation (Chandler et al. 2000; Samish et al. 2004). More recently, Webster et al. (2017) reported that field populations of $R$. microplus were highly variable in their susceptibility to $M$. anisopliae. The mechanism behind this variability was not examined, but Metarhizium spp. entomopathogenic fungi produce a subtilisinlike serine protease which is a virulence factor because it degrades the tick's cuticle (St. Leger et al. 1989). Golo et al. (2015) recently showed that increased levels of expression and production of this enzyme by the fungus are not correlated with increased mortality of tick larvae from a laboratory strain of ticks. However, inhibitors of subtilisin-like proteases have been described in tick haemolymph (reviewed by Blisnik et al. 2017) and the silkworm Bombyx mori has an haemolymph inhibitor that inhibits fungal proteases (Eguchi et al. 1994). Ticks from field strains respond to different stressors by varying levels of gene expression, including of genes encoding for protease inhibitors (Maruyama et al. 2017) and this finding may explain the findings of Webster et al. (2017). Entomopathogenic nematodes are also being studied for biological control of the cattle tick (Monteiro et al. 2013), associated, or not, with herbal plants (Monteiro et al. 2014) or entomopathogenic fungi (Monteiro et al. 2013). Lastly, the use of Ixodiphagus hookeri (Howard), a Hymenoptera parasitoid, for tick control is also being considered because the female wasps oviposit their eggs into internal cavities of ticks and, upon uptake of the nymph's first blood meal, egg development initiates. These Ixodiphagus species appear to be restricted to ticks thus eliminating any non-target effects (Samish et al. 2004). 
The pathogens for ticks are, however, not specific for this parasite. Therefore, as with all agents of biological control, non-target effects are of concern; this matter and examples of unexpected effects have been reviewed by several authors (Barbercheck and Millar 2000; Dolinski and Moino 2006; Louda et al. 2003; Lynch and Thomas 2000). In addition, intensive stocking of pastures due to feeding practices can increase density of tick populations, thus requiring inundative applications of biological control agents. It is felt that while this practice does affect a wide range of nontargets, increasing risk of non-target effects, these effects are always transient (Lynch and Thomas 2000). As informed in the introduction section, it must be kept in mind that acaricides and natural products employed in tick control can affect entomopathogens because they too are not specific for R. microplus.

\section{Host skin microbiota, skin volatiles and tick behaviour}

Specificity of vectors of disease for vertebrates is dictated by the repertoire of semiochemicals that derive from body emissions (breath, sweat, sebum, tears, saliva, urine, faeces, etc.) (Smallegange et al. 2011). These emissions contain amino acids, short chain fatty acids and hormones, precursors that are transformed into volatile odoriferous substances by bacterial enzymes present on the host's skin (Smallegange et al. 2011). Volatiles produced by microbes colonizing hosts affect behaviours of arthropods (Davis et al. 2013; Yoder et al. 1998). Relatively few studies address host semiochemicals for ticks, including those of microbial origin. Sick hedgehogs contain more indole in their faeces than healthy hedgehogs and the tick Ixodes hexagonus Leach seeks these sick hosts by means of this cue possibly because, when weakened by disease, they are more suitable for blood meals and for reservoirs of tick-borne disease (Bunnell et al. 2011). The generic semiochemicals $\mathrm{NH}_{3}, \mathrm{CO}_{2}$ and $\mathrm{H}_{2} \mathrm{~S}$, and 1-octen-3-ol (an attraction-aggregation-attachment pheromone for ticks found on the tick cuticle and on the skin of humans) are host attractants for at least Amblyomma sp. (Sonenshine 2006); squalene from host skin and blood was the first host-derived substance shown to affect behaviour of the ticks Dermacentor variabilis (Say) and Amblyomma americanum Linnaeus (Yoder et al. 1998). Furthermore, bacteria can transform squalene into other volatiles such as nerylacetone (geranylacetone) that affects behaviour of several blood sucking arthropods (Vale et al. 2012). Headspace sampling of lipid extracts from skin of an undetermined bovine breed (probably a taurine breed since the study was conducted in Germany) showed that benzoic acid, 2-ethylhexanoic acid, hexanoic acid, 2-nitrophenol, 1-octen-3-ol, pentanoic acid, and pyruvate affect questing behaviour of $R$. microplus and Ixodes ricinus Murray (Osterkamp et al. 1999). Another study showed that many species of hard ticks are attracted to contents of the bovine rumen and that they possess olfactory receptor cells for carboxylic acid, phenol and indole, all end-products of rumen fermentation (Donzé et al. 2004). The study of semiochemical production by tick-resistant and tick-susceptible breeds of bovines and their skin microbiota demands many more efforts until full understanding of host-tick chemical interactions is reached. The knowledge generated by these studies may offer at least three new fronts for control of $R$. microplus: treatment of animals with repellent semiochemicals and/or of pastures with poison decoys based on attractant semiochemicals; probiotic interventions in host skin; vaccination against skin microbiota producing attractant semiochemicals.

Interfering in the microbiota of R. microplus: potential for impacts on its performance and vectorial competence

At the time of writing of this chapter, only two studies had examined aspects of the microbiota of $R$. microplus: one demonstrated that cultures of the microbiota of different anatomical niches of the tick (gut, ovary, salivary glands and Genés organ of females) contained compounds with 
anti-biofilm and trichomonicidal properties (Zimmer et al. 2013); another studied assessed the overall microbiota with pyrosequencing and determined that $R$. microplus harbours Wolbachia, Coxiella, and Borrelia besides Staphylococcus aureus, Staphylococcus chromogenes, Streptococcus dysgalactiae, Staphylococcus sciuri, Serratia marcescens, Corynebacterium glutamicum and Finegoldia magna (Andreotti et al. 2011).

Current research indicates that the microbiota of several hematophagous arthropods affects their competence as vectors (Barletta et al. 2017; Dennison et al. 2014; Ramirez et al. 2014; Zhang et al. 2015). Importantly, it also affects their performance in the field regarding, for example, seasonality, lifespan, reproduction and attraction to sources of blood (Coon et al. 2016; Novakova et al. 2017; Nunes et al. 2016; Zhang et al. 2015). Another aspect of the microbiota of hematophagous arthropods that may direct towards useful control technologies is that it's affected by the vectors' diet (Nunes et al. 2016). While this finding applies to hematophagous vectors that have developmental stages that also feed on plants, it is reasonable to assume the composition of skin and blood of the bovine host, including its microbial composition, can also affect the microbial composition of $R$. microplus. Furthermore, all of these findings indicate that it is possible to affect the fitness and vectorial competence of $R$. microplus by manipulating its microbiota. Clearly, especially in view of current advances in sequencing technologies and bioinformatics, this is a field that must urgently be addressed for cattle ticks. Indeed, paratransgenesis with genetically engineered components of microbiota of other hematophagous vectors and pests is being studied and adopted to control them or the diseases caused by the pathogens they transmit (Gilbert et al. 2016; Mancini et al. 2016; Tikhe et al. 2016).

\section{On-host ecology of R. microplus: changing host immunity}

\section{Tick vaccines}

Tick vaccines are addressed in a companion chapter in this book; this chapter will examine vaccines in the context of manipulating on-host ecology. Immunobiological control of ticks was first demonstrated by William Trager in 1939. Trager (1939) tested various tick tissues and showed that salivary glands were the most efficient to induce immunity that affected blood feeding and reproduction of $D$. andersoni. In 1986, Johnston et al. (1986) demonstrated that immunisation of cattle with a crude extract of adult female $R$. microplus protected cattle against tick challenge. They also report the first observation of 'red ticks' which are due to blood leaking from their gut tissues into their body cavities in ticks fed on vaccinated animals. The authors tested both the soluble and insoluble (pellet) fraction of the crude tick extracts, and both provided protection indicating that both membrane-associated and secreted tick porteins are capable of inducing a protective response. Tick saliva is composed of numerous pharmacological molecules that are used by the parasite to promote their attachment to host skin, to destroy the skin matrix and form the blood feeding pool, and to inhibit coagulation and modulate the inflammatory and immune responses of their hosts (reviewed by Francischetti et al. 2009). One opportunity for sustainable tick control offered by the ecology of $R$. microplus is the fact that, due to its approximately threeweek monoxen life cycle and to the number of generations that a single tick can produce during a production animal's time in service, the bovine host has time to mount an acquired secondary immune response and thus neutralize these salivary mediators of parasitism that are produced during the parasitic stage. This response indeed seems to be efficiently recruited in genetically tick-resistant, indicine cattle because after exposure to few infestations, the antibody response of the indicine Nelore breed of cattle recognizes many more types of salivary proteins than the antibody response of Holsteins, a tick-susceptible taurine breed that carries significantly higher 
ticks loads that consequently deliver larger amounts of salivary proteins to this host (Garcia et al. 2017). This finding suggests that if anti-tick vaccines induce saliva-neutralizing antibodies these will modify the host-tick interface. However, studies to date have shown that these numerous compounds may prevent efficient activation of humoral and cellular immunity (Kazimírová and Štibrániová 2013), a phenomenon which could negatively affect vaccination success. Moreover, the large families of salivary protein found in ticks may compensate for loss of function and represent an escape mechanism from their hosts' immune response (Guerrero et al. 2012b; Kiss et al. 2012). Thus, the jury is still out on whether using tick salivary gland proteins (exposed antigens) or non-exposed (concealed antigens) is the best way forward for a tick vaccine.

Currently, only two vector-directed vaccines have been commercialized globally in Latin America, Gavac $^{\circledR}$ (Heber Biotec SA, Playa la Habana, Cuba) and Go Tick ${ }^{\circledR}$ (Limor de Colombia, Bogota, Colombia). Of these two products, the antigen (Bm86) used in $\mathrm{Gavac}^{\circledR}$ is the best described. This antigen is based on a midgut protein isolated from $R$. microplus for which its efficacy in lowering tick burdens on cattle has been sufficiently demonstrated in both controlled and field vaccination trials. Gavac ${ }^{\circledR}$ was used recently in a countrywide two year vaccination program (including 18 states) in the Republic of Venezuela (South America). In this program over 1.9 million head of cattle were immunized with Gavac $^{\oplus}$ that resulted in a $83.7 \%$ reduction in acaricide use, as well as an estimated $81.5 \%$ cost saving on traditional chemical control. Moreover, in Cuban trials using the Gavac $^{\circledR}$ antigen and $\sim 260,000$ head of cattle, a $\sim 60 \%$ reduction in acaricide use and lowered incidence of disease (transmission of Babesia and Anaplasma spp.) was recorded along with significant savings on production costs ( 6 million US\$) (De la Fuente et al. 2007). However, this vaccine has not been implemented with success as genetic differences of the Bm86 antigen from different geographic areas is believed to greatly influence the variability in vaccine efficiency of Bm86 (Garcia-Garcia et al. 2000; Parizi et al. 2009). The global patent on Bm86 has since expired and new tick antigens are in dire need to develop a $2^{\text {nd }}$ generation tick vaccine.

Modelling studies are lacking, but necessary to predict the level of efficacy that tick vaccines should attain (i.e. maximum number of off-host and on-host, feeding ticks compatible with productivity and animal well-being and health, as well as with maintaining enzootic stability of tick-borne diseases) and how long after vaccination it will take for tick populations in pastures to be reduced to levels acceptable for good production gains and for animal well-being. One study, however, has modelled the impact of a tick vaccine used in white-tailed deer on the size of tick populations infesting both this host and unvaccinated cattle when they share grazing areas and sustain cattle tick populations (Busch et al. 2014). Modelling predicted that vaccination of deer alone during three consecutive years could result in the reduction of populations of $R$. microplus by a factor of 60 and that, since the tick persisted where habitats are highly fragmented, the presence of well-connected, large vegetation patches will be essential for tick control where deer and cattle share the habitat (Estrada-Peña et al. 2014).

A last endeavour that is in dire need for the development of globally protective tick vaccines is that of understanding the genetic diversity of $R$. microplus. The protection of Bm86 ranging from $0-100 \%$ shows that there must be a genetic driver behind protective responses to vaccination. Tick vaccines will need to be multivalent, if not also multi-component formulations. Conventional genes used for phylogenetics (16S and 12S rRNA, COI, ITS 1 and 2) have been used to date to show that $R$. microplus contained a cryptic species and displayed some population structure between distant geographical areas (Burger et al. 2014, Low et al. 2015) but additional markers to fully understand population structures at farm level and smaller geographical areas are in dire need. In this regard, a number of microsatellites have been develped for R. microplus (Busch et al. 2014; 
Isabel K.F. de Miranda Santos et al.

Chigagure et al. 2000; Koffi et al. 2006), but these have not been able to provide insight into population structure. Next-generation sequening approaches may be the way forward.

\section{Breeding for resistance to ticks}

Among the alternatives for tick control in cattle, breeding animals for resistance to ticks is the most effective way to control this ectoparasite. This method is cost-effective since there is no cost with chemicals for tick control, and it is environmentally sound, since it does not pollute the environment, and does not harm men or cattle (Frisch et al. 2000; Utech and Wharton 1982). The selection for individual cattle observed as being genetically more resistant to infestations with $R$. microplus may be one of the most efficient alternatives for tick control, but it is not a widely adopted practice in current production systems mainly due to the difficulty in evaluating this phenotype. Selection for resistance is efficient because there is genetic variability not only among individuals of different breeds, but also among individuals of the same breed, allowing the animals to differ substantially in their tick loads, where some are more resistant and others more susceptible to infestations.

As stated in the introduction, it is well known that indicine (zebu) cattle (B. indicus) are naturally more resistant to tick infestations than taurine cattle (B. taurus) (Ibelli et al. 2012; Silva et al. 2007; Utech and Wharton, 1982; Wambura et al. 1998). This fact is mainly due to the evolutionary history to which these different subspecies have been subjected over the years. Both subspecies began to be domesticated about 10,000 years ago (Bradley et al. 1998; Chen et al. 2010), but in very different ecological, including climatic conditions. During their evolution, indicine cattle were submitted to high levels of environmental stress present in Asia and more recently in Africa, Oceania and the Americas, causing them to evolve physiologically according to the conditions imposed on them in the tropics (Hanotte et al. 2002; Hansen 2004; Harris 1966) and this included the selective pressure of ticks on their hosts. As stated above, the cattle tick R. microplus tick originated in Asia. It was later introduced to regions with climates established between $32^{\circ}$ north and $32^{\circ}$ south parallels, such as Australia, Mexico, Central America, South America and Africa, which are considered to be favourable for the development and maintenance of this species of tick. Thus, it is possible to conclude that over the thousands of years of coexistence and coevolution, indicine cattle that were naturally more susceptible to infestations were eliminated in response to the pressure of natural selection in the shaping of this subspecies. On the other hand, European breeds did not undergo selective pressure by this parasite until they were introduced relatively recently to new habitats by European colonization ventures. Interestingly, the African N'Dama, which is a taurine breed, carries low tick loads (Mattioli et al. 2000), emphasizing the role of natural selection in generating hosts that are resistant to ticks.

Besides differences in the composition of local (Carvalho et al. 2010a,b; Constantinoiu et al. 2010; Franzin et al. 2017; Piper et al. 2010) and systemic (Carvalho et al. 2008) immune responses to tick bites, the genetic composition of indicine and taurine bovines has been shown to correlate with coat characteristics (Ibelli et al. 2012), tongue morphology (Veríssimo et al. 2015), and activity of sweat glands (Allen 1962; Schleger and O'Kelly 1986). Denser and longer coats can protect ticks from sun rays and desiccation, a coarser tongue can be more effective in removal of larvae by licking and skin glands can provide different compounds and amounts of compounds in sweat that directly or indirectly affect skin physiology and/or tick behaviour.

The role of the host's coat characteristics in resistance to infestations is reinforced by some of the following facts: short haired cattle have fewer ticks than longhaired cattle (Fraga et al. 2003; Hüe 
et al. 2014; Marufu et al. 2011; Veríssimo et al. 2002); animals that carry the SLICK haplotype that results in shorter coats, besides being more heat tolerant (Davis et al. 2017; Dikmen et al. 2014) carry lower tick loads (Hüe et al. 2014). Short hair likely provides an unfavourable environment for tick larvae and even other stages on cattle. A difference of $40 \%$ fewer ticks was found between a shorn and unshorn side in cattle and the shorter the hair, the lower the tick infestation (C.J. Veríssimo, unpublished observations). Besides differences in tongue morphology (Veríssimo et al. 2015), differences in the area of the skin were also identified as being correlated with the increased resistance of the animals to tick infestations (Meltzer 1996). Thickness of host skin appears to play an important role in resistance to tick infestations (Foster et al. 2007), as well as colour of the coat (Gasparin et al. 2007). Thus, the association between the heritability of coat characteristics and tick loads indicates that the practice of shearing dairy cattle with long, dense coats could be a useful practice on some farms to control infestations while the results of breeding are underway.

The advent of artificial selection has directed breeding programs over the years to attend specific goals for meat and milk supplies. With the increase of the world population, production systems began to suffer even greater pressure for efficiency and increasingly productive animals began to be selected without taking into account the other desirable characteristics that can also be selected for. As a result, there has been an increase in the use of taurine breeds, since in Europe these animals became specialized in meat and milk supplies through the use of dedicated breeding programs (O'Neill et al. 2010). In addition, the intensive use of a few breeding herds with greater production potential, identified by increasingly accurate methods, has led to a reduction of genetic diversity (Gibbs et al. 2009) and, consequently, there was a lack of adaptive or robust characteristics, including resistance to tick infestations.

According to Wagland (1975), when cattle have not been exposed to $R$. microplus, resistance against this parasite is an acquired phenomenon in both indicine and taurine breeds, both types of cattle being susceptible to primary infestation with $R$. microplus larvae. In addition, much evidence indicates that a large part of the variation of this characteristic is under genetic control. The estimated heritability for resistance of bovines to infestations, observed in different studies, representing the total variance attributable to the mean effects of the genes, varies from 0.03 to 0.49 (Budeli et al. 2009; Madalena et al. 1985).

The identification of animals considered genetically more resistant to infestations by $R$. microplus ticks to become parents of the next generations is not an easy task. According to Utech et al. (1978) resistance to tick infestations is characterized as the ability of cattle to limit the number of adult ticks attached to them. Therefore, the evaluation of the infestation occurs mainly through the repeated evaluation of the phenotype, after natural and/or artificial infestation, mainly through quantitative techniques, where the number of engorged females present is counted throughout the body of the animal or only in a specific region defined by researchers, either manually or through images associated with computer programs. In addition, for the results to be considered reliable, it is necessary to control the different effects that influence the degree of infestation of the animals, especially when comparing groups of animals from different herds and properties. In this case, the most common effects tested for in statistical analyses are: farm, sex, year of birth, month or season of birth, management group, date of infestation evaluation, racial composition, climate, as well as genetic effects (Ayres et al. 2015; Biegelmeyer et al. 2015; Ibelli et al. 2012; Martinez et al. 2006; Passafaro et al. 2015).

Several efforts have been made to identify and validate molecular markers that can be used to select individuals with genotypes that make them more resistant to tick infestations. Marker 
assisted selection (MAS) has been increasingly explored mainly because the identification of animals presenting desirable features through the evaluation of a phenotype, besides being difficult to measure as previously mentioned, is economically unfeasible in extensive breeding systems. One of the main advantages of MAS is the precocity in the selection of genetically resistant individuals due to its ability to predict the genetic value of the animals. In other words, it is possible to carry out a pre-selection with very young animals or even embryos without having to wait for the ideal age for the expression of the phenotype, having as main consequences a reduction of the generation interval and a considerable increase in the genetic progress of the herd.

With genome-wide association studies that concomitantly involve a large number of markers spread throughout the DNA (in the case of cattle, currently more than 770,000 markers), it has become possible to identify associations between regions of the genome and mechanisms of resistance and susceptibility to tick infestations, which act together but are hitherto unknown. This genomic strategy contributes substantially to a better understanding of the fundamental physiological processes that involve the expression of this characteristic. Indeed, different loci of DNA, including those associated with BoLA, the bovine MHC complex, and the integrin alpha 11 gene have already been identified as being associated with the mechanisms that involve the resistance of animals to tick infestations (Acosta-Rodríguez et al. 2005; Martinez et al. 2006; PortoNeto et al. 2010; Untalan et al. 2007). It is likely that most of these markers will explain a very small proportion of the variation of characteristic of interest, thus making it impossible to select animals based on few markers. In the case of resistance to tick infestations, besides being a quantitative trait it is also a very complex trait, influenced by several mechanisms of small effect that act together to confer overall resistance to the host.

According to Goddard and Hayes (2009), selection by genotype has been used successfully mainly when it comes to phenotypes influenced by few genes and with high heritability. Therefore, it is necessary that the selection of individuals considered genetically resistant to tick infestations be carried out through a panel of molecular markers that, even if considered of low predictive value when evaluated alone, together result in a higher proportion of the explained variance, thus improving the precision of the predictions (Porto-Neto et al. 2011). Furthermore, regardless of the level of heritability of tick loads, whether low or moderate, it has been pointed out (Davis 1993) that it is similar to the heritability of growth traits and milk production seen in cattle from tick-free, temperate regions and that present considerable genetic progress within selection programs. This allows one to suggest that it is possible to obtain genetic gain for the progeny for resistance to infestations with R. microplus ticks.

As an alternative to selection programs of animals genetically resistant to tick infestations, another way of increasing resistance to ticks in herds would be the adoption of mating systems between different breeds, known as crossbreeding. Crossbreeding is the main strategy applied to exploit the differences between breeds and the main advantage, besides the heterosis observed in progeny performance, is the complementarity between them (Passafaro et al. 2015). In this context, from 1996 onwards in Brazil, Girolando cattle have been notable in the Brazilian dairy scenario, mainly due to their complementarity, i.e. the high productive capacity of the Holstein breed and the high adaptation to tropical conditions of the Gir breed, including low levels of infestation by ticks. Great genetic variety is observed in the progeny of these crosses, thus facilitating the identification of extreme phenotypes, and consequently the selection of the individuals that are best suited to the production systems. 
Despite many controversies in the literature, different studies have shown that productive potentials of animals are not related to resistance to tick infestations and that it is possible to develop increasingly resistant breeds without negatively influencing the productive potential of the animals (Shyma et al. 2015; Turner et al. 2010).

The question remains if and how selecting large numbers of animals for genetic resistance to $R$. microplus will affect this parasite's ecology. As mentioned in this chapter, genetic variability and effective population size seem to be reduced among ticks feeding on less permissive hosts like deer. The apparent equilibrium reached by ticks and their zebu hosts on the Indian subcontinent thousands of years ago indicates that it will also be the case after MAS generates a large population of tick-resistant cattle.

\section{Tick-borne pathogens and tick control}

As mentioned previously in this chapter, the cattle tick is a vector for haemoparasites that have a huge negative impact on production. Under the current options for tick control and prevention of tick-borne diseases, maintaining loads of feeding $R$. microplus ticks below a certain level increases the risk of enzootic instability for tick-borne diseases because herd immunity is weak or entirely absent due when low or no exposure to the vector occurs; when infected ticks are eventually introduced, a large population of infected vectors is rapidly generated. Thus, any measure employed to control $R$. microplus, must take into account the need to maintain a good immunity against the haemoparasites that it transmits. Climatic factors can also affect tick loads and, hence, enzootic stability for tick-borne diseases.

Besides enzootic stability being afforded directly by exposure to infected ticks and ensuing immunity against the tick-borne pathogen, another interesting possibility for the origin of this stability is immunity against tick saliva itself. Tick saliva is a Trojan horse for pathogens and enhances their transmission (Francischetti et al. 2009); immunity against its components through frequent exposure to large tick loads may weaken the pathogen's delivery system and the tick's payload. This hypothesis has been examined and proved for Leishmania brasiliensis and Borrelia burgdorferi, two pathogens transmitted by blood-sucking arthropods (Dai et al. 2009; Gomes et al. 2016; Narasimhan et al. 2016). On the other hand, the inflammatory response against arthropod bites has been shown to enhance transmission of a vector-borne virus (Pingen et al. 2016), therefore the role of immunity to the cattle tick's salivary proteins and pathogen transmission to cattle must be examined, including immunity induced by anti-tick vaccines.

Interestingly, the haemoparasites themselves can affect tick biology. For example, Anaplasma marginale, a rickettsial pathogen transmitted by $R$. microplus, down-regulates expression of this tick's immune-related genes (Rosa et al. 2016) and theoretically could render ticks more vulnerable to entomopathogens. However, the consequences of this down-regulation for tick innate defences against entomopathogens has not been examined. Conversely, the evolution of this pathogen is shaped by the performance of populations of its tick vectors undergoing different seasonal dynamics (i.e. high temperature and medium rainfall, constant high temperature and rainfall, or low and inadequate rainfall) because variability of the pathogen's genes encoding four major surface protein 1 repeats and other genetic structures (e.g. microsatellites) evolves with the different ecoregion clusters of ticks (Estrada-Peña et al. 2009). The ecoregion cluster that undergoes low and inadequate rainfall is not an effective vector of $A$. marginale. Since major surface protein 1 mediates interactions with components of host immunity, the ecological factors that shape the genetic composition, the size and the seasonal dynamics of tick populations can 
have far-reaching consequences for adapting pathogens to vectors and, consequently, hosts. It will be interesting to examine if the introduction of a large number of genetically resistant indicine hosts will have an equivalent effect upon adaptation of $A$. marginale to surviving populations of R. microplus that low and inadequate rainfall has.

\section{Conclusions and new research venues}

The complexity of the biology of $R$. microplus, the demands of producers and consumers, and the failure of a single component approach, namely, acaricides, to control ticks after a relatively short period of use, indicate that a multi-component and integrated strategy will have to be adopted if tick infestations in production animals are to be maintained at levels compatible with animal wellbeing and health and profitable and sustainable production. The quest for controlling cattle ticks without poisons entails increasing scientific knowledge about the on-host and off-host ecology of this parasite in order to identify opportunities and develop technologies that control infestations sustainably.

This chapter points to new opportunities for alternatives to acaricides for control of the cattle tick that require research on many topics, some of which were approached in this chapter and are summarized here:

- natural products with acaricide and/or repellent activities: high-throughput screening platforms, mechanisms of activity on ticks, chemical modifications and delivery systems;

- evolution and speciation of of $R$. microplus and $R$. australis for insights into their biology and factors for reproductive success;

- evaluation of tick aggregation on hosts and impact of targeted selective treatment on tick control and emergence of resistance to acaricides;

- vaccines: antigen discovery platforms, new antigens, adjuvants and delivery systems;

- breeding cattle for resistance to tick infestations while maintaining good productivity;

- impact of host immunity and genotypes on tick genotypes and biology;

- impact of host microbiota on tick behaviour;

- impact of host genotype on composition of host microbiota;

- impact of tick microbiota on tick performance;

- impact of effective population sizes of ticks to generate ticks resistant to interventions;

- breeding pasture grasses for both nutritional value, agronomical value and impact on tick biology;

- impact of immunity against tick saliva on immunity to tick-borne pathogens;

- modelling to determine levels of infestations in pastures and on hosts compatible with productivity;

- systematic evaluation of production systems in tick infestations;

- the role of tick innate immunity against entomopathogens and tick-borne pathogens;

- breeding programmes with marker assisted selection of animals with both low tick loads and high productivity;

- delivering knowledge about tick biology to producers in order to increase compliance to control methods.

\section{Acknowledgements}

The authors are indebted to their funding agencies for supporting part of the research described in this chapter. Specifically, IKFMS, GRG, PSO, CJV, LK and LR acknowledge the support of the Fundação de Amparo à Pesquisa do Estado de São Paulo - FAPESP, grant numbers 12/50814-1, 
12/13708-9, 12/06374-7, 09/53645-3, 12/03191-9, 11/08274-7, and 99/12628-5; IKFMS and MPS acknowledge the support of the Conselho Nacional de Desenvolvimento Científico e Tecnológico - CNPq, grant number 559603/2009-6. CM-O acknowledges the support of the National Research Foundation of South Africa.

\section{References}

Abbas RZ, Zaman MA, Colwell DD, Gilleard J and lqbal Z (2014) Acaricide resistance in cattle ticks and approaches to its management: the state of play. Vet Parasitol 203: 6-20.

Aboelhadid SM, Kamel AA, Arafa WM and Shokier KA (2013) Effect of Allium sativum and Allium cepa oils on different stages of Boophilus annulatus. Parasitol Res 112: 1883-1890.

Acosta-Rodríguez R, Alonso-Morales R, Balladares S, Flores-Aguilar H, García-Vazquez Z and Gorodezky C (2005) Analysis of BoLA class II microsatellites in cattle infested with Boophilus microplus ticks: class II is probably associated with susceptibility. Vet Parasitol 127: 313-321.

Allen TE (1962) Responses of Zebu, Jersey and Zebu x Jersey cross-bred heifers to rising temperature with particular reference to sweating. Aust J Agricul Res 13: 165-179.

Alvarenga LC, Paiva PCA, Banys VL, Collao-Saens EA, Rabelo AMG and Rezende CAP (2004) Alteração da carga de carrapatos de bovinos sob a ingestão de diferentes níveis do resíduo do beneficiamento do alho. Ciênc Agrotec 28: 906-912.

Anadon A, Martinez-Larranaga MR and Martinez MA (2009) Use and abuse of pyrethrins and synthetic pyrethroids in veterinary medicine. Vet J 182: 7-20.

Andreotti R, Pérez de León AA, Dowd SE, Guerrero FD, Bendele KG and Scoles GA (2011) Assessment of bacterial diversity in the cattle tick Rhipicephalus (Boophilus) microplus through tag-encoded pyrosequencing. BMC Microbiol 11: 6.

Andreotti R, Garcia MV, Cunha RC and Barros JC (2013) Protective action of Tagetes minuta (Asteraceae) essential oil in the control of Rhipicephalus microplus (Canestrini, 1887) (Acari: Ixodidae) in cattle pen trial. Vet Parasitol 197: 341-345.

Agnolin CA, Olivo CJ, Leal MLR, Beck RCR, Meinerz GR, Parra CLC, Machado PR, Foletto V, Bem CM and Nicolodi PRSJ (2010) Eficácia da citronela [Cymbopogon nardus (L.) Rendle] no controle de ectoparasitas de bovinos. Rev Bras Plantas Med 12: 482-487.

Apanaskevich DA and Oliver JH Jr. (2014) Life cycles and natural history of ticks. In: Sonenshine DE and Roe MR (eds.) Biology of ticks (2 ${ }^{\text {nd }}$ Ed.). Oxford University Press, New York, NY, USA.

Aurnheimer RCM, Costa Pereira MAV, Vita GF and Damas SL (2012) Eficácia in vitro de Ruta graveolens, nas formas fitoterápica e homeopática, para o controle de carrapatos. Ars Vet 28: 122-127.

Ayres DR, Pereira RJ, Boligon AA, Baldi F and Roso VM and Albuquerque LG (2015) Genetic parameters and investigation of genotype $\times$ environment interactions in Nellore $\times$ Hereford crossbred for resistance to cattle ticks in different regions of Brazil. J Appl Genet 56: 107-113.

Baffi MA, De Souza GRL and Vieira CU (2007) Identification of point mutations in putative carboxylesterase and their association with acaricide resistance in Rhipicephalus (Boophilus) microplus. Vet Parasitol 148: 301-309.

Bahiense TC and Bittencourt VR (2004) Laboratory evaluation of the compatibility and the synergism between the entomopathogenic fungus Beauveria bassiana and deltamethrin to resistant strains of Boophilus microplus. Ann $\mathrm{N}$ Y Acad Sci 1026: 319-322.

Bai SH and Ogbourne S (2016) Eco-toxicological effects of the avermectin family with a focus on abamectin and ivermectin. Chemosphere 154: 204-214.

Balashov YS (1972) Bloodsucking ticks (Ixodoidea) - vectors of diseases of man and animals. Ann Entomol Soc America 54: 421.

Barbercheck ME and Millar LC (2000) Environmental impacts of entomopathogenic nematodes used for biological control in soil. In: Follet PA and Duan JJ (eds.) Nontarget effects of biological control. Kluwer Academic Publishers, Boston, MA, USA. 
Barletta AB, Nascimento-Silva MC, Talyuli OA, Oliveira JH, Pereira LO, Oliveira PL and Sorgine MH (2017) Microbiota activates IMD pathway and limits Sindbis infection in Aedes aegypti. Parasite Vectors 10: 103.

Baron S, Van der Merwe NA, Madder M and Maritz-Olivier C (2015) SNP analysis infers that recombination is involved in the evolution of amitraz resistance in Rhipicephalus microplus. PLoS ONE 10: e0131341.

Barrero RA, Guerrero FD, Black M, McCooke J, Chapman B, Schilkey F, Pérez de León AA, Miller RJ, Bruns S, Dobry J, Mikhaylenko G, Stormo K, Bell C, Tao Q, Bogden R, Moolhuijzen PM, Hunter A and Bellgard MI (2017) Geneenriched draft genome of the cattle tick Rhipicephalus microplus: assembly by the hybrid Pacific Biosciences/ Illumina approach enabled analysis of the highly repetitive genome. Int J Parasitol 47: 569-583.

Baxter GD and Barker SC (1998) Acetylcholinesterase CDNA of the cattle tick, Boophilus microplus: characterisation and role in organophosphate resistance. Insect Biochem Mol Biol 28: 581-589.

Bendele KG, Guerrero FD, Miller RJ, Li AY, Barrero RA, Moolhuijzen PM, Black M, McCooke JK, Meyer J, Hill CA and Bellgard MI (2015) Acetylcholinesterase 1 in populations of organophosphate-resistant North American strains of the cattle tick, Rhipicephalus microplus (Acari: Ixodidae). Parasitol Res 114: 3027-3040.

Benelli G, Pavela R, Canale A and Mehlhorn H (2016) Tick repellents and acaricides of botanical origin: a green roadmap to control tick-borne diseases? Parasitol Res 115: 2545-2560.

Berk Z, Laurenson YC, Forbes AB and Kyriazakis I (2016) Modelling the consequences of targeted selective treatment strategies on performance and emergence of anthelmintic resistance amongst grazing calves. Int J Parasitol Drugs Drug Resist 6: 258-271.

Biegelmeyer P, Nizoli LQ, Da Silva SS, Dos Santos TR, Dionello NJ, Gulias-Gomes CC and Cardoso FF (2015) Bovine genetic resistance effects on biological traits of Rhipicephalus (Boophilus) microplus. Vet Parasitol 208: 231-237.

Bianchi MW, Barré N and Messad S (2003). Factors related to cattle infestation level and resistance to acaricides in Boophilus microplus tick populations in New Caledonia. Vet Parasitol 112: 75-89.

Blisnick AA, Foulon T and Bonnet SI (2017) Serine protease inhibitors in ticks: an overview of their role in tick biology and tick-borne pathogen transmission. Front Cell Infect Microbiol 7: 199.

Bloomquist JR (2003) Chloride channels as tools for developing selective insecticides. Insect Biochem Physiol 54: 145-156.

Booth TF (1989) Effects of biogenic amines and adrenergic drugs on oviposition in the cattle tick Boophilus: evidence for octopaminergic innervation of the oviduct. Exp Appl Acarol 7: 259-266.

Borges LMF, Sousa LAD and Barbosa CS (2011) Perspectives for the use of plant extracts to control the cattle tick Rhipicephalus (Boophilus) microplus. Rev Bras Parasitol Vet 20: 89-96.

Bradley DG, Loftus RT, Cunningham P and MacHugh DE (1998) Genetics and domestic cattle origins. Evol Anthropol Issues News Rev 6: 79-86.

Brito LG, De Oliveira Nery L, Da Silva Barbieri F, Huacca MEF, Dos Santos Pereira S, Da Silva RR, De Freitas Fernanades CC and De Sena Oliveira MC (2017) Molecular quantitative assay for esterase-mediated organophosphate resistance in Rhipicephalus microplus. Ticks Tick Borne Dis 8: 725-732.

Brovini CN, Furlong J and Chagas ACS (2003) Influence of climatic factors in the biology and behavior of Boophilus microplus engorged females in the field. Biosci J 19: 71-76.

Budeli MA, Nephawe KA, Norris D, Selapa NW, Bergh L and Maiwashe A (2009) Genetic parameters estimates for tick resistance in Bonsmara cattle. S Afr J Anim Sci 39: 321-327.

Bunnell T, Hanisch K, Hardege JD and Breithaupt T (2011) The fecal odor of sick hedgehogs (Erinaceus europaeus) mediates olfactory attraction of the tick Ixodes hexagonus. J Chem Ecol 37: 340-347.

Burger TD, Shao R and Barjer SC (2014) Phylogenetic analysis of mitochondrial genome sequences indicates that the cattle tick, Rhipicephalus (Boophilus) microplus, contains a cryptic species. Mol Phylogenet Evol 76: 241-253.

Busch JD, Stone NE, Nottingham R, Araya-Anchetta A, Lewis J, Hochhalter C, Giles JR, Gruendike J, Freeman J, Buckmeier G, Bodine D, Duhaime R, Miller RJ, Davey RB, Olafson PU, Scoles GA and Wagner DM (2014) Widespread movement of invasive cattle fever ticks (Rhipicephalus microplus) in southern Texas leads to shared local infestations on cattle and deer. Parasite Vectors 7: 188.

Calabrese JM, Brunner JL and Ostfeld RS (2011) Partitioning the aggregation of parasites on hosts into intrinsic and extrinsic components via an extended Poisson-gamma mixture model. PLoS ONE 6: e29215. 
Camargo MG, Marciano AF, Sá FA, Perinotto WMS, Quinelato S, Gôlo PS, Angelo IC, Prata MCA and Bittencourt VREP (2014) Commercial formulation of Metarhizium anisopliae for the control of Rhipicephalus microplus in a pen study. Vet Parasitol 205: 271-276.

Camargo MG, Nogueira MRS, Marciano AF, Perinotto WMS, Coutinho-Rodrigues CJB, Scott FB, Angelo IC, Prata MCA and Bittencourt VREP (2016) Metarhizium anisopliae for controlling Rhipicephalus microplus tick under field conditions. Vet Parasitol 223: 38-42.

Campos RNS, Bacci L, Araújo APA, Blank AF, Arrigoni-Blank MF, Santos GRA and Roner MNB (2012) Óleos essenciais de plantas medicinais e aromáticas no controle do carrapato Rhipicephalus microplus. Arch Zootec 61: 67-78

Campos Pereira M and Labruna MB (2008) Rhipicephalus (Boophilus) microplus. In: Campos Pereira M, Labruna MB, Szabó MPJ and Klafke GM (eds.) Rhipicephalus (Boophilus) microplus: biologia, controle e resistência. Medicina Veterinária, São Paulo, SP, Brazil.

Canevari JT, Mangold AJ, Guglielmone AA and Nava S (2017) Population dynamics of the cattle tick Rhipicephalus (Boophilus) microplus in a subtropical sub-humid region of Argentina for use in the design of control strategies. Medical and Veterinary Entomology 31: 6-14.

Carvalho WA, Bechara GH, Moré DD, Ferreira BR, Da Silva JS and De Miranda Santos IK (2008) Rhipicephalus (Boophilus) microplus: distinct acute phase proteins vary during infestations according to the genetic composition of the bovine hosts, Bos taurus and Bos indicus. Exp Parasitol 118: 587-591.

Carvalho WA, Franzin AM, Abatepaulo AR, De Oliveira CJ, Moré DD, Da Silva JS, Ferreira BR and De Miranda Santos IK (2010a) Modulation of cutaneous inflammation induced by ticks in contrasting phenotypes of infestation in bovines. Vet Parasitol 167: 260-273.

Carvalho WA, Maruyama SR, Franzin AM, Abatepaulo AR, Anderson JM, Ferreira BR, Ribeiro JM, Moré DD, Augusto Mendes Maia A, Valenzuela JG, Garcia GR and De Miranda Santos IK (2010b) Rhipicephalus (Boophilus) microplus: clotting time in tick-infested skin varies according to local inflammation and gene expression patterns in tick salivary glands. Exp Parasitol 124: 428-435.

Castro KNC, Ishikawa MM, Catto JB, Castro MM and Motta IS (2009) Avaliação in vitro do extrato do pinheiro brasileiro para controle do carrapato dos bovinos. Rev Bras Agroecol 4: 2575-2578.

Castro KNC, Lima DF, Wolschick D, Andrade IM, Santos RCS, Santos FJS, Veras LMC and Costa-Júnior LMC (2016) In vitro effects of Pilocarpus microphyllus extracts and pilocarpine hydrochloride on Rhipicephalus (Boophilus) microplus. Vet Parasitol 25: 248-253.

Catto JB, Bianchin I and Saito ML (2009) Efeito acaricida in vitro de extratos de plantas do pantanal no carrapato de bovinos Rhipicephalus (Boophilus) microplus. Research and Development Bulletin 26 Embrapa Gado de Corte, Campo Grande, MS, Brazil.

Cerny V and De La Cruz J (1971) Development and survival of the tick Boophilus microplus (Can.) in laboratory and under natural conditions of Cuba. Folia Parasitol (Praha) 18: 73-78.

Chagas ACS, Passos WM, Prates HT, Leite RC, Furlong J and Fortes ICP (2002) Efeito acaricida de óleos essenciais e concentrados emulsionáveis de Eucalyptus spp. em Boophilus microplus. Braz J Vet Res An Sci 39: 247-253.

Chagas ACS, Oliveira MCS, Giglioti R, Calura FH, Ferrenzini J, Forim MR and Barros ATM (2010) Efficacy evaluation of a commercial neem cake for control of Haematobia irritans on Nelore cattle. Rev Bras Parasitol Vet 19: 217-221.

Chagas ACS and Rabelo MD (2012) Método para detecção de substâncias com atividade repelente sobre larvas do carrapato Rhipicephalus (Boophilus) microplus: revisão e recomendações. Research and Development Bulletin 106 Embrapa Pecuária Sudeste, São Carlos, SP, Brasil.

Chagas ACS, Domingues LC, Fantatto RR, Giglioti R, Oliveira MCS, Oliveira DH, Mano RA and Jacob RG (2014) In vitro and in vivo acaricide action of juvenoid analogs produced from the chemical modifiction of Cymbopogon spp. and Corymbia citriodora essential oil on the cattle tick Rhipicephalus (Boophilus) microplus. Vet Parasitol 205: 277-284.

Chagas ACS, Oliveira MCS, Santana RCM, Bizzo HR, Gama PE and Chaves FCM (2016) Efficacy of 11 Brazilian essential oils on lethality of the cattle tick Rhipicephalus (Boophilus) microplus. Ticks Tick Borne Dis 7: 427-432.

Chandler D, Davidson G, Pell JK, Ball BV, Shaw K and Sunderland KD (2000) Fungal biocontrol of Acari. Biocontrol Sci Technol 10:357-384. 
Chen AC, He H and Davey RB (2007) Mutations in a putative octopamine receptor gene in amitraz-resistant cattle ticks. Vet Parasitol 148: 379-383.

Chen S, Lin BZ, Baig M, Mitra B, Lopes RJ, Santos AM, Magee DA, Azevedo M, Tarroso P, Sasazaki S, Ostrowski S, Mahgoub O, Chaudhuri TK, Zhang YP, Costa V, Royo LJ, Goyache F, Luikart G, Boivin N, Fuller DQ, Mannen H, Bradley DG and Beja-Pereira A (2010) Zebu cattle are an exclusive legacy of the South Asia Neolithic. Mol Biol and Evol 27: 1-6.

Chevillon C, Ducornez S, Meeüs T, Koffi BB, Gaia H, Delathière JM and Barré N (2007) Accumulation of acaricide resistance mechanisms in Rhipicephalus (Boophilus) microplus (Acari: Ixodidae) populations from New Caledonia Island. Vet Parasitol 147: 276-288.

Chevillon C, De Garine-Wichatitsky M, Barré N, Ducornez S and De Meeûs T (2013) Understanding the genetic, demographical and/or ecological processes at play in invasions: lessons from the southern cattle tick Rhipicephalus microplus (Acari: Ixodidae). Exp Appl Acarol 59: 203-218.

Chigagure NN, Baxter GD and Barker SC (2000) Microsatellite loci of the cattle tick Boophilus microplus (Acari: Ixodidae). Exp Appl Acarol 24: 951-956.

Constantinoiu CC, Jackson LA, Jorgensen WK, Lew-Tabor AE, Piper EK, Mayer DG, Venus B and Jonsson NN (2010) Local immune response against larvae of Rhipicephalus (Boophilus) microplus in Bos taurus indicus and Bos taurus taurus cattle. Int J Parasitol 40: 865-875.

Coon KL, Brown MR and Strand MR (2016) Gut bacteria differentially affect egg production in the anautogenous mosquito Aedes aegypti and facultatively autogenous mosquito Aedes atropalpus (Diptera: Culicidae). Parasite Vectors 9: 375 .

Corson MS, Teel PD and Grant WE (2001) Influence of acaricide resistance on cattle-fever tick (Boophilus spp.) infestations in semi-arid thornshrublands: a simulation approach. Exp Appl Acarol 25: 171-184.

Costa-Júnior LM and Furlong J (2011) Efficiency of sulphur in garlic extract and non-sulphur homeopathy in the control of the cattle tick Rhipicephalus (Boophilus) microplus. Med Vet Entomol 25: 7-11.

Costa-Júnior LM, Miller RJ, Alves PB, Blank AF, Li AY and León AP (2016) Acaricidal efficacies of Lippia gracilis essential oil and its phytochemicals against organophosphate-resistant and susceptible strains of Rhipicephalus (Boophilus) microplus. Vet Parasitol 228: 60-64.

Cruz EM, Costa-Júnior LM, Pinto JÁ, Santos DA, Araújo AS and Blank AF (2013) Acaricidal activity of Lippia gracilis essential oil and its major constituents on the tick Rhipicephalus (Boophilus) microplus. Vet Parasitol 195: 198-202.

Cunha AP, Bello ACP, Leite RC, Oliveira PR, Martins JR, Ribeiro ACL, Domingues LN, Freitas CMV, Bastianetto E, Wanderley RPB and Rosa RCD (2008) Efeito da adubação com ureia em pastagem, sobre Rhipicephalus (Boophilus) microplus (Acari: Ixodidae). Rev Bras Parasitol Vet 17: 64- 68.

Cunha AP, Bello ACP, Domingues LN, Martins JR, Oliveira PR, Freitas CMV, Bastianetto E, Silva MX and Leite RC (2010) Effects of urea on the cattle tick Rhipicephalus (Boophilus) microplus (Acari: Ixodidae). Vet Parasitol 174: 300-304.

Dai J, Wang P, Adusumilli S, Booth CJ, Narasimhan S, Anguita J and Fikrig E (2009) Antibodies against a tick protein, Salp15, protect mice from the Lyme disease agent. Cell Host Microbe 6: 482-492.

Davis GP (1993) Genetic-parameters for tropical beef-cattle in northern Australia: a review. Aust J Agric Res 44: 179-198.

Davis TS, Crippen TL, Hofstetter RW and Tomberlin JK (2013) Microbial volatile emissions as insect semiochemicals. J Chem Ecol 39: 840-859.

Davis SR, Spelman RJ and Littlejohn MD (2017) Breeding heat tolerant dairy cattle: the case for introgression of the 'slick' prolactin receptor variant into dairy breeds. J Anim Sci 95: 1788-1800.

De la Fuente J, Almazán C, Canales M, Pérez de la Lastra JM, Kocan KM and Willadsen P (2007) A ten-year review of commercial vaccine performance for control of tick infestations on cattle. Anim Health Res Ver 8: 23-28.

De Meeüs T, Koffi BB, Barré N, De Garine-Wichatitsky M and Chevillon C (2010) Swift sympatric adaptation of a species of cattle tick to a new deer host in New Caledonia. Infect Genet Evol 10: 976-983

Dennison NJ, Jupatanakul N and Dimopoulos G (2014) The mosquito microbiota influences vector competence for human pathogens. Curr Opin Insect Sci 3: 6-13.

De Paula PH and Furlong J (2002) The drop-off behavior of engorged females of the cattle tick, Boophilus microplus. Ciência Rural 32: 627-631. 
Dikmen S, Khan FA, Huson HJ, Sonstegard TS, Moss JI, Dahl GE and Hansen PJ (2014) The SLICK hair locus derived from Senepol cattle confers thermotolerance to intensively managed lactating Holstein cows. J Dairy Sci 97: 5508-5520.

Dolinski C and Moino A Jr. (2006) Utilização de nematoides entomopatogênicos nativos ou exóticos: o perigo das introduções. Nematol Bras, Brasilia 30: 139-149.

Domingos A, Antunes S, Borges L and Estólio do Rosário V (2013) Approaches towards tick and tick-borne diseases control. Rev Soc Bras MedTrop 46: 265-269.

Domingues LF, Fantatto RR, Batista JH, Rabelo MD, Politi FAS, Giglioti R, Oliveira MCS, Chaves FCM and Chagas ACS (2014) In vitro activity of 13 essential oils on the cattle tick Rhipicephalus (Boophilus) microplus and on the sheep nematode Haemonchus contortus in Brazil. Planta Med 80: P2B4.

Dong K (2007) Insect sodium channels and insecticide resistance. Invert Neurosci 7: 17-30.

Donzé G, McMahon C and Guerin PM (2004) Rumen metabolites serve ticks to exploit large mammals. J Exp Biol 207: 4283-4289.

Drummond RO, Ernst SE, Trevino JL, Gladney WJ and Graham OH (1973) Boophilus annulatus and Boophilus microplus: laboratory test of insecticides. J Econ Entomol 66: 130-133.

Dutta S, Godara R, Katoch R, Yadav A, Katoch M and Singh NK (2017) Detection of amitraz and malathion resistance in field populations of Rhipicephalus (Boophilus) microplus (Acari: Ixodidae) in Jammu region of India. Exp Appl Acarol 71: 291-301.

Eguchi M, Itoh M, Nishino K, Shibata H, Tanaka T, Kamei-Hayashi K and Hara S (1994) Amino acid sequence of an inhibitor from the silkworm (Bombyx mori) hemolymph against fungal protease. J Biochem 115: 881-884.

Ellse $L$ and Wall $R$ (2014) The use of essential oils in veterinary ectoparasite control: a review. Med Vet Entomol 28: 233-243.

Estrada-Peña A, Bouattour A, Camicas JL, Guglielmone A, Horak I, Jongejan F, Latif A, Pegram R and Walker AR (2006) The known distribution and ecological preferences of the tick subgenus Boophilus (Acari: Ixodidae) in Africa and Latin America. Exp Appl Acarol 38: 219-235.

Estrada-Peña A, Naranjo V, Acevedo-Whitehouse K, Mangold AJ, Kocan KM and De La Fuente J (2009) Phylogeographic analysis reveals association of tick-borne pathogen, Anaplasma marginale, MSP1 a sequences with ecological traits affecting tick vector performance. BMC Biol 7:57.

Estrada-Peña A, Venzal JM, Nava S, Mangold A, Guglielmone AA, Labruna MB and De la Fuente J (2012) Reinstatement of Rhipicephalus (Boophilus) australis (Acari: Ixodidae) with redescription of the adult and larval stages. J Med Entomol 49: 794-802.

Estrada-Peña A and Salman M (2013) Current limitations in the control and spread of ticks that affect livestock: a review. Agriculture 3: 221-235.

Estrada-Peña A, Carreón C, Almazán C and De la Fuente J (2014) Modeling the impact of climate and landscape on the efficacy of white tailed deer vaccination for cattle tick control in northeastern Mexico. PLoS ONE 9: e102905.

Estrada-Peña A, De la Fuente J and Cabezas-Cruz A (2017) Functional redundancy and ecological innovation shape the circulation of tick-transmitted pathogens. Front Cell Infect Microbiol 7: 234.

Farenhorst M, Knols BG, Thomas MB, Howard AF, Takken W, Rowland M and N'Guessan R (2010) Synergy in efficacy of fungal entomopathogens and permethrin against West African insecticide-resistant Anopheles gambiae mosquitoes. PLOS ONE 5: e12081.

Farias MPO, Sousa DP, Arruda AC, Wanderley AG, Teixeira WC, Alves LC and Faustino MAG (2009) Potencial acaricida do óleo de andiroba Carapa guianensis Aubl. Sobre fêmeas adultas ingurgitadas de Anocentor nitens Neumann, 1897 e Rhipicephalus sanguineus Latreille, 1806. Arq Bras Med Vet Zootec 61: 877-882.

Farias MPO, Wanderley AG, Alves LC and Faustino MAG (2012) Cálculo da Cl50 (concentração inibitória média) e CL50 (concentração letal média) do óleo da semente de andiroba (Carapa guianensis, Aubl.) sobre Rhipicephalus (Boophilus) microplus (Canestrini, 1887), Anocentor nitens (Neumann, 1897) e Rhipicephalus sanguineus (Latreille, 1806) (Acari: Ixodidae). Arq Inst Biol 79: 255-261.

Faza AP, Pinto IS, Fonseca I, Antunes GR, Monteiro CM, Daemon E, Muniz Mde S, Martins MF, Furlong J and Prata MC (2013) A new approach to characterization of the resistance of populations of Rhipicephalus microplus (Acari: Ixodidae) to organophosphate and pyrethroid in the state of Minas Gerais, Brazil. Exp Parasitol 134: 519-523. 
Fernandes FF, Freitas EPS, Costa AC and Silva IG (2005) Larvicidal potential of Sapindus saponaria to control the cattle tick Boophilus microplus. Pesq Agropec Bras 40: 1243-1245.

Fernandez-Ruvalcaba M, Torre FP, Cruz-Vasquez C and Garcia-Vasquez Z (2004) Anti-tick effects of Melinis minutiflora and Andropogon gayanus grasses on plots experimentally infested with Boophilus microplus larvae. Exp Appl Acarol 32: 293-299.

Ferrarini SR, Duarte O, Rosa ZG, Rolim V, Lima VLE, Von Poser G and Ribeiro VLS (2008) Acaricidal activity of limonene, limonene oxide and $\beta$-amino alcohol derivatives on Rhipicephalus (Boophilus) microplus. Vet Parasitol 157: 149-153.

Ferreira LL, Oliveira Filho JG, Mascarin GM, León AAP and Borges LMF (2017) In vitro repellency of DEET and $\beta$-citronellol against the ticks Rhipicephalus sanguineus sensu lato and Amblyomma sculptum. Vet Parasitol 239: 42-45.

Ferreira GF, Freitas TM, Gonçalves CL, Mendes JF, Vieira JN, Villareal JP and Nascente OS (2016) Antiparasitic drugs: in vitro tests against nematophagous fungi. Braz J Biol 76: 990-993.

Flores-Fernández JM, Padilla-Camberos E, Castillo Herrera GA and Martínez-Velázquez M (2016) Adulticidal and oviposition- and hatching-altering activities of essential oil from Mexican oregano leaves (Lippia graveolens H.B.K.) against the cattle tick Rhipicephalus microplus (Acari: Ixodidae). Trop Biomed 33: 290-294.

Foster A, Jackson A and D'Alteiro GL (2007) Skin diseases of South American camelids. In Pract 29: 216-222.

Fraga AB, Alencar MM, Figueiredo LA, Razook AG and Cyrillo JNSG (2003) Análise de fatores genéticos e ambientais que afetam a infestação de fêmeas bovinos da raça Caracu por carrapatos (Boophilus microplus). Rev Bras Zootec 32: 1578-1586.

Fragoso-Sanchez H, Garcia-Vazquez Z, Tapia-Perez A, Ortiz-Najera A, Rosario-Cruz R and Rodriguez-Vivas I (2011) Response of Mexican Rhipicephalus (Boophilus) microplus ticks to selection by amitraz and genetic analysis of attained resistance. J Entomol 8: 218-228.

Francischetti IM, Sa-Nunes A, Mans BJ, Santos IM and Ribeiro JM (2009) The role of saliva in tick feeding. Frontier Biosci 14: 2051-2088.

Franzin AM, Maruyama SR, Garcia GR, Oliveira RP, Ribeiro JM, Bishop R, Maia AA, Moré DD, Ferreira BR and Santos IK (2017) Immune and biochemical responses in skin differ between bovine hosts genetically susceptible and resistant to the cattle tick Rhipicephalus microplus. Parasite Vectors 10: 51.

Frisch JE, O'Neill CJ and Kelly MJ (2000) Using genetics to control cattle parasites - the Rockhampton experience. Int J Parasitol 30: 253-264.

Furlong J, Brovini CN and Chagas ACS (2002a) Behavior of Boophilus microplus larvae in Pennisetum purpureum grass. Biosci J 18: 23-31.

Furlong J, Costa-Júnior LM, Chagas ACS and Reis ES (2002b) Cl50 e CL90 dos extratos alcoólico e aquoso de nim indiano (Azadirachta indica) em larvas de Boophilus microplus. In: Proceedings of the $12^{\text {th }}$ Brasilian Congress of Veterinary Parasitology, September 1-5, 2002, Rio de Janeiro, Brasil.

Gandhi PR, Jayaseelan C, Mary RR, Mathivanan D and Suseem SR (2017) Acaricidal, pediculicidal and larvicidal activity of synthesized $\mathrm{ZnO}$ nanoparticles using Momordica charantia leaf extract against blood feeding parasites. Exp Parasitol 181: 47-56.

Gantz VM, Jasinskiene N, Tatarenkova O, Fazekas A, Macias VM, Bier E and James AA (2015) Highly efficient Cas9mediated gene drive for population modification of the malaria vector mosquito Anopheles stephensi. Proc Natl Acad Sci USA 112: 6736-6743.

Garcia GR, Maruyama SR, Nelson KT, Ribeiro JM, Gardinassi LG, Maia AA, Ferreira BR, Kooyman FN and De Miranda Santos IKF (2017) Immune recognition of salivary proteins from the cattle tick Rhipicephalus microplus differs according to the genotype of the bovine host. Parasite Vectors 10: 144.

García-García JC, Montero C, Redondo M, Vargas M, Canales M, Boue O, Rodríguez M, Joglar M, Machado H, González IL, Valdés M, Méndez L and De la Fuente J (2000) Control of ticks resistant to immunization with Bm86 in cattle vaccinated with the recombinant antigen Bm95 isolated from the cattle tick, Boophilus microplus. Vaccine 18: 2275-2287.

Garris Gl, Popham TW and Zimmerman RH (1990) Boophilus microplus (Acari: Ixodidae): oviposition, egg viability, and larval longevity in grass and wooded environments of Puerto Rico. Environ Entomol 19: 66-75. 
Gasparin G, Miyata M and Coutinho LL (2007) Mapping of quantitative trait loci controlling tick [Rhipicephalus (Boophilus) microplus] resistance on bovine chromosomes 5, 7 and 14. Anim Genet 38: 453-459.

Gaur RS, Sangwan AK, Sangwan N and Kumar S (2016) Acaricide resistance in Rhipicephalus (Boophilus) microplus and Hyalomma anatolicum collected from Haryana and Rajasthan states of India. Exp Appl Acarol 69: 487-500.

Gibbs RA, Taylor JF, Van Tassell CP, Barendse W, Eversole KA, Gill CA, Green RD, Hamernik DL, Kappes SM, Lien S, Matukumalli LK, McEwan JC, Nazareth LV, Schnabel RD, Weinstock GM, Wheeler DA, Ajmone-Marsan P, Boettcher PJ, Caetano AR, Garcia JF, Hanotte O, Mariani P, Skow LC, Sonstegard TS, Williams JL, Diallo B, Hailemariam L, Martinez ML, Morris CA, Silva LO, Spelman RJ, Mulatu W, Zhao K, Abbey CA, Agaba M, Araujo FR, Bunch RJ, Burton J, Gorni C, Olivier H, Harrison BE, Luff B, Machado MA, Mwakaya J, Plastow G, Sim W, Smith T, Thomas MB, Valentini A, Williams P, Womack J, Woolliams JA, Liu Y, Qin X, Worley KC, Gao C, Jiang H, Moore SS, Ren Y, Song XZ, Bustamante CD, Hernandez RD, Muzny DM, Patil S, San Lucas A, Fu Q, Kent MP, Vega R, Matukumalli A, McWilliam S, Sclep G, Bryc K, Choi J, Gao H, Grefenstette JJ, Murdoch B, Stella A, Villa-Angulo R, Wright M, Aerts J, Jann O, Negrini R, Goddard ME, Hayes BJ, Bradley DG, Barbosa da Silva M, Lau LP, Liu GE, Lynn DJ, Panzitta F, Dodds KG and The Bovine HapMap Consortium (2009) Genome-wide survey of SNP variation uncovers the genetic structure of cattle breeds. Science 324: 528-532.

Giglioti R, Rotim MR, Oliveira HN, Chagas ACS, Ferrezini J, Brito LG, Falcoski TORS, Albuquerque LG and Oliveira MCS (2011) In vitro acaricidal activity of neem (Azadirachta indica) seed extracts with known azadirachtin concentrations against Rhipicephalus microplus. Vet Parasitol 181: 309-315.

Gilbert JA, Medlock J, Townsend JP, Aksoy S, Ndeffo Mbah M and Galvani AP (2016) Determinants of human African trypanosomiasis elimination via paratransgenesis. PLoS Negl Trop Dis 10: e0004465.

Goddard ME and Hayes BJ (2009) Mapping genes for complex traits in domestic animals and their use in breeding programmes. Nat Rev Genet 10: 381-391.

Golo PS, Santos HA, Perinotto WM, Quinelato S, Angelo IC, Camargo MG, Sá FA, Massard CL, Fernandes ÉK, Roberts DW and Bittencourt VR (2015) The influence of conidial Pr1 protease on pathogenicity potential of Metarhizium anisopliae senso latu to ticks. Parasitol Res 114: 2309-2315.

Gomes R, Cavalcanti K, Teixeira C, Carvalho AM, Mattos PS, Cristal JR, Muniz AC, Miranda JC, De Oliveira Cl and Barral A (2016) Immunity to Lutzomyia whitmani saliva protects against experimental Leishmania braziliensis infection. PLoS Negl Trop Dis 10: e0005078.

Grisi L, Leite RC, Martins JR, Barros AT, Andreotti R, Cançado PH, León AA, Pereira JB and Villela HS (2014) Reassessment of the potential economic impact of cattle parasites in Brazil. Rev Bras Parasitol Vet 23: 150-156.

Groh KJ, Carvalho RN, Chipman JK, Denslow ND, Halder M, Murphy CA, Roelofs D, Rolaki A, Schirmer K and Watanabe KH (2015) Development and application of the adverse outcome pathway framework for understanding and predicting chronic toxicity: II. A focus on growth impairment in fish. Chemosphere 120: 778-792.

Gross AD, Temeyer KB, Day TA, Pérez de León AA, Kimber MJ and Coats JR (2015) Pharmacological characterization of a tyramine receptor from the southern cattle tick, Rhipicephalus (Boophilus) microplus. Insect Biochem Mol Biol 63: 47-53.

Guerrero FD, Davey RB and Miller RJ (2001) Use of an allele-specific polymerase chain reaction assay to genotype pyrethroid resistant strains of Boophilus microplus (Acari: Ixodidae). J Med Entomol 38: 44-50.

Guerrero FD, Lovis L and Martins JR (2012a) Acaricide resistance mechanisms in Rhipicephalus (Boophilus) microplus. Rev Bras Parasitol Vet 21: 1-6.

Guerrero FD, Miller RJ and Pérez de León AA (2012b) Cattle tick vaccines: many candidate antigens, but will a commercially viable product emerge? Int J Parasitol 42: 421-427.

Hammond A, Galizi R, Kyrou K, Simoni A, Siniscalchi C, Katsanos D, Gribble M, Baker D, Marois E, Russell S, Burt A, Windbichler N, Crisanti A and Nolan T (2016) A CRISPR-Cas9 gene drive system targeting female reproduction in the malaria mosquito vector Anopheles gambiae. Nat Biotechnol 34: 78-83.

Hanotte O, Bradley DG, Ochieng JW, Verjee Y, Hill EW and Rege JE (2002) African pastoralism: genetic imprints of origins and migrations. Science 296: 336-339.

Hansen PJ (2004) Physiological and cellular adaptations of zebu cattle to thermal stress. Anim Reprod Sci 82: 349-360.

Harris M (1966) The cultural ecology of India's sacred cattle. Cur Anthropol 7: 51-59. 
He H, Chen AC, Davey RB, Ivie GW and George JE (1999) Identification of a point mutation in the para-type sodium channel gene from a pyrethroid-resistant cattle tick. Biochem Biophysic Res Comm 261: 558-561.

Heath A and Levot GW (2015) Parasiticide resistance in flies, lice and ticks in New Zealand and Australia: mechanisms, prevalence and prevention. N Z Vet J 63: 199-210.

Hernández Al, Cianzio D and Olson TA (2002) Physiological performance and grazing behavior of Senepol, Brahman and Holstein heifers in Puerto Rico. In: Proceedings of the Senepol Symposium, November 8-10, 2002, St. Croix, USVI.

Hernandez RH, He H, Chen AC, Ivy GW, George JE and Wagner GG (1999) Cloning and sequencing of a putative acetylcholinesterase from Boophilus microplus (Acari: Ixodidae). J Med Entomol 36: 764-770.

Hernandez R, He H, Chen AC, Waghela SD, Ivie GW, George JE and Wagner GG (2000) Identification of a point mutation in an esterase gene in different populations of the southern cattle tick, Boophilus microplus. Insect Biochem Mol Biol 30: 969-977.

Hernandez R, Guerrero FD, George JE, and Wagner GG (2002) Allele frequency and gene expression of a putative carboxylesterase-encoding gene in a pyrethroid resistant strain of the tick Boophilus microplus. Insect Biochem Mol Biol 32: 1009-1016.

Higa LO, Garcia MV, Barros JC, Koller WW and Andreotti R (2016) Evaluation of Rhipicephalus (Boophilus) microplus (Acari: Ixodidae) resistance to different acaricide formulations using samples from Brazilian properties. Rev Bras Parasitol Vet 25: 163-171.

Hoogstraal $\mathrm{H}$ and Aeschlimann A (1982) Tick-host specificity. Bul Soc Ent Suisse 55: 5-32.

Hoogstraal H and Kim KC (1985) Tick and mammal's coevolution with emphasis on Haemaphysalis. In: Kim KC (ed) Coevolution of parasitic arthropods and mammals. John Wiley \& Sons, New York, NY, USA.

Hüe T, Horlin JC, Teurlai M and Naves M (2014) Comparison of tick resistance of crossbred Senepol x Limousin to purebred Limousin cattle. Trop Anim Health Prod 46: 447-453.

Hüe T, Cauquil L, Hzounda Fokou JB, Jazet Dongmo PM, Bakarnga-Via I and Menut C (2015) Acaricidal activity of five essential oils of Ocimum species on Rhipicephalus (Boophilus) microplus larvae. Parasitol Res 114: 91-99.

Ibelli AMG, Ribeiro ARB, Giglioti R, Regitano LCA, Alencar MM, Chagas ACS, Paco AL, Oliveira HN, Duarte JMS and Oliveira MCS (2012) Resistance of cattle of various genetic groups to the tick Rhipicephalus microplus and the relationship with coat traits. Vet Parasitol 186: 425-430.

Jamroz RC, Guerrero FD, Pruett JH, Oehler DD and Miller RJ (2000) Molecular and biochemical survey of acaricide resistance mechanisms in larvae from Mexican strains of the southern cattle tick, Boophilus microplus. J Insect Physiol 46: 685-695.

Jongejan F and Uilenberg G (2004) The global importance of ticks. Parasitology 129: S3-S14.

Jonsson NN and Matschoss AL (1998) Attitudes and practices of Queensland dairy farmers to the control of the cattle tick, Boophilus microplus. Aust Vet J 76: 746-751.

Jonsson NN, Davis R and De Witt M (2001) An estimate of the economic effects of cattle tick (Boophilus microplus) infestation on Queensland dairy farms. Aust Vet J 79: 826-831.

Jonsson NN (2006) The productivity effects of cattle tick (Boophilus microplus) infestation on cattle, with particular reference to Bos indicus cattle and their crosses. Vet Parasitol 137: 1-10.

Jonsson NN and Hope M (2007) Progress in the epidemiology and diagnosis of amitraz resistance in the cattle tick Boophilus microplus. Vet Parasitol 146: 193-198.

Jonsson NN, Cutullè C, Corley SW and Seddon JM (2010) Identification of a mutation in the para-sodium channel gene of the cattle tick Rhipicephalus microplus associated with resistance to flumethrin but not to cypermethrin. Int J Parasitol 40: 1659-1664.

Johnston LA, Kemp DH and Pearson RD (1986) Immunization of cattle against Boophilus microplus using extracts derived from adult female ticks: effects of induced immunity on tick populations. Int J Parasitol 16: 27-34.

Jyoti NA, Singh H, Singh NK and Rath SS (2016a) Fenvalerate resistance status in Rhipicephalus (Boophilus) microplus (Acari: Ixodidae) from Punjab, India. J Parasit Dis 40: 694-698

Jyoti NA, Singh NK, Singh H and Rath SS (2016b) Multiple mutations in the acetylcholinesterase 3 gene associated with organophosphate resistance in Rhipicephalus (Boophilus) microplus ticks from Punjab, India. Vet Parasitol 216: 108-117. 
Kaaya GP, Samish M, Hedimbi M, Gindin G and Glazer I (2011) Control of tick populations by spraying Metarhizium anisopliae conidia on cattle under field conditions. Exp App Acarol 55: 273-281.

Kaaya GP and Hedimbi M (2012) The use of entomopathogenic fungi, Beauveria bassiana and Metarhizium anisopliae, as bio-pesticides for tick control. Intern J Agricult Sci 2: 245-250.

Kazimírová M and Štibrániová I (2013) Tick salivary compounds: their role in modulation of host defences and pathogen transmission. Front Cell Infect Microbiol 3: 43.

Kiss T, Cadar D and Spinu M (2012) Tick prevention at a crossroad: new and renewed solutions. Vet Parasitol 187: 357-366.

Klafke G, Webster A, Dall Agnol B, Pradel E, Silva J, De la Canal LH, Becker M, Osório MF, Mansson M, Barreto R, Scheffer R, Souza UA, Corassini VB, Dos Santos J, Reck J and Martins JR (2017) Multiple resistance to acaricides in field populations of Rhipicephalus microplus from Rio Grande do Sul state, Southern Brazil. Ticks Tick Borne Dis 8: 73-80.

Klompen JSH, Black IV WC, Keirans JE and Oliver JH Jr. (1996) Evolution of ticks. Annu Rev Entomol 41: 141-161.

Koffi BB, Meeuis T, Barre N, Durand P, Arnathau C and Chevillon C (2006) Founder effects, inbreeding and effective sizes of the southern cattle tick: the effect of transmission dynamics and implications for pest management. Mol Ecol 15: 4603-4611.

Korta E, Bakkali A, Berrueta LA, Gallo B, Vicente F and Bogdanov S (2003) Determination of amitraz and other acaricide residues in beeswax. Analytica Chimica Acta 475: 97-103.

Kumar KGA, Sharma AK, Kumar S, Ray DD, Rawat AKS, Srivastava S and Ghosh S (2016) Comparative in vitro anti-tick efficacy of commercially available products and newly developed phyto-formulations against field collected and resistant tick lines of Rhipicephalus (Boophilus) microplus. J Parasitic Dis 40: 1590-1596.

Labruna MB (2008). As gerações anuais. In: Campos Pereira M, Labruna MB, Szabó MPJ and Klafke GM (eds.) Rhipicephalus (Boophilus) microplus: biologia, controle e resistência. Medicina Veterinária, São Paulo, SP, Brazil.

Lara FA, Pohl PC, Gandara AC, Ferreira J da S, Nascimento-Silva MC, Bechara GH, Sorgine MH, Almeida IC, Vaz Ida S Jr. and Oliveira PL (2015) ATP binding cassette transporter mediates both heme and pesticide detoxification in tick midgut cells. PLOS ONE 10: e0134779.

Leal B, Thomas DB and Dearth R (2017) Cattle fever tick, Rhipicephalus (Boophilus) microplus (Acari: Ixodidae): potential control on pastures by the application of urea fertilizer. Vet Parasitol 241: 39-42.

Lees K and Bowman AS (2007) Tick neurobiology: recent advances and the post-genomic era. Invert Neurosci 7: 183-198.

Li AY, Davey RB, Miller RJ and George JE (2003) Resistance to coumaphos and diazinon in Boophilus microplus (Acari: Ixodidae) and evidence for the involvement of an oxidative detoxification mechanism. J Med Entomol 40: 482-490.

Li AY, Davey RB, Miller RJ and George JE (2004) Detection and characterization of amitraz resistance in the southern cattle tick, Boophilus microplus (Acari: Ixodidae). J Med Entomol 41: 193-200.

Li AY, Davey RB, Miller RJ and George JE (2005) Mode of inheritance of amitraz resistance in a Brazilian strain of the southern cattle tick, Boophilus microplus (Acari: Ixodidae). Exp Appl Acarol 37: 183-198.

Li AY, Chen AC, Miller RJ, Davey RB and George JE (2007) Acaricide resistance and synergism between permethrin and amitraz against susceptible and resistant strains of Boophilus microplus (Acari: Ixodidae). Pest Manag Sci 63: 882-889.

Li H, Cheng F, Wei Y, Lydy MJ and You J (2017) Global occurrence of pyrethroid insecticides in sediment and the associated toxicological effects on benthic invertebrates: an overview. J Hazard Mater 324(B): 258-271.

Lima AS, Carvalho JF, Peixoto MG, Blank AF, Borges LMF and Costa-Junior LM (2016) Assessment of the repellent effect of Lippia alba essential oil and major monoterpenes on the cattle tick Rhipicephalus microplus. Med Vet Entomol 30: $73-77$.

Lively T (2013) Technical trade barriers facing U.S. meat exports. Choices 28: 1-3.

Lopes FA, Broglio-Micheletti SMF, Andrade ABF and Santos MJM (2004) Efeito do óleo de nim (Azadirachta indica A. Juss) na infestação de carrapatos Boophilus microplus, Canestrini, 1887 (Acarina: Ixodidae), em bovinos mestiços leiteiros pertencentes à fazenda São Luiz/CECA/UFAL. In: Proceedings of ZOOTEC 2004, May 28-31, 2004, Brasília, DF, Brasil.

Louda SM, Pemberton RW, Johnson MT and Follet PA (2003) Nontarget effects - the Achille heel of biological control? Retrospective analyses to reduce risk associated with biological control. Annu Rev Entomol 48: 365-396.

Lovis L, Perret LJ, Fellay JM, Kaminsky R, Betschart B and Sager H (2011) A new in vitro test to evaluate the resistance level against acaricides of the cattle tick, Rhipicephalus (Boophilus) microplus. Vet Parasitol 182: 269-280. 
Lovis L, Reggi J, Berggoetz M, Betschart B and Sager H (2013a) Determination of acaricide resistance in Rhipicephalus (Boophilus) microplus (Acari: Ixodidae) field populations of Argentina, South Africa, and Australia with the Larval Tarsal Test. J Med Entomol 50: 326-323.

Lovis L, Mendes MC, Perret JL, Martins JR, Bouvier J, Betschart B and Sager H (2013b) Use of the Larval Tarsal Test to determine acaricide resistance in Rhipicephalus (Boophilus) microplus Brazilian field populations. Vet Parasitol 191: 323-331.

Low VL, Tay ST, Kho KL, Koh FX, Tan TK, Lim YAL, Ong BL, Panchadcharam C, Norma-Rashid Y and Sofian-Azirun M (2015) Molecular characterisation of the tick Rhipicephalus microplus in Malaysia: new insights into the cryptic diversity and distinct genetic assemblages throughout the world. Parasite Vectors 8: 341.

Lynch LD and Thomas MB (2000) Nontarget effects in the biological control of insects with insects, nematodes and microbial agentes: the evidence. Biocontrol News and Information. 21: 117-130.

Lysyk TJ (2008) Effects of ambient temperature and cattle skin temperature on engorgement of Dermacentor andersoni. J Med Entomol 45: 1000-1006.

Macedo F, Marsico ET, Conte-Júnior CA, De Almeida Furtado L, Brasil TF and Pereira Netto AD (2015) Short communication: macrocyclic lactone residues in butter from Brazilian markets. J Dairy Sci 98: 3695-3700.

Machado AF, Silva AC, Ribeiro HCT, Procópio ARL, Pinheiro CCS, Martins JRS and Silva WC (2013) Atividade biológica de extratos acetato de etila, etanólico e aquoso de timbó (Lonchocarpus floribundus) sobre o carrapato bovino. Acta Amaz 43: 135-142.

MacHugh DE, Shriver MD, Loftus RT, Cunningham P and Bradley DG (1997) Microsatellite DNA variation and the evolution, domestication and phylogeography of taurine and zebu cattle (Bos taurus and Bos indicus). Genetics 146: 1071-1086.

Maciel WG, Lopes WD, Gomes LV, Cruz BC, Felippelli G, Santos IB, Borges FA, Gonçalves WA Junior, Scarpa AB, Nicaretta $\mathrm{JE}$, Bastos TS and Da Costa AJ (2016) Susceptibility of Rhipicephalus (Boophilus) microplus to fluazuron $(2.5 \mathrm{mg} / \mathrm{kg}$ ) and a combination of novaluron $(2.0 \mathrm{mg} / \mathrm{kg})+$ eprinomectin $(0.36 \mathrm{mg} / \mathrm{kg})$ in field studies in Brazil. Prev Vet Med 135: 74-86.

Madalena FE, Teodoro RL, Lemos AM and Oliveira GP (1985) Causes of variation of field burdens of cattle ticks ( $B$. microplus). Rev Bras Genet 8: 361-375.

Mancini MV, Spaccapelo R, Damiani C, Accoti A, Tallarita M, Petraglia E, Rossi P, Cappelli A, Capone A, Peruzzi G, Valzano M, Picciolini M, Diabaté A, Facchinelli L, Ricci I and Favia G (2016) Paratransgenesis to control malaria vectors: a semi-field pilot study. Parasite Vectors 9: 140.

Martinez ML, Machado MA, Nascimento CS, Silva MVGB, Teodoro RL, Furlong J, Prata MCA, Campos AL, Guimarães MFM, Azevedo ALS, Pires MFA and Verneque RS (2006) Association of BoLA-DRB3.2 alleles with tick (Boophilus microplus) resistance in cattle. Genet Mol Res 5: 514-524.

Martinez-Velazquez M, Rosario-Cruz R, Castillo-Herrera G, Flores-Fernandez JM, Alvarez AH and Lugo-Cervantes E (2011) Acaricidal effect of essential oils from Lippia graveolens (Lamiales:Verbenaceae), Rosmarinus officin)alis (Lamiaceae), and Allium sativum (Liliales: Liliaceae) against Rhipicephalus (Boophilus) microplus (Acari: Ixodidae). J Med Entomol 48: 822-827.

Martins JR, Evans DE, Ceresér VH and Corrêa BL (2002) Partial strategic tick control within a herd of European breed cattle in the state of Rio Grande do Sul, southern Brazil. Exp Appl Acarol 27: 241-251.

Martins RM (2006) Estudo in vitro da ação acaricida do óleo essencial da gramínea Citronela de Java (Cymbopogon winterianus Jowitt) no carrapato Boophilus microplus. Rev Bras Plantas Med 8: 71-78.

Martins RM and González FHD (2007) Uso del aceite de citronela de Java (Cymbopogon winterianus Jowitt) (Panicoidideae) como acaricida frente a la garrapata Boophilus microplus Casnestrini (Acari:Ixodidae). Rev Bras Plantas Med 9: 1-8.

Marufu MC, Qokweni L, Chimonyo M and Dzama K (2011) Relationships between tick counts and coat characteristics in Nguni and Bonsmara cattle reared on semiarid rangelands in South Africa. Ticks Tick Borne Dis 2: 172-177.

Maruyama SR, Anatriello E, Anderson JM, Ribeiro JM, Brandão LG, Valenzuela JG, Ferreira BR, Garcia GR, Szabó MP, Patel S, Bishop R and De Miranda-Santos IK (2010) The expression of genes coding for distinct types of glycinerich proteins varies according to the biology of three metastriate ticks, Rhipicephalus (Boophilus) microplus, Rhipicephalus sanguineus and Amblyomma cajennense. BMC Genomics 11: 363. 
Maruyama SR, Garcia GR, Teixeira FR, Brandão LG, Anderson JM, Ribeiro JMC, Valenzuela JG, Horackova J, Veríssimo CJ, Katiki LM, Banin TM, Zangirolamo AF, Gardinassi LG, Ferreira BR and De Miranda-Santos IKF (2017) Mining a differential sialotranscriptome of Rhipicephalus microplus guides antigen discovery to formulate a vaccine that reduces tick infestations. Parasite Vectors 10: 206.

Matos RS, Melo DR, Monteiro CMO, Zeringóta V, Senra TOS, Calmon R, Maturano R, Prata MCA and Daemon E (2014) Determination of the susceptibility of unengorged larvae and engorged females of Rhipicephalus microplus (Acari: Ixodidae) to different methods of dissolving thymol. Parasitol Res 113: 669-673.

Mattioli RC and Cassama M (1995) Comparison of characteristics of life cycle in female ticks collected on N'Dama and zebu cattle. Trop Anim Health Prod 27: 150-154.

Mattioli RC, Pandey VS, Murray M and Fitzpatrick JL (2000) Immunogenetic influences on tick resistance in African cattle with particular reference to trypanotolerant N'Dama (Bos taurus) and trypanosusceptible Gobra zebu (Bos indicus) cattle. Acta Trop 75: 263-277.

Marshall JM, Buchman A, Sánchez CHM and Akbari OS (2017) Overcoming evolved resistance to population-suppressing homing-based gene drives. Sci Rep 7: 3776.

Melo V, Prata MCA, Silva MR, Daemon E, Silva LS, Guimarães FG, Mendonça AE, Folly E, Vilela FMP, Amaral LH, Cabral LM and Amaral MPH (2014) Acaricidal properties of the formulations based on essential oils from Cymbopogon winterianus and Syzygium aromaticum plants. Parasitol Res 113: 4431-4437.

Meltzer MI (1996) A possible explanation of the apparent breed-related resistance in cattle to bont tick (Amblyomma hebraeum) infestations. Vet Parasitol 67: 275-279.

Miller RJ, Davey RJ and George JE (1999) Characterization of pyrethroid resistance and susceptibility to coumaphos in Mexican Boophilus microplus (Acari: Ixodidae). J Med Entomol 36: 533-538.

Molento MB, Fortes FS, Buzatti A, Kloster FS, Sprenger LK, Coimbra E and Soares LD (2013) Partial selective treatment of Rhipicephalus microplus and breed resistance variation in beef cows in Rio Grande do Sul, Brazil. Vet Parasitol 192: 234-239.

Monteiro CMO, Araújo LXA, Matos RS, Golo PS, Angelo IC, Perinotto WMS, Rodrigues CAC, Furlong J, Bittencourt VREP and Prata MCA (2013) Association between entomopathogenic nematodes and fungi for control of Rhipicephalus microplus (Acari: Ixodidae). Parasitol Res 112: 3645-3651.

Monteiro CMO, Araújo LX, Gomes GA, Senra TOSS, Calmon F, Daemon E, Carvalho MG, Bittencourt VREP, Furlong J and Prata MCA (2014) Entomopathogenic nematodes associated with essential oil of Lippia sidoides for control of Rhipicephalus microplus (Acari: Ixodidae). Parasitol Res 113: 189-195.

Monteiro IN, Monteiro OS, Costa-Junior LM, Lima AS, Andrade EHA, Maia JGS and Mouchrek Filho VE (2017) Chemical composition and acaricide activity of an essential oil from a rare chemotype of Cinnamomum verum Presl on Rhipicephalus microplus (Acari: Ixodidae). Vet Parasitol 238: 54-57.

Morel N, Signorini ML, Mangold AJ, Guglielmone AA and Nava S (2017) Strategic control of Rhipicephalus (Boophilus) microplus infestation on beef cattle grazed in Panicum maximum grasses in a subtropical semi-arid region of Argentina. Prev Vet Med 144: 179-183.

Morgan JAT, Corley SW, Jackson LA, Lew-Tabor AE, Moolhuijzen PM and Jonsson NN (2009) Identification of a mutation in the para-sodium channel gene of the cattle tick Rhipicephalus (Boophilus) microplus associated with resistance to synthetic pyrethroid acaricides. Int J Parasitol 39: 775-779.

Narasimhan S, Deponte K, Marcantonio N, Liang X, Royce TE, Nelson KF, Booth CJ, Koski B, Anderson JF, Kantor F and Fikrig E (2016) Immunity against Ixodes scapularis salivary proteins expressed within 24 hours of attachment thwarts tick feeding and impairs Borrelia transmission. PLoS ONE 2: e451.

Nari A (1995) Strategies for the control of one-host ticks and relationship with tick-borne diseases in South America. Vet Parasitol 57: 153-165.

Nasuti C, Coman MM, Olek RA, Fiorini D, Verdenelli MC, Cecchini C, Silvi S, Fedeli D and Gabbianelli R (2016) Changes on fecal microbiota in rats exposed to permethrin during postnatal development. Environ Sci Pollut Res Int 23: 10930-10937. 
Nava S, Mastropaolo M, Guglielmone AA and Mangold AJ (2013) Effect of deforestation and introduction of exotic grasses as livestock forage on the population dynamics of the cattle tick Rhipicephalus (Boophilus) microplus (Acari: Ixodidae) in northern Argentina. Res Vet Sci 95: 1046-1054.

Novakova E, Woodhams DC, Rodríguez-Ruano SM, Brucker RM, Leff JW, Maharaj A, Amir A, Knight R and Scott J (2017) Mosquito microbiome dynamics, a background for prevalence and seasonality of West Nile virus. Front Microbiol 8: 526.

Novelino MAS, Daemon E and Soares GLG (2007) Avaliação da atividade repelente do timol, mentol, salicilato de metila e ácido salicílico sobre larvas de Boophilus microplus (Canestrini, 1887) (Acari: Ixodidae). Arq Bras Med Vet Zootec 59: 700-704.

Nunes RD, Ventura-Martins G, Moretti DM, Medeiros-Castro P, Rocha-Santos C, Daumas-Filho CR, Bittencourt-Cunha PR, Martins-Cardoso K, Cudischevitch CO, Menna-Barreto RF, Oliveira JH, Gusmão DS, Alves Lemos FJ, Alviano DS, Oliveira PL, Lowenberger C, Majerowicz D, Oliveira RM, Mesquita RD, Atella GC and Silva-Neto MA (2016) Polyphenolrich diets exacerbate AMPK-mediated autophagy, decreasing proliferation of mosquito midgut microbiota, and extending vector lifespan. PLoS Negl Trop Dis 10: e0005034.

Nyangiwe N, Matthee C, Horak I and Matthee S (2013) First record of the pantropical blue tick Rhipicephalus microplus in Namibia. Exp Appl Acarol 6: 503-507.

Oliveira GP, Costa RP, De Mello RP and Meneguelli CA (1974) Estudo ecológico da fase não parasítica do Boophilus microplus (Canestrini, 1887) (Acarina, Ixodidae) no Estado do Rio de Janeiro. Arq Univ Fed Rur 4: 1-10.

Olivo CJ, Agnolin CA, Parra CLC, Vogel FSF, Richards NSPS, Pellegrini LG, Webe A, Pivoto F and Araújo L (2013) Efeito do óleo de eucalipto (Corymbia citriodora) no controle do carrapato bovino. Ciênc Rural 43: 331-337.

O'Neill CJ, Swain DL and Kadarmideen HN (2010) Evolutionary process of Bos taurus cattle in favourable versus unfavourable environments and its implications for genetic selection. Evol Appl 3: 422-433.

Osterkamp J, Wahl U, Schmalfuss G and Haas W (1999) Host-odour recognition in two tick species is coded in a blend of vertebrate volatiles. J Comp Physiol(A) 185: 59-67.

Pang Y-P, Brimijoin S, Ragsdale DW, Zhu KY and Suranyi R (2012) Novel and viable acetylcholinesterase target site for developing effective and environmentally safe insecticides. Curr Drug Targets 13: 471-482.

Parizi LF, Pohl PC, Masuda A and Vaz Ida S Jr. (2009) New approaches toward anti-Rhipicephalus (Boophilus) microplus tick vaccines. Rev Bras Parasitol Vet 18:1-7.

Passafaro TL, Carrera JP, Dos Santos LL, Raidan FS, Dos Santos DC, Cardoso EP, Leite RC and Toral FL (2015) Genetic analysis of resistance to ticks, gastrointestinal nematodes and Eimeria spp. in Nellore cattle. Vet Parasitol 210: 224-234.

Peixoto MG, Costa-Júnior LM, Blank AF, Lima AS, Menezes TSA, Santos DA, Alves PB, Cavalcanti SCH, Bacci L and ArrigoniBlank MF (2015) Acaricidal activity of essential oils from Lippia alba genotypes and its major components carvone, limonene, and citral against Rhipicephalus microplus. Vet Parasitol 210: 118-122.

Pereira SG, De Araújo SA, Guilhon GMSP, Santos LS and Junior LMC (2017) In vitro acaricidal activity of Crescentia cujete L. fruit pulp against Rhipicephalus microplus. Parasitol Res 116: 1487-1493.

Picinin LTA, Toaldo IM, Hoff RB, Souza FN, Leite MO, Fonseca LM, Diniz AS, Silva MX, Haddad JPA, Cerqueira MMOP and Luiz MTB (2016) Climate conditions associated with the occurrence of pyrethroid residues in bulk milk tank. Arq Bras Med Vet Zootec 68: 1721-1726.

Pingen M, Bryden SR, Pondeville E, Schnettler E, Kohl A, Merits A, Fazakerley JK, Graham GJ and McKimmie CS (2016) Host inflammatory response to mosquito bites enhances the severity of arbovirus infection. Immunity 44: 14551469.

Piper EK, Jackson LA, Bielefeldt-Ohmann H, Gondro C, Lew-Tabor AE and Jonsson NN (2010) Tick-susceptible Bos taurus cattle display an increased cellular response at the site of larval Rhipicephalus (Boophilus) microplus attachment, compared with tick-resistant Bos indicus cattle. Int J Parasitol 40: 431-441.

Pires JEP (2006) Efeito dos extratos aquoso e etanólico de planta Simarouba versicolor St. Hill in vitro sobre larvas e teleóginas de carrapatos Boophilus microplus Canestrini, 1887 e Rhipicephalus sanguineus Latreille, 1806. PhD thesis, Universidade Federal do Piauí, Teresina, Piauí, Brazilië. 
Pohl PC, Carvalho DD, Daffre S, Vaz Ida S Jr and Masuda A (2014) In vitro establishment of ivermectin-resistant Rhipicephalus microplus cell line and the contribution of $A B C$ transporters on the resistance mechanism. Vet Parasitol 204: 316-322.

Porto Neto LR, Bunch RJ, Harrison BE, Prayaga KC and Barendse W (2010) Haplotypes that include the integrin alpha 11 gene are associated with tick burden in cattle. BMC Genet 11: 55 .

Porto Neto LR, Jonsson NN, D'Occhio MJ and Barendse W (2011) Molecular genetic approaches for identifying the basis of variation in resistance to tick infestation in cattle. Vet Parasitol 180: 165-172.

Prates HT, Leite RC, Craveiro AA and Oliveira AB (1998) Identification of some chemical components of the essential oil from Molasses Grass (Melinis minutiflora Beauv.) and their activity against cattle-tick (Boophilus microplus). J Braz Chem Soc 9: 193-197.

Pruett JH (2002) Comparative inhibition kinetics for acetylcholinesterases extracted from organophosphate resistant and susceptible strains of Boophilus microplus (Acari: Ixodidae). J Econ Entomol 95: 1239-1244.

Pruett JH, Guerrero FD and Hernandez R (2002) Isolation and identification of an esterase from a Mexican strain of Boophilus microplus (Acari: Ixodidae). J Econ Entomol 95: 1001-1007.

Qi Z and Wei Z (2017) Microbial flora analysis for the degradation of beta-cypermethrin. Environ Sci Pollut Res Int 24: 6554-6562.

Qin Y, Jatamunua F, Zhang J, Li Y, Han Y, Zou N, Shan J, Jiang Y and Pan C (2017) Analysis of sulfonamides, tilmicosin and avermectins residues in typical animal matrices with multi-plug filtration cleanup by liquid chromatographytandem mass spectrometry detection. J Chromatogr B Analyt Technol Biomed Life Sci 1053: 27-33.

Ramirez JL, Short SM, Bahia AC, Saraiva RG, Dong Y, Kang S, Tripathi A, Mlambo G and Dimopoulos G (2014) Chromobacterium Csp_P reduces malaria and dengue infection in vector mosquitoes and has entomopathogenic and in vitro anti-pathogen activities. PLoS Pathog 10: e1004398.

Reck J, Klafke GM, Webster A, Dall'Agnol B, Scheffer R, Souza UA, Corassini VB, Vargas R, Dos Santos JS and Martins JR (2014) First report of fluazuron resistance in Rhipicephalus microplus: a field tick population resistant to six classes of acaricides. Vet Parasitol 201: 128-136.

Robbertse L, Baron S, Van der Merwe NA, Madder M, Stoltsz WH and Maritz-Olivier C (2016) Genetic diversity, acaricide resistance status and evolutionary potential of a Rhipicephalus microplus population from a disease-controlled cattle farming area in South Africa. Ticks Tick Borne Dis 7: 595-603.

Ribeiro VLS, Santos JC, Martins JR, Schripsema J, Siqueira IR, Von Poser G and Apel MA (2011) Acaricidal properties of the essenctial oil and precocene II obtained from Calea serrata (Asteraceae) on the cattle tick Rhipicephalus (Boophilus) microplus (Acari: Ixodidae). Vet Parasitol 179: 195-198.

Rocha CMBM, Oliveira PR, Leite RCL, Cardoso DL, Calic SB and Furlong J (2006) Percepção dos produtores de leite do município de Passos, MG, sobre o carrapato Boophilus microplus (Acari: Ixodidae), 2001. Ciência Rural 36: 1235-1242.

Rodríguez-Hidalgo R, Pérez-Otáñez X, Garcés-Carrera S, Vanwambeke SO, Madder M and Benítez-Ortiz W (2017) The current status of resistance to alpha-cypermethrin, ivermectin, and amitraz of the cattle tick (Rhipicephalus microplus) in Ecuador. PLoS ONE 12: e0174652.

Rodríguez-Vivas RI, Pérez-Cogollo LC, Rosado-Aguilar JA, Ojeda-Chi MM, Trinidad-Martinez I, Miller RJ, Li AY, De León AP, Guerrero F and Klafke G (2014) Rhipicephalus (Boophilus) microplus resistant to acaricides and ivermectin in cattle farms of Mexico. Rev Bras Parasitol Vet 23: 113-122.

Roel AR (2001) Utilização de plantas com propriedades inseticidas: uma contribuição para o desenvolvimento rural sustentável. Revista Internacional de Desenvolvimento Local 1: 43-50.

Romo M, Ruvalcaba MF, Velázquez VMH, Chora GP, Garcia LPL and Miranda JO (2013) Evaluation of natural origin products for the control of Rhipicephalus (Boophilus) microplus (Acari: Ixodidae) on cattle artificially infested. Basic Res J Agricul Sci Rev 2: 64-79.

Rosa RD, Capelli-Peixoto J, Mesquita RD, Kalil SP, Pohl PC, Braz GR, Fogaça AC and Daffre S (2016) Exploring the immune signalling pathway-related genes of the cattle tick Rhipicephalus microplus: from molecular characterization to transcriptional profile upon microbial challenge. Dev Comp Immunol 59: 1-14. 
Rosado-Aguilar JA, Rodríguez-Vivas RI, Borges-Argaez R and Arjona-Cambranes KA (2017) Acaricidal activity of Havardia albicans and Caesalpinia gaumeri methanolic leaf extracts on Rhipicephalus microplus and its toxicity to laboratory animals. Exp Appl Acarol 71: 345-354.

Rosario-Cruz R, Almazan C, Miller RJ, Dominguez-Garcia DI, Hernandez-Ortiz R and De la Fuente J (2009) Genetic basis and impact of tick acaricide resistance. Front Biosci (Landmark Ed) 14:2657-2665.

Saldivar L, Guerrero FD, Miller RJ, Bendele KG, Gondro C and Brayton KA (2008) Microarray analysis of acaricide-inducible gene expression in the southern cattle tick, Rhipicephalus (Boophilus) microplus. Insect Mol Biol 17: 597-606.

Samish M, Ginsberg H and Glazer I (2004) Biological control of ticks. Parasitol 129: S389-403.

Santos FCC, Vogel FSF and Monteiro SG (2012) Efeito de diferentes concentrações de óleo e tintura de citronela sobre os parâmetros reprodutivos de teleóginas de Rhipicephalus (Boophilus) microplus. Semina-Ciênc Agrar 33: 1141-1148.

Santos FCC, Vogel FSF, Roll VFB and Monteiro SG (2013) In vitro effect of the association of citronella, Santa Maria herb (Chenopodium ambrosioides) and quassia tincture on cattle tick Rhipicephalus (Boophilus) microplus. Ciênc An Bras 14: 113-119.

Santos TRB, Klafke GM, Pappen FG, Nizoli LQ, Biegelmeyer P and Farias NAR (2013) Comparison of three larval bioassays to evaluate susceptibility of Rhipicephalus (Boophilus) microplus to amitraz. Rev Bras Parasitol Vet 22: 495-501.

Schleger AV and O'Kelly JC (1986) Esterase activity in the bovine sweat gland: genetic differences and the effect of temperature. Acta Anat (Basel) 125: 229-232.

Schröder CH, Pinhel MF and Mendonça AO (2016) The Brazilian strategy for monitoring persistent organic pollutants in food obtained from animals. Sci Total Environ 15(573): 1370-1379.

Seifert GW (1971) Ectoparasitic and endoparasitic effects on growth rates of zebu crossbred and British cattle in field. Aust J Agric Res 22: 839-850.

Senra TOS, Zeringóta V, Monteiro CMO, Calmon F, Maturano R, Gomes GA, Faza A, Carvalho MGC and Daemon E (2013) Assessment of the acaricidal activity of carvacrol, (E)-cinnamaldehido, trans-anethole, and linalool on larvae of Rhipicephalus microplus and Dermacentor nitens (Acari: Ixodidae). Parasitol Res 112: 1461-1466.

Shaw RD and Malcom HÁ (1964) Resistance of Boophilus microplus to organophosphorus insecticides. Vet Rec 7: 210-211.

Shaw RD (1966) Culture of an organophosphorus resistant strain of Boophilus microplus (Can.) and an assessment of its resistance spectrum. Bull Entomol Res 56: 389-405.

Short NJ, Floyd RB, Norval RA and Sutherst RW (1989) Survival and behaviour of unfed stages of the ticks Rhipicephalus appendiculatus, Boophilus decoloratus and Boophilus microplus under field conditions in Zimbabwe. Exp Appl Acarol 6: 215-236.

Shyma KP, Gupta JP and Singh V (2015) Breeding strategies for tick resistance in tropical cattle: a sustainable approach for tick control. J Parasit Dis 39: 1-6.

Smallegange RC, Verhulst NO and Takken W (2011) Sweaty skin: an invitation to bite? Trends Parasitol 27: 143-148.

Silva AM, Alencar MM, Regitano LCA, Oliveira MCS and Barioni Júnior W (2007) Artificial infestation of Boophilus microplus in beef cattle heifers of four genetic groups. Gen Mol Biol 30: 1150-1155.

Silva Filho ML, Silva LB, Fernandes RM and Lopes GS (2013) Efeito do extrato aquoso e etanólico do angico preto sobre larvas de Rhipicephalus (Boophilus) microplus. Arq Bras Med Vet Zootec 65: 637-644.

Silva WJ, Silva WC and Borges LMF (2002) Avaliação de duas formulações comerciais de Azadirachta indica (Meliacea) sobre fêmeas de Boophilus microplus (Acari: Ixodidae). In: Proceedings of the $12^{\text {th }}$ Brazilian Congress of Veterinary Parasitology, Rio de Janeiro, September 1-5, 2002, Rio de Janeiro, Brazil.

Singh RK, Sanyal PK, Patel NK, Sarkar AK, Santra AK, Pal S and Mandal SC (2010) Fungus-benzimidazole interactions: a prerequisite to deploying egg-parasitic fungi Paecilomyces lilacinus and Verticillium chlamydosporium as biocontrol agents against fascioliasis and amphistomiasis in ruminant livestock. J Helminthol 84: 123-131.

Soares AMS, Penha TA, Araújo SA, Cruz EMO, Blank AF and Costa-Junior LM (2016) Assessment of different Lippia sidoides genotypes regarding their acaricidal activity against Rhipicephalus (Boophilus) microplus. Braz J Vet Parasitol 25: 401-406.

Soderlund DM and Knipple DC (2003) The molecular biology of knockdown resistance to pyrethroid insecticides. Insect Biochem Molec 33: 563-577.

Sonenshine DE (2006) Tick pheromones and their use in tick control. Annu Rev Entomol 51: 557-580. 
Srivastava R, Ghosh S, Mandal DB, Azhahianambi P, Singhal PS, Pandey NN and Swarup D (2008) Efficacy of Azadirachta indica extracts against Boophilus microplus. Parasitol Res 104: 149-153.

St Leger RJ, Butt TM, Staples RC and Roberts DW (1989) Synthesis of proteins including a cuticle-degrading protease during differentiation of the entomopathogenic fungus Metarhizium anisopliae. Exp Mycol 13: 253-262.

Stone BF and Haydock KP (1962) A method for measuring the acaricide susceptibility of the cattle tick Boophilus microplus (Can.). Bull Entomol Res 53: 563-578.

Sutherst RW, Jones RJ and Schnitzerling HJ (1982) Tropical legumes of the genus Stylosanthes immobilize and kill cattle ticks. Nature 295: 320-321.

Temeyer KB, Davey RB and Chen AC (2004) Identification of a third Boophilus microplus (Acari: Ixodidae) CDNA presumptively encoding an acetylcholinesterase. J Med Entomol 41:259-268.

Temeyer KB, Pruett JH and Olafson PU (2010) Baculovirus expression, biochemical characterization and organophosphate sensitivity of rBmAChE1, rBmAChE2, and rBmAChE3 of Rhipicephalus (Boophilus) microplus. Vet Parasitol 172: 114-121.

Terassani E, Santos HJ, Silva ID, Cardoso BK, Souza SGH and Gazim ZC (2012) Effect of Azadirachta indica extract on Rhipicephalus (Boophilus) microplus. Arqu Ciênc Vet Zool UNIPAR 15: 197-200.

Thompson KC, Roa EJ and Romero NR (1978) Anti-tick grasses as the basis for developing practical tropical tick control packages. Trop Anim Health Prod 10: 179-182.

Tikhe CV, Martin TM, Howells A, Delatte J and Husseneder C (2016) Assessment of genetically engineered Trabulsiella odontotermitis as a'Trojan Horse' for paratransgenesis in termites. BMC Microbiol 16: 202.

Tønnensen MH, Penzhorn BL, Bryson NR, Stoltsz WH and Masibigiri T (2004) Displacement of Boophilus decoloratus by Boophilus microplus in the Soutpansberg region, Limpopo Province, South Africa. Exp Appl Acarol 32: 199-208.

Trager W (1939) Acquired immunity to ticks. J Parasitol 25: 57-81.

Turner LB, Harrison BE, Bunch RJ, Porto Neto LR, Li YT and Barendse W (2010) A genome wide association study of tick burden and milk composition in cattle. Anim Prod Sci 50: 235-245.

Untalan PM, Pruett JH and Steelman CD (2007) Association of the bovine leukocyte antigen major histocompatibility complex class II DRB3*4401 allele with host resistance to the Lone Star tick, Amblyomma americanum. Vet Parasitol 145: 190-195.

Urdaz-Rodríguez J, Fosgate F, Alleman AR, Rae O, Donovan A, Binford M, Zaragoza A and Melendez P (2012) Association between ecological factors and the presence of Rhipicephalus (Boophilus) microplus larvae in Puerto Rico. Exp Appl Acarol 58: 145-157.

Utech KGW, Wharton RH and Kerr JD (1978) Resistance to Boophilus microplus (Canestrini) in different breeds of cattle. Aust J Agric Res 29: 885-895.

Utech KB and Wharton RH (1982) Breeding for resistance to Boophilus microplus in Australian Illawarra shorthorn and Brahman $\times$ Australian Illawarra shorthorn cattle. Aust Vet J 58: 41-46.

Vale GA, Hall DR, Chamisa A and Torr SJ (2012) Towards an early warning system for Rhodesian sleeping sickness in savannah areas: man-like traps for tsetse flies. PLoS Negl Trop Dis 6: e1978.

Valente M, Barranco A and Sellaive-Villaroel AB (2007) Eficácia do extrato aquoso de Azadiracta indica no controle de Boophilus microplus em bovino. Arqu Bras Med Vet Zoot 59: 1341-1343.

Valente PP, Amorim JM, Castilho RO, Leite RC and Ribeiro MFB (2014) In vitro acaricidal efficacy of plant extracts from Brazilian flora and isolated substances against Rhipicephalus microplus (Acari: Ixodidae). Parasitol Res 113: 417-423.

Valente PP, Moreira GHFA, Serafini MF, Facury-Filho EJ, Carvalho AU, Faraco AAG, Castilho RO and Ribeiro MFB (2017) In vivo efficacy of a biotherapic and eugenol formulation against Rhipicephalus microplus. Parasitol Res 116: 929-938.

Van Wyk RDJ, Baron S and Maritz-Olivier C (2016) An integrative approach to understanding pyrethroid resistance in Rhipicephalus microplus and R. decoloratus ticks. Ticks Tick Borne Dis 7: 586-594.

Veríssimo CJ, Silva RG, Oliveira AAD, Ribeiro WR and Rocha UF (1997) Contagens de ínstares do carrapato Boophilus microplus em bovinos mestiços. Bol Ind An 54: 21-26.

Veríssimo CJ, Nicolau VL, Cardoso VL and Pinheiro MG (2002) Haircoat characteristics and tick infestation on Gyr (Zebu) and crossbred (Holstein x Gyr) cattle. Arch Zootec 51: 389-392.

Veríssimo CJ (2013) Controle biológico do carrapato no Brasil. Rev Ed Contin CRMV-SP 11: 14-23.

Pests and vector-borne diseases in the livestock industry 
Veríssimo CJ and D'Agostino SM (2014) Predation of Tropidurus torquatus lizard on Rhipicephalus (Boophilus) microplus ticks. In: Proceedings of the $18^{\text {rd }}$ Brazilian congress of Veterinary Parasitology, Gramado, October 21-24, 2014, Rio Grande do Sul, Brazil.

Veríssimo CJ, D'Agostino SM, Pessoa FF, De Toledo LM and Santos IK (2015) Length and density of filiform tongue papillae: differences between tick-susceptible and resistant cattle may affect tick loads. Parasite Vectors 8: 594.

Veríssimo CJ (2015) Fatores que afetam a fase de vida livre de carrapatos. Controle de carrapatos nas pastagens. Instituto de Zootecnia, Nova Odessa, SP, Brasil. Available at: https://tinyurl.com/y8qewtkm.

Vieira JN, Maia FS Filho, Ferreira GF, Mendes JF, Gonçalves CL, Villela MM, Pereira DI and Nascente OS (2016) In vitro susceptibility of nematophagous fungi to antiparasitic drugs: interactions and implications for biological control. Braz J Biol 77: 476-479.

Wagland BM (1975) Host resistance to cattle tick (Boophilus microplus) in Brahman (Bos indicus) cattle. I. Response of previously unexposed cattle to four infestations with 20,000 larvae. Aust J Agric Res 26: 1073-1078.

Wambura PN, Gwakisa PS, Silayo RS and Rugaimukamu EA (1998) Breed-associated resistance to tick infestation in Bos indicus and their crosses with Bos taurus. Vet Parasitol 77: 63-70.

Wanderley LF, Batista KLR, Carvalho JF, Lima ADS, Landulfo GA, Soares AMDS and Costa LM Jr. (2017) The first assessment of the stress inducible defense of Leucaena leucocephala with acaricidal potential effect against Rhipicephalus (Boophilus) microplus (Acari: Ixodidae). Rev Bras Parasitol Vet 26: 171-176.

Wanzala W, Hassanali A, Mukabana WR and Takken W (2014) Repellent activities of essential oils of some plants used traditionally to control the brown ear tick, Rhipicephalus appendiculatus. J Parasitol Res, Article ID: 434506.

Webster A, Pradel E, Souza UA, Martins JR, Reck J, Schrank A and Klafke G. (2017) Does the effect of a Metarhizium anisopliae isolate on Rhipicephalus microplus depend on the tick population evaluated? Ticks Tick Borne Dis 8: 270-274.

Wharton RH, Harley KLS, Wilkinson PR, Utech KB and Kelley BM (1969) A comparison of cattle tick control by pasture spelling, planned dipping, and tick resistant cattle. Austral J Agricul Res 20: 783-797.

Wharton RH and Utech KBW (1970) The relation between engorgement and dropping of Boophilus microplus (Canestrini) (Ixodidae) to the assessment of tick numbers on cattle. J Aust Ent Soc 9: 171-182.

Werner KM and Moran K (2008) Effects of pyrethroid insecticides on aquatic organisms. In: Gan J (ed.) Synthetic pyrethroids. American Chemical Society, Washington, DC, USA.

Yoder J, Atwood A and Stevens B (1998) Attraction to squalene by ticks (Acari: Ixodidae): first demonstration of a hostderived attractant. Int J Acarol 24: 159-164.

Yu FH, Yarov-Yarovoy V, Gutman GA and Catterall WA (2005) Overview of molecular relationships in the voltage-gated ion channel superfamily. Pharmacol Rev 57: 387-395.

Zhang X, Crippen TL, Coates CJ, Wood TK and Tomberlin JK (2015) Effect of quorum sensing by Staphylococcus epidermidis on the attraction response of female adult yellow fever mosquitoes, Aedes aegypti aegypti (Linnaeus) (Diptera: Culicidae), to a blood-feeding source. PLOS ONE 10: e0143950.

Zeringóta V, Senra TO, Calmon F, Maturano R, Faza AP, Catunda-Junior FE, Monteiro CM, De Carvalho MG and Daemon E (2013) Repellent activity of eugenol on larvae of Rhipicephalus microplus and Dermacentor nitens (Acari: Ixodidae). Parasitol Res 112: 2675-2679.

Zimmer KR, Seixas A, Conceição JM, Zvoboda DA, Barros MP, Tasca T, Macedo AJ and Termignoni C (2013) Cattle tickassociated bacteria exert anti-biofilm and anti-Tritrichomonas foetus activities. Vet Microbiol 164: 171-176.

Zoubiri S and Baaliouamer A (2014) Potentiality of plants as source of insecticide principles. J Saudi Chem Soc 18: 925-938. 


\title{
5. Sheep myiasis: a one health perspective
}

\author{
Douglas D. Colwell ${ }^{1 *}$ and Richard Wall ${ }^{2}$ \\ ${ }^{1}$ Agriculture and Agri-Food Canada, Lethbridge Research and Development Centre, $54031^{\text {st }}$ Ave. S., \\ Lethbridge, AB, T1J 4B1, Canada; ${ }^{2}$ University of Bristol, Life Sciences Building, 24 Tyndall Avenue, Bristol \\ BS8 1TQI, United Kingdom; doug.colwell@agr.gc.ca
}

\begin{abstract}
Myiasis (the invasion of internal and external tissues by fly larvae) results in major welfare and economic problems for sheep husbandry in many areas of the world. Here myiasis of sheep is reviewed with a summary of the most important species involved and their lifecycles and a discussion of how using a One Health approach can result in a more robust and responsive industry to reduce the impact of myiasis on sheep production.
\end{abstract}

Keywords: life history, fly larvae, One Health, production, sheep

\section{Introduction}

The production landscape for livestock husbandry is changing, with increasing human population, population movement, greater urbanization, changes in landscape management, changes in climate, greater affluence driving increased demand for meat in many parts of the world and a greater emphasis on food security and resilience - all imposing ever new pressures on the patterns and processes of animal production (Davendra 2001; Godber and Wall 2014). Nevertheless, pests and parasites remain a persistent constraint to effective livestock production (Colwell et al. 2011). One globally important category of insect-mediated damage, particularly in small ruminant production, is myiasis - the invasion of internal and external tissues by the larvae of dipterous flies (Wall 2012). These larvae belong to the families Calliphoridae, Sarcophagidae and Oestridae and invade superficial sites, often cutaneous, as well as internal sites such as those of the nasopharyngeal region (Hall and Wall 1995). In the former two families of flies only a relatively small number of species are characteristically involved in myiasis and many of these are facultative myiasis agents; in contrast all species of the Oestridae are obligate agents of myiasis. Two species of facultative blowflies are of particular economic importance in sheep rearing areas of the world: Lucilia sericata (Meigen) and Lucilia cuprina (Wiedemann) (Foster et al. 1975; McLeod 1995; Snoep 2002; Tenquist and Wright 1976), one species of sarcopaghid fly, the obligate parasite, Wohlfahrtia magnifica (Schiner) (Sotiraki and Hall 2012) and one species of nose bot fly Oestrus ovis L. (AnguloValadez et al. 2011; Cepeda-Palacios et al. 2011). Myisasis by the obligate screwworms, Cochliomyia hominivorax (Coquerel) and Chrysomya bezziana (Villeneuve) may also be locally important in parts of the new and old-world respectively. Myiasis by these species presents a continual challenge in a wide variety of husbandry systems in different parts of the world (Jacquiet et al. 2016; Vasconcelos et al. 2016).

Management of myiasis is effected primarily by the use of topical and systemic insecticides, such as organophosphates, pyrethroids, insect growth regulators and macrocyclic lactones (Bisdorff and Wall 2008). However, it has been suggested that future sustainable production of animal protein, as well as other by-products such as wool, will require 'One Health' thinking which integrates the usual thought processes about animal health with concepts of production which are more holistic and sustainable, including consideration of animal welfare, human health and environmental impacts (Rushton and Bruce 2017). These concepts will be highlighted in this review. 


\section{Biology of Oestrus ovis}

Oestrus ovis causes a cavitary myiasis within the upper reaches of the respiratory tract in sheep. The female flies are among the oestrids that keep their eggs within a modified common oviduct where they hatch into first instar larvae and where they are retained until the gravid fly can find a suitable host (Cepeda-Palacios et al. 2011). On these flies the tarsal sensilla are relatively sparse as they do not use them in host location or selection (Colwell and Berry 1993). From the oviduct first instars are ejected, in packages, directly onto the host's nostrils with precision. Each larval packet consists of a group of longitudinally arranged larvae that become activated as they contact with the air and high host temperature relative to the ambient air temperature. The larvae quickly colonize the nasal cavity overcoming the early host defensive reactions such as sneezing and rubbing against close objects. The first instars can persist for a variable period of less than 10 days to more than 100 days depending on the local conditions (D.D. Colwell, unpublished data). Inside the host's nasal cavity, the larvae continue growing but can go into hypobiosis (arrested development) depending on a combination of intrinsic conditions and external environmental signals.

As with other oestrids, intrapuparial development is highly dependent on the temperature that regulates the metamorphosis process (Cepeda-Palacios and Scholl 2000). Approximately 243 degree-days are required for development in male flies and 279 degree-days for females. Individuals can withstand transitory temperatures of as high as $45^{\circ} \mathrm{C}$ for short periods during the day. However, weight losses are accelerated resulting in delayed eclosion (seven weeks or more) when pupae are exposed to persistent high temperatures. Under these conditions, weak adults and morphological defects may occur. It appears that high temperatures are deleterious because unavoidable puparial weight losses are increased. During this period, weight losses are also important for adult survival, since lightweight flies (i.e. $60 \mathrm{mg}$ or less) die within a few days under laboratory and field conditions. Mortality of developing individuals during the intrapuparial period may be significant with rates varying between $41-100 \%$, depending on the rearing temperature. Under laboratory conditions, females emerge $22 \mathrm{~d}$ and males $21 \mathrm{~d}$ after pupariation (Colwell, unpublished). Alternating high and low temperatures during the day seem to enhance survival in a laboratory. Increasing temperature during early hours of the day may be an appropriate signal for emergence since most flies emerge during the morning. During the intra-puparial period, normally $82 \%$ of larval weight is lost. At emergence, water and fat reserves are between $76.5 \pm 12.3 \mathrm{mg}$ in females and $75.4 \pm 11.6 \mathrm{mg}$ in males. Thus, during both larval-pupal and pupal-adult metamorphoses, individuals lose almost $77 \%$ of their water and $65 \%$ of their fat reserves which were acquired during the larval stage. As with most other oestrids the adult mouthparts are vestigal and adults do not feed (Wood 2006). Furthermore, the gut in in the imago is barely evident (Martin-Vega et al. 2018) and apparently has no known function.

\section{Biology of Wohlfahrtia magnifica}

Myiasis associated with larvae of by W. magnifica is common throughout the Mediterranean, in eastern Europe and extending into China. In locations with high temperatures and low humidity, W. magnifica is often a more significant cause of myiasis in livestock than other myiasis species, such as L. sericata (Hall 1997; Hall and Farkas 2000).

Locations on the body infested include the mucous membranes, especially the head and body orifices. The genitalia of both female and male hosts are specially attacked. Numerous studies have shown that approximately $87 \%$ of infestations to occur in the genitalia (Farkas et al. 1997; 
see also Ruiz Martinez et al. 1987, 1991). It should be noted that larvae of W. magnifica are not able to develop in carrion. Established infestations tend to be attractive to gravid females and, as a result, infestations frequently occur with larvae at all stages of development (Farkas et al. 1997). Larvae moult from first to second instars within 6-8 h (Ruiz Martinez et al. 1989), undergo a second moult to the third instar, then 5-7 days after larvaposition larvae drop from the infested tissue and burrow into the ground where pupariation takes place. The pupa may either continue to develop and emerge as an adult, or, in response to shortening day length $(<14.5 \mathrm{~h})$ (Ternovoy 1978), will enter diapause from which it is reactivated under natural conditions after a period of 3-4 months of cooling. Flies are active from May to October (Ruiz Martinez and Mira 1994) but are most numerous in the warmer summer months.

Myiasis by W. magnifica (known as wohlfahrtiosis) occurs, particularly along the trade routes through which considerable numbers of livestock are transported. The annual prevalence of the infestation in sheep and goats tends to be higher in Eastern Europe, where infestation ranges from $23 \%$ to slightly over $50 \%$ (e.g. Bulgaria, $23-41 \%$, Nedelchev 1988 ; the former USSR, $30-50 \%$, Pokidov and Goncharov 1971). Generally the prevalence is lower in the Mediterranean basin where Spain (Ruiz Martinez et al. 1987), Italy (Giangaspero et al. 2010), Greece (Sotiraki et al. 2003) and Israel (Hadani et al. 1971) have reported infestations ranging from $>1$ to $15 \%$ ). Hungary tends to have intermediate infestation prevalences of nearly 40\% (Farkas et al. 1997). In Europe, W. magnifica has been a problem among imported breeds of sheep, such as Merino, Romney, Polwarth and Corriedale in Romania and Hungary, which are very susceptible to infestation (Farkas et al. 1997; Lehrer and Verstraeten 1991). The cause of variation in breed tolerance is unknown and requires further study. Ruiz Martnez et al. (1993b) have discussed the role of defensive behaviours of some autochthonous breeds of sheep in Spain which are successful in avoiding infestations in up to almost $75 \%$ of larviposition attempts by females of $W$. magnifica.

Current research has shown that there are at least two genetic lineages of $W$. magnifica, one in Spain and France and the other in all countries to the east of these (Hall et al. 2009a,b). The distribution of the species appears to be dynamic, in part because of host animal movement. Examples include the outbreaks seen since 1999 in Greece and Morocco where W. magnifica has caused significant health and welfare issues in livestock, particularly in sheep, which are the basis of much of the local dairy industry (El Abrak et al. 2002; Sotiraki et al. 2003, 2005a,b). The fly is able to larviposit in either sheep or goats and the main host in each area is associated with the numbers of animals present and the husbandry practices (Sotiraki et al. 2010). In areas where small ruminant production is strongly milk-oriented, prevention and control of wohlfahrtiosis can be problematic, especially for lactating females in sheep herds, because of the long milk withdrawal periods for many of the insecticidal products, such as macrocyclic lactones (Sotiraki et al. 2010) although use of eprinomectin, with a zero withdrawal period for milk, has not been evaluated.

\section{Biology of Lucilia spp.}

The two fly species of Lucilia that are primarily responsible for the infestation of sheep (known as fly strike) are L. cuprina and L. sericata (Hall and Wall 1995). In general, L. cuprina is found in tropical, sub-tropical and arid habitats, such as much of Australia, and L. sericata in more cooltemperate habitats, for example in Europe, but considerable overlap and regional variations in behaviour may exist. Both species may be important in myiasis in New Zealand and South Africa. Adult females deposit batches of around 200 eggs in the wool. After hatching, first instars migrate down the wool fibres toward the skin. Feeding is assisted by mouth hooks, which abrade the skin surface and proteolytic enzymes, which initiate external breakdown of the host's tissue (Evans 
1936). The mature third instars cease feeding and enter a dispersal phase, in which they migrate away from the strike focus, drop from the host and pupate in the soil, prior to adult emergence. At the host skin surface temperatures, eggs will hatch after 12-24 hours, moult to second instars after 12-18 hours and moult to third instars at about 30 hours later (Wall et al. 1992). Thus, they will feed for a total of 3-4 days before leaving the host to pupate in the soil. Pupation usually requires one to several weeks, depending on ambient temperature.

A number of other species, such as Calliphora stygia (Fabricius) and Calliphora augur (Fabricius) may occur in sheep strikes in Australia, while in Europe Lucilia caesar L., Lucilia illustris (Meigen), Calliphora vomitoria L., Calliphora vicina Robineau-Desvoidy, and Protophormia terraenovae (Protophormia terraenovae) may also occasionally be found (Wall 2012). In Bulgaria, Chrysomya albiceps (Wiedemann) has also been reported as causing myiasis of sheep (Nedelchev 1988). Phormia regina (Meigen) causes livestock myiasis in North America, but it does not appear to do so in Europe (Hall and Wall 1995).

Fly strike in sheep is associated with a series of predisposing conditions such as soiling of the fleece, usually by urine and faeces (and associated bacterial contamination of these sites), endoparasitic worm burdens, fleece length and humidity, which promote oviposition and larval survival (French and Morgan 1996; French et al. 1995). As a result of soiling, breech strikes are particularly common (Broughan and Wall 2007). The larvae of Lucilia remain on the skin surface causing cutaneous damage and only rarely move toward deeper tissue. Death may result, usually after multiple oviposition, and secondary effects include toxaemia (Guerrini 1988). Lucilia spp. may also be found at wound sites, in necrotic tissues in cases of foot rot caused by bacterial infection by two gram-negative, anaerobic bacteria: Fusobacterium necrophorum and Dichelobacter nodosus.

The cost of fly strike can be high, for example costing the Australian economy almost AUS\$ 280 million per year (2011 figures at http://www.wool.com.au). This figure includes losses from mortality, production losses, labour and insecticides. For example, in Queensland, 79\% of flocks were shown to be affected by strike, with between 0.5 and $1.1 \%$ of animals struck annually (Ward 2001a), while in New South Wales between 60 and $90 \%$ of flocks were shown to be affected with a flock prevalence of 1-2\% (Wardhaugh and Morton 1990). Similarly, in Europe, blowfly strike was shown to affect $75 \%$ of farms in the UK, where an average of $1.4 \%$ of ewes and $2.8 \%$ of lambs were struck (Bisdorff et al. 2006). Taking into account factors such as mortality, insecticide costs and hide damage, the economic cost of fly strike in the UK has been estimated to be about $£ 3,104,000$ per year, with an estimated range of $£ 1,768,000$ to $£ 4,439,000$ per year (Bennett and ljpelaar 2003). In the Netherlands, a study of 164 farms found that $52 \%$ reported at least one case of myiasis with, overall, $2.9 \%$ of the sheep struck (Snoep et al. 2002).

Management practices that can help to reduce blowfly strike are strategic shearing of the fleece, reduction of the causes of diarrhoea by good grazing management and treatment of the diseases that predispose sheep to the disorder (i.e. gastrointestinal nematodes) and insecticide use. The effectiveness of these techniques can be extremely dependent on the timing and seasonal effects of temperature and rainfall. The reduction of conformational susceptibility to strike by Lucilia spp., through removal of skin folds and faecally-soiled wool may also be brought about by mechanical means. The removal of faecally soiled wool (dagging), and the regular shearing of wool from around the breech (crutching), may both reduce susceptibility to strike by eliminating suitable oviposition sites. Similarly strike susceptibility is reduced in ewes following annual shearing and by removal of the tail in lambs (French et al. 1994). Surgical removal of skin folds around the breech, the 'Mules' operation, has also been used for Merino sheep in Australia and New Zealand (Windsor 
et al. 2016). The scar tissue formed following this procedure results in a smooth denuded area of skin, reducing faecal soiling and the development of potential oviposition sites (Windsor et al. 2016). However, the welfare implications have resulted in considerable opposition to the practice and attempts to phase it out (Elkington and Mahony 2007; Phillips 2009). Alternative methods of removing wool and wrinkles from the hindquarters of sheep, including the topical application of quarternary ammonium compounds, phenols, caustic soda and plastic clips have been used, but have largely been rejected for reasons of animal welfare. In the long term, the breeding of sheep without wrinkles or wool on their hindquarters offers the most likely approach for this method of control (Palmer 2004; Phillips 2009). In Australia, susceptibility to fly strike in Merino sheep has an intermediate heritability (Raadsma 1991), and there is a positive genetic correlation $(>0.9)$ between fly strike and fleece-rot. Breeding explicitly for lower strike susceptibility has not been adopted in Europe, and the potential for increasing breed tolerance is an important consideration.

Alternative interventions considered for the management of fly strike include monitoring and raised awareness of the weather conditions to enable farmers to strategically treat their sheep with insecticides, or to inspect them regularly and treat affected animals more quickly (Phillips 2009). The use of plant oils such as tea tree, fenugreek, celery, radish and mustard, have yet to be proven effective at a commercial scale (Khater and Khater 2009). Trapping flies to reduce population levels has been attempted in both Australia and the UK and may make a valuable contribution to local fly population management (Broughan and Wall 2006, 2007).

Genetic control through the development of a lethal female strain initially took place in the 1980s but eventually failed (Foster et al. 1993). This was later followed by development of a transgenic strain that was lethal to females (Li et al. 2014) but was developed with North American strains which may well limit the effectiveness of this approach in any campaign in Australia.

Vaccination for the control of Lucilia spp. has also been an area of intensive research over the last 20 years but has not advanced far, probably because of the complex relationship with its host and because the infection proceeds so rapidly (reviewed by Elkinton and Mahoney 2007; Sandeman et al. 2014). Recent work on both a draft genome and a transcriptome of $L$. cuprina have been described by Anstead et al. (2016). This development may advance the prospects for development of vaccine strategies as the discovery and ability to follow-up on the use of new antigens can now proceed quickly. In addition, the draft genome might also contribute to the identification of novel approaches for the control of these extremely important flies.

\section{Screwworm flies}

The obligate screwworm flies C. hominivorax (New World screwworm) and C. bezziana (Old World screwworm) oviposit batches of 200-300 eggs around the edges of wounds of any type or the orifices of the body, and the emergent larvae can invade healthy, living tissues. As the larvae feed, they burrow deeply into the host's tissues so that only the posterior segment and spiracles are exposed. Infestation results in progressive liquefaction, necrosis and haemorrhage and the production of extensive internal lesions. Death may be rapid following multiple infestation. Both species are largely tropical and sub-tropical in their distribution and C. bezziana is not present in Australia or New Zealand. As a result, although they have the potential to have a large impact, they tend to not be important to any large extent in major sheep production areas.

Of note, however, is that fact that sterile insect technique (SIT) has been used with particular success against C. hominivorax in North and Central America (Scott et al. 2017). SIT involves the 
release of irradiated sterile males into a wild population. Eggs fertilized by the sperm of the irradiated males fail to hatch. If enough irradiated males are released and obtain a large proportion of the matings with fertile females, with continued release of sterile males, the population of wild insects is eventually driven to extinction. In addition to the highly successful eradication campaigns in North and Central America, in 1988, C. hominivorax was discovered close to Tripoli in Libya. This was the first known established population of this species outside the Americas. The fly quickly spread to affect animals in about $25,000 \mathrm{~km}^{2}$. In 1989, there were about 150 cases of myiasis by C. hominivorax but by 1990 , a total of 12,068 confirmed cases of screwworm fly myiasis were recorded and, at its peak, almost 3,000 cases were seen in a single month. It was estimated that if unchecked the infestation could cost the Libyan livestock industry about US\$30 million per year and the North African region approximately US\$280 million per year (Lindquist et al. 1992). This concern lead to the application of a successful international control programme to eradicate the fly from northern Africa, using technology and expertise developed during the eradication campaigns in north and central America. Concerns have been raised with regard to the impact of strain differences and there has been some controversy over the impact of weather on the outcome (Gutierrez and Ponti 2014) but these issues have not proved major obstacles to successful implementation.

For C. bezziana there has been little use of SIT to control populations and it has been suggested that perhaps strain differences between the released flies and the target populations have contributed to the lack of success (Morgan et al. 2012).

\section{Discussion - myiasis management in a One Health context}

Sheep are important contributors to both food and financial security, particularly in rural communities in marginal environments. Given the infestation rates described above, the negative impact of myiasis on sheep production can be substantial; effective disease management is therefore important. At its most fundamental, the ability of livestock owners to manage myiasis can be seen to be driven by a number of factors: the availability of effective prophylactic and therapeutic treatments, the residual duration of existing treatments (which determines how often they need to be applied), the ability of producers to predict the incidence of the disease (so that treatments can be applied at the right time) and the cost of prevention (insecticides and labour) relative to the value of the production animals. Myiasis management is likely, therefore, to be a particular issue for small flock owners and where low economic returns from sheep husbandry necessarily result in the low availability of investment for prevention.

However, a strictly economic evaluation of myiasis management often ignores the important, but difficult to quantify, wider context; the historical practice of thinking about the control of specific diseases in isolation may in fact have contributed to our inability to manage them effectively in the long-term (Rushton and Bruce 2017). We are increasingly aware of the importance of considering animal production within its social and economic context and the complexity of the systems within which it operates (Godber and Wall 2014). For example, despite arguments that raising livestock for meat is inefficient and environmentally damaging (Goldstein et al. 2017), livestock production is likely to continue to be an essential component of sustainable food security for many nations, particularly in low income areas and in marginal habitats unsuitable for crop production (Godber and Wall 2014). The availability of high quality food contributes directly to human health and wellbeing; animal products account for approximately one third of global human protein consumption and, hence, animal production is likely to remain important in the foreseeable future (Röös et al. 2017). Animal disease management, therefore, should be 
viewed within a comprehensive One Health framework in which animal health and animal welfare together with human economic and social aspects of the production of sheep and their products must inevitably be included (Godber et al. 2016).

For example, the susceptibility of animals to myiasis may be affected by the immune response of the host, which is in turn affected by its nutritional status and underlying infection by other parasites - for example infection by and clinical response to gastrointestinal worms is a major predisposing factor for fly strike. Nutritional status in turn is affected by factors such as the local availability of high quality fodder. A lack of accessible feed has traditionally been overcome by herding livestock long distances, but this can lead to reduced condition, over-grazing and environmental degradation. Both grazing quality and production of supplementary feed are likely to be compromised in the future as a result of reduced precipitation associated with climate change. Furthermore the use of insecticides such as organophosphates, pyrethroids or macrocyclic lactones for myiasis management may also have long-term negative impacts on the environment and human health (Beynon 2012a,b). All of these factors are interlinked and an attempt to simply focus on one component is inevitably compromised. Hence, in the future, whole-farm systems analysis will be required to provide further understanding of the complex interaction of biophysical and socioeconomic factors relating to animal production, within which disease such as myiasis, should be included.

\section{References}

Angulo-Valadez CE, Ascencio F, Jacquiet P, Dorchies $P$ and Cepeda-Palacios $R$ (2011) Sheep and goat immune responses to nose bot infestation: a review. Med Vet Entomol 25: 117-125.

Anstead CA, Batterham P, Korhonen PK, Young Hall RS, Bowles VM, Richards S, Scott MJ and Gasser RB (2016) A blow to the fly - Lucilia cuprina draft genome and transcriptome to support advances in biology and biotechnology. Biotech Adv 34: 605-620.

Bennett R and ljpelaar JI (2003) Economic assessment of livestock diseases in Great Britain. Final report to the Department for Environment, Food and Rural Affairs, University of Reading, Reading, UK.

Beynon SA (2012a) Potential environmental consequences of administration of anthelmintics to sheep. Vet Parasitol 189: $113-124$.

Beynon SA (2012b) Potential environmental consequences of administration of ectoparasiticides to sheep. Vet Parasitol 189: $125-135$.

Bisdorff B and Wall R (2008) Control and management of sheep mange and pediculosis in the UK. Vet Parasitol 155: 120-126.

Bisdorff B, Milnes A and Wall R (2006) Prevalence and regional distribution of scab, lice and blowfly strike in sheep in Great Britain. Vet Rec 158: 749-752.

Broughan JM and Wall R (2007) Fly abundance and climate as determinants of sheep blowfly strike incidence. Med Vet Entomol 21: 231-238.

Broughan JM and Wall R (2006) Control of sheep blowfly strike using fly-traps. Vet Parasitol 135: 57-63.

Cepeda-Palacios R and Scholl PJ (2000) Intra-puparial development in Oestrus ovis (Diptera: Oestridae). J Med Entomol 37: 239-245.

Cepeda-Palacios R, Angulo-Valadez CE, Scholl PJ, Ramírez-Orduña R, Jacquiet Ph and Dorchies Ph (2011) Ecobiology of the sheep nose bot fly (Oestrus ovis L.): a review. Revue Méd Vét 162: 503-507.

Colwell DD and Berry NM (1993) Tarsal sensilla of warble flies (Hypoderma bovis and H. lineatum (Diptera: Oestridae). Ann Entomol Soc Am 86: 756-765.

Colwell DD, Dantes-Torres F and Otranto D (2011) Vector-borne parasitic zoonoses: emerging scenarios and new perspectives. Vet Parasitol 182: 14-21. 
Devendra C (2001) Small ruminants: imperatives for productivity enhancement, improved livelihoods and rural growth - a review. Asian-Australasian J Anim Sci 14: 1483-1496.

El Abrak A, Tabarani A and Zro K (2002) Dossier myiases animals. Bulln Epidem Vet 9: 1-3.

Elkington RA and Mahony TJ (2007) A blowfly strike vaccine requires an understanding of host - pathogen interactions. Vaccine 51: 33-45.

Evans AC (1936) The physiology of the sheep blowfly Lucilia sericata (Meigen). Trans R Entomol Soc 85: 363-377.

Farkas R, Hall MJR and Kelemen F (1997) Wound myiasis of sheep in Hungary. Vet Parasitol 69: 133-144.

Foster GG, Kitching RL, Vogt WG and Whitten MJ (1975) Sheep blowfly and its control: in the pastoral ecosystem in Australia. Proc Ecol Soc Aust 9: 213-229.

Foster GG, Weller GL, James WJ, Paschalidis KM and McKenzie LJ (1993) Advances in sheep blowfly genetic control in Australia. In: FAO/IAEA (eds.) Proceedings of FAO/IAEA International Symposium Management of Insect Pests: Nuclear and Related Molecular and Genetic Techniques, October 19-23, 1992, Vienna, Austria, pp. 299-312.

French NP and Morgan KL (1996) Role of neonatal and maternal risk factors in the faecal soiling of lambs. Vet Rec 139: 460-465.

French NP, Wall R and Morgan KL (1994) Lamb tail docking: a controlled field study of the effects of tail amputation on health and productivity. Vet Rec 134: 463-467.

French NP, Wall R and Morgan KL (1995) The seasonal pattern of sheep blowfly strike in England and Wales. Med Vet Entomol 9: 1-8.

Giangaspero A, Traversa D, Trentinic R, Scalad A and Otranto D (2010) Traumatic myiasis by Wohlfahrtia magnifica in Italy. Vet Parasitol 175(1-2): 109-112.

Godber OF and Wall R (2014) Livestock husbandry and food security: their vulnerability to population growth and climate change. Glob Change Bio 20: 3092-3102.

Godber OF, Laroussi BF, Chentouf M and Wall R (2016) Intensification of Mediterranean goat production systems: a case study in northern Morocco. Agriculture 6: 16.

Goldstein B, Moses R, Simmmons N and Birkved M (2017) Potential to curb the environmental burdens of American beef consumption using a novel plant-based substitute. PLoS ONE 12: e0189029.

Guerrini VH (1988) Ammonia toxicity and alkalosis in sheep infested by Lucilia cuprina larvae. Int J Parasitol 18: 79-81.

Gutierrez AP and Ponti L (2014) The new world screwworm: prospective distribution and role of weather in eradication. Agricult Forest Entomol 16: 158-173.

Hadani A, Rabinsky R, Shimshoni A and Vishinsky Y (1971) Myiasis caused by Wohlfahrtia magnifica (Schiner) in sheep on the Golan Heights. Refuah Vet 28: 25-33.

Hall MJ (1997) Traumatic myiasis of sheep in Europe: a review. Parassitologia 39: 409-413.

Hall MJ and Farkas R (2000) Traumatic myiasis of humans and animals. In: Papp L and Darvas B (eds.) Manual of Palearctic Diptera. Science Herald, Budapest, Hungary, $978 \mathrm{pp}$.

Hall MJR, Adams ZJO, Wyatt NP, Testa JM, Edge W, Nikolausz M, Farkas R and Ready PD (2009) Morphological and mitochondrial DNA characters for identification and phylogenetic analysis of the myiasis-causing flesh fly Wohlfahrtia magnifica and its relatives, with a description of Wohlfahrtia monegrosensis sp. n. Wyatt \& Hall. Med Vet Entomol 23 (Suppl 1): 59-71.

Hall M and Wall R (1995) Myiasis of humans and domestic-animals. Adv Parasitol 35: 257-334.

Jacquiet P, Alzieu J-P, Lienard E, Grisez C, Prevot F, Bergeaud E, Boushira E, Franc M and Dorchies P (2016) Evolutions epidemioloiques et nouvelles contraintes dans la lutte contre les myiases ovines. Bull Acad Vet France 169: 46-53.

Khater HF and Khater DF (2009) The insecticidal activity of four medicinal plants against the blowfly Lucilia sericata (Diptera: Calliphoridae). Int J Dermatol 48: 492-497.

Lehrer AZ and Verstraeten C (1991) Expansion parasitologique et geographgique de Wohlfahrtia magnifica (Schiner) (Diptera: Sarcophagidae) en Romanie. Bull Rech Agronom Gembloux 26: 563-567.

Li F, Wantuch HA, Linger RJ, Belikoff EJ and Scott MJ (2014) Transgenic sexing system for genetic control of the Australian sheep blowfly Lucilia cuprina. Insect Biochem Mol Biol 51: 80-88.

Lindquist DA, Abusowa M and Hall MJR (1992) The New World screwworm fly in Lybya: a review of its introduction and eradication. Med Vet Entomol 6: 2-8. 
Martin-Vega D, Garbout A, Ahmed F, Ferrer LM, Lucientes J, Colwell DD and Hall MJR (2018) Micro-computed tomography visualization of the vestigial alimentary canal in adult oestrid flies. Med Vet Entomol 32: 378-382.

McLeod RS (1995) Costs of major parasites to the Australian livestock industries. Int J Parasitol 25: 1363-1367.

Morgan ER, Clare EL, Jefferikes R and Stevens JR (2012) Parasite epidemiology in a changing world: can molecular phylogeography help us tell the wood from the trees? Parasitology 139: 1924-1938.

Nedelchev NK (1988) Distribution and causes of myiasis among farm animals. Vet Sbirka 86: 33-35.

Palmer J (2004) The mulesing debate. Aust Vet J 82: 663.

Phillips CJC (2009) A review of mulesing and other methods to control flystrike (cutaneous myiasis) in sheep. Anim Welfare 18: 113-121.

Pokidov II and Goncharov AP (1971) Problems of the control of wohlfahrtiosis in sheep. Veterinariya 7:25-31.

Raadsma HW (1991) Resistance to fleece rot and flystrike. In: Axford RFE and Owen JB (eds.) Breeding for disease resistance in farm animals. CAB International, Wallingford, UK, pp. 263-290.

Röös E, Bajzel B, Smith P, Patel M, Little D and Garnett T (2017) Greedy or needy? Land use and climate impacts of food in 2050 under different livestock futures. Global Env Change 47: 1-12.

Ruiz Martinez I, Cruz SMD, Rodriguez R, Lopez DM, Parra MS and Navio FA (1987) Myiasis caused by Wohlfahrtia magnifica in southern Spain. Isr J Vet Med 43: 34-41.

Ruiz Martinez I and Mira MC (1994). Biogeografía de la mosca Wohlfahrtia magnifica (Schiner 1862) en el sur de Espasna (Diptera, Sarcophagidae). Bol Real Soc Espasnola de Historia Nat (Seccíon Biológica) 91: 123-128.

Ruiz Martinez I, Perez Jimenez JM and Cruz Mira M (1993). Epidemiology of wohlfahrtiosis in sheep and goats. Invest Agrar Prod Sanid Anim 8: 299-311.

Ruiz Martinez I, Soler Cruz MD, Benitez Rodriguez R, Perez Jimenez JM and Diaz Lopez M (1989) Postembryonic development of Wohlfahrtia magnifica (Schiner, 1862) (Diptera: Sarcophagidae). J Parasitol 75: 531-539.

Ruiz Martinez I, Soler Cruz MD, Benitez Rodriguez R, Perez Jimenez JM and Diaz Lopez M (1991) Myiasis caused by Wohlfahrtia magnifica in sheep and goats in southern Spain. II. Effect of age, body region and sex on larval infestation. Isr J Vet Med 46: 64-68.

Rushton J and Bruce M (2017) Using a One Health approach to assess the impact of parasitic disease in livestock: how does it add value? Parasitology 144: 15-25.

Sandeman RM, Bowles VM and Colwell DD (2014) The immunobiology of myiasis infections - whatever happened to vaccination? Parasite Immunol 36: 605-615.

Scott MJ, Concha C, Welch JB, Phillips PL and Skoda SR (2017) Review of research advances in the screwworm eradication program over the past 25 years. Ento Exp Appl 164: 226-230.

Snoep JJ, Sol J, Sampimon OC, Roeters N, Elbers ARW, Scholten HW and Borgsteede FHM (2002) Myiasis in sheep in the Netherlands. Vet Parasitol 106: 357-363.

Sotiraki S, Farkas R and Hall MJ (2010) Fleshflies in the flesh: epidemiology, population genetics and control of outbreaks of traumatic myiasis in the Mediterranean Basin. Vet Parasitol 174: 12-18.

Sotiraki S, Stefanakis A and Hall MJ (2003) Assessment of cypermethrin and doramectin for controlling wohlfartiosis in Crete. Vet Parasitol 116: 327-332.

Sotiraki S, Stefanakis A, Hall MJR and Graf JF (2005a) Field trial of the efficacy of dicyclanil for the prevention of wohlfahrtiosis of sheep. Vet Record 156: 37-40.

Sotiraki S, Stefanakis A, Hall MJR, Farkas R and Graf JF (2005b) Wohlfahrtiosis in sheep and the role of dicyclanil in its prevention. Vet Parasitol 131: 107-117.

Sotiraki S and Hall MJR (2012) A review of comparative aspects of myiasis in goats and sheep in Europe. Small Ruminant Research 103: 75-83.

Tenquist JD and Wright DF (1976) The distribution, prevalence, and economic importance of blowfly strike in sheep. New Zeal J Exp Agr 4: 291-295.

Ternovoy VI (1978) A study of the diapause in Wohlfahrtia magnifica (Diptera Sarcophagidae). Rev Entomol URSS 57: 328-332.

Vasconcelos TC, Macedo JTSA, Silva A, Silva MMN, Bittencourt TCC, Santos MVB, Costa JN and Pedroso PMO (2016) Oestrose: uma parasitose emertente em pequenos ruminantes no Nordeste do Brasil. Pesq Vet Bras 36: 925-929. 
Wall R (2012) Ovine cutaneous myiasis: effects on production and control. Vet Parasitol 189: 44-51.

Wall R, French N and Morgan KL (1992) Effects of temperature on the development and abundance of the sheep blowfly Lucilia sericata (Diptera, Calliphoridae). Bull Entomol Res 82: 125-131.

Wardhaugh KG and Morton R (1990) The incidence of flystrike in sheep in relation to weather conditions, sheep husbandry, and the abundance of the Australian sheep blowfly, Lucilia cuprina (Weidmann) (Diptera, Calliphoridae). Australian J Agricult Research 41: 1155-1167.

Windsor PA, Lomax S and White P (2016) Progress in pain management to improve small ruminant farm welfare. Small Rum Res 142: 55-57.

Wood DM (2006) Morphology of adult oestrids. In: Colwell D, Hall MJR and Scholl PJ (eds.) The oestrid flies: biology, host-parasite relationships, impact and management. CABI, Cambridge, UK, 359 pp. 
Case studies of vector borne diseases in livestock 


\title{
6. Integrated control of trypanosomosis
}

\begin{abstract}
Geoffrey Gimonneau ${ }^{1,2^{*}}$, Jean-Baptiste Rayaisse ${ }^{2}$ and Jérémy Bouyer $3,4,5,6$
${ }^{1}$ CIRAD, UMR INTERTRYP, Département BIOS TA A-17/G Campus International de Baillarguet 34398 Montpellier Cedex 5, France; ${ }^{2}$ Centre International de Recherche-développement sur l'Élevage en Zone Subhumide, BP 454, Bobo-Dioulasso, Burkina Faso; ${ }^{3}$ Unité Mixte de Recherche ASTRE 'Animal, Santé, Territoires, Risques et Ecosystèmes', Campus international de Baillarguet, Centre de Coopération Internationale en Recherche Agronomique pour le Développement (CIRAD), 34398 Montpellier, France; ${ }^{4}$ National Institute for Tsetse and Trypanosomosis Control and Eradication, Livestock Development Sector, Ministry of Agriculture, Addis Ababa, Ethiopia; ${ }^{5}$ Pan-African Tsetse and Trypanosomosis Eradication Campaign coordination office, Rural Economy and Agriculture Department, African Union Commission, P.O. Box 3243, Addis Ababa, Ethiopia; ${ }^{6}$ ASTRE, University of Montpellier, CIRAD, INRA, Montpellier, France; geoffrey.gimonneau@cirad.fr
\end{abstract}

\section{Abstract}

In many parts of Africa, tsetse eradication is impossible due to political, environmental or economic circumstances. In these situations, African animal trypanosomosis control relies on communities or farmer-based control, implemented at a local scale in accordance to the ecoepidemiological context and the cattle rearing system to be sustainable. Management of the African animal trypanosomosis requires integrated controls strategies that combine the use of more than one locally-based tool and where possible, needs to be assisted by veterinarians and other animal health professionals. Several tsetse control methods based on insecticide treated cattle (i.e. pour-on, manual spraying, community bath) and insecticide treated target (traps and screens impregnated with insecticides) are available and should be complemented with diagnostic tests and medication (active trypanocides with prophylactic and/or therapeutic action). However, their adoption is mainly dependent on the engagement of communities, farmers and herders. Indeed, the adoption of a locally-adapted control strategy will depend on farmers socio-technical networks, the cost-effectiveness of the control activities, as well as the time and cost for implementation. In general, insecticide treated cattle methods are the most suitable and acceptable for farmers, because they protect a private good i.e. cattle, whereas insecticide treated targets are generally considered to provide a public good. Nonetheless, selection of the most appropriate tools requires consideration of local disease epidemiology (including host-parasite coevolution), local environmental and socio-economic constraints. The active involvement of communities, farmers and herders is essential from the beginning of the conception of innovative control strategies, and the cost of local integrated pest management should be reduced as much as possible, to be adopted as an acceptable and sustainable animal production cost.

Keywords: African animal trypanosomosis, cattle rearing system, epidemiological cycle, Glossina, integrated management, vector control

\section{Introduction}

Tsetse flies are the major vectors of African animal trypanosomosis (AAT), a disease of economic importance to the livestock production in Africa (Bouyer et al. 2015; Itard et al. 2003). AAT is considered among the greatest constraints to livestock production in sub-Saharan Africa and its economic cost has been estimated at USD 4.75 billion per year (Van den Bossche et al. 2010). To date, vector borne diseases of cattle are mainly controlled through prophylactic and curative 
drugs in livestock. This approach is no longer sustainable, because of the increasing development of drug resistance (Geerts et al. 2001).

In 2000, the African Heads of State and Government decided to increase efforts to address the tsetse and trypanosomosis problem on the African continent and created the Pan-African Tsetse and Trypanosomiasis Eradication Campaign (Kabayo 2002). This initiative aims to encourage tsetse eradication projects throughout Africa, based on the area-wide integrated pest management principles (AW-IPM), see Vreysen et al. (2007) and (2013) for more details. The eradication of tsetse populations is considered the most cost-effective option when successful and sustainable (Kgori et al. 2006; Vreysen et al. 2000). However, successful examples of sustainable tsetse eradication cover less than $2 \%$ of the total infested area in Africa (estimated around 10 million $\mathrm{km}^{2}$ ). Several conditions must be met to achieve this goal: strong political support, feasibility studies, preoperational agreement (governmental and departmental agreements, ethical committee) and planning, mass rearing facilities (if the sterile insect technique is included in the IPM strategy) and a comprehensive eradication campaign (Vreysen et al. 2007). For more details on eradication, see Chapter 14, 'Genetic control of vectors' (Bouyer and Marois 2018). According to an extensive study of the published successes and failures (Bouyer et al. 2010; Vreysen et al. 2013), a decision diagram for tsetse control, taking into account the cost-efficiency of each technique has been proposed (Bouyer et al. 2013).

In most of cases (cases IA and IB in Figure 1), tsetse control will have to be conducted by the beneficiaries themselves in a sustainable way, since tsetse eradication at a regional scale would not be feasible. In this context, it is only necessary to achieve a reduction in the relative density of tsetse flies below the transmission threshold (Bouyer et al. 2013). This local integrated pest management (L-IPM) would be considered as a'production cost' and therefore it would be essential to minimize it as much as possible. Several tools will be needed to implement effective local integrated control strategies for trypanosomosis disease management by either communities or farmers and, in close association with veterinary services. It should include tsetse control methods such as insecticide-treated cattle (ITC; e.g. pour-on, spray and dip) and insecticide-treated targets (ITT; e.g. traps and screens impregnated with insecticides) (Vreysen et al. 2013), but also diagnostic tests for trypanosomes and medications (prophylactic and/or therapeutic trypanocides). Before any intervention, a baseline data collection should be performed in order to select the most appropriate tools, according to the local disease epidemiology, host-parasite coevolution factors and environmental and socio-economic constraints. For tsetse-transmitted AAT, the objective is to use vector control as the primary method to reduce the incidence of the most important trypanosome species for cattle, namely Trypanosoma congolense Broden and Trypanosoma vivax Ziemann (Trypanosomatidae), and thus to prevent or minimize establishment and spreading of strains resistant to trypanocides within the cattle population. Also, the use of insecticides needs to be carefully managed to reduce costs, and the risk of insecticide resistance in tsetse (although this has not been yet observed) but also in other vectors that could be exposed such as ticks in insecticide-treated cattle (Eisler et al. 2003). Therefore, a sustainable AAT control system requires careful over-sight to both minimize costs and avoid resistance development in both the vector and the parasite whilst ensuring effective disease control.

Moreover, the success of AAT control will be strongly dependent on the communities, farmers and herders implication. Indeed, the adoption of the proposed control strategy will depend on their socio-technical networks, and the cost-effectiveness derived from the control activities, as well as the time and cost it will require (Bouyer et al. 2011a; Hargrove 2003; Kamuanga et al. 2001a,b). The perception of the benefits will depend on the establishment and understanding of a sustainable 


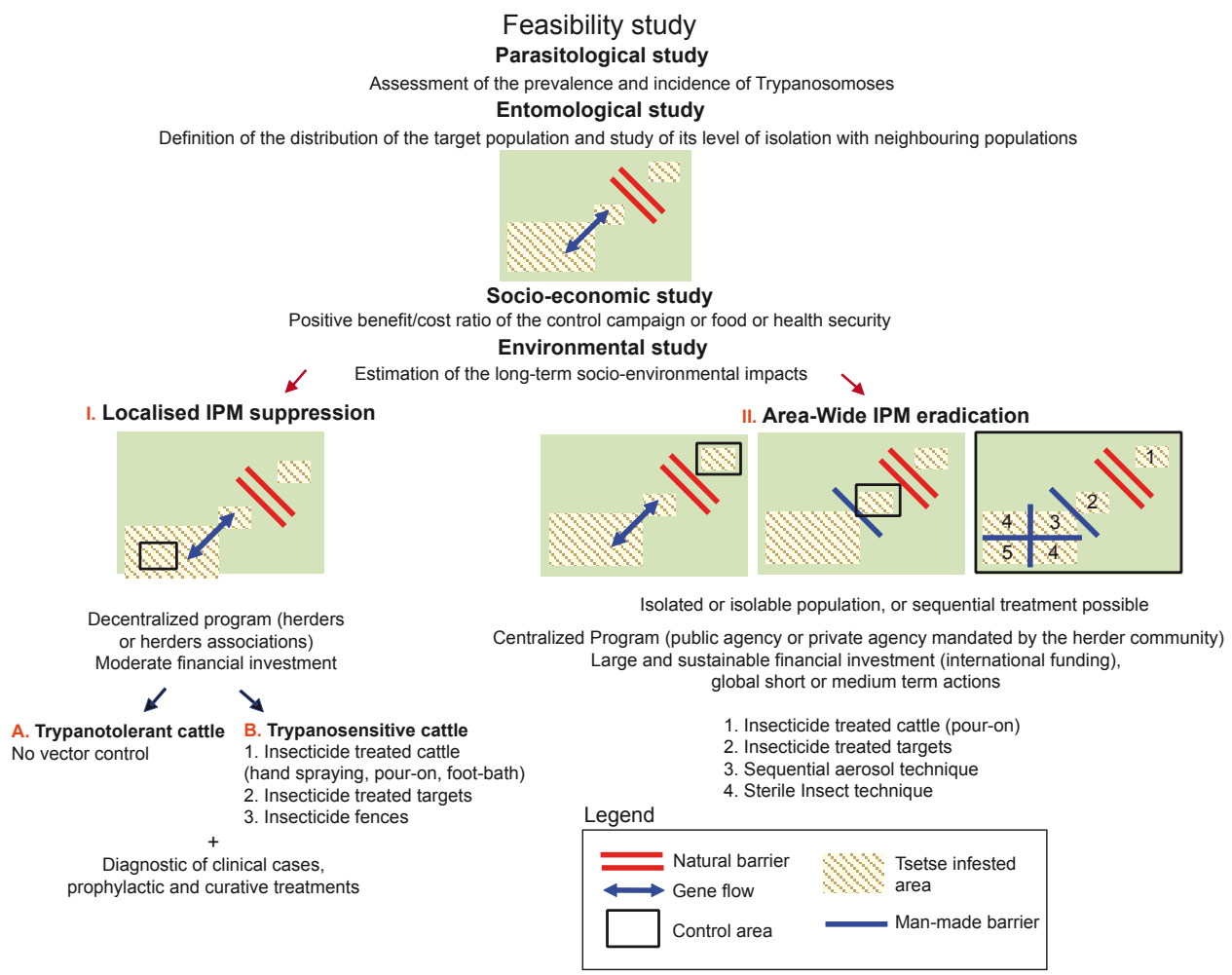

Figure 1. Decision diagram for tsetse control operation (Bouyer et al. 2013, with permission).

production plan (i.e. strategy including the management of health, nutritional and zootechnical constraints). In order to select the best L-IPM strategies in the different contexts, it is important to identify baseline indicators such as the eco-epidemiological setting, the rearing system and the cattle breed. According to these pieces of information, L-IPM implementation would be refined in association to the socio-technical networks involved (Bouyer et al. 2013).

This chapter will focus on the control of tsetse-transmitted African animal trypanosomosis (AAT) with a recommended framework for situations where farmer-based vector control is the only sustainable way of controlling the disease.

\section{Vector control methods relevant to African animal trypanosomosis control in cattle}

\section{Insecticide treated cattle}

This method is based on the treatment of cattle with insecticide formulations (mainly based on pyrethroids) using a wide range of techniques (pour-on, spraying, whole body dips / baths and sprays/ showers). The treated cattle then act as very attractive and lethal baits for tsetse and ticks due to their odour, movement and size. 


\section{Pour-on formulations}

The main advantages of pour-on formulations over other techniques is that no equipment and particularly no water are needed to treat cattle. Application is quick and easy, because the product is deposited on the back line of the animal and diffuses over the whole body (Figure 2A). However, pour-on formulation is more expensive than emulsifiable concentrate formulation of insecticides, due to large dose of active material per $\mathrm{kg}$ of animal weight. It is also more suitable for small rather than large herds, as treatment requires a lot of time. Moreover, pour-on formulations have a more important potential impact on the environment than spraying of insecticides (Vale et al. 2004).

\section{Manual spraying}

Manual spraying of emulsifiable concentrate formulations of pyrethroids is much cheaper (based on the cost of treatment per animal) than pour-on (lower amount of active ingredient required and emulsifiable concentrate cheaper than oil formulations), and presents a similar persistency. Insecticide solution is sprayed over the whole body of cattle (Figure $2 \mathrm{~B}$ ). The main hindrance of pour-on and manual spraying techniques is the treatment time $(\sim 5 \mathrm{~min}$ per animal) because the animal must be immobilized for treatment. For large herds $(>100)$, the treatment time becomes

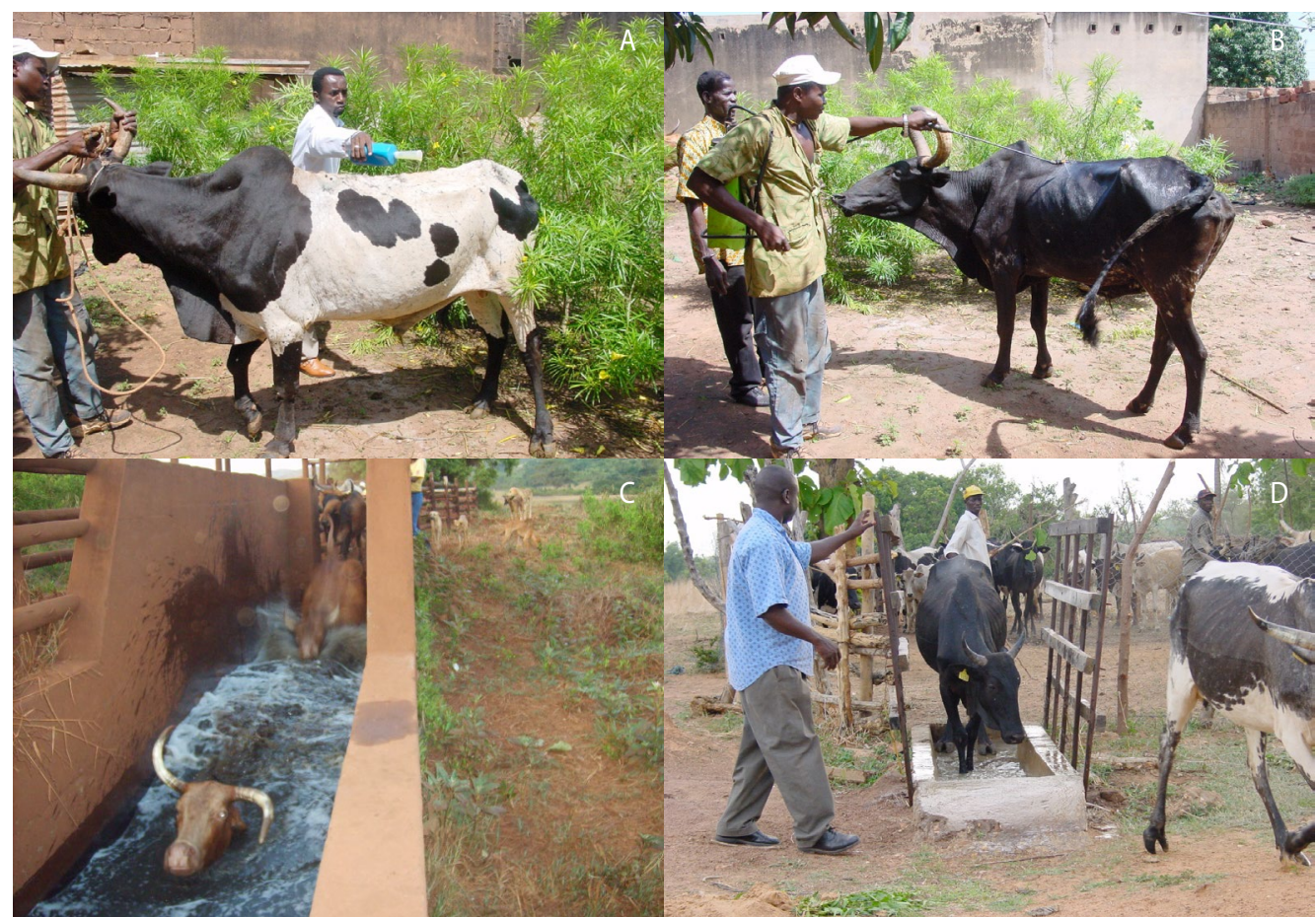

Figure 2. Insecticide treated cattle application methods. (A) Treatment of a zebu bull using a flumethrin pour on in Burkina Faso (photo by J. Bouyer). (B) Treatment of a zebu bull using a hand sprayer containing $0.005 \%$ alphacypermethrin in Burkina Faso (photo by J. Bouyer). (C) Treatment of zebu in community bath in South Africa (photo by J. Ntshangase). (D) Treatment of a zebu using a footbath containing $0.005 \%$ alphacypermethrin in Burkina Faso (photo by J. Bouyer). 
very long. It can be reduced by handling the animals in a 'vaccination corridor' or cattle 'race' (with or without mechanical spray jet races) and/or by the simultaneous administration of other prophylactic or therapeutic disease controls. Moreover to be effective, the pour-on or manual spraying must be repeated every 2 weeks or monthly depending on the product used and tsetse species and density (Bouyer et al. 2007, 2008; Torr et al. 2007).

\section{Community bath}

Community baths (or dip tanks) are routinely used to treat cattle against ticks (vectors of East Coast fever) in some places like the Kwazulu-Natal region of South Africa. Cattle cross a community bath (generally more than $1.5 \mathrm{~m}$ deep) filled with an emulsifiable insecticide solution, resulting in the whole body impregnation in a short time (Figure 2C). The drawback of this method is that the dip tanks must be emptied when insecticides must be changed, with associated environmental hazards and high costs. Dip tanks are also used by individual owners in large and modern farms.

However, in general, dip tanks are very expensive to build and to use and are not appropriate for poor farmers. In addition, inappropriate management of residual insecticides can lead to hazards for individual and health risks for farmers.

\section{Restricted spraying of insecticides}

Restricting spraying reduces the quantity and cost of insecticides used and improves administration to only the lower parts of the cattle body (belly or lower abdomen, and legs) or by application using a footbath (Stachurski and Lancelot 2006), which is a cheap and fast way to treat cattle (Figure 2D).

This restricted treatment has the additional benefit of controlling the tick Amblyomma variegatum Fabricius (Acari: Ixodidae) for which it was first designed (Stachurski 2006; Stachurski and Lancelot 2006) as well as tsetse flies. Its principle is based on behavioural and ecological studies on $A$. variegatum, whose invasion process includes a temporary fixation in the inter-digital areas before they can reach their preferred sites on the body (Stachurski 2006). Similarly, this vector control method is very efficient against tsetse because their feeding behaviour mainly focuses on legs and belly of cattle (Torr and Hargrove 1998; Torr et al. 2007; Vale et al. 1999). Moreover, restricted application of insecticides reduces the environmental impact of ITC, particularly for dung fauna (Vale et al. 2004).

A very important parameter of restricting spraying is that 60 cattle can be treated within 8 min with a footbath versus 120 min for a full, hand-operated spray. For farmers, time savings are often more important than direct costs of these treatments, especially during the rainy season when they are very busy in cultivating their fields. The most important drawback of footbaths is that they are fixed installations and thus not appropriate for transhumant herds. Also, they require strong technical skills to correctly dose the insecticide and require community management (insecticide control, individual contribution) for sharing the use of the footbath that can be burdensome and problematic in some situations. A summary of ITC advantages and disadvantages is presented in Table 1. 
Table 1. Summary of insecticide-treated cattle (ITC) advantages and disadvantages.

\begin{tabular}{|c|c|c|}
\hline & Advantages & Disadvantages \\
\hline ITT overall & $\begin{array}{l}\text { cheap } \\
\text { private good } \\
\text { protection against ticks } \\
\text { cost sharing of public } \\
\text { facilities }\end{array}$ & $\begin{array}{l}\text { requires the treatment of a large proportion of cattle to } \\
\text { reduce tsetse densities } \\
\text { no wild fauna } \\
\text { partial protection (need trypanocides treatment) }\end{array}$ \\
\hline Pour-on & $\begin{array}{l}\text { no equipment, no water } \\
\text { quick application }\end{array}$ & $\begin{array}{l}\text { cost } \\
\text { environmental impact }\end{array}$ \\
\hline Manual spraying & cheap & $\begin{array}{l}\text { treatment time } \\
\text { need animal immobilisation } \\
\text { low persistency ( } 2-3 \text { weeks) } \\
\text { environmental impact }\end{array}$ \\
\hline Community baths & treatment time & $\begin{array}{l}\text { expensive to built } \\
\text { management } \\
\text { environmental impact }\end{array}$ \\
\hline Restricted spraying & $\begin{array}{l}\text { cheap } \\
\text { reduced environmental } \\
\text { impact }\end{array}$ & $\begin{array}{l}\text { need animal immobilisation } \\
\text { repeated weekly } \\
\text { treatment time }\end{array}$ \\
\hline Footbath & $\begin{array}{l}\text { cheap } \\
\text { very quick treatment time } \\
\text { reduced environmental } \\
\text { impact }\end{array}$ & $\begin{array}{l}\text { low persistency (repeated application up to } 10 \text { times/month) } \\
\text { fixed installation (not for pastoralists) } \\
\text { community management constraints }\end{array}$ \\
\hline
\end{tabular}

\section{Insecticide treated targets and traps}

Tsetse traps and screens (Figure 3) impregnated with insecticides (known as insecticide treated targets), particularly pyrethroids, have been used as control methods since the 1970s, due to their user friendliness, low cost and efficacy (Cuisance et al. 1991; Laveissière et al. 1980). They can quickly decrease tsetse densities by up to $99 \%$ and therefore interrupt parasite transmission. Tsetse trapping has the additional advantage of being a good surveillance tool. Many models of traps and screens are available, and they must be selected specifically for the target species in order to function efficiently (Table 2; Figure 3).

Screens are simple devices (Figure 3B), originally constituted of 1 square meter of fabric impregnated with pyrethroids. They present the advantage to be environmentally friendly since insecticides are not dispersed in the environment, which limits their impact on non-targeted fauna, although a temporary impact on insectivorous birds densities and the disturbance of wild mammals have been observed during area-wide campaigns (De Garine-Wichatitsky et al. 2001). Their efficacy and persistence depends on the active ingredient, its formulation, and its concentration (200 to $400 \mathrm{mg} / \mathrm{m}^{2}$ of cloth), and also on the nature of the cloth (density of fibres, presence of an anti-UV protective agent, type of fabric, thickness), and can last up to one year in certain conditions. 


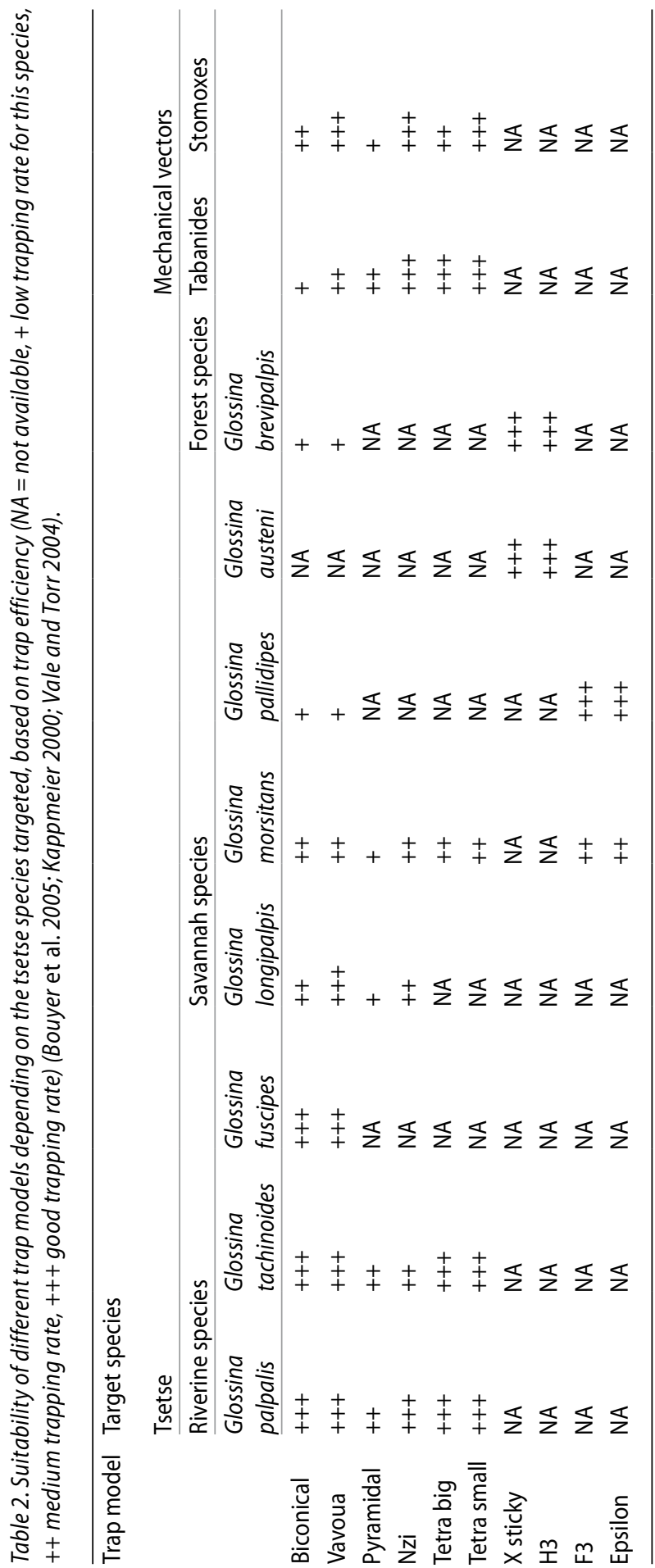




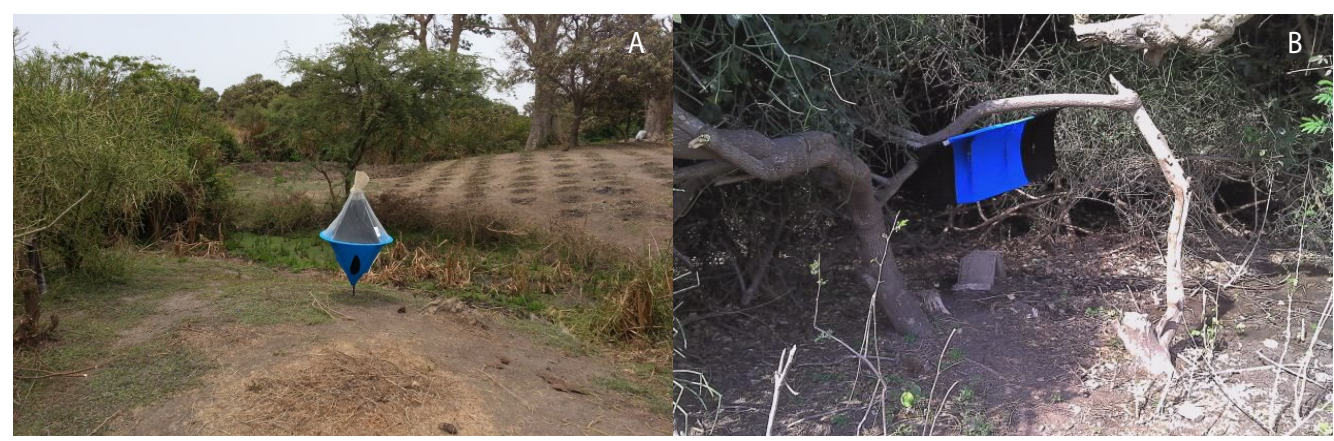

Figure 3. (A) Biconical trap set to monitor Glossina palpalis gambiensis Vanderplank (Diptera, Glossinidae) densities in Senegal (photo by G. Gimonneau). (B) Screen impregnated with deltamethrin, used to control G. p. gambiensis in Senegal (photo A.G. Mbaye).

Traps attractiveness can be boosted by olfactive attractants (octenol, acetone, metacresol, cow urine), especially against savannah species (Vale 1980), but results for riverine species are not so evident (Rayaisse et al. 2010). The use of olfactive attractant requires technical capacities and therefore is more adapted to AW-IPM programs than to farmer based control. Moreover, the cost effectiveness of attractants still needs to be proved.

The density of targets (trap or screen) placements to obtain the desired effect is context dependent and depends on the target tsetse species, and particularly the density of the vegetation. It is generally necessary to use higher densities of screens against riverine species in forest environments (density of $30 / \mathrm{km}$ of river or more) than against savannah species in open environments $\left(1-5 / \mathrm{km}^{2}\right)$.

The main drawbacks are the vulnerability of target material to fire, theft (especially for traps using metal frame) and floods. The insecticide persistence time may also be an issue, if it is too short and requires regular target replacements with associated cost. Moreover, there is a limited adoption of targets by farmers or communities due to a lack of sensitization on the use of these tools and also their availability on the market. A summary of ITT advantages and disadvantages is presented in Table 3.

\section{Innovations and gaps}

\section{Tiny targets}

The design of the target can have substantial effect on the cost-effectiveness in a vector control campaign and the optimal design is always dependent on the species targeted. Recently, smaller screens have been developed, that could lead to important implications for the costs of tsetse control programs (Esterhuizen et al. 2011). It was recently highlighted experimentally that reducing the target size to $1 / 16^{\text {th }}$ of normal $1 \times 1$ m size (i.e. $25 \times 25 \mathrm{~cm}$ ), reduce catches of Glossina fuscipes fuscipes Newstead (Diptera, Glossinidae) only by half in average, suggesting a better costeffectiveness (Lindh et al. 2009). Similar results were obtained for Glossina palpalis gambiensis Vanderplank and Glossina tachinoides Westwood reducing the size of the current $1 \times 1 \mathrm{~m}$ blackblue-black target to horizontal design of around $50 \mathrm{~cm}$ and replacing black clothes by netting 
Table 3. Summary of insecticide-treated targets (ITT) advantages and disadvantages.

\begin{tabular}{|c|c|c|}
\hline & Advantages & Disadvantages \\
\hline ITT overall & $\begin{array}{l}\text { simple, easy to set up and efficient } \\
\text { low environmental impact } \\
\text { cheap }\end{array}$ & $\begin{array}{l}\text { public good } \\
\text { community management } \\
\text { cost of deployment } \\
\text { exposed to vandalism, theft }\end{array}$ \\
\hline Screens & $\begin{array}{l}\text { cheap } \\
\text { easy and fast to set up }\end{array}$ & could induce behavioural resistance \\
\hline Traps & $\begin{array}{l}\text { strong sociological impact (visualisation of } \\
\text { flies in the cage) }\end{array}$ & theft of iron pickets (not for all traps) \\
\hline Small targets & cheaper & $\begin{array}{l}\text { increased density } \\
\text { low visibility in dense vegetation }\end{array}$ \\
\hline
\end{tabular}

will improve the cost effectiveness six-fold for G. p. gambiensis and G. tachinoides (Rayaisse et al. 2011). Table 4 gives the relative indexes (proportion of catches relative to black-blue-black $1 \times 1 \mathrm{~m}$ standard targets) of small targets for G. p. gambiensis for different models and size.

Although there is evidence that reducing the target size will reduce its cost, it is not so clear if in any situation, this tool will be more cost-effective than other methods. Since the index for smaller targets is below 1 , their use requires an increase in their density by 1 /index (Table 3 ). Therefore, their cost effectiveness must be carefully assessed before implementation in control programs

Table 4. Detransformed daily mean catches (transformed means in bracket) of male and female Glossina palpalis gambiensis in Folonzo, Burkina Faso (Rayaisse et al. 2011). ${ }^{1,2}$

\begin{tabular}{llllllllll}
\hline Size $(\mathrm{m} \times \mathrm{m})$ & Material & Shape & Rep & Male & Index & Female & Index & Total & Index \\
& & & & & & & & & \\
$0.5 \times 0.75$ & NBIN & V & 14 & $3.0(0.60)$ & 0.57 & $3.3(0.63)$ & 0.46 & $6.5(0.88)$ & 0.50 \\
$0.75 \times 0.5$ & NBIN & H & 14 & $4.3(0.72)$ & 0.82 & $5.6(0.82)$ & 0.78 & $10.3(1.05)$ & 0.79 \\
$0.25 \times 0.5$ & BkBIN & V & 14 & $0.6(0.21)$ & 0.12 & $0.9(0.27)$ & 0.12 & $1.3(0.37)^{* * *}$ & 0.10 \\
$0.5 \times 0.25$ & BkBIN & H & 14 & $2.8(0.58)$ & 0.54 & $1.7(0.43)$ & 0.24 & $4.5(0.74)$ & 0.35 \\
$0.25 \times 0.5$ & NBIN & V & 14 & $0.3(0.10)^{* * *}$ & 0.05 & $0.5(0.16)^{* * *}$ & 0.06 & $0.7(0.24)$ & 0.06 \\
$0.5 \times 0.25$ & NBIN & H & 14 & $2.2(0.51)$ & 0.42 & $1.4(0.37)$ & 0.19 & $3.5(0.65)$ & 0.27 \\
& & & sed & 0.085 & & 0.084 & & 0.090 & \\
$0.25 \times 0.5$ & BBkBIBk & V & 24 & $0.9(0.29)^{* * *}$ & 0.32 & $0.8(0.25)^{* * *}$ & 0.25 & $1.8(0.44)^{* * *}$ & 0.28 \\
$0.25 \times 0.25$ & BkBIBk & V & 24 & $0.3(0.12)^{* * *}$ & 0.11 & $0.2(0.08)^{* * *}$ & 0.07 & $0.5(0.18)^{* * *}$ & 0.08 \\
$1 \times 1$ & NBIN & V & 24 & $3.2(0.62)$ & 1.09 & $4.6(0.75)$ & 1.47 & $7.9(0.95)$ & 1.25 \\
$0.25 \times 0.5$ & NBIN & V & 24 & $0.7(0.24)^{* * *}$ & 0.26 & $0.6(0.19)^{* * *}$ & 0.18 & $1.4(0.37)^{* * *}$ & 0.21 \\
$0.25 \times 0.25$ & NBIN & V & 24 & $0.1(0.05)^{* * *}$ & 0.04 & $0.1(0.05)^{* * *}$ & 0.04 & $0.2(0.09)^{* * *}$ & 0.04 \\
& & & sed & 0.068 & & 0.066 & & 0.075 & \\
\hline
\end{tabular}

${ }^{1}$ Catches followed by ${ }^{* * *}$ differ from the control at 0.001 level. Catch index is the mean catch of a target expressed as a proportion of that of the standard, which is $1 \times 1 \mathrm{BkBIBk}$.

${ }^{2} \mathrm{Bk}=$ black; $\mathrm{BI}=$ blueH = horizontal; $; \mathrm{N}=$ net; Rep. = replicates; sed = standard error deviation; $\mathrm{V}=$ vertical. 
according to the environment and species targeted. For $G$. fuscipes fuscipes, small targets seems more interesting than classical one, since small targets have indexes of almost 1 (Lindh et al. 2009). A recent study evaluated the cost using small target against this species in Uganda and there was reduces by $48 \%$ from USD 179 to USD 85.4 per $\mathrm{km}^{2}$ (Shaw et al. 2015). For some tsetse species such as flies of the morsitans group (Diptera, Glossinidae), small targets have been proven to be inefficient (Torr et al. 2011).

Although small target is an important innovation, the knowledge gap here, is a lack of a fine tuned cost-benefit study of small targets to determine if this technology can reduce the cost of ITT in different contexts. Also, when applied by the beneficiaries themselves, to what extent this might impact on the will of communities to take responsibility over targets.

\section{Insecticide treated fences}

Insecticide treated fences are very efficient in areas where dairy cows are confined in zerograzing units (food and water supplied to them). The use of mosquito netting impregnated with a pyrethroid (Figure 4) can reduce significantly the incidence of trypanosomosis (Bauer et al. 2006).

This technique was first designed for tsetse control and the principle is to surround zero-grazing units by insecticide-treated mosquito netting at a height of $150 \mathrm{~cm}$. Indeed, behavioural studies have highlighted that tsetse usually attack their hosts at a height of less than $100 \mathrm{~cm}$ above ground level to feed on the legs (Bauer et al. 2006). In some situations, insecticide treated fences can also have a significant effect on vector of human diseases. In Ghana, impregnated mosquito nets were used to protect cattle pens but also reduced significantly mosquito malaria vectors (Anopheles gambiae Giles and Anopheles ziemanni Grunberg; Diptera: Culicidae) (Maia et al. 2012). In Ghana, treated fences have been proved to be very efficient to protect pigs pen against tsetse (Bauer et al. 2011). It was also successfully used in Guinea in older non active human African trypanosomosis (HAT) foci to reduce vector densities and prevent HAT transmission (Kagbadouno et al. 2011). In some conditions, it can also offer a good protection against biting flies, Stomoxys spp. (Diptera: Muscidae) (Maia et al. 2010), which favours its adoption since animals were less disturbed by flies.

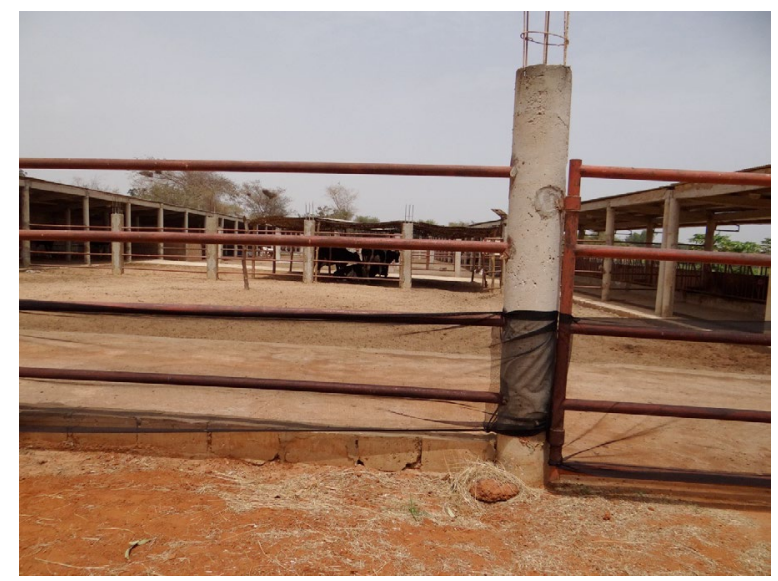

Figure 4. Protection of cattle with impregnated mosquito net in a modern farm in Senegal (photo by J. Bouyer). 
However, in La Reunion Island, it failed to reduce the density of Stomoxys spp., probably because the cattle pens were not surrounded completely with mosquito netting (Bouyer et al. 2011b).

The residual gap for this technology is that most of the trials were organized by comparing fully small protected and small control pens, whereas in some farms the reality is quite different with a calculated total length of fence of $1.2 \mathrm{~km}$. Therefore, according to the fence price (i.e. $€ 160$ for a roll of $100 \mathrm{~m}$ length $\times 1 \mathrm{~m}$ height in Senegal), it would require $€ 1,920$ to surround all the cattle pens, whereas in the same way, 5 impregnated targets would probably have similar impact on tsetse density. Further research is thus required to define the best areas to protect with either insecticidal fences or screens which will be between the tsetse resting sites in the vegetation and the cattle, in order to reduce the cost of fencing needed to protect a farm.

\section{Combination of repellent and insecticides}

Recently, several combinations of repellents and insecticides have been developed in order to improve the effect of formulations in the increasing context of insecticide resistance, especially against mosquitoes (Faulde and Nehring 2012). Today, although no insecticide resistance has been identified in tsetse, combination of different insecticides and molecules can significantly increase the formulation effect (Gimonneau et al. 2016). Application of repellents on cattle to reduce trypanosomosis incidence was tested, and it was concluded that the repellent technology was not sufficiently efficient under natural tsetse challenge to merit commercial development (Bett et al. 2010). Moreover, we think that pushing tsetse towards other animals or herds is not a good principle for tsetse control, even if the repellent technology is efficient. This is because the protection of an individual animal or herd should not increase the risk for other animals at a community level, especially since the poorer farmers in that community might not be able to afford the repellent product. Another option is to associate repellents with insecticides. A new pour-on containing two insecticides, a pyrethroid synergist and a repellent have recently been developed and compared to a standard pour-on (i.e. based on one pyrethroid) (Gimonneau et al. 2016). Results showed in the laboratory that the repellent and insecticides formulation was significantly more efficient than the classical formulation with a longer knockdown effect ( 37 and 28 days, respectively; Figure 5A). More importantly, it significantly increased the cattle protection against tsetse bites (Figure $5 B$ ). In the field, this combination has been proved to be very effective against ticks with a complete elimination three days after application and in the same time a significant reduction in trypanosomosis prevalence and increased in packed-cell volume (PCV).

This new pour-on formulation, combining insecticides and repellent, was highly effective against AAT and ticks, with a longer persistence than other pour-on products on the market. It offered immediate effect on ticks and low treatment frequency to maintain a low ticks infestation and trypanosomosis prevalence. Moreover, this new insecticide formulation represented the first one to provide a partial individual protection against tsetse bites and AAT (Hargrove et al. 2000). Although the efficacy will probably not be the same in various contexts and environments (Hargrove et al. 2003), it was therefore a great innovation for farmers for the control of ticks and AAT. Actually, providing individual protection of cattle against AAT and ticks might increase the adoption of the control technique by farmers, as opposed to collective protection (Bouyer et al. 2011a). In sites where AAT coexists with HAT, this tool might represent another weapon against tsetse within a one-health perspective, as it has been suggested earlier that treating cattle could help controlling HAT through a reduction of tsetse densities (Ndeledje et al. 2013). 

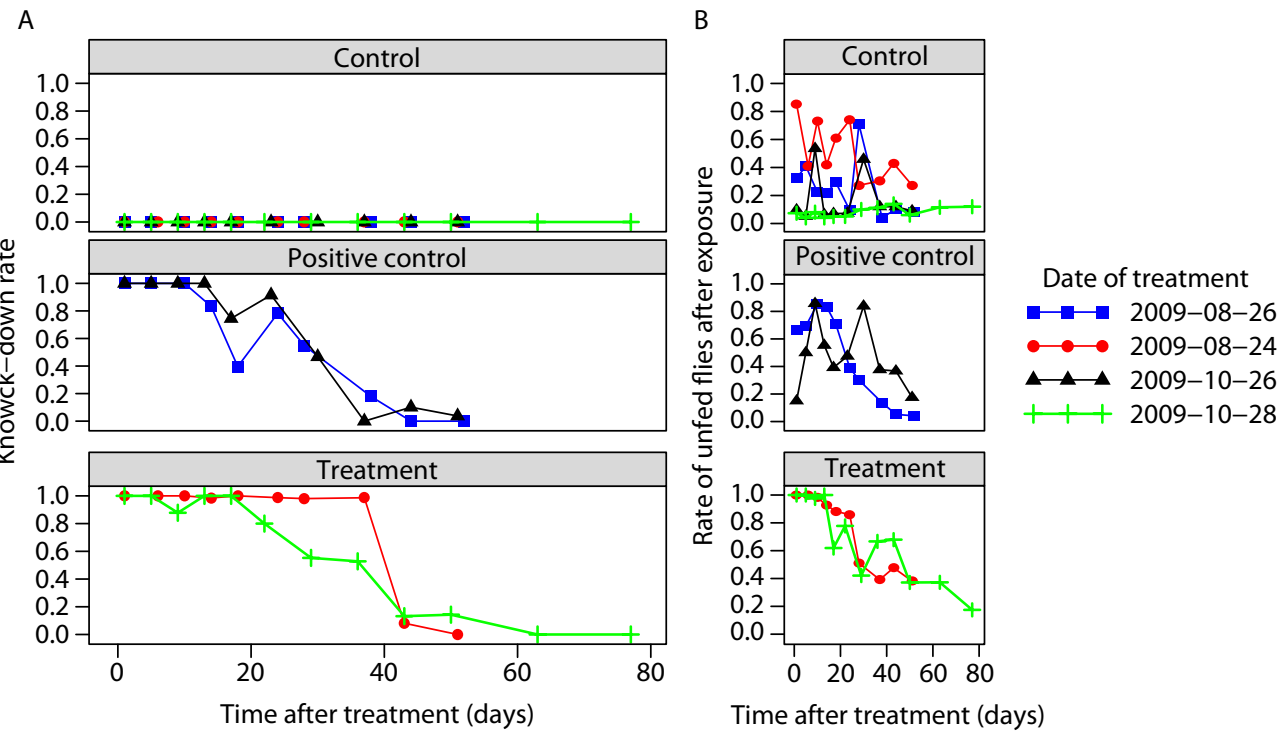

Figure 5. (A) Knock-down rate of Glossina palpalis gambiensis in function of the time after treatment. (B) Rate of unfed G. palpalis gambiensis, giving an estimation of the protective effect of treatment, in function of the time after treatment.

One of the main constraints for farmers could be the price but a formulation for water suspension has been developed, decreasing the total amount of insecticide used and the treatment price. However, its impact regarding knock-down rate and disease or biting protection will have to be tested. Another knowledge gap is that no data is available on the toxicity, residuals and ecotoxicity of this combination.

\section{Restricted application of insecticides}

Socio-economic studies on restricted application of insecticides to cattle have revealed that treatment time and usage by the community were the most important constraints for the adoption of partial spraying and footbaths respectively (Bouyer et al. 2011a; Selby 2010). Socioeconomic studies identifying the types of setting in which corridors or footbaths (and the maximal distances between farms and communal corridors or footbaths) are acceptable for sustainable use of the technique are needed to further improve the adoption of these techniques. It would also be important to develop with the stakeholders, acceptable methods for sharing the costs between farmers. Actually, there is no recommendation on how to use this technique taking into consideration the herder constraints and their habits, particularly in the case of traditional farmers.

Moreover, regarding footbaths, the dosage of the insecticide is a tricky issue for traditional farmers, when it is done using an abacus. A simpler system would be needed to reduce the technical problems associated with this dosage of insecticides. 
Finally, because restricted application focused to the lower parts of the body, it would be very useful to quantify the insecticide residuals in milk.

\section{Quality control and evaluation procedure for targets}

There is a real need to develop a standard quality control procedure and evaluation for targets in order to improve the reliability, efficacy and persistency of the different batches of targets sent by a given manufacturer.

According to the literature, two tools have been developed to evaluate targets but with several drawbacks. The first method consists in the restriction of a fly in the head of a glass tube by a loose-fitting piston carrying a sample of insecticide (Kernaghan and Johnston 1962). The fly is handled only when first introduced into the tube; it then remains in the same tube during the holding period. This method is time-consuming because the test is performed on one fly at a time and several flies are needed to obtain robust statistics (i.e. minimum of 30 flies). Moreover, injuries could be inflicted to the flies, generating biases in the results. Also, the fly stays in the same tube during the observation period which represents a bias since insecticides could remain in the tube. The second apparatus is a ' $T$ ' shape flight tunnel where flies are attracted to the light where the netting is set (Torr 1985). The fly collides with the netting, set obliquely across the flight-path, and it then flies towards a second, stronger, light source. In this system, there is no possibility to control the time of exposure of the fly that is a very important criterion for insecticide bioassays. Moreover, the apparatus configuration allows some flies to reach the second light source without entering into contact with the net (Torr 1985). Anyway, these two tools seem not to be used by the researchers community as there is no mention of these in the literature, may be due to a lack of reproducibility.

In target manufactures, quality control procedures seem to be absent. Huge differences in persistency are observed in the field between batches that impact the success of vector control. A solution would be that the manufacturers should use exactly the same production process for a given trap or screen model, or that they should be able to test these different properties for each change in the production process and provide data, such as trapping indexes relative to a reference trap and persistency in the field.

\section{Impact of farming systems and tick-borne diseases on the adoption of tsetse control methods}

Even if socio-economic surveys have already been conducted to study the adoption of tsetse control techniques by farmers, it would be important to study accurately the perception and adoption rate of the different techniques available to control tsetse in different farming systems. Actually, farmers belonging to different farming systems (traditional/modern, sedentary/ transhumant) have different perceptions of their cattle (production tool vs money savings) and socio-economic networks (Bouyer et al. 2015). Moreover, the risk level in case of an innovation is not the same (much higher for traditional farmers) and will probably impact the adoption process (Bouyer et al. 2011a).

It appears also important to study the impact of ticks and tick-borne diseases on the adoption of tsetse control techniques. Because ITC based on pyrethroids directly impact ticks and tick-borne diseases, the adoption process is facilitated. This aspect should not be overlooked because it can lead to situations where the farmers prefer to keep their usual control technique even if it is working only against ticks than to adopt a new one that is working both against tsetse and ticks 
(see below the example of ITC in Uganda). It is thus very important to sensitize the farmers on the likely impact on ticks when a new strategy to control tsetse is proposed. These should be studied in each African region (at least western and eastern), since important local specificities might be observed.

\section{AAT control strategies including vector control with preventive and/or curative treatment of cattle}

\section{Description of the different epidemiological settings}

In this part, we consider only AAT or Nagana, caused by T. congolense, T. vivax and Trypanosoma brucei brucei (Plimmer \& Bradford) transmitted to cattle by tsetse, and mechanically transmitted for T. vivax beyond the tsetse belt. Mechanically transmitted Animal trypanosomosis or Surra caused by Trypanosoma evansi (Steel) worldwide and by T. vivax mainly in South America is not considered. For Nagana, and with a special focus on T. congolense and T. vivax, three major ecoepidemiological cycles related to cyclical transmission by tsetse have been described, and one mechanical cycle occurring at the limit of the tsetse distribution area (Pagabeleguem et al. 2012; Van den Bossche et al. 2010).

\section{Sylvatic trypanosomosis}

In sylvatic trypanosomosis settings, trypanosomes are transmitted by tsetse to the trypanotolerant wild fauna. Cattle are supposed to be absent in these areas but sometimes enter this system for illicit grazing purposes (and/or transhumance) and are then infected with highly virulent strains.

\section{Interface trypanosomosis}

Interface trypanosomoses occur at the edge or interface of agro-pastoral areas and protected areas. Tsetse diversity and abundance is high at this interface (border effect), and virulent strains are transmitted from wild fauna to cattle, leading to acute infections in the latter, with a high mortality rate, or even epidemic situations.

\section{Endemic trypanosomosis}

In endemic trypanosomosis, no or very scarce wild fauna is involved and cattle are the main host for tsetse. Tsetse density, lifespan and diversity tends to reduce (Van den Bossche et al. 2010). The virulence of trypanosome strains is reduced by their circulation within the cattle compartment, although virulent strains can be imported from the former areas by transhumant herds. In this setting, cattle are used to 'live with the disease'.

\section{Mechanical trypanosomosis}

Mechanical trypanosomosis occurs at the limit of tsetse distribution, within the range of movement of transhumant herds. In West Africa, trypanosomes are imported by cattle transhuming into the tsetse belt and locally transmitted to resident herds by mechanical vectors (T. vivax only). In East Africa, T. vivax is well established in sedentary cattle whereas tsetse flies are absent, but numerous movements of cattle occur and might also allow the importation of trypanosomes (Ahmed et al. 2016). The relationship between mechanical trypanosomosis and cyclical trypanosomosis is not so clear and need further investigation (Tadesse et al. 2011). 


\section{Description of the different cattle rearing systems}

In this section, 3 types of cattle rearing systems will be considered as important to take into consideration when selecting the best control options:

1. Transhumant rearing systems, where herders drive their cattle to remote grazing areas (up to several hundred kilometres), especially during the dry season. Animals are mainly local breeds, more or less trypanotolerant depending on AAT pressure. Inputs to improve productivity are very low. Animals may encounter tsetse eco-epidemiological contexts that are different from those in their area of origin. Therefore, they will probably move between tsetse free and infested area with different eco-epidemiological cycles, linking them and importing trypanosome strains from one to the other. Controlling this genetic flow is a very important challenge (see below).

The two other rearing systems can be considered as sedentary systems, which are generally exposed to one epidemiological cycle only:

2. Agro-pastoral system/mixed farming systems, where farmers have two main activities: agriculture and cattle rearing. Crop residues are generally used to rear cattle in association with the surrounding environment, i.e. natural pastures within a range of 5 to $10 \mathrm{~km}$. Cattle are mainly local trypanotolerant breeds or more or less crossbred with trypanosusceptible breeds depending on AAT pressure. Inputs are low to medium. Different situations could be observed between farmers according to the time and efforts they spend in different activities, i.e. cropping or cattle rearing but they will be considered here together because the proposed AAT strategies will be the same.

3. Zero grazing units are modern farms, generally in peri-urban areas where animals are mainly fed with forage or agro industrial subproducts. Farmers rear improved local breeds (zebu), sometimes cross-breed with exotic cattle or even pure exotic cattle. Animals are considered as a production tool and generally have high economic value in comparison to previous systems. Inputs are generally high in order to maintain these breed under high parasitological pressure in combination to high productive pressure.

\section{Proposal of a general framework for farmer-based AAT Control}

Proposals presented in this section are mainly based on argument present in this chapter and other peer reviewed documents (Bouyer et al. 2010, 2013; Vale and Torr 2004; Van den Bossche and Delespaux 2011; Vreysen et al. 2013).

A general decision framework depending on the type of farming system and epidemiological cycles is presented in Figure 6 from Bouyer et al. 2013. The integrated packages are developed below.

\section{Sylvatic $A A T$ (Al and BI)}

This epidemiological cycle is encountered in protected areas only, where cattle rearing should be discouraged. In this situation, ecotourism, ranching of wild fauna or game and hunting reserves should be encouraged instead. 


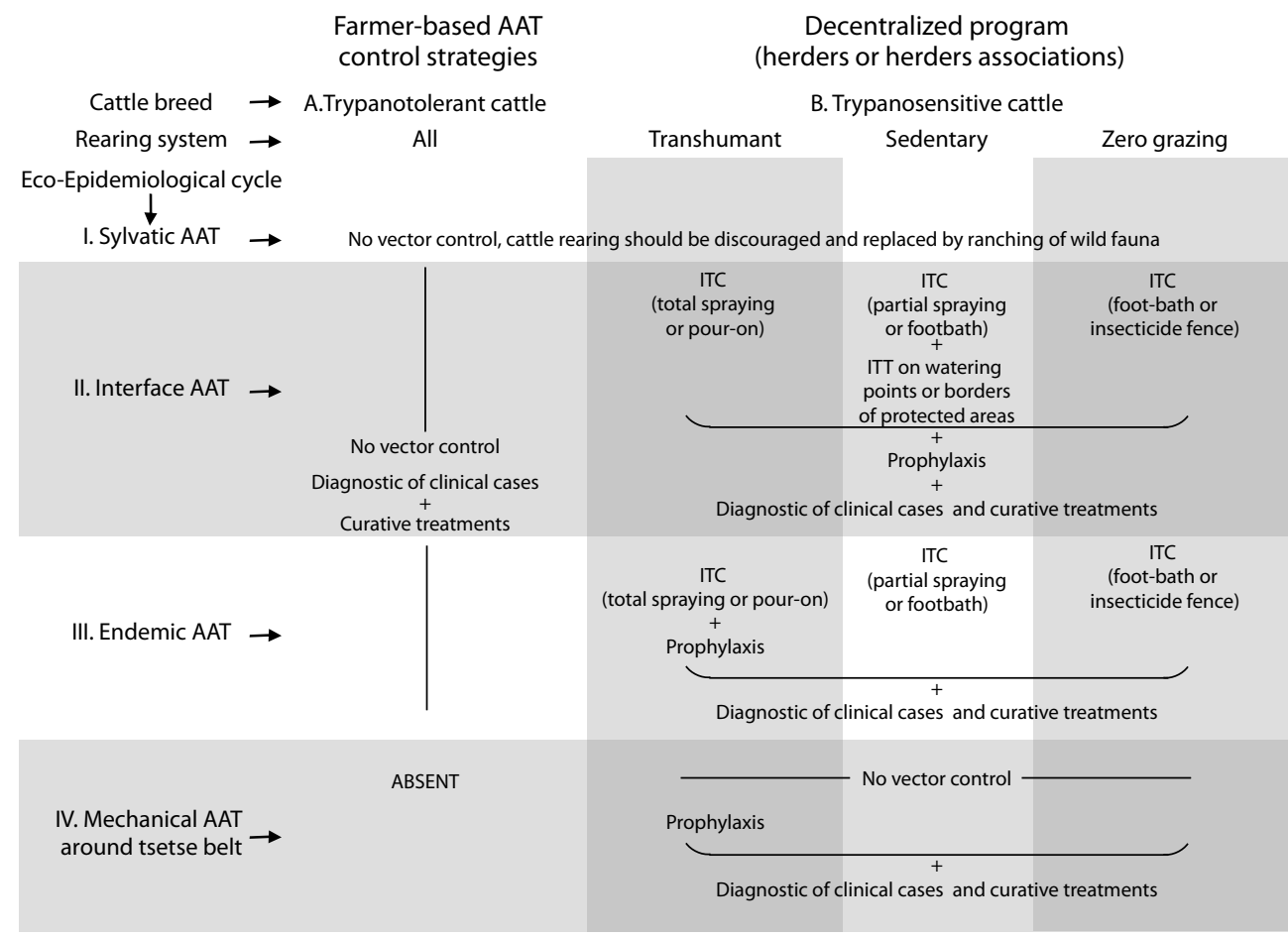

Figure 6. Decision framework proposing different strategies integrating vector control and the use of trypanocides, depending on the type of farming system (columns) and epidemiological cycles (rows) (ITC = insecticide treated cattle; ITT = insecticide treated targets; AAT = animal African trypanosomosis) (Bouyer et al. 2013, with permission).

\section{Trypanotolerant cattle (All and AIII)}

In systems involving trypanotolerant cattle, vector control is not cost-effective. Trypanotolerance is defined here as the ability to limit parasitaemia and anaemia and remain productive in enzootic areas (Dayo et al. 2012). Therefore, clinical cases are rare and generally caused by a loss of immunity (other disease or insufficient food). These clinical cases should be diagnosed and treated using trypanocide drug treatments but prophylactic treatments should be avoided in order to reduce the development of drug-resistant strains of trypanosomes. Moreover, it might prevent the establishment of a protective immunity in young cattle that must be infected at an early age to ensure maintenance of tolerance. The relationship between trypanosomes and trypanotolerant cattle is more or less at equilibrium, and interventions should in general be limited to avoid breaking this equilibrium. This strategy has the additional advantage to allow the long term conservation of trypanotolerant breeds. 
Trypanosensitive cattle (BII to BIV)

\author{
Interface AAT (BII)
}

Interface AAT is probably the case where the economic impact of AAT on cattle rearing systems is the highest, because virulent strains are transmitted from wild fauna to trypanosensitive cattle on a regular basis. Here, the use of different vector control strategies and the use of trypanocides (prophylactic and curative trypanocides) are necessary and cost-effective, in order to reduce the incidence of AAT, the morbidity and mortality of cattle, and the development of resistant strains of trypanosomes.

ITC is the method of choice because it protects an individual good instead of a public one and it is also the most cost-effective (Shaw et al. 2013). The type of ITC must be differentiated depending on the rearing systems:

For transhumant herds, fixed structures like footbaths are not suitable, and water is difficult to transport. Therefore, it should be recommended to reduce the treatment frequency as much as possible by the use of pour-on or total spraying (if water is available) of the cattle.

For sedentary herds, fixed structures are convenient. Restricted application of insecticides using partial spraying (every two weeks) or footbaths (weekly) should be recommended: the treatment corridor or the footbath respectively can be easily built at the exit of the cattle' pen, with a door allowing to pass cattle through it when desired, in order to reduce treatment time as much as possible.

For zero-grazing herds, insecticide fences can be used around the cattle pens, or footbaths placed between the pen and the grazing area in order to facilitate treatment.

However, ITC is generally not sufficient to protect cattle totally and it should be completed by ITT especially at watering points when riverine tsetse species are involved. The use of impregnated targets or traps set every $100 \mathrm{~m}$ along $1 \mathrm{~km}$ of the river on each side of the water points will then be enough to suppress the tsetse population at this site (Knols et al. 1993; Willemse 1991). Based on several tsetse control programs, target barriers will never be maintained by the farmers themselves; therefore authorities managing game or hunting reserves should be sensitized to use part of the benefits generated by tourism to maintain these barriers.

\title{
Endemic AAT (BIII)
}

In this system, the same ITC strategy as described for interface AAT (BII) should be recommended but ITT is no more required. Indeed, the strains circulating here are less pathogenic. Moreover, due to environmental degradation, tsetse distribution is much more fragmented and densities are generally low. Because the tsetse feed mainly on domestic animals due to the absence of wild fauna, ITC is likely to have a more important impact on tsetse.

In endemic AAT, the relationship between trypanosomes and trypanotolerant cattle is more or less at equilibrium, so prophylactic treatments should be limited. However, importation of virulent strains from the sylvatic and interface cycles is possible because of transhumant herds. Clinical cases should be quickly diagnosed and treated immediately using curative trypanocides. 


\section{Mechanical AAT (BIV)}

In mechanical AAT, tsetse are absent and vector control approaches are generally not effective. In this system, AAT occurs as epidemics so prophylactic treatments may not be cost-effective, except in the case of transhumant herds. If animals come from a tsetse free area, it is recommended to use prophylactic drugs and curative ones just before their return.

This regimen is intended to avoid losing animals during transhumance through areas with other epidemiological cycles, and to avoid importing trypanosomes into this tsetse-free area and thus prevent the establishment of mechanical transmission.

\section{Case studies of vector control approaches}

\section{Restricted application of insecticides using footbaths around Bobo Dioulasso, Burkina Faso}

This case study is based on three peer reviewed articles (Bouyer et al. 2007, 2009, 2011a). The epidemiological cycle is the endemic AAT cycle and the type of cattle rearing system is an agropastoral rearing system (situation BIII).

In Dafinso (Burkina Faso, 15 km north of Bobo- Dioulasso), 68 cattle of a herd of 96 cattle (i.e. 71\%) were treated with a footbath containing a pyrethroid formulation. The effect of this restricted insecticide treatment of cattle was observed on released cohorts of reared, irradiated and marked tsetse flies (Figure 7A).

Footbath treatment significantly reduced the mean lifespan of released flies (G. p. gambiensis and G. tachinoides) from 4.7 days $(95 \% \mathrm{Cl} 3.4-7.5)$ to 1.7 days (95\% Cl 1.3-2.4). The apparent densities of wild flies at the water point frequented by the footbath-treated cattle herds reduced quickly in Dafinso (Bouyer et al. 2007), with an estimated daily mortality rate related to this exposure of $0.39(95 \% \mathrm{Cl} 0.19-0.54)$, which was much higher than needed to reduce a wild tsetse population (0.03) (Hargrove 2003).

An one-year survey (May 2005-June 2006) was conducted in the same site in order to compare the incidence of AAT in a footbath-treated cattle herd (48 footbath-treated animals with a suspension of pyrethroids administered every 2 days by farmer itself with a control one (69 cattle not treated), watered at another river section in the same area (Bouyer et al. 2009). From farmers' viewpoint, the main justification of the footbath treatment was to prevent infestation by $A$. variegatum so, they stopped using the footbath when tick infestation decreased, after the end of the rainy season, i.e. the 7 October 2005. This scenario was monitored and data used to assess whether tsetse-control measure implemented during the rainy season only were sufficient to limit tsetse populations and trypanosome transmission during the dry season, when riparian flies are usually still active and numerous enough to have a great epidemiological importance. During the survey, cattle were sampled for identification of the three trypanosome species occurring in the area using PCR ( $T$. vivax, $T$. brucei sensu lato and $T$. congolense savannah type). The study can thus be considered as a comparison of an integrated AAT control strategy combining vector control and curative treatment of clinical cases with the control group only receiving curative treatment of clinical cases but no vector control.

The initial prevalence of trypanosomosis was $6.6 \%(n=91)$, i.e. $0.0 \%$ for $T$. brucei, $4.4 \%$ for $T$. congolense and $2.2 \%$ for T. vivax (no difference between the two herds, Fisher's exact test, $P=0.69$ ). 
A

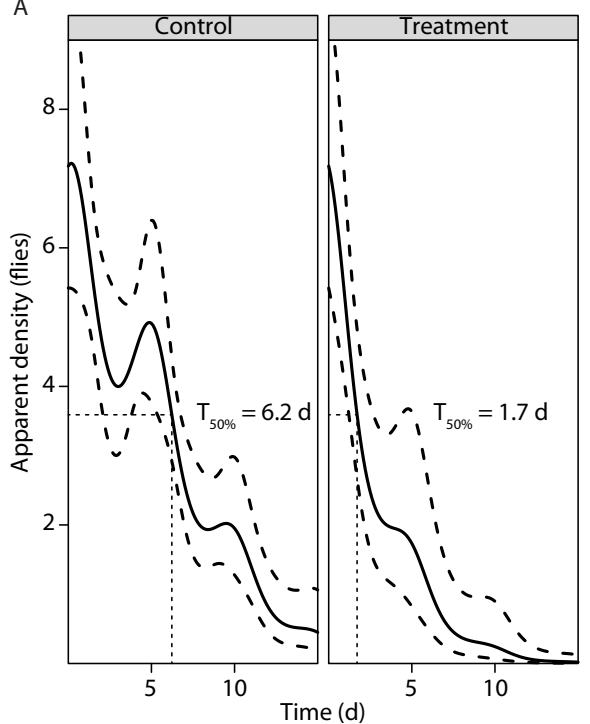

B

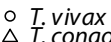

$\triangle T$. congolense

* T. brucei

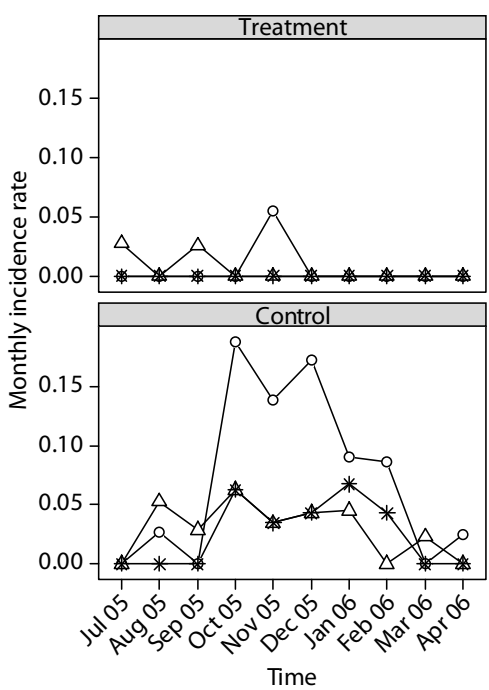

Figure 7. (A) Effect of the exposition to footbath-treated cattle on the time corresponding to a $50 \%$ reduction (T50\%) of the initial apparent density of released, irradiated tsetse flies in Dafinso (Burkina Faso) during the early rainy season 2005 (points: observed data; the solid line was estimated from a negative-binomial model fitted to the points; dashed lines: 95\% confidence limits) (Bouyer et al. 2007, with permission). (B) Observed incidence rate of cattle trypanosomosis in Dafinso (Burkina Faso) according to Trypanosoma species and treatment category (Bouyer et al. 2009, with permission).

The overall PCV was $28.3 \%$ with no significant difference between the 2 groups (Welch 2-sample t-test, $P=0.18)$. Pyrethroid administration with the footbath resulted in a significant reduction of the monthly trypanosomosis incidence rate $(P=0.003)$. This effect lasted during the dry season, after the treatment period, and no new AAT infection was recorded in the treated herd (Figure 7B). The overall incidence of cattle trypanosomosis was reduced $(P=0.01)$ from 0.76 (control group) to 0.11 (footbath treated group). A positive effect of the footbath treatment on packed-cell volume was also observed $(P<0.001$, see Bouyer et al. 2009) which proved a significant improvement of animal health (Bouyer et al. 2009).

These field studies demonstrated that restricted application of insecticides using footbaths allowed a significant reduction of tsetse populations, leading to a breakdown of trypanosome transmission cycle. Moreover, it reduced treatment cost and time by more than $90 \%$ compared with other techniques such as spraying of the full body of cattle. These results were also attributed to environmental conditions favourable to tsetse control in this area. Indeed, the scarcity of wild hosts but also the landscape fragmentation has favoured a fast reduction of tsetse subpopulations by reducing the speed of re-invasion of the cleared water points.

Unfortunately, these good results were not sustained. A socio-economic survey conducted in the same study site in 2008 at the beginning of the rainy season revealed that farmers have 
abandoned the use of footbaths (Bouyer et al. 2011a). The main raison given was technical problems encountered to treat animals (whereas they did not during the experimental period), i.e. cattle were reluctant to pass in the footbaths. Later and in other sites, other farmers built more traditional cattle pens (with wood) instead of using metallic wire which greatly improved the treatment conditions. Moreover, footbaths manager were young educated people that have to count cattle during each treatment in order to calculate the price to pay for each herd (in order to buy new insecticides) and it conferred to them a strategic position which changed the former social relationships that farmers could not accept.

Therefore, the experience of a communal use of footbaths in this site was a failure despite the clear benefits associated to the technique, and an one year follow up by technicians offering long-term technical advice to the farmers. The main adoption factor was related to the economic dynamism of farmer associations. Actually, in another group of farmers using a similar farming system than the one studied here, but with more active farmer associations, a large proportion of the farmers adopted the technique (Bouyer et al. 2011a).

\section{ITC in Uganda: the SOS program}

This case study is based on several peer reviewed articles but two main data sources focusing on vector control and animal trypanosomosis were used (Morton 2010; Selby 2010). The Stamp Out Sleeping Sickness (SOS) program was a human health program, initiated mainly to fight sleeping sickness, which is not the priority of this work but it is a good case study for three reasons:

1. it combined mass treatment of cattle with curative drugs and restricted insecticide treatment of cattle (partial spraying);

2. the size of the initiative in terms of the number of cattle treated $(>180,000)$;

3. the inclusion of the cattle farmers as one of the partners, together with researchers, Ugandan public authorities and the private sector (Ceva Santé Animale and IKARE).

In October 2006, an intervention was initiated to arrest the northerly advance through Uganda of the zoonotic parasite Trypanosoma brucei rhodesiense (Stephens \& Fantham). The main goal of the SOS campaign was to target the cattle reservoir of $T$. brucei rhodesiense (agent of the acute form of sleeping sickness), in an areas of approximately $8,000 \mathrm{~km}^{2}$ by treating $>180,000$ head of cattle (Selby 2010).

The field operations were conducted during 4 years between 2006 and 2010, and Morton (2010) reported the treatment of 'almost 180,000 cattle - belonging to around 50,000 households with trypanocidal drugs and insecticidal sprays over five districts'. In this context, we will focus on the involvement of farmers in treating their cattle and the sustainability of the results and also in establishing whether or not this mass treatment of cattle has improved animal health, by controlling $T$. congolense and $T$. vivax, which were also present.

The control operations were subdivided in four rounds of treatments of cattle over 5 districts with trypanocides (isometamidium chloride and diminazene aceturate) and pyrethroid spray. Objectives of the first control session (November 2006) were to treat $86 \%$ of the estimated cattle population and to sensitise the farmers to pursue treatments on their own with support from District Veterinary Offices. In one month, 178,000 head of cattle were treated, corresponding to more than $100 \%$ of the initially estimated cattle population, but more probably to $\sim 55 \%$ of the actual one (Selby 2010). The second round of control operations presented a lower coverage and dropped to approximately $30 \%$ of animals treated with strong variations of coverage observed 
within and between districts despite provision of pyrethroid for two more rounds during the first round. Therefore, the third round (April/May 2007) was supervised by people from the Makerere University and was more successful $(\sim 80 \%)$. This low coverage was attributed to lack of communication about the insecticide efficacy because herders used other products, and distrust of the programme objectives (fear of taxation of cattle). Finally, a fourth round was implemented in a specific area where substantial cases of $T$. brucei rhodesiense were found both in cattle and human populations. Only 30,000 cattle were treated. In the other 5 districts, a new control strategy was implemented by five veterinarians sent to the field and in charge of sensitizing farmers and distributing products. Because of different problems lowering the farmers' investment capacities, this situation was not sustainable for veterinarian earning not enough money. Therefore, they started to sell different products, especially amitraz which is not efficient against tsetse. This product was considered more efficient against ticks by farmers due to its strong and visual knockdown effect on ticks whereas this effect was not visible with pyrethroids (the ticks become dry but remain on cattle; F. Stachurski, personal communication). Moreover, the initial price of the insecticide during the interventions was $0.02 \mathrm{US} \$ /$ treatment due to reduced or subsidized prices by the private partners, whereas the actual cost of the wholesale drugs was closer to 0.06 US\$/ treatment (Shaw et al. 2013). This data on farmers' awareness and practice of regular spraying have been judged inconclusive. Indeed, by June $2009,45 \%$ of cattle keepers had sprayed their animals in the last month' which was not dissimilar to the $40 \%$ who reported spraying their animals monthly in 2006 before the SOS project (Morton 2010).

Prior to the SOS project, the prevalence of T. b. brucei within the cattle reservoir was estimated by PCR at $15.57 \%$ (T. brucei rhodesiense as $0.81 \%$ ) (Selby 2010). This prevalence was then monitored in 23 locations at three, nine and 18 months after the beginning of SOS program. A consistent number of cattle was sampled in the 23 sites during the survey ( $>1700$ heads of cattle). The prevalence of $T$. brucei. s.l. dropped from 15 to $3 \%$ three months post intervention, but then returned at nine month and exceeded the baseline at 18 months (Figure 8).

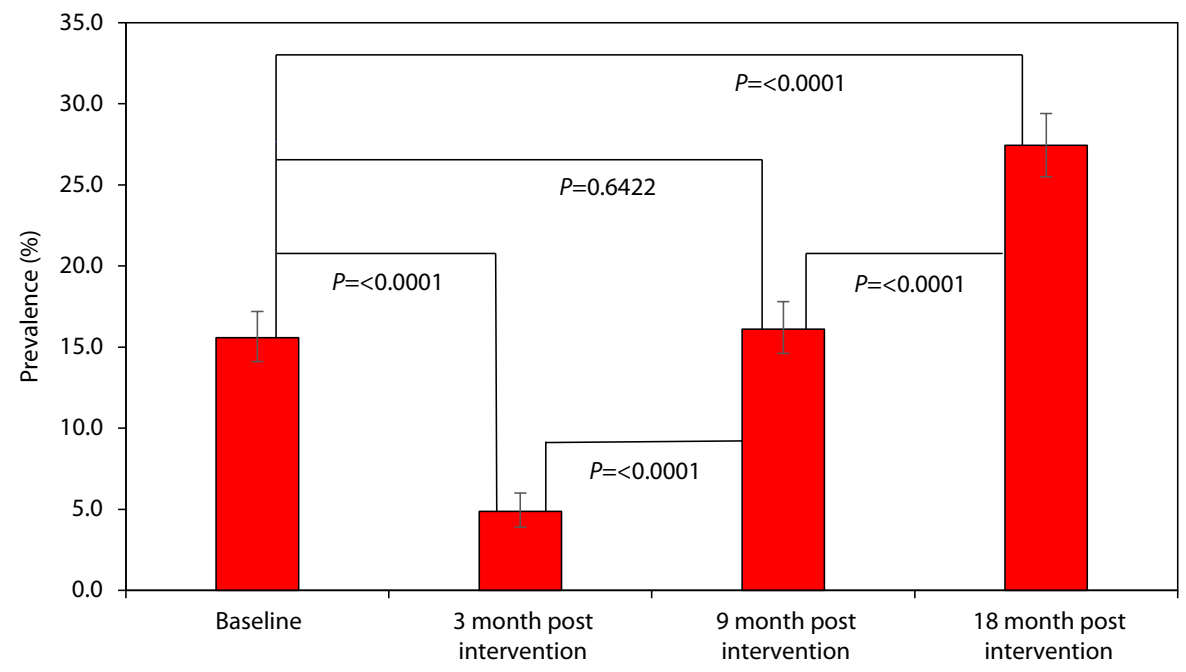

Figure 8. Prevalence of Trypanosoma brucei. s.I. during the SOS operation program (Selby 2010, with permission). 
A similar pattern was observed for T. brucei rhodesiense. This increase in prevalence was attributed to the absence of sustained vector control. Although T. congolense and T. vivax were not directly studied during this program, some data was available before the beginning of the program and highlight that $T$. congolense was the most prevalent trypanosome species with $43 \%$ (of the animals tested; 15/35), followed by T. brucei and T. vivax with 34\% (12/35) and 23\% (8/35) respectively (Cox et al. 2010). This high prevalence indicated that animal trypanosomosis was a serious problem in this country. Therefore, the SOS program has probably lead to similar reduction in prevalence level in T. congolense and T. vivax as suggested by the increase of PCV in cattle.

Interview of the personnel implicated in the SOS program point out several reasons for the disappointing results such as poor community involvement; absence of crush in treatment sites (technical problem) and transport of animals to and from the treatment site (technical problem).

Therefore, the main conclusions of this program are that:

- Treating cattle with a partial spray of pyrethroid associated with an injection of diminazene did not succeed to control the transmission of T. brucei. s.l. in a sustainable way. This result is probably the consequence of a failure in the suppression of $G$. fuscipes fuscipes, the exclusive vector of this trypanosome species in the area. Because resistance to trypanocides has been previously highlighted (Matovu et al. 1997), it could partially explain the result. However, the temporary significant reduction of its prevalence 3 months after mass-treatment shows that T. brucei was at least partially susceptible to diminazene.

- The reduction in pyrethroid treatment cost due to the restricted application and subvention did not ensure good adoption by farmers.

- The technical problems related to the treatment sites and the associated time loss for farmers is probably responsible for the lack of commitment. It proves that even for traditional farmers, 'time is money'. Therefore the technical approaches could significantly be improved.

- The potential for improved control of human sleeping sickness was not considered important enough by the farmers to change their habits, and the apparent efficiency of the insecticide treatment against ticks seemed more important (since both molecules were probably as efficient but amitraze effect was more visible).

\section{ITT in the Loos Islands of Guinea}

This case study is based on two peer reviewed articles (Kagbadouno et al. 2009, 2011). In Guinea, the ministry of health has launched a control program against G. palpalis gambiensis (Diptera, Glossinidae) on three islands: Fotoba, Room and Kassa islands. Before the start of control operations, baseline entomological data were collected and revealed that tsetse populations from the Loos Islands presented a low rate of genetics exchange with flies from the mainland and also between islands (Camara et al. 2006; Solano et al. 2009). Therefore, these apparent isolated populations could be targeted using an area-wide integrated strategy with very low risk of reinvasion.

Different control operations were implemented on each island:

- in Fotoba island, impregnated targets were used at a density of 30 and then 60 targets $/ \mathrm{km}^{2}$;

- in Room island, ground spraying and impregnated targets at a density of 30 targets $/ \mathrm{km}^{2}$ and then 60 targets $/ \mathrm{km}^{2}$ were used;

- in Kassa island, ground spraying, pour-on treatment of pigs (the major species bred on Kassa), fencing of pig pens with impregnated nets and targets were used at a density of 30 and then 60 targets $/ \mathrm{km}^{2}$. 
Before control operation, average tsetse apparent densities were 10.33, 3 and 1.16 flies/trap/day in Kassa, Room and Fotoba, respectively. Three years after the beginning of control operations, tsetse apparent reduction was $100 \%$, indicating that populations have been suppressed. This objective was achieved more or less faster according to measures implemented. The fully integrated strategy on Kassa allowed a very fast reduction of tsetse densities (>98\% within 6 months) and similar results were obtained in Room island (>97\% within 6 months; Figure 9).

The surrounding of pig pens with insecticide mosquito net proved particularly efficient on Kassa. However, on Fotoba using only blue targets set at the density of $30 / \mathrm{km}^{2}$ was not sufficient to reduce tsetse densities below $50 \%$ and target density was therefore increased to $60 / \mathrm{km}^{2}$ after one year of control, which allowed a further reduction of densities to undetectable levels within 6 months. The success of control operation was also due to strong community sensitization and participation. The farmers appeared cooperative and the targets and insecticide mosquito nets were well maintained. However, all these techniques were provided free of charge by the national program, and there was no transfer of the control operations to the farmers. Apart from ground spraying, all other techniques used during this control campaign would be suitable for farmerbased vector control. The main limitation was that targets were considered as public goods, and thus pour-on and insecticide mosquito nets would probably have better chances to be adopted. On these islands, the programme was not sustainable and tsetse densities recovered within a few years after the end of the intervention of national authorities.

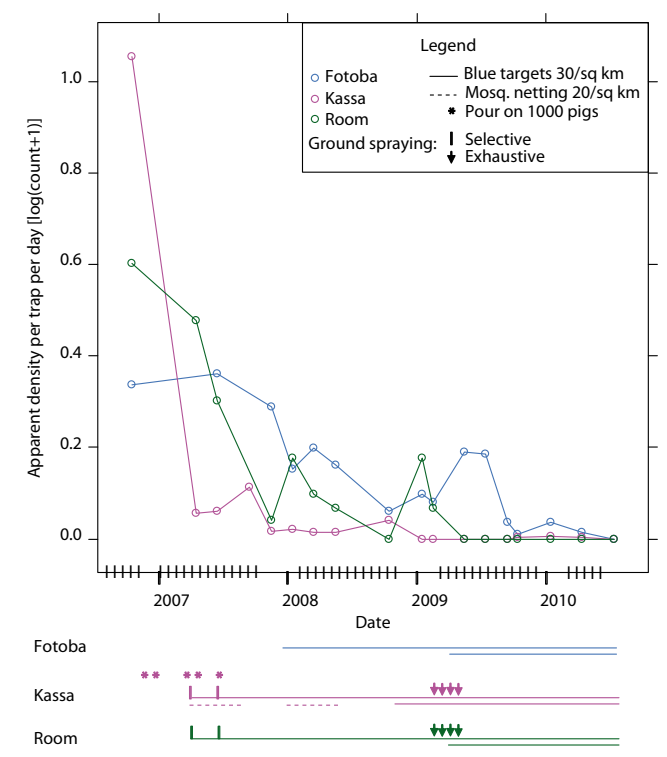

Figure 9. Evolution of tsetse apparent density in Loos Islands according to different control operations (Kagbadouno et al. 2011). 


\section{Use of fencing to protect zero grazing units in western Kenya}

This case study is based on the peer reviewed article from Bauer et al. (2006). The epidemiological cycle is the endemic AAT cycle and the type of cattle rearing system is zero grazing units (situation BIII).

In Kenya, $80 \%$ of 3 million dairy cows are the property of small-scale farmers, of which a large part are confined to zero grazing units with all food and water provided to the animals. The farmers use trypanocidal drugs (diminazene aceturate for therapy or isometamidium chloride for prophylaxis (Bauer et al. 2006) but they still experience trypanosomosis cases (adults mortalities, abortions, stillbirths and a reduction of milk production). Four tsetse species occur in western Kenya: $G$. fuscipes fuscipes, G. pallidipes, Glossina brevipalpis Newstead and Glossina swynertoni Austen at generally low densities (<1 fly per trap per day) (case IIIB of Figure 9), except in Busia District along fringing vegetation, which borders the shores of Lake Victoria, where the densities can be up to 30 per trap per day and in protected areas (Cecchi et al. 2015) (case IIB of Figure 9).

In this context, Bauer et al. (2006) tested the impact of surrounding the zero-grazing units with insecticide-treated mosquito netting to a height of $150 \mathrm{~cm}$ to protect the cows against tsetse and AAT. Fifty-seven randomly selected dairy units were protected with netting and another 42 randomly selected units served as controls. The dominant cattle breeds in the trial were HolsteinFriesian and Ayrshire, which are highly trypano sensitive breeds. The authors used black mosquito netting, impregnated with pyrethroid. At the start of the experiment, all cattle (except those in late-stage pregnancy) were given a therapeutic dose of diminazene aceturate $(3.5 \mathrm{mg} / \mathrm{kg})$ and also during the trial in case of positive diagnosis of AAT or hematocrit below $25 \%$.

The insecticide-treated netting significantly reduced the risk of trypanosome infection in cattle and significantly increased the mean haematocrit (from $27.6 \pm 0.6$ to $29.7 \pm 0.4 ; P<0.05$ ) (Bauer et al. 2006).

Just after setting the net, no more flies were detected in the protected pens and cows remained calm during milking. Farmers also reported a reduction in the number of biting flies, mosquitoes and house flies in their homesteads. However, no differences in milk production between the experimental and control groups were observed despite immediate increases in milk production in individual cows, probably because of other constraints (possibly food).

The authors observed that the netting did not last longer than 2 months due to destruction by strong wind or animal movements. Also many farmers let their cattle graze freely outside the units during the day, despite technical advice, resulting in exposure of animals to habitats suitable for tsetse. Although these problems led to increased AAT incidence in comparison to animals under continuous protection, it still offered an advantage in comparison to a system with no protection.

In conclusion, fencing farms with insecticidal netting can provide efficient and cost effective protection of zero-grazed cattle but its adoption by farmers is not yet warranted. Indeed, it requires modification of the rearing system, especially for free-grazing system and only a minority of the farmers accepted to change their habits. In this case, the benefit was probably lower because the cattle will be only partially protected. Moreover, in this trial, the pens were totally surrounded with insecticide-treated netting, which is sometime not possible in modern farms (see above). Fencing cattle pens will benefit farmers as they will spend less money for trypanocidal drugs and in the same time, it will limit the emergence of drug resistance of the exposed trypanosome populations. 


\section{Conclusions}

The aim of this review was to present the different vector control methods available for farmers in the fight against AAT. To be sustainable, integrated control strategies of trypanosomosis must be context dependent, mainly based on the environment, cattle breed and rearing system. More importantly, the success of farmer based control operation is highly dependent on the costeffectiveness and user friendliness of tools proposed, which will increase their acceptability in the community. As we have seen in different study cases, sensitisation and implication of farmers is one of the key to succeed in control operations. Moreover, new vector control technologies such as tiny targets, impregnated fences or restricted treatments must be more widely adopted by stakeholders and in the same time important effort must be done by veterinarian offices to train community farmer in their use. The availability of new pour-on treatment offering individual protection against AAT is a new promising development. However, this is not a warranty for success and the different tools proposed should be adapted to each particular situation and rearing system in collaboration with the farmers (co-building of innovative control strategy) (see chapter on vector control acceptability in this book). In the context of increasing resistance of trypanosomes to both to diminazene and isometamidium there is a urgent need to implement a control strategy including vector control, particularly in the sites where these resistances have a high prevalence.

\section{References}

Ahmed SK, Rahman AH, Hassan MA, Salih SEM, Paone M and Cecchi G (2016) An atlas of tsetse and bovine trypanosomosis in Sudan. Parasite Vectors 9: 194.

Bauer B, Gitau D, Oloo FP and Karanja SM (2006) Evaluation of a preliminary trial to protect zero-grazed dairy cattle with insecticide-treated mosquito netting in western Kenya. Trop Anim Health Prod 38: 29-34.

Bauer B, Holzgrefe B, Mahama Cl, Baumann MP, Mehlitz D and Clausen PH (2011) Managing tsetse transmitted trypanosomosis by insecticide treated nets - an affordable and sustainable method for resource poor pig farmers in Ghana. PLoS Negl Trop Dis 5: e1343.

Bett B, Randolph TF, Irungu P, Nyamwaro SO, Kitala P, Gathuma J, Grace D, Vale G, Hargrove J and McDermott J (2010) Field trial of a synthetic tsetse-repellent technology developed for the control of bovine trypanosomosis in Kenya. Prev Vet Med 97: 220-227.

Bouyer F, Bouyer J, Seck MT, Sall B, Dicko AH, Lancelot R and Chia E (2015) Importance of vector-borne infections in different production systems: bovine trypanosomosis and the innovation dynamics of livestock producers in Senegal. Rev Sci Tech Off Int Epiz 34: 213-225.

Bouyer F, Hamadou S, Adakal H, Lancelot R, Stachurski F, Belem AMG and Bouyer J (2011a) Restricted application of insecticides: a promising tsetse control technique, but what do the farmers think of it? PLoS Negl Trop Dis 5: e1276.

Bouyer J and Marois E (2018) Genetic control of vectors. In: Garros C, Bouyer J, Takken W and Smallegange RC (eds.) Pests and vector-borne diseases in the livestock industry. Ecology and Control of Vector-borne diseases. Vol. 5. Wageningen Academic Publishers, Wageningen, the Netherlands, pp. 435-451.

Bouyer J, Bouyer F, Donadeu M, Rowan T and Napier G (2013) Community- and farmer-based management of animal African trypanosomosis in cattle. Trends Parasitol 29: 519-522.

Bouyer J, Grimaud Y, Pannequin M, Esnault $O$ and Desquesnes M (2011b) Importance épidémiologique et contrôle des stomoxes à la Réunion. Bull Epidémiol 43: 53-58.

Bouyer J, Solano P, Cuisance D, Itard J, Frézil J-L and Authié E (2010) Trypanosomosis: control methods. In: Lefèvre P-C, Blancou J, Chermette R and Uilenberg G (eds.) Infectious and parasitic diseases of livestock. Éditions Lavoisier (Tec \& Doc), Paris, France, pp. 1936-1943.

Bouyer J, Stachurski F, Gouro AS and Lancelot R (2008) On-station cattle insecticide treatment against tsetse flies using a footbath. Rev Elev Méd Vét Pays Trop 61: 153-160. 
Bouyer J, Stachurski F, Gouro AS and Lancelot R (2009) Control of bovine trypanosomosis by restricted application of insecticides to cattle using footbaths. Vet Parasitol 161: 187-193.

Bouyer J, Stachurski F, Kaboré I, Bauer B and Lancelot R (2007) Tsetse control in cattle from pyrethroid footbaths. Prev Vet Med 78: 223-238.

Camara M, Caro-Riaño H, Ravel S, Dujardin J-P, Hervouet J-P, De Meeüs T, Kagbadouno MS, Bouyer J and Solano P (2006) Genetic and morphometric evidence for population isolation of Glossina palpalis gambiensis (Diptera: Glossinidae) on the Loos Islands, Guinea. J Med Entomol 43: 853-860.

Cecchi G, Paone M, Argilés Herrero R, Vreysen MJB and Mattioli RC (2015) Developing a continental atlas of the distribution and trypanosomal infection of tsetse flies (Glossina species). Parasite Vectors 8: 284.

Cox AP, Tosas O, Tilley A, Picozzi K, Coleman P, Hide G and Welburn SC (2010) Constraints to estimating the prevalence of trypanosome infections in East African zebu cattle. Parasite Vectors 3: 82.

Cuisance D, Cailton P, Kota Guinza A, Ndokoué F, Pounekrozou E and Demba D (1991) Lutte contre Glossina fuscipes fuscipes par piégeage chez les éleveurs Mbororo de République Centrafricaine. Rev Elev Méd Vét Pays Trop 44: 81-89.

Dayo GK, Gautier M, Berthier D, Poivey JP, Sidibe I, Bengaly Z, Eggen A, Boichard D and Thevenon S (2012) Association studies in QTL regions linked to bovine trypanotolerance in a West African crossbred population. Anim Genet 43: 123-132.

De Garine-Wichatitsky M, Cheke RA and Lazaro D (2001) Effects of tsetse targets on mammals and birds in Kasungu National Park, Malawi. Biodivers Conserv 10: 869-891.

Eisler MC, Torr SJ, Coleman PG, Machila N and Morton JF (2003) Integrated control of vector-borne diseases of livestock - pyrethroids: panacea or poison? Trends Parasitol 19: 341-345.

Esterhuizen J, Rayaisse JB, Tirados I, Mpiana S, Solano P, Vale GA, Lehane MJ and Torr SJ (2011) Improving the cost-effectiveness of visual devices for the control of riverine tsetse flies, the major vectors of human African Trypanosomiasis. PLoS Negl Trop Dis 5: e1257.

Faulde MK and Nehring O (2012) Synergistic insecticidal and repellent effects of combined pyrethroid and repellentimpregnated bed nets using a novel long-lasting polymer-coating multi-layer technique. Parasitol Res 111: 755-765.

Geerts S, Holmes PH, Eisler MC and Diall O (2001) African bovine trypanosomiasis: the problem of drug resistance. Trends Parasitol 17: 25-28.

Gimonneau G, Alioum Y, Abdoulmoumini M, Zoli A, Cene B, Adakal H and Bouyer J (2016) Insecticide and repellent mixture pour-on protects cattle against animal trypanosomosis. PLoS Negl Trop Dis 10: e0005248.

Hargrove JW (2003) Optimized simulation of the control of tsetse flies Gloissina pallidipes and G-m. morsitans (Diptera : Glossinidae) using odour-baited targets in Zimbabwe. Bul Entomol Res 93: 19-29.

Hargrove JW, Omolo S, Msalilwa JSI and Fox B (2000) Insecticide-treated cattle for tsetse control: the power and the problems. Med Vet Ent 14: 123-130.

Hargrove JW, Torr SJ and Kindness HM (2003) Insecticide-treated cattle against tsetse (Diptera: Glossinidae): what governs success? Bull Entomol Res 93: 203-217.

Itard J, Cuisance D and Tacher G (2003) Trypanosomoses: historique - répartition géographique. In: Lefèvre P-C, Blancou $J$ and Chermette R (eds.) Principales maladies infectieuses et parasitaires du bétail. Europe et Régions Chaudes, Lavoisier, Paris, France, pp. 1607-1615.

Kabayo JP (2002) Aiming to eliminate tsetse from Africa. Trends Parasitol 18: 473-475.

Kagbadouno M, Camara M, Bouyer J, Courtin F, Onikoyamou M, Schofield C and Solano P (2011) Progress towards the eradication of tsetse from the Loos Islands, Guinea. Parasite Vectors 4: 18.

Kagbadouno M, Camara M, Bouyer J, Hervouet JP, Courtin F, Jamonneau V, Morifaso O, Kaba D and Solano P (2009) Tsetse elimination: its interest and feasibility in the historical sleeping sickness focus of Loos Islands, Guinea. Parasite 16: 29-35.

Kamuanga M, Sigue H, Swallow B, Bauer B and d'leteren G (2001a) Farmers' perceptions of the impacts of tsetse and trypanosomosis control on livestock production: evidence from southern Burkina Faso. Trop Anim Health Prod 33: 141-153.

Kamuanga M, Swallow BM, Sigué H and Bauer B (2001b) Evaluating contingent and actual contributions to a local public good: tsetse control in the Yale agro-pastoral zone, Burkina Faso. Ecological Economics 39: 115-130. 
Kernaghan RJ and Johnston MRL (1962) A method of determining insecticide persistence in tsetse fly control operations. Bul World Health Org 26: 139-141.

Kgori PM, Modo S and Torr SJ (2006) The use of aerial spraying to eliminate tsetse from the Okavango Delta of Botswana. Acta Trop 99: 184-199.

Knols BGJ, Willemse L, Flint S, and Mate A (1993). A trial to control the tsetse fly, Glossina morsitans centralis, with low densities of odour-baited targets in west Zambia. Med Vet Entomol 7: 161-169.

Laveissière C, Couret D and Kiénou J-P (1980) Lutte contre les glossines riveraines à l'aide de pièges biconiques imprégnés d'insecticides, en zone de savane humide: 1. Description du milieu, du matériel et de la méthode. Cahiers ORSTOM Série Entomol Méd Parasitol 18: 201-207.

Lindh JM, Torr SJ, Vale GA and Lehane MJ (2009) Improving the cost-effectiveness of artificial visual baits for controlling the tsetse fly Glossina fuscipes fuscipes. PLoS Negl Trop Dis 3: e474.

Maia M, Clausen PH, Mehlitz D, Garms R and Bauer B (2010) Protection of confined cattle against biting and nuisance flies (Muscidae: Diptera) with insecticide-treated nets in the Ghanaian forest zone at Kumasi. Parasitol Res 106: 1307-1313.

Maia MF, Abonuusum A, Lorenz LM, Clausen PH, Bauer B, Garms R and Kruppa T (2012) The effect of deltamethrintreated net fencing around cattle enclosures on outdoor-biting mosquitoes in Kumasi, Ghana. PLoS ONE 7: e45794.

Matovu E, Iten M, Enyaru JC, Schmid C, Lubega GW, Brun Rand Kaminsky R (1997) Susceptibility of Ugandan Trypanosoma brucei rhodesiense isolated from man and animal reservoirs to diminazene, isometamidium and melarsoprol. Trop Med \& Intern Health 2: 13-18.

Morton J (2010) The innovation trajectory of sleeping sickness control in Uganda. Research knowledge in its context. Research Into Use Programme, UK Department for International Development, London, UK, 43 pp. Available at: https://tinyurl.com/y74lhd6j.

Ndeledje N, Bouyer J, Stachurski F, Grimaud P, Belem AMG, Mbaïndingatoloum FM, Bengaly Z, Cecchi G and Lancelot R (2013) Treating cattle to protect people? Impact of footbath insecticide treatment on tsetse density in Chad. PLoS ONE 8: e67580.

Pagabeleguem S, Sangaré M, Bengaly Z, Akoudjin M, Belem AMG and Bouyer J (2012) Climate, cattle rearing systems and African animal trypanosomosis risk in Burkina Faso. PLoS ONE 7: e49762.

Rayaisse JB, Esterhuizen J, Tirados I, Kaba D, Salou E, Diarrassouba A, Vale GA, Lehane MJ, Torr SJ and Solano P (2011) Towards an optimal design of target for tsetse control: comparisons of novel targets for the control of palpalis group tsetse in West Africa. PLoS Negl Trop Dis 5: e1332.

Rayaisse JB, Tirados I, Kaba D, Dewhirst SY, Logan JG, Diarrassouba A, Salou E, Omolo MO, Solano P, Lehane MJ, Pickett JA, Vale GA, Torr SJ and Esterhuizen J (2010) Prospects for the development of odour baits to control the tsetse flies Glossina tachinoides and G. palpalis s.l. PLoS Negl Trop Dis 4: e632.

Selby R (2010) Limiting the northerly advance of Trypanosoma brucei rhodesiense in post conflict Uganda. PhD thesis, University of Edinburgh, Edinburgh, UK.

Shaw APM, Tirados I, Mangwiro CTN, Esterhuizen J, Lehane MJ, Torr SJ and Kovacic V (2015) Costs of using 'Tiny Targets' to control Glossina fuscipes fuscipes, a vector of Gambiense sleeping sickness in Arua district of Uganda. PLoS Negl Trop Dis 9: e0003624.

Shaw APM, Torr SJ, Waiswa C, Cecchi G, Wint GRW, Mattioli RC and Robinson TP (2013) Estimating the costs of tsetse control options: an example for Uganda. Prev Vet Med 110: 290-303.

Solano P, Ravel S, Bouyer J, Camara M, Kagbadouno MS, Dyer N, Gardes L, Herault D, Donnelly MJ and De Meeûs T (2009) The population structure of Glossina palpalis gambiensis from island and continental locations in coastal Guinea. PLoS Negl Trop Dis 3: e392.

Stachurski F (2006) Attachment kinetics of the adult tick Amblyomma variegatum to cattle. Med Vet Entomol 20:317-324.

Stachurski F and Lancelot R (2006) Footbath acaricide treatment to control cattle infestation by the tick Amblyomma variegatum. Med Vet Entomol 20: 402-412.

Tadesse A, Hadgu E, Mekbib B, Abebe R and Mekuria S (2011) Mechanically transmitted bovine Trypanosomosis in Tselemty Woreda, western Tigray, northern Ethiopia. Agri J 6: 10-13. 
Torr SJ (1985) The susceptibility of Glossina pallidipes Austen (Diptera, Glossinidae) to insecticide deposits on targets. Bul Entomol Res 75: 451-458.

Torr SJ, Chamisa A, Vale GA, Lehane MJ and Lindh JM (2011) Responses of tsetse flies, Glossina morsitans morsitans and Glossina pallidipes, to baits of various size. Med Vet Entomol 25: 365-369.

Torr SJ and Hargrove JW (1998) Factors affecting the landing and feeding responses of the tsetse fly Glossina pallidipes to a stationary ox. Med Vet Entomol 12: 196-207.

Torr SJ, Maudlin I and Vale GA (2007) Less is more: restricted application of insecticide to cattle to improve the cost and efficacy of tsetse control. Med Vet Entomol 21: 53-64.

Vale G and Torr S (2004) Development of bait technology to control tsetse. In: Maudlin I, Holmes P and Miles M (eds.) The Trypanosomiases. CABI Publishing, Wallingford, pp. 509-523.

Vale GA (1980) Field studies of the responses of tsetse flies (Glossinidae) and other diptera to carbon-dioxide, acetone and other chemicals. Bul Entomol Res 70: 563-570.

Vale GA, Grant IF, Dewhurst CF and Aigreau D (2004) Biological and chemical assays of pyrethroids in cattle dung. Bul Entomol Res 94: 273-282.

Vale GA, Mutika G and Lovemore DF (1999) Insecticide-treated cattle for controlling tsetse flies (Diptera: Glossinidae): some questions answered, many posed. Bul Entomol Res 89: 569-578.

Van den Bossche P, De la Rocque S, Hendrickx G and Bouyer J (2010) A changing environment and the epidemiology of tsetse-transmitted livestock trypanosomiasis. Trends Parasitol 26: 236-243.

Van den Bossche P and Delespaux V (2011) Options for the control of tsetse-transmitted livestock trypanosomosis. An epidemiological perspective. Vet Parasitol 181: 37-42.

Vreysen MJB, Robinson AS and Hendrichs J (2007) Area-wide control of insect pests. From research to field implementation. Springer, Dordrecht, the Netherlands.

Vreysen MJB, Saleh KM, Ali MY, Abdulla AM, Zhu ZR, Juma KG, Dyck VA, Msangi AR, Mkonyi PA and Feldmann HU (2000) Glossina austeni (Diptera: Glossinidae) eradicated on the Island of Unguja, Zanzibar, using the sterile insect technique. J Econ Entomol 93: 123-135.

Vreysen MJB, Seck MT, Sall B and Bouyer J (2013) Tsetse flies: their biology and control using area-wide integrated pest management approaches. J Invertebr Pathol 112, Suppl. 1: S15-S25.

Willemse LPM (1991) A trial of odour baited targets to control the tsetse fly, Glossina morsitans centralis (Diptera: Glossinidae) in west Zambia. Bull Entomol Res 81: 351-357. 


\title{
7. Prevention and control of tick-borne anaplasmosis, cowdriosis and babesiosis in the cattle industry
}

\author{
Martin Pfeffer ${ }^{*}$, Nina Król and Anna Obiegala \\ Institute of Animal Hygiene and Veterinary Public Health, Veterinary Faculty, University of Leipzig, An \\ den Tierkliniken 1, 04103 Leipzig, Germany; pfeffer@vetmed.uni-leipzig.de
}

\begin{abstract}
Ticks have a tremendous impact on cattle production worldwide. Economic losses caused by tick infestation are due to direct effects like less milk production and less gain in body weight, costs for application of control measures, which in most cases is based on the application of acaricides, and the losses due to tick-borne diseases. A variety of tick-borne pathogens are responsible for significant losses due to morbidity and mortality, veterinary and diagnostic costs, vaccines and trade restrictions. In this chapter we describe tick-borne diseases of cattle caused by three different groups of pathogens in order to emphasize the challenges currently faced in their control. Antigenic and genetic heterogeneity of each of these pathogens is challenging the attempts of specifically controlling the related disease, and precise diagnostic or vaccine development is hampered. As different tick species are involved as the main transmitting vectors, the development of tick vaccines is likewise challenging. With the exception of the Bm86 gut antigen used against the two main Rhipicephalus tick species transmitting Babesia bovis and Babesia bigemina, this strategy is thus far not working with any other tick species. Currently the best protection of the cattle industry against tick-borne diseases is a combination of various measures, which have to be adapted to the particular situation and to consider all possibilities including chemical tick control, grazing management, and breeding for host resistance to ticks.
\end{abstract}

Keywords: ticks, Anaplasma, Babesia, Ehrlichia, age-dependent disease, control

\section{Bovine anaplasmosis and tick-borne fever}

\section{Aetiology}

Bovine anaplasmosis (BA) is an infectious but non-contagious tick-borne disease caused by obligate intracellular bacteria of blood cells of the genus Anaplasma, family Anaplasmataceae, order Rickettsiales, phylum Proteobacteria (Aubry and Geale 2011; Battilani et al. 2017; Dumler et al. 2001; Kocan et al. 2015). The aetiological agent of bovine anaplasmosis is Anaplasma marginale (an intraerythrocytic pathogen) which is responsible for the majority of outbreaks of clinical disease in cattle but does not cause disease in humans. A second less pathogenic species, the intraerythrocytic Anaplasma centrale, causes benign infections. Other organisms, like Anaplasma phagocytophilum (an intragranulocytic bacterium) and Anaplasma bovis (infecting monocytes) infect cattle but clinical cases are very rare. A. phagocytophilum causes tick-borne fever in domestic and wild ruminants only in Europe (Hoar et al. 2008).

\section{Epidemiology and transmission}

Bovine anaplasmoses caused by $A$. marginale and $A$. centrale occur worldwide, especially in subtropical and tropical regions. BA is found in the Americas, Europe, Asia, Africa and Australia (Kocan et al. 2010; McCallon 1973; Rar and Golovljova 2011). Climate changes, affecting movement 
of vector populations, as well as the transportation of cattle from endemic to non-endemic areas influence the spread of the disease (Kocan et al. 2010).

The life cycle of Anaplasma spp. involves ixodid ticks and vertebrates (Rikihisa 1991). A. marginale is well known to infect domestic ruminants but also wild ruminants such as water buffalo (Bubalus bubalis), American bison (Bison bison) or mule deer (Odocoileus hemmionus) (Aubry and Geale 2011). A. centrale was previously thought to infect only cattle but has recently been considered to infect wild ruminants as well (Hosseini-Vasoukolaei et al. 2014; Khumalo et al. 2016). Apart from ruminants, A. bovis was detected among other mammals such as dogs, cats, raccoons, or cottontail rabbits (Atif 2016; Uilenberg 1993). Finally, hosts for A. phagocytophilum - which is widely distributed in Europe, Asia and North America - include mammals (carnivores, ruminants, rodents, insectivores and humans), birds and reptiles (Stuen et al. 2013).

There are over 20 tick species mainly of the Rhipicephalus and Dermacentor genera, e.g. Rhipicephalus microplus (Canestrini) which serve as vector for A. marginale (Dikmans 1950; Ewing 1981; Kocan et al. 2003). The pathogen is transmitted transstadially but transovarial transmission has not been demonstrated (Kocan et al. 1981; Stich et al. 1989). Consequently, a mammalian host is needed as reservoir for A. marginale. Cattle that overcome the acute infection become persistently infected, thus may represent such a reservoir (Kocan et al. 2003). Mechanical transmission seems to be important especially where biological carriers do not occur, by bloodcontaminated fomites or by insects (e.g. Tabanus spp. and Stomoxys spp.) (Dikmans 1950; Ewing 1981; Hawkins et al. 1982). Transplacental transmission of $A$. marginale was also documented and persistently infected calves may serve as an additional reservoir host for $A$. marginale (Kocan et al. 2015). For A. centrale only one tick species has been proven as vector - Rhipicephalus simus (Koch) (Potgieter and Van Rensburg 1987). The risk of transmission of $A$. centrale by $R$. microplus is limited, that is why $A$. centrale is used as a live vaccine in $R$. microplus infested areas. However, DNA of this pathogen has also been found in engorged and questing Haemaphysalis punctata Canestrini \& Fanzago and Amblyomma ticks collected from cattle and sheep (Palomar et al. 2015; Teshale et al. 2015). A. bovis is suspected to be transmitted by several tick species, e.g. Rhipicephalus sanguineus (Latreille) sensu lato, Rhipicephalus appendiculatus, Amblyomma variegatum (Fabricius), Hyalomma spp. and Haemaphysalis spp. (Harrus et al. 2011). In the case of A. phagocytophilum, the main vectors are Ixodes ricinus in Europe, Ixodes persulcatus (Schulze) in Asia, Ixodes scapularis Say, Ixodes pacificus Cooley \& Kohls and Ixodes spinipalpis Hadwen \& Nuttall, in North America (Woldehiwet 2010). Additionally, A. phagocytophilum has been detected in other tick species but their role as vector is unclear (i.e. Dermacentor reticulatus Fabricius, Dermacentor variabilis Say, Dermacentor occidentalis, Amblyomma americanum (Linnaeus), or Haemaphysalis concinna Koch) (Clark 2012; Holden et al. 2003; Paulauskas et al. 2012, Tomanovic et al. 2013). A. phagocytophilum is transmitted transstadially in Ixodes ticks (Ogden et al. 1998; Stuen et al. 2013) but transovarial transmission of $A$. phagocytophilum has been observed only in Dermacentor albipictus Packard ticks. Thus mammalian hosts are important as reservoir hosts for $A$. phagocytophilum as well (Baldridge et al. 2009).

\section{Ecology and genetics}

A. phagocytophilum is a single species but it is occurring as many different genetic variants within ecological niches. When in 2001 the reorganization of the genera within the Rickettsiales took place (Dumler et al. 2001), three species, i.e. Ehrlichia equi, Ehrlichia phagocytophilum, and the agent of the human granulocytic ehrlichiosis were merged to the species A. phagocytophilum. The rationale behind this restructuring was the genetic similarities (mainly of the 16S rRNA gene). The 
past 15 years has brought an enormous gain in genetic sequencing information, mainly because of the tearing pace with which next generation sequencing platforms became available and more cost efficient (Dugat et al. 2016a). This progression demonstrated a rather diverse genetic heterogeneity, which is described for rodent-associated $A$. phagocytophilum strains (Rosso et al. 2017), a cluster consisting of sequence types found in red deer, cattle and sheep, while roe deer, shrew and rodent-associated sequence types are not found in A. phagocytophilum strains recovered from humans, domestic or farm animals (Huhn et al. 2014). Using a different set of genes we found a similar situation in a rather small farm in Germany where in a 15 ha area roe deerassociated sequence types were found which were distinct from the types found in cattle grazing at the same pastures (Figure 1; Silaghi et al. 2017a). Besides the great gain in the knowledge of distinct genetic lineages and ecotypes differing in pathogenicity and host predilection (Battilani et al. 2017), there is a great challenge to learn about the different transmission cycles and pathogenic mechanisms in various mammalian hosts (Dugat et al. 2015; Stephenson and Foley 2016). It would be awarding when either multi locus sequence typing or genome wide single nucleotide polymorphism analyses will get to the point where genetic markers are identified that are associated with particular hosts or a particular pathogenicity (Dugat et al. 2016b).

Host association of found A. phagocytophilum 16S rRNA gene variants:

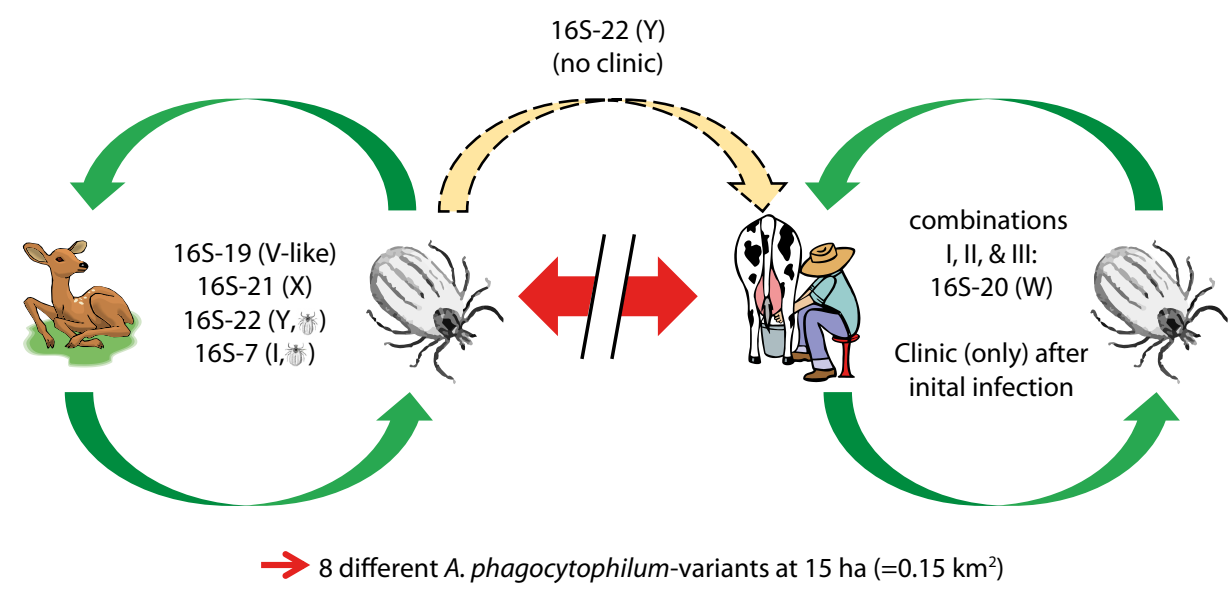

Figure 1. (Co-)circulation of different gene variants of Anaplasma phagocytophilum in a herd in Germany. Genetic characterization is based on partial 165 rRNA-, msp2-, msp4-, and groEL-gene sequences (Silaghi et al. 2017a). Although the herd is grazing only on very small pastures, cattle were found to be infected only with A. phagocytophilum variants of a particular 165 rRNA gene variant ('20') in various combinations with the other genes. There was only one finding of a 165 rRNA 22-variant detected in a cow but this animal did not show typical clinical signs of tick-borne fever. This picture summarizes the distinct circulation of cattle-associated (and sickening) A. phagocytophilum variants with other wildlife-associated A. phagocytophilum variants. 


\section{Clinical signs and diagnosis}

\section{Diagnosis of tick-borne fever}

Recently, guidelines for the direct detection of Anaplasma spp. in diagnosis and epidemiological studies have been reviewed by a consortium of experts in this field (Silaghi et al. 2017b). Haematological and biochemical parameters show anaemia, leucopenia, thrombocytopenia, and increase of aspartate aminotransferase and alanine aminotransferase enzymes but they are only indicative for an infection. Blue intracytoplasmic inclusion microcolonies (morulae) may be seen in granulocytes and monocytes in the acute phase of disease after Giemsa or Romanowsky staining of blood smears (Atif 2015). To confirm the infection, serological tests are commonly used, such as indirect fluorescent antibody (IFA), enzyme-linked immunosorbent assay (ELISA), complement fixation tests, and counter-current immunoelectrophoresis tests (Bakken and Dumler 2008). Confirmatory molecular biological methods such as conventional, nested, and real-time PCRs are established for the detection of partial sequences of $16 \mathrm{~S}$ rRNA, msp4, groEL, ankA and the p44 genes (Alberti et al. 2005; Chen et al. 1994; Courtney et al. 2004; Sainz et al. 2015; Silaghi et al. 2017b). PCR may be performed using DNA extracted from blood and from spleen samples. Splenomegaly accompanied with subscapular bleeding is the most important post-mortem finding in roe deer, sheep, and reindeer (Stuen et al. 2003).

\section{Diagnosis of bovine anaplasmosis}

It is possible to find A. marginale in Giemsa-stained blood smears during the acute phase of the disease. However, this method is not suitable for the detection of pre-symptomatic or carrier animals. This is why additional serologic analysis of antibodies with confirmation by molecular detection should always be considered. Several serological tests have been employed such as complement fixation tests, the capillary agglutination assay, the card agglutination test (CAT), IFA and ELISA. Currently, the most popular tests used are the cELISA and the CAT (OIE 2017a). PCR methods may be performed additionally. As mentioned earlier, recovered cattle are considered persistently infected as well as transplacentally infected calves. Here the latter methods are useful to identify carrier animals (Silaghi et al. 2017b).

\section{Clinical tick-borne fever}

Though tick-borne fever may be transmitted to wild as well as to domestic ruminants, clinical signs are mostly observed in domestic animals as sheep and cows. Clinical signs in roe deer and reindeer are rather seldom (Atif 2015). Clinical signs may be reduced weight gain, fever, sudden drop in milk production, still birth, low fertility, anorexia, coughing, abortions and dullness. Usually the disease is easily treatable with antibiotics. The immunosuppressive effect of the infection itself (by reducing the number of immunologically active granulocytes) may lead to secondary infections (Woldehiwet 2010). It may be self-limiting as well and animals normally recover within 14 days. Simultaneous secondary infections as tick pyemia, pasteurellosis, and septicemic listeriosis may lead to severe symptoms. Lethal outcomes of tick-borne fever are very rarely reported (Atif 2015).

\section{Clinical bovine anaplasmosis}

A. marginale usually causes subacute to chronic cases of bovine anaplasmosis. Acute cases are sometimes described in old cattle, especially in dairy cows of non-indigenous breeds. Acute cases display unspecific symptoms such as anorexia, fever, diarrhoea, swollen preparotid lymph nodes, 
incoordination, severe anaemia, jaundice, pale mucous membranes, brownish urine, and mortality without hemoglobinaemia. Fever is indicative for the onset of disease with a variable duration of 1 to 44 days (Aubry and Gaele 2011).

\section{Prevention and control}

\section{Vector control and prevention of iatrogenic transmission}

Tick control is very expensive due to repeated applications which are necessary to maintain acaricide protection. Repeated application of acaricides may lead to resistant tick populations, which could lead to a susceptible population of cattle in endemic areas (Kocan et al. 2000). And, even though a transmission via ticks could be ruled out due to this treatment, iatrogenic transmission would still be possible. To avoid iatrogenic transmission, proper hygienic usage of instruments and equipment is indispensable. Another option would be to keep the cattle indoors so that they would not have access to fields where ticks are present and able to complete their life cycle (Aubry and Gaele 2011). This option is, however, not possible for all sectors of cattle production (Jonsson et al. 1998). In the dairy cattle industry, only few acaricides are licensed and an economic drawback is the waiting time for milk (to ensure complete breakdown of the acaricides), making these products only suitable for heifers up to the date they give birth to their calves. The most cost efficient way to prevent economical loss due to A. phagocytophilum infection is a proper grazing management. The aim is to get the heifers out on the pastures and to get them infected before they give birth. This does not help to prevent subsequent infection(s) with $A$. phagocytophilum with the identical or another genetic makeup, but the cows do not get sick, do not suffer and milk production is not affected (Silaghi et al. 2017a). It is different with A. marginale infections as the aim here should be an identification and elimination of persistently infected animals in order to remediate the herd and then to prevent reinfection by the strategies listed above.

\section{Vaccines}

\section{Bovine anaplasmosis}

Vaccines are a cost-effective way to control and prevent bovine anaplasmosis. Though vaccines exist against bovine anaplasmosis, none of them prevents from persistent infection and cattle become reservoir for A. marginale (Kocan et al. 2003). This is why sterile immunity vaccines should be developed. However this is really hard to accomplish because there are a lot of antigenically different strains circulating.

\section{Tick-borne fever}

The main problem associated with the development of effective vaccine against $A$. phagocytophilum is the lack of cross-protection studies, selection of suitable conserved antigens and antigenic variation against diverse genotypes (Stuen et al. 2013). The identification of three invasin proteins OmpA, Asp14, and AipA is involved in the development of suitable vaccines (Seidman et al. 2015). Furthermore, anti-tick vaccines would be a solution not only against Anaplasma spp. but also against other tick-borne agents such as Babesia spp. (see below). These vaccines would be sustainable and would prevent local skin lesions caused by high tick burden (De la Fuente et al. 2006, 2011; Graf et al. 2004). Understanding vector-pathogen interactions and immunization of animal reservoirs as well as of high-risk animals would be important for the control of tick and tick-borne pathogens. 


\section{Cowdriosis - an infection with Ehrlichia ruminantium in livestock}

\section{Aetiology}

Cowdriosis is a notifiable disease to the World Organisation for Animal Health (OIE 2017b). In 1925 the causative agent was first described as Rickettsia ruminantium (Cowdry 1925a,b). Later it was renamed to Cowdria ruminantium (Moshkovski 1947) from which the name cowdriosis arose. Phylogenetic studies from the early 1990s revealed the evolutionary relationships of the pathogen to the family Anaplasmataceae so the pathogen was reclassified and finally called Ehrlichia ruminantium (Dumler et al. 2001). This pathogen is tick-borne and transmitted by Amblyomma ticks. Further, it invades endothelial cells in cattle, sheep, goats and wild ruminants with a frequently fatal outcome. Economically speaking, cowdriosis which is often called 'heartwater', is a serious problem especially in sub-Saharan Africa and its offshore islands. It is also known to occur in the Caribbean islands and is of economic concern for the farmers as the disease is highly lethal for their livestock. In Africa and the Caribbean, non-native livestock are more susceptible to infection than traditional stock breeds which are more resistant (Simpson et al. 1987). This leads to the problem that farmers in endemic areas do not have a chance to improve their herds to modern high-yielding breeds (Allsopp 2015). Most farmers are poor because of several infectious diseases affecting their livestock and therefore are not able or willing to pay for diagnosis methods concerning heartwater. Because of the lack of diagnostic, it is far not possible to estimate the real economic damage cowdriosis is causing. To date, only a few studies quantified the animal production loss with 48 million US dollars annually solely in South Africa (Minjauw et al. 2000; Mukhebi et al. 1999).

\section{Epidemiology and transmission}

E. ruminantium originates from southern Africa (Allsopp et al. 2005). To date, over fifteen different natural reservoir hosts are known. Most of them are wild-living ruminants such as giraffes, steenboks and African buffalos (Allsopp 2010). In Africa, heartwater is endemic in livestock wherever the main vector Amblyomma spp. is prevalent. It is known that all Amblyomma ticks may transmit the pathogen. However, Amblyomma hebraeum Koch and A. variegatum are the most important species involved in the live cycle of E. ruminantium (Bezuidenhout 1987). Amblyomma spp. are ticks with three developmental stages. Each stadium needs to feed on a host and may become infected with E. ruminantium two to four days after feeding (Bezuidenhout 1988; Camus and Barré 1992). Amblyomma males, which feed several times on different hosts, may transmit the pathogen intra-stadially by feeding first on an infected reservoir and then on a susceptible host. Transstadial transmission from nymphs developing to adults occurs. However, transovarial transmission seems unlikely. In general, infected ticks do not lose their infectivity after feeding on a host. However, larvae and nymphs just become infectious in the following instar (Bezuidenhout 1987). A. variegatum was introduced into the Caribbean through heartwater infected cattle (Maillard and Maillard 1998). Endemic areas are also present on islands of Madagascar, Mauritius, La Reunion, Grande Comore and São Tomé (Du Plessis et al. 1989; Provost and Bezuidenhout 1987). The African cattle egret (Bubulcus ibis) is actively involved in the transport of $A$. variegatum. $B$. ibis migrates in America and therefore $A$. variegatum and thus E. ruminantium may also spread to another continent. The infection rate of E. ruminantium in cattle is depending on the vector which, in turn, is depending on the weather conditions, mainly humidity and temperature. The infection rate in ticks varies in endemic areas between 12-41\% (Esemu et al. 2013; Gueye et al. 1993; Molia et al. 2008; Peter et al. 1999). Animals treated for heartwater may retain the pathogen for up to one year and spread it to their vectors. 


\section{Clinical signs and diagnosis}

\section{Ante-mortem diagnosis}

There are two practical possibilities to diagnose heartwater; firstly via serological and secondly via molecular biologic testing. Current serological tests are based on the detection of antibodies against the immune-dominant $E$. ruminantium outer membrane protein MAP1 via an ELISA (Van Vliet et al. 1995). However, false positive and false negative results are possible. PCRs were designed for the purpose of identifying E. ruminantium. The target region is consisting of sequences from two overlapping genes within the PCS20 region. Conventional, nested, quantitative real-time and loop-mediated isothermal amplification (LAMP) PCR approaches have been developed. While LAMP is the less sensitive test, the real-time approach yields the highest sensitivity (Martinez et al. 2004; Nakao et al. 2010; Steyn et al. 2008; Van Heerden et al. 2004).

\section{Pathology and post-mortem pathology}

Because of logistic problems and high costs, a full necropsy is rarely performed. Only animals of high personal and financial value are examined. Classic post-mortem symptoms are hydrothorax and hydropericardium, oedema of lungs and brain. All signs may be absent but Ehrlichia colonies are nevertheless present in the cytoplasm of brain endothelial cells but only in animals which were not previously treated with tetracycline (Allsopp 2015).

\section{Clinic}

After the inoculation of E. ruminantium to a susceptible host, the incubation period is usually less than two weeks long. Clinical signs are diverse from peracute to inapparent depending on the livestock breed. Angora and Boer goats are highly susceptible and thus the disease has mostly a peracute clinical course. A vast variety of clinical signs makes it difficult to diagnose the disease (Allsopp 2015). The classic clinical signs are loss of appetite, high body temperature, convulsions, anorexia, recumbency and death (Du Plessis et al. 1983).

\section{Prevention and control}

\section{Tick control}

In order to prevent heartwater in livestock, it is important to protect animals from their high and frequent tick burden by the use of acaricides. Either applied directly on individual cattle or via foot bath for a group of animals. However, intensive tick control needs intensive and efficient management to succeed. Earlier it was regularly used but two upcoming factors led to stop this treatment. Firstly, due to the lack of ticks, the natural challenge was missing in livestock leading to a loss of naturally developed immunity against E. ruminantium. Mistakes and breakdowns in the intensive tick control rapidly led to massive animal losses due to heartwater and other tickborne diseases (Allsopp 2015). Secondly, ticks started to develop resistance against acaricides (Bezuidenhout and Bigalke 1987). Nowadays, the tick burden is still strategically and selectively controlled so that their natural immunity against $E$. ruminantium is preserved. Heavy rainfall may lead to the increase of tick populations and new livestock with a different immunity status may be introduced to endemic areas so that chemical tick control will never be a sufficient solution for this problem (Tice et al. 1998). However a recombinant vaccine against ticks could be a choice in future (Parizi et al. 2012). 


\section{Usage of resistant stock}

Some cattle breeds are more resistant against E. ruminantium than others. Generally, Bos taurus is less resistant than Bos indicus (zebus) e.g. zebus become infected but the outcome is less severe (Uilenberg 1983). However it also depends when and where B. taurus was first introduced, African breeds are more resilient than European ones. Unfortunately, resistance against E. ruminantium is negatively correlated with reproduction success in livestock. Attempts to breed high resistant goats had no positive outcome thus far (Camus et al. 1996).

\section{Antibiotic treatment}

Acute infection is often treated with oxytetracycline. When introducing new animals to the herd, farmers have to administer antibiotics prophylactically. A treatment of $20 \mathrm{mg} / \mathrm{kg}$ is recommended (Allsopp et al. 2005). Angora goats are highly susceptible to heartwater. This is why farmers in South Africa administer routinely a prophylactic dose of $3 \mathrm{mg} / \mathrm{kg}$. This treatment which has to be repeated every two weeks is very expensive because of the high costs for the antibiotics and for logistical reasons (Allsopp 2015). Moreover there is a risk that antibiotic resistance will develop in these animals. For the long term, a vaccine needs to be developed (Purnell et al. 1989).

\section{Immunization of livestock}

As outlined above, highlighting the limited options for tick control, the only solution to eradicate heartwater in livestock is a protective vaccine. The most popular use in endemic areas in Africa is, however, not a vaccine but it is a cryopreserved preparation of sheep blood (Neitz and Alexander 1945) containing virulent infective E. ruminantium organisms, which are intravenously administered. The temperatures of the animals have to be measured daily and antibiotics are administered when clinical signs occur. However, the blood vaccine must be held at a certain temperature below freezing immediately before application because the bacteria lose the infectivity rapidly once thawed (Brayton et al. 2003). It is a logistical challenge to administer the 'vaccine' and measure the body temperature regularly of each animal in the herd. This vaccination concept mimics the situation of a stable enzootic circulation of the pathogen, which likewise leads to immunity against a local antigenic variant of the pathogen with the slight difference that a developing clinic cannot be immediately treated as infections may occur any time and not after a certain time point, i.e. the vaccination. The efficiency is moreover highly variable because there are numerous immunotypes of $E$. ruminantium circulating in the field and cross-protection ranges from total to minimal (Collins et al. 2003).

\section{New vaccine development}

Three types of vaccines are currently under development: (1) an attenuated vaccine; (2) an inactivated vaccine; and (3) a recombinant vaccine.

\section{Attenuated vaccine development}

This approach is based on an a-virulent live pathogen which is not causing any clinical signs but activates immunity against virulent forms of E. ruminantium. Three strains have been attenuated: (1) the West Africa strain (Senegal); (2) the South Africa strain (Welgevonden); and (3) the Guadeloupe strain (Gardel). The South African (Welgevonden) vaccine provides $100 \%$ protection against $E$. ruminantium in its endemic area for merino sheep and boer goats with one homologous strain 
and four heterologous strains (Zweygarth et al. 2005). However, this vaccine is less satisfactory in Angora goats. These are highly susceptible for E. ruminantium and some of them needed to be treated after vaccination (Zweygarth et al. 2008).

\section{Inactivated vaccine development}

The inactivated vaccine is produced from elementary bodies partially purified from a bovine endothelial cell culture. The chemically inactivated bacteria are formulated with a suitable adjuvant. Currently-tested vaccines can protect 50-100\% from death after a lethal needle challenge (see below) but after a heterologous challenge they are not so effective (Faburay et al. 2007; Mahan et al. 2003). The reason is that the cross-protection is weak because of the immunogenic diversity amongst $E$. ruminantium strains circulating in the wild. The different genotypes mutate and recombine to generate further diversity (Adakal et al. 2009; Allsopp and Allsopp 2007). To resolve this problem, one can make vaccines for the genotypes common in a certain region which was already done with some positive effect (Adakal et al. 2010). The general problem with infection experiments is that needle infection is less infectious than natural infection by ticks (Collins et al. 2003; Pretorius et al. 2008). This is why experimental infections with tick challenge are needed.

\section{Recombinant vaccine development}

It is crucial to develop a recombinant vaccine because of its high protection and safety. Moreover it would be easier and cheaper to store in comparison to an attenuated or an inactivated vaccine. Nevertheless the relevant genes of E. ruminantium have to be identified first. Thus far, eleven genes have been identified which encode for protein products inducing cytokine responses similar to the immune response induced by intact E. ruminantium cells (Liebenberg et al. 2012). Epitope mapping of these products is conducted with the intention of developing a multi-epitope vaccine (Allsopp 2015). If this vaccine is accomplished, it could stop outbreaks in non-endemic areas. Previous tests had a variable outcome and a lot of different experiments did not lead to a satisfactory solution thus far.

\section{Babesiosis (Piroplasmosis)}

\section{Aetiology}

Babesiosis is a malaria-like disease of mammals caused by intra-erythrocytic protozoan parasites (Figure 2) of the genus Babesia, family Babesidae, order Piroplasmida, phylum Apicomplexa, and transmitted by ticks (Birkenheuer 2014; Homer et al. 2000; Schnittger et al. 2012). Diseases similar to babesiosis can be caused by Theileria species (mainly Theileria parva and Theileria annulata) belonging to the same order. Theileriidae are however different from Babesiidae as they can also infect white blood cells and endothelial cells. Both pathogens - because of the shape of their trophic forms - are also called piroplasms.

There are three principal Babesia species that cause economically important bovine babesiosis (BB): B. bovis and B. bigemina in tropical and subtropical regions of the world, and Babesia divergens in temperate climates (Bock et al. 2004; De Vos et al. 2004). Other species that can infect cattle include Babesia major, Babesia ovata, Babesia occultans and Babesia jakimovi. 


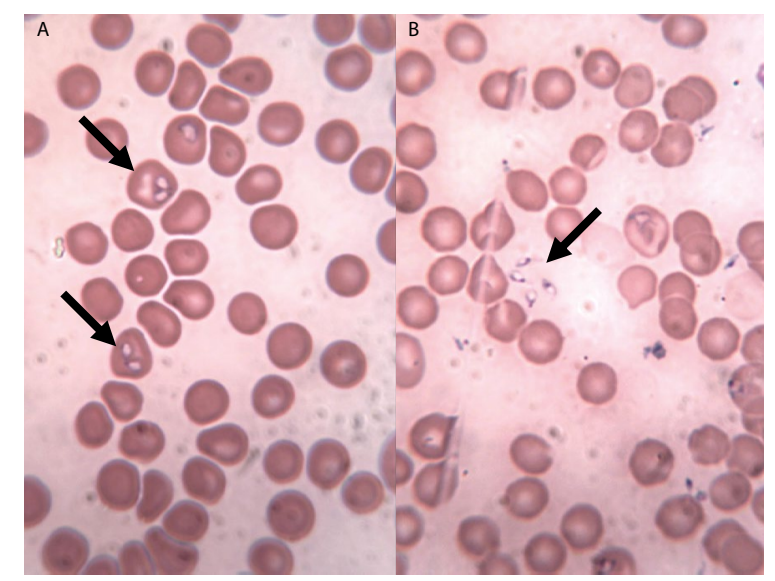

Figure 2. Typical appearance of Babesia spp. in erythrocytes of a blood smear from an infected dog. (A) Typical double pear-shaped merozoite in erythrocytes. (B) Released merozoites after the burst of the infected erythrocyte. Please note that Babesia spp. cannot be differentiated based on morphological criteria neither in unstained (here) nor Giemsa-stained blood smears.

\section{Epidemiology and transmission}

Bovine babesiosis is primarily maintained by sub-clinically infected individuals that have not completely recovered from disease. But infection depends on many factors: reservoir animals, abundance of vectors, susceptibility of hosts, suitable tick habitat (Perry 1996). Rates of mortality and morbidity vary immensely and are influenced by such factors as vaccination status, previous exposure to the parasite, and prevailing treatments. An important factor to establish endemic stability is the age of the animal during the first exposure (Mahoney and Mirre 1979; Mahoney and Ross 1972). Calves have natural resistance during the first 6-9 months and usually remain asymptomatic and develop a long-term immunity (Callow 1984; Dalgliesh 1993). However if their exposure to the pathogen at young age is interrupted (e.g. because of decrease of tick population caused by acaricide treatment, season of unfavourable weather conditions, etc.) or immunologynaïve specimens are presented, outbreaks of disease can occur in endemic areas. The epizootical situation can also be changed by introducing competent vectors into new areas.

Bovine babesiosis can be found anywhere where tick vectors are present, but especially in tropical and subtropical climates (Bock et al. 2004; De Vos et al. 2004). B. bovis and B. bigemina are widely distributed and particularly important in Africa, Asia, Australia, Central and South America, and southern parts of Europe. These two pathogens are very often found in the same general geographic regions but $B$. bigemina is more widespread and common in Africa than $B$. bovis due to a wider spectrum of vectors (De Vos 1979; Friedhoff 1988; McCosker 1981; Uilenberg 1995). These parasites were formerly enzootic all over southern USA but now, thanks to Rhipicephalus (Boophilus) eradication programs established in 1906, are found only in buffer zone along the Mexican border (Pegram et al. 2000, Pérez de León et al. 2012). B. divergens is economically important in Europe including Spain, the United Kingdom, and northern parts of European continent. B. major occurs mostly in Northwest Africa and Asia but can be found also in Europe; $B$. 
ovata is located in eastern Asia; B. occultans has been found in Africa, and B. jakimovi in Siberia. The main vector for B. bigemina are Rhipicephalus ticks, as R. microplus (formerly Boophilus microplus) and Rhipicephalus annulatus but also Rhipicephalus decoloratus, Rhipicephalus geigyi, Rhipicephalus evertsi are involved in maintaining this parasite. Also B. bovis is mainly transmitted by R. microplus, R. annulatus, and R. geigyi (Bouattour et al. 1999; Caeiro 1999; Ndi et al. 1991; Pipano 1995; Sahibi et al. 1998; Sayin et al. 1996). These pathogens can be passed transovarially, however tick life stages involved in maintaining the infection among cattle are different for Babesia species (Callow 1979 and 1984; Callow and Hoyte 1961; Dalgliesh and Stewart 1982, 1983; Mahoney and Mirre 1979). Parasites of $B$. bovis become infective in 2-3 days after larval tick attaches and can be carried by larvae, however B. bovis does not persist after larval stage in $R$. microplus. In the case of B. bigemina, piroplasms mature in around 9 days after larvae attachment, and thus it is transmitted only by nymphs and adults. B. divergens is closely associated with $I$. ricinus ticks which can be also vectors for B. jakimovi (Friedhoff 1988). All active stages of I. ricinus can carry B. divergens (which can sustain in tick population for four years even when vertebrate hosts are absent).

\section{Clinical signs and diagnosis}

As bovine babesiosis is transmitted by ticks, the first clinical signs of infection appear usually after 2-3 weeks after ticks infestation (Callow 1984; De Vos et al. 2000; Wright and Goodger 1988). Symptoms vary with the age of the animal but most of the cases are observed in adults. Infections caused by different Babesia species are similar but $B$. bovis is more virulent than the other piroplasms. Susceptibility to BB varies with the breeds of cattle. Bos indicus are more resistant than B. taurus (Bock et al. 1997, 1999a,b). It is a result of the evolutionary relationship between $B$. indicus cattle, Rhipicephalus spp. ticks and Babesia spp. parasites (Dalgliesh 1993).

Typically, B. bigemina infection leads to high fever $\left(>40{ }^{\circ} \mathrm{C}\right)$, anorexia, haemolysis, anaemia accompanied by haemoglobinemia and haemoglobinuria, hence the common name 'redwater' (Ahmed 2002; Callow 1984; Dalgliesh et al. 1995; De Vos and Potgieter 1994; Singleton 1974; Wright and Goodger 1988; Wright et al. 1989). Also mucous membranes become pale and heart frequency increases. In subacute cases, jaundice and diarrhoea or constipation are noted. Pregnant cows may abort and bulls may show reduced fertility. Animals behaviour is changed as well: inappetence, general weakness, reluctance to move, depression and/or separation from the herd are usually observed. Central nervous system (CNS) signs are uncommon in this infection. Usually some of the infected animals die but those which survive generally recover fully. Symptoms of infections with $B$. bovis are similar but often more severe. Infected erythrocytes can be sequestered in brain capillaries causing neurological signs (like incoordination, teeth grinding, hyperaesthesia, nystagmus, circling, head pressing, aggression, mania, convulsions and/or paralysis). Most of the cows affected with CNS signs die. However, haemoglobinuria and haemoglobinemia are rare. In case of $B$. divergens the course of the disease can be mild to severe; mild fever, anorexia and uneventful recovery are common. CNS symptoms are sporadic, but may occur if anaemia causes brain anoxia. B. ovata is mildly pathogenic and B. major is non-pathogenic in most cases.

Post-mortem lesions are mostly associated with an intravascular haemolysis, anaemia and jaundice (De Vos and Potgieter 1994). Characteristic necropsy of typical BB is light red, watery blood water and mucous membranes and carcasses are paler than normal (which can be masked by icterus). The spleen is invariably enlarged with dark and pulpy, friable consistency. The liver is usually swollen, with orange-brown coloration, gall bladder is distended containing thick, granular bile. The kidneys appear darker (dark red, brown/black), and bladder may contain reddish-brown 
urine. Oedema of lungs is possible. Heart can be flabby and petechiae and/or ecchymoses may be observed.

Babesiosis can be diagnosed with several methods (Bock et al. 2004). Most common are: identification of the piroplasms in blood smear, serological tests and PCR. Microscopic examinations with Giemsa-stained thin blood films from capillary blood are a traditional method of identifying the agent (Böse et al. 1995; Callow et al. 1993). This approach is the cheapest but because of morphological variability of piroplasms, the differentiation of species is more difficult and not always precise. Parasites are found mostly during acute infections but not when parasitaemia is very low. They are present within red blood cells and all divisional stages can be found at the same time (Figure 2). Serology tests are more specific (Böse et al. 1995; De Vos et al. 2000). This method is now the most often used for surveillance and export certifications. Two tests are commonly used to detect antibodies: IFA and ELISA. Unfortunately, cross-reactions in particular between B. bovis and $B$. bigemina, can complicate the results of tests and accurate differentiation can be sometimes a particular problem. Complement fixation has been used and agglutination assays have been described. PCR assays are much more sensitive than the mentioned methods and more specific than blood smears. They can easily detect and differentiate Babesia species not only in cattle but also in vectors (Böse et al. 1995). Several molecular techniques have been described but because of costs they are not so common in large-scale testing.

\section{Prevention and control}

Complete elimination of tick vectors for Babesia spp. leading to eradication of bovine babesiosis is the perfect, permanent solution, but it is rarely possible because of practical and economic issues. It was successfully achieved in the USA, where BB was eradicated (Pegram et al. 2000). Permanent and temporary cattle tick fever quarantine areas along the border to Mexico are in place in order to keep this status (Pérez de León et al. 2012; http://www.tahc.texas.gov/animal_health/cattle/\#ticks). The incidence of the disease can be reduced by control of ticks (Bock et al. 2004; De Vos 1979; De Vos and Potgieter 1983). It can be accomplished by treatment with acaricides, minimizing the exposure of cattle to ticks. Destroying tick habitats by modification of the environment could also bring desirable result but it can be difficult and almost unrealistic in areas where tick populations are well established. The best approach, as for control of anaplasmosis and cowdriosis discussed above, is the use of integrated methods: strategic and regular use of repellents, application of vaccines and use of tick-resistance breeds (Norval et al. 1992; Perry et al. 1998).

As cattle develop long-lasting immunity after a single infection with Babesia spp., vaccination is used to immunize the animals (Bock and De Vos 2001; Callow 1984; Callow et al. 1997; De Vos and Jorgensen 1992; Gray et al. 1989; Pipano 1995). Even though live-attenuated strains of both $B$. bovis and $B$. bigemina that are used for this purpose have reduced virulence, there are some safety issue as hypersensitivity reactions to blood proteins, potential for virulence in adult specimens or possible contamination with other pathogens. Therefore the recommendation is to vaccine only calves aged 3-9 months to minimize the risk of reactions. If there is a need to immunize older cows, they should be observed daily for three weeks after treatment. Because of abortion risk, pregnant cows should not be vaccinated.

Vaccination with tick antigens may be a safe alternative to the application of acaricides. However, this strategy thus far only works with a few tick species. The Bm86 gut antigen (from Boophilus, now $R$. microplus) is a commercially available tick vaccine which significantly reduces the number of engorging females and thus the reproducing capacity of $R$. microplus and in part of R. annulatus, 
the two main vectors for B. bovis and B. bigemina (De la Fuente et al. 2007; De Vos et al. 2001). This vaccine is often used together with low dose acaricide treatment to enhance efficacy (De la Fuente 1999, 2007; Miller et al. 2012; Pérez de León et al. 2012, 2014).

During an outbreak of disease, procedures depend on the number of affected animals, availability and costs of drugs, vaccines, acaricides and tests. To limit loss, some actions are recommended: separation of sick animals from the herd and treatment, confirmation of the diagnosis, prevention of uninfected cows from tick exposure. The immediate vaccination of all unaffected animals and the usage of prophylactic treatment programs have to be considered.

\section{Concluding remarks}

More emphasis has to be put on understanding the immunology related to tick-borne infections in cattle, as this is a prerequisite for the development of specific vaccines. In addition, the genetic basis of host resistance associated with particular vector tick species has to be deciphered thus allowing further development of anti-tick vaccines beyond the Bm86 gut antigen used against $R$. microplus and $R$. annulatus. The key to successful tick and tick-borne disease control is a combination of measures involving the use of acaricides and a (age-dependent) grazing management, in order to balance the effort and financial burden towards animal health and the cattle industry's benefit.

\section{Acknowledgements}

The work of MP and AO is done under the frame of EurNegVec COST Action TD1303.

\section{References}

Adakal H, Meyer DF, Carasco-Lacombe C, Pinarello V, Allegre F, Huber K, Stachurski F, Morand S, Martinez D, Lefrançois T, Vachiéry $N$ and Frutos R (2009) MLST scheme of Ehrlichia ruminantium: genomic stasis and recombination in strains from Burkina-Faso. Infect Genet Evol 9: 1320-1328.

Adakal H, Stachurski F, Konkobo M, Zoungrana S, Meyer DF, Pinarello V, Aprelon R, Marcelino I, Alves PM, Martinez D, Lefrançois T and Vachiéry N (2010) Efficiency of inactivated vaccines against heartwater in Burkina Faso: impact of Ehrlichia ruminantium genetic diversity. Vaccine 28: 4573-4580.

Ahmed JS (2002) The role of cytokines in immunity and immunopathogenesis of pirolasmoses. Parasitol Res 88: S48-S50. Alberti A, Zobba R, Chessa B, Addis MF, Sparagano O, Pinna Parpaglia ML, Cubeddu T, Pintori G, Pittau M (2005) Equine and canine Anaplasma phagocytophilum strains isolated on the island of Sardinia (Italy) are phylogenetically related to pathogenic strains from the United States. Appl Environ Microbiol 71: 6418-6422.

Allsopp BA, Bezuidenhout SM and Prozesky L (2005) Heartwater. In: Coetzer JAW and Tustin RC (eds.) Infectious diseases of livestock. Vol. 1. Oxford University Press, Oxford, UK, pp. 507-535.

Allsopp MT and Allsopp BA (2007) Extensive genetic recombination occurs in the field between different genotypes of Ehrlichia ruminantium. Vet Microbiol 124(1-2): 58-65.

Allsopp BA (2010) Natural history of Ehrlichia ruminantium. Vet Parasitol 167: 123-135.

Allsopp BA (2015) Heartwater-Ehrlichia ruminantium infection. Rev Sci Tech Off Int Epiz 34: 557-558.

Atif FA (2015) Anaplasma marginale and Anaplasma phagocytophilum: Rickettsiales pathogens of veterinary and public health significance. Parasitol Res 114: 3941-57.

Atif FA (2016) Alpha proteobacteria of genus Anaplasma (Rickettsiales: Anaplasmataceae): epidemiology and characteristics of Anaplasma species related to veterinary and public health importance. Parasitol 143: 659-685.

Aubry P and Geale DW (2011) A review of bovine anaplasmosis. Trans Emerg Dis 58: 1-30.

Bakken JS and Dumler S (2008) Human granulocytic anaplasmosis. Infect Dis Clin North Am 22: 433-448. 
Baldridge GD, Scoles GA, Burkhardt NY, Schloeder B, Kurtti TJ, Munderloh UG (2009) Transovarial transmission of Francisella-like endosymbionts and Anaplasma phagocytophilum variants in Dermacentor albipictus (Acari: Ixodidae). J Med Entomol 46: 625-632.

Battilani M, De Arcangeli S, Balboni A and Dondi F (2017) Genetic diversity and molecular epidemiology of Anaplasma. Infect Genet Evol 49: 195-211.

Bezuidenhout JD (1987) Natural transmission of heartwater. Onderstepoort J Vet Res 54: 349-351.

Bezuidenhout JD (1988) Certain aspects of the transmissionof heartwater, the occurrence of the organism in ticks and in vitro culture. PhD-thesis, University of Pretoria, Pretoria, South Africa.

Bezuidenhout JD and Bigalke RD (1987) The control of heartwater by means of tick control. Onderstepoort J Vet Res 54: 525-528.

Birkenheuer AJ (2014) Babesiosis. In: Sykes JE (ed.) Canine and feline infectious diseases. Elsevier, St. Louis, MO, USA, pp. 727-738.

Bock RE and De Vos AJ (2001) Immunity following use of Australian tick fever vaccine: a review of the evidence. Aust Vet J 79: 832-839.

Bock RE, De Vos AJ, Kingston TG and McLellan DJ (1997) Effect of breed of cattle on innate resistance to infection with Babesia bovis, B. bigemina and Anaplasma marginale. Aust Vet J 75: 337-340.

Bock RE, Jackson L, De Vos A and Jorgensen W (2004) Babesiosis of cattle. Parasitol 129: 247-269.

Bock RE, Kingston TG and De Vos AJ (1999a) Effect of breed of cattle on transmission rate and innate resistance to infection with Babesia bovis and B. bigemina transmitted by Boophilus microplus. Aust Vet J 77: 461-464.

Bock RE, Kingston TG, Standfast NF and De Vos AJ (1999b) Effect of cattle breed on innate resistance to inoculations of Babesia bigemina. Aust Vet J 77: 465-466.

Bouattour A, Darghouth MA and Daoud A (1999) Distribution and ecology of ticks (Acari: Ixodidae) infesting livestock in Tunisia: an overview of eight years field collections. Parassitologia 41: S5-S10.

Böse R, Jorgensen WK, Dalgliesh RJ, Friedhoff KT and De Vos AJ (1995) Current state and future trends in the diagnosis of babesiosis. Vet Parasitol 57: 61-74.

Brayton KA, Collins NE, Van Strijp F and Allsopp BA (2003) Preparation of Ehrlichia ruminantium challenge material for quantifiable and reproducible challenge in mice and sheep. Vet Parasitol 112(1-2): 63-73.

Caeiro V (1999) General review of tick species present in Portugal. Parassitologia 41: 11-15.

Callow LL (1979) Some aspects of the epidemiology and control of bovine babesiosis in Australia. J S Afr Vet Assoc 50: 353-356.

Callow LL (1984) Animal health in Australia, protozoal and Rickettsial diseases. Vol. 5. Animal Health in Australia, Australian Bureau of Animal Health, Canberra, Australia, pp. 121-160.

Callow LL, Dalgliesh RJ and De Vos AJ (1997) Development of effective living vaccines against bovine babesiosis - the longest field trial? Intern J Parasitol 27: 747-767.

Callow LL and Hoyte HMD (1961) Transmission experiments using Babesia bigemina, Theileria mutans, Borrelia sp. and the tick cattle Boophilus microplus. Aust Vet J 37: 381-390.

Callow LL, Rogers RJ, De Vos AJ (1993) Tick-borne diseases: cattle pathology and serology. In: Corner LA and Bagust TJ (eds) Australian Standard Diagnostic Techniques for Animal Diseases. CSIRO Information Services, East Melbourne, Australia, pp. 1-16.

Camus $\mathrm{E}$ and Barré $\mathrm{N}$ (1992) The role of Amblyomma variegatum in the transmission of heartwater with special reference to Guadeloupe. Ann New York Acad Sci 653: 33-41.

Camus E, Maillard JC, Ruff G, Pepin L, Naves M and Matheron G (1996) Genetic resistance of Creole goats to cowdriosis in Guadeloupe. Status in 1995. Ann New York Acad Sci 791: 46-53.

Chen SM, Dumler JS, Bakken JS and Walker DH (1994) Identification of a granulocytotropic Ehrlichia species as the etiologic agent of human disease. J Clin Microbiol 32: 589-595.

Clark KL (2012) Anaplasma phagocytophilum in small mammals and ticks in northeast Florida. J Vector Ecol 37: 262-268.

Collins NE, Pretorius A, Van Kleef M, Brayton KA, Allsopp MT, Zweygarth E and Allsopp BA (2003) Development of improved attenuated and nucleic acid vaccines for heartwater. Dev. Biol. 114: 121-136. 
Courtney JW, Kostelnik LM, Zeidner NS and Massung RF (2004) Multiplex real-time PCR for detection of Anaplasma phagocytophilum and Borrelia burgdorferi. J Clin Microbiol 42: 3164-3168.

Cowdry EV (1925a) Studies on the etiology of heartwater. Observation of a rickettsia, Rickettsia ruminantium (n. sp.), in the tissues of infected animals. J Experim Med 42: 231-252.

Cowdry EV (1925b) Studies on the etiology of heartwater. Rickettsia ruminantium (n. sp.) in the tissues of ticks transmitting the disease. J Experim Med 42: 253-274.

Dalgliesh RJ (1993) Babesiosis. In: Warren SK (ed.) Immunology and molecular biology of parasite infections. Blackwell, Oxford, UK, pp. 352-383.

Dalgliesh RJ, Molloy JB, Jorgensen WK and Bock RE (1995) Do parasite antigens on erythrocytes determine host-parasite relationships in Babesia infections in cattle? Lessons from Malaria. In: Coons L and Rothchild M (eds.) Proceedings of the $2^{\text {nd }}$ International Conference on tick-borne pathogens at the host-vector interface: a global perspective, United Litho, Johannesburg, South Africa.

Dalgliesh RJ and Stewart NP (1982) Some effects of time, temperature and feeding on infection rates with Babesia bovis and Babesia bigemina in Boophilus microplus larvae. Int J Parasitol 12: 323-326.

Dalgliesh RJ and Stewart NP (1983) The use of tick transmission by Boophilus microplus to isolate pure strains of Babesia bovis, Babesia bigemina and Anaplasma marginale from cattle with mixed infections. Vet Parasitol 13: 317-323.

De la Fuente J, Almazán C, Canales M, De la Lastra JMP, Kocan KM and Willadsen P (2007) A ten-year review of commercial vaccine performance for control of tick infestations on cattle. Anim Health Res Rev 8: 23-28.

De la Fuente J, Canales M and Kocan KM (2006) The importance of protein glycoylation in development of novel tick vaccine strategies. Parasite Immunol 28: 687-688.

De la Fuente J, Moreno-Cid JA, Canales M, Villar M, De la Lastra JMP, Kocan KM, Galindo RC, Almazán C and Blonin EF (2011) Targeting arthropod subolesin/akirin for the development of a universal vaccine of vector infestation and pathogen transmission. Vet Parasitol 181: 17-22.

De la Fuente J, Rodriguez M, Montero C, Redondo M, García-García JC, Méndez L, Serrano E, Valdés M, Enríquez A, Canales M, Ramos E, Boué O, Machado H, Lleonart R (1999) Vaccination against ticks (Boophilus spp.): the experience with the Bm86-based vaccine Gavac. Genet Anal 15: 143-148.

De Vos AJ (1979) Epidemiology and control of bovine babesiosis in South Africa. J South African Vet Assoc 50: 357-362.

De Vos AJ and Jorgensen WK (1992) Protection of cattle against babesiosis in tropical and subtropical countries with a live, frozen vaccine. In: Fivaz BH, Petney TN and Horak IG (eds.) Tick vector biology, medical and veterinary aspects. Springer Verlag, London, UK, pp. 159-174.

De Vos AJ and Potgieter FT (1983) The effect of tick control on the epidemiology of bovine babesiosis. Onderstepoort J Vet Res 50: 3-5.

De Vos AJ and Potgieter FT (1994) Bovine babesiosis. In: Coetzer JAW, Thomson GR and Tustin RC (eds.) Infectious diseases of livestock. Oxford University Press, Capetown, South Africa, pp. 278-294.

De Vos AJ, De Waal DT and Jackson LA (2004) Bovine babesiosis. In: Coetzer JAW and Tustin RC (eds.) Infectious diseases of livestock. Vol. 1. Oxford University Press, Capetown, South Africa, pp. 406-424.

De Vos AJ, Jorgensen WK and Mollow JB (2000) Bovine babesiosis. In: Office International des Epizootic (eds.) Manual of standards for diagnostic tests and vaccines ( $4^{\text {th }}$ Ed.). Office International des Epizootic, Paris, France, pp. 412-422.

De Vos S, Zeinstra L, Taoufik O, Willadsen P and Jongejan F (2001) Evidence for the utility of the Bm86 antigen from Boophilus microplus in vaccination against other tick species. Exp Appl Acarol 25: 245-261.

Dikmans G (1950) The transmission of anaplasmosis. Am J Vet Res 11: 5-16.

Dugat T, Haciane D, Durand B, Lagrée AC, Haddad N and Boulouis HJ (2016b) Identification of a potential marker of Anaplasma phagocytophilum associated with cattle abortion. Transbound Emerg Dis 64: e1-e3.

Dugat T, Lagrée AC, Maillard R, Boulouis HJ and Haddad N (2015) Opening the black box of Anaplasma phagocytophilum diversity: current situation and future perspectives. Front Cell Infect Microbiol 5:61.

Dugat T, Rossignol MN, Rué O, Loux V, Marthey S, Morolda M, Silaghi C, Höper D, Fröhlich J, Pfeffer M, Zweygarth E, Lagrée AC, Boulouis HJ and Haddad N (2016a) Draft Anaplasma phagocytophilum genome sequences from five cows, two horses, and one roe deer collected in Europe. Genome Announc 4: e00950-16. 
Dumler JS, Barbet AF, Bekker CP, Dasch GA, Palmer GH, Ray SC, Rikihisa Y and Rurangirwa FR (2001) Reorganization of genera in the families Rickettsiaceae and Anaplasmataceae in the order Rickettsiales: unification of some species of Ehrlichia with Anaplasma, Cowdria with Ehrlichia and Ehrlichia with Neorickettsia, descriptions of six new species combinations and designation of Ehrlichia equi and 'HGE agent' as subjective synonyms of Ehrlichia phagocytophila. Int J Syst Evol Microbiol 51: 2145-2165.

Du Plessis JL, Jansen BC and Prozesky L (1983) Heartwater in Angora goats. Immunity subsequent to artificial infection and treatment. Onderstepoort J Vet Res 50: 137-143.

Du Plessis JL, Van Gas L, Olivier JA and Bezuidenhout JD (1989) The heterogenicity of Cowdria ruminantium stocks: crossimmunity and serology in sheep and pathogenicity to mice. Onderstepoort J Vet Res 56: 195-201.

Esemu SN, Besong WO, Ndip RN and Ndip LM (2013) Prevalence of Ehrlichia ruminantium in adult Amblyomma variegatum collected from cattle in Cameroon. Exp Appl Acarol 59: 377-387.

Ewing SA (1981) Transmission of Anaplasma marginale by arthropods. Proceedings of the $7^{\text {th }}$ National Anaplasmosis Conference. Starkville, MS, USA, pp. 425-434.

Faburay B, Geysen D, Ceesay A, Marcelino I, Alves PM, Taoufik A, Postigo M, Bell-Sakyi L and Jongejan F (2007) Immunisation of sheep against heartwater in the Gambia using inactivated and attenuated Ehrlichia ruminantium vaccines. Vaccine 25: 7939-7947.

Friedhoff KT (1988) Transmission of Babesia. In: Ristic M (ed.) Babesiosis of domestic animals and man. CRC Press, Boca Raton, FL, USA, pp. 23-52.

Graf JF, Gogolewski R, Leach-Bing N, Sabatini GA, Molento MB, Bordin EL and Arantes GJ (2004) Tick control: an industry point of view. Parasitol 129: S427-42.

Gray JS, Langley RJ, Brophy PO and Gannon P (1989) Vaccination against bovine babesiosis with drug-controlled live parasites. Vet Rec 125: 369-372.

Gueye A, Mbengue M and Diouf A (1993) Épidémiologie de la cowdriose au Sénégal. Étude de la transmission et du taux d'infection d'Amblyomma variegatum (Fabricius, 1794) dans la région des Niayes. Rev Elev Méd Vét Pays Trop 46: 441-447.

Harrus S, Perlman-Avrahami A, Mumcuoglu KY, Morick D, Eyal O and Baneth G (2011) Molecular detection of Ehrlichia canis, Anaplasma bovis, Anaplasma platys, Candidatus Midichloria mitochondrii and Babesia canis vogeli in ticks from Israel. Clin Microbiol Infect 17: 459-463.

Hawkins JA, Love JN and Hidalgo RJ (1982) Mechanical transmission of anaplasmosis by tabanids (Diptera: Tabanidae). Am J Vet Res 43: 732-734.

Hoar BR, Nieto NC, Phodes DM and Foley JE (2008) Evaluation of sequential coinfection with Anaplasma phagocytophilum and Anaplasma marginale in cattle. Am J Vet Res 69: 1171-1178.

Holden K, Boothby JT, Anand S and Massung RF (2003) Detection of Borrelia burgdorferi, Ehrlichia chaffeensis, and Anaplasma phagocytophilum in ticks (Acari: Ixodidae) from a coastal region of California. J Med Entomol 40:534-539.

Homer MJ, Aguilar-Delfin I, Telford SR, Krause PJ and Persing DH (2000) Babesiosis. Clin Microbiol Rev 13: 451-469.

Hosseini-Vasoukolaei N, Oshaghi MA, Shayan P, Vatandoost H, Babamahmoudi F, Yaghoobi-Ershadi MR, Telmadarraiy Z and Mohtarami F (2014) Anaplasma infection in ticks, livestock and human in Ghaemshahr, Mazandaran Province, Iran. J Arthropod Borne Dis 8: 204-211.

Huhn C, Winter C, Wolfsperger T, Wüppenhorst N, Smrdel KS, Skuballa J, Pfäffle M, Petney T, Silaghi C, Dyachenko V, Pantchev N, Straubinger RK, Schaarschmidt-Kiener D, Ganter M, Aardema ML and Von Loewenich F (2014) Analysis of the population structure of Anaplasma phagocytophilum using multilocus sequence typing. PLoS ONE 9: e93725.

Jonsson NN, Mayer DG, Matschoss AL, Green PE and Ansell J (1998) Production effects of cattle tick (Boophilus microplus) infestation of high yielding dairy cows. Vet Parasitol 78: 65-77.

Khumalo ZT, Catanese HN, Liesching N, Hove P, Collins NE, Chaisi ME, Gebremedhin AH, Oosthuizen MC and Brayton KA (2016) Characterization of Anaplasma marginale subspecies centrale strains using msp1aS genotyping reveals a wildlife reservoir. J Clin Microbiol 54: 2501-2512.

Kocan KM, Blouin EF and Barbet AF (2000) Anaplasmosis control: past, present, and future. Ann New York Acad Sci 916: 501-509. 
Kocan KM, De la Fuente J, Blouin EF, Coetzee JF and Ewing SA (2010) The natural history of Anaplasma marginale. Vet Parasitol 167: 95-107.

Kocan KM, De la Fuente J, Guglielmone AA and Meléndez RD (2003) Antigens and alternatives for control of Anaplasma marginale infection in cattle. Clin Microbiol Rev 16:698-712.

Kocan KM, De la Fuente J and Cabezas-Cruz A (2015) The genus Anaplasma: new challenges after reclassification. Rev Sci Tech 34: 577-586.

Kocan KM, Hair JA, Ewing SA and Stratton LG (1981) Transmission of Anaplasma marginale Theiler by Dermacentor andersoni Stiles and Dermacentor variabilis Say. Am J Vet Res 42: 15-18.

Liebenberg J, Pretorius A, Faber FE, Collins NE, Allsopp BA and Van Kleef M (2012) Identification of Ehrlichia ruminantium proteins that activate cellular immune responses using a reverse vaccinology strategy. Vet Immunol Immunopathol 145(1-2): 340-349.

Mahan SM, Barbet AF and Burridge MJ (2003) Development of improved vaccines for heartwater. Dev. Biol. 114: 137-145.

Mahoney DF and Mirre GB (1979) Note on the transmission of Babesia bovis (syn. B. argentina) by the one-host tick, Boophilus microplus. Res Vet Sci 26: 253-254.

Mahoney DF and Ross DR (1972) Epizootiological factors in the control of bovine babesiosis. Aust Vet J 48: 292-298.

Maillard JC and Maillard N (1998) Historique dupeuplement bovin et de l'introduction de la tique Amblyomma variegatum dans les îles françaises des Antilles: synthèse bibliographique. Ethnozootechnie 1: 19-36.

Martinez D, Vachiéry N, Stachurski F, Kandassamy Y, Raliniaina M, Aprelon R and Gueye A (2004) Nested PCR for detection and genotyping of Ehrlichia ruminantium: use in genetic diversity analysis. Ann New York Acad Sci 1026: 106-113.

McCallon BR (1973) Prevalence and economic aspects of anaplasmosis. Proceedings of the $6^{\text {th }}$ National Anaplasmosis Conference. Las Vegas, NV, USA, pp. 1-3.

McCosker PJ (1981) The global importance of babesiosis. In: Ristic M and Kreier JP (eds.) Babesiosis. Academic Press, New York, NY, USA, pp. 1-24.

Miller R, Estrada-Peña A, Almazán C, Allen A, Jory L, Yeater K, Messenger M, Ellis D, Pérez de León AA (2012) Exploring the use of an anti-tick vaccine as a tool for the integrated eradication of the cattle fever tick, Rhipicephalus (Boophilus) annulatus. Vaccine 30: 5682-5687.

Minjauw B, Kruska RL, Odero A, Randolph TF, McDermott JJ, Mahan SM and Perry BD (2000) Economic impact assessment of Cowdria ruminantium infection and its control in southern Africa. In: Proceedings of the $9^{\text {th }}$ Symposium of the International Society for Veterinary Epidemiology and Economics, Breckenridge, CO, USA, pp. 645. Available at: www.sciquest.org.nz/elibrary/edition/5415.

Molia S, Frebling M, Vachiéry N, Pinarello V, Petitclerc M, Rousteau A, Martinez D and Lefrançois T (2008) Amblyomma variegatum in cattle in Marie Galante, French Antilles: prevalence, control measures, and infection by Ehrlichia ruminantium. Vet Parasitol 153(3-4): 338-346.

Moshkovski SD (1947) Comments by readers. Science 106: 62.

Mukhebi AW, Chamboko T, O'Callaghan CJ, Peter TF, Kruska RL, Medley GF, Mahan SM and Perry BD (1999) An assessment of the economic impact of heartwater (Cowdria ruminantium infection) and its control in Zimbabwe. Prev Vet Med 39: 173-189.

Nakao R, Stromdahl EY, Magona JW, Faburay B, Namangala B, Malele I, Inoue N, Geysen D, Kajino K, Jongejan F and Sugimoto C (2010) Development of loopmediated isothermal amplification (LAMP) assays for rapid detection of Ehrlichia ruminantium. BMC Microbiol 10: 296.

Ndi C, Bayemi P, Ekue FN and Tarounga B (1991) Preliminary observations on ticks and tick-borne diseases in the north west province of Cameroon. I. Babesiosis and anaplasmosis. Rev Elev Med Vet Pays Trop 44: 263-265.

Neitz WO and Alexander RA (1945) Immunization of cattle against heartwater and the control of the tick-borne diseases, redwater, gallsickness and heartwater. Onderstepoort J Vet Sci Anim Ind 20: 137-158.

Norval RAI, Perry BD and Hargreaves SK (1992) Tick and tick-borne disease control in Zimbabwe: what might the future hold? Zimbabwe Vet J 23: 1-15.

Ogden NH, Bown K, Horrocks BK, Woldehiwet Z, Bennett M (1998) Granulo-cytic Ehrlichia infection in ixodid ticks and mammals in woodlands and uplands of the U.K. Med Vet Entomol 12: 423-429. 
Palomar AM, Portillo A, Santibáñez P, Mazuelas D, Roncero L., García-Álvarez L, Santibáñez S, Gutiérrez Ó and Oteo JA (2015) Detection of tick-borne Anaplasma bovis, Anaplasma phagocytophilum and Anaplasma centrale in Spain. Med Vet Entomol 29: 349-353.

Parizi LF, Githaka NW, Logullo C, Konnai S, Masuda A, Ohashi K and Da Silva Vaz I Jr (2012) The quest for a universal vaccine against ticks: cross-immunity insights. Vet J 194: 158-165.

Paulauskas A, Radzijevskaja J, Rosef O (2012) Molecular detection and characterization of Anaplasma phagocytophilum strains. Comp Immunol Microbiol Infect Dis 35: 187-195.

Pegram RG, Wilson DD and Hannsen JW (2000) Past and present national tick control programs. Why they succeed or fail. Ann New York Acad Sci 916: 546-554.

Pérez de León AA, Teel PD, Auclair AN, Messenger MT, Guerrero FD, Schuster G and Miller RJ (2012) Integrated strategy for sustainable cattle fever tick eradication in USA is required to mitigate the impact of global change. Front Physiol 3: 195.

Pérez de León AA, Vannier E, Almazán C and Krause PJ (2014) Tick-borne protozoa. In: Sonenshine DE and Roe RM (eds.) Biology of ticks. Vol. 2. Oxford University Press, New York, NY, USA, pp. 147-179.

Perry BD (1996) Epidemiological indicators and their application to the control of tick-borne diseases. Manual on tick and tick-borne disease control, FAO, Rome, Italy.

Perry BD, Chamboko T, Mahan SM, Medley GF, Minjauw B, O'Callaghan CJ and Peter TF (1998) The economics of integrated tick and tick borne disease control on commercial farms in Zimbabwe. Zimbabwe Vet J 29: 21-29.

Peter TF, Perry BD, O'Callaghan CJ, Medley GF, Mlambo G, Barbet AF and Mahan SM (1999) Prevalence of Cowdria ruminantium infection in Amblyomma hebraeum ticks from heartwater-endemic areas of Zimbabwe. Epidemiol Infect 123: 309-316.

Pipano E (1995) Live vaccines against hemoparasitic diseases in livestock. Vet Parasitol 57: 213-231.

Potgieter FT and Van Rensburg L (1987) Tick transmission of Anaplasma centrale. Onderstepoort J Vet Res 54: 5-7.

Pretorius A, Van Kleef M, Collins NE, Tshikudo N, Louw E, Faber FE, Van Strijp MF and Allsopp BA (2008) A heterologous prime/boost immunisation strategy protects against virulent $E$. ruminantium Welgevonden needle challenge but not against tick challenge. Vaccine 26: 4363-4371.

Provost A and Bezuidenhout JD (1987) The historical background and global importance of heartwater. Onderstepoort J Vet Res 54: 165-169.

Purnell RE, Gunter TD and Schroder J (1989) Development of a prophylactic regime using long-acting tetracycline for the control of redwater and heartwater in susceptible cattle moved into an endemic area. Trop Anim Health Prod 21: 11-19.

Rar V and Golovljova I (2011) Anaplasma, Ehrlichia, and 'Candidatus Neoehrlichia' bacteria: pathogenicity, biodiversity, and molecular genetic characteristics, a review. Infec Gen Evol 11: 1842-1861.

Rikihisa Y (1991) The tribe Ehrlichieae and Ehrlichial diseases. Clin Microbiol Rev 4: 286-308.

Rosso F, Tagliapietra V, Baráková I, Derdáková M, Konečný A, Hauffe HC and Rizzoli A (2017) Prevalence and genetic variability of Anaplasma phagocytophilum in wild rodents from the Italian alps. Parasit Vectors 10:293.

Sahibi H, Rhalem A, Berrag B and Goff WL (1998) Bovine babesiosis. Seroprevalence and ticks associated with cattle from two different regions of Morocco. Ann New York Acad Sci 849: 213-218.

Sainz Á, Roura X, Miró G, Estrada-Peña A, Kohn B, Harrus S and Solano-Gallego L (2015) Guideline for veterinary practitioners on canine ehrlichiosis and anaplasmosis in Europe. Parasit Vectors 8: 75.

Sayin F, Dincer S, Karaer Z, Cakmak A, Inci A, Yukari BA, Eren H, Friedhoff KT and Muller I (1996) Studies on seroprevalence of Babesia infection of cattle in Turkey. In: Özcel MA and Alkan MZ (eds.) Acta parasitologica Turcica. Proceedings of the VIII International Congress of Parasitology - New Dimensions in Parasitology. Izmir, Turkish Society for Parasitology 20, Suppl. 1: 505-516.

Schnittger L, Rodriguez AE, Florin-Christensen M and Morrison DA (2012) Babesia: a world emerging. Infect Genet Evol 12: 1788-1809.

Seidman D, Hebert KS, Truchan HK, Miller DP, Tegels BK, Marconi RT and Carlyon JA (2015) Essential domains of Anaplasma phagocytophilum invasins utilized to infect mammalian host cells. PLoS Pathogens 11: e1004669. 
Silaghi C, Nieder M, Sauter-Louis C, Knubben-Schweizer G, Pfister K and Pfeffer M (2017a) Epidemiology, genetic variants and clinical course of natural infections with Anaplasma phagocytophilum in a dairy cattle herd. Parasit Vectors 11: 20 .

Silaghi C, Santos AS, Gomes J, Christova I, Matei IA, Walder G, Domingos A, Bell-Sakyi L, Sprong H, Von Loewenich FD, Oteo JA, De la Fuente J and Dumler JS (2017b) Guidelines for the direct detection of Anaplasma spp. in diagnosis and epidemiological studies. Vector-Borne Zoonot Dis 17: 12-22.

Simpson BC, Lindsay MS, Morris JR, Muirhead FS, Pollock A, Prichard SG, Stanley HG, Thirlwell GR, Hunter AG and Bradley $J(1987)$ Protection of cattle against heartwater in Botswana: comparative efficacy of different methods against natural and blood-derived challenges. Vet Rec 120: 135-138.

Singleton EF (1974) The effect of heat on reproductive function in the bull. PhD thesis, University of Queensland, Brisbane, Australia.

Stephenson $\mathrm{N}$ and Foley J (2016) Parallelisms and contrasts in the diverse ecologies of the Anaplasma phagocytophilum and Borrelia burgdorferi complexes of bacteria in the far western United States. Vet Sci 2: 26.

Steyn HC, Pretorius A, McCrindle CM, Steinmann CM and Van Kleef M (2008) A quantitative real-time PCR assay for Ehrlichia ruminantium using pCS20. Vet Microbiol 131(3-4): 258-265.

Stich RW, Kocan KM, Palmer GH, Ewing SA, Hair JA and Barron SJ (1989) Transstadial and attempted transovarial transmission of Anaplasma marginale by Dermacentor variabilis. Am J Vet Res 50: 1377-1380.

Stuen S, Granquist EG and Silaghi C (2013) Anaplasma phagocytophilum - a widespread multi-host pathogen with highly adaptive strategies. Front Cell Infect Microbiol 22: 31.

Stuen S, Nevland S and Moum T (2003) Fatal cases of Tick-Borne Fever (TBF) in sheep caused by several 16S rRNA gene variants of Anaplasma phagocytophilum. Ann New York Acad Sci 990: 433-434.

Teshale S, Geysen D, Ameni G, Asfaw Y and Berkvens D (2015) Improved molecular detection of Ehrlichia and Anaplasma species applied to Amblyomma ticks collected from cattle and sheep in Ethiopia. Ticks Tick Borne Dis 6: 1-7.

Tice GA, Bryson NR, Stewart CG, Du Plessis B and De Waal DT (1998) The absence of clinical disease in cattle in communal grazing areas where farmers are changing from an intensive dipping programme to one of endemic stability to tick-borne diseases. Onderstepoort J Vet Res 65: 169-175.

Tomanović S, Chochlakis D, Radulovic Z, Milutinovic M, Cakic S, Mihaljica D, Tselentis Y, Psaroulaki A (2013) Analysis of pathogen co-occurrence in host-seeking adult hard ticks from Serbia. Exp Appl Acarol 59: 367-376.

Uilenberg G (1983) Heartwater (Cowdria ruminantium infection): current status. Adv Vet Sci Comp Med 27: 427-480.

Uilenberg G (1993) Other ehrlichioses of ruminants. In: Woldenhiwet $Z$ and Ristic M (eds.) Rickettsial and Chlamydial diseases of domestic animals. Pergamon Press, Elmsford, NY, USA, pp. 269-279.

Uilenberg G (1995) International collaborative research: significance of tick-borne hemoparasitic diseases to world animal health. Vet Parasitol 57: 19-41.

Van Heerden H, Steyn HC, Allsopp MT, Zweygarth E, Josemans Al and Allsopp BA (2004) Characterization of the pCS20 region of different Ehrlichia ruminantium isolates. Vet Microbiol 101: 279-291.

Van Vliet AH, Van der Zeijst BA, Camus E, Mahan SM, Martinez D and Jongejan F (1995) Use of a specific immunogenic region on the Cowdria ruminantium MAP1 protein in a serological assay. J Cli. Microbiol 33: 2405-2410.

Woldehiwet Z (2010) The natural history of Anaplasma phagocytophilum. Vet Parasitol 167: 108-122.

World Organisation for Animal Health (OIE) (2017a) Manual of diagnostic tests and vaccines for terrestrial animals 2017. Bovine anaplasmosis. Available at: http://www.oie.int/en/international-standard-setting/terrestrial-manual/ access-online.

World Organisation for Animal Health (OIE) (2017b) Manual of diagnostic tests and vaccines for terrestrial animals 2017. Heartwater. Available at: http://www.oie.int/en/international-standard-setting/terrestrial-manual/access-online.

Wright IG and Goodger BV (1988) Pathogenesis of babesiosis. In: Ristic M (ed.) Babesiosis of domestic animals and man. CRC Press, Boca Raton, FL, pp. 99-118.

Wright IG, Goodger BV, Buffington GD, Clark IA, Parrodi F and Waltisbuhl DJ (1989) Immunopathophysiology of babesial infections. Transact Royal Soc Trop Med Hyg 83: S11-S13. 
Martin Pfeffer et al.

Zweygarth E, Josemans Al and Steyn HC (2008) Experimental use of the attenuated Ehrlichia ruminantium (Welgevonden) vaccine in Merino sheep and Angora goats. Vaccine 26, Suppl. 6: G34-G39.

Zweygarth E, Josemans Al, Van Strijp MF, Lopez-Rebollar L, Van Kleef M and Allsopp BA (2005) An attenuated Ehrlichia ruminantium (Welgevonden stock) vaccine protects small ruminants against virulent heartwater challenge. Vaccine 23: $1695-1702$. 


\title{
8. Mosquito-borne diseases in the livestock industry
}

\author{
Nonito Pagès ${ }^{1 *}$ and Lee W. Cohnstaedt ${ }^{*}$ \\ ${ }^{1}$ CIRAD/INRA UMR ASTRE (Animal, Santé, Territoires, Risques et Ecosystèmes), CRVC - Centre for Research \\ and surveillance on Vector-borne diseases in the Caribbean, Domaine Duclos-Prise d'Eau, 97170-Petit \\ Bourg, Guadeloupe, France; ${ }^{2}$ United States Department of Agriculture, Agricultural Research Service, \\ Arthropod-borne Animal Diseases Research Unit, 1515 College Ave, Manhattan, KS 66502, USA; \\ nonito.pages@cirad.fr; lee.cohnstaedt@ars.usda.gov
}

\begin{abstract}
Mosquito bites may result in increased stress and pain, which reduces livestock fitness, weight gain, and animal welfare. Furthermore, mosquito feeding may also result in pathogen transmission between livestock reservoirs (epizootics) and incidentally humans (zoonotic diseases). Not all mosquito species are disease vectors and not all individuals within a species will become infected post exposure and these important differences between mosquito genera and species are discussed. The epidemiology (hosts, environment, pathogen, and mosquito vectors) of most significant and frequent pathogens are explained, with particular emphasis on the viruses Rift Valley fever, Japanese encephalitis, West Nile, and the equine encephalitis (Western equine, Eastern equine, and Venezuelan equine). Increased globalization and anthropogenic landscape modification has resulted in widespread emergence and re-emergence of pathogens in old and new habitats. Furthermore, viral adaptation and global climate change will place more animal and human populations at risk of these pathogens.
\end{abstract}

Keywords: Rift Valley fever, Japanese encephalitis, West Nile, equine encephalitis viruses

\section{Introduction}

Mosquitoes are insects that belong to the order Diptera (two-winged) and family Culicidae. The first mosquito ancestors probably appeared during the Triassic period, 200-245 million years ago (Reidenbach et al. 2009). Since then, the Culicidae probably co-evolved with terrestrial vertebrates along the Jurassic period to date. Fossil record is sparse for Culicidae origin, however Culicidae fossils were recovered from the Early Cretaceous (Rasnitsyn and Quicke 2002).

Ancestrial mosquitoes evolved to become vectors of disease-producing microorganisms because they developed blood feeding abilities. First insights on the role of mosquitoes as vectors of disease-producing pathogens date from the end of the $19^{\text {th }}$ century. A pioneering study of Sir Patrick Manson demonstrated that mosquitoes became infected with microfialriae while bloodfeeding on infected patients. This represented the beginning of a period referred as the age of vector biology (Eldridge 1992). During the $20^{\text {th }}$ century the scientific community realized mosquitoes were the most important group of arthropods from the standpoint of public and veterinary health.

Mosquitoes are present worldwide except Antarctica. They occur in a large variety of environments requiring a minimum level of standing water for immature stages (larvae). Their special adaptation mechanisms allowed mosquitoes to adapt and colonize almost every aquatic habitat as breeding sites from sea level to above elevations of 3,000 meters. They are found either in temporary or permanent water sources, irrespective to the pollution, size and nature of the water body. 


\section{Systematics and taxonomy}

Mosquitoes are members of the family Culicidae; one of the best characterized groups from the standpoint of morphological taxonomy (Munstermann and Conn 1997). Since Edwards (Edwards 1932) the family Culicidae was suggested to include three subfamilies of monophiletic origin: Anophelinae, Culicinae, and Toxorhynchitinae. Harbach and Kitching (1998), through cladistic phylogenetics, suggested the family Culicidae was composed of two valid subfamilies of monophyletic origin, Anophelinae and Culicinae, and ten tribes. Nowadays, the family Culicidae comprises 3,549 formally described mosquito species that are classified in two subfamilies, 11 tribes and 112 genera (http://mosquito-taxonomic-inventory.info). However, Wilkerson et al. (2015) proposed to restore the generic structure of tribe Aedini to its status prior to the year 2000, representing a considerable reduction of the number of genera. It is expected that a significant number of species still lack a formal description, mainly from tropical areas characterized by high mosquito diversity, but low surveillance efforts.

Relationships of lower taxonomic entities are challenging as well. The boundaries between subspecies, species complexes, strains or populations are sometimes difficult to define. Mosquito species identification based exclusively on external characters has limitations due to intraspecific variability of external phenotypic characters across geographic areas. This concerns several species, being evident for Culex pipiens s.s. synonymies described globally and for difficult species complexes characterization within Anopheles genus. Congruence between genetics and morphology is sometimes compromised as genetic characterization often suggests interspecific differences for isomorphic (true sibling) species. In addition, incomplete reproductive barriers between recently diverged species can lead to gene flow, known as genetic introgression (Fontaine et al. 2015). Mosquito sibling species could have different capacity to transmit pathogens. Therefore the species definition becomes a paramount concept and sometimes the within-species genetic plasticity is diffuse and species delineation becomes challenging.

Complementary methods should be combined to improve mosquito species identification. Ideally, a critical assessment of morphological, ecological, and molecular (genetic) data would ensure better discrimination capacity. Early attempts included the study of polytene chromosomes, isoenzyme analyses and genomic hybridization methods (Munstermann and Conn 1997). However, the standardisation of the polymerase chain reaction technique and Sanger DNA sequencing represented an essential breakthrough. More recently, the advent of next-generation sequencing methods allowed entomologists to obtain genomic and transcriptomic data to infer insect phylogenetics (Behura 2015). This represents a powerful approach to study insect systematics and evolution.

\section{Life cycle and morphology}

Mosquitoes have evolved with highly specialized anatomical characters to exploit particular ecological niches and resources. Mosquitoes are holometabolous insects and undergo a renewed growth to adult state through a complete metamorphosis. Their life cycle has four stages: the egg, four larval instars, pupae, and adult (Figure 1).

A female can lay from few to hundreds (over 300) eggs depending on the mosquito species and the presence of stressors. Some species require specific larval habitats whereas others have larger plasticity for site selection. Egg-laying patterns vary according to mosquito genera. Females of Culex, Mansonia, Culiseta and Uranotaenia deposit egg-rafts upon surface of the water (Figure 1A). 


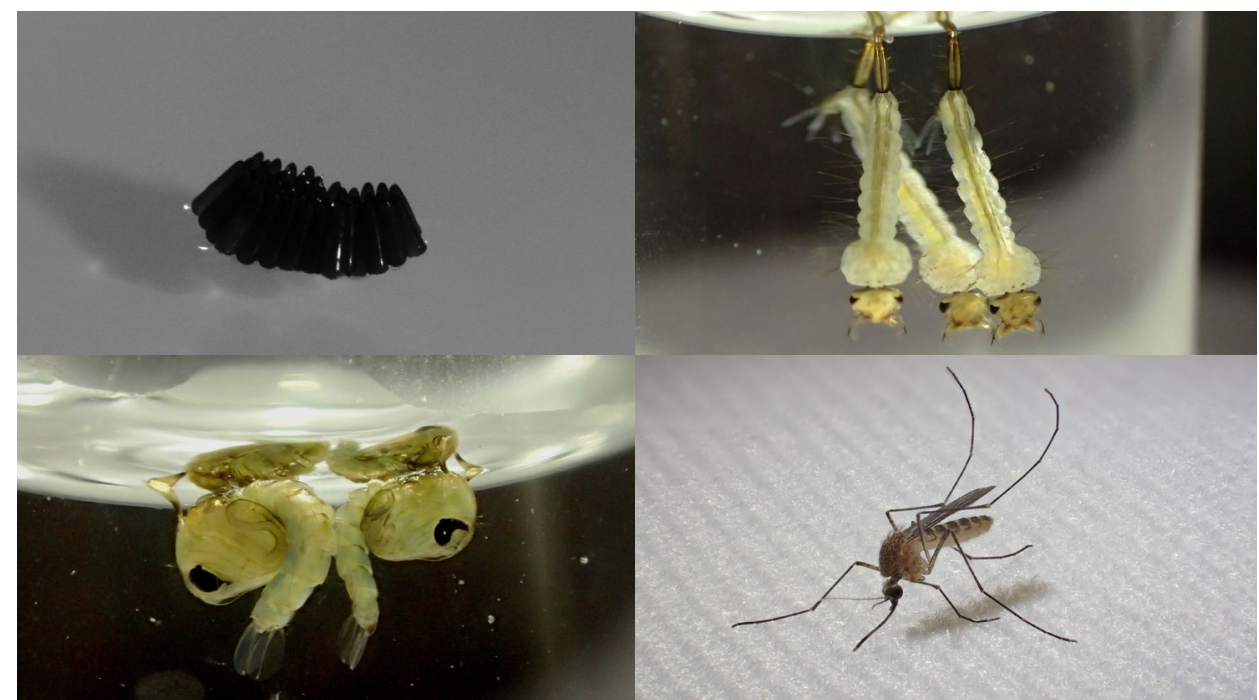

Figure 1. Culex quinquefasciatus (A) egg raft; (B) larval stages; (C) pupae; and (D) adult female (photos by Lee W. Cohnstaedt).

Anopheles deposit the eggs, individually, on the surface of water. Aedes and Psorophora deposit the eggs in moist surfaces where the egg can undergo a seasonal dormancy awaiting for flooding (Clements 1992). Eggs are soft and white when laid and darken in few hours.

Aquatic larvae (Figure 1B) hatch from the egg. The structure of larvae is fully adapted to live and feed in the water. Mosquito larvae have three body-regions, the head, thorax and abdomen. The larvae moults four times during its life-time and ecdyses by shedding its cuticle. In the first three moults the larvae becomes larger with little morphological change whereas in the fourth it evolves to pupae stage.

The pupae are aquatic and have a characteristic comma-shape (Figure 1C). The anterior part, cephalothorax, is enlarged and contains the respiratory organs, two horns or trumpets. The posterior part, abdomen, has eight segments with a pair of paddles in the last segment. During the pupal stage the adult mosquito structures are under developed and completed by the final molt and ecdyses.

The body of an adult mosquito (Figure 1D) is slender and has three distinct regions, the head, thorax and abdomen. Thin legs and narrow elongate wings are inserted into the thorax. Scales and setae present on the body surface provide characteristic patterns used to differentiate some mosquito species.

The head is attached to the thorax with a slender neck. Two large compound eyes occupy most of the lateral part of the head. Antennae has sexual dimorphism, each flagellomer (but the first) has short and sparse basal hairs in females but longer and bushy in males giving a plumose appearance. Antennae are usually subequal to proboscis length except in Deinocerites and Galindomyia genera. The form and function of mouthparts of adult and larvae diverge considerably. Adult 
mouthparts are complex and consist on an elongated proboscis and a pair of maxillary palpus. Male mosquitoes, and females of Malaya and Toxorhinchites genera, are not bloodsuckers. Their mouthparts are highly specialized to feed exclusively on nectar or plant juices.

The thorax is composed of three segments, prothorax, mesothorax and metathorax. The mesothorax and metathorax contain a pair of lateral spiracles. The prothorax is usually greatly reduced to two lobes just behind the head. The mesothorax is the most developed segment, greatly enlarged to hold the wing muscles responsible of fore wings movement and flight. The wings are narrow and long with a characteristic and simple vein pattern. Scales are present along veins and the wing hind margin (fringe). The metathorax holds the hind wings, called halteres, which are reduced and modified into gyroscopic structures for flight control. The thin legs are attached to the thorax by coxae and contain the femur, tibiae and 5 tarsi, the last with claws and a central pad called empodium.

The abdomen is divided into 10 segments, the first is joined basally to the thorax and last segments taper distally. Each segment has a tergal (tergites) and sternal (sternites) plates that usually present a characteristic scale pattern. Male abdomen is characterized by the presence of two large genital claspers (parameres) that project from the distal part of the abdomen (segment IX). Male segment $X$ (proticter) is a flattened anal lobe with a ventral position at the base of the parameres. Few hours after adult emergence from pupae, whole male genitalia beyond segment VII rotates $180^{\circ} \mathrm{C}$ to mature. In females, segment $9^{\text {th }}$ has the cerci and postgenital lobe. The characters present in external male genitalia are outstanding diagnostic features for species identification because of their generic and specific variations.

Mosquito systematics and morphology are essential to identify mosquito species because not all mosquitoes are competent or able to transmit pathogens. Pathogen transmission is mosquito species specific, which means competent mosquito vectors for one virus does not necessarily make that species competent for other viruses. Mosquito population management measures are often customized to target specific mosquito species which are pests or disease vectors. On farm treatments typically target the larval habitats, adult biting times, and adult resting areas. Therefore proper mosquito management requires mosquito species identification to assess an area's potential disease transmission risk and most effective treatment options.

\section{Mosquito-borne diseases important to livestock}

Mosquitoes have the potential to transmit a large variety of pathogens to livestock. Nevertheless, mosquitoes are able to reduce the livestock fitness as well without pathogen transmission. Mosquitoes can stress livestock during their landing, probing and biting activity, and later through biting side effects causing irritation and allergic reactions. This affects animal behaviour and can lead to skin laceration and hair loss that might promote bacterial secondary infection. The final output for livestock might include weight loss or a reduction in weight gain, even reduction in milk production has been reported for cattle (Foster and Walker 2002).

Natural transmission of a pathogen by mosquitoes requires that a mosquito bites and feeds on an infected (and amplifying) host, the pathogen then multiplies within the mosquito until being released into the mosquito saliva while re-feeding into a new non-infected susceptible host. For a particular mosquito-borne pathogen, few (or a single) mosquito species need to be competent in order to have biological transmission of the pathogen. Vector competence refers to the mosquito susceptibility to infection during blood-feeding and ability to transmit the pathogen during 
posterior re-feeding. Vector competence ranges from competent, with higher or lower level of competence, to completely refractory species. However, other behavioural and ecological traits might have more relevance to disease emergence, such as lifespan, host feeding preferences, biting rate, population size, extrinsic incubation period of the pathogen, which are incorporated into vectorial capacity (Smith et al. 2012).

Three mosquito genera, Aedes, Culex and Anopheles are well known for having species that transmit pathogens causing diseases of upmost importance (Wilkerson et al. 2015). Overall, mosquitoes have the potential to transmit a large array of pathogens of different aetiology to vertebrates, including livestock. Among them, filarial nematodes, parasites, and viruses. Most important nonhuman vertebrate filarial nematodes transmitted by mosquitos belong to Onchocercidae family. Representative genera are Wuchereria and Brugia that infect non-human primates, Dirofilaria infecting carnivores (cause of dog heartworm), Dipetalonema causing arterial fialariasis of camelid livestock (Duvallet and Boireau 2015), Setaria infecting several livestock species (cattle, equine), Oswaldofilaria infecting reptiles, Foleyella that infects amphibians and chamaleons and Cardiofilaria that infect birds. Among mosquito-transmitted parasites, non-human malaria caused by genus Plasmodium infects a significant range of vertebrates: non-human primates are infected by 29 species of Plasmodium, birds by 30 species, rodents by 12 species and reptiles by 29 species. Of these, avian malaria caused by Plasmodium has the potential to infect domestic fowls (Plasmodium gallinaceum; Williams 2005). However, avian malaria epizootics are rarely reported and usually associated to zoological collections, remote islands introduction events, and domestic birds outside their original geographic range exposed to sylvatic cycles (Lapointe et al. 2012). Mosquito-borne viruses are amongst the most important endemic and emerging pathogen diseases in a global context affecting livestock, humans and other vertebrates. The most important viruses transmitted to vertebrates belong to Bunyaviridae, Faviviridae and Togaviridae families. Among them, a selected group of mosquito-transmitted viral diseases is reviewed: Rift Valley fever (Bunyaviridae), Japanese encephalitis and West Nile fever (Flaviviridae), and Equine encephalitis (Togaviridae). These are all emerging or re-emerging diseases in the $21^{\text {st }}$ century of utmost importance for veterinary and public health, due to their zoonotic potential.

\section{Rift Valley fever}

Rift Valley fever virus (RVFV) is an arbovirus of the genus Phlebovirus, family Bunyaviridae. RVFV is the causative agent of Rift Valley fever (RVF), a zoonotic disease primarily transmitted by mosquitoes affecting both ruminants and humans. The disease is amongst the most important viral zoonoses in Africa, epizootics are characterized by high ruminant mortality and mass abortions. Large outbreaks produce major economic losses associated to livestock mortality and trade interdiction. Human infections usually occur after epizootic outbreaks. Therefore, RVF epizootics have the potential to cause high economic burden to veterinary and public health.

RVF was historically prevalent in sub-Saharan Africa and more recently spread to the Middle East. RVF was described in 1930 in the Rift Valley region of Kenya when deaths and abortions were detected around Lake Naivasha (Daubney and Hudson 1932; Lapointe et al. 2012). In South Africa, 1950-1951, a large epizootic caused an estimated 100,000 deaths and 500,000 abortions in sheep (Weiss 1957). During the last 4 decades RVF's distribution spread beyond the traditional enzootic area, southwestern Africa, and was reported in northeastern (Egypt, 1977) and western (Mauritania and Senegal, 1987) Africa. Nowadays RVF has been reported in most continental African countries, including some islands in the Pacific Ocean (Madagascar, Mayotte, and Comoros). Nevertheless, the most relevant event has been the first emergence of RVFV outside Africa, into the Arabian 
Peninsula. There, Saudi Arabia and Yemen experienced a large outbreak in 2000-2001 affecting livestock and humans (Balkhy and Memish 2003), with estimates of 40,000 animal deaths and 8,000-10,000 ruminant abortions in Saudi Arabia (Al-Afaleq and Hussein 2011). In 2007, RVF affected half the regions of Tanzania with estimated direct losses over $\$ 4$ and $\$ 2$ million for cattle and sheep mortality, respectively (Sindato et al. 2012). The public health impact of RVF has been evidenced periodically as well. Human case-fatality rate was estimated to be $<1 \%$ before 1970 (Gubler 2002) and data suggested about $0.3 \%$ (of 200,000 cases) in Egypt for 1977-1978 epidemic (Meegan et al. 1979). However, in Saudi Arabia, 14\% case-fatality rate was reported in the 2000 epidemic (Balkhy and Memish 2003). Later, about 32\% case-fatality rate (698 cases) was detected in the 2007 epidemic in Sudan (WHO 2008). Previous data is derived from officially reported cases, therefore underreporting would be expected.

The recent increase of RVF outbreaks reported and wide geographic expansion have evidenced that the disease is a potential threat virtually worldwide (Rolin et al. 2013). Particularly, the possibility for illegal livestock trade after RVF introductions into Egypt, the Arabian Peninsula and certain Indian Ocean islands highlights the high risk of RVF introduction into Europe (Chevalier et al. 2010). Recent outbreaks in Mauritania and detection of RVFV seropositive camels in Morocco reinforce the introduction threat towards Europe (El-Harrak et al. 2011; El Mamy et al. 2011).

\section{Hosts}

Several wild and domestic ruminants are susceptible to RVFV infection. Ruminant infection provokes neonatal mortality, and increased abortion or foetal malformations (Mansfield et al. 2015). The disease susceptibility and clinical manifestations largely depends on host species and age. World-wide Artiodacyla (even toed-ungulates such as cattle, sheep, goats, deer, elk, moose, etc.) will likely play the most significant role as both transmission and amplification reservoir hosts (Golnar et al. 2014). Sheep and new-born lambs are the most susceptible species while cattle and camels are less susceptible. Mortality rate is apparently age-dependent as well, thus infected new-born lambs could reach mortality rate over $90 \%$, whereas adults either die (10-30\% mortality rate) or develop a mild infection (Bird et al. 2009). Adult ( $>3$ months) sheep and lamb symptoms range from only vomiting to additional clinical signs, including fever with depression, blood muco-purulent nasal discharge or haemorrhagic diarrhoea. Adult goats have frequent abortions (80\%) but mild infections with low mortality (Gerdes 2004). Calves develop acute illness (fever, diarrhoea, dyspnea) and mortality rate ranges from $10-70 \%$. Infected pregnant ruminants have frequent abortions, ranging from 40-100\% (Mansfield et al. 2015). Not all rodents develop viremia sufficient to infect mosquitoes ( $<10^{4.7} \mathrm{PFU} / \mathrm{ml}$; Golnar et al. 2014), but some common rodents such as mice (Mus musculus) and the brown rat (Rattus norvegicus) do (Olive et al. 2012). Humans are susceptible to RVFV infection and usually considered dead-end hosts. Human infection tends to be asymptomatic or with mild flu-like symptoms, however infection can lead to significant morbidity and mortality. Direct transmission exists from infected ruminants to other (healthy) ruminants or humans. Although mosquitoes usually transmit the virus to ruminants, livestock epizootics might lead to human infections through direct contact with livestock infected body fluids, tissues, and unpaturized milk (Balkhy and Memish 2003). Thus, certain professionals as farmers, veterinarians, slaughterhouse employees and butchers who are regularly exposed to ruminant body fluids and tissues are more at risk. 


\section{Vectors}

Mosquitoes are the primary vectors of RVFV. The virus has been isolated in ticks and several nonmosquito Nematocera, but their contribution to RVFV transmission cycles is uncertain. Worldwide, more than 65 mosquito species among different genera are considered potential RVFV vectors (reviewed by Golnar et al. 2014). However, species in the genera Aedes and Culex are of upmost importance, beyond those in genera Anopheles, Coquillettidia, Eretmapodite, Mansonia, and Ochlerotatus.

Experimental infections confirmed several endemic mosquito species from Africa, EU and USA are potential vectors of RVFV. In Europe the ubiquitous Culex pipiens Linnaeus and Aedes vexans Meigen, in the USA Culex tarsalis Coquillett, Ae. vexans and Ochlerotatus taeniorhynchus Wiedemann (Iranpour et al. 2011; Moutailler et al. 2008; Turell et al. 2008, 2010). The invasive species Aedes albopictus Skuse, successfully established in the EU and USA, is able to transmit RVFV and might pose an additional risk (Turell et al. 1988). However, the drivers that modulate RVF vector competence are still not fully understood. Environmental factors, as temperature, that affect viral transmission are not fully characterized and show varying effects according to mosquito species (Brustolin et al. 2017; Turell et al. 1985). RVFV infection in dormant eggs (diapause) has been reported in Aedes mcintoshi Huang (Linthicum et al. 1985). Transovarial (vertical) RVFV transmission is expected to occur in other Aedes species. This mechanism would allow RVFV over-wintering in latent eggs over inter-epizootic periods (Linthicum et al. 1985). Thereafter, environmentally favourable conditions (flooding) would induce egg-hatching, larvae development and emergence of RVFV-infected adult mosquitoes.

\section{Epidemiology, cycle}

The diverse susceptible ruminants and vertical transmission mechanisms in mosquitoes (transovarial) and ruminants (transplacental) add complexity to RVFV epidemiological cycle. Nonruminant vertebrates (rodents, bats) might be infected, but their epidemiological role remains unclear.

In Africa, several bioclimatic environments such as dambos, semi-arid and irrigation regions are suitable for RVF emergence. In eastern and southern Africa, RVFV usually occurs in dambos regions, characterized by shallow depressions suitable for mosquito breeding when flooded. In dambos regions, RVFV re-emerges periodically every 5-15 years coinciding with the El Niño Southern Occilation (ENSO) or suitable heavy rains (Anyamba et al. 2010). ENSO provides heavy rains to flood dambos and consequently massive hatching of quiescent Aedes eggs. Increased severity and frequency of RVFV outbreaks in East Africa was suggested as climate models expected more frequent and intense extreme rainfall events (Chevalier et al. 2010). Contrarily, RVF transmission cycles in semi-arid regions of western Africa are correlated with years of rainfall deficit (Pepin et al. 2010) and less frequently to heavy rainfall. In semi-arid regions, viral persistence in mosquitoes, unknown wildlife reservoir or livestock movement from endemic regions might lead to epizootics. Viral transmission occurs year-round in irrigation regions, including areas in the Nile Delta and Senegal River basin with paddy cultures. Dam facilitates irrigation and flooding, thus creating permanent water bodies that are ideal breeding environments for Culex mosquitoes. Similar breeding environments are present in Wadi, temporary rivers from arid areas (i.e. Arabia Saudi, Yemen) where temporary shallow water bodies (ponds) remain once water flowing is interrupted. More recently, temperate and mountainous areas in Madagascar have been shown to be suitable ecosystems to support RVFV epizootics (Chevalier et al. 2011). Wild ruminants have been suggested 
to contribute to RVF epidemiology in certain areas, but their role and importance still remains unclear (Olive et al. 2012). Some of the mechanisms behind transmission, spread, and persistence are poorly known for the complex RVFV cycle.

Viremic host movement is the most likely mechanism for RVFV introduction and dissemination into a naive region, particularly domestic livestock, including camels. Sheep and goat trade from African disease-endemic regions into the Middle East might be the best mechanism for RVF introduction into Middle East and the Mediterranean basin. Similarly, sheep trade between sub-Saharan Africa and Northern Africa increase RVF incursion risk into the Mediterranean basin (Chevalier et al. 2005). In addition, higher risk is expected during religious festivals in Mecca, Saudi Arabia, and Muslim feasts in northern Africa when millions of small ruminants are imported from sub-Saharan Africa (Chevalier et al. 2010). Therefore, livestock trade represents low (but not negligible) risk of RVFV introduction into Europe and North America. Both regions established trade bans impeding livestock import from RVFV-endemic countries and prohibited livestock shipment from RVF-free countries travelling through infected regions (Chevalier et al. 2010; Kasari et al. 2008). However, risk of introduction into EU via legal or illegal imported ruminant trade would remain high during epizootic periods (EFSA 2005).

Northern Egypt epizootics raised concerns over RVF emergence in USA and EU for having suitable bioclimatic environments and potential vector species, making RVFV transmission ecologically feasible. Presence of competent mosquitoes in Europe, including C. pipiens s.l. and Ae. vexans, suggests RVFV might spread and lead to epidemics in case of viral emergence (Moutailler et al. 2008). Besides, the current climatic scenario supports feasibility of incursion of exotic competent species into Europe (Gale et al. 2010). In addition, presence of RVF in northern Africa and Middle East increases risk of virus-carrying vector incursion events into Europe. During epizootics, two independent pathways might support mosquito incursion. First pathway consists on introduction from northern Africa to Spain, assuming that, under favourable conditions, mosquitoes would overcome the short distance to reach Spain. Second pathway consists on the introduction through Turkey (Albayrak and Ozan 2012). However, the possibility of virus-carrying vector introduction through intercontinental air and ship cargo is less probable, but not negligible.

\section{Vaccines}

Live and inactivated licensed RVFV vaccines are available for livestock. Contrarily, there is no licensed vaccine or antiviral treatment for human use. The Smithburn live attenuated vaccine is recommended by the Food and Agriculture Organization (FAO). It has been widely used in Africa, has low production costs and could reach life-long immunity. The vaccine protects sheep, goat and cattle from RVFV -induced abortions. However, vaccine side-effects include reversion to virulence with the potential for co-infection between vaccine and wild strains. Contrarily, RVFV inactivated vaccine has higher production costs and induces a lower protection, requiring double inoculation and periodic boosts to reach desired immunity thresholds. Other live attenuated vaccines include MP-12 and Clone 13 vaccines. MP12, obtained by reverse genetics, has partial license in the USA and the Clone 13, naturally attenuated, is licensed in South Africa and Zimbabwe (Muller et al. 1995; OIE). Besides live attenuated and inactivated vaccines, recombinant vaccines are on the pipeline, either using poxvirus, vaccinia or chimp adenovirus. Interestingly pox virus based vaccines, Clone 13 and MP12 offer DIVA (differentiating vaccinated from infected animals) potential. Virus like particles and DNA vaccines still offer poor immunogenic capacity (reviewed in Mansfield et al. 2015). Vaccination of ruminants is expected to be the best preventive strategy as human infection is mostly produced by direct contact with infected ruminants. Very often, 
the disease is already well established among livestock population by the onset of human cases. Interestingly, new approaches target the concept of 'One Health' vaccine and seek to license the same vaccine for both ruminants and humans, being a highly attractive approach (Warimwe et al. 2016).

\section{Japanese encephalitis}

Japanese encephalitis virus (JEV) is a major cause of encephalitis in Asia. JEV belongs to the genus Flavivirus, family Flaviviridae. The virus is the nominal member of the JEV serological complex. The serocomplex includes viruses producing important morbidity and mortality: Alfuy, Cacipacore, Koutango, Kunjin (KUNV), JEV, Murray Valley encephalitis, St. Louis encephalitis, Usutu, West Nile (WNV) and Yaounde viruses.

JEV was first isolated from the brain of a fatal encephalitis human case in 1934, Japan (Mitamura et al. 1936) and 4 years later from Culex tritaeniorhynchus Giles mosquitoes. The geographic range of JEV includes temperate and tropical regions of Asia including eastern, southern and southeastern Asia, Papua New Guinea and North of Australia. JEV epidemics were reported periodically in Japan, Korea, and Taiwan every 2-3 years until the mid-1960s, when epidemics ceased due to vaccination and use of pesticides. Since then, major epidemics occurred in Vietnam (1965), Thailand (1969-70), West Bengal (1973), lowland Nepal (1978) and Sri Lanka from 1985 to 1987 (reviewed in Van den Hurk et al. 2009). Most recent JEV reports demonstrate the wide geographical range where the disease could be active, ranging from northern Australia (Hanna et al. 1999) to Pakistan (Igarashi et al. 1994). At the time immunization programs were implemented, the detection of major outbreaks continued. For example, in 2005 an outbreak reported 5,737 human cases with 1,344 deaths in India (Parida et al. 2006). However, JEV national immunization programs might lead to decrease the incidence of the pathogen and number of cases, as experienced in Republic of Korea (Nett et al. 2009). There are at least four genotypes of JEV (Mackenzie et al. 2004). Genotype I strains have been isolated from northern Thailand, Cambodia and Korea. Genotype II typically from Southern Thailand, Malaysia, Indonesia, and Australia. Genotype III has been reported from temperate areas of Asia, including Japan, Korea, China, Taiwan, Philippines, India, and Sri Lanka. Genotype IV was isolated in Indonesia.

\section{Hosts}

Birds are the host reservoirs of the JEV enzootic cycle. About 90 bird species have been found seropositive or infected with JEV (Rodrigues et al. 1981). However, wading ardeid water birds (e.g. herons and egrets) are considered the primary JEV enzootic hosts, with the potential to contribute to epizootics and epidemics (Soman et al. 1977). Nevertheless, other birds present in anthropogenic environments have been related with JEV transmission cycles. Domestic birds, chicken, and ducks, might produce a viremia sufficient to infect mosquitoes, but their contribution to JEV transmission still needs to be evaluated (Lord et al. 2015). Pigs are the only known mammal that serves as JEV amplifyfing hosts because they develop high although not prolonged viremia ( 3-5 days) without clinical symptoms (Ricklin et al. 2016). Although generally asymptomatic, still births and fetal abortions are reported in sows, and aspermia in boars (Takashima et al. 1988). Domestic pig rearing is a relevant risk factor for JEV human transmission because of, backyard pig farming brings the virus closer to human settlements. High seroprevalence rates have been detected in other mammals, but they likely play a minor role for JEV transmission. The primary enzootic JEV vector, C. tritaeniorhynchus, feeds on pigs when available (Pennington and Phelps 1968), however it prefers to feed on cattle when available (Mitchell et al. 1973; Reuben et al. 1992). 
Interestingly, JEV infected cattle develop a viremia too low to infect mosquitoes. Hence abundant presence of bovines might deflect vectors, such as $C$. tritaeniorhynchus, from pigs with the potential to confer passive zooprophilaxis against JEV (Mwandawiro et al. 2000). Horses and humans are susceptible to JEV infection and can develop central nervous system (CNS) symptoms, but are dead-end hosts. Most JEV human infections are asymptomatic, however, symptomatic cases can lead up to $25 \%$ case-fatality rate. Clinical signs range from mild-fever to meningoencephalitis and surviving people can suffer permanent neurological sequelae. JEV is estimated to be the most important cause of viral encephalitis in eastern and southern Asia, with an annual incidence in the range of 50,000-175,000 reported human infections (Ricklin et al. 2016).

\section{Vectors}

More than 30 mosquito species of the genera Aedes, Anopheles, Armigeres, Culex and Mansonia have been described as potential JEV vectors (Le Flohic et al. 2013). However, Culex mosquitoes are the most competent and primary JEV vectors. Culex species inhabiting paddy areas, especially species in the Culex vishnui subgroup are the major enzootic vectors. The primary enzootic vector is C. tritaeniorhynchus (Van den Hurk et al. 2009), a species inhabiting similar ecosystems to C. tarsalis (western USA) and Culex annulirostris Theobald (Australia) both primary vectors of related JEV serogroup viruses in their respective geographic areas. Secondary vectors have been described, including Culex gelidus Theobald, Culex fuscocephala Theobald and C. annulirostris from which the virus has been detected (Peiris et al. 1992; Ritchie et al. 1997; Vythilingam et al. 1997).

Experimental infections have confirmed that C. tritaeniorhynchus is an efficient JEV vector (Takahashi 1976; Weng et al. 2000). Several other Culex species might have the potential to transmit JEV, including C. pipiens var. molestus, Culex pseudovishnui Colless, C. gelidus, C. fuscocephala, C. annulirostris, and Culex sitiens Wiedemann (reviewed in Van den Hurk et al. 2009).

Most JEV vectors do not have restricted or special feeding behaviour and are usually opportunistic blood feeders. Consequently, feeding preferences might bias according to host availability. In Asia, most Culex vectors of JEV present minor preference to feed on humans (2-6\% of blood meals) compared to cattle and pigs. However, an increased availability of humans sleeping outdoors during the hot season would increase the human blood-feeding rate (Reuben et al. 1992). To date, vector control lacks effectiveness against JEV in the absence of innovative mosquito control strategies.

\section{Epidemiology, cycle}

The climatic pattern of JEV-endemic areas influences the epidemiological cycle of the disease. In temperate areas, large epidemics occur during the warmer months, following a summer seasonality (Mackenzie et al. 2004). The length of viraemia in birds and pigs would be too short to maintain JEV during inter-epizootic or inter-epidemic periods. However, JEV vector-free transmission between pigs through direct contact has been described (Ricklin et al. 2016). This proved pigs are highly susceptible to oronasal infection suggesting pig to pig JEV transmission in temperate areas is possible during vector free season. In tropical areas, sporadic cases occur periodically throughout the year, as mosquito vectors, and epizootics or epidemics are associated to the monsoon season (Van den Hurk et al. 2009). The virus is maintained in nature in an enzootic transmission cycle between water birds and mosquitoes, primarily wading ardeid water birds and paddy-rice breeding mosquitoes such as $C$. tritaeniorhynchus. Pigs became the main component of JEV domestic cycle in eastern and southeastern Asia as a result of intensive backyard farming 
and broad development of pig farming industrialization (Le Flohic et al. 2013). Livestock (pigs) proximity to human settings represents the main component for JEV spill-over towards humans. JEV periodically shifts towards pigs, equines and humans.

The specific mechanisms leading to JEV emergence and establishment into new areas are still poorly known. JEV emergence through Asia has been related to a rapid increase of human population with an exponential increase of pig rearing and rice production systems during the last decades (Erlanger et al. 2009). However, these factors might contribute to the emergence and establishment of JEV transmission cycles in peridomestic environments rather than contributing to viral emergence into new areas. Non-migratory birds might promote JEV spread at regional scale, whereas migratory birds might promote large scale spread and viral emergence into new areas. In addition, overseas passive transport of infected vectors by air currents was suggested to be the mechanism for JEV introduction in northern Australia from Papua New Guinea, with a minimal oceanic distance of $160 \mathrm{~km}$ (Johansen et al. 2000). The species C. annulirostris, JEV vector in northern Australia, has an estimated flying range over $500 \mathrm{~km}$ (Kay and Farrow 2000). Therefore, C. annulirostris was expected to be able to overcome the minimal oceanic distance (about $160 \mathrm{~km}$ ) separating Papua New Guinea from Northern Australia. Similarly, windblown passive transport was described for C. tritaeniorhynchus in China (Ming et al. 1993) and India (Reynolds et al. 1996). Finally, international travellers are unlikely to contribute to JEV dispersion for being dead-end hosts with short and low viremia levels. The transport of vectors though trade might not be important due to the bio-ecology of JEV vectors and similarly via passenger airplanes.

\section{Vaccines}

Live attenuated JEV vaccines are licensed for human and animal use, however multiple doses are required to boost immunity. There is no antiviral treatment for JEV infections. Prevention might be achieved using an integrated strategy targeting vaccination, vector control, and husbandry (Igarashi 2002). Pig vaccination might be a potential control strategy to limit JEV epizootics/ epidemics. In addition, vaccination protects sows against abortion. Unfortunately, vaccination is not widely used as the high turnover in pig population would require annual vaccination and maternal antibodies interfere with piglet immunization (Igarashi 2002). However, to reduce equine encephalitis caused by JEV, Japan applied yearly vaccination of horses since 1948 (Goto 1976). Similarly, mandatory vaccination of race-horses is required at some Asiatic countries. The impossibility to reduce the spread of JEV despite the presence of effective vaccines for decades is of special veterinary and public health concern. However, successful control programmes have been implemented in Japan and South Korea. These countries decreased JEV incidence through pig and human vaccination programs, changes in agricultural practices and relocation of pig rearing away from human settlements (Erlanger et al. 2009).

\section{West Nile fever}

WNV is the causal agent of West Nile fever. The virus was first isolated from a human case in 1937 in Uganda, East Africa (Smithburn et al. 1940). WNV is an enveloped, single-stranded RNA virus of the Flavivirus genus, family Flaviviridae (Kramer et al. 2008). The virus is serologically classified within the Japanese encephalitis serocomplex. Based on phylogenetic analyses two major WNV lineages have been described, lineage 1 and 2, despite up to seven genetic lineages have been proposed (Rizzoli et al. 2015). Lineage 1 is prevalent worldwide and has virulent and attenuated viral isolates. Lineage 1 consists of two clades, clade $1 \mathrm{a}$ and $1 \mathrm{~b}$. Clade $1 \mathrm{a}$ is present in Africa, Europe, Middle East, Asia and the American continent. Clade 1b is present in Australia and locally 
known as Kunjin virus. Lineage 2 was historically prevalent in sub-Saharan Africa and Madagascar causing asymptomatic infections. However, virulent isolates have been detected in Europe in the $21^{\text {st }}$ century (Blitvich 2008).

Until the mid-1990s, WNV usually produced asymptomatic infections and sporadic outbreaks across rural landscapes in Africa, Asia and Australia, with few cases of neurological disease (Blitvich 2008). During that period, WNV was not considered a major threat to animal or humans. However, the pattern changed by mid-1990s with an increase of the frequency, geographic range and severity of the outbreaks, with a rise of neurologic disease incidence in horses and humans. Between 1996 and 2000 four major epidemics were present in urban environments in Romania, the Volga Delta, Israel and Northeastern USA. In Israel (1997-1999) severe disease and death was first reported in domestic birds, affecting hundreds of young geese. However, the most significant event was the first introduction of WNV in the Western Hemisphere, causing an initial outbreak in New York City, 1999. There, high wild bird mortality (thousands) preceded a severe outbreak with equine and human encephalitis. The strain introduced, WN-NY99, isolated from a flamingo in the Bronx Zoo belonged to Lineage 1 (Lanciotti et al. 1999). Genetic data of isolates suggested strain WN-NY99 could be introduced from the Middle East, but to date, the mode of introduction still remains unclear (Blitvich 2008). Since the introduction of WNV in Eastern USA, New York, the virus became established and experienced an unprecedented dissemination all over the Americas. The virus successfully spread into Canada (2001), Mexico (2002) and some areas of the Caribbean (2002), Central (2003) and South (2004) America. In the USA WNV became endemic and recurrent outbreaks have been affecting birds, equines and humans for 16 consecutive years, representing the largest epidemic of WNV in the world.

In Australia, infection with WNV Kunjin strain usually occurs sporadically and encephalitis cases are rare. However, in 2011, a WNV (Kunjin strain) outbreak occurred among horses in New South Wales. Estimations suggested up to 1,000 horses affected, with $10-15 \%$ case-fatality rate (Frost et al. 2012). The isolated strain had new aminoacid substitutions that might explain enhanced virulence in horses.

\section{Hosts}

A large variety of bird species has been shown to be susceptible to WNV infection. Evidence for WNV infection has been detected in $>300$ species of birds (Blitvich 2008). Birds are considered the most important WNV amplifying hosts, they develop a high viremia that allows the infection of susceptible mosquitoes (Chancey et al. 2015). Passeriformes are considered to be the main WNV reservoir hosts. Among Passeriformes, house sparrow was suggested to have a primary role to WNV cycle in North America and Europe (Blitvich 2008). Bird disease causes neurologic signs as ataxia, paralysis, incoordination, and non-neurologic signs including depression lethargy ruffled feathers, weight loss and myocarditis (Blitvich 2008).

Equines and humans are susceptible to WNV infection and may suffer severe disease or even death. However, they are considered dead-end or incidental hosts as they develop a viremia too low to infect mosquitoes, without contribution to WNV cycle. In horses, most WNV infections are asymptomatic, however WNV caused extensive morbidity and mortality in horses during recent outbreaks in Europe, Israel and USA (Blitvich 2008). The illness usually lasts 3 weeks and is characterized by fever and a range of neurologic signs including ataxia, muscular weakness and amaurosis. WNV has important CNS tropism in horses, consequently, important mortality is associated to symptomatic (illness) cases. Most WNV infections in humans are asymptomatic. 
However, in recent urban outbreaks in Europe, Israel and USA about 20\% infections cause West Nile fever, a mild flu-like illness (Blitvich 2008). The illness lasts for 2-5 days with non-specific symptoms with most common fever, headache, myalgia, fatigue, weakness, vomiting and diarrhea. Severe neuroinvasive disease is present in about $1 / 150$ infections, leading to encephalitis, meningitis, with a fatality rate $\sim 10 \%$.

Other non-avian vertebrates have been reported to be susceptible to WNV infection but disease occurrence is less common. For example, naturally-acquired WNV infection has been documented in a wide range of mammalian species. Experimentally infected reptiles and amphibians have proven to be susceptible to WNV infection, sometimes with viremia sufficient to infect susceptible mosquitoes (Blitvich 2008). High viremia expected to support vertebrate-mosquito WNV transmission has been reported in other non-avian vertebrates, including brown lemur (Lemur fulvus), lake frogs (Rana rinibunda), hamsters, fox squirrels (Sciurus niger), eastern gray squirrels (Sciurus carolinensis), eastern cottontail rabbits (Sylvilagus floridanus) and eastern chipmunks (Tamias striatus; Chancey et al. 2015).

The WNV transmission between susceptible hosts in the absence of mosquito vectors has been suggested (Kipp et al. 2006). Direct WNV transmission by fecal-oral route between viremic and susceptible birds, or oral consumption of WNV infected meat by some carnivore mammals (Austgen et al. 2004) and reptiles (Klenk et al. 2004) might be possible (Blitvich 2008).

\section{Vectors}

Mosquitoes are the vectors that support the WNV transmission cycle. The primary WNV amplifying vectors are mosquitoes of the genus Culex. Several Culex species support WNV enzootic circulation between birds and modulate the seasonal shift of the virus between birds and non-bird vertebrates (Rizzoli et al. 2015). However WNV has been isolated in species of 11 other genera and at least 65 mosquito species in the USA have been implicated in WNV transmission according to the CDC. Worldwide, the range mosquito species that act as primary WNV amplification vectors is diverse: in Europe and Africa, C. pipiens, Culex univitattus Theobald and Culex antennatus Becker; in Asia, mainly species in the Culex vishnui complex, as C. pseudovishnui Colless, C. vishnui and $C$. tritaeniorhynchus; in Australia, C. annulirostris; in the USA, C. pipiens and Culex restuans Theobald in the northeast, C. tarsalis in the west and Culex quinquefasciatus Say in the south (Blitvich 2008). In Central and South America, it remains uncertain which particular species act as primary vector, available information is scarce due to the apparent asymptomatic circulation.

Laboratory infection of mosquitoes with WNV provides useful information to identify potential vectors as well. In USA, C. pipiens, Culex nigripalpus Theobald and C. quinquefasciatus were extensively characterized and confirmed to transmit WNV. In Europe, C. pipiens, Culex modestus Ficalbi and Ae. albopictus showed to be competent species for WNV. In Australia, several species were described to be highly competent for WNV, including C. annulirostris, C. quinquefasciatus, C. gelidus, or moderate competent, including Aedes notoscriptus Skuse, Aedes vigilax Skuse and Aedes procax Skuse.

Viral overwintering in adult mosquitoes was described in February 2000 in New York city when WNV was isolated from overwintering C. pipiens mosquitoes (Nasci et al. 2001). 


\section{Epidemiology, cycle}

WNV is considered an ecological generalist for its capacity to infect a wide range of mosquitoes and birds, a characteristic contributing to the pathogen global expansion and current distribution (Kramer et al. 2008). The virus is maintained in nature in an enzootic transmission cycle between mosquitoes and birds. Ornitophilic mosquitoes are the amplification or enzootic vectors that contribute to WNV enzootic cycle. Moreover, bridge vectors have the potential to guide the occasional WNV epizootic spill-over and infect non-bird vertebrates. Bridge vectors are mosquitoes with general feeding habits, hence they can infect non-bird vertebrates while refeeding on them after feeding upon a viraemic bird. For example, the temporal shift on C. pipiens feeding behaviour in north-eastern USA was related to a high increase of human WNV cases. In late spring, C. pipiens bites preferably American robins (primary hosts), that are abundant. By late summer, they bite mainly humans as migration decreases American robin density (Blitvich 2008). In the tropics, warmer temperatures support longer WNV transmission seasons compared to temperate regions. In addition, warm temperatures would lead to higher transmission intensity as well, as a result of higher biting rate and increased mosquito and virus development rates (Kilpatrick 2011).

The epidemiology of WNV transmission cycles is complex and influenced by several intrinsic and extrinsic factors. Best known intrinsic factors include the variable competence of different species and populations of vectors and hosts, the differential fitness of viral strains for vector and host infection, and the vector and host life-history parameters (development time, longevity and mosquito feeding behaviour). Best known extrinsic factors includes temperature, rainfall, landscape usage and density of hosts and vectors. Temperature is considered to be a critical parameter modulating the susceptibility of mosquitos to WNV infection. For example, C. pipiens and $\mathrm{C}$. tarsalis were more competent at high temperature $\left(28-30{ }^{\circ} \mathrm{C}\right)$ than at lower $\left(14-22{ }^{\circ} \mathrm{C}\right)$ in laboratory studies. In addition, periods of high (above-average) summer temperatures were experienced in some of the major WNV epidemics: Romania (1996), Russia (1999) and USA between 2002 and 2004. The long-distance dispersal of WNV has been associated to viremic migratory birds in the New World (Rappole et al. 2000). Migratory routes might provide important clues into the global dynamics of WNV large-scale spread. Once WNV introduced into a disease-free area, non-directional movement of infected mosquitoes and residential or synanthropic birds would disperse the virus locally (Kramer et al. 2008). After introduction and local spread, several mechanisms might lead to WNV inter-annual persistence in enzootic transmission cycles either in temperate and tropical areas. Enzootic cycle could be sustained through a basal (low-level) horizontal transmission between mosquitoes and birds, horizontal (faecal-oral) transmission between birds, persistent infection in certain bird tissues, and vertical transmission in mosquitoes.

\section{Vaccines}

Several licensed vaccines are available and widely used for horses due to the impact of WNV to the horse industry. Avian WNV vaccines are used in zoos and domestic geese (Malkinson et al. 2001; Okeson et al. 2007). To date no licensed vaccine is available for humans, however promising candidate approaches are under study (Rizzoli et al. 2015).

\section{Equine encephalitis}

Several viruses of the genus Alphavirus, family Togaviridae, produce highly neurovirulent disease in horses and humans. In the western hemisphere, the most commonly reported alphaviruses isolated in horses and humans are Eastern, Western and Venezuelan equine encephalitis. The 
three alphaviruses are related, but genetically and antigenically different. Other alphaviruses are present around the world with an epizootic, zoonotic or epidemic potential. Equines are important in Latin America for agriculture and transport, therefore, equine encephalitis are a social and economic concern (Weaver et al. 2004).

Eastern equine encephalitis virus (EEEV) was isolated in horses from eastern USA in 1933 (Giltner and Shahan 1933). In South America, few EEEV epizootics were reported in horses before 2000. However, in 2008-2009, large outbreaks in Central and South America were reported (Long 2014). High mortality was reported in north-eastern Brazil outbreaks with case-fatality rate of $73 \%$ from 229 infected horses (Silva et al. 2011).

Western equine encephalitis virus (WEEV) was isolated from the brain of a horse with encephalitis from western USA in 1930, California State (Meyer et al. 1931). However, reports of WEEV outbreaks in horses have been scarce and limited during the last decade.

Venezuelan equine encephalitis virus (VEEV) is one of the most important human and veterinary pathogens of the American continent. VEEV has the potential for a rapid spread causing explosive outbreaks with high equine mortality rates. VEEV was described to cause disease in equine livestock, horses, donkeys and mules, in northern South America during the 1930s (Weaver et al. 2004). Since then, large VEEV outbreaks occurred periodically with inter-epidemic periods of about 10 years, until a major outbreak spread to central and North America. During 19691972 VEEV spread into Central America, Mexico and the southern USA (Texas) provoking tens of thousands of equine and human cases. Of special remark were some of the largest outbreaks recorded in central Colombia during the 1960 s with estimated 200,000 human cases and 100,000 equine deaths (Weaver et al. 2004). After this major epizootic, no activity was reported during the following 2 decades (1973-1992; Weaver et al. 2004). Then, one of the largest epizootics occurred in Venezuela, in 1995, with estimated 75,000-100,000 human infections.

\section{Hosts, symptomatology}

Birds are the enzootic hosts of EEEV. However, the detection of snakes naturally infected with EEEV in North America provided evidence for their involvement as non-avian hosts, with the potential to facilitate viral maintenance as over-wintering reservoirs (Bingham et al. 2012).

EEEV infection in horses causes a deterioration of mentation associated to fore-brain damage and results in an extremely low survivorship (Silva et al. 2011). Humans are susceptible to EEEV, but act as dead-end hosts. Human infection can lead to disease and might include chills, fever, malaise and brain swelling. EEEV causes probably the most virulent human infection of the three equine encephalitis alphaviruses. Human symptomatic cases develop an acute viral infection that can lead to case-fatality rates ranging 30-70\% (Silverman et al. 2013), and survivors often show residual neurologic sequelae (Villari et al. 1995).

WEEV infection in young animals produces clinical CNS disease. Horses usually recover from infection and case-fatality rate ranges 20-30\%. Most WEEV human infections tend to be asymptomatic or cause mild disease, case fatality-rate is estimated 3-7\% (Arechiga-Ceballos and Aguilar-Setien 2015). However, WEEV infections in children produce clinical signs more frequently, with the potential to evolve to CNS clinical disease. 
Equine and human VEEV-infection ranges from asymptomatic to acute encephalitis. When infection produces disease in equine, symptoms include fever, tachycardia, depression and anorexia with the potential to evolve towards encephalitis 5-10 days after infection. Experimental infections reported a VEEV mean equine peak viraemia of $10^{5}$ suckling mouse $\mathrm{LD}_{50}$ per $\mathrm{ml}$ serum, but sometimes exceeding $10^{7}$ (Wang et al. 2001). In epizootics, rate estimates for equine mortality range $19-83 \%$, whereas less frequent in humans with neurologic disease in $4-14 \%$ of detected cases (Weaver et al. 2004). However, VEEV might affect more severely children that tend to develop more frequently fatal encephalitis or permanent neurologic sequelae. In addition, the virus has the potential to cross the trans-placental barrier and is able to infect foetus causing birth defects, abortions and stillbirths. Human VEEV infections are usually symptomatic, including fever, chills, malaise and severe headache that can be accompanied by tachycardia, nausea, vomiting and diarrhoea. Less frequently, infection leads to CNS symptoms and sometimes death ( $<1 \%$ of cases). Non-human primates and a range of other vertebrates are susceptible to VEEV infection and suffer fatal disease: rabbits, dogs, goats, sheep, guinea pigs and laboratory rodents (hamster and mice). However, there is strong evidence for bats to serve as natural reservoirs and contribute to enzootic and epizootic VEEV dissemination (Weaver et al. 2004).

\section{Vectors}

The known enzootic vectors of EEEV, WEEV and VEEV are primarily Culex mosquitoes of the Spissipes sections. However, in North America, Culiseta melanura (Coquillett) would act as the primary enzootic vector of EEEV maintaining the virus among avian hosts (Molaei et al. 2015). Known EEEV bridge vectors includes the species Ae. vexans, Coquillettidia perturbans Walker, Ochlerotatus canadensis Theobald and Ochlerotatus sollicitans Walker. These species have an opportunistic feeding behaviour and are involved in the transmission of EEEV in epidemic or epizootic transmission cycles (Armstrong and Andreadis 2010). The opportunistic feeding pattern of Culex erraticus Dyar \& Knab suggested its involvement in the bridging potential of EEEV toward horses and humans (Estep et al. 2011).

The WEEV enzootic vector is $C$. tarsalis, a species associated to irrigated cultures and stream drainage in the western USA. In the genus Culex, mosquitoes in the Melanoconion and Spissipes sections constitute the whole subgenus Melanoconion. The ecology of species in the subgenus Melanoconion still remains poorly understood. It is primarily associated to tropical forests and swamps where they feed on sylvatic animals and occasionally on humans and domestic animals. The known WEEV bridge vectors in USA include Ochlerotatus melanimon Dyar, (California), Aedes dorsalis Meigen, (Utah and New Mexico) and Aedes campestris Dyar \& Knab (New Mexico).

The main VEE enzootic vectors are 0 . taeniorhynchus, Psorophora confinnis Lynch Arribálzaga and Anopheles aquasalis Curry which were highly abundant during the Venezuela and Colombia outbreaks and abundant mosquito populations coincided with unusual heavy rains through July, August, and September. The VEE viral adaptation to the salt marsh species 0 . taeniorhynchus has been primarily related with epizootic or epidemic outbreaks (Weaver et al. 2004). Other mosquito species such as $P$. confinnis and $O$. sollicitans have been related to VEEV epizootics as well. The VEEV bridge vectors in the USA likely are Psorophora columbiae (Dyar \& Knab) and Ae. albopictus both of which are common and highly abundant in the southern USA in agricultural and urban areas respectively (Moncayo et al. 2008; Moore and Mitchell 1997). 


\section{Epidemiology, cycle}

Equine encephalitis produced by alphaviruses are typically maintained in nature in enzootic cycles. Periodical shifts in the host reservoirs and mosquito vectors lead to pathogen spill-over from enzootic towards epizootic cycle. During the spill-over towards the epizootic cycle, the EEEV, WEEV, and VEEV have the potential to transmit the virus to equines and humans.

Viral transmission between ornitophilic mosquitoes and birds supports EEEV and WEEV enzootic cycles. Birds, Passerines among others, act as natural reservoirs and amplifying hosts. Therefore, in temperate areas, EEEV and WEEV might overwinter in enzootic hosts or be reintroduced by migratory birds. Freshwater swamps are suitable environments for EEEV transmission cycles (Long 2014). Equines and humans are considered dead-end hosts for EEEV and WEEV as infections produce a viremia too low to infect mosquitoes.

The VEEV enzootic cycle involves primarily sylvatic rodents (spiny and cotton rats) as reservoir hosts (Carrara et al. 2005). The epizootic transmission cycle of VEEV is characterized by the role of equines as efficient amplification hosts. However, some subtypes of VEEV are not able to use equines as epizootic reservoir hosts. In addition, humans develop high viremia titter and might serve as hosts as well. Other vertebrates are susceptible to infection, but major outbreaks have not been reported in the absence of equines (Weaver et al. 2004).

\section{Vaccines}

A licensed vaccine is available to protect horses from EEEV, but requires several vaccinations per year. Proper vaccination prevents horse infection, however horse fatalities are periodically reported in North America in non-immunized animals. In addition, EEEV horse vaccination induces cross-immunity for WEEV. There is currently no licensed human vaccine or antiviral treatment for EEEV. Horses are the primary reservoir host for VEEV epidemics, and are consequently a primary vaccination target for Public Health prevention strategy in Central and South America (Weaver et al. 2004). Several VEEV vaccine strategies have been developed, recombinant subunit, inactivated, live-attenuated or DNA vaccines. Interestingly, compounds with VEEV and WEEV (but not EEEV) anti-viral activity have been recently discovered (Chung et al. 2014).

The recent emergence of EEEV in disease-free areas of USA and Panama (Carrera et al. 2013) evidenced the strategic need to develop a trivalent-alphavirus (EEEV, VEEV, WEEV) vaccine to be licensed for human use. In addition, there is rising need to develop new vaccines inducing proper and long immunity in naïve and young horses.

\section{Emergence of mosquito-borne diseases}

The emergence and spread of mosquito-borne pathogens continues to pose significant veterinary and public health risk worldwide. The introduction of a given pathogen into a naïve population has the potential to produce significant morbidity and mortality, and might lead to a major health crisis. The emergence and spread of such pathogens is the output of a complex interplay of ecologic, environmental, climatic and anthropogenic factors. Therefore, to predict the emergence of mosquito-borne diseases remains difficult.

Past and recent epidemics provide insights into the potential emergence of new pathogens transmitted by mosquitoes. Anthropogenic landscape modification towards urbanized and 
agricultural habitats provide new ecological niches for pathogens. Landscape modification has the potential to bring together mosquitoes, amplifying hosts, livestock, and humans. Hence, increased risks of pathogen shift from enzootic cycle towards livestock and/or humans, as seen for JEV in Asia and WNV in USA. As an example, an increase of WNV incidence was correlated with urbanization and agriculture in the USA (Bowden et al. 2011).

Globalization linked to rapid transportation systems for trade and travellers has definitively overcome biogeographic barriers and political boundaries favouring large scale mosquito and pathogen introduction. The introduction of major mosquito-borne disease vectors has been already reported from different regions of the globe. The most significant mosquito species with reported introductions includes C. pipiens, C. quinquefasciatus, Ae. albopictus or Aedes aegypti Linnaeus (Kilpatrick 2011). Nevertheless, wind-borne dispersal of virus carrying vectors has been reported as spreading mechanism for some arboviral diseases, including bluetongue (Culicoides) and Japanese encephalitis (mosquitoes). Large-scale overseas migration of mosquitoes might be feasible during tropical low-pressure systems present in cyclonic or monsoonal weather events (Chapman et al. 1997).

At risk areas for mosquito-borne virus introduction can be predicted based on the presence of mosquito vector species, competent amplifying and reservoir host species, and favourable climatic conditions. Viral threat and risk assessment modelling have determined three likely pathways of virus introduction: (1) imported infected animals; (2) infected humans; or (3) infected mosquito vectors. These pathways can be accidental or intentional as an act of bioterrorism. Additional methods have been mentioned previously in this chapters, such as wind-borne or migratory birds or animals, but these are less likely for between continent introductions. After introduction and establishment, the viruses would be largely impossible to eliminate because of the wild mammal and avian host reservoir densities and distributions, as was seen with West Nile virus introduction to North and South America. Therefore prevention and surveillance are vital to prevent or limit introductions.

RVFV was largely limited to Africa with a single outbreak in the Arabian Peninsula caused by the transportation of infected animals. RVFV network modelling which can simulate mosquito, host, and human population interactions has described past transmission dynamics in South Africa (Xue et al. 2012) and theoretical transmission in the USA (Xue et al. 2013). After an RVF introduction mitigation would be difficult due to the extensive animal movement between and within USA farms (Scogligo et al. 2016). Europe has the same challenges with competent mosquito vector species, abundant animal reservoirs, and favourable climatic conditions with the added risk of close proximity and animal trade with African countries (Rolin et al. 2013).

The JEV geographic range has largely been limited to South East Asia, but North and South America, and Europe all have competent mosquito species and abundant animal reservoirs. Additionally in the last 20 years, increases in feral swine abundance and distribution on all three continents has increased the presence of this JEV amplifying host. The increase in feral swine was modelled in the USA and this suggests in the next 30-50 years most counties nationwide could be inhabited by feral swine (Snow et al. 2017). Mathematical modelling of JEV spread in the USA has demonstrated how JEV will spread geographically by birds and maintained locally by swine throughout the eastern USA (Riad et al. 2016). JEV may ultimately have the same geographic distribution as the related WNV, which is already globally distributed (reviewed by Chancey et al. 2015 and Ciota and Kramer 2013) and continues to spread to new countries because of the high number of competent mosquitoe species and avian reservoirs. 
VEEV is an exception to the previously listed viruses because although competent vector species are highly abundant in the southern USA, this virus was not maintained after a 1972 introduction in Texas. However, a major VEE competent mosquito species Ae. albopictus, was not introduced to the USA until 1987 (Moore and Mitchell 1997). This vector species is highly abundant in the USA, South America, and Europe and could pose a serious threat to humans and livestock if VEEV is subsequently introduced based strictly on mosquito and host animal distributions (Benedict et al. 2007).

In arboviruses, viral adaptation to new hosts and vectors represents a major threat for disease emergence. Known viruses with historically low burden for animal and/or public health can shift into new emergent epizootic and/or epidemic strains through viral adaptation. For diseases having enzootic and epizootic (or epidemic) transmission cycles, the spill over of the virus from enzootic cycle into epizootic vectors and hosts is a pre-requisite for disease emergence. For example, phylogenetic studies evidenced enzootic VEEV strains mutate to a convergent epizootic phenotype generating the epizootic strains. Comparative genomics suggested virus evolved to infect new host and mosquito species leading to VEEV emergence, with a special focus on E2 envelope glycoprotein mutations (Weaver et al. 2004). Similarly mutations in WNV lineages 1 and 2 , increased nueroinvasion and virulence compared to the other six lineages and consequently explain the North American invasion (Beasley et al. 2002; Donadieu et al. 2013). Additionally, a mutation in the E1 envelope protein (E1-A226V) of Chikungunya virus was responsible for an increase in viral infectivity and transmission in Ae. albopictus (Tsetsarkin et al. 2007). The emergent new viral strain adapted to Ae. albopictus was the origin of large-scale epidemics even in regions lacking the typical vector (Ae. aegypti). When a virus is introduced into a new area, such mechanisms might lead to the establishment of new, sometimes improved, transmission cycles.

Global climate change will undoubtedly affect the dynamics of mosquito-borne diseases through modulation of important abiotic factors. Ambient temperature is a major factor regulating viral replication and mosquito development. Hence, above-average temperatures would increase viral replication and evolution rates. The mosquito would increase growth rate, shorten interval between mosquito bloodmeals and reduce the pathogen extrinsic incubation period. In addition, a temperature increase can enhance the vector competence of mosquitoes for certain pathogens (Kilpatrick et al. 2008). Therefore, the temperature increase would increase the magnitude of pathogen transmission and outbreaks. In addition, the rise of temperature in temperate regions might elongate the effective transmission season and increase the areas suitable for the establishment of mosquito transmitted diseases. However, temperature above a certain threshold would slow mosquito activity and reduce larval survival. Global warming might modulate precipitation patterns, however it is not obvious how a change in precipitation would affect the final transmission output. As an example, above-average rainfall would increase mosquito abundance, however heavy rainfall can flush ditches and drainage channels used by Culex larvae reducing local population density (Paz and Semenza 2013). Below-average rainfall and drought would reduce larval breeding places and probably reduce mosquito populations. However, droughts might bring wild hosts vertebrates in closer contact with mosquitoes around scarce water sources, thus enhancing the enzootic transmission cycle.

The diseases listed before, RVFV, EEEV, WEEV, VEEV, JEV and WNV, evidenced the capacity of mosquito-borne viruses to spread and colonize into previous disease-free areas. Better understanding of the particular disease dynamics would contribute to improve preparedness and better approaches to implement more effective mosquito-borne disease control strategies. 


\section{References}

Al-Afaleq Al and Hussein MF (2011) The status of Rift Valley fever in animals in Saudi Arabia: a mini review. Vector Borne Zoonotic Dis 11: 1513-1520.

Albayrak H and Ozan E (2012) The investigation of pestivirus and Rift Valley fever virus infections in aborted ruminant foetuses in the Blacksea region in Turkey. Kafkas Univ Vet Fak Derg 18: 457-461.

Anyamba A, Linthicum KJ, Small J, Britch SC, Pak E, De la Rocque S, Formenty P, Hightower AW, Breiman RF, Chretien JP, Tucker CJ, Schnabel D, Sang R, Haagsma K, Latham M, Lewandowski HB, Magdi SO, Mohamed MA, Nguku PM, Reynes JM and Swanepoel R (2010) Prediction, assessment of the Rift Valley fever activity in East and Southern Africa 2006-2008 and possible vector control strategies. Am J Trop Med Hyg 83: 43-51.

Arechiga-Ceballos N and Aguilar-Setien A (2015) Alphaviral equine encephalomyelitis (Eastern, Western and Venezuelan). Rev Sci Tech 34: 491-501.

Armstrong PM and Andreadis TG (2010) Eastern equine encephalitis virus in mosquitoes and their role as bridge vectors. Emerg Infect Dis 16: 1869-1874.

Austgen LE, Bowen RA, Bunning ML, Davis BS, Mitchell CJ and Chang GJ (2004) Experimental infection of cats and dogs with West Nile virus. Emerg Infect Dis 10: 82-86.

Balkhy HH and Memish ZA (2003) Rift Valley fever: an uninvited zoonosis in the Arabian peninsula. Int J Antimicrob Agents 21: 153-157.

Beasley DW, Li L, Suderman MT and Barrett AD (2002) Mouse neuroinvasive phenotype of West Nile strains varies depending upon virus serotype. Virology 296: 17-23.

Behura SK (2015) Insect phylogenomics. Insect Mol Biol 24: 403-411.

Benedict MQ, Levine RS, Hawley WA and Lounibos LP (2007) Spread of the tiger: global risk of invasion by the mosquito Aedes albopictus. Vector-Borne Zoonotic Dis 7: 76-85.

Bingham AM, Graham SP, Burkett-Cadena ND, White GS, Hassan HK and Unnasch TR (2012) Detection of eastern equine encephalomyelitis virus RNA in North American snakes. Am J Trop Med Hyg 87: 1140-1144.

Bird BH, Ksiazek TG, Nichol ST and Maclachlan NJ (2009) Rift Valley fever virus. J Am Vet Med Assoc 234: 883-893.

Blitvich BJ (2008) Transmission dynamics and changing epidemiology of West Nile virus. Anim Health Res Rev 9: 71-86.

Bowden SE, Magori K and Drake JM (2011) Regional differences in the association between land cover and West Nile virus disease incidence in humans in the United States. Am J Trop Med Hyg 84: 234-238.

Brustolin $M$, Talavera S, Nuñez A, Santamaría C, Rivas R, Pujol N, Valle $M$, Verdún $M$, Brun $A$, Pagès $N$ and Busquets $N$ (2017) Rift Valley fever virus and European mosquitoes: vector competence of Culex pipiens and Stegomyia albopicta (=Aedes albopictus). Med Vet Ent 31:365-372.

Carrara AS, Gonzales G, Ferro C, Tamayo M, Aronson J, Paessler S, Anishchenko M, Boshell J and Weaver SC (2005) Venezuelan equine encephalitis virus infection of spiny rats. Emerg Infect Dis 11: 663-669.

Carrera JP, Forrester N, Wang E, Vittor AY, Haddow AD, Lopez-Verges S, Abadia I, Castano E, Sosa N, Baez C, Estripeaut D, Diaz Y, Beltran D, Cisneros J, Cedeno HG, Travassos da Rosa AP, Hernandez H, Martinez-Torres AO, Tesh RB and Weaver SC (2013) Eastern equine encephalitis in Latin America. N Engl J Med 369: 732-744.

Chancey C, Grinev A, Volkova E and Rios M (2015) The global ecology and epidemiology of West Nile virus. Biomed Res Int 2015: 376230.

Chapman HF, Hughes JM, Kay BH and Ritchie SA (1997) The role of population genetics in mosquito control. Arbovirus Res Aust 7: 36-40.

Chevalier V, De la Rocque S, Baldet T, Vial L and Roger F (2005) Epidemiological processes involved in the emergence of vectorborne diseases: West Nile fever, Rift Valley fever, Japanese encephalitis and Crimean-Congo haemorrhagic fever. Rev Sci Tech 23: 535-555.

Chevalier V, Pepin M, Plee L and Lancelot R (2010) Rift Valley fever - a threat for Europe? Euro Surveill 15: 19506.

Chevalier V, Rakotondrafara T, Jourdan M, Heraud JM, Andriamanivo HR, Durand B, Ravaomanana J, Rollin PE and Rakotondravao R (2011) An unexpected recurrent transmission of Rift Valley fever virus in cattle in a temperate and mountainous area of Madagascar. PLoS Negl Trop Dis 5: e1423. 
Chung DH, Jonsson CB, Tower NA, Chu YK, Sahin E, Golden JE, Noah JW, Schroeder CE, Sotsky JB, Sosa MI, Cramer DE, McKellip SN, Rasmussen L, White EL, Schmaljohn CS, Julander JG, Smith JM, Filone CM, Connor JH, Sakurai Y and Davey RA (2014) Discovery of a novel compound with anti-venezuelan equine encephalitis virus activity that targets the nonstructural protein 2. PLoS Pathog 10: e1004213.

Ciota AT and Kramer LD (2013) Vector-virus interactions and transmission dynamics of West Nile virus. Viruses 5: 3021-3047.

Clements AN (1992) The biology of mosquitoes: development, nutrition, and reproduction. Chapman \& Hall, London, UK.

Daubney R and Hudson JR (1932) Rift Valley fever. Lancet 219: 611-612.

Donadieu E, Bahuon C, Lowenski S, Zientara S, Coulpier M and Lecollinet S (2013) Differential virulence and pathogenesis of West Nile virus. Viruses 5: 2856-2880.

Duvallet $\mathrm{G}$ and Boireau P (2015) Other vector-borne parasitic diseases: animal helminthiases, bovine besnoitiosis and malaria. Rev Sci Tech 34: 651-658.

Edwards FW (1932) Genera insectorum. Diptera, Fam. Culicidae, Fascicle 194. Desmet-Verteneuil, Brussels, Belgium, $258 \mathrm{pp}$.

European Food Safety Authority (EFSA) (2005) The risk of a Rift Valley fever incursion and its persistence within the community. EFSA Journal 238: 1-128.

El-Harrak M, Martin-Folgar R, Llorente F, Fernandez-Pacheco P, Brun A, Figuerola J and Jimenez-Clavero MA (2011) Rift Valley and West Nile virus antibodies in camels, North Africa. Emerg Infect Dis 17: 2372-2374.

El Mamy AB, Baba MO, Barry Y, Isselmou K, Dia ML, El Kory MO, Diop M, Lo MM, Thiongane Y, Bengoumi M, Puech L, Plee L, Claes F, De la Rocque S and Doumbia B (2011) Unexpected Rift Valley fever outbreak, northern Mauritania. Emerg Infect Dis 17: 1894-1896.

Eldridge BF (1992) Patrick Manson and the discovery age of vector biology. J Am Mosq Control Assoc 8: 215-220.

Erlanger TE, Weiss S, Keiser J, Utzinger J and Wiedenmayer K (2009) Past, present, and future of Japanese encephalitis. Emerg Infect Dis 15: 1-7.

Estep LK, McClure CJ, Burkett-Cadena ND, Hassan HK, Hicks TL, Unnasch TR and Hill GE (2011) A multi-year study of mosquito feeding patterns on avian hosts in a southeastern focus of eastern equine encephalitis virus. Am J Trop Med Hyg 84: 718-726.

Fontaine MC, Pease JB, Steele A, Waterhouse RM, Neafsey DE, Sharakhov IV, Jiang X, Hall AB, Catteruccia F, Kakani E, Mitchell SN, Wu YC, Smith HA, Love RR, Lawniczak MK, Slotman MA, Emrich SJ, Hahn MW and Besansky NJ (2015) Mosquito genomics. Extensive introgression in a malaria vector species complex revealed by phylogenomics. Science 347: 1258524.

Foster WA and Walker ED (2002) Mosquitoes (Culicidae). In: Mullen G and Durden L (eds.) Medical and veterinary entomology. Academic Press, Amsterdam, the Netherlands.

Frost MJ, Zhang J, Edmonds JH, Prow NA, Gu X, Davis R, Hornitzky C, Arzey KE, Finlaison D, Hick P, Read A, HobsonPeters J, May FJ, Doggett SL, Haniotis J, Russell RC, Hall RA, Khromykh AA and Kirkland PD (2012) Characterization of virulent West Nile virus Kunjin strain, Australia, 2011. Emerg Infect Dis 18: 792-800.

Gale P, Brouwer A, Ramnial V, Kelly L, Kosmider R, Fooks AR and Snary EL (2010) Assessing the impact of climate change on vector-borne viruses in the EU through the elicitation of expert opinion. Epidemiol Infect 138: 214-225.

Gerdes GH (2004) Rift Valley fever. Rev Sci Tech 23: 613-623.

Giltner LT and Shahan MS (1933) The 1933 outbreak of infectious equine encephalomyelitis in the eastern states. N Am Vet 14: 25-27.

Golnar AJ, Turell MJ, LaBeaud AD, Kading RC and Hamer GL (2014) Predicting the mosquito species and vertebrate species involved in the theoretical transmission of Rift Valley fever virus in the United States. PLoS Negl Trop Dis 8: e3163.

Goto H (1976) Efficacy of Japanese encephalitis vaccine in horses. Equine Vet J 8: 126-127.

Gubler DJ (2002) The global emergence/resurgence of arboviral diseases as public health problems. Arch Med Res 33: 330-342. 
Hanna JN, Ritchie SA, Phillips DA, Lee JM, Hills SL, Van den Hurk AF, Pyke AT, Johansen CA and Mackenzie JS (1999) Japanese encephalitis in north Queensland, Australia, 1998. Med J Aust 170: 533-536.

Harbach RE and Kitching IJ (1998) Phylogeny and classification of the Culicidae (Diptera). Sys Entomol 23: 327-370.

Igarashi A (2002) Control of Japanese encephalitis in Japan: immunization of humans and animals, and vector control. Curr Top Microbiol Immunol 267: 139-152.

Igarashi A, Tanaka M, Morita K, Takasu T, Ahmed A, Akram DS and Waqar MA (1994) Detection of West Nile and Japanese encephalitis viral genome sequences in cerebrospinal fluid from acute encephalitis cases in Karachi, Pakistan. Microbiol Immunol 38: 827-830.

Iranpour M, Turell MJ and Lindsay LR (2011) Potential for Canadian mosquitoes to transmit Rift Valley fever virus. J Am Mosq Control Assoc 27: 363-369.

Johansen CA, Van den Hurk AF, Ritchie SA, Zborowski P, Nisbet DJ, Paru R, Bockarie MJ, Macdonald J, Drew AC, Khromykh TI and Mackenzie JS (2000) Isolation of Japanese encephalitis virus from mosquitoes (Diptera: Culicidae) collected in the Western Province of Papua New Guinea, 1997-1998. Am J Trop Med Hyg 62: 631-638.

Kasari TR, Carr DA, Lynn TV and Weaver JT (2008) Evaluation of pathways for release of Rift Valley fever virus into domestic ruminant livestock, ruminant wildlife, and human populations in the continental United States. J Am Vet Med Assoc 232: 514-529.

Kay BH and Farrow RA (2000) Mosquito (Diptera: Culicidae) dispersal: implications for the epidemiology of Japanese and Murray Valley encephalitis viruses in Australia. J Med Entomol 37: 797-801.

Kilpatrick AM (2011) Globalization, land use, and the invasion of West Nile virus. Science 334: 323-327.

Kilpatrick AM, Meola MA, Moudy RM and Kramer LD (2008) Temperature, viral genetics, and the transmission of West Nile virus by Culex pipiens mosquitoes. PLoS Pathog 4: e1000092.

Kipp AM, Lehman JA, Bowen RA, Fox PE, Stephens MR, Klenk K, Komar N and Bunning ML (2006) West Nile virus quantification in feces of experimentally infected American and fish crows. Am J Trop Med Hyg 75: 688-690.

Klenk K, Snow J, Morgan K, Bowen R, Stephens M, Foster F, Gordy P, Beckett S, Komar N, Gubler D and Bunning M (2004) Alligators as West Nile virus amplifiers. Emerg Infect Dis 10: 2150-2155.

Kramer LD, Styer LM and Ebel GD (2008) A global perspective on the epidemiology of West Nile virus. Annu Rev Entomol 53: 61-81.

Lanciotti, R.S., Roehrig, J.T., Deubel, V., Smith, J., Parker, M., Steele, K., Crise B, Volpe KE, Crabtree MB, Scherret JH, Hall RA, MacKenzie JS, Cropp CB, Panigrahy B, Ostlund E, Schmitt B, Malkinson M, Banet C, Weissman J, Komar N, Savage HM, Stone W, McNamara T and Gubler DJ (1999) Origin of the West Nile virus responsible for an outbreak of encephalitis in the northeastern United States. Science 286: 2333-2337.

Lapointe DA, Atkinson CT and Samuel MD (2012) Ecology and conservation biology of avian malaria. Ann NY Acad Sci 1249: 211-226.

Le Flohic G, Porphyre V, Barbazan P and Gonzalez JP (2013) Review of climate, landscape, and viral genetics as drivers of the Japanese encephalitis virus ecology. PLoS Negl Trop Dis 7: e2208.

Linthicum KJ, Davies FG, Kairo A and Bailey CL (1985) Rift Valley fever virus (family Bunyaviridae, genus Phlebovirus). Isolations from Diptera collected during an inter-epizootic period in Kenya. J Hyg 95: 197-209.

Long MT (2014) West nile virus and equine encephalitis viruses: new perspectives. Vet Clin North Am Equine Pract 30: 523-542.

Lord JS, Gurley ES and Pulliam JR (2015) Rethinking Japanese Encephalitis virus transmission: a framework for implicating host and vector species. PLoS Negl Trop Dis 9: e0004074.

Mackenzie JS, Gubler DJ and Petersen LR (2004) Emerging flaviviruses: the spread and resurgence of Japanese encephalitis, West Nile and Dengue viruses. Nat Med 10: S98-S109.

Malkinson M, Banet C, Khinich Y, Samina I, Pokamunski S and Weisman Y (2001) Use of live and inactivated vaccines in the control of West Nile fever in domestic geese. Ann NY Acad Sci 951: 255-261.

Mansfield KL, Banyard AC, McElhinney L, Johnson N, Horton DL, Hernandez-Triana LM and Fooks AR (2015) Rift Valley fever virus: a review of diagnosis and vaccination, and implications for emergence in Europe. Vaccine 33: 5520-5531.

Meegan JM, Hoogstraal H and Moussa MI (1979) An epizootic of Rift Valley fever in Egypt in 1977. Vet Rec 105: 124-125. 
Meyer KF, Haring CM and Howitt B (1931) The etiology of epizootic Encephalomyelitis of horses in the San Joaquin Valley, 1930. Science 74: 227-228.

Ming JG, Jin H, Riley JR, Reynolds DR, Smith AD, Wang RL, Cheng JY and Cheng XN (1993) Autumn southward 'return' migration of the mosquito Culex tritaeniorhynchus in China. Med Vet Entomol 7: 323-327.

Mitamura T, Kitaoka M, Watanabe M, Okuba K, Tenjin S, Yamada S, Mori K and Asada J (1936) Study on Japanese encephalitis virus. Animal experiments and mosquito transmission experiments. Kansai lji 1: 260-261.

Mitchell CJ, Chen PS and Boreham PF (1973) Host-feeding patterns and behaviour of 4 Culex species in an endemic area of Japanese encephalitis. Bull World Health Organ 49: 293-299.

Moncayo AC, Lanzaro G, Kang W, Orozco A, Ulloa A, Arredondo-Jiménez J and Weaver SC (2008) Vector competence of eastern and western forms of Psorophora columbiae (Diptera: Culicidae) mosquitoes for enzootic and epizootic Venezuelan equine encephalitis virus. Am J Trop Med Hyg 78: 413-421.

Molaei G, Armstrong PM, Graham AC, Kramer LD and Andreadis TG (2015) Insights into the recent emergence and expansion of eastern equine encephalitis virus in a new focus in the Northern New England USA. Parasit Vectors 8: 516.

Moore CG and Mitchell CJ (1997) Aedes albopictus in the United States: ten-year presence and public health implications. Emerg Infect Dis 3: 329-334.

Moutailler S, Krida G, Schaffner F, Vazeille M and Failloux AB (2008) Potential vectors of Rift Valley fever virus in the Mediterranean region. Vector Borne Zoonotic Dis 8: 749-753.

Muller R, Saluzzo JF, Lopez N, Dreier T, Turell M, Smith J and Bouloy M (1995) Characterization of clone 13, a naturally attenuated avirulent isolate of Rift Valley fever virus, which is altered in the small segment. Am J Trop Med Hyg 53: 405-411.

Munstermann LE and Conn JE (1997) Systematics of mosquito disease vectors (Diptera, Culicidae): impact of molecular biology and cladistic analysis. Annu Rev Entomol 42: 351-369.

Mwandawiro C, Boots M, Tuno N, Suwonkerd W, Tsuda Y and Takagi M (2000) Heterogeneity in the host preference of Japanese encephalitis vectors in Chiang Mai, northern Thailand. Trans R Soc Trop Med Hyg 94: 238-242.

Nasci RS, Savage HM, White DJ, Miller JR, Cropp BC, Godsey MS, Kerst AJ, Bennett P, Gottfried K and Lanciotti RS (2001) West Nile virus in overwintering Culex mosquitoes, New York City, 2000. Emerg Infect Dis 7: 742-744.

Nett RJ, Campbell GL and Reisen WK (2009) Potential for the emergence of Japanese encephalitis virus in California. Vector Borne Zoonotic Dis 9: 511-517.

Okeson DM, Llizo SY, Miller CL and Glaser AL (2007) Antibody response of five bird species after vaccination with a killed West Nile virus vaccine. Journal of Zoo and Wildlife Medicine 38: 240-244.

Olive MM, Goodman SM and Reynes JM (2012) The role of wild mammals in the maintenance of Rift Valley fever virus. J Wildl Dis 48: 241-266.

Parida M, Dash PK, Tripathi NK, Sannarangaiah S, Saxena P, Agarwal S, Sahni AK, Singh SP, Rathi AK, Bhargava R, Abhyankar A, Verma SK, Rao PV and Sekhar K (2006) Japanese encephalitis outbreak, India, 2005. Emerg Infect Dis 12: 1427-1430.

Paz S and Semenza JC (2013) Environmental drivers of West Nile fever epidemiology in Europe and Western Asia - a review. Int J Environ Res Public Health 10: 3543-3562.

Peiris JS, Amerasinghe FP, Amerasinghe PH, Ratnayake CB, Karunaratne SH and Tsai TF (1992) Japanese encephalitis in Sri Lanka - the study of an epidemic: vector incrimination, porcine infection and human disease. Trans R Soc Trop Med Hyg 86: 307-313.

Pennington NE and Phelps CA (1968) Identification of the host range of Culex tritaeniorhynchus mosquitoes on Okinawa, ryukyu islands. J Med Entomol 5: 483-487.

Pepin M, Bouloy M, Bird BH, Kemp A and Paweska J (2010) Rift Valley fever virus (Bunyaviridae: Phlebovirus): an update on pathogenesis, molecular epidemiology, vectors, diagnostics and prevention. Vet Res 41:61.

Rappole JH, Derrickson SR and Hubalek Z (2000) Migratory birds and spread of West Nile virus in the western hemisphere. Emerg Infect Dis 6: 310-328.

Rasnitsyn AP and Quicke DLJ (eds.) (2002) History of insects. Kluwer, Dordrecht, the Netherlands, 517 pp. 
Reidenbach KR, Cook S, Bertone MA, Harbach RE, Wiegmann BM and Besansky NJ (2009) Phylogenetic analysis and temporal diversification of mosquitoes (Diptera: Culicidae) based on nuclear genes and morphology. BMC Evol Biol 9: 298.

Reuben R, Thenmozhi V, Samuel PP, Gajanana A and Mani TR (1992) Mosquito blood feeding patterns as a factor in the epidemiology of Japanese encephalitis in southern India. Am J Trop Med Hyg 46: 654-663.

Reynolds DR, Smith AD, Mukhopadhyay S, Chowdhury AK, De BK, Nath PS, Mondal SK and Das BK (1996) Atmospheric transport of mosquitoes in northeast India. Med Vet Entomol 10: 185-186.

Riad MH, Scoglio CM, McVey DS and Cohnstaedt LW (2016) An individual-level network model for a hypothetical outbreak of Japanese encephalitis in the USA. Stoch Environ Res Risk Assess 31(2): 353-367.

Ricklin ME, Garcia-Nicolas O, Brechbuhl D, Python S, Zumkehr B, Nougairede A, Charrel RN, Posthaus H, Oevermann $A$ and Summerfield A (2016) Vector-free transmission and persistence of Japanese encephalitis virus in pigs. Nat Commun 7: 10832.

Ritchie SA, Phillips D, Broom A, Mackenzie J, Poidinger M and Van den Hurk A (1997) Isolation of Japanese encephalitis virus from Culex annulirostris in Australia. Am J Trop Med Hyg 56: 80-84.

Rizzoli A, Jimenez-Clavero MA, Barzon L, Cordioli P, Figuerola J, Koraka P, Martina B, Moreno A, Nowotny N, Pardigon N, Sanders N, Ulbert S and Tenorio A (2015) The challenge of West Nile virus in Europe: knowledge gaps and research priorities. Euro Surveill 20:21135.

Rodrigues FM, Guttikar SN and Pinto BD (1981) Prevalence of antibodies to Japanese encephalitis and West Nile viruses among wild birds in the Krishna-Godavari Delta, Andhra Pradesh, India. Trans R Soc Trop Med Hyg 75: 258-262.

Rolin Al, Berrang-Ford L and Kulkarni MA (2013) The risk of Rift Valley fever virus introduction and establishment in the United States and European Union. Emerg Microbes Infect 2: e81.

Scoglio CM, Bosca C, Riad MH, Sahneh FD, Britch SC, Cohnstaedt LW and Linthicum KJ (2016) Biologically informed individual-based network model for Rift Valley fever in the US and evaluation of mitigation strategies. PLOS ONE 11: e0162759.

Silva ML, Galiza GJ, Dantas AF, Oliveira RN, lamamoto K, Achkar SM and Riet-Correa F (2011) Outbreaks of eastern equine encephalitis in northeastern Brazil. J Vet Diagn Invest 23: 570-575.

Silverman MA, Misasi J, Smole S, Feldman HA, Cohen AB, Santagata S, McManus M and Ahmed AA (2013) Eastern equine encephalitis in children, Massachusetts and New Hampshire, USA, 1970-2010. Emerg Infect Dis 19: 194-201.

Sindato C, Karimuribo E and Mboera EG (2012) The epidemiology and socio-economicimpact of Rift Valley fever epidemics in Tanzania: a review. Ondersterpoort J Vet Res 79: E1.

Smith DL, Battle KE, Hay SI, Barker CM, Scott TW and McKenzie FE (2012) Ross, Macdonald, and a theory for the dynamics and control of mosquito-transmitted pathogens. PLoS Pathog 8: e1002588.

Smithburn KC, Hughes TP, Burke AW and Paul JH (1940) A neurotropic virus isolated from the blood of a native of Uganda. Am J Trop Med Hyg 20: 471-473.

Snow NP, Jarzyna MA and VerCauteren KC (2017) Interpreting and predicting the spread of invasive wild pigs. J Appl Ecol 54(6): 2022-2032.

Soman RS, Rodrigues FM, Guttikar SN and Guru PY (1977) Experimental viraemia and transmission of Japanese encephalitis virus by mosquitoes in ardeid birds. Indian J Med Res 66: 709-718.

Takahashi M (1976) The effects of environmental and physiological conditions of Culex tritaeniorhynchus on the pattern of transmission of Japanese encephalitis virus. J Med Entomol 13: 275-284.

Takashima I, Watanabe T, Ouchi N and Hashimoto N (1988) Ecological studies of Japanese encephalitis virus in Hokkaido: interepidemic outbreaks of swine abortion and evidence for the virus to overwinter locally. Am J Trop Med Hyg 38: 420-427.

Tsetsarkin KA, Vanlandingham DL, McGee CE and Higgs S (2007) A single mutation in chikungunya virus affects vector specificity and epidemic potential. PLoS Pathog 3: e201.

Turell MJ, Bailey CL and Beaman JR (1988) Vector competence of a Houston, Texas strain of Aedes albopictus for Rift Valley fever virus. J Am Mosq Control Assoc 4: 94-96.

Turell MJ, Dohm DJ, Mores CN, Terracina L, Wallette DL Jr., Hribar LJ, Pecor JE and Blow JA (2008) Potential for North American mosquitoes to transmit Rift Valley fever virus. J Am Mosq Control Assoc 24: 502-507. 
Turell MJ, Rossi CA and Bailey CL (1985) Effect of extrinsic incubation temperature on the ability of Aedes taeniorhynchus and Culex pipiens to transmit Rift Valley fever virus. Am J Trop Med Hyg 34: 1211-1218.

Turell MJ, Wilson WC and Bennett KE (2010) Potential for North American mosquitoes (Diptera: Culicidae) to transmit rift valley fever virus. J Med Entomol 47: 884-889.

Van den Hurk AF, Ritchie SA and Mackenzie JS (2009) Ecology and geographical expansion of Japanese encephalitis virus. Annu Rev Entomol 54: 17-35.

Villari P, Spielman A, Komar N, McDowell M and Timperi RJ (1995) The economic burden imposed by a residual case of eastern encephalitis. Am J Trop Med Hyg 52: 8-13.

Vythilingam I, Oda K, Mahadevan S, Abdullah G, Thim CS, Hong CC, Vijayamalar B, Sinniah M and Igarashi A (1997) Abundance, parity, and Japanese encephalitis virus infection of mosquitoes (Diptera:Culicidae) in Sepang District, Malaysia. J Med Entomol 34: 257-262.

Wang E, Bowen RA, Medina G, Powers AM, Kang W, Chandler LM, Shope RE and Weaver SC (2001) Virulence and viremia characteristics of 1992 epizootic subtype IC Venezuelan equine encephalitis viruses and closely related enzootic subtype ID strains. Am J Trop Med Hyg 65: 64-69.

Warimwe GM, Gesharisha J, Carr BV, Otieno S, Otingah K, Wright D, Charleston B, Okoth E, Elena LG, Lorenzo G, Ayman el B, Alharbi NK, Al-Dubaib MA, Brun A, Gilbert SC, Nene V and Hill AV (2016) Chimpanzee adenovirus vaccine provides multispecies protection against Rift Valley fever. Sci Rep 6: 20617.

Weaver SC, Ferro C, Barrera R, Boshell J and Navarro JC (2004) Venezuelan equine encephalitis. Annu Rev Entomol 49: 141-174.

Weiss KE (1957) Rift Valley fever - a review. Bull Epizoot Dis Afr 5: 431-458.

Weng MH, Lien JC, Lin CC and Yao CW (2000) Vector competence of Culex pipiens molestus (Diptera: Culicidae) from Taiwan for a sympatric strain of Japanese encephalitis virus. J Med Entomol 37: 780-783.

Wilkerson RC, Linton YM, Fonseca DM, Schultz TR, Price DC and Strickman DA (2015) Making mosquito taxonomy useful: a stable classification of tribe aedini that balances utility with current knowledge of evolutionary relationships. PLOS ONE 10: e0133602.

Williams RB (2005) Avian malaria: clinical and chemical pathology of Plasmodium gallinaceum in the domesticated fowl Gallus gallus. Avian Pathol 34: 29-47.

World Health Organisation (WHO), 2008. Report update 5: Rift Valley fever in Sudan. WHO, Geneve, Switzerland. Available at: http://www.who.int/csr/don/2008_01_22/en.

Xue L, Cohnstaedt LW, Scott HM and Scoglio C (2013) A hierarchical network approach for modeling rift valley fever epidemics with applications in North America. PLoS ONE 85: e62049.

Xue L, Scott HM, Cohnstaedt LW and Scoglio C. (2012) A network-based meta-population approach to model Rift Valley fever epidemics. J. Theoretical Biol. 306: 129-144. 


\title{
9. Case studies of vector-borne diseases in livestock: bluetongue virus
}

\author{
Miguel Á. Miranda \\ Laboratory of Zoology, University of the Balearic Islands, Carretera de Valldemossa Km 7.5, 07122 Palma \\ de Mallorca, Spain; ma.miranda@uib.es
}

\begin{abstract}
Biting midges of the genus Culicoides (Diptera; Ceratopogonidae) are the main vectors of several arboviral diseases such as bluetongue, African horse sickness and epizootic haemorrhagic disease among others. Culicoides females acquire the virus from mammal hosts by haematophagous feeding and they transmit the virus to the following host after the completion of the extrinsic incubation period. Despite the importance of Culicoides during the last two decades after several waves of bluetongue outbreaks in regions such as Europe, there is still a lack of effective control methods against the vectors. In addition, little information is also available about crucial aspects of their bioecology that will lead to a better use of control methods. Here we review the major control methods developed for control of Culicoides spp. Those methods also need monitoring tools to estimate the abundance and seasonality of adult and larvae Culicoides populations. Control methods reviewed here include mechanical methods, chemical methods including 'in vitro' trials, semifield and field trials, as well as biological, genetic and biotechnological control methods. At the moment of writing this review, insecticide pour-on treatments as well as the use of vector-proof establishment appear to be the most efficacious method to protect domestic animals against Culicoides feeding activity.
\end{abstract}

Keywords: Culicoides, vector, control, bluetongue, monitoring

\section{Introduction}

Bluetongue is a non-contagious arboviral disease caused by a double stranded RNA virus from the family Reoviridae and genus Orbivirus. Bluetongue virus (BTV) to date includes 27 serotypes (Hofmann et al. 2008; Jenckel et al. 2015; Maan et al. 2011; Schwartz-cornil et al. 2008) and is a notifiable disease of ruminants in the OIE due to its potential health impact on susceptible hosts and the economic impact on the trade of animals and animal products (OIE 2016). BTV is generally present in numerous tropical and temperate regions between $40^{\circ} \mathrm{N}$ to $35^{\circ} \mathrm{S}$ (Walton 2003), and periodical epidemics occur in regions adjacent to BTV's endemic range, with outbreaks recorded up to the $58^{\circ} \mathrm{N}$ latitude (Wilson and Mellor 2009). Europe lies outside of the endemic range of BTV but has suffered multiple serious incursions of BTV since 1998 with circulation of serotypes $1,2,4,8,9,16$ and 27 detected and some of them are still circulating in Europe after more than a decade (EFSA 2008).

BTV infects domestic as well as wild ruminants (Falconi et al. 2011; MacLachlan 1994; Niedbalski 2015; Taylor 1986) but clinical signs are usually evident only in domestic animals, such as sheep. BTV is transmitted biologically almost exclusively by the bites of haematophagous females of several species of the genus Culicoides (Diptera; Ceratopogonidae) biting midges (Mellor et al. 2000) and this was first demonstrated in South Africa by Du Toit in 1943 (Du Toit 1944). Alternative routes of transmission include semen (Mellor and Boorman 1995; Walton et al. 1984) and vertical 
transmission (VT) through the placenta (De Clercq et al. 2008; Desmecht et al. 2008; Saegerman et al. 2011).

Worldwide it is estimated that ca. 20 species of Culicoides are considered to be epidemiologically important vectors of the virus (Mellor 1990; Meiswinkel et al. 1994, 2004), but the list of proven vectors will probably increase in the following decade, as vector competence has been tested on a limited number of species and virus field isolations are also scarce (Purse et al. 2015).

Culicoides are able to transmit 66 viruses, 15 species of protozoa and 26 species of filarial nematodes (Borkent 2004). Bluetongue, African horse sickness and epizootic haemorrhagic disease viruses are currently considered the most clinically important Culicoides-borne pathogens and all are members of the family Reoviridae (Mellor et al. 2000). Culicoides spp. are also vectors of the Schmallenberg virus that affects cattle and sheep (Gibbens 2012). Other important viruses are Akabane virus and the human pathogen Oropouche virus which belong to the family Peribunyaviridae (Meiswinkel et al. 1994; Mellor et al. 2000). Culicoides are also well known for being biological vectors of protozoans and nematodes (Fallis and Bennett 1961; Linley 1985; Mellor et al. 2000; Meiswinkel et al. 1994). Avian protozoa included in Trypanosomatidae have received increased attention over the last few years (Bobeva et al. 2013; Svobodová et al. 2007; Synek et al. 2013) as has the group of Haemosporidians (Ferraguti et al. 2013). Culicoides spp. are also recognised for producing serious allergenic skin reactions ('sweet itch') in horses (Baker and Quinn 1978; Baker et al. 2015; Braverman et al. 1983; Riek 1954).

\section{Bluetongue virus episystems}

BTV distribution is related to the presence of particular species of Culicoides in a given region that, including environmental components, BTV serotypes, hosts and socioeconomic factors, define a particular episystem (Maclachlan and Osburn 2006; Tabachnick 2004, 2010). In general, BTV episystems are found throughout temperate and tropical areas of the world and the global distribution of vectors is wider than that of BTV (Maclachlan and Mayo 2013). Among environmental factors, temperature is the one that largely determines BTV competent vector species distribution (Maclachlan and Osburn 2006; Wittman and Baylis 2000).

Vertebrate hosts of BTV include wild and domestic species of ruminants. The incidence of BTV may depend on immunity of the population, breeds affected and serotype involved (Dungu et al. 2004). In fact, BTV is particularly pathogenic to some breeds of sheep, such as the European breeds (i.e. Merina). Pathogeny has also been recorded in some naturally infected wild species such as white tail deer (Taylor 1986), llamas (Lama glama) (Meyer et al. 2009), bighorn sheep (Ovis canadensis) and mouflon (Ovis aries musimon) (Fernández-Pacheco et al. 2008). Other species of wild ruminants, such as red deer (Cervus elaphus), roe deer (Capreolus capreolus), fallow deer (Dama dama), arruí (Ammotragus lervia) and ibex (Capra pyrenaica) do not show clinical signs but BTV has been extensively detected in populations of these species (EFSA 2017; Falconi et al. 2011; García et al. 2009; Ruiz-Fons et al. 2008). In general domestic bovines show few clinical signs (MacLachlan 1994), with the exception of some serotypes, as seen during the BTV-8 outbreak in northern Europe in 2006/2007 (Niedbalski 2015).

Apart from the BTV episystems described worldwide, periodic incursions of BTV into new territories have also been recorded, in particular the intensive waves of BTV across Europe from late '90s (Wilson and Mellor 2008, 2009). Two decades after these incursions, some serotypes of BTV (i.e. BTV 8 and BTV 3) are still circulating in Europe, which strongly suggests that in some areas the 
virus could already be considered more endemic than epidemic. The recent discovery of outbreaks of BTV-8 in France after a 10-year period absence of clinical reports (Sailleau et al. 2017) indicates the potential endemic presence of BTV in northern Europe (EFSA 2017).

\section{Cycle of transmission of bluetongue virus}

The cycle of transmission of BTV starts when a non-infective female Culicoides feeds on a BTV infected animal. Blood feeding is necessary in anautogenous species of Culicoides for females to obtain protein for subsequent egg development. Infection of the vector occurs when females bite through the skin of the vertebrate and feed on blood that contains viral particles of BTV that are able to infect the cells of the posterior mid-gut of the insect, due to particular receptors that the virus recognises. If the virus fails to recognise the cell receptors, then the vector will be poorly competent for BTV. A series of barriers to BTV infection have been identified in Culicoides and are summarised in Mellor et al. (2000). When the virus succeeds in invading the gut cells, replication of the virus allows infection of the haemocoel and different tissues of the vectors. Accordingly, the salivary glands become infected and a second replicative cycle takes place leaving virus particles in the saliva ready for further transmission to the next susceptible host (Beaty and Marquardt 1996; Mellor et al. 2000). The period required for the completion of the virus replication cycle in the vector is called the extrinsic incubation period (EIP). The EIP is a temperature-dependent process; Wittman et al. (2002) reported minimum temperatures of 9 to $15^{\circ} \mathrm{C}$ for virus replication and an optimal EIP temperature of ca. $28^{\circ} \mathrm{C}$ for BTV. In general, the EIP has been found to last 20 to 26 days at $15{ }^{\circ} \mathrm{C}$ and 5 to 7 days at $30^{\circ} \mathrm{C}$ (Carpenter et al. 2011; Foster et al. 1963; Luedke et al. 1967; Wittman et al. 2002). Temperatures below $15^{\circ} \mathrm{C}$ generally inhibit the EIP (Wittman et al. 2002); however, recent models obtained from outbreak analysis in Andalusia (Spain) have shown that for some species, such as Culicoides imicola Kieffer, EIP limiting temperatures could be as high as $21^{\circ} \mathrm{C}$ (Napp et al. 2016). Carpenter et al. (2011) proposed that EIP can be estimated from the temperature using degree day models. Once the EIP is completed in female Culicoides, it is currently presumed that they remain infectious for life. When a BTV-infected female bites an uninfected susceptible vertebrate then the cycle of transmission vector to host is completed and there is evidence that Culicoides saliva delays the immune response of the host against the inoculated virus particles (Pages et al. 2014). The replication of BTV in targeted tissues, such as the lymphatic nodules and endothelial tissue of the vertebrate, after inoculation has been well documented (Gibbs and Greiner 1994). The virus invades several organs and cells of the blood stream, particularly erythrocytes and leucocytes (MacLachlan 1994). The viremia in the vertebrate host is variable according to the host species, lasting longer in cattle than sheep according to the lifespan of BTV-infected red blood cells (Bonneau et al. 2002; Gubbins et al. 2008; Koumbati et al. 1999; MacLachlan et al. 1990, 1991; Sellers 1980). In general, OIE (2016) considers a viremia of 60 days for BTV-infected ruminants and after the viremic period, vertebrate hosts are not infective for vectors.

The persistence of BTV from one year to another ('overwintering') is of particular relevance in temperate areas were transmission stops during winter due to low temperatures for EIP and the absence of activity of adult Culicoides. Several mechanisms have been proposed to explain the inter-seasonal reoccurrence of BTV. VT in vectors accounts for the transmission of the virus from reproductive females to immature phases and the subsequent infection of larvae, pupae and emerged adults. One of the most recognised mechanisms for VT is transovarial transmission (TOT), where the germinal tissue of the female vector is infected with the virus through all developing stages to adult. This mechanism has been described for other arboviruses such as La Crosse virus, dengue virus and West Nile virus which are transmitted by mosquitoes but not biting 
midges (Lequime and Lambrechts 2014). In the case of BTV, all studies indicate that TOT is highly improbable in Culicoides spp. While White et al. (2005) found BTV nucleic acid in field-collected larvae of Culicoides sonorensis and Culicoides crepuscularis in North America, a more complete study conducted by Osborne et al. (2015) failed to detect BTV-17 by RT-qPCR in larvae and pupae reared from adults of $C$. sonorensis infected by feeding them using BTV-spiked blood. Furthermore, analysis of more than 2000 larvae collected in seasonally BTV-affected farms in California did not show any presence of BTV. Another method of TOT detection is the analysis of nulliparous females; transovarial transmission could also be found by detecting virus particles in nulliparous females. To date there are no published studies demonstrating the presence of BTV in nulliparous females; however, some evidence with unclear epidemiological significance has been reported for other arboviruses, including Schmallenberg virus (Orthobunyaviridae) where field collected nulliparous females of the Obsoletus complex (Culicoides obsoletus (Meigen) and Culicoides scoticus Downes $\&$ Kettle) and Culicoides punctatus (Meigen) gave sub-transmissible infection values by RT-qPCR (Larska et al. 2013).

\section{Bluetongue virus vectors}

\section{Taxonomy of Culicoides spp.}

BTV is transmitted by insects of the genus Culicoides Latreille, 1809, that belongs to the family Ceratopogonidae and subfamily Ceratopogoninae which includes 5 tribes: Ceratopogonini, Culicoidini, Hetyeromyiini, Palpomyiini and Sphaeromiini. The subfamily contains 34 genus (McAlpine et al. 1981). The tribe Culicoidini includes the genus Culicoides which to date consists of 32 subgenera (Borkent 2015). The subgenus Avaritia Fox, 1955, in particular includes many species of veterinary importance due to their role as vectors of arboviruses.

To date, the genus Culicoides comprises 21.5\% of Ceratopogonid species (Harrup et al. 2015), including 1343 extant species and 44 extinct species (Borkent 2004). The origin of the group has been recorded in the Baltic amber of the Cretaceous, making it as old as 90 million years (Borkent 1996, 2015; Sontag and Szadziewski 2011; Szadziewski and Grogan Jr. 1998).

Tools for approaching Culicoides taxonomy have been extensively reviewed by Harrup et al. (2015). In summary, the most common is the use of morphological characters, particularly those of adults. The pigmentation pattern of the wing is widely used to distinguish species and has been revealed to be very useful in vector surveillance programmes at national level. Good examples of wing atlas of Culicoides for Palearctic species can be found in Mathieu et al. (2010), González and Goldarazena (2011) and Australasian species in Dyce et al. (2007).

Apart from the wing patterns, species of Culicoides can also be identified using other morphology characters that require slide mounting. Male Culicoides can be identified using characteristics of their genitalia, while females can be identified by using a cluster of characteristics such as eyes, ocelli, antennal sensilla distribution, palpi and spermathecae. In recent years, morphological identification has improved due to the development of on-line tools such as IIKC (Mathieu et al. 2010). A complete listing of resources for Culicoides taxonomy can be found at Harrup et al. (2015).

Biting midges species of the genus Culicoides are present on all continents except Antarctica. They are also absent from New Zealand, Iceland and the Hawaiian islands (Mellor et al. 2000). Confirmed vectors of BTV based on field isolation of the virus, completion of EIP in laboratory infection, evidence of full dissemination within field collected individuals and significant abundance in 
proximity to susceptible hosts were recently revised by Purse et al. (2015). The following Culicoides species, listed by continent, are known to transmit BTV:

- Africa: Culicoides (Avaritia) imicola Kieffer 1913 (Meiswinkel et al. 1994).

- Asia: Culicoides (Avaritia) fulvus Sen \& Das Gupta 1959 and Culicoides (Remnia) schultzei (Enderlein) 1908 in Asia (Tabachnick 2004).

- Australia: Culicoides (Avaritia) wadai Kitaoka 1980, Culicoides (Avaritia) brevitarsis Kieffer 1917, Culicoides (Avaritia) actoni Smith 1929, Culicoides brevipalpis Delfinado 1961 and C. fulvus Bezzi 1927 (Kirkland 2004).

- Europe: C. (Avaritia) imicola, C. (Avaritia) obsoletus (Meigen, 1818) C. (Avaritia) scoticus Downes \& Kettle, 1952, Culicoides (Avaritia) dewulfi Goethebuer 1936, Culicoides (Avaritia) chiopterus (Meigen, 1830), Culicoides (Culicoides) pulicaris (Linnaeus, 1758) have been involved in field virus transmission, meanwhile the species Culicoides (Culicoides) newsteadi Austen, 1921, Culicoides (Culicoides) lupicaris Downes \& Kettle, 1952 and C. punctatus (Meigen, 1804) are considered potential vectors due to laboratory evidence (Goffredo et al. 2015; Harrup et al. 2015; Meiswinkel et al. 2007; Mellor 2004; Savini et al. 2005; Tabachnick 2004).

- North America: C. (Monoculicoides) sonorensis Wirth \& Jones 1957 (Holbrook et al. 2000).

- South, Central America and the Caribbean: Culicoides (Hoffmania) insignis Lutz 1913 (Tanya et al. 1992).

Culicoides taxonomy includes species complexes and groups, which are challenging for the taxonomist. These categories lack systematic recognition and are not applied consistently by authors. In general, it is neither easy nor even possible to separate females in these groups of cryptic species using only morphological characteristics. In this case, molecular taxonomy has become a useful means of separating cryptic species, even if its regular use in national surveillance programmes is difficult. Despite some of the categories having no taxonomic significance (Harrup et al. 2015), groups and complexes are commonly used by authors to organise cryptic species. Some examples are found within the Obsoletus group, which includes the species $C$. obsoletus, C. scoticus, C. dewulfi and C. chiopterus. The former two species are considered the Obsoletus complex and females are highly isomorphic. There are several works, particularly in Europe, which have explored the feasibility of different molecular methods for separating species included in the Obsoletus group (Deblauwe et al. 2012; Garros et al. 2014). Similar works have also been conducted with other groups, such as the Pulicaris group (Mordue et al. 2007; Pagès et al. 2009). In recent years, the use of DNA Barcoding has also been promoted to complete the taxonomy of some of the groups (Ander et al. 2013), as well as the use of other techniques based on wing biometrics (Henni et al. 2014; Muñoz-Muñoz et al. 2011, 2014).

\section{Bioecology}

Life cycle

Culicoides are holometabolous insects and adults are in general 2-3 $\mathrm{mm}$ in size. The Culicoides life cycle includes egg, four larvae instar, the pupa and adult. Time for development is temperaturedependent, requiring $34-56$ days at $20^{\circ} \mathrm{C}, 15-21$ days at $25^{\circ} \mathrm{C}$ and only $11-16$ days at $28{ }^{\circ} \mathrm{C}$ for $\mathrm{C}$. imicola according to Veronesi et al. (2009), while Barceló \& Miranda estimated an average of 28 days at $25^{\circ} \mathrm{C}$ for six common Culicoides species from Mediterranean Europe. The lifespan of adults is species- and temperature-dependent. In general, it has been described between 20 days and 3 months (Barceló and Miranda 2018; Goffredo et al. 2004; Mellor et al. 2000; Purse et al. 2015; Veronesi et al. 2009). 
Females and males feed on nectar and sugar substrates (Meiswinkel et al. 2004; Mellor et al. 2000). Females that are anautogenous require blood to lay eggs; however, some autogenous species such as Culicoides circumscriptus (Becker 1961) and Culicoides impunctatus (Boorman and Goddard 1970) do not require blood for the first oviposition. Eggs are usually laid in wet substrates or organic enriched soil (i.e. C. imicola) (Braverman et al. 1974) that could be highly variable and is species-dependent. Coetzer et al. (1994) proposed four major habitats for immature Culicoides: (1) water-land interfaces, which are common for species that breed in water ponds shorelines, such as C. sonorensis (Pfannenstiel et al. 2015). In this case, pupae are able to float and keep motility on a water-land surface; (2) dung pats, e.g. Culicoides bolitinos in elephant dung pats in Africa (Meiswinkel 1989), and C. dewulfi in cattle dung in Europe (Campbell and Pelham-Clinton 1960); (3) tree cavities, plants and cracks (Kitaoka 1975; Nevill and Dyce 1994; Obona and Dominiak 2014; Pappas and Pappas 1990; Rioux et al. 1959); and (4) decaying plant material, fruits and fungi (Braverman et al. 1974; Buxton 1960; Estrada et al. 2011; Mellor and Pitzolis 1979; Uslu and Dik 2007b, Zimmer et al. 2008). Each female could lay between 30 and 200 eggs per batch (Barceló and Miranda 2018; Braverman and Linley 1994; Nevill 1967; Veronesi et al. 2009,). Eggs are 250 to 500 microns pale in colour at the beginning and change to brown when they become harder in contact with the air. Egg development takes ca. 2 days at $25^{\circ} \mathrm{C}$ (Barceló and Miranda 2018; Beaty and Marquardt 1996; Nevill 1967). Larval development has four instars, from L1 (0.5 mm length) to $L 4$ (5 mm length). The development of the four larvae instars also depends on the temperature. Veronesi et al. (2009) obtained up to 21 days for larval development of C. imicola at $20^{\circ} \mathrm{C}$ while Barceló and Miranda (2018) obtained an average of 17 days at $25^{\circ} \mathrm{C}$. The pupae, a non-feeding stage that develops in 1 to 3 days to adults, are approximately $5 \mathrm{~mm}$ in size (Barceló and Miranda 2018; Veronesi et al. 2009).

\section{Feeding and daily activity}

Adult Culicoides are common in rural areas, associated with domestic animals, such as sheep, cattle, horses, and goats. Many species are also present in natural areas, for example those associated with big mammals in Africa (i.e. C. bolitinos) (Meiswinkel et al. 2000; Verhoef et al. 2014) and wild fauna such as deer (Acevedo et al. 2010; Ruiz-Fons et al. 2014; Smith and Stallknecht 1996; Talavera et al. 2015). Some species are common in beaches and mangrove environments such as Culicoides furens (Wirth and Blanton 1956), and golf courses, such as C. impunctatus (Stuart et al. 1996) the latter becoming a serious pest for golfers in Scotland.

With regards to feeding, most of the Culicoides of veterinary importance feed on mammals (Bartsch et al. 2009; Calvo et al. 2012) but bird feeders are also common and in fact some species, show an opportunistic behaviour obtaining blood from different types of hosts (Martínez-de la Puente et al. 2014). Some Culicoides species feed also on invertebrates (i.e. mosquitoes) (Chhilar and Chaudhry 2010; Ma et al. 2013). Interestingly, only a few species appear to have human host feeding preference in the absence of other hosts, such as C. impunctatus (Blackwell et al. 2004) in Europe and C. furens in North America (Linley and Davies 1971).

Host preferences have been explored in recent years by using molecular analysis on blood-fed females, for example using Cytochrome $b$ gene (Calvo et al. 2012) and DNA barcoding techniques (Martínez-de la Puente et al. 2012). Other researchers have approached feeding behaviour studies by comparing captures in ultraviolet (UV) light traps located among different hosts. Elbers and Meiswinkel (2014) showed Culicoides spp. to be 9.3 times more attracted to cow than sheep. Viennet et al. (2013) showed horses to be more attractive to Culicoides spp., accounting for up to $95 \%$ of Culicoides collected in drop traps baited with different hosts including sheep, goat, 
cow and chicken. In addition, feeding preferences are reflected in the anatomy of antennae of Culicoides spp., with ornithophilic species displaying different shapes in some antennal segments and in number and distribution of antennal sensilla (Isberg et al. 2013).

The majority of Culicoides species display crepuscular activity (i.e. feeding, mating) but some species display activity during the day, for example Culicoides hollensis (Koch and Axtell 1979a) and Culicoides heliophilus Edwards. Activity of adult Culicoides also varies according to weather conditions. Thus, depending on the temperature and sunlight, some species shift their behaviour from crepuscular to diurnal as was recorded for C. sonorensis by Mayo et al. (2014a) in California cattle farms and for C. obsoletus/scoticus, C. dewulfi and C. chiopterus in France (Balenghien et al. (2008). Diurnal activity of BTV-infected adults may underestimate the attacking rate to host of Culicoides when population abundance is only measured by light traps (Carpenter et al. 2008).

In general, Culicoides species are considered to be exophilic and feed outdoors on preferred hosts (Mellor et al. 2000). However, indoor activity has been recorded for C. obsoletus/scoticus, C. dewulfi and C. chiopterus in Belgium (Deken et al. 2007; Zimmer et al. 2008), France (Baldet et al. 2008), the Netherlands (Meiswinkel et al. 2008) and the UK (Baylis et al. 2010). The possible role of the indoor activity for adult Culicoides overwintering during the inter-seasonal period of BTV still needs further clarification.

Biting midges are considered weak flyers (few kilometres) and their activity depends on temperature and other variables such us wind speed and rain. In general, flight activity is observed between 12 to $35^{\circ} \mathrm{C}$ (Walton and Osburn 1992). However, Viennet et al. (2012) observed activity of Culicoides in France at $4.8^{\circ} \mathrm{C}$ using drop traps. Active flight has been described to be inhibited when wind is more than $3 \mathrm{~m} / \mathrm{s}$ (Alba et al. 2004; Braverman and Chechik 1996; Carpenter et al. 2008; Murray 1987; Sanders et al. 2011b; Sellers 1991). Culicoides' flight at significant altitudes has also been documented by several authors. Using a net suspended from a tethered balloon over southern England, Sanders et al. (2011a) recorded adult Culicoides flying $200 \mathrm{~m}$ above land.

Culicoides can also disperse passively over a long distance carried by air streams that transport adults as aerial plankton (Braverman and Chechik 1996; Sellers et al. 1983). This type of dispersal has been described by several authors as the main mechanism of BTV incursion into new areas, as for example in Portugal (Sellers et al. 1978), Israel (Braverman and Chechik 1996; Sellers 1984), Florida (Sellers and Maarouf 1989), New South Wales in Australia (Bishop et al. 1995, 2000), Balearic Islands (Alba et al. 2004), Greece and Bulgaria (Ducheyne et al. 2007), Sweden (Agren et al. 2010) and the UK (Burgin et al. 2013; Gubbins et al. 2010). Movement of Culicoides spp. from one territory to another has been evidenced (Jacquet et al. 2016) by using population genetic analysis and showing that populations of $C$. imicola detected in 2008 in Pyréneés-Orientales (France) originated from Corsica and not from the nearest population located at less than $80 \mathrm{~km}$ in northeast Spain. Although apparently a less explored mechanism of incursion, introduction of BTV-infected adult Culicoides by transport of commodities was also addressed by several authors after the outbreak of BTV-8 in northern Europe (Mintiens et al. 2008; Napp et al. 2009, 2013).

\section{Seasonality of the major vector species}

The seasonal pattern of biting midges is influenced by climate and specific factors, such as environmental factors related to breeding sites (Purse et al. 2015). It is also species-dependent and even considering the same species the seasonality can vary depending on the climatological conditions. In general, it is considered that a cold climate (i.e. those in the northern latitudes) 
corresponds to short seasonal activity, while a warmer climate (i.e. in southern latitudes) allows for longer seasonal activity of adults. Humidity and temperature appear to be the main regulating factors for immature stage development and adult activity of biting midges. Seasonality of the vectors is closely linked to BTV occurrence and spread.

In southern Europe adults of C. imicola generally appear in low numbers in April/May and peak in September/October. In general, no adults of this species are captured from January to March (see Baldet et al. 2004; Calistri et al. 2003; Calvete et al. 2006; Capela et al. 1993, 2003; Conte et al. 2003; Foxi and Delrio 2010; Foxi et al. 2011; Goffredo and Meiswinkel 2004; Goffredo et al. 2004; Miranda et al. 2004; Ortega et al. 1999; Patakakis 2004; Rawlings et al. 1997; Sarto I Monteys and Saiz-Ardanaz 2003). The peak of abundance of the Obsoletus group generally occurs in the summer in southern Europe (Calistri et al. 2003; Capela et al. 1993, 2003; Conte et al. 2003; Foxi and Delrio 2010; Foxi et al. 2011; Goffredo and Meiswinkel 2004; Miranda et al. 2004; Ortega et al. 1999). Adults of this group are also commonly found during winter (González et al. 2013; Romón et al. 2012). In northern Europe Culicoides activity starts in May, peaking in July-August and lasting until November (i.e. C. obsoletus/C. scoticus, C. dewulfi and C. chiopterus). In northerly areas of Europe it is common for adult Culicoides activity to be absent during the winter (Ander et al. 2012; Balczun et al. 2009; Brugger and Rubel 2013; Burylova and Milushina 1993; Clausen et al. 2009; Kiel et al. 2009; Meiswinkel et al. 2014; Santiago-Alarcon et al. 2013; Searle et al. 2014; Takken et al. 2008; Vorsprach et al. 2009). Other examples of vector seasonality can be found in Africa (Senegal) where C. imicola has been shown to peak in February (Diarra et al. 2014); Australia where C. brevitarsis is most abundant in summer (Bishop et al. 1995); Asia (Korea) where Culicoides arakawae was more abundant in July and C. punctatus in August (Kim et al. 2014); North America (USA) where C. sonorensis can be present all year round peaking in August or in September (Gerry et al. 2001; Mayo et al. 2014a); and South America (Brazil) where C. pusillus and C. insignis adults were captured in every month of the year and peaked in the rainy season (Carvalho and Silva 2014).

\section{Females parity status as a measure of risk of BTV transmission}

In anautogenous species the parity of females is a critical epidemiological factor. Classically we distinguish three gonotrophic or parity status in biting midges females. The identification of these stages in Culicoides spp. was first described by Dyce (1969). Nulliparous females are those that have no pigmentation in the abdomen and thus coloration is whitish-greyish. A nulliparous female is unfed and has not yet completed a gonotrophic cycle and consequently its epidemiological relevance to transmission is nil since blood from a potentially infected animal has not been ingested. Gravid females are those that hold eggs in the abdomen and they can easily be identified by using a stereomicroscope. Finally, parous females are those that have completed the full gonotrophic cycle, that is, feeding blood from a host, then develop, mature and oviposit eggs. Parous females are identified by a pale reddish colour in the abdomen due to the blood remnants, and they are the most important for BTV transmission, as they have completed a full gonotrophic cycle and therefore potentially the EIP if they have fed on a viremic host. Parous females should therefore be selected for analysing virus presence via RT-qPCR since biologically they are the most relevant for identifying potential and proven vectors. It is common to include a fourth category, blood fed females, from which the age cannot be established by age-grading techniques. This category does not represent a true phase of the gonotrophic cycle, because fresh blood in the abdomen prevents the observer from distinguishing between nulliparous and parous females, but it is of great importance when the biting rate is to be estimated or blood fed females are captured for bloodmeal analysis to understand host preferences. The method of age grading developed by Dyce (1969) has been found to have some limitations. Braverman and Mumcuoglu 
(2009) found that old nulliparous females of $C$. imicola obtained in emergence traps also displayed pigmented abdomens. According to the authors, $23 \%$ of the females identified as parous were indeed old nulliparous females that had developed a reddish pigment in the abdomen. This fact was also confirmed by Harrup et al. (2013) when nulliparous females of $C$. obsoletus captured by soil emergence traps also showed pigmented abdomens. Despite these limitations, which should be taken into account particularly during key transmission seasons, observation of the colour of the abdomen is still the most accepted method for determining the parity status of Culicoides females.

Information on the parity status of females is of great importance, since currently some of the regulations, for example at EU level, Regulation (EC) 1266/2007 (EC 2007), are based on the detection of the activity of parous females during the year to establish periods of null/low risk of transmission of BTV which are defined as seasonal vector free period (SVFP) (EFSA Panel on Animal Health and Welfare 2007). The activity of adult biting midges is measured for this purpose by UV light traps (see section below) and it is therefore necessary to include age grading of females in national surveillance programmes across EU countries aiming to determine SVFP consistently. The parity status of Culicoides female populations changes throughout the year, the nulliparous being the dominant class early in the year; when the population starts declining, parous females are relatively more abundant. Examples of this shift in the relative prevalence of the different parity states of females has been observed for C. sonorensis by Lysyk (2007) in Canada, Mayo et al. (2014a) in California and Meiswinkel et al. (2014) in the Netherlands.

\section{Monitoring Culicoides spp.}

\section{Adults}

Adult Culicoides can be sampled by using a series of devices depending on the aim of the study; the main methods, utilised are summarised below. Many of the methods explained in this section are also used to test the efficacy of control measures (i.e. insecticides) on either adults or immature stages.

\section{Light traps}

Light traps are the most common method for capturing adult Culicoides. Traps are usually equipped with UV light and a suction fan. Several models are available, some of them commercially. The Miniature CDC trap (Model 1212, J.W. Hock, Gainesville, FL, USA) and the Onderstepoort type trap (ARC, Institute for Agricultural Engineering, Pretoria, South Africa) are the most commonly used in published studies. Other models such as the BG Sentinel (Biogents, Regensburg, Germany) were initially designed for Aedes mosquitoes, but a modified model provided with UV light has been used for monitoring Culicoides spp. in countries such as Germany (Hörbrand and Geier 2009; Kiel et al. 2009; Vorsprach et al. 2009). The advantages of using UV light traps are that they are easy to operate, and can be used as a routine system for monitoring Culicoides populations. Light traps have been used in numerous national surveillance programmes for Culicoides elsewhere, particularly in Europe since the incursion of BTV in the late 90s (Calvete et al. 2006; Capela et al. 2003; Goffredo et al. 2015; Kiel et al. 2009; Meiswinkel et al. 2014; Patakakis 2004; Venail et al. 2012). In countries such as the USA, light traps (miniature CDC) are used in combination with carbon dioxide $\left(\mathrm{CO}_{2}\right)$ for sampling populations of $C$. sonorensis, and frequently light is not used but only $\mathrm{CO}_{2}$ as an attractant (Gerry and Mullens 2000; Smith and Mullens 2003). 
Subsampling methods are available when large quantities of insects are collected and the analysis of the whole sample will be too labour intensive to be logistically feasible (Van Ark and Meiswinkel 1992). Light traps are also useful for collecting large quantities of adults that would be very convenient for molecular diagnostic of BTV in vectors (Goffredo et al. 2015; Savini et al. 2005) or when specimens are required for vector competence studies or bionomy studies (Barceló and Miranda 2018; Del Río López et al. 2012; Paweska and Venter 2004; Venter et al. 1999, 2006, 2010). In the latter case, Culicoides are collected alive by modifying the characteristics of the collector bottle. Comparison of different types of traps has been performed in several countries, taking into account different population abundances. Miniature CDC traps and Onderstepoort traps appear to be the best options in terms of Culicoides species composition and abundance in Spain (Del Río et al. 2009, 2013) and South Africa (Venter et al. 2009), while in Germany, the BG Sentinel (Biogents) was found to perform better than the above-mentioned models (Probst et al. 2015)

Despite light traps being the most common method for adult Culicoides surveillance, they present a series of limitations. When compared to other methods of collection, for example animal-baited traps, light traps appear to be biased to certain species. Carpenter et al. (2008) demonstrated a bias in light trap collections when compared to collections in a drop trap containing Poll Dorset sheep; underestimating the abundance of $C$. chiopterus and overestimating the abundance of $C$. obsoletus (Carpenter et al. 2008). Viennet et al. (2011) demonstrated that light traps were overestimating C. obsoletus biting rate as well and underestimating the Culicoides brunnicans Edwards when compared to drop traps and sticky traps on sheep. Gerry et al. (2009) also demonstrated that Culicoides species composition captured using a miniature CDC trap was different to that obtained when adult Culicoides were collected by directly vacuuming sheep; in this case, C. obsoletus and Culicoides parroti biting rates were underestimated. In addition, Culicoides collected in light traps is influenced by the proportion of Culicoides in different physiological states in the surrounding area (Rigot and Gilbert 2012). Light traps also collect males and gravid females in addition to hostseeking females, while other methods such as drop traps collect mainly host-seeking females. Different Culicoides species composition can also be obtained when alternative methods to light traps are used, such as passive suction traps and truck traps (Sanders et al. 2011b, 2012). In addition, light traps usually capture large amounts of other insects which are of little interest as regards BTV transmission (Del Río et al. 2014). Even if nets are placed around the light to avoid capturing larger insects (i.e. moths), some groups are similar in size to Culicoides and are easily captured in the collecting bottles (i.e. Cecidomydae). Those insects increase the difficulty of handling procedures, of sorting samples and substantially increase the time consumed by sorting and identifying collected Culicoides specimens.

\section{Drop traps}

Drop traps consist of a method to collect Culicoides based on placing animals (the live bait) under a canvas that is folded and unfolded in a systematic way to that for collecting host-seeking Culicoides. This method has been widely used to estimate the biting rate of Culicoides in France (Viennet et al. 2011), Denmark and the UK (Carpenter et al. 2008), as well as to demonstrate the efficacy of insecticides (Weiher et al. 2014). The main advantages of this method is that it targets the host-seeking population, and blood-fed individuals are collected in higher numbers when compared to other sampling method such as light traps. However, the main disadvantages are that the technique is labour intensive and requires significant infrastructure (i.e. individual pens and animals) to achieve comparable results. 


\section{Sticky traps}

Sticky traps consist of a surface that is covered with a glue-like substance that retains adult Culicoides that land on it. They have only been used on a few occasions, for example for assessing different attraction to semiochemicals (Bishop et al. 2008; Blackwell et al. 1994a), attraction to colours and host (Koch and Axtell 1979b; Thompson et al. 2014; Viennet et al. 2011; Votýpka et al. 2009), dispersal and distribution of adults (Kline and Axtell 1977; Service 1969; Zimmerman and Turner 1984). However, none of the current systems based on sticky traps are a viable alternative to the use of light traps for general monitoring and large-scale surveillance objectives. The main advantage of sticky traps is their low price and ease of use, and in the absence of stimuli, traps act as a passive sampling method. However, once insects have been captured in the glue, morphological identifications, as well as subsequent analysis for virus, blood and molecular taxonomy are hampered by the difficulties in removing the insect from the glue.

\section{Truck traps}

Truck traps are vehicle-mounted traps, usually in the roof of the vehicle, which are used to collect insects while the vehicle is circulating at low speed. The main advantage of this type of trap is to collect insects that are active at the particular moment of sampling. Therefore, bias due to stimuli (i.e. light, semiochemicals) is avoided. Truck traps have been used in several studies for collecting Culicoides fauna and for assessing the diel pattern activity of different species; such studies have been carried out by Kettle et al. (1998) in Australia and by (Sanders et al. 2012) in the UK. Both found that Culicoides activity peaked at dusk.

\section{Aerial sampling}

Several methods are available for sampling adult Culicoides at high altitudes. These methods usually determine the passive dispersal of adult Culicoides due to wind transport as aerial plankton, which has been described as one of the main mechanisms of incursion of the BTV into territories (Braverman and Chechik 1996; Sellers et al. 1983). Methods for high altitude aerial sampling of Culicoides include tethered balloons, which have been used in Australia where Johansen et al. (2003) captured adults of Culicoides bundyensis Lee \& Reye and Culicoides histrio Johannson at heights between 70 and $150 \mathrm{~m}$ when assessing the risk of entry of infected vectors of Japanese encephalitis from New Guinea. Similarly, adult biting midges were recorded between 170 and 200 $\mathrm{m}$ by Sanders et al. (2011a) also using a tethered balloon over southern Britain.

\section{Suction traps}

Suction traps consist of vertical tubes, generally $12 \mathrm{~m}$ long, provided with an electrical fan at the base of the tube, that backdraw insects into a collecting bottle (i.e. Rothamsted trap; Rothamsted Research, Harpenden, UK) (Macaulay et al. 1988). Suction traps have been proposed as a relatively unbiased collection method for Culicoides since collections allow for the exploration of insect fauna at different heights, unbiased sex ratios, diel patterns as well as the comparison of captures with meteorological conditions. In the work by Service (1971) Culicoides fauna was sampled from 23 to $550 \mathrm{~cm}$ in a forest in southern England and adults from the Obsoletus group were the most abundant, showing a peak of activity at dusk between 20:00 and 21:00 h and a decreasing amount of captures according to height of the suction traps. (Fassotte et al. 2008) analysed samples from suction traps placed in two locations of Belgium during the BTV outbreak in 2006. Nine species of Culicoides were collected, including those of subgenus Avaritia which are the suspected vector 
of BTV in the region. In a more recent study, Sanders et al. (2011b) explored the seasonality and influence of meteorological conditions on the flight activity of Culicoides fauna in 12 different locations in England by using $12.2 \mathrm{~m}$ aerial Rothamsted suction traps.

\section{Vacuuming}

Vacuuming of adult Culicoides directly from animals is one of the most preferred methods when information is required about the species that are associated with a particular host. In comparison to light traps, direct sampling on animals would avoid bias due to position of traps and different responses of Culicoides spp. to the wavelength of light used. Usually an adapted cleaning hand vacuum is used on animals with ease of replacing tubes for each sampling, but backpack aspirators are also used, normally for capturing insects from surfaces. Aspirators are frequently used in combination with other systems such as drop traps, where insects are collected after the animal has been exposed for a time to field Culicoides and avoiding human presence that may affect host-seeking behaviour of the vectors. Aspiration of Culicoides is commonly used when assessing biting rate (Carpenter et al. 2008; Elbers and Meiswinkel 2014; Gerry et al. 2009) and species composition in relation to particular hosts (Sáenz and Greiner 1994; Viennet et al. 2011), when testing insecticide efficacy (Liebisch and Liebisch 2008; Lincoln et al. 2015; Weiher et al. 2014) or when comparing with other sampling tools such as light traps (Scheffer et al. 2012). The main disadvantage of using aspirators is the amount of time required and the need for infrastructure to immobilise animals during sampling.

\section{Breeding site sampling}

\section{Direct collection of substrate}

The usual method for studying Culicoides breeding sites is to collect substrate samples and subsequently analyse them in the laboratory. Two methods are frequently used: the first uses flotation properties, i.e. direct extraction of larvae using a saturated salt solution (i.e. $\mathrm{MgSO}_{4}$ ). This method was used by Mayo et al. (2014b) for extracting larvae from waste-water lagoons in cattle farms in California. Extracted larvae could therefore be directly identified using morphology or molecular taxonomy (Schwenkenbecher and Mordue 2009). This direct method of extraction is also used when some analysis is intended to be performed directly on larvae, as for example when assessing for the presence of virus in larvae with the aim of demonstrating VT (Osborne et al. 2015; White et al. 2005). Collected larvae can also subsequently be fed using nematodes (Kettle et al. 1975) until adults emerge.

The second method of sampling larval breeding sites directly consists of collecting breeding site samples (i.e. mud, dung pats, litter, etc.) and placing these directly into containers until adults emerge. Examples of the use of this method for studying breeding sites can be found elsewhere, for example in the UK (Blackwell et al. 1994b, 1999; Boorman and Goddard 1970; Buxton 1960; Campbell and Pelham-Clinton 1960; Harrup et al. 2013; Kettle 1961); Cyprus (Mellor and Pitzolis 1979); Italy (Foxi and Delrio 2010); Germany (Lühken et al. 2015; Steinke et al. 2014); Spain (González et al. 2013); Belgium (Zimmer et al. 2010, 2013, 2014); Ireland (Thompson et al. 2013); France (Ninio et al. 2011); Denmark (Kirkeby et al. 2009); Turkey (Uslu and Dik 2006, 2007a, 2010); Russia (Glushchenko and Mirzaeva 2008); Israel (Birley and Braverman 1987; Braverman et al. 1974); North America (Kline and Greiner 1985; Mayo et al. 2014b; Mullens 1989; Mullens and Lip 2014; Wirth and Hubert 1962); South America (Carrasquilla et al. 2010) and Australia (Bishop et al. 1996; Brei et al. 2003; Cribb et al. 2003). 


\section{Emergence traps}

Emergence traps are directly placed in the field, on top of the suspected breeding sites and allow for the capture of all insects that develop and emerge from the targeted substrate. This method provides data on the time of emergence of adults in relation to the type of substrate and the climatological factors, as well as the sex ratio. Some examples of this method were found in the work by Nielsen (1963) in Denmark; Dipeolu and Ogunrinade (1977); Campbell and PelhamClinton (1960) and Harrup et al. (2013) in UK; Braverman and Mumcuoglu (2009) in Israel and González et al. (2013) in Spain.

\section{Traps as a decision tools}

From 1998 onwards, and due to the widespread epidemics of BTV in Europe, measurement of the abundance of Culicoides has been a key issue for the determination of the SVFP (EFSA 2007). Based on EU regulations, animal movement was allowed in the absence of Culicoides adults in the field or no activity demonstrated by captures of UV suction light/traps. A standard number of 5 parous/females trap/night was set as the limit to declare the end of the SVFP. This approach was developed due to practical issues surrounding animal movement, but was shown to have poor biological value in places where Culicoides are active all year around (i.e. southern Europe). The length of the SVFP is region dependent according to climatic conditions that are the main drivers of biting midges activities. In consequence, it has been showed that some regions in Europe with warm winters lack a period in which vector activity completely ceases (i.e. some parts of southern Europe). This is the case for example for C. imicola in southern regions of Spain (Calvete et al. 2006; Miranda et al. 2004; Ortega et al. 1997), as well as for C. obsoletus/C. scoticus in regions of southern (Foxi and Delrio 2010; Foxi et al. 2011) and northern Europe (Balczun et al. 2009; Clausen et al. 2009; Kiel et al. 2009; Santiago-Alarcon et al. 2013; Vorsprach et al. 2009). In other regions with colder winters the SVFP can extend for more than 150 days (Searle et al. 2014). However dependent upon intra-annual conditions, the activity of low numbers of nulliparous females can be detected during winter in many northern European countries (Ander et al. 2012; Meiswinkel et al. 2014; Searle et al. 2014; Takken et al. 2008). The concept of SVFP for defining trade windows was revised by EFSA (2008) and a more biologically sound version based on a 'low period of transmission' was proposed. This is based on the knowledge of the seasonal dynamics of the major vector species in particular areas (i.e. for at least 3 years) and therefore the estimation of the period when BTV transmission is not possible due to low vector activity or low temperatures that preclude the EIP from taking place. To date, the regulation at EU level (Regulation (EC) 1266/2007, Annex V; EC 2007) includes the definition of SVFP which takes into account the Culicoides species composition, the abundance of vectors, the presence of virus circulating and the environmental factor that favours virus transmission. Long-term data on Culicoides seasonality combined with environmental data would therefore provide more secure estimates of the periods when BTV transmission is biologically very unlikely. This concept was reviewed by Searle et al. (2014) aiming to develop models to obtain SVFP from environmental drivers and phenological metrics. According to the authors, the different bioecologies of the species included in the Avaritia subgenus limited their ability to predict the SVFP, due to the availability of group-level data for these species from the study area.

\section{Control of Culicoides spp.}

Vector control is one of the cornerstones for managing vector-borne diseases, particularly if vaccines or disease treatments are not available or resistance to them is in place. Reducing the 
vector population has the potential to suppress the rate of transmission of a particular arbovirus and to prevent its spread into new areas. While there are several examples of vector-borne diseases where vector control techniques have been widely developed and applied in field conditions to reduce transmission/incidence (i.e. malaria, dengue, trypanosomiasis), control of Culicoides-borne diseases does not make maximum use of techniques to reduce adult and/or immature Culicoides populations of veterinary importance.

The limitations of biting midge control are principally due to their size, biology, activity and lack of importance from a human diseases point of view. These factors have strongly hampered research on this group when compared to other vector such as mosquitoes. Furthermore, and in line with Mullens et al. (2015), research in this field also appears limited because of the monetary cost and difficulty of field trials, as well as the substantial lack of interdisciplinary connection among the diverse range of groups involved in the management of vector-borne diseases.

Paradoxically, Culicoides vector control would be an essential aspect of the management of diseases such as bluetongue in situations when vaccines for a particular serotype are not available, several serotypes are circulating, the serotype/strain is low pathogenic and/or movement restrictions and protection of animals from vector bites are the only way to reduce transmission. In addition, vector control could also be appropriate under emergency outbreak situations when rapid action is needed or where vaccines are not affordable (Harrup et al. 2016). However, to date, and despite the increase in publications on Culicoides control trials during the last decade, there are still no examples of biting midge control being applied at field level to prevent and/or manage BTV epidemics. Currently it is highly impractical to control Culicoides at an environmental level due to the ubiquity and complexity of breeding sites, the small size of adults, the lack of knowledge about activity and resting places, together with the limited availability of officially approved commercial biocides for adult control. The majority of testing and regulatory approval has focused on testing topical veterinary insecticides and repellents. However, little information is available regarding the efficiency of topical insecticides and repellents or the efficiency rate required to stop or reduce BTV transmission (Mullens et al. 2015).

Here we review the state-of-the-art of Culicoides control, considering the general classification of control methods as they appear in previous summary works (Carpenter et al. 2008; EFSA 2008; Harrup et al. 2016; Maclachlan and Mayo 2013; Mullens et al. 2015; Pfannenstiel et al. 2015). Therefore, we consider the following types of control: mechanical, chemical, biological, genetic and biotechnological.

\section{Mechanical control}

\section{Habitat modification and source reduction}

Breeding sites of Culicoides spp. include different types of habitats, from soils enriched with organic matter, litter, rotten vegetables, to dung pats, manure heap and farm associated wastewater lagoons (see above) (Mellor et al. 2000; Purse et al. 2015). Breeding sites could therefore only be controlled for those species whose habitats are clearly demarcated and where feasible removal or modification is logistically possible. Breeding sites for some of the species in Europe, such as $C$. imicola, has been recorded around farm premises (Braverman et al. 1974). This species is known to breed in the surroundings of farms taking advantage of water leaks and humid soil environments associated with farming practices (Mellor and Pitzolis 1979). Removing those potential breeding sites would theoretically result in decreasing the abundance of biting midges around the farm. 
When compared to other BTV vector species, such as those included in the Obsoletus complex (C. obsoletus/C. scoticus), breeding site control appears unfeasible due to the wide variety and ubiquity of breeding habitats, from farms to deciduous forests. In fact, Harrup et al. (2014) showed that muck heaps covered with tarpaulins had no effect on the overall vector population on UK farms. For other BTV vector species described in Europe, such as C. pulicaris, there are no examples of mechanical control of breeding sites. In the case of Culicoides vector species, which breed in dung habitats, the mechanical disturbance of cow-pats had no effect on the populations of $C$. chiopterus, C. dewulfi and C. scoticus, according to the work conducted by Lühken et al. (2014a). On the other hand, flooded cow-pats showed an effect on Culicoides larvae survival (Lühken et al. 2014b); however, its operational feasibility at field level remains unknown.

In North America, C. sonorensis is considered the major vector species for BTV. Breeding sites are located in manure-polluted and silty mud at the margins of habitats such as dairy wastewater ponds as reviewed by Pfannenstiel et al. (2015). Control of breeding sites was explored by Mayo et al. (2014b), comparing the effect of removal of breeding sites for $C$. sonorensis on farms in California. The study showed that the removing of waste-water lagoon had no effect on the overall population of adults collected at farm level. According to the authors, alternative breeding sites were present around the farm premises and remained largely uncontrolled. Other species such as C. furens, a human biting salt marsh midge of the Americas, was controlled in the '70s by continuous flooding procedures (Kettle 1969).

All these studies on different species demonstrate that there are still inadequate farming procedures (i.e. cleaning and removal of manure; composting) which may have an impact on Culicoides larvae and pupae populations. Breeding site control seems feasible a priori in farms that contain clustered biting midges breeding sites, such as C. imicola. However, the patchiness of farms (i.e. Mediterranean basin agriculture) and the wide variety of potential breeding sites even at farm level, makes it labour intensive and impractical for the farmer to control them. In places with more homogeneous landscapes (i.e. farms in California), the presence of vast areas containing natural breeding sites apart from those located at farm level also precludes any attempt at larval control.

\section{Screening of doors and windows}

Since most of the species of Culicoides are considered exophilic, protecting animals inside stables and the screening of windows and doors with anti-biting midges nets would seem to be a practical strategy for protecting animals from bites, particularly at dusk. In South Africa Meiswinkel et al. (2000) showed that protecting horses inside gauzed-stables reduced by 14-fold the presence of C. imicola and C. bolitinos (major vectors of AHSV) when compared to the outside; however, an assessment of the efficacy of these results under an epidemic scenario of Orbivirus (i.e. BTV and AHSV) transmission is still pending. In Australia, Doherty et al. (2004) tested a combination of roof and walls for protecting cattle against the bites of C. brevitarsis, C. wadai, C. actoni and C. fulvus. Pens provided with a high roof and walls showed fewer captures of $C$. brevitarsis in light traps when compared to the other combinations of pens. However, this relative protection was not sufficient to prevent contact between midges and animals, and particularly with other species such as C. wadai. Therefore, the reduction of the Culicoides rate of attack was difficult to estimate. In the UK, Baylis et al. (2010) measured the abundance of biting midges (i.e. C. obsoletus) using Onderstepoort UV light traps; densities were shown to be 3 to 4 times lower inside stables where cattle were housed at night. However, as in the previous work conducted in Australia, the impact of those farming practices in the case of a BTV epidemic scenario is unknown. In Switzerland, a significant reduction in biting midges inside horse stables (from 98 to 65\%) was achieved by 
Lincoln et al. (2015) testing polypropylene nets (Ultravent ${ }^{\circledR}$ Bemisia TIP 250. Micrometric mesh of $0.1825 \mathrm{~mm}^{2}$, Texinov, France) only. The combination of nets and fans (ZOO no. 1400; B+M Hausund Agrotech, Densbüren, Switzerland) did not provide further protection.

Stabling with or without screenings in door and windows appears to be a cost-effective and feasible strategy for reducing biting pressure on animals. Stabling without screening would not, however, be as efficient as screens because we know that some Culicoides vector species exhibit some indoor behaviour depending on the time of the year and climatological conditions (Baldet et al. 2008). Screening of stables, without compromising ventilation (Meiswinkel et al. 2000), would therefore be recommended in any scenario where the aim is to decrease vector biting activity on domestic animals, even if the impact on the rate of transmission of BTV is unknown.

\section{Chemical control}

\section{Repellents}

Repellents are designed to prevent contact between biting insects and the host. They have been well studied and developed for other biting insects, such as mosquitoes (Debboun and Strickman 2013; Pates and Curtis 2005). However, little development has been made on specific repellents against Culicoides, especially for those intended to be used in domestic animals to reduce BTV transmission. For example, in Europe there are no officially registered repellents against Culicoides licensed to be used on domestic animals and only human products based on DEET (N,N-diethylmeta-toluamide) are commercially available. Most of the products applied to high-value species, such as horses, are products mainly intended for general 'biting insects'. Some topical insecticides also show a repellent effect, but there is usually a lack of data about its impact on the biting rate of Culicoides.

Plant-derived repellents have been tested on a limited number of Culicoides species. AG1000t, a plant-derived extract from Meliaceae, has been shown to be efficacious against $C$. imicola in Israel lasting up to 4 hours, as well as pyrethroid T (type II pyrethroid containing alfa-ciano, 3-phenoxybenzyl moiety fly toxicant with $2 \%$ a.i.) (Braverman and Chivoz-Ginzburg 1997, 1998). Another plant-derived repellent, the Mosiguard, a lemon-scented repellent formulation based on Eucalyptus maculata var citriodora, has been demonstrated to attract rather than repel C. imicola when used in impregnated nets placed in light traps (Braverman et al. 1999). Similar studies (Braverman et al. 2000) have also been conducted for the primary vector of BTV in North America C. sonorensis. Moreover, Eucalyptus-based repellents ( $p$-menthane-3,8-diol) have also been tested in humans against $C$. impunctatus in Scotland, showing a similar effectiveness ( $10 \mathrm{~h}$ of protection) when compared with DEET (Trigg 1996). Similarly, a Neem repellent (extracted from Azadirachta indica A. Juss; Meliaceae) for human protection against $C$. impunctatus also showed an antilanding and anti-feeding effect on adults of a colony of Culicoides nubeculosus and field-collected C. impunctatus (Blackwell et al. 2004). Other examples of tested repellents against human-biting Culicoides were found in Scotland, where Logan et al. (2009) tested human-derived odours against C. impunctatus; in Australia where Greive et al. (2010) tested Melaleuca oil against C. ornatus and C. immaculatus and in Colombia where para-mentano-3,8-diol and limonaria oil was tested against Culicoides pachymerus Lutz (Santamaría et al. 2012). None of the current plant-derived repellents are routinely used against Culicoides and no information is available about their use in a BTV outbreak scenario. Harrup et al. (2016) mentioned that in farms in rural India, Neem leaves are regularly burned to repel biting Culicoides, but no data is available about its efficacy. 
Less information is available on testing repellents for protecting domestic animals compared to that of human repellents. Due to the difficulties in performing trials using animals, collections in UV light traps as surrogates for host animals have been used together with laboratory trials using Y-tube tests. González et al. (2014) in Spain and Venter et al. (2011) in South Africa tested a mixture of octanoic, decanoic and nonanoic fatty acids impregnated in a mesh on UV light traps and showed that fewer Culicoides spp. were collected when compared with control traps. Also in South Africa, Venter et al. (2014) obtained no repellent effect against any of the gonotrophic stages of $C$. imicola females when testing commercial peel-stick patches containing citronella and lemon eucalyptus oil on UV light traps. Few tests have been directly conducted on animals. Reeves et al. (2010) tested the repellent effect of insecticide (Y-TEX: $2.5 \%$ permethrin and 2.5\% piperonyl butoxide, $12 \mathrm{ml} / \mathrm{sheep}$ ) separately and in combination with insecticide impregnated ear-tags (PYthon: 10\% zeta-cypermethrin $9.8 \mathrm{~g} /$ tag and $20 \%$ piperonyl butoxide). A test was conducted on sheep that were treated and exposed to $C$. sonorensis laboratory-reared adults by using feeding tubes in the axillary area. According to the authors, both the ear-tag and the insecticide were effective in decreasing feeding of $C$. sonorensis for up to 4 weeks, with insecticide treatments being cheaper and quicker in giving protection. Nevertheless, since efficacy data were not provided from this semi-field trial, it is difficult to assess the practical application of the results in a field scenario. The following two examples include trials conducted at field level. Robin et al. (2015) found no repellent effect on the feeding rate of Culicoides spp. when a 1\% pour-on solution of a deltamethrin insecticide ('Spot on'; Zoetis UK, London, UK) was tested on horses in UK. This work was carried out using animals as bait for Culicoides; however, the direct effect of the repellent was not measured directly on animals but on Onderstepoort traps placed near the animals. In a similar trial, Lincoln et al. (2015) also obtained low efficacy of protection against Culicoides spp. bites after applying a spray solution composed of permethrin insecticide and DEET (Flymax, $6 \mathrm{mg}$ / $\mathrm{ml}$ permethrin and $20 \mathrm{mg} / \mathrm{ml} \mathrm{DEET}$; Audevard Ltd., Clichy, France); as in the previous work, efficacy was tested by using UV light traps as a proxy for animals. Baker et al. (2015) tested the effect on colony adults of $C$. nubeculosus of a series of commercially available repellents applied to mesh nets evaluated using WHO cone tests. The repellents tested were NAF Off Citronella (citronella oil $<1.5 \%$ w/w. Greencoat Ltd t/a Natural Animal Feeds, Monmouth, UK); NAF Off DEET POWER (DEET $<20 \%$ w/w; Greencoat Ltd t/a Natural Animal Feeds, Monmouth, UK) and NAF Off Extra Effect (citriodiol1\% w/w; Greencoat Ltd t/a Natural Animal Feeds, Monmouth, UK). None of the repellents tested showed efficacy in decreasing the engorged rate of females, showing no spatial repellence in any of the conducted test.

From the examples provided in this section it is obvious that much more research is needed in the field of Culicoides repellents. Efficacy of repellents should be tested in field conditions, where different species composition and therefore behaviour and biology of biting midges may challenge the efficacy of the compound. Trials performed 'in vitro', semi-field or using UV light traps as a proxy for animals are of great value for prior screening of possible repellent active ingredients. However, as in other areas of Culicoides control, field test of repellents are still needed, whilst, more importantly, taking into account different epidemiological scenarios in relation to BTV transmission.

\section{Insecticides}

Chemical control of Culicoides spp. using insecticides has developed much more during the last decade in terms of trials and protocols than control with repellents. Here we divide insecticides into adulticides and larvicides. Adulticides are also divided into systemic insecticides, topical treatments and environmental applications. Topical treatments could also be divided into studies 
conducted 'in vitro', i.e. using colony-reared Culicoides and exposing adults to insecticides using different techniques (i.e. WHO tubes, hair clippers from treated animals); studies conducted in 'semi-field' conditions, i.e. exposing adult Culicoides directly to animals; and finally field tests, where the efficacy of insecticides is measured directly on animals and takes account of environmental conditions.

\section{Adulticides}

Control of adult Culicoides represents serious problems since there is limited information about the efficacy of control methods that could be used on both animals and the environment. Little information is available about resting sites and shelters, which makes it difficult to select targets for biocide treatments. Protection of animals using pour-on insecticides is currently the most explored method, however there is a great variability of results in the literature. Environmental application of insecticides against Culicoides is also limited due to the current regulations on environmental protection and due to the above-mentioned lack of information about where to target the treatments.

\section{Systemic insecticides}

Systemic insecticides such as ivermectin (macrolactone) have been tested against $C$. brevitarsis, the main vector of BTV in Australia where Standfast et al. (1985) reported 99\% of mortality in females at 10 days after treatment and $40 \%$ of mortality at 18 days post-treatment. However, when ivermectin was used on adults of a colony of Culicoides varipennis (Coquillet), there was no significant effect on their survival when females were blood-fed on calves treated with a commercial formulation of ivermectin $(200 \mathrm{pg} / \mathrm{kg}$; Ivomec, Merck and Co., Inc., Rahway, NJ, USA) (Holbrook 1994). When colony-reared C. varipennis females were fed through an artificial membrane with blood containing 0-1.0 microgram $/ \mathrm{ml}$ of ivermectin, significant mortality was observed at $48 \mathrm{~h}$ post-feeding; however, the recommended dosage in animals $(200 \mu \mathrm{g} / \mathrm{kg}$ ) would have no effect on the mortality of biting midges due to the low concentration of serum (Holbrook and Mullens 1994). Ivermectin is widely used to treat parasitic infestations such as scabies (Dhana et al. 2018), pediculosis and myasis (Fox 2006), helminths of medical and veterinary importance (Barrett et al. 2016), and parasites of aquaculture fishes (Horsberg 2012). However, its use for routine field control of Culicoides and other blood-feeding insects is far from being effective.

\section{Topical treatments}

\section{Wind tunnel assays}

Floore (1985) tested nine insecticides against adult Culicoides spp. in a wind tunnel. Five of these insecticides were organophosphates (malathion, naled, fenitrothion, fenthion and clorpyrifos), whose use in Europe today is highly restricted in animals and the environment. The other insecticides tested were pyrethroids (resmethrin, phenotrin and permethrin) and a combination of malathion/resmethrin (90:1). The pyrethroids showed the best results with $100 \%$ knockdown $1 \mathrm{~h}$ post-treatment.

\section{In vitro assays using WHO protocol}

Several authors have tested the efficacy of insecticides against Culicoides, adapting WHO testing protocols initially developed for mosquitoes. The protocol includes the assessment of the 
mortality of adult Culicoides by estimating LD50 and LD90 when they make contact with separate insecticide-impregnated papers of different concentrations inside WHO plastic tubes (See Venail et al. 2011 for further details). Braverman et al. (2004) tested the susceptibility of field collected C. imicola nulliparous females to lambda-cyhalothrin $(125 \mathrm{mg} / \mathrm{ml}$. Syngenta) using the WHO protocol. The tested insecticides showed high efficacy against C. imicola (LD50: 0.0098). In France Venail et al. $(2011,2015)$ pioneered the use of the adapted WHO protocol for Culicoides. When tested against colony-reared adults of $C$. nubeculosus and field-collected adults of $C$. obsoletus and C. imicola, they obtained high mortality (i.e. 100\%) after $1 \mathrm{~h}$ exposure to concentrations of $0.0025 \%$ deltamethrin impregnated paper. A similar study was conducted by Del Río et al. (2013) in Spain, showing that field collected adults of C. obsoletus were highly susceptible to doses above $0.001 \%$ of deltamethrin. Populations tested in Spain proved to be 3.5 times more sensitive to deltamethrin compared to previous trials by Venail et al. (2011) in France. One explanation could be the different pressure of insecticide treatments on a rural scale and the level of exposure of populations from Spain and France. Also using WHO protocols, Onuike et al. (2015) carried out a very comprehensive trial on the killing effect, oviposition and feeding at different temperatures $\left(25,20,15\right.$ and $\left.10{ }^{\circ} \mathrm{C}\right)$ of different concentrations of deltamethrin-impregnated papers on colony-reared adults of $C$. nubeculosus, similar to the trial conducted by Venail et al. (2011). The concentration of $0.05 \%$ showed the highest mortality (90\%) and a knock-down effect was observed after $1 \mathrm{~h}$ of exposure; however, at lower concentrations of insecticide, midges recovered activity $24 \mathrm{~h}$ post-treatment. No significant effect on mortality was obtained at different temperatures, except at $10{ }^{\circ} \mathrm{C}$ where higher mortality was observed. The effect of low temperature on the killing effect of insecticides remains unclear. Del Río et al. (2013) also showed that Culicoides that were chilled down showed different rates of mortality compared to non-chilled adults. In this case, chilled adults showed fewer mortality effects when compared to non-chilled ones. In the study by Onuike et al. (2015) no effect of the insecticide was detected on the feeding behaviour, however, oviposition was affected since adults that were exposed to the insecticide laid fewer eggs. It is therefore important to understand the effect of insecticides, not only in the mortality of adult Culicoides, but in other activities such as mating, feeding, and oviposition. If insecticides affect some of these activities, besides lifespan, it might help decrease abundance and biting pressure on domestic animals. In general, results derived from the use of the WHO protocol for testing insecticides against Culicoides show that widely used insecticides such as pyrethroids have a significant effect on mortality. This high mortality shown in laboratory results needs further exploration at the field level.

\section{In vitro assays exposing adults to treated hair}

The technique of testing insecticides by exposing adult Culicoides to insecticide-treated hair or wool clippings enables more information to be obtained about the dose needed and the part of the body to be treated in order to achieve a satisfactory killing effect. Results obtained could be considered more similar to 'field' conditions. However, this technique shares the same limitations as the WHO, i.e. adults are usually enclosed and therefore close and continuous contact with the impregnated substrate (i.e. paper, hair, wool) may overestimate the killing effect of the tested dose. Movement of adults in field conditions and landing on preferred body parts of the animal may prevent the strong killing effects of some of the selected insecticides. Most of the testing on animal hair and wool has been conducted in USA and Europe.

Hair clippers collected from the dorsum of goats treated with Permethrin ( $65 \%$ a.i.) effectively reduced engorgement of colony-reared $C$. varipennis females for 40 days and the effect lasted significantly more than hair clippers collected from head and belly (Mullens 1993). In a similar study, Mullens et al. (2000) tested 5\% a.i. permethrin (Boss ${ }^{\circledR}$; Merck, Darmstadt, Germany) and 27\% 
a.i. pirimiphos methyl using treated hair clippers collected 3, 7, 14, 27, 28, 42 and 56 days from the dorsum, side and belly of treated and untreated calves. Permethrin applied to the dorsum and sides showed a relevant toxic effect on adult midges and reduced the engorged rate up to 14 days after treatment, while pirimiphos methyl showed no reduction at any time. Hair collected from the side and the belly showed less efficacy than the hair collected from the back when tested in vitro. The work conducted in Germany by Schmahl et al. (2008) could not be considered a 'pure' trial on testing insecticides on hair or wool. On the contrary, they used wood plates as a proxy for the surface of stable walls where the microencapsulated insecticide Oxyfly ${ }^{\mathrm{TM}}$ (lambda-cyhalothrin; Novartis, Basel, Switzerland) is usually applied. Therefore, the aim of this type of treatment is to kill adult Culicoides when they make contact with the surface of the stable. In fact, Schmahl et al. (2008) showed that after two weeks of insecticide post-treatment, field-collected adults of C. obsoletus and C. pulicaris died after contact with the plates for 9-20 s. Despite the fact that insecticide application inside stables and transport vehicles is a highly recommended practice during BTV epidemic scenarios (i.e. UE), and particularly after the BTV epidemics in Europe, there are no publications or indications about the efficacy of those measures in reducing the risk of attack of biting midges. The work by Schmahl et al. (2008) may indicate that treatment of walls inside premises was highly effective, but the study did not provide any indication in terms of efficacy on mortality.

An example of the different efficacy of insecticides according to the method of testing (lab vs field) is given by Carpenter et al. (2007). The study conducted in UK assessed two insecticides: Coopers' Spot $\mathrm{On}^{\mathrm{TM}}\left(1.25 \%\right.$ w/v high-cis cypermethrin-based pour-on at $450 \mathrm{mg} / \mathrm{m}^{2}$; ScheringPlough Animal Health, Harefield, UK) and deltamethrin ( $1 \%$ at $\left.60 \mathrm{mg} / \mathrm{m}^{2}\right)$. Hair clips were collected from sheep and cattle that were treated with both insecticides by pour-on method. High mortality was previously obtained in adults tested in the laboratory; however, a lower killing effect was observed on adults exposed to hair clippers collected from legs and the belly of sheep. In the case of cattle, Coopers' Spot On significantly killed adults Culicoides seven days after treatment. The difference in results between the laboratory method and the hair clippers is an explanatory example of the different efficacy of pour-on insecticides according to the nature of diffusion of the active ingredient across the fleece and hair of animals. Apparently, pour-on insecticides diffuse less in sheep fleece compared to hair of other animals. In addition, and considering the results from Mullens (1993) and Mullens et al. (2000), the diffusion of pour-on insecticides also differs according to the animal body part, with the dorsum more protected than belly and legs.

Ear tags are insecticide-impregnated material that diffuse insecticide on the hair of animals in the same way as the pour-on insecticides. Collecting hair clippers and exposing adult Culicoides to them is also a method for testing the extension of insecticide to the body parts of animals. Liebisch and Liebisch (2008) conducted a trial in Germany where 1 or 2 ear-tags per animal (Flectron ${ }^{\circledast}$ Flytags, $1.067 \mathrm{~g}$ cypermethrin per ear-tag; Fort Dodge Animal Health, ParsippanyTroy Hills, NJ, USA) were tested. They performed an in vitro test where field-collected Culicoides spp. were exposed to hair clippers from heifers and dairy cows. Either hair collected from the dorsal line or the ventral abdomen showed toxic activity for Culicoides adults for 14 days when one ear-tag was used, and 21 days when 2 were used. However, these interesting results lack standardised measures for assessing the killing effect of an insecticide. In fact, it is important that researchers report parameters such as LD50 and LD90 in order to make comparable results among studies. This will allow for clearly identifying doses and active ingredients to be applied in field testing. In subsequent trials, Schmahl et al. (2009) tested pour-on insecticides Butox ${ }^{\circledR} 7.5$ (7.5 g deltamethrin per litre of ready-to-use solution; Intervet International B.V., Boxmeer, the Netherlands), Versatrine ${ }^{\circledast}(1 \mathrm{~g}$ deltamethrin per $100 \mathrm{ml}$ excipient; Schering-Plough Vétérinaire, 
Levallois-Perret, France), Flypor ${ }^{\circledR}$ (4\% w/v of permethrin ready-to-use solution; Novartis), Arkofly ${ }^{\circledR}$ ( $6 \mathrm{~g}$ fenvalerate per $100 \mathrm{ml}$ of the ready-to-use spray; Novartis) and Acadrex ${ }^{\circledR} 60$ (6 g fenvalerate within $100 \mathrm{ml}$ excipient solution; Novartis) on cattle and sheep. After treatment, hair clippers were collected just from feet of animals at intervals of 7, 14, 21, 28 and 35 days. Unidentified adult Culicoides collected from the field were exposed to the hair clippers in Petri dishes. All products proved to be efficacious for killing adults even 35 days after treatment. As mentioned before, neither the Culicoides species nor the standard indexes of mortality (i.e. LD50-LD90; \% mortality) were estimated in this series of works and therefore comparisons with other studies are difficult. In addition, unlike other studies (i.e. Carpenter et al. 2007), the authors did not report any limitation of diffusion of the product across the hair of the animals and, considering the testing conditions, close contact between adults and hair clippers in enclosed Petri dishes may overestimate the effect of the tested insecticides. Papadopoulos et al. (2009) tested two pour-on insecticides: Dysect Cattle Pour-On (15 g/l alphacypermethrin) on cattle and Dysect Sheep Pour-On (12.5 g/l alphacypermethrin) on sheep. As in previous studies, hair clippers were collected from leg, belly and back of animals and colony-reared C. nubeculosus adults were exposed to them. Hair clippers demonstrated a keep-killing effect of almost $100 \%$ after 21 days of pour-on treatments. The same methodology (Papadopoulos et al. 2010) was applied when testing cypermethrin (Deosect Spray, $5.0 \% \mathrm{w} / \mathrm{v}$; Fort Dodge Animal Health) on horses. In this case, hair clippers were collected from face, legs, back and hindquarters. Authors reported a decreasing killing effect, of almost $80 \%$ at day 7 to $50 \%$ at day 35 post-treatment. Differences in the killing effect according to the origin of hair clippers was also found and those collected from the back of the horse showed the highest killing effect. As in previous studies, it seems rather obvious that the diffusion of pour-on insecticides is not homogeneous on the body of animals, depending on the type of hair and its nature. This makes it difficult to rely on pour-on treatments for the full protection of animals against Culicoides biting activity. Treatment of animals with pour-on insecticides has been a common recommended practice for reducing the risk of BTV transmission, particularly in animals moving from BTV-affected zones to BTV-free zones (EFSA 2008). It is well known that these practices have been implemented by the different veterinary authorities in BTV-affected European countries. However, there are currently no studies quantifying the reduction of BTV transmission risk when applying this type of protection. If we consider that according to Mellor et al. (2000) one single bite of an infected female could lead to a successful BTV infection, it is evident that pour-on insecticides do not provide full protection and consequently a certain level of risk of BTV transmission still remains.

\section{Semi-field assays}

We could consider semi-field testing of pour-on insecticides when tests are conducted directly on animals and do not use adult Culicoides spp. exposed to hair clippers. Operationally this type of trial is more difficult to put into place, since it requires special infrastructure to keep animals enclosed while tests are running. Compared to hair or fleece clippers, tests performed directly on animals give more information about the diffusion of pour-on insecticides according to the particular physiology of each animal species. Another clear limitation of this technique is the manipulation of adult Culicoides out of full laboratory conditions. This may result in undesired mortality of adults thus limiting the results obtained. Due to the limitations mentioned above, few trials has been completed using this technique.

In France, Venail et al. (2011) treated shorn sheep with Butox ${ }^{\circledast} 7.5$ Pour On (7.5\% w/v deltamethrin; Intervet International B.V.) and tested its killing effect on colony-reared nulliparous females of $C$. nubeculosus directly exposed to the skin of the animals at 1, 4, 6, and $13 \mathrm{~d}$ after treatment. The maximum killing effect was achieved on day 4 post-treatment and only reached $45 \%$ efficacy. The 
product was estimated to last a maximum of $10 \mathrm{~d}$. This study conducted by Venail et al. (2011), as well as others using the hair clipping technique (Carpenter et al. 2007; Papadopoulos et al. 2009, 2010), showed that the main limitation of using pour-on insecticides is the diffusion of active ingredients on the hair/fleece of domestic animals that may leave parts of the body of animals exposed to Culicoides biting activity. If we consider that different species of Culicoides show a preference for different body parts of the animal (i.e. belly, face, legs, ears), complete protection of animals is unlikely to be achieved by using pour-on insecticides only. Indeed, in a recent review conducted by EFSA (2017), the maximum protection achieved by pour-on insecticides was considered to be up to $86 \%$ efficacy. Furthermore, it is not known if repeated pour-on treatments would promote insecticide resistance in Culicoides. The mechanism could therefore be similar to that already observed in mosquitoes exposed to insecticide-treated nets for protecting humans against malaria transmission (Enayati and Hemingway 2010).

\section{Pour-on insecticides}

The most commonly used methods for protecting animals in the field from blood-feeding insects and ectoparasites are the pour-on treatments. These involve treating the back of animals with insecticides that then diffuse to the other parts of the animal. Field testing of pour-on products reveals the performance of insecticides in real conditions, where adult Culicoides are not manipulated from field to laboratory and behaviour and insecticide sensitivity traits of colony-reared adults are avoided. Here we divide field tests into those that include pour-on insecticide and ear-tags. Ear-tags are not included in the section about insecticide-impregnated materials because their mode of action is similar to the pour-on insecticides. We also consider the environmental application of insecticides, both adulticides and larvicides, as well as the use of systemic insecticides.

Mullens et al. (2000) treated the dorsum of calves with 5\% permethrin and the belly with $0.2 \%$ permethrin (Atroban 11\% EC; Schering-Plough Animal Health, Union, NJ, USA). Animals were exposed to field $C$. sonorensis that were vacuumed from animals after allowing vector-feeding activity. Treatment performed in the dorsum of the animals failed to provide protection, i.e. to decrease female engorging rate. In contrast, treatment applied to the belly of the calves succeeded in reducing the feeding rate, particularly because $C$. sonorensis shows a preference for the belly and legs of animals, demonstrating that species-specific feeding behaviour should be taken into account when pour-on insecticides are aimed at biting midge feeding. Curiously, these results are contrary to those obtained exposing Culicoides varipennis to impregnated dorsum hair-clippers that showed the highest efficacy. In a subsequent trial, Mullens et al. (2001) found no decrease in the seroconversion rates of BTV on a cattle farm in California when protection against $C$. sonorensis bites was the intention behind six periodical treatments on the belly of heifers with permethrin $(0.2 \%$ Atroban, $11 \%$ EC). On the contrary, Doherty et al. (2004) effectively protected steers against C. brevitarsis and C. wadai by treating separately with fenvalerate ( $1 \%$ a.i.) and 'Flyaway' (12 g/l permethrin, $50 \mathrm{~g} / \mathrm{l} \mathrm{n}$-octyl bicycloheptene diethyltoluamide, $25 \mathrm{~g} / \mathrm{l}$ dicarboximide, $25 \mathrm{~g} / \mathrm{l}$ piperonyl butoxide, $20 \mathrm{~g} / \mathrm{l}$ dibutyl phthalate and $10 \mathrm{~g} / \mathrm{l}$ lavender oil) on the back, rump, flanks and head of animals. A permethrin pour-on insecticide intended for cattle (Tectonik ${ }^{\otimes} 36 \mathrm{~g} / \mathrm{l}$; doses $10 \mathrm{ml} / 100$ $\mathrm{kg}$ ) was tested in horses in the Netherlands by De Raat et al. (2008). The effect on the Culicoides population was assessed by aspiration of adults from a tent trap (drop trap) and the proportion of blood-fed adults was compared between treated and untreated horses. The drop traps (see section on (ulicoides capture methods) have the advantage of capturing adults without damaging them and avoiding bias due to capture devices such as UV light traps. Species C. obsoletus and C. pulicaris were the most abundant in the study, and despite the fact that a reduction in the 
adults was observed, the selected insecticide failed to reduce the number of blood-fed adults between treated and untreated horses. Limited diffusion of the insecticide to other parts of the body from the application placed on the back of animals may explain the lack of efficacy and provide proof that the distribution of the insecticide on the whole body of the animal is a limiting aspect. This was demonstrated by Mullens et al. (2010) when they carried out a trial on sheep that were treated with $7.5 \%$ deltamethrin (Butox 7.5) not only on the backline, but on face, ears and belly assuring a better distribution of the insecticide. The level of efficacy was measured by vacuuming adult Culicoides from animals using enclosed drop traps. This method of treatment proved to be efficacious since no blood-engorged Culicoides were collected from the animal. In this case, the particular application of the insecticide and the low pressure of biting attack due to low populations of biting midges Obsoletus complex and C. parroti may lead to a higher level of protection. Griffioen et al. (2011) used the same technique as Mullens et al. (2010), testing Tectonik $3.6 \%$ permethrin pour-on solution $(1 \mathrm{ml} / 10 \mathrm{~kg}$ bodyweight; Virbac Animal Health, Barneveld, the Netherlands) on sheep from the Netherlands. In this case a $50 \%$ efficacy rate was reported in reducing number of Culicoides as well as proportion of engorged females on the treated sheep. In Germany Weiher et al. (2014) also conducted a drop trap test of pour-on insecticide applied to sheep (Butox ${ }^{\circledR}, 7.5 \mathrm{mg}$ deltamethrin/ml; Intervet International B.V.). Interestingly, the lowest efficacy on engorged adults was found after $24 \mathrm{~h}(44.7 \%)$, while the maximum efficacy was found after three weeks (94.6\%). The total efficacy of the trial conducted over 5 weeks was $86.4 \%$. According to the authors, low efficacy of the product during the first $24 \mathrm{~h}$ after treatment and insufficient spread to different parts of the animal body (i.e. legs, feet, belly, face) are considered the main limitations of pour-on insecticide treatments.

Field testing of pour-on insecticides using drop traps has been demonstrated to be an appropriate technique for testing the protection of animals against unmanaged field populations of Culicoides. However, differences in the methodology followed by the different authors may lead to noncomparable data. The frequency of days for collecting biting midges and particularly the time between sampling and the moment of application of the pour-on insecticide on animals appeared to be the main differences. On the other hand, the drop trap technique could be limited by the number of animals required for the experiment, since the field setting is labour-intensive and timeconsuming. The animals are usually tested in pair, one treated and one untreated, and although repetitions are conducted, each sampling could be unique due to animal and environmental conditions of the particular time of sampling. Using more than two animals at the same time does not seem very feasible due to the labour and infrastructure required. In field testing of pour-on insecticides it is particularly important to show changes in the efficacy from the first days after application to the end of the trial. If animals are less protected from Culicoides feeding activity during the first days after pour-on application, this should be taken into account to estimate the level of protection of animals.

\section{Insecticide-impregnated ear tags}

Insecticide-impregnated ear-tags are commercially available and represent an alternative to pour-on treatments. Examples of efficacy testing of ear-tags are found mainly in Europe. In North Germany, Liebisch and Liebisch (2008) tested the efficacy of placing one and two eartags (Flectron ${ }^{\circledast}$ Flytags, 1,067 g cypermethrin per ear tag; Fort Dodge Animal Health) per animal in heifers and dairy cows. Midge abundance was measured by suction light traps and direct aspiration from the skin of the animal. According to authors, C. obsoletus, C. pulicaris and C. dewulfi were the dominant species. The conclusions of the study are limited since no specific result is given about the decrease in feeding activity of midges on tested animals. Apparently, the toxic 
efficacy duration was estimated at 14 days when using one ear-tag and up to 21 days for two eartags. This result was also confirmed in vitro by exposing field-collected Culicoides to hair clippers. In another trial conducted in Germany by Bauer et al. (2009), protection of bulls inside a pen by using insecticide-treated ear-tags (Auriplak ${ }^{\circledR}$; Virbac, Barneveld, the Netherlands) containing 1.2 $\mathrm{g}$ of permethrin, as well as five pour-on treatments with deltamethrin (Butox $7.5 ; 750 \mathrm{mg} / 100 \mathrm{ml}$ ), failed to reduce the number of engorged midges captured by BG-Sentinel traps placed inside the bull pens.

\section{Environmental application of adulticides}

There are only a few studies reported about the control of Culicoides spp. in field conditions, which is an indication of the difficulty of controlling them in the environment due to their small size and the general lack of information about resting sites. Before the universal ban, DDT was used in several countries for the control of insect nuisance, including biting midges (Kettle 1969). In Scotland, Kettle (1949) failed to control the human blood feeder C. impunctatus with environmental DDT barrier-spraying. This method was developed during the Second World War for controlling mosquitoes in several parts of the world. After the introduction of BTV in Sardinia, Satta et al. (2004) performed a trial using a micro-encapsulated pyrethroid-based product, (composition per $100 \mathrm{~g}$ : cypermethrin $11 \mathrm{~g}$, esbiothrin $1 \mathrm{~g}$, piperonyl butoxide $11 \mathrm{~g}$, coformulants $77 \mathrm{~g}$; Mycrocip, ICF, Cremona, Italy) and assessed the efficacy of field treatments in collections from Onderstepoort traps in two treatment sites and one control. Treatment showed no efficacy in decreasing C. imicola abundance in comparison to the control site. Even though adulticides have showed high efficacy when tested in laboratory conditions (i.e. WHO test), their application in the field is a long way from being successfully implemented. Apart from the lack of knowledge as to where to apply those adulticides, current regulations on their use in the environment may also preclude general use.

\section{Larvicides}

The control of immature stages, such as larvae, is one of the most important aspects of vector control programmes (i.e. mosquitoes and black flies) since effective reduction of the population can be achieved. However, due to the life cycle of Culicoides and in particular the heterogeneity and nature of breeding sites, larvicides have generally been of limited application. In the USA, trials have been conducted for controlling larvae of species that cause a nuisance in environments such as beaches and salt marshes. Clements and Rogers (1968) succeeded in controlling larvae of C. furens, C. melleus and Culicoides mississippiensis Hoffman; however, none of the products that were used in 1968 would be generally approved for environmental use nowadays. In Florida, the efficacy of four organophosphate compounds (chlorpyrifos, temephos, fenthion and malathion) were tested in the laboratory against $C$. furens and $C$. mississippiensis larvae from salt marsh areas (Kline et al. 1985b). Chlorpyrifos showed the highest efficacy, followed by temephos, fenthion and malathion, either applying the insecticides to larvae placed in estuarine water with and without soil substrate. Despite the efficacy showed by those organophosphate insecticides, environmental concerns these days preclude their use in most environments. In Europe, C. impunctatus has also been a targeted species for larvae control. Kettle et al. (1956) performed several field trials against C. impunctatus in Scotland, considering that this species was found in the soil during all seasons of the year. Several concentrations of DDT and $\gamma$-benzene hexachloride $(\mathrm{BCH})$ (both in wettable powder water-miscible concentrate, oil solution and dust), as well as Aldrin (wettable powder 23.3\% w), dieldrin (wettable powder $10 \% \mathrm{w}$.) and malathion (wettable powders $25 \%$ w and emulsifiable liquid $60 \% \mathrm{w} / \mathrm{v}$ ), chlordane (water-miscible concentrate $55 \% \mathrm{w} / \mathrm{v}$ ) and malathion were applied 
in several plots aiming to control larval populations for several months. The maximum efficacy achieved by DDT and BCH was 77 and 25\%, respectively; meanwhile for the others insecticides, only malathion reached an efficacy of $50 \%$, while chlordane and aldrin were around $30 \%$ and dieldrin 20\%. In a similar trial, Kettle et al. (1959) again tested DDT and BCH for larval control and showed the former to be more efficacious than the latter. We should make it clear that none of the products tested during the late' 50 s would be used nowadays for environmental applications against Culicoides larvae due to their residual effect on biodiversity (bioaccumulation) and highly non-selective killing effect. Since the 1960s there has been a shortage of scientific publications on control of Culicoides larvae. Some larvicide tests with more environmentally friendly products were found in India, where the plant-based repellent AV/FRC/18 (M/S Myurvet Ltd., Mathura, Uttar Pradesh, India) showed a larvicidal effect against C. peregrinus in laboratory and field trials (Naraladker et al. 2011).

Apart from regular larvicides, the use of the so-called Biorational Insecticides such as insect growth regulators (IGR) have scarcely been tested for most of the species of Culicoides. Laboratory testing of IGRs against field-collected larvae of C. varipennis (Apperson and Yows, 1976) included the following products: Dimilin ${ }^{\circledR}$ (TH-6040; 1-(4chlorophenyl)-3 (2,6-diflurobenzoyl) urea; Thompson-Hayward Co., Kansas City, KS, USA); Methoprene (Altosid ${ }^{\circledast}$ (Isopropyl 11-methoxy-3,7 11-trimethyldodeca-2, 4-dienoate; Zoecon Corp., New York, NY, USA); R-20458 (84-ethylphenyl-6, 7-epoxy geranyl ether; Stauffer Chem. Co., New York, NY, USA); Mon-858 (2,6, di-tert-butyl-4-(a, a-dimethylbenzyl) phenol; Monsanto Chem. Co., St. Louis, MO, USA); HE-24108 (3-butyn-2-yl N(p-chlorophenyl) carbamate; Hercules Powder Co., Wilmington, DE, USA); RO-20-3600 (6,7-epoxy3-methyl-7-ethyl-1-(3,4- (methyl-enedioxy)-phenoxy)-2- cis/trans-octene; Hoffman La Roche Co., Basel, Switzerland). Results showed that dimilin and methoprene had a $90 \%$ efficacy in inhibiting emergence at $0.5 \mathrm{ppm}$ and $1 \mathrm{ppm}$ respectively. R-20458 showed $100 \%$ efficacy, but at $10 \mathrm{ppm}$. The other compounds tested showed no efficacy and it was concluded that compared to the effect caused in mosquitoes, IGR appeared to be of low efficacy in controlling Culicoides (Apperson and Yows, 1976). In a subsequent study, Takahashi et al. (1985) showed that Altosid $10 \mathrm{~F}$ (methoprene $10 \%$ slow release formulation) had little effect on the last larval stage of $C$. circumscriptus, reaching the maximum of efficacy ( $89.6 \%$ mortality at $15 \mathrm{ppm})$ against the pupal stage, while at lower concentrations ( 0.5 to 0.001$)$ an effect on adult emergence due to deformity was observed. Dimilin ${ }^{\circledR}$ (diflubenzuron wettable powder) proved to be more efficacious $\left(\mathrm{IC}_{50}: 0.0094 \mathrm{ppm}\right.$ methoprene vs 0.0052 ppm Dimilin), reaching $100 \%$ of mortality in larvae at 0.04 ppm.

In summary, there is hardly any literature on larvicides against Culicoides larvae. The control of some species of major importance, such as C. imicola, seems to be feasible due to the highly localised semi-moistened breeding sites at farm level, usually associated with water dripping from irrigation pipes (Foxi and Delrio 2010; Mellor and Pitzolis 1979). However, despite the discrete localisation of breeding sites, to date there are no specific larvicide products recommended for use in such environments and therefore larvae control still remains largely unexplored.

\section{Insecticide-treated materials}

The majority of insecticide-treated materials are nets (insecticide treated nets; ITN) which are intended to prevent the entrance of Culicoides into stables and therefore protect animals against bites. Culicoides are small insects $(1-3 \mathrm{~mm})$ and therefore the usual nets used for mosquito screens do not prevent the passage of adult Culicoides. For example, untreated nets with $1.6 \mathrm{~mm}^{2} \mathrm{mesh}$ reduced Culicoides by $56 \%$ and nets with $0.9 \mathrm{~mm}^{2}$ pass reduced Culicoides by $95 \%$ (Porter 1959). Impregnated nets should have a sufficiently wide mesh (no less than $1.6 \mathrm{~mm}$ ) to ensure proper 
ventilation of the stable and avoid passage of Culicoides due to the killing effect of the insecticide. Its efficacy will depend on the type and dose of insecticide, the surface to protect, as well as endo- and exophilic behaviour (indoor/outdoor) of the Culicoides species. In many cases, ITNs have been tested using UV light traps as stimuli for attracting biting midges. Traps are an easy-tohandle sampling tool and could be considered as a type of semi-field testing scenario. In addition, this technique allows for the testing of an appropriate number of replicates and control position and rotation. However, traps can bias species composition, sex ratio and physiological status of Culicoides when compared to other means, such as drop traps (Carpenter et al. 2008; Viennet et al. 2011). Another testing method is to compare captures inside and outside a stable by placing traps in both places. In this case, attraction into the stable is assumed to be caused by the presence of the animal. This allows for an estimate of the coefficient of protection from intrusion (CPI; percentage comparing inside captures with no-mesh and mesh) (Baker et al. 2015).

Several studies on ITNs were conducted in the' 70 s and ' 80 s. Nets treated with an organophosphate insecticide were tested against $C$. mississippiensis and $C$. furens showing a $>90 \%$ mortality during the first $30 \mathrm{~min}$ after treatment (Dukes and Axtell 1976; Kline and Roberts 1981). Subsequently, Kline et al. (1985b) tested chlorpyrifos, fenthion, malathion and propoxur treated screens against C. mississippiensis, a salt-marsh midge and nuisance species for humans in Florida. These authors reported propoxur as the most effective ( 35 days of efficacy), followed by chlorpyrifos, malathion and fenthion. Del Río et al. (2014b) conducted tests with ITNs using only UV light traps (no animals) and manually impregnating blue shading nets (polyethylene, fibre wideness: $1 \mathrm{~mm}$, gap between fibres: $2 \mathrm{~mm}$ ) with 1 I of cypermethrin solution 1\%. The ITNs were placed around a cylinder of metal grating ( $1.5 \mathrm{~m}$ high and $1 \mathrm{~m}$ wide) and an Onderstepoort light trap was placed in the middle of the cylinder. Results obtained in this study showed no statistical difference when the abundance of $C$. imicola captured in the trap with ITN and the control trap was compared. Despite the insecticide, no difference in mortality of biting midges after $24 \mathrm{~h}$ was found between ITN and the control net. According to the authors, midges passed through the net without coming into contact with the ITNs or if they did make contact, this was too short to be affected by the insecticide, unlike the non-targeted species (i.e. Diptera: Homoptera) which were knocked-down when coming into contact with ITN. In a subsequent study (Del Río et al. 2014b) tested the killing effect of a commercial polyethylene net (ZeroVector ${ }^{\circledR}$ Durable Lining; Dart Association, Lausanne, Switzerland) impregnated with $4.4 \mathrm{~g} / \mathrm{kg} \pm 15 \%$ of deltamethrin using the Culicoides adapted WHO protocol. The $100 \%$ mortality in field-collected Culicoides was obtained after an exposure of 17 minutes to the ITN. The nets were therefore tested in the field following the same protocol as explained in Del Río et al. (2014a). Results from the field test showed that ITN did not impede the passage of adult Culicoides, but did affect them as the mortality rate of Culicoides was significantly higher in adults collected in the ITN trap $(84.9 \pm 10.5 \%)$ compared to those from the untreated net (72.3 $\pm 5.9 \%)$. Similar results were obtained by Page et al. (2014) testing high density polyethylene nets manually impregnated with alphacypermethrin $\left(20-40 \mathrm{mg} / \mathrm{m}^{2}\right)$. In this case, nets placed around Onderstepoort black light traps did not show any repellent effect on Culicoides spp. but reduced biting midges captures by 7.2 times when compared to the untreated net. An in vitro test of the ITN showed $100 \%$ mortality in field-collected C. imicola after exposing them for 1 to 3 min.

Testing of ITNs for Culicoides control using animals is included in the works by Bauer et al. (2009), Calvete et al. (2010) and Baker et al. (2015). In Germany, Bauer et al. (2009) tested insecticide-treated mosquito fences ( $100 \mathrm{mg} / \mathrm{m}^{2}$ deltamethrin, $180 \mathrm{~cm}$ height, $1 \times 2 \mathrm{~mm}$ mesh) around bull pens for reducing the biting rate of Culicoides. Efficacy was measured with BG-Sentinel traps that showed no significant difference with control. According to the authors, biting midges avoided the fence by flying over it. A manually impregnated cypermethrin ITN ( $2.6 \mathrm{~m}$ height; $0.5 \mathrm{~g} / \mathrm{l}$ cypermethrin) 
was used by Calvete et al. (2010) in Spain to protect yearly ewe pens. Efficacy was measured using miniature CDC UV light traps inside and outside the pens. The ITN showed an efficacy of 50 to 78.8\% and no protection against C. imicola. As in the case of Bauer et al. (2009) adult Culicoides avoided contact with the insecticide-impregnated canvas.

Baker et al. (2015) included a new approach using the WHO cone test. Like the WHO tube test, the cone test is designed to assess the effect of insecticides on mosquito specimens which are exposed to insecticides applied to different substrates. This test is frequently used to test the effect of intradomiciliary residual spray on the population of a particular region. The method, as in the case of WHO test tubes, also allows for the detection of resistance to insecticides. The work by Baker et al. (2015) included the WHO cone testing of seven insecticides: (1) Agropharm's Dairy Fly Spray (pyrethrins including cinerins 0.25\% w/w; Agropharm Ltd., Penn, UK); (2) Degrain Insectaclear C (cypermethrin $0.1 \%$ w/w; Lodi, Kingswinford, UK); (3) Fly Free Zone (permethrin $0.1 \% \mathrm{w} / \mathrm{w}$; tetramethrin $0.04 \% \mathrm{w} / \mathrm{w}$. Fly Away Ltd, Stourbridge, UK); (4) Protector C (cypermethrin $0.09 \%$ w/w; Agropharm Ltd); (5) Strikeback Insect Killing Spray (cypermethrin $0.01 \%$ w/w; Group 55, Preston, UK); (6) Tri-Tec $14^{\oplus}$ (cypermethrin 0.15\% w/w; pyrethrins $0.2 \%$ w/w; LS Sales (Farnham) Ltd, Bloxham, UK); and (7) Insecticide Ultrashield EX (permethrin 0.5\% w/w; pyrethrins $0.1 \% \mathrm{w} / \mathrm{w}$; W.F. Young, Inc., East Longmeadow, MA, USA). Insecticides were impregnated on a black polyvinylcoated polyester mesh (Fine Mesh Metals; PetMesh, Telford, UK) (1.6 mm aperture; $1.6 \mathrm{~mm}$ thickness). Colony-reared adults of $C$. nubeculosus were exposed at 1, 7 and $14 \mathrm{~d}$ after insecticide treatment of the mesh. After the in vitro trial, a field test of the most efficacious insecticide was carried out. In this case, Tri-Tec $14^{\circledR}$ showed a $100 \%$ killing effect following the WHO cone test and was firstly tested on the semi-field by comparing captures obtained in UV MiniCDC light traps placed in ITN screened wooden cages in a method similar to that of Del Río (2014a,b). Secondly TriTec $14^{\oplus}$ manually sprayed ITN was tested in field conditions by screening the windows and doors of a horse stable. The CPI was measured according to captures of adults biting midges recorded outside and inside the stable. A CPI of $88 \%$ was obtained for the untreated mesh and $100 \%$ for the treated one in wooden frames. The CPI recorded in stables was 71 and $96 \%$ for untreated and treated mesh, respectively. According to the authors, either untreated-mesh or ITN provided significant protection against Culicoides.

The use of ITNs appears to be one of the most promising methods for protecting animals against the biting activity of Culicoides. Despite the variety of results obtained by testing different types of ITNs, in general impregnated mesh can provide a method of protecting high-value animals (i.e. horses) depending on the type of mesh and the selected dose of insecticide.

\section{Use of ITNs in vector-proof establishments}

EFSA (2017) recently reviewed the efficacy of vector-proof establishments (VPE) and repellents/ insecticides. VPE are stables regulated at EU level after the high number of outbreaks of BTV registered in Europe after 1998. The VPE were intended to allow animal movement (particularly high-value animals) while decreasing the risk of BTV transmission. Specific criteria for the VPE are included in Annex II of the Regulation (EC) 1266/2007 (EC 2007) as amended by Commission Regulation (EC) No 456/2012 (EC 2012), and also appear in the OIE Terrestrial Animal Health Code (OIE, 2014). The main method for protecting animals inside VPE is the use of ITNs. All stable openings must be protected by ITNs (mesh size no greater than $1.6 \mathrm{~mm}^{2}$ ), and the insecticides to be used are determined by the competent authority and should follow manufacturers' recommendations. In order to assess the efficacy of VPE, a comparison of captures of Culicoides spp. by using UV light traps (CDC and Onderstepoort types) inside vs outside of VPE is mandatory. Despite the fact that 
there is no scientific literature on VPE, EFSA (2017) assessed its performance, showing that in Italy from 2009 to 2012 a mean reduction of $99.7 \%$ of Culicoides population inside VPE was achieved. When protection provided by VPE is compared to that of pour-on repellents/insecticides, EFSA (2017) concluded that protection provided by pour-on insecticides could be considered up to $86 \%$ effective, meanwhile protection due to VPE could reach $96 \%$. This result confirms the use of VPE for protecting high-value animals (i.e. horses, stallion bulls, etc.), when specific measures are taken to prevent Culicoides movement into the stables. However, there is still a lack of implementation of ITN on a routine basis for preventing Culicoides activity in stables and surroundings of less valuable domestic species.

\section{Biological control}

Biological control includes the use of natural enemies, including predators, parasitoids, entomopathogens or products for controlling insects pest and harmful insects. Work conducted in this area for controlling Culicoides has focused mainly on the use of entomopathogenic fungi and parasitic infection by nematodes.

Fungal and Oomycete infecting Culicomorpha (Simuliidae, Ceratopogonidae and Chironomidae) have been reviewed by De Souza et al. (2014). Wright and Easton (1996) found Lagenidium giganteum Couch infecting natural populations of larvae of Culicoides molestus Skuse in Australia, with rates from 2.4 to $33 \%$ of infection. Exposure of $L 4$ larvae to the fungus resulted in $31 \%$ mortality at $20^{\circ} \mathrm{C}$. Unkles et al. (2004) tested seven strains of the mosquito pathogen fungus Culicinomyces clavisporus against colony-reared C. nubeculosus larvae. The killing effect was dosedependent and was delayed until 72-96 h post-treatment. Ansari et al. (2010) tested the effect of four species of entomopathogenic fungi: Metarhizium anisopliae (Metchnikoff) Sorokin (10 strains), Beauveria bassiana (Balsamo) Vuillemin (3 strains), Paecilomyces fumosoroseus (Wize) (5 strains), and Lecanicillium longisporum (Starke, Karapapa, Bainbr. and Heale) (1 strain) on egghatching and larval susceptibility of colonised C. nubeculosus. Results indicated no effect on egghatching, and $M$. anisopliae was the most effective fungal species showing mortality in larvae from 81 to $100 \%$ independent of the strain used. In a subsequent study, Ansari et al. (2011) tested the effect of different isolated strains of $M$. anisopliae, B. bassiana, I. fumosorosea and L. longisporum on colony-reared adults of $C$. nubeculosus by exposing them to conidia at a rate of $10^{11} / \mathrm{m}^{2}$. All fungal strains used in the trial decreased C. nubeculosus adult survival, M. anisopliae (strain V275. F52; Novozymes, Bagsværd, Denmark) being the most effective among them reaching Lethal Time 90 (LT90) at 3.26 days. Application of dry conidia at a dose of $1.5 \times 10^{8} / \mathrm{m}^{2}$ on a substrate of dry tissue paper appeared to be the most effective in killing adult Culicoides and in all cases; adults showed fungal development. In a similar study conducted in Australia, Nicholas and McCorkell (2014) tested four isolates of $M$. anisopliae (dose $0.6 \mathrm{~g}$ conidia $/ \mathrm{m}^{2}$ ) against $C$. brevitarsis adults reared from dung collected from the field. They also tested the $\%$ of emergence of adults from dung that was treated with $M$. anisopliae conidia doses of $0.25 \mathrm{~g}, 0.5 \mathrm{~g}$, and $1 \mathrm{~g}$ conidia/ $\mathrm{kg}$ dung. A significant killing effect on adults was observed at day eight for all isolates; however, $100 \%$ of mortality was not reached for any of them. Conidia at different doses applied to dung proved to be effective in decreasing the emergence rate of adults compared to control; however, not all doses and isolates were equally effective. Narladkar et al. (2015) also tested in vitro M. anisopliae and B. bassiana isolates against adults, field-collected larvae and free living larvae in farm-associated drainage channels of C. peregrinus in India. Higher mortality was attributed to M. anisopliae; however the effect lasted only 7 days. In the case of adults, the authors state that infection acquired in the resting place was efficacious for killing midges in $24 \mathrm{~h}$, although no measures about the efficacy are included in the study. Screening of possible Oomycete species as killing agents for 
Culicoides subimmaculatus larvae was explored by Stephen and Kurtböke (2011) in the mangroves of Queensland, Australia. They tested six isolates including the fungal species Pythium prolatum, Halophytophthora batemanensis, Phytophthora gonapodyides and Halophytophthora spp. None of the tested fungi appeared to be pathogenic to midge larvae.

The Sporulaceae bacteria Bacillus thuringiensis var. israelensis (Bti) is an entomopathogenic bacteria that has been widely used in larvae control for more than 40 years (Bravo et al. 2011). However, the same strain has proved to be highly ineffective when applied to Culicoides larvae control, including those species that breed in semi-aquatic habitats, such as C. sonorensis, Culicoides occidentalis Wirth and Jones, C. mississippiensis and Culicoides guttipennis (Coq.) (Kelson et al. 1980; Lacey and Kline 1983), as well as those breeding in wet substrates (i.e. mud) such as C. impunctatus (Blackwell and King 1997). As a result, recent research on biting midge control using Bti is limited and remains largely anecdotal. Interestingly, Bti has proved to be effective in controlling groups taxonomically related to Ceratopogonidae, such as non-biting midges included in the family Chironomidae (Larget and De Barjac 1981; Priest 1992). The reason why Bti is not able to lethally affect the midgut of Culicoides larvae remains unclear. Possible explanations related to the chitinase activity of Bti on the peritrophic membrane of midge's midgut still remain unexplored (Sampson and Gooday 1998).

Entomopathogenic viruses in Culicoides have been scarcely isolated and never applied for the control of either larvae or adults in field conditions. The most common group of pathogenic viruses found in Culicoides are those belonging to the Iridescent viruses (IIVs) (family Iridoviridae; genus (ridovirus), the name refers to the iridescent blue colour they produce in the body of infected larvae and a complete review of those recorded in Culicoides spp. can be found in Williams (2008). Iridescent viruses are non-occluded icosahedral particles around $130 \mathrm{~nm}$ diameter that contain a dsDNA genome of approximately 140-210 kbp (Williams et al. 2008). The mechanism of transmission of Iridescent virus remains unclear, but the mediation of parasitic nematodes or parasitoids are among the suspected paths (Mullens et al. 1999; Williams 2008). Mullens et al. (1999) found between 1 and $28 \%$ infection on C. sonorensis larvae infected in California; Chapman et al. (1968) found infected larvae of Culicoides arboricola Root \& Hffm., C. guttipennis from tree holes of Louisiana (USA); Rieb (1982) found 1\% infection from Culicoides odibilis Austen, Culicoides cubitalis Edwards and Culicoides clastrieri Callot, Kremer \& Deduitin France and Fukuda et al. (2002) found $4.7 \%$ infection in larvae of Culicoides barbosai Wirth \& Blanton in Florida.

Other parasites such as Microsporidia Nosema-like species have been found in salt-marsh Culicoides (Kline et al. 1985a) and in C. barbosai in Florida (Fukuda et al. 2002); Vavraria sp. on Culicoides edeni (Wirth \& Blanton) (Atkinson 1990) and Plistophora sp. on larvae of C. nanus Root \& Hffm in Louisiana (Chapman et al. 1968).

The family of nematodes Mermithidae is one of the most researched groups of parasites of Culicoides and other aquatic and semi-aquatic Diptera. A complete review of Mermithidae parasitism in Ceratopogonidae can be found in Mullens et al. (2008). The vast majority of work on this group has been conducted in the USA, where in particular the species Heleidomermis magnapapula Poinar and Mullens has been found parasitising several species of biting midges, such as C. sonorensis (up to $69 \%$ parasitism), Culicoides lahontan Wirth \& Blanton, Culicoides boydi Wirth \& Mullens and Culicoides cacticola Wirth \& Hubert (Mullens et al. 1997; Paine and Mullens 1994; Poinar and Mullens 1987) and Heleidomermis vivipara Rubtzov has been found parasitising Culicoides spp. (up to $51 \%$ ). 
Several attempts have been made to improve rearing techniques of $H$. magnapapula for its use in biological control programmes of C. sonorensis. Mullens and Velten (1994) studied its life history parameters under laboratory conditions; Luhring and Mullens (1997) determined that 900 infective preparasite stages of $H$. magnapapula were the optimal preparasite/host ratio for 650-700 C. sonorensis larvae; Mullens et al. (1995) determined the thermal limits and development and Mullens and Luhring (1996) showed the effect on the parasite infectivity according to salinity and dairy waste in water. Another species that has been extensively studied is Heleidomermis catalonensis n. sp., which could be detected in adults of $C$. circumscriptus as the parasite causes the development of intersexes with morphological abnormalities (Muñoz-Muñoz et al. 2016; Poinar and Sarto i Monteys 2008).

There are a few examples of the use of $H$. magnapapula in field or semi-field trials. A semi-field trial was conducted by Mullens et al. (2008) in California. Pre-parasite forms of the nematode were inoculated to controlled slurry ponds where eggs of $C$. sonorensis were also inoculated. After 21 days they found an $84 \%$ reduction in adults emerging from the treated pond. Despite the good results in larvae infectivity, adults usually show a low level of parasitism (Mullens et al. 2008; Paine and Mullens 1994), despite the fact that exposure of fourth-instar host larvae to the parasite may result in up to $17 \%$ parasitism in adults according to Mullens and Velten (1994). This factor, added to the lack of a massive rearing of the nematode, limits its use in successful biological control programmes. Further discussion on the limitations of the genus Heleidomermis for biological control can be found in (Mullens et al. 2008).

Heritable endosymbiotic bacteria such as Wolbachia have been studied in-depth over the last decade due to their potential for being implemented in control programmes against mosquitoes (Iturbe-Ormaetxe et al. 2011). Heritable bacteria may influence host longevity, virus- host interaction and reproduction compatibility, among other aspects. There are several studies showing positive detection of endosymbiotic bacteria in Culicoides spp. For example, Wolbachia was detected in Culicoides paraflavescens Wirth \& Hubert in Japan (Nakamura et al. 2009) and Cardinium and Wolbachia were detected from 16 Culicoides spp. in Australia using qPCR (Mee et al. 2015), including the vectors of the Avaritia subgenus, such as C. wadai, C. brevitarsis and C. imicola. All species were positive with low levels of infection to Cardinium, while $10 \mathrm{spp}$. were positive to Wolbachia and 9 showed coinfection Wolbachia-Cardinium. The genus Cardinium was also detected in C. punctatus with a prevalence of 0.960 and in C. pulicaris with 0.256 of prevalence in the UK (Lewis et al. 2014). In Israel, the maternally inherited symbiont Candidatus Cardinium hertigii (Bacteroidetes) was detected in adults Culicoides with a prevalence of $50.7 \%$ in C. imicola, $31.4 \%$ in C. oxystoma, and $0 \%$ in C. schultzei, the prevalence being correlated to land surface of the environment where samples were collected (Morag et al. 2012). However, this symbiont was reported to have no effect on the survival and size of C. imicola (Morag et al. 2013).

\section{Genetic control}

Genetic control applied to vector insects relies on a thorough knowledge of the genetic mechanism that regulates the vector competence and therefore the resistance or susceptibility of the vector to particular pathogens (i.e. arboviruses) and strains. Prior to the era of development of molecular techniques such as PCR and sequencing, (Tabachnick 1991) selected isofemale lines that showed high or low levels of infection for BTV-11 in trials performed using colony-reared adults of $C$. sonorensis. Families that were homogenously resistant and susceptible to BTV were obtained and the author hypothesised that a single controlling locus based primarily on the rapid response to selection and a second locus controlled the degree of infection. The application of genomic 
and transcriptomic techniques to other vectors, such as mosquitoes (Kean et al. 2015), has increased the interest in its application to other vector groups, such as biting midges. The study of transcriptome of $C$. sonorensis by using RNA-sequencing has allowed for a greater understanding of the functionality of the vectors' genetics in modulating arboviral infection.

Campbell and Wilson (2002) identified mid-gut transcripts in EHDV blood-fed infected C. sonorensis while Campbell et al. (2005) described midgut cDNA library identifying transcripts associated with midgut and salivary glands with anti-haemostatic and immunomodulatory functions. Subsequent work by Nayduch et al. (2014a) described the transcriptome for colonyreared females of $C$. sonorensis, elucidating the genetic basis for sugar and blood feeding as well as vitellogenesis. Measuring gene dynamics through transcriptome analysis is expected to provide key information for better understanding vector competence as well developing new possible control tools (Nayduch et al. 2014b). However, as in many fields of research associated with Culicoides, the availability of only two species ( $C$. sonorensis and $C$. nubeculosus) colonies and the notable failure to be able to colonise other major vector species such as $C$. imicola (Veronesi et al. 2009) is a significant limitation for progressing with potential genetic control measures for Culicoides. In addition, there is a significant limitation when results obtained from colony-reared adults are extrapolated to field populations (Nayduch et al. 2014b).

Another genetic strategy that has been considered is the use of RNA interference (RNAi), which causes innate immune responses to arbovirus replication. RNAi has been identified by Schnettler et al. (2013); in particular, the exogenous small interfering RNA (siRNA) was found in C. sonorensisderived KC cells, in a similar pathway proven to have antiviral function in mosquitoes. In subsequent work, Mills et al. (2015) succeeded in inducing environmental RNAi by injecting intrathoracically double-stranded RNA (dsRNA) in colony-reared females of $C$. sonorensis.

Other genetic control types currently explored in mosquitoes and inducing sterility in populations, such as 'release of insects carrying a dominant lethal genetic system', 'incompatible insect technique' and 'sterile insect technique' (Alphey 2014), remain vastly unexplored in Culicoides. The main reasons are the limitation on mass-producing adult Culicoides due to the lack of colonies available worldwide and the high cost in terms of time and research compared to other arbovirus control methods such as development of vaccines.

\section{Biotechnological control: semiochemicals}

Semiochemicals in the form of volatile compound are essential in the biology of Culicoides. Olfaction of volatiles compounds is involved in the detection of hosts and therefore closely linked to triggering feeding activity of Culicoides, as well as detection of oviposition sites (for review see Logan and Cook 2010). One of the most widespread practices in monitoring and controlling vector and pest insects is the use of pheromones and kairomones, many of them commercially produced. While this is true for some groups of vectors, such as mosquitoes, where several examples of kairomone-based products are available (Takken and Kline 1989), products and molecules available for Culicoides are still at the experimental level and far from being commercially available.

Carbon dioxide has been identified as one of the most important odour cues that vectors follow to locate host, usually detected by the sensilla coeloconica in the antennae and the basiconica in the palpi. Kairomones based on $\mathrm{CO}_{2}$ are commonly used for capturing and monitoring groups of vectors such as mosquitoes; however, in the case of Culicoides, results are contradictory among different studies. Carbon dioxide is routinely use for the monitoring of $C$. sonorensis in the USA 
(Smith and Mullens 2003), but in general $\mathrm{CO}_{2}$ alone has little effect on increasing captures of other species such as C. imicola and C. obsoletus (Gerry et al. 2009; Harrup et al. 2012).

Other commonly tested kairomones either in laboratory (olfactometer) or field studies include 1-octen-3-ol (Bhasin et al. 2000a; Blackwell et al. 1996; Braverman et al. 2000; Mands et al. 2004), L-(+)-lactic acid Kline (Bhasin et al. 2000b; Kline et al. 2007, 2012; Romón et al. 2012), butanone (Bhasin et al. 2000b), acetone (Bhasin et al. 2000a, 2001; Kline et al. 2012; Romón et al. 2012), 1-hexen-ol, phenol and dimethyl disulfide (Kline et al. 2012); benzaldehyde, 6-methyl-5-hepten2one, octanal, 2-ethylhexanol, limonene, dihydromyrcenol, nonanal, linalool, (E)-2-nonenal, menthol, naphthalene, decanal, geranylacetone and alpha-isomethylionone (Logan et al. 2009); decanal, 2-phenylethanal, 1-octen-3-ol, 2-ethylhexanol, 3-methylindole, phenol, 3-ethylphenol (Isberg et al. 2016). None of the above-cited kairomones showed a strong effect in attracting males or females of Culicoides, particularly when tested in field conditions. In those cases the kairomones were tested at field level comparing standard UV traps against kairomone-baited UV traps. None of the compounds showed a significant increment in Culicoides spp. captures compared to the control.

Different attractant effects have been observed in the case of 1-octen-3-ol enantiomers (Kline et al. 2007) where natural (R)-(+) isomer of octenol was more attractive to Culicoides spp. than the synthetic one. Similar results were found by Harrup et al. (2012) using suction traps baited with (R) enantiomer of 1-octen-3-ol with $\mathrm{CO}_{2}$. Several combinations of the above-mentioned kairomones have been used in traps (usually commercial ones) with the aim of increasing Culicoides captures and preventing biting pressure on the human population. Trials carried out using two commercial traps - ABC Pro insect suction traps (Clarke Mosquito Products Inc., Roselle, IL, USA) baited with $\mathrm{CO}_{2}$ and a 4:1:8 octenol/phenol mixture of 1-octen-3-ol: 3-n-propylphenol:4-methylphenol, and a Mosquito Magnet ${ }^{\oplus}$ trap (American Biophysics Corporation, East Greenwich, Rhode Island, USA) baited with $\mathrm{CO}_{2}$ and racemic 1-octen-3-ol - failed in decreasing biting midge attacks in Florida (Cilek and Hallmon 2005; Cilek et al. 2003). However, in a subsequent work, (Lloyd et al. 2008) showed promising results in monitoring populations of $C$. furens, $C$. barbosai and $C$. mississippiensis in Florida by using continuous trapping efforts with a propane-based mosquito magnet (MM) (American Biophysics Corporation), MM-FreedomH (Freedom) and MM-Liberty PlusH (Liberty Plus) baited with $\mathrm{CO}_{2}$ (propane combustion), heat, moisture, and also 1-octen-3-ol (octenol) in the case of the Liberty trap.

In the USA the Mosquito Magnet baited with $\mathrm{CO}_{2}(0.5 \mathrm{l} / \mathrm{min})$ and a mixture of 1-octen-3-ol: 3-n-propylphenol: 4-methylphenol (4:1:8) $(\approx 5 \mathrm{mg} / \mathrm{h})$ reduced the number of Culicoides in the area by 2.3 to $70.6 \%$ (Cilek 2003; Cilek and Hallmon 2005). The same trap was used in Scotland baited as well with $\mathrm{CO}_{2}(0.5 \mathrm{l} / \mathrm{min}), 1$-octen-3-ol $(6-8 \mathrm{mg} / \mathrm{h})$ and hair extracts from different animals (sheep, calf, deer, pony, buffalo). The number of Culicoides collected was higher for some extracts such as the buffalo one. Despite there is an extensive body of research about this topic, no field trials have been published regarding the use of semiochemical baited traps for the reduction of local adult Culicoides population with the aim of reducing transmission under an epizootic scenario. The application of such technology seems to be very much restricted to human protection in specific places such as golf courses where Culicoides may represent a serious nuisance (Mands et al. 2004).

\section{Future perspectives on control of Culicoides spp.}

Culicoides control is still a pending task for medical and veterinary entomologists. Immature stages are probably the most challenging to control due to the variety of habitats they occupy, our limited 
knowledge about the bioecology of these habitats and the lack of chemical/biological tools that effectively could reduce egg, larval and pupal populations. Some species such as $C$. sonorensis in the USA appear a priori the easiest to control by managing farm associated water-pools. However, we also know that this species is capable of breeding in high numbers in natural habitat sites that escape to the control at farm-level. Other examples on applied source modifications of breeding sites (i.e. dung removal/destruction; mechanical covering of breeding sites) remain limited to experimental level and have never been placed in a systematic control programme. Biological control should be one of the priorities when thinking of control of immatures of Culicoides. Some attempts have been conducted in using entomopathogenic nematodes and fungi for control of larvae and the results seem to be promising in the near future. In regard to adults, the use of pour-on insecticides has been extensively researched during the last decade, however, results are far from being consistent and different results of efficacy have been achieved based on the active ingredient test, the host species and the targeted Culicoides spp. Understanding the hostseeking and feeding behaviour seems a key aspect of the Culicoides biology to better use contact insecticides intended to protect hosts against bites. It is also worth mentioning that despite the progress in using topical insecticides, little progress has been achieved in understanding the impact of such topical treatments in the decrease of BTV transmission among animals. Other potential methods for controlling vectors, such as genetic techniques are still far from being applied on Culicoides and probably this technique would be first developed for other vectors such as mosquitoes (Alphey 2014; Alphey and Alphey 2014). The use of semiochemicals for monitoring and trapping of Culicoides is another area of interest that remains vastly unexplored when compared to other insects of economic or health importance. Despite the research during the last decade on potential attractants for Culicoides based on known attractive compounds for other vector species, the reality is that currently there are no standard compounds available for Culicoides. The nature of the compounds, different specific response of Culicoides spp. and concentration seems to be the main limitations. Further research should therefore be focused on understanding the chemical ecology of Culicoides spp. and possible applications for mass trapping in premises on farms or specific attractants for Culicoides spp. that would avoid larger captures of non-targeted insects in light traps for instance. Specific attractants would allow also to improve monitoring precisely in periods when risk of BTV transmission is higher. In the same sense, little is known about repellents, besides those that are of general use such as DEET. Protection of animals from biting midges by using repellents still deserves more attention from researchers, although they are already widely used for protecting animals during transport, there is no evidence of the effect of such repellents in decreasing the risk of BTV transmission. In summary, future progress on Culicoides spp. control could only be conceived by substantially increasing our knowledge of the basic bioecology at species level of this group of vectors.

\section{References}

Acevedo P, Ruiz-Fons F, Estrada R, Márquez AL, Miranda MA, Gortázar C, Lucientes J, Luz Marquez A, Angel Miranda M, Gortazar C and Lucientes J (2010) A broad assessment of factors determining Culicoides imicola abundance: modelling the present and forecasting its future in climate change scenarios. PLoS ONE 5: e14236.

Agren, ECC, Burgin L, Lewerin SS, Gloster J and Elvander M (2010) Possible means of introduction of bluetongue virus serotype 8 (BTV-8) to Sweden in August 2008: comparison of results from two models for atmospheric transport of the Culicoides vector. Vet Rec 167: 484-488.

Alba A, Casal J and Domingo M (2004) Possible introduction of bluetongue into the Balearic Islands, Spain, in 2000, via air streams. Vet Rec 155: 460.

Alphey L (2014) Genetic control of mosquitoes. Annu Rev Entomol 59: 205-224. 
Alphey L and Alphey N (2014) Five things to know about genetically modified (gm) insects for vector control. PLoS Pathog 10: 3-5.

Ander M, Meiswinkel R and Chirico J (2012) Seasonal dynamics of biting midges (Diptera: Ceratopogonidae: Culicoides), the potential vectors of bluetongue virus, in Sweden. Vet Parasitol 184: 59-67.

Ander M, Troell Kand Chirico J (2013) Barcoding of biting midges in the genus Culicoides: a tool for species determination. Med Vet Entomol 27: 323-331.

Ansari MA, Carpenter S and Butt TM (2010) Susceptibility of Culicoides biting midge larvae to the insect-pathogenic fungus, Metarhizium anisopliae: prospects for bluetongue vector control. Acta Trop 113: 1-6.

Ansari MA, Pope EC, Carpenter S, Scholte EJ and Butt TM (2011) Entomopathogenic fungus as a biological control for an important vector of livestock disease: the Culicoides biting midge. PLoS ONE 6(1): e16108.

Atkinson CT (1990) Fine structure and sporogonic development of a Vavraia sp. (Microsporida: Pleistophoridae) in the biting midge Culicoides edeni (Diptera: Ceratopogonidae). J Invertebr Pathol 55: 105-111.

Baker KP and Quinn PJ (1978) A report on clinical aspects and histopathology of sweet itch. Equine Vet J 10: 243-248.

Baker T, Carpenter S, Gubbins S, Newton R, Lo lacono G, Wood J and Harrup LE (2015) Can insecticide-treated netting provide protection for equids from Culicoides biting midges in the United Kingdom? Parasit Vectors 8: 604.

Balczun C, Vorsprach B, Meiser CK and Schaub GA (2009) Changes of the abundance of Culicoides obsoletus s.s. and Culicoides scoticus in Southwest Germany identified by a PCR-based differentiation. Parasitol Res 105: 345-349.

Baldet T, Delécolle JC, Cêtre-Sossah C, Mathieu B, Meiswinkel R and Gerbier G (2008) Indoor activity of Culicoides associated with livestock in the bluetongue virus (BTV) affected region of northern France during autumn 2006. Prev Vet Med 87: 84-97.

Baldet T, Delécolle JC, Mathieu B, De la Rocque S and Roger F (2004) Entomological surveillance of bluetongue in France in 2002. Vet Ital 40: 227.

Balenghien T, Cêtre-Sossah C, Grillet C, Delécolle J-C, Mathieu B and Baldet T (2008) Diurnal activity of potential bluetongue vectors in northern Europe. Vet Rec 162: 323-324.

Barceló C and Miranda MA (2018) Bionomics of livestock-associated Culicoides (biting midge) bluetongue virus vectors under laboratory conditions. Med Vet Entomol 32: 216-225.

Barrett J, Broderick C, Soulsby H, Wade P and Newsholme W (2016) Subcutaneous ivermectin use in the treatment of severe Strongyloides stercoralis infection: two case reports and a discussion of the literature. J Antimicrob Chemother 71: 220-225.

Bartsch S, Bauer B, Wiemann A, Clausen PH and Steuber S (2009) Feeding patterns of biting midges of the Culicoides obsoletus and Culicoides pulicaris groups on selected farms in Brandenburg, Germany. Parasitol Res 105: 373-380.

Bauer B, Jandowsky A, Schein E, Mehlitz D and Clausen PH (2009) An appraisal of current and new techniques intended to protect bulls against Culicoides and other haematophagous nematocera: the case of Schmergow, Brandenburg, Germany. Parasitol Res 105: 359-365.

Baylis M, Parkin H, Kreppel K, Carpenter S, Mellor PS and Mcintyre KM (2010) Evaluation of housing as a means to protect cattle from Culicoides biting midges, the vectors of bluetongue virus. Med Vet Entomol 24: 38-45.

Beaty BJ and Marquardt WC (1996) The biology of disease vectors. University Press of Colorado, Boulder, CO, USA.

Becker $P, 1961$. Observation on the life cycle and immature satage of Culicoides circumscriptus Kieff. (Diptera: Ceratopogonidae). Proc R Soc Edinburgh 67: 363-386.

Bhasin A, Mordue AJ and Mordue W (2000a) Electrophysiological and behavioural identification of host kairomones as olfactory cues for Culicoides impunctatus and C. nubeculosus. Physiol Entomol 25: 6-16.

Bhasin A, Mordue AJ and Mordue W (2000b) Responses of the biting midge Culicoides impunctatus to acetone, $\mathrm{CO}_{2}$ and 1-octen-3-ol in a wind tunnel. Med Vet Entomol 14:300-307.

Bhasin A, Mordue AJ and Mordue W (2001) Field studies on efficacy of host odour baits for the biting midge Culicoides impunctatus in Scotland. Med Vet Entomol 15: 147-156.

Birley MH and Braverman Y (1987) Diversity andassociation among Culicoides (Diptera: Ceratopogonidae) fauna in Israel. MH Isr J Entomol 21: 39-50. 
Bishop AL, McKenzie HJH, Barchia IM and Harris AM (1996) Effect of temperature regimes on the development, survival and emergence of Culicoides brevitarsis Kieffer (Diptera: Ceratopogonidae) in bovine dung. Aust J Entomol 35: 361-368.

Bishop AL, Barchia IM and Spohr LJ (2000) Models for the dispersal in Australia of the arbovirus vector, Culicoides brevitarsis kieffer (Diptera: Ceratopogonidae). Prev Vet Med 47: 243-254.

Bishop AL, Kirkland PD, McKenzie HJ, Spohr LJ, Barchia IM and Muller MJ (1995) Distribution and seasonal movements of Culicoides brevitarsis Kieffer (Diptera: Ceratopogonidae) at the southern limits of its distribution in New South Wales and their correlation with arboviruses affecting livestock. Aust J Entomol 34: 289-298.

Bishop AL, McKenzie HJ and Spohr LJ (2008) Attraction of Culicoides brevitarsis Kieffer (Diptera: Ceratopogonidae) and Culex annulirostris Skuse (Diptera: Culicidae) to simulated visual and chemical stimuli from cattle. Aust J Entomol 47: 121-127.

Blackwell A, Dyer C, Mordue AJ, Wadhams LJ and Mordue W (1994a) Field and laboratory evidence for a volatile pheromone produced by parous females of the Scottish biting midge, Culicoides impunctatus. Physiol Entomol 19: 251-257.

Blackwell A, Dyer C, Mordue AJ, Wadhams LJ and Mordue W (1996) The role of 1-octen-3-ol as a host-odour attractant for the biting midge, Culicoides impunctatus Goetghebuer, and interactions of 1-octen-3-ol with a volatile pheromone produced by parous female midges. Physiol Entomol 21: 15-19.

Blackwell A, Evans KA, Strang RHC and Cole M (2004) Toward development of neem-based repellents against the Scottish Highland biting midge Culicoides impunctatus. Med Vet Entomol 18: 449-452.

Blackwell A and King FC (1997) The vertical distribution of Culicoides impunctatus larvae. Med Vet Entomol 11: 45-48.

Blackwell A, Young MR and Mordue W (1994b) The microhabitat of Culicoides impunctatus (Diptera: Ceratopogonidae) larvae in Scotland. Bull Entomol Res 84: 295-301.

Blackwell A, Lock KA, Marshall B, Boag B and Gordon SC (1999) The spatial distribution of larvae of Culicoides impunctatus biting midges. Med Vet Entomol 13: 362-371.

Bobeva A, Zehtindjiev P, Bensch S and Radrova J (2013) A survey of biting midges of the genus Culicoides Latreille, 1809 (Diptera: Ceratopogonidae) in NE Bulgaria, with respect to transmission of avian haemosporidians. Acta Parasitol 58: 585-591.

Bonneau KR, DeMaula CD, Mullens BA and MacLachlan NJ (2002) Duration of viraemia infectious to Culicoides sonorensis in bluetongue virus-infected cattle and sheep. Vet Microbiol 88: 115-125.

Boorman J and Goddard P (1970) Observations on the biology of Culicoides impunctatus Goetgh. (Dipt., Ceratopogonidae) in southern England. Bull Ent Res 60: 189-198.

Borkent A (1996) Biting midges from Upper Cretaceous New Jersey amber (Ceratopogonidae: Diptera). American Museum of Natural History, New York, NY, USA.

Borkent A (2004) The biting midges, the Ceratopogonidae (Diptera). In: Marquardt WC (ed.) Biology of disease vectors. Elsevier Academic Press, New York, NY, USA, pp. 113-126.

Borkent A (2015) The subgeneric classification of species of Culicoides - thoughts and a warning. Available at: https:// tinyurl.com/y8robxo5.

Braverman Y and Chechik F (1996) Air streams and the introduction of animal diseases borne on Culicoides (Diptera, Ceratopogonidae) into Israel. Rev Sci Tech 15: 1037-1052.

Braverman Y and Chivoz-Ginzburg A (1997) Repellency of synthetic and plant-derived preparations for Culicoides imicola. Med Vet Entomol 11:355-360.

Braverman Y and Chizov-Ginzburg A (1998) Duration of repellency of various synthetic and plant-derived preparations for Culicoides imicola, the vector of African horse sickness virus. Arch Virol Suppl 14: 165-174.

Braverman Y, Chizov-Ginzburg A and Mullens BA (1999) Mosquito repellent attracts Culicoides imicola (Diptera: Ceratopogonidae). J Med Entomol 36: 113-115.

Braverman Y, Chizov-Ginzburg A, Pener H and Wilamowski A (2004) Susceptibility and repellency of Culicoides imicola and Culex pipiens to lambda-cyhalothrin. Vet Ital 40: 336-339.

Braverman Y, Galun R and Ziv M (1974) Breeding sites of some Culicoides species (Diptera, Ceratopogonidae) in Israel. Mosq News 34: 303-308. 
Braverman Y and Linley JR (1994) Fecundity and proportions of gravid females in populations of the bluetongue vector Culicoides imicola (Diptera: Ceratopogonidae) and several other species in Israel. J Med Entomol 31: 838-843.

Braverman Y and Mumcuoglu K (2009) Newly emerged nulliparous Culicoides imicola Kieffer (Diptera: Ceratopogonidae) with pigmented abdomen. Vet Parasitol 160: 356-358.

Braverman Y, Ungar-Waron H, Frith K, Adler H, Danieli Y, Baker KP and Quinn PJ (1983) Epidemiological and immunological studies of sweet itch in horses in Israel. Vet Rec 112: 521-524.

Braverman Y, Wegis MC and Mullens BA (2000) Response of Culicoides sonorensis (Diptera: Ceratopogonidae) to 1-octen3-ol and three plant-derived repellent formulations in the field. J Am Mosq Control Assoc 16: 158-163.

Bravo A, Likitvivatanavong S, Gill SS and Soberón M (2011) Bacillus thuringiensis: a story of a successful bioinsecticide. Insect Biochem Mol Biol 41: 423-431.

Brei B, Cribb BW and Merritt DJ (2003) Effects of seawater components on immature Culicoides molestus (Skuse) (Diptera: Ceratopogonidae). Aust J Entomol 42: 119-123.

Brugger K and Rubel F (2013) Bluetongue disease risk assessment based on observed and projected Culicoides obsoletus spp. vector densities. PLoS ONE 8: e60330.

Burgin LE, Gloster J, Sanders C, Mellor PS, Gubbins S and Carpenter S (2013) Investigating incursions of bluetongue virus using a model of long-distance Culicoides biting midge dispersal. Transbound Emerg Dis 60: 263-272.

Burylova A and Milushina A (1993) Phenology and seasonal variation of biting midges in the Upper Kama River region. Entomol Rev 72: 21-23.

Buxton PA (1960) British Diptera associated with fungi. III. Flies of all families reared from about 150 species of fungi. Entomol Mon Mag 96: 61-94.

Calistri P, Goffredo M, Caporale V and Meiswinkel R (2003) The distribution of Culicoides imicola in Italy: application and evaluation of current Mediterranean models based on climate. J Vet Med B Infect Dis Vet Public Health 50: 132-138.

Calvete C, Estrada R, Miranda MA, Del Río R, Borràs D, Beldrón FJ, Martínez A, Calvo AJ and Lucientes J (2010) Protection of livestock against bluetongue virus vector Culicoides imicola using insecticide-treated netting in open areas. Med Vet Entomol 24: 169-175.

Calvete C, Miranda MA, Estrada R, Borras D, Collantes F, Moreno N and Lucientes J (2006) Spatial distribution of Culicoides imicola, the main vector of bluetongue virus, in Spain. Vet Rec 158: 130-131.

Calvo JH, Berzal B, Calvete C, Miranda MA, Estrada R and Lucientes J (2012) Host feeding patterns of Culicoides species (Diptera: Ceratopogonidae) within the Picos de Europa National Park in northern Spain. Bull Entomol Res 102: 692-697.

Campbell CL, Vandyke KA, Letchworth GJ, Drolet BS, Hanekamp and Wilson WC (2005) Midgut and salivary gland transcriptomas of the arbovirus vector Culicoides sonorensis (Diptera: Ceratopogonidae). Insect Mol Biol 14: 121-136.

Campbell CL and Wilson WC (2002) Differentially expressed midgut transcripts in Culicoides sonorensis (Diptera: Ceratopogonidae) following Orbivirus (Reoviridae) oral feeding. Insect Mol Biol 11: 595-604.

Campbell JA and Pelham-Clinton BA (1960) A taxonomic review of the British species of Culicoides Latreille (Diptera, Ceratopogonidae). Proc R Soc Edinburgh 67: 181-302.

Capela R, Purse BV, Pena I, Wittman EJ, Margarita Y, Capela M, Romão L, Mellor PS and Baylis M (2003) Spatial distribution of Culicoides species in Portugal in relation to the transmission of African horse sickness and bluetongue viruses. Med Vet Entomol 17: 165-177.

Capela R, Sousa C, Pena I and Caeiro V (1993) Preliminary note on the ditribution and ecology of Culicoides imicola in Portugal. Med Vet Entomol 7: 23-26.

Carpenter S, Mellor PS and Torr SJ (2008) Control techniques for Culicoides biting midges and their application in the U.K. and northwestern Palaearctic. Med Vet Entomol 22: 175-187.

Carpenter S, Mellor P and Torr S (2007) Bluetongue and midge control. Vet Rec 161: 633.

Carpenter S, Szmaragd C, Barber J, Labuschagne K, Gubbins S and Mellor P (2008) An assessment of Culicoides surveillance techniques in northern Europe: have we underestimated a potential bluetongue virus vector? J Appl Ecol 45: 1237-1245.

Carpenter S, Wilson A, Barber J, Veronesi E, Mellor P, Venter G and Gubbins S (2011) Temperature dependence of the extrinsic incubation period of orbiviruses in Culicoides biting midges. PLoS ONE 6: e27987. 
Carrasquilla MC, Guhl F, Zipa Y, Ferro C, Pardo RH, Cabrera OL and Santamaría E (2010) Breeding sites of Culicoides pachymerus Lutz in the Magdalena River basin, Colombia. Mem. Inst Oswaldo Cruz 105: 216-219.

Carvalho LPC and Silva FS (2014) Seasonal abundance of livestock-associated Culicoides species in northeastern Brazil. Med Vet Entomol 28: 228-231.

Chapman HC, Petersen JJ, Woodard DB and Clark TB (1968) New records of parasites of Ceratopogonidae. Mosq News 28: $122-123$.

Chhilar JS and Chaudhry S (2010) First report of a biting midge Culicoides anophelis parasitzing mosquito Anopheles stephensi from north-western India J Exp Sci 1:3-6.

Cilek JE and Hallmon CF (2005) The effectiveness of the Mosquito Magnet trap for reducing biting midge (Diptera: Ceratopogonidae) populations in coastal residential backyards. J Am Mosq Control Assoc 21: 218-221.

Cilek JE, Kline DL and Hallmon CF (2003) Evaluation of a novel removal trap system to reduce biting midge (Diptera: Ceratopogonidae) populations in Florida backyards. J Vector Ecol 28: 23-30.

Clausen PH, Stephan A, Bartsch S, Jandowsky A, Hoffmann-Köhler P, Schein E, Mehlitz D and Bauer B (2009) Seasonal dynamics of biting midges (Diptera: Ceratopogonidae, Culicoides spp.) on dairy farms of Central Germany during the 2007/2008 epidemic of bluetongue. Parasitol Res 105: 381-386.

Clements BW Jr. and Rogers AJ (1968) Tests of larvicides for control of salt-marsh sand flies (Culicoides), 1967. Mosq News 28: 529-534.

De Clercq K, Vandenbussche F, Vandemeulebroucke E, Vanbinst T, De Leeuw I, Verheyden B, Goris N, Mintiens K, Méroc E, Herr C, Hooybergs J, Houdart P, Sustronck B, De Deken R, Maquet G, Bughin J, Saulmont M, Lebrun M, Bertels G and Miry C (2008) Transplacental bluetongue infection in cattle. Vet Rec 162(17): 564.

Coetzer JAW, Thomson GR and Tustin RC (1994) Infectious diseases of livestock with special reference to southern Africa. Oxford University Press, Cape Town, South Africa.

Conte A, Giovannini A, Savini L, Goffredo M, Calistri P and Meiswinkel R (2003) The effect of climate on the presence of Culicoides imicola in Italy. J Vet Med B Infect Dis Vet Public Health 50: 139-147.

Cribb BW, Brei B, Ridley AW and Merritt DJ (2003) Occurrence of immature Culicoides molestus (Skuse) (Diptera: Ceratopogonidae) in relation to habitat characteristics. Aus J Entomol 42: 114-118.

Debboun M and Strickman D (2013) Insect repellents and associated personal protection for a reduction in human disease. Med Vet Entomol 27: 1-9.

Deblauwe I, De Witte JC, De Deken G, De Deken R, Madder M, Van Erk S, Hoza FA, Lathouwers D and Geysen D (2012) A new tool for the molecular identification of Culicoides species of the Obsoletus group: the glass slide microarray approach. Med Vet Entomol 26: 83-91.

De Souza Jl, Gleason FH, Ansari MA, López Lastra CC, Garcia JJ, Pires-Zottarelli CLA and Marano AV (2014) Fungal and oomycete parasites of Chironomidae, Ceratopogonidae and Simuliidae (Culicomorpha, Diptera). Fungal Biol Rev 28: $13-23$.

Del Río R, Barceló C, Lucientes J and Miranda MA (2014a) Detrimental effect of cypermethrin treated nets on Culicoides populations (Diptera; Ceratopogonidae) and non-targeted fauna in livestock farms. Vet Parasitol 199: 230-234.

Del Río R, Barceló C, Paredes-Esquivel C, Lucientes J and Miranda MA (2014b) Susceptibility of Culicoides species biting midges to deltamethrin-treated nets as determined under laboratory and field conditions in the Balearic Islands, Spain. Med Vet Entomol 28: 414-420.

Del Río R, Monerris M, Miquel M, Borràs D, Calvete C, Estrada R, Lucientes J and Miranda MA (2013) Collection of Culicoides spp. with four light trap models during different seasons in the Balearic Islands. Vet Parasitol 195: 150-156.

Del Río R, Monerris M, Miranda MA, Calvete CC, Borras D, Miquel M, Estrada R and Lucientes J (2009) Two years of Culicoides trap comparison in the Balearic Islands. Rev Élev Méd Vét Pays Trop 62: 97.

Del Río R, Venail R, Calvete C, Barceló C, Baldet T, Lucientes J, Miranda MA, Del Río R, Venail R, Calvete C, Barcelo C, Baldet T, Lucientes J and Miranda MA (2013) Sensitivity of Culicoides obsoletus (Meigen) (Diptera: Ceratopogonidae) to deltamethrin determined by an adapted WHO standard susceptibility test. Parasitology 141: 542-546.

Del Río López R, Miranda MA, Paredes-Esquivel C, Lucientes J, Calvete C, Estrada R and Venter GJ (2012) Recovery rates of bluetongue virus serotypes 1, 2, 4 and 8 Spanish strains from orally infected Culicoides imicola in South Africa. Med Vet Entomol 26: 162-167. 
Desmecht D, Vanden Bergh R, Sartelet A, Leclerc M, Mignot C, Misse F, Sudraud C, Berthemin S, Jolly S, Mousset B, Linden A, Coignoul F and Cassart D (2008) Evidence for transplacental transmission of the current wild-type strain of bluetongue virus serotype 8 in cattle. Vet Rec 163: 50-52.

Dhana A, Yen H, Okhovat J-P, Cho E, Keum N and Khumalo NP (2018) Ivermectin versus permethrin in the treatment of scabies: a systematic review and meta-analysis of randomized controlled trials. J Am Acad Dermatol 78: 194-198.

Diarra M, Fall M, Fall MG, Diop A, Seck MT, Garros C, Balenghien T, Allene X, Rakotoarivony I, Lancelot R, Mall I, Bakhoum MT, Dosum AM, Ndao M, Bouyer J and Guis H (2014) Seasonal dynamics of Culicoides (Diptera: Ceratopogonidae) biting midges, potential vectors of African horse sickness and bluetongue viruses in the Niayes area of Senegal. Parasit Vectors 7: 147.

Dipeolu OO and Ogunrinade AF (1977) Studies on Culicoides species of Nigeria. VII. The biology of some Nigerian Culicoides species. Z Parasitenkd 51: 289-298.

Doherty WM, Bishop AL, Melville LF, Johnson SJ, Bellis GA and Hunt NT (2004) Protection of cattle from Culicoides spp. in Australia by shelter and chemical treatments. Vet Ital 40: 320-323.

Ducheyne E, De Deken R, Bécu S, Codina B, Nomikou K, Mangana-Vougiaki O, Georgiev G, Purse BV and Hendickx G (2007) Quantifying the wind dispersal of Culicoides species in Greece and Bulgaria. Geospat Health 1: 177-189.

Dukes JC and Axtell RC (1976) Residual effectiveness of insecticide-treated screens for control of sand flies, Culicoides furens (Poey) (Diptera: Ceratopogonidae). Mosq News 36: 488-491.

Dungu B, Gerdes T and Smit T (2004) The use of vaccination in the control of bluetongue in southern Africa. Vet Ital 40: 616-622.

Du Toit RM (1944). The transmission of bluetongue and horse-sickness by Culicoides. Onderstepoort J Vet Science Animal Industry 19: 7-16.

Dyce AL (1969) The recognition of nulliparous and parous Culicoides (Diptera: Ceratopogonidae) without dissection. J Aus Enthomol Soc 8: 11-15.

Dyce AL, Bellis GA and Muller JM (2007) Pictorial atlas of Australian culicoides wings (Diptera: Ceratopogonidae). Australian Biological Resources Study, Canberra, Australia.

Elbers ARW and Meiswinkel R (2014) Culicoides (Diptera: Ceratopogonidae) host preferences and biting rates in the Netherlands: comparing cattle, sheep and the black-light suction trap. Vet Parasitol 205: 330-337.

Enayati A and Hemingway J (2010) Malaria management: past, present, and future. Annu Rev Entomol 55: 569-591.

Estrada R, Carmona VJ, Alarcón-Elbal PM, Miranda MA, Borrás D, Roche ML, Tamarit A, Navarro J and Lucientes J (2011) Primera cita De Culicoides Paolae Boorman, 1996 (Diptera, Ceratopogonidae) Para La Península Ibérica Y Aportaciones Sobre Su Distribución. Bol Soc Entomol Aragon 49: 217-221.

European Commission (EC) (2007) Commission Regulation (EC) No 1266/2007 of 26 October 2007 on implementing rules for Council Directive 2000/75/EC as regards the control, monitoring, surveillance and restrictions on movements of certain animals of susceptible species in relation to bluetongue. European Commission, Brussels, Belgium.

European Food Safety Authority (EFSA) (2007) Scientific Opinion of the EFSA Panel on Animal Health and Welfare (AHAW) on request from the Commission on bluetongue vectors and vaccines. EFSA J 5: 479.

European Commission (EC) (2012) Commission Regulation (EC) No 456/2012 of 30 May 2012 amending Regulation (EC) No 1266/2007 on implementing rules for Council Directive 2000/75/EC as regards the control, monitoring, surveillance and restrictions on movements of certain animals of susceptible species in relation to bluetongue. OJ L141: 7-13.

European Food Safety Authority (EFSA) (2008) Bluetongue scientific opinion of the Panel on Animal Health and Welfare. EFSA J 735: 1-70.

European Food Safety Authority (EFSA) (2017) Bluetongue: control, surveillance and safe movement of animals. EFSA J 15: 4698.

Falconi C, López-Olvera JR and Gortázar C (2011) BTV infection in wild ruminants, with emphasis on red deer: a review. Vet Microbiol 151(3-4): 209-219.

Fallis AM and Bennett GF (1961) Ceratopogonidae as intermediate hosts for Haemoproteus and other parasites. Mosq News 21:21-28. 
Fassotte C, Delécolle JC, Cors R, Defrance T, De Deken T, Haubruge E and Losson B (2008) Culicoides trapping with Rothamsted suction traps before and during the bluetongue epidemic of 2006 in Belgium. Prev Vet Med 87: 74-83.

Fernández-Pacheco P, Fernández-Pinero J, Agüero M and Jiménez-Clavero MA (2008) Bluetongue virus serotype 1 in wild mouflons in Spain. Vet Rec 162: 659.

Ferraguti M, Martínez-de la Puente J, Ruiz S, Soriguer R and Figuerola J (2013) On the study of the transmission networks of blood parasites from SW Spain: diversity of avian haemosporidians in the biting midge Culicoides circumscriptus and wild birds. Parasite Vectors 6: 208.

Floore TG (1985) Laboratory wind tunnel tests of nine insecticides against adult Culicoides species (Diptera: Ceratopogonidae). Florida Entomol 68: 678-682.

Foster NM, Jones RH and McCrory BR (1963) Preliminary investigations on insect transmission of bluetongue virus in sheep. Am J Vet Res 24: 1195-1200.

Fox LM (2006) Ivermectin: uses and impact 20 years on. Curr Opin Infect Dis 19: 588-593.

Foxi C and Delrio G (2010) Larval habitats and seasonal abundance of Culicoides biting midges found in association with sheep in northern Sardinia, Italy. Med Vet Entomol 24: 199-209.

Foxi C, Pinna M, Monteys VSI and Delrio G (2011) An updated checklist of the Culicoides latreille (Diptera: Ceratopogonidae) of Sardinia (Italy), and seasonality in proven and potential vectors for bluetongue virus (BTV). Proc Entomol Soc Washingt 113: 403-416.

Fukuda T, Kline DL and Day JE (2002) An iridescent virus and a microsporidium in the biting midge Culicoides barbosai from Florida. J Am Mosq Control Assoc 18: 128-130.

García I, Napp S, Casal J, Perea A, Allepuz A, Alba A, Carbonero A and Arenas A (2009) Bluetongue epidemiology in wild ruminants from Southern Spain. Eur J Wildl Res 55: 173-178.

Garros C, Balenghien T, Carpenter S, Delécolle JC, Meiswinkel R, Pédarrieu A, Rakotoarivony I, Gardès L, Golding N, Barber J, Miranda M, Borràs D, Goffredo M, Monaco F, Pagès N, Sghaier S, Hammami S, Calvo JH, Lucientes J, Geysen D, De Deken G, Monteys V, Schwenkenbecher J, Kampen H, Hoffmann B, Lehmann K, Werner D, Baldet T, Lancelot R and Cêtre-Sossah C (2014) Towards the PCR-based identification of Palaearctic Culicoides biting midges (Diptera: Ceratopogonidae): results from an international ring trial targeting four species of the subgenus Avaritia. Parasit Vectors 7: 223.

Gerry AC and Mullens BA (2000) Seasonal abundance and survivorship of Culicoides sonorensis (Diptera: Ceratopogonidae) at a southern California dairy, with reference to potential bluetongue virus transmission and persistence. J Med Entomol 37: 675-688.

Gerry AC, Mullens BA, Maclachlan BJ and Mecham JO (2001) Seasonal transmission of bluetongue virus by Culicoides sonorensis (Diptera: Ceratopogonidae) at a southern California dairy and evaluation of vectorial capacity as a predictor of bluetongue virus transmission. J Med Entomol 38: 197-209.

Gerry AC, Sarto i Monteys V, Moreno Vidal JO, Francino O and Mullens BA (2009) Biting rates of Culicoides midges (Diptera: Ceratopogonidae) on sheep in northeastern Spain in relation to midge capture using UV light and carbon dioxide-baited traps. J Med Entomol 46: 615-624.

Gibbens N (2012) Schmallenberg virus: a novel viral disease in northern Europe. Vet Rec 170: 58.

Gibbs EP and Greiner E (1994) The epidemiology of Bluetongue. Comp Immunol Microbiol Infect Dis 17: 207-220.

Glushchenko NP and Mirzaeva AG (2008) Larval habitats and species composition of bloodsucking midges (Diptera: Ceratopogonidae) in mountain landscapes of South Siberia and the Russian far East. Russ Entomol J 17: 63-70.

Goffredo M, Catalani M, Federici V, Portanti O, Marini V, Mancini G, Quaglia M, Santilli A, Teodori L and Savini G (2015) Vector species of Culicoides midges implicated in the 2012-2014 Bluetongue epidemics in Italy. Vet Ital 51:131-138.

Goffredo M and Meiswinkel R (2004) Entomological surveillance of bluetongue in Italy: methods of capture, catch analysis and identification of Culicoides biting midges. Vet Ital 40: 260-265.

Goffredo M, Romeo G, Monaco F, Di Gennaro A and Savini G (2004) Laboratory survival and blood feeding response of wild-caught Culicoides obsoletus Complex (Diptera: Ceratopogonidae) through natural and artificial membranes. Vet Ital 40: 282-285. 
González M, Venter GJ, López S, Iturrondobeitia JC and Goldarazena A (2014) Laboratory and field evaluations of chemical and plant-derived potential repellents against Culicoides biting midges in northern Spain. Med Vet Entomol 28: 421-431.

González M, Baldet T, Delécolle JC, Romón P and Goldarazena A (2013) Monitoring of Culicoides Latreille (Diptera : Ceratopogonidae) after BTV outbreaks, in sheep farms and natural habitats from the Basque Country (northern Spain). Entomol Soc Washington 115: 48-69.

González M and Goldarazena A (2011) El género Culicoides en el País Vasco. Guía práctica para su identificación y control. Servicio Central de Publicaciones del Gobierno Vasco, Bilbao, Spain.

González M, López S, Mullens BA, Baldet T and Goldarazena A (2013) A survey of Culicoides developmental sites on a farm in northern Spain, with a brief review of immature habitats of European species. Vet Parasitol 191: 81-93.

Greive, KA, Staton JA, Miller PF, Peters BA and Oppenheim VMJ (2010) Development of Melaleuca oils as effective natural-based personal insect repellents. Aus J Entomol 49: 40-48.

Griffioen K, Van Gemst DBJ, Pieterse MC, Jacobs F and Sloet van Oldruitenborgh-Oosterbaan MM (2011) Culicoides species associated with sheep in the Netherlands and the effect of a permethrin insecticide. Vet J 190: 230-235.

Gubbins S, Carpenter S, Baylis M, Wood JLN and Mellor PS (2008) Assessing the risk of bluetongue to UK livestock: uncertainty and sensitivity analyses of a temperature-dependent model for the basic reproduction number. J R Soc Interface 5: 363-371.

Gubbins S, Szmaragd C, Burgin L, Wilson A, Volkova V, Gloster J and Gunn GJ (2010) Assessing the consequences of an incursion of a vector-borne disease. I. Identifying feasible incursion scenarios for bluetongue in Scotland. Epidemics 2: 148-154.

Harrup LE, Bellis GA, Balenghien T and Garros C (2015) Culicoides Latreille (Diptera: Ceratopogonidae) taxonomy: current challenges and future directions. Infect Genet Evol 30: 249-266.

Harrup LE, Gubbins S, Barber J, Denison E, Mellor PS, Purse BV and Carpenter S (2014) Does covering of farm-associated Culicoides larval habitat reduce adult populations in the United Kingdom? Vet Parasitol 201: 137-145.

Harrup LE, Logan JG, Cook JI, Golding N, Birkett MA, Pickett JA, Sanders CA, Barber J, Rogers DJ, Mellor PS, Purse BV and Carpenter S (2012) Collection of Culicoides (Diptera: Ceratopogonidae) using $\mathrm{CO}_{2}$ and enantiomers of 1-octen-3-ol in the United Kingdom. J Med Entomol 49: 112-121.

Harrup LE, Miranda MA and Carpenter S (2016) Advances in control techniques for Culicoides and future prospects. Vet Ital 52: 247-264.

Harrup LE, Purse BV, Golding N, Mellor PS and Carpenter S (2013) Larval development and emergence sites of farmassociated Culicoides in the United Kingdom. Med Vet Entomol 27: 441-449.

Henni LH, Sauvage F, Ninio C, Depaquit J and Augot D (2014) Wing geometry as a tool for discrimination of Obsoletus group (Diptera: Ceratopogonidae: Culicoides) in France. Infect Genet Evol 21: 110-117.

Hofmann MA, Renzullo S, Mader M, Chaignat V, Worwa G and Thuer B (2008) Genetic characterization of toggenburg orbivirus, a new bluetongue virus, from goats, Switzerland. Emerg Infect Dis 14: 1855-1861.

Holbrook FR (1994) Survival, fecundity, and egg fertility of Culicoides variipennis (Diptera: Ceratopogonidae) fed on calves inoculated with ivermectin. J Am Mosq Control Assoc 10: 7-9.

Holbrook FR and Mullens BA (1994) Effects of ivermectin on survival, fecundity, and egg fertility in Culicoides variipennis (Diptera:Ceratopogonidae). J Am Mosq Control Assoc 10: 70-73.

Holbrook FR, Tabachnick WJ, Schmidtmann ET, McKinnon CN, Bobian RJ and Grogan WL (2000) Sympatry in the Culicoides variipennis complex (Diptera: Ceratopogonidae): a taxonomic reassessment. J Med Entomol 37: 65-76.

Hörbrand T and Geier M (2009) Monitoring of Culicoides at nine locations in Southern Germany (2007-2008). Parasitol Res 105: 387-392.

Horsberg TE (2012) Avermectin use in aquaculture. Curr Pharm Biotechnol 13: 1095-1102.

Isberg E, Bray DP, Birgersson G, Hillbur Y and Ignell R (2016) Identification of cattle-derived volatiles that modulate the behavioral response of the biting midge Culicoides nubeculosus. J Chem Ecol 42: 24-32.

Isberg E, Hillbur Y and Ignell R (2013) Comparative study of antennal and maxillary palp olfactory sensilla of female biting midges (Diptera: Ceratopogonidae: Culicoides) in the context of host preference and phylogeny. J Med Entomol 50: 485-492. 
Iturbe-Ormaetxe I, Walker T and O' Neill SL (2011) Wolbachia and the biological control of mosquito-borne disease. EMBO Rep 12: 508-518.

Jacquet S, Huber K, Pagès N, Talavera S, Burgin LE, Carpenter S, Sanders C, Dicko AH, Djerbal M, Goffredo M, Lhor Y, Lucientes J, Miranda-Chueca MA, Pereira Da Fonseca I, Ramilo DW, Setier-Rio M-L, Bouyer J, Chevillon C, Balenghien T, Guis H and Garros C (2016) Range expansion of the Bluetongue vector, Culicoides imicola, in continental France likely due to rare wind-transport events. Sci Rep 6: 27247.

Jenckel M, Bréard E, Schulz C, Sailleau C, Viarouge C, Hoffmann B, Höper D, Beer M and Zientara S (2015) Complete coding genome sequence of putative novel bluetongue virus serotype 27. Genome Announc 3: 16-17.

Johansen CA, Farrow RA, Morrisen A, Foley P, Bellis G, Van Den Hurk AF, Montgomery B, Mackenzie JS and Ritchie SA (2003) Collection of wind-borne haematophagous insects in the Torres Strait, Australia. Med Vet Entomol 17: 102-109.

Kean J, Rainey SM, McFarlane M, Donald CL, Schnettler E, Kohl A and Pondeville E (2015) Fighting arbovirus transmission: natural and engineered control of vector competence in Aedes mosquitoes. Insects 6: 236-278.

Kelson RV, Colwell AE and McClusky DK (1980) Studies of Culicoides occidentalis at Borax Lake, California. In: Grant $C D$ (ed.) Proceedings and papers of the $48^{\text {th }}$ Annual Conference of the Californian Mosquito and Vector Control Association, Inc. CMVCA Press, Sacramento, CA, USA, pp. 130-135.

Kettle DS (1949) An attempt to control Culicoides impunctatus Goetghebuer in Scotland by barrier-spraying. An Trop Med Parasitol 43: 284-296.

Kettle DS (1961) A study of the association between moorland vegetation and breeding sites of Culicoides (Diptera, Ceratopogonidae). Bull Entomol Res 52: 381-411.

Kettle DS (1969) The ecology and control of blood-sucking ceratopogonids. Acta Trop 26: 235-248.

Kettle DS, Edwards PB and Barnes A (1998) Factors affecting numbers of Culicoides in truck traps in coastal Queensland. Med Vet Entomol 12: 367-377.

Kettle DS, Nash RH and Hopkins BA (1956) Field tests with larvicides against Culicoides impunctatus Goetgh. in Scotland. Bull Entomol Res 47: 553-573.

Kettle DS, Parish RH and Parish J (1959) Further observations the persistence of larvicides against Culicoides and discussion on the interpretation of population changes in the untreated plots. Bull Entomol Res 50: 63-80.

Kettle DS, Wild CH and Elson MM (1975) A new technique for rearing individual Culicoides larvae (Diptera: Ceratopogonidae). J Med Entomol 12: 263-264.

Kiel E, Liebisch G, Focke R and Liebisch A (2009) Monitoring of Culicoides at 20 locations in northwest Germany. Parasitol Res 105: 351-357.

Kim HC, Bellis GA, Kim M, Klein TA, Chong S and Park J (2014) Seasonal abundance of Culicoides (Diptera: Ceratopogonidae) collected by Mosquito Magnet ${ }^{\oplus}$ in Northern Gyeonggi-do (Province), Korea. Korean J Parasitol 52: 57-62.

Kirkeby C, Bødker R, Stockmarr A and Enøe C (2009) Association between land cover and Culicoides (Diptera: Ceratopogonidae) breeding sites on four Danish cattle farms. Entomol Fenn 20: 228-232.

Kirkland PD (2004) Bluetongue viruses, vectors and surveillance in Australia - the current situation and unique features. Vet Ital 40: 47-50.

Kitaoka S (1975) Five new species of Culicoides (Diptera: Ceratopogonidae) of the Nansei Islands. Natl Inst Anim Health $\mathrm{Q}$ (Tokyo) 15: 192-200.

Kline DL, Allan SA, Bernier UR and Welch CH (2007) Evaluation of the enantiomers of 1-octen-3-ol and 1-octyn-3-ol as attractants for mosquitoes associated with a freshwater swamp in Florida, U.S.A. Med Vet Entomol 21: 323-331.

Kline DL and Axtell RC (1977) Distribution of Culicoides hollensis, C. furens and C. bermudensis in relation to plant cover in a North Carolina salt marsh (Diptera: Ceratopogonidae). J Med Entomol 13: 545-552.

Kline DL, Bernier UR and Hogsette JA (2012) Efficacy of three attractant blends tested in combination with carbon dioxide against natural populations of mosquitoes and biting flies at the lower suwannee wildlife refuge. J Am Mosq Control Assoc 28: 123-127.

Kline DL and Greiner EC (1985) Observations on larval habitats of suspected Culicoides vectors of bluetongue virus in Florida. Prog Clin Biol Res 178: 221-227. 
Kline DL, Kelly JF and Ellis EA (1985a) A Nosema-type microsporidian infection in larvae of Culicoides spp. from salt marshes in Florida. J Invertebr Pathol 45: 60-65.

Kline DL and Roberts RH (1981) Effectiveness of Chloropyrifos, Fenthion, Malation, and Proporxur as screen treatments for control of Culicoides mississippiensis. J Econ Entomol 74: 331-333.

Kline DL, Wood JR, Roberts RH and Baldwin KF (1985b) Laboratory evaluation of four organophosphate compounds as larvicides against field collected salt marsh Culicoides spp. (Diptera: Ceratopogonidae). J Am Mosq Control Assoc 1: $48-50$.

Koch HG and Axtell RC (1979a) Correlation of hourly suction trap collections of Culicoides furens and C. hollensis (Diptera: Ceratopogonidae) with wind, temperature and habitat. J Med Entomol 15: 500-505.

Koch HG and Axtell RC (1979b) Attraction of Culicoides furens and C. hollensis (Diptera: Ceratopogonidae) to animal hosts in a salt marsh habitat. J Med Entomol 15: 494-499.

Koumbati M, Managa O, Nimikou K, Mellor PS and Papadopoulos O (1999) Duration of bluetongue viremia and serologycal responses in experimentally infected European breeds of sheeps and goats. Vet Microbiol 64: 277-285.

Lacey LA and Kline DL (1983) Laboratory bioassay of Bacillus thuringiensis (H-14) against Culicoides spp. and Leptoconops spp.(Ceratopogonidae). Mosq News 43: 502-503.

Larget I and De Barjac H (1981) Specificity and active principle of Bacillus thuringiensis var. israelensis. Bull Soc Pathol Exot Filiales 74: 216-227.

Larska M, Lechowski L, Grochowska M and Zmudziński JF (2013) Detection of the Schmallenberg virus in nulliparous Culicoides obsoletus/scoticus complex and C. punctatus - the possibility of transovarial virus transmission in the midge population and of a new vector. Vet Microbiol 166: 467-473.

Lequime S and Lambrechts L (2014) Vertical transmission of arboviruses in mosquitoes: a historical perspective. Infect Genet Evol 28: 681-690.

Lewis SE, Rice A, Hurst GDD and Baylis M (2014) First detection of endosymbiotic bacteria in biting midges Culicoides pulicaris and Culicoides punctatus, important palaearctic vectors of bluetongue virus. Med Vet Entomol 28: 453-456.

Liebisch $\mathrm{G}$ and Liebisch A (2008) Efficacy of Flectron-eartags (cypermethrin) for control of midges (Culicoides) as the vectors of bluetongue virus in cattle: field studies and biossays. Dtsch Tierarztl Wochenschr 115: 220-230.

Lincoln VJ, Page PC, Kopp C, Mathis A, Von Niederhäusern R, Burger D and Herholz C (2015) Protection of horses against Culicoides biting midges in different housing systems in Switzerland. Vet Parasitol 210: 206-214.

Linley JR (1985) Biting midges (Diptera: Ceratopogonidae) as vectors of nonviral animal pathogens. J Med Entomol 22: 589-599.

Linley JR and Davies JB (1971) Sandflies and tourism in Florida and the Bahamas and Caribbean Area. J Econ Entomol 64: 264-278.

Lloyd AM, Kline DL, Hogsette JA, Kaufman PE and Allan SA (2008) Evaluation of two commercial traps for the collection of Culicoides (Diptera: Ceratopogonidae). J Am Mosq Control Assoc 24: 253-262.

Logan J and Cook J (2010) Understanding and exploiting olfaction for the surveillance and control of Culicoides biting midges. In: Takken W and Knols BG (eds.) Olfaction in vector-host interacttions. Ecology and Control of Vectorborne diseases. Vol. 2. Wageningen Academic Publishers, Wageningen, the Netherlands, pp. 217-246.

Logan JG, Seal NJ, Cook JI, Stanczyk NM, Birkett MA, Clark SJ, Gezan SA, Wadhams LJ, Pickett JA and Mordue AJ (2009) Identification of human-derived volatile chemicals that interfere with attraction of the Scottish biting midge and their potential use as repellents. J Med Entomol 46: 208-219.

Losson B, Mignon B, Paternostre J, Madder M, De Deken R, De Deken G, Deblauwe I, Fassotte C, Cors R, Defrance T, Delécolle JC, Baldet T, Haubruge E, Frédéric F, Bortels J and Simonon G (2007) Biting midges overwintering in Belgium 9: 451-452.

Luedke AJ, Jones RH and Jochim MM (1967) Transmission of bluetongue between sheep and cattle by Culicoides variipennis. Am J Vet Res 28: 457-460.

Lühken R, Kiel E and Steinke S (2014a) Impact of mechanical disturbance on the emergence of Culicoides from cowpats. Parasitol Res 113: 1283-1287.

Lühken R, Kiel E and Steinke S (2015) Topsoil conditions correlate with the emergence rates of Culicoides chiopterus and Culicoides dewulfi (Diptera : Ceratopogonidae) from cowpats. Parasitol Res 114: 1113-1117. 
Lühken R, Steinke S, Wittmann A and Kiel E (2014b) Impact of flooding on the immature stages of dung-breeding Culicoides in Northern Europe. Vet Parasitol 205(1-2): 289-294.

Luhring KA and Mullens BA (1997) Improved rearing methods for Heleidomermis magnapapula (Nematoda: Mermithidae), a larval parasite of Culicoides variipennis sonorensis (Diptera: Ceratopogonidae). J Med Entomol 34: 704-709.

Lysyk TJ (2007) Seasonal abundance, parity, and survival of adult Culicoides sonorensis (Diptera: Ceratopogonidae) in Southern Alberta, Canada. J Med Entomol 44: 959-969.

Ma Y, Xu J, Yang Z, Wang X, Lin Z, Zhao W, Wang Y, Li X and Shi H (2013) A video clip of the biting midge Culicoides anophelis ingesting blood from an engorged Anopheles mosquito in Hainan, China. Parasit Vectors 6: 326.

Maan S, Maan NS, Nomikou K, Batten C, Antony F, Belaganahalli MN, Samy AM, Reda AA, Al-Rashid SA and El Batel M (2011) Novel bluetongue virus serotype from Kuwait. Emerg Infect Dis 17: 886.

Macaulay EDM, Tatchell GM and Taylor LR (1988). The Rothamsted insect survey '12-metre' suction trap. Bull Entomol Res 78: 121-128.

MacLachlan NJ (1994) The pathogenesis and immunology of bluetongue virus infection of ruminants. Comp Immunol Microbiol Infect Dis 17: 197-206.

MacLachlan NJ, Barratt-Boyes SM, Brewer AW and Stott JL (1991) Bluetongue virus infection of cattle. In: Walton $\mathrm{TE}$, Osburn BI (eds.) Bluetongue, African horse sickness, and related orbiviruses: proceedings of the second international symposium. CRC Press, Boca Raton, FL, USA, pp. 17-21.

MacLachlan NJ, Jagels G, Rossitto PV, Moore PF and Heidner HW (1990) The pathogenesis of experimental bluetongue virus infection of calves. Vet Pathol 27: 223.

Maclachlan NJ and Mayo CE (2013) Potential strategies for control of bluetongue, a globally emerging, Culicoidestransmitted viral disease of ruminant livestock and wild life. Antiviral Res 99: 79-90.

Maclachlan NJ and Osburn BI (2006) Public health impact of bluetongue virus infection. J Am Vet Med Assoc 228: 1-4.

Mands V, Kline DL and Blackwell A (2004) Culicoides midge trap enhancement with animal odour baits in Scotland. Med Vet Entomol 18: 336-342.

Martínez-De la Puente J, Figuerola J and Soriguer R (2014) Fur or feather? Feeding preferences of species of Culicoides biting midges in Europe. Trends Parasitol 31: 16-22.

Martínez-De la Puente J, Martínez J, Ferraguti M, Morales-De la Nuez A, Castro N and Figuerola J (2012) Genetic characterization and molecular identification of the bloodmeal sources of the potential bluetongue vector Culicoides obsoletus in the Canary Islands, Spain. Parasit Vectors 5: 147.

Mathieu B, Cêtre-Sossah C and Garros C (2010). IIKC: an interactive identification key for female Culicoides (Diptera: Ceratopogonidae) from the West Palearctic region. In: Morrison DA (ed.) Tools for identifying biodiversity: progress and problems. Oxford University Press, Oxford, UK, pp. 201-205.

Mayo CE, Mullens BA, Reisen WK, Osborne CJ, Gibbs EPJ, Gardner IA and MacLachlan NJ (2014a) Seasonal and interseasonal dynamics of bluetongue virus infection of dairy cattle and Culicoides sonorensis midges in northern California - implications for virus overwintering in temperate zones. PLoS ONE 9: e106975.

Mayo CE, Osborne CJ, Mullens BA, Gerry AC, Gardner IA, Reisen WK, Barker CM and MacLachlan NJ (2014b) Seasonal variation and impact of waste-water lagoons as larval habitat on the population dynamics of Culicoides sonorensis (Diptera: Ceratpogonidae) at two dairy farms in northern California. PLoS ONE 9: e89633.

McAlpine JF, Peterson BV, Shewell GE, Teskey HJ, Vockeroth JR and Wood DM (1981) Manual of nearctic diptera. Vol 1. Research Branch, Agriculture Canada, Ottawa, Canada.

Mee, PT, Weeks AR, Walker PJ, Hoffmann AA and Duchemin JB (2015) Detection of low-level Cardinium and Wolbachia infections in Culicoides. Appl Environ Microbiol 81: 6177-6188.

Meiswinkel R (1989) Afrotropical Culicoides: a redescription of C. (Avaritia) imicola Kieffer, 1913 (Diptera:Ceratopogonidae) with description of the closely allied C. (A.) bolitinos sp. nov. reared from the dung of the African Buffalo, blue wildebeest and cattle. Onderstepoort J Vet Res 56: 23-39.

Meiswinkel R, Baylis M and Labuschagne K (2000) Stabling and the protection of horses from Culicoides bolitinos (Diptera: Ceratopogonidae), a recently identified vector of African horse sickness. Bull Entomol Res 90: 509-515. 
Meiswinkel R, Goffredo M, Dijkstra EGM, Van der Ven IJK, Baldet T and Elbers A (2008) Endophily in Culicoides associated with BTV-infected cattle in the province of Limburg, south-eastern Netherlands, 2006. Prev Vet Med 87: 182-195.

Meiswinkel R, Gomulski LM, Delécolle JC, Goffredo M and Gasperi G (2004) The taxonomy of Culicoides vector complexes - unfinished business. Vet Ital 40: 151.

Meiswinkel R, Venter GJ and Nevill EM (1994). Vectors: Culicoides spp. In: Coetzer JAW, Thomson GR and Tustin RC (eds.) Infectious diseases of livestock, with special reference to Southern Africa. Oxford University Press, Cape Town, South Africa, pp. 93-136.

Meiswinkel R, Van Rijn P, Leijs P and Goffredo M (2007) Potential new Culicoides vector of bluetongue virus in northern Europe. Vet Rec 161: 564-565.

Meiswinkel R, Scolamacchia F, Dik M, Mudde J, Dijkstra E, Van der Ven IJK, and Elbers ARW (2014) The Mondrian matrix: Culicoides biting midge abundance and seasonal incidence during the 2006-2008 epidemic of bluetongue in the Netherlands. Med Vet Entomol 28: 10-20.

Meiswinkel R, Venter GJ and Nevill EM (1994) Vectors: Culicoides spp. In: Coetzer JAW, Thomson GR and Tustin RC (eds.) Infectious diseases of livestock with special reference to southern Africa. Oxford University Press, Cape Town, South Africa, pp. 93-136.

Mellor PS (1990) The replication of bluetongue virus in Culicoides vectors. Curr Top Microbiol Immunol 162: 143-161.

Mellor PS (2004) Infection of the vectors and bluetongue epidemiology in Europe. Vet Ital 40: 164-167.

Mellor PS and Boorman J (1995) The transmission and geographical spread of African horse sickness and bluetongue viruses. Ann Trop Med Parasitol 89: 1-15.

Mellor PS, Boorman J and Baylis M (2000) Culicoides biting midges: their role as arbovirus vectors. Annu Rev Entomol 45: 307-340.

Mellor PS and Pitzolis G (1979) Observation on breeding sites and light trap collections of Culicoides during an outbreak of bluetongue in Cyprus. Bull Entomol Res 69: 234.

Meyer G, Lacroux C, Léger S, S. T, Goyeau K, Deplanche M and Lemaire M (2009) Lethal bluetongue virus serotype 1 infection in llamas. Emerg Infect Dis 15: 608-610.

Mills MK, Nayduch D and Michel K (2015) Inducing RNA interference in the arbovirus vector, Culicoides sonorensis. Insect Mol Biol 24: 105-114.

Mintiens K, Méroc E, Mellor PS, Staubach C, Gerbier G, Elbers ARW, Hendrickx G and De Clercq k (2008) Possible routes of introduction of bluetongue virus serotype 8 into the epicentre of the 2006 epidemic in north-western Europe. Prev Vet Med 87: 131-144.

Miranda MA, Rincon C and Borras D (2004) Seasonal abundance of Culicoides imicola and C. obsoletus in the Balearic islands. Vet Ital 40: 292-295.

Morag N, Klement E, Saroya Y, Lensky I and Gottlieb Y (2012) Prevalence of the symbiont Cardinium in Culicoides (Diptera: Ceratopogonidae) vector species is associated with land surface temperature. FASEB J 26: 4025-4034.

Morag N, Mullens BA and Gottlieb Y (2013) Assessment of survival and body size variation in Culicoides imicola (Diptera: Ceratopogonidae) as a function of Cardinium (Bacteriodetes) infection status. Appl Environ Microbiol 79(20): 62606263.

Mordue AJ, Dallas JF, Nolan DV and Logan JG (2007) Novel methods for the identification and control of Culicoides midges as vectors of emerging diseases. Ecol Control Vector-Borne Dis 1: 307-325.

Mullens BA (1989) A quantitative survey of Culicoides variipennis (Diptera: Ceratopogonidae) in dairy wastewater ponds in southern California. J Med Entomol 26: 559-565.

Mullens BA (1993) In vitro assay for permethrin persistence and interference with bloodfeeding of Culicoides (Diptera: Ceratopogonidae) on animals. J Am Mosq Control Assoc 9: 256-259.

Mullens BA, Gerry AC, Monteys VSI, Pinna M and González A (2010) Field studies on Culicoides (Diptera: Ceratopogonidae) activity and response to deltamethrin applications to sheep in northeastern Spain. J Med Entomol 47: 106-110.

Mullens BA, Gerry AC and Velten RK (2001) Failure of a permethrin treatment regime to protect cattle against bluetongue virus. J Med Entomol 38: 760-762.

Mullens BA and Lip K-S (2014) Larval population dynamics of Culicoides variipennis (Diptera: Ceratopogonidae) in Southern California. J Med Entomol 24: 566-574. 
Mullens BA and Luhring KA (1996) Salinity and pollution effects oil survival and infectivity of Heleidomermis magnapapula (Stichosomida: Mermithidae) for Culicoides variipennis sonorensis (Diptera: Ceratopogonidae). Env Entomol 25: 1202-1208.

Mullens BA, Luhring KA and Breidenbaugh MS (1997) Experimental host range studies with Heleidomermis magnapapula (Mermithidae), a parasite of Culicoides variipennis (Ceratopogonidae). J Am Mosq Control Assoc 13: 398-401.

Mullens BA, Mcdermott EG and Gerry AC (2015) Progress and knowledge gaps in Culicoides ecology and control. Vet Ital 51:313-323.

Mullens BA, Paine EO and Velten RK (1995) Temperature effects on survival and development of Heleidomermis magnapapula in the laboratory. J Nematol 27: 29-35.

Mullens BA, Sarto Monteys V and Przhiboro AA (2008) Mermithid parasitism in Ceratopogonidae: a literature review and critical assessment of host impact and potential for biological control of Culicoides. Russ Entomol J 17: 87-113.

Mullens BA and Velten RK (1994) Laboratory culture and life history of Heleidomermis magnapapula in its host, Culicoides variipennis (Diptera: Ceratopogonidae). J Nematol 26: 1-10.

Mullens BA, Velten RK and Federici BA (1999) Iridescent virus infection in Culicoides variipennis sonorensis and interactions with the mermithid parasite Heleidomermis magnapapula. J Invertebr Pathol 73: 231-233.

Mullens BA, Velten RK, Gerry AC, Braverman Y and Endris RG (2000) Feeding and survival of Culicoides sonorensis on cattle treated with permethrin or pirimiphos-methyl. Med Vet Entomol 14:313-320.

Muñoz-Muñoz F, Ramoneda J, Pagès N, Pujol N and Talavera S (2016) Is the morphology of Culicoides intersexes parasitized by mermithid nematodes a parasite adaptation? A morphometric approach to Culicoides circumscriptus (Diptera: Ceratopogonidae). J Invertebr Pathol 135: 1-9.

Muñoz-Muñoz F, Talavera S, Carpenter S, Nielsen SA, Werner D and Pagès N (2014) Phenotypic differentiation and phylogenetic signal of wing shape in western European biting midges, Culicoides spp., of the subgenus Avaritia. Med Vet Entomol 28: 319-329.

Muñoz-Muñoz F, Talavera S and Pagès N (2011) Geometric morphometrics of the wing in the subgenus Culicoides (Diptera: Ceratopogonidae): from practical implications to evolutionary interpretations. J Med Entomol 48: 129-139.

Murray MD (1987) Local dispersal of biting-midge Culicoides brevitarsis Kieffer (Diptera: Ceratopogonidea) in southeastern Australia. Aust J Zool 35: 559-573.

Nakamura Y, Kawai S, Yukuhiro F, Ito S, Gotoh T, Kisimoto R, Yanase T, Matsumoto Y, Kageyama D and Noda H (2009) Prevalence of Cardinium bacteria in planthoppers and spider mites and taxonomic revision of 'Candidatus Cardinium hertigii' based on detection of a new Cardinium group from biting midges. Appl Environ Microbiol 75: 6757-6763.

Napp S, Allepuz A, Alba A and Casal J (2009) Introduction of infected Culicoides by means of transport or commodities. Rev Elev Med Vet Pays Trop 62: 112.

Napp S, Allepuz A, Purse BV, Casal J, Garcia-Bocanegra I, Burgin LE and Searle KR (2016) Understanding spatio-temporal variability in the reproduction ratio of the bluetongue (BTV-1) epidemic in Southern Spain (Andalusia) in 2007 using epidemic trees. PLoS ONE 11: e0151151.

Napp S, García-Bocanegra I, Pagès N, Allepuz A, Alba A and Casal J (2013) Assessment of the risk of a bluetongue outbreak in Europe caused by Culicoides midges introduced through intracontinental transport and trade networks. Med Vet Entomol 27: 19-28.

Naraladker BW, Khillare BS, Kalwaghe ST, Chghure GM, Ravikanth K and Maini S (2011) Effectiveness of phytogenic fly repellant product against dipteran flies. International Res J Pharmacy Pharmacol 1: 70-74.

Narladkar BW, Shivpuje PR and Harke PC (2015) Fungal biological control agents for integrated management of Culicoides spp. (Diptera: Ceratopogonidae) of livestock. Vet World 8: 156-163.

Nayduch D, Cohnstaedt LW, Saski C, Lawson D, Kersey P, Fife M and Carpenter S (2014) Studying Culicoides vectors of BTV in the post-genomic era: resources, bottlenecks to progress and future directions. Virus Res 182: 43-49.

Nayduch D, Lee MB and Saski CA (2014) The reference transcriptome of the adult female biting midge (Culicoides sonorensis) and differential gene expression profiling during teneral, blood, and sucrose feeding conditions. PLoS ONE 9: e98123. 
Nevill EM (1967) Biological studies on some South African Culicoides species (Diptera: Ceratopogonidae) and the morphology of their immature stages. University of Pretoria, Pretoria, South Africa.

Nevill H and Dyce AL (1994) Afrotropical Culicoides: description and comparison of the pupae of seven species of the Similis supergroup (Diptera: Ceratopogonidae). Onderstepoort J Vet Res 61: 85-106.

Nicholas AH and McCorkell B (2014) Evaluation of Metarhizium anisopliae for the control of Culicoides brevitarsis Kieffer (Diptera: Ceratopogonidae), the principal vector of bluetongue virus in Australia. J Vector Ecol 39: 213-218.

Niedbalski W (2015) Bluetongue in Europe and the role of wildlife in the epidemiology of disease. Pol J Vet Sci 18: 455-461.

Nielsen BO (1963) The biting midges of Lyngby Aamose. Natura Jutlandica 10: 5-46.

Ninio C, Augot D, Dufour B and Depaquit J (2011) Emergence of Culicoides obsoletus from indoor and outdoor breeding sites. Vet Parasitol 183: 125-129.

Obona J and Dominiak P (2014) Biting midges (Diptera: Ceratopogonidae) in tree hole habitats in Slovakia. Oceanol Hydrobiol Stud 43: 61-65.

Onuike AL, Ikpeze $\mathrm{OO}$ and Ngenegbo UC (2015) Effects of deltamethrin on mortality, feeding behaviour and oviposition in the UK Culicoides species and at UK environmental temperature. Int J Vet Sci 4: 175-182.

Ortega MD, Holbrook FR and Lloyd JE (1999) Seasonal distribution and relationship to temperature and precipitation of the most abundant species of Culicoides in five provinces of Andalusia, Spain. J Am Mosq Control Assoc 15: 391-399.

Ortega MD, Lloyd JE and Holbrook FR (1997) Seasonal and geographical distribution of Culicoides imicola Kieffer (Diptera: Ceratopogonidae) in southwestern Spain. J Am Mosq Control Assoc 13: 227-232.

Osborne CJ, Mayo CE, Mullens BA, McDermott EG, Gerry AC, Reisen WK and MacLachlan NJ (2015) Lack of evidence for laboratory and natural vertical transmission of bluetongue virus in Culicoides sonorensis (Diptera: Ceratopogonidae). J Med Entomol 52: 274-277.

Page PC, Labuschagne K, Venter GJ, Schoeman JP and Guthrie AJ (2014) Field and in vitro insecticidal efficacy of alphacypermethrin-treated high density polyethylene mesh against Culicoides biting midges in South Africa. Vet Parasitol 203(1-2): 184-188.

Pages N, Bréard E, Urien C, Talavera S, Viarouge C, Lorca-Oro C, Jouneau L, Charley B, Zientara S, Bensaid A, Solanes D, Pujols J and Schwartz-Cornil I (2014) Culicoides midge bites modulate the host response and impact on bluetongue virus infection in sheep. PLoS ONE 9: e83683.

Pagès N, Muñoz-Muñoz F, Talavera S, Sarto V, Lorca C and Núñez JI (2009) Identification of cryptic species of Culicoides (Diptera: Ceratopogonidae) in the subgenus Culicoides and development of species-specific PCR assays based on barcode regions. Vet Parasitol 165: 298-310.

Paine EO and Mullens BA (1994) Distribution, seasonal occurrence, and patterns of parasitism of Heleidomermis magnapapula (Nematoda: Mermithidae), a parasite of Culicoides variipennis (Diptera: Ceratopogonidae) in California. Environ Entomol 23: 154-160.

Papadopoulos E, Bartram D, Carpenter S, Mellor P and Wall R (2009) Efficacy of alphacypermethrin applied to cattle and sheep against the biting midge Culicoides nubeculosus. Vet Parasitol 163: 110-114.

Papadopoulos E, Rowlinson M, Bartram D, Carpenter S, Mellor P and Wall R (2010) Treatment of horses with cypermethrin against the biting flies Culicoides nubeculosus, Aedes aegypti and Culex quinquefasciatus. Vet Parasitol 169: 165-171.

Pappas CD and Pappas LG (1990) Habitat pH characteristics of tree hole Culicoides (Diptera: Ceratopogonidae). J Am Mosq Control Assoc 6: 99-100.

Patakakis MJ (2004) Culicoides imicola in Greece. Vet Ital 40: 233.

Pates $\mathrm{H}$ and Curtis C (2005) Mosquito behavior and vector control. Annu Rev Entomol 50: 53-70.

Paweska JT and Venter GJ (2004) Vector competence of Culicoides species and the seroprevalence of homologous neutralizing antibody in horses for six serotypes of equine encephalosis virus (EEV) in South Africa. Med Vet Entomol 18: 398-407.

Pfannenstiel RS, Mullens BA, Ruder MG, Zurek L, Cohnstaedt LW and Nayduch D (2015) Management of North American Culicoides biting midges: current knowledge and research needs. Vector Borne Zoonotic Dis 15: 374-384.

Poinar GO and Mullens BA (1987) Heleidomermis magnapapula (Mermithidae: Nematoda) parasitizing Culicoides variipennis (Ceratopogonidae: Diptera) in California. Rev Nématol 10: 387-391. 
Poinar GO and Sarto i Monteys V (2008) Mermithids (Nematoda: Mermithidae) of biting midges (Diptera: Ceratopogonidae): Heleidomermis cataloniensis n. sp. from Culicoides circumscriptus Kieffer in Spain and a species of Cretacimermis Poinar, 2001 from a ceratopogonid in Burmese amber. Syst Parasitol 69: 13-21.

Porter JE (1959) Some effects of screens in retarding entry of the common salt-marsh sand fly Culicoides furens (Poey) (Diptera: Heleidae). Mosq News 19: 159-163.

Priest FG (1992) Biological control of mosquitoes and other biting flies by Bacillus sphaericus and Bacillus thuringiensis. J Appl Bacteriol 72: 357-369.

Probst C, Gethmann JM, Kampen H, Werner D and Conraths FJ (2015) Parasitol Res 114: 4717-4724.

Purse BV, Carpenter S, Venter GJ, Bellis G and Mullens BA (2015) Bionomics of temperate and tropical Culicoides midges: knowledge gaps and consequences for transmission of Culicoides-borne viruses. Annu Rev Entomol 60: 373-392.

De Raat IJ, Van den Boom R, Van Poppel M and Sloet van Oldruitenborgh-Oosterbaan MM (2008) The effect of a topical insecticide containing permethrin on the number of Culicoides midges caught near horses with and without insect bite hypersensitivity in the Netherlands. T Diergeneeskd 133: 838-842.

Rawlings P, Pro MJ, Pena I, Ortega MD and Capela R (1997) Spatial and seasonal distribution of Culicoides imicola in Iberia in relation to the transmission of African horse sickness virus. Med Vet Entomol 11: 49-57.

Reeves WK, Lloyd JE, Stobart R, Stith C, Miller MM, Bennett KE and Johnson G (2010) Control of Culicoides sonorensis (Diptera: Ceratopogonidae) blood feeding on sheep with long-lasting repellent pesticides. J Am Mosq Control Assoc 26: 302-305.

Rieb JP (1982) Contribution à la connaissance de l'écologie et de la biologie des Cératopogonidés (Diptera, Nematocera). $\mathrm{PhD}$ thesis, University Louis Pasteur, Strasbourg, France, $395 \mathrm{pp}$.

Riek RF (1954) Studies on allergic dermatitis (Queensland itch) of the horse: the aetiology of the disease. Aust J Agric Res 5: 109-129.

Rigot T and Gilbert m (2012) Quantifying the spatial dependence of Culicoides midge samples collected by Onderstepoort-type blacklight traps: an experimental approach to infer the range of attraction of light traps. Med Vet Entomol 26: 152-161.

Rioux JA, Descous S and Pech J (1959) A new tree-hole Ceratopogonidae: Culicoides haranti n. sp. (Diptera, Heleidae). Ann Parasitol Hum Comp 34: 432-438.

Robin M, Archer D, McGowan C, Garros C, Gardès L and Baylis M (2015) Repellent effect of topical deltamethrin on blood feeding by Culicoides on horses. Vet Rec 176: 574.

Romón P, Higuera M, Delécolle JC, Baldet T, Aduriz G and Goldarazena A (2012) Phenology and attraction of potential Culicoides vectors of bluetongue virus in Basque Country (northern Spain). Vet Parasitol 186: 415-424.

Ruiz-Fons F, Reyes-Garcia AR, Alcaide V and Gortazar C (2008) Spatial and temporal evolution of bluetongue virus in wild ruminants, Spain. Emerg Infect Dis 14: 951-953.

Ruiz-Fons F, Sánchez-Matamoros A, Gortázar C and Sánchez-Vizcaíno JM (2014) The role of wildlife in bluetongue virus maintenance in Europe: lessons learned after the natural infection in Spain. Virus Res 182: 50-58.

Saegerman C, Bolkaerts B, Baricalla C, Raes M, Wiggers L, De Leeuw I, Vandenbussche F, Zimmer JY, Haubruge E, Cassart D, De Clercq K and Kirschvink N (2011) The impact of naturally-occurring, trans-placental bluetongue virus serotype-8 infection on reproductive performance in sheep. Vet J 187: 72-80.

Sáenz MR and Greiner EC (1994) Culicoides aspirated from cattle in Costa Rica, Honduras, Panama and Puerto Rico, and their role as potential vectors of bluetongue viruses. Regional Bluetongue Team. Med Vet Entomol 8: 15-19.

Sailleau C, Bréard E, Viarouge C, Vitour D, Romey A, Garnier A, Fablet A, Lowenski S, Gorna K, Caignard G, Pagneux $C$ and Zientara S (2017) Re-emergence of bluetongue virus serotype 8 in France, 2015. Transbound Emerg Dis 64: 998-1000.

Sampson MN and Gooday GW (1998) Involvement of chitinases of Bacillus thuringiensis during pathogenesis in insects. Microbiology 144: 2189-2194.

Sanders CJ, Gubbins S, Mellor PS, Barber J, Golding N, Harrup LE and Carpenter ST (2012) Investigation of diel activity of Culicoides biting midges (Diptera: Ceratopogonidae) in the United Kingdom by using a vehicle-mounted trap. J Med Entomol 49: 757-765. 
Sanders CJ, Selby R, Carpenter S and Reynolds DR (2011a) High-altitude flight of Culicoides biting midges. Vet Rec 169: 208.

Sanders CJ, Shortall CR, Gubbins S, Burgin L, Gloster J, Harrington R, Reynolds DR, Mellor PS and Carpenter S (2011b) Influence of season and meteorological parameters on flight activity of Culicoides biting midges. J Appl Ecol 48: $1355-1364$.

Santamaría E, Cabrera OL, Zipa Y and Pardo RH (2012) Eficacia en campo de un repelente con base en para-mentano3,8-diol y aceite de limonaria contra Culicoides pachymerus (Diptera: Ceratopogonidae) en Colombia. Biomédica 32: $457-460$.

Santiago-Alarcon D, Havelka P, Pineda E, Segelbacher G and Schaefer HM (2013) Urban forests as hubs for novel zoonosis: blood meal analysis, seasonal variation in Culicoides (Diptera: Ceratopogonidae) vectors, and avian haemosporidians. Parasitology 140: 1799-1810.

Sarto I Monteys V and Saiz-Ardanaz M (2003) Culicoides midges in Catalonia (Spain), with special reference to likely bluetongue virus vectors. Med Vet Entomol 17: 288-293.

Satta G, Goffredo M, Sanna S, Vento L, Cubeddu GP and Mascherpa E (2004) Field disinfestation trials against Culicoides in north-west Sardinia. Vet Ital 40: 329-335.

Savini G, Goffredo M, Monaco F, Di Gennaro A, Cafiero MA, Baldi L, De Santis P, Meiswinkel R and Caporale V (2005) Bluetongue virus isolations from midges belonging to the Obsoletus complex (Culicoides, Diptera: Ceratopogonidae) in Italy. Vet Rec 157: 133-139.

Scheffer EG, Venter GJ, Labuschagne K, Page PC, Mullens BA, MacLachlan NJ, Osterrieder N and Guthrie AJ (2012) Comparison of two trapping methods for Culicoides biting midges and determination of African horse sickness virus prevalence in midge populations at Onderstepoort, South Africa. Vet Parasitol 185: 265-273.

Schmahl G, Klimpel S, Walldorf V, Schumacher B, Jatzlau A, Al-Quraishy S and Mehlhorn H (2009) Effects of permethrin (FlyporA (R)) and fenvalerate (AcadrexA (R) 60, ArkoflyA (R)) on Culicoides species-the vector of Bluetongue virus. Parasitol Res 104: 815-820.

Schmahl G, Walldorf V, Klimpel S, Al-Quraishy S and Mehlhorn H (2008) Efficacy of Oxyfly ${ }^{\text {TM }}$ on Culicoides species - the vectors of Bluetongue virus - and other insects. Parasitol Res 103: 1101-1103.

Schnettler E, Ratinier M, Watson M, Shaw AE, McFarlane M, Varela M, Elliott RM, Palmarini M and Kohl A (2013) RNA interference targets arbovirus replication in Culicoides cells. J Virol 87: 2441-2454.

Schwartz-Cornil I, Mertens PPC, Contreras V, Hemati B, Pascale F, Bréard E, Mellor PS, James MacLachlan N and Zientara $S$ (2008) Bluetongue virus: virology, pathogenesis and immunity. Vet Res 39: 46.

Schwenkenbecher J and Mordue A (2009) Discrimination of Culicoides midge larvae using multiplex polymerase chain reaction assays based on DNA sequence variation at the mitochondrial cytochrome C. J Med 46: 610-614.

Searle KR, Barber J, Stubbins F, Labuschagne K, Carpenter S, Butler A, Denison E, Sers C, Mellor PS, Wilson A, Nelson N, Gubbins S and Purse BV (2014) Environmental drivers of Culicoides phenology: how important is species-specific variation when determining disease policy? PLoS ONE 9: e111876.

Sellers RF (1980) Weather, host and vector - their interplay in the spread of insect borne animal virus diseases. J Hyg 81: 189-196.

Sellers RF (1984) Bluetongue in Africa, the Mediterranean region and Near East - disease, virus and vectors. Prev Vet Med 2: 371-378.

Sellers RF (1991) Weather, Culicoides, and the distribution and spread of bluetongue and African horse sickness viruses. In: Walton TE and Osburn BI (eds.) Bluetongue, African horse sickness, and related orbiviruses: proceedings of the second international symposium. Paris, France, pp. 17-21.

Sellers RF and Maarouf AR (1989) Trajectory analysis and bluetongue virus serotype 2 in Florida 1982. Can J Vet Res 53: 100-102.

Sellers RF, Pedgley DE and Tucker MR (1978) Possible windborne spread of bluetongue to Portugal, June-July 1956. J Hyg 81: 189-196.

Sellers RF, Rees WHG, Gillett JD, Boorman JPT and Rainey RC (1983) Seasonal variations in spread of arthropodborne disease agents of man and animals: implications for control [and discussion]. Philos Trans R Soc B Biol Sci 302: 485-495. 
Service MW (1969) Studies on the biting habits of Culicoides impunctatus Goetghebuer, C. obsoletus (Meigen) and C. punctatus (Meigen) (Diptera: Ceratopogonidae) in Southern England. Proc R Entomol Soc London Ser A Gen Entomol 44: 1-10.

Service MW (1971) Adult flight activities of some British Culicoides species. J Med Entomol 8: 605-609.

Smith $\mathrm{H}$ and Mullens BA (2003) Seasonal activity, size, and parity of Culicoides occidentalis (Diptera: Ceratopogonidae) in a coastal southern California salt marsh. J Med Entomol 40: 352-355.

Smith KE and Stallknecht DE (1996) Culicoides (Diptera: Ceratopogonidae) collected during epizootics of hemorrhagic disease among captive white-tailed deer. J Med Entomol 33: 507-510.

Sontag E and Szadziewski R (2011) Biting midges (Diptera: Ceratopogonidae) in Eocene Baltic amber from the Rovno region (Ukraine). Polish J Entomol 80: 779-800.

Standfast HA, Muller MJ and Wilson DD (1985) Mortality of Culicoides brevitarsis fed on cattle treated with ivermectin. Prog Clin Biol Res 178: 611-616.

Steinke S, Lühken R and Kiel E (2014) Assessment of the abundance of Culicoides chiopterus and Culicoides dewulfi in bovine dung: a comparison of larvae extraction techniques and emergence traps. Vet Parasitol 205: 255-262.

Stephen K and Kurtböke DI (2011) Screening of oomycete fungi for their potential role in reducing the biting midge (Diptera: Ceratopogonidae) Iarval populations in Hervey Bay, Queensland, Australia. Int J Environ Res Public Health 8: 1560-1574.

Stuart AE, Evans A, Brooks C, Simpson MJ, Cloughley JB, Maclntosh DF, Stuart CL, Blackwell A and Kettle DS (1996) The biting midge of the West Highlands: fifty years of research. Scott Med J 41: 143-146.

Svobodová M, Zídkova L, Čepička I, Oborník M, Lukeš J and Votýpka J (2007) Sergeia podlipaevi gen. nov., sp. nov. (Typanosomatidae, Kinetoplastida), a parasite of biting midges (Ceratopogonidae, Diptera). Int J Syst Evol Microbiol 57: 423-432.

Synek P, Munclinger P, Albrecht T and Votýpka J (2013) Avian haemosporidians in haematophagous insects in the Czech Republic. Parasitol Res 112: 839-845.

Szadziewski R and Grogan WL Jr. (1998) Biting midges from Dominican amber. III. Species of the tribes Culicoidini and Ceratopogonini (Diptera: Ceratopogonidae). Insecta mundi 12: 39-52.

Tabachnick WJ (1991) Genetic control of oral susceptibility to infection of Culicoides variipennis with bluetongue virus. Am J Trop Med Hyg 45: 666-671.

Tabachnick WJ (2004) Culicoides and the global epidemiology of bluetongue virus infection. Vet Ital 40: 144-150.

Tabachnick WJ (2010) Challenges in predicting climate and environmental effects on vector-borne disease episystems in a changing world. J Exp Biol 213: 946-954.

Takahashi K, Yagi K and Hattori K (1985) The effects of two insect growth regulators on the biting midges, Culicoides circumscriptus Kieffer (Diptera: Ceratopogonidae). Med Entomol Zool 36: 353-355.

Takken W and Kline DL (1989) Carbon dioxide and 1-octen-3-ol as mosquito attractans. J Am Mosq Control Assoc 5: 311-316.

Takken W, Verhulst N, Scholte EJ, Jacobs F, Jongema Y and Van Lammeren R (2008) The phenology and population dynamics of Culicoides spp. in different ecosystems in the Netherlands. Prev Vet Med 87: 41-54.

Talavera S, Muñoz-Muñoz F, Durán M, Verdún M, Soler-Membrives A, Oleaga Á, Arenas A, Ruiz-Fons F, Estrada R and Pagès N (2015) Culicoides species communities associated with wild ruminant ecosystems in Spain: tracking the way to determine potential bridge vectors for arboviruses. PLoS ONE 10: e0141667.

Tanya VN, Greiner EC and Gibbs EP (1992) Evaluation of Culicoides insignis (Diptera: Ceratopogonidae) as a vector of bluetongue virus. Vet Microbiol 32: 1-14.

Taylor WP (1986) The epidemiology of bluetongue. Rev Sci Tech l'Office Int des Epizoot 5: 351-356.

Thompson GM, Jess S, Gordon AW and Murchie AK (2014) Sticky-trapping biting midges (Culicoides spp.) alighting on cattle and sheep: effects of trap colour and evidence for host preference. Parasitol Res 113: 3085-3094.

Thompson GM, Jess S and Murchie AK (2013) Differential emergence of Culicoides (Diptera: Ceratopogonidae) from on-farm breeding substrates in Northern Ireland. Parasitology 140: 699-708.

Trigg JK (1996) Evaluation of a eucalyptus-based repellent against Culicoides impunctatus (Diptera: Ceratopogonidae) in Scotland. J Am Mosq Control Assoc 12: 329-330. 
Unkles SE, Marriott C, Kinghorn JR, Panter C and Blackwell A (2004) Efficacy of the entomopathogenic fungus, Culicinomyces clavisporus against larvae of the biting midge, Culicoides nubeculosus (Diptera: Ceratopogonidae). Biocontrol Sci Technol 14: 397-401.

Uslu U and Dik B (2006) Vertical distribution of Culicoides larvae and pupae. Med Vet Entomol 20: 350-352.

Uslu U and Dik B (2007a) Description of breeding sites of Culicoides species (Diptera: Ceratopogonidae) in Turkey. Parasite 14: 173-177.

Uslu U and Dik B (2010) Chemical characteristics of breeding sites of Culicoides species (Diptera: Ceratopogonidae). Vet Parasitol 169: 178-184.

Uslu U and Dik B (2007b) Description of breeding sites of Culicoides species (Diptera: Ceratopogonidae) in Turkey. Parasite 14: 176-177.

Van Ark H and Meiswinkel R (1992) Subsampling of large light trap catches of Culicoides (Diptera: Ceratopogonidae). Onderstepoort J Vet Res 59: 183-189.

Venail R, Balenghien T, Guis H, Tran A, Setier-Rio ML, Delécolle JC, Mathieu B, Cêtre-Sossah C, Martinez D and Languille $J$ (2012) Assessing diversity and abundance of vector populations at a national scale: example of Culicoides surveillance in France after bluetongue virus emergence. In: Mehlhorn $\mathrm{H}$ (ed.) Arthropods as vectors of emerging diseases. Parasitology Research Monographs. Vol 3. Springer, Berlin, Heidelberg, Germany, pp. 77-102.

Venail R, Lhoir J, Fall M, Del Río R, Talavera S, Labuschagne K, Miranda M, Pagès N, Venter G, Rakotoarivony I, Allène X, Scheid B, Gardès L, Gimonneau G, Lancelot R, Garros C, Cêtre-Sossah C, Balenghien T, Carpenter S and Baldet T (2015) How do species, population and active ingredient influence insecticide susceptibility in Culicoides biting midges (Diptera: Ceratopogonidae) of veterinary importance? Parasit Vectors 8: 439.

Venail R, Mathieu B, Setier-Rio ML, Borba C, Alexandre M, Viudes G, Garros C, Allene X, Carpenter S and Baldet T (2011) Laboratory and field-based tests of deltamethrin insecticides against adult Culicoides biting midges. J Med Entomol 48: 351-357.

Venter GJ, Groenewald DM, Paweska JT, Venter EH and Howell PG (1999) Vector competence of selected South African Culicoides species for the Bryanston serotype of equine encephalosis virus. Med Vet Entomol 13: 393-400.

Venter GJ, Labuschagne K, Boikanyo SNB and Morey L (2014) Assessment of the repellent effect of citronella and lemon eucalyptus oil against South African Culicoides species. J S Afr Vet Assoc 85: 1-5.

Venter GJ, Labuschagne K, Boikanyo SNB, Morey L and Snyman MG (2011) The repellent effect of organic fatty acids on Culicoides midges as determined with suction light traps in South Africa. Vet Parasitol 181: 365-369.

Venter GJ, Labuschagne K, Hermanides KG, Boikanyo SNB, Majatladi DM and Morey L (2009) Comparison of the efficiency of five suction light traps under field conditions in South Africa for the collection of Culicoides species. Vet Parasitol 166: 299-307.

Venter GJ, Mellor PS and Paweska JT (2006) Oral susceptibility of South African stock-associated Culicoides species to bluetongue virus. Med Vet Entomol 20: 329-334.

Venter GJ, Wright IM and Paweska JT (2010) A comparison of the susceptibility of the biting midge Culicoides imicola to infection with recent and historical isolates of African horse sickness virus. Med Vet Entomol 24: 324-328.

Verhoef FA, Venter GJ and Weldon CW (2014) Thermal limits of two biting midges, Culicoides imicola Kieffer and C. bolitinos Meiswinkel (Diptera: Ceratopogonidae). Parasit Vectors 7: 384.

Veronesi E, Venter GJ, Labuschagne K, Mellor PS and Carpenter S (2009) Life-history parameters of Culicoides (Avaritia) imicola Kieffer in the laboratory at different rearing temperatures. Vet Parasitol 163: 370-373.

Viennet E, Garros C, Gardès L, Rakotoarivony I, Allène X, Lancelot R, Crochet D, Moulia C, Baldet T and Balenghien T (2013) Host preferences of palaearctic Culicoides biting midges: implications for transmission of orbiviruses. Med Vet Entomol 27: 255-266.

Viennet E, Garros C, Lancelot R, Allene X, Gardes L, Rakotoarivony I, Crochet D, Delecolle J-C, Moulia C, Baldet T and Balenghien T (2011) Assessment of vector/host contact: comparison of animal-baited traps and UV-light/suction trap for collecting Culicoides biting midges (Diptera: Ceratopogonidae), vectors of Orbiviruses. Parasit Vectors 4: 119.

Vorsprach B, Meiser CK, Werner D, Balczun C and Schaub GA (2009) Monitoring of Ceratopogonidae in Southwest Germany. Parasitol Res 105: 337-344. 
Votýpka J, Synek P and SvobodovÁ M (2009) Endophagy of biting midges attacking cavity-nesting birds. Med Vet Entomol 23: 277-280.

Walton TE (2003) The history of bluetongue and a current global overview. Vet Ital 40: 31-38.

Walton TE, Barber TL, Jones RH and Luedke AJ (1984) Epizootiology of bluetongue virus: transmission cycle, vectors, and serotypic distribution in the Americas. Prev Vet Med 2(1-4): 379-388.

Walton TE and Osburn BI (1992) Bluetongue, African horse sickness, and related orbiviruses: proceedings of the second international symposium. CRC Press, Boca Raton, FL, USA.

Weiher W, Bauer B, Mehlitz D, Nijh of AM and Clausen P-H (2014) Field trials assessing deltamethrin (Butox ${ }^{\circledR}$ ) treatments of sheep against Culicoides species. Parasitol Res 113: 2641-2645.

White DM, Wilson WC, Blair CD and Beaty BJ (2005) Studies on overwintering of bluetongue viruses in insects. J Gen Virol 86: 453-462.

Williams T (2008) Natural invertebrate hosts of iridoviruses (Iridoviridae). Neotrop Entomol 37: 615-632.

Wilson A and Mellor P (2008) Bluetongue in Europe: vectors, epidemiology and climate change. Parasitol Res 103: S69-S77.

Wilson AJ and Mellor PS (2009) Bluetongue in Europe: past, present and future. Philos Trans R Soc Lond B Biol Sci 364: 2669-2681.

Wirth WW and Blanton FS (1956) A new species of salt-marsh sand fly from Florida, the Bahamas, Panama and Ecuador: its distribution and taxonomic differentiation from Culicoides furens (Poey) (Diptera, Heleidae). Florida Entomol 39: 157-162.

Wirth WW and Hubert AA (1962) The species of Culicoides related to piliferus root and Hoffman in Eastern North America (Diptera, Ceratopogonidae). Ann Entomol Soc Am 55: 182-195.

Wittman EJ. and Baylis M (2000) Climate change: effects on Culicoides-transmited viruses and implications for the UK. Vet J 160: 107-117.

Wittman EJ, Mellor PS and Baylis M (2002) Effect of temperature on the transmision of arboviruses by the biting midge, Culicoides sonorensis. Med Vet Entomol 16: 147-156.

World Organisation for Animal Health (OIE) (2016) Bluetongue virus. OIE, Paris, France.

Wright PJ and Easton CS (1996) Natural incidence of Lagenidium giganteum Couch (Oomycetes: Lagenidiales) infecting the biting midge Culicoides molestus (Skuse) (Diptera: Ceratopogonidae). Aust J Entomol 35: 131-134.

Zimmer J-Y, Brostaux Y, Haubruge E and Francis F (2014) Larval development sites of the main Culicoides species (Diptera: Ceratopogonidae) in northern Europe and distribution of coprophilic species larvae in Belgian pastures. Vet Parasitol 205: 676-686.

Zimmer J-Y, Claude Saegerman C, Losson B and Haubruge E (2010) Breeding sites of bluetongue virus vectors, Belgium. Emerg Infect Dis 16: 575-576.

Zimmer J-Y, Haubruge E, Francis F, Bortels J, Simonon G, Losson B, Mignon B, Paternostre J, De Deken R, De Deken G, Deblauwe I, Fassotte C, Cors R and Defrance T (2008) Breeding sites of bluetongue vectors in northern Europe. Vet Rec 162: 13.

Zimmer J-Y, Losson B and Haubruge E (2008) Biologie et Ecologie des Culicoides (Diptera), vecteurs de la FCO. Entomol Faun 61: 53-57.

Zimmer JY, Saegerman C, Losson B, Beckers Y, Haubruge E and Francis F (2013) Chemical composition of silage residues sustaining the larval development of the Culicoides obsoletus/Culicoides scoticus species (Diptera: Ceratopogonidae). Vet Parasitol 191: 197-201.

Zimmerman RH and Turner EC (1984) Dispersal and gonotrophic age of Culicoides variipennis (Diptera: Ceratopogonidae) at an isolated site in southwestern Virginia, USA. J Med Entomol 21: 527-535. 
State of the art interventions 


\section{Public-private partnership enabled use of anti-tick vaccine for integrated cattle fever tick eradication in the USA}

Adalberto A. Pérez de León ${ }^{1 *}$, Suman Mahan ${ }^{2}$, Matthew Messenger ${ }^{3}$, Dee Ellis ${ }^{4}$, Kevin Varner ${ }^{5}$, Andy Schwartz ${ }^{6}$, Dan Baca ${ }^{5}$, Renato Andreotti ${ }^{7}$, Manuel Rodríguez Valle ${ }^{8}$, Rodrigo Rosario Cruz ${ }^{9}$, Delia Inés Domínguez García ${ }^{9}$, Myrna Comas Pagan ${ }^{10}$, Carmen Oliver Canabal ${ }^{10}$, Jose Urdaz ${ }^{11}$, Francisco Collazo Mattei $^{12}$, Fred Soltero ${ }^{13}$, Felix Guerrero ${ }^{1}$ and Robert J. Miller ${ }^{14}$

${ }^{1}$ United States Department of Agriculture-Agricultural Research Service, Knipling-Bushland United States Livestock Insects Research Laboratory, and Veterinary Pest Genomics Center, 2700 Fredericksburg Rd., Kerrville, TX 78028, USA; ${ }^{2}$ Zoetis, Veterinary Medicine Research and Development, 333 Portage Street, KZO-300-329.4SW, Kalamazoo, MI 49007, USA; ${ }^{3}$ United States Department of Agriculture-Animal and Plant Health Inspection Service, Plant Protection and Quarantine, 4700 River Road, Unit 140, Riverdale, MD 20737, USA; ${ }^{4}$ Institute for Infectious Animal Diseases, Texas A\&M University System, 1500 Research Parkway, Suite B270, College Station, TX 77843-2129, USA; ${ }^{5}$ United States Department of AgricultureAnimal and Plant Health Inspection Service, Veterinary Services, San Jacinto Blvd., Austin, TX 78701, USA; ${ }^{6}$ Texas Animal Health Commission, 2105 Kramer Lane, Austin TX 78758-4013, USA; ${ }^{7}$ Embrapa Gado de Corte, Av. Rádio Maia, No. 830, Zona Rural, CEP 79106-550 Campo Grande, MS, Brazil; ${ }^{8}$ The University of Queensland, Queensland Alliance for Agriculture and Food Innovation, 306 Carmody Rd., Bldg. 80, Level 3; St. Lucia, Qld 4072, Australia; ' Laboratorio de Investigación en Biotecnología, Salud y Ambiente de la Unidad Académica de Ciencias Naturales de la Universidad Autonoma de Guerrero. Campus el Shalako, Petaquillas, Guerrero, C.P. 39105, Mexico; ${ }^{10}$ Department of Agriculture of Puerto Rico, P.O. Box 10163, San Juan 00908-1163, Puerto Rico; ${ }^{11}$ United States Department of Agriculture-Animal and Plant Health Inspection Service, Veterinary Services, 2150 Centre Ave. Bldg. B, MS-3E13, Ft. Collins, CO 80526, USA; ${ }^{12}$ United States Department of Agriculture-Animal and Plant Health Inspection Service, Veterinary Services, 8100 NW 15 th Place Gainesville, FL 32606, USA; ${ }^{13}$ United States Department of AgricultureAnimal and Plant Health Inspection Service, Veterinary Services, 654 Munoz Rivera Ave. Plaza Bldg. Suite 700 San Juan 00918, Puerto Rico; ${ }^{14}$ United States Department of Agriculture-Agricultural Research Service, Cattle Fever Tick Research Laboratory, 22675 North Moorefield Rd. MAB 6419, Edinburg, TX 78541, USA; beto.perezdeleon@ars.usda.gov

\section{Abstract}

Rhipicephalus (Boophilus) microplus and Rhipicephalus annulatus are invasive tick species and vectors of microbes causing bovine babesiosis and anaplasmosis that were declared eradicated from the USA in 1943 through efforts of the Cattle Fever Tick Eradication Program. These tick disease vectors remain established and affect livestock health and production in other countries located in tropical and subtropical parts of the world. R. microplus is considered the most economically important external parasite of livestock where it is established. Synthetic acaricides are used intensely to kill $R$. microplus and $R$. annulatus, but this leads eventually to the problem of acaricide resistance and other associated undesired effects. Novel and safer technologies that can be integrated with existing control methods are required to manage $R$. microplus and $R$. annulatus populations and associated diseases sustainably. In the case of the USA, the need for a systems approach was identified to keep the national cattle herd free of bovine babesiosis through the integrated use of technologies, including anti-tick vaccines, to eliminate outbreaks of $R$. microplus and $R$. annulatus. Anti-tick vaccines containing the recombinant antigen Bm86 are veterinary biologics used together with veterinary pharmaceuticals such as acaricides to enhance livestock protection where populations of $R$. microplus and $R$. annulatus are established. But, access to $\mathrm{Gavac}^{\mathrm{TM}}$, the only anti-tick vaccine commercially available and used to control $R$. microplus and $R$. 
annulatus, is limited to certain national veterinary products markets, excluding the USA. Efforts of a public-private partnership that developed, and obtained an experimental use permit issued to the animal health company Zoetis for a novel Bm86-based vaccine formulation to be integrated as part of operations by the Cattle Fever Tick Eradication Program are described here. Statutes more than 100 years old governing operations of the Cattle Fever Tick Eradication Program were adapted to eliminate $R$. microplus and $R$. annulatus infestations in cattle and mitigate the risk of future tick outbreaks in the Permanent Quarantine Zone in south Texas on the border with Mexico by adding immunization with the Bm86-based vaccine as part of the operational protocol. This achievement enabled the experimental use of the Zoetis $\mathrm{Bm}-86$ based vaccine to immunize beef and dairy cattle as part of the research project for integrated control of the southern cattle fever tick in Puerto Rico. Our collective work documenting anti-cattle tick vaccine discovery research is described to illustrate how international cooperation supported research on integrated management for the Cattle Fever Tick Eradication Program. Public-private partnerships may be a way to develop novel anti-tick vaccines in other parts of the world for use as part of integrated $R$. microplus management strategies.

Keywords: cattle ticks, cattle, ectoparasite, disease vector, immunization

\section{Introduction}

Ticks and tick-borne diseases affect animal health and the productivity of livestock (Brites-Neto et al. 2015; De la Fuente et al. 2008). The ticks Rhipicephalus (Boophilus) microplus (Canestrini) and Rhipicephalus annulatus (Say) affect livestock health and production (Brake and Pérez de León 2012; Reck et al. 2009), and are vectors of microbes that cause bovine babesiosis and anaplasmosis in countries where they are established, which are located in tropical and subtropical parts of the world (Aubry and Geale 2011; Pérez de León et al. 2014a). R. microplus is more invasive than $R$. annulatus, and it is considered the most economically important external parasite of livestock wherever it is established (Jongejan and Uilenberg 2004; Sonenshine and Roe 2014). It has been estimated that in Brazil, which is the country with the largest commercial cattle herd including around 219 million head in 2016 (USDA 2016b), R. microplus causes annual losses totaling around USD 3 billion (Grisi et al. 2014). Producers in Mexico, which has the $8^{\text {th }}$ largest national bovine herd in the world with 16 million head (USDA 2016b), suffer annual losses estimated at USD 573 million due to the effects of $R$. microplus parasitism on livestock (Rodriguez-Vivas et al. 2017). Bovine babesiosis, caused principally by Babesia bovis (Babes) or Babesia bigemina (Smith and Kilborne), is the most financially important arthropod-borne disease of cattle worldwide (Bock et al. 2008).

Chemicals synthetized by humans that kill ticks, commonly known as acaricides, are used predominantly for tick control, and in particular to manage R. microplus populations (Graf et al. 2004; Kiss et al. 2012), and to mitigate the health and economic burden of bovine babesiosis (Drummond 1983). However, intense use selects for acaricide resistance (Dominguez García et al. 2010; Guerrero et al. 2014a; Higa Lde et al. 2016). Of concern is the increased frequency of R. microplus populations reported to be resistant to multiple classes of acaricides (Abbas et al. 2014; Klafke et al. 2017; Miller et al. 2013; Rosario-Cruz et al. 2009). In the case of endectocides, that is molecules with endo- and ectoparasiticidal activities, the frequency to treat primarily gastrointestinal infections in cattle and other livestock may be selecting for resistance in $R$. microplus against molecules like ivermectin (Alegría-López et al. 2015; Rodriguez-Vivas et al. $2014 a, b)$. This situation highlights the urgency to innovate technologies that can be used together to overcome the problem with resistance to synthetic acaricides, and the undesired effects of acaricide overuse (Hlatshwayo and Mbati 2005; Lopez-Arias et al. 2015). 
Tick control and the management of tick-borne diseases is a complex issue (De Meneghi et al. 2016). Integrated tick management approaches have been proposed, and some have been applied recently to R. microplus in several countries (Barnard et al. 1988; Jonsson 2004; Mondal et al. 2013; Pérez de León 2014b; Rodríguez-Vivas et al. 2016; Suarez et al. 2016). The history of integrated $R$. microplus management includes efforts that ranged from combining acaricide use with alternative methods to control tick numbers relative to an injury level threshold, to approaches that resulted in tick eradication (Angus 1996; Bram and Gray 1983; FAO 1989). In addition to a sound understanding of the ecological processes that regulate tick populations (Schmidtmann 1994), control or eradication efforts must consider economic and social aspects to be successful (De la Fuente 2015; Pegram et al. 2000; Walker 2011). Models have also been used to anticipate the effects of different management interventions, or to document the benefits of integrated approaches (Elder and Morris 1986; Hernandez et al. 2000; Norton et al. 1983; Popham and Garris 1991; Wang et al. 2016).

\section{Anti-tick vaccine for integrated cattle fever tick eradication in USA}

The challenge to manage the populations of tick disease vectors affecting cattle like $R$. microplus and $R$. annulatus can be viewed as a continuum of options that can range from doing nothing about it to establishing national eradication programs as it was attempted in several countries before (Pegram et al. 2000). Table 1 lists some characteristics of parameters related to control or eradication as intervention strategies to mitigate the impact of tick infestations in cattle. Aspects related to global change complicate efforts to keep national areas or countries encompassing suitable habitat free of $R$. microplus invasion or re-invasion in the long term (Benavides Ortiz et al. 2016; George 2008; Giles et al. 2014; White et al. 2003). However, even the strategy to maintain the USA free of the invasive cattle fever ticks requires adaptation to be able to deal with an evolving world (Esteve-Gassent et al. 2014; Pérez de León et al. 2012).

The elimination of bovine babesiosis was the main driver to establish the Cattle Fever Tick Eradication Program in the USA, which started operations in 1907 (Graham and Hourrigan 1977). As a result, the USA was declared free of $R$. microplus and $R$. annulatus in 1943 with the exception of a Permanent Quarantine Zone, also known as the Systematic Area, in south Texas along the border with Mexico (Bram et al. 2002). This Permanent Quarantine Zone starts in Del Rio, extends

Table 1. Suggested parameters to consider for spectrum of strategies, using control and eradication as examples, to manage populations of the southern cattle fever tick, Rhipicephalus microplus, which can mitigate the burden of bovine babesiosis and anaplasmosis on cattle herds.

\begin{tabular}{|c|c|c|}
\hline Parameter & Control & Eradication $^{1}$ \\
\hline Tolerance for infestation & economic threshold & zero \\
\hline Surveillance & optional, or reactive & constant \\
\hline Quarantine & no & obligatory; sanctioned by laws \\
\hline Treatment & optional & obligatory \\
\hline Cost & variable & $\begin{array}{l}\text { although initial inputs can be considerable, the return } \\
\text { on investment long-term is high }\end{array}$ \\
\hline Duration & seasonal & requires continuous commitment \\
\hline
\end{tabular}

\footnotetext{
${ }^{1}$ Known operations of the U.S. Cattle Fever Tick Eradication Program exemplify eradication parameters.
} 
southeast for $700 \mathrm{~km}$ to Brownsville varying in width between 0.4 to $16 \mathrm{~km}$, and serves as a buffer zone under constant surveillance by state and federal inspectors to prevent dispersal into the Free Area of $R$. microplus and R. annulatus from parts of Mexico where they are endemic (Miller et al. 2007). Tick-infested cattle in the Permanent Quarantine Zone undergo systematic acaricide treatment and the premises are quarantined for 6-9 months until the outbreak is eliminated (Davey et al. 2012; Miller et al. 2005). Outbreaks of R. microplus and $R$. annulatus have been detected in the USA outside the Permanent Quarantine Zone since 1968 (Graham and Hourrigan 1977). This situation is managed through Temporary Preventative Quarantine Areas created to eliminate tick outbreaks detected in the Free Area (see maps in Lohmeyer et al. 2011 and Pérez de León 2012 for details).

Alternative approaches were taken recently in other parts of the world to deal with cattle ticks and associated diseases. In New Caledonia, the approach to eradicate bovine babesiosis was chemosterilization of the infections through the use of the babesiocidal drug imidocarb without having to totally eliminate populations of what was then considered to be R. microplus, and now reclassified to be Rhipicephalus australis (Fuller) (Barré et al. 2011; Estrada Peña et al. 2012). A regionalization approach was taken in Australia to deal with populations of what is now considered to be $R$. australis whereby biosecurity zones are established in Queensland to manage the risk associated with cattle tick infestation (Barker et al. 2014; State of Queensland 2016a). Queensland State Regulations require that the person, who is the owner or occupier of infested land, takes action to eradicate $R$. australis and to comply with the stated way or procedure for eradication (State of Queensland 2016b).

Technologies considered as alternatives for use with acaricides include essential oils, biocontrol agents, anti-tick vaccines, ecological measures (pasture vacation whereby cattle are removed from grazing land to starve host-seeking tick larvae, brush cleaning in the pasture, induction of engorged female detachment in unfavourable environment to reduce pasture infestation), and the use of cattle breeds and crosses resistant to tick infestation (Biegelmeyer et al. 2015; Costa-Junior et al. 2016; Fernandes et al. 2012; Goolsby et al. 2016; Mapholi et al. 2016; Martinez-Velazquez et al. 2011; Redondo et al. 1999; Rodriguez Valle et al. 2004). Regulatory and commercial intricacies influence the availability of those technologies across national and regional veterinary medicines markets. Essential oil-based acaricidal products remain to be widely available commercially for practical use (Andreotti et al. 2013). Biocontrol products based on acaropathogenic fungi are emerging in certain markets (Bharadwaj and Stafford 2010; Camargo et al. 2016). Access to the only commercially available anti- $R$. microplus vaccine worldwide is limited to certain national animal health markets (De la Fuente et al. 2007). Investment decisions based on economic principles determine research, development, and marketing of an anti-tick vaccine by an animal health company in a particular national market (Guerrero et al. 2012). The benefit of integrating the use of some of these technologies has been documented (Bautista-Garfias and Martínez-lbañez 2012; Rodriguez Valle et al. 2004; Suarez et al. 2016; Webster et al. 2015).

Our collaboration on anti-tick vaccine discovery research involving international partners was established after a public workshop convened in April 2009 by the United States Department of Agriculture - Agricultural Research Service and the Center for Ecoepidemiology, Yale University School of Medicine in McAllen, Texas where the One Health concept, that is the collaborative effort of multiple disciplines to attain optimal health for people, animals and our environment, was applied to identify research gaps regarding bovine and human babesioses in the USA (Androetti et al. 2012; Guerrero et al. 2014b; Pérez de León et al. 2010). Integrated approaches for sustainable cattle fever tick eradication in the USA, and the overarching theme of anti-tick vaccine use in the 
context of bovine and human babesioses were among the topics of focused discussions during the workshop. We developed the concept of sustainable tick eradication to reflect the need for a systems approach in agricultural research whereby the principle of precision agriculture was adapted to integrate technologies, including anti-tick vaccines, which translate into resilient management practices to keep the USA free of $R$. microplus and $R$. annulatus in the context of shifting ecological, economical, and societal paradigms (Miller et al. 2012; Pérez de León et al. 2012; Pound et al. 2010). Dialogue among diverse stakeholder groups at the workshop generated discussion on the possibility to address those challenges through public-private partnerships to promote innovation in agricultural research on ticks and tick-borne diseases.

Public-private partnerships involve an agreement between a public agency (federal, state or local) and a private sector entity to join forces to deliver science-based solutions to agricultural problems addressing unmet societal technological needs (Chatelain and Don 2009; USDA 2016a). Adaptation of the public-private partnership business model for product development was identified as a strategy that could achieve technological solutions for the Cattle Fever Eradication Program. Regarding anti-tick vaccines, one of the research gaps identified was the need to conduct studies to determine whether the treatment of cattle with Gavac ${ }^{\mathrm{TM}}$ (Heber Biotec, Habana, Cuba), another Bm86-based vaccine formulation, or a vaccine formulation containing novel antigen(s) could be used safely in the Permanent Quarantine Zone together with other technologies to manage outbreaks by invasive populations of $R$. microplus and $R$. annulatus according to requirements established by state and federal regulatory agencies. Bm86-based products represent the first generation of anti-tick vaccines to be commercialized (Rodriguez et al. 1995b; Willadsen et al. 1995). The opportunity to research existing and emerging anti-tick vaccine technology according to the One Health perspective to deal with ticks and tick-borne diseases of public health and veterinary importance was a factor that facilitated the public-private partnership delivering a new Bm86-based vaccine formulation for experimental use in cattle in the USA and Puerto Rico (Évora et al. 2017; Rodríguez-Mallon et al. 2013). This is another example of how the One Health approach has expanded to try to overcome barriers for the development and commercialization of novel global health vaccines, and public-private partnerships have been identified as a mechanism to achieve that goal (Benfield 2016; King et al. 2004; Monath 2013).

\section{Past experiences developing Bm86-based vaccines}

Pioneer work on the possibility to control tick infestations in livestock through immunization led a group of Australian scientists to discover that the recombinant version of a midgut glycoprotein called Bm86, from the tick species now recognized as $R$. australis, elicited an immune response that reduced tick infestations on cattle, which opened the way for the development of a vaccine that was commercially released as TickGard ${ }^{\circledR}$ (Intervet, Bendigo East, Victoria, Australia) in 1994 (Allen and Humphreys 1979; Trager 1939; Willadsen et al. 1995). Similar efforts undertaken in Cuba after the patent with the Bm86 gene sequence was published around 1988 resulted in the commercialization in 1993 of another Bm86-based vaccine marketed as Gavac ${ }^{\mathrm{TM}}$ (Rodriguez Valle et al. 1994, 1995b; Willadsen 2008a), which was registered in Colombia, Dominican Republic, Brazil, and Mexico (Canales et al. 1997; Rodriguez Valle et al. 1995a). TickGard ${ }^{\circledR}$ disappeared from the market about a decade after its commercial release for reasons described by Playford (2005) and De la Fuente et al. (2007). It must be noted that in the case of Bm86-based vaccines, the knock down effect against $R$. microplus is observed over time after continued vaccine usage in the entire herd, which requires enforcement of product use compliance through education of producers and animal health personnel. This is discussed below in the context of our research efforts involving the integrated use of an anti-tick vaccine for $R$. microplus and $R$. annulatus eradication in the USA. 
Vaccine development is an arduous process that requires resources (Chambers et al. 2016; Giese 2013). Vaccines for use in domestic animals and wildlife are developed and approved by regulatory agencies for sale by animal health companies (Heldens et al. 2008; Marshall 2014). National agencies ensure that veterinary vaccines meet purity, safety, and efficacy regulations required for market authorization (Banks 2014; Henderson and Lewis 2014). Because economic context drives corporate investment in their commercialization (BBSRC 2015; Benfield 2016), the protection of intellectual property for vaccine-based innovations through patents ensuring freedom to operate is an important consideration to make in the decision making process to develop vaccines (Durell 2016; Farmer and Grund 2014; Krol et al. 2016).

Several publications have outlined the stages in the development of Bm86-based vaccines commercialized before (Canales et al. 1997; De la Fuente et al. 1999; De la Fuente et al. 1998; Jittapalapong et al. 2006; Rodriguez-Mallon 2016; Willadsen 2008b; Willadsen et al. 1992, 1995). In the case of TickGard ${ }^{\circledR}$, it took 14 years and testing in thousands of cattle to complete demonstration of feasibility, documentation of efficacy in field trials, completion of product registration, and post-marketing product surveillance to complete the commercialization process (Willadsen 2008b; Willadsen et al. 1995). In addition to access to the R. microplus genome (Barrero et al. 2017; Bellgard et al. 2012), the advent of next-generation sequencing platforms enabled strategies that analyse genomic data through bioinformatics such as immunogenomics, reverse vaccinology, and vaccinomics to accelerate the identification of potentially immunoprotective $R$. microplus polypeptides, which helped prioritize the production of recombinant tick proteins for in vivo testing (De la Fuente and Merino 2013; Guerrero et al. 2012; Lew-Tabor and Rodriguez Valle 2016; Santos et al. 2004).

Beyond the identification of a highly efficacious antigen through discovery research and the selection of an adjuvant that improves the immunological response (Guerrero et al. 2014b; Petermann et al. 2017; Rodriguez-Mallon et al. 2015; Shah et al. 2017), economic considerations related to cost-effective production bioprocesses, estimation of the potential market, and pricing of the vaccine product relative to acaricides significantly influence the decision to develop a novel anti-R. microplus vaccine formulation for commercial use (Canales et al. 2010). An incentive for animal health companies to partner with academic institutions and public agencies is having access to technological platforms that can be developed into a product, including vaccines, rapidly (Rippke 2105; Yarbrough 2016). Despite the testing in cattle of several recombinant proteins regarded as candidate antigens since the commercialization of TickGard ${ }^{\circledR}$ and Gavac $^{\mathrm{TM}}$ (Almazan et al. 2010; De la Fuente et al. 2016; Fang and Pung 2011; Lew-Tabor and Rodriguez Valle 2016; Olds et al. 2013), it appears that a gap between basic research and translation to novel therapeutics, also known as the 'valley of death', may have occurred in the anti-tick vaccine scientific field (Collins et al. 2016; Guerrero et al. 2012; Plotkin et al. 2015). For example, the antigen pP0 was reported to afford $96 \%$ efficacy against $R$. microplus infesting cattle (Rodriguez-Mallon et al. 2015), but we were unaware of a commercial vaccine based on that technology as of this writing. However, the future looks promising because consortia have been established to overcome the challenge to obtain regulatory and marketing approval for novel vaccines to protect livestock against cattle ticks (Schetters et al. 2016), and diverse host species from tick vectors of zoonotic diseases (Sprong et al. 2014). 


\section{USA experience developing Bm86-based vaccine through public-private partnership}

Regulatory requirements for veterinary biologics in the USA made impractical the importation of Gavac $^{\text {TM }}$ manufactured in Cuba for use by the Cattle Fever Tick Eradication Program. We sought international collaboration with more experienced research groups as a way to accelerate our anti-tick vaccine discovery research efforts initiated in 2009. Research outcomes are described here to illustrate how international cooperation resulted in the experimental use of a new Bm86based vaccine formulation in beef cattle in the USA, and in beef and dairy cattle in Puerto Rico. We experienced some of the vicissitudes associated with the development and adoption of anti-tick vaccine technology based on the Bm86 recombinant antigen in the 1990s to be able to understand aspects of the critical path that animal health companies take into consideration to decide if financial investment (Woodcock 2014), and the commitment of other corporate resources is warranted to seek regulatory approval of a novel anti- $R$. microplus vaccine. Regulatory aspects influencing attempts to develop a public-private partnership that could enable the translation of our anti-tick vaccine discovery research findings are described below. The research and development program between public agencies and Zoetis that delivered a novel experimental Bm86-based vaccine in the USA provides another case study of how public-private partnerships can create value for society and corporations, among other ways, by enhancing the sustainability of animal agriculture (Black 2012; Hendriks et al. 2013).

Resource constraints in vaccine development require the identification of rational approaches that are likely to succeed in prioritizing molecules, like those discovered in ticks, for testing as antigens (Giersing et al. 2016; Guerrero et al. 2012). Following examples of landscape analyses applied to vaccines (Geels et al. 2015; Stephens 2014), notable publications in the scientific literature are cited here in the context of key considerations that influenced decisions by the public-private partnership established to obtain approval by the USA agency regulating veterinary biologics for experimental use of an anti-tick vaccine in cattle. It is elemental to reach consensus on a mutually acceptable Research and Development to License Approval (R\&D2LA) plan as soon as the proper agreement(s) covering the interaction between an academic or public anti-tick vaccine discovery research group and a team from an animal health company are in place. The R\&D2LA plan should outline activities and milestones for each step in the Research and Development phases to be able to inform go or no-go decisions on the project. It has been argued that 'failing early' in vaccine studies allows more effective use of limited resources (Benfield 2016). Sources on strategy and requirements to develop a safe and effective vaccine to protect animals against tick infestation, which can help structure a R\&D2LA include the publications by Willadsen et al. (1995), Willadsen (2008a), Rodríguez-Mallon (2016), and the guidelines by the International Cooperation on Harmonization of Technical Requirements for Registration of Biological Veterinary Medicinal Products (VICH 2016).

It is productive to select as early as possible an expression system for the recombinant version of the candidate antigen that maximizes immunogenicity and is scalable for industrial production. Research on expression systems keeps advancing the science of heterologous protein production for veterinary immunotherapeutics and prophylactics (Drugmand et al. 2012; Legastelois et al. 2016; MacDonald 2015). For example, redesign of the bioprocess to produce the recombinant Bm86 antigen in the yeast Pichia pastoris (Phaff) resulted in cost savings (Canales 2010). Other biotechnological advances are optimizing further the use of the $P$. pastoris expression system (Liu et al. 2016; Shen et al. 2016). It is important for health companies to determine sooner than later, among other things, if the recombinant antigen production cost will be profitable and 
whether the sale price for the vaccine product is competitive (Lee and McGlone 2010; Schwartz and Mahmoud 2016).

Adjuvant selection is an important aspect of the vaccine formulation process because adjuvants are substances that can enhance, accelerate, prolong, or modulate the immune response to an antigen (Olafsdottir et al. 2016; Petermann et al. 2017; Shah et al. 2017). A new formulation of the TickGARD Plus product (Intervet) that included a different adjuvant was introduced in 2002 in the Australian animal health market and was reported to decrease $R$. australis, counts by $70 \%$ when used according to label instructions (Hunter 2002; Willadsen 2008a). Further development work, including adjuvant formulation, resulted in the launch of GavacPlus containing the oilbased adjuvant Montanide 888 (Rodriguez Valle et al. 2001; Vargas et al. 2010). Other oil-based adjuvants have been developed that can stimulate humoral- and cell-mediated immune responses in ruminants, which can be used to formulate parasite antigens (Kalyanasundaram et al. 2015; Khorasani 2016). A way anti-tick vaccine researchers in academic institutions and public agencies can benefit from partnering with industry is through confidential access to corporate knowledge of adjuvants approved as safe by the regulatory agencies that can maximize the efficacy of a development candidate antigen (Boué et al. 1999; Guerrero et al. 2012; Shah et al. 2017).

Commercial and non-commercial forces influence the perception of the attributes that would differentiate a new vaccine developed for sale in markets encompassing tropical and subtropical regions of the world to protect cattle under field conditions against the direct and indirect health and productivity effects caused by $R$. microplus (Guerrero et al. 2012; Playford 2005; Willadsen 2008a). Reaching consensus on an acceptable level of efficacy to make a go/no-go decision in the development process of an anti- $R$. microplus vaccine may require significant discussion among the parties involved in a public-private partnership. The current paradigm to assess the efficacy of a vaccine against $R$. microplus in pen trials, controlled field trials, and subsequently under field conditions is based on the experience to commercialize TickGard and Gavac ${ }^{\mathrm{TM}}$ (De la Fuente et al. 1999, 2007; Willadsen 2008b; Wong and Opdebeeck et al. 1989). Efficacy assessment depends on the capacity to evaluate the host immune response, and the ability to quantitate host protection afforded by a vaccine formulation against the development of ticks during the parasitic phase of their life cycle.

In vitro tick feeding techniques provide a way to begin assessing protective effects by testing blood or serum from cattle immunized with a candidate vaccine formulation (Antunes et al. 2014; De la Vega 2004; Inokuma and Kemp 1998; Lew-Tabor et al. 2014). Host systems other than cattle can be used to begin screening candidate antigens in vivo (Galay et al. 2016). The influence of the genetic background on bovine to bovine variation in response to vaccination with a particular tick antigen should be considered as soon as possible (Acosta-Rodriguez et al. 2005; Jonsson et al. 2014; Piper et al. 2016; Sitte et al. 2002). Tick vaccine efficacy can also have implications on the transmission of tick-borne diseases, including bovine babesiosis in the case of $R$. microplus and $R$. annulatus (De la Fuente et al. 1998, 2007; Merino et al. 2013). Procedures to calculate the efficacy of candidate antigens to develop vaccines against one- and three-host ticks were reviewed recently (Aguirre Ade et al. 2015; Cunha 2013; Rodríguez-Mallon 2016).

Having a plan helps the working team make informed science-based decisions, which will help maximize the chances of fulfilling the R\&D2LA. One way this can be achieved is by elaborating a Target Product Profile (Lee and Burke 2010; Pramod 2016). A 'wish-list' of optimistic, realistic, and minimal goals for the anti-tick vaccine development project we embarked on was presented to 
stakeholders in 2012 to communicate the framework of our strategy and to manage expectations regarding the potential end results of our efforts (Figure 1).

Successful commercialization of a an anti-tick vaccine will depend upon the demand for the product, the size of the market that needs the product to make it a profitable venture, and continued regulatory compliance. Ultimately, the goal would be to produce the vaccine cheaply so that the end user is able to afford it. With the understanding that biologics like anti-tick vaccines and pharmaceuticals such as acaricides are dissimilar classes of veterinary products, a nice-to-have attribute that would contribute to the commercial success of a new vaccine targeting $R$. microplus would be a strong knock-down effect. One way to achieve that could be through a polyvalent vaccine including 'concealed' and 'exposed' tick antigens that alter larval development or the blood feeding process, which could be perceived as a faster effect controlling ticks infesting cattle (Nuttall et al. 2006; Parizi et al. 2012; Riding et al. 1994). The combination with standard acaricide applications during initial use of a Bm86-based vaccine helps potentiate its long-term effect because over time acaricide treatments decrease due to the reduced tick population caused by the protective effect of the vaccine at the cattle herd level (Canales et al. 1997; Floyd et al. 1995; Lodos et al. 2000; Suarez et al. 2016). Acaricidal products and anti-R. microplus vaccines should have their attributes differentiated in marketing materials highlighting their distinct nature as pharmaceuticals and biologics, respectively, to ensure consumers understand how they must be used to protect cattle against tick infestation and the risk of bovine babesiosis and anaplasmosis transmission.

\section{Anti-tick vaccine for integrated $R$. microplus and $R$. annulatus eradication in the USA}

Because the anti-tick vaccine technology would have to be registered in the USA before a company could distribute, or might sell it for administration to cattle, it was determined early on in the process that outlining a critical path for registration and the need for production domestically

\begin{tabular}{|c|c|c|}
\hline \multicolumn{3}{|c|}{ Strategic Evaluation Framework for Use of Tick Vaccine by CFTEP } \\
\hline Attribute & Have to have & Nice to have \\
\hline Indication & Cattle & Cattle \& deer \\
\hline Efficacy & $\begin{array}{l}\text { R. annulatus: }>95 \% \\
\text { R. microplus: } \geq 50 \%\end{array}$ & $\begin{array}{l}\text { R. annulatus: } 99 \% \\
\text { R. microplus: }>95 \%\end{array}$ \\
\hline Composition & Bm86 + novel adjuvant & Polyvalent + nanoadjuvant \\
\hline Immunization schedule & $\begin{array}{l}0,4,7 \text { weeks, then boost } \\
\text { every } 6 \text { months }\end{array}$ & $\begin{array}{l}0,6 \text { months, then boost } \\
\text { annually }\end{array}$ \\
\hline Delivery system & Injection & Needleless \\
\hline
\end{tabular}

Walking the critical path to cross the valley of death

Figure 1. Framework for Target Product Profile concept applied to meet the need of USA stakeholders to use antitick vaccine in the Cattle Fever Tick Eradication Program that was presented at the $58^{\text {th }}$ Texas A\&M University Beef Cattle Short Course. 
would need to be considered if a Bm86-based, or any other anti-tick vaccine showed potential as a tool for integrated tick eradication (Pérez de León et al. 2010, 2012). Additionally, it was anticipated that assessing this potential would require the conduct of trials in the USA before an anti-tick vaccine were to be licensed for use as an aid in the prevention of $R$. microplus infestation among cattle in the Permanent Quarantine Zone. The fruitful public-private partnership described here enabled stall tests with Gavac $^{\mathrm{TM}}$ against strains of $R$. microplus and $R$. annulatus causing outbreaks in the Permanent Quarantine Zone, which had been colonized at the USDA-ARS Cattle Fever Tick Research Laboratory (Miller et al. 2012). Because we didn't have access to TickGardPlus (Vargas et al. 2010), we adhered to instructions in the product label to vaccinate cattle with $\mathrm{Gavac}^{\mathrm{TM}}$, which required an initial injection followed by two booster immunizations at 5 and 7 weeks thereafter. Subsequently, instructions in the Gavac ${ }^{\mathrm{TM}}$ label recommended revaccination every 6 months to maintain effective antibody titres. The paradigm to assess anti-tick vaccine efficacy against one-host ticks like $R$. microplus and $R$. annulatus is founded on the experience with commercial Bm86-based vaccines evaluated in stall tests where an algorithm is applied to assess the effect of vaccination on the ability of females infesting immunized cattle to complete the parasitic phase of their life cycle on the host, their fecundity, i.e. the amount of eggs laid by engorged females that dropped off the host, and fertility, i.e. the amount of larvae hatching from a subsample of the eggs laid, relative to the same parameters evaluated in a control group of non-vaccinated animals infested similarly (Cunha et al. 2013; Rodríguez-Mallon 2016).

For the R. annulatus study, an initial tick infestation in cattle was established 2 weeks after the second booster injection. To get a sense of duration of immunity, cattle were infested again 5 months after the second booster injection. Gavac ${ }^{\mathrm{TM}}$ was highly efficacious against the $R$. annulatus Texas outbreak strain with a vaccine efficacy of 99.9 and $91.4 \%$, two weeks and 5.5 months after the second booster, respectively (Miller et al. 2012). These findings confirmed previous reports of the high efficacy of Bm-86 based vaccines against $R$. annulatus (Fragoso et al. 1998; Pipano et al. 2003). Lower efficacy was documented with Gavac ${ }^{\mathrm{TM}}$ against the $R$. microplus Texas outbreak strain. In this experiment, cattle were infested with ticks 2 weeks after the second booster injection as it was done for $R$. annulatus. However, 6 months after the second boost vaccination, cattle received a third injection and were infested again 2 weeks thereafter. The efficacy of Gavac $^{\mathrm{TM}}$ against the R. microplus Texas outbreak strain was 27 and $23 \%$ after the second and third booster injections, respectively (R.J. Miller et al. unpublished data). Differences in protein degradation machinery involved in blood meal digestion correlate with the higher efficacy of Bm-86 vaccines against $R$. annulatus as compared to R. microplus (Popara et al. 2013).

Our work with Gavac ${ }^{\mathrm{TM}}$ prompted adaptation of the Estrada-Peña and Venzal (2006) model in a way that allowed us to run simulations based on measurement of tick habitat suitability taking into account tick development under various abiotic conditions, vegetation, weather, and host animal density for the Permanent Quarantine Zone. In this simulation model a prediction of the tick population expected under different levels of control achieved by vaccination was estimated based on an initial input of 100 engorged females into a tick-free environment (Miller et al. 2012). Simulations were completed for 10 years, 100 times each. The model estimated that with control greater than $40 \%$, eradication would be maintained for $R$. annulatus in the northern part of the Permanent Quarantine Zone (Figure 2), where this species is the predominant cause of outbreaks (Lohmeyer et al. 2011). For R. microplus, however, $80 \%$ control would be required using an antitick vaccine alone to maintain eradication whereas $40 \%$ control would reduce the tick burden by over 50\% (Figure 3; R.J. Miller et al. unpublished data). Researchers are developing and testing models for the combined effect of treatments including acaricides plus vaccination in cattle that also contemplate the effect of integrated interventions to mitigate the risk of tick infestation in 


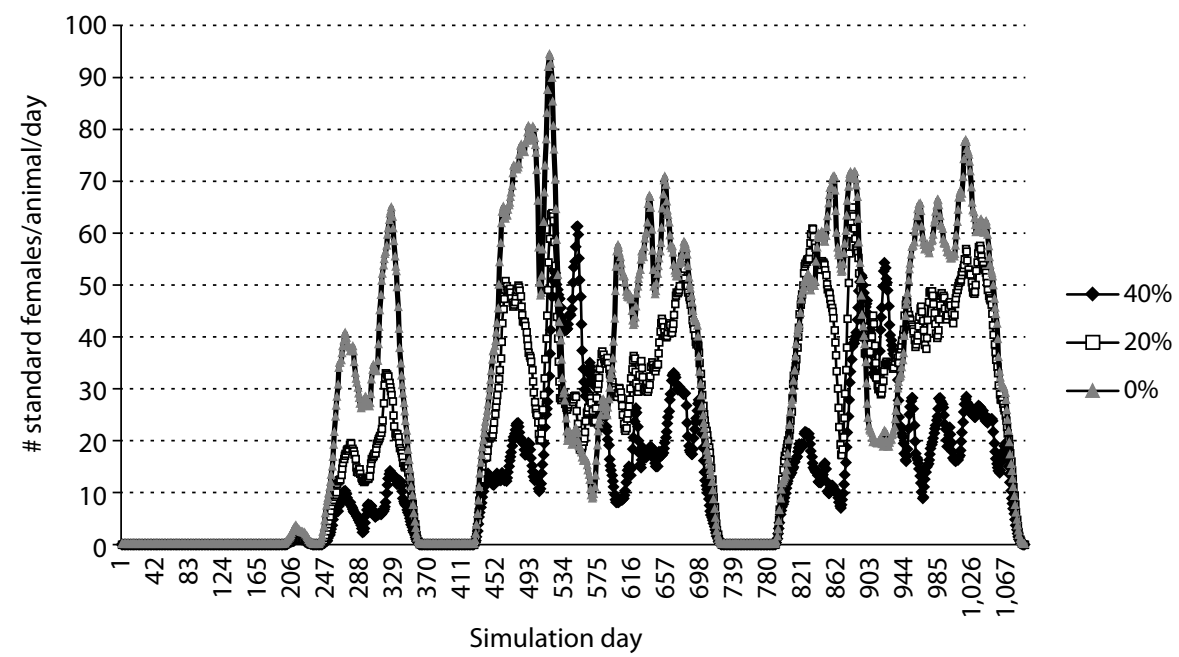

Figure 2. Simulation of the effect of anti-tick vaccine at different levels of efficacy against Rhipicephalus annulatus outbreak populations in the Permanent Quarantine Zone in south Texas (adapted from Miller et al. 2012). Simulation results are shown for 100 engorged $R$. annulatus invading the northwestern half of the Permanent Quarantine Zone where the ticks had been eradicated. The results are an average of 100 simulations over 10 years at various levels of control achieved by vaccination. The first year shows an increasing population with stabilization over years 2 and 3 . A similar seasonal population trend occurs from year 3 until year 10, which are not shown. The model predicted that efficacy greater than $40 \%$ achieved with an anti-tick vaccine prevented the establishment of R. annulatus populations for the entire simulation period mainly due to the climate in the northwestern half of the Permanent Quarantine Zone assuming initial tick-free status (see map in Lohmeyer et al. 2011).

white-tailed deer, which is an abundant wildlife species in the Permanent Quarantine Zone that complicates eradication efforts in the USA (Estrada Peña et al. 2014; Wang et al. 2016).

The experience testing Gavac ${ }^{\mathrm{TM}}$ in the USA was a breakthrough that enabled the partnership with Zoetis to test a novel Bm86-based vaccine in efforts to meet the needs of the Cattle Fever Tick Eradication Program for technologies that could be integrated to existing operational protocols described above. Following stall tests documenting efficacy equivalent to $\operatorname{Gavac}^{\mathrm{TM}}$, we were able to conduct a field safety test that involved around 200 beef cattle in two ranches located in the Permanent Quarantine Zone. No adverse reactions associated with vaccination were observed in cattle. Evaluation of the efficacy and safety data by the USDA Center for Veterinary Biologics resulted in the issuance of an experimental use permit for the Zoetis Bm86-based vaccine. The data generated indicated that a booster immunization could be applied 28 days after the initial vaccination, and subsequent injections administered every 6 months thereafter.

In 2015, the Texas Animal Health Commission had initiated the process to facilitate the use of the novel Bm86-based vaccine in the Cattle Fever Tick Eradication Program. Efficacy of the Zoetis Bm86-based vaccine in stalls tests conducted at the USDA-ARS Cattle Fever Tick Research Laboratory was 98 and $40 \%$ versus $R$. annulatus and R. microplus, respectively (R.J. Miller et al. 


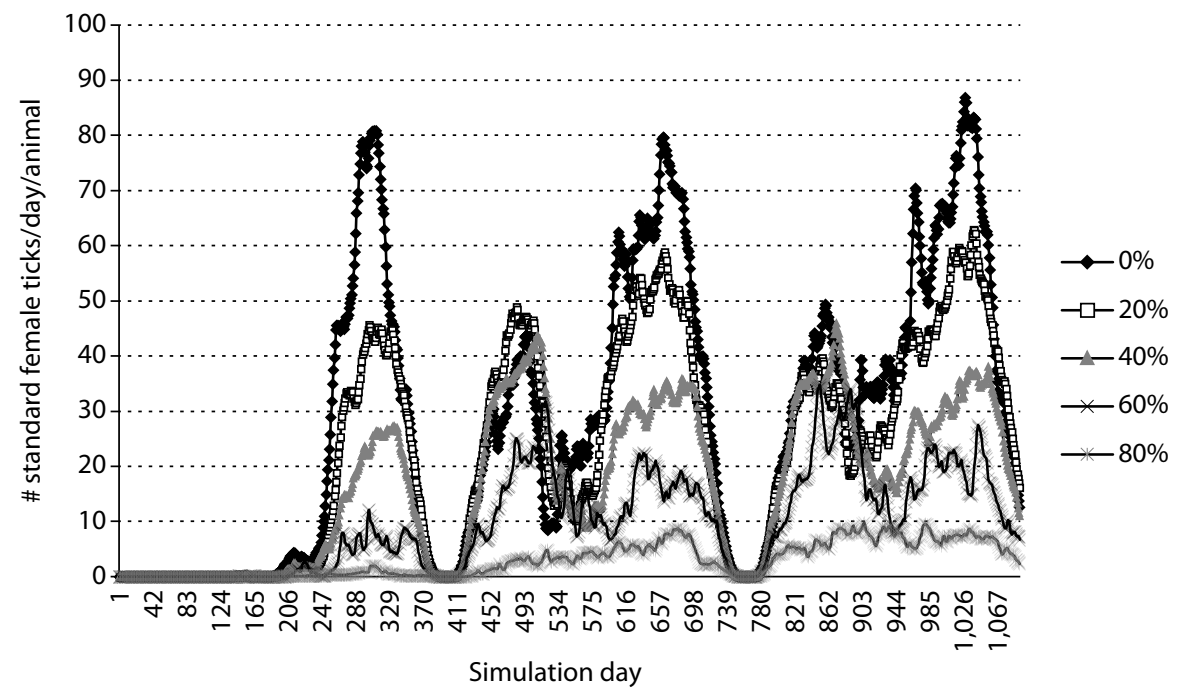

Figure 3. Simulation of the effect of anti-tick vaccine at different levels of efficacy against Rhipicephalus microplus outbreak populations in the Permanent Quarantine Zone in south Texas (adapted from Miller et al. 2012). Simulation results are shown for 100 engorged R. microplus invading the southeastern half of the Permanent Quarantine Zone where the ticks had been eradicated. The results are an average of 100 simulations over 10 years at various levels of control achieved by vaccination. The first year shows an increasing population with stabilization over years 2 and 3. A similar seasonal population trend occurs from year 3 until year 10, which are not shown. The model predicted that efficacy greater than $80 \%$ maintained eradication, whereas $40 \%$ control would reduce the tick burden $50 \%$, for the entire simulation period mainly due to the climate in the southeastern half of the Permanent Quarantine Zone assuming initial tick-free status (see map in Lohmeyer et al. 2011).

unpublished data). During its 395 $5^{\text {th }}$ Meeting on 24 May 2016, the Texas Animal Health Commission adopted rules calling for the administration of the vaccine to beef cattle residing in the Permanent Quarantine Zone (http://tinyurl.com/ybhza59t). This was a significant event in the history of the Cattle Fever Tick Eradication Program because statutes more than 100 years old were adapted to integrate the use of an anti-tick vaccine as part of its operations. The first doses of the Zoetis Bm86-based vaccine arrived in Texas by June 2016. At the same time, plans were formalized to train Program personnel on the use of the anti-tick vaccine as part of an integrated eradication strategy. Public hearings in south Texas were conducted in September 2016 to educate cattle producers on how the anti-tick vaccine technology would be used, and the purpose of adapting official protocols to realize the full potential of this technology to promote raising cattle in the Permanent Quarantine Zone.

\section{Puerto Rico experience meeting the challenge for integrated R. microplus control using anti-tick vaccine in dairy cattle operations}

Livestock in Puerto Rico was re-infested with R. microplus after eradication efforts in the island were terminated before every tick in the entire island was eliminated (Cortés et al. 2005; UrdazRodriguez et al. 2012). Morbidity and mortality due to bovine babesiosis and anaplasmosis in 
the dairy cattle herd of Puerto Rico became such a problem that livestock producers requested technical assistance from state and federal agencies to address the situation (Urdaz-Rodriguez et al. 2009a, 2009b). Members of our partnership were invited to visit Puerto Rico in 2012 to evaluate the problem with R. microplus and associated diseases in the island, and discuss the burden of tick and tick-borne diseases on animal health in the Caribbean. The possibility to conduct research and generate science-based information that could serve as the basis for integrated tick management was identified as an opportunity to control $R$. microplus in a progressive manner.

With the support of the livestock industry of Puerto Rico and USDA-Animal and Plant Health Inspection Service-Veterinary Services, an agreement was established in 2014 between the Puerto Rico Department of Agriculture and the USDA-Agricultural Research Service to conduct the research project for integrated control of the southern cattle fever tick in Puerto Rico. The objective of this project was to create science-based knowledge to integrate technologies for sustainable control of $R$. microplus infestations (for details see page 4 in: http://tinyurl.com/y7nr8j96). Originally, four commercial farms were selected to represent the main dairy farming areas of Puerto Rico. An additional research site was a commercial beef cattle ranch in the southwest quadrant of the island. Our approach addressed food safety and environmental health concerns with the ecological impact, and residue levels of synthetic acaricides in dairy cattle products. The project implemented good acaricide management practices through the acknowledgement of parasite economic thresholds prior to treatment and the use of novel pesticide formulations containing natural products, which were labelled to treat lactating cows and their environment. Dairy and beef cattle producers in Puerto Rico had the opportunity to explore the use of the Zoetis Bm86based vaccine and safer acaricides as part of an experimental integrated tick control protocol as it was done before with other Bm86-based vaccines in lactating dairy cows (Jonsson et al. 2000; Rodríguez-Valle et al. 2004). Participating producers were able to mitigate the economic impact of $R$. microplus in their cattle operations as a result of concerted efforts taking place between the animal health industry, and federal and state regulatory agencies. No adverse reactions were observed in association with the experimental use in Puerto Rico of the Zoetis Bm86-based vaccine in beef cattle (R.J. Miller et al. unpublished data). A study was completed documenting the safe use of the vaccine in dairy cattle under field conditions (R.J. Miller et al. unpublished data).

\section{Brief update of anti-R. microplus vaccine discovery research efforts in Brazil and Mexico}

International collaboration contributed to our ability to deliver an anti-tick vaccine for the Cattle Fever Tick Eradication Program. In this section we describe briefly anti-tick vaccine discovery research by our international collaborators with emphasis on recent efforts in Mexico and Brazil. The only known test comparing the efficacy of TickGard $^{\circledR}$ and $\mathrm{Gavac}^{\mathrm{TM}}$ in a pen trial was done in Brazil where efficacy of 46 and $49 \%$, respectively, was documented with the two vaccine products against $R$. microplus infesting cattle (Andreotti 2006). The hypothesis that recombinant Bm86 from a local strain would enhance protection against $R$. microplus infesting cattle in the same area was tested in a pen trial (Cunha et al. 2012). However, aspects involving vaccine formulation or host factor polymorphisms may have caused the lesser than expected efficacy obtained in cattle immunized with the recombinant version of the Bm86 antigen based on partial genetic information from the local $R$. microplus strain. Novel protective antigens that could be used alone or formulated together have been discovered and they offer the opportunity to develop a highly efficacious vaccine against $R$. microplus (Andreotti et al. 2012; Parizi et al. 2012; Prudencio et al. 2010). Reverse vaccinology approaches have shown positive results using a peptide derived from the ATAQ protein against $R$. microplus (Aguirre Ade et al. 2016). Transcriptomics translational 
research helped advance the project to the early development stage. The apparent genetic diversity of $R$. microplus populations in Brazil should be addressed in terms of efficacy when developing a new vaccine for an animal health market that involves the largest commercial cattle herd in the world (Csordas et al. 2016).

The use of $\mathrm{Gavac}^{\mathrm{TM}}$ and its contribution as a tool in integrated cattle fever tick management was documented in Mexico (Bautista-Garfias and Martínez-lbañez 2012; Domínguez García et al. 2016; Redondo et al. 1999). Anti-cattle fever tick vaccine research and development efforts have increased in Mexico in part due to issues regarding the availability of Gavac ${ }^{\mathrm{TM}}$ in the national veterinary products market. Alternative recombinant proteins potentially useful for development to control infestations caused by R. microplus have been identified (Almazan et al. 2012; Ramirez Rodriguez et al. 2016). The economic impact of multiple acaricide resistance in Mexico highlights the need for an anti- $R$. microplus vaccine that is commercially available (Dominguez García et al. 2010; Rosario-Cruz et al. 2009). An experimental vaccine decreased the local $R$. microplus population for 7 months when tested in Mexico under field conditions where $90 \%$ of the cattle herd was immunized; however, the identity of the antigen tested was not revealed by the investigators publishing their research results (Palacios-Bautista 2014). Applying the subunit vaccine approach (Willadsen 1990), a recombinant peptide derived from the subolesin gene sequence showed $79 \%$ reduction on tick numbers in immunized cattle with the effect observed on knock-down because no significant reduction was detected on female tick weight and individual egg mass (Almazán et al. 2010; Dominguez García et al. 2015).

The opportunity exists to adapt what was learned from our experience to try to incorporate the latest science and technology to develop an anti- $R$. microplus vaccine through public-private partnerships for the Brazilian and Mexican animal health markets. Mexico and Brazil are working to establish enabling environments for public-private partnerships that enhance sustainable animal production (Bassi et al. 2015; Hopper et al. 2012). International collaborations help the United States Department of Agriculture - Agricultural Research Service contribute to global food security.

\section{Acknowledgements}

We thank those that had the audacity to challenge us to conduct anti-tick vaccine discovery research at the USDA-ARS Knipling-Bushland USA Livestock Insects Research Laboratory and the Cattle Fever Tick Research Laboratory in support of the U.S. Cattle Fever Tick Eradication Program. The support and collaboration of industry colleagues, initially with Pfizer Animal Health, and then Zoetis is greatly appreciated. Now we understand when efforts have to stay on the critical path for development, and focused to be pragmatic and meet mutual interests of the public-private partnership. All vaccine discovery research with cattle at the USDA-ARS Cattle Fever Tick Research Laboratory was conducted according to protocols reviewed and approved by the Institutional Animal Care and Use Committee. We are deeply grateful to all the staff at the USDA-ARS KniplingBushland U.S. Livestock Insects Research Laboratory and the Cattle Fever Tick Research Laboratory, APHIS-VS, TAHC, and Zoetis that assisted with the research and development of the novel Bm86based vaccine. This would have never been possible without the support of livestock producers in the USA and Puerto Rico. 


\section{References}

Abbas RZ, Zaman MA, Colwell DD, Gilleard J and Iqbal Z (2014) Acaricide resistance in cattle ticks and approaches to its management: the state of play. Vet Parasitol 203: 6-20.

Acosta-Rodriguez R, Alonso-Morales R, Balladares S, Flores-Aguilar H, Garcia-Vazquez Z and Gorodezky C (2005) Analysis of Bola class II microsatellites in cattle infested with Boophilus microplus ticks: class II is probably associated with susceptibility. Vet Parasitol 127: 313-321.

Aguirre Ade A, Garcia MV, Szabo MP, Barros JC and Andreotti R (2015) Formula to evaluate efficacy of vaccines and systemic substances against three-host ticks. Int J Parasitol 45: 357-359.

Aguirre Ade A, Lobo FP, Cunha RC, Garcia MV and Andreotti R (2016) Design of the ataq peptide and its evaluation as an immunogen to develop a Rhipicephalus vaccine. Vet Parasitol 221: 30-38.

Alegría-López MA, Rodríguez-Vivas, R.I., Torres-Acosta, J.F., Ojeda-Chi, M.M., Rosado-Aguilar, J.A. (2015) Use of ivermectin as endoparasiticide in tropical cattle herds generates resistance in gastrointestinal nematodes and the tick Rhipicephalus microplus (Acari: Ixodidae). J Med Entomol 52: 214-221.

Allen JR and Humphreys SJ (1979) Immunisation of guinea pigs and cattle against ticks. Nature 280: 491-493.

Almazan C, Lagunes R, Villar M, Canales M, Rosario-Cruz R, Jongejan F and De la Fuente J (2010) Identification and characterization of Rhipicephalus (Boophilus) microplus candidate protective antigens for the control of cattle tick infestations. Parasitol Res 106: 471-479.

Almazan C, Moreno-Cantu O, Moreno-Cid JA, Galindo RC, Canales M, Villar M and De la Fuente J (2012) Control of tick infestations in cattle vaccinated with bacterial membranes containing surface-exposed tick protective antigens. Vaccine 30: 265-272.

Andreotti R (2006) Performance of two Bm86 antigen vaccine formulation against tick using crossbreed bovines in stall test. Rev Bras Parasitol Vet 5: 3.

Andreotti R, Cunha RC, Soares MA, Guerrero FD, Leite FP and De Leon AA (2012) Protective immunity against tick infestation in cattle vaccinated with recombinant trypsin inhibitor of Rhipicephalus microplus. Vaccine 30:6678-6685.

Andreotti R, Garcia MV, Cunha RC and Barros JC (2013) Protective action of tagetes minuta (asteraceae) essential oil in the control of Rhipicephalus microplus (Canestrini, 1887) (Acari: Ixodidae) in a cattle pen trial. Vet Parasitol 197: 341-345.

Angus BM (1996) The history of the cattle tick Boophilus microplus in Australia and achievements in its control. Int J Parasitol 26: 1341-1355.

Antunes S, Merino O, Mosqueda J, Moreno-Cid JA, Bell-Sakyi L, Fragkoudis R, Weisheit S, Perez de la Lastra JM, Alberdi P, Domingos A and De la Fuente J (2014) Tick capillary feeding for the study of proteins involved in tick-pathogen interactions as potential antigens for the control of tick infestation and pathogen infection. Parasit Vectors 7: 42.

Aubry P and Geale DW (2011) A review of bovine anaplasmosis. Transbound Emerg Dis 58: 1-30.

Banks R (2014) Veterinary vaccines: EU regulatory requirements. In: Giese M (ed.) Molecular vaccines. Springer-Verlag, Wien, Austria, pp. 859-865.

Barker SC, Walker AR and Campelo D (2014) A list of the 70 species of Australian ticks; diagnostic guides to and species accounts of Ixodes holocyclus (Paralysis tick), Ixodes cornuatus (southern paralysis tick) and Rhipicephalus australis (Australian cattle tick); and consideration of the place of Australia in the evolution of ticks with comments on four controversial ideas. Int J Parasitol 44: 941-953.

Barnard DR, Mount GA, Kochanek HG, Haile DG and Garris GI (1988) Management of the lone star tick in recreation areas. Agricultural Handbook no 682. U.S. Department of Agriculture, Springfield, VA, USA, pp. 33.

Barré N, Happold J, Delathiere JM, Desoutter D, Salery M, De Vos A, Marchal C, Perrot R, Grailles M and Mortelecque A (2011) A campaign to eradicate bovine babesiosis from New Caledonia. Ticks Tick Borne Dis 2: 55-61.

Barrero RA, Guerrero FD, Black M, McCooke J, Chapman B, Schilkey F, Pérez de León AA, Miller RJ, Bruns S, Dobry J, Mikhaylenko G, Stormo K, Bell C, Tao Q, Bogden R, Moolhuijzen PM, Hunter A and Bellgard MI (2017) Geneenriched draft genome of the cattle tick Rhipicephalus microplus: assembly by the hybrid Pacific Biosciences/ Illumina approach enabled analysis of the highly repetitive genome. Int J Parasitol 47: 569-583.

Bassi NSS, Silva CL and Santoyo A (2015) Technology transfer: an interdisciplinary process. Eur Sci J 2: 314-324. 
Bautista-Garfias CR and Martínez-lbañez F (2012) Experiences on the control of cattle tick Rhipicephalus (Boophilus) microplus in Mexico. In: Woldemeskel M (ed.) Ticks: management, disease control and the environment, BBSRC veterinary vaccinology strategy 2015-2020. Nova Science Publishers, Hauppauge, NY, USA, pp. 205-216.

Biotechnology and Biological Sciences Research Council (BBSRC) (2015) Veterinary vaccinology strategy 2015-2020. BBSRC, Swindon, UK. Available at: https://tinyurl.com/ycjk2poh.

Bellgard MI, Moolhuijzen PM, Guerrero FD, Schibeci D, Rodriguez-Valle M, Peterson DG, Dowd SE, Barrero R, Hunter A, Miller RJ and Lew-Tabor AE (2012) Cattletickbase: an integrated internet-based bioinformatics resource for Rhipicephalus (Boophilus) microplus. Int J Parasitol 42: 161-169.

Benfield C (2016) One vaccinology? Overcoming challenges in vaccine development. Vet Rec 179: 508-509.

Benavides Ortiz E, Romero Prada J and Villamil Jiménez LC (2016). Las garrapatas del ganado bovino y los agentes de enfermedad que transmiten en escenarios epidemiológicos de cambio climático: guía para el manejo de garrapatas y adaptación al cambio climático. Instituto Interamericano de Cooperación para la Agricultura, Costa Rica, 98 pp.

Bharadwaj A and Stafford KC $3^{\text {rd }}$ (2010) Evaluation of Metarhizium anisopliae strain F52 (hypocreales: Clavicipitaceae) for control of Ixodes scapularis (Acari: Ixodidae). J Med Entomol 47: 862-867.

Biegelmeyer P, Nizoli LQ, Da Silva SS, Dos Santos TR, Dionello NJ, Gulias-Gomes CC and Cardoso FF (2015) Bovine genetic resistance effects on biological traits of Rhipicephalus (Boophilus) microplus. Vet Parasitol 208: 231-237.

Black PF (2012) Good governance of animal health systems and public-private partnerships: an Australian case study. Rev Sci Tech 31: 699-708.

Bock ER, Jackson AL, De Vos JA and Jorgensen KW (2008) Babesia of cattle. In: Nuttall ASBAP (ed.) Ticks: biology, disease and control. Cambridge University Press, New York, NY, USA, pp. 281-307.

Boué O, Redondo M, Montero C, Rodriguez M and De la Fuente J (1999) Reproductive and safety assessment of vaccination with Gavac $^{(\mathrm{Tm})}$ against the cattle tick (Boophilus microplus). Theriogenology 51: 1547-1554.

Brake DK and Perez de Leon AA (2012) Immunoregulation of bovine macrophages by factors in the salivary glands of Rhipicephalus microplus. Parasit Vectors 5: 38.

Bram RA and Gray JH (1983) Eradication - an alternative to tick control. Ticks and tick-borne diseases. FAO Anim Health Prod Paper 36: 54-59.

Bram RA, George JE, Reichar RE and Tabaciinic WJ (2002) Threat of foreign arthropod-borne pathogens to livestock in the United States. J Med Entomol 39: 405-416.

Brites-Neto J, Duarte KM and Martins TF (2015) Tick-borne infections in human and animal population worldwide. Vet World 8: 301-315.

Camargo MG, Nogueira MR, Marciano AF, Perinotto WM, Coutinho-Rodrigues CJ, Scott FB, Angelo IC, Prata MC and Bittencourt VR (2016) Metarhizium anisopliae for controlling Rhipicephalus microplus ticks under field conditions. Vet Parasitol 223: 38-42.

Canales M, Enriquez A, Ramos E, Cabrera D, Dandie H, Soto A, Falcon V, Rodriguez M and De la Fuente J (1997) Largescale production in Pichia pastoris of the recombinant vaccine Gavac ${ }^{(\mathrm{Tm})}$ against cattle tick. Vaccine 15: 414-422.

Canales M, Moreno-Cid JA, Almazán C, Villar M and De la Fuente J (2010) Bioprocess design and economics of recombinant Bm86/Bm95 antigen production for anti-tick vaccines. Biochem Eng J 52: 79-90.

Chambers MA, Graham SP and La Ragione RM (2016) Challenges in veterinary vaccine development and immunization. Methods Mol Biol 1404: 3-35.

Chatelain E and Don R (2009) Antiparasitic antibacterial drug discovery. In: Selzer PM (ed.) Drug discovery for neglected diseases. Wiley-Blackwell, Hoboken, NJ, USA.

Collins JM, Reizes $O$ and Dempsey MK (2016) Healthcare commercialization programs: improving the efficiency of translating healthcare innovations from academia into practice. IEEE J Transl Eng Health Med 4: 3500107.

Cortés M, Pantoja A, Robles W and Pantoja J (2005) Tick incidence in Puerto Rico. J Agric Univ PR 89: 59-66.

Costa-Junior LM, Miller RJ, Alves PB, Blank AF, Li AY and Pérez de León AA (2016) Acaricidal efficacies of Lippia gracilis essential oil and its phytochemicals against organophosphate-resistant and susceptible strains of Rhipicephalus (Boophilus) microplus. Vet Parasitol 228: 60-64.

Csordas BG, Garcia MV, Cunha RC, Giachetto PF, Blecha IM and Andreotti R (2016) New insights from molecular characterization of the tick Rhipicephalus (Boophilus) microplus in Brazil. Rev Bras Parasitol Vet 25: 317-326. 
Cunha RC, Pérez de León AA, Leite FP, Pinto Lda S, Dos Santos Júnior AG and Andreotti R (2012) Bovine immunoprotection against Rhipicephalus (Boophilus) microplus with recombinant Bm86-Campo Grande antigen. Rev Bras Parasitol Vet 21: 254-262.

Cunha RC, Andreotti R, Garcia MV, Aguirre Ade A and Leitão A (2013) Calculation of the efficacy of vaccines against tick infestations on cattle. Rev Bras Parasitol Vet 22: 571-578.

Davey RB, Pound JM, Klavons JA, Lohmeyer KH, Freeman JM and Olafson PU (2012) Analysis of doramectin in the serum of repeatedly treated pastured cattle used to predict the probability of cattle fever ticks (Acari: Ixodidae) feeding to repletion. Exp Appl Acarol 56: 365-374.

De la Fuente J, Almazan C, Canales M, Perez de la Lastra JM, Kocan KM and Willadsen P (2007) A ten-year review of commercial vaccine performance for control of tick infestations on cattle. Anim Health Res Rev 8: 23-28.

De la Fuente J, Estrada-Pena A, Venzal JM, Kocan KM and Sonenshine DE (2008) Overview: ticks as vectors of pathogens that cause disease in humans and animals. Front Biosci 13: 6938-6946.

De la Fuente J, Kocan KM and Contreras M (2015) Prevention and control strategies for ticks and pathogen transmission. Rev Sci Tech 34: 249-264.

De la Fuente J, Kopacek P, Lew-Tabor A and Maritz-Olivier C (2016) Strategies for new and improved vaccines against ticks and tick-borne diseases. Parasite Immunol 38: 754-769.

De la Fuente J and Merino O (2013) Vaccinomics, the new road to tick vaccines. Vaccine 31: 5923-5929.

De la Fuente J, Rodriguez M, Montero C, Redondo M, Garcia-Garcia JC, Mendez L, Serrano E, Valdes M, Enriquez A, Canales M, Ramos E, Boue O, Machado H and Lleonart R (1999) Vaccination against ticks (Boophilus spp.): the experience with the Bm86-based vaccine Gavac ${ }^{(\mathrm{m})}$. Gen Anal Biomol Engin 15: 143-148.

De la Fuente J, Rodriguez M, Redondo M, Montero C, Garcia-Garcia JC, Mendez L, Serrano E, Valdes M, Enriquez A, Canales M, Ramos E, Boue O, Machado H, Lleonart R, De Armas CA, Rey S, Rodriguez JL, Artiles M and Garcia L (1998) Field studies and cost-effectiveness analysis of vaccination with Gavac (TM) against the cattle tick Boophilus microplus. Vaccine 16: 366-373.

De la Vega R, Camejo, A and Fonseca AH (2004) An automatic system to feed ticks through membranes. Rev Salud Anim 26: 202-205.

De Meneghi D, Stachurski F and Adakal H (2016) Experiences in tick control by acaricide in the traditional cattle sector in Zambia and Burkina Faso: possible environmental and public health implications. Front Public Health 9(4): 239.

Dominguez García DI, Lagunes Quintanilla R, Martinez Velazquez M, Quiróz Romero H and Rosario-Cruz R (2015) Diseño y expression de un péptido recombinante potencialmente útil contra las infestaciones por garrapatas del ganado bovino. Entomol Mex 2: 88-92.

Dominguez García DI, Rosario-Cruz R, Almazán-García C, Saltijeral Oaxaca JA, De la Fuente J (2010) Boophilus microplus: biological and molecular aspects of acaricides resistance and their impact on animal health. Trop Subtrop Agroecosyst 12: 181-192.

Domínguez García DI, Torres Agatón F and Rosario-Cruz R (2016) Economic evaluation of tick (Rhipicephalus microplus) control in Mexico. Rev Iberoamericana Cienc Biol Agro 5: 43-52.

Drugmand JC, Schneider YJ and Agathos SN (2012) Insect cells as factories for biomanufacturing. Biotechnol Adv 30: 1140-1157.

Drummond RO (1983) Tick-borne livestock diseases and their vectors. Chemical control of ticks. Wld Anim Rev (FAO) 36: $28-33$.

Durell K (2016) Vaccines and IP rights: a multifaceted relationship. Methods Mol Biol 1404: 791-811.

Elder JK and Morris RS (1986) The use of decision analysis to compare cattle tick control strategies under conditions of risk. Prev Vet Med 3: 12.

Esteve-Gassent MD, Pérez de León AA, Romero-Salas D, Feria-Arroyo TP, Patino R, Castro-Arellano I, Gordillo-Pérez G, Auclair A, Goolsby J, Rodriguez-Vivas RI and Estrada-Franco JG (2014) Pathogenic landscape of transboundary zoonotic diseases in the Mexico-US border along the Rio Grande. Front Publ Health 2: 177.

Estrada-Peña A and Venzal JM (2006) High-resolution predictive mapping for Boophilus annulatus and B. microplus (Acari: Ixodidae) in Mexico and Southern Texas. Vet Parasitol 142: 350-358. 
Estrada-Peña A, Venzal JM, Nava S, Mangold A, Guglielmone AA, Labruna MB, De la Fuente J (2012) Reinstatement of Rhipicephalus (Boophilus) australis (Acari: Ixodidae) with redescription of the adult and larval stages. J Med Entomol 49: 794-802.

Estrada-Peña A, Carreón D, Almazán C and De la Fuente J (2014) Modeling the impact of climate and landscape on the efficacy of white tailed deer vaccination for cattle tick control in northeastern Mexico. PLoS ONE 9: e102905.

Évora PM, Sanches GS, Guerrero FD, Pérez de León AA and Bechara GH (2017) Immunogenic potential of Rhipicephalus (Boophilus) microplus aquaporin 1 against Rhipicephalus sanguineus in domestic dogs. Rev Bras Parasitol Vet 26: 60-66.

Fang QQ and Pung OJ (2011) Anti-tick vaccine development: status and perspectives. In: Liu T and Kang L (ed.) Recent advances in entomological research. Springer-Verlag, Berlin, Germany, pp. 359-375.

Food and Agriculture Organisation (FAO) (1989) The eradication of ticks. FAO Anim Health Prod Paper 75: 332.

Farmer SJ and Grund M (2014) Patentability of vaccines: a practical perspective. In: Giese M (ed.) Molecular vaccines. Springer-Verlag, Wien, Austria, pp. 807-821.

Fernandes EK, Bittencourt VR and Roberts DW (2012) Perspectives on the potential of entomopathogenic fungi in biological control of ticks. Exp Parasitol 130: 300-305.

Floyd RB, Sutherst RW and Hungerford J (1995) Modelling the field efficacy of a genetically engineered vaccine against the cattle tick, Boophilus microplus. Int J Parasitol 25: 285-291.

Fragoso H, Rad PH, Ortiz M, Rodriguez Valle M, Redondo M, Herrera L and De la Fuente J (1998) Protection against Boophilus annulatus infestations in cattle vaccinated with the B. microplus Bm86-containing vaccine Gavac. Vaccine 16: 1990-1992.

Galay RL, Miyata T, Umemiya-Shirafuji R, Mochizuki M, Fujisaki K and Tanaka T (2016) Host immunization with recombinant proteins to screen antigens for tick control. Methods Mol Biol 1404: 261-273.

Geels MJ, Thogersen RL, Guzman CA, Ho MM, Verreck F, Collin N, Robertson JS, McConkey SJ, Kaufmann SH and Leroy $O$ (2015) Transvac research infrastructure - results and lessons learned from the European network of vaccine research and development. Vaccine 33: 5481-5487.

George JE (2008) The effects of global change on the threat of exotic arthropods and arthropod-borne pathogens to livestock in the United States. Ann N Y Acad Sci 1149: 249-254.

Giersing BK, Modjarrad K, Kaslow DC, Moorthy VS, WHO Product Development for Vaccines Advisory Committee, WHO Product Development for Vaccines Product Development Advisory Committee (2016) Report from the World Health Organization's product development for vaccines advisory committee (PDVAC) meeting Geneva, $7-9^{\text {th }}$ Sep 2015. Vaccine 34: 2865-2869.

Giese M (2013) From Pasteur to personalized vaccines. In: Giese M (ed.) Molecular vaccines. Springer-Verlag, Wien, Austria, pp. 3-18.

Giles JR, Peterson AT, Busch JD, Olafson PU, Scoles GA, Davey RB, Pound JM, Kammlah DM, Lohmeyer KH and Wagner DM (2014) Invasive potential of cattle fever ticks in the southern United States. Parasit Vectors 7: 189.

Goolsby J, Guerrero F, Gaskin JF, Bendele KG, Azhahianambi P, Amalin D, Flores-Cruz M, Kashefi J, Smith L, Saini RK, Racelis A and Pérez de León AA (2016) Molecular comparison of cattle fever ticks from native and introduced ranges with insights into optimal search areas for classical biological control agents. Southwestern Entomologist 41: 595-604.

Graf JF, Gogolewski R, Leach-Bing N, Sabatini GA, Molento MB, Bordin EL and Arantes GJ (2004) Tick control: an industry point of view. Parasitol 129: S427-42.

Graham OH and Hourrigan JL (1977) Eradication programs for the arthropod parasites of livestock. J Med Entomol 13: 629-658.

Grisi L, Leite RC, Martins JR, Barros AT, Andreotti R, Cancado PH, Leon AA, Pereira JB and Villela HS (2014) Reassessment of the potential economic impact of cattle parasites in Brazil. Rev Bras Parasitol Vet 23: 150-156.

Guerrero F, Pérez de León AA, Rodriguez-Vivas R, Jonsson N, Miller R and Andreotti R (2014a) Acaricide research and development, resistance and resistance monitoring. In: Sonenhine DE and Roe RM (eds.) Biology of ticks ( $2^{\text {nd }} \mathrm{Ed}$.) Vol. 2. Oxford University Press, New York, NY, USA, pp. 353-381. 
Guerrero FD, Andreotti R, Bendele KG, Cunha RC, Miller RJ, Yeater K and Pérez de León AA (2014b) Rhipicephalus (Boophilus) microplus aquaporin as an effective vaccine antigen to protect against cattle tick infestations. Parasit Vectors 7: 475.

Guerrero FD, Miller RJ and Pérez de León AA (2012) Cattle tick vaccines: many candidate antigens, but will a commercially viable product emerge? Int J Parasitol 42: 421-427.

Heldens JG, Patel JR, Chanter N, Ten Thij GJ, Gravendijck M, Schijns VE, Langen A and Schetters TP (2008) Veterinary vaccine development from an industrial perspective. Vet J 178: 7-20.

Henderson LM and Lewis A (2014) Licensing and permitting of veterinary vaccines in the USA: US regulatory requirements. In: Giese M (ed.) Molecular vaccines. Springer-Verlag, Wien, Austria, pp. 851-858.

Hendriks J, Holleman M, Hamidi A, Beurret M and Boog C (2013) Vaccinology capacity building in Europe for innovative platforms serving emerging markets. Hum Vaccin Immunother 9: 932-936.

Hernandez AF, Teel PD, Corson MS and Grant WE (2000) Simulation of rotational grazing to evaluate integrated pest management strategies for Boophilus microplus (Acari: Ixodidae) in Venezuela. Vet Parasitol 92: 139-149.

Higa Lde O, Garcia MV, Barros JC, Koller WW and Andreotti R (2016) Evaluation of Rhipicephalus (Boophilus) microplus (Acari: Ixodidae) resistance to different acaricide formulations using samples from Brazilian properties. Rev Bras Parasitol Vet 25: 163-171.

Hlatshwayo M and Mbati PA (2005) A survey of tick control methods used by resource-poor farmers in the Qwa-Qwa area of the eastern free state province, South Africa. Onderstepoort J Vet Res 72: 245-249.

Hopper M, Salles Almeida J, Garcia M (2012) Public-private partnerships to enhance SPS capacity: what can we learn from this collaborative approach? Joint document of the Standards and Trade Development Facility and the InterAmerican Development Bank. Available at: https://tinyurl.com/y75t53sa.

Hunter J (2002) Tickgard Plus relaunched to fight cattle ticks. Aust Vet J 80: 394.

Inokuma H and Kemp DH (1998) Establishment of Boophilus microplus infected with Babesia bigemina by using in vitro tube feeding technique. J Vet Med Sci 60: 509-512.

Jittapalapong S, Thanasilp S, Kaewmongkol G and Sirinarukmitr T (2006) The progress and process of the development of anti-tick vaccine against cattle ticks (Boophilus microplus) in Thailand. Kasetsart J (Nat Sci) 40: 276-283.

Jongejan F and Uilenberg G (2004) The global importance of ticks. Parasitol 129: S3-S14.

Jonsson N (2004) Integrated control programs for ticks on cattle. An examination of some possible components. FAO Animal Production and Health Paper, University of Queensland, Brisbane, Australia.

Jonsson NN, Matschoss AL, Pepper P, Green PE, Albrecht MS, Hungerford J and Ansell J (2000) Evaluation of TickGard(plus), a novel vaccine against Boophilus microplus, in lactating Holstein-Friesian cows. Vet Parasitol 88: 275-285.

Jonsson NN, Piper EK and Constantiniou CC (2014) Host resistance in cattle to infestation with the cattle tick Rhipicephalus microplus. Parasite Immunol 36: 553-559.

Kalyanasundaram A, Jawahar S, Ilangopathy M, Palavesam A and Raman M (2015) Comparative immunoprophylactic efficacy of Haemonchus contortus recombinant enolase ( $\mathrm{rHcENO}$ ) and Con a purified native glycoproteins in sheep. Exp Parasitol 154: 98-107.

Khorasani AMO, Soleimanjahi $\mathrm{H}$, Keyvanfar $\mathrm{H}$ and Mahravani $\mathrm{H}$ (2016) Evaluation of the efficacy of a new oil-based adjuvant ISA 61 VG FMD vaccine as a potential vaccine for cattle. Iran J Vet Res 17: 8-12.

King LJ, Marano N and Hughes JM (2004) New partnerships between animal health services and public health agencies. Rev Sci Tech 23: 717-725.

Kiss T, Cadar D and Spinu M (2012) Tick prevention at a crossroad: new and renewed solutions. Vet Parasitol 187: 357-366.

Klafke G, Webster A, Dall Agnol B, Pradel E, Silva J, De la Canal LH, Becker M, Osorio MF, Mansson M, Barreto R, Scheffer R, Souza UA, Corassini VB, Dos Santos J, Reck J and Martins JR (2017) Multiple resistance to acaricides in field populations of Rhipicephalus microplus from Rio Grande do Sul State, Southern Brazil. Ticks Tick Borne Dis 8: 73-80.

Krol AC, Abu-Shaar M and Brady P (2016) From the bench to the pharmacy: protecting innovation during vaccine development and commercialization. Methods Mol Biol 1404: 813-834.

Lee BY and Burke DS (2010) Constructing target product profiles (TPPS) to help vaccines overcome post-approval obstacles. Vaccine 28: 2806-2809.

Lee BY and McGlone SM (2010) Pricing of new vaccines. Hum Vaccin 6: 619-629. 
Legastelois I, Buffin S, Peubez I, Mignon C, Sodoyer R and Werle B (2016) Non-conventional expression systems for the production of vaccine proteins and immunotherapeutic molecules. Hum Vaccin Immunother 13(4): 947-961.

Lew-Tabor AE, Bruyeres AG, Zhang B and Rodriguez Valle M (2014) Rhipicephalus (Boophilus) microplus tick in vitro feeding methods for functional (DSRNA) and vaccine candidate (antibody) screening. Ticks Tick Borne Dis 5: 500-510.

Lew-Tabor AE and Rodriguez Valle M (2016) A review of reverse vaccinology approaches for the development of vaccines against ticks and tick borne diseases. Ticks Tick Borne Dis 7: 573-585.

Liu WC, Gong T, Wang QH, Liang X, Chen JJ and Zhu P (2016) Scaling-up fermentation of Pichia pastoris to demonstrationscale using new methanol-feeding strategy and increased air pressure instead of pure oxygen supplement. Sci Rep 6: 18439 .

Lodos J, Boue $O$ and De la Fuente J (2000) A model to simulate the effect of vaccination against Boophilus ticks on cattle. Vet Parasitol 87: 315-326.

Lohmeyer KH, Pound JM, May MA, Kammlah DM and Davey RB (2011) Distribution of Rhipicephalus (Boophilus) microplus and Rhipicephalus (Boophilus) annulatus (Acari: Ixodidae) infestations detected in the United States along the Texas/ Mexico border. J Med Entomol 48: 770-774.

Lopez-Arias A, Villar-Argaiz D, Chaparro-Gutierrez JJ, Miller RJ, Pérez de León AA (2015) Reduced efficacy of commercial acaricides against populations of resistant cattle tick Rhipicephalus microplus from two municipalities of Antioquia, Colombia. Environ Health Insights 8: 71-80.

MacDonald J, Doshi K, Dussault M, Hall JC, Holbrook L, Jones G, Kaldis A, Klima CL, Macdonald P, McAllister T, McLean MD, Potter A, Richman A, Shearer H, Yarosh O, Yoo HS, Topp E and Menassa R (2015) Bringing plant-based veterinary vaccines to market: managing regulatory and commercial hurdles. Biotechnol Adv 33: 1572-1581.

Mapholi NO, Maiwashe A, Matika O, Riggio V, Bishop SC, MacNeil MD, Banga C, Taylor JF and Dzama K (2016) Genomewide association study of tick resistance in South African nguni cattle. Ticks Tick Borne Dis 7: 487-497.

Marshall V (2014) United States Food and Drug Administration: regulation of vaccines. In: Giese M (ed.) Molecular vaccines. Springer-Verlag, Wien, Austria, pp. 839-844.

Martinez-Velazquez M, Castillo-Herrera GA, Rosario-Cruz R, Flores-Fernandez JM, Lopez-Ramirez J, Hernandez-Gutierrez $R$ and Lugo-Cervantes Edel C (2011) Acaricidal effect and chemical composition of essential oils extracted from Cuminum cyminum, Pimenta dioica and Ocimum basilicum against the cattle tick Rhipicephalus (Boophilus) microplus (Acari: Ixodidae). Parasitol Res 108: 481-487.

Merino O, Alberdi P, Perez de la Lastra JM and De la Fuente J (2013) Tick vaccines and the control of tick-borne pathogens. Front Cell Infect Microbiol 3: 30.

Miller RJ, Davey RB and George JE (2005) First report of organophosphate-resistant Boophilus microplus (Acari: Ixodidae) within the United States. J Med Entomol 42: 912-917.

Miller RJ, Davey RB and George JE (2007) First report of permethrin-resistant Boophilus microplus (Acari: Ixodidae) collected within the United States. J Med Entomol 44: 308-315.

Miller R, Estrada-Pena A, Almazan C, Allen A, Jory L, Yeater K, Messenger M, Ellis D and Pérez de León AA (2012) Exploring the use of an anti-tick vaccine as a tool for the integrated eradication of the cattle fever tick, Rhipicephalus (Boophilus) annulatus. Vaccine 30: 5682-5687.

Miller RJ, Almazan C, Ortiz-Estrada M, Davey RB, George JE and Pérez de León AA (2013) First report of fipronil resistance in Rhipicephalus (Boophilus) microplus of Mexico. Vet Parasitol 191: 97-101.

Monath TP (2013) Vaccines against diseases transmitted from animals to humans: a one health paradigm. Vaccine 31: 5321-5338.

Mondal DB, Sarma K and Saravanan M (2013) Upcoming of the integrated tick control program of ruminants with special emphasis on livestock farming system in India. Ticks Tick Borne Dis 4: 1-10.

Norton GA, Sutherst RW and Maywald GF (1983) A framework for integrating control methods against the cattle tick, Boophilus microplus in Australia. J Appl Ecol 20: 489-505.

Nuttall PA, Trimnell AR, Kazimirova M and Labuda M (2006) Exposed and concealed antigens as vaccine targets for controlling ticks and tick-borne diseases. Parasite Immunol 28: 155-163. 
Olafsdottir TALM, Nookaew I, Andersen P, Maertzdorf J, Persson J, Christensen D, Zhang Y, Anderson J, Khoomrung S, Sen P, Agger EM, Coler R, Carter D, Meinke A, Rappuoli R, Kaufmann SH, Reed SG and Harandi AM (2016) Comparative systems analyses reveal molecular signatures of clinically tested vaccine adjuvants. Sci Rep 6: 39097.

Olds C, Bishop R and Daubenberger C (2013) Anti-tick vaccines for the control of ticks affecting livestock. In: Giese M (eds.) Molecular vaccines. Springer, Vienna, Austria, pp. 295-311.

Palacios-Bautista G, Domínguez-García, DI, Ortiz-Estrada M, López-Velázquez JC, Rosario-Cruz R (2014) Post-vaccination evaluation of the cattle tick Rhipicephalus (Boophilus) microplus population in a farm from iguala Guerrero, Mexico. Rev Cien Biol Agro Tuxpan 2: 150-156.

Parizi LF, Reck J Jr., Oldiges DP, Guizzo MG, Seixas A, Logullo C, De Oliveira PL, Termignoni C, Martins JR and Vaz Ida S Jr. (2012) Multi-antigenic vaccine against the cattle tick Rhipicephalus (Boophilus) microplus: a field evaluation. Vaccine 30: 6912-6917.

Pegram RG, Wilson DD and Hansen JW (2000) Past and present national tick control programs. Why they succeed or fail. Ann N Y Acad Sci 916: 546-554.

Pérez de León A, Strickman DA, Knowles DP, Fish D, Thacker E, De la Fuente J, Krause PJ, Wikel SK, Miller RS, Wagner GG, Almazán C, Hillman R, Messenger MT, Ugstad PO, Duhaime RA, Teel PD, Ortega-Santos A, Hewitt DG, Bowers EJ, Bent SJ, Cochran MH, McElwain TF, Scoles GA, Suarez CE, Davey R, Howell Freeman JM, Lohmeyer K, Li AY, Guerrero FD, Kammlah DM, Phillips P and Pound JM (2010) One health approach to identify research needs in bovine and human babesioses: workshop report. Parasit Vectors 3: 36-47.

Pérez de León AA, Teel PD, Auclair AN, Messenger MT, Guerrero FD, Schuster G and Miller RJ (2012) Integrated strategy for sustainable cattle fever tick eradication in USA is required to mitigate the impact of global change. Front Physiol 3: 195.

Pérez de León AA, Teel PD, Li A, Ponnusamy L and Roe RM (2014b) Advancing integrated tick management to mitigate burden of tick-borne diseases. Outlooks Pest Manag 25: 382-389.

Pérez de León AA, Vannier E, Almazán C and Krause PJ (2014a) Tick-borne protozoa. In: Sonenhine DE and Roe RM (eds.) Biology of ticks (2 ${ }^{\text {nd }}$ Ed.). Oxford University Press, New York, NY, USA, pp. 147-179.

Petermann J, Bonnefond R, Mermoud I, Rantoen D, Meynard L, Munro C, Lua LHL and Hüe T (2017) Evaluation of three adjuvants with respect to both adverse effects and the efficacy of antibody production to the Bm86 protein. Exp Appl Acarol 72(3): 303-315.

Pipano E, Alekceev E, Galker F, Fish L, Samish M and Shkap V (2003) Immunity against Boophilus annulatus induced by the Bm86 (Tick-Gard) vaccine. Exp Appl Acarol 29: 141-149.

Piper EK, Jonsson NN, Gondro C, Vance ME, Lew-Tabor A and Jackson LA (2016) Peripheral cellular and humoral responses to infestation with the cattle tick Rhipicephalus microplus in Santa-Gertrudis cattle. Parasite Immunol 39(1): e12402.

Pound JM, George JE, Kammlah DM, Lohmeyer KH and Davey RB (2010) Evidence for role of white-tailed deer (Artiodactyla: Cervidae) in epizootiology of cattle ticks and southern cattle ticks (Acari: Ixodidae) in reinfestations along the Texas/Mexico border in south Texas: a review and update. J Econ Entomol 103: 211-218.

Playford M, Rabiee AR, Lean IJ and Ritchie M (2005) Review of research needs for cattle tick control, phases I and II. Meat and Livestock Australia, Locked Bag 991, North Sydney, NSW 2059.

Plotkin SA, Mahmoud AA and Farrar J (2015) Establishing a global vaccine-development fund. N Engl J Med 373: 297-300.

Popara M, Villar M, Mateos-Hernandez L, De Mera IG, Marina A, Del Valle M, Almazan C, Domingos A and De la Fuente $J$ (2013) Lesser protein degradation machinery correlates with higher Bm86 tick vaccine efficacy in Rhipicephalus annulatus when compared to Rhipicephalus microplus. Vaccine 31: 4728-4735.

Popham TW and Garris GI (1991) Considerations when modeling alternative eradication strategies for Boophilus microplus (Canestrini) (Acari: Ixodidae) in Puerto Rico. J Agric Entomol 8: 271-289.

Pramod K, Tahir MA, Charoo NA, Ansari SH and Ali J (2016) Pharmaceutical product development: a quality by design approach. Int J Pharm Investig 6: 129-138.

Prudencio CR, Marra AO, Cardoso R and Goulart LR (2010) Recombinant peptides as new immunogens for the control of the bovine tick, Rhipicephalus (Boophilus) microplus. Vet Parasitol 172: 122-131.

Ramirez Rodriguez PB, Rosario Cruz R, Dominguez Garcia DI, Hernandez Gutierrez R, Lagunes Quintanilla RE, Ortuno Sahagun D, Gonzalez Castillo C, Gutierrez Ortega A, Herrera Rodriguez SE, Vallejo Cardona A and Martinez 
Velazquez M (2016) Identification of immunogenic proteins from ovarian tissue and recognized in larval extracts of Rhipicephalus (Boophilus) microplus, through an immunoproteomic approach. Exp Parasitol 170: 227-235.

Reck J Jr., Berger M, Terra RM, Marks FS, Da Silva Vaz I Jr., Guimaraes JA and Termignoni C (2009) Systemic alterations of bovine hemostasis due to Rhipicephalus (Boophilus) microplus infestation. Res Vet Sci 86: 56-62.

Redondo M, Fragoso H, Ortiz M, Montero C, Lona J, Medellin JA, Fria R, Hernandez V, Franco R, Machado H, Rodriguez $M$ and De la Fuente J (1999) Integrated control of acaricide-resistant Boophilus microplus populations on grazing cattle in Mexico using vaccination with Gavac ${ }^{(\mathrm{Tm})}$ and amidine treatments. Exp Appl Acarol 23: 841-849.

Riding GA, Jarmey J, McKenna RV, Pearson R, Cobon GS and Willadsen P (1994) A protective 'concealed' antigen from Boophilus microplus. Purification, localization, and possible function. J Immunol 153: 5158-5166.

Rippke B (2105) Regulatory perspective on trends in veterinary biologics. In: Proceedings of the VICH 5 Conference, October 28-29, 2015, Tokyo, Japan. Available at: http://www.vichsec.org/conference-documents.html.

Rodriguez-Mallon A (2016) Developing anti-tick vaccines. In: Thomas S (ed.) Vaccine design. Springer, New York, NY, USA, pp. 243-259.

Rodriguez-Mallon A, Encinosa PE, Mendez-Perez L, Bello Y, Rodriguez Fernandez R, Garay H, Cabrales A, Mendez L, Borroto $C$ and Estrada MP (2015) High efficacy of a 20 amino acid peptide of the acidic ribosomal protein p0 against the cattle tick, Rhipicephalus microplus. Ticks Tick Borne Dis 6: 530-537.

Rodríguez-Mallon A, Bechara G, Zacarias R, Benavides-Ortiz E, Soto-Rivas J, Gómez-Ramírez A, Jaimes-Olaya J and Estrada-García M (2013) Inhibition of Ehrlichia canis and Babesia can is transmission among ticks fed together on dogs vaccinated with Bm86 antigen. Open J Anim Sci 3: 24-32.

Rodriguez Valle M, Massard CL, Da Fonseca AH, Ramos NF, Machado H, Labarta V and De la Fuente J (1995a) Effect of vaccination with a recombinant $\mathrm{Bm} 86$ antigen preparation on natural infestations of Boophilus microplus in grazing dairy and beef pure and cross-bred cattle in Brazil. Vaccine 13: 1804-1808.

Rodriguez Valle M, Mendez L, Valdez M, Redondo M, Espinosa CM, Vargas M, Cruz RL, Barrios HP, Seoane G, Ramirez ES, Boue O, Vigil JL, Machado H, Nordelo CB and Pineiro MJ (2004) Integrated control of Boophilus microplus ticks in Cuba based on vaccination with the anti-tick vaccine Gavac ${ }^{(\mathrm{rm})}$. Exp Appl Acarol 34: 375-382.

Rodriguez Valle M, Montero C, Machado H and Joglar M (2001) The evaluation of yeast derivatives as adjuvants for the immune response to the Bm86 antigen in cattle. BMC Biotechnol 1: 2 .

Rodriguez Valle M, Penichet ML, Mouris AE, Labarta V, Luaces LL, Rubiera R, Cordoves C, Sanchez PA, Ramos E, Soto A, Canales M, Pelenzuela D, Triguero A, Lleonart R, Herrera L and De la Fuente J (1995b) Control of Boophilus microplus populations in grazing cattle vaccinated with a recombinant Bm86 antigen preparation. Vet Parasitol 57: 339-349.

Rodriguez Valle M, Rubiera R, Penichet M, Montesinos R, Cremata J, Falcon V, Sanchez G, Bringas R, Cordoves C, Valdes M, Lleonart R, Herrera L and De la Fuente J (1994) High-level expression of the B. microplus Bm86 antigen in the yeast Pichia pastoris forming highly immunogenic particles for cattle. J Biotechnol 33: 135-146.

Rodriguez-Vivas RI, Grisi L, Pérez de León AA, Silva Villela H, Torres-Acosta JFJ, Fragoso Sanchez H, Romero Salas D, Rosario Cruz R, Saldierna F and Garcia Carrasco D (2017) Potential economic impact assessment for cattle parasites in Mexico. Rev Mex Cienc Pec 8: 61-74.

Rodriguez-Vivas RI, Miller RJ, Ojeda-Chi MM, Rosado-Aguilar JA, Trinidad-Martinez IC and Perez de Leon AA (2014a) Acaricide and ivermectin resistance in a field population of Rhipicephalus microplus (Acari: Ixodidae) collected from red deer (Cervus elaphus) in the Mexican tropics. Vet Parasitol 200: 179-188.

Rodriguez-Vivas RI, Perez-Cogollo LC, Rosado-Aguilar JA, Ojeda-Chi MM, Trinidad-Martinez I, Miller RJ, Li AY, De Leon AP, Guerrero F and Klafke G (2014b) Rhipicephalus (Boophilus) microplus resistant to acaricides and ivermectin in cattle farms of Mexico. Rev Bras Parasitol Vet 23: 113-122.

Rodríguez-Vivas RI, Rosado-Aguilar JA, Ojeda-Chi MM, Pérez-Cogollo LC, Trinidad-Martínez I and Bolio-González ME (2016) Control integrado de garrapatas en la ganadería bovina. Ecosist Recurs Agro 1: 295-308.

Rosario-Cruz R, Almazan C, Miller RJ, Dominguez-Garcia DI, Hernandez-Ortiz R, De la Fuente J (2009) Genetic basis and impact of tick acaricide resistance. Front Biosci 14: 2657-2665.

Santos I, Ribeiro JMC, Kashino SS, Carvalho WA, Ferreira BR, Da Silva JS, Szabo MP, Bechara GH, Gershwin LJ (2004) Immunogenomics for development of anti-tick vaccines. Rev Bras Parasitol Vet 13: 135-139. 
Schetters T, Bishop R, Crampton M, Kopáček P, Lew-Tabor A, Maritz-Olivier C, Miller R, Mosqueda J, Patarroyo J, Rodriguez-Valle M, Scoles $G$ and De la Fuente J (2016) Cattle tick vaccine researchers join forces in CATVAC. Parasit Vectors 9: 105-111.

Schmidtmann ET (1994) Ecologically based strategies for controlling ticks. In: Sonenshine DE, Mather and TN (eds.) Ecological dynamics of tick-borne zoonoses. Oxford University Press, New York, NY, USA, pp. 240-280.

Schwartz JL and Mahmoud A (2016) When not all that counts can be counted: economic evaluations and the value of vaccination. Health Aff 35: 208-211.

Shah RR, Hassett KJ and Brito LA (2017) Overview of vaccine adjuvants: introduction, history, and current status. Methods Mol Biol 1494: 1-13.

Shen W, Xue Y, Liu Y, Kong C, Wang X, Huang M, Cai M, Zhou X, Zhang Y and Zhou M (2016) A novel methanol-free Pichia pastoris system for recombinant protein expression. Microb Cell Fact 15: 178.

Sitte K, Brinkworth R, East IJ and Jazwinska EC (2002) A single amino acid deletion in the antigen binding site of boladrb3 is predicted to affect peptide binding. Veterinary Immunology and Immunopathology 85: 129-135.

Sonenshine DE and Roe RM (2014) Biology of ticks ( $2^{\text {nd }}$ Ed.). Oxford University Press, New York, NY, USA.

Sprong H, Trentelman J, Seemann I, Grubhoffer L, Rego RO, Hajdusek O, Kopacek P, Sima R, Nijhof AM, Anguita J, Winter P, Rotter B, Havlikova S, Klempa B, Schetters TP and Hovius JW (2014) Antidote: anti-tick vaccines to prevent tickborne diseases in Europe. Parasit Vectors 7: 77.

State of Queensland (2016a) Queensland biosecurity manual. Queensland Government, Brisbane, Australia. Available at: http://tinyurl.com/y8kqnpsy.

State of Queensland (2016b) Biosecurity regulation. Queensland Government, Brisbane, Australia. Available at: https:// tinyurl.com/yce4houz.

Stephens $P$ (2014) Vaccine R\&D: past performance is no guide to the future. Vaccine 32: 2139-2142.

Suarez M, Rubi J, Pérez D, Cordova V, Salazar Y, Vielma A, Barrios F, Gil CA, Segura N, Carrillo Y, Cartaya R, Palacios M, Rubio E, Escalona C, Ramirez C, Basulto Baker R, Machado H, Sordo Y, Bermudes J, Vargas M, Montero, C, Cruz A, Puente P, Rodriguez JL, Mantilla E, Oliva O, Smith E, Castillo A, Ramos B, Ramirez Y, Abad Z, Morales A, Gonzalez EM, Hernandez A, Ceballo Y, Callard D, Cardoso A, Navarro M, Gonzalez JL, Pina R, Cueto M, Borroto C, Pimentel E, Carpio Y, Estrada MP (2016) High impact and effectiveness of Gavac vaccine in the national program for control of bovine ticks Rhipicephalus microplus in Venezuela. Livestock Science 187: 48-52.

Trager W (1939) Acquired immunity to ticks. J Parasitol 25: 57-81.

Urdaz-Rodriguez J, Fosgate G, Alleman AR, Rae O, Donovan A, Binford M, Zaragoza A and Melendez P (2012) Association between ecological factors and the presence of Rhipicephalus (Boophilus) microplus larvae in Puerto Rico. Exp Appl Acarol 58: 145-157.

Urdaz-Rodriguez JH, Fosgate GT, Waghela SD, Alleman AR, Rae DO, Donovan GA and Melendez P (2009a) Seroprevalence estimation and management factors associated with high herd seropositivity for Babesia bovis in commercial dairy farms of Puerto Rico. Trop Anim Health Prod 41: 1465-1473.

Urdaz-Rodríguez JH, Fosgate GT, Alleman AR, Rae DO, Donovan GA and Melendez P (2009b) Seroprevalence estimation and management factors associated with high herd seropositivity for Anaplasma marginale in commercial dairy farms of Puerto Rico. Trop Anim Health Prod 41: 1439-1448.

United States Department of Agriculture (USDA) (2016a) Forming partnerships. USDA - Agricultural Research Service, Beltsville, MD, USA. Available at: https://tinyurl.com/ydh3nxcj.

United States Department of Agriculture (USDA) (2016b) Livestock and poultry: world markets and trade. USDA Foreign Agricultural Service, Washington, DC, USA. Available at: https://tinyurl.com/n2wdnuk.

Vargas M, Montero C, Sanchez D, Perez D, Valdes M, Alfonso A, Joglar M, Machado H, Rodriguez E, Mendez L, Lleonart R, Suarez M, Fernandez E, Estrada MP, Rodriguez-Mallon A and Farnos O (2010) Two initial vaccinations with the Bm86based GavacPlus vaccine against Rhipicephalus (Boophilus) microplus induce similar reproductive suppression to three initial vaccinations under production conditions. BMC Vet Res 6: 43.

VICH (2016) Guidelines biologicals. VICH, Brussels, Belgium. Available at: https://tinyurl.com/y7u8xu48.

Walker AR (2011) Eradication and control of livestock ticks: biological, economic and social perspectives. Parasitol 138: 945-959. 
Wang HH, Teel PD, Grant WE, Schuster G and Pérez de León AA (2016) Simulated interactions of white-tailed deer (Odocoileus virginianus), climate variation and habitat heterogeneity on southern cattle tick (Rhipicephalus (Boophilus) microplus) eradication methods in South Texas, USA. Ecol Model 342: 82-96.

Webster A, Reck J, Santi L, Souza UA, Dall'Agnol B, Klafke GM, Beys-da-Silva WO, Martins JR and Schrank A (2015) Integrated control of an acaricide-resistant strain of the cattle tick Rhipicephalus microplus by applying Metarhizium anisopliae associated with cypermethrin and chlorpyriphos under field conditions. Vet Parasitol 207: 302-308.

White N, Sutherst RW, Hall N and Whish-Wilson P (2003) The vulnerability of the Australian beef industry to impacts of the cattle tick (Boophilus microplus) under climate change. Clim Change 61: 157-190.

Willadsen P (1990) Perspectives for subunit vaccines for the control of ticks. Parassitologia 32: 195-200.

Willadsen P (2008a) The development of a new or improved vaccine against Boophilus microplus: opportunities for R\&D investment. Meat \& Livestock Australia, North Sydney NSW, Australia. Available at: https://tinyurl.com/yc3k9vxc.

Willadsen P (2008b) Anti-tick vaccines. In: Bowman AS and Nuttall PA (eds.) Ticks: biology, disease and control. Cambridge University Press, Cambridge, UK, pp. 424-446.

Willadsen P, Bird P, Cobon GS and Hungerford J (1995) Commercialisation of a recombinant vaccine against Boophilus microplus. Parasitol 110: S43-50.

Willadsen P, Kemp DH, Cobon GS and Wright IG (1992) Successful vaccination against Boophilus microplus and Babesia bovis using recombinant antigens. Mem Inst Oswaldo Cruz 87: 289-294.

Wong JY and Opdebeeck JP (1989) Protective efficacy of antigens solubilized from gut membranes of the cattle tick, Boophilus microplus. Immunol 66: 149-155.

Woodcock J (2014) Paving the critical path of drug development: the CDER perspective. Nat Rev Drug Discov 13: 783-784.

Yarbrough C (2016) Why animal health is the next big growth area. Life Science Leader, Erie, PA, USA. Available at: https://tinyurl.com/ybbmensc. 


\title{
11. Biological control with parasitoids
}

\author{
Erika T. Machtinger ${ }^{1 *}$ and Christopher J. Geden ${ }^{2}$ \\ 1Penn State University, Department of Entomology, 501 ASI Building, University Park, State College, PA \\ 16082, USA; ${ }^{2}$ United States Department of Agriculture, Agricultural Research Service, Center for Medical, \\ Agricultural and Veterinary Entomology, 1600 SW 23 ${ }^{\text {rd }}$ Drive, Gainesville, FL 32608, USA; etm10@psu.edu
}

\begin{abstract}
House flies, Musca domestica L., and stable flies, Stomoxys calcitrans (L.), are common pests in livestock, poultry, and equine facilities. Biological control of these 'filth flies' with pupal parasitoids can be used in conjunction with other control methods as part of an integrated fly management program. The principal filth fly parasitoids include members of the genera Muscidifurax and Spalangia in the hymenopteran family Pteromalidae, as well as others in the family Encyrtidae. Many of these parasitoids are native globally and have been introduced in many areas worldwide. Filth fly parasitoids are generally present in all habitats where suitable hosts can be found, including those associated with poultry, cattle, equine, swine, and other animal operations. They are also commonly occurring in refuse and forensic situations. Naturally occurring populations of parasitoids are typically insufficient to manage fly populations because of the flies' shorter development time and higher fecundity. Augmentation of natural parasitoid populations by releasing commercially-produced parasitoids can increase fly control. Here we review the biology of these biological control agents, and discuss the prospects for their successful use in managing filth fly populations in a variety of animal facilities.
\end{abstract}

Keywords: house fly, stable fly, Musca, Stomoxys, Pteromalidae, Encyrtidae, Muscidifurax, Spalangia, poultry, cattle, equine, swine

\section{Introduction}

House flies, M. domestica L., and stable flies, Stomoxys calcitrans (L.), (Diptera: Muscidae) belong to the group commonly known as 'filth flies.' These flies are two of the most common pests on livestock, poultry, and equine facilities. They are often present in high numbers on farms, developing in manure and decomposing organic matter such as wet feed, silage, rotting hay, and soiled bedding (Machtinger and Geden 2013; Malik et al. 2007; Meyer and Petersen 1983; Pitzer et al. 2011 a; Talley et al. 2009; Weinhold and Taylor 2013). House flies are associated with numerous pathogens that cause disease in humans and animals, and have been implicated in the movement of antibiotic resistant bacteria (Ghosh and Zurek 2015; Graczyk et al. 2001; Zurek and Ghosh 2014). Stable flies are obligate blood feeders that inflict painful bites. They have been estimated to cost the USA agricultural economy over $\$ 2$ billion per year in production losses in the cattle sector alone (Taylor et al. 2012).

An integrated pest management (IPM) program involving elements of cultural, mechanical, and biological control has the potential to significantly reduce reliance on chemical insecticides for management of filth flies. Commercially available pupal parasitoids (Hymenoptera: Pteromalidae) are the most common biological control agents used for filth fly management, and their use is increasing (Machtinger et al. 2013; USDA 2006). However, because the development of naturally occurring parasitoids is slower than their fly hosts, natural populations are typically insufficient to manage flies at acceptable levels. Augmentation of natural populations with commercially produced parasitoids can address this issue, and be effective in suppressing fly populations when 
coupled with other management methods (McKay et al. 2007; Skovgård and Nachman 2004). Though a vast body of literature exists on fly parasitoids, this work has not been thoroughly reviewed in decades, with the exception of a recent article on their potential use in equine facilities (Machtinger et al. 2015b). Here we review the biology of these biological control agents, and discuss the prospects for their successful use in managing filth fly populations in a variety of animal facilities.

\section{Taxonomy}

Chalcidoidea is one of the most diverse hymenopteran superfamilies. Species in this group range in size from the smallest known insect, Dicopomorpha echmepterygis Mockford at just $0.139 \mathrm{~mm}$, to species measuring over $30 \mathrm{~mm}$. There are approximately 22,000 described species of Chalcids in 2,100 genera, though chalicidoid diversity may range from 60,000 to 100,000 species worldwide (Gibson 2009). Members of this superfamily are largely parasitic with the primary dipteran parasitoids found in the families Encyrtidae and Pteromalidae.

Encyrtidae currently includes 496 genera and 3,735 species placed in 2 subfamilies; Encyrtinae with 353 recorded genera and 2,920 species, and Tetracneminae with 107 genera and 815 species. Tachinaephagus zealandicus Ashmead is the only encyrtid that is commonly associated with filth flies.

Pteromalidae is one of the largest families in Chalcidoidea, including 31 subfamilies, 588 genera, and 3,506 species. Members of family are morphologically and ecologically diverse, with dipteran parasitoids found primarily in the subfamilies Pteromalinae and Spalangiinae. The Pteromalinae is the largest subfamily and includes 320 described genera worldwide. Important filth fly parasitoids in this subfamily include Muscidifurax raptor Girault and Sanders, Muscidifurax zaraptor Kogan and Legner, Muscidifurax raptorellus Kogan and Legner, Urolepis rufipes (Ashmead), Nasonia vitripennis (Walker), and Trichomalopsis sarcophagae (Gahan). The Spalangiinae includes two genera, Spalangia Latreille 1805 and Playaspalangia Yoshimoto 1976 (Gibson 2009). The former genus includes the economically important and common species Spalangia cameroni Perkins, Spalangia endius Walker, Spalangia nigra Latreille, and Spalangia nigroaenea Curtis. A number of excellent keys are available for identifying the species that are typically encountered on livestock facilities (Gibson 2000, 2009; Gibson and Floate 2001; Rueda and Axtell 1985a). The guide by Rueda and Axtell (1985a) is long out of print, but the Gibson (2000) key is available online.

\section{Life history of pupal parasitoids}

Most of the common fly parasitoids belong to the Pteromalidae, a highly diverse family that includes the full range of life histories found throughout the superfamily Chalcidoidea. The majority of Pteromalids are idiobionts, developing as ectoparasitoids of larvae and pupae in many insect orders including Lepidoptera, Diptera, Coleoptera, and Hymenoptera. A number of Pteromalids are endoparasitoids, while other species are koinobionts. Some pteromalids are predatory rather than parasitic, and there are even a few phytophagous (gall-forming) species (http://www.nhm.ac.uk/chalcidoids).

The majority of the common ectoparasitic pupal parasitoids of filth flies have similar life histories (Table 1). After a female parasitoid locates a suitable host puparium, she penetrates through the puparial wall with her ovipositor, and deposits an egg on the external surface of the host pupa in the space between the host puparium and pupal cuticle (Gerling and Legner 1968) (Figure 1). 


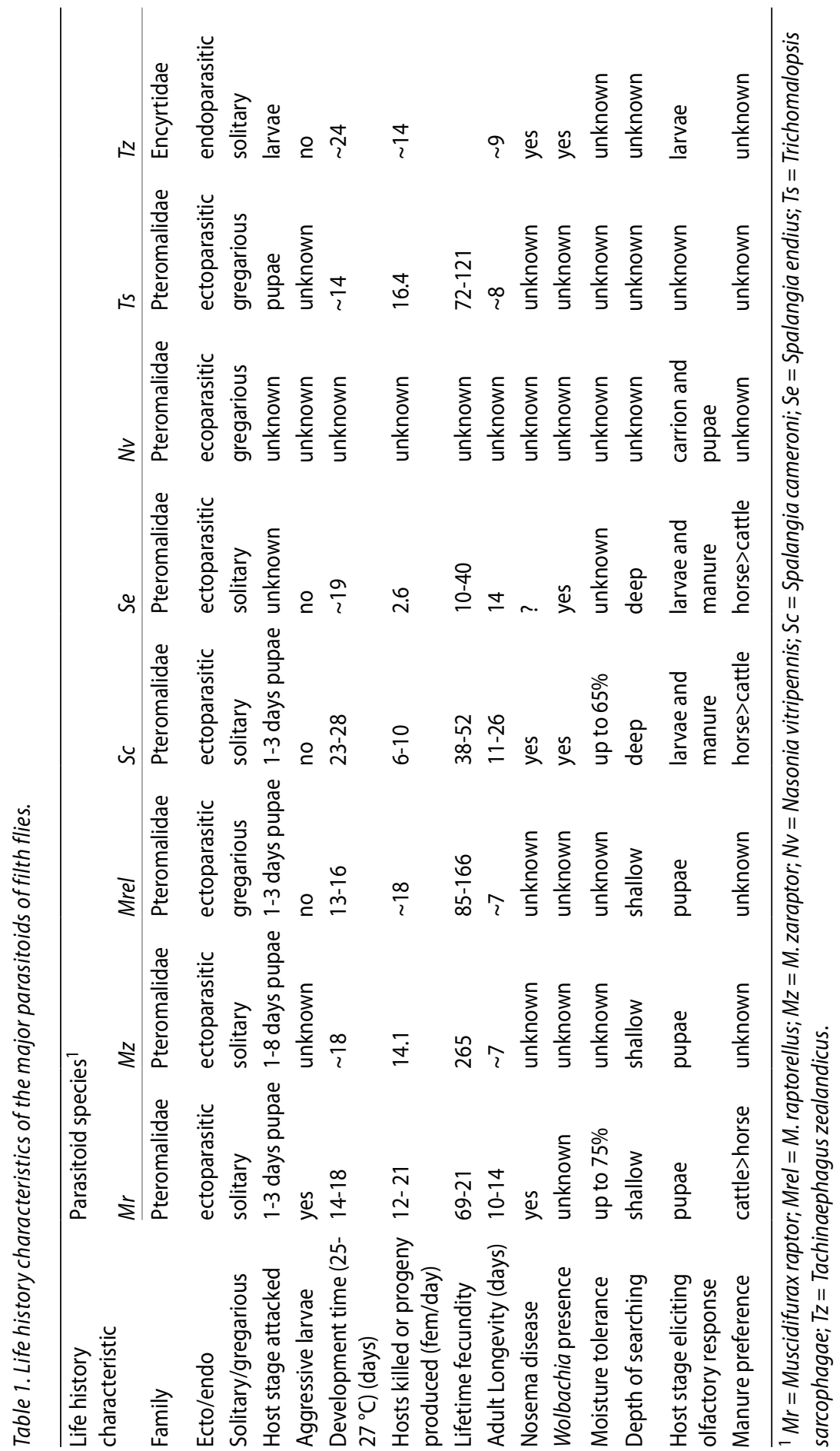




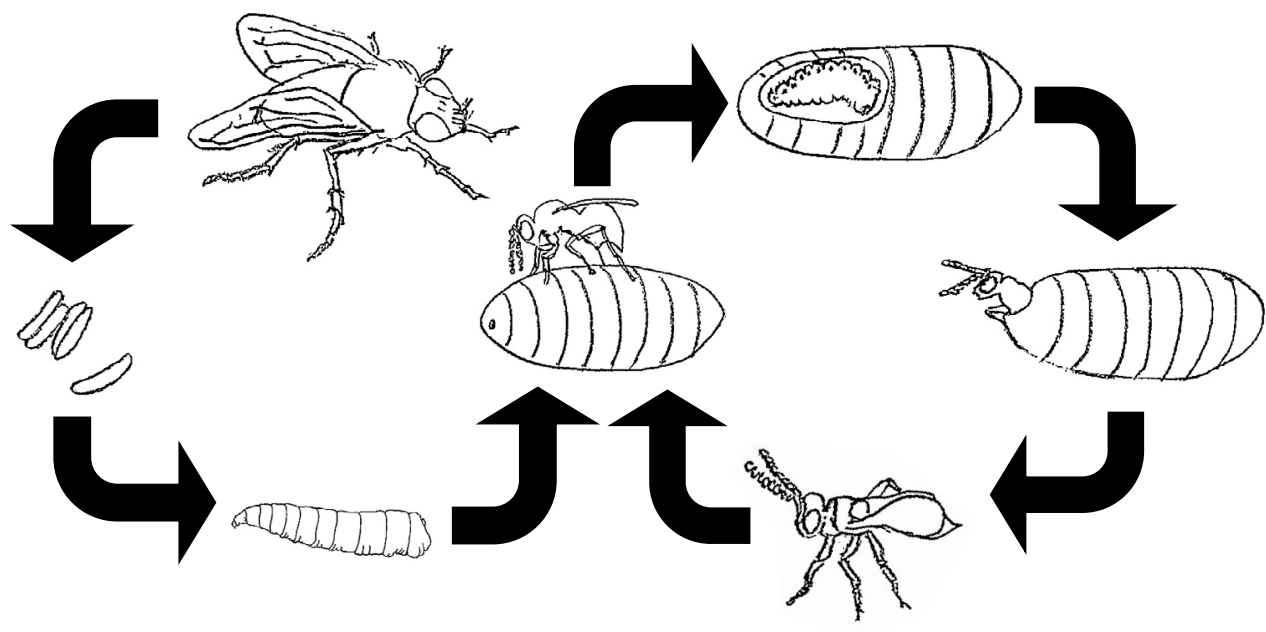

Figure 1. The life cycle and reproductive cycle of filth flies and several of the common species of filth fly parasitoids. Female flies (top left) lay eggs in suitable media that develop and pupariate. When a female parasitoid locates a suitable fly puparia (center) she assesses the host for suitability, and if deemed acceptable will feed and oviposit in the developing puparia, killing the fly and breaking the fly life cycle. Parasitoid larvae feed externally on the fly, mature within the host puparium (top right), emerge and continue the cycle (drawing by Erika Machtinger).

Adults of both sexes may feed from the fluid surrounding the fly pupa at this time as well. For solitary species, a single egg is laid while gregarious species will lay many eggs, depending on the suitability and quality of the host. Host age and size can influence the success of parasitism, and this can vary over the lifetime of the female as her ovipositor ages and accrues damage (Broski and King 2017). Three larval instars are completed prior to pupation (Figure 2). Pupation occurs within the host puparium and, following eclosion, adults must chew an exit hole to escape. Under normal temperatures during seasons with the greatest pest fly activity, development from egg to adult takes 14-30 days. This is of course dependent on species, temperature, sex, host, environmental factors, and even biotypes within species (Birkemoe et al. 2012).

Filth fly parasitoids have a haploid-diploid mechanism of sex determination that is common throughout the parasitic Hymenoptera (Heimpel and de Boer 2008). This arrhenotokous development is defined as that in which fertilized (diploid) eggs normally develop into females and unfertilized (haploid) eggs normally develop into males. However, some species present thelytokous development where males are absent or rare, and females reproduce asexually. This reproductive strategy is a result of the maternally inherited bacteria Wolbachia spp. Wolbachia has been removed from uniparental parasitoid species such as Muscidifurax uniraptor Kogan and Legner that exhibit thelytokous development by treatment with heat and antibiotics, generating arrhentokous strains (Gottlieb et al. 2002; Stouthamer et al. 1990). Other consequences of Wolbachia infection have been reproductive isolation between closely related species that may possess different Wolbachia strains, but viable hybrids can be produced if the symbionts are eliminated from these species (Breeuwer and Werren 1993, 1995). In some cases, different strains of Wolbachia may be present in populations of the same species of parasitoids, yet their function 

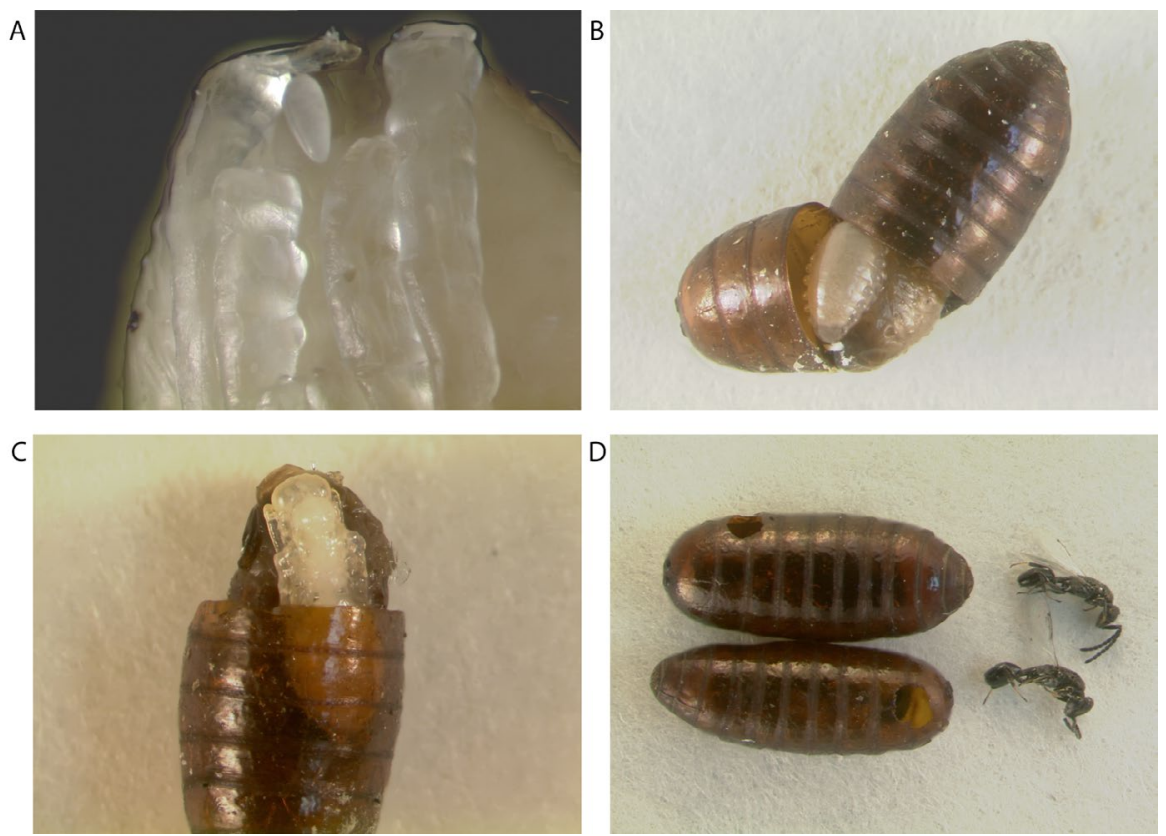

Figure 2. Preimaginal and adult stages of development of Spalangia cameroni (photos by Erika Machtinger and Ashley Church). (A) Parasitoid egg deposited on the host pupa. (B) Third instar. (C) Pupa of S. cameroni. (D) Emerged adult parasitoids and emergence holes.

in the biology of the species is not currently known (Machtinger et al. 2016a). The literature on Wolbachia is vast, complex, and changing rapidly (De Oliviera et al. 2015; Werren et al. 2008).

\section{Specific life histories of common and commercially available filth fly parasitoids}

\section{Muscidifurax raptor}

M. raptor (Figure $3 \mathrm{~A}$ ) is a very common solitary pupal parasitoid of filth flies in many animal facilities worldwide. As with all insects, development and life history characteristics are temperature dependent. However, at $25^{\circ} \mathrm{C}$ the greatest number of progeny produced per female per day is an average of 21.7 (Lysyk 2000). Reported lifetime fecundity ranges from 69-211 progeny (Geden et al. 1992a; Lysyk 2000; Morgan et al. 1989). At $25^{\circ} \mathrm{C}$, M. raptor males emerge as adults in $\approx 17$ days, with females emerging in 19 days (Geden 1997). Adult longevity ranges from 38 days at $15^{\circ} \mathrm{C}$ to 10 days at $33^{\circ} \mathrm{C}$ (Lysyk 2001a).

\section{Muscidifurax zaraptor}

Although the recovery of naturally occurring $M$. zaraptor (Figure $3 \mathrm{~B}$ ) is less frequent than $M$. raptor in most regions, these species have very similar biologies, and $M$. zaraptor may be the most frequently produced pteromalid parasitoid on a commercial scale. Progeny production per female per day at $25^{\circ} \mathrm{C}$ is documented at an average of 14.1 (Lysyk 2001a). Lifetime fecundity is approximately 265 offspring (Coats 1976), and this species is highly competitive against other 

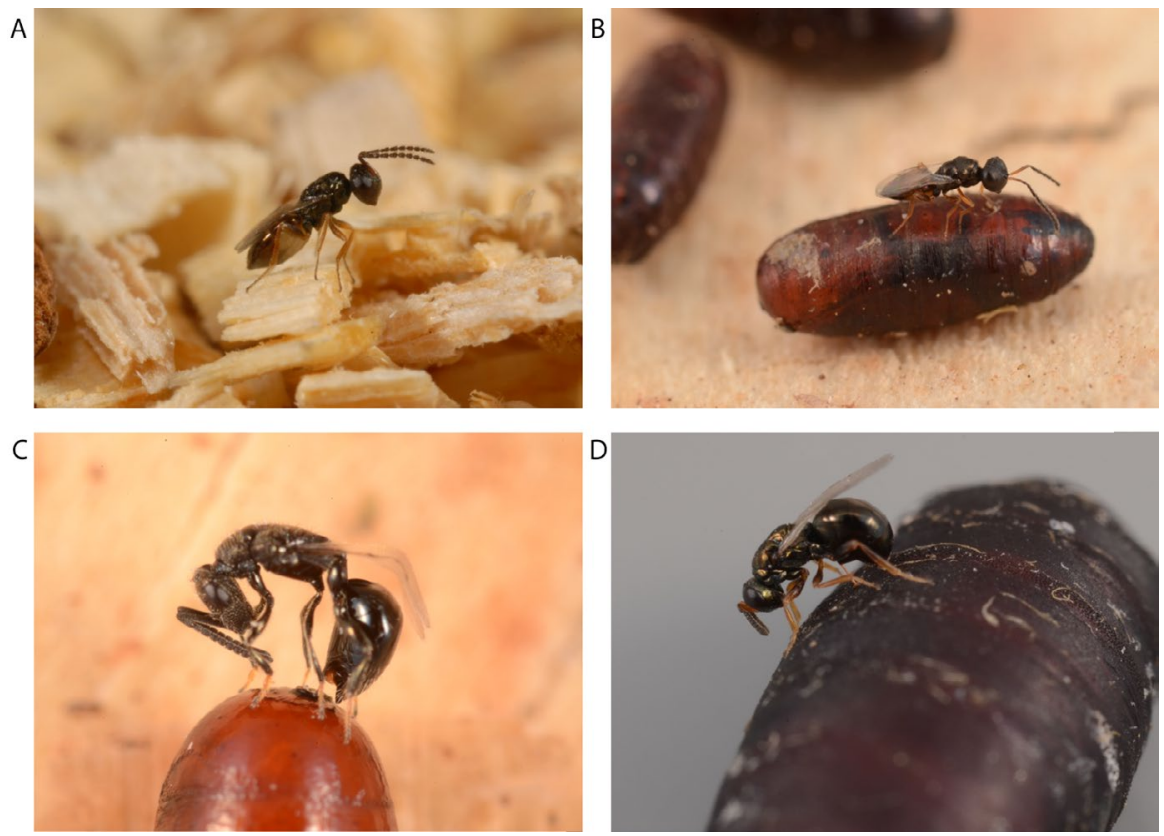

Figure 3. Filth fly parasitoids (photos by Lyle Buss). (A) Muscidifurax raptor. (B) Muscidifurax zaraptor. (C) Spalangia cameroni. (D) The jewel wasp, Nasonia vitripennis.

parasitoids. Female adults emerge slightly later than males, but emergence occurs at $\sim 18$ days (Lysyk 2001a). Adult longevity ranges from 7.7 days at $15^{\circ} \mathrm{C}$ to 11.8 days at $20^{\circ} \mathrm{C}$, but then drops to 6.5 days or less with subsequent increasing temperatures (Lysyk 2001a).

\section{Muscidifurax raptorellus}

M. raptorellus is a prolific parasitoid. This species is gregarious, producing 3-8 progeny from a single host. Lysyk (2001b) found daily fecundity per female was maximized at $25^{\circ} \mathrm{C}$ with an average of 18.2 offspring produced. At the same temperature, total lifetime fecundity ranges from 85-166 progeny, and adults of both sexes emerge at $\sim 16$ days (Lysyk $2001 \mathrm{~b}$; Petersen and Currey 1996). Longevity ranges from a high of 11.1 days at $15^{\circ} \mathrm{C}$ to 5.1 days at $33^{\circ} \mathrm{C}$ (Lysyk 2001b).

\section{Spalangia cameroni}

S. cameroni (Figure 3C) is a common solitary parasitoid. At $23^{\circ} \mathrm{C}$, Moon et al. (1982) found S. cameroni to emerge with an initial average reproductive capacity of a mean 12.9 progeny per female per day that declined $22 \%$ or more in subsequent days. Machtinger et al. (2016a) found progeny production differed by colony age, ranging from an average of 4.8 progeny per female per day on day 1 to 7.0 on day 5. S. cameroni has an average total lifetime fecundity ranging from 38 to 52 (Machtinger et al. 2016a; Morgan et al. 1989; Skovgard and Nachman 2016). Adult parasitoid emergence occurs at ca. $27 d$, with males emerging slightly earlier than females 
(Geden 1997). Longevity can differ by colony age with individuals from one colony reported to live a maximum of 26 days at $26^{\circ} \mathrm{C}$, with those of another surviving only 11 days at the same temperature (Machtinger et al. 2016a).

Spalangia endius

S. endius is another solitary parasitoid. Progeny production averages 2.6 progeny per female per day at $27.8^{\circ} \mathrm{C}$. Lifetime fecundity ranges from 10-40 progeny per female (Ables and Shepard 1974a; Morgan et al. 1976). Adult emergence occurs in ca. 19 days at $26{ }^{\circ} \mathrm{C}$ (De Araujo et al. 2012). Median female mortality is under 4 days, with maximum longevity calculated at 14 days at $27.8^{\circ} \mathrm{C}$ (Morgan et al. 1976).

Nasonia vitripennis

$N$. vitripennis (Walker) (Figure 3D) is gregarious, widely distributed in North America, and easy to rear. Females can live about two weeks (Davies 1975) and produce 115 progeny (Wylie 1966). Although sold commercially by at least one insectary, this is a generalist that is common in carrion and calliphorid systems (Peters 2010), but rare within livestock facilities (Legner 1967b). Despite its low cost and ready availability, N. vitripennis is ineffective for augmentative biocontrol in livestock systems (Kaufman et al. 2001; McKay and Galloway 1999). Even in laboratory tests, this species was unable to locate hosts in any of the test substrates unless they were placed directly on the surface (Frederickx et al. 2014). Ironically, N. vitripennis has become a model system for studying parasitoids in general, and it is the most-investigated of all the parasitoids associated with muscoid flies. The complete genome of this species has been sequenced, and this will undoubtedly provide invaluable information for future work with the more economically important Muscidifurax and Spalangia relatives (Werren et al. 2010).

Trichomalopsis sarcophagae

In Alberta, Canada, Spalangia spp. are rare whereas another species, T. sarcophagae Gahan is very common (Floate et al. 1999; Lysyk 1995). T. sarcophagae is a gregarious ectoparasitoid of house flies and stable flies that typically produces 4-8 parasitoids per host with a maximum lifelong production of 72-121 progeny per female (Lysyk 1998). It is one of the most cold-tolerant of the filth fly parasitoids (Floate and Skovgård 2004), which may account for its abundance in Canada. Females live for about 8 days at $25^{\circ} \mathrm{C}$, with most progeny in a cohort produced long before the $\mathrm{LT}_{50}$ is reached (Lysyk 1998). This species is easy to rear, has been produced commercially, and has been used with some success in augmentation programs in cattle feedlots (Floate 2003).

\section{Life histories of other species of interest}

Many surveys throughout the world have identified small proportions of Trichopria species in field collections. This genus belongs to the Diapriidae and the parasitoids that comprise it are endoparasitic koinobionts that can either be solitary or gregarious (Jamil et al. 1997; Masner and Garcia 2002; Morgan et al. 1990; Taley and Thakare 1979). Trichopria stomoxydis attacks stable fly pupae, but its low fecundity and short lifespan rule out use for biocontrol (Morgan et al. 1990). In contrast, $T$. nigra can kill $>10$ stable fly pupae and produce $>50$ progeny per day (Geden and Moon 2009). Unlike the pteromalids, T. nigra readily attacks and parasitizes newly formed pupae (Geden and Moon 2009) and can produce nearly 90 parasitoids per stable fly host pupa (Ferrero 2008). 
Development of T. nigra as a biocontrol agent has been limited by the inability of this species to develop in house flies, the host that is preferred by commercial insectaries.

T. zealandicus Ashmead is a larval encyrtid parasitoid that was first described in Australia (Ashmead 1904). It received considerable attention in the 1960 s and was imported and released onto farms in Denmark and California (Legner and Olton 1968; Mourier and Hannine 1971). Although Olton and Legner (1974) were able to produce T. zealandicus in house fly hosts, it has almost never been recovered from house flies in field surveys of parasitoids (reviewed in Geden and Skovgård 2014). Subsequent studies have found that this species performs best in calliphorid and sarcophagid hosts and poorly on house flies (Ferreira de Almeida et al. 2002; Geden and Moon 2009). Although T. zealandicus is capable of killing large numbers of filth fly larvae of various species, the natural history of this species appears to affiliate it with carrion/blow fly/flesh flies, rather than livestock/ manure/filth flies.

\section{Dispersal}

Dispersal to areas that may harbor developing hosts is necessary for a female parasitoid to reproduce under natural conditions. Movement of parasitoids from release points is also critical for effective parasitoid use in augmentation programs. Dispersal information for filth fly parasitoids is limited to a handful of studies, but the general trend indicates that the distances travelled by individuals from a release point is minimal. Published dispersal distances for Muscidifurax spp. vary with the study location and species examined. M. zaraptor were found to move no more than $8 \mathrm{~m}$ on feedlots (Pawson and Petersen 1988), and Smith et al. (1989) observed M. raptor to move less than $30 \mathrm{~m}$ from release points on a dairy. Tobin and Pitts (1999) found parasitism by M. raptorellus did not occur greater than $6 \mathrm{~m}$ away from release sites in a high rise poultry unit, regardless of release rate. In this case the majority of parasitized puparia were recovered within $2 \mathrm{~m}$ from the release point. Lysyk (1996) recovered M. raptorellus $22.5 \mathrm{~m}$ from their release location, and Petersen and Cawthra (1995) recovered M. raptorellus from puparia located on the perimeter of feedlot pens, approximately $48 \mathrm{~m}$ from the release point. In contrast, Floate et al. (2000) recovered M. raptorellus from puparia $100 \mathrm{~m}$ from the release point in confined cattle facilities with high numbers of released parasitoids $(>200,000)$, this being significantly further than previous studies. The dispersal range of $S$. cameroni has been evaluated in indoor swine facilities in Denmark, where Skovgård (2002) recovered the majority of released females $3 \mathrm{~m}$ from the release point, though recoveries were made up to $9 \mathrm{~m}$ away. In a pasture situation, Machtinger et al. (2015c) found that the majority of released $S$. cameroni parasitized hosts $<5 \mathrm{~m}$ from the release point. Taken together, the available evidence underscores the need to make releases as close as possible to known fly breeding sites when using parasitoids in an augmentation program.

Environmental and physiological effects may influence the rate and distance of parasitoid dispersal after adult emergence. At $15^{\circ} \mathrm{C}$, flight activity of $N$. vitripennis and S. nigra decreased to a minimum, whereas maximum activity was observed at $28^{\circ} \mathrm{C}$ (Abraham 1978). Smith et al. (1989) reported that flight in $M$. raptor and $U$. rufipes was initiated at $12{ }^{\circ} \mathrm{C}$ and reached a peak at $25^{\circ} \mathrm{C}$. Along with temperature, female age has been found to influence dispersal and movement in pteromalids. Newly emerged N. vitripennis females flew and dispersed widely compared to older females that had been parasitizing hosts for several days (Wylie 1958). King (1990a) found that the majority of $S$. cameroni females do not mate upon emergence, but instead disperse within 3 $\mathrm{h}$ to seek hosts and mate at new locations, which suggests a strong dispersal instinct. Nagel and Pimentel (1963) suggested that $N$. vitripennis deprived of hosts disperse faster than those given access to hosts. Davies and King (1975) observed that N. vitripennis females that were exposed to 
hosts for the previous $24 \mathrm{~h}$ were less likely to fly than females that fed only on sucrose without host exposure. In contrast, King (1993) found no effect of host presence on flight in N. vitripennis. Other factors that may influence dispersal such as inter- and intraspecific interactions, daylight, and humidity have not been evaluated in filth fly parasitoids.

\section{Microhabitat and host selection}

Upon emergence from host puparia, parasitoids need to locate suitable hosts for reproduction. Due to the ephemeral nature and patchy distribution of some of the habitats associated with developing filth flies, initially host-location is often long-range. This is particularly true when hosts and their parasitoids have developed in isolated resources such as manure in pastures. Females of many hymenopteran parasitoids use olfactory cues to locate hosts (Elzen et al. 1983; Read et al. 1970; Vinson 1975, 1976, 1981). Long-range host location by parasitoids is assumed to be determined by volatile semiochemicals originating from a parasitoid host, organisms associated with the host or host habitat or a combination of these factors (Vinson 1976). Successful parasitism can be divided into four basic steps; host habitat location, host location, host acceptance, and host suitability (Vinson 1976).

\section{Host habitat location}

Parasitoids often have preferences for certain habitats that increase the likelihood of finding hosts. Habitat location is likely achieved by identification of attractive long-range semiochemicals. Hosts may be chosen because they are available in a searched habitat, not necessarily because they are preferred (Cushman 1926; Townes 1960). Parasitism can vary in response to moisture (Geden 1999; Legner 1977; Smith and Rutz 1991b, 1991c), light (Smith and Rutz 1991b), temperature (Pawson and Petersen 1990), and accessibility of hosts (Geden 2002; Legner 1977; Pitzer et al. 2011 b; Rueda and Axtell 1985c). Greene et al. (1989) determined that the rates of parasitism of filth flies can also vary depending on the habitat and substrate preference.

Evaluations of habitat preferences have been conducted in selected animal facilities. On dairy farms, M. raptor has been found more often around drainage areas and the edge of the animal facilities than in shavings in calf hutches (Olbrich and King 2003). M. raptor was found primarily outdoors in spilled feed, straw, and manure piles (Skovgård and Jespersen 2000; Smith and Rutz 1991b). S. cameroni was recovered primarily indoors and preferred loose substrates such as straw and calf bedding on dairy farms (Smith and Rutz 1991b, 1991c). Skovgård and Jespersen (2000) corroborated these findings and reported S. cameroni most frequently indoors on Danish pig and cattle farms. S. cameroni preferred calf hutches with shavings on dairies in Illinois (Olbrich and King 2003).

Beef cattle feedlots consist entirely of outdoor fly breeding sites that include manure in piles and empty pens, as well as along various edges and under feed bunks. Silage and spilled feed in and around feed bunks provide additional breeding materials (Meyer and Petersen 1983). Petersen and Meyer (1983b) mapped parasitism patterns in these various habitats and found only small differences, with most of the parasitism attributed to S. nigroaenea and Muscidifurax spp. S. cameroni has been recovered primarily from soiled pine shavings and hay on equine facilities (Machtinger et al. 2016b). This preference was further supported by increased progeny production in these substrates in laboratory studies (Machtinger and Geden 2013). 
Abiotic habitat features can influence the preference of parasitoids, although some species display a degree of plasticity in habitat selection. S. endius preferred dry poultry manure at about $45 \%$ moisture, although it would search at broader moisture levels when hosts were limited (Geden 1999). Preferred substrate moisture levels of S. cameroni were $45-65 \%$ in poultry manure, but when hosts were limited this species was somewhat flexible, parasitizing hosts in substrates with up to $75 \%$ moisture (Geden 1999). S. cameroni has demonstrated habitat preferences when hosts are abundant (Geden 1999; Machtinger and Geden 2013). When fewer numbers of hosts were present per female parasitoid, however, this species exhibited diminished habitat discrimination, inducing greater total host mortality in common equine substrates. This suggests $\mathrm{S}$. cameroni has a flexible niche breadth that widens when hosts are in short supply.

Laboratory behaviour studies show genus level preferences for manure from different animals. S. endius and $S$. cameroni prefer equine manure over bovine manure in olfactometer experiments (Machtinger and Geden 2015). Conversely, M. raptor and M. zaraptor were found to prefer bovine manure over equine manure (Machtinger and Geden 2015).

\section{Host location and interspecific competition}

Semiochemicals can be used by parasitoids for short-range host location, although early experiments observing the effect of chemical stimuli on pteromalid parasitoids delivered conflicting results. In studies investigating chemical cues on host selection by $N$. vitripennis, Laing (1937) found individuals to be positively attracted to cues from decaying meat, a common habitat for hosts of this species. However, subsequent studies found that N. vitripennis was not attracted to host habitat alone, but instead by a combination of carrion and hosts (Edwards 1954; Wylie 1958). In the laboratory, M. zaraptor was strongly attracted to odours of house fly puparia, however female wasps were not attracted to manure mixed with decomposing organic matter, as would be found where developing flies are present (McKay and Broce 2003). Fresh manure was found to be slightly repellent to $M$. zaraptor, suggesting that habitat was not the main cue for this species in host location, but rather semiochemicals emitted from host puparia. Similar attraction to host puparial odours over habitat odours (equine manure) has been found with M. raptor (Machtinger et al. 2015a). Conversely, virgin S. cameroni females responded strongly to combined odours of house fly puparia with chicken manure, the host development habitat (King 1990a). When comparisons were made between host development substrate with larvae and substrate with puparia, S. cameroni strongly preferred odours of larval house flies in their development habitat than those of puparia and habitat (Machtinger et al. 2015a). Although these were close-range studies in the laboratory, the responses show that even closely related parasitoids may address the problem of host location in different ways, and this may be important in considering multiple species for augmentative biocontrol releases.

Differential host-seeking behaviour can be observed when considering the depth at which hostseeking occurs within a substrate. Muscidifurax spp., including M. raptor and M. zaraptor, generally search for host fly pupae in the top $3 \mathrm{~cm}$ or less of the host development substrate (Geden 2002; Legner 1977; Rueda and Axtell 1985c). The competitive searching ability of $M$. raptorellus has not been extensively investigated, but it was found to be ineffective at locating and parasitizing pupae in soiled shavings from an equine farm under laboratory conditions (Pitzer et al. 2011b). S. endius searches uniformly for hosts throughout the substrate, parasitizing hosts at depths of 5-10 cm (Geden 2002; Rueda and Axtell 1985c), much deeper than Muscidifurax spp. Additionally, S. endius was found to be as successful as $S$. cameroni at locating and parasitizing hosts in pine shavings soiled with equine waste (Pitzer et al. 2011b). Under interspecific competition pressure, 
S. cameroni females may search deeper for hosts in host development substrates than Muscidifurax spp., with depths of up to $10 \mathrm{~cm}$ reported (Rueda and Axtell 1985c), although King (1997) observed little change in the searching behaviour of M. raptor and S. cameroni when they were alone or in combination. S. cameroni was found to be effective at locating and parasitizing house fly hosts that had pupated in soiled shavings from an equine farm (Pitzer et al. 2011b), as well as other common equine-generated substrates (Machtinger and Geden 2013). The type and degree of compaction of bedding types may also influence the ability of parasitoids to locate hosts, even in species that are intrinsically prone to searching deeper in host habitats (Geden 2002; King et al. 2014).

Parasitoids can avoid competition with other species by concentrating their searching behaviour in different sub-habitats, seeking out different host stages, parasitizing different host ages, and altering foraging depth as discussed above. Larvae of solitary Muscidifurax species also have aggressive first instars that explore within the host puparium after hatching and kill supernumeraries of their own or other species, including the gregarious $M$. raptorellus (Geden et al. 2014; Wylie 1972). Adults of these species can also detect and avoid parasitizing hosts that are already occupied by conspecifics (Wylie 1971). These mechanisms mitigate the risks of M. raptor and M. zaraptor sharing a host that can only provide resources to produce a single individual and give them a distinct advantage over Spalangia spp. Subsequently, solitary Muscidifurax are typically the victors when they are forced to compete for hosts with S. cameroni or S. endius (Ables and Shepard 1974a; Propp and Morgan 1983; Wylie 1972).

\section{Host acceptance and suitability}

House fly and stable fly hosts are of nearly equal suitability for all of the common pteromalid filth fly parasitoids (Geden and Moon 2009; Geden et al. 2006). Nevertheless, in several laboratory studies house fly pupae produced slightly more parasitoids than stable flies. House fly pupae are larger than stable fly pupae, and host size alone can influence parasitoid behaviour and life history parameters (King 1988, 1990a, 1990b, 1991). Machtinger and Geden (2013) did not find broad host preferences of S. cameroni, however there were differences in the effectiveness of S. cameroni parasitizing hosts in six equine substrates. Mandeville and Mullens (1990b) found that M. zaraptor preferred house fly hosts over the smaller pupae of Fannia canicularis (L.) in the laboratory.

Under field conditions, the distribution of different potential hosts reflects the microhabitat preferences of the flies. For example, immatures of house flies and stable flies occur in different proportions in many different sub-habitats on cattle feedlots (Meyer and Petersen 1983), and F. canicularis pupate deeper in poultry manure than house flies. Parasitism patterns in the field may therefore reflect preferences of the parasitoids for different hosts, different habitats, or a combination of both. Petersen and Meyer (1983a) found that stable fly pupae in Nebraska feedlots were parasitized most frequently by S. nigroaenea, whereas $M$. raptor was the dominant species in house fly hosts. There was no evidence for host preferences (Fannia versus house fly) by Muscidifurax spp. or Splangia spp. in California poultry houses (Mandeville and Mullens 1990a), and Skovgård and Jespersen (1999) saw little differences in proportions of parasitoid species attacking house flies and stable flies on Danish pig and cattle farms. Similarly, Olbrich and King (2003) found little evidence for parasitoid preferences after accounting for differential habitat and seasonal distribution of house and stable fly hosts. Most of the common filth fly parasitoids are opportunistic species that will sting and develop on a wide range of hosts outside the guild of filth flies. The known host list for S. cameroni, for example, contains about 50 species and includes a tachinid parasitoid of silkworms (http://www.nhm.ac.uk/chalcidoids). M. raptor is known to 
parasitize seven families of flies, including fruit flies, sepsids, and syrphids in addition to the more 'usual' muscids, anthomyiids, calliphorids, and sarcophagids (http://www.nhm.ac.uk/chalcidoids). It is perhaps not surprising that host preferences by such parasitoids are muted and flexible.

\section{Parasitoid abundance and distribution}

\section{Monitoring methods}

Parasitoids can be monitored by collecting wild fly pupae and holding them for parasitoid emergence or by placement/retrieval of laboratory-reared pupae ('sentinel pupae'). Both methods have advantages, limitations, and biases for sampling different species (reviewed in Petersen 1986a; Petersen and Watson 1992). Collection of wild pupae perhaps provides the most realistic estimate of relative species abundance, but this method is time-consuming, removes potentially suitable hosts for future parasitism, and makes it difficult to monitor the same locations over time. Because it is highly dependent on the size and distribution of the fly population, sample sizes are generally unequal and tend to over-represent hot spots where pupae are locally abundant. Sentinel pupae allow more accurate assessments of host mortality, can be placed consistently in known locations and numbers, and ensure that hosts are always available for parasitoids to discover. However, this method inevitably positions the hosts in less natural presentations without accompanying larval kairomones, and does not collect parasitoids that parasitize fly larvae (e.g. Tachinaephagus zealandicus) or newly formed pupae (some Trichopria spp.).

The two monitoring methods provide substantially different pictures of relative abundance of parasitoid species. The most conspicuous difference is in the relative proportions of Muscidifurax and Spalangia species. Host-seeking by Muscidifurax spp. focuses near the surface of the substrate using pupal cues, whereas Spalangia spp. search more deeply and rely on host larval odours to find pupae (Geden 2002; Machtinger et al. 2015a). This is likely a mechanism to avoid interspecific competition. Therefore, it is not surprising that sentinels give a larger estimate of relative abundance of Muscidifurax than do collections of wild pupae (Gibson and Floate 2004; Meyer and Petersen 1982; Meyer et al. 1990; Petersen and Watson 1992; Rutz and Axtell 1980). In a review of natural parasitism assessments worldwide (Table 2-5), Spalangia spp. compose $78.3 \%$ of species recovered when naturally developing hosts are collected. In contrast, traditional sentinels recovered just $48.8 \%$ Spalangia spp. across all habitats analysed. Mean total parasitism collected from naturally occurring puparia is $34.9 \%$, more than double that found using sentinel puparia (16.2\%). Recently a third option that combines some of the advantages of sentinels and wild collections have been evaluated (author CJG, unpublished data). This method involves the deployment of hosts in rearing medium while they are still larvae and recovering them several days after pupation. Collections made using the larval 'modified sentinel' method yield large numbers of parasitoids and give relative abundance estimates similar to those made from wild pupal collections (Table 6). This method would also detect parasitoids that attack fly larvae and newly formed puparia. 


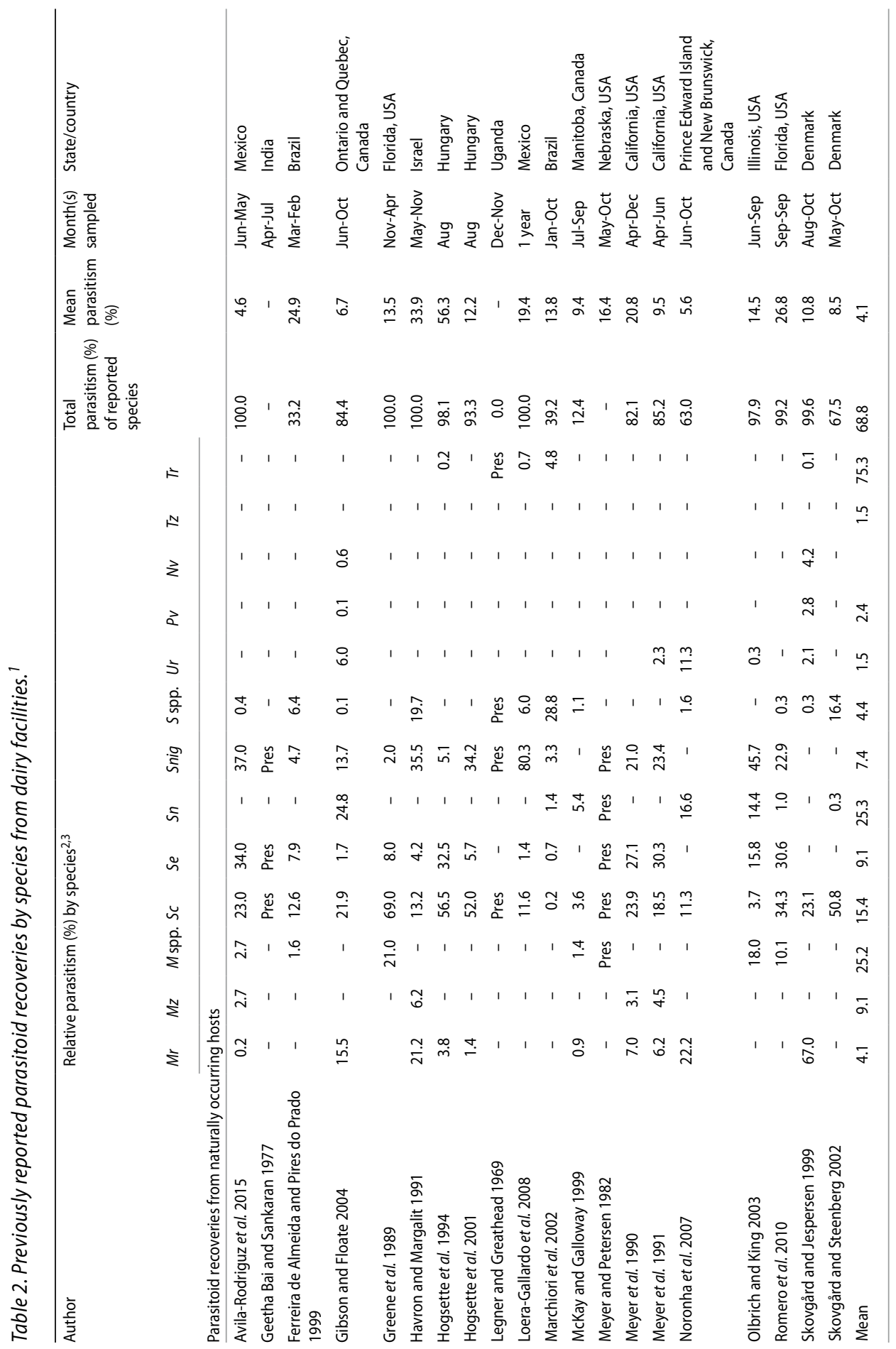




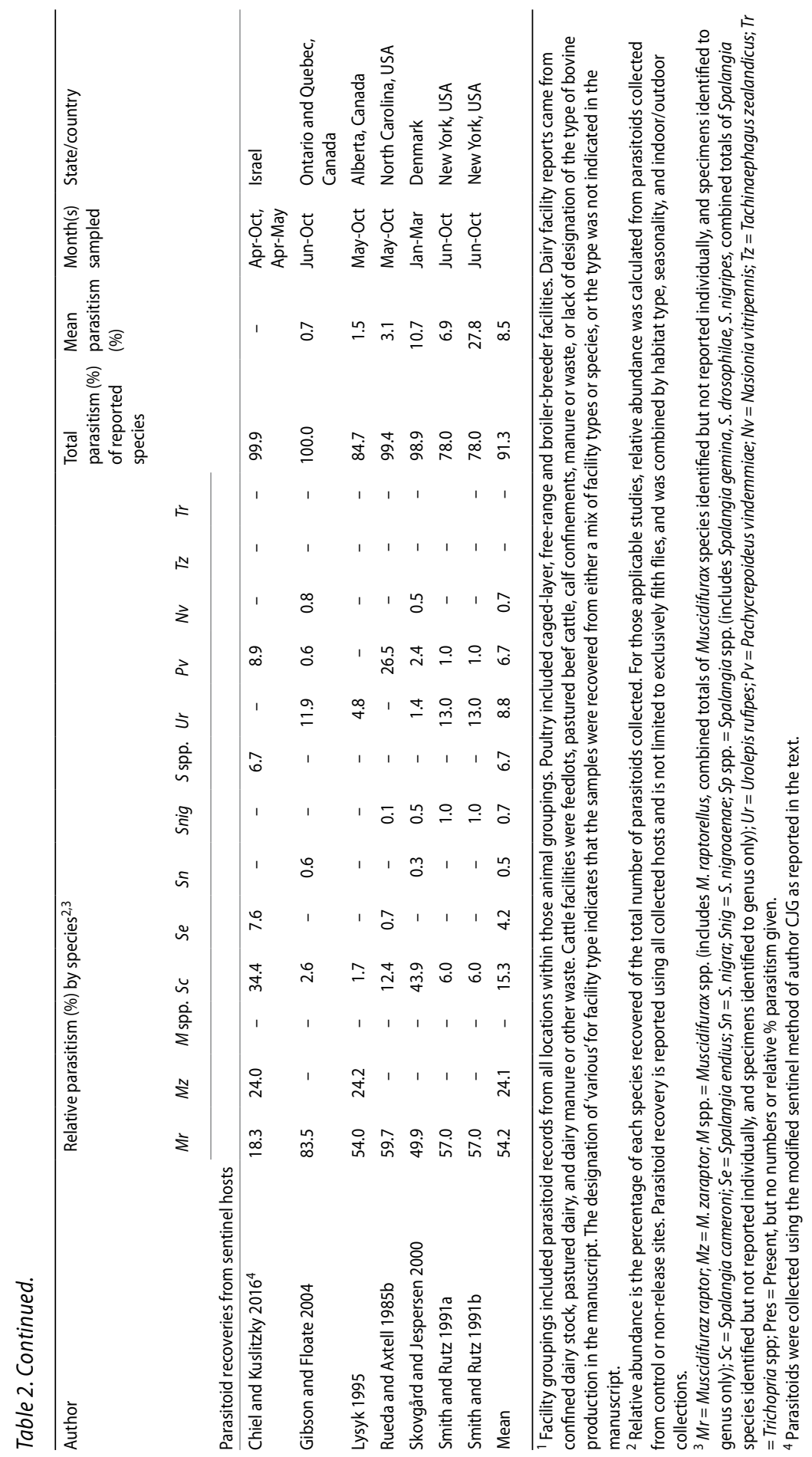




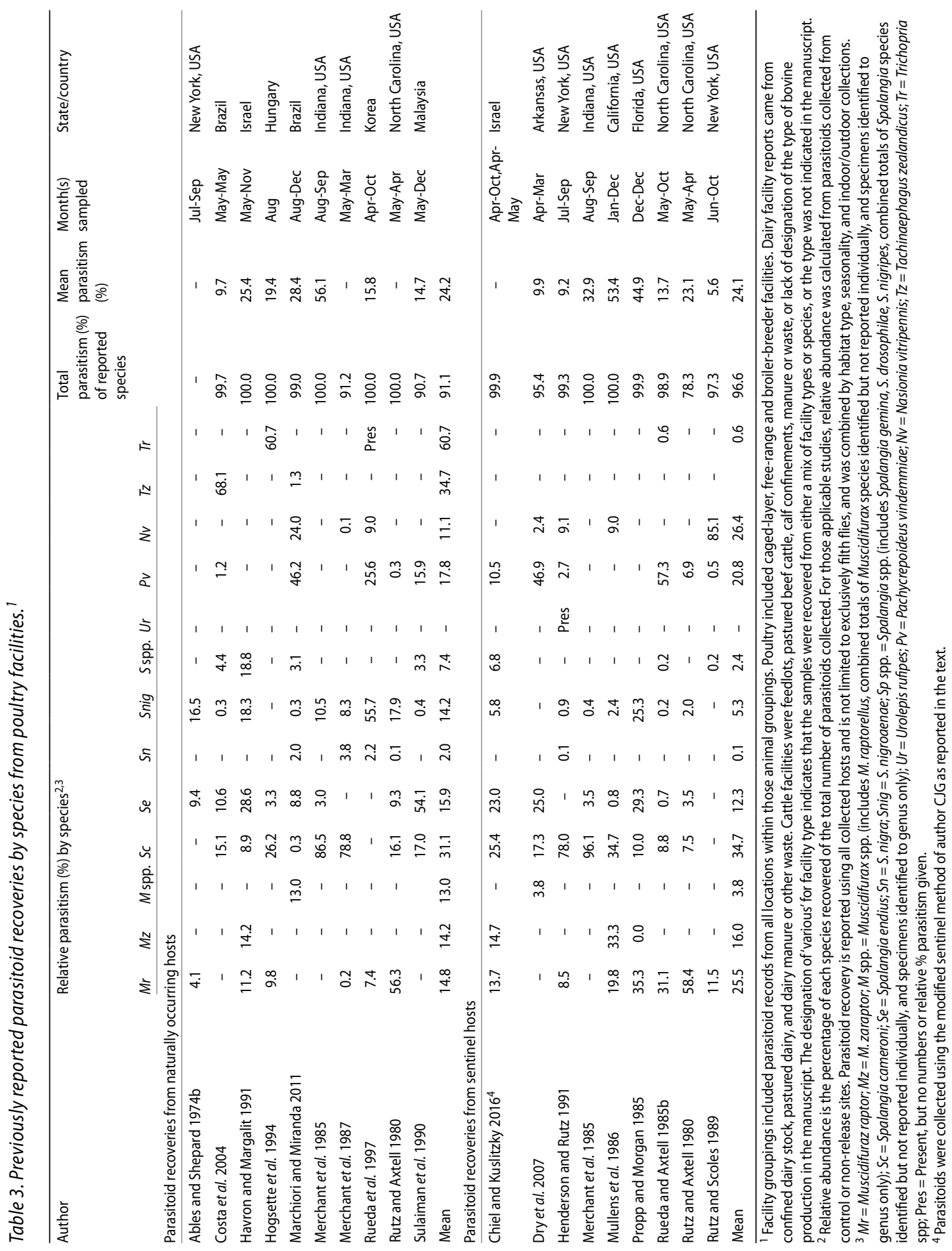




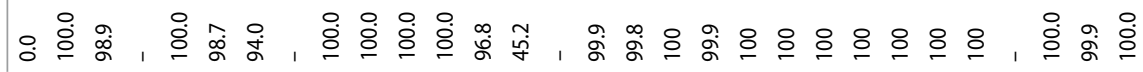

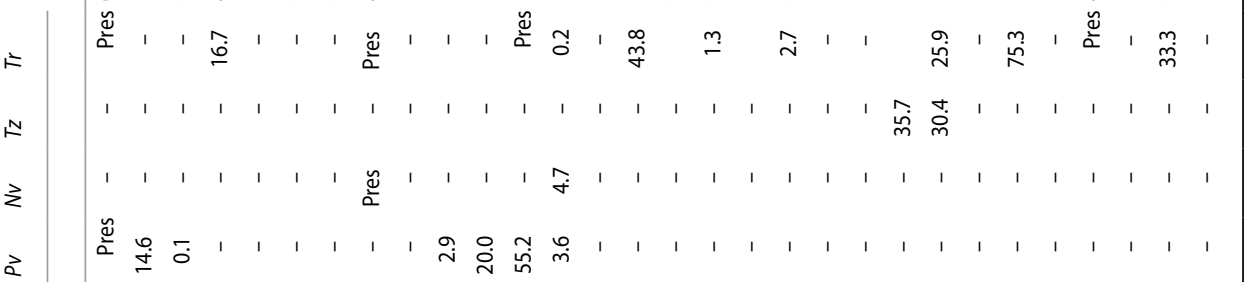

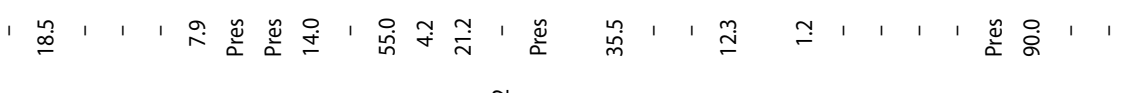




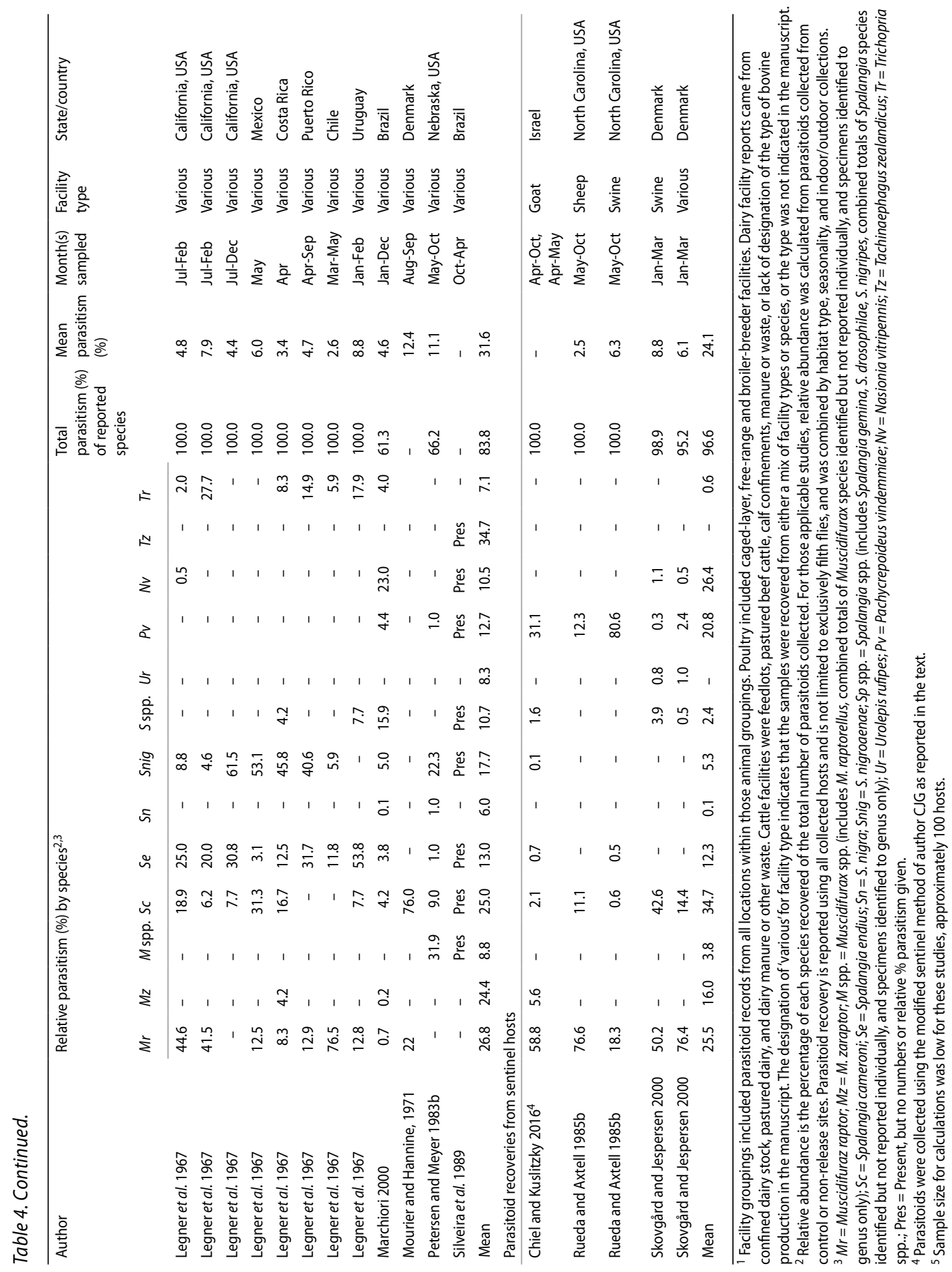




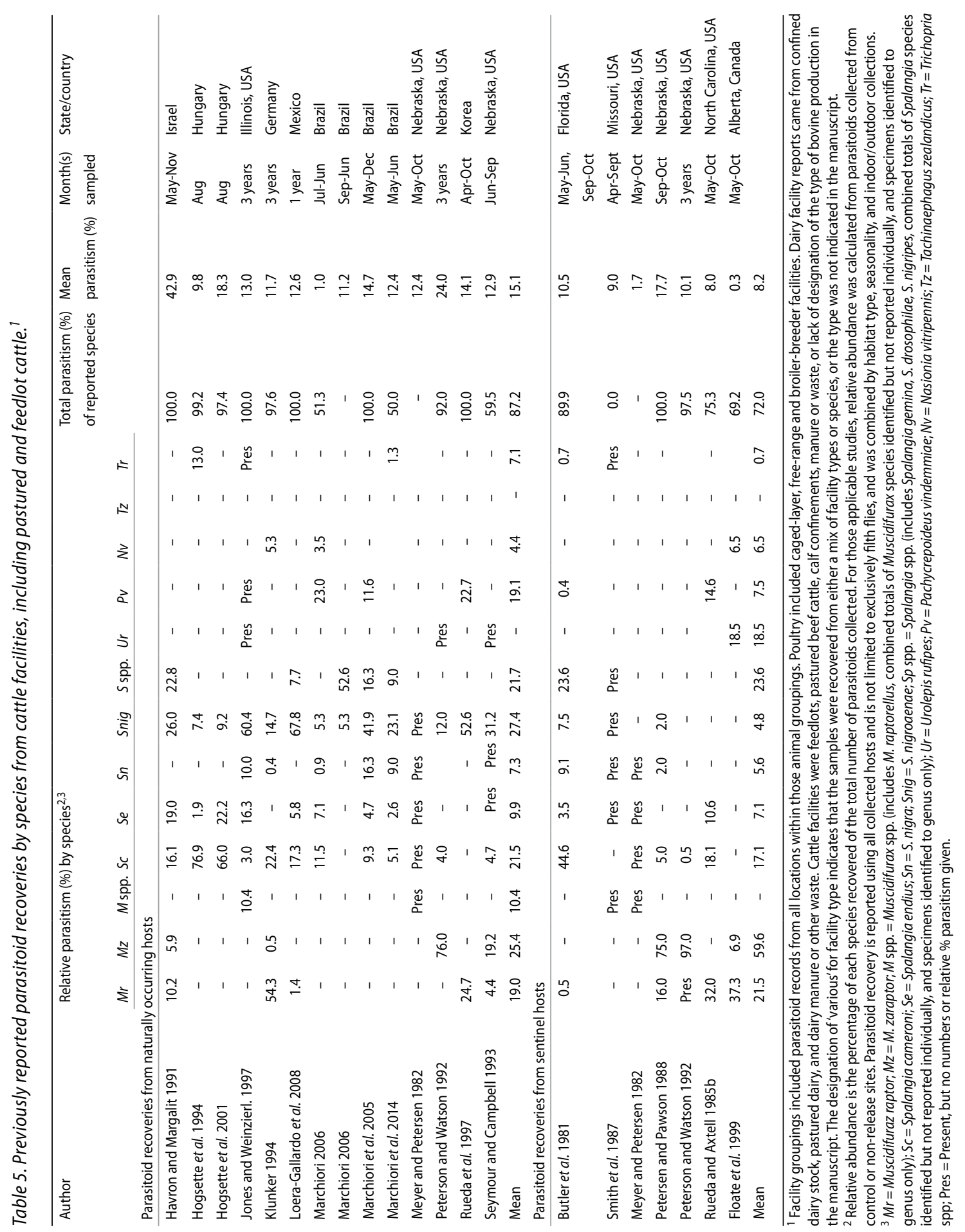


Table 6. Percent relative abundance of house fly parasitoids collected using different methods on a Florida dairy (previously unpublished data).

\begin{tabular}{lcccc}
\hline Species & Wild pupae & $\begin{array}{l}\text { S0-pupae } \\
\text { conventional } \\
\text { sentinel bags }\end{array}$ & Pupae & Larvae \\
\cline { 3 - 4 } & & & 53.1 & 17.7 \\
Muscidifurax raptor & 13.1 & 20.2 & 6.6 & 20.1 \\
Spalangia cameroni & 37.8 & 2.7 & 7.7 & 46.1 \\
Spalangia endius & 28.6 & 14.2 & 0.4 & 2.3 \\
Spalangia nigra & 0.5 & 0.0 & 0.2 & 6.7 \\
Spalangia nigroaenea & 19.6 & 0.0 & 1.0 & 1.6 \\
Spalangia drosophilae & 0.0 & 0.0 & 30.9 & 5.5 \\
Pachycrepoideus vindemmiae & 0.5 & 62.8 & 15.9 & 76.8 \\
\% total Spalangia & 86.5 & 16.9 & 3,690 & 14,040 \\
Total no. parasitoids & 444 & 549 & & \\
\hline
\end{tabular}

\section{Natural parasitism}

Pteromalid parasitoids occur naturally in all areas where suitable hosts can be found including poultry, cattle, equine, swine, and other animal operations as well as refuse and forensic situations. However, because most facilities produce an abundance of attractive breeding substrates for fly development and as fly hosts have faster development times than their parasitoids, natural parasitism of house and stable fly puparia is generally low, ranging from $0.25-56.3 \%$ in published natural parasitism studies (Table 2-5). Across all studies, mean parasitism is $22.7 \%$, although differences in methodologies may make comparisons difficult. Determinations of natural parasitism can be influenced by sampling method and size, region, time of year, and other biotic and abiotic factors, and many published studies reported parasitism from limited sampling events, sometimes as few as one. Collections can also vary from year to year on the same farm, so data on relative abundance of species made from a single year are not necessarily indicative of overall relative abundance at a given location.

Analysis of previously published literature on natural parasitism has revealed slight differences by animal facility type. Mean total parasitism at dairy facilities is $38.7 \%$ (Table 2), and $24.2 \%$ at poultry facilities (Table 3). Slightly lower parasitism has been found in swine (Table 4), cattle (Table 5), and equine facilities (Table 4) with 16.6, 11.7, and 10.0\% parasitism rates, respectively. These differences may be a result of the influence of husbandry practices on host presence and location among animal facilities, as well as parasitoid preferences for different microhabitat types.

\section{Distribution}

Filth flies and their parasitoids are likely of African origin, evolving over the Ethiopian region (Legner and Greathead 1969). The principal filth fly parasitoids are common and have been introduced in many areas worldwide, potentially disseminated accidentally with their fly hosts as humans travelled across the globe. Most species occur, in varying relative proportions, on all continents except Antarctica (Boucek 1963). Overall, S. cameroni has been reported from the widest geographic range with accounts from 71 countries (http://www.nhm.ac.uk/chalcidoids). 
Other species with high frequencies include $S$. endius reported from 65 countries, $P$. vindemmiae 63 , S. nigroaenea 62, and M. raptor 46. S. nigra, T. zealandicus, M. zaraptor from, U. rufipes have country records of $39,27,9$, and 8 , respectively. Surveys of natural parasitism have recovered many of the common parasitoids in Africa (Kenya, Mauritius, South Africa, Uganda), Asia (China, India, Israel, Korea, Malaysia, Philippines, Thailand), Europe (Denmark, Germany, Hungary, Norway), North America (Canada, USA including Puerto Rico, Costa Rica), Oceania (American Samoa, Australia, New Zealand), and South America (Brazil, Chile, Uruguay) (Table 2-5 and references therein), suggesting that parasitoids spread with their fly hosts long ago.

\section{Overwintering}

The relationship between temperature and life history parameters is well documented for most species over the range of temperatures typically encountered when flies are active (Ables and Sheppard 1976; Ables et al. 1976; Geden 1996, 1997; Lysyk 2000, 2001a, 2000b; Mann et al. 1990b; Skovgård and Nachman 2015). Parasitoid populations in warmer regions of the world track those of their hosts, with both responding similarly to changing temperatures. Overwintering in cold climates, where fly populations are nearly absent for several months of the year, poses challenges for parasitoids. Mourier (1971) observed that pupae parasitized by S. cameroni and M. raptor survived the winter in outdoor manure piles in Denmark where temperatures reached $-10^{\circ} \mathrm{C}$. The developmental threshold for several species is around $13^{\circ} \mathrm{C}$, and immature stages can survive prolonged exposures of $5-10{ }^{\circ} \mathrm{C}$ (Guzman and Petersen 1986a, 1986b; Lysyk 2004). Guzman and Petersen (1986a) did not find evidence for diapause in S. cameroni or M. zaraptor, but both species had better tolerance of freezing conditions in the laboratory if they were cold-acclimated by exposure to $10^{\circ} \mathrm{C}$ before being subjected to freezing. Immature M. raptor, M. zaraptor, S. cameroni, S. nigroaenea, S. nigra, and U. rufipes all survived, to varying degrees, Nebraska winters in outdoor silage piles (Petersen and Meyer 1983b). S. cameroni was not observed to survive outdoor winter conditions in Alberta, Canada (mean daily temperature, $-3.5^{\circ} \mathrm{C}$ ), but this observation was made of lab-reared individuals that were placed in the field without a period of cold-acclimation (Floate and Skovgård 2004). Variable, albeit low, winter survival rates in Canada were observed with $M$. raptor, M. zaraptor, M. raptorellus, N. vitripennis, U. rufipes, and T. sarcophagae. Winter survival of M. raptor, S. cameroni, and U. rufipes inside Danish dairy (mean temperature, $8.6^{\circ} \mathrm{C}$ ) and pig (mean temperature, $15.4^{\circ} \mathrm{C}$ ) barns was substantially higher than at outdoor Alberta sites. Parasitoids that began the winter as older larvae or pupae had higher survival in both locations than young individuals (Floate and Skovgård 2004).

\section{Use of pupal parasitoids in augmentative biological control programs}

Flies have shorter development times and higher fecundity than their parasitoids. Natural parasitoid populations therefore always lag behind their hosts and cannot provide adequate suppression once fly population sizes become problematic. Natural enemies can be conserved by minimizing insecticide usage (discussed below) and keeping breeding substrates as dry as possible. Even under ideal conditions, multiple augmentative parasitoid releases are usually needed to get maximum benefit from them as biocontrol agents.

Commercial availability of pupal parasitoids has facilitated augmentative releases for control of filth flies. Evaluations of the effectiveness of releases have had mixed results. Filth fly populations have been reduced in some situations (Crespo et al. 1998, 2002; Geden et al. 1992c; Morgan and Patterson 1990; Petersen and Cawthra 1995; Skovgård and Nachman 2004) but not in others (Andress and Campbell 1994; Kaufman et al. 2001; McKay and Galloway 1999; Meyer et al. 1990; 
Morgan 1980; Weinzierl and Jones 1998). These disparities may have been partially due to environmental factors and variables that affect host or parasitoid abundance and distribution (Skovgård 2004), and/or biological characteristics of the parasitoid species used. Recent research suggests that parasitoids commonly used as biological control agents may have preferences for specific animal manures (Machtinger and Geden 2015), and that these preferences are not easily manipulated or influenced by post-emergencepost-emergence learning (Taylor et al. 2016). Commercial colony quality, microhabitat preferences, and a lack of understanding of the timing and methods of release could have also influenced the success of releases (Patterson and Rutz 1986; Peterson et al. 1983). Additionally, existing active parasitoid populations may interfere with the establishment or function of a newly released species (Legner et al. 1990). The biological system in the release location is an important consideration for an augmentative biological control program, including the type of animal facility, physical characteristics of structures, and husbandry choices being implemented by operators.

\section{Mass rearing and quality control}

Common parasitoids used in augmentative control programs for filth flies are sold commercially, but there is minimal understanding of how long-term mass-rearing might alter dispersal and host-seeking of these parasitoids. Pteromalid and other filth fly parasitoids have been shown to experience inbreeding depression after prolonged colony rearing that could negatively affect dispersal and host-seeking. Historically, inbreeding depression in haplodiploid parasitoids has been considered minimal. In theory, detrimental recessive genes will be expressed in males and deleted (Antolin 1999; Bruckner 1978; Crozier 1985; Werren 1993). However, Henter (2003) found substantial inbreeding depression in Uscana semifumipennis Girault (Hymenoptera: Trichogrammatidae) after only four generations. Legner (1979) found a significant decline in longevity after M. raptor was in colony production for 110 generations. Declines in searching ability were observed by Geden et al. (1992a) in M. raptor after only two generations. Declines in longevity and fecundity were also observed in these studies on M. raptor, though, although Nosema disease was thought to be primarily responsible for these declines in the latter study. Machtinger et al. (2016a) found that several colonies of S. cameroni collected from the same location on a dairy in Florida in different years differed significantly in several life history parameters including longevity, progeny production, and host seeking ability. This supports that new colonies should be started with as broad a genetic background as possible.

Large-scale production of parasitoids presents a variety of operational and quality control challenges (Figure 4). The most fundamental of these is the difficulty of preventing fast-developing, high-fecundity species such as Muscidifurax spp. and N. vitripennis from invading and overtaking colonies of slower and less fecund Spalangia species. Species purity requires constant monitoring because parasitoid contaminants can cause a collapse in slower developing species after just a few generations. Isolating different species in separate buildings is critical for those operations that wish to rear multiple parasitoid species. Employee and equipment traffic between the buildings should be kept as close to zero as possible. The host fly production part of the operation should also be kept strictly separate, with rearing trays and harvesting procedures protected from stray oviposition by rogue parasitoids from outdoors or other rearing areas.

Pest fly population increases follow seasonal cycles in most parts of the world. This requires insectaries to adjust production targets upward and downward to meet the needs of stakeholders without overproducing products during times of low demand. Host pupae can be stockpiled in anticipation of rising parasitoid production schedules by refrigerating pupae that have been 


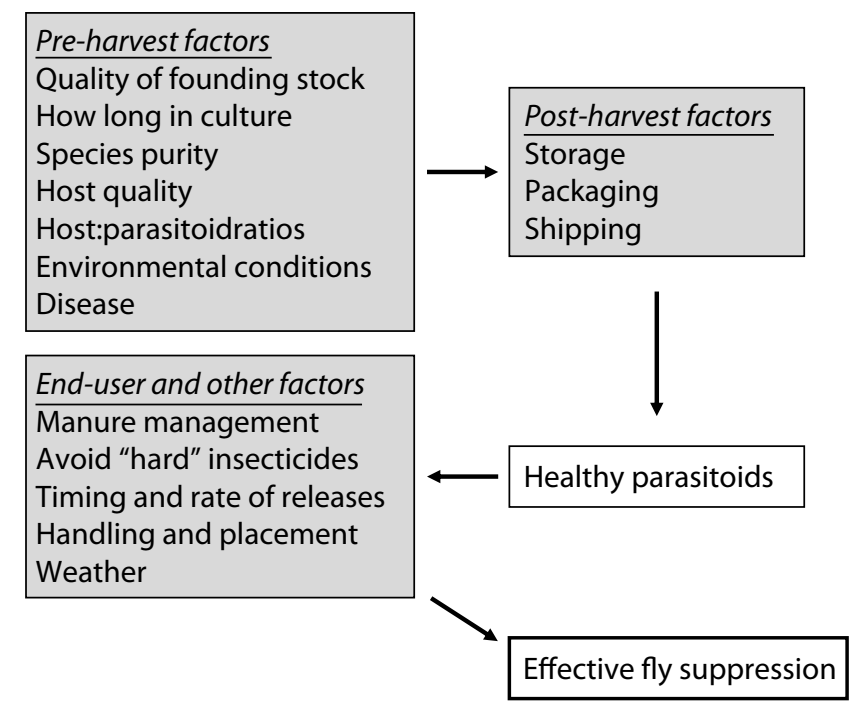

Figure 4. Establishing new colonies of parasitoids for commercial purposes, and their successful use, requires several steps in a long chain of events.

killed by heat shock (Geden and Kaufman 2007). Parasitized pupae can also be stockpiled for several months under cool storage conditions (Lysyk 2004). Rearing temperatures can be raised to accelerate development, but only within limits - very high temperatures can result in parasitoid sex ratios that are distorted toward males (Birkemoe et al. 2012). Host:parasitoid ratios can be adjusted seasonally, but need to be kept in balance to avoid the extremes of under-parasitizing hosts, which 'wastes' host resources, and over-parasitizing hosts, which results in high mortality and poor efficiency of host use (Legner 1967a; Mann et al. 1990a; Skovgard and Nachman 2015)

Pathogens that occur at low to moderate levels in nature can cause debilitating diseases under the pressures of mass production. This is especially true of Nosema disease, which affects many species of pteromalid parasitoids as well as T. zealandicus (Dry et al. 1999; Geden et al. 2003; ZchoriFein et al. 1992). The high efficiency of maternal transmission coupled with the opportunities for horizontal transmission under crowded conditions results in rapid spread and amplification of disease (Geden et al. 1995). The best way to avoid disease problems is to start with uninfected founding populations, but infected colonies can be treated with drug and heat therapies if necessary (Boohene et al. 2003).

\section{Release rates}

Because of the difficulty in conducting filth fly dose-response studies in the field, little published information is available on parasitoid release rates needed to provide satisfactory management. The numbers of parasitoids needed for a given situation depends on the size of the fly population, the species of parasitoid(s) released, the number and type of animals on the facility, weather conditions, manure management practices, insecticide use patterns, and other factors. Here it 
may be best to consult commercial insectaries that have been supplying parasitoids to producers for decades. In Table 7 we have tried to arrive at consensus recommended rates for a variety of situations using the information provided by four companies in the USA (IPM Laboratories, Locke, NY; Beneficial Insectary, Redding, CA; Rincon-Vitova Insectaries, Ventura, CA; Spalding Laboratories, Reno, NV) and Europe (Koppert, Berkel en Rodenrijs, the Netherlands). With the exception of Koppert, which produces only M. raptorellus $\left(B_{I O P A R^{\mathrm{TM}}}\right)$, the other insectaries generally provide mixes of species that can include $M$. raptor, M. zaraptor, M. raptorellus, S. cameroni, and S. endius. Release rates are presented on a parasitoids-per-animal basis, with recommended intervals of one to two weeks between releases. These figures are consistent with published studies and provide a starting point that allows the end-user to scale up or down depending on the needs of the local situation.

Large cattle feedlots represent a special and challenging case simply because of the sheer numbers of animals involved. In the USA, feedlots of over 32,000 animals now account for $40 \%$ of the industry (USDA 2016). A herd of this size, which occupies 92-184 acres of pen space, would require 6-13 million parasitoids per release if the guidelines in Table 7 are followed. The efficacy of parasitoid releases on such a massive scale has never been assessed. It should also be noted that all of these recommendations are only for confined production systems, where animals and associated fly habitats are concentrated and relatively stable. House flies and stable flies are the dominant species in such systems. Pastured animals, whose droppings are dispersed over large areas, mainly produce horn flies (Haematobia irritans (L.)) and face flies (Musca autumnalis DeGeer), neither of which is a good candidate for augmentative parasitoid releases.

\section{Compatibility with insecticide use}

Parasitoid releases are most effective when they are incorporated into an overall IPM program that, with the exception of organic farms, can include insecticide use. Applications of residual insecticides are often made as premise treatments to the walls and ceilings of animal housing where flies rest and congregate. Adult parasitoids are at risk of experiencing negative consequences from these treatments when they contact treated surfaces, particularly around windows where dispersing individuals are frequently seen. (Skovgård 2002). Parasitoid susceptibility to premise

Table 7. Recommended release rates of parasitoids for a multiple animal facility types using information provided by five distribution companies. ${ }^{1}$

\begin{tabular}{lc}
\hline Animal type & Parasitoids per animal (1-2 week intervals) \\
& $250-500$ \\
Dairy cows & $500-1000$ \\
Calves & $200-400$ \\
Beef cattle & $300-700$ \\
Pigs & $250-1000$ \\
Horses & $2-8$ \\
Poultry & \\
\hline Sources: http://www.ipmlabs.com/animal-fly-management/dairy-fly-parasites/biological-controls; http:// \\
www.ipmlabs.com/animal-fly-management/poultry-fly-parasites/biological-controls; https://greenmethods. \\
com/calculator; http://www.rinconvitova.com/catalog_fly_control.pdf; https://www.spalding-labs.com/orders/ \\
place/step1.aspx?Customer=new; https://www.koppert.com/pests/stable-fly/product-against/biopar.
\end{tabular}


treatments varies by species and active ingredients, such that few generalizations can be made, except that $S$. cameroni is slightly more tolerant than other species to several toxicants (Geden et al. 1992b; Scott et al. 1988, 1991b). Relative susceptibility of immature parasitoids within host puparia differs substantially from that of adults (Scott et al. 1991a). Taken together, the results indicate that space sprays with natural pyrethrins are the safest option for premise treatments. Baits containing neonicotinoids or spinosad have become a mainstay of fly management in the past decade and should be compatible with parasitoids if they are used properly (Burgess and King 2015). Recent work has shown that baits should not be scattered on the surface of active larval breeding sites or around windowsills because parasitoids are highly susceptible to the active ingredients in these products (Burgess and King 2015, 2016; Burgess et al. 2016).

\section{Efficacy of parasitoid releases on different animal facilities}

Many evaluations have been conducted to assess the effectiveness of parasitoid releases for controlling pest flies. Results have varied by animal facility type, region, facility design, and husbandry practices, as well as with environmental influences, parasitoid species, and other unaccounted for biotic and abiotic factors. There are numerous opportunities for further study and refinement to improve the effectiveness of these biological control agents in different animal rearing situations. An overview of efficacy trials in different animal systems follows.

\section{Poultry}

Perhaps the earliest attempt to use augmentative releases of parasitoids for fly management was by Legner and Brydon (1966), who released S. endius, S. cameroni, S. nigroaenea, and M. uniraptor on California poultry ranches. Parasitism levels rose significantly after the releases but the effect on adult fly populations was modest, potentially a result of adult fly migration from other facilities. This work also raised concerns about the potentially harmful effects to parasitoids caused by use of 'nonselective toxicants' targeting the flies (Legner and Brydon 1966).

The poultry system is well suited to parasitoid use because of the relative stability of the habitat and the potential for beetle and mite predators to assist in suppressing fly populations (Geden 1990). Morgan et al. (1975a) achieved nearly $100 \%$ control of flies in an experimental poultry system in Florida after releasing S. endius. In subsequent studies these authors developed massrearing methods (Morgan et al. 1978), conducted simulations to determine release timing and rates (Weidhaas et al. 1977), and confirmed the effectiveness of S. endius releases in both experimental and commercial poultry facilities (Morgan and Patterson 1990; Morgan et al. 1975b, 1981a, 1981b). $M$. raptor and $M$. raptorellus, either alone or in combination, have been effective in poultry systems in North Carolina, Argentina, New York, and Alberta (Canada) (Al-Ani et al. 2012; Crespo et al. 1998; Kaufman et al. 2002; Rutz and Axtell 1979, 1981). M. raptorellus has also been paired with S. cameroni to increase the niche breadth of the released parasitoids in Arkansas and Florida (Geden and Hogsette 2006; McKay et al. 2007). Tobin and Pitts (1999) observed that M. raptorellus did not disperse far from the point of release and suggested that this species should be used for localized 'hot spots' of fly breeding. Nasonia vitripennis does not appear to be effective in poultry systems, nor dairy, cattle feedlot, or swine systems (Andress and Campbell 1994; Birkemoe et al. 2004b; Kaufman et al. 2001; McKay and Galloway 1999; Meyer et al. 1990; Stage and Petersen 1981). 


\section{Dairy cattle}

Dairy and poultry operations share the advantages of having defined fly-breeding sites, some measure of habitat constancy, and protection from the elements. Morgan and Patterson (1977) observed high (>90\%) levels of parasitism in feed troughs on a small Florida dairy when S. endius were released at rates equivalent to ca. 2,400 parasitoids per cow. Meyer et al. (1990) released mixtures of species (mostly M. zaraptor) on California dairies at rates of about 500 parasitoids per cow and observed 'negligible' increases in parasitism and no impact on adult fly populations. Miller et al. (1993) also reported little impact following releases of M. raptor on Maryland dairy farms, but the numbers of parasitoids released were relatively low. In contrast, releases of either M. raptor alone (Geden et al. 1992C), or in combination with M. raptorellus (Kaufman et al. 2012), at rates of 500-1000 parasitoids per calf were highly effective in inflicting mortality on fly immatures. On Danish dairies, where S. cameroni is often the dominant species, releases of this species at 100200 parasitoids per $\mathrm{m}^{2}$ of indoor pen space (about 225-850 per animal) provided high kill rates of house fly and stable fly pupae (Skovgård 2004; Skovgård and Nachman 2004).

\section{Beef cattle}

Cattle feedlots are a more challenging environment for parasitoid use. Fly breeding sites are varied, spread over large areas, often disturbed, and exposed to the elements. An early smallscale study in Florida using S. endius releases was encouraging (Morgan 1980). Weekly releases of Florida S. endius on Nebraska feedlots at rates of about 200-430 parasitoids per animal resulted in no increase in parasitism, this being attributed to possible incompatibility of the Florida parasitoids with Nebraska conditions (Petersen et al. 1983). Similarly, Andress and Campbell (1994) observed no effect on stable fly parasitism in Nebraska feedlots following weekly releases of mixed species (M. zaraptor, N. vitripennis, S. endius, and S. nigroaenea) at ca. 200-550 parasitoids per animal. Releases of locally-sourced $M$. zaraptor and $M$. raptorellus were successful in increasing fly mortality in a variety of Nebraska trials, even at fairly low release rates (Petersen and Cawthra 1995; Petersen and Curry 1996; Petersen et al. 1992a, 1995). Releases of M. zaraptor were also augmented by placing caches of freeze-killed fly pupae in the feedlots to provide hosts for parasitoid regeneration (Petersen 1986b; Petersen and Pawson 1991; Petersen et al. 1992b). Jones and Weinzierl (1997) conducted pre-release surveys of Illinois feedlots and subsequently increased fly mortality by releasing the locally dominant species, S. nigroaenea (Weinzierl and Jones 1998). Natural parasitism is low in Alberta feedlots (Lysyk 1995), but significant increases were seen during releases of M. raptorellus and locally-sourced T. sarcophagae (Floate 2003; Floate et al. 2000).

\section{Other livestock and equine facilities}

Little information has been published on the outcome of parasitoid releases in other production systems. Releases of $S$. endius on a small Florida pig facility decreased house fly numbers by $37 \%$, but large numbers of immigrating flies may have masked the effect of the releases on fly mortality (Morgan 1980). In Norway, where S. cameroni is the dominant species on pig farms (Birkemoe 2004a), releases of $N$. vitripennis were ineffective (Birkemoe et al. 2004b). Releases of S. cameroni on Norwegian pig farms at rates of 94-243 parasitoids per $\mathrm{m}^{2}$ of increased parasitism of house flies and stable flies but had little observable effect on fly populations (Birkemoe et al. 2009). Releases of Danish S. cameroni at rates of about 75-250 per $\mathrm{m}^{2}$ of pen space were partially successful at suppressing both house flies and stable flies (Skovgård and Nachman 2004). In S. cameroni releases conducted inNorway, parasitism in the target fly population was unaffected by whether 
parasitized pupae were scattered by hand or placed in release stations (Birkemoe and Oyrehagen 2010). S. cameroni and S. endius have been the dominant species recovered on equine farms (J.A. Hogsette, unpublished data; Hogsette 1981; Pitzer et al. 2011a; D.W. Watson, unpublished data). Research studies on efficacy of parasitoids in equine systems are sorely needed. To our knowledge, the only data available are from cases where parasitoids were released as part of broader management efforts (Hogsette 1981; Merritt et al. 1981, 1983).

\section{Summary}

Parasitoids have been used for control of flies on livestock and poultry farms for 50 years. Although augmentative releases are not always effective, our examination of the literature, personal experience, and discussions with insectary owners highlight several pertinent principles for using parasitoids successfully as part of an IPM program. They may be summarized as follows:

1. Manure and other breeding sites should be removed often and kept as dry as possible to conserve resident parasitoids.

2. Insecticide use should be limited to pyrethrin space sprays or baits that are placed where parasitoids are unlikely to contact them.

3. Parasitoid releases should begin early in the fly season (before flies are a problem) and sustained until fly populations begin to drop at the end of the season.

4. Occasional space sprays and baits should be used to keep adult fly populations from reaching outbreak levels that parasitoids cannot manage. Organic producers may wish to consider using large sticky ribbons, non-toxic baited traps and microbials such as Beauveria bassiana.

5. Releases need to be made in sufficient quantities to be effective. The guidelines in Table 7 are a good starting point.

6. Releases should be made by placing the parasitoids (deployed as parasitized hosts) as close to known fly larval development sites as possible.

7. When deploying parasitized pupae for releases, place them in a manner that protects them from damage, rodents, and direct sunlight (if in a hot climate).

8. When choosing a commercial insectary, ask which species will be provided and avoid products that include N. vitripennis.

9. When making releases, hold back a small sample from each batch and examine them a week later for emerged parasitoids or exit holes in pupae to confirm that they arrived in good condition.

10. Keep records of the numbers of parasitoids released and make notes on the status of the fly populations for future use. If possible, use available standard fly monitoring tools to quantify fly populations over time. Such tools include spot cards, Scudder grids, and baited traps.

\section{References}

Ables JR and Shepard M (1974a) Responses and competition of the parasitoids Spalangia endius and Muscidifurax raptor (Hymenoptera: Pteromalidae) at different densities of house fly pupae. Canadian Entomol 106: 825-830.

Ables JR and Shepard M (1974b) Hymenopterous parasitoids associated with poultry manaure. Environ Entomol 3: 884-886.

Ables JR and Shepard M (1976) Influence of temperature on oviposition by the parasitoids Spalangia endius and Muscidifurax rator. Environ Entomol 5: 511-513.

Ables JR, Shepard M and Holman JR (1976) Development of the parasitoids Spalangia endius and Muscidifurax raptor in relation to constant and variable temperature: Simulation and validation. Environ Entomol 5: 329-332.

Abraham R (1978) Die parasitierung on Phormia regina (Dipt.) durch Nasonia vitripennis (Hym., Pteromalidae) in Abbangigkeit von Temperatur und relative Luftfeuchtigkeit im Freiland. Mitt Dtsch Ges Allg Angew Entomol 1: 277-280. 
Al-Ani LM, Khan A, Ganowarden L and Al-Rawi B (2012) Biological control of house flies using indogenous ptermalid parasitoids in egg-layer facilities in Alberta. J Tikrit Univ Agric. 12: 191-204. Available at: http://iasj.net/ iasj?func=fulltextandald $=29709$.

Andress ER and Campbell, JB (1994) Innundative releases of pteromalid parasitoids (Hymenoptera: Pteromalidae) for the control of stable flies, Stomoxys calcitrans (L.) (Diptera: Muscidae) at confined cattle installations in west central Nebraska. J Econ Entomol 87: 714-722.

Antolin MF (1999) A genetic perspective on mating systems and sex ratios of parasitoid wasps. Res Pop Ecol 41: 29-37.

Apiwarthnasorn C (2012) Literature review of parasitoids of filth flies in Thailand: a list of species with brief notes on bionomics of common species. Southeast Asian J Trop Med Publ HIth 43: 48-54.

Ashmead WH (1904) Classification of the chalcid flies of the superfamily Chalcidoidea, with descriptions of new species in the Carnegie Museum, collected in South America by Herbert H. Smith. Publications of the Carnegie museum, serial no. 21. Carnegie Institute, Pittsburg, CA, USA.

Avila-Rodrigues V, Martinez-Lopez R, Nava-Camberos U and Sosa-Rubio MM (2015) Parasitoides de pupas (Hymenoptera: Pteromalidae) de moscas (Diptera: Muscidae) en establos lecheros de Jalisco, México. Southwestern Entomol 40: $141-150$.

Birkemoe T and Øyrehagen H (2010) Parasitism of the house fly parasitoid Spalangia cameroni on Norwegian pig farms: local effect of release method. BioControl 55: 583-591.

Birkemoe T, Soleng A and Aak A (2009) Biological control of Musca domestica and Stomoxys calcitrans by mass releases of the parasitoid Spalangia cameroni on two Norwegian pig farms. BioControl 54: 425-436.

Birkemoe T, Soleng A and Riddervold KW (2004a) Abundance of parasitoid Hymenoptera on pupae of Musca domestica and Stomoxys calcitrans (Diptera, Muscidae) on pig farms in Vestfold, Norway. Norwegian J Entomol 51: 159-164.

Birkemoe T, Soleng A and Riddervold KW (2004b) Use of the parasitoid wasp Nasonia vitripennis (Walker, 1836) in the control of Musca domestica L., 1758 and Stomoxys calcitrans (L., 1758) on two Norwegian pig farms. Norwegian J Entomol 51: 165-173.

Birkemoe T, Soleng A and Skovgård H (2012) Life history parameters of two geographically separated populations of Spalangia cameroni, a microhymenopteran pupal parasitoid of muscoid flies. BioControl 57: 375-385.

Boohene CK, Geden CJ and Becnel JJ (2003) Evaluation of remediation methods for Nosema disease in Muscidifurax raptor (Hymenoptera: Pteromalidae). Environ. Entomol. 32: 1146-1153.

Boucek Z (1963) A taxonomic study in Spalangia Latr. (Hymenoptera, Chalcidoidea). Acta Entomol. Mus. Nat. Pragae 35: 429-512.

Breeuwer JAJ and Werren JH (1993) Cytoplasmic incompatibility and bacterial density in Nasonia vitripennis. Genetics 135: 565-574.

Breeuwer JAJ and Werren JH (1995) Hybrid breakdown between two haplodiploid species: the role of nuclear and cytoplasmic genes. Evol 49: 705-717.

Broski, SA and King BH (2017) Effects of size and age of the host Musca domestica (Diptera: Muscidae) on production of the parasitoid wasp Spalangia endius (Hymenoptera: Pteromalidae). J Econ Entomol 110: 282-287.

Bruckner D (1978) Why are there inbreeding effects in haplodiploid systems? Evol 32: 546-548.

Burgess ER and King BH (2015) Compatibility of the parasitoid wasp Spalangia endius (Hymenoptera: Pteromalidae) and insecticides against Musca domestica (Diptera: Muscidae) as evaluated by a new index. J Econ Entomol 108: 986-992.

Burgess ER and King BH (2016) Behavior and survival of the filth fly parasitoids Spalangia endius and Urolepis rufipes (Hymenoptera: Pteromalidae) in response to three granular house fly baits and components. Environ Entomol 6: 1496-1504.

Burgess ER, Kremer A, Elsawa SF and King BH (2016) Sublethal effects of imidacloprid exposure on Spalangia endius, a pupal parasitoid of filth flies. BioControl 62: 53-60.

Butler JF, Escher R and Hogsette JA (1981) Natural parasite levels in house flies, stable flies and horn flies in Florida. In: Patterson RS, Koehler PG, Morgan PB and Harris RL (eds.) Status of biological control of filth flies. Proceedings a workshop, February 4-5 1981, University of Florida, Gainesville, FL, USA.

Chiel E and Kuzlitsky W (2016) Diversity and abundance of house fly pupal parasitoids in Israel, with first records of two Spalangia species. Environ Entomol 45: 283-291. 
Coats SA (1976) Life cycle and behavior of Muscidifurax zaraptor (Hymenoptera: Pteromalidae). Annals Entomol Soc Amer 69: 772-780.

Costa VA, Berti Filho ES and Silveira-Neto S (2004) Parasitoids (Hymenoptera: Chalcidoidea) de moscas synantropicas (Diptera: Muscidae) em aviarios de Echapora, SP. Arq Inst Biol Sao Paolo 71: 203-209.

Crespo DC, Lecuona PE and Hogsette JA (1998) Biological control: an important component in integrated management of Musca domestica (Diptera: Muscidae) in caged layer poultry houses in Buenos Aires, Argentina. Biol Control 13: 16-24.

Crespo DC, Lecuona PE and Hogsette JA (2002) Strategies for controlling house fly populations resistant to cyromazine. Neotrop Entomol 31: 141-147.

Crozier RH (1985) Adaptive consequences of male haploidy. In: Helle W and Sabelis MW (eds.) Spider mites: their biology, natural enemies, and control, vol. 1a. Elsevier, Amsterdam, the Netherlands.

Cushman RA (1926) Location of individual hosts versus systematic relation of host species as a determining factor in parasite attack. Proc Entomol Soc Wash 28: 5-6.

Davies I (1975) A study of the effect of diet on the lifespan of Nasonia vitripennis (Walk.) (Hymenoptera, Pteromalidae). J Gerontol 30: 294-298.

Davies I and King PE (1975) A morphometric and cytochemical analysis of aging changes in the flight muscle of Nasonia vitripennis. Mech Ageing Devt 4: 459-468.

De Araújo DF, Krüger RF and Ribeiro PB (2012) Phenology of Spalangia endius Walker (Hymenoptera, Pteromalidae) in pupae of Musca domestica Linnaeus (Diptera, Muscidae) under laboratory conditions. Rev Bras Entomol 56: 504-507.

De Oliviera CD, Gonçalves DS, Baton LA, Shimabukuro PHF, Carvalho FD and Moreira LA (2015) Broader prevalence of Wolbachia in insects including potential human disease vectors. Bull Entomol Res 105: 305-315.

Dry FE, Steinkraus DC and McNew RW (1999) Microsporidia (Protozoa: Microspora) in pteromalids (Hymenoptera: Pteromalidae) in broiler-breeder houses of northwest Arkansas. Biol Control 16: 164-169.

Dry FE, Steinkraus DC, Steelman CD, McNew RW and McKay T (2007) Survey of parasitoids (Hymenoptera: Pteromalidae) of house fly (Diptera: Muscidae) pupae from broiler-breeder poultry houses in northwest Arkansas. Biocontrol Sci Technol 17: 757-763.

Edwards RL (1954) The host-finding and oviposition behavior of Mormoniella vitripennis (Walker) (Hym. pteromalidae), a parasite of muscoid flies. Behav 7: 88-112.

Elzen GW, Williams HJ and Vinson SB (1983) Response by the parasitoid Campoletis sonorensis (Hymenoptera:Ichneumonidae) to chemicals (synomones) in plants: implications for host habitat location. Environ Entomol 12: 1873-1877.

Ferreira de Almeida MA and Pires do Prado A (1999) Aleachara spp. (Coleoptera: Staphilinidae) and pupal parasitoids (Hymenoptera: Pteromalidae) attacking symbovine fly pupae (Diptera: Muscidae, Sarcophagidae and Otitidae) in Southeastern Brazil. Biol Control 14: 77-83.

Ferreira de Almeida MA, Pires do Prado A and Geden CJ (2002) Influence of temperature on development time and longevity of Tachinaephagus zealandicus (Hymenoptera: Encyrtidae), and effects of nutrition and emergence order on longevity. Environ Entomol 31:375-380.

Ferrero KM (2008) Life history, host choice, and behavioral plasticity of Trichopria nigra (Hymenoptera: Diapriidae), a parasitoid of higher Diptera. MSc thesis, University of Florida, Gainesville, FL, USA. Available at: http://ufdc.ufl.edu/ UFE0021395/00001.

Floate KD (2003) Field trials of Trichomalopsis sarcophagae (Hymenoptera: Pteromalidae) in cattle feedlots: a potential biocontrol agent of filth flies (Diptera: Muscidae). Canad Entomol 135: 599-608.

Floate KD, Coghlin P and Gibson GAP (2000) Dispersal of the filth fly parasitoid Muscidifurax raptorellus (Hymenoptera: pteromalidae) following mass releases in cattle confinements. Biol Control 18: 172-178.

Floate KD, Khan B and Gibson GAP (1999) Hymenopterous parasitoids of filth fly (Diptera: Muscidae) pupae in cattle feedlots. Can. Entomol. 131: 347-362.

Floate, KD and Skovgard H (2004) Winter survival of nuisance fly parasitoids (Hymenoptera:Pteromalidae) in Canada and Denmark. Bull Entomol Res 94: 331-340.

Frederickx C, Dekeirsschieter J, Verheggen FJ and Haubruge E (2014) Depth and type of substrate influence the ability of Nasonia vitripennis to locate a host. J Insect Sci 14(58): 1-12. 
Geden CJ, Rutz DA, Scott JG and Long SJ (1992b) Susceptibility of house flies (Diptera: Muscidae) and five pupal parasitoids (Hymenoptera: Pteromalidae) to abamectin and seven commercial insecticides. J Econ Entomol 85: 435-440.

Geden CJ (1990) The role of coleopteran and acarine predators in house fly population regulation in poultry production facilities. In: Rutz DA and Patterson RS (eds.) Biocontrol of arthropods affecting livestock and poultry. Westview Press, Boulder, CO, USA.

Geden CJ (1996) Modeling host attacks and progeny production of Spalangia gemina, S. cameroni and Muscidifurax raptor (Hymenoptera: Pteromalidae) at constant and variable temperatures. Biol Control 7: 172-178.

Geden CJ (1997) Development models for the filth fly parasitoids Spalangia gemina, S. cameroni, and Muscidifurax raptor (Hymenoptera: Pteromalidae) under constant and variable temperatures. Biol Control 9: 185-192.

Geden CJ (1999) Host location by house fly (Diptera: Muscidae) parasitoids in poultry manure at different moisture levels and host densities. Environ Entomol 28: 755-760.

Geden CJ (2002) Effect of habitat depth on host location by five species of parasitoids (Hymenoptera: Pteromalidae, Chalcididae) of house flies, Musca domestica L. (Diptera: Muscidae) in three types of substrates. Environ Entomol 31: 411-417.

Geden CJ and Hogsette JA (2006) Suppression of house flies (Diptera: Muscidae) in Florida poultry houses by sustained releases of Muscidifurax raptorellus and Spalangia cameroni (Hymenoptera: Pteromalidae). Environ Entomol 35: 75-82.

Geden CJ and Kaufman PE (2007) Development of Spalangia cameroni and Muscidifurax raptor on live house fly pupae and pupae killed by heat shock, irradiation, and cold. Environ Entomol 36:34-39.

Geden CJ and Moon RD (2009) Host ranges of gregarious muscoid fly parasitoids: Muscidifurax raptorellus (Kogan and Legner) (Hymenoptera: Pteromlaidae), Tachinaephagus zealandicus Ashmead (Hymenopter: Encyrtidae), and Trichopria nigra (Nees) (Hymenoptera: Diapriidae). Environ Entomol 38: 700-707.

Geden CJ and Skovgård H (2014) Status of Tachinaephagus zealandicus (Hymenoptera:Encyrtidae), a larval parasitoid of muscoid flies, in the eastern U.S. and Denmark. J Vector Ecol 39: 453-456.

Geden CJ, Ferreira de Almeida MA and Pires do Prado A (2003) Effects of Nosema disease on fitness of the parasitoid Tachinaephagus zealandicus (Hymenoptera: Encyrtidae). Environ Entomol 32:1139-1145.

Geden CJ, Johnson D, Kaufman PE and Boohene CK (2014) Competition between the filth fly parasitoids Muscidifurax raptor and M. raptorellus (Hymenoptera: Pteromalidae). J Vector Ecol 39: 278-287.

Geden CJ, Long SJ, Rutz DA and Becnel JJ (1995) Nosema disease of the parasitoid Muscidifurax raptor (Hymenoptera: Pteromalidae): prevalence, patterns of transmission, management, and impact. Biol Control 5: 607-614.

Geden CJ, Rutz DA, Miller RW and Steinkraus DC (1992c) Suppression of house flies (Diptera: Muscidae) on New York and Maryland dairies using releases of Muscidifurax raptor (Hymenoptera: Pteromalidae) in an integrated management program. Environ Entomol 21: 1419-1426.

Geden CJ, Smith L, Long SJ and Rutz DA (1992a) Rapid deterioration of searching behavior, host destruction, and fecundity of the parasitoid Muscidifurax raptor (Hymenoptera: Pteromalidae) in culture. Annals Entomol Soc Amer 85: 179-187.

Geetha Bai M and Sankaran T (1977) Parasites, predators, and other arthropods associated with Musca domestica and other flies breeding in bovine manure. Entomophaga 22: 163-167.

Gerling D and Legner EF (1968) Developmental history and reproduction of Spalangia cameroni, parasite of synanthropic flies. Annals Entomol Soc Amer 61: 1436-1443

Ghosh A and Zurek L (2015) Fresh steam-flaked corn in cattle feedlots is an important site for fecal coliform contamination by house flies. J Food Prot 3: 448-627.

Gibson GAP (2000) Illustrated key to the native and introduced chalcidoid parasitoids of filth flies in America north of Mexico (Hymenoptera: Chalcidoidea). Available at: https://tinyurl.com/y9aq77hr.

Gibson GAP (2009) Revision of New World Spalangiinae (Hymenoptera: pteromalidae). Zootaxa 2259: 1-159.

Gibson GAP and Floate KD (2004) Filth fly parasitoids on dairy farms in Ontario and Quebec, Canada. Canad Entomol 136: 407-417.

Gibson, GAP and Floate K (2001) Species of Trichomalopsis (Hymenoptera: Pteromalidae) associated with filth flies (Diptera: Muscidae) in North America. Canad Entomol 133: 49-85.

Gottlieb Y, Zchori-Fein E, Werren JH and Karr TL (2002) Diploidy restoration in Wolbachia-infected Muscidifurax uniraptor (Hymenoptera: Pteromalidae). J Invertebr Pathol 81: 166-174. 
Graczyk TK, Knight R, Gilman RH and Cranfield MR (2001) The role of non-biting flies in the epidemiology of human infectious diseases. Microb Infect 3: 231-235.

Greene GL, Hogsette JA and Patterson RS (1989) Parasites that attack stable fly and house fly (Diptera: Muscidae) pupae during the winter on dairies in northwestern Florida. J Econ Entomol 82: 412-415.

Guo Y, Hogsette JA, Greene GL and Jones CJ (1997) Survey report on pupal parasitoids of filth flies in livestock and poultry facilities in China. Chinese J Biol Control 13: 106-109.

Guzman DR and Petersen JJ (1986a) Cold acclimation and prolonged low temperature survival of filth fly parasites (Hymenoptera: Pteromalidae). Environ Entomol 15: 936-942.

Guzman DR and Petersen JJ (1986b) Overwintering of filth fly parasites (Hymenoptera: Pteromalidae) in open silage in Eastern Nebraska. Environ Entomol 15: 1296-1300.

Havron A and Margalit J (1991) Pupal parasitoids (Hymenoptera: Pteromalidae) of muscoid filth flies in Israel. Med Vet Entomol 5: 267-275.

Heimpel GE and De Boer JG (2008) Sex determination in the Hymenoptera. Ann Rev Entomol 53: 209-230.

Henderson CE and Rutz DA (1991) Species composition of parasitoids attacking house flies (Musca domestica) in high rise poultry farms in New York State. J Agric Entomol 8: 51-57.

Henter $\mathrm{HJ}$ (2003) Inbreeding depression and haplodiploidy: experimental measures in a parasitoid and comparisons across diploid and haplodiploid insect taxa. Evol 57: 1793-1803.

Hogsette JA (1981) Fly control by composting at a south Florida equine facility. In: Patterson RS, Koehler PG, Morgan PB and Harris RL (eds.) Status of biological control of filth flies. Proceedings a workshop, Feb 4-5 1981, University of Florida, Gainsville, FL, USA.

Hogsette JA, Farkas R and Coler RR (1994) Hymenopteran pupal parasites recovered from house fly and stable fly pupae collected on livestock and poultry facilities in northern and central Hungary. Environ Entomol 23: 778-781.

Hogsette JA, Farkas R and Thuroczy C (2001) Hymenopteran pupal parasitoids recovered from house fly and stable fly (Diptera: Muscidae) pupae collected on livestock facilities in southern and eastern Hungary. Environ Entomol 30: 107-111.

Jamil K, Jyothi KN, Prasuna AL, Murty USN and Persoons CJ (1997) Trichopria khandalus (Hym., Diapriidae): a new biocontrol agent for the uzifly Exorista bombysis (Dipt., Tachinidae). J Appl Entomol 121: 99-102.

Jones CJ and Weinzierl RA (1997) Geographic and temporal variation in pteromalid (Hymenoptera: Pteromalidae) parasitism of stable fly and house fly (Diptera: Muscidae) collected from Illinois cattle fedlots. Environ Entomol 26: 421-432.

Kaufman PE, Burgess M, Rutz DA and Glenister C (2002) Population dynamics of manure inhabiting arthropods under an integrated pest management (IPM) program in New York poultry facilities. J Appl Poult Res 11: 90-103.

Kaufman PE, Long SJ, Rutz DA and Waldron JK (2001) Parasitism rates of Muscidifurax raptorellus and Nasonia vitripennis (Hymenoptera: Pteromalidae) after individual and paired releases in New York poultry facilities. J Econ Entomol 94: 593-598.

Kaufman PE, Strong C, Waldron JK and Rutz DA (2012) Individual and combined releases of Muscidifurax raptor and M. raptorellus (Hymenoptera: Pteromalidae) as a biological control tactic targeting house flies in dairy calf facilities. J Med Entomol 49: 1059-1066.

King BH (1988) Sex ratio manipulation in response to host size by the parasitoid wasp Spalangia cameroni: a laboratory study. Evol 42: 1190-1198.

King BH (1990a) Sex ratio manipulation by the parasitoid wasp Spalangia cameroni in response to host age: a test of the host-size model. Evol Ecol 4: 149-156.

King BH (1990b) Interspecific differences in host (Diptera:Muscidae) size and species usage among parasitoid wasps (Hymenoptera: Pteromalidae) in a poultry house. Environ Entomol 19: 1519-1522.

King BH (1991) A field study of host size effects on sex ratio of the parasitoid wasp Spalangia cameroni. Am Midl Nat 125: 10-17.

King BH (1993) Flight activity in the parasitoid wasp Nasonia vitripennis (Hymenoptera: pteromalidae). J Insect Behav 6: 313-321.

King BH (1997) Effects of age and burial of house fly (Diptera: Muscidae) pupae on parasitism by Spalangia cameroni and Muscidifurax raptor (Hymenoptera: Pteromaildae). Environ Entomol 26: 410-415. 
King BH, Colyott KL and Chesney AR (2014) Livestock bedding effects on two species of parasitoid wasps of filth flies. J Insect Sci 14(185): 1-6.

Klunker R (1994) Zum Auftreten von Pupariumparasitoiden als natürliche Feinde von Stallfliegen. Appl Parasitol 35: 36-50.

Laing J (1937) Host finding by insect parasites. I. Observations on the finding of hosts by Alysia manducator, Mormoniella vitripennis and Trichogramma evanescens. J Anim Ecol 6: 298-317.

Legner EF (1967a) Behavior changes the reproduction of Spalangia cameroni, S. endius, Muscidifurax raptor, and Nasonia vitripennis (Hymenoptera: Pteromalidae) at increasing fly host densities. Annals Entomol Soc Amer 62: 128-133.

Legner EF (1967b) The status of Nasonia vitripennis (Walker) as a natural parasite of the house fly, Musca domestica L. Canad Entomol 99: 308-309.

Legner EF (1977) Temperature, humidity and depth of habitat influencing host destruction and fecundity of muscoid fly parasites. Entomophaga 22: 199-206.

Legner EF (1979) Prolonged culture and inbreeding effects on reproductive rates of 2 pteromalid parasites of muscoid flies. Annals Entomol Soc Amer 72: 114-118.

Legner EF and Brydon HW (1966) Suppression of dung-inhabiting fly populations by pupal parasities. Annals Entomol Soc Amer 59: 638-651.

Legner EF and Greathead DJ (1969) Parasitism of pupae in east African populations of Musca domestica and Stomoxys calcitrans. Annals Entomol Soc Amer 62: 128-133.

Legner EF and Olton GS (1968) Activity of parasites from Diptera: Musca domestica, Stomoxys calcitrans and species of Fannia, Muscina, and Ophyra. Il. At sites in the Eastern Hemisphere and Pacific area. Annals Entomol Soc Amer 61: 1306-1314.

Legner EF and Olton GS (1971) Distribution and relative abundance of dipterous pupae and their parasitoids in accumulations of domestic animal manure in the southwestern United States. Hilgardia 40: 505-535.

Legner EF, Bay EC and White EB (1967) Activity of parasites from Diptera: Musca domestica, Stomoxys calcitrans, Fannia canicularis and F. femoralis at sites on the Western Hemisphere. Annals Entomol Soc Amer 60: 461-468.

Legner EF, McKeen WD and Warkentin RW (1990) Inoculation of three pteromalid wasp species (Hymenoptera: Pteromalidae) increases parasitism and mortality of Musca domestica L. pupae in poultry manure. Bull Soc Vector Ecol 15: 49-155.

Loera-Gallardo J, Luna-Salas JF and Gibson GAP (2008) First report of pupal parasitoids of filth-breeding flies (Diptera) from bovine manure in northeastern Mexico. Canad Entomol 140: 682-689.

Lysyk TJ (1995) Parasitoids (Hymenoptera: Pteromalidae, Ichneumonidae) of filth fly (Diptera: Muscidae) pupae at dairies in Alberta. J Econ Entomol 88: 659-665.

LysykTJ (1996) Development of biological control methods for stable flies in feedlots. Final Report (Grant no.920100) Alberta Agricultural Research Institute, Edmonton, Alberta, Canada, 106 pp.

LysykTJ (1998) Relationships between temperature and life history parameters of Trichomalopsis sarcophagae (Hymenoptera: Pteromalidae). Environ Entomol 27: 488-498.

Lysyk TJ (2000) Relationships between temperature and life history parameters of Muscidifurax raptor (Hymenoptera: Pteromalidae). Environ Entomol 29: 596-605.

Lysyk TJ (2001a) Relationships between temperature and life history parameters of Muscidifurax zaraptor (Hymenoptera: Pteromalidae). Environ Entomol 30: 147-156.

Lysyk TJ (2001b) Relationships between temperature and life history parameters of Muscidifurax raptorellus (Hymenoptera: Pteromalidae). Environ Entomol 30: 982-992.

Lysyk TJ (2004) Effects of cold storage on development and survival of three species of parasitoids (Hymenoptera: Pteromalidae) of house fly, Musca domestica L. Environ Entomol 33: 823-831.

Machtinger ET and Geden CJ (2013) Host location by Spalangia cameroni in equine associated substrates. Biol Control 65: 130-134.

Machtinger ET and Geden CJ (2015) Comparison of the olfactory preferences of four filth fly pupal parasitoid species (Hymenoptera: Pteromalidae) for hosts in equine and bovine manure. Environ Entomol 44: 1417-1424.

Machtinger ET, Leppla NC and Sanders C (2013) Pest management perceptions and practices for equine farms in north and central Florida (IFAS publication number ENY-2028). University of Florida, Gainesville, FL, USA. Available at: http://edis. ifas.ufl.edu/pdffiles/IN/IN98300.pdf. 
Machtinger ET, Geden CJ and Leppla NC (2015c) The effect of linear distance on the parasitism of house fly (Diptera: Muscidae) hosts by Spalangia cameroni (Hymenoptera: Pteromalida). PLoS ONE 10(6): e0129105.

Machtinger ET, Geden CJ, Kaufman PE and House AM (2015b) Use of pupal parasitoids as biological control agents of filth flies on equine facilities. J Integr Pest Mgmt, 6(1), 16 DOI: http://dx.doi.org/10.1093/jipm/pmv015.

Machtinger ET, Geden CJ, LoVullo ED and Shirk PD (2016a) Impacts of extended laboratory rearing on female fitness in the parasitoid Spalangia cameroni (Hymenoptera: Pteromalidae) with an analysis of Wolbachia strains in Florida colonies. Annals Entomol Soc Amer 109: 176-182.

Machtinger ET, Geden CJ, Teal PE and Leppla NC (2015a) Comparison of host-seeking strategies used by the filth fly pupal parasitoids Spalangia cameroni and Muscidifurax raptor (Hymenoptera: Pteromalidae). Environ Entomol 44: 330-337.

Machtinger ET, Leppla NC and Hogsette JA (2016b) An assessment of the seasonal abundance and species composition of filth flies and their parasitoids on Florida equine farms to improve biological control. Neotrop Entomol 45: 433-440.

Malik A, Singh N and Satya S (2007) House fly (Musca domestica): a review of control strategies for a challenging pest. J Environ Sci HIth B 42: 453-469.

Mandeville DA and Mullens BA (1990b) Host preference and learning in Muscidifurax zaraptor (Hymenoptera: Pteromalidae). Annals Entomol Soc Amer 83: 1203-1209.

Mandeville JD and Mullens BA (1990a) Host species and size as factors in parasitism by Muscidifurax spp. and Spalangia spp. (Hymenoptera: Pteromalidae) in the field. Annals Entomol Soc Amer 83: 1074-1083.

Mann JA, Axtell RC and Stinner RE (1990b) Temperature-dependent development and parasitism rates of four species of Pteromalidae (Hymenoptera) parasitoids of house fly (Musca domestica) pupae. Med Vet Entomol 4: 245-253.

Mann JA, Stinner RE and Axtell RC (1990a) Parasitism of house fly (Musca domestica) pupae by four species of Pteromalidae (Hymenoptera): effects of host-parasitoid densities and host distribution. Med Vet Entomol 4:235-243.

Marchiori CH (2000) Parasitóides de estágios imaturos de dípteros sinantrópicos coletados em vários ambientes em Itumbiara-GO. Acta Scientiarum 22: 655-661.

Marchiori CH (2006) Microimenópteros de Musca domestica L. (Diptera: Muscidae) coletados em diferentes substratos em Itumbiara, Goiás. Arq Bras Med Vet Zootec 58: 447-449.

Marchiori CH and Miranda JM (2011) Occurencia natural de parasitoides de piteros coletados em granjas de aves poedeiras em Goiás. Rev Bras Zoocien 13: 103-108.

Marchiori CH, Borges LMF and Ferreira LL (2014) Parasitoids of dipterous collected in cattle dung in the regions Southern and Central of Goiás, Brazil. Adv Entomol 2: 20-23.

Marchiori CH, Calas ER and Dias KGS (2002) Parasitóides de Diptera coletados em fezes bovinas em vários tempos de exposição em Itumbiara, Goiás, Brasil. Arq Inst Biol 69: 37-42.

Marchiori CH, Silva Filho OM and Milhomen MEV (2004) Espécies de Spalangia (Hymenoptera: Pteromalidae) coletadas em fezes de búfalos em Itumbiara, Goiás, Brasil. Biotemas 17: 197-204.

Marchiori CH, Silva Filho OM, Borges MO and Melo MF (2005) Parasitóides da família Pteromalidae (Hymenoptera: Pteromalidae) coletados em fezes de gado bovino em uma propriedade rural do município de Panamá, Goiás. Arq Bras Vet Zootec 57, Suppl. 2: 270-272.

Masner L and Garcia JL (2002) The genera of Diapriinae (Hymenoptera: Diapriidae) in the new world. Bull Amer Mus Nat Hist 268: 1-138.

Mckay T and Broce AB (2003) Response of Muscidifurax zaraptor (Hymenoptera: pteromalidae) to olfactory stimuli from hosts and their habitat. J Kansas Entomol Soc 76: 31-37.

McKay T and Galloway TD (1999) Survey and release of parasitoids (Hymenoptera) attacking house and stable flies (Diptera: Muscidae) in dairy operations. Canad Entomol 131: 743-756.

McKay T, Steelman CD, Brazil SM and Szalanski AL (2007) Sustained mass release of pupal parasitoids (Hymenoptera: Pteromalidae) for control of Hydrotaea aenescens and Musca domestica (Diptera: Muscidae) in broiler-breeder poultry houses in Arkansas. J Agric Urban Entomol 24: 67-85.

Merchant ME, Flanders RV and Williams RE (1985) Sampling method comparisons for estimation of parasitism of Musca domestica pupae in accumulated poultry manure. J Econ Entomol 78: 1299-1303.

Merchant ME, Flanders RV and Williams RE (1987) Seasonal abundance and parasitism of house fly (Diptera: Muscidae) pupae in enclosed, shallow-pit poultry houses in Indiana. Environ Entomol 716-721. 
Merritt RW, Gersabeck EF and Kennedy MK (1981) The contribution of Spalangia endius and Muscidifurax raptor to a stable fly management program on Mackinac Island, Michigan:a question of effort. In: Patterson RS, Koehler PG, Morgan PB and Harris RL (eds.) Status of biological control of filth flies. Proceedings a workshop, Feb 4-5 1981. University of Florida, Gainesville, FL, USA.

Merritt RW, Kennedy MK and Gersabeck EF (1983) Integrated pest management of nuisance and biting flies in a Michigan resort; dealing with secondary pest outbreaks. In: Frankie GW and Koehler CS (eds.) Urban entomology, interdisciplinary perspectives. Praeger, New York, NY, USA.

Meyer JA and Petersen JJ (1982) Sampling house fly and stable fly pupal parasites on beef feedlots and dairies in eastern Nebraska. Southwest Entomol 7: 119-124.

Meyer JA and Petersen JJ (1983) Characterization and seasonal distribution of breeding sites of stable flies and house flies (Diptera: Muscidae) on eastern Nebraska feedlots and dairies. J Econ Entomol 76: 103-108.

Meyer JA, Mullens BA, Cyr TL and Stokes C (1990) Commercial and naturally occurring fly parasitoids (Hymenoptera: Pteromalidae) as biological control agents of stable flies and house flies (Diptera: Muscidae) on California dairies. J Econ Entomol 83: 799-806.

Meyer JA, Shutz TA, Collar C and Mullens BA (1991) Relative abundance of stable fly and house fly (Diptera: Muscidae) pupal parasites (Hymenoptera: Pteromalidae; Coleoptera: Staphylinidae) on confinement dairies in California. Environ Entomol 20: 915-921.

Miller RW, Rutz DA, Pickens LG and Geden CJ (1993) Evaluation of traps and the parasitoid Muscidifurax raptor (Girault and Sanders) to manage house flies and stable flies on dairy farms. J Agr Entomol 10: 9-19.

Moon RD, Berry IL and Petersen JJ (1982) Reproduction of Spalangia cameroni Perkins (Hymenoptera: Pteromalidae) on stable fly (Diptera: Muscidae) in the laboratory. J Kansas Entomol Soc 55: 77-85.

Morgan PB (1980) Sustained releases of Spalangia endius Walker (Hymenoptera: Pteromalidae) for the control of Musca domestica L. and Stomoxys calcitrans (L.) (Diptera: Muscidae). J Kansas Entomol Soc 53: 367-372.

Morgan PB and Patterson RS (1975) Field parasitization of house flies by natural populations of Pachycrepoideus vindemiae (Rondani), Muscidifurax raptor Girault and Sanders, and Spalangia nigroaenea Curtis. Fla Ent 58: 202.

Morgan PB and Patterson RS (1977) Sustained releases of Spalangia endius to parasitize field populations of three species of filth breeding flies. J Econ Entomol 70: 450-452.

Morgan PB and Patterson RS (1990) Efficiency of target formulations of pesticides plus augmentative releases of Spalangia endius Walker (Hymenoptera: Pteromalidae) to suppress populations of Musca domestica L. (Diptera: Muscidae) at poultry ranches in the southeastern United States. In: Rutz DA and Patterson RS (eds.) Biocontrol of arthropods affecting livestock and poultry. Westview Press, Boulder, CO, USA.

Morgan PB, Hogsette JA and Patterson RS (1990) Life history of Trichopria stomoxydis (Hymenoptera: Proctotrupidae: Diapriidae) a gregarious endoparasite of Stomoxys calcitrans from Zimbabwe, Africa. Florida Entomol 73: 496-502.

Morgan PB, Hoyer H and Patterson RS (1989) Life history of Spalangia cameroni (Hymenoptera: Pteromalidae), a microhymenopteran pupal parasite of muscoid flies (Diptera: Muscidae). J Kansas Entomol Soc 62: 381-386.

Morgan PB, LaBrecque GC and Patterson RS (1978) Mass culturing the microhymenopteran parasite Spalangia endius (Hymenoptera: Pteromalidae). J Med Entomol 14: 671-673.

Morgan PB, Patterson RS and LaBrecque GC (1976) Host-parasitoid relationship of the house fly, Musca domestica L., and the protelean parasitoid Spalangia endius (Hymenoptera: Pteromalidae and Diptera: Muscidae). J Kansas Entomol Soc 4: 483-488.

Morgan PB, Patterson RS, LaBrecque GC, Weidhaas DE and Benton A (1975a) Suppression of a field population of houseflies with Spalangia endius. Science 189: 388-389.

Morgan PB, Patterson RS, LaBrecque GC, Weidhaas DE, Benton A and Whitfield T (1975b) Rearing and release of the house fly pupal parasite Spalangia endius Walker. Environ Entomol 4:609-611.

Morgan PB, Weidhaas DE and Patterson RS (1981a) Programmed releases of Spalangia endius and Muscidifurax raptor (Hymenoptera: Pteromalidae) against estimated populations of Musca domestica (Diptera: Muscidae). J Med Entomol 18: 158-166. 
Morgan PB, Weidhaas DE and Patterson RS (1981b) Host-parasite relationship: augmentative releases of Spalangia endius Walker used in conjunction with population modeling to suppress field populations of Musca domestica $\mathrm{L}$. (Hymenoptera: Pteromalidae and Diptera: Muscidae). J Kansas Entomol Soc 54: 496-504.

Mourier H (1971) Seasonal occurrence of pupal parasitoids from the housefly Musca domestica (Diptera) in Denmark. Videnskabelige Meddelelser fra Dansk Naturhistorisk Forening 134: 109-118.

Mourier $\mathrm{H}$ and Hannine S (1971) Survey of the importance of natural enemies of houseflies in Denmark. Danish Pest Infestation Laboratory Annual Report 1970: 51-52.

Mullens BA, Meyer JA and Mandeville JD (1986) Seasonal and diel activity of filth fly parasites (Hymenoptera: Pteromalidae) in caged-layer manure in southern California. Environ Entomol 15: 56-60.

Nagel WP and Pimentel D (1963) Some ecological attributes of a pteromalid parasite and its housefly host. Canad Entomol 95: 208-213.

Noronha C, Gibson GAP and Floate KD (2007) Hymenopterous parasitoids of house fly and stable fly puparia in Prince Edward Island and New Brunswick, Canada. Canad Entomol 139: 748-750.

Olbrich DL and King BH (2003) Host and habitat use by parasitoids (Hymenoptera: Pteromalidae) of house fly and stable fly (Diptera: Muscidae) pupae. Great Lakes Entomol 36: 179-190.

Olton GS and Legner EF (1974) Biology of Tachinaephagus zealandicus (Hymenoptera: Encyrtidae), parasitoid of synanthropic Diptera. Canad Entomol 106: 785-800.

Patterson RS and Rutz DA (1986) Biological control of muscoid flies. Entomological Society of America, Annapolis, MD, USA, $174 \mathrm{pp}$.

Pawson BM and Petersen JJ (1988) Dispersal of Muscidifurax zaraptor (Hymenoptera: Pteromalidae), a filth fly parasitoid, at dairies in eastern Nebraska. Environ Entomol 17: 398-402.

Pawson BM and Petersen JJ (1990) Temperature preference and effects of photoperiod on oviposition behavior of five pteromalid wasps (Hymenoptera:Pteromalidae) using house fly (Diptera: Muscidae) pupae as hosts. Environ Entomol 19: $1452-1456$.

Peters RS (2010) Host range and offspring quantities in natural populations of Nasonia vitriennis (Walker, 1836) (Hymenotera: Chalcidoidea: Pteromalidae). J Hymen Res 19: 128-138.

Petersen JJ (1986a) Evaluating the impact of pteromalid parasites on filth fly populations associated with confined livestock installations. Misc Publ Entomol Soc Amer 61: 52-56.

Petersen JJ (1986b) Augmentation of early season releases of filth fly (Diptera: Muscidae) parasites (Hymenoptera: Pteromalidae) with freeze-killed hosts. Environ Entomol 15: 590-593.

Petersen JJ and BM Pawson (1988) Early season dispersal of Muscidifurax zaraptor (Hymenoptera: Pteromalidae) utilizing freeze-killed housefly pupae as hosts. Med Vet Entomol 2: 137-140.

Petersen JJ and Currey DM (1996) Timing and releases of gregarious Muscidifurax raptorellus (Hymenoptera: Pteromalidae) to control flies associated with confined beef cattle. J Agr Entomol 13:55-63.

Petersen JJ and Meyer JA (1983a) Observations on the overwintering pupal parasities of filth flies associated with open silage in eastern Nebraska. Southwest Entomol 3: 219-225.

Petersen JJ and Meyer JA (1983b) Host preference and seasonal distribution of pteromlalid parasites (Hymenoptera: Pteromalidae) of stable flies and house flies (Diptera: Muscidae) associated with confined livestock in eastern Nebraska. Environ Entomol 12: 567-571.

Petersen JJ and Pawson BM (1991) Early season introduction, population increase, and movement of the filth fly parasite Muscidifurax zaraptor (Hymenoptera: Pteromalidae). Environ Entomol 20: 1155-1159.

Petersen JJ, Meyer JA, Stage DA and Morgan PB (1983) Evaluation of sequential releases of Spalangia endius (Hymenoptera: Pteromalidae) for control of house flies and stable flies (Diptera: Muscidae) associated with confined livestock in eastern Nebraska. J Econ Entomol 76: 283-286.

Petersen JJ, Watson DW and Cawthra JK (1995) Comparative effectiveness of three release rates for a pteromalid parasitoid (Hymenoptera) of house flies (Diptera) in beef cattle feedlots. Biol Control 5: 561-565.

Petersen JJ, Watson DW and Pawson BM (1992a) Evaluation of Muscidifurax zaraptor and Pachycrepoideus vindemiae (Hymenoptera: Pteromalidae) for controlling flies associated with confined beef cattle. Biol Control 2: 44-50. 
Petersen JJ, Watson DW and Pawson BM (1992b) Evaluation of field propagation of Muscidifurax zaraptor (Hymenoptera: Pteromalidae) for control of flies associated with confined beef cattle. J Econ Entomol 85: 451-455.

Petersen JJ and Cawthra JK (1995) Release of a gregarious Muscidifurax species (Hymenoptera: Pteromalidae) for the control of filth flies associated with confined beef cattle. Biol Control 5: 279-284.

Petersen JJ and Watson DW (1992) Comparison of sentinel and naturally occurring fly pupae to measure field parasitism by pteromalid parasitoids (Hymenoptera). Biol Control 2: 244-248.

Pitzer JB, Kaufman PE, Geden CJ and Hogsette JA (2011b) The ability of selected pupal parasitoids (Hymenoptera: Pteromalidae) to locate stable fly hosts in soiled equine bedding substrate. Environ Entomol 40: 88-93.

Pitzer JB, Kaufman PE, Hogsette JA Geden CJ and TenBroeck SH (2011a) Seasonal abundance of stable flies and filth fly pupal parasitoids (Hymenoptera: Pteromalidae) at Florida equine facilities. J Econ Entomol 104: 1108-1115.

Propp GD and Morgan PB (1983) Multiparasitism of house fly, Musca domestica, pupae by Spalangia endius and Muscidifurax raptor (Hymenoptera: Pteromalidae). Environ Entomol 12: 1232-1238.

Propp GD and Morgan PB (1985) Effect of host distribution on parasitoidism of house fly (Diptera: Muscidae) pupae by Spalangia spp. and Muscidifurax raptor (Hymenoptera: Pteromalidae). Canad Entomol 117: 515-542.

Read DP, Paul PF and Root RB (1970) Habitat selection by aphid parasite Delia rapae (Charip brassicae, Hymenoptera: (Cynipidae). Canad Entomol 102: 1567-1578.

Romero A, Hogsette JA and Coronado A (2010) Distribution and abundance of natural parasitoid (Hymenoptera: Pteromalidae) populations of house flies and stable flies (Diptera: Muscidae) at the University of Florida Dairy Research Unit. Neotrop Entomol 39: 424-429.

Rueda LM and Axtell RC (1985a) Guide to common species of pupal parasites (Hymenoptera: Pteromalidae) of the house fly and other muscoid flies associated with poultry and livestock manure. North Carolina Agricultural Research Service, North Carolina State University, Raleigh, NC, USA, 88 pp.

Rueda LM and Axtell RC (1985b) Comparison of hymenopterous parasites of house fly, Musca domestica (Diptera, Muscidae), pupae in different livestock and poultry production systems. Environ Entomol 14: 217-222.

Rueda LM and Axtell RC (1985c) Effect of depth of house fly pupae in poultry manure on parasitism by six species of Pteromalidae (Hymenoptera). J Entomol Sci 20: 444-449.

Rueda LM, Roh PU and Ryu JL (1997) Pupal parasitoids (Hymenoptera: Pteromalidae) of filth flies (Diptera, Muscidae, Calliphoridae) breeding in refuse and poultry and livestock manure in South Korea. J Med Entomol 34: 82-85.

Rutz DA and Axtell RC (1979) Sustained releases of Muscidifurax raptor (Hymenoptera: Pteromalidae) for house fly (Musca domestica L.) control in two types of caged-layer houses. Environ Entomol 8: 1105-1110.

Rutz DA and Axtell RC (1980) House fly parasites (Hymenoptera: Pteromalidae) associated with poultry manure in North Carolina. Environ Entomol 9: 175-180.

Rutz DA and Axtell RC (1981) House fly (Musca domestica) control in broiler-breeder poultry houses by pupal parasites (Hymenoptera: Pteromalidae): Indigenous parasite species and releases of Muscidifurax raptor. Environ Entomol 10: 343-345.

Rutz DA and Scoles GA (1989) Occurrence and seasonal abundance of parasitoids attacking muscoid flies (Diptera: Muscidae) in caged-layer poultry facilities in New York. Environ Entomol 18: 51-55.

Scott JG, Geden CJ, Rutz DA and Liu N (1991a) Comparative toxicity of seven insecticides to immature stages of Musca domestica (Diptera: Muscidae) and two of its important biologican control agents: Muscidifurax raptor and Spalangia cameroni (Hymenoptera: Pteromalidae). J Econ Entomol 84: 776-779.

Scott JG, Dong K, Geden CJ and Rutz DA (1991b) Evaluation of the biochemical basis of insecticide selectivity between host and parasitoid species. Environ Entomol 19: 1722-1725.

Scott JG, Rutz DA and Walcott J (1988) Comparative toxicities of seven insecticides to adult Spalangia cameroni Perkins. J Agric Entomol 5: 139-145.

Seymour RC and Campbell JB (1993) Predators and parasitoids of house flies and stable flies (Diptera: Muscidae) in cattle confinements in west central Nebraska. Environ Entomol 22: 212-219.

Silveira GAR, Madeira NG, De Azeredo-Espin AL and Pavan C (1989) Levantamento de microhimenópteros parasitóides de dípteros de importância, médico-veterinária no Brasil. Mem Inst Oswaldo Cruz 84, Suppl. 4: 505-510. 
Skovgård H (2002) Dispersal of the filth fly parasitoid Spalangia cameroni (Hymenoptera: pteromalidae) in a swine facility using fluorescent dust marking and sentinel pupal bags. Environ Entomol 31:425-431.

Skovgård H (2004) Sustained releases of the pupal parasitoid Spalangia cameroni (Hymenoptera: Pteromalidae) for control of house flies, Musca domestica and stable flies Stomoxys calcitrans (Diptera: Muscidae) on dairy farms in Denmark. Biol Control 30: 288-297.

Skovgård H and Nachman G (2004) Biological control of house flies Musca domestica and stable flies Stomoxys calcitrans (Diptera: Muscidae) by means of inundative releases of Spalangia cameroni (Hymenoptera: Pteromalidae). Bull Entomol Res 94: 555-567.

Skovgård H and Nachman G (2015) Temperature-dependent functional response of Spalangia cameroni (Hymenoptera: Pteromalidae), a parasitoid of Stomoxys calcitrans (Diptera: Muscidae). Environ Entomol 44: 90-99.

Skovgård H and Nachman G (2016) Temperature- and age-dependent survival, development, and oviposition rates of the pupal parasitoid Spalangia cameroni (Hymenoptera: Pteromalidae). Environ Entomol 45: 1063-1075.

Skovgård $\mathrm{H}$ and Steenberg $\mathrm{T}$ (2002) Activity of pupal parasitoids of the stable fly Stomoxys calcitrans and prevalence of entomopathogenic fungi in the stable fly and the house fly, Musca domestica. BioControl 47: 45-60.

Skovgård H and Jespersen JB (1999) Activity and relative abundance of hymenopterous parasitoids that attack puparia of Musca domestica and Stomoxys calcitrans (Diptera: Muscidae) on confined pig and cattle farms in Denmark. Bull Entomol Res 89: 263-269.

Skovgård $\mathrm{H}$ and Jespersen JB (2000) Seasonal and spatial activity of hymenopterous pupal parasitoids (Pteromalidae and Ichneumonidae) of the house fly (Diptera: Muscidae) on Danish pig and cattle farms. Environ Entomol 29: 630-637.

Smith JP, Hall RD and Thomas GD (1987) Field parasitism of the stable fly (Diptera: Muscidae). Annals Entomol Soc Amer 80: 391-397.

Smith L and Rutz DA (1991a) Seasonal and relative abundance of hymenopterous parasitoids attacking house fly pupae at dairy farms in Central New York. Environ Entomol 20: 661-668.

Smith L and Rutz DA (1991b) Microhabitat associations of hymenopterous parasitoids that attack house fly pupae at dairy farms in Central New York. Environ Entomol 20:675-684.

Smith L and Rutz DA (1991c) Relationship of microhabitat to incidence of house fly (Diptera: Muscidae) immatures and their parasitoids at dairy farms in Central New York. Environ Entomol 20: 669-674.

Smith L, Rutz DA and Scoles GA (1989) Influence of habitat and temperature on dispersal behavior of two pteromalid parasitoids of houseflies during an inundative release at a dairy barn. Med Vet Entomol 3: 169-178.

Stage DA and Petersen JJ (1981) Mass release of pupal parasites for control of stable flies and house flies in confined feedlots in Nebraska. In: Patterson RS, Koehler PG, Morgan PB and Harris RL (eds.) Status of biological control of filth flies. Proceedings a workshop, Feb 4-5 1981, University of Florida, Gainesville, FL, USA.

Stouthamer R, Luck RF and Hamilton WD (1990) Antibiotics cause parthenogenetic Trichogramma (Hymenoptera/ Trichogrammatidae) to revert to sex. Proc Natl Acad Sci USA 87: 2424-2427.

Sulaiman SB, Omar B, Omar S, Jeffrey J, Ghauth I and Busparani V (1990) Survey of microhymenoptera (Hymenoptera: Chalcidoidea) parasitizing filth flies (Diptera: Muscidae, Calliphoridae) breeding in refuse and poultry farms in peninsular Malaysia. J Med Entomol 27: 851-855.

Taley YM and Thakare KR (1979) Biology of seven new hymenopterous parasitoids of Atherigona soccata Rondani. Indian J Agric Sci 59: 344-354.

Talley J, Broce A and Zurek L (2009) Characterization of stable fly (Diptera: Muscidae) larval developmental habitat at round hay bale feeding sites. J Med Entomol 46: 1310-1319.

Taylor C, Machtinger ET and Geden CJ (2016) Manure preferences and postemergence learning of two of common filth fly parasitoids, Spalangia cameroni and Muscidifurax raptor (Hymenoptera: Pteromalidae). PLoS ONE 11: e0167893.

Taylor DB, Moon RD and Mark DR (2012) Economic impact of stable flies (Diptera: Muscidae) on dairy and beef cattle production. J Med Entomol 49: 198-209.

Tobin PC and Pitts CW (1999) Dispersal of Muscidifurax raptorellus Kogan and Legner (Hymenoptera: Pteromalidae) in a high-rise poultry facility. Biol Control 16: 68-72.

Townes H (1960) Host selection patterns in some Neartic ichneumonids. Proc 11 ${ }^{\text {th }}$ Int Congr Entomol 2: 738-741. 
United States Department of Agriculture (USDA) (2016) Economics Research Service - cattle and beef. Available at: https:// tinyurl.com/ydcekmpt.

United States Department of Agriculture (USDA) (2006) Equine 2005, Part II: changes in the US equine industry, 1998-2005. Available at: https://tinyurl.com/yb8ftrgt.

Vinson SB (1975) Biochemical convolution between parasitoids and their hosts. In: Price PW (ed.) Evolutionary strategies of parasitic insects and mites. Plenum Press, New York, NY, USA, pp. 14-48.

Vinson SB (1976) Host selection by insect parasitoids. Annu Rev Entomol 21: 109-134.

Vinson SB (1981) Habitat location. In: Nordlund DA, Lewis WJ and Jones RL (eds.) Semiochemicals: their role in pest control. Wiley, New York, NY, USA.

Weidhaas DE, Haile DG, Morgan PB and LaBrecque GC (1977) A model to simulate control of house flies with a pupal parasite, Spalangia endius. Environ Entomol 6: 489-500.

Weinzierl RA and Jones CJ (1998) Releases of Spalangia nigroaenea and Muscidifurax zaraptor (Hymenoptera: Pteromalidae) increase rates of parasitism and total mortality of stable fly and house fly (Diptera: Muscidae) pupae in Illinois cattle feedlots. J Econ Entomol 91: 1114-1121.

Werren JH (1993) The evolution of inbreeding in haplodiploid organisms. In: Thornhill N (ed.) The natural history of inbreeding and outbreeding: theoretical and empirical perspectives. University of Chicago Press, Chicago, IL, USA, pp. 42-59.

Werren JH, Baldo L and Clark ME (2008) Wolbachia: master manipulators of invertebrate biology. Nature Rev Microbiol 6: 741-751.

Werren JH, Richards S, Desjardins CA, Niehuis O, Gadan J, Colbourne, JK and The Nasonia Genome Working Group (2010) Functional and evolutionary insights from the genomes of three parasitoid Nasonia species. Science 327: 343-348.

Wienhold BJ and Taylor DB (2013) Substrate properties of stable fly (Diptera: Muscidae) developmental sites associated with round bale hay feeding sites in eastern Nebraska. Environ Entomol 41:213-221.

Wylie HG (1958) Factors that affect host finding by Nasonia vitripennis (Walker) (Hymenoptera: Pteromalidae). Canad Entomol 90:597-608.

Wylie HG (1966) Survival and reproduction of Nasonia vitripennis (Walk.) at different host population densities. Canad Entomol 98: 275-281.

Wylie HG (1971) Oviposition restraint of Muscidifurax zaraptor (Hymenoptera: Pteromalidae) on parasitized housefly pupae. Canad Entomol 103: 1537-1544

Wylie HG (1972) Larval competition among three hymenopterous parasite species on multiparasitized housefly (Diptera: Muscidae) pupae. Canad Entomol 104: 1181-1190.

Zchori-Fein E, Geden CJ and Rutz DA (1992) Microsporidioses of pteromalid parasitoids of muscoid flies. J Invertebr Pathol 60:292-298.

Zurek $L$ and Ghosh A (2014) Insects represent a link between food animal farms and the urban environment for antibiotic resistance traits. Appl Environ Microbiol 80: 3562-3567. 


\title{
12. Biological control of livestock pests: entomopathogens
}

\author{
Emma N.I. Weeks ${ }^{1 *}$, Erika T. Machtinger ${ }^{2}$, Diana Leemon ${ }^{3}$ and Christopher J. Geden ${ }^{4}$ \\ ${ }^{1}$ University of Florida, Entomology and Nematology Dept., 970 Natural Area Drive, Gainesville, FL 32653, \\ USA; ${ }^{2}$ Penn State University, Department of Entomology, 501 ASI Building, University Park, State College, \\ PA 16082, USA; ${ }^{3}$ Department of Agriculture and Fisheries (Queensland), Agri-Science Queensland, \\ Ecosciences Precinct, GPO Box 267, Brisbane, Queensland, 4001, Australia; ${ }^{4}$ United States Department of \\ Agriculture, Agricultural Research Service, Center for Medical, Agricultural and Veterinary Entomology, \\ 1600 SW 23 ${ }^{\text {rd }}$ Drive, Gainesville, FL 32608, USA; eniweeks@ufl.edu
}

\begin{abstract}
Interest in biological methods for livestock and poultry pest management is largely motivated by the development of resistance to most of the available synthetic pesticides by the major pests. There also has been a marked increase in organic systems, and those that promote animal welfare by reducing animal densities and allowing greater freedom of movement. Such systems, especially organic operations, are in need of new tools and strategies to manage pest problems. This chapter reviews the status of entomopathogenic fungi, viruses, and nematodes for management of pests of livestock and poultry production. The pests covered here are limited to the research that has been conducted to date, but include biting and nuisance flies, ticks, poultry ectoparasites, and litter beetles. Many of the same pests affect horses as well, although little work has been done in this area. The fungal pathogens Beauveria bassiana and Metarhizium anisopliae s.l. have received the most attention and have been evaluated against most of the major livestock and poultry pests. These pathogens have the greatest potential for practical use, but more work is needed to identify efficacious isolates and develop formulations for both on- and off-host use. The slow kill rate of these pathogens has been considered a liability in the past, but a growing body of work has demonstrated important sublethal effects of infection that includes reduced feeding, movement, and pathogen transmission. Two pathogens of house fly, the fungus Entomophthora muscae and salivary gland hypertrophy virus, are effective under some conditions but have biological characteristics that have limited their development as practical management tools. Entomopathogenic nematodes (Steinernema and Heterorhabditis spp.) also have potential but are limited by environmental constraints, especially temperature and substrate moisture. Discovery of, or selection for, strains with wider environmental tolerance could broaden the range of situations where they can be used effectively.
\end{abstract}

Keywords: Beauveria bassiana, biological control, entomopathenic fungi, entomopathogenic nematodes, Entomopthora muscae, Metarhizium anisopliae, salivary gland hypertrophy virus

\section{Introduction}

There is a shared imperative for the development of alternative management options for pests of crop, horticultural, and livestock systems. The issues associated with resistance to conventional chemical-based pesticides, along with safety concerns regarding residues in the environment and on food, are common across agricultural production. Despite the potential in this area, progress in the development of entomopathogenic biological control agents (BCAs) for pests of livestock and other animal production systems has lagged behind that for crop and horticultural pests. One reason for this lag is that research that includes animals is more expensive than crop pest work. The animals must be fed, housed, and have access to veterinary services. Moreover, animal studies 
in nearly all countries now require animal use/ethics permits or approvals to conduct the research that require additional time and resources.

The life stage of the livestock pest can generate additional challenges for entomopathogenic biological control if the optimal stage for treatment is on the animal (e.g. cattle tick, poultry ectoparasites, sheep lice, and sheep blowflies). The surface temperatures of many animals can be too high for the germination and growth of entomopathogenic fungi (EPF) for much of each day, especially during the summer. In addition, formulation and application technologies might be more limited. Aqueous formulations may not be as effective as oil-based formulations for carrying fungal spores into an oily or greasy animal coat, and mineral oils may not be as acceptable as vegetable oils in formulations that are to be applied directly to the animals.

Despite the extra challenges associated with developing entomopathogenic BCAs for livestock, poultry, and equine pests there have been encouraging results in many areas. Advances in control of off-host pests, such as litter beetles and nuisance flies, have been particularly promising. In these cases, the BCAs do not have to be applied to the animal surface, but can be applied to the environment within the animal facility.

In areas where the application of synthetic pesticides is restricted, such as in livestock facilities close to or within urban areas, or detrimental, such as in rural environments close to sensitive ecosystems, efficacious entomopathogenic BCAs may provide a highly desirable alternative. Furthermore, the use of entomopathogenic BCAs has the potential to aid in managing insecticide resistance in conventional animal production systems. Therefore, BCAs may provide or assist in effective management of the pest, preventing economic losses due to pest pressure and controlling the potential spread of zoonotic pathogens to urban neighbors, whilst protecting the environment through reductions in synthetic pesticide pollution and consequential nontarget effects. Moreover, there is a growing market in organic production that sorely needs pest management tools. Biological control is a key facet of both organic production and integrated pest management (IPM), the well-recognized approach for sustainable arthropod management through the integration of all the available techniques and tools to minimize losses to pests in an economical manner.

In the next sections, livestock pests that are currently under investigation as targets of biological control using entomopathogens will be outlined, followed by discussion of the potential for the use of fungi, viruses, and nematodes in their management. The research surrounding the use of Bacillus thuringiensis Berliner (Bacillales: Bacillaceae) will not be discussed in this chapter as there are ample other reviews on the topic (Pinnock 1994; Sanchis and Bourguet 2008).

\section{Livestock pests and parasites of greatest economic concern}

This section will provide background information on the livestock pests of economic concern that will be covered in the following sections of this chapter. The goal of this section is not to provide an exhaustive review of all livestock, poultry, and equine pests, but to introduce the key pests that have received attention in the development of entomopathogens for pest control in animal facilities to date. 


\section{Mosquitoes}

Most research on mosquito (Diptera: Culicidae) control is done from a public health perspective. However, improved management of mosquitoes on livestock and other animal facilities can have both animal welfare and public health benefits. High numbers of mosquitoes are a nuisance to animals. Pest avoidance behaviour results in lower product yields including less milk from dairy cows, and lower weights in beef cattle, sheep, and poultry (Byford et al. 1992; Minár et al. 1979). When biting flies (mosquitoes and horse flies) were excluded from dairy cows using repellent, milk production increased by $6.2 \%$, and the milk fat content by $11.8 \%$ (Minár et al. 1979). Furthermore, mosquitoes can transmit pathogens such as fowl pox virus, Rift Valley fever virus, West Nile virus, and other viruses that cause encephalitis including eastern equine encephalitis, western equine encephalitis, and St. Louis encephalitis, which are a danger both to animals and people in the locality, such as farm workers and neighbours.

\section{Biting midges}

Biting midges of the genus Culicoides (Diptera: Ceratopogonidae) are pests of livestock, poultry, equines, and humans worldwide (Mellor et al. 2000). Culicoides are blood-feeding flies that are a nuisance in areas, such as the Scottish highlands, where numbers are high. The bites of Culicoides cause an allergic response in horses, a type of acute dermatitis known as 'sweet itch,' and they can vector protozoa and filarial worms to birds, humans, and other animals (Mellor et al. 2000). However, their pest status is mainly due to their ability to transmit over 50 viruses including several of global importance to livestock and other animals such as bluetongue virus, Akabane virus, Schmallenberg virus, African horse sickness virus, and epizootic haemorrhagic disease virus (Mellor et al. 2000).

\section{House flies, stable flies and Fannia spp.}

House flies (Musca domestica L.), lesser house flies (Fannia canicularis L.) and other Fannia spp., and stable flies (Stomoxys calcitrans (L.)) (Diptera: Muscidae) are synanthropic pests that are common in a variety of animal facilities. These flies frequently aggregate in great numbers on animal facilities, developing in decomposing organic matter and animal waste. Fannia spp. are not considered to be significant pathogen transmitters, but can be nuisance pests in high numbers. Over 100 pathogens can be transmitted mechanically by house flies, increasing the risk of disease in animals and their caretakers (Malik et al. 2007). Stable flies are obligate blood feeders, and can transmit pathogens causing equine infectious anaemia (Greenburg 1971), equine stomach nematode worms (Habronema spp.) (Conn et al. 2007; Naem 2007), anaplasmosis (Potgieter et al. 1981), and arthropod hypersensitivity and pruritus (Gortel 1998). Both species of fly can cause losses to production and to the physical condition of livestock. Additionally, farm owners may be more vulnerable to legal challenges when their operations produce high filth fly populations in suburban areas.

\section{Sheep blowflies}

Adult sheep blowflies (Diptera: Calliphoridae) can be domestic nuisances or carry diseases, although in this capacity they are not of great importance. However, the larval stage of these flies can inflict enormous damage and suffering upon domestic animals (Norris 1959). Calliphorid larvae are responsible for myiasis, or the infestation of the living tissues, of animals in all important stock raising countries in the world. In most sheep raising countries, the genus Lucilia represents a 
group of non-specific blowflies that breed in carrion, but are also capable of initiating a cutaneous myiasis of living sheep termed fly strike (Norris 1959). Lucilia cuprina Wiedemann and L. sericata Meigen are responsible for considerable production losses in the wool industry around the world.

\section{Tsetse flies}

Tsetse flies (Diptera: Glossinidae) feed exclusively on vertebrate blood and transmit protozoan parasites of the genus Trypanosoma, which cause human and animal trypanosomosis, known as sleeping sickness or nagana, respectively, in tropical Africa (Maniania et al. 2002). Trypanosoma brucei, Trypanosoma congolense, and Trypanosoma vivax are the most important agents of nagana. Several domestic animals including camels, horses, mules, cattle, sheep, goats, and pigs are susceptible to this disease, which often manifests as anaemia, weakness, reduced muscle mass, enlargement of internal organs and lymph nodes, fever, and diarrhoea. Most organ systems can become infected, and death is common in infected domestic stock.

\section{Horn flies and buffalo flies}

Haematobia (Diptera: Muscidae) is a genus containing several species, but the two of most economic significance in livestock production are the horn fly (Haematobia irritans irritans (L.)) and the buffalo fly (Haematobia irritans exigua (de Meijere)) (Foil and Hogsette 1994). The horn fly has a wider distribution and is found almost throughout the world, including Europe, North Africa, Asia Minor, and the Americas, while the buffalo fly is found through Asia and Oceania (Foil and Hogsette 1994). Most of the biology and control of these two flies is similar. Therefore, the term 'horn fly' will be used to refer to both flies for the rest of this review. Horn flies are one of the most economically important ectoparasites of pastured cattle, feeding exclusively on the blood of their host. Each adult can take in up to 20 blood meals a day causing stress, behavioural changes, and reductions in milk yield and weight gains (Campbell 1976). In addition, horn flies can vector the nematode Stephanofilaria stilesi, causing lesions that weaken cattle and result in hide damage (Bawer et al. 2014).

\section{Ticks}

Ticks are obligate bloodsucking arthropods found on vertebrates in almost every region of the world. Ticks and tick-borne diseases are one of the most important causes of losses to the livestock industry, primarily cattle and small ruminant production, in tropical and sub-tropical areas (Jongejan and Uilenberg 1994). The importance of ticks is due to their ability to transmit a wide range of pathogenic microorganisms, such as protozoa, rickettsiae, spirochaetes, and viruses, together with the direct effects of anaemia, weight loss, and damage to the skin (Jongejan and Uilenberg 1994). There are at least 840 tick species in two major families, namely the Ixodidae or 'hard' ticks (so called by virtue of their hard dorsal shield) and the Argasidae or 'soft' ticks (due to their flexible leathery cuticle). The family Ixodidae comprises approximately $80 \%$ of all tick species, including the species of greatest economic importance to livestock production. The seven main genera of Ixodid ticks are: Boophilus, Dermacentor, Haemaphysalis, Hyalomma, Ixodes, Rhipicephalus and Amblyomma. A number of species can vector diseases, such as anaplasmosis, theileriosis, and babesiosis, which are responsible for significant production losses through mortality and morbidity if not controlled. However, argasid ticks also play a significant role as vectors of diseases, especially in poultry. 


\section{Poultry ectoparasites}

Most research on poultry ectoparasites has concentrated on species that are found consistently on the birds, especially the northern fowl mite, Ornithonyssus sylviarum (Canestrini and Fanzago) and the chicken body louse, Menacanthus stramineus (Nitzsch) (Axtell 1999; Axtell and Arends 1990). Permanent ectoparasites are challenging for fungal biopesticide implementation because of difficulties in treating birds directly and the high temperatures on the skin of the animals, which typically has a negative effect on conidial viability. Intervention with fungal BCAs is better suited to pests that live off the hosts except while feeding, as agents can be applied to off-host harbourages. This group includes bed bugs (Cimex lectularius L.), fowl ticks (Argus persicus Oken), and the chicken red mite, Dermanyssus gallinae (De Geer). All are blood-feeders that can cause significant economic damage and have deleterious effects on the bird welfare when population densities are high.

\section{Litter beetles}

The lesser mealworm, Alphitobius diaperinus (Panzer), is perhaps the most costly pest of poultry throughout the world (Dinev 2013; Dunford and Kaufman 2006; Turner 1986; Wang et al. 2014). The direct contact between these insects and the litter as well as their feeding on dead and sick broiler chickens make $A$. diaperinus effective reservoirs of many avian disease agents and parasites, including some food-borne diseases (Axtell and Arends 1990; Chernaki-Leffer et al. 2010; Moraes et al. 2016; Watson et al. 2000). Because of this ability to transmit pathogens that cause disease, a large population of lesser mealworms poses a significant threat to the production of safe chicken meat. Moreover, the birds will consume large numbers of this pest in preference to their specially formulated diet, which can lead to reductions in bird weight (Despins and Axtell 1995). Lesser mealworm larvae also cause damage to poultry facilities when they burrow into and destroy the insulation materials of broiler houses (Geden and Axtell 1988; Vaughan et al. 1984).

\section{Entomopathogenic hyphomycetes}

\section{Introduction}

EPF, or fungi that kill insects and closely related arthropods, actively invade their host (Figure 1). This process involves conidial adhesion, conidial germination (Figure 2), appressoria formation, and finally penetration from the appressoria through a combination of mechanical pressure and secretion of hydrolytic enzymes that digest the cuticle (Arruda et al. 2005; Butt et al. 1995, 2016; Charnley and St. Leger 1991). Once inside the host, the fungal pathogen proliferates within the haemocoel as hyphal bodies (yeast-like propagules) (Samson et al. 1988). Hyphal bodies are quickly disseminated throughout insect fluids, thus evading host immune cells. Several toxic metabolites may be produced by the pathogen during this stage. For Metarhizium anisopliae (Metch.) Sorokin (Hypocreales: Clavicipitaceae) these toxins are primarily destruxins, for Beauveria bassiana (Bals.-Criv.) Vuill. (Hypocreales: Clavicipitaceae) some isolates may produce beauvericin, bassianolide, and/or oosporein (Kershaw et al. 1999). The toxins appear to have various effects on the host, usually leading to paralysis. However, there is disagreement over the importance of the toxins, as there appears to be variation in the exact way in which EPF kill the host (Butt 1990). Death of the host can be facilitated by a combination of actions including nutrient depletion, physical blockage including organ invasion, and systemic poisoning from pathogen-produced toxins (Charnley 1989; Inglis et al. 2001). Once the host dies, the fungus undergoes saprophytic mycelial growth within the host. If the external humidity is high enough, the fungus will emerge 


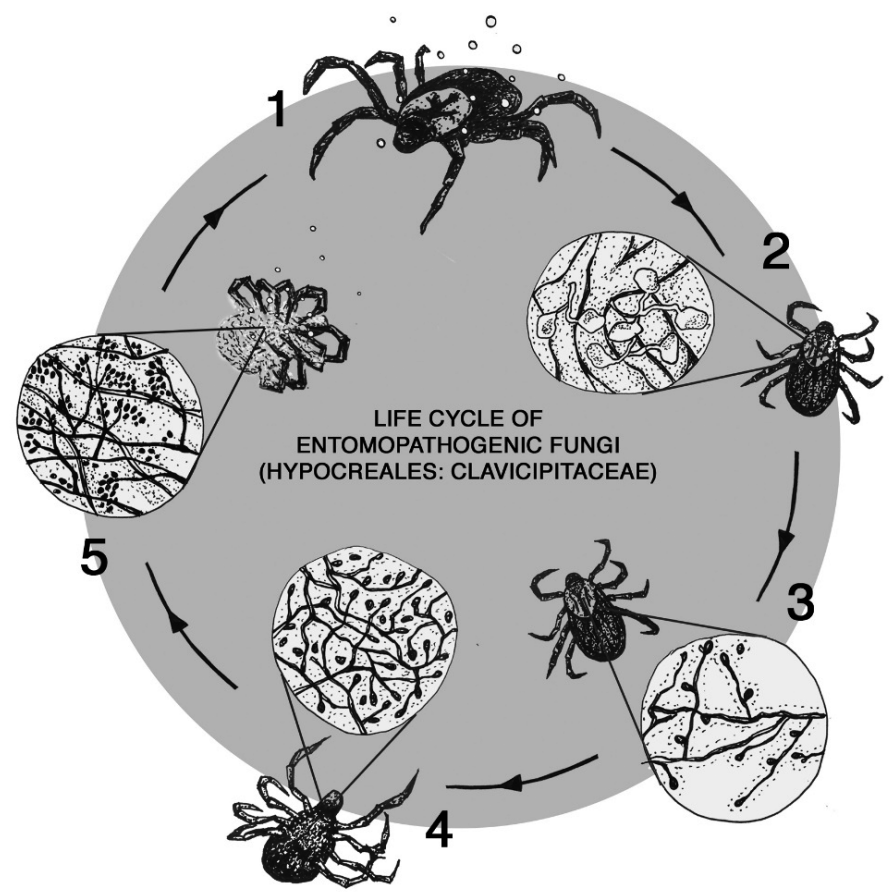

1. Spores or conidia are released from the cadaver of an infected host and persist in the host environment until they come into contact with the cuticle of a suitable new host. The hydrophobic conidia then adhere to the waxy cuticle.

2. The conidium germinates and forms an appressoria, which penetrates the host cuticle through the application of mechanical pressure and production of hydrolytic enzymes.

3. Once inside, the fungus grows through the host as hyphal bodies (yeast-like propagules). Toxic metabolites are produced at this stage destruxins by Metarhizium anisopliae and beauvericin, bassinolide and/or oosporein by Beauveria bassiana.

4. The host dies in $\mathbf{4}$ to $\mathbf{3 0}$ days depending on the organism. Death is facilitated by a combination of nutrient depletion, organ blockage due to hyphal growth, and systemic poisoning due to the toxins.

5. Once the host is dead, the fungus undergoes saprophytic mycelial growth throughout the cadaver until it reaches the cuticle. When the environmental humidity is sufficient, the fungus will emerge back out of the cadaver and sporulate, producing conidia that can be released to disseminate the infection.

Figure 1. Life cycle of entomopathogenic fungi (Hypocreales: Clavicipitaceae) in cattle tick, Rhipicephalus microplus Canestrini (Parasitiformes: Ixodidae) (illustrated by Laura Jayne Weeks, Cornwall, UK).

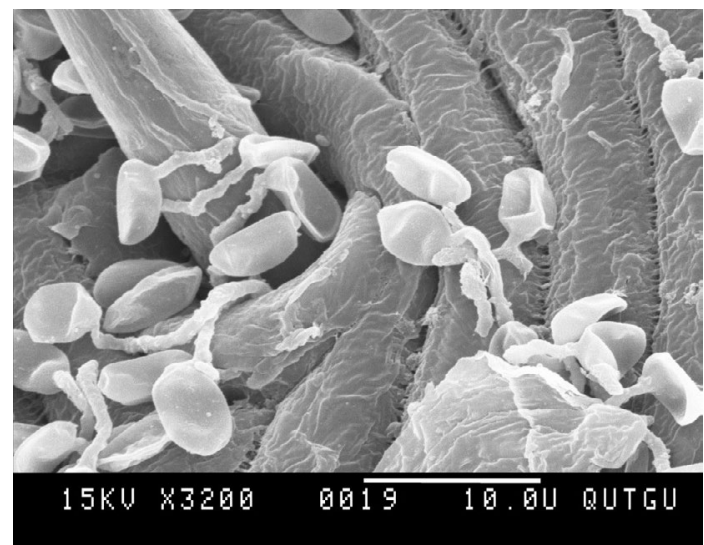

Figure 2. Germination of Metarhizium anisopliae (Metsch.) Sorok conidia on the surface of a cattle tick, Rhipicephalus microplus Canestrini (Parasitiformes: Ixodidae) (photo by Diana Leemon, Department of Agriculture and Fisheries, Queensland, Australia). 
back out of the host and sporulate on the exterior of the host cadaver (Charnley 1989; Samson et al. 1988) producing conidia that are then dispersed from the infected host to uninfected hosts.

EPF can have multiple infection routes. In dry conditions or on a solid substrate they produce aerial conidia, but in liquid, they produce blastospores. When conidia germinate, they have been observed to produce a structure (appressorium), which allows the penetration of the host cuticle through mechanical and chemical (enzymatic) forces, while blastospores appear to be limited to mechanical entry. Blastospores can enter the gut and penetrate directly into the haemocoel whereas conidia in the gut cause stress induced mortality. Blastospores also germinate faster, and store for longer. Therefore, their use should be considered for aquatic applications such as for the treatment of mosquito larvae (Alkhaibari et al. 2016).

There are many variables that are associated with changes in entomopathogenic efficacy including fungal species and isolate, host species and strain, applied dose, and application method. The host species and its cuticular make-up and environment are important; some arthropods have a dry or heavily sclerotized cuticle, which might prevent germination or penetration, respectively. In those arthropods, such as ticks, where direct invasion through the cuticle occurs, dose has a critical impact on the effect; the more conidia exposed to the cuticle, the more that successfully attach (Leemon and Jonsson 2012b). Increasing the dose not only increases tick mortality but also shortens the time to death. One study demonstrated that using three different methods, dipping, surface treatment and spraying, all resulted in a dose dependent effect leading to faster mortality (Bharadwaj and Stafford 2012).

Although effective in the laboratory, the efficacy of EPF for pest control in field trials has been inconsistent due to variations in environmental conditions. Acharya et al. (2015b,c) demonstrated reduced persistence of $B$. bassiana products in the field compared with in the laboratory. Under field conditions conidial viability declined steadily after the first month to less than $20 \%$ by 11 weeks (Acharya et al. 2015c). An appropriate formulation is needed to maintain viability while ensuring an even spread of hydrophobic conidia and enhancing persistence of conidia following exposure to the environment, especially ultraviolet (UV) light and high temperatures (Alves et al. 1998; Morley-Davis et al. 1996; Reis et al. 2008). Oil-based formulations have been shown to reduce damage due to UV radiation compared with aqueous formulations (Alves et al. 1998).

Application methods for livestock, poultry, and equine pest management may be off- or onhost depending on the pest species and life stage targeted. Different application methods will have different environmental considerations, with on-host applications being complicated by the host microclimate and conditions, including temperature, humidity, $\mathrm{pH}$, and, skin secretions and microflora that may enhance or limit germination (Polar et al. 2008). The preferred habitat of the pest species and its lifecycle must be considered when applying EPF for management (Polar et al. 2008). For example, dipping or spraying animals will have little effect on an ear-dwelling tick, and spraying pastures will have limited impact on ticks that quest in brush. Similarly, direct applications to hens would be less effective than habitat treatments for management of poultry ectoparasites that spend much of their time off the host such as bed bugs and poultry red mites.

Although there have been many studies testing the effect of EPF on a wide range of crop, urban, and livestock pests, the studies are often not comparable due to the diversity in fungal isolates that are tested and methods that are used for the assessment. For example, the method of application of a fungus to the target organism in the laboratory is often through dipping in fungal suspensions. However, this method is likely to produce unrealistically high mortalities compared 
with a field application. Although dipping provides a fast and reliable way to screen isolates for pathogenicity in the laboratory, subsequent studies must be conducted under natural conditions to determine the true virulence of the pathogen (Nchu et al. 2010). Different methods of exposure result in a variation of conidial distribution and some areas, particularly joints, mouthparts, setae, and spiracles, are easier for the fungus to penetrate (Arruda et al. 2005; Leemon and Jonsson, 2008). Studies have demonstrated that dipping in fungal suspensions can also block the spiracles resulting in higher background mortality (Soarés 1982).

A substantial number of mycopesticides have now been developed and the active agent in the majority of the products are specific strains of B. bassiana and M. anisopliae (De Faria and Wraight 2007). Few of these formulated products have been tested on livestock and other animal pests, with most of the research and development focused on crop systems. Although the term 'entomopathogenic' refers to causing disease and death in insects, other arthropods, such as ticks and mites also are affected. This is perhaps testament to one of the disadvantages in the use of $E P F$, even though effects on survival may vary by system, some pathogens such as $B$. bassiana are not species- or even order-specific in the laboratory. However, some studies have suggested that the specific environmental requirements of each isolate render them more specific in the field (Fargues and Remaudiere 1977; Goettel et al. 1990; Polar et al. 2008).

It is important to note that the M. anisopliae lineage was revised in 2009 into four species: $M$. anisopliae, Metarhizium robertsii, Metarhizium pingshaense, and Metarhizium brunneum (Bischoff et al. 2009). Many isolates of M. brunneum have been researched for biocontrol with some strains being developed as mycopesticides, however many of the articles detailing this research were published prior to 2009 when these isolates were classified as M. anisopliae. Therefore, we refer to all M. anisopliae isolates as $M$. anisopliae sensu lato (s.l.) unless they were clearly identified as $M$. brunneum in the published literature.

\section{Mosquitoes and biting midges}

\section{Biting midges (Culicoides)}

Studies on the efficacy of EPF for livestock pest control have been conducted on two Culicoides species, Culicoides nubeculosus Meigen (Ansari et al. 2010, 2011; Unkles et al. 2004) and Culicoides brevitarsis Kieffer (Nicholas and McCorkell 2014). These investigations have focused on five pathogens: B. bassiana (Ansari et al. 2010, 2011), the ascomycetous hyphomycete Culicinomyces clavisporus Couch, Romney and B. Rao (not assigned to order or family) (Unkles et al. 2004), Isaria fumosorosea Wize (Hypocreales: Cordycipitaceae) (Ansari et al. 2010, 2011), Lecanicillium longisporum Zare and Gams (Hypocreales: Cordycipitaceae) (Ansari et al. 2010, 2011), and M. anisopliae s.l. (Ansari et al. 2010, 2011; Nicholas and McCorkell, 2014). Results of some of these studies are summarized below. (Note: at the time that some of the research was completed, Isaria fumosorosea was referred to as Paecilomyces fumosoroseus).

C. clavisporus is an EPF with proven activity against mosquitoes (Goettel et al. 1984; Sweeney 1985) and Culicoides biting midges (Unkles et al. 2004). Seven isolates of $C$. clavisporus were tested for efficacy against $C$. nubeculosus larvae, and were found to cause mortality following 3 to 4 days. Lethal concentrations to $50 \%$ mortality ranged from $3.2 \times 10^{5}$ to $1.1 \times 10^{6}$ conidia per $\mathrm{ml}$ distilled water at 96 hours (Unkles et al. 2004). This pathogen causes death following ingestion of conidia, which germinate in the gut. Once the gut is penetrated, the hyphae grow in the haemocoel before bursting out of the dead larvae and infecting other individuals. 
M. anisopliae s.l. also has been tested with C. nubeculosus, and has been found to cause mortality in larvae (Ansari et al. 2010) and adults (Ansari et al. 2011). Likewise, B. bassiana, I. fumosorosea, and L. longisporum all resulted in reductions in survival compared to an untreated control (Ansari et al. 2010, 2011). In larvae, M. anisopliae s.l. is much more effective with almost 80 to $100 \%$ mortality with all 10 isolates tested compared to less than $40 \%$ with all other species and isolates (Ansari et al. 2010). Although each larval instar was tested, there were no differences in the efficacy of each pathogen isolate or species by life stage (Ansari et al. 2010). In adults, the results were less contrasting. The lethal time to 50\% mortality was shortest with M. anisopliae s.l. (1.42 days) and longest with I. fumosorosea (3.22 days) (Ansari et al. 2011). None of the pathogens had any effect on egg hatching (Ansari et al. 2010). Unfortunately, infected larvae did not sporulate (Ansari et al. 2010), and so fungal populations in breeding sites would be unlikely to self-propagate to infect future generations.

In an Australian species, Culicoides brevitarsis Kieffer, four isolates of M. anisopliae s.l. (ARI Ma01, 03,04 , and 06 ) were shown to cause mortality in larvae and adults. Assays in cattle dung as a breeding site resulted in 98.5\% reduction in adult emergence (Nicholas and McCorkell 2014). The four isolates had varying effectiveness with $\mathrm{MaO} 4$ resulting in significantly less adult mortality after 8 days compared to the other three. Interestingly, in a semi-field study where treated cattle dung pats remained in the field for 6 days as sentinels for fly oviposition before being returned to the laboratory for development, the number of adults was significantly higher in M. anisopliae s.l. isolate Ma06 (Nicholas and McCorkell 2014). Targeting breeding sites of this dung-breeding species would be easier than for other Culicoides species, where the preferred breeding sites are many, hard to access, or unknown. However, there are concerns about the treatment of dung pats due to potential effects on non-target organisms such as dung beetles (Nicholas and McCorkell 2014).

\section{Mosquitoes}

Many studies have been conducted on the effect of EPF on mosquitoes, particularly the three main genera: Aedes, Anopheles, and Culex. Although not all the mosquito species tested are pests of commercial animal facilities, the results will be mentioned here, as some of the general effects of the fungus on mortality, behaviour, fertility and fecundity are likely to be transferable to livestock pests.

M. anisopliae s.l. and B. bassiana have been shown to be efficacious against: Aedes aegypti L. (Alkhaibari et al. 2016; Clark et al. 1968; Daoust and Roberts 1982; Darbro et al. 2012; GarcíaMunguía et al. 2011; Gomes et al. 2015; Kirubakaran et al. 2015; Lules et al. 2012; Miranpuri and Khachatourians 1990; Paula et al. 2011a,b; Reyes-Villanueva et al. 2011), Anopheles gambiae Giles (Blanford et al. 2012a; Bukhari et al. 2011; Howard et al. 2010; Mnyone et al. 2010, 2011; Scholte et al. 2005), Anopheles messeae Fall (Khodyrev et al. 2013), Anopheles stephensi Liston (Blanford et al. 2005, 2012b; Bukhari et al. 2011; Daoust and Roberts, 1982; George et al. 2011), Culex quinquefasciatus Say (Gayathri et al. 2010; Kirubakaran et al. 2015), and Culex pipiens pipiens L. (Clark et al. 1968; Daoust and Roberts 1982; Khodyrev et al. 2013).

In a comprehensive study by Clark et al. (1968) that tested multiple isolates of several species against multiple host species, $B$. bassiana was shown to be efficacious against Aedes nigromaculis Ludlow, Aedes sierrensis Ludlow, Anopheles albimanus Wiedemann, and Culex tarsalis Coquillett. Other fungal pathogens that have been tested against mosquitoes include $C$. clavisporus (Goettel et al. 1984; Sweeney 1985), Isaria farinosa (Holmsk.) Fr. (Blanford et al. 2012b), Metarhizium acridium 
(Driver and Milner) J.F. Bisch., Rehner and Humber (Blanford et al. 2012b; George et al. 2011), M. robertsii J.F. Bisch., Rehner and Humber (Khodyrev et al. 2013), and I. fumosorosa (Wize) (Gayathri et al. 2010).

Studies have been completed with adult and larval mosquitoes. Bukhari et al. (2011) found that larval An. gambiae and An. stephensi exposed to B. bassiana and M. anisopliae s.l. conidia had reduced survival and so less pupation, but this effect was dependent upon the formulation used. Unformulated EPF had reduced efficacy by day 2 in water (Bukhari et al. 2011). EPF formulated in carriers were found to persist longer in water, killing larvae after 7 days. A study on B. bassiana with C. quinquefasciatus found developmental abnormalities in the wing resulting from larvae raised in fungal-treated water as well as adults that failed to eclose successfully from the pupae (Gayathri et al. 2010). Different species were found to be more effectively treated with different formulations. For example, C. pipiens pipiens and An. messeae were most susceptible to water suspensions and dry conidia, respectively (Khodyrev et al. 2013). This result is not surprising given that dry conidia float and Anopheles spp. feed at the surface, whereas Culex spp. feed within the water column. Larval feeding behaviour should be considered when formulating products for application to larval breeding sites. Metarhizium also has been shown to infect mosquito eggs and reduce larval hatch rate in a dose-dependent manner (Lules et al. 2012).

Target insects typically are treated with conidia, but conidia have been less successful for treating mosquito larvae in aquatic environments. Dry conidia applied to the water surface attached to the siphon and larvae died from asphyxiation due to perispiracular infections (Clark et al. 1968; Kirubakaran et al. 2015). However, Clark et al. (1968) found that one-day post moult larvae were no longer susceptible to the conidial formulations because the larvae were able to evade attachment. Studies with Ae. aegypti larvae have demonstrated that in the aquatic environment fungal blastospores kill faster than conidia, typically within 12 to 24 hours (Alkhairbari et al. 2016; Miranpuri and Khachatourians 1990). This fast action is related to the dual mode of action, as the blastospores adhere to and penetrate the cuticle as well as being ingested and penetrating the gut (Alkhairbari et al. 2016). Gene expression studies revealed that both pathogen and host could detect each other. Pathogenicity genes were upregulated in blastosprores exposed to larvae and immune and defence genes were upregulated in mosquito larvae exposed to blastospores (Alkhairbari et al. 2016).

As with all other systems, fungal isolate can have a substantial impact on virulence, with apparently contradictory results in different studies. Blanford et al. (2012b) compared Metarhizium, Beauveria and Isaria spp., and found I. farinosa was the least effective at reducing the survival of An. stephensi. (Note that at the time that some of the research was completed, Isaria farinosa was referred to as Paecilomyces farinosus). As well as genetic virulence, isolates vary in their host specificity and hosts vary in susceptibility. Clark et al. (1968) tested multiple species of Aedes, Anopheles and Culex larvae with EPF and found that while Anopheles and Culex were readily infected, Aedes appeared to be more resistant to infection. It was noted that the fungus took longer to penetrate the siphon of the Aedes spp. tested, and if the fungus was unable to occupy more than one-fourth of the siphon then the individual would moult and emerge as the next instar unaffected (Clark et al. 1968).

Fungal virulence also can be substantially influenced by host passage (Daoust and Roberts 1982; Reyes-Villanueva et al. 2011), formulation, and application technique. In a study by Daoust and Roberts (1982), a single passage through a host increased pathogenicity by 1.63 to 2.45 times compared to unpassaged isolates. Culturing EPF on artificial media gradually reduces the 
production of toxins that are believed to be partially responsible for killing infected arthropods (Reyes-Villanueva et al. 2011).

Life stage (Miranpuri and Khachatourians 1990; Mnyone et al. 2011), feeding status (Mnyone et al. 2011; Paula et al. 2011a), co-infection status, and resistance status can all impact host sensitivity to entomopathogens. Larval mosquitoes become less susceptible to EPF with each moult (Miranpuri and Khachatourians 1990). In contrast, adult mosquitoes increased in sensitivity with age (Mnyone et al. 2011). As adult mosquitoes senesce, increased susceptibility to fungal pathogens is likely due to a weakened cuticle and reduced immune function (Mnyone et al. 2011). Non blood-fed (or sugar-fed only) mosquitoes were significantly more susceptible to EPF than all blood-fed categories (Mnyone et al. 2011; Paula et al. 2011a). This likely was due to a lack of nutritional reserves for mounting an immune response (Mnyone et al. 2011) or upregulated immune function (Paula et al. 2011a). However, by 72 to 96 hours post-blood-feeding susceptibility was equal to that of a sugar fed mosquito (Mnyone et al. 2011; Paula et al. 2011a). In addition, as with all other pest management systems, environmental conditions, such as temperature, $\mathrm{pH}$, and relative humidity, can influence the viability of conidia and germination rate and thus pathogenicity to mosquitoes.

Although mortality is relatively slow compared to synthetic pesticides, fungal infection has been demonstrated to cause a range of pre- and sublethal effects on mosquitoes including reductions in feeding, fecundity, motility, and predator avoidance, coupled with an increase in metabolic rate and changes in development (Blanford et al. 2012b; Darbro et al. 2012; George et al. 2011; Reyes-Villanueva et al. 2011). Changes in host location and feeding behaviour due to fungal infections by B. bassiana and M. anisopliae s.l. have been demonstrated in species of the genera Aedes, Anopheles, and Culex (Blanford et al. 2012b; Darbro et al. 2012; George et al. 2011). Sublethal effects of $B$. bassiana on Ae. aegypti included a reduction in mosquito-human contact by $30 \%$, a reduction in fecundity from 75 to 45 eggs/female/lifetime (Darbro et al. 2012). Reduction in fecundity also was observed with Ae. aegypti and M. anisopliae s.l. with average egg production declining from 35 eggs in untreated mosquitoes to $<1$ egg following exposure to a high-virulence isolate (Reyes-Villanueva et al. 2011). When mosquitoes were offered the opportunity to feed in a 10-minute period, those previously exposed to EPF had decreased activity from $90 \%$ active to approximately $40 \%$ active by day 10 , and were significantly less active compared with controls by day 4 (Darbro et al. 2012). After day 4, there was a difference in landing and after day 10 in blood-feeding. In a semi-field large cage study, survival was reduced by 60 to $95 \%$ (Darbro et al. 2012). Although effect varied by trial in the semi-field study, blood-feeding was reduced by $80 \%$ in one trial, yet there was no impact on fecundity. Feeding propensity also was reduced in An. stephensi treated with Beauveria and Metarhizium (Blanford et al. 2012b). When biting risk was calculated as a product of survival and feeding propensity, this risk was zero for most isolates of Beauveria (Blanford et al. 2012b). Reductions in host location and feeding behaviour due to fungal infection have been shown to be correlated with reduced electroantennagram and electropalpagram recordings. Single cell recordings from maxillary palps demonstrated that fungal infection resulted in impaired response in neurons that respond to host location cues such as carbon dioxide and 1-octen-3-ol (George et al. 2011). Therefore, fungal infection would reduce the ability of a mosquito to locate a host, thus reducing survival, reproduction and pathogen transmission in field situations.

Although studies have not been conducted on the impact of fungal infection on transmission of animal pathogens, several studies have demonstrated that in addition to longevity and other sublethal effects there is a reduction in susceptibility to human parasites and viral pathogens in fungus-infected individuals (Blanford et al. 2005; Dong et al. 2012). Reduction in the development 
of Plasmodium parasites in EPF-infected Anopheles has been demonstrated by Blanford et al. (2005) to potentially decrease malaria risk 80 -fold. As well as a decrease in survival, EPF-infected mosquitoes were less likely to develop infective stage sporozoites in the salivary glands, $8 \%$ compared to $35 \%$ in the malaria only control (Blanford et al. 2005). Furthermore, two-fold reduced dengue virus replication in the midgut of Ae. aegypti (Dong et al. 2012) was demonstrated to be due to activation of the immune system following fungal exposure, specifically the Toll (anti-gram positive bacteria, fungi, and viruses) and the anti-viral JAK/STAT (janus kinase signal transducer and activator of transcription) pathways (Dong et al. 2012).

\section{Application techniques for mosquito and biting midge management with EPF}

Application represents an important limitation for the implementation of EPF in the management of blood feeding flies such as mosquitoes and midges. The use of treated resting sites has been suggested (Howard et al. 2010; Mnyone et al. 2010; Scholte et al. 2005), but careful research is needed to ensure these sites are at least as attractive as the naturally available resting sites. Formulation is also important to maximize duration of efficacy. Germination rates of B. bassiana and $M$. anisopliae s.I. conidia applied to netting, such as the material used for insecticide-treated curtains in Africa, decreased over 7 days, from 77 to $62 \%$ (B. bassiana) and 36 to 2\% (M. anisopliae) (Howard et al. 2010). Other studies also have found decreased conidial viability on polyester netting, compared to mud panels and cotton cloth (Mnyone et al. 2010). However, in both studies despite a reduction in viability and germination, residual efficacy remained high for up to one month (Howard et al. 2010; Mnyone et al. 2010).

Several studies have demonstrated that autodissemination of an EPF is possible during mating behaviour (García-Munguía et al. 2011; Reyes-Villanueva et al. 2011). Virgin B. bassiana treated Ae. aegypti males can transfer conidia to females, resulting in a high $(90 \%)$ mortality rate (GarcíaMunguía et al. 2011). Of these females, 40 to 67\% were infected but not inseminated. Therefore, they were infected during courtship and mating attempts but mating did not occur or was unsuccessful. This fungus also reduced fecundity in surviving females, from 42 eggs/female in the control to 2 eggs/female in the fungal treatment, i.e. females that acquired conidia from males. Dissemination of $M$. anisopliae s.l. also has been demonstrated, all Ae. aegypti females confined with a male exposed to a highly virulent isolate died within 15 days (Reyes-Villanueva et al. 2011). Only $10 \%$ of these were inseminated, compared to an insemination rate of $46 \%$ in controls (Reyes-Villanueva et al. 2011). As mosquitoes in animal facilities will be dispersed throughout the environment, development of an autodissemination device could be highly beneficial.

Using products with different modes of action in combination is one technique employed in IPM to delay the development of resistance and enhance control program effectiveness. EPF with proven efficacy with mosquitoes have been shown to be compatible for use with other pesticides, such as imidacloprid (Paula et al. 2011 b) and neem (Gomes et al. 2015). The main active ingredient of neem, azadirachtin, acts as a growth regulator of mosquito larvae leading to incomplete moulting and a weakened immune system. Consequently, when the two biopesticides were applied together mortality in Ae. aegypti was increased even at sublethal conidial concentrations (Gomes et al. 2015). In adults, a 6- or 12-hour exposure resulted in significantly higher mortality in the combined imidacloprid and fungus treatment than either insecticideal agent alone (Paula et al. 2011b).

In summary, EPF are effective at reducing survival in mosquitoes and biting midges. Decreasing the longevity of these vectors will reduce their feeding and, therefore, the number of blood meals, so that host-vector contact is reduced. This reduction can dramatically influence disease 
transmission. In addition, mosquitoes have been shown to be less susceptible to human pathogens when already infected with a fungus, which likely translates to a similar relationship with animal pathogens.

\section{Other biting and nuisance flies}

House flies, stable flies, and Fannia spp.

House flies ( $M$. domestica), stable flies (S. calcitrans), and lesser house flies (F. canicularis) and others in the Fannia spp. group are common pests and vectors with a global distribution in animal facilities. Continuing resistance to available insecticides by these 'filth flies' has made the need for alternative control methods critical. The generalist EPF B. bassiana and M. anisopliae s.l. are promising candidates for control of these flies (Figure 3). Dresner (1950) suggested the potential of $B$. bassiana as a BCA against pest flies. Since then, an abundance of literature has documented the potential efficacy of these fungal species to infect and kill immature and adult filth flies under laboratory (Angel-Sahagún et al. 2005; Barson et al. 1994; Geden et al. 1995; Hall et al. 1972; Kaufman et al. 2008; Lecuona et al. 2005; Watson et al. 1995; Weeks et al. 2017), and field conditions (Kaufman et al. 2005; Watson et al. 1996).

B. bassiana and M. anisopliae s.l. are soil-dwelling fungal pathogens that are naturally found in filth fly populations. Steinkraus et al. (1990) first reported B. bassiana infections at low prevalence $(\sim 1 \%)$ in house flies from New York state dairy farms. Prevalence of $B$. bassiana infection of house flies on poultry facilities in Argentina was between 0.4 and $1.4 \%$ (Siri et al. 2005). Natural $B$. bassiana infection of stable flies was less than $1 \%$ in dairy and pig farms in Denmark (Skovgård and Steenberg, 2002). There have been no reports on the natural prevalence of M. anisopliae s.l. in filth fly systems. Filth flies live in a diverse range of septic environments including excreta, garbage, animal carcasses, wet animal feed, decaying vegetation, and other waste, and therefore, it is not surprising that these flies encounter EPF. In the presence of $B$. bassiana and $M$. anisopliae s.l. flies can pick up $4 \times 10^{4}$ to $1 \times 10^{5}$ conidia in 30 minutes to 4 hours (Anderson et al. 2011; Renn et al. 1999).

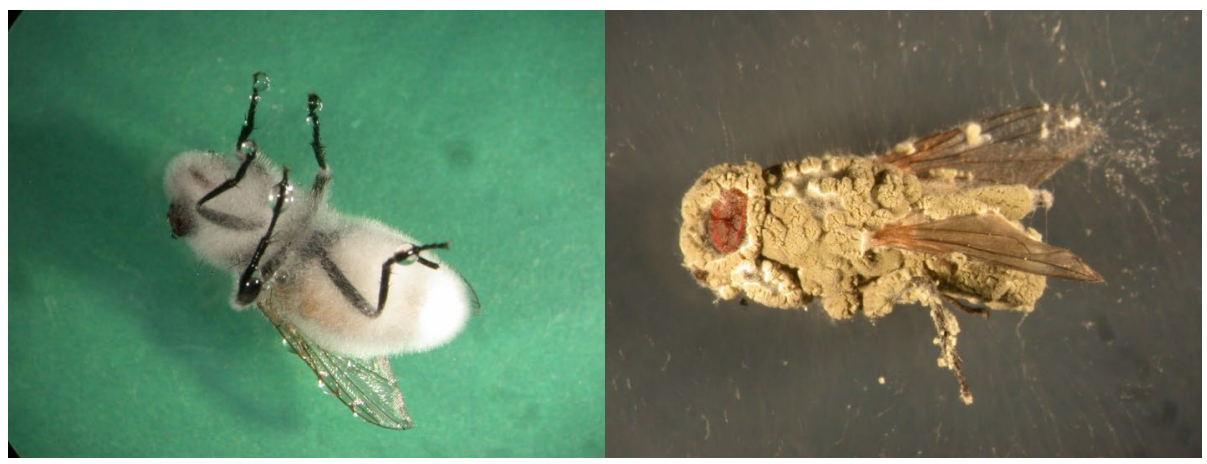

Figure 3. Entomopathogenic fungi (Hypocreales: Clavicipitaceae) on the house fly, Musca domestica L. (Diptera: Muscidae). Left photo shows a Beauveria bassiana (Bals.-Criv.) Vuill. infected cadaver and right photo shows a Metarhizium anisopliae (Metsch.) Sorok cadaver sporulating (photos by Diana Leemon, Department of Agriculture and Fisheries, Queensland, Australia). 
B. bassiana and M. anisopliae s.l. have been tested against flies with various life stages, ages and physiological characteristics. The age of house fly adults does not affect pathogenicity of $B$. bassiana or M. anisopliae s.l. (Kaufman et al. 2008; Rizzo 1977). However, differences were seen in the virulence of the EPF to male and female flies (Anderson et al. 2013). Female house flies had shorter lifespans than either males or controls after treatment with B. bassiana (Lecuona et al. 2005). Acharya et al. (2015a) found females died at the same rate when treated with either B. bassiana or M. anisopliae s.l., whereas males survived longer. Furthermore, EPF infection also reduced the reproductive fitness of house fly populations by more than $90 \%$ per capita lifetime fecundity and decreased egg viability (Acharya et al. 2015a). Horizontal transfer of conidia has been recorded in house fly adults. Females were infected with M. anisopliae s.l. after being confined with fungus-treated males (Cárcamo et al. 2015).

Both B. bassiana and M. anisopliae s.l. have been tested against all life stages of several species of filth flies. Low or no susceptibility to fungal infection has been demonstrated by some stages of immature stable flies (Moraes et al. 2008). Resistance to fungal infection by stable fly larvae and pupae has been observed (Moraes et al. 2008), although high doses of M. anisopliae s.l. conidia caused egg mortality and infections in larvae emerging from treated eggs. Machtinger et al. (2016) also found decreased emergence from house fly and stable fly eggs treated with some commercial formulations of B. bassiana and M. anisopliae s.l., and Weeks et al. (2017) found increased mortality in adult flies exposed to these same products. However, mortality may have been caused by, or assisted by, the formulation rather than infection by the fungal conidia.

The effects of EPF on immature house flies have been variable. In some cases, only limited pathogenic effects on house fly larvae by B. bassiana have been observed (Geden et al. 1995; Lecuona et al. 2005). In others, increased mortality after fungal exposure has been demonstrated. Mature house fly larvae were susceptible to $B$. bassiana, forming normal puparia but failing to produce adult flies (Barson et al. 1994; Darwish and Zayed, 2002; Steinkraus et al. 1990). Bernardi et al. (2006) observed reduced emergence when house fly larvae were treated with M. anisopliae s.l., however B. bassiana treatment did not influence the survival of treated puparia. Similarly, Fernandes et al. (2013) found that house fly larvae were more susceptible to M. anisopliae s.I. than B. bassiana. Younger larvae may be more susceptible than older larvae, potentially because of cuticular properties (Boucias and Pendland 1991). The responses of immature filth flies to fungal infection may also depend on the virulence of the fungal isolate, assay method, fly colony variability, conidial doses, substrate used, and culture methods. Larval resistance to fungal infection may be related to the strong immunological defences that a fly needs to cope with the diversity of microorganisms found in the developmental environment of filth breeding flies (Nayduch and Joyner 2013).

Environmental conditions can affect the virulence of B. bassiana and M. anisopliae s.l. to filth flies. Temperature and relative humidity affect germination of conidia (Arthurs and Thomas 2001; Sivasankaran et al. 1998). Relative humidity was not found to be a key factor in infection of house fly larvae by M. anisopliae s.l., but was influential in the efficacy of B. bassiana (Sharififard et al. 2011). In other studies, temperatures of $30{ }^{\circ} \mathrm{C}$ and relative humidity over $90 \%$ were the most effective conditions for virulence of $B$. bassiana against house fly adults (Mishra et al. 2015). However, these conditions may be isolate specific (El Damir 2006; Mishra and Malik 2012).

Other environmental conditions influencing effectiveness of EPF include exposure to UV light, dust, and particulate debris (Leland and Behle 2005; Watson et al. 1995). It has been suggested that dust, dander, and other debris from animals and facility management can coat and inactivate conidial applications (Watson et al. 1995). Therefore, presence of high levels of particulate debris is a serious 
consideration for application of B. bassiana or M. anisopliae s.l. in livestock, poultry, or equine facilities. Rapid declines in fungal spray virulence in poultry houses was observed after treatment (Acharya et al. 2015b), which may also have been a result of excessive ammonia in the basement areas.

Individual fly or fly population differences may influence efficacy of fungal control. Conidial germination of B. bassiana and M. anisopliae s.l. can be triggered by insect cuticle components (ElSayed et al. 1993), and house flies can also affect fungal persistence by both deactivating conidia with antifungal effects of excreta and vomit, and by physically removing conidia by grooming (Acharya et al. 2015a; Moraes et al. 2015). Behavioural modifications have been observed in house flies after fungal infection. Anderson et al. (2013) found house flies preferentially rested in areas of high temperature after fungal exposure. This 'behavioural fever' nominally benefited fitness of the flies by reducing pathogen virulence, effectively extending fly survival to enable females to oviposit, although infected females were less fecund after treatment. Other behavioural changes such as reduced feeding and increased grooming also have been noted (Tamilam et al. 2010).

Various application methods for B. bassiana and M. anisopliae s.I. have been examined in laboratory settings including topical applications, baits, and residual sprays and dusts. Aqueous and dust formulations can be applied directly on target flies or as a spray into the environment. Topical application was found to be more virulent than oral applications in house fly adults and larvae (Sharififard et al. 2011). Aqueous applications to plywood were less effective than dusts (Watson et al. 1995), although this may have been due to absorption of the liquid solution by the wood base (Blanford et al. 2012a). However, other studies found dust applications caused faster kill times than liquid applications to house flies (Geden et al. 1995). Direct residue spraying on concrete and wood has been attempted, but as with plywood surfaces, these substrates can negatively influence infection rates and conidia persistence (Blanford et al. 2012a). Sprays of B. bassiana applied to plastic sheeting and then hung in poultry facilities slowed fungal degradation over time, and minimized overall treated surface area (Acharya et al. 2015b). Larvae of Fannia femoralis (Stein) and F. canicularis were both susceptible to direct application of B. bassiana (Hall et al. 1972). In this study, mortality increased to $87 \%$ when B. bassiana was passed through the host 3 times, suggesting that the orthopteran-derived fungus adapted to the new host.

House flies have been shown to be susceptible to sugar baits formulated with fungal conidia in the laboratory (Geden et al. 1995; Lecuona et al. 2005; Machtinger et al. 2016; Renn et al. 1999; Watson et al. 1995). Mortality reached $100 \%$ generally within 6 to 10 days, depending on the assay methods as well as isolate and dose of the EPF. Exposure of adult house flies to M. anisopliae s.l. baits resulted in high levels of mortality within 8 days (Hong and Hai 2012). It has been suggested that using baits could provide an alternative to chemical control, slow insecticide resistance, and reduce control costs. Because some isolates of both B. bassiana and M. anisopliae s.l. are generalist EPF with low selectivity, there are potential risks to natural enemies (Zimmer et al. 2010). Therefore, the use of baits could minimize the risks to beneficial arthropods (Vega et al. 1995). However, there have been no published field studies assessing the efficacy of baits for control of filth flies.

Different $B$. bassiana and $M$. anisopliae s.l. isolates have variable time requirements to achieve high mortality in filth flies, so there has been some interest in the potential synergism between EPF and bacterial pathogens or insecticides. Spray applications of a combination of B. bassiana and $B$. thuringiensis were more effective as a house fly larvicide than $B$. bassiana on its own, which was not effective at controlling larval populations or inhibiting adult emergence (Mwamburi et al. 2009). Sharififard et al. (2011) found synergistic interactions between combinations of $M$. anisopliae s.l. and sublethal doses of the insecticide spinosad for both adult and larval house flies. 
This combination also led to a reduced level of fungal inoculum needed to cause high levels of mortality in the test groups. Combinations of sublethal doses of six different insecticides with $B$. bassiana conidia caused a higher mortality than when the fungus was used alone, except with bifenthrin and fipronil (Farooq and Freed 2016). In several combinations, adult longevity and fecundity, egg hatching, percentage pupation, and pupal weight were all reduced. The duration of the larval and pupal stages were prolonged as well. Sublethal doses of synthetic insecticides can act as physiological stressors or behaviour modifiers, weakening the immune response to pathogen infection (Inglis et al. 2001).

Significant differences in virulence have been noted among isolates of B. bassiana and $M$. anisopliae s.l. This may be due to different levels of enzymes produced by the different isolates (St. Leger et al. 1986), and fungal adaptation to specific hosts. Isolate differences have been noted in house fly adult and larval mortality (Mishra and Malik 2012; Sharififard et al. 2011). Highly variable pathogenicity of both species of fungus was observed with stable flies (López-Sánchez et al. 2012). House flies were highly susceptible to both B. bassiana and M. anisopliae s.l., but only one isolate of $B$. bassiana was highly pathogenic to stable flies. Anderson et al. (2011) found some M. anisopliae s.l. isolates to be less virulent than B. bassiana, but attributed differences to use of orthopteranderived isolates, and suggested that isolates may adapt to their current host and lose plasticity for affecting other arthropods. Therefore, it has been suggested that the search for EPF against filth flies should include isolates from the target insect (Poprawski et al. 1985; Steinkraus et al. 1991).

Relatively few field studies have been conducted evaluating EPF as filth fly BCAs. An IPM program including a spray formulation of $B$. bassiana in conjunction with pupal parasitoid releases was successful in caged-layer poultry in New York state (Kaufman et al. 2005). The number of fly larvae recovered from the treated facilities was less than half the number recovered from pyrethrin treated facilities, moreover there was a greater recovery of beneficial insects. Watson et al. (1996) observed reduced adult house fly numbers after spray applications of $B$. bassiana in calf hutches. Applications of $M$. anisopliae s.l. reduced populations of house flies in treated poultry sheds twofold compared to the control sheds (Fernandes et al. 2013). Conversely, weekly applications of $B$. bassiana were inadequate to achieve effective larval control in poultry houses in South Africa (Mwamburi et al. 2009).

Despite the potential of EPF as BCAs of filth flies that has been demonstrated in laboratory studies there are several challenges relating to environmental stress and suitable formulation and application methods to address for EPF to be competitive with synthetic insecticides. The potential that has been demonstrated in laboratory studies suggests there should be more field studies to evaluate both B. bassiana and M. anisopliae s.l. as BCAs for filth flies.

\section{Sheep blowflies}

L. cuprina is the most prevalent and important cause of fly strike in Australia, accounting for more than $90 \%$ of all fly strike on susceptible sheep (Vogt and Woodburn 1979), while L. sericata is the most important fly strike species in Northern Europe (Wall et al. 1992). Over the last 100 years, wide ranges of chemicals and insecticides have been used to control blowfly strike, but the development of resistance and concerns around toxic residues in the fleece has limited their use.

There is considerable potential for alternative non-chemical controls such as EPF for supplementary blowfly control, but little work has been conducted in this area. There is one report in the literature involving fungal control of $L$. sericata (Wright et al. 2004) and two reports investigating EPF against 
L. cuprina (Leemon and Jonsson 2012a, 2012b). Wright et al. (2004) screened an isolate of $M$. anisopliae s.l. against adult females using a variety of methods for exposing the flies to conidia: topical application, immersion, and tarsal contact. They achieved high levels of infection with immersion and tarsal contact and concluded that M. anisopliae s.l. may be an effective BCA for L. sericata if the insects can be attracted to and induced to alight on a treated surface, and if the appropriate duration of contact is achieved.

When adult L. cuprina were fed M. anisopliae s.I. conidia mixed with food the adult flies died after an initial latency period of 3 to 5 days depending on isolate though regardless of dose (Leemon and Jonsson 2012a). Therefore, there is a minimum time to adult L. cuprina death irrespective of conidial dose. Light and scanning electron microscopy investigations indicated that the most likely route of entry for the fungus is through the buccal cavity (Leemon and Jonsson, 2012b). Leemon and Jonsson (2012a) concluded that although an attractant trap baited with a mixture of $M$. anisopliae s.l. conidia and food might be the best method for applying a fungus to adult blowflies it would be unlikely to be as effective as traditional traps using chemicals. Conidia in outdoor traps would likely rapidly lose viability due to high temperatures and humidity. Furthermore, the time lag from fungus uptake to death might mean that flies in the traps would die from heat and dehydration before the fungus could kill them. Due to this time lag to death and concerns that flies would have time to initiate strike before death, Leemon and Jonsson (2012b) concluded that further development of M. anisopliae for the control of adult L. cuprina was not warranted.

Ideally, blowfly larvae need to be prevented from reaching the damaging third instar stage. Although third instar L. cuprina larvae are highly susceptible to a number of isolates of $B$. bassiana and $M$. anisopliae s.l. (D. Leemon, unpublished data), applying fungal conidia to areas where sheep blowfly drop from the sheep to pupate after fly strike is not practical or economical. D. Leemon (unpublished data) screened isolates of $B$. bassiana and M. anisopliae s.l. against first instar L. cuprina larvae using two bioassays. First instar larvae were susceptible to most of the $M$. anisopliae s.l. isolates and highly susceptible to selected B. bassiana isolates. This unpublished research implies that EPF have potential for further investigations into developing prophylactic and remedial fleece treatments for blowfly strike.

\section{Horn flies}

Horn flies are one of the most economically important ectoparasites of pastured cattle, feeding exclusively on the blood of their host with control largely reliant upon chemical insecticides, applied as sprays, dusts, pour-ons, feed additives, ear tags and boluses. However, control failure due to the development of resistance to the available chemical classes has occurred in many regions. Natural infections of horn flies by EPF have been reported, albeit rarely (Holderman et al. 2017).

A number of studies have investigated EPF as a potential alternative management tool for the horn fly (Angel-Sahagún et al. 2005; Bawer et al. 2014; Galindo-Velasco et al. 2015; Holderman et al. 2017; Leemon 2012; Lohmeyer and Miller, 2006; Mochi et al. 2009, 2010a,b). Most of these studies involved laboratory evaluations of different isolates or formulations of M. anisopliae s.l., B. bassiana, I. fumosorosa or I. farinosa against different stages of horn fly (Angel-Sahagún et al. 2005; Bawer et al. 2014; Holderman et al. 2017; Lohmeyer and Miller, 2006; Mochi et al. 2010a,b). Generally, isolates of M. anisopliae and B. bassiana are the most virulent for horn flies, but mortality after treatment, as with other flies, is slow. As adult flies are able to oviposit 3-5 days post eclosion a rapid death rate is important for their control. To accelerate mortality, Holderman et al. (2017) 
passaged a horn fly-derived isolate of $B$. bassiana (EN1) back through adult horn flies. However, after seven passages they did not find any increase in virulence from this selection process. Although horn flies can begin ovipositing before a fungal infection can cause death, they only deposit small numbers of eggs in multiple batches through the adult stage of their life cycle (Foil and Hogsette 1994). Thus even if a fungal infection does not prevent the first batch of eggs, if should still reduce fecundity by killing the adult before subsequent oviposition events.

The direct application of EPF to cattle for horn fly control has been reported (Galindo-Velasco et al. 2015; Leemon 2012; Mochi et al. 2009). Mochi et al. (2009) applied an isolate of M. anisopliae s.l. to cattle to target the immature stages in the manure, and the adult stage on the animals. They were able to kill larval horn flies through the oral administration of M. anisopliae s.l. conidia microencapsulated in sodium alginate. This microencapsulated formulation maintained the conidial viability during passage through the bovine gastrointestinal tract so that the fungus remained active against horn fly larvae in the manure. Direct application of an aqueous formulation of M. anisopliae s.l. to cattle reduced adult horn fly populations (Mochi et al. 2009). Leemon (2012) carried out a preliminary evaluation of the effect of two different formulations of M. anisopliae s.l. on natural horn fly populations on pastured cattle. Cattle sprayed once with an emulsified oil-based $M$. anisopliae s.l. formulation resulted in an initial rapid decrease in flies. However, after three weeks the average population on the cattle had increased to $>300 \mathrm{flies} / \mathrm{side} /$ animal, although the number on the untreated cattle was still higher at $>500$ flies/side/animal. Another group of cattle were treated with M. anisopliae s.l. conidia in pure vegetable oil applied to limited areas on the backs of cattle. The application was similar to what might be achieved with a 'pour on' strategy or even self-application through a back rubber. The 'pour on' was applied three times per week over three weeks during which time the fly numbers decreased to fewer than 50 flies/side/animal compared to $>500$ flies/side/animal on untreated animals. This level of control was maintained for up to a week after the applications ceased.

Five isolates of M. anisopliae s.l. and three isolates of $I$. fumosorosea formulated in an aqueous suspension were applied to the backs of individually caged cattle (Galindo-Velasco et al. 2015). Seven of these isolates had previously been evaluated against immature and adult horn flies by Angel-Sahagún et al. (2005). The M. anisopliae s.l. isolates achieved between 94 and 100\% reduction in flies over the 13 days of direct counts, while the I. fumosorosea isolates achieved between 90 and $98 \%$ reduction in flies over this period. During the same period, flies decreased on untreated cattle between 19 and $29 \%$ only. In addition, Galindo-Velasco et al. (2015) found a reduction in the emergence of the next generation of adult flies from the manure of cattle treated with the fungal isolates.

\section{Tsetse flies}

Tsetse flies are a group of several species in the Glossina genus, which transmit several pathogens that cause livestock and human diseases in tropical Africa. The application of insecticides was initially an overwhelming success for tsetse fly control. However, the cost and pesticide toxicity effects in treated animals have motivated the search for safer and less costly alternatives. $B$. bassiana has been isolated from two different naturally infected Glossina species (Glossina pallidipes Austen and Glossina fuscipes Newstead) (Maniania et al. 2002), thus EPF have been investigated as potential alternative controls for tsetse flies. The primary aim of much of this research has been to find suitable fungal isolates and methods of application so that enough conidia can be autodisseminated to kill tsetse flies (Maniania et al. 2002). 
Maniania (1998) developed and tested a device for infecting natural populations of G. pallidipes, Glossina longipennis Corti, and Glossina fuscipes fuscipes, with M. anisopliae s.l. The device permitted the passage and subsequent contamination of all three species with dry fungal conidia held within an infection chamber. Subsequently, the efficacy of these contamination devices were tested against $G$. fuscipes fuscipes under field conditions (Maniania et al. 2006). The promising results of this trial along with further research to confirm the transfer of M. anisopliae s.l. conidia between adult tsetse flies (Maniania and Ekesi 2013) provide optimism for fungal control of Glossina spp., particularly if the adherence of conidia to the cuticle can be improved through formulation (Maniania and Ekesi 2013).

\section{Ticks}

Many fungi attack and kill ticks (Samish et al. 2004), and so the use of EPF has been investigated in many tick pests of livestock, including hard (Ixodidae; many studies see below) and soft ticks (Argasidae et al. 2001). The majority of these studies have focused on B. bassiana and $M$. anisopliae s.l., which have been isolated from wild ticks (Da Costa et al. 2001), indicating a natural occurrence in the environment and interaction with ticks (Figure 4). However, there have been other studies evaluating the effects of Aspergillus ochraceus G. Wilh (Eurotiales: Trichocomaceae) (Estrada-Peña et al. 1990), Lecanicillium psalliotae (Treschew) Zare and Gams (Pirali-Kheirabadi et al. 2007), Metarhizium flavoviridae Gams and Roszypal (Gindin et al. 2002; Samish et al. 2001), P. fumosoroseus (Gindin et al. 2002; Samish et al. 2001), and Verticillium lecanii (Zimm.) Viégas (Hypocreales: Cordycipitaceae) (Gindin et al. 2002).

In general, these other pathogens have not shown as much acaricidal promise as B. bassiana and M. anisopliae s.l. For example, treatment with M. flavoviridae, V. lecanii, or $P$. fumosoroseus did not reduce survival in Rhipicephalus sanguineus Latreille (Gindin et al. 2002; Samish et al. 2001), and the latter two species also had no effect on Hyalomma excavatum Stoker and Marmion or Rhipicephalus annulatus Say (Gindin et al. 2002). Two isolates of L. psalliotae isolated from Ixodes spp. were tested on $R$. annulatus, one of the two isolates showed some promise for control of

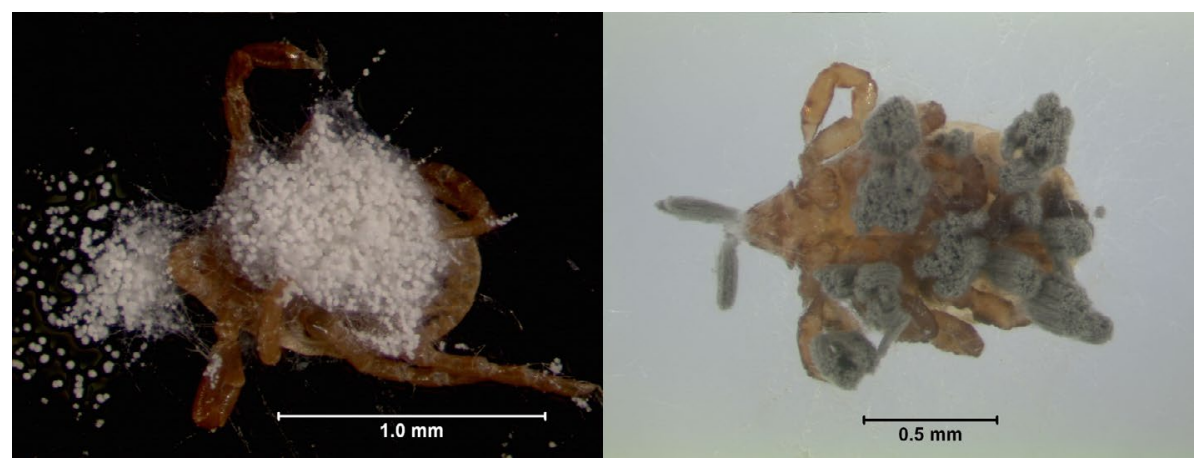

Figure 4. Infection of the brown dog tick Rhipicephalus sanguineus Latrielle (Parasitiformes: Ixodidae) with entomopathogenic fungi (Hypocreales: Clavicipitaceae). Left photo shows a Beauveria bassiana (Bals.-Criv.) Vuill. infected cadaver and right photo shows a Metarhizium anisopliae (Metsch.) Sorok cadaver sporulating (photos by Lyle Buss, University of Florida, FL, USA). 
this species (Pirali-Kheirabadi et al. 2007). There are several existing reviews that have covered the extensive number of tick species that have been tested with B. bassiana and M. anisopliae (Fernandez and Bittencourt 2008; Polar et al. 2008).

The process of infection of the cattle tick Rhipicephalus (Boophilus) microplus Canestrini by M. anisopliae s.l. has been described in detail (Arruda et al. 2005; Bittencourt et al. 1999; Leemon and Jonsson 2012b). Other studies have described M. anisopliae s.l. or B. bassiana infecting other species of ticks (Garcia et al. 2005; Kirkland et al. 2004a,b). Scanning electron microscope images show conidia adhering to the tick surface and germinating, and hyphae proliferating across the tick surface. Bittencourt et al. (1999) concluded that penetration of the tick was primarily through the cuticle, and showed germinating conidia producing both germ tubes and appressoria. Leemon and Jonsson (2012a) showed dissolution of the tick cuticle around the penetrating hyphae leading to destruction of the tick cuticle in a time series of micrographs. Arruda et al. (2005) also noted that hyphae grew extensively between the cuticle layers causing cuticle disruption. Although conidia can attach anywhere, there are certain preferred sites. On ticks, these are usually at body junctures such as where the legs join to the body (Arruda et al. 2005). Germination of conidia has been shown to be influenced by lipids extracted from tick cuticle and mammalian skin (Ment et al. 2010, 2012). Lipids extracted from susceptible ticks and calf skin, hair, and ear secretions, stimulated germination of conidia and the formation of appressoria. In contrast, lipids extracted from tick strains known to be more resistant to EPF appeared to inhibit conidial germination (Ment et al. 2012).

There are several biotic factors that influence pathogenicity of an EPF including fungal species and isolate (Samish et al. 2001), tick species and strain (Perinotto et al. 2012), tick development stage, tick feeding status (Cafarchia et al. 2015; Samish et al. 2001), and host species. Samish et al. (2001) treated all life stages with five isolates of two species and found large variability in effectiveness. For example, three isolates of M. anisopliae s.l. resulted in 20, 33 and $100 \%$ mortality in adult female $R$. sanguineus (Samish et al. 2001). Furthermore, Perinotto et al. (2012) found significant differences in mortality due to EPF in larvae of two distinct tick populations collected from two farms in Rio de Janeiro state (Brazil). In general, resistance to infection by EPF increases with increasing age from larvae to nymph to adult. In adult studies, low mortality is often observed even when the ticks are followed for a month or more post exposure. On the other hand, mortality in larvae is often relatively high after a week (Pirali-Kheirabadi et al. 2007), and nymphs typically survive for around 14 days (Nchu et al. 2010). Studies with $R$. sanguineus have tested both unfed and fed larvae and found increased mortality due to both B. bassiana and M. anisopliae s.l. in unfed larvae in a shorter period (Cafarchia et al. 2015; Samish et al. 2001). For example, the most effective M. anisopliae isolate tested on unfed larvae resulted in $96 \%$ mortality in 7 days, whereas fed larvae resulted in $83 \%$ mortality in 10 days (Samish et al. 2001). However, in nymphs and adult females of the same species the opposite was true, in that unfed nymphs and adult females had lower mortality after exposure than their fed counterparts (Cafarchia et al. 2015). For example, $95 \%$ of $B$. bassiana treated fed adult females were dead by day 10 , whereas only $40 \%$ of treated unfed adult females died in the same period (Cafarchia et al. 2015).

In attempts to increase virulence and time to mortality, researchers have investigated the use of EPF isolated from the target pest (Cafarchia et al. 2015; Perinotto et al. 2012). Cafarchia et al. (2015) isolated $B$. bassiana from $R$. sanguineus and found it to cause significant mortality after 5 days (i.e. $60 \%$ in engorged females, $55 \%$ in unfed larvae, and $28 \%$ in engorged nymphs), which is fast for EPF induced mortality on ticks. Additionally, significant reductions on egg laying and hatching, and larval moulting were reported. However, in general, increased virulence with tick-derived 
fungal isolates has not been demonstrated in studies that have compared them with other isolates (Polar et al. 2008).

Abiotic factors are also important including temperature, humidity, UV radiation, and formulation of the fungus. High temperatures and UV radiation, as well as low humidity can all result in reduced viability and germination of the fungus, which is particularly relevant in field studies. Polar et al. (2005) investigated two isolates of $B$. bassiana with the goal of finding an isolate with a higher temperature tolerance, which should improve its efficacy when applied to cattle where the temperature is known to fluctuate from 28 to $40{ }^{\circ} \mathrm{C}$ (Monty and Garbareno, 1978). They found that one isolate had a higher temperature tolerance, and while in the laboratory $\left(\right.$ at $28{ }^{\circ} \mathrm{C}$ ) they performed similarly, when applied to cattle in the field (at 30 to $35^{\circ} \mathrm{C}$ ) the high-temperature tolerant isolate reduced the $R$. microplus population by half compared to the isolate with lower temperature tolerance (Polar et al. 2005).

A few studies have evaluated application of formulated conidia to cattle to control ticks (Bittencourt et al. 1999; Correia et al. 1998; Kaaya 2000; Leemon et al. 2008; Polar et al. 2005). Although some tick control was achieved in these studies the efficacy was much less than had been observed in laboratory studies of EPF applied to ticks (Castineiras et al. 1987; Frazzon et al. 2000; Gindin et al. 2001; Leemon and Jonsson 2008; Monteiro et al. 1998; Mwangi et al. 1995; Onofre et al. 2001; Polar et al. 2005; Sewify and Habib, 2001). The difference is most likely due to the abiotic factors experienced within the cattle coat.

Kaaya et al. (1996) applied B. bassiana and M. anisopliae s.l. to rabbits for control of Amblyomma variegatum Fabricius and to cattle for control of Rhipicephalus appendiculatus Neumann and showed increased tick mortality. Furthermore, they demonstrated decreased engorgement weight, egg hatching, resulting in a decrease in eggs per tick ( $R$. appendiculatus) from 4,327 in the control to 660 and 41 in the B. bassiana and M. anisopliae s.l. treated cattle, respectively (Kaaya et al. 1996). In recovery experiments, Kaaya et al. (1996) also found viable EPF in washings of cattle ears and sand samples weeks and months after application, respectively. A study in calf pens in Brazil found that $M$. anisopliae was as effective, but no more than, deltamethrin at controlling deltamethrin-resistant $R$. microplus (Bahiense et al. 2008). It was noted in that study that while laboratory efficacy of the fungus against the ticks was $96 \%$, efficacy in the field dropped to $32 \%$, likely due to environmental factors (Bahiense et al. 2008).

Product formulation is crucial for enhancing conidial adhesion and maintaining conidial viability in the field. Reis et al. (2008) tested three formulations of B. bassiana and M. anisopliae s.l. and found the most complex formulation of an emulsifiable concentrate with polymerized cellulose gel to result in the highest mortality in exposed $R$. sanguineus. In an on-host study, Rot et al. (2013) found that compared to an unformulated control, the formulations (i.e. oil and starch-sucrose) significantly reduced the number of engorged nymphs and moulted adults. Both tick cuticle and conidia are hydrophobic. Therefore, a water-based solution does not promote adhesion to the cuticle. Conversely, an oil-based formulation has an affinity for the cuticle, promoting conidial adhesion, penetration, and mortality. A comparison of aqueous suspensions and oilbased formulations of the same fungal isolates of B. bassiana and M. anisopliae s.l. showed that formulating with oil enhanced efficacy against $R$. microplus larvae (Camargo et al. 2012). Almost $100 \%$ mortality on larvae was observed by day 5 with an oil-based formulation of $M$. anisopliae, whereas there was still only approximately $10 \%$ mortality in the aqueous suspension treatment by day 10 (Camargo et al. 2012). A similar result was not seen with B. bassiana until day 20, when $100 \%$ of the oil-formulation treated larvae were dead, and mortality in the aqueous solution was 
at about 30\% (Camargo et al. 2012). An estimate of the cumulative effect on engorged females, eggs, and larvae revealed a control success of $3,11,17$, and $100 \%$ for the aqueous $B$. bassiana and $M$. anisopliae s.l. followed by the oil-formulated B. bassiana and $M$. anisopliae, respectively (Camargo et al. 2012). Due to the high reproductive potential of ticks, in the case of the two M. anisopliae solutions, this is the difference between 0 survivors (oil-formulation) and 136,000 survivors (aqueous solution) (Camargo et al. 2012).

Due to a shortage of products formulated and labelled for tick control, numbering three worldwide according to De Faria and Wraight (2007), there has been limited research testing such products. One granulated product containing M. brunneum Petch strain F52 has been tested on Ixodes scapularis Say (Behle et al. 2013; Bharadwaj and Stafford 2012), unfed and fed nymphs showed 56 and 74\% mortality (Behle et al. 2013). It was thought that the application of granules versus a spray formulation would be more effective at penetrating leaf litter. The leaf litter is not only where the ticks of this species reside, but also provides a microclimate of high humidity and lower UV exposure and temperatures for improved conidial survival. Furthermore, as a three-host tick that feeds on small animals as an immature, I. scapularis would be more exposed to fungus present within the leaf litter where its potential small mammal hosts reside. However, some researchers believe that variability in results between experiments with granular formulation is due to the scattered distribution leading to some ticks contacting a granule and some not (Bharadwaj and Stafford 2012).

Mortality of ticks infected with fungus is slow, usually ranging from one week to a month. The time limiting factor is unclear, but could provide clues as to the barriers to tick infection. Ticks are long-lived creatures that have high fecundity, so any impacts on their fitness could be beneficial to management. Transstadial effects of infection have been demonstrated to occur with $M$. anisopliae s.l. and $M$. flavoviridae between treated $R$. sanguineus larvae to the nymph stage, and nymph to the adult stage (Samish et al. 2001). Larvae that did not die following treatment showed a decrease in successful moulting and an increase in mortality following moulting (Samish et al. 2001). Sublethal effects of fungal treatments, such as decreased oviposition and incubation periods as well as reduced overall fecundity of females, have been observed in many studies (Gindin et al. 2001, 2002; Kaaya et al. 1996; Mwangi et al. 1995; Perinotto et al. 2012; Pirali-Kheirabadi et al. 2007; Rot et al. 2013; Samish et al. 2001). Females that survived M. anisopliae s.l. treatment retained only 7 to $8 \%$ of their egg laying capacity and of the eggs laid B. bassiana and M. anisopliae s.l. reduced egg hatching by 70 to $98 \%$ (Gindin et al. 2001). In one study with three different pathogens, $M$. anisopliae S.I., B. bassiana, and L. passaliotae, there was an 88,78 , and $60 \%$ reduction in reproductive efficacy in $R$. annulatus, respectively (Pirali-Kheirabadi et al. 2007). Therefore, in contrast to most other arthropod eggs, tick eggs are highly susceptible to EPF. Another species of Metarhizium, $M$. favoviridae, caused a reduction in egg laying but had no effect on survival of treated female $R$. sanguineus (Samish et al. 2001). The process of egg invasion by M. anisopliae has been studied in detail by Garcia et al. (2005). It was shown that germination occurred after 18 hours and by 5 days $>90 \%$ of the germinated conidia had penetrated the egg. Sporulation was visible on infected eggs by day 9 , and although no histopathological damage was observed in the egg, there was a $54 \%$ reduction in hatching from infected eggs (Garcia et al. 2005).

In summary, EPF have been demonstrated to be efficacious for most of the species of tick tested to some extent, but careful selection of EPF species and isolates to the tick species and life stage to be targeted is important. In particular, for the use of EPF to control ticks, the sublethal effects should not be overlooked. Due to the high reproductive capacity of surviving adult females, any impacts on their fecundity and fertility can drastically reduce populations. In mosquitoes, EPF 
infection limits pathogen transmission (Blanford et al. 2005; Dong et al. 2012). Similar studies with tick-borne pathogens would be beneficial although hindered by the complications with maintaining and feeding ticks in the laboratory. As with the use of EPF in all livestock systems, product formulation is critical to maximize efficacy, and residual effect, if EPF are to be successfully incorporated into a pest management program.

\section{Poultry ectoparasites}

Poultry red mite, D. gallinae, is a good candidate for management with EPF. Mites of this species are occasional and nocturnal feeders on the birds. They spend most of their time in nest boxes, under feeders, and in cracks and crevices of the housing (Pritchard et al. 2015; Sparagano et al. 2014, 2018). Because unfed mites can survive for up to nine months (Nordenfors et al. 1999), mite populations in harborages can remain high even when the intervals between flocks are long. Steenberg and Kilpinen (2003) first observed susceptibility of the mites to fungal pathogens, and noted that one isolate of $B$. bassiana caused high mortality by day 5 after exposure. Steenberg et al. (2006) compared five isolates of B. bassiana and M. anisopliae s.l., and identified an isolate of $B$. bassiana for further testing. The same authors reported that desiccant dusts, especially a modified diatomaceous earth product, had a synergistic effect on efficacy of $B$. bassiana and suggested that such combinations have good mite management potential (Steenberg and Kilpinen 2014). However, behavioural studies revealed that both desiccant dusts and B. bassiana conidia are repellent to $D$. gallinae (Kilpinen and Steenberg 2016). Repellency to desiccant dusts was product specific and concentration dependent and the addition of $B$. bassiana conidia had a synergistic effect, making the combination even more repellent than either agent was alone.

Kaoud (2010) observed partial control of mites when 'built-up litter' was treated with B. bassiana at about $3 \times 10^{6}$ conidia per gram of litter. A virulent isolate of $B$. bassiana has been found in field populations of mites that produces conidia from the mite cadavers (Immediato et al. 2015). Several isolates of M. anisopliae s.l. also are known to be virulent for the mites in the laboratory (Tavassoli et al. 2008). Partial control was achieved under field conditions by spraying hens and cages in a commercial egg production facility. The facility was treated with three isolates of $M$. anisopliae in sunflower oil at $10^{9}$ conidia per $\mathrm{ml}$ (Tavassoli et al. 2011). As D. gallinae aggregate (Entrekin and Oliver 1982), it might be possible to autodisseminate EPF by combining the use of an EPF with a mite monitoring device, such as an automated mite counter (Mul et al. 2015). Autodissemination of EPF has been demonstrated in other livestock pests (Reyes-Villanueva et al. 2011; Vega et al. 1995). Mites would drop into the counter from the perch, be counted by the sensor, and then exposed to fungus before being released back into the cage to infect their conspecifics.

Little is known about the biological control potential of fungal pathogens for other poultry ectoparasites. Rassette et al. (2012) found that treatment of roosters with B. bassiana only provided satisfactory control of northern fowl mites, 0 . sylviarum, when weekly applications of extremely high doses $\left(3 \times 10^{11}\right.$ conidia per bird) were used. Similarly, Mullens et al. (2012) found that $O$. sylviarum were susceptible to $B$. bassiana when tests were conducted in glass tubes but observed little mortality when conidia were applied directly on the birds. Sewify and Habib (2001) evaluated a local isolate of M. anisopliae s.l. against fowl ticks (A. persicus) in Egypt. Treatment of the sackcloth harbourage where ticks congregate, with conidia in sunflower oil at $10^{7}$ conidia per $\mathrm{ml}$, provided $100 \%$ control for three weeks in poultry houses. Engorged females were more susceptible than unfed ticks, and V245 was the most virulent of the three isolates of $M$. anisopliae s.l. tested (Pourseyed et al. 2010). 
Bed bugs that were briefly exposed to paper or cloth treated with $3 \times 10^{6} \mathrm{M}$. anisopliae s.l. conidia per $\mathrm{cm}^{2}$ died within five days and transmitted conidia horizontally to untreated bugs (Barbarin et al. 2012). Ulrich et al. $(2014,2015)$ cautioned that M. anisopliae s.l. treatments for bed bugs could be limited by the need for high humidity required to kill infected bugs and by the potential of bed bug defensive secretions to inhibit fungal growth. A new commercial formulation of B. bassiana (Aprehend ${ }^{\mathrm{TM}}$; ConidioTec, PA, USA) has been developed that appears to have potential for managing insecticide-resistant bed bugs (Barbarin et al. 2017). When deltamethrin-resistant bed bugs were briefly ( 15 minutes) exposed to cloth treated with the product at approximately $4 \times 10^{6}$ conidia per $\mathrm{cm}^{2}$, nearly $100 \%$ mortality was observed by day 14 . In contrast, exposure to deltamethrin-treated cloth had no effect on survival of the three populations of resistant bugs that were tested.

The poultry industry is changing rapidly throughout the world, with increased prevalence of small ('backyard') flocks and cage-free systems for commercial egg production (Matthews and Sumner 2015). Moreover, many of the owners of such systems are trying to run them as organic operations. Because the diversity of ectoparasites in cage-free systems is higher than in conventional commercial operations (Horn et al. 2016; Murillo and Mullens, 2016), future poultry IPM programs will need to re-examine management practices for pests that were long considered to be of lesser importance.

\section{Litter beetles}

Insecticidal control of $A$. diaperinus is challenging because of the location of beetles deep in the manure and litter, and the presence of pupae in protected sites such as soil floors or insulation (Axtell and Arends 1990; Geden and Axtell 1987). These challenges are exacerbated by resistance to many of the conventionally used products for beetle control (Hamm et al. 2006). For this reason, there has long been a search for alternative management practices including mechanical barriers to protect insulation (Geden and Carlson 2001), litter treatment with diatomaceous earth (Lambkin et al. 2012), essential oils (Silva et al. 2007; Szczepanik et al. 2015; Wang et al. 2014), heat/ cold (Salin et al. 1998), lime (Wolf et al. 2015), and EPF.

There have been several reports of B. bassiana and M. anisopliae s.l. causing natural infections of $A$. diaperinus (Alves et al. 2004; Geden et al. 1998; Rezende et al. 2009; Rhode et al. 2006; Steinkraus et al. 1991). These EPF have shown a wide range of virulence to larval and adult $A$. diaperinus (Figure 5 ), with the susceptibility of the larvae consistently greater than that of the adults (Geden et al. 1998; Rezende et al. 2009). Gindin et al. (2009) noted that the degree of virulence to the beetle depends less on the genus of the fungus (Beauveria or Metarhizium) but more on the specific isolates.

High mortality rates of $A$. diaperinus were observed in efficacy tests of $B$. bassiana isolates under laboratory and field conditions, however there was concern that the increasing levels of ammonia in the litter during broiler grow-out cycles or in caged-layer houses may have a fungistatic effect on these treatments (Crawford et al. 1998; Geden and Steinkraus 2003). Nevertheless, the promising results with early instars could mean that the fungal treatments will have had their greatest effect before ammonia levels build up. Aleksandro et al. (2006) and Rhode et al. (2006) inoculated different stages of lesser mealworm with fungal suspensions and found that the early life-stages were the most susceptible. In general, the relative resistance to EPF increases with advancing development through the $A$. diaperinus life cycle from young larvae, then older larvae, to the older well-sclerotized adults (Gindin et al. 2009). Although mature adults showed the highest resistance, 


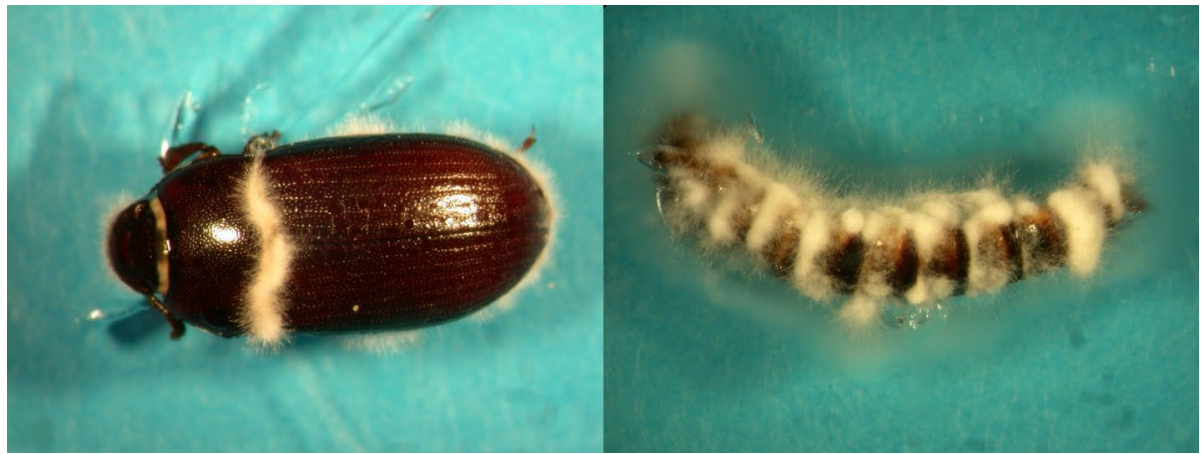

Figure 5. Entomopathogenic fungi (Hypocreales: Clavicipitaceae) infection in the lesser mealworm, Alphitobius diaperinus (Panzer) (Coleoptera: Tenebrionidae). Left photo shows a Beauveria bassiana (Bals.-Criv.) Vuill. infected adult and right photo shows an infected larva sporulating (photos by Diana Leemon, Department of Agriculture and Fisheries, Queensland, Australia).

the newly emerged adults are still susceptible to fungal infection. Gindin et al. (2009) achieved $100 \%$ mortality with early instar larvae $(3-4 \mathrm{~mm})$ within 3 days through passive contact with low levels $\left(2.5 \times 10^{3}\right.$ per conidia $\left.\mathrm{cm}^{2}\right)$ of a M. anisopliae s.l. isolate.

Gindin et al. (2009) tested two different formulations (a water-based spray and an oil-based spray) of $M$. anisopliae s.l. conidia against $A$. diaperinus eggs and neonate larvae under simulated broiler house conditions. They achieved $93 \%$ control using the water-based spray. It is interesting that they found the water-based formulation was significantly more effective than the oil-based formulation, as most other studies have found the opposite.

Crawford et al. (1998) found that exposing larvae to pine wood shavings treated with B. bassiana in a liquid suspension resulted in $>90 \%$ mortality. In contrast, Alves et al. (2008) found that a powder-formulated EPF applied to litter was much more effective against lesser mealworm than liquid formulations. This is supported by Steinkraus et al. (1991) who reported that B. bassiana formulations with corn starch were more effective against lesser mealworm than B. bassiana formulations with water when applied to used poultry litter. Alves et al. (2008) demonstrated that a dry powder formulation of $B$. bassiana was more efficient against the beetle when applied to poultry house soil rather than poultry litter under laboratory conditions. Furthermore, they noted that the natural ecosystem for $B$. bassiana is soil, and poultry houses with soil floors provide appropriate temperature and moisture conditions for the fungus. In addition, the fungus can remain in the soil in dead insects ready to infect the following generations of lesser mealworms.

Alves et al. (2015) built on the demonstrated potential of a fungal control for A. diaperinus with a successful broiler house field trial. A soil application of $B$. bassiana in an emulsifiable oil-type formulation resulted in 56 and $73 \%$ reduction in the A. diaperinus population at 96 and 146 days post application, respectively. In addition, they noted that a large number of dead beetles with signs of fungus infection were found outside the treated broiler shed. This was atypical and the observation was made over the time of the trial exclusively on the perimeter of the treated shed. They were able to use molecular techniques to confirm that these beetles had died from infection by the $B$. bassiana isolate applied in the treatment and not from any other naturally occurring 
isolate. This study demonstrated that even a single application of a fungal control was able to control the populations of $A$. diaperinus for up to 150 days effectively.

The body of work on the control of A. diaperinus by EPF to date indicates that mycoinsecticides have strong potential for management of this challenging pest. Some fruitful areas for further investigation might include powder formulations and more frequent broiler shed treatments.

\section{Entomophthora muscae group fungi}

Another fungal pathogen that has been considered for filth fly management is the Entomophthora muscae (Entomophthorales: Entomophthoraceae) species complex, which includes E. muscae (Cohn) Fresen. and Entomophthora schizophorae S. Keller and Wilding (Pinnock and Mullens 2007). These species are members of the order Entomophthorales, a group that has generally larger and more fragile conidia, faster germination times, a stronger tendency to produce conspicuous epizootics, and a narrower host range than the group that includes Beauveria and Metarhizium (Hyphomycetes) (Pell et al. 2001). Known hosts of the E. muscae complex include the following flies (Diptera): M. domestica (house fly; Muscidae), Fannia spp. (Fanniidae), Ophyra aenescens Wiedemann (black dump fly; Muscidae), Scathophaga stercoraria L. (yellow dung fly; Scathophagidae), Pollenia rudis Fabricius (cluster fly; Calliphoridae), Delia spp. (onion fly, cabbage maggot fly; Anthomyiidae), Psila rosae Fabricius (carrot fly; Psilinae), and others (Gryganskyi et al. 2013; Mullens et al. 1987a; Pell et al. 2001).

Transmission occurs primarily between infected and healthy adult flies. Shortly before death, infected flies undergo a behaviour change that causes them to anchor themselves via a rhizoid structure to conspicuous elevated objects in the fly habitat (Krasnoff et al. 1995). Such hijacking of host behaviour to produce 'zombie' hosts is common in the Entomophthorales (reviewed in Gryganskyi et al. 2017; Roy et al. 2006). Several hours after host death, conidiophores appear on the surface of the cuticle, which forcibly discharge the large and sticky conidia (Kalsbeek et al. 2001a; Six and Mullens 1996a). Healthy flies can encounter the conidia by chance in the environment. In addition, male flies are attracted to the swollen cadavers and acquire conidia, which they, in turn, can transfer to healthy females during mating attempts (Watson and Petersen 1993) (Figure 6). The conidia germinate quickly on the new host, enter the hemocoel, and fungal replication results in fly death by starvation 6 to 8 days later (Hansen and De Fine Licht 2017). Infected flies are unable to develop their ovaries and are thus rendered sterile by the infection.

Natural patterns of E. muscae prevalence are well established, with massive epizootics often occurring in the fall, when temperatures are moderate and fly populations still high (Mullens et al. 1987a; Six and Mullens, 1996b; Steinkraus et al. 1993; Watson and Petersen, 1993). The fungus can overwinter in temperate regions by low rates of transmission in overwintering hosts (Eilenberg et al. 2013). Daily high temperatures in excess of $28^{\circ} \mathrm{C}$ are correlated with low infection rates (Six and Mullens $1996 \mathrm{~b}$ ). Flies are able to exploit the high-temperature sensitivity of $E$. muscae by inducing 'behavioural fever,' where flies selectively rest in local areas of higher temperatures to elevate their body temperature to mitigate the infection (Kalsbeek et al. 2001b; Watson et al. 1993).

The spectacular epizootics of E. muscae in the fall have prompted efforts to induce higher infections rates earlier in the season. However, the $E$. muscae-house fly system poses challenges for such inductions. Unlike the conidia of Beauveria and Metarhizium, E. muscae conidia are fragile and cannot be stockpiled for later use (Kalsbeek et al. 2001a). This limitation requires that fungal introductions be done using either live infected flies or cadavers of very recently killed 


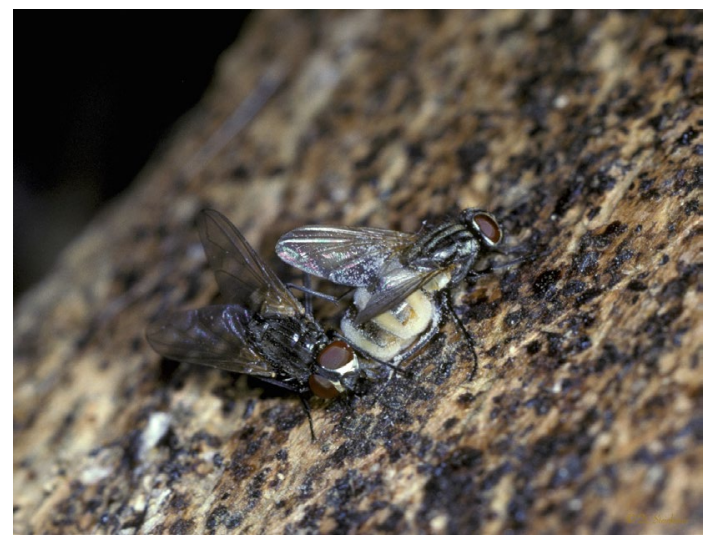

Figure 6. Healthy male house fly, Musca domestica L. (Diptera: Muscidae), probing the cadaver of a female fly infected with Entomophthora muscae (Entomophthorales: Entomophthoraceae) (photo by Donald C. Steinkraus, University of Arkansas, $A R, U S A)$.

flies. Although methods for mass production of infections have been developed (Mullens 1986), attempts to induce epizootics by introducing E. muscae into field populations have had limited success to date. Early season disease introduction on New York dairy farms via release of infected live flies or cadaver placement resulted in only small increases in overall prevalence of infections, and such increases required the presence of large resident fly populations (Geden et al. 1993; Steinkraus et al. 1993). Six and Mullens (1996b) observed zero to minimal increases in infection following release of flies infected with E. schizophorae on California dairy farms. Successful exploitation of this important natural entomopathogen will require technical breakthroughs in the areas of fungal propagation and storage.

\section{Salivary gland hypertrophy virus}

House fly salivary gland hypertrophy virus (MdSGHV) belongs to the small and recently described family Hytrosaviridae (Kariithi et al. 2017a). These are double-stranded circular DNA viruses with a genome of 120 to $190 \mathrm{kbp}$ that form enveloped, non-occluded, rod-shaped virions (Jehle et al. 2013). Their most conspicuous biological characteristic is that they replicate in host salivary glands, generally resulting in obvious hypertrophy of the glands and sterility of female hosts (Lietze et al. 2011b) (Figure 7). The family consists of two well-studied viruses, one found in adult house flies (MdSGHV) and the other in adult tsetse flies (GpSGHV); other suspected family members include viruses in Merodon equestris Fabricius (Diptera: Syrphidae) and Diachasmimorpha longicaudata Ashmead (Hymenoptera: Braconidae) (Jehle et al. 2013; Kariithi et al. 2017b). GpSGHV infects many Glossina spp., but is not believed to have potential as a BCA for that target insect (AbdAlla et al. 2011). Rather, viral infections jeopardize the health of tsetse mass-rearing operations used in sterile insect technique programs in Africa, and most of the research is focused on the development of ways to reduce the effects of infections on colony fitness (Abd-Alla et al. 2011, 2013). 

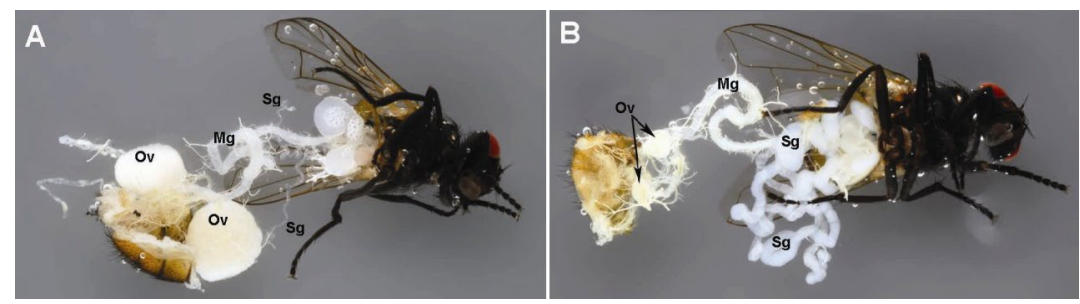

Figure 7. Salivary gland hypertrophy virus (SGHV) infection in a house fly, Musca domestica L. (Diptera: Muscidae). (A) a healthy fly, and (B) a fly infected with SGHV (photo by Lyle Buss, University of Florida, FL, USA). Note the underdeveloped ovaries (Ov) and overdeveloped salivary glands $(\mathrm{Sg})$ in the infected fly ( $\mathrm{Mg}=$ midgut).

House flies are infected with MdSGHV globally, with prevalence rates usually of less than $2 \%$ (Geden et al. 2008, 2011a; Lietze et al. 2011b, 2013; Prompiboon et al. 2010). The virus appears to be specific to house flies, as other filth flies either have no natural infections, are refractory altogether, or produce light infections without the characteristic salivary gland hypertrophy (Geden et al. 2011a, 2011b). Although the salivary glands are the site of maximum virus replication, virus particles can be found in other tissues such as fat body, ovaries, and tracheole cells (Lietze et al. 2011a). Additional replication occurs in the corpora allata/corpora cardiacum complex (Kariithi et al. 2017a). Infection effects extend beyond salivary gland hypertrophy and include failure of female flies to develop eggs and refusal to mate (Lietze et al. 2007). The genome of MdSGHV has been sequenced (Garcia-Maruniak et al. 2008), and recent research is revealing possible mechanisms for the observed reproductive effects of infection (Kariithi et al. 2017a).

The primary route of infection is thought to be via shared food sources between healthy and infected flies (Lietze et al. 2009, 2013). Flies can be infected in the laboratory by presenting them with food items collected from farms with high infection rates (Geden et al. 2008). Infected flies deposit virus particles onto food at a rate of about 100 million virus particles per hour (Geden et al. 2008), and the crop as well as the salivary glands of infected flies contain infectious virions (Lietze et al. 2009). Some viable virus particles also survive the digestive tract and are passed out in the faeces of infected flies (Lietze et al. 2009).

If the primary route of infection was per os, then it would be expected that infective baits could be used to increase and spread natural infections. This has proven to be difficult to achieve in practice. When fly populations were infused with infected flies to produce an overall infection rate of $40 \%$, infections declined steadily over the next 10 generations (Lietze et al. 2011c). Infection rates of flies that are given contaminated food in the laboratory are modest, even when massive numbers of virus particles are used in the bait (Geden et al. 2008, 2011a; Lietze et al. 2009). This refractoriness to oral infection may be due to the protection afforded by the peritrophic matrix of adult flies. Newly emerged adult flies, which have not yet developed a mature peritrophic matrix and do not feed voluntarily, are much more susceptible to per os infection than are flies that are 24 hours old (Prombiboon et al. 2010). Boucias et al. (2015) were able to reverse refractoriness to infection in older flies by first having them imbibe chemicals that disrupted the integrity of the peritrophic matrix. The results suggest that flies are maximally susceptible to oral infection only during a brief window after adult eclosion, when they would not normally be feeding. This presents a severe limitation on the potential for baits to deliver the virus and initiate epizootics. A more simple approach may be the use of virus sprays on hot spots of adult activity. Homogenates 
of infected individuals are highly effective for infecting flies by direct contact (Geden et al. 2011a). Massive numbers of infected flies can be produced in the laboratory using this contactcontamination method, perhaps offering an effective alternative to baits for initiating epizootics in the field. Lietze et al. (2013) suggested that the effectiveness of such sprays could be synergized by use of abrasives to compromise the integrity of fly cuticle.

\title{
Entomopathogenic nematodes
}

\section{Introduction}

Entomopathogenic nematodes (EPN) in the families Steinernematidae and Heterorhabditidae (Order: Rhabditida) have a long history of use and have been reviewed extensively on several occasions (Atwa 2014; Gaugler 2002; Georgis et al. 2006; Kaya and Gaugler 1993). The unique feature of these parasites is that they serve as hosts and vectors of bacterial symbionts in the genera Xenorhabdus and Photorhabdus (Enterobacteriales: Enterobacteriaceae), respectively (Boemare 2002; Forst et al. 1997). The following overview is a brief summary based on these sources. Infective juveniles enter the host either through natural openings (Steinernema spp.) or via penetration of host cuticle (Heterorhabditis spp.) and release their symbionts into the host haemolymph (Figure 8 ). The symbionts replicate rapidly and kill the host by septicaemia within a few days. EPN develop and reproduce within the dead host, followed by a mass exodus of a new generation of infective juveniles (third stage larvae) from the cadaver (Figure 9). This free-living

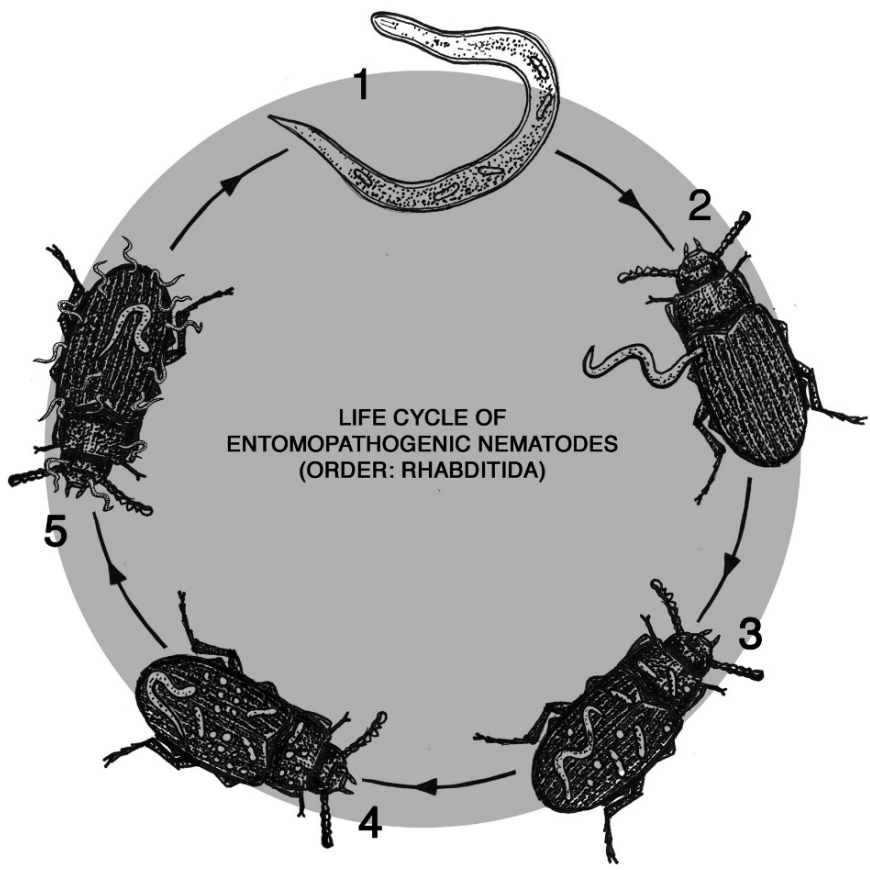

\author{
1. An entomopathogenic nematode in \\ its free-living stage, infective juvenile \\ (third stage larva), will ambush or \\ actively search for a host; within its gut \\ are symbiotic bacteria of the genera \\ Xenorhabdus (Steinernema spp. \\ nematodes) or Photorhabdus \\ (Heterorhabditis spp. nematodes). \\ 2. When the nematode finds a suitable \\ host it enters either via natural \\ openings (Steinernema spp.) or \\ penetrates the host cuticle \\ (Heterorhabditis spp.). \\ 3. Once inside the host, the nematodes \\ release their bacterial symbionts into \\ the haemolymph. These bacteria \\ replicate, rapidly producing molecules \\ that modify the host immune system \\ and toxins that result in host death. \\ 4. Once the host is dead, the symbiotic \\ bacteria produce antibiotic molecules \\ that will prevent microorganisms in the \\ environment from decomposing the \\ cadaver. \\ 5. The nematodes then use the \\ cadaver as a food source to provide \\ the necessary nutrients for \\ reproduction. Steinernema spp. mate \\ and Heterorhabditis spp. are \\ hermaphrodites. The symbiotic bacteria \\ hermaphrodites. The symbiotic bacteria
colonize the juvenile nematodes before \\ they emerge from the cadaver to begin \\ their free-living stage.
}

Figure 8. Life cycle of entomopathogenic nematodes (order: Rhabditida) in lesser mealworm Alphitobius diaperinus (Panzer) adult beetles (illustrated by Laura Jayne Weeks, Cornwall, UK). 


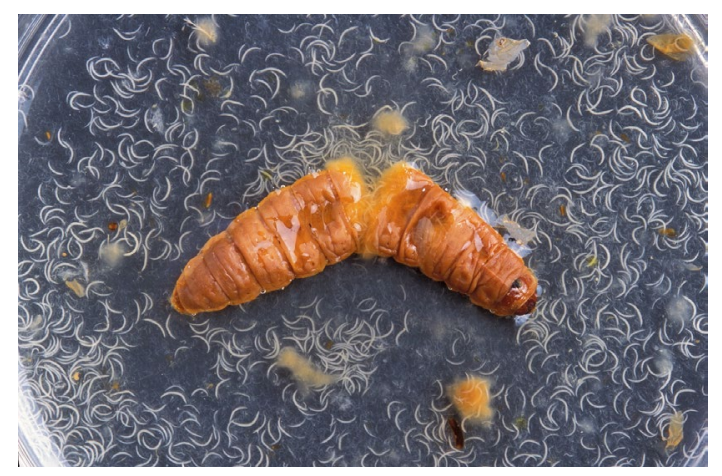

Figure 9. Entomopathogenic nematodes, Heterorhabditis bacteriophora (Khan, Brooks and Hirschmann) (Rhabditida: Heterorhabditidae), exiting from the larva of a dissected greater wax moth, Galleria mellonella (L.) (Lepidoptera: Pyralidae) (photo by: Peggy Greb, USDA-ARS, FL, USA).

stage is tolerant of a wide range of conditions and can survive prolonged intervals while waiting for hosts. Hosts are located by either waiting for a passing host ('ambusher' species) or by active search ('cruisers'). EPN possess a number of qualities that make them attractive candidates as BCAs. They often have a wide host range, can be easily mass-produced at low cost, have a long storage life (especially Steinernema spp.), can be selected for desirable traits, are compatible with certain insecticides, are safe for vertebrates, and are easily applied in the field.

\section{House flies}

Other than a series of laboratory studies on EPN infecting sheep strike flies (Molyneux et al. 1983, 1986; Töth et al. 2005), the majority of work with these parasites for filth management has concentrated on house flies. Geden et al. (1986) observed that fly larvae and adults were highly susceptible to Steinernema carpocapsae (Weiser) (Rhabditida: Steinernematidae) and Heterorhabditis bacteriophora (Khan, Brooks and Hirschmann) (Rhabditida: Heterorhabditidae) but not to Steinernema glaseri (Steiner) when insects were confined on treated filter paper. (Note: at the time that the work was done, the first two species were referred to as Steinernema feltiae (Filipjev) and Heterorhabditis heliothidis Khan, Brooks and Hirschman, respectively).

Larval susceptibility was lower when EPN were placed in fly larval rearing medium and even lower when placed in poultry manure. Although the results suggested that poultry manure was hostile to nematode survival, a field study in Canada found that manure treatment with $H$. heliothidis was effective in small poultry barns (Belton et al. 1987). Several additional investigations confirmed that nematode survival in poultry manure was poor (Archana et al. 2017; Georgis et al. 1987). Mullens et al. (1987b) found no parasitism in the field after applying eight to nine million $S$. feltiae or $\mathrm{H}$. heliothidis per square meter of poultry manure.

The reasons for EPN failure in poultry manure are uncertain, but other animal manure types may be more hospitable. Taylor et al. (1998) screened $>35$ strains of Steinenema and Heterohabditis spp. and conducted more extensive testing with four species in cattle manure. The resulting lethal concentration to $50 \%$ mortality $\left(\mathrm{LC}_{50}\right)$ in manure was only reduced by about $50 \%$ compared with 
filter paper assays. Moreover, nematodes persisted in the treated manure for at least 10 weeks after application (Taylor et al. 1998). Field application of S. feltiae to pig manure resulted in fly control that was as good as or superior to control in barns where methomyl bait was used (Renn 1998). Furthermore, protecting S. feltiae and H. megidis Poinar, Jackson and Klein in calcium alginate capsules allowed treatments to be made to poultry manure without unacceptable nematode mortality (Renn 1995). Because EPN species and strains vary in their virulence for fly larvae (Taylor et al. 1998; Wojciechowska et al. 2013), it may be possible to develop these parasites into a management tool by a combination of selecting superior strains and formulating them in ways that maximize their ability to survive in manure habitats.

Another approach for using EPN for fly management is to target adult flies (Renn et al. 1999). However, development of an effective delivery system is critical. Geden et al. (1986) observed high adult mortality in forced-contact Petri dish assays. In contrast, mortality was $<70 \%$ when flies were housed with pads moistened with an EPN-based suspension in $5 \%$ sucrose, even at the high rate of 100,000 EPN per ml of bait. Renn and Wright (2000) found that EPN delivery in a 0.25 $M$ sucrose solution to adult flies varied depending on the type of substrate on which the bait was presented. For example, the lethal dose to $50 \%$ mortality $\left(\mathrm{LD}_{50}\right)$ for $S$. feltiae was about 200 times lower on cotton poplin than on perlite. Baits containing S. feltiae and supplemented with the fly pheromone $z$-9-tricosene were more effective at managing flies than insecticide baits on a pig farm in the UK (Renn 1998).

\section{Ticks}

EPN are not restricted in action to insects, as many studies have demonstrated their potential for tick control (reviewed by Samish 2004; Samish and Glazer, 2001; Samish et al. 2008). Although the lethal doses are similar for all arthropods, the time to mortality appears to be longer with ticks than with insects (Samish 2004). Unpublished observations have shown the EPN to penetrate the tick at natural apertures including the mouthparts, spiracles, anus, and genital pore (Kocan et al. 1998; Samish 2004). In an insect, the symbiotic bacteria replicate in the haemocoel, killing the host and providing a suitable environment for the growth and reproduction of the nematodes. While injection of a single EPN can cause mortality (Glazer and Samish 1993), injection of axenic nematodes is non-pathogenic (Samish and Glazer, 2001). Once inside the tick, the symbiotic bacteria cause host death but there is no continuation of the life cycle as most juvenile nematodes die after entry (Hill 1998; Kocan et al. 1998; Mauléon et al. 1993; Samish and Glazer 2001). Consequently, the tick is unlike insects in that it is a dead-end host.

There are many factors that influence the efficacy that EPN would have in a given system including host species (Hartelt et al. 2008; Mauléon et al. 1993), host life stage (Kaaya et al. 2000; Mauléon et al. 1993), nematode species and strain (Mauléon et al. 1993; Samish et al. 1999a), and nematode dose or concentration (Hartelt et al. 2008; Vasconcelos et al. 2004). Mauléon et al. (1993) tested 17 isolates of two genera of nematodes (Steinernema and Heterorhabditis) against three tick species: $R$. annulatus, $A$. variegatum, and $R$. microplus. There were no pathogenic effects of any of the 17 nematode isolates on any life stage of $A$. variegatum or $R$. microplus. The refractory ticks survived and moulted to the next stage even when the EPN were injected. In contrast, $R$. annulatus was sensitive to EPN, with $100 \%$ mortality occurring within 8 days with all 17 isolates (Mauléon et al. 1993). Other studies have reported some infection in A. variegatum (Kaaya et al. 2000) and $R$. microplus (Vasconcelos et al. 2004) but infection rates were low even at high doses and, therefore, they still were considered to be resistant. For example, while 1000 larvae/dish infected $100 \% R$. annulatus, 5000 larvae/dish were needed to kill $50 \%$ A. variegatum and 2,500 larvae/dish to kill 
$50 \%$ R. microplus (and 25,000 larvae/dish to kill 100\%) (Vasconcelos et al. 2004). Comparisons of the cuticle thickness and genital aperture diameter were not able to explain the differences between the two very similar Rhipicephalus species. Another potential explanation might be related to cuticular secretions.

Rhipicephalus annulatus were especially susceptible when ovipositing compared to engorged pre-ovipositional females, which is most likely because the point of entry is typically the genital pore (Samish 2004). Mauléon et al. (1993) found that Heterorhabditis spp. were in general more pathogenic than Steinernema spp. to ovipositing $R$. annulatus. The lethal time to $50 \%$ mortality was 20 to 40 hours in tests with Heterorhabditis. The lethal time to $50 \%$ mortality in ovipositing females exposed to Steinernerma was 30 to 60 hours (Mauléon et al. 1993). Unfed females are even less susceptible (Kaaya et al. 2000); although others have reported contrasting results in this life stage (Samish 2004). Tick larvae and nymphs are much less susceptible than adults are to EPN, likely due to the lack of a genital pore in immatures (Kaaya et al. 2000; Mauléon et al. 1993; Samish et al. 1999a).

Environmental effects can cause variability between laboratory and field studies. EPN species and strains that do well in laboratory studies can do poorly under field conditions and vice versa, likely due to environmental conditions. For example, while Heterorhabditis species were generally more effective in the laboratory (i.e. in Petri dishes) (Samish et al. 1999a), Steinernerma species were more effective under field conditions (i.e. in soil) (Samish et al. 1999b). These differences are likely due to a combination of the nematode and tick preferences for host searching and aggregation, respectively. Steinernerma spp. appear to prefer the top soil layers $(<2 \mathrm{~cm})$, whereas Heterorhabditis spp. are present throughout the top $6 \mathrm{~cm}$ (Alekseev et al. 2006). However, this behaviour was strongly affected by the type of soil, with moist sandy loam soils harbouring the most nematodes (Alekseev et al. 2006). Soil moisture and temperature are important for tick control using EPN in field systems, and tolerance to extremes varies by isolate (Kocan et al. 1998; Samish and Glazer 2001). The optimal temperature range for EPN activity was 25 to $28{ }^{\circ} \mathrm{C}$ with good activity between 20 and $30^{\circ} \mathrm{C}$ (Zhioua et al. 1995). Providing shade to the soil through cloth or leaf litter also increased EPN efficacy, as did regular irrigation to maintain moisture levels (Zangi 2003).

As with EPF, EPN cause sublethal effects on fertility and fecundity in ticks. Although there were no significant changes in pre-ovipositional, ovipositional period, larval hatching percentage or reproductive efficiency, there was a significant reduction in egg mass, from $112 \mathrm{mg}$ in the control to approximately $80 \mathrm{mg}$ in R. microplus exposed to S. glaseri. (Vasconcelos et al. 2004). Conversely, Hill (1998) noted that EPN-infected I. scapularis females produced fewer egg masses than uninfected ticks ( $<1 \%$ compared to $84 \%$ ), and that fewer larvae hatched ( $25 \%$ compared to $100 \%$ ), yet in that study there was no effect on egg mass. The effect of EPN infection on egg mass was demonstrated to vary by EPN isolate in a study on Amblyomma americanum L. (Kocan et al. 1998); some isolates had no effect while others had a dose-dependent effect.

In a semi-field study comprised of buckets of sand with pebbles and leaves on the surface in a greenhouse under field conditions, a Mexican strain of $S$. carpocapsae was found to be highly effective against $R$. annulatus. This study compared eight strains, with the Mexican strain resulting in $90 \%$ mortality in 10 days at a rate of $50 \mathrm{EPN}$ per $\mathrm{cm}^{2}$ (Samish et al. 1999b). Economic analysis has revealed that 100 to 150 EPN per $\mathrm{cm}^{2}$ is economically feasible for insect pests (Georgis and Manweiler 1994; Martin 1997). Therefore, EPN show potential for sustainable tick management as they can be applied to tick habitats with suitable microclimates at a rate that is effective and 
economically viable. More field studies are needed to determine the most effective strain and species of EPN for different tick species and to identify the environmental parameters that are most favourable to nematodes in natural tick habitats.

\section{Litter beetles}

EPN offer a potential management strategy for litter beetles, especially in broiler and turkey houses, where beetle populations are highly aggregated in locations such as under the feeders (Chernaki-Leffer et al. 2005; Lambkin et al. 2007, 2008; Safrit and Axtell 1984). Geden et al. (1985) found that adult and larval A. diaperinus were susceptible to $S$. carpocapsae and H. bacteriophora in a variety of substrates, and that mature larvae were particularly susceptible to EPN-treated soil from poultry houses. Field tests with S. carpocapsae applied to the soil floors of cleaned-out North Carolina poultry houses at $10^{6} \mathrm{EPN}$ per $\mathrm{m}^{2}$ were partially successful, but control only lasted for about seven weeks after birds were placed in the houses (Geden et al. 1987). As with the fly tests by the same authors mentioned previously, these species were referred to as S. feltiae and $H$. heliothidis at the time of publication. Application of Heterorhabditis indica Poinar, Karunakar and David to litter below feeders provided greater control than application to soil in the same locations (Rodrigueiro et al. 2008). Szalanski et al. (2004) screened an additional 12 strains of S. feltiae and S. carpocapsae in poultry litter and identified the Pye and Mexican strains, respectively, as being particularly virulent for adult beetles. Alves et al. (2012) evaluated 14 species and strains and found that Steinernema arenarium (Artyukhovshy) was the most virulent in soiled litter. A commercial product containing S. feltiae (Owinema SC; Owiplant Ltd. Horticultural Enterprise, Poznań, Poland) had higher virulence than four other EPN preparations against beetle larvae in sawdust litter (Pezowicz et al. 2013), and this product outperformed three others in tests where EPN were combined with silver, gold, and copper nanoparticles (Kucharska et al. 2016). Larval cadavers of $A$. diaperinus produce 7 to $20 \%$ as many new infective juvenile EPN as the much-larger larvae of the wax moth, Galleria mellonella L. (Lepidoptera: Pyralidae) (Costa et al. 2007).

The success of EPN treatment depends on environmental conditions as well as strain characteristics. Gray and Johnson (1983) reported low survival of S. carpocapsae in soil held at $>30^{\circ} \mathrm{C}$. Under hot summer conditions in North Carolina (mean $26.4{ }^{\circ} \mathrm{C}$ ), no S. feltiae were detected in the soil of treated houses three weeks post-treatment (Geden and Axtell 1988). Laboratory bioassays of treated soil showed zero infection of $A$. diaperinus two weeks after being held at temperatures $>24^{\circ} \mathrm{C}$ (Geden and Axtell 1988). Similar temperature sensitivities have been observed for other Steinernema and for Heterorhabditis spp. (Alves et al. 2012). Another consideration is the degree of faecal loading in poultry litter, because 'pure' poultry manure is known to be harmful for nematode survival (Georgis et al. 1987). EPN efficacy against $A$. diaperinus was higher in unsoiled or lightly soiled rice hull litter compared with litter from a broiler farm three weeks after placement of birds (Del Valle et al. 2016). Similarly, Pezowicz et al. (2013) found that infections with S. feltiae were much higher in unsoiled or one-week-old pine sawdust litter than in three-week-old litter collected from a commercial broiler farm. Successful use of EPN as an operational tool for lesser mealworm control will require accommodating or altering the sensitivity of these parasites to high temperatures and high faecal loading of the litter. Further improvements could be made by combining EPN applications with the aggregation pheromone of the beetle (Cossé and Zilkowski 2015; Hassemer et al. 2016). 


\section{Integrated pest management and the use of combined biological control agents}

IPM is the use of multiple tools or techniques for sustainable management of pests. The goal of developing an IPM program is to maintain the pest below the economic threshold above which unacceptable damage occurs. In order for an IPM program to be sustainable, efforts must be made to minimize environmental impacts and costs due to damage and inputs, and delay the development of arthropod resistance to the available tools in order to prolong their effective use. The use of individual and multiple BCAs as part of an IPM program has the advantage that it reduces the need for widespread pesticide applications, which can be costly, and has the propensity to delay insecticide resistance development. Typically, even generalist BCAs are more host specific, and consequently have less environmental impact than synthetic pesticide applications. However, it is important that when using BCAs that all aspects of IPM are considered. For example, pest populations still need to be monitored, such as they are when applying synthetic pesticides, to allow for targeted applications and to determine the success of treatments.

Although IPM is more effective if implemented before resistance develops, it is usually the development of resistance that drives applicators towards alternatives to synthetic pesticides. Studies with insecticide resistant strains of mosquitoes have shown they were 2 to 4 times more susceptible to the EPF B. bassiana and M. anisopliae than susceptible strains (Howard et al. 2010). The slow mortality associated with EPF for mosquito control suggests they may be more suitable for resistance management. It is desirable to allow the susceptible individuals to mate before death in order to ensure a new generation of a mixed resistance profile (Howard et al. 2010; Reed et al. 2009).

Another technique to delay resistance development and optimize the impact of BCAs is to use a combination of active ingredients. EPF have been shown to be compatible for use with other pesticides, such as B. bassiana and acetamiprid, emamectin, imidacloprid, and lufenuron (Farooq and Freed 2016), or M. anisopliae and deltamethrin (Bahiense et al. 2008), imidacloprid (Paula et al. 2011b), neem (Gomes et al. 2015), and spinosad (Sharififard et al. 2011). Compatability between EPNs and imidacloprid (Koppenhöfer et al. 2000) and clorfenvinfos (Reis-Menini et al. 2008) also has been demonstrated. However, emergence of nematodes from the cuticle of $R$. microplus was dependent upon the acaricide dose, indicating that high doses disrupted the nematode life cycle (Reis-Menini et al. 2008). Compatability between desiccant dusts and B. bassiana was demonstrated in the poultry mite system (Steenberg and Kilpinen 2014).

Other BCAs also can be compatible for combined treatments. Larval control studies with the house fly, $M$. domestica showed compatibility of $B$. bassiana and the bacterial entomopathogen B. thuringiensis (Mwamburi et al. 2009). Other studies have demonstrated the compatibility and synergistic effects of $B$. thuringiensis and EPNs (Koppenhöfer and Kaya 1997). Certain species of EPN and EPF are also compatible, and in some cases, result in additive mortality effects on the host (Shapiro-llan et al. 2004). Female R. microplus treated with EPF and EPN laid egg masses with a lower weight and fewer larvae hatched from the eggs (Monteiro et al. 2013). However, the effects varied depending on the EPF, and EPN species. Although there is limited research on EPF and EPN combinations in the livestock pest system, there are many examples in other systems. For example, the nematode $H$. indica and the fungus $M$. anisopliae caused $20 \%$ mortality individually, but greater than $40 \%$ mortality in combination against the pecan weevil, Curculio caryae Horn (Coleoptera: Curculionidae) (Shapiro-Ilan et al. 2004). Additive effects also were observed with the nematode S. carpocapsae and B. bassiana and the entomopathogenic bacteria Serratia marcescens Bizio (Enterobacteriales: Enterobacteriaciae) (Shapiro-Ilan et al. 2004). However, many 
species appear to be antagonistic (Shapiro-Ilan et al. 2004) with some combinations resulting in the failure to produce progeny in both BCAs (Babercheck and Kaya 1990). One explanation for this variation in compatibility is that the synergistic bacteria of some EPN species are detrimental to some EPF species. For example, Photorhabdus luminescens (Thomas and Poinar) (Enterobacteriales: Enterobacteriaceae) was antagonistic to both M. anisopliae and B. bassiana, reducing fungal growth and conidial production (Ansari et al. 2005). Similarly, fungal extracts had antibacterial properties. However, the fungi appeared to have no effect on nematode survival or mortality (Ansari et al. 2005). On the contrary, Xenorhabdus poinarii (Akhurst) Akhurst and Boemare (Enterobacteriales: Enterobacteriaceae) was not antagonistic to any EPF species (Ansari et al. 2005). As these two bacterial genera are associated with different nematode genera, i.e. Xenorhabdus spp. with Steinernema spp. and Photorhabdus spp. with Heterorhabditis spp., this indicates that the combination of Steinernema spp. EPN and EPF hold greater promise than the alternative for livestock pest management.

Environmental risks associated with BCAs are typically considered to be less severe than those with synthetic pesticides. In general, BCAs are somewhat host specific if not by species then by isolate or due to abiotic constraints such as the temperature and humidity of the environment. Studies on non-target organisms are few and are often aimed at beneficial organisms providing natural management through predation, parasitism, or ecosystem services. Negative effects on vertebrates are unlikely due to the action of EPF through cuticle penetration and the limiting effect of higher temperatures $\left(>35^{\circ} \mathrm{C}\right)$ on EPF growth. For example, studies have shown no negative effects on chickens (Haas and Alves 2010). Viable conidia are observed in chickens faeces by 24 hours and for 24 hours after oral exposure ceases. Fish embryos were found to be susceptible to B. bassiana and M. anisopliae conidial attachment, which often resulted in rupture and or death (Genther and Middaugh 1995). Although negative interactions with invertebrates are more likely, EPF have been found to be compatible with beneficial insects including parasitoids and predators (Geden et al. 1995; Kaufman et al. 2005; Nielsen et al. 2005). However, due to the known efficacy of EPF against Coleoptera, there are concerns about dung treatment due to potential effects on dung beetles (Nicholas and McCorkell, 2014), which provide ecosystem services and reduce breeding sites for filth breeding flies. EPN appear to be even more host specific, with potential environmental risks being classified as temporary and remote to moderate (Ehlers and Hokkanen 1996).

Potential impacts of BCAs on non-target organisms could be further reduced by targeted applications, informed by careful monitoring, and the use of directed control methods such as autodissemination and lure-and-kill. The potential for autodissemination of EPF has been demonstrated with mosquitoes and house flies (Reyes-Villanueva et al. 2011; Vega et al. 1995). Lure-and-kill methods and autodissemination devices typically incorporate a baited trap that lures arthropods in and either kills them with a BCA or releases them after inoculation with a BCA to infect their conspecifics.

In conclusion, although some risks are associated with their use, particularly with invertebrates, careful selection of species and isolates along with targeted applications will reduce the potential for environmental damage. BCAs make a valuable and safe addition to IPM programs when used in combination with synthetic pesticides or other tactics (including multiple BCAs). Their use reduces the need for extensive pesticide application, which has positive impacts on environmental pollution, and should slow the development of resistance. 


\section{Summary and outlook}

Many of the entomopathogens discussed in this chapter show promise as BCAs for livestock pests. Although the time to mortality is often slow compared to chemical pesticides, pathogens can have additional sublethal effects that limit fecundity and damaging behaviour such as host location, blood feeding, and pathogen transmission. These additional benefits need to be considered as part of the broad picture of entomopathogen efficacy, of which kill rate is only one facet.

Challenges remain in widespread use of BCAs for pest control in animal facilities. Much of the variation in efficacy seen between studies, particularly with the fungal pathogens, is likely due to the isolate selected and its handling prior to use. Different isolates can vary widely in their virulence to different hosts and environmental tolerance, making selection of the appropriate isolate for the system crucial. Similarly, it is clear that recent passage through the host is necessary to maintain virulence, at least for EPF. Future research on isolate selection to aid development of EPF for commercial use may increase specificity and virulence, and/or identify critical factors that could be added to artificial media to maintain virulence without host passage. Partnerships with commercial producers of pathogens are also needed to ensure that those isolates that look promising in the laboratory have growth and storage characteristics that will favour development as commercial products. There is also a critical need for more field or near-field studies to demonstrate proof-of-concept. A rich body of laboratory-based research with pathogens is available but comparatively few projects have tested them under field conditions.

Careful formulation of pathogens, especially fungi, is critical to retain virulence and viability in the harsh and variable environmental conditions of the field. An effective formulation, whether liquid, dust, or other method of application, should protect conidia from UV and temperature degradation, as well as provide a suitable situation for germination. Furthermore, the formulation needs to adhere well to the target surface or substrate. Different formulations are needed for on-host or off-host applications; on-host applications are the more challenging due to the need to protect the conidia and the animal from side effects due to the formulation. Bait formulations remain largely unexplored and warrant further development. Baits are particularly promising for highly mobile pests such as flies and have the additional benefit of positioning the products in ways that minimize unwanted effects on parasitoids and predators.

Livestock and poultry production is changing to align with global needs and desires. As the human population continues to grow, our need for animal products continues to increase as well, and this has driven commercial production practices towards systems with high animal densities over the last half-century. Current pest management practices that rely heavily on chemical insecticides have been developed to meet the needs of high-density production. However, these housing and pest management practices are at odds with the growing desire for more sustainable and organic production systems, and much attention is being placed on animal welfare in farming operations. Entomopathogens can be expected to play an increasing role in developing IPM programs for both conventional and more sustainable and organic production systems. Innovations in strain selections, formulation and application technology are still needed to improve the efficacy and cost-effectiveness of these BCAs before they are widely adopted in livestock systems. 


\section{References}

Abd-Alla AM, Kariithi HM, Mohamed AH, Lapiz E, Parker AG and Vreysen, MJ (2013) Managing hytrosavirus infections in Glossina pallidipes colonies: feeding regime affects the prevalence of salivary gland hypertrophy syndrome. PLoS ONE 8: e61875.

Abd-Alla AM, Parker AG, Vreysen MJ and Bergoin M (2011) Tsetse salivary gland hypertrophy virus: hope or hindrance for tsetse control? PLoS Neglect Trop Dis 5: e1220.

Acharya N, Rajotte EG, Jenkins NE and Thomas MB (2015a) Potential for biocontrol of house flies, Musca domestica, using fungal biopesticides. Biocontrol Sci Techn 25: 513-524.

Acharya N, Rajotte EG, Jenkins NE and Thomas MB (2015b) Influence of biotic and abiotic factors on the persistence of a Beauveria bassiana biopesticide in laboratory and high-rise poultry house settings. Biocontrol Sci Techn 25: 1317-1332.

Acharya N, Seliga RA, Rajotte EG, Jenkins NE and Thomas MB (2015c) Persistence and efficacy of a Beauveria bassiana biopesticide against the house fly, Musca domestica, on typical structural substrates of poultry houses. Biocontrol Sci Techn 25: 697-715.

Aleksandro SD, Quintal APN, Monteiro SG, Doyle RL, Santurio JM and Bittencourt VREP (2006) Action of the fungus Beauveria bassiana, strain 986, over the biologic cycle of the Alphitobius diaperinus beetle in laboratory. Cienc Rural 36: 1944-1947.

Alekseev E, Glazer I and Samish M (2006) Effect of soil texture and moisture on the activity of entomopathogenic nematodes against female Boophilus annulatus ticks. BioControl 51: 507-518.

Alkhaibari AM, Carolino AT, Yavasoglu SI, Maffeis T, Mattoso TC, Bull JC, Samuels RI and Butt TM (2016) Metarhizium brunneum blastospore pathogenesis in Aedes aegypti larvae: attack on several fronts accelerates mortality. PLoS Pathog 12: e1005715.

Alves LFA, Alves VS, Bressan DF, Neves PMOJ and Alves SB (2004) Natural occurrence of Metarhizium anisopliae (Metsch.) Sorok. on adults of the lesser mealworm (Alphitobius diaperinus) (Panzer) (Coleoptera: Tenebrionidae) in poultry houses in Cascavel, PR, Brazil. Neotrop Entomol 33: 793-795.

Alves LFA, Neves PMOJ, Oliveira RC and Oliveira DGP (2008) Factors to be considered in the use of Beauveria bassiana for the control of Alphitobius diaperinus on poultry farms. Arq Inst Biol 75: 13-20.

Alves LFA, Oliveira DGP, Lambkin T, Bonini AK, Alves V, Pinto FGS and Scur MC (2015) Beauveria bassiana applied to broiler chicken houses as biocontrol of Alphitobius diaperinus (Panzer) (Coleoptera: Tenebrionidae), as avian pathogens vector. Braz J Poultry Sci 17: 459-466.

Alves RT, Bateman RP, Prior C and Leather SR (1998) Effects of simulated solar radiation on conidial germination of Metarhizium anisopliae. Crop Prot 17: 675-679.

Alves VS, Neves PMJ, Alves LFA, Moino A and Holz N (2012) Entomopathogenic nematodes (Rhabditida: Heterorhabditidae and Steinernematidae) screening for lesser mealworm Alphitobius diaperinus (Coleoptera: Tenebrionidae) control. Rev Colomb Entomol 38: 66-80.

Anderson RD, Bell AS, Blanford S, Paaijmans KP and Thomas MB (2011) Comparative growth kinetics and virulence of four different isolates of entomopathogenic fungi in the house fly (Musca domestica L.). J Invertebr Pathol 107: 179-184.

Anderson RD, Blanford S and Thomas MB (2013) House flies delay fungal infection by fevering: at a cost. Ecol Entomol 38: 1-10.

Angel-Sahagún CA, Lezama-Gutiérrez R, Molina-Ochoa J, Galindo-Velascío E, López-Edwards M, Rebolledo-Dominguez O, Cruz-Vázquez C, Reyes-Velázquez WP, Skoda SR and Foste JE (2005) Susceptibility of biological stages of the horn fly, Haematobia irritans, to entomopathogenic fungi (Hyphomycetes). J Insect Sci 5: 50.

Ansari MA, Carpenter S and Butt TM (2010) Susceptibility of Culicoides larvae to the insect-pathogenic fungus, Metarhizium anisopliae: prospects for bluetongue vector control. Acta Trop 113: 1-6.

Ansari MA, Pope EC, Carpenter S, Scholte E-J and Butt TM (2011) Entomopathogenic fungi as a biological control for an important vector of livestock disease: the Culicoides biting midge. PLoS ONE 6: e16108.

Ansari MA, Tirry L and Moens M (2005) Antagonism between entomopathogenic fungi and bacterial symbionts of entomopathogenic nematodes. BioControl 50: 465-475. 
Archana M, D'Souza PE and Patil J (2017) Efficacy of entomopathogenic nematodes (Rhabditida: Steinernematidae and Heterorhabditidae) on developmental stages of house fly, Musca domestica. J Parasit Dis 41: 782-794.

Arruda W, Lübeck I, Schrank A and Henning Vainstein M (2005) Morphological alterations of Metarhizium anisopliae during penetration of Boophilus microplus ticks. Exp Appl Acarol 37: 231-244.

Arthurs S and Thomas MB (2001) Effect of temperature and relative humidity on sporulation of Metarhizium anisopliae var. acridum in mycosed cadavers of Schistocera gregaria. J Invertebr Pathol 78: 59-65.

Atwa AA (2014) Entomopathogenic nematodes as biopesticides. In: Sahayaraj K (ed.) Basic and applied aspects of biopesticides. Springer, New York, NY, USA.

Axtell RC (1999) Poultry integrated pest management: status and future. Integrated Pest Manag Rev 4: 53-73.

Axtell RC and Arends JJ (1990) Ecology and management of arthropod pests of poultry. Annu Rev Entomol 35: 101-126.

Babercheck ME and Kaya HK (1990) Interactions between Beauveria bassiana and the entomogenous nematodes, Steinernema feltiae and Heterorhabditis heliothidis. J Invertebr Pathol 22: 225-234.

Bahiense TC, Fernandes, EKK, Angelo IDC, Perinotto WMS and Bittencourt VREP (2008) Performance of Metarhizium anisopliae and its combination with deltamethrin against pyrethroid-resistance strain of Boophilus microplus in a stall test. Ann NY Acad Sci 1149: 242-245.

Barbarin AM, Bellicanta GS, Osborne JA, Schal C and Jenkins NE (2017) Susceptibility of insecticide-resistant bed bugs (Cimex lectularius) to infection by fungal biopesticide. Pest Manag Sci 73: 1568-1573.

Barbarin AM, Jenkins NE, Rajotte EG and Thomas MB (2012) A preliminary evaluation of the potential of Beauveria bassiana for bed bug control. J Invetebr Pathol 111: 82-85.

Barson G, Renn N and Bywater A (1994) Laboratory evaluation of six species of entomopathogenic fungi for the control of the house fly (Musca domestica L.), a pest of intensive animal units. J Invertebr Pathol 64: 107-113.

Bawer MD, D'Souza PR and Pradeep BS (2014). Biocontrol of Haemotobia irritans horn flies with entomomopathogenic fungi (Beauveria bassiana and Metarhizium anisopliae). J Biol Control 28: 112-114.

Behle RW, Jackson MA and Flor-Weiler LB (2013) Efficacy of a granular formulation containing Metarhizium brunneum F52 (Hypocreales: Clavicipitaceae) microsclerotia against nymphs of Ixodes scapularis (Acari: Ixodidae). J Econ Entomol 106: 57-63.

Belton T, Rutherford TA, Trotter DB and Webster JM (1987) Heterorhabditis heliothidis: a potential biological control agent of house flies in caged-layer poultry barns. J Nematol 19: 263-266.

Bernardi E, Pinto DM, Do Nascimento JS, Ribeiro PB and Da Silva Cl (2006) Efeito dos fungos entomopatogênicos Metarhizium anisopliae e Beauveria bassiana sobre o desenvolvimento de Musca domestica L. (Diptera: Muscidae) em laboratório. Arq Inst Biol 73: 127-129.

Bharadwaj A and Stafford KC (2012) Susceptibility of Ixodes scapularis (Acari: Ixodidae) to Metarhizium brunneum F52 (Hypocreales: Clavicipitaceae) using three exposure assays in the laboratory. J Econ Entomol 105: 222-231.

Bischoff JF, Rehner SA and Humber RA (2009) A multilocus phylogeny of the Metarhizium anisopliae lineage. Mycologia 101: 512-530.

Bittencourt VREP, Mascarenhas AG and Faccini JLH (1999) The penetration of the fungus Metarhizium anisopliae on Boophilus microplus in experimental conditions. Cienc Rural 29: 351-354.

Blanford S, Chan BHK, Jenkins N, Sim D, Turner RJ, Read AF and Thomas MB (2005) Fungal pathogen reduces potential for malaria transmission. Science 308: 1638-1641.

Blanford S, Jenkins NE, Christian R, Chan BHK, Nardini L, Osae M, Koekemoer L, Coetzee M, Read AF and Thomas MB (2012a) Storage and persistence of a candidate fungal biopesticide for use against adult malaria vectors. Malaria J 11: 354 .

Blanford S, Jenkins NE, Read AF and Thomas MB (2012b) Evaluating the lethal and pre-lethal effects of a range of fungi against adult Anopheles stephensi mosquitoes. Malaria J 11: 365 .

Boemare NO (2002) Systematics of Photorhabdus and Xenorhabdus. In: Gaugler R (ed.) Entomopathogenic nematology. Centre for Agriculture and Bioscience International (CABI), Oxfordshire, UK, pp. 35-56.

Boucias D and Pendland J (1991) Attachment of mycopathogens to cuticle. In: Cole GT and Hock HC (eds.) The fungal spore and disease initiation in plants and animals. Plenum, New York, NY, pp. 101-127. 
Boucias D, Baniszewski J, Prompiboon P, Lietze V-U and Geden CJ (2015) Enhancement of the Musca domestica hytrosavirus infection with peritrophic matrix disruptive chemistries. J Invertebr Pathol 125: 35-43.

Bukhari T, Takken W and Koenraadt JM (2011) Development of Metarhizium anisopliae and Beauveria bassiana formulations for control of malaria mosquito larvae. Parasite Vector 4: 23.

Butt T (1990) Fungal infection processes. In: Osmond G (ed.) $5^{\text {th }}$ International Colloquium on Invertebrate Pathology and Microbial Control, Adelaide, Australia, August 1990, Department of Entomology, University of Adelaide, Adelaide, Australia, p. 121.

Butt T, Coates C, Dubovskiy I and Ratcliffe N (2016) Entomopathogenic fungi: new insights into host-pathogen interactions. Adv Genet 94: 307-364.

Butt T, Ibrahim L, Clark SJ and Beckett A (1995) The germination behaviour of Metarhizium anisopliae on the surface of aphid and flea beetle cuticles. Mycol Res 99: 945-950.

Byford RL, Craig ME and Crosby BL (1992) Review of ectoparasites and their effect on cattle production. J Anim Sci 70: 597-602.

Cafarchia C, Immediato D, latta R, Ramos RAN, Lia RP, Poretta D, Figuerefo LA, Dantas-Torres F and Otranto D (2015) Native strains of Beauveria bassiana for the control of Rhipicephalus sanguineus sensu lato. Parasite Vector 8: 80 .

Camargo MG, Golo PS, Angelo IC, Perinotto WMS, Sá FA, Quinelato S and Bittencourt VREP (2012) Effect of oil-based formulations of acaripathogenic fungi to control Rhipicephalus microplus ticks under laboratory conditions. Vet Parasitol 188: 140-147.

Campbell JB (1976) Effects of horn fly control on cows as expressed by increased weaning weights of calves. J Econ Entomol 69: 711-712.

Cárcamo MC, Felchicher F, Duarte JP, Bernardi E and Ribeiro PB (2015) Horizontal transmission of Beauveria bassiana (Hypocreales: Cordycipitaceae) and Metarhizium anisopliae (Hypocreales: Clavicipitaceae) in Musca domestica (Diptera: Muscidae). J Econ Entomol 108: 1579-1586.

Castineiras A, Jimeno G and Sosa ML (1987) Efecto de Beauveria bassiana, Metarhizium anisopliae (Fungi Imperfecti) y Pheidole megacephala (Hymenoptera: Formicidae) contra huevos de Boophilus microplus (Acarina: Ixodidae). Rev Salud Anim 9: 288-293.

Charnley AK (1989) Mechanisms of fungal pathogenesis in insects. In: Whipps JM and Lumsden RD (eds.) Biotechnology of fungi for improving plant growth: symposium of the British Mycological Society held at the University of Sussex. Cambridge University Press, Cambridge, UK.

Charnley AK and St. Leger RJ (1991) The role of cuticle-degrading enzymes in fungal pathogenesis in insects. In: Cole GT and Hoch HC (eds.) The fungal spore and disease initiation in plants and animals. Plenum Press, New York, NY, USA, pp. 267-286.

Chernaki-Leffer AM, Almeida LM, Sosa-Gómez DR, Anjos A and Vogado KM (2005) Populational fluctuation and spatial distribution of Alphitobius diaperinus (Panzer) (Coleoptera; Tenebrionidae) in a poultry house, Cascavel, Parana state, Brazil. Braz J Biol 67: 209-213.

Chernaki-Leffer AM, Kuttel J, Martins LM, Pedroso AC, Astolfi-Ferreira CS, Ferreira F and Ferreira AJP (2010) Vectorial competence of larvae and adults of Alphitobius diaperinus in the transmission of Salmonella enteritidis in poultry. Vector-Borne Zoonot 10: 48-487.

Clark TB, Kellen WR, Fukuda T and Lindegren JE (1968) Field and laboratory studies on the pathogenicity of the fungus Beauveria bassiana to three genera of mosquitoes. J Invertebr Pathol 11: 1-7.

Conn DB, Weaver J, Tamang L and Graczyk TK (2007) Synanthropic flies as vectors of Cryptosporidum and Giardia among livestock and wildlife in a multispecies agricultural complex. Vector-Borne Zoonot 7: 643-651.

Correia A, Fiorin AC, Monteiro AC and Verissimo CJ (1998) Effects of Metarhizium anisopliae on the tick Boophilus microplus (Acari: Ixodidae) in stabled cattle. J Invertebr Pathol 71: 189-191.

Cossé AA and Zilkowski BW (2015) Behavioral responses of lesser mealworm beetles, Alphitobius diaperinus, (Coleoptera: Tenebrionidae) to pheromone components using a wind tunnel dual choice walking bioassay. J Insect Behav 28: 202-210.

Costa JCR, Dias RJP and Morenz MJF (2007) Determining the adaptation potential of entomopathogenic nematode multiplication of Heterorhabditis riobravus and Steinernema carpocapsae (Rhabditida: Heterorhabditidae, 
Steinernematidae) in larvae of Alphitobius diaperinus (Coleoptera: Tenebrionidae) and Galleria mellonella (Lepidoptera: Pyralidae). Parasitol Res 102: 139-144.

Crawford PJ, Brooks WM and Arends JJ (1998) Efficacy of field-isolated strains of Beauveria bassiana (Moniliales: Moniliaceae) as microbial control agents of the lesser mealworm (Coleoptera: Tenebrionidae). J Econ Entomol 91: 1295-1301.

Da Costa GL, Sarquis MIM, De Moraes AML and Bittencourt VREP (2001) Isolation of Beauveria bassiana and Metarhizium anisopliae var. anisopliae from Boophilus microplus tick (Canestrini, 1887), in Rio de Janeiro State, Brazil. Mycopathol 154: 207-209.

Daoust RA and Roberts DW (1982) Virulence of natural and insect passaged strains of Metarhizium anisopliae to mosquito larvae. J Invertebr Pathol 40: 107-117.

Darbro JM, Johnson PH, Thomas MB, Ritchie SA, Kay BH and Ryan PA (2012) Effects of Beauveria bassiana on survival, blood-feeding success, and fecundity of Aedes aegypti in laboratory and semi-field conditions. Am JTrop Med Hyg 86: 656-664.

Darwish E and Zayed A (2002) Pathogenicity of two entomopathogenic hyphomycetes, Beauveria bassiana and Metarhizium anisopliae, to the housefly Musca domestica L. J Egypt Soc Parasitol 32: 785-796.

De Faria MR and Wraight SP (2007) Mycoinsecticides and mycoacaricides: a comprehensive list with worldwide coverage and international classification of formulation types. Biol Control 43: 237-256.

Del Valle EE, Frizzo LS, Malmierca M, Zbrun MV, Lax P and Doucet ME (2016) Biological control of Alphitobius diaperinus with Steinernema rarum CUL and Heterorhabditis bacteriophora SMC and feasibility of application in rice hull. J Pest Sci 89: 161-170.

Despins JL and Axtell RC (1995) Feeding behavior and growth of broiler chicks fed larvae of the darkling beetle, Alphitobius diaperinus. Poultry Sci 74: 331-336.

Dinev I (2013) The darkling beetle (Alphitobius diaperinus) - a health hazard for broiler chicken production. Trakia J Sci 11:1-4.

Dong Y, Morton JC Jr., Ramirez JL, Souza-Neto JA and Dimopoulos G (2012) The entomopathogenic fungus Beauveria bassiana activate toll and JAK-STAT pathway-controlled effector genes and anti-dengue activity in Aedes aegypti. Insect Biochem Molec 42: 126-132.

Dresner E (1950) The toxic effect of Beauveria bassiana (Bals) Vuill, on insects. J NY Entomol Soc 58: 269-278.

Dunford JC and Kaufman PE (2006) Lesser mealworm, litter beetle, Alphitobius diaperinus (Panzer) (Insecta: Coleoptera: Tenebrionidae). In: UF/IFAS featured creatures. Entomology and Nematology Department, Institute of Food and Agricultural Sciences, University of Florida, FL, USA. Available at: https://tinyurl.com/cbvzzhz.

Ehlers R-U and Hokkanen HMT (1996) Insect biocontrol with non-endemic entomopathogenic-nematodes (Steinernema and Heterorhabditis spp.): conclusions and recommendations of a combined OECD and COST workshop on scientific and regulatory policy issues. Biocontrol Sci Techn 6: 295-302.

Eilenberg J, Thomsen $L$ and Jensen AB (2013) A third way for entomophthoralean fungi to survive the winter: slow disease transmission between individuals of the hibernating host. Insects 4: 392-403.

El Damir M (2006) Comparison of house fly forewing and artificial media for examining environmental effects on conidial germination of entomopathogenic fungi. J Biol Sci 6: 286-293.

El-Sayed GN, Ignoffo CM, Leathers TD and Gupta SC (1993) Effects of cuticle source and concentration on the expression of hydrolytic enzymes by an entomopathogenic fungus, Nomuraea rileyi. Mycopathologia 122: 149-152.

Entrekin DL and Oliver JH (1982) Aggregation of the chicken mite, Dermanyssus gallinae (Acari: Dermanyssidae). J Med Entomol 19: 671-678.

Estrada-Peña A, González J and Casasolas A (1990) The activity of Aspergillus ochraceus (fungi) on replete females of Rhipicephalus sanguineus (Acari: Ixodidae) in natural and experimental conditions. Folia Parasitol 37: 331-336.

Fargues J and Remaudiere G (1977) Considerations on the specificity of entomopathogenic fungi. Mycopathologia 62: 31-37.

Farooq M and Freed S (2016) Lethal and sublethal effects of mixtures of entomopathogenic fungi and synthetic insecticides on biological aspects of Musca domestica L. Türk Entomol Derg 40: 211-225. 
Fernandes EG, Valério HM, Borges MAZ, Mascarin GM, Silva CE and Van der Sand ST (2013) Selection of fungi for the control of Musca domestica in aviaries. Biocontrol Sci Techn 23: 1256-1266.

Fernandez EKK and Bittencourt VREP (2008) Entomopathogenic fungi against South American tick species. Exp Appl Acarol 46: 71-93.

Foil LD and Hogsette JA (1994) Biology and control of tabanids, stable flies and horn flies. Rev Sci Tech Off Int Epiz 13: $1125-1158$.

Forst S, Dowds B, Boemare N and Stackebrandt E (1997) Xenorhabdus and Photorhabdus spp.: bugs that kill bugs. Annu Rev Microbiol 51: 47-72.

Frazzon APG, Da Silva Vaz I, Masuda A, Schrank A and Vainstein MH (2000) In vitro assessment of Metarhizium anisopliae isolates to control the cattle tick Boophilus microplus. Vet Parasitol 94: 117-125.

Galindo-Velasco E, Lezama-Gutiérrez R, Cruz-Vázquez C, Pescador-Rubio A, Angel-Sahagún CA, Ojeda-Chi MM, Rodríguez-Vivas RI and Contreras-Lara D (2015) Efficacy of entomopathogenic fungi (Ascomycetes: Hypocreales) against adult Haematobia irritans (Diptera: Muscidae) under stable conditions in the Mexican dry tropics. Vet Parasitol 209: 173-178.

Garcia MV, Monteiro AC, Szabo MJP, Prette N and Bechara GH (2005) Mechanism of infection and colonization of Rhipicephalus sanguineus eggs by Metarhizium anisopliae as revealed by scanning electron microscopy and histopathology. Braz J Microbiol 36: 368-372.

Garcia-Maruniak A, Maruniak JE, Farmerie W and Boucias DG (2008) Sequence analysis of a non-classified, non-occluded DNA virus that causes salivary gland hypertrophy of Musca domestica, MdSGHV. Virology 377: 184-196.

García-Munguía AM, Garza-Hernández JA, Rebollar-Tellez EA, Rodríguez-Pérez MA and Reyes-Villanueva F (2011) Transmission of Beauveria bassiana from male to female Aedes aegypti mosquitoes. Parasite Vector 4: 24 .

Gaugler R (ed.) (2002) Entomopathogenic nematology. Centre for Agriculture and Bioscience International (CABI), Oxfordshire, UK.

Gayathri G, Balasubramanian C, Vinyaga Moorthi P and Kubendren T (2010) Larvicidal potential of Beauveria bassiana (Balsamo) Vuillemin and Paecilomyces fumosoroseus (Wize) Brown and Smith on Culex quinquefasciatus (Say). J Biopestic 3: 147-151.

Geden CJ and Axtell RC (1987) Factors affecting climbing and tunneling behavior of the lesser mealworm, Alphitobius diaperinus (Coleoptera: Tenebrionidae). J Econ Entomol 80: 1197-1204.

Geden CJ and Axtell RC (1988) Effect of temperature on nematode (Steinernema feltiae) treatment of soil for control of lesser mealworm in turkey houses. J Econ Entomol 81: 800-803.

Geden CJ and Carlson DA (2001) A mechanical barrier for preventing climbing behavior by lesser mealworm and hide beetle (Coleoptera: Tenebrionidae, Dermestidae) larvae in poultry houses. J Econ Entomol 94: 1610-1616.

Geden CJ and Steinkraus DC (2003) Evaluation of three formulations of Beauveria bassiana for control of lesser mealworm and hide beetle in Georgia poultry houses. J Econ Entomol 96: 1602-1607.

Geden CJ, Arends JJ and Axtell RC (1987) Field trials of Steinernema feltiae (Nematoda: Steinernematidae) for control of Alphitobius diaperinus (Ceoleoptera: Tenebrionidae) in commercial broiler and turkey houses. J Econ Entomol 80: 136-141.

Geden CJ, Arrends JJ, Rutz DA and Steinkraus DC (1998) Laboratory evaluation of Beauveria bassiana (Moniliales: Moniliaceae) against the lesser mealworm, Alphitobius diaperinus (Coleoptera: Tenebrionidae), in poultry litter, soil, and a pupal trap. Biol Control 20: 71-77.

Geden CJ, Axtell RC and Brooks WM (1985) Susceptibility of the lesser mealworm, Alphitobius diaperinus (Coleoptera: Tenebrionidae) to the entomogenous nematodes Steinernema feltiae, S. glaseri (Steinernematidae) and Heterorhabditis heliothidis (Heterorhabditidae). J Entomol Sci 20: 331-339.

Geden CJ, Axtell RC and Brooks WM (1986) Susceptibility of the house fly, Musca domestica (Diptera: Muscidae) to the entomogenous nematodes Steinernema feltiae, S. glaseri (Steinernematidae) and Heterorhabditis heliothidis (Heterorhabditidae). J Med Entomol 23: 326-332.

Geden CJ, Lietze V-U and Boucias DG (2008) Seasonal prevalence and transmission of salivary gland hypertrophy virus of house flies (Diptera: Muscidae). J Med Entomol 45: 42-51. 
Geden CJ, Maruniak AG, Lietze V-U, Maruniak J and Boucias DG (2011b) Impact of house fly salivary gland hypertrophy virus (MdSGHV) on a heterologous host, stable fly (Stomoxys calcitrans). J Med Entomol 48: 1128-1135.

Geden CJ, Rutz DA and Steinkraus DC (1995) Virulence of different isolates and formulation of Beauveria bassiana for house flies and the parasitoid Muscidifurax raptor. Biol Control 5: 615-621.

Geden CJ, Steenberg T, Lietze V-U and Boucias DG (2011a) Salivary gland hypertrophy virus of house flies in Denmark: prevalence, host range, and comparison with a Florida isolate. JVector Ecol 36: 231-238.

Geden CJ, Steinkraus, DC and Rutz DA (1993) Evaluation of two methods for release of Entomophthora muscae (Entomophthorales: Entomophthoraceae) to infect house flies (Diptera: Muscidae) on dairy farms. Environ Entomol 20: 1201-1208.

Genther FJ and Middaugh DP (1995) Nontarget testing of an insect control fungus: effects of Metarhizium anisopliae on developing embryos of the inland silverfish Menidia beryllina. Dis Aquat Org 22: 163-171.

George J, Blanford S, Domingue MJ, Thomas MB, Read AF and Baler TC (2011) Reduction in host-finding behavior in fungus-infected mosquitoes is correlated with reduction in olfactory receptor neuron responsiveness. Malaria J 10: 219.

Georgis R and Manweiler SA (1994) Entomopathogenic nematodes: a developing biological control technology. Agricult Zool Rev 6: 63-94.

Georgis R, Koppenhöfer AM, Lacey LA, Bélair G, Duncan LW, Grewal PS, Samish M, Tan L, Torr P and Van Tol RW (2006) Successes and failures in the use of parasitic nematodes for pest control. Biol Control 38: 103-123.

Georgis R, Mullens BA and Meyer JA (1987) Survival and movement of insect parasitic nematodes in poultry manure and their infectivity against Musca domestica. J Nematol 19:292-295.

Gindin G, Glazer I, Mishoutchenko A and Samish M (2009) Entomopathogenic fungi as a potential control agent against the lesser mealworm, Alphitobius diaperinus, in broiler houses. Biol Control 54: 549-558.

Gindin G, Samish M, Alekseev E and Glazer I (2001) The susceptibility of Boophilus annulatus (Ixodidae) ticks to entomopathogenic fungi. Biocontrol Sci Techn 11:111-118.

Gindin G, Samish M, Zangi G, Mishoutchenko A and Glazer I (2002) The susceptibility of different species and stages of ticks to entomopathogenic fungi. Exp Appl Acarol 28: 283-288.

Glazer I and Samish M (1993) Suitability of Boophilus annulatus repleted female ticks as hosts of the nematode Steinernema carpocapsae. Exp J Invert Pathol 61: 220-222.

Goettel MS, Poprawski TJ, Vandenberg JD, Li Z and Roberts DW (1990) Safety to nontarget invertebrates of fungal biocontrol agents. In: Laird M, Lacey LA and Davidson EW (eds.) Safety of microbial insecticides. CRC Press, Boca Raton, FL, USA.

Goettel MS, Sigler L and Carmichael JW (1984) Studies on the mosquito pathogenic Hyphomyces Culicinomyces clavisporus. Mycologia 76: 614-625.

Gomes SA, Paula AR, Ribeiro A, Moraes COP, Santos JWAB, Silva CP and Samuels RL (2015) Neem oil increases the efficiency of the entomopathogenic fungus Metarhizium anisopliae for the control of Aedes aegypti (Diptera: Culicidae) larvae. Parasite Vector 8: 669.

Gortel K (1998) Equine parasitic hypersensitivity - a review. Equine Pract 20: 14-16.

Gray PA and Johnson DT (1983) Survival of the nematode Neoaplectana carpocapsae in relation to soil temperature, moisture and time. J Georgia Entomol Soc 18: 454-460.

Greenburg B (1971) Flies and disease, volume I: ecology, classification and biotic associations. Princeton University Press, Princeton, NJ, USA.

Gryganskyi AP, Humber R, Stajich JE, Mullens BA, Anishchenko IM and Vilgalys R (2013) Sequential utilization of hosts from different fly families by genetically distinct, sympatric populations within the Entomophthora muscae species complex. PLOS ONE 8: e71168.

Gryganskyi AP, Mullens BA, Gajdeczka M, Rehner SA, Vilgalys, R and Hajek AE (2017) Hijacked: co-option of host behavior by entomophthoralean fungi. PLoS Pathogens 13: e1006274.

Haas J and Alves LFA (2010) Safety of Beauveria bassiana (Bals.) Vuill. to Gallus domesticus L. Braz Arch Biol Technol 53: 465-471. 
Hall IM, Dulmage HTM and Arakawa KY (1972) Laboratory tests with entomogenous bacteria and the fungus Beauveria bassiana against the little house fly species Fannia canicularis and F. femoralis. Environ Entomol 1: 105-107.

Hamm RL, Kaufman PE, Reasor CA, Rutz DA and Scott JG (2006) Resistance to cyfluthrin and tetrachlorvinphos in the lesser mealworm, Alphitobius diaperinus, collected from the eastern United States. Pest Manag Sci 62: 673-677.

Hansen AN and De Fine Licht HH (2017) Logistic growth of the host-specific obligate insect pathogenic fungus Entomophthora muscae in house flies (Musca domestica). J Appl Entomol 141: 583-586.

Hartelt K, Wurst E, Collatz J, Zimmermann G, Kleespies RG, Oehme RM, Kimmig P, Steidle JLM and Mackenstedt U (2008) Biological control of the tick Ixodes ricinus with entompathogenic fungus and nematodes: preliminary results from laboratory experiments. Int J Med Microbiol 298: 314-320.

Hassemer MJ, Sant'Ana J, Borges M, Withall D, Pickett JA, De Oliveira MW, Laumann RA, Birkett MA and Blassioli-Moraes MC (2016) Revisiting the male-produced aggregation pheromone of the lesser mealworm, Alphitobius diaperinus (Coleoptera, Tenebrionidae): identification of a six-component pheromone from a Brazilian population. J Agr Food Chem 64: 6809-6818.

Hill DE (1998) Entomopathogenic nematodes as control agents of developmental stages of the black-legged tick, Ixodes scapularis. J Parasitol 84: 1124-1127.

Holderman CJ, Wood LA, Geden CJ and Kaufman PE (2017) Discovery, development, and evaluation of a horn flyisolated (Diptera: Muscidae) Beauveria bassiana (Hypocreales: Cordyciptaceae) strain from Florida, USA. J Insect Sci 17: 1-6.

Hong J and Hai J (2012) Entomopathogenic fungus for biological control of vectors. Chin J Hyg Insect Equip 18: 374-379.

Horn TB, Körbes JH, Granich J, Senter M and Ferla NJ (2016) Influence of laying hen systems on the mite fauna (Acari) community of commercial poultry farms in southern Brazil. Parasitol Res 115: 355-366.

Howard AFV, Koenraadt CJM, Farenhorst M, Knols BGJ and Takken W (2010) Pyrethroid resistance in Anopheles gambiae leads to increased susceptibility to the entomopathogenic fungi Metarhizium anisopliae and Beauveria bassiana. Malaria J 9: 168.

Immediato D, Camarda A, latta R, Puttilli MR, Ramos RAN, Di Paola G, Giangaspero A, Otranto D and Cafarchia C (2015) Laboratory evaluation of a native strain of Beauveria bassiana for controlling Dermanyssus gallinae (De Geer, 1778) (Acari: Dermanyssidae). Vet Parasitol 212: 478-482.

Inglis GD, Goettel MS, Butt TM and Strasser H (2001) Use of hyphomycetous fungi for managing insect pests. In: Butt TM, Jackson $\mathrm{C}$ and Magan $\mathrm{N}$ (eds.) Fungi as biocontrol agents: progress, problems and potential. Centre for Agriculture and Bioscience International (CABI), Oxfordshire, UK.

Jehle JA, Abd-Alla AM and Wang Y (2013) Phylogeny and evolution of Hytrosaviridae. J Invertebr Pathol 112: S62-S67. Jongejan F and Uilenberg G (1994) Ticks and control methods. Rev Sci Tech Off Int Epiz 13: 1201-1226.

Kaaya GP (2000) Laboratory and field evaluation of entomogenous fungi for tick control. Ann NY Acad Sci 916: 559-564. Kaaya GP, Mwangi EN and Ouna EA (1996) Prospects for biological control of livestock ticks, Rhipicephlaus appendiculatus and Amblyomma variegatum, using the entomogenous fungi Beauveria bassiana and Metarhizium anisopliae. J Invertebr Pathol 67: 15-20.

Kaaya GP, Samish M and Glazer I (2000) Laboratory evaluation of pathogenicity of entomopathogenic nematodes to African tick species. Ann NY Acad Sci 916: 303-308.

Kalsbeek V, Mullens BA and Jespersen JB (2001b) Field studies of Entomophthora (Zygomycetes: Entomophthorales) induced behavioral fever in Musca domestica (Diptera: Muscidae) in Denmark. Biol Control 21: 264-273.

Kalsbeek V, Pell, JK and Steenberg T (2001a) Sporulation by Entomophthora schizophorae (Zygomycetes: Entomophthorales) from housefly cadavers and the persistence of primary conidia at constant temperatures and relative humidities. J Invertebr Pathol 77: 149-157.

Kaoud HA (2010) Susceptibility of poultry red mites to entomopathogens. Int J Poultry Sci 9: 259-263.

Kariithi HM, Meki IK, Boucias DG and Abd-Alla AM (2017a) Hytrosaviruses: current status and perspective. Curr Opin Insect Sci 22: 71-78.

Kariithi HM, Yao X, Yu F, Teal PE, Verhoeven CP and Boucias DG (2017b) Responses of the housefly, Musca domestica, to the Hytrosavirus replication: impacts on host's vitellogenesis and immunity. Front Microbiol 8: 583. 
Kaufman PE, Reasor C, Rutz D, Ketzis J and Arends J (2005) Evaluation of Beauveria bassiana applications against adult house fly, Musca domestica, in commercial caged-layer poultry facilities in New York State. Biol Control 33: 360-367.

Kaufman PE, Wood LA, Goldberg JA, Long SJ and Rutz DA (2008) Host age and pathogen exposure level as factors in the susceptibility of the house fly, Musca domestica (Diptera: Muscidae) to Beauveria bassiana. Biocontrol Sci Techn 18: 841-847.

Kaya HK and Gaugler R (1993) Entomopathogenic nematodes. Annu Rev Entomol 38: 181-206.

Kershaw MJ, Moorhouse ER, Bateman R, Reynolds SE and Charnley AK (1999) The role of destruxins in the pathogenicity of Metarhizium anisopliae for three species of insect. J Invertebr Pathol 74: 213-223.

Khodyrev VP, Dubovskiy IM, Kryukov VY and Glupov VV (2013) Susceptibility of Anopheles messeae Fall. and Culex pipiens L. larvae to entomopathogenic fungi Metarhizium. Contemp Probl Ecol 7: 334-337.

Kilpinen $O$ and Steenberg T (2016) Repellent activity of desiccant dusts and conidia of the entomopathogenic fungus Beauveria bassiana when tested against poultry red mites (Dermanyssus gallinae) in laboratory experiments. Exp Appl Acarol 70: 329-341.

Kirkland B, Cho E-M and Keyhani NO (2004a) Differential susceptibility of Amblyomma maculatum and Amblyomma americanum (Acari: Ixodidea) to the entomopathogenic fungi Beauveria bassiana and Metarhizium anisopliae. Biol Control 31: 414-421.

Kirkland BH, Westwood GS and Keyhani NO (2004b) Pathogenicity of entomopathogenic fungi Beauveria bassiana and Metarhizium anisopliae to Ixodidae tick species Dermacentor variabilis, Rhipicephalus sanguineus, and Ixodes scapularis. J Med Entomol 41: 705-711.

Kirubakaran SA, Haripriya R, Naveenraj D and Thirumalaivasan P (2015) Virulence of Metarhizium anisopliae soil and commercial isolates against Culex quniquefasciatus Say, a vector of Bancroftian filariasis, and Aedes aegypti L., a vector of dengue fever. Eur J Biotechnol Biosci 3: 39-47.

Kocan KM, Blouin EF, Pidherney MS, Claypool PL, Samish M and Glazer I (1998) Entomopathogenic nematodes as a potential biological control method for ticks. Ann NY Acad Sci 849: 355-356.

Koppenhöfer AM and Kaya HK (1997) Additive and synergistic interaction between entomopathogenic nematodes and Bacillus thuringiensis for scarab grub control. Biol Control 8: 131-137.

Koppenhöfer AM, Grewal PS and Kaya HK (2000) Synergism of imidacloprid and entomopathogenic nematodes against white grubs: the mechanism. Entomol Exp Appl 94: 283-293.

Krasnoff SB, Watson DW, Gibson DM and Kwan EC (1995) Behavioral effects of the entomopathogenic fungus, Entomophthora muscae on its host Musca domestica: postural changes in dying hosts and gated pattern of mortality. J Insect Physiol 41: 895-903.

Kucharska K, Zajdel B, Pezowicz E, Jarmul-Pietraszczyk J, Mazurkiewicz A and Tumialis D (2016) Control of the lesser mealworm Alphitobius diaperinus using entomopathogenic nematodes (EPNs) combined with nanoparticles. Ann Warsaw U Life Sci - SGGW, Anim Sci 55: 57-67.

Lambkin TA, Kopittke RA, Rice SJ, Bartlett JS and Zalucki MP (2007) Distributions of lesser mealworm (Coleoptera: Tenebrionidae) in litter of a compacted earth floor broiler house in subtropical Queensland, Australia. J Econ Entomol 100: 1136-1146.

Lambkin TA, Kopittke RA, Rice SJ, Bartlett JS and Zalucki MP (2008) Factors affecting localized abundance and distribution of lesser mealworm in earth-floor broiler houses in subtropical Australia. J Econ Entomol 101: 61-67.

Lambkin TA, Swain AJ, Rice SJ, Bartlett JS and Zalucki MP (2012) Field assessments of control agents for lesser mealworm (Coleoptera: Tenebrionidae) using litter sampling. J Econ Entomol 105: 979-987.

Lecuona RE, Turica M, Tarocco F and Crespo DC (2005) Microbial control of Musca domestica (Diptera: Muscidae) with selected strains of Beauveria bassiana. J Med Entomol 42: 332-336.

Leemon D (2012) Fungal biopesticides for cattle tick and buffalo fly control. Report B. AHE.0193. Meat and Livestock Australia, North Sydney NSW, Australia. Available at: www.mla.com.au.

Leemon DM and Jonsson NN (2008) Laboratory studies on Australian isolates of Metarhizium anisopliae as a biopesticide for the cattle tick Boophilius microplus. J Invertebr Pathol 97: 40-49.

Leemon DM and Jonsson NN (2012a) Comparison of bioassay responses to the potential fungal biopesticide Metarhizium anisopliae in Rhipicephalus (Boophilus) microplus and Lucilia cuprina. Vet Parasitol 185: 236-247. 
Leemon DM and Jonsson NN (2012b) Comparative studies on the invasion of cattle ticks (Rhipicephalus (Boophilus) microplus and sheep blowflies Lucilia cuprina by Metarhizium anisopliae. J Invertebr Pathol 109: 248-259.

Leemon DM, Turner LB and Jonsson NN (2008) Pen studies on the control of cattle tick (Rhipicephlus (Boophilus) microplus) with Metarhizium anisopliae (Sorokin). Vet Parasitol 156: 248-260.

Leland JE and Behle RW (2005) Coating Beauveria bassiana with lignin for protection from solar radiation and effects on pathogenicity to Lygus lineolaris (Heteroptera: Miridae) Biocontrol Sci Techn 15: 309-320.

Lietze V, Geden CJ, Blackburn P and Boucias DG (2007) Effects of salivary gland hypertrophy virus on the reproductive behavior of the housefly, Musca domestica. Appl Environ Microb 73: 6811-6818.

Lietze VU, Abd-Alla AM, Vreysen MJ, Geden CJ and Boucias DG (2011b) Salivary gland hypertrophy viruses: a novel group of insect pathogenic viruses. Annu Rev Entomol 56: 63-80.

Lietze VU, Geden CJ, Doyle MA and Boucias DG (2011c) Transmission dynamics and persistence of MdSGHV in laboratory house fly (Musca domestica) populations. Appl Environ Microb 78: 311-317.

Lietze VU, Keesling JE, Lee JA, Vallejo CR, Geden CJ and Boucias DG (2013) Muscavirus (MdSGHV) disease dynamics in house fly populations - how is this virus transmitted and has it potential as a biological control agent? J Invetebr Pathol 112: S40-S43.

Lietze VU, Salem TZ, Prompiboon P and Boucias DG (2011a) Tissue tropism of the Musca domestica salivary gland hypertrophy virus. Virus Res 155: 20-27.

Lietze V-U, Simms K, Salem TZ, Geden CJ and Boucias DG (2009) Transmission of MdSGHV among adult house flies, Musca domestica (Diptera: Muscidae), occurs via oral secretions and excreta. J Invertebr Pathol 101: 49-55.

Lohmeyer KH and Miller JA (2006) Pathogenicity of three formulations of entomopathogenic fungi for control of adult Haematobia irritans (Diptera: Muscidae). J Econ Entomol 99: 1943-1947.

López-Sánchez J, Cruz-Vázquez C, Lezama-Gutiérrez R and Ramos-Parra M (2012) The effect of entomopathogenic fungi upon adults of Stomoxys calcitrans and Musca domestica (Diptera: Muscidae). Biocontrol Sci Techn 22: 969-973.

Lules RN, D'Alessandro WB and Luz C (2012) Effects of Metarhizium anisopliae conidia mixed with soil against the eggs of Aedes aegypti. Parasitol Res 110: 1579-1582.

Machtinger ET, Weeks ENI, Geden CJ and Kaufman PE (2016) House fly (Musca domestica) (Diptera: Muscidae) mortality after exposure to commercial fungal formulations in sugar bait. Biocontrol Sci Techn 26: 1444-1450.

Malik A, Singh N and Satya S (2007) House fly (Musca domestica): a review of control strategies for a challenging pest. J Environ Sci Health B 42: 453-469.

Maniania NK (1998) A device for infecting adult tsetse flies, Glossina spp., with entomopathogenic fungus in the field. Biol Control 11: 248-254.

Maniania NK and Ekesi S (2013) The use of entomopathogenic fungi in the control of tsetse flies. J Invertebr Pathol 112: S83-S88.

Maniania NK, Ekesi S, Odulaja A, Okech MA and Nadel DJ (2006) Prospects of a fungus-contamination device for the control of tsetse fly Glossina fuscipes fuscipes. Biocontrol Sci Techn 1692: 129-139.

Maniania NK, Laveissiere C, Odulaja A, Ekesi S and Herren H (2002) Entomopathogenic fungi as potential biocontrol agents for tsetse flies. In: Upadhyay R (ed.) Advances in microbial control of insect pests. Kluwer Academic/Plenum Publishers, Dordrecht, the Netherlands, pp. 145-163.

Martin W (1997) Using entomopathogenic nematodes to control insects during stand establishment. Hortic Sci 32: 196-200.

Matthews WA and Sumner DA (2015) Effects of housing system on the costs of commercial egg production. Poultry Sci 94: 552-557.

Mauléon H, Barré N and Panoma S (1993) Pathogenicity of 17 isolates of entomophagous nematodes (Steinernematidae and Heterorhabditidae) for the ticks Amblyomma variegatum (Fabricius), Boophilus microplus (Canestrini) and Boophilus annulatus (Say). Exp Appl Acarol 17: 831-838.

Mellor PS, Boorman J and Baylis M (2000) Culicoides biting midges: their role as arbovirus vectors. Annu Rev Entomol 45: 307-340. 
Ment D, Churchill AC, Belausov E, Glazer I, Rehner SA, Rot A, Donzelli BG and Samish M (2012) Resistant ticks inhibit Metarhizium infection prior to haemocoel invasion by reducing fungal viability on the cuticle surface. Environ Microbiol 14: 1570-1583.

Ment D, Gindin G, Soroker G, Glazer I, Rot A and Samish M (2010) Metarhizium anisopliae conidial responses to lipids from tick cuticle and tick mammalian host surface. J Invertebr Pathol 103: 132-139.

Minár J, Ríha J and Lamatová Z (1979) Losses in milking qualities of dairy cattle caused by mosquitoes and horseflies and reduction of such losses due to use of diethyltoluamide repellent. Folia Parasitol 26: 285-288.

Miranpuri GS and Khachatourians GG (1990) Larvicidal activity of blastospores and conidiospores of Beauveria bassiana (strain GK 2016) against groups of Aedes aegypti. Vet Parasitol 37: 155-162.

Mishra S and Malik A (2012) Comparative evaluation of five Beauveria isolates for housefly (Musca domestica L.) control and growth optimization of selected strain. Parasitol Res 111: 1937-1945.

Mishra S, Kumar P and Malik A (2015) Effect of temperature and humidity on pathogenicity of native Beauveria bassiana isolate against Musca domestica L. J Paras Dis 39: 697-704.

Mnyone LL, Kirby MJ, Lwetoijera DW, Mpingwa MW, Simfukwe ET, Knols BGJ, Takken W and Russell TL (2010) Tools for delivering entomopathogenic fungi to malaria mosquitoes: effect of delivery surfaces on fungal efficacy and persistence. Malaria J 9: 246.

Mnyone LL, Kirby MJ, Mpingwa MW, Lwetoijera DW, Knols BGJ, Takken W, Koenraadt CJM and Russell TL (2011) Infection of Anopheles gambiae mosquitoes with entomopathogenic fungi: effect of host age and blood-feeding status. Parasitol Res 108: 317-322.

Mochi DA, Monteiro AC, Ribeiro MAC and Yoshida L (2010a) Efficiency of entomopathogenic fungi in the control of eggs and larvae of the horn fly Haematobia irritans (Diptera: Muscidae). Vet Parasitol 167: 62-66.

Mochi DA, Monteiro AC, Ribeiro MAC and Yoshida L (2010b). Entomopathogenic fungal activity against pupae and adult Haematobia irritans (Diptera: Muscidae). Vet Parasitol 168: 105-110.

Mochi DA, Monteiro AC, Simi LD and Sampaio AAM (2009) Susceptibility of adult and larval stages of the horn fly, Haemotobia irritans, to the entomopathogenic fungus Metarhizium anisopliae under field conditions. Vet Parasitol 166: 136-143.

Molyneux AS (1986) Heterorhabditis spp. and Steinernema (=Neoaplectana) spp.: temperature, and aspects of behavior and infectivity. Exp Parasitol 62: 169-180.

Molyneux AS, Bedding RA and Akhurst RJ (1983) Susceptibility of the sheep blowfly Lucilia cuprina to various Heterrhabditis spp., Neoaplectana spp., and an undescribed steinernematid (Nematoda). J Invertebr Pathol 42: 1-7.

Monteiro CMO, Arújo LX, Matos RS, Golo PS, Angelo IC, Perinotto WMS, Rodrigues CAC, Furlong J, Bittencourt VREP and Prata MCA (2013) Association between entomopathogenic nematodes and fungi for the control of Rhipicephalus microplus (Acari: Ixodidae). Parasitol Res 112: 3645-3651.

Monteiro SG, Bittencourt VR, Daemon E and Faccini JL (1998) Pathogenicity under laboratory conditions of the fungi Beauveria bassiana and Metarhizium anisopliae on larvae of the tick Rhipicephalus sanguineus (Acari: Ixodidae). Braz JVet Parasitol 7: 113-116.

Monty DE and Garbareno MS (1978) Behavioral and physiologic responses of Holstein-Freisian cows to high environmental temperatures and artificial cooling in Arizona. Am J Vet Res 39: 877-882.

Moraes APR, Angelo IC, Fernandes EKK, Bittencourt VREP and Bittencourt AJ (2008) Virulence of Metarhizium anisopliae to eggs and immature stages of Stomoxys calcitrans. Ann NY Acad Sci 1149: 384-387.

Moraes APR, De Salles CMC, Bittencourt VREP and Bittencourt AJ (2015) Antimicrobial activity of Stomoxys calcitrans against Beauveria bassiana s.l. isolates. Rev Bras Parasitol Vet 24: 331-339.

Moraes D, Andrade MA, Duarte SC, Bastos TS, Arnhold E, Jayme VDS and Nunes IA (2016) Phenotypic and molecular detection of Salmonella sp. on growing, rearing and production phases in a commercial group of laying hens. Pesqui Vet Brasil 36: 503-508.

Morley-Davis J, Moore D and Prior C (1996) Screening of Metarhizium and Beauveria spp. conidia with exposure to simulated sunlight and a range of temperatures. Mycol Res 100: 31-38.

Mul MF, Van Riel JW, Meerburg BG, Dicke M, George DR and Groot Koerkamp PWG (2015) Validation of an automated mite counter for Demanyssus gallinae in experimental laying hen cages. Exp Appl Acarol 66: 589-603. 
Mullens BA (1986) A method for infecting large numbers of Musca domestica (Diptera: Muscidae) with Entomophthora muscae (Entomophthorales: Entomophthoraceae). J Med Entomol 23: 457-458.

Mullens BA, Meyer JA and Georgis R (1987b) Field tests of insect-parasitic nematodes against larvae of manure-breeding flies on caged-layer poultry facilities. J Econ Entomol 80: 438-442.

Mullens BA, Rodriguez JL and Meyer JA (1987a) An epizootiological study of Entomophthora muscae in muscoid fly populations on Southern California poultry facilities, with emphasis on Musca domestica. Hilgardia 55: 1-41.

Mullens BA, Soto D, Martin CD, Callaham BL and Gerry AC (2012) Northern fowl mite (Ornithonyssus sylviarum) control evaluations using liquid formulations of diatomaceous earth, kaolin, sulfur, azadirachtin, and Beauveria bassiana on caged laying hens. J Appl Poultry Res 21:111-116.

Murillo AC and Mullens BA (2016) Diversity and prevalence of ectoparasites on backyard chicken flocks in California. J Med Entomol 53: 707-711.

Mwamburi LA, Laing MD and Miller R (2009) Interaction between Beauveria bassiana and Bacillus thuringiensis var. israelensis for the control of house fly larvae and adults in poultry houses. Poultry Sci 88: 2307-2314.

Mwangi EN, Kaaya EN and Essumen S (1995) Experimental infections of the tick Rhipicephalus appendiculatus with entomopathogenic fungi, Beauveria bassiana and Metarhizium anisopliae, and natural infections of some ticks with bacteria and fungi. J Afr Zoo 109: 151-160.

Naem S (2007) First description of the horse stomach worm, Habronema muscae (Spirurida: Habronematidae) by scanning electron microscopy. Parasitol Res 101: 427-432.

Nayduch D and Joyner C (2013) Expression of lysozyme in the life history of the house fly (Musca domestica L.). J Med Entomol 50: 847-852.

Nchu F, Maniania NK, Hassanali A and Eloff KN (2010) Optimizing modes of inoculation of Rhipicephalus ticks (Acari: Ixodidae) with a mitisporic entomopathogenic fungus in the laboratory. Exp Appl Acarol 51: 373-382.

Nicholas AH and McCorkell B (2014) Evaluation of Metarhizium anisopliae for the control of Culicoides brevitarsis Kieffer (Diptera: Ceratopogonidae), the principal vector of bluetongue virus in Australia. J Vector Ecol 39: 213-218.

Nielsen CK, Skovgaard and Steenberg T (2005) Effect of Metarhizium anisopliae (Deuteromycotina: Hyphomycetes) on survival and reproduction of the filth fly parasitoid, Spalangia cameroni (Hymenoptera: Pteromalidae). Environmental Entomology 34: 133-139.

Nordenfors H, Höglund J and Uggla A (1999) Effects of temperature and humidity on oviposition, molting, and longevity of Dermanyssus gallinae (Acari: Dermanyssidae). J Med Entomol 36: 68-72.

Norris KR (1959) The ecology of sheep blowflies in Australia. In: Keast A, Crocker RL and Christian CS (eds.) Biogeography and ecology in Australia. Springer, Dordrecht, the Netherlands, pp. 514-544.

Onofre SB, Miniuk CM, de Barros NM and Azevedo JL (2001) Pathogenicity of four strains of entomopathogenic fungi against the bovine tick Boophilus microplus. Am J Vet Res 62: 1478-1480.

Paula AR, Carolino AT, Paula CO and Samuals RI (2011b) The combination of the entomopathogenic fungus Metarhizium anisopliae with the insecticide imidacloprid increases virulence against the dengue vector Aedes aegypti (Diptera: Culicidae). Parasite Vector 4: 8.

Paula AR, Carolino AT, Silva CP and Samuals RI (2011a) Susceptibility of adult female Aedes aegypti (Diptera: Culicidae) to the entomopathogenic fungus Metarhizium anisopliae is modified following blood feeding. Parasite Vector 4: 91 .

Pell JK, Eilenberg J, Hajek AE and Steinkraus DC (2001) Biology, ecology and pest management potential of Entomophthorales. In: Butt TM, Jackson CW and Magan N (eds.) Fungi as biocontrol agents: progress, problems and potential. Centre for Agriculture and Bioscience International (CABI), Oxfordshire, UK, pp. 71-153.

Perinotto WMS, Angelo IC, Golo PS, Quinelato S, Sá FA and Bittencourt VREP (2012) Susceptibility of different populations of ticks to entomopathogenic fungi. Exp Parasitol 130: 257-260.

Pezowicz EL, Mazurkiewicz AN and Tumialis DO (2013) Sensitivity of imago and larvae of the lesser mealworm Alphitobius diaperinus (Panzer 1797) in a sawdust litter to selected species and strains of Steinernematidae and Heterorhabditidae under laboratory conditions. Ann Warsaw U Life Sci - SGGW Anim Sci 52: 161-166.

Pinnock DE (1994) The use of Bacillus thuringiensis for control of pests of livestock. Agr Ecosyst Environ 49: 59-63.

Pests and vector-borne diseases in the livestock industry 
Pinnock DE and Mullens BA (2007). Application and evaluation of entomopathogens for control of livestock and poultry pests. In Lacey LA and Kaya HK (eds) Field manual of techniques in invertebrate pathology. Springer Science and Business Media BV, Dordrecht, Netherlands, pp. 713-734.

Pirali-Kheirabadi K, Haddadzadeh H, Razzaghi-Abyanch M, Bokaie S, Zare R, Ghazavi M and Shams-Ghahfarokhi M (2007) Biological control of Rhipicephalus (Boophilus) annulatus by different strains of Metarhizium anisopliae, Beauveria bassiana and Lecanicillium psalliotae fungi. Parasitol Res 100: 1297-1302.

Polar P, Aquino de Muro M, Kairo MTK, Moore D, Pegram R, John S-A and Roach-Benn C (2005) Thermal characteristics of Metarhizium anisopliae isolates important for the development of biological pesticides for the control of cattle ticks. Vet Parasitol 134: 159-167.

Polar P, Moore D, Kairo MTK and Ramsubhag A (2008) Topically applied myco-acaricides for the control of cattle ticks: overcoming challenges. Exp Appl Acarol 46: 119-148.

Poprawski TJ, Marchal M and Robert PH (1985) Comparative susceptibility of Otiorrhynchus sulcatus and Sitona lineatus (Coleoptera: Curculionidae) early stages to five entomopathogenic Hyphomycetes. Environ Entomol 12: 247-253.

Potgieter FT, Sutherland B and Biggs HC (1981) Attempts to transmit Anaplasma marginale with Hippobosca rufipes and Stomoxys calcitrans. J Vet Res 48: 119-122.

Pourseyed SH, Tavassoli M, Bernousi I and Mardani K (2010) Metarhizium anisopliae (Ascomycota: Hypocreales): an effective alternative to chemical acaricides against different developmental stages of fowl tick Argas persicus (Acari: Argasidae). Vet Parasitol 172: 305-310.

Pritchard J, Kuste T, Sparagano O and Tomley F (2015) Understanding the biology and control of the poultry red mite Dermanyssus gallinae: a review. Avian Pathol 44: 143-153.

Prompiboon P, Lietze V-U, Denton JSS, Geden CJ, Steenberg T and Boucias DG (2010) Musca domestica salivary gland hypertrophy virus, a globally distributed insect virus that infects and sterilizes female houseflies. Appl Environ Microbiol 76: 994-998.

Rassette MS, Pierpont El, Wahl T and Berres M (2012) Use of Beauveria bassiana to control northern fowl mites (Ornithonyssus sylviarum) on roosters in an agricultural research facility. J Am Assoc Lab Anim 50: 910-915.

Reed AF, Lynch PA and Thomas MB (2009) How to make evolution-proof insecticides for malaria control. PLoS Biol 7: e10000058.

Reis RC, Fernandez EK and Bittencourt VR (2008) Fungal formulations to control Rhipicephalus sanguineus engorged females. Ann NY Acad Sci 1149: 239-241.

Reis-Menini CMR, Prata MCA, Furlong J and Silva ER (2008) Compatability between the entomopathogenic nematode Steinernema glaseri (Rhabditida: Steinernematidae) and an acaricide in the control of Rhipicephalus (Boophilus) microplus (Acari: Ixodidae). Parasitol Res 103: 1391-1396.

Renn N (1995) Mortality of immature houseflies (Musca domestica L.) in artificial diet and chicken manure after exposure to encapsulated entomopathogenic nematodes (Rhabditida: Steinernematidae, Heterorhabditidae). Biocontrol Sci Techn 5: 349-359.

Renn N (1998) The efficacy of entomopathogenic nematodes for controlling housefly infestations of intensive pig units. Med Vet Entomol 12: 46-51.

Renn N and Wright E (2000) The effect of artificial substrates on the pathogenicity of Steinernema feltiae (Rhabditida: Steinernematidae) to adult Musca domestica (Diptera: Muscidae). Nematol 2: 217-222.

Renn N, Bywater AF and Barson G (1999) A bait formulated with Metarhizium anisopliae for the control of Musca domestica L. (Diptera: Muscidae) assessed in large-scale laboratory enclosures. J Appl Entomol 123: 309-314.

Reyes-Villanueva F, Garza-Hernadez JA, Garcia-Munguia AM, Tamez-Guerra P, Howard AFV and Rodriguez-Perez MA (2011) Dissemination of Metarhizium anisopliae of low and high virulence by mating behavior in Aedes aegypti. Parasite Vector 4: 171.

Rezende SRF, Curvello FA, Fraga ME, Reis RCS, Castiho AMC and Agostinho TP (2009) Control of the Alphitobius diaperinus (Panzer) (Coleoptera: Tenebrionidae) with entomopathogenic fungi. Braz J Poultry Sci 11: 121-127.

Rhode C, Alves LF, Neves PMOJ, Alves SB, Da Silva ERL and De Almeida JEM (2006) Selection of Beauveria bassiana (Bals.) Vuill. against Metarhizium anisopliae (Metsch.) Sorok. isolates against Alphitobius diaperinus (Panzer) (Coleoptera: Tenebrionidae). Neotrop Entomol 35: 231-240. 
Rizzo DC (1977) Age of three dipteran hosts as a factor governing the pathogenicity of Beauveria bassiana and Metarhizium anisopliae. J Invertebr Pathol 3: 127-130.

Rodrigueiro TS, Ginarete CM, Leite LG, Tavares FM, Goulart RM and Giacometti FH (2008) Eficiência de Heterorhabditis indica IBCB-N05 (Rhabditida: Heterorhabditidae) no controle de Alphitobius diaperinus (Coleoptera:Tenebrionidae) sob comedouros de granja avícola. Arq Inst Biol 75: 279-284.

Rot A, Gindin G, Ment D, Mishoutchenko A, Glazer I and Samish M (2013). On-host control of the brown dog tick Rhipicephalus sanguineus Latrielle (Acari: Ixodidae) by Metarhizium brunneum (Hypocreales: Clavicipitaceae). Vet Parasitol 193: 229-237.

Roy HE, Steinkraus DC, Eilenberg J, Hajek AE and Pell JK (2006) Bizarre interactions and endgames: entomopathogenic fungi and their arthropod hosts. Annu Rev Entomol 51:331-357.

Safrit RD and Axtell RC (1984) Evaluations of sampling methods for darkling beetles (Alphitobius diaperinus) in litter of turkey and broiler houses. Poult Sci 63: 2368-2375

Salin C, Vernon P and Vannier G (1998) The supercooling and high temperature stupor points of the adult lesser mealworm Alphitobius diaperinus (Coleoptera: Tenebrionidae). J Stored Prod Res 34: 385-394.

Samish M (2004) Biological control of ticks. Parasitol 129: S389-S403.

Samish M and Glazer I (2001) Entomopathogenic nematodes for the biocontrol of ticks. Trends Parasitol 17: 368-371.

Samish M, Alekseev E and Glazer I (1999a) Interaction between ticks (Acari: Ixodidae) and pathogenic nematodes (Nematoda): susceptibility of ticks species at various developmental stages. J Med Entomol 36: 733-740.

Samish M, Alekseev EA and Glazer I (1999b) Efficacy of entomopathogenic nematode strains against engorged Boophilus annulatus females (Acari: Ixodidae) under simulated field conditions. J Med Entomol 36: 727-732.

Samish M, Gindin G, Alekseev E and Glazer I (2001) Pathogenicity of entomopathogenic fungi to different developmental stages of Rhipicephalus sanguineus (Acari: Ixodidae). J Parasitol 87: 1355-1359.

Samish M, Ginsberg H and Glazer I (2004) Biological control of ticks. Parasitol 129: 389-403.

Samish M, Ginsberg H and Glazer I (2008) Anti-tick biological control agents: assessment and future perspectives. In: Bowman AS and Nuttall PA (eds.) Ticks: biology, disease and control. Cambridge University Press, Cambridge, UK, pp. 447-469.

Samson RA, Evans HC and Latge J-P (1988) Atlas of entomopathogenic fungi. Springer-Verlag, Berlin, Germany.

Sanchis V and Bourguet D (2008) Bacillus thuringiensis: applications in agriculture and insect resistance management - a review. Agron Sustain Dev 28: 11-20.

Scholte E-J, Ng'habi K, Kihonda J, Takken W, Paaijmans K, Abdulla S, Killeen GF and Knols BGJ (2005) An entomopathogenic fungus for control of adult African malaria mosquitoes. Science 308: 1641-1642.

Sewify GH and Habib SM (2001) Biological control of the tick fowl Argas persicargas persicus by the entomopathogenic fungi Beauveria bassiana and Metarhizium anisopliae. Anz Schadl J Pest Sci 74: 121-123.

Shapiro-llan DI, Jackson M, Reilly CC and Hotchkiss MW (2004) Effects of combining an entomopathogenic fungi or bacterium with entomopathogenic nematodes on mortality of Curculio caryae (Coleoptera: Curculionidae). Biol Control 30: 119-126.

Sharififard M, Mossadegh MS, Vazirianzadhe B and Zarei Mahmoudabadi A (2011) Laboratory pathogenicity of entomopathogenic fungi, Beauveria bassiana (Bals.) Vuill. and Metarhizium anisopliae (Metch.) Sorok. to larvae and adult of house fly, Musca domestica L. (Diptera: Muscidae). Asian J Biol Sci 4: 128-137.

Silva GS, Michels MG, Toma SB, Terra FE, Soares VE and Costa AJ (2007) Effectiveness of the compound chlorpyrifos+ cypermethrin+citronellal against Alphitobius diaperinus: laboratory analysis and residue determination in carcasses. Rev Bras Cienc Avic 9: 157-160.

Siri A, Scorsetti AC, Dikgolz VE and Lopez LC (2005) Natural infections caused by the fungus Beauveria bassiana as a pathogen of Musca domestica in the neotropic. BioControl 50: 937-940.

Sivasankaran P, Easwaramoorthy S and David H (1998) Influence of temperature and relative humidity on the growth, sporulation and pathogenicity of Beauveria bassiana. J Biol Control 12: 71-76.

Six DL and Mullens BA (1996a). Distance of conidial discharge of Entomophthora muscae and Entomophthora schizophorae (Zygomycotina: Entomophthorales). J Invetebr Pathol 67: 253-258. 
Six DL and Mullens BA (1996b) Seasonal prevalence of Entomophthora muscae and introduction of Entomophthora schizophorae (Zygomycotina: Entomophthorales) in Musca domestica (Diptera: Muscidae) populations on California dairies. Biol Control 6: 315-323.

Skovgård $\mathrm{H}$ and Steenberg $\mathrm{T}$ (2002) Activity of pupal parasitoids of the stable fly Stomoxys calcitrans and prevalence of entomopathogenic fungi in the stable fly and the house fly Musca domestica in Denmark. BioControl 47: 45-60.

Soarés GG Jr. (1982) Pathogenesis of infection by the hyphomycetous fungus, Tolypocladium cylindrosporum in Aedes sierrensis and Culex tarsalis [Dip.: Culicidae]. Biol Control 27: 283-299.

Sparagano O, Di Domenico D, Venturelli C, Papadopoulos E, Smallegange RC and Giangaspero A (2018) Arthropod pests in the poultry industry. In: Garros C, Bouyer J, Takken W and Smallegange RC (eds.) Pests and vector-borne diseases in the livestock industry. Ecology and Control of Vector-borne diseases. Vol. 5. Wageningen Academic Publishers, Wageningen, the Netherlands, pp. 17-53.

Sparagano OA, George DR, Harrington DWJ and Giangaspero A (2014) Significance and control of the poultry red mite, Dermanyssus gallinae. Annu Rev Entomol 59: 447-466.

St. Leger RJ, Charnley AK and Cooper RM (1986) Cuticle degrading enzymes of entomopathogenic fungi; mechanisms of interaction between pathogen enzymes and insect cuticle. J Invertebr Pathol 47: 295-302.

Steenberg T and Kilpinen 0 (2014) Synergistic interaction between the fungus Beauveria bassiana and desiccant dusts applied against poultry red mites (Dermanyssus gallinae). Exp Appl Acarol 62: 511-524.

Steenberg T, Kilpinen $O$ and Moore D (2006) Fungi control of the poultry red mite, Dermanyssus gallinae. In: Hansen L and Steenberg T (eds.) Implementation of biocontrol in practice in temperate regions - present and near future. Aarhus Universitet, Det Jordbrugsvidenskabelige Fakultet, Tjele, DIAS report No. 119, pp. 71-73.

Steinkraus DC, Geden CJ and Rutz DA (1990) First report of the natural occurrence of Beauveria bassiana (Moniliales: Moniliaceae) in Musca domestica (Diptera: Muscidae). J Med Entomol 27: 309-312.

Steinkraus DC, Geden CJ and Rutz DA (1991). Susceptibility of lesser mealworm (Coleoptera: Tenebrionidae) to Beauveria bassiana (Moniliales: Moniliaceae): effects of host stage, substrate, formulation, and host passage. J Med Entomol 28: 314-321.

Steinkraus DC, Geden CJ and Rutz DA (1993) Prevalence of Entomophthora muscae (Cohn) Fresenius (Zygomycetes: Entomophthoraceae) in house flies (Diptera: Muscidae) on dairy farms in New York and induction of epizootics. Biol Control 3: 93-100.

Sweeney AW (1985) The potential of the fungus Culicinomyces clavisporus as a biocontrol agent for medically important Diptera. In: Laird M and Miles JW (eds.) Integrated mosquito control methodologies. Vol. 2. Academic Press, Waltham, MA, USA.

Szalanski AL, Palmer TW, Mckay T and Steelman CD (2004) Infectivity of Steinernema spp. (Nematoda: Steinernematidae) to adult litter beetles, Alphitobius diaperinus (Coleoptera: Tenebrionidae) in the laboratory. Biocontrol Sci Techn 14(1): 81-85.

Szczepanik M, Orszewska P and Szołyga B (2015) Synergistic effect of anethole on the activity of abamectin against the lesser mealworm (Alphitobius diaperinus Panzer). Prog Plant Prot 55: 1891-1894.

Tamilam TV, Ponnudurai G, Harikrishnan TJ and Balasubramanian GA (2010) Efficacy of thermocole coated with spores of Metarhizium anisopliae and Beauveria bassiana against adult Musca domestica. J Vet Parasitol 24: 155-158.

Tavassoli M, Allymehr M, Pourseyed SH, Ownag A, Bernousi I, Mardani K, Ghorbanzadegan M and Shokrpoor S (2011) Field bioassay of Metarhizium anisopliae strains to control the poultry red mite Dermanyssus gallinae. Vet Parasitol 178: 374-378.

Tavassoli M, Ownag A, Pourseyed SH and Mardani K (2008) Laboratory evaluation of three strains of the entomopathogenic fungus Metarhizium anisopliae for controlling Dermanyssus gallinae. Avian Pathol 37(3): 259-263.

Taylor DB, Szalanski AL, Adams BJ and Peterson RD (1998) Susceptibility of house fly (Diptera: Muscidae) larvae to entomopathogenic nematodes (Rhabditida: Heterorhabditidae, Steinernematidae). Environ Entomol 27: 1514-1519.

Töth EM, Márialigeti, K, Fodor A, Lucskai A and Farkas R (2005) Evaluation of efficacy of entomopathogenic nematodes against larvae of Lucilia sericata (Meigen, 1826) (Diptera: Calliphoridae). Acta Vet Hung 53: 65-71.

Turner EC (1986) Structural and litter pests. Poult Sci 65: 644-648 
Ulrich KR, Feldlaufer MF, Kramer M and Leger RJS (2015) Inhibition of the entomopathogenic fungus Metarhizium anisopliae sensu lato in vitro by the bed bug defensive secretions (E)-2-hexenal and (E)-2-octenal. BioControl 60: 517-526.

Ulrich KR, Feldlaufer MF, Kramer M and St. Leger RJ (2014). Exposure of bed bugs to Metarhizium anisopliae at different humidities. J Econ Entomol 107: 2190-2195.

Unkles SE, Marriott C, Kinghorn JR, Panter C and Blackwell A (2004) Efficacy of the entomopathogenic fungus Culicinomyces clavisporus against larvae of the biting midge, Culicoides nubeculosus (Diptera: Ceratopogonidae). Biocontrol Sci Techn 14: 397-401.

Vasconcelos V de O, Furlong J, De Freitas GM, Dolinski C, Aguillera MM, Rodrigues RCD and Prata M (2004) Steinernema glaseri Santa Rosa strain (Rhabditida: Steinernematidae) and Heterorhabditis bacteriophora CCA strain (Rhabditida: Heterorhabditidae) as biological control agents of Boophilus microplus (Acari: Ixodidae). Parasitol Res 94: 201-206.

Vaughan JA, Turner EC Jr. and Ruszler PL (1984) Infestation and damage of poultry house insulation by the lesser mealworm, Alphitobius diaperinus (Panzer). Poult Sci 63: 1094-1100.

Vega FE, Dowd PF and Bartlet RJ (1995) Dissemination of microbial agents using an autoinoculating device and several insect species as vectors. Biol Control 5: 545-552.

Vogt WG and Woodburn TL (1979) Ecology, distribution and importance of sheep myiasis flies in Australia. In: Proceedings of the National Symposium on Sheep Blowfly and Flystrike in Sheep. New South Wales Department of Agriculture, Sydney, Australia, p. 23.

Wall R, French NP and Morgan KL (1992) Effects of temperature on the development and abundance of the sheep blowfly Lucilia sericata (Diptera: Calliophoridae). Bull Entomol Res 82: 125-131.

Wang X, Li Q, Shen L, Yang J, Cheng H, Jiang S, Jiang C and Wang H (2014) Fumigant, contact, and repellent activities of essential oils against the darkling beetle, Alphitobius diaperinus. J Insect Sci 14: 75.

Watson DW and Petersen JJ (1993) Seasonal activity of Entomophthora muscae (Zygomycetes: Entomophthorales) in Musca domestica L. (Diptera: Muscidae) with reference to temperature and relative humidity. Biol Control 3: 182-190.

Watson DW, Geden CJ, Long SJ and Rutz DA (1995) Efficacy of Beauveria bassiana for controlling the house fly and stable fly (Diptera: Muscidae). Biol Control 5: 405-411.

Watson DW, Guy JS and Stringham SM (2000) Limited transmission of turkey coronavirus in young turkeys by adult Alphitobius diaperinus (Coleoptera: Tenebrionidae). J Med Entomol 37: 480-483.

Watson DW, Mullens BA and Petersen JJ (1993) Behavioral fever response of Musca domestica (Diptera: Muscidae) to infection by Entomophthora muscae (Zygomycetes: Entomophthorales). J Invertebr Pathol 61: 10-16.

Watson DW, Rutz DA and Long SJ (1996) Beauveria bassiana and sawdust bedding for the management of the house fly, Musca domestica (Diptera: Muscidae) in calf hutches. Biol Control 7: 221-227.

Weeks ENI, Machtinger ET, Gezan SA, Kaufman PE and Geden CJ (2017) Effects of four commercial fungal formulations on mortality and sporulation in house flies (Musca domestica) and stable flies (Stomoxys calcitrans). Med Vet Entomol 31: 15-22.

Wojciechowska I, Kamionek M and Morytz B (2013) Studies on the potential use of entomopathogenic nematodes for biological control of animals in the stables. Ann Warsaw U Life Sci - SGGW Anim Sci 52: 227-236.

Wolf J, Potrich M, Lozano ER, Gouvea A and Pegorini CS (2015) Combined physical and chemical methods to control lesser mealworm beetles under laboratory conditions. Poult Sci 94: 1145-1149.

Wright C, Brooks A and Wall R (2004) Toxicity of the entomopathogenic fungus, Metarhizium anisopliae (Deuteromycotina: Hyphomycetes) to adult females of the blowfly Lucilia sericata (Diptera: Calliphoridae). Pest Manage Sci 60: 639-644.

Zangi G (2003) Tick control by means of entomopathogenic nematodes and fungi. MSc-thesis, Hebrew University of Jerusalem, Israel.

Zhioua E, Lebrun RA, Ginsberg HS and Aeschliman A (1995) Pathogenicity of Steinernema carpocapsae and S. glaseri (Nematoda: Steinernematidae) to Ixodes scapularis (Acari: Ixodidae). J Med Entomol 32: 900-905.

Zimmer CR, Cárcamo MC, Ribeiro PB and Nascimento JS (2010) Ação do fungo Metarhizium anisopliae sobre desenvolvimento do díptero Muscina stabulans em laboratório. Arq Bras Med Vet Zootec 62: 1142-1147. 


\title{
13. Semiochemical tools for a new generation of livestock pest control
}

\author{
Victor A. Brugman ${ }^{1,2^{*}}$, Renate C. Smallegange ${ }^{3}$ and James G. Logan ${ }^{2}$ \\ ${ }^{1}$ Evolution Biotechnologies, Colworth Science Park, Sharnbrook, Bedford, MK44 1LZ, United Kingdom; \\ ${ }^{2}$ London School of Hygiene and Tropical Medicine, Keppel Street, London, WC1E 7HT, United Kingdom; \\ ${ }^{3}$ Laboratory of Entomology, Wageningen University \& Research, P.O. Box 16, 6700 AA Wageningen, the \\ Netherlands; vab@evolutionbiotech.com
}

\begin{abstract}
Arthropod-borne diseases and ectoparasite infestation of livestock remain a serious veterinary and economic problem worldwide. Furthermore, nuisance biting by haematophagous arthropods can result in a significant disruption to livestock behaviour and production. Existing tools for managing pest arthropods have so far not been successful in providing elimination or effective control of many pest arthropod species. The rapid emergence and re-emergence of pathogens and the on-going burden of those currently circulating mean that the need to develop new tools and interventions for pest management is urgent. A vast array of host-derived, arthropod-derived and environmental semiochemicals regulate the behaviour of pest arthropods and, therefore, many of these present promising targets for control interventions. This chapter details the principles of semiochemical-based approaches to arthropod surveillance and control and the major arthropod behavioural targets (host-seeking, sugar-feeding and oviposition/larviposition behaviour) that need to be understood to enable successful interventions to take place. The chapter then reviews current progress towards semiochemical-based approaches for the surveillance and control of major arthropod pests: tsetse flies (Diptera: Glossinidae), mosquitoes (Diptera: Culicidae), biting midges (Diptera: Ceratopogonidae), sand flies (Diptera: Phlebotominae), black flies (Diptera: Simuliidae), horse flies (Diptera: Tabanidae), horn flies and stable flies (Diptera: Muscidae), and ticks (Ixodida: Ixodoidea).
\end{abstract}

Keywords: arthropod, behaviour, ectoparasite, haematophagous, livestock, attractants, repellents, surveillance

\section{Introduction to semiochemicals}

The highly specialised olfactory system possessed by haematophagous (blood-feeding) arthropods is an important sensory mechanism, central to their ability to respond to the environment. The detection of olfactory cues, or semiochemicals (behaviour-modifying chemicals), in tandem with visual, heat and moisture-based stimuli enables arthropods to identify conspecifics, sugar and blood meal sources as well as favourable sites for breeding. Due to their importance in mediating arthropod survival, the exploitation of semiochemical cues offers promise for the management and control of livestock pest species of economic and veterinary importance.

Semiochemicals convey information to and between arthropods, inducing a physiological and/ or behavioural change in the recipient organism (Nordlund and Lewis 1976). These key survival parameters include the location, suitability and physiological state of other members of the same species, the presence of predators and resources including prey, plants and appropriate breeding habitats (Pickett et al. 1998). 
Semiochemicals can be classified into two primary groups depending on whether they mediate information transfer between members of the same species (intraspecific communication) or between different species (interspecific communication). The first group, pheromones, play a wide role in facilitating key behaviours across arthropod groups. Among these, some of the most important are the releaser pheromones which generate immediate and essential behavioural responses involved with reproductive, alarm and aggregative functions (Shorey 1973; Wilson and Bossert 1963). The second group, allelochemicals, can be further subdivided into allomones, kairomones and synomones based on their function (Nordlund and Lewis 1976). Allomones benefit the producing organism at the expense of the receiver; an example of these are compounds that are naturally produced by vertebrate hosts such as humans (Logan et al. 2008, 2009), horses (Creton et al. 2016), cattle (Birkett et al. 2004), waterbuck (Bett et al. 2015) and birds (Douglas et al. 2005) that function as insect repellents. Kairomones provide a benefit exclusively to the receiver of the signal; these include volatile compounds produced by vertebrates which stimulate the host location process in biting arthropods (Gibson and Torr 1999). Finally, synomones provide a beneficial effect to both the producing organism and the receiver. Important examples of synomones are plant-produced volatile compounds that attract sugar-feeding arthropods, providing nectar whilst facilitating the pollination of the plant (Milet-Pinheiro et al. 2013).

The first section of this chapter details the key principles underlying the exploitation of semiochemicals for the surveillance and control of important livestock pest arthropods. The second section evaluates current understanding of the semiochemicals involved in the regulation of the behaviour of these pest groups, with a focus on existing evidence and prospects for the semiochemical-based management of these pests. Although the role of semiochemicals has been identified in many pest arthropods, this chapter will focus on the following key groups: tsetse flies (Diptera: Glossinidae), mosquitoes (Diptera: Culicidae), biting midges (Diptera: Ceratopogonidae), sand flies (Diptera: Phlebotominae), black flies (Diptera: Simuliidae), horse flies (Diptera: Tabanidae), horn and stable flies (Diptera: Muscidae), and ticks (Ixodida: Ixodoidea).

\section{Arthropod detection and laboratory identification of semiochemicals}

The arthropod olfactory system shows a remarkable degree of sensitivity and specificity, enabling the organism to detect low concentrations of important semiochemicals against a complex background of other volatile compounds. Semiochemical detection by arthropods is principally via the antennae, with additional involvement of the maxillary palps, proboscis and other parts of the body (such as the Haller's organ in ticks), depending on the arthropod group. The surface of these sensory organs are equipped with sensilla, cuticular structures housing one or several neurones depending on arthropod species and sensillum type. Pores located on the sensilla allow the semiochemical molecules to enter, whereupon odorant binding proteins transport them across the sensillum lymph to olfactory receptors located on the dendrites of olfactory receptor neurones (ORNs) (Mclver 1982; Zhou et al. 2004). Appropriate stimulation of the ORNs leads to direct stimulation of the arthropod central nervous system resulting in the observed physiological or behavioural response (Hansson 2002; Zhou et al. 2004).

Vertebrates produce many thousands of different semiochemicals, presenting a challenge to the identification of individual chemicals. Currently available laboratory methods offer high levels of sensitivity and specificity for the identification and characterisation of individual semiochemicals. When implemented correctly, the identification of semiochemicals that produce an attractive, aggregative or repellent effect can provide important insights into the regulation of interactions 
between arthropods, their hosts and the environment and can lead to the development of novel methods of exploiting these interactions for the purposes of control (Agelopoulos et al. 1999).

The process of identifying the role of semiochemicals in modulating arthropod behaviour can broadly be separated into five steps: (1) sample collection (isolation) from the source; (2) preparation of the sample; (3) identification of individual compounds; (4) testing the arthropod's electrophysiological response to compounds; and (5) testing the arthropod's behavioural response to compounds. Obtaining a high-quality sample of an appropriate volume is an important starting point, although a major influence on experimental output will be the logistics of volatile collection from the source. For example, the collection of skin volatile samples by dynamic headspace sampling (air entrainment) from a live animal will require it to be restrained for some time, which may present a challenge. Early semiochemical identification studies most commonly used solventbased collection methods which involved the washing of samples using methanol or ethanol (e.g. Lowther et al. 1964). Solvent-free methods have largely replaced these approaches and include vacuum distillation (e.g. Bentley et al. 1979) and most recently solid phase microextraction (e.g. Syed and Leal 2009) alongside air entrainment (Birkett et al. 2004), to collect samples for arthropod olfaction studies.

Once the sample has been obtained, gas chromatography (GC) and coupled GC-mass spectrometry (GC-MS) are the most commonly used methods to separate, identify and quantify individual semiochemicals from a sample (Pickett 1990). Tentative identification of a compound by GC-MS is followed by confirmation by GC co-injection. Once individual components have been identified, their ability to produce an electrophysiological response in the target arthropod can be tested using GC-electroantennography (GC-EAG) or, at the finer scale, by GC-single-cell recordings (GCSRC) (Adham 1982; Qiu and Van Loon 2010). EAG measures the sum of electrical potentials created by activated ORNs on the insect antenna, while SRC measures the action potential generated by ORNs within a single sensilla on the insect antenna (Olsson and Hansson 2013). To test whether this physiological response translates into a behavioural response in the arthropod, olfactometers can be used. An example is the Y-tube assay. Y-tubes were initially designed for use with agricultural pests (Mclndoo 1926) and, along with other olfactometers, are now widely used to compare the upwind movement of haematophagous arthropods towards host-derived kairomones. An arthropod is placed at the base of the Y-tube olfactometer and is presented with a two-way choice of odours carried by a clean air stream; a preference for one odour over the other will cause upwind movement into the appropriate arm of the olfactometer. In an extension of this approach, three- (Lacroix et al. 2005) and four-way olfactometers (Vet et al. 1983) facilitate the simultaneous comparison of more than two odours. These designs allow an arthropod to be placed in a central chamber wherein it is exposed to multiple odours (Figure 1). Working to a similar principle, wind tunnels are larger designs frequently used to investigate arthropod flight behaviour in response to semiochemicals. The arthropod is released at one end of the wind tunnel, whilst one or multiple odours are released from the other end, mixing in the chamber in between (Figure 1). The 3D dynamics of upwind flight behaviour can be tracked using video cameras (Spitzen et al. 2013) and the arthropod collected at the odour-end of the tunnel. Modified designs comprising one to three stacked wind tunnels can be used to facilitate the simultaneous release of multiple arthropods (Posey et al. 1998; Verhulst et al. 2010). Once a behavioural response has been established in the laboratory, a promising semiochemical attractant or repellent compound (or blend of compounds) can be evaluated in the field (Smallegange and Takken 2010). 
Victor A. Brugman et al.

A
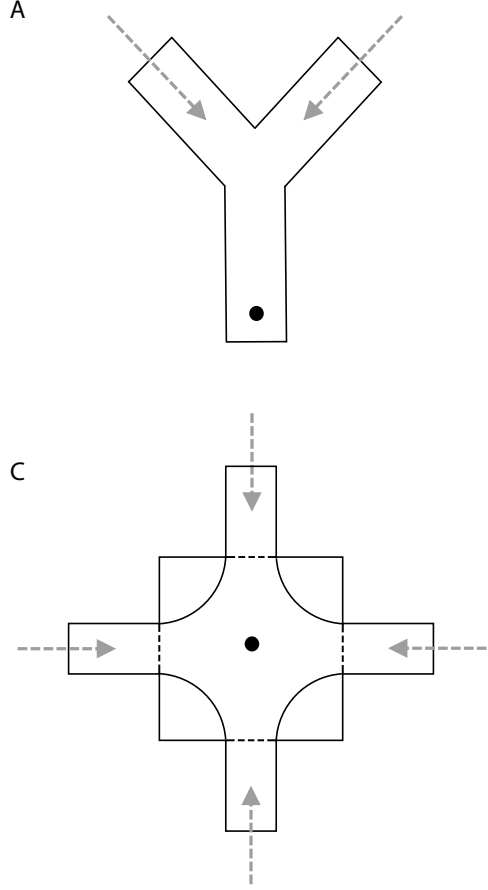

Direction of airflow
B

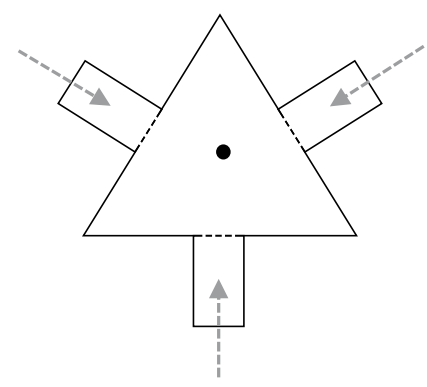

D

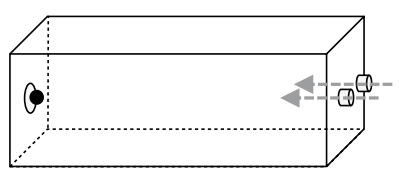

- Arthropod starting point

Figure 1. Arthropod olfactometers. Schematic diagrams illustrating (A) a two-way olfactometer (or Y-tube), (B) a three-way olfactometer, $(C)$ a four-way olfactometer, and $(D)$ a wind tunnel in $3 D$; direction of airflow and the starting point for the arthropod are indicated.

\section{Approaches to exploiting semiochemicals for arthropod control}

Semiochemicals play an important role in the life cycle of arthropods and each behaviour in which they are involved offers a target for intervention strategies. Three of the most important sources of semiochemicals influencing arthropod behaviour are vertebrate hosts, the environment and conspecifics, and compounds from these sources can be used to exploit the behaviours they regulate (Figure 2 ).

\section{Exploiting vertebrate host-derived semiochemicals}

The exploitation of vertebrate host-derived semiochemicals (kairomones and allomones) is based on the targeted use of compounds naturally produced by hosts in their breath, body, or sometimes from their faeces or urine. These compounds are often produced directly by the host or by closelyassociated communities of commensal organisms such as skin bacteria (Smallegange et al. 2011; Verhulst et al. 2011). These semiochemicals may either serve as attractant cues which stimulate an arthropod and are used to locate its host, or as repellent cues which prevent an arthropod 
Semiochemical source

Exploitable arthropod behaviour

Approaches to exploiting target behaviours

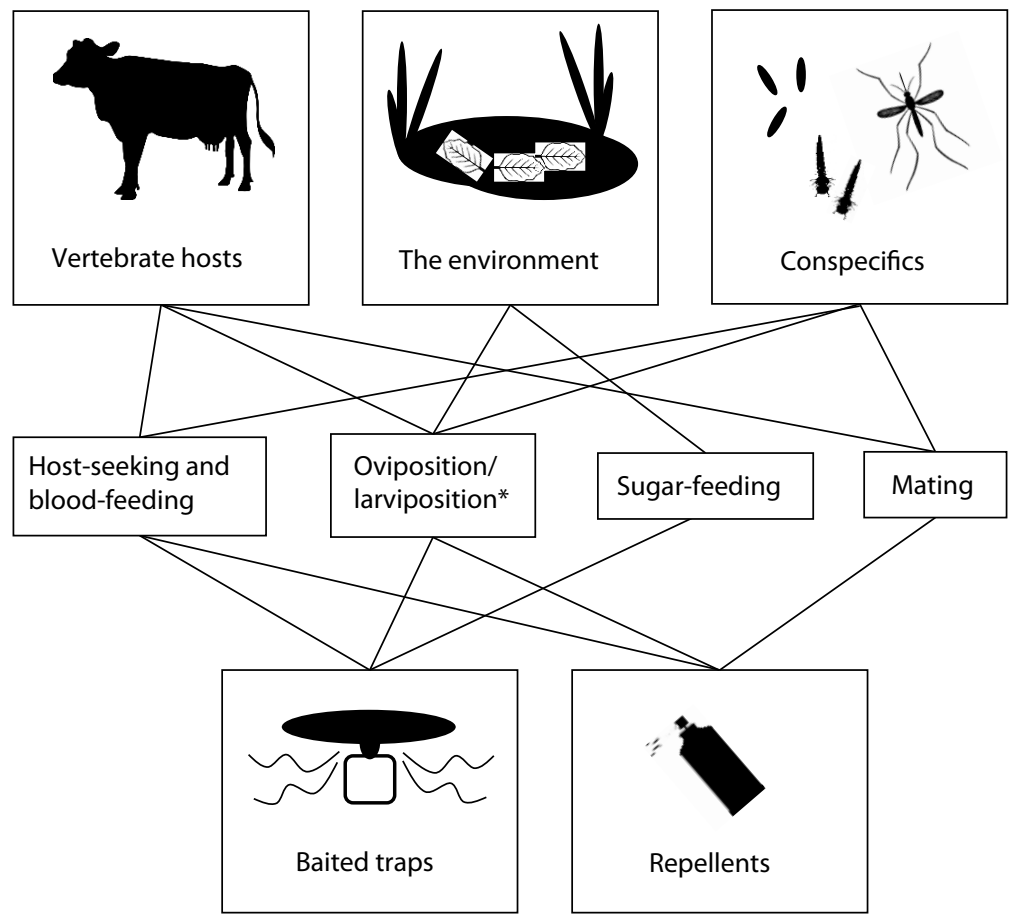

Figure 2. The role of semiochemicals in regulating arthropod behaviour, illustrated using mosquitoes. The major sources of semiochemicals involved in arthropod biology, relevant arthropod behaviours that these regulate, and the major approaches used to exploit each behaviour for the purposes of surveillance and control. *Note that larviposition does not occur in mosquitoes but is a characteristic of other groups such as tsetse flies (Glossina spp.).

from detecting or landing on the host, even after the host has been located. The combination of attractive and repellent semiochemicals produced by individual hosts plays an important role in mediating differential attractiveness to biting arthropods (Logan and Birkett 2007).

\section{Host-derived attractants}

A wide range of host-derived kairomones are involved in the host-seeking behaviour of pest arthropods (Gibson and Torr 1999). Host location begins with the activation of host-seeking behaviour, followed by long-range upwind anemotaxis towards the host. Short-range orientation and landing is guided by both host kairomones and visual cues such as colour, patterns, shape and movement, following which a blood meal can be taken or larvae deposited in the host (for example, in the case of deer bot flies of the genus (ephenemyia). A more detailed evaluation of the role of specific semiochemicals involved in the host-seeking behaviour of various pest arthropods follows in the second half of the chapter.

Semiochemicals that attract pest arthropods are widely used to bait artificial traps as part of arthropod and pathogen surveillance operations and in lure-and-kill approaches to control target 
pest populations. These baited traps mimic the olfactory cues produced by a vertebrate host and are frequently partnered with attractive visual or thermal cues depending on the target species. The use of generalist semiochemical attractants such as carbon dioxide $\left(\mathrm{CO}_{2}\right)$, produced in the breath of vertebrates, may increase the number and species diversity of specimens collected (e.g. Carestia and Savage 1967). Where one arthropod species needs to be more effectively targeted, $\mathrm{CO}_{2}$ may be combined or replaced with other host-specific semiochemicals. For example, the addition of the cattle-derived compound 1-octen-3-ol (henceforth, octenol) attracts mammalfeeding species across arthropod groups including the Culicidae, Ceratopogonidae and Simuliidae (Atwood and Meisch 1993; Burkett et al. 2001; Mands et al. 2004). Considerable evidence has shown that many semiochemicals work synergistically or additively with $\mathrm{CO}_{2}$ (e.g. Van Loon et al. 2015) to produce an attractive effect; this has led to many options for the delivery of $\mathrm{CO}_{2}$ in remote field situations where traditional gas canisters pose a logistical challenge. These include dry ice (Newhouse and Chamberlain 1966), and, usefully, the fermentation of yeast using refined sugar sources (Smallegange et al. 2010) or molasses (Mweresa et al. 2014). The compound 2-butanone has also been used as a replacement for $\mathrm{CO}_{2}$ in the field (Homan et al. 2016), as it has been shown to produce similar electrophysiological responses to $\mathrm{CO}_{2}$ in laboratory assays (Turner et al. 2011).

In recent years, blends of multiple semiochemicals at defined concentrations have been optimised to more effectively collect a targeted range of species. Indeed, recent success with semiochemical blends is such that some can attract the target species in greater numbers than the complete odour of the whole-host, as demonstrated for the collection of the major malaria vector Anopheles gambiae Giles s.s. (Okumu et al. 2010; Qiu et al. 2007). This high specificity and collection efficiency provides multiple practical advantages: (1) limited resources (time, effort and finances) for surveillance are maximised by avoiding the need to sort through large volumes of non-target specimens, essential when a rapid answer to a sampling question is required; (2) this precludes the need for further costly and time-consuming identifications of other kairomones from a given host; and (3) a trapping system that can collect large volumes of a pest species even in the presence of the real host offers exciting prospects for reducing nuisance biting and pathogen transmission to the host by the target arthropod.

Some mosquito arbovirus surveillance studies have combined the attractive effect of host kairomone-baited traps with a sugar source. Collected mosquitoes are allowed to feed on sugarcoated substrates such as simple cotton pads or Whatman ${ }^{\circledR}$ FTA $^{\oplus}$ cards. These substrates can then be tested for the presence of target virus nucleic acid. This approach has successfully identified the presence of several viruses including Ross River, Barmah Forest and Chikungunya viruses as part of surveillance activities (Girod et al. 2016; Hall-Mendelin et al. 2010).

\section{Host-derived repellents}

Repellents are defined as chemicals that result in the movement of a host-seeking arthropod away from its source (Dethier et al. 1960). There are several potential modes of action of repellents, and for some repellents, although their mode of action is not fully understood, these are likely to involve at least three pathways. These include interference with the ability of the olfactory neurones to detect semiochemicals (Ditzen et al. 2008), direct detection of the repellent by olfactory receptor neurones (Stanczyk et al. 2010; Syed and Leal 2008; Xu et al. 2014) and inhibition of P450 enzymes (Jaramillo Ramirez et al. 2012).

Whilst host-derived volatile semiochemical attractants are important in enabling pest arthropods to locate their hosts, vertebrates also produce semiochemicals that repel. Novel repellent 
compounds have been isolated from a diverse range of vertebrates including humans, livestock, amphibians and fish (Bailey et al. 2006; Bett et al. 2015; Birkett et al. 2004; Creton et al. 2016; Logan et al. 2008, 2009; Menger et al. 2014; Williams et al. 2006). Thus, vertebrate hosts have the potential to provide an extensive source of novel repellent compounds that could be an essential addition to the synthetic repellents currently available. The importance of developing new repellents is highlighted by the finding that repeated exposure of mosquitoes to the widely used synthetic compound DEET (N,N-diethyl-3-methylbenzamide) reduces their sensitivity to it (Stanczyk et al. 2013). Spatial repellents are the most appealing for development. These repellents, some of which have been identified from human hosts (Logan et al. 2008), work by the release of volatile compounds into the air which disrupt arthropod behaviour at a distance from the host, and provide promise as a means for creating arthropod-free 'zones' in which hosts are protected from biting (Achee et al. 2012; Ogoma et al. 2014). Because of their volatility, they require formulation to maintain longevity of efficacy.

\section{Approaches to repellent use}

There are multiple ways in which repellents, synthetic or host-derived, can be deployed to prevent biting of animals. These include: (1) direct application of the repellent onto the animal; (2) impregnating wearable items such as collars with repellent; (3) impregnating screening materials with repellent; (4) deploying the repellent directly to resting areas for pest species; and (5) feed-through approaches. Direct-application repellents are one of the most commonly used approaches for arthropod bite prevention by humans, however their large-scale use with animals presents considerable logistical challenges. These formulations must first and foremost be safe for extended skin/hide exposure, and must be easy to apply (for example as a pour-on). However, even with these criteria fulfilled, repeated reapplication of the repellent is unlikely to be feasible for large numbers of animals in a commercial setting. Impregnating items such as collars and turnout rugs for horses with repellent is an alternative approach, although once again is not feasible for large numbers of animals. Screening animal housing with repellent-treated netting may offer protection for a larger number of housed livestock or poultry. This approach has been investigated for protecting stabled horses from indoor-biting Culicoides species (Page et al. 2009). At a larger scale, repellent-treated netting has been investigated as a means to protect a given outdoor area from biting activity (Schreck and Kline 1983). Application of a repellent formulation to areas where pest species rest can also offer protection to livestock. For example, wax beads impregnated with catnip oil and spread directly onto soil and manure areas in cattle feedlots resulted in a $99 \%$ repellency of stable flies in treated areas compared with untreated areas, for up to three hours (Zhu et al. 2010).

Feed-through approaches offer exciting prospects for arthropod control and repellent in commercially-important animals. These approaches involve the inclusion of specific compounds in the diet of the target animal. Current approaches are insecticidal rather than repellent, whereby compounds such as benzamides, insect growth regulators or avermectins are included with the feed, remain active in the faeces of the animal, and inhibit the growth and development of arthropods which use dung as an oviposition substrate (Axtell and Edwards 1983; Miller and Corley 1980; Miller et al. 1980). This method can therefore effectively target stable flies, horn flies and house flies (Stomoxys spp., Haematobia spp. and Musca spp.). Furthermore, the use of feed-through insecticides in wild rodent populations may also be effective against sand flies (Phlebotomus spp.) developing inside rodent burrows (Mascari et al. 2007, 2008, 2012). Feed-through repellents are in contrast far less explored; this approach involves the inclusion of compounds that, instead of exerting a killing effect, would work to alter the attractiveness of the host to a biting arthropod, 
for example through the production of natural repellent compounds. At present however, there is little published research into the use of feed-through repellents. Salmon (Salmo salar L.) fed a diet containing natural essential oils showed reduced sea lice (Lepeophtheirus salmonis Krøyer) infestation relative to the control (Jensen et al. 2014), although the mechanism responsible for this reduction was not clear. Limited evidence indicates that the inclusion of some plant essential oils in the diet of ruminants can alter the animals' bacterial flora and the activity of certain enzymes (see Greathead (2003) for review), although any effect on semiochemical profile has not been investigated.

\section{Key considerations for repellents}

As is the case with attractants, repellent compounds may show either a broad or narrow range of specificity against pest species. It is, therefore, important to test newly identified host-derived repellents for their effect against the range of relevant pests associated with a given habitat before deployment, to ensure the correct product is utilised. The importance of this has been shown for synthetic and plant-derived repellents. For example, DEET has a proven repellent effect against a broad range of species groups including mosquitoes, Culicoides biting midges, phlebotomine sand flies and black flies (e.g. Coleman et al. 1993; Tawatsin et al. 2006; Trigg 1996). In contrast, field trials showed that an oil of lemon Eucalyptus-based repellent (the active ingredient is p-menthane-3,8diol), repellent against mosquitoes, increased the number of Culicoides imicola Kieffer collected in artificial traps relative to the DEET control trap (Braverman et al. 1999). This demonstrates that repellents active against one species may have an unwanted attractive effect on other species.

A key consideration is the period of time over which a repellent remains effective before reapplication is required (the protection time). Formulations that rapidly lose their efficacy and require frequent reapplication are unlikely to be logistically or economically viable as management solutions. The inherent volatility of host-derived compounds presents a challenge for sustained release as after application they can be rapidly lost through evaporation. Higher concentrations of the active compound in the formulation are commonly used to increase protection time, whilst alternative strategies aim to control the release rate of the active compound. This can be achieved through microencapsulation of the active compound or by including other compounds into the formulation to reduce evaporation rates. Microencapsulation works by coating the repellent molecules with a film to produce small capsules. The resulting formulation results in a significant reduction in evaporative loss of the repellent whilst maintaining a consistent release rate (Solomon et al. 2012), although environmental conditions can influence the release rate depending on the capsule structure and material used (Zhu et al. 2014). Compounds trialled as repellent additives include synthetic musks such as vanillin (4-hydroxy-3-methyoxy-benzaldehyde): a 1:3 ratio of DEET to vanillin increased the protection time against Aedes aegypti Linnaeus by $176 \%$ (Khan et al. 1975). More recently, the in silico rational design and synthesis of novel synthetic repellents based on structures exhibiting lower volatility provide a promising approach in the search for new long-lasting repellents (lovinella et al. 2014). At present, most repellents are designed for human skin. More studies are needed to measure efficacy and to develop better, long-lasting formulations that can be applied onto animals.

\section{Host-specific differences in semiochemical profile}

The phenomenon that certain hosts are fed upon or parasitized at a greater rate than others is a widely-observed phenomenon. These differences occur both between individuals of the same species (intraspecific variation) and between different host species (interspecific variation). The 
reasons underlying these differences in host preference from the perspective of the arthropod remain to be fully elucidated but appear to be an interplay between intrinsic (genetic), extrinsic (environmental) and ecological (e.g. host availability within the community) factors that vary according to setting (Chaves et al. 2010; Lyimo and Ferguson 2009; McBride et al. 2014). Nonetheless, the role of semiochemicals in mediating differential host attraction has been confirmed for a variety of pest groups including mosquitoes (Logan et al. 2008; Qiu et al. 2006; Syed and Leal 2009; Verhulst et al. 2011), Culicoides biting midges (Logan et al. 2009), black flies (Schofield and Sutcliffe 1996; Young et al. 2015), tsetse flies (Torr et al. 2006), stable flies and horn flies (Birkett et al. 2004; Torr et al. 2006). It is worth noting, however, that the relative importance of olfactory, visual and other host cues, in tandem with the impacts of host defensive behaviour (Darbro and Harrington 2007; Mullens et al. 2006; Torr and Mangwiro 2000) varies considerably between pest groups.

Each host produces a large number of different volatile semiochemicals. For example, over one thousand volatiles from the human body have been identified (De Lacy Costello et al. 2014). In a recent study, Young et al. (2015) identified a total of 1261 volatiles from three humans but only $29(2.3 \%)$ were common to all three. Further complication is added when considering the role of the physiological and disease status of a host. For example, traps baited with the aged urine of a pregnant mare collected 6.4 times more horse flies than unbaited traps, and more than traps baited with the aged urine from a stallion, gelding or mare that was not pregnant (Krčmar and Lajos 2011). Pregnant women have similarly been shown to be more attractive to the major malaria vector Anopheles gambiae s.l. (Lindsay et al. 2000). Mice infected with Plasmodium chabaudii attract more Anopheles stephensi Liston when infectious (De Moraes et al. 2014), cattle infected with trypanosomes attract more tsetse than uninfected cattle (Baylis and Mbwabi 1995; Baylis and Nambiro 1993), dogs displaying clinical signs of infection with Leishmania infantum (presenting as visceral leishmaniasis) emitted a distinct volatile profile to infected dogs without clinical symptoms and healthy dogs (Magalhães-Junior et al. 2014), and children harbouring the gametocyte stage of Plasmodium falciparum (the stage transmissible to mosquitoes) were more attractive to An. gambiae s.s. than both uninfected children and those with the non-transmissible parasite stages (Lacroix et al. 2005). Furthermore, birds infected with avian malaria (P. relictum) attract more host-seeking Culex pipiens s.l. mosquitoes than those that are uninfected (Cornet et al. 2013a, 2013b). During preening, birds spread antimicrobial secretions from the uropygial gland over their bodies (Martín-Vivaldi et al. 2010). House sparrows (Passer domesticus L.) with low avian malaria parasite loads show a greater antimicrobial activity in uropygial gland secretions than individuals with higher parasite loads (Magallanes et al. 2016). Although uropygial gland secretions have been identified as attractants for several arthropods including black flies (Fallis and Smith 1964) and Culex mosquitoes (Russell and Hunter 2005), evidence for this attractive effect is equivocal (Martínez-de la Puente et al. 2011). A greater antimicrobial activity in these secretions has been suggested to impact the bacterial composition of feathers and skin (Magallanes et al. 2016). An altered bacterial composition could result in a reduced production of olfactory stimuli, attracting fewer biting arthropods and thus providing an evolutionary advantage to these individual birds.

The attractive and repellent properties of semiochemicals can be harnessed in an integrated push-pull strategy (also known as a stimulo-deterrent diversionary strategy) to pest control (Agelopoulos et al. 1999; Cook et al. 2007). This method utilises repellents to exert a 'push' effect away from the host in concert with attractants that draw the pests into a trap where they would be removed from the population (lure-and-kill traps). This approach has proven success in controlling 
a range of agricultural pests but at present remains far less explored for haematophagous pest arthropods.

The involvement of host genetics in the susceptibility of livestock to various diseases and ectoparasite infestations has been established (see Bishop and Morris (2007) for a review). Additionally, a recent twin study comparing the responses of Ae. aegypti to the odours of monozygotic and dizygotic twins provides compelling evidence for heritability in human attractiveness to mosquitoes (Fernández-Grandon et al. 2015). Confirmation of the involvement of semiochemicals in influencing the attractiveness of cattle to the horn fly Haematobia irritans L. (Birkett et al. 2004; Jensen et al. 2004) has led to the novel use of sniffer dogs to detect sheep susceptible to fly strike (caused by infestation with blow flies e.g. of the genus Lucilia) (Greeff et al. 2013). Dogs were trained to sniff wool samples from 10 susceptible and 10 resistant Merino ewes (as assessed by the occurrence of fly strike) and could identify resistant animals with an accuracy of $82 \%$. This provides important prospects for the selective breeding of resistant sheep, as well as other livestock, based on their semiochemical profile.

\section{Semiochemicals involved in sugar feeding}

The consumption of carbohydrate in the form of sugar is an essential dietary component for many pest arthropods. Sugar feeding has been widely observed in the Culicidae (Foster 1995), Ceratopogonidae (Kaufmann et al. 2015), Phlebotominae and Simuliidae (Lewis and Domoney 1966), with more limited evidence for the occurrence of natural sugar feeding in the Tabanidae (Magnarelli and Anderson 1981), Muscidae (Mohr et al. 2011; Taylor and Berkebile 2008) and Glossinidae (Solano et al. 2015). The nectar in flowering plants provides one of the most readily available sources of sugar, whilst other sources such as fermenting fruit may also be utilised (Foster 1995; Joseph 1970; Müller et al. 2011b).

Plants produce a wide range of semiochemicals (phytochemicals) which mediate plant-arthropod interactions including defence against predation and the attraction of sugar-feeding pollinators (Knudsen et al. 1993; Pare and Tumlinson 1999; Pichersky and Gershenzon 2002). Attractive plant semiochemicals induce physiological and behavioural responses in arthropods in a similar manner to that of host-derived kairomones, to facilitate the location of sugar sources (Jhumur et al. 2006, 2008). Plants produce unique emission profiles that display both interspecific and intraspecific variation that may be influenced by factors such as geography (Knudsen 2002). These will, in turn, influence the arthropods with which plants will interact. Identifying the semiochemicals involved in mediating these interactions may enable their use as lures to collect pest arthropods for surveillance and control.

A commonly used phytochemical is phenyl acetaldehyde, produced by plants of the genus Silene and which is attractive to several mosquito species (Jhumur et al. 2006, 2008). Baiting an artificial trap with phenyl acetaldehyde and allowing mosquitoes to feed on a sugar-soaked substrate resulted in the detection of West Nile virus (Flaviviridae: Flavivirus) circulation in the USA (Lothrop et al. 2012). This approach relies on the expectoration of arthropod-borne viruses in the saliva of mosquitoes during sugar feeding, in a similar manner to the detection of arboviruses in sugarpads fed upon by mosquitoes attracted by host-derived kairomones, as described above.

The deployment of attractive toxic sugar baits (ATSBs) currently shows promise as a lure-and-kill approach, attracting sugar-feeding arthropods to feed on a sugar source laced with an insecticide or toxin such as spinosad or boric acid. This approach has been commonly used against non- 
haematophagous pests of livestock such as the house fly (Musca domestica L.) (e.g. Butler et al. 2007) but has more recently been adapted for haematophagous species groups. The ATSB may either be sprayed directly onto vegetation or offered as part of a 'bait station', the latter of which requires an attractant such as phenyl acetaldehyde. ATSB-mediated population reduction has been achieved in field trials targeting several important mosquito genera in diverse ecological settings (e.g. Khallaayoune et al. 2013; Müller and Schlein 2008; Müller et al. 2008, 2010a, 2010c; Stewart et al. 2013) and there is emerging evidence for their potential use in the control of phlebotomine sand flies (Müller and Schlein 2011; Qualls et al. 2015; Schlein and Müller 2010) and Culicoides (Snyder et al. 2016).

Environmental acceptability is an important consideration in the use of ATSBs (Khallaayoune et al. 2013; Revay et al. 2014). The consumption of toxic sugar sources by non-target organisms, such as pollinators, may have a detrimental effect on the ecosystem. Approaches to reducing feeding on the toxic sugar sources by non-target organisms include: (1) the installation of a grate of defined size over the sugar source, which will prevent larger sugar feeders such as honey bees from accessing the sugar; (2) application of free ATSB to non-flowering vegetation rather than flowering species to minimise feeding by non-target organisms attracted to the flowering plants (Revay et al. 2014); and (3) avoiding incorporating visual cues utilised by many non-target pollinators in ATSB bait stations. Future work to identify the semiochemicals involved in the location of sugar sources in pest arthropods would provide even greater specificity for bait station setups.

\section{Oviposition cues}

The selection of oviposition or larviposition sites of optimal quality will determine the survival chances of the next generation and is therefore of particular importance to pest arthropods as the majority do not exhibit brood care (McCall and Cameron 1995). The selection of oviposition sites is governed by a variety of visual, olfactory and tactile cues (McCall 2002), with thermal cues from the host an additional consideration when considering species such as blow flies and bot flies which lay eggs or larvae directly onto the host (Hall et al. 1995). Semiochemicals have been identified that are involved in the regulation of oviposition/larviposition site selection in both aquatic (mosquitoes and black flies) and terrestrial (Phlebotomine sand flies, tsetse flies and stable flies) pest groups (Seenivasagan and Vijayaraghavan 2010).

There are several different sources for oviposition/larviposition semiochemicals. These include habitat-associated odours, odours produced by other organisms within the habitat and those produced by conspecific eggs and larvae. For species larvipositing directly into the vertebrate host, host-derived kairomones are key both for the selection of suitable hosts and for the selection of specific regions of the host. For example, deer bot flies use host odours including $\mathrm{CO}_{2}$ and octenol to locate their hosts before selectively larvipositing into the nostrils (Cephenemyia jellisoni Townsend) or lips and lower muzzle (Cephenemyia apicata Bennett \& Sabrosky) of deer (Anderson 2001).

Habitat-associated odours are a complex mixture of compounds which may exert their effect over a short or long range and either attract or repel different species depending on their concentration (McCall 2002). Oviposition semiochemicals have been studied in particular detail for mosquitoes: Culex species, for example, show a preference for ovipositing in aquatic habitats containing high levels of organic matter (Allan et al. 2005) whilst the soil-associated compound cedrol has been shown to attract gravid Anopheles species (Killeen et al. 2014). Environmental microorganisms are considered primary sources of these habitat-associated volatiles. Bacteria (Beehler et al. 1994; 
Ponnusamy et al. 2008), fungi (Geetha et al. 2003) and algae (Torres-Estrada et al. 2007) have been associated with oviposition site selection in several mosquito genera, whilst the bacterial composition of animal faeces influences the oviposition of stable flies (Stomoxys calcitrans L.) (Jeanbourquin and Guerin 2007b; Romero et al. 2006) and the sandfly Lutzomyia longipalpis Lutz and Neiva (Dougherty et al. 1995). Habitat-associated semiochemicals may also indicate the unsuitability of a habitat due to the presence of parasites or predators. For example, the presence of predatory Notonecta species has been shown to repel oviposition by Culex spp. even after the removal of the predators from the water (Blaustein et al. 2004, 2005) and exposure to the odours of damaged conspecifics reduces activity levels of Culex pipiens and An. gambiae ( $\mathrm{M}$ form) larvae, a response which reduces detectability to predators (Gimonneau et al. 2010; Sih 1986).

The eggs of Culex mosquitoes produce an apical droplet containing the compound erythro-6acetoxy-5-hexadecanolide (Laurence and Pickett 1982, 1985). Habitats containing this compound are preferentially chosen for oviposition by Culex pipiens form molestus Forskal, Culex tarsalis Coquillett, and Culex quinquefasciatus Say (Bruno and Laurence 1979). Similarly, the accessory gland of the eggs of L. longipalpis produce dodecanoic acid (Dougherty and Hamilton 1997; Dougherty et al. 1994), which, together with the compounds hexenal and 2-methyl-2-butanol isolated from rabbit faeces, attract ovipositing females (Dougherty et al. 1993). Female Simulium damnosum Theobold s.l. black flies will preferentially oviposit on substrates in lotic habitats where other eggs have recently been laid, mediated by pheromones produced by the eggs during development (McCall 1995; McCall et al. 1994, 1997a).

Larval pheromones produced by mosquitoes can also indicate the suitability of a habitat for oviposition (Sumba et al. 2008). The compound heneicosane, for example, acts as a dosedependent oviposition attractant for Ae. aegypti (Seenivasagan et al. 2009). Exudates from larval tsetse flies include the compounds n-pentadecane (in Glossina morsitans morsitans Westwood) and $\mathrm{n}$-dodecane (in Glossina morsitans centralis Machado) and mediate aggregative larviposition (Leonard and Saini 1993; Saini et al. 1996).

The involvement of semiochemicals in the selection of sites for oviposition/larviposition has provided an extensive array of tools for the collection of pest arthropods. Oviposition attractants can be used to bait artificial traps and will selectively target the gravid segment of the arthropod population. Traps baited with oviposition attractants can be used in an integrated approach together with traps targeting the host-seeking segment of the population to increase the fraction of the arthropod population collected, or alone in lure-and-kill approaches to reduce pest populations. Selective collection of the gravid population is useful when conducting surveillance for vector-borne pathogens. This is because the gravid females of haematophagous arthropods (except those individuals with egg batches produced autogenously, such as $C x$. pipiens form molestus) will have necessarily taken a blood meal prior to oviposition and therefore have a higher chance of exposure to the target pathogen. Other compounds may be used to deter pest arthropods from ovipositing. For example, application of catnip oil-impregnated wax beads was found to reduce stable fly oviposition by up to $98 \%$ depending on the concentration used (Zhu et al. 2014).

\section{Semiochemicals in the surveillance and control of key arthropod livestock pests}

The first section of this chapter has highlighted the importance of semiochemicals in regulating the interaction of pest arthropods with their hosts and environment, and the ways in which these interactions can be exploited for surveillance and control by attracting or repelling. This section 
details semiochemical-based approaches that have been successfully exploited for surveillance and control of major groups of livestock pest arthropods including tsetse flies, mosquitoes, biting midges, sand flies, black flies, horse flies, horn and stable flies and ticks. For those pest groups for which few examples of practical success currently exist, promising developments in the exploitation of semiochemicals are described.

\section{Tsetse flies (Diptera: Glossinidae)}

Tsetse flies (Glossina spp.) are haematophagous biting flies that are important vectors of the protozoan parasite Trypanosoma spp. in sub-Saharan Africa, responsible for human African trypanosomosis (sleeping sickness), and the counterpart cattle disease, animal African trypanosomosis (nagana) (Kabayo 2002). The resulting medical and veterinary impact and economic repercussions for cattle production make the control of tsetse a priority in the region.

Tsetse reproduction occurs by adenotrophic viviparity, in which a single fertilised egg hatches and undergoes three developmental instars within the female before being larviposited onto the ground (Tobe and Langley 1978). The larva immediately burrows into the soil where it pupates and later emerges as an adult, approximately 30 days after initial fertilisation of the egg. The result of this unusual nutritional and time investment in reproduction is a relatively low reproduction rate in comparison with other blood-feeding Dipterans, and therefore interventions that remove just $3 \%$ of adult females per day are able to eliminate the population (Hargrove 1988). Tsetse are obligately haematophagous and both sexes need to take regular blood meals (approximately every three days). Together with the unavailability of the immature stages as targets for control (eggs and larvae develop inside the female, pupae develop underground), interventions exploiting host-seeking and feeding behaviour are recognised as the principal means of population control.

Tsetse species are broadly classified into three groups: (1) the Morsitans group, associated with savannah habitats and of principle importance in the epidemiology of nagana; (2) the Palpalis group associated with riverine habitats and of key importance in the epidemiology of human trypanosomosis; and (3) the lesser-studied Fusca group associated with densely forested regions of central Africa (Torr and Solano 2010). Whilst members of all groups use visual cues such as colour and movement to locate their hosts, the relative importance of olfactory cues varies both within and between species groups (see Torr and Solano (2010) for a comprehensive review). Broadly speaking, current evidence indicates that host-derived kairomones are of greatest importance in the host-seeking behaviour of the Morsitans group.

Carbon dioxide is the key olfactory cue for tsetse: it is the sole semiochemical involved in the initial activation of host-seeking activity, and is required to initiate long-range upwind flight activity towards a host (Bursell 1983; Torr 1988). Other host-derived kairomones such as octenol, acetone, butanone and the phenols 4-methylphenol and 3-propylphenol are produced by the large mammalian ungulate hosts of the Morsitans group such as the ox (Bursell et al. 1988; Hassanali et al. 1986; Torr et al. 1995; Vale 1980; Vale and Hall 1985). These compounds work in a dose-dependent manner in synergy with $\mathrm{CO}_{2}$ to aid in long-range host-seeking responses (Torr 1990; Torr and Mangwiro 1996; Vale and Hall 1985). However, traps baited with synthetic blends of these compounds collect significantly fewer tsetse than those baited with whole host odour. This indicates the role of other, unidentified kairomones (Hargrove et al. 1995; Torr et al. 1995, 2006). Landing behaviour onto the host is mainly influenced by visual cues, however, $\mathrm{CO}_{2}$ together with as yet unidentified skin odours also plays a role (Torr 1989; Vale and Hall 1985; Warnes 1995). 
The first successful attempts to control tsetse were targeted towards an experimentally introduced population of G. m. morsitans and Glossina pallidipes Austen on Antelope island in Lake Karima, Zimbabwe in the 1980s (Vale et al. 1986). Six beta traps (which killed or sterilised captured insects) baited with $\mathrm{CO}_{2}$ and acetone were initially deployed across the $4.5 \mathrm{~km}^{2}$ island for two years, resulting in a population reduction of 90 and $99 \%$ of G. m. morsitans and G. pallidipes, respectively. In the following year, the beta traps were replaced with 20 black cloth target traps baited with acetone and octenol and treated with the insecticides dieldrin or deltamethrin. These traps eliminated the remaining populations of both species in 11 weeks (G. pallidipes) and nine months (G. m. morsitans) (Vale et al. 1986). The success of this trial was followed by a larger-scale deployment of deltamethrin-treated black cloth targets baited with octenol and acetone and placed at a density of 4 per $\mathrm{km}^{2}$ over a $1000 \mathrm{~km}^{2}$ area of the Zambezi valley in Zimbabwe (Vale et al. 1988). Eight months of trapping induced an estimated $99.99 \%$ reduction of the tsetse population in the area. This trapping approach showed similar success in Zambia and, over the course of one year, G. m. centralis was eliminated from a large $\left(>500 \mathrm{~km}^{2}\right)$ area of western Zambia using these traps alone (Willemse 1991). Subsequent optimisation of the odour bait led to the incorporation of attractive phenols into a standardised lure containing acetone $(\sim 100 \mathrm{mg} / \mathrm{h}$ release rate), octenol ( 0.5 mg/h), 4-methylphenol ( 1 mg/h) and 3-propylphenol ( 0.1 mg/h) (Torr et al. 1997).

Host kairomones have been shown to play some role in the host-seeking behaviour of members of the Palpapis group. The addition of acetone and octenol to artificial traps was shown to double catches of Glossina palpalis palpalis Robineau-Desvoidy (Cheke and Garms 1988). More recently, a synthetic blend of 3-propylphenol, octenol, 4-methylphenol and acetone (the 'POCA' blend) enhanced trap collections of Glossina palpalis gambiensis Vanderplank, and the addition of the POCA blend or synthetic cattle odour (acetone, 1-octen-3-ol, 4-methylphenol and 3-n-propylphenol with $\mathrm{CO}_{2}$ ) to artificial traps collected significantly more Glossina tachinoides Westwood than natural cattle odour alone (Rayaisse et al. 2010). The recognition that the blood meal hosts of the Palpalis group vary more widely than those of the Morsitans group is likely to play an important role in the relative importance of different host-derived kairomones. Therefore, the identification of novel compounds from less-studied but highly utilised blood meal hosts such as reptiles (Clausen et al. 1998; Küpper et al. 1990; Späth 2000) may offer new compounds to enhance the attractiveness of existing lures to riverine species (Rayaisse et al. 2010).

Odour-baited traps for tsetse continue to play an important role as a population monitoring tool and as a part of integrated control for tsetse, for example in the creation of barriers to the recolonization of tsetse-free sites from adjacent intervention-free areas (Meyer et al. 2016; Torr and Solano 2010; Vreysen et al. 2013). Other components of integrated control include aerial spraying of aerosolised deltamethrin (Adam et al. 2013), insecticide-treated cattle (Gimonneau et al. 2016; Ndeledje et al. 2013) (this offers the additional advantage of the insecticide killing ticks thus enhancing acceptability to farmers; Eisler et al. 2003) and the sterile insect technique (Vreysen et al. 2000, 2014).

\section{Mosquitoes (Diptera: Culicidae)}

Mosquitoes are a group of important nuisance biting insects many of which also serve as vectors for a vast number of pathogens of humans and veterinary importance worldwide. These pathogens include viruses, parasites and bacteria (Clements 2012). Mosquito-borne pathogens of particular veterinary importance include Rift Valley fever virus (RVFV) (Bunyaviridae: Phlebovirus) which can result in severe disease and abortion in livestock as well as zoonotic infection in humans when heavy rainfall provides optimum conditions for Aedes and Culex vector species (Davies et al. 
1985) and West Nile virus, maintained in bird-mosquito-bird enzootic cycles (Work et al. 1955) but which infects a wide range of economically important incidental vertebrate hosts such as horses (Autorino et al. 2002) and farmed alligators (Miller et al. 2003). The appearance and rapid spread of the West Nile virus in North America (Hayes et al. 2005) and the widespread emergence of the zika virus (Fauci and Morens 2016) highlights the need for developing novel, effective methods of mosquito control.

The olfactory regulation of mosquito behaviour has been the focus of considerable research effort and has formed the basis of much of the available knowledge of semiochemical interactions between haematophagous arthropods, their hosts and the environment. Several reviews have highlighted the importance of semiochemicals in the host-seeking (Cardé and Gibson 2010), oviposition (Navarro-Silva et al. 2009) and sugar-feeding (Nyasembe and Torto 2014) behaviour of mosquitoes and how they offer new routes for control. The literature on semiochemical regulation of mosquito-host interactions shows considerable bias towards human-biting mosquito species that vector pathogens of humans. Nonetheless, the role of livestock and other animals as intermediate or amplifying hosts for many pathogens is clear. Therefore, continued research to increase our understanding of the semiochemical basis for the interactions between mosquitoes and non-human hosts remains paramount.

\section{Host-derived kairomones as mosquito attractants}

The role of $\mathrm{CO}_{2}$ in the activation and long-range anemotaxic behaviour of host-seeking mosquitoes is well established in laboratory and field experiments, with the addition of $\mathrm{CO}_{2}$ to artificial traps generally used to increase the species diversity and abundance of mosquitoes in collections (Carestia and Savage 1967; Jawara et al. 2009; Qiu et al. 2007; Smallegange et al. 2010). A large number of other host-derived attractive compounds including octenol, ammonia and fatty acids have been identified as being attractive to mosquitoes (e.g. Bosch et al. 2000; Geier et al. 1999; Hall et al. 2011; Smallegange and Takken 2010; Smallegange et al. 2005, 2009). Some compounds such as octenol are common to several different host species but others, such as L-lactic acid, are produced only by certain hosts (Dekker et al. 2002), and differential attraction to these may reflect the intrinsic preference of mosquitoes to feed on certain host groups over others (Takken and Verhulst 2013). The choice of attractant is a key consideration when choosing traps to target important mosquito species groups for surveillance or control.

Many host-derived kairomones work most effectively as attractants in synergy or additively with each other and/or $\mathrm{CO}_{2}$ (Dekker et al. 2005; Geier and Boeckh 1999; Kline et al. 1990; Smallegange et al. 2005; Van Loon et al. 2015), although some chemical combinations may also show antagonistic effects (Logan et al. 2010b; Owino et al. 2015). Although the addition of $\mathrm{CO}_{2}$ and compounds such as octenol or lactic acid to artificial traps increase trap collections they generally do not match the attractive effect of a live host (e.g. Canyon and Hii 1997). This presents a challenge to the use of attractants to estimate natural biting rates and to their use in mass, or removal-trapping, schemes (Day and Sjogren 1994) when there are hosts present in the area.

To overcome this challenge for anthropophilic mosquito vectors, blends of different synthetic odours originally identified as being produced by humans have been developed as mosquito attractants. A blend of ammonia, lactic acid and 12 carboxylic acids was identified in laboratory assays to be highly attractive to An. gambiae s.s. (Smallegange et al. 2005). Subsequent semi-field studies demonstrated the attraction of An. gambiae s.s. to foot odour blends in Mosquito Magnet $\mathrm{X}(\mathrm{MMX})$ traps (American Biophysics Corp., North Kingstown, RI, USA) and this was followed by 
successful field studies demonstrating that a blend of the synthetic compounds ammonia, L-lactic acid, 3-methylbutanoic acid offered with $\mathrm{CO}_{2}$ in the MMX traps could be more attractive than a human bait to a range of Anopheles, Mansonia and Culex species (Qiu et al. 2007). The components of this blend underwent refinement and field testing (Okumu et al. 2010; Van Loon et al. 2015) before the standardised Mbita (MB5) blend was produced, consisting of ammonia (25\%); L-(+)lactic-acid (88-92\%); tetradecanoic acid (16\%); 3-methyl-1-butanol (0.01\%) and butan-1-amine (0.001\%) (Mukabana et al. 2012; Menger et al. 2014). This blend has been shown to be effective in collecting equal or greater numbers of the major malaria vectors An. gambiae s.s., Anopheles arabiensis Patton and Anopheles funestus Giles s.s. than human odour in the field (Busula et al. 2015). Placed in a solar-powered optimised version of the Suna trap (BioGents, Regensburg, Germany), this blend, without $\mathrm{CO}_{2}$ but including 2-butanone, was also used in a recent two-year trapping trial targeted at reducing malaria incidence on Rusinga island, Lake Victoria, Kenya (Homan et al. 2016; Hiscox et al. 2012, 2016). Traps were installed at a total of 4,358 households in a stepped-wedge, cluster-randomised design between 2013 and 2015, with clinical malaria the primary outcome and malaria prevalence in humans and Anopheles spp. densities as secondary outcomes. Over the course of the trial, the prevalence of clinical malaria was reduced by $29.8 \%$ and An. funestus densities were significantly lower in intervention clusters as compared to non-intervention clusters (Homan et al. 2016). Although the authors highlight that a low clinical incidence of malaria at the start of the trial made it difficult to estimate the efficacy of the intervention based on clinical incidence data, the reduction in Anopheline density shows that odour-baited traps may be beneficial as part of an integrated vector control program.

Whilst impressive progress has been made in optimising traps for vectors of human malaria, there is a need to identify semiochemicals involved in the attraction of mosquito species involved in the transmission of key livestock diseases such as RVFV. Floodwater Aedes spp. are the primary (maintenance) vectors of RVFV, with other genera, most importantly Culex, involved as secondary (amplifying) vectors (Pepin et al. 2010). Including odours collected from sheep skin with $\mathrm{CO}_{2}-$ baited CDC light traps increased catch rates of both primary and secondary vectors of RVFV as compared to the CDC trap with or without $\mathrm{CO}_{2}$ (Tchouassi et al. 2012). A subsequent study found that the addition of skin odours collected from a cow, donkey, goat or sheep to CDC light traps with the lightbulb removed each significantly increased the catch of Aedes mcintoshi Huang and Aedes ochraceus Theobald (Tchouassi et al. 2013), two species considered to be primary RVFV vectors in Kenya (Sang et al. 2010). This significant increase in catch was not observed by addition of human skin odours (Tchouassi et al. 2013). The same study then identified four volatile aldehydes common to all the mammalian hosts, heptanal, octanal, nonanal and decanal, which produced electrophysiological responses in several RVFV vectors. Blends of these compounds in CDC light traps without the light bulbs increased catches of RVFV vectors to a greater degree than addition of the compounds individually, with a 2:0.5:0.1:0.1 blend of heptanal, octanal, nonanal and decanal, used with $\mathrm{CO}_{2}$, showing the greatest performance by increasing catch rates of primary RVFV vectors by three-fold compared to the control. These results highlight the promise of using semiochemicals to improve capture rates of key livestock disease vectors, although further optimisation and testing will be required to assess whether this blend could be used in concerted efforts to reduce vector populations.

The commercially-available BG-lure, deployed in BG-Sentinel traps (Biogents) is also widely used in mosquito surveillance activities. The lure consists of a blend of lactic acid, ammonia and caproic (hexanoic) acid and is effective at targeting key invasive vectors including Ae. aegypti, Aedes albopictus Skuse and Aedes polynesiensis Marks (Farajollahi et al. 2009; Lühken et al. 2014; Macielde-Freitas et al. 2006; Schmaedick et al. 2008). The BG-Sentinel can collect Ae. aegypti (Kröckel et 
al. 2006) and, in modified form, Anopheles darlingi Root (Gama et al. 2013), in similar number to human landing catches. This shows promise as a replacement for the gold-standard of human landing catches, which has ethical considerations as a collection method. A recent field study in Kenya furthermore found a combination of $\mathrm{CO}_{2}$ and caproic acid alone collected 2.2-fold more Ae. aegypti than the $\mathrm{BG}$-lure blend plus $\mathrm{CO}_{2}$, adding complexity to the current understanding of how these lures function in the field (Owino et al. 2015). Live mice have also been used to bait BG-Sentinel traps for surveillance purposes, showing success in capturing both male and female Ae. albopictus (Lacroix et al. 2009; Le Goff et al. 2016) and indicating that mouse-derived volatiles could in future be used in improved bait formulations.

Semiochemicals associated with oviposition sites are widely used in arbovirus surveillance for mosquitoes. Gravid traps collect mosquitoes as they attempt to lay their eggs in a container of water (Reiter 1983). This approach is useful in arbovirus surveillance as it targets older segments of the population which will have taken a blood meal prior to collection and therefore may have been exposed to the pathogen of interest. Other simple ovitraps are designed to encourage mosquitoes to oviposit in them. These traps enable the collection of the eggs and larvae of mosquitoes, particularly invasive Aedes species (e.g. Hasty et al. 2015) although they are not effective for all species (Andreadis et al. 2001). These traps have also been used to determine the occurrence of transovarial (vertical) dengue virus transmission in wild mosquito populations (Lee and Rohani 2005; Le Goff et al. 2011). Various semiochemicals are used in these traps. For example, traps baited with infusions of dehydrated cashew leaves (Anacardium occidentale) can be used to attract ovipositing female Ae. aegypti at an equivalent rate to existing infusions (Santos et al. 2010). This compound has the additional benefit that it has a more pleasant smell than other commonly used compounds, and thus would be more acceptable for use in and around households.

\section{Lure-and-kill strategies for control}

Lure-and-kill strategies have shown promise for controlling populations of different mosquito species in areas where they pose a disease transmission or nuisance biting risk. These approaches utilise semiochemicals that regulate oviposition, host-seeking or sugar-feeding behaviour to attract adult mosquitoes into a trap where they are either killed by contact with an insecticide or killed by desiccation in adult traps. Additionally, emerging larvae that hatch in oviposition traps may be killed by larvicides including entomopathogens such as Bacillus thuringiensis var. israelensis (Bti) (e.g. Barbosa et al. 2010). Lethal ovitraps combine the standard baited ovitrap design with an insecticide to kill ovipositing females. This approach, using a $10 \%$ hay infusion-water solution has been extensively trialled to target Ae. aegypti as part of efforts to control the Dengue virus. Initial laboratory trials using a paper strip impregnated with deltamethrin in ovitraps achieved control of up to $98 \%$ (Zeichner and Perich 1999). This approach showed subsequent success in the field to control Ae. aegypti in Thailand (Sithiprasasna et al. 2003) and Brazil (Perich et al. 2003), although neither study eliminated the local mosquito population. Subsequent trials in Australia showed uncertain impacts of lethal ovitraps on Ae. aegypti (Rapley et al. 2009). More limited attempts have been targeted at other species groups: a $300 \mathrm{mg}$ dose of dried microcapsules containing 6-acetoxy-5-hexadecanolide, the compound present in the apical droplets of $C x$. quinquefasciatus, together with the insecticide tempehos, were able to attract and kill gravid $C x$. pipiens form molestus in the laboratory (Michaelakis et al. 2007).

Host-derived attractive kairomones have also been used in lure-and-kill mass trapping approaches to reduce mosquito populations, with variable success. Various types of the commercial Mosquito Magnet (MM) trap (American Biophysics Corp.) have been used with some success. This trap works 
on a counterflow system producing $\mathrm{CO}_{2}$ through the combustion of a gas such as propane whilst creating a suction force to collect attracted insects into an internal collection net. One control attempt used Mosquito Magnet Pro (MMP) traps at residential properties plagued by nuisance biting of the tree-hole species Ochlerotatus sierrensis Ludlow in Salt Lake City (Hougaard and Dickson 1999). Success was gauged by way of follow-up calls to local residents, who indicated that biting rates had decreased. Kline (2006) subsequently reported up to $90 \%$ reductions in the population of Ochlerotatus taeniorhynchus Wiedemann over three years through the use of multiple MMP traps baited with octenol on an island off the coast of Florida. Initial mosquito densities were so high that the MMP traps were modified to collect into $11.5 \mathrm{~L}$ barrels; after 16 months of use the standard collection net could again be used.

More recently, some success has been demonstrated with the use of BG-Sentinel traps for mass trapping. Running multiple BG-Sentinel traps baited with $\mathrm{BG}$-lure, without $\mathrm{CO}_{2}$, in three urban sites in Cesena, Italy, Englbrecht et al. (2015) resulted in between 64 and $87 \%$ fewer Ae. albopictus being collected by human landing catch as compared to control sites without the traps. The effectiveness of mass-trapping may be density-dependent and thus varies seasonally in nontemperate regions: Ae. aegypti populations targeted with BG-Sentinels in Manaus, Brazil were significantly reduced only in the wet, not the dry season (Degener et al. 2014) and Ae. aegypti in Cairns, Australia targeted with lethal ovitraps and BG-Sentinels showed significant population reduction only in the wet season (Rapley et al. 2009).

Although these studies show some evidence of successful mass-trapping, other attempts using baited traps including MMs (Smith et al. 2010) and MosquiTRAPs (Ecovec Ltda, Belo Horizonte, MG, Brazil) that use a grass infusion oviposition attractant with a sticky substrate for collection (Degener et al. 2015), have not been successful. A thorough understanding of the host-seeking and dispersal behaviour of local mosquitoes is essential when considering mass trapping approaches as this will (1) ensure the correct trap and lure combination is used for the target species; and (2) enable an understanding of whether on-going trapping may be required to maintain control in a given area due to immigration of mosquitoes from neighbouring non-intervention areas.

Lure-and-kill strategies employing ATSBs have also shown promise in field application for controlling a range of different mosquito species. The bulk of early work was conducted in Israel but has since been successfully deployed to other parts of the world to target a wide range of species. In Israel, ten-fold reductions in the populations of Anopheles claviger Meigen were effected using ATSB stations (Müller and Schlein 2008), direct application of ATSBs to vegetation reduced Culex pipiens s.l. populations to less than $7 \%$ of pre-treatment numbers (Müller et al. 2010d) and ATSB stations reduced populations of Anopheles sergentii Theobald and Aedes caspius Pallas to less than a tenth and a third of pre-treatment levels, respectively (Müller et al. 2008). ATSBs targeting malaria vectors in Africa have also shown success; for example, An. gambiae s.l. populations were reduced by $90 \%$ in Mali (Müller et al. 2010a) and An. sergentii populations were reduced by between 95.2 to $98.6 \%$, regardless of the presence of alternative sugar sources (Beier et al. 2012). Culex quinquefasciatus and Ae. aegypti populations in a field trial in Morocco were reduced by over $70 \%$ (Khallaayoune et al. 2013) and reductions in a variety of genera including Culex, Aedes, Uranotaenia, Culiseta were achieved in trials in Florida, USA (Qualls et al. 2014; Revay et al. 2014). The advantage of exploiting sugar feeding is that local fruits can be used as fermenting agents (Müller et al. 2010b) offering a cheap and easily accessible source for baiting traps and one that is likely to be specific to local mosquito species. 


\section{Culicoides biting midges (Diptera: Ceratopogonidae)}

Culicoides biting midges are the most important genus within the Ceratopogonidae for their role as nuisance biters of animals and humans and their status as vectors of important livestock pathogens including bluetongue (BTV), Schmallenberg and African horse sickness viruses (Mellor et al. 2000; Veronesi et al. 2013). Biting rates as high as 635 midges per minute on a human arm have been recorded for the highland midge, Culicoides impunctatus Goetghebuer (Carpenter et al. 2005) and the biting of Culicoides spp. can trigger the allergic condition sweet itch in horses (Mellor and McCaig 1974). Recent climatic changes such as warmer winters may be permitting greater overwintering survival rates of Palearctic Culicoides species, which may in turn facilitate the overwintering of viruses such as BTV (Meiswinkel et al. 2008). The small size of Culicoides may additionally enable them to be passively transported over long distances by the wind, a mechanism implicated in the range expansion of competent vector species such as $C$. imicola Kieffer across Europe (Jacquet et al. 2016; Purse et al. 2005).

Attempts to control Culicoides populations via the application of insecticides have proved unsuccessful (Kettle 1996) and current approaches to reducing biting rates involve short-term protection via the application of topical repellents and insecticides (Carpenter et al. 2008; Reeves et al. 2010), or the stabling or screening of livestock at peak biting times (Baker et al. 2015; Meiswinkel et al. 2000), the latter approach depending in large part on the local species primarily displaying exophagy (outdoor biting). Thus, an unmet need remains for the development of novel methods of control.

Temperature and humidity combined with visual and olfactory cues enable Culicoides to find and land on their hosts (Logan et al. 2010a). Most studies on the olfactory responses of Culicoides have utilised Culicoides nubeculosus Meigen due to the ease with which it can be colonised (Boorman 1974), or field populations of C. impunctatus due to the high densities encountered close to its natural habitats. C. impunctatus possess olfactory chemosensilla on the antennae and maxillary palps used to detect semiochemicals such as $\mathrm{CO}_{2}$ (Bhasin et al. 2000, 2001). The attraction of host-seeking Culicoides spp. to $\mathrm{CO}_{2}$ has been demonstrated in both laboratory assays (Bhasin et al. 2000) and in field collections where its inclusion into traps can increase the number of specimens collected (Bishop et al. 2008; Gerry et al. 2009; Harrup et al. 2012; Kline et al. 1994; Mullens and Gerry 1998; Ritchie et al. 1994). Octenol also attracts Culicoides through synergism with $\mathrm{CO}_{2}$ (Bishop et al. 2008; Blackwell et al. 1996; Kline et al. 1994; Ritchie et al. 1994) but this attractant effect does not apply to all species (Gerry et al. 2009; Kline et al. 1994). Furthermore, (R)1-octen-3-ol enantiomers were found to collect a greater number of Culicoides spp. in the United Kingdom than other lure combinations (Harrup et al. 2012), indicating a greater complexity with regards to the olfactory responses to key compounds. There is also evidence of synergism between octenol and an aggregation pheromone produced by parous female C. impunctatus (Blackwell et al. 1994, 1996). This pheromone causes the aggregation of other female $C$. impunctatus onto a blood-feeding host. The reasons for this remain unknown but are hypothesised to be related to reduced chances of individual mortality during blood-feeding or the signalling of a suitable host in an environment where hosts are infrequently encountered. When an extract of parous female C. impunctatus was added to traps together with octenol, an additive effect on catch rates was observed (Blackwell et al. 1996).

Synergistic attractive effects of acetone, butanone and lactic acid and cow urine with either $\mathrm{CO}_{2}$ and octenol have also been identified for C. impunctatus (Bhasin et al. 2000, 2001). Mands et al. (2004) used odour extracts from the hairs of five mammals and paired them with $\mathrm{CO}_{2}$ and octenol 
in a MMP trap. Samples from water buffalo produced the greatest dose-dependent increases in the catch rates of $C$. impunctatus and C. pulicaris L. in Scotland. Most recently, 23 volatile compounds were identified from the urine and hair of cattle, of which 17 were tested using GC-EAG and behavioural assays with $\mathrm{CO}_{2}$ using $C$. nubeculosus (Isberg et al. 2016). Dose-dependent behavioural effects were observed for sixteen of the compounds: seven elicited attraction in colony insects, five inhibited host-seeking activity relative to the control and four caused both attraction and inhibition. One of the inhibitory compounds was 6-methyl-5-hepten-2-one, previously identified as having a repellent effect to C. impunctatus (Logan et al. 2009), Ae. aegypti (Logan et al. 2008), An. gambiae s.s. (Menger et al. 2014; Qiu et al. 2011) and the horn fly, H. irritans (Jensen et al. 2004). Together with geranylacetone, this compound offers promise for use as an effective topical repellent (Logan et al. 2009).

Attempts to control populations of Culicoides via mass trapping with artificial semiochemicalbaited traps have shown some success in trials in the USA. Day et al. (2001) reported that trapping using insecticide-treated fabric targets baited with $\mathrm{CO}_{2}$ and octenol reduced populations of the coastal species $C$. furens Poey in Florida and on an island in the Bahamas. Subsequent attempts to reduce biting midge populations from residential properties using odour-baited traps showed variable success. ABC PRO suction traps (Clarke Mosquito Products Inc., Roselle, IL, USA) baited with carbon dioxide (500 $\mathrm{ml} / \mathrm{min}$ ) and a 4:1:8 mixture of octenol, 3-propylphenol, and 4-methylphenol $(5.39 \pm 0.54 \mathrm{mg} / \mathrm{h})$ reduced populations of five Culicoides species over the study period in the backyards of properties in north-western Florida, although the observed reduction was highly variable, ranging from 2.3 to $70.6 \%$ (Cilek et al. 2003). A similar experimental setup using MM traps (American Biophysics Corp.) resulted in population control of between 4.2 and $85.3 \%$ for the three species of Culicoides collected (Cilek and Hallmon 2005).

Taken together, there remains considerable work to be done to understand the semiochemicalmediated interactions of Culicoides species. For example, there is currently no available information on the semiochemicals involved in oviposition behaviour. However, the identification of novel repellent compounds and novel compounds that serve as attractants offer hope for the development of an effective push-pull control system to be implemented in the future.

\section{Phlebotomine sand flies (Diptera: Phlebotominae)}

Phlebotomine sand flies are haematophagous pests of significant medical and veterinary importance (Alexander and Maroli 2003). The blood-feeding behaviour of females of Phlebotomus spp. in the Old World and Lutzomyia spp. in the New World (World Health Organization 2010) have led to their key role as vectors for Leishmania spp. and their involvement in the transmission of several human pathogenic viruses (Depaquit et al. 2010) and the bacterium Bartonella bacilliformis which infects humans and domestic and wild animals (Breitschwerdt and Kordick 2000; Noguchi et al. 1929). Sand fly control is effected primarily by the application of insecticides although there is increasing interest in the development of novel semiochemical-based tools in light of the emergence of insecticide resistance and the continued importance of leishmaniasis in many parts of the world (Alexander and Maroli 2003; Alvar et al. 2012).

The majority of work on the chemical ecology of sand flies has been focused on L. longipalpis (see Bray et al. (2010b) for a recent review). Carbon dioxide acts as a dose-dependent attractive cue to enable both males and females to locate their human or animal hosts for mating and feeding, respectively (Pinto et al. 2001) and is regularly used to increase catch rates for sand fly surveillance, primarily in CDC light traps (Müller et al. 2011a). Other host-derived kairomones 
such as benzaldehyde, 4-hydroxy-4-methyl-2-pentanone and 4-methyl-2-pentanone, originally isolated from a fox (Vulpes vulpes L.), have been shown to play a role in host-seeking behaviour in laboratory assays (Dougherty et al. 1999), but their importance in the field is relatively poorly understood. Baiting sticky traps with octenol did not increase field collections of Phlebotomus argentipes Annandale \& Brunetti (Cameron et al. 1991).

Although oviposition pheromones have been identified which attract gravid females (Dougherty and Hamilton 1997), the greatest interest has been afforded to the use of ATSBs and the manipulation of the male sex pheromone in L. longipalpis. The use of ATSBs for sand fly control relies on the requirement of adults of both sexes to sugar feed. A mixture of fermented fruit juice and sugar shown previously to be attractive to mosquitoes (Müller and Schlein 2008) and containing the toxin boric acid, was either sprayed on vegetation, coated onto netting or presented in bait stations in Israel (Müller and Schlein 2011). Decreases of 95, 88 and $60 \%$ in the local population of Phlebotomus papatasi Scopoli relative to the controls were observed for the three respective treatments. Similar effects were obtained in Morocco by the presentation of ATSBs containing the toxin dinotefuran in bait stations or through direct spraying onto vegetation (Qualls et al. 2015). Local populations of P. papatasi and P. sergenti Parrot were reduced by 76.9 to 83.2\%. Importantly, non-toxic dye-marked applications showed a low uptake of the ATSBs by nontarget organisms was likely, indicating the species-specificity of this approach to sand fly control.

Male sand flies produce terpene sex pheromones from abdominal glands (Lane and Ward 1984). The addition of a synthetic version of the natural compound, ( \pm )-9-methylgermacrene- $B$, to CDC light traps resulted in significantly higher catches of $L$. longipalpis than unbaited control traps (Bray et al. 2009), although the use of this compound to enhance sticky trap collections collected more males but not females relative to unbaited controls (Bray et al. 2010a). Subsequent work incorporated this pheromone into a long-lasting delivery mechanism in experimental chicken sheds in Brazil and was attractive to female L. longipalpis for up to ten weeks and males for up to 12 weeks (Bray et al. 2014). Taken together with evidence showing that this pheromone can significantly increase the number of female sand flies attracted to insecticide-treated chicken sheds (Bray et al. 2010a), this indicates that optimised lure-and-kill approaches for sand flies could be an effective control measure in future.

\section{Black flies (Diptera: Simuliidae)}

The control of simulids has taken place primarily for the targeted elimination of human onchocerciasis caused by Onchocerca volvulus. An integrated approach combining the treatment of human infection using ivermectin (Amazigo and Boatin 2006) with larviciding of lotic habitats using either Bti serotype $\mathrm{H} 14$ or the organophosphate temephos has been effective at achieving elimination in some key areas (Traoré et al. 2009). Nevertheless, there remains a need for the effective collection of anthropophilic and zoophilic simulid species for pathogen surveillance and to monitor nuisance biting populations. Therefore, although several compounds associated with aggregative oviposition have been identified as highlighted previously, with some suggestion that these could be used in baited traps (McCall et al. 1994, 1997a, 1997b), recent focus has been on the semiochemicals involved in collecting host-seeking onchocerciasis vectors, with more limited research into topical repellents to reduce attack rates on livestock.

The bites of simulids can cause considerable skin irritation due to their salivary components, and in rare cases this may lead to fatal anaphylactic shock (simuliotoxicosis) in animals (Schnellbacher et al. 2012). Considerable losses to cattle milk and meat production can also result from simulid 
biting (Sarıözkan et al. 2014). The biting of horses by simulids may have significant veterinary and financial repercussions as horses become distressed and unrideable; particular distress may be caused by targeted feeding by simulids on the inner ear (Fallis 1964). At present, few repellents show proven field efficacy against simulid biting on horses. However, one recent trial using a synthetic blend of compounds originally isolated from the cheek gland secretions of viverrine mammals including the European badger Meles meles Linnée has shown some success for topical use (Creton et al. 2016). Applying $5 \mathrm{ml}$ of a 3\% slow-release formulation containing two compounds to the inner surface of the ear of horses achieved up to $98 \%$ protection from wild simulid biting over a ten-hour field study.

Several semiochemicals have been identified as providing attractive olfactory stimuli to the Simuliidae. Early trapping experiments showed that $\mathrm{CO}_{2}$ is an attractive host kairomone to simulids: its addition to sticky (Fallis et al. 1967), fan-based (Fallis and Raybould 1975) and silhouette traps (Sutcliffe et al. 1994) has been shown to increase the number of specimens collected. Other early studies implicated host odours as a source of attraction to simulids. For example, it was possible to attract the ornithophilic species Simulium euryadminiculum Davies from around a recentlydeceased carcass of its principal host, the common loon Gavia immer Brunnich (Lowther et al. 1964). Furthermore, the dried museum skins of the loon and the patch of ground where skin washings of the carcass had taken place also attracted S. euryadminiculum. The source of attraction was later identified as a product of the uropygial gland, secretions from which are spread over the feathers during preening (Fallis and Smith 1964); their attractive effect to simulids have been confirmed in more recent field experiments (Weinandt et al. 2012). Human sweat also serves as an attractant for S. damnosum when added to traps in the form of worn clothing, although the specific components responsible for attraction could not be isolated (Thompson 1976, 1977). Furthermore, whole-body cattle odour was shown to enhance the attraction of Simulium arcticum Malloch to silhouette traps (Sutcliffe et al. 1994) although octenol was found to only provide slight, non-significant, increases in catch rates for this species (Sutcliffe et al. 1994) and for other zoophilic species (Atwood and Meisch 1993). Recent behavioural work using Y-tube assays showed that Simulium ochraceum Walker s.l. showed significant attraction to octenol but that S. damnosum s.l. did not (Young et al. 2015). Taken together, these data show that the response of simulids to hostderived odours is not uniform and is likely to reflect differences in host preference.

Recent work focussed on the collection of human 0 . volvulus vectors in Mexico led to the development of the Esperanza window trap, a novel design utilising visual and olfactory cues as attractants and a sticky substrate to collect alighting insects. When baited with $\mathrm{CO}_{2}$ plus the BGlure (Biogents), the trap collected a similar number of $S$. ochraceum s.l. to a human landing catch (Rodríguez-Pérez et al. 2013). A modified version of the Esperanza trap was subsequently found to display similarly promising results for collecting the major African vector of human onchocerciasis, S. damnosum s.l., in Burkina Faso (Toé et al. 2014). This simulid is a vector for Onchocerca ochengi between cattle, although as hide damage is the primary issue resulting from cattle infection (Bwangamoi 1969), the importance of this trap lies with providing a promising replacement for the human landing catch, currently the gold-standard approach used for surveillance of human onchocerciasis vectors. The identification of additional human-derived semiochemicals including acetophenone and ammonium bicarbonate as attractants for both S. ochraceum s.l. and S. damnosum s.l. in the field (Young et al. 2015) provides further promise for the optimisation of semiochemical-baited simulid traps. Most recently, a study using EAG and Y-tube assays identified five compounds: octenol, 2-heptanone, acetophenone, 1-octanol and naphthalene, as attractive to Simulium vittatum Zetterstedt s.l. (Verocai et al. 2016). This simulid species is a vector of vesicular stomatitis virus to several livestock species (Cupp et al. 1992; Mead et al. 2004). Interestingly, 
low concentrations of naphthalene were previously found to have a repellent effect on Musca autumnalis De Geer in a wind tunnel assay (Birkett et al. 2004). This indicates the necessity for further work to determine whether these differences reflect a species-specific difference, dosedependency, or a combination of both.

\section{Horse flies (Diptera: Tabanidae)}

The Tabanidae are a large group of flying Dipterans which include several species groups responsible for considerable biting nuisance of livestock and humans. In addition to the pain and irritation inflicted directly by persistent biting activity (Foil and Hogsette 1994), tabanids are biological and mechanical vectors for many viruses, bacteria and parasitic pathogens (see Baldacchino et al. (2014a) for a recent review) such as the equine infectious anaemia virus (Maresca et al. 2012) and Besnoitia besnoiti, responsible for bovine besnoitiosis (Álvarez-García et al. 2013; Jacquiet et al. 2010), two re-emerging pathogens in Europe. Few effective methods for control or management of biting nuisance exist although an increased understanding of the olfactory cues governing behaviour provide promise for improved control methods.

Tabanids are predominantly diurnal and possess large eyes to utilise optical cues such as polarised light (polarotaxis), shape and colour to orientate to the environment and towards blood-meal hosts (Egri et al. 2012; Horváth et al. 2008, 2010; Kriska et al. 2009). However, in common with other haematophagous arthropods they possess olfactory sensilla located in the antennae and maxillary palps that enable the detection of olfactory cues involved in host-seeking (Ivanov 2007; Parashar et al. 1994).

Carbon dioxide is a dose-dependent attractant for many tabanids including Tabanus spp., Atylotus spp. and Hybomitra spp. (Baldacchino et al. 2013a), and has been used extensively to increase the numbers collected in artificial traps (Cilek and Olson 2008; Hayes et al. 1993; Kline et al. 1991; Leprince et al. 1994; McElligott and Mclver 1987; Phelps and Vale 1976; Vale 1980). Octenol also is a proven attractant for tabanids (French and Kline 1989; Takken and Kline 1989) and its addition to traps has been demonstrated to increase catch rates by 2-9 times relative to unbaited control traps (Baldacchino et al. 2014b; Krčmar 2005; Mihok and Mulye 2010; Mihok et al. 2007), although a dose-dependent effect has not yet been characterised (Mihok and Mulye 2010). Indeed, the efficiency of the widely-used Nzi trap, which works primarily on the basis of visual cues, can be doubled from 45 to $91 \%$ by the addition of octenol (Mohamed-Ahmed et al. 2007). Two phenols, 4-methylphenol and 3-propylphenol, identified as key attractants to tsetse (Owaga et al. 1988), also serve as attractants for tabanids. Adding these compounds to traps can increase catch rates in a dose-dependent manner (Baldacchino et al. 2014b).

Whilst the specific compounds of aged urine have been shown to be useful attractants for tabanids, a superior attractive effect may be obtained by the use of the complete aged urine itself (Baldacchino et al. 2014b; Djiteye et al. 1998; Krčmar 2005; Krčmar and Lajos 2011; Mihok and Lange 2012). Livestock urine consists of a mixture of compounds including ketones, phenols and fatty alcohols, with at least 29 compounds having been identified as constituents to date (Baldacchino et al. 2014b). Current evidence indicates that attraction to aged urine is reliant on a complex synergism between known and unknown compounds within the urine. Indeed, traps baited with aged urine generally catch more tabanids than those using isolated compounds. For example, canopy traps baited with a 5:3:2 blend of octenol, acetone and ammonia collected significantly fewer Atylotus loewianus Villeuneuve than those baited with aged donkey urine (Krčmar et al. 2010). Similarly, Atylotus quadrifarius Loew was collected in significantly greater number in traps 
with aged horse urine than those with octenol alone (Baldacchino et al. 2014b). Aged urine does not have a uniform attractive effect on all species, however, indicating the complexity of the olfactory environment within this group of pests (Baldacchino et al. 2014a). Furthermore, the urine of different species elicits different EAG responses (Baldacchino et al. 2013a) and trapping efficacy in the field (Krčmar et al. 2006, 2010), adding further complexity when considering their use in settings where multiple biting species are present. Nevertheless, these advances in the understanding of the semiochemical biology of tabanids offer some promise for the reduction of biting populations around afflicted livestock holdings (Baldacchino et al. 2014c).

\section{Horn and stable flies (Diptera: Muscidae)}

Horn flies (H. irritans) and stable flies (S. calcitrans) are important biting pests of livestock. In addition to direct skin and hide damage (Guglielmone et al. 1999), losses to livestock production can be severe. The cattle industry is particularly affected as the stress caused by biting activity may reduce meat and milk yields (Byford et al. 1992; Taylor et al. 2012). Stable flies may additionally play a role as vectors of livestock pathogens (Baldacchino et al. 2013b). Although chemical insecticides applied directly to the animal may afford some protection, resistance in both horn and stable flies has been reported (Cilek and Greene 1994; Guerrero et al. 1997; Weinzierl et al. 1990) and therefore there is a clear need for novel methods of managing these pest populations (Logan and Birkett 2007; Oyarzún et al. 2008).

Horn and stable flies are attracted by $\mathrm{CO}_{2}$ and cattle odours (Dalton et al. 1978; Oyarzún et al. 2009; Schofield and Brady 1997; Vale 1980; Vale and Hall 1985). Electrophysiological analysis of the responses of $S$. calcitrans to the rumen digesta of cattle identified responses to 30 compounds, with three, dimethyl trisulphide, butanoic acid and $p$-cresol producing attractive responses in wind tunnel assays (Jeanbourquin and Guerin 2007a). Interestingly, whilst octenol is well-described as an attractant for $H$. irritans (Oyarzún et al. 2009), and elicits electrophysiological responses in S. calcitrans (Jeanbourquin and Guerin 2007a; Schofield et al. 1995), its ability to serve as an attractant in behavioural and field studies with $S$. calcitrans is less consistent. For example, whilst some studies found that the addition of octenol to artificial traps increased S. calcitrans catch rates (Holloway and Phelps 1991; Mihok et al. 1995, 2007), others found no significant increases in field catches (Cilek 1999; Mullens et al. 1995) or no orientation or attraction behaviour in laboratory assays (Alzogaray and Carlson 2000; Jeanbourquin and Guerin 2007a). The addition of other volatile phenols attractive to tsetse and tabanids, including 4-methylphenol and 3-propylphenol, can also increase catch rates (Cilek 1999).

Cattle display intraspecific variation in biting fly loads, in part mediated by semiochemicals (Birkett et al. 2004; Jensen et al. 2004; Torr et al. 2006). Birkett et al. (2004) identified 23 compounds from air entrainments of Holsten-Friesian heifers and their urine followed by coupled GC-EAG and GC-MS. Two compounds (octenol and 6-methyl-5-hepten-2-one) were attractive and three (naphthalene, linalool and propyl butanoate) were repellent in laboratory behavioural assays with horn flies. When a slow-release formulation of 6-methyl-5-hepten-2-one was applied to heifers associated with high fly loads, the whole herd showed a reduction in fly load (Birkett et al. 2004). Subsequent research isolating semiochemicals from Chilean Holstein-Friesian heifers identified a further two semiochemicals involved in mediating horn fly-host interactions (Oyarzún et al. 2009). The compound 2-decanone acted as a repellent to $H$. irritans in behavioural assays whilst 2-undecanone was attractive to this species, although these compounds have yet to be trialled in a field setting. The ability of the plant-derived volatiles from lemongrass (Cymbopogon citratus) oil to repel $S$. calcitrans in the laboratory and catnip (Nepeta cataria) oil-impregnated wax beads 
to act as an oviposition deterrent to S. calcitrans in both laboratory and field trials (Zhu et al. 2010, 2014) has shown the potential of using semiochemical repellents to controlling biting flies at their source. Overall therefore, there is promise for the development of push-pull strategies for horn and stable fly control, based on the use of repellent semiochemicals applied directly to susceptible hosts or oviposition sites, and attractant semiochemicals placed in baited traps nearby. Used in concert with breeding programs identifying and selectively breeding resistant individuals (for example using sniffer dogs; Greeff et al. 2013), this could lead to improved livestock welfare and production.

\section{Ticks (Ixodida: Ixodoidea)}

Ticks are important arachnid livestock pests, responsible for direct damage to hosts during feeding and for the transmission of a wide range of important pathogens (Jongejan and Uilenberg 2004). The combined direct and indirect effects of tick infestations and tick-borne diseases result in significant economic losses in livestock production systems in many parts of the world (Jonsson et al. 2001; Kivaria 2006; Ocaido et al. 2009).

The application of chemical acaricides is the main approach to livestock tick control, but this can be expensive and resistance to acaricides such as deltamethrin has been reported (Bianchi et al. 2003). Several semiochemicals have been identified which play a role in key behaviours in the tick life cycle and therefore offer the prospect for exploitation.

Four different classes of pheromones are produced by ticks to modify the behaviour of conspecifics (Sonenshine 2004), and each provides exploitable options for control. Arrestment, or assembly, pheromones cause ticks to cluster in the environment to enhance survival and improve mating success (Sonenshine 1985). Attraction-aggregation-attachment (AAA) pheromones are secreted by feeding males and attract host-seeking (questing) male and female ticks and cause them to form clusters of feeding individuals on a host. Sex pheromones are emitted by one sex and are involved in mate attraction and selection. Primer pheromones are poorly understood relative to the other classes but are thought to be involved in mediating physiological functions such as fecundity for the purpose of enhancing survival in response to stressors such as overcrowding (Khalil 1984).

Ticks also respond to host-derived kairomones including $\mathrm{CO}_{2}$, ammonia and the skin-derived lipid squalene during questing behaviour (Garcia 1962; Haggart and Davis 1980; Yoder et al. 1998). Hostderived kairomones are detected using chemoreceptors on the Haller's organ, a sensory accessory located on the tarsi of the forelegs (Sonenshine et al. 1986). The collection of ticks in $\mathrm{CO}_{2}$-baited traps has been demonstrated (Wilson et al. 1972) although manual collection methods such as blanket dragging remain the most common approaches to collecting ticks for ecological and surveillance studies. Ticks will cluster in areas of vegetation containing secretions from preferred hosts in order to maximise chances of future encounters. For example, Ixodes neitzi Clifford, Walker \& Keirans clusters onto twigs marked with the ante-orbital gland secretions of its preferred host, the Klipspringer antelope (Oreotragus oreotragus Zimmermann) (Rechav et al. 1978).

There have been various attempts to exploit semiochemicals for the control of ticks. Approaches using arrestment pheromones can increase the aggregation of ticks in the environment, where they can then be exposed to an acaricide. One recent control approach combined a 25:1:1 ratio of the arrestment pheromones guanine, adenine and xanthine into sprayable oil droplets containing deltamethrin. When applied to vegetation, the pheromone mixture induced aggregation of Ixodes 
scapularis Say at the oil droplets, thus exposing them to the acaricide; mortality rates of up to $95 \%$ were reported (Allan et al. 2001; Sonenshine 2004).

Semiochemical-mediated disruption of mating behaviour by using so-called 'confusants' is another approach to control. This method has the potential to impact tick populations by reducing the chances of successful mating occurring and thus reducing the chances of successful oviposition and the number of offspring that are produced. Confusants take advantage of the excitation response of males when exposed to female-derived sex pheromones. These pheromones cause males to stop feeding and detach in order to seek females by walking over the host. The host has been treated with an acaricide and therefore the male ticks become exposed to a greater, lethal, dose of acaricide than when attached and feeding. For example, application of a microencapsulated mixture of the sex pheromone 2,6-dichlorophenol and the acaricide propoxur to dogs infested with Dermacentor variabilis Say resulted in reduced mating success, high kill rates of male ticks and an oviposition rate reduced by over $90 \%$ (Sonenshine et al. 1985). However, some sex pheromones show evidence for species-specificity (Louly et al. 2008) and therefore this approach would need to be modified to include the appropriate pheromone for the relevant tick species in different areas.

Several lure-and-kill devices utilising various pheromones as attractants together with chemical acaricides have been trialled for ticks. Early approaches showed that applying an aggregationattachment pheromone from fed male Amblyomma maculatum Koch with the acaricide isobenzam to the back of cattle caused attraction and killing of female ticks that were released onto the cattle (Gladney et al. 1974). In a similar approach, extracts from fed male Amblyomma hebraeum Koch were combined with toxaphene, resulting in an attractive killing effect on nymphal and adult ticks (Rechav and Whitehead 1978). In experimental testing of a device called the 'tick decoy', beads containing the mounting sex pheromone cholesteryl oleate and the acaricide proxopur were applied to rabbits infested with D. variabilis. When excited by application of 2,6-dichlorophenol, males began female-seeking activity and attempted to mount the beads; this resulted in kill rates as high as $89 \%$ for male D. variabilis within 30 minutes (Hamilton and Sonenshine 1989). The cholesteryl oleate was important in ensuring the males were exposed for a prolonged time period to the acaricide. Similar positive results were observed with this approach using $D$. variabilisinfested cattle (Sonenshine et al. 1992) and Hyalomma dromedarii Koch on camels (AbdelRahman et al. 1998). A similar but modified approach has also been used to target African bont ticks Amblyomma variegatum Fabricius and Amblyomma hebraeum Koch. A mixture of the AAA compounds o-nitrophenol, methyl salicylate and 2,6-dichlorophenol together with the attractant phenylacetaldehyde and a chemical acaricide were impregnated onto plastic tags that were then affixed onto the tails of cattle. Tick aggregations formed around the tags, became exposed to the acaricide and were killed. Control rates of the ticks using these tags ranged between 87.5 and 99.3\% for treatment groups in field trials in Zimbabwe (Norval et al. 1996).

Although these lure-and-kill approaches work to protect hosts from ticks on the animal, they do not prevent initial attachment and in some cases ticks are still able to briefly feed on the animal, therefore potentially exposing it to a pathogen. Therefore, a lure-and-kill approach has recently been trialled which targets tick populations in the environment. Ticks were lured onto filter paper containing a mixture of 2,6-dichlorophenol plus a 25:1:1:1 ratio of guanine, xanthine, adenine and hematin, with dry ice to provide $\mathrm{CO}_{2}$. Finally, the filter paper was impregnated with deltamethrin. In simulated field trials larval and nymphal ticks of Rhipicephalus sanguineus Latreille were attracted to the paper and subsequently killed, although the highest rate of attraction was only $40 \%$ of released ticks. Nonetheless, the optimisation of such an environmental lure-and-kill approach could work together with lure-and-kill approaches on the host to effect greater control 
of target tick populations. Importantly, research on tick pheromones has shown the possibilities of using sexual pheromones to disrupt arthropod behaviour highlighting the need to explore this in more detail in other important livestock pest groups.

\section{Future developments}

It is clear that the need for developing novel and effective approaches to reduce the impact of biting arthropods is essential in light of the emergence of new pathogens, increasing pressure on livestock systems in response to a growing human population and the observed increase in insecticide resistance worldwide.

Some of the most promising approaches lie in understanding the genetics involved in making certain individuals more or less attractive to biting arthropods, a phenomenon that has now been recognised in several vertebrate species to date. With this knowledge, the genetic structure of livestock populations can be manipulated using selective breeding programs to decrease the number of 'attractive' individuals and increase the number of 'unattractive', or resistant, individuals. The ability to detect fly-resistant animals, for example using sniffer dogs (Greeff et al. 2013), offers an important practical step towards this goal. However, such programs require continual assessment as arthropods are able to rapidly adapt to changes in their environment which may include the semiochemical profile of available hosts.

Understanding the specific chemical components of attractive and resistant semiochemical profiles will furthermore enable the development of novel repellent compounds. Spatial repellents would provide the most effective coverage from an animal perspective, enabling the protection of a group of animals from a single application. The identification of altered semiochemical profiles in infected animals and humans presents an exciting opportunity for the diagnosis of disease. If the production of a particular semiochemical is associated with a disease state prior to the appearance of the pathogen in currently available tests, this could offer a far more rapid opportunity for early diagnosis, resulting in earlier and more effective treatment or the appropriate removal of a susceptible host from the population.

An emerging area of promise lies with increasing our understanding of the role of semiochemicals in the learning behaviour and memory capacity of arthropods. Evidence of learned behaviour has been demonstrated in different life stages of several pest insects. For example, in both adult tsetse and mosquitoes, prior feeding on a particular host increases the likelihood of this host being selected for a subsequent blood meal (Bouyer et al. 2007; Mwandawiro et al. 2000). Larval Culex restuans Theobald exposed to salamander (Ambystoma tigrinum Green) odour plus crushed conspecifics subsequently responded to the salamander odour alone with a threat response (reduced movement) (Ferrari et al. 2008). Larvae not previously conditioned in this manner did not respond to these odours. Furthermore, associative learning (learning the association between two stimuli; see Vinauger et al. (2016) for a recent review) has been observed in mosquitoes of the genera Aedes (Vinauger et al. 2014), Anopheles (Chilaka et al. 2012; Vantaux et al. 2014) and Culex (Tomberlin et al. 2006).

The impact, whether help or hindrance, of vector learned behaviour on surveillance and control tools remains yet to be fully understood. However, there is evidence for reduced repellent efficacy of DEET upon second exposure in Ae. aegypti mosquitoes (Stanczyk et al. 2013), and this species will also preferentially oviposit into water in which they were reared, even when the water contained repellent (Kaur et al. 2003). Whilst trapping will remove individuals from the population, therefore 
precluding the chance for learning behaviour for captured individuals, how about the effect on individuals attracted but unsuccessfully captured? Are these mosquitoes able to associate a particular attractant or trap with a non-reward and therefore learn to avoid the trap in future? Vinauger et al. (2014) showed Ae. aegypti could retain the positive association between L-lactic acid and a blood reward for 24 hours, so could a negative association be retained for a similar or longer period? Conversely, could you 'prime' the mosquito population in an area to feed on an attractive sugar bait, then spray the toxic version soon after to achieve a greater killing effect due to higher sugar feeding rates? It is clear therefore that surveillance and control tools need to be continually revaluated as new evidence emerges in this area.

Furthermore, currently available evidence on the semiochemical biology of arthropods and their hosts is heavily biased to certain systems, such as tsetse and cattle, whilst other systems remain relatively unexplored. The identification of semiochemical-mediated interactions in nonmammalian hosts including amphibians (Williams et al. 2006) and fish (Bailey et al. 2006; Hastie et al. 2013) has demonstrated that semiochemicals are likely to be involved in a vast array of arthropodhost interactions across the animal kingdom. Nonetheless, the overwhelming focus of research has been on economically-important livestock and humans. Exploring the volatile components of a wide range of host species may provide novel volatiles for managing pest populations as part of surveillance and control programs. Finally, research gaps lie in our understanding of the sexual chemoecology of pest arthropods which may offer useful new approaches to disrupting pest arthropod behaviour beyond the involvement of host semiochemicals.

\section{Synthesis and conclusions}

A wide array of semiochemicals is involved in the behavioural regulation of arthropod pests across their life stages and in their interactions between members of their own species, their hosts and the environment. Current evidence demonstrates that certain host-derived compounds such as $\mathrm{CO}_{2}$, octenol and the phenolic compounds contained in dung and urine (4-methylphenol and 3-propylphenol) act as generalist, although not uniform, attractants to several pest arthropod groups. In like manner, other host kairomones such as 6-methyl-5-hepten-2-one may serve as repellents to more than one pest group. Continuing to improve our understanding of the role of these and other semiochemicals in regulating the behaviour of multiple arthropod pests is useful as livestock are not generally exposed to a single group of biting pests. A push-pull system which targets multiple pests therefore offers broader protection for livestock but also increases acceptability to the end user, in this case the farmers, as shown in the preferable use of insecticidetreated cattle for tsetse control.

The optimal use of semiochemicals for control of pest arthropods is likely to be via the use of an integrated control approach comprising novel repellents and lure-and-kill traps, used in tandem with existing methods such as larval source reduction for mosquitoes and sanitation of feedlots for stable flies and house flies. Despite limitations and logistical challenges, recent studies continue to show promise in bringing our understanding of arthropod-host semiochemical interactions from the laboratory to practical field application. Continuing to enhance our knowledge of the semiochemical environment integral to the behaviour of arthropods will enable their improved exploitation for the control of key pest species in future. 


\section{References}

Abdel-Rahman MS, Fahmy MM and Aggour MG (1998) Trials for control of ixodid ticks using pheromone acaricide tick decoys. J Egypt Soc Parasitol 28: 551-557.

Achee NL, Bangs MJ, Farlow R, Killeen GF, Lindsay S, Logan JG, Moore SJ, Rowland M, Sweeney K, Torr SJ, Zwiebel LJ and Grieco JP (2012) Spatial repellents: from discovery and development to evidence-based validation. Malar J 11: 164.

Adam Y, Cecchi G, Kgori PM, Marcotty T, Mahama Cl, Abavana M, Anderson B, Paone M, Mattioli R and Bouyer J (2013) The sequential aerosol technique: a major component in an integrated strategy of intervention against riverine tsetse in Ghana. PLoS Negl Trop Dis 7: e2135.

Adham LJW (1982) Coupled gas chromatography - single cell recording: a new technique for use in the analysis of insect pheromones. Zeitschrift für Naturforsch C 37: 947-952.

Agelopoulos N, Birkett MA, Hick AJ, Hooper AM, Pickett JA, Pow EM, Smart LE, Smiley DWM, Wadhams LJ and Woodcock CM (1999) Exploiting semiochemicals in insect control. Pestic Sci 55: 225-235.

Alexander B and Maroli M (2003) Control of phlebotomine sandflies. Med Vet Entomol 17: 1-18.

Allan SA, Bernier UR and Kline DL (2005) Evaluation of oviposition substrates and organic infusions on collection of Culex in Florida. J Am Mosq Control Assoc 21: 268-273.

Allan SA, Sonenshine DE and Burridge MJ (2001) Tick pheromones and uses thereof. Patent US6331297B1. Available at: https://patents.google.com/patent/US6331297B1/en.

Alvar J, Vélez ID, Bern C, Herrero M, Desjeux P, Cano J, Jannin J, Den Boer M and the WHO Leishmaniasis Control Team (2012) Leishmaniasis worldwide and global estimates of its incidence. PLoS ONE 7: e35671.

Álvarez-García G, Frey CF, Mora LMO and Schares G (2013) A century of bovine besnoitiosis: an unknown disease reemerging in Europe. Trends Parasitol 29: 407-415.

Alzogaray RA and Carlson DA (2000) Evaluation of Stomoxys calcitrans (Diptera: Muscidae) behavioral response to human and related odors in a triple cage olfactometer with insect traps. J Med Entomol 37: 308-315.

Amazigo U and Boatin B (2006) The future of onchocerciasis control in Africa. Lancet 368: 1946-1947.

Anderson JR (2001) Larviposition by nasopharyngeal bot fly parasites of Columbian black-tailed deer: a correction. Med Vet Entomol 15: 438-442.

Andreadis TG, Anderson JF, Munstermann LE, Wolfe RJ and Florin DA (2001) Discovery, distribution, and abundance of the newly introduced mosquito Ochlerotatus japonicus (Diptera: Culicidae) in Connecticut, USA. J Med Entomol 38: 774-779.

Atwood DW and Meisch MV (1993) Evaluation of 1-octen-3-ol and carbon dioxide as black fly (Diptera: Simuliidae) attractants in Arkansas. J Am Mosq Control Assoc 9: 143-146.

Autorino GL, Battisti A, Deubel V, Ferrari G, Forletta R, Giovannini A, Lelli R, Murri S and Scicluna MT (2002) West Nile virus epidemic in horses, Tuscany region, Italy. Emerg Infect Dis 8: 1372-1378.

Axtell RC and Edwards TD (1983) Efficacy and nontarget effects of larvadex as a feed additive for controlling house flies in caged-layer poultry manure. Poult Sci 62: 2371-2377.

Bailey RJE, Birkett MA, Ingvarsdóttir A, Mordue (Luntz) AJ, Mordue W, Shea BO, Pickett JA and Wadhams LJ (2006) The role of semiochemicals in host location and non-host avoidance by salmon louse (Lepeophtheirus salmonis) copepodids. Can J Fish Aquat Sci 63: 448-456.

Baker T, Carpenter S, Gubbins S, Newton R, lacono G Lo, Wood J and Harrup LE (2015) Can insecticide-treated netting provide protection for Equids from Culicoides biting midges in the United Kingdom? Parasit Vectors 8: 604.

Baldacchino F, Cadier J, Porciani A, Buatois B, Dormont L and Jay-Robert P (2013a) Behavioural and electrophysiological responses of females of two species of tabanid to volatiles in urine of different mammals. Med Vet Entomol 27:77-85.

Baldacchino F, Desquesnes M, Mihok S, Foil LD, Duvallet G and Jittapalapong S (2014a) Tabanids: neglected subjects of research, but important vectors of disease agents! Infect Genet Evol 28: 596-615.

Baldacchino F, Manon S, Puech L, Buatois B, Dormont L and Jay-Robert P (2014b) Olfactory and behavioural responses of tabanid horseflies to octenol, phenols and aged horse urine. Med Vet Entomol 28: 201-209.

Baldacchino F, Muenworn V, Desquesnes M, Desoli F, Charoenviriyaphap T and Duvallet G (2013b) Transmission of pathogens by Stomoxys flies (Diptera, Muscidae): a review. Parasite 20: 26. 
Baldacchino F, Puech L, Manon S, Hertzog LR and Jay-Robert P (2014c) Biting behaviour of Tabanidae on cattle in mountainous summer pastures, Pyrenees, France, and effects of weather variables. Bull Entomol Res 104: 471-479.

Barbosa RMR, Regis L, Vasconcelos R and Leal WS (2010) Culex mosquitoes (Diptera: Culicidae) egg laying in traps loaded with Bacillus thuringiensis variety israelensis and baited with skatole. J Med Entomol 47: 345-348.

Baylis M and Mbwabi AL (1995) Feeding behaviour of tsetse flies (Glossina pallidipes Austen) on Trypanosoma-infected oxen in Kenya. Parasitol 110: 297-305.

Baylis M and Nambiro CO (1993) The effect of cattle infection by Trypanosoma congolense on the attraction, and feeding success, of the tsetse fly Glossina pallidipes. Parasitol 106: 357-361.

Beehler JW, Millar JG and Mulla MS (1994) Protein hydrolysates and associated bacterial contaminants as oviposition attractants for the mosquito Culex quinquefasciatus. Med Vet Entomol 8: 381-385.

Beier JC, Müller GC, Gu W, Arheart KL and Schlein Y (2012) Attractive toxic sugar bait (ATSB) methods decimate populations of Anopheles malaria vectors in arid environments regardless of the local availability of favoured sugarsource blossoms. Malar J 11: 31.

Bentley MD, McDaniel IN, Yatagai M, Lee H-P and Maynard R (1979) p-Cresol: an oviposition attractant of Aedes triseriatus. Environ Entomol 8: 206-209.

Bett MK, Saini RK and Hassanali A (2015) Repellency of tsetse-refractory waterbuck (Kobus defassa) body odour to Glossina pallidipes (Diptera: Glossinidae): assessment of relative contribution of different classes and individual constituents. Acta Trop 146: 17-24.

Bhasin A, Mordue AJ and Mordue W (2000) Electrophysiological and behavioural identification of host kairomones as olfactory cues for Culicoides impunctatus and C. nubeculosus. Physiol Entomol 25: 6-16.

Bhasin A, Mordue AJ and Mordue W (2001) Field studies on efficacy of host odour baits for the biting midge Culicoides impunctatus in Scotland. Med Vet Entomol 15: 147-156.

Bianchi MW, Barré N and Messad S (2003) Factors related to cattle infestation level and resistance to acaricides in Boophilus microplus tick populations in New Caledonia. Vet Parasitol 112: 75-89.

Birkett MA, Agelopoulos N, Jensen KMV, Jespersen JB, Pickett JA, Prijs HJ, Thomas G, Trapman JJ, Wadhams LJ and Woodcock CM (2004) The role of volatile semiochemicals in mediating host location and selection by nuisance and disease-transmitting cattle flies. Med Vet Entomol 18: 313-322.

Bishop AL, McKenzie HJ and Spohr LJ (2008) Attraction of Culicoides brevitarsis Kieffer (Diptera: Ceratopogonidae) and Culex annulirostris Skuse (Diptera: Culicidae) to simulated visual and chemical stimuli from cattle. Aust J Entomol 47: 121-127.

Bishop SC and Morris CA (2007) Genetics of disease resistance in sheep and goats. Small Rumin Res 70: 48-59.

Blackwell A, Dyer C, Mordue AJ, Wadhams LJ and Mordue W (1994) Field and laboratory evidence for a volatile pheromone produced by parous females of the Scottish biting midge, Culicoides impunctatus. Physiol Entomol 19: 251-257.

Blackwell A, Dyer C, Mordue AJ, Wadhams LJ and Mordue W (1996) The role of 1-octen-3-ol as a host-odour attractant for the biting midge, Culicoides impunctatus Goetghebuer, and interactions of 1-octen-3-ol with a volatile pheromone produced by parous female midges. Physiol Entomol 21: 15-19.

Blaustein L, Blaustein J and Chase J (2005) Chemical detection of the predator Notonecta irrorata by ovipositing Culex mosquitoes. JVector Ecol 30: 299-301.

Blaustein L, Kiflawi M, Eitam A, Mangel M and Cohen JE (2004) Oviposition habitat selection in response to risk of predation in temporary pools: mode of detection and consistency across experimental venue. Oecologia 138: 300-305.

Boorman J (1974) The maintenance of laboratory colonies of Culicoides variipennis (Coq.), C. nubeculosus (Mg.) and C. riethi Kieff. (Diptera, Ceratopogonidae). Bull Entomol Res 64: 371-377.

Bosch OJ, Geier M and Boeckh J (2000) Contribution of fatty acids to olfactory host finding of female Aedes aegypti. Chem Senses 25: 323-330.

Bouyer J, Pruvot M, Bengaly Z, Guerin PM and Lancelot R (2007) Learning influences host choice in tsetse. Biol Lett 3: 113-117. 
Braverman Y, Chizov-Ginzburg A and Mullens BA (1999) Mosquito repellent attracts Culicoides imicola (Diptera: Ceratopogonidae). J Med Entomol 36: 113-115.

Bray DP, Alves GB, Dorval ME, Brazil RP and Hamilton JGC (2010a) Synthetic sex pheromone attracts the leishmaniasis vector Lutzomyia longipalpis to experimental chicken sheds treated with insecticide. Parasit Vectors 3: 16.

Bray DP, Bandi KK, Brazil RP, Oliveira AG and Hamilton JGC (2009) Synthetic sex pheromone attracts the leishmaniasis vector Lutzomyia longipalpis (Diptera: Psychodidae) to traps in the field. J Med Entomol 46: 428-434.

Bray DP, Carter V, Alves GB, Brazil RP, Bandi KK and Hamilton JGC (2014) Synthetic sex pheromone in a long-lasting lure attracts the visceral leishmaniasis vector, Lutzomyia longipalpis, for up to 12 weeks in Brazil. PLoS Negl Trop Dis 8: e2723.

Bray DP, Ward RD and Hamilton JGC (2010b) The chemical ecology of sandflies. In: Takken W and Knols BGJ (eds.) Olfaction in vector-host interactions. Wageningen Academic Publishers, Wageningen, the Netherlands.

Breitschwerdt EB and Kordick DL (2000) Bartonella infection in animals: carriership, reservoir potential, pathogenicity, and zoonotic potential for human infection. Clin Microbiol Rev 13: 428-438.

Bruno DW and Laurence BR (1979) The influence of the apical droplet of Culex egg rafts on oviposition of Culex pipiens fatigans (Diptera: Culicidae). J Med Entomol 16: 300-305.

Burkett DA, Lee WJ, Lee KW, Kim HC, Lee HI, Lee JS, Shin EH, Wirtz RA, Cho HW, Claborn DM, Coleman RE and Klein TA (2001) Light, carbon dioxide, and octenol-baited mosquito trap and host-seeking activity evaluations for mosquitoes in a malarious area of the Republic of Korea. J Am Mosq Control Assoc 17: 196-205.

Bursell E (1983) Effects of host odour on the behaviour of tsetse. Insect Sci Appl 5: 345-349.

Bursell E, Gough AJE, Beevor PS, Cork A, Hall DR and Vale GA (1988) Identification of components of cattle urine attractive to tsetse fies, Glossina spp. (Diptera: Glossinidae). Bull Entomol Res 78: 281-291.

Busula AO, Takken W, Loy DE, Hahn BH, Mukabana WR and Verhulst NO (2015) Mosquito host preferences affect their response to synthetic and natural odour blends. Malar J 14: 133.

Butler SM, Gerry AC and Mullens BA (2007) House fly (Diptera: Muscidae) activity near baits containing (Z)-9-tricosene and efficacy of commercial toxic fly baits on a southern California dairy. J Econ Entomol 100: 1489-1495.

Bwangamoi O (1969) Dermatitis in cattle caused by Onchocerca ochengi Bwangamoi, 1969, and the effect of the adult filaria on the finished leather. Bull des Epizoot en Afrique 17: 435-445.

Byford RL, Craig ME and Crosby BL (1992) A review of ectoparasites and their effect on cattle production. J Anim Sci 70: 597-602.

Cameron MM, Amerasinghe FP and Lane RP (1991) The field response of Sri Lankan sandflies and mosquitoes to synthetic cattle-derived attractants. Parassitologia 33: 119-126.

Canyon DV and Hii JLK (1997) Efficacy of carbon dioxide, 1-octen-3-ol, and lactic acid in modified Fay-Prince traps as compared to man-landing catch of Aedes aegypti. J Am Mosq Control Assoc 13: 66-70.

Cardé RT and Gibson G (2010) Host finding by female mosquitoes: mechanisms of orientation to host odours and other cues. In: Takken W and Knols BGJ (eds.) Olfaction in vector-host interactions. Wageningen Academic Publishers, Wageningen, the Netherlands.

Carestia RR and Savage LB (1967) Effectiveness of carbon dioxide as a mosquito attractant in the CDC miniature light trap. Mosq News 27: 90-92.

Carpenter S, Eyres K, McEndrick I, Smith L, Turner J, Mordue W and Mordue AJ (2005) Repellent efficiency of BayRepel against Culicoides impunctatus (Diptera: Ceratopogonidae). Parasitol Res 95: 427-429.

Carpenter S, Mellor PS and Torr SJ (2008) Control techniques for Culicoides biting midges and their application in the UK and northwestern Palaearctic. Med Vet Entomol 22: 175-187.

Chaves LF, Harrington LC, Keogh CL, Nguyen AM and Kitron UD (2010) Blood feeding patterns of mosquitoes: random or structured? Front Zool 7: 3 .

Cheke RA and Garms R (1988) Trials of compounds to enhance trap catches of Glossina palpalis palpalis in Liberia. Med Vet Entomol 2: 199-200.

Chilaka N, Perkins E and Tripet F (2012) Visual and olfactory associative learning in the malaria vector Anopheles gambiae sensu stricto. Malar J 11: 27. 
Cilek JE (1999) Evaluation of various substances to increase adult Stomoxys calcitrans (Diptera: Muscidae) collections on alsynite cylinder traps in north Florida. J Med Entomol 36: 605-609.

Cilek JE and Greene GL (1994) Stable fly (Diptera: Muscidae) insecticide resistance in Kansas cattle feedlots. J Econ Entomol 87: 275-279.

Cilek JE and Hallmon CF (2005) The effectiveness of the Mosquito Magnet trap for reducing biting midge (Diptera: Ceratopogonidae) populations in coastal residential backyards. J Am Mosq Control Assoc 21: 218-221.

Cilek JE, Kline DL and Hallmon CF (2003) Evaluation of a novel removal trap system to reduce biting midge (Diptera: Ceratopogonidae) populations in Florida backyards. J Vector Ecol 28: 23-30.

Cilek JE and Olson MA (2008) Effects of carbon dioxide, an octenol/phenol mixture, and their combination on Tabanidae (Diptera) collections from French 2-tier box traps. J Med Entomol 45: 638-642.

Clausen PH, Adeyemi I, Bauer B, Breloeer M, Salchow F and Staak C (1998) Host preferences of tsetse (Diptera: Glossinidae) based on bloodmeal identifications. Med Vet Entomol 12: 169-180.

Clements AN (2012) The biology of mosquitoes, vol. 3. Transmission of viruses and interactions with bacteria. CAB International, Wallingford, UK.

Coleman RE, Robert LL, Roberts LW, Glass JA, Seeley DC, Laughinghouse A, Perkins PV and Wirtz RA (1993) Laboratory evaluation of repellents against four Anopheline mosquitoes (Diptera: Culicidae) and two Phlebotomine sand flies (Diptera: Psychodidae). J Med Entomol 30: 499-502.

Cook SM, Khan ZR and Pickett JA (2007) The use of push-pull strategies in integrated pest management. Annu Rev Entomol 52: 375-400.

Cornet S, Nicot A, Rivero A and Gandon S (2013a) Both infected and uninfected mosquitoes are attracted toward malaria infected birds. Malar J 12: 179.

Cornet S, Nicot A, Rivero A and Gandon S (2013b) Malaria infection increases bird attractiveness to uninfected mosquitoes. Ecol Lett 16: 323-329.

Creton B, Pageat P, Robejean M, Lafont-Lecuelle C and Cozzi A (2016) Protection of horse ears against Simulid parasitism: efficacy of a mammal semiochemical solution over 10 hours. Vet Parasitol 227: 15-19.

Cupp EW, Mare CJ, Cupp MS and Ramberg FB (1992) Biological transmission of vesicular stomatitis virus (New Jersey) by Simulium vittatum (Diptera: Simuliidae). J Med Entomol 29: 137-140.

Dalton LW, Kinzer HG, Reeves JM and Atmatí JW (1978) Host location in the horn fly: roles of heat, $\mathrm{CO}_{2}$, water vapor, and cow-produced odors in attraction. Southwest Entomol 3: 147-153.

Darbro JM and Harrington LC (2007) Avian defensive behaviour and blood-feeding success of the West Nile vector mosquito, Culex pipiens. Behav Ecol 18: 750-757.

Davies FG, Linthicum KJ and James AD (1985) Rainfall and epizootic Rift Valley fever. Bull World Health Organ 63: 941-943.

Day JF, Duxbury CV, Glasscock S and Paganessi JE (2001) Removal trapping for the control of coastal Culicoides populations. Tech Bull Florida Mosq Control Assoc 3: 15-16.

Day JF and Sjogren RD (1994) Vector control by removal trapping. Am J Trop Med Hyg 50: 126-133.

Degener CM, De Ázara TMF, Roque RA, Rösner S, Rocha ESO, Kroon EG, Codeço CT, Nobre AA, Ohly JJ, Geier M and Eiras ÁE (2015) Mass trapping with MosquiTRAPs does not reduce Aedes aegypti abundance. Mem Inst Oswaldo Cruz 110:517-527.

Degener CM, Eiras ÁE, Ázara TMF, Roque RA, Rösner S, Codeço CT, Nobre AA, Rocha ESO, Kroon EG, Ohly JJ and Geier M (2014) Evaluation of the effectiveness of mass trapping with BG-Sentinel traps for Dengue vector control: A cluster randomized controlled trial in Manaus, Brazil. J Med Entomol 51: 408-420.

Dekker T, Geier M and Cardé RT (2005) Carbon dioxide instantly sensitizes female yellow fever mosquitoes to human skin odours. J Exp Biol 208: 2963-2972.

Dekker T, Steib B, Cardé RT and Geier M (2002) L-lactic acid: a human-signifying host cue for the anthropophilic mosquito Anopheles gambiae. Med Vet Entomol 16: 91-98.

Depaquit J, Grandadam M, Fouque F, Andry PE and Peyrefitte C (2010) Arthropod-borne viruses transmitted by Phlebotomine sandflies in Europe: a review. Euro Surveill 15: 19507.

Dethier VG, Browne BL and Smith CN (1960) The designation of chemicals in terms of the responses they elicit from insects. J Econ Entomol 53: 134-136. 
Ditzen M, Pellegrino M and Vosshall LB (2008) Insect odorant receptors are molecular targets of the insect repellent DEET. Science 319(5871): 1838-1842.

Djiteye A, Diarra M, Ouattara I and Traore D (1998) Comparison of the efficacy of different traps and attractants for tabanidae and Stomoxys in Mali. J Protozool Res 8: 263-273.

Dougherty MJ, Guerin P and Ward RD (1995) Identification of oviposition attractants for the sandfly Lutzomyia longipalpis (Diptera: Psychodidae) present in volatiles of faeces from vertebrates. Physiol Entomol 20: 23-32.

Dougherty MJ, Guerin PM, Ward RD and Hamilton JGC (1999) Behavioural and electrophysiological responses of the phlebotomine sandfly Lutzomyia longipalpis (Diptera: Psychodidae) when exposed to canid host odour kairomones. Physiol Entomol 24: 251-262.

Dougherty MJ and Hamilton JGC (1997) Dodecanoic acid is the oviposition pheromone of Lutzomyia longipalpis. J Chem Ecol 23: 2657-2671.

Dougherty MJ, Hamilton JGC and Ward RD (1993) Semiochemical mediation of oviposition by the phlebotomine sandfly Lutzomyia longipalpis. Med Vet Entomol 7: 219-224.

Dougherty MJ, Hamilton JGC and Ward RD (1994) Isolation of oviposition pheromone from the eggs of the sandfly Lutzomyia longipalpis. Med Vet Entomol 8(2): 119-124.

Douglas HD, Co JE, Jones TH, Conner WE and Day JF (2005) Chemical odorant of colonial seabird repels mosquitoes. J Med Entomol 42: 647-651.

Egri Á, Blahó M, Sándor A, Kriska G, Gyurkovszky M, Farkas R and Horváth G (2012) New kind of polarotaxis governed by degree of polarization: attraction of tabanid flies to differently polarizing host animals and water surfaces. Naturwissenschaften 99: 407-416.

Eisler MC, Torr SJ, Coleman PG, Machila N, and Morton JF (2003) Integrated control of vector-borne diseases of livestock - Pyrethroids: Panacea or poison? Trends Parasitol 19: 341-345.

Englbrecht C, Gordon S, Venturelli C, Rose A and Geier M (2015) Evaluation of BG-Sentinel Trap as a management tool to reduce Aedes albopictus nuisance in an urban environment in Italy. J Am Mosq Control Assoc 31: 16-25.

Fallis AM (1964) Feeding and related behavior of female Simuliidae (Diptera). Exp Parasitol 15: 439-470.

Fallis AM, Bennett GF, Griggs G and Allen T (1967) Collecting Simulium venustum female in fan traps and on silhouettes with the aid of carbon dioxide. Can J Zool 45: 1011-1017.

Fallis AM and Raybould JN (1975) Response of two African simuliids to silhouettes and carbon dioxide. J Med Entomol 12: 349-351.

Fallis AM and Smith SM (1964) Ether extracts from birds and $\mathrm{CO}_{2}$ as attractants for some ornithophilic simuliids. Can J Zool 42: 723-730.

Farajollahi A, Kesavaraju B, Price DC, Williams GM, Healy SP, Gaugler R and Nelder MP (2009) Field efficacy of BGSentinel and industry-standard traps for Aedes albopictus (Diptera: Culicidae) and West Nile virus surveillance. J Med Entomol 46: 919-925.

Fauci AS and Morens DM (2016) Zika virus in the Americas - yet another arbovirus threat. N Engl J Med 374: 601-604.

Fernández-Grandon GM, Gezan SA, Armour JAL, Pickett JA and Logan JG (2015) Heritability of attractiveness to mosquitoes. PLOS ONE 10: e0122716.

Ferrari MCO, Messier F and Chivers DP (2008) Threat-sensitive learning of predators by larval mosquitoes Culex restuans. Behav Ecol Sociobiol 62: 1079-1083.

Foil LD and Hogsette JA (1994) Biology and control of tabanids, stable flies and horn flies. Rev Sci Tech 13: 1125-1158.

Foster WA (1995) Mosquito sugar feeding and reproductive energetics. Annu Rev Entomol 40: 443-474.

French FE and Kline DL (1989) 1-octen-3-ol, an effective attractant for Tabanidae (Diptera). J Med Entomol 26: 459-461.

Gama RA, Maria da Silva I, Geier M and Eiras ÁE (2013) Development of the BG-Malaria trap as an alternative to humanlanding catches for the capture of Anopheles darlingi. Mem Inst Oswaldo Cruz 108: 763-771.

Garcia R (1962) Carbon dioxide as an attractant for certain ticks (Acarina: Argasidae and Ixodidae). Ann Entomol Soc Am 55: 605-606.

Geetha I, Paily KP, Padmanaban V and Balaraman K (2003) Oviposition response of the mosquito, Culex quinquefasciatus to the secondary metabolite(s) of the fungus, Trichoderma viride. Mem Inst Oswaldo Cruz 98: 223-226. 
Geier M and Boeckh J (1999) A new Y-tube olfactometer for mosquitoes to measure the attractiveness of host odours. Entomol Exp Appl 92: 9-19.

Geier M, Bosch OJ and Boeckh J (1999) Ammonia as an attractive component of host odour for the yellow fever mosquito, Aedes aegypti. Chem Senses 24: 647-653.

Gerry AC, Sarto i Monteys V, Moreno Vidal JO, Francino O and Mullens BA (2009) Biting rates of Culicoides midges (Diptera: Ceratopogonidae) on sheep in northeastern Spain in relation to midge capture using UV light and carbon dioxide-baited traps. J Med Entomol 46: 615-624.

Gibson G and Torr SJ (1999) Visual and olfactory responses of haematophagous Diptera to host stimuli. Med Vet Entomol 13: 2-23.

Gimonneau G, Alioum Y, Abdoulmoumini M, Zoli A, Cene B, Adakal H and Bouyer J (2016) Insecticide and repellent mixture pour-on protects cattle against animal trypanosomosis. PLoS Negl Trop Dis 10: e0005248.

Gimonneau G, Bouyer J, Morand S, Besansky NJ, Diabate A and Simard F (2010) A behavioral mechanism underlying ecological divergence in the malaria mosquito Anopheles gambiae. Behav Ecol 21: 1087-1092.

Girod R, Guidez A, Carinci R, Issaly J, Gaborit P, Ferrero E, Ardillon V, Fontaine A, Dusfour I and Briolant S (2016) Detection of Chikungunya virus circulation using sugar-baited traps during a major outbreak in French Guiana. PLoS Negl Trop Dis 10: e0004876.

Gladney WJ, Grabbe RR, Ernst SE and Oehler DD (1974) The Gulf coast tick: evidence of a pheromone produced by males. J Med Entomol 11: 303-306.

Greathead H (2003) Plants and plant extracts for improving animal productivity. Proc Nutr Soc 62: 279-290.

Greeff JC, Biggs A, Grewar W and Crumblin P (2013) Dogs can differentiate between odours from sheep that are resistant or susceptible to breech strike. Proc Twent Conf Assoc Adv Anim Breed Genet 20: 397-400.

Guerrero FD, Jamroz RC, Kammlah D and Kunz SE (1997) Toxicological and molecular characterization of pyrethroidresistant horn flies, Haematobia irritans: identification of kdr and super-kdr point mutations. Insect Biochem Mol Biol 27: 745-755.

Guglielmone AA, Gimeno E, Idiart J, Fisher WF, Volpogni MM, Quaino O, Anziani OS, Flores SG and Warnke O (1999) Skin lesions and cattle hide damage from Haematobia irritans infestations. Med Vet Entomol 13: 324-329.

Haggart DA and Davis EE (1980) Ammonia-sensitive neurones on the first tarsi of the tick, Rhipicephalus sanguineus. J Insect Physiol 26: 517-523.

Hall-Mendelin S, Ritchie SA, Johansen CA, Zborowski P, Cortis G, Dandridge S, Hall RA, and Van den Hurk AF (2010) Exploiting mosquito sugar feeding to detect mosquito-borne pathogens. Proc Natl Acad Sci 107: 11255-11259.

Hall DR, Beevor PS, Cork A, Nesbitt BF and Vale GA (2011) 1-Octen-3-ol. A potent olfactory stimulant and attractant for tsetse isolated from cattle odours. Int J Trop Insect Sci 5: 335-339.

Hall MJ, Farkas R, Kelemen F, Hosier MJ and El-Khoga JM (1995) Orientation of agents of wound myiasis to hosts and artificial stimuli in Hungary. Med Vet Entomol 9: 77-84.

Hamilton JGC and Sonenshine DE (1989) Methods and apparatus for controlling arthropod populations. Patent US4884361A. Available at: https://patents.google.com/patent/US4884361A/en.

Hansson BS (2002) A bug's smell - research into insect olfaction. Trends Neurosci 25: 270-274.

Hargrove JW (1988) Tsetse: the limits to population growth. Med Vet Entomol 2: 203-217.

Hargrove JW, Holloway MTP, Vale GA, Gough AJE and Hall DR (1995) Catches of tsetse (Glossina spp.) (Diptera: Glossinidae) from traps and targets baited with large doses of natural and synthetic host odor. Bull Entomol Res 85: 215-227.

Harrup LE, Logan JG, Cook Jl, Golding N, Birkett MA, Pickett JA, Sanders C, Barber J, Rogers DJ, Mellor PS, Purse BV and Carpenter S (2012) Collection of Culicoides (Diptera: Ceratopogonidae) using $\mathrm{CO}_{2}$ and enantiomers of 1-octen-3-ol in the United Kingdom. J Med Entomol 49: 112-121.

Hassanali A, McDowell PG, Owaga MLA and Saini RK (1986) Identification of tsetse attractants from excretory products of a wild host animal, Syncerus caffer. Insect Sci Appl 7: 5-9.

Hastie LC, Wallace C, Birkett MA, Douglas A, Jones O, Mordue (Luntz) AJ, Ritchie G, Pickett JA, Webster JL and Bowman AS (2013) Prevalence and infection intensity of sea lice (Lepeophtheirus salmonis) on Atlantic salmon (Salmo salar) host is reduced by the non-host compound 2-aminoacetophenone. Aquaculture 410-411: 179-183. 
Hasty JM, Yang P, Oshiro P, Nakasone L and Whelen C (2015) Mosquito surveillance program using ovitraps detected Aedes aegypti at the Honolulu International Airport in 2012. Proc Hawaiin Entomol Soc 47: 1-11.

Hayes RO, Doane OW, Sakolsky G and Berrick S (1993) Evaluation of attractants in traps for Greenhead fly (Diptera: Tabanidae) collections on a Cape-Cod, Massachusetts, salt-marsh. J Am Mosq Control Assoc 9: 436-440.

Hayes EB, Komar N, Nasci RS, Montgomery SP, O'Leary DR and Campbell GL (2005) Epidemiology and transmission dynamics of West Nile virus disease. Emerg Infect Dis 11:1167-1173.

Hiscox A, Homan T, Mweresa CK, Maire N, Di Pasquale A, Masiga D, Oria PA, Alaii J, Leeuwis C, Mukabana WR, Takken W and Smith TA (2016) Mass mosquito trapping for malaria control in western Kenya: study protocol for a stepped wedge cluster-randomised trial. Trials 17: 356.

Hiscox A, Maire N, Kiche I, Silkey M, Homan T, Oria P, Mweresa CK, Otieno B, Ayugi M, Bousema T, Sawa P, Alaii J, Smith T, Leeuwis C, Mukabana WR and Takken W (2012) The SolarMal Project: innovative mosquito trapping technology for malaria control. Malar J: S15.

Holloway MTP and Phelps RJ (1991) The responses of Stomoxys spp. (Diptera: Muscidae) to traps and artificial host odours in the field. Bull Entomol Res 81: 51-55.

Homan T, Hiscox A, Mweresa CK, Masiga D, Mukabana WR, Oria P, Maire N, Di Pasquale A, Silkey M, Alaii J and Bousema $T$ (2016) The effect of mass mosquito trapping on malaria transmission and disease burden (SolarMal): a steppedwedge cluster-randomised trial. Lancet 388: 207-211.

Horváth G, Blahó M, Kriska G, Hegedüs R, Gerics B, Farkas R and Akesson S (2010) An unexpected advantage of whiteness in horses: the most horsefly-proof horse has a depolarizing white coat. Proc R Soc B Biol Sci 277: 1643-1650.

Horváth G, Majer J, Horváth L, Szivák I and Kriska G (2008) Ventral polarization vision in tabanids: horseflies and deerflies (Diptera: Tabanidae) are attracted to horizontally polarized light. Naturwissenschaften 95: 1093-1100.

Hougaard BR and Dickson SL (1999) The Mosquito Magnet: a new tool in controlling tree hole mosquitoes. Proc $52^{\text {nd }}$ Annu Meet Utah Mosq Abat Assoc Park City UT: 4-8.

lovinella I, Pelosi P and Conti B (2014) A rationale to design longer lasting mosquito repellents. Parasitol Res 113: $1813-1820$.

Isberg E, Bray DP, Birgersson G, Hillbur Y and Ignell R (2016) Identification of cattle-derived volatiles that modulate the behavioral response of the biting midge Culicoides nubeculosus. J Chem Ecol 42: 24-32.

Ivanov VP (2007) Investigation of the sensory organs on antennae of the horseflies Hybomitra bimaculata and Tabanus bovinus (Diptera: Tabanidae) by scanning electron microscope. Parazitologiia 41: 372-380.

Jacquet $S$, Huber K, Pagès N, Talavera S, Burgin LE, Carpenter S, Sanders C, Dicko AH, Djerbal M, Goffredo M, Lhor $Y$, Lucientes J, Miranda-Chueca MA, Pereira da Fonseca I, Ramilo DW, Setier-Rio M-L, Bouyer J, Chevillon C, Balenghien T, Guis H and Garros C (2016) Range expansion of the Bluetongue vector, Culicoides imicola, in continental France likely due to rare wind-transport events. Sci Rep 6: 27247.

Jacquiet P, Liénard E and Franc M (2010) Bovine besnoitiosis: epidemiological and clinical aspects. Vet Parasitol 174: 30-36.

Jaramillo Ramirez GI, Logan JG, Loza-Reyes E, Stashenko E and Moores GD (2012) Repellents inhibit P450 enzymes in Stegomyia (Aedes) aegypti. PLoS ONE 7: e48698.

Jawara M, Smallegange RC, Jeffries D, Nwakanma DC, Awolola TS, Knols BGJ, Takken W and Conway DJ (2009) Optimizing odor-baited trap methods for collecting mosquitoes during the malaria season in The Gambia. PLoS ONE 4: e8167.

Jeanbourquin P and Guerin PM (2007a) Sensory and behavioural responses of the stable fly Stomoxys calcitrans to rumen volatiles. Med Vet Entomol 21:217-224.

Jeanbourquin P and Guerin PM (2007b) Chemostimuli implicated in selection of oviposition substrates by the stable fly Stomoxys calcitrans. Med Vet Entomol 21: 209-216.

Jensen KMV, Jespersen JB, Birkett MA, Pickett JA, Thomas G, Wadhams LJ and Woodcock CM (2004) Variation in the load of the horn fly, Haematobia irritans, in cattle herds is determined by the presence or absence of individual heifers. Med Vet Entomol 18: 275-280.

Jensen LB, Provan F, Larssen E, Bron JE and Obach A (2014) Reducing sea lice (Lepeophtheirus salmonis) infestation of farmed Atlantic salmon (Salmo salar L.) through functional feeds. Aquac Nutr 21: 983-993. 
Jhumur US, Dötterl S and Jürgens A (2006) Naïve and conditioned responses of Culex pipiens pipiens biotype molestus (Diptera: Culicidae) to flower odors. J Med Entomol 43: 1164-1170.

Jhumur US, Dötterl S and Jürgens A (2008) Floral odors of Silene otites: their variability and attractiveness to mosquitoes. J Chem Ecol 34: 14-25.

Jongejan F and Uilenberg G (2004) The global importance of ticks. Parasitol 129: S3-S14.

Jonsson NN, Davis R and de Witt M (2001) An estimate of the economic effects of cattle tick (Boophilus microplus) infestation on Queensland dairy farms. Aust Vet J 79: 826-831.

Joseph SR (1970) Fruit feeding of mosquitoes in nature. Proc Annu Meet New Jersey Mosq Exterm 57: 125-131.

Kabayo JP (2002) Aiming to eliminate tsetse from Africa. Trends Parasitol 18: 473-475.

Kaufmann C, Mathis A and Vorburger C (2015) Sugar-feeding behaviour and longevity of European Culicoides biting midges. Med Vet Entomol 29: 17-25.

Kaur JS, Lai YL and Giger AD (2003) Learning and memory in the mosquito Aedes aegypti shown by conditioning against oviposition deterrence. Med Vet Entomol 17: 457-460.

Kettle DS (1996) The Scottish midge, Culicoides impunctatus Goetghebuer (Diptera: Ceratopogonidae), an early attempt (1945-1958) to control this intractable pest. Mem Entomol Soc Washingt 18: 134-139.

Khalil GM (1984) Fecundity-reducing pheromone in Argas (Persicargas) arboreus (Ixodoidea: Argasidae). Parasitol 88: 395.

Khallaayoune K, Qualls WA, Revay EE, Allan SA, Arheart KL, Kravchenko VD, Xue RD, Schlein Y, Beier JC and Muller GC (2013) Attractive toxic sugar baits: control of mosquitoes with the low-risk active ingredient dinotefuran and potential impacts on nontarget organisms in Morocco. Environ Entomol 42: 1040-1045.

Khan AA, Maibach HI and Skidmore DL (1975) Addition of vanillin to mosquito repellents to increase protection time. Mosq News 35: 223-225.

Killeen GF, Okal MN, Herrera-Varela M, Borg-Karlson A-K, Torto B, Lindsay SW and Fillinger U (2014) Characterizing, controlling and eliminating residual malaria transmission. Malar J 13: 330.

Kivaria FM (2006) Estimated direct economic costs associated with tick-borne diseases on cattle in Tanzania. Trop Anim Health Prod 38: 291-299.

Kline DL (2006) Traps and trapping techniques for adult mosquito control. J Am Mosq Control Assoc 22: 490-496.

Kline DL, Dame DA and Meisch MV (1991) Evaluation of 1-octen-3-ol and carbon dioxide as attractants for mosquitoes associated with irrigated rice fields in Arkansas. J Am Mosq Control Assoc 7: 165-169.

Kline DL, Hagan DV and Wood JR (1994) Culicoides responses to 1-octen-3-ol and carbon dioxide in salt marshes near Sea Island, Georgia, U.S.A. Med Vet Entomol 8: 25-30.

Kline DL, Takken W, Wood JR and Carlson DA (1990) Field studies on the potential of butanone, carbon dioxide, honey extract, 1-octen-3-ol, L-lactic acid and phenols as attractants for mosquitoes. Med Vet Entomol 4:383-391.

Knudsen JT (2002) Variation in floral scent composition within and between populations of Geonoma macrostachys (Arecaceae) in the western Amazon. Am J Bot 89: 1772-1778.

Knudsen JT, Tollsten L and Bergström LG (1993) Floral scents - a checklist of volatile compounds isolated by head-space techniques. Phytochemistry 33: 253-280.

Krčmar S (2005) Response of horse flies (Diptera, Tabanidae) to different olfactory attractants. Biologia (Bratisl) 60:611-613.

Krčmar S and Lajos P (2011) Response of horse flies to aged equine urine (Diptera: Tabanidae). Entomol Gen 33: 245-250.

Krčmar S, Mikuška A and Merdić E (2006) Response of Tabanidae (Diptera) to different natural attractants. J Vector Ecol 31: 262-265.

Krčmar S, Mikuška A and Radolić V (2010) Comparison of sampling tabanids (Diptera: Tabanidae) by four different potential attractants. J Appl Entomol 134: 608-613.

Kriska G, Bernáth B, Farkas R and Horváth G (2009) Degrees of polarization of reflected light eliciting polarotaxis in dragonflies (Odonata), mayflies (Ephemeroptera) and tabanid flies (Tabanidae). J Insect Physiol 55: 1167-1173.

Kröckel U, Rose A, Eiras ÁE and Geier M (2006) New tools for surveillance of adult yellow fever mosquitoes: comparison of trap catches with human landing rates in an urban environment. J Am Mosq Control Assoc 22: 229-238.

Küpper W, Staak C, Kröber T and Späth J (1990) Natural hosts of Glossina tachinoides (Diptera: Glossinidae) in northern Côte d'Ivoire. Trop Med Parasitol 41:217-218. 
Lacroix R, Delatte H, Hue T and Reiter P (2009) Dispersal and survival of male and female Aedes albopictus (Diptera: Culicidae) on Réunion Island. J Med Entomol 46: 1117-1124.

Lacroix R, Mukabana WRR, Gouagna LC and Koella JC (2005) Malaria infection increases attractiveness of humans to mosquitoes. PLoS Biol 3: e298.

De Lacy Costello B, Amann A, Al-Kateb H, Flynn C, Filipiak W, Khalid T, Osborne D and Ratcliffe NM (2014) A review of the volatiles from the healthy human body. J Breath Res 8: 14001.

Lane RP and Ward RD (1984) The morphology and possible function of abdominal patches in males of two forms of Lutzomyia longipalpis (Diptera: Phlebotominae). Ser Entomol Medicale Parasitol 22: 245-249.

Laurence BR and Pickett JA (1982) Erythro-6-acetoxy-5-hexadecanolide, the major component of a mosquito oviposition attractant pheromone. J Chem Soc Chem Commun 1:59-60.

Laurence BR and Pickett JA (1985) An oviposition attractant pheromone in Culex quinquefasciatus Say (Diptera: Culicidae). Bull Entomol Res 75: 283.

Lee HL and Rohani A (2005) Transovarial transmission of Dengue virus in Aedes aegypti and Aedes albopictus in relation to Dengue outbreak in an urban area in Malaysia. Dengue Bull 29: 106.

Le Goff G, Damiens D, Payet L, Ruttee A-H, Jean F, Lebon C, Dehecq J-S and Gouagna L-C (2016) Enhancement of the BG-sentinel trap with varying number of mice for field sampling of male and female Aedes albopictus mosquitoes. Parasit Vectors 9: 514.

Le Goff G, Revollo J, Guerra M, Cruz M, Barja Simon Z, Roca Y, Vargas Florès J and Hervé JP (2011) Natural vertical transmission of dengue viruses by Aedes aegypti in Bolivia. Parasite 18: 277-280.

Leonard DE and Saini RK (1993) Semiochemicals from anal exudate of larvae of tsetse flies Glossina morsitans morsitans Westwood and G. morsitans centralis Machado attract gravid females. J Chem Ecol 19: 2039-2046.

Leprince DJ, Hribar LJ and Foil LD (1994) Responses of horse flies (Diptera: Tabanidae) to Jersey bullocks and canopy traps baited with ammonia, octenol, and carbon dioxide. J Med Entomol 31: 729-731.

Lewis DJ and Domoney CR (1966) Sugar meals in Phlebotominae and Simuliidae (Diptera). Proc R Entomol Soc London Ser A, Gen Entomol 41: 175-179.

Lindsay S, Ansell J, Selman C, Cox V, Hamilton K and Walraven G (2000) Effect of pregnancy on exposure to malaria mosquitoes. Lancet 355: 1972.

Logan JG and Birkett MA (2007) Semiochemicals for biting fly control: their identification and exploitation. Pest Manag Sci 63: 647-657.

Logan JG, Birkett MA, Clark SJ, Powers S, Seal NJ, Wadhams LJ, Mordue (Luntz) AJ and Pickett JA (2008) Identification of human-derived volatile chemicals that interfere with attraction of Aedes aegypti mosquitoes. J Chem Ecol 34: 308-322.

Logan JG, Cook Jl, Mordue J and Kline DL (2010a) Understanding and exploiting olfaction for the surveillance and control of Culicoides biting midges. In: Takken W and Knols BG (eds.) Olfaction in vector-host interactions. Wageningen Academic Publishers, Wageningen, the Netherlands.

Logan JG, Seal NJ, Cook JI, Stanczyk NM, Birkett MA, Clark SJ, Gezan SA, Wadhams LJ, Pickett JA and Mordue AJ (2009) Identification of human-derived volatile chemicals that interfere with attraction of the Scottish biting midge and their potential use as repellents. J Med Entomol 46: 208-219.

Logan JG, Stanczyk NM, Hassanali A, Kemei J, Santana AEG, Ribeiro KAL, Pickett JA and Mordue (Luntz) AJ (2010b) Armin-cage testing of natural human-derived mosquito repellents. Malar J 9: 239.

Van Loon JJA, Smallegange RC, Bukovinszkiné-Kiss G, Jacobs F, De Rijk M, Mukabana WR, Verhulst NO, Menger DJ and Takken W (2015) Mosquito attraction: crucial role of carbon dioxide in formulation of a five-component blend of human-derived volatiles. J Chem Ecol 41:567-573.

Lothrop HD, Wheeler SS, Fang Y and Reisen WK (2012) Use of scented sugar bait stations to track mosquito-borne arbovirus transmission in California. J Med Entomol 49: 1466-1472.

Louly CCB, Silveira DDN, Soares SF, Ferri PH, De Melo ACC and Borges LMF (2008) More about the role of 2,6-dichlorophenol in tick courtship: identification and olfactometer bioassay in Amblyomma cajennense and Rhipicephalus sanguineus. Mem Inst Oswaldo Cruz 103: 60-65. 
Lowther JK, Wood DM, Anderson RC, Bennett GF, Davies DM and Peterson BV (1964) Specificity of a black fly, Simulium euryadminiculum Davies, toward its host, the common loon. Can Entomol 96: 911-913.

Lühken R, Pfitzner WP, Börstler J, Garms R, Huber K, Schork N, Steinke S, Kiel E, Becker N, Tannich E and Krüger A (2014) Field evaluation of four widely used mosquito traps in Central Europe. Parasit Vectors 7: 268.

Lyimo IN and Ferguson HM (2009) Ecological and evolutionary determinants of host species choice in mosquito vectors. Trends Parasitol 25: 189-196.

Maciel-de-Freitas R, Eiras ÁE, and Lourenço-de-Oliveira R (2006) Field evaluation of effectiveness of the BG-Sentinel, a new trap for capturing adult Aedes aegypti (Diptera: Culicidae). Mem Inst Oswaldo Cruz 101: 321-325.

Magalhães-Junior JT, Mesquita PRR, Dos Santos Oliveira WF, Oliveira FS, Franke CR, De Medeiros Rodrigues F, De Andrade $J B$, and Barrouin-Melo SM (2014) Identification of biomarkers in the hair of dogs: new diagnostic possibilities in the study and control of visceral leishmaniasis. Anal Bioanal Chem 406: 6691-6700.

Magallanes S, Møller AP, García-Longoria L, De Lope F and Marzal A (2016) Volume and antimicrobial activity of secretions of the uropygial gland are correlated with malaria infection in house sparrows. Parasit Vectors 9: 232.

Magnarelli LA and Anderson JF (1981) Sugar feeding by female tabanids (Diptera: Tabanidae) and its relation to gonotrophic activity. J Med Entomol 18: 429-433.

Mands V, Kline DL and Blackwell A (2004) Culicoides midge trap enhancement with animal odour baits in Scotland. Med Vet Entomol 18: 336-342.

Maresca C, Scoccia E, Faccenda L, Zema J and Costarelli S (2012) Equine infectious anemia: active surveillance in central Italy 2007-2009. J Equine Vet Sci 32: 596-598.

Martín-Vivaldi M, Peña A, Peralta-Sánchez JM, Sánchez L, Ananou S, Ruiz-Rodríguez M, and Soler JJ (2010) Antimicrobial chemicals in hoopoe preen secretions are produced by symbiotic bacteria. Proc R Soc B Biol Sci 277: 123-130.

Martínez-de la Puente J, Rivero-de Aguilar J, Cerro S Del, Argüello A and Merino S (2011) Do secretions from the uropygial gland of birds attract biting midges and black flies? Parasitol Res 109: 1715-1718.

Mascari TM, Mitchell MA, Rowton ED and Foil LD (2007) Laboratory evaluation of diflubenzuron as a feed-through for control of immature sand flies (Diptera: Psychodidae). J Med Entomol 44: 171-174.

Mascari TM, Mitchell MA, Rowton ED and Foil LD (2008) Ivermectin as a rodent feed-through insecticide for control of immature sand flies (Diptera: Psychodidae). J Am Mosq Control Assoc 24: 323-326.

Mascari TM, Stout RW and Foil LD (2012) Evaluation of three feed-through insecticides using two rodent and two sand fly species as models. J Am Mosq Control Assoc 28: 260-262.

McBride CS, Baier F, Omondi AB, Spitzer SA, Lutomiah J, Sang R, Ignell R and Vosshall LB (2014) Evolution of mosquito preference for humans linked to an odorant receptor. Nature 515: 222-227.

McCall PJ (1995) Oviposition aggregation pheromone in the Simulium damnosum complex. Med Vet Entomol 9: 101-108.

McCall PJ (2002) Chemoecology of oviposition in insects of medical and veterinary importance. In: Hilker M and Meiners $\mathrm{T}$ (eds.) Chemoecology of insect eggs and egg deposition. Blackwell Publishing Ltd., Oxford, UK.

McCall PJ and Cameron MM (1995) Oviposition pheromones in insect vectors. Parasitol Today 11: 352-355.

McCall PJ, Heath RR, Dueben BD and Wilson MD (1997a) Oviposition pheromone in the Simulium damnosum complex: biological activity of chemical fractions from gravid ovaries. Physiol Entomol 22: 224-230.

McCall PJ, Trees AJ, Walsh JF and Molyneux DH (1994) Aggregated oviposition in the Simulium damnosum complex is mediated by eggs in a laboratory bioassay. Med Vet Entomol 8: 76-80.

McCall PJ, Wilson MD, Dueben BD, De Clare Bronsvoort BM and Heath RR (1997b) Similarity in oviposition aggregation pheromone composition within the Simulium damnosum (Diptera: Simuliidae) species complex. Bull Entomol Res 87: 609-616.

McElligott PE and Mclver SB (1987) Range of attractiveness of carbon dioxide to Hybomitra spp. (Diptera: Tabanidae). J Am Mosq Control Assoc 3: 655-656.

McIndoo NE (1926) An insect olfactometer. J Econ Entomol 19: 545-571.

Mclver SB (1982) Sensilla of mosquitoes (Diptera: Culicidae). J Med Entomol 19: 489-535.

Mead DG, Gray EW, Noblet R, Murphy MD, Howerth EW and Stallknecht DE (2004) Biological transmission of vesicular stomatitis virus (New Jersey serotype) by Simulium vittatum (Diptera: Simuliidae) to domestic swine (Sus scrofa). J Med Entomol 41: 78-82. 
Meiswinkel R, Baldet T, De Deken R, Takken W, Delécolle JC and Mellor PS (2008) The 2006 outbreak of bluetongue in northern Europe - the entomological perspective. Prev Vet Med 87: 55-63.

Meiswinkel R, Baylis M, and Labuschagne K (2000) Stabling and the protection of horses from Culicoides bolitinos (Diptera: Ceratopogonidae), a recently identified vector of African horse sickness. Bull Entomol Res 90: 509-515.

Mellor PS, Boorman J and Baylis M (2000) Culicoides biting midges: their role as arbovirus vectors. Annu Rev Entomol 45: 307-340.

Mellor PS and McCaig J (1974) The probable cause of 'sweet itch' in England. Vet Rec 95: 411-415.

Menger DJ, Van Loon JJA and Takken W (2014) Assessing the efficacy of candidate mosquito repellents against the background of an attractive source that mimics a human host. Med Vet Entomol 28: 407-413.

Meyer A, Holt HR, Selby R and Guitian J (2016) Past and ongoing tsetse and animal trypanosomiasis control operations in five African countries: a systematic review. PLoS Negl Trop Dis 10: e0005247.

Michaelakis A, Mihou AP, Koliopoulos G and Couladouros EA (2007) Attract-and-kill strategy. Laboratory studies on hatched larvae of Culex pipiens. Pest Manag Sci 63: 954-959.

Mihok S, Carlson DA and Ndegwa PN (2007) Tsetse and other biting fly responses to Nzi traps baited with octenol, phenols and acetone. Med Vet Entomol 21: 70-84.

Mihok S, Kang'ethe EK and Kamau GK (1995) Trials of traps and attractants for Stomoxys spp. (Diptera: Muscidae). J Med Entomol 32: 283-289.

Mihok S and Lange K (2012) Synergism between ammonia and phenols for Hybomitra tabanids in northern and temperate Canada. Med Vet Entomol 26: 282-290.

Mihok S and Mulye H (2010) Responses of tabanids to Nzi traps baited with octenol, cow urine and phenols in Canada. Med Vet Entomol 24: 266-272.

Milet-Pinheiro P, Ayasse M, Dobson HEM, Schlindwein C, Francke W and Dötterl S (2013) The chemical basis of host-plant recognition in a specialized bee pollinator. J Chem Ecol 39: 1347-1360.

Miller RW and Corley C (1980) Feed-through efficacy of CGA-19255 and CGA-72662 against manure-breeding flies and other arthropods and residues in feces, eggs, and tissues of laying hens. Southwest Entomol 5: 144-148.

Miller JA, Kunz SE, Oehler DD and Miller RW (1980) Larvicidal activity of Merck MK-933, an avermectin, against the horn fly, stable fly, face fly, and house fly. J Econ Entomol 74: 608-611.

Miller DL, Mauel MJ, Baldwin C, Burtle G, Ingram D, Hines ME and Frazier KS (2003) West Nile virus in farmed alligators. Emerg Infect Dis 9: 794-799.

Mohamed-Ahmed MM, Abdulla MA and Mohamed Y (2007) Trapability of periurban populations of horseflies (Diptera: Tabanidae) in Khartoum State, Sudan. J Sci Technol 8: 46-64.

Mohr RM, Mullens BA and Gerry AC (2011) Diel patterns of female host seeking, male swarming, and sugar feeding in Fannia conspicua (Diptera: Muscidae) in southern California. J Med Entomol 48: 188-195.

De Moraes CM, Stanczyk NM, Betz HS, Pulido H, Sim DG, Read AF and Mescher MC (2014) Malaria-induced changes in host odors enhance mosquito attraction. Proc Natl Acad Sci 111: 11079-11084.

Mukabana WR, Mweresa CK, Otieno B, Omusula P, Smallegange RC, Van Loon JJA and Takken W (2012) A novel synthetic odorant blend for trapping of malaria and other African mosquito species. J Chem Ecol 38: 235-244.

Mullens BA and Gerry AC (1998) Comparison of bait cattle and carbon dioxide-baited suction traps for collecting Culicoides variipennis sonorensis (Diptera: Ceratopogonidae) and Culex quinquefasciatus (Diptera: Culicidae). J Med Entomol 35: 245-250.

Mullens BA, Lii KS, Mao Y, Meyer JA, Peterson NG, and Szijj CE (2006) Behavioural responses of dairy cattle to the stable fly, Stomoxys calcitrans, in an open field environment. Med Vet Entomol 20: 122-137.

Mullens BA, Peterson N, Dada CE and Velten RK (1995) Octenol fails to lure stable fly to insecticide. Calif Agric 49: 16-18.

Müller GC, Beier JC, Traore SF, Toure MB, Traore MM, Bah S, Doumbia S and Schlein Y (2010a) Successful field trial of attractive toxic sugar bait (ATSB) plant-spraying methods against malaria vectors in the Anopheles gambiae complex in Mali, West Africa. Malar J 9: 210.

Müller GC, Beier JC, Traore SF, Toure MB, Traore MM, Bah S, Doumbia S and Schlein Y (2010b) Field experiments of Anopheles gambiae attraction to local fruits/seedpods and flowering plants in Mali to optimize strategies for malaria vector control in Africa using attractive toxic sugar bait methods. Malar J 9: 262. 
Müller GC, Junnila A, Qualls W, Revay EE, Kline DL, Allan S, Schlein Y and Xue R (2010c) Control of Culex quinquefasciatus in a storm drain system in Florida using attractive toxic sugar baits. Med Vet Entomol 24: 346-351.

Müller GC, Junnila A, Schlein Y and Ller GCM (2010d) Effective control of adult Culex pipiens by spraying an attractive toxic sugar bait solution in the vegetation near larval habitats. J Med Entomol 47: 63-66.

Müller GC, Kravchenko VD and Schlein Y (2008) Decline of Anopheles sergentii and Aedes caspius populations following presentation of attractive toxic (spinosad) sugar bait stations in an oasis. J Am Mosq Control Assoc 24: 147-149.

Müller GC, Revay EE and Beier JC (2011a) Simplified and improved monitoring traps for sampling sand flies. J Vector Ecol 36: 454-457.

Müller GC and Schlein Y (2008) Efficacy of toxic sugar baits against adult cistern-dwelling Anopheles claviger. Trans R Soc Trop Med Hyg 102: 480-484.

Müller GC and Schlein Y (2011) Different methods of using attractive sugar baits (ATSB) for the control of Phlebotomus papatasi. J Vector Ecol 36: S64-S70.

Müller GC, Xue RD and Schlein Y (2011b) Differential attraction of Aedes albopictus in the field to flowers, fruits and honeydew. Acta Trop 118: 45-49.

Mwandawiro C, Boots M, Tuno N, Suwonkerd W, Tsuda Y and Takagi M (2000) Heterogeneity in the host preference of Japanese encephalitis vectors in Chiang Mai, northern Thailand. Trans R Soc Trop Med Hyg 94: 238-242.

Mweresa CK, Omusula P, Otieno B, Van Loon JJA, Takken W and Mukabana WR (2014) Molasses as a source of carbon dioxide for attracting the malaria mosquitoes Anopheles gambiae, and Anopheles funestus. Malar J 13: 160.

Navarro-Silva MA, Marques FA and Duque LJE (2009) Review of semiochemicals that mediate the oviposition of mosquitoes: a possible sustainable tool for the control and monitoring of Culicidae. Rev Bras Entomol 53: 1-6.

Ndeledje N, Bouyer J, Stachurski F, Grimaud P, Belem AMG, Molélé Mbaïndingatoloum M, Bengaly Z, Oumar Alfaroukh I, Cecchi G and Lancelot R (2013) Treating cattle to protect people? Impact of footbath insecticide treatment on tsetse density in Chad. PLoS ONE 8: e67580.

Newhouse VF and Chamberlain RW (1966) Use of dry ice to increase mosquito catches of the CDC miniature light trap. Mosq News 26: 30-35.

Noguchi H, Shannon RC, Tilden EB and Tyler JR (1929) Etiology of Oroya fever: XIV. The insect vectors of Carrion's disease. J Exp Med 49: 993-1008.

Nordlund DA and Lewis WJ (1976) Terminology of chemical releasing stimuli in intraspecific and interspecific interactions. J Chem Ecol 2: 211-220.

Norval RAI, Sonenshine DE, Allan SA and Burridge MJ (1996) Efficacy of pheromone-acaricide-impregnated tail-tag decoys for controlling the bont tick, Amblyomma hebraeum (Acari: Ixodidae), on cattle in Zimbabwe. Exp Appl Acarol 20: 31-46.

Nyasembe VO and Torto B (2014) Volatile phytochemicals as mosquito semiochemicals. Phytochem Lett 8: 196-201.

Ocaido M, Muwazi RT and Opuda JA (2009) Economic impact of ticks and tick-borne diseases on cattle production systems around Lake Mburo National Park in South Western Uganda. Trop Anim Health Prod 41: 731-779.

Ogoma SB, Ngonyani H, Simfukwe ET, Mseka A, Moore J, Maia MF, Moore SJ and Lorenz LM (2014) The mode of action of spatial repellents and their impact on vectorial capacity of Anopheles gambiae sensu stricto. PLoS ONE 9: e1 10433.

Okumu FO, Killeen GF, Ogoma S, Biswaro L, Smallegange RC, Mbeyela E, Titus E, Munk C, Ngonyani H, Takken W, Mshinda $\mathrm{H}$, Mukabana WR and Moore SJ (2010) Development and field evaluation of a synthetic mosquito lure that is more attractive than humans. PLoS ONE 5: e8951.

Olsson SB and Hansson BS (2013) Electroantennogram and single sensillum recording in insect antennae. In: Touhara $\mathrm{K}$ (ed.) Pheromone signaling: methods and protocols. Humana Press, New York, NY, USA.

Owaga MLA, Hassanali A and McDowell PG (1988) The role of 4-cresol and 3-n-propylphenol in the attraction of tsetse flies to buffalo urine. Insect Sci Appl 9: 95-100.

Owino EA, Sang R, Sole CL, Pirk C, Mbogo C and Torto B (2015) An improved odor bait for monitoring populations of Aedes aegypti vectors of dengue and chikungunya viruses in Kenya. Parasit Vectors 8: 253.

Oyarzún MP, Palma R, Alberti E, Hormazabal E, Pardo F, Birkett MA and Quiroz A (2009) Olfactory response of Haematobia irritans (Diptera: Muscidae) to cattle-derived volatile compounds. J Med Entomol 46: 1320-1326. 
Oyarzún MP, Quiroz A and Birkett MA (2008) Insecticide resistance in the horn fly: alternative control strategies. Med Vet Entomol 22: 188-202.

Page PC, Labuschagne K, Nurton JP, Venter GJ and Guthrie AJ (2009) Duration of repellency of N,N-diethyl-3methylbenzamide, citronella oil and cypermethrin against Culicoides species when applied to polyester mesh. Vet Parasitol 163: 105-109.

Parashar BD, Chauhan RS, Prakash S and Rao KM (1994) Mechanotactile and olfactory antennal sensilla in four species of female tabanids (Diptera). Bolletino di Zool 61: 121-128.

Pare PW and Tumlinson JH (1999) Plant volatiles as a defense against insect herbivores. Plant Physiol 121: 325-332.

Pepin M, Bouloy M, Bird BH, Kemp A and Paweska J (2010) Rift Valley fever virus (Bunyaviridae: Phlebovirus): an update on pathogenesis, molecular epidemiology, vectors, diagnostics and prevention. Vet Res 41:61.

Perich MJ, Kardec A, Braga IA, Portal IF, Burge R, Zeichner BC, Brogdon WA and Wirtz RA (2003) Field evaluation of a lethal ovitrap against dengue vectors in Brazil. Med Vet Entomol 17: 205-210.

Phelps RJ and Vale GA (1976) Studies on the local distribution and on the methods of host location of some Rhodesian Tabanidae (Diptera). J Entomol Soc South Afr 39: 67-81.

Pichersky E and Gershenzon J (2002) The formation and function of plant volatiles: perfumes for pollinator attraction and defense. Curr Opin Plant Biol 5: 237-243.

Pickett JA (1990) Gas chromatography-mass spectrometry in insect pheromone identification: three extreme case histories. In: McCaffery AR and Wilson ID (eds.) Chromatography and isolation of insect hormones and pheromones. Springer, Boston, MA, USA.

Pickett JA, Wadhams LJ and Woodcock CM (1998) Insect supersense: mate and host location by insects as model systems for exploiting olfactory interactions. Biochem 20: 8-13.

Pinto MC, Campbell-Lendrum DH, Lozovei AL, Teodoro U and Davies CR (2001) Phlebotomine sandfly responses to carbon dioxide and human odour in the field. Med Vet Entomol 15: 132-139.

Ponnusamy L, Xu N, Nojima S, Wesson DM, Schal C and Apperson CS (2008) Identification of bacteria and bacteriaassociated chemical cues that mediate oviposition site preferences by Aedes aegypti. Proc Natl Acad Sci 105: 9262-9267.

Posey KH, Barnard DR and Schreck CE (1998) Triple cage olfactometer for evaluating mosquito (Diptera: Culicidae) attraction responses. J Med Entomol 35: 330-334.

Purse BV, Mellor PS, Rogers DJ, Samuel AR, Mertens PPC and Baylis M (2005) Climate change and the recent emergence of bluetongue in Europe. Nat Rev Microbiol 3: 171-181.

Qiu YT and Van Loon JJA (2010) Olfactory physiology of blood-feeding vector mosquitoes. In: Takken W and Knols BGJ (eds.) Olfaction in vector-host interactions. Wageningen Academic Publishers, Wageningen, the Netherlands, pp. 39-61.

Qiu YT, Smallegange RC and Van Loon JJA (2006) Interindividual variation in the attractiveness of human odours to the malaria mosquito Anopheles gambiae ss. Med Vet Entomol 20: 280-287.

Qiu YT, Smallegange RC, Van Loon JJA and Takken W (2011) Behavioural responses of Anopheles gambiae sensu stricto to components of human breath, sweat and urine depend on mixture composition and concentration. Med Vet Entomol 25: 247-255.

Qiu YT, Smallegange RC, Ter BCJF, Spitzen J, Van Loon JJA, Jawara M, Milligan P, Galimard AM, Van Beek TA, Knols BGJ and Takken W (2007) Attractiveness of MM-X traps baited with human or synthetic odor to mosquitoes (Diptera: Culicidae) in The Gambia. J Med Entomol 44: 970-983.

Qualls WA, Müller GC, Khallaayoune K, Revay EE, Zhioua E, Kravchenko VD, Arheart KL, Xue RD, Schlein Y, Hausmann A and Kline DL (2015) Control of sand flies with attractive toxic sugar baits (ATSB) and potential impact on non-target organisms in Morocco. Parasit Vectors 8: 87.

Qualls WA, Müller GC, Revay EE, Allan SA, Arheart KL, Beier JC, Smith ML, Scott JM, Kravchenko VD, Hausmann A, Yefremova ZA and Xue RD (2014) Evaluation of attractive toxic sugar bait (ATSB)-Barrier for control of vector and nuisance mosquitoes and its effect on non-target organisms in sub-tropical environments in Florida. Acta Trop 131: 104-110. 
Rapley LP, Johnson PH, Williams CR, Silcock RM, Larkman M, Long SA, Russell RC and Ritchie SA (2009) A lethal ovitrapbased mass for dengue control in Australia: II. Impact on trapping scheme populations of the mosquito Aedes aegypti. Med Vet Entomol 23: 303-316.

Rayaisse JB, Tirados I, Kaba D, Dewhirst SY, Logan JG, Diarrassouba A, Salou E, Omolo MO, Solano P, Lehane MJ, Pickett JA, Vale GA, Torr SJ and Esterhuizen J (2010) Prospects for the development of odour baits to control the tsetse flies Glossina tachinoides and G. palpalis s.l. PLoS Negl Trop Dis 4: e632.

Rechav Y, Norval RAl, Tannock J and Colborne J (1978) Attraction of the tick Ixodes neitzi to twigs marked by the klipspringer antelope. Nature 275:310-311.

Rechav Y and Whitehead GB (1978) Field trials with pheromone-acaricide mixtures for control of Amblyomma bebraeum. $J$ Econ Entomol 71: 149-151.

Reeves WK, Lloyd JE, Stobart R, Stith C, Miller MM, Bennett KE and Johnson G (2010) Control of Culicoides sonorensis (Diptera: Ceratopogonidae) blood feeding on sheep with long-lasting repellent pesticides. J Am Mosq Control Assoc 26: 302-305.

Reiter P (1983) A portable battery-powered trap for collecting gravid Culex mosquitoes. Mosq News 43: 496-498.

Revay EE, Müller GC, Qualls WA, Kline DL, Naranjo DP, Arheart KL, Kravchenko VD, Yefremova Z, Hausmann A, Beier JC, Schlein Y and Xue RD (2014) Control of Aedes albopictus with attractive toxic sugar baits (ATSB) and potential impact on non-target organisms in St. Augustine, Florida. Parasitol Res 113: 73-79.

Ritchie SA, Van Essen PH, Kemme JA, Kay BH and Allaway D (1994) Response of biting midges (Diptera: Ceratopogonidae) to carbon dioxide, octenol, and light in southeastern Queensland, Australia. J Med Entomol 31: 645-648.

Rodríguez-Pérez MA, Adeleke MA, Burkett-Cadena ND, Garza-Hernández JA, Reyes-Villanueva F, Cupp EW, Toé L, Salinas-Carmona MC, Rodríguez-Ramírez AD, Katholi CR and Unnasch TR (2013) Development of a novel trap for the collection of black flies of the Simulium ochraceum complex (CR Lazzari, Ed). PLoS ONE 8: e76814.

Romero A, Broce A and Zurek L (2006) Role of bacteria in the oviposition behaviour and larval development of stable flies. Med Vet Entomol 20: 115-121.

Russell CB and Hunter FF (2005) Attraction of Culex pipiens/restuans (Diptera: Culicidae) mosquitoes to bird uropygial gland odors at two elevations in the Niagara region of Ontario. J Med Entomol 42: 301-305.

Saini RK, Hassanali A, Andoke J, Ahuya P and Ouma WP (1996) Identification of major components of larviposition pheromone from larvae of tsetse flies Glossina morsitans morsitans Westwood Glossina morsitans centralis Machado. J Chem Ecol 22: 1211-1220.

Sang R, Kioko E, Lutomiah J, Warigia M, Ochieng C, O'Guinn M, Lee JS, Koka H, Godsey M, Hoel D, Hanafi H, Miller B, Schnabel D, Breiman RF and Richardson J (2010) Rift Valley fever virus epidemic in Kenya, 2006/2007: The entomologic investigations. Am J Trop Med Hyg 83: 28-37.

Santos E, Correia J, Muniz L, Meiado Mand Albuquerque C (2010) Oviposition activity of Aedes aegypti L. (Diptera:Culicidae) in response to different organic infusions. Neotrop Entomol 39: 299-302.

Sarıözkan S, İnci A, Yıldırım A, Düzlü Ö, Gray EW and Adler PH (2014) Economic losses during an outbreak of Simulium (Wilhelmia) species (Diptera: Simuliidae) in the Cappadocia region of Turkey. Turkiye Parazitol Derg 38: 116-119.

Schlein Y and Müller GC (2010) Experimental control of Phlebotomus papatasi by spraying attractive toxic sugar bait (ATSB) on vegetation. Trans R Soc Trop Med Hyg 104: 766-771.

Schmaedick MA, Ball TS, Burkot TR and Gurr NE (2008) Evaluation of three traps for sampling Aedes polynesiensis and other mosquito species in American Samoa. J Am Mosq Control Assoc 24: 319-322.

Schnellbacher RW, Holder K, Morgan T, Foil L, Beaufrere H, Nevarez J and Tully Jr. TN (2012) Avian simuliotoxicosis: outbreak in Louisiana. Avian Dis 56: 616-620.

Schofield S and Brady OJ (1997) Effects of carbon dioxide, acetone and 1-octen-3-ol on the flight responses of the stable fly, Stomoxys calcitrans, in a wind tunnel. Physiol Entomol 22: 380-386.

Schofield S, Cork A and Brady J (1995) Electroantennogram responses of the stable fly, Stomoxys calcitrans, to components of host odour. Physiol Entomol 20: 273-280.

Schofield SW and Sutcliffe JF (1996) Human individuals vary in attractiveness for host-seeking black flies (Diptera: Simuliidae) based on exhaled carbon dioxide. J Med Entomol 33: 102-108. 
Schreck CE and Kline DL (1983) Area protection by use of repellent-treated netting against Culicoides biting midges. Mosq News 43: 338-342.

Seenivasagan T, Sharma KR, Sekhar K, Ganesan K, Prakash S and Vijayaraghavan R (2009) Electroantennogram, flight orientation, and oviposition responses of Aedes aegypti to the oviposition pheromone $n$-heneicosane. Parasitol Res 104: 827-833.

Seenivasagan T and Vijayaraghavan R (2010) Oviposition pheromones in haematophagous insects. Vitam Horm 83: $597-630$.

Shorey HH (1973) Behavioral responses to insect pheromones. Annu Rev Entomol 18: 349-380.

Sih A (1986) Antipredator responses and the perception of danger by mosquito larvae. Ecology 67: 434-441.

Sithiprasasna R, Mahapibul P, Noigamol C, Perich MJ, Zeichner BC, Burge B, Norris SL, Jones JW, Schleich SS and Coleman RE (2003) Field evaluation of a lethal ovitrap for the control of Aedes aegypti (Diptera: Culicidae) in Thailand. J Med Entomol 40: 455-462.

Smallegange RC, Qiu YT, Bukovinszkiné-Kiss G, Van Loon JJA and Takken W (2009) The effect of aliphatic carboxylic acids on olfaction-based host-seeking of the malaria mosquito Anopheles gambiae sensu stricto. J Chem Ecol 35: 933-943.

Smallegange RC, Qiu YT, Van Loon JJA and Takken W (2005) Synergism between ammonia, lactic acid and carboxylic acids as kairomones in the host-seeking behaviour of the malaria mosquito Anopheles gambiae sensu stricto (Diptera: Culicidae). Chem Senses 30: 145-152.

Smallegange RC, Schmied WH, Van Roey KJ, Verhulst NO, Spitzen J, Mukabana WR and Takken W (2010) Sugar-fermenting yeast as an organic source of carbon dioxide to attract the malaria mosquito Anopheles gambiae. Malar J 9: 292.

Smallegange RC and Takken W (2010) Host-seeking behaviour of mosquitoes: responses to olfactory stimuli in the laboratory. In: Takken W and Knols BGJ (eds.) Olfaction in vector-host interactions. Wageningen Academic Publishers, Wageningen, the Netherlands.

Smallegange RC, Verhulst NO and Takken W (2011) Sweaty skin: an invitation to bite? Trends Parasitol 27: 143-148.

Smith JP, Cope EH, Walsh JD and Hendrickson CD (2010) Ineffectiveness of mass trapping for mosquito control in St. Andrews State Park, Panama City Beach, Florida. J Am Mosq Control Assoc 26: 43-49.

Snyder D, Cernicchiaro N, Allan SA and Cohnstaedt LW (2016) Insecticidal sugar baits for adult biting midges. Med Vet Entomol 30: 209-217.

Solano P, Salou E, Rayaisse JB, Ravel S, Gimonneau G, Traore I and Bouyer J (2015) Do tsetse flies only feed on blood? Infect Genet Evol 36: 184-189.

Solomon B, Sahle FF, Gebre-Mariam T, Asres K and Neubert RHH (2012) Microencapsulation of citronella oil for mosquitorepellent application: formulation and in vitro permeation studies. Eur J Pharm Biopharm 80: 61-66.

Sonenshine DE (1985) Pheromones and other semiochemicals of the Acari. Annu Rev Entomol 30: 1-28.

Sonenshine DE (2004) Pheromones and other semiochemicals of ticks and their use in tick control. Parasitology 129: S405-S425.

Sonenshine DE, Hamilton JGC and Lusby WR (1992) Use of cholesteryl esters as mounting sex pheromones in combination with 2,6-dichlorophenol and pesticides to control populations of hard ticks. Patent US5149526 A. Available at: https://www.google.com/patents/US5149526.

Sonenshine DE, Taylor D and Carson KA (1986) Chemically mediated behavior in Acari: adapations for finding hosts and mates. J Chem Ecol 12: 1091-1108.

Sonenshine DE, Taylor D and Corrigan G (1985) Studies to evaluate the effectiveness of sex pheromone-impregnated formulations for control of populations of the American dog tick, Dermacentor variabilis (Say) (Acari: Ixodidae). Exp Appl Acarol 1:23-34.

Späth J (2000) Feeding patterns of three sympatric tsetse species (Glossina spp.) (Diptera: Glossinidae) in the preforest zone of Cote d'Ivoire. Acta Trop 75: 109-118.

Spitzen J, Spoor CW, Grieco F, Ter Braak C, Beeuwkes J, Van Brugge SP, Kranenbarg S, Noldus LPJJ, Van Leeuwen JL and Takken W (2013) A 3D analysis of flight behavior of Anopheles gambiae sensu stricto malaria mosquitoes in response to human odor and heat. PLoS ONE 8: e62995.

Stanczyk NM, Brookfield JFY, Field LM and Logan JG (2013) Aedes aegypti mosquitoes exhibit decreased repellency by DEET following previous exposure. PLOS ONE 8: e54438. 
Stanczyk NM, Brookfield JFY, Ignell R, Logan JG and Field LM (2010) Behavioral insensitivity to DEET in Aedes aegypti is a genetically determined trait residing in changes in sensillum function. Proc Natl Acad Sci 107: 8575-8580.

Stewart ZP, Oxborough RM, Tungu PK, Kirby MJ, Rowland MW and Irish SR (2013) Indoor application of attractive toxic sugar bait (ATSB) in combination with mosquito nets for control of pyrethroid-resistant mosquitoes. PLoS ONE 8: e84168.

Sumba LA, Ogbunugafor CB, Deng AL and Hassanali A (2008) Regulation of oviposition in Anopheles gambiae s.s.: Role of inter- and intra-specific signals. J Chem Ecol 34: 1430-1436.

Sutcliffe JF, Shemanchuk JA and McKeown DB (1994) Preliminary survey of odours that attract the black fly, Simulium arcticum (Malloch) (IIS-10.11) (Diptera: Simuliidae) to its cattle hosts in the Athabasca region of Alberta. Insect Sci Appl 4: 487-494.

Syed $Z$ and Leal WS (2008) Mosquitoes smell and avoid the insect repellent DEET. Proc Natl Acad Sci 105: 13598-13603.

Syed $Z$ and Leal WS (2009) Acute olfactory response of Culex mosquitoes to a human- and bird-derived attractant. Proc Natl Acad Sci 106: 18803-18808.

Takken W and Kline DL (1989) Carbon dioxide and 1-octen-3-ol as mosquito attractants. J Am Mosq Control Assoc 5:311-316.

Takken W and Verhulst NO (2013) Host preferences of blood-feeding mosquitoes. Annu Rev Entomol 58: 433-453.

Tawatsin A, Thavara U, Chansang U, Chavalittumrong P, Boonruad T, Wongsinkongman P, Bansidhi J and Mulla MS (2006) Field evaluation of deet, Repel Care, and three plant based essential oil repellents against mosquitoes, black flies (Diptera: Simuliidae) and land leeches (Arhynchobdellida: Haemadipsidae) in Thailand. J Am Mosq Control Assoc 22: 306-313.

Taylor DB and Berkebile DR (2008) Sugar feeding in adult stable flies. Environ Entomol 37: 625-629.

Taylor DB, Moon RD and Mark DR (2012) Economic impact of stable flies (Diptera: Muscidae) on dairy and beef cattle production. J Med Entomol 49: 198-209.

Tchouassi DP, Sang R, Sole CL, Bastos ADS, Mithoefer K and Torto B (2012) Sheep skin odor improves trap captures of mosquito vectors of Rift Valley fever. PLoS Negl Trop Dis 6: e1879.

Tchouassi DP, Sang R, Sole CL, Bastos ADS, Teal PEA, Borgemeister C and Torto B (2013) Common host-derived chemicals increase catches of disease-transmitting mosquitoes and can improve early warning systems for Rift Valley fever virus. PLoS Negl Trop Dis 7: e2007.

Thompson BH (1976) Studies on the attraction of Simulium damnosum s.I. (Diptera: Simuliidae) to its hosts. I. The relative importance of sight, exhaled breath, and smell. Tropenmed Parasitol 27: 455-473.

Thompson BH (1977) Studies on the attraction of Simulium damnosum s.l. (Diptera: Simuliidae) to its hosts. II. The nature of substances on the human skin responsible for attractant olfactory stimuli. Tropenmed Parasitol 28: 83-90.

Tobe SS and Langley PA (1978) Reproductive physiology of Glossina. Annu Rev Entomol 23: 283-307.

Toé LD, Koala L, Burkett-Cadena ND, Traoré BM, Sanfo M, Kambiré SR, Cupp EW, Traoré S, Yameogo L, Boakye D, Rodríguez-Pérez MA and Unnasch TR (2014) Optimization of the Esperanza window trap for the collection of the African onchocerciasis vector Simulium damnosum sensu lato. Acta Trop 137: 39-43.

Tomberlin JK, Rains GC, Allan SA, Sanford MR and Lewis WJ (2006) Associative learning of odor with food- or blood-meal by Culex quinquefasciatus Say (Diptera: Culicidae). Naturwissenschaften 93: 551-556.

Torr SJ (1988) The activation of resting tsetse flies (Glossina) in response to visual and olfactory stimuli in the field. Physiol Entomol 13: 315-325.

Torr SJ (1989) The host-orientated behaviour of tsetse flies (Glossina): the interaction of visual and olfactory stimuli. Physiol Entomol 14: 325-340.

Torr SJ (1990) Dose responses of tsetse flies (Glossina) to carbon dioxide, acetone and octenol in the field. Physiol Entomol 15: 93-103.

Torr SJ, Hall DR, Phelps RJ and Vale GA (1997) Methods for dispensing odour attractants for tsetse flies (Diptera: Glossinidae). Bull Entomol Res 87: 299.

Torr SJ, Hall DR and Smith JL (1995) Responses of tsetse flies (Diptera: Glossinidae) to natural and synthetic ox odors. Bull Entomol Res 85: 157-166. 
Torr SJ and Mangwiro TNC (1996) Upwind flight of tsetse (Glossina spp.) in response to natural and synthetic host odour in the field. Physiol Entomol 21: 143-150.

Torr SJ and Mangwiro TNC (2000) Interactions between cattle and biting flies: effects on the feeding rate of tsetse. Med Vet Entomol 14: 400-409.

Torr SJ, Mangwiro TNC and Hall DR (2006) The effects of host physiology on the attraction of tsetse (Diptera: Glossinidae) and Stomoxys (Diptera: Muscidae) to cattle. Bull Entomol Res 96: 71-84.

Torr SJ and Solano P (2010) Olfaction in Glossina - host interactions: a tale of two tsetse. In: Takken W and Knols BGJ (eds.) Olfaction in vector-host interactions. Wageningen Academic Publishers, Wageningen, the Netherlands.

Torres-Estrada JL, Meza-Alvarez RA, Cruz-López L, Rodríguez MH and Arredondo-Jiménez Jl (2007) Attraction of gravid Anopheles pseudopunctipennis females to oviposition substrates by Spirogyra majuscula (Zygnematales: Zygnmataceae) algae under laboratory conditions. J Am Mosq Control Assoc 23: 18-23.

Traoré S, Wilson MD, Sima A, Barro T, Diallo A, Aké A, Coulibaly S, Cheke RA, Meyer RRF, Mas J, McCall PJ, Post RJ, Zouré H, Noma M, Yaméogo L, Sékétéli AV and Amazigo UV (2009) The elimination of the onchocerciasis vector from the island of Bioko as a result of larviciding by the WHO African Programme for Onchocerciasis Control. Acta Trop 111:211-218.

Trigg JK (1996) Evaluation of a Eucalyptus-based repellent against Culicoides impunctatus (Diptera: Ceratopogonidae) in Scotland. J Am Mosq Control Assoc 12: 329-330.

Turner SL, Li N, Guda T, Githure J, Cardé RT and Ray A (2011) Ultra-prolonged activation of $\mathrm{CO}_{2}$-sensing neurons disorients mosquitoes. Nature 474: 87-91.

Vale GA (1980) Field studies of the responses of tsetse flies (Glossinidae) and other Diptera to carbon dioxide, acetone and other chemicals. Bull Entomol Res 70: 563-570.

Vale GA and Hall DR (1985) The role of 1-octen-3-ol, acetone and carbon dioxide in the attraction of tsetse flies, Glossina spp. (Diptera: Glossinidae), to ox odour. Bull Entomol Res 75: 209.

Vale GA, Hargrove JW, Cockbill GF and Phelps RJ (1986) Field trials of baits to control populations of Glossina morsitans morsitans Westwood and G. pallidipes Austen (Diptera: Glossinidae). Bull Entomol Res 76: 179.

Vale GA, Lovemore DF, Flint S and Cockbill GF (1988) Odour-baited targets to control tsetse flies, Glossina spp. (Diptera: Glossinidae), in Zimbabwe. Bull Entomol Res 78: 31-49.

Vantaux A, Lefèvre T, Dabiré KR and Cohuet A (2014) Individual experience affects host choice in malaria vector mosquitoes. Parasit Vectors 7: 249.

Verhulst NO, Andriessen R, Groenhagen U, Kiss GB, Schulz S, Takken W, Van Loon JJA, Schraa G and Smallegange RC (2010) Differential attraction of malaria mosquitoes to volatile blends produced by human skin bacteria. PLoS ONE 5: e15829.

Verhulst NO, Qiu YT, Beijleveld H, Maliepaard C, Knights D, Schulz S, Berg-Lyons D, Lauber CL, Verduijn W, Haasnoot GW, Mumm R, Bouwmeester HJ, Claas FHJ, Dicke M, Van Loon JJA, Takken W, Knight R and Smallegange RC (2011) Composition of human skin microbiota affects attractiveness to malaria mosquitoes. PLoS ONE 6: e28991.

Verocai GG, McGaha Jr. TW, Iburg JP, Katholi CR, Cupp EW, Noblet R and Unnasch TR (2016) Identification of semiochemicals attractive to Simulium vittatum (IS-7). Med Vet Entomol 31: 140-149.

Veronesi E, Henstock M, Gubbins S, Batten C, Manley R, Barber J, Hoffmann B, Beer M, Attoui H, Mertens PPC and Carpenter $S$ (2013) Implicating Culicoides biting midges as vectors of Schmallenberg virus using semi-quantitative RT-PCR. PLOS ONE 8: e57747.

Vet LEM, Van Lenteren JC, Heymans M and Meelis E (1983) An airflow olfactometer for measuring olfactory responses of hymenopterous parasitoids and other small insects. Physiol Entomol 8: 97-106.

Vinauger C, Lahondère C, Cohuet A, Lazzari CR and Riffell JA (2016) Learning and memory in disease vector insects. Trends Parasitol 32: 761-771.

Vinauger C, Lutz EK and Riffell JA (2014) Olfactory learning and memory in the disease vector mosquito Aedes aegypti. J Exp Biol 217: 2321-2330.

Vreysen MJ, Saleh KM, Ali MY, Abdulla AM, Zhu ZR, Juma KG, Dyck VA, Msangi AR, Mkonyi PA and Feldmann HU (2000) Glossina austeni (Diptera: Glossinidae) eradicated on the island of Unguja, Zanzibar, using the sterile insect technique. J Econ Entomol 93: 123-135. 
Vreysen MJB, Saleh K, Mramba F, Parker A, Feldmann U, Dyck VA, Msangi A and Bouyer J (2014) Sterile insects to enhance agricultural development: the case of sustainable tsetse eradication on Unguja Island, Zanzibar, using an area-wide integrated pest management approach. PLoS Negl Trop Dis 8: e2857.

Vreysen MJB, Seck MT, Sall B and Bouyer J (2013) Tsetse flies: Their biology and control using area-wide integrated pest management approaches. J Invertebr Pathol 112: S15-S25.

Warnes ML (1995) Field studies on the effect of cattle skin secretion on the behaviour of tsetse. Med Vet Entomol 9: 284-288.

Weinandt ML, Meyer M, Strand M and Lindsay AR (2012) Cues used by the black fly, Simulium annulus, for attraction to the common loon (Gavia immer). J Vector Ecol 37: 359-364.

Weinzierl RA, Schmidt CD, Faulkner DB, Cmarik GF and Zinn GD (1990) Chronology of permethrin resistance in a southern Illinois population of the horn fly (Diptera: Muscidae) during and after selection by pyrethroid use. J Econ Entomol 83: 690-697.

Willemse LPM (1991) A trial of odour baited targets to control the tsetse fly, Glossina morsitans centralis (Diptera: Glossinidae) in west Zambia. Bull Entomol Res 81: 351-357.

Williams CR, Smith BPC, Best SM and Tyler MJ (2006) Mosquito repellents in frog skin. Biol Lett 2: 242-245.

Wilson EO and Bossert WH (1963) Chemical communication among animals. Recent Prog Horm Res 19: 673-716.

Wilson JG, Kinzer DR, Sauer JR and Hair JA (1972) Chemo-attraction in the lone star tick (Acarina: Ixodidae): 1. Response of different developmental stages to carbon dioxide administered via traps. J Med Entomol 9:245-252.

Work TH, Hurlbut HS and Taylor RM (1955) Indigenous wild birds of the Nile-delta as potential West Nile virus circulating reservoirs. Am J Trop Med Hyg 4: 872-888.

World Health Organization (2010) Control of the leishmaniases. World Health Organ Tech Rep Ser 978: 22-26.

Xu P, Choo Y-M, De la Rosa A and Leal WS (2014) Mosquito odorant receptor for DEET and methyl jasmonate. Proc Natl Acad Sci 111: 16592-16597.

Yoder JA, Atwood AD and Stevens BW (1998) Attraction to squalene by ticks (Acari: Ixodidae): first demonstration of a host-derived attractant. Int J Acarol 24: 143-147.

Young RM, Burkett-Cadena ND, McGaha TW, Rodríguez-Pérez MA, Toé LD, Adeleke MA, Sanfo M, Soungalo T, Katholi CR, Noblet R, Fadamiro H, Torres-Estrada JL, Salinas-Carmona MC, Baker B, Unnasch TR and Cupp EW (2015) Identification of human semiochemicals attractive to the major vectors of Onchocerciasis. PLoS Negl Trop Dis 9: e3450.

Zeichner BC and Perich MJ (1999) Laboratory testing of a lethal ovitrap for Aedes aegypti. Med Vet Entomol 13: 234-238.

Zhou JJ, Huang W, Zhang GA, Pickett JA and Field LM (2004) 'Plus-C' odorant-binding protein genes in two Drosophila species and the malaria mosquito Anopheles gambiae. Gene 327: 117-129.

Zhu JJ, Dunlap CA, Behle RW, Berkebile DR and Wienhold B (2010) Repellency of a wax-based catnip-oil formulation against stable flies. J Agric Food Chem 58: 12320-12326.

Zhu JJ, Wienhold BJ, Wehrle J, Davis D, Chen H, Taylor D, Friesen K and Zurek L (2014) Efficacy and longevity of newly developed catnip oil microcapsules against stable fly oviposition and larval growth. Med Vet Entomol 28: 222-227. 


\title{
14. Genetic control of vectors
}

\begin{abstract}
Jérémy Bouyer ${ }^{1,2,3^{*}}$ and Eric Marois ${ }^{3,4}$
'Unité Mixte de Recherche ASTRE 'Animal, Santé, Territoires, Risques et Ecosystèmes', Campus international de Baillarguet, Centre de Coopération Internationale en Recherche Agronomique pour le Développement (CIRAD), 34398 Montpellier, France; ${ }^{2}$ ASTRE, University of Montpellier, CIRAD, INRA, Montpellier, France; ${ }^{3}$ Insect Pest Control Laboratory, Joint FAO/IAEA Programme of Nuclear Techniques in Food and Agriculture, 1400 Vienna, Austria; ${ }^{4}$ INSERM U963, CNRS UPR9022, Université de Strasbourg, Institut de Biologie Moléculaire et Cellulaire, Strasbourg, France; jeremy.bouyer@cirad.fr
\end{abstract}

\begin{abstract}
In a context of tighter regulations on approved insecticide molecules, the spread of insecticide resistance in insect vectors of human and animal diseases and the introduction of exotic vectors to new territories call for the development of new pest control methods and strategies. New genetic control methods, related to the ancestral sterile insect technique (SIT), show particular promise and are being developed in response to increasing health and agricultural challenges. These include the use of symbionts like Wolbachia and the use of transgenic insect strains, some of which incorporate gene editing techniques that can lead to transgene spread (gene drive). Here we present the principles, associated opportunities and risks, as well as the degree of advancement of these various techniques for a subset of livestock pests and disease vectors including screwworms, tsetse, mosquitoes and stomoxes. We then present some case studies on recent improvements in the use of the SIT in tsetse and the release of insects carrying a dominant lethal gene, symbiontbased approaches and gene drive in mosquitoes. Finally, we call to speed up the development of genetic control, within a rigorous benefit-risk analysis framework including international public consultation.
\end{abstract}

Keywords: sterile insect technique, incompatible insect technique, gene drive, pathogen interference, wolbachia

\section{Introduction}

Awareness of the toxicity of insecticides to living organisms and ecosystems is leading a growing number of countries to reduce the number of approved molecules (Bouyer 2015). For example, in 2014, the European Union was authorising four classes of vector control insecticides only, and only three new classes are being developed by industrials for operational use no earlier than 2019 (McBeath 2015). Moreover, resistance to pyrethroids, the class most commonly used against insects, is spreading, which could result in its disuse in the short-term. This is particularly true for mosquito vectors of malaria, the most important vector-borne disease causing fatalities globally), for which resistance has been observed in all major vector species throughout Africa (Ranson et al. 2011).

These regulations are being tightened in spite of a growing pressure from insect vectors of human and animal diseases. This increased pressure is explained by greater resistance to insecticides, but also by global factors such as climate change or the growth of global trade. These global factors foster the invasion of new territories by exotic vectors. For example, the tiger mosquito Aedes albopictus (Skuse), which is native to Asia and a major vector of human arboviruses, caused indigenous cases of dengue, chikungunya and Zika in Europe (Akiner et al. 2016; Benedict et al. 2007; Rezza et al. 2007). Innovations are therefore urgently required in the field of vector control. 
Recently, the 2009/128/EC Directive on sustainable use of pesticides (EC, 2009) was adapted to European vectors of animal diseases and SIT appeared as the main alternative biological control method (Durel et al. 2015). More generally, genetic control appears as one of the main alternatives to chemical control (Bourtzis et al. 2016; McGraw and O'Neill 2013; Suckling et al. 2014).

\section{Principles, opportunities and risks associated to genetic control}

Genetic control consists in the large-scale rearing of insects - whether genetically modified or not - with the subsequent release of males in order for them to sterilise wild females or to transfer modifications to the progeny of wild females that are either lethal or impede the ability to transmit the pathogen. The genetic modifications correspond to random mutations or to transgenes, whereby foreign genetic material is incorporated in an organism. One variant consists in establishing novel insect-bacterial symbiotic associations which cause the death of embryos in females inseminated by the released males (cytoplasmic incompatibility) or block the transmission of the pathogen (pathogen interference). Symbionts could potentially also be genetically modified to prevent the infection of the vector, an approach called paratransgenesis.

The sterile insect technique (SIT) is the first and ancestral method from which other genetic control methods were derived: the males are irradiated and transmit sperm cells carrying many random lethal mutations to the females they inseminate. It is thus a birth control method that impacts the next generation. This technique has been used successfully in the control of a number of insect pests or vectors, such as species of fruit flies in Mexico (Enkerlin et al. 2015), the new world screwworm from the Americas and Libya (Vargas-Terán et al. 1994; Wyss 2006) and the tsetse fly in Africa (Vreysen et al. 2000). It is generally considered a biological control method in Europe, exempted from the regulation on genetically modified organisms (EFSA 2013), although no specific regulation is available for disease vectors. In the case of plant pests, SIT is also regarded as a biological control technique, the sterile males being assimilated to beneficial organisms (FAO 2015).

The use of genetically modified male insects to transmit a modification to females has the advantage of avoiding the need for irradiation ${ }^{1}$ but presents other potential risks associated with the release of transgenes in the ecosystem. Genetic modifications under consideration could block the ability to transmit a pathogen, turn females into inoffensive males (Adelman and Tu 2016), skew the sex ratio in favour of males (Galizi et al. 2014, 2016) or even destroy the targeted insect population. However, genetically modified mosquitoes trigger public reluctance because of their potential biological risks, including the possibility of uncontrolled transfer of transgenes to non-targeted insects (EFSA 2013). The risk of horizontal transfer depends on the employed genetic mechanisms, and in particular on their potential for dissemination, but it is overall very low (Alphey 2014). It is even lower for transgenes than for symbionts (Loreto and Wallau 2016a).

The use of transgenic strains for vector control interventions is a recent field of research that has mainly focussed on mosquitoes. Many different mechanisms can be envisioned for control (Alphey 2014) that range from non-diffusive technologies like the release of insects carrying a dominant lethal gene (RIDL) (Lacroix et al. 2012) or the use of transgenic genetic sexing strains that can be

\footnotetext{
${ }^{1}$ Irradiation is often based on the use of radioactive sources but can also be conducted using safer X-ray machines. Besides, irradiation is helpful in treating the blood used to feed mosquito vector colonies to prevent vertical transmission of pathogens, and could be used to secure the mass-rearing of vectors.
} 
irradiated before release to apply SIT, which have already been developed in Cochliomyia (Concha et al. 2016), to diffusive techniques like transposable elements and gene drive.

Considering the costs, the more diffusive the technology, the less costly it will be since the initial number of insects to release decreases. Techniques that are not diffusive like the SIT and RIDL are the most expensive because they necessitate the continuous release of males if the target population is not eliminated. When genetic modifications and transfected symbionts confer fitness costs which can reduce their diffusion in field conditions, a high initial frequency must be imposed in the target population which is also associated to high costs (Hoffmann et al. 2011). Moreover, the diffusion of genetic modifications is also limited by intrinsic dispersal capacities of the target species, which can be very low, for example in the case of Aedes species (outside occasional passive transport via human transportation, which will generally be insufficient to establish a modification carrying a fitness cost in a new population). Releases will then have to be carried out all other the target populations and the associated costs will be proportional to the target areas although scale savings are of course possible (Barclay et al. 2011). Finally, the use of transgenic insects is generally associated with intellectual property and commercial interests that can highly increase their cost, contrary to SIT.

Whatever the selected technology, genetic control requires the mass rearing, handling and release of sterile or modified insects, which can impact on their quality. Aerial release is recommended to ensure homogeneous distribution of the released insects (Mubarqui et al. 2014). It is thereafter critical to assess the competitiveness, survival and dispersal of the released males in the environmental conditions of the target area in order to make sure that they will be able to compete with their wild counterparts (Bellini et al. 2013; Sow et al. 2012). Finally, the released insects will have to show a similar behaviour as their wild counterparts (Vreysen et al. 2011). Below we develop examples of these technologies including SIT, RIDL, Wolbachia-based and gene drive approaches against insect pests and disease vectors.

\section{Degree of advancement of the various genetic control methods against vector species}

The degree of advancement of the various gene control technologies varies according to the vector group (Bourtzis et al. 2016). Table 1 gives an overview of the development of each technique at the time of writing in four families of vectors, ranging from the concept stage to the large scale operational control campaigns.

\section{The sterile insect technique}

SIT is the most developed technology for the insect families considered (see below a case study with tsetse flies). It has been used on an operational scale only against screwworms and tsetse flies, despite promising trials against Stomoxys calcitrans (L.) within an integrated management strategy in the USA Virgin Islands more than three decades ago ( $>99 \%$ of suppression of the target population) (Patterson et al. 1981). It might be useful to reconsider its use in areas like La Réunion island where other techniques failed to achieve a sustainable control of stomoxes (Bouyer et al. 2011). In mosquitoes, the technique is actively being developed against Aedes vectors of major arboviruses like dengue, chikungunya and Zika but also against Anopheles vectors of malaria and it will probably become available at the operational scale within the next five years (Bourtzis et al. 2016). 
Table 1. Degree of advancement of various genetic control methods against four families of vectors and livestock pest insects.

\begin{tabular}{|c|c|c|c|c|}
\hline Technology & Screwworm & Tsetse & Mosquito & Stomoxes \\
\hline $\begin{array}{l}\text { Sterile insect } \\
\text { technique (SIT) }\end{array}$ & operational & operational & pilot field trials & pilot field trials \\
\hline $\begin{array}{l}\text { Incompatible insect } \\
\text { technique (IIT) }\end{array}$ & - & concept (Wolbachia) & pilot field trials & - \\
\hline $\begin{array}{l}\text { Replacement } \\
\text { (Wolbachia) }\end{array}$ & - & - & $\begin{array}{l}\text { pilot field trials, in } \\
\text { course of extension }\end{array}$ & - \\
\hline Combined SIT/IIT & - & - & pilot field trials & - \\
\hline Paratransgenesis & - & $\begin{array}{l}\text { laboratory } \\
\text { evaluation (Sodalis) }\end{array}$ & $\begin{array}{l}\text { semi-field trials } \\
\text { (Asaia) }\end{array}$ & - \\
\hline $\begin{array}{l}\text { Gene silencing using } \\
\text { RNA interference } \\
\text { (RNAi) }\end{array}$ & - & $\begin{array}{l}\text { laboratory } \\
\text { evaluation }\end{array}$ & $\begin{array}{l}\text { laboratory } \\
\text { evaluation }\end{array}$ & - \\
\hline $\begin{array}{l}\text { Release of insects } \\
\text { carrying a dominant } \\
\text { lethal gene (RIDL) }\end{array}$ & $\begin{array}{l}\text { laboratory } \\
\text { evaluation (genetic } \\
\text { sexing strain) }\end{array}$ & - & $\begin{array}{l}\text { pilot field trials, in } \\
\text { course of extension }\end{array}$ & $\begin{array}{l}\text { laboratory } \\
\text { evaluation }\end{array}$ \\
\hline Gene drive & - & - & $\begin{array}{l}\text { laboratory } \\
\text { evaluation }\end{array}$ & - \\
\hline References & $\begin{array}{l}\text { Concha et al. 2016; } \\
\text { Vargas-Terán et al. } \\
\text { 1994; Wyss } 2006\end{array}$ & $\begin{array}{l}\text { Bourtzis et al. 2016; } \\
\text { Dicko et al. 2014; } \\
\text { Vreysen et al. 2000; } \\
\text { Walshe et al. 2009; } \\
\text { Whyard et al. } 2015\end{array}$ & $\begin{array}{l}\text { Alphey 2014; } \\
\text { Bourtzis et al. 2016; } \\
\text { Gantz et al. 2015; } \\
\text { Hammond et al. } \\
\text { 2016; Haut Conseil } \\
\text { des Biotechnologies } \\
\text { 2017; Mancini et al. } \\
2016\end{array}$ & $\begin{array}{l}\text { O'Brochta et al. } \\
\text { 2000; Patterson et } \\
\text { al. } 1981\end{array}$ \\
\hline
\end{tabular}

When considering applications of SIT to insect vectors of human disease (as opposed to agricultural pests, for which there is no such concern), special attention must be dedicated to the risk of introducing radiation-induced mutations in the natural insect population. Mutations in mosquito immune genes could render them hyper-susceptible to a human pathogen they can vector; therefore, $100 \%$ sterility of the released males must be guaranteed to exclude the possibility of accidentally introducing mutations that could elevate the vector competence of the mosquito population, especially when a strategy of long term reduction is selected, whereby a fraction of the initial population is preserved and might be modified by the intervention.

\section{The incompatible insect technique}

The incompatible insect technique (IIT) is an approach akin to SIT based on cytoplasmic incompatibility conferred by certain strains of Wolbachia bacteria, and that can be uni or bi- 
directional ${ }^{2}$. This technique has been mostly developed against Aedes mosquitoes where it is currently tested in pilot field trials to reduce target populations (O'Connor et al. 2012). Wolbachia induces cytoplasmic incompatibility $(\mathrm{Cl})$, i.e. embryonic lethality corresponding to a sperm-egg incompatibility occurring when infected males mate either with uninfected females or with females infected with different, incompatible Wolbachia strains. This technique was tested successfully in laboratory cages in La Réunion, with the introduction of highly competitive infected Culex quinquefasciatus Say males resulting in a demographic crash (Atyame et al. 2011). It is presently being tested in a pilot trial in French Polynesia against Aedes polynesiensis (Marks) (H. Bossin, personal communication; O'Connor et al. 2012).

One of the important risks associated to this strategy is to release, in addition to the intended males, a few females infected with the Wolbachia strain used for control that may quickly establish the Wolbachia infection in the target population, thus 'immunizing' this population against further IIT interventions. To curb this risk, it could be beneficial to combine IIT with SIT. Actually, a low irradiation dose suffices to ensure complete sterilization of females, which as a result cannot establish the Wolbachia infection in the target population, while male sterility is ensured by the combination of Wolbachia-induced $\mathrm{Cl}$ and irradiation (Bourtzis et al. 2016). This strategy is presently tested in a pilot trial in Thailand against Aedes aegypti (P. Kittayapong, personal communication) and in China against Ae. albopictus (Zhang et al. 2015).

\section{Population replacement using Wolbachia}

Transinfection consists in transferring specific bacteria from their natural host to a new host. In the case of the Wolbachia endosymbiont, this can result in stable novel infections causing cytoplasmic incompatibility and pathogen interference, two properties that can be exploited in vector control. The pathogen interference (PI) strategy has been used successfully to modify local Ae. aegypti populations in pilot trials in Australia (Hoffmann et al. 2011), even if additional studies will be necessary to demonstrate the epidemiological impact of this modification, particularly on Dengue transmission (see below). A variant of $\mathrm{PI}$ is to genetically modify a symbiont (paratransgenesis) to prevent the transmission of pathogens. This has been successfully tested in laboratory conditions on Sodalis symbionts of tsetse resulting in fly resistance to trypanosomes (Aksoy et al. 2008) but never in field conditions at the time of writing. More recently, large cage tests have demonstrated the ability of genetically modified Asaia bacteria to spread in Anopheles stephensi Liston and Anopheles gambiae Giles mosquito populations, confirming that using Asaia to deliver antimalarial compounds in the mosquito is a valid perspective (Mancini et al. 2016).

\section{Gene silencing using RNA interference}

Silencing specific gene expression through RNA interference in larvae proved to be efficient in the lab to kill Ae. aegypti females by targeting the female-specific isoform of a sex determination gene (doublesex), concomitantly with testis-expressed genes, which produced a population of mosquitoes that was both highly male-biased and sterile (Whyard et al. 2015). In these experiments, double-stranded RNA was produced in bacteria and delivered to the larvae by feeding, a costeffective gene silencing method that is potentially compatible with mass rearing with a built-in sex sorting component. The main challenge with this approach will be the homogeneous delivery of the RNAi-triggering molecules under large-scale mass rearing conditions to achieve $100 \%$

\footnotetext{
${ }^{2}$ Cytoplasmic incompatibility is unidirectional when released males are sterilizing for wild females, and bidirectional when, in addition, wild males would be sterilizing for accidentally released females.
} 
efficiency, but the possibility to produce large amounts of double-stranded RNA using bacteria or yeast makes it a promising option in the near future. Much work is however still needed to explore the field competitiveness of such males, especially when they are produced in massrearing conditions, before it becomes available for operational use.

It has also been suggested that the delivery of RNAi elements to mosquitoes through bacteria, algae or yeasts might also be used to silence essential genes to induce a specific larvicidal effect (Kumar et al. 2013).

\section{Release of insects carrying a dominant lethal gene}

RIDL consists in releasing mosquito males that have been genetically engineered to carry a dominant lethal gene (Figure 1).

The repression of the lethal gene using tetracycline as a dietary supplement has been the subject of criticism. Transgenic Ae. aegypti larvae are rescued from lethality by a diet supplemented with this antibiotic, but even in its absence, up to $3-4 \%$ of transgenic larvae survive to adulthood (Phuc et al. 2007). However, the massive fitness cost conferred by the transgene will lead to its rapid disappearance in the natural environment. The main associated drawback is that this might cause a partial modification of the target population through the 'pollution' of the local strains with some of the genetic background of the transgenic strain (from Asian origin, and colonized 14 years

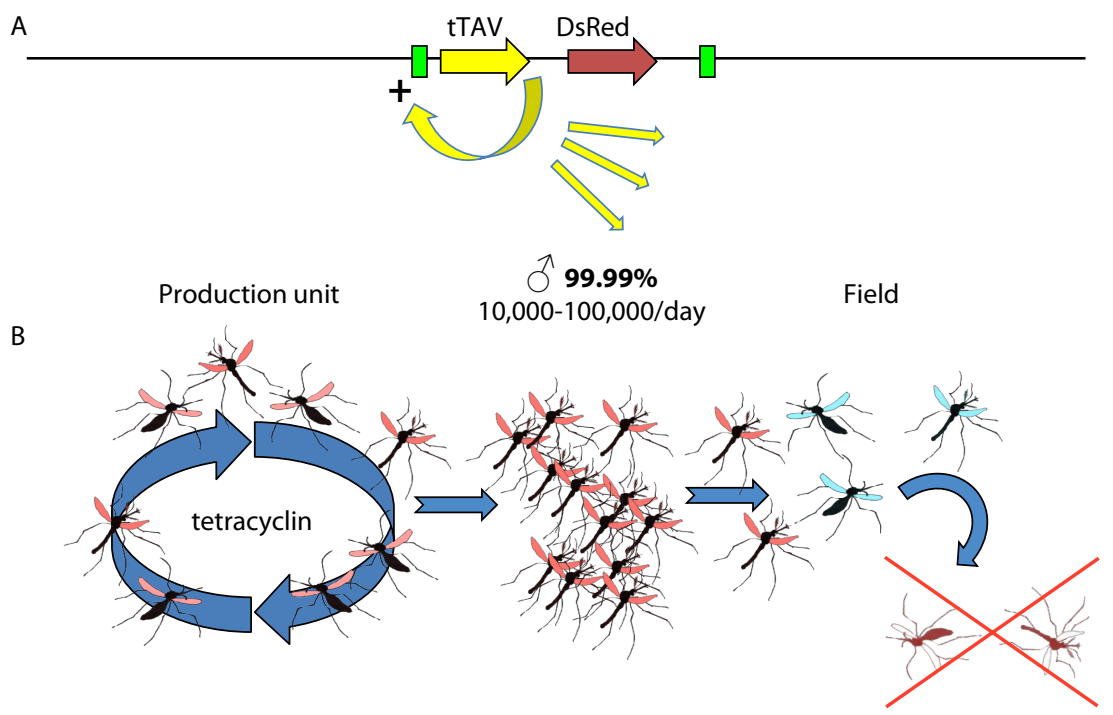

Figure 1. Principle of the release of insects carrying a dominant lethal gene (RIDL). (A) scheme of the transgene. The tetracycline activator variant (TTAV) protein binds to its own promoter, activates its own transcription and perturbs overall gene expression in the cells, resulting in mosquito death, unless tetracycline that binds and inactivates TTAV is provided. (B) During mass rearing in the production unit, mosquitoes develop normally in the presence of tetracycline. For an intervention, males are sorted at the pupal stage (based on the smaller size of male pupae). Once released, they mate with wild females whose progeny will die due to unrestricted tTAV activity. 
ago), with unforeseen effects on vector competence for example. Field trials of this technique are presented below.

\section{Gene drive}

The most diffusive technology at the time of writing is gene drive based on the clustered regularly interspaced short palindromic repeats (CRISPR)/CRISPR-associated protein 9 (Cas9) system, which has the potential to invade a full target population from only one or few released individuals (see below) (Adelman and Tu 2016). Gene drive is based on the super-Mendelian inheritance of a homing endonuclease-like gene specifically designed to home into, and disrupt, a specific locus (Burt 2003; Windbichler et al. 2011). On cleaving its specific genomic sequence on the homologous (non-transgenic) chromosome in a heterozygous individual, this selfish transgene can replicate by homing with an efficiency reaching 90 to $100 \%$ (Figure 2). This underlies its remarkable selective advantage and spread in the target insect population.

A gene drive construct can be designed to carry another sequence (cargo) in its insertion locus. After mating with a released gene drive transgenic male, almost all the offspring of a wild female would thus inherit the construct, theoretically leading to its invasion of the entire population of the target species within a few generations (Esvelt et al. 2014). Although this concept is relatively old, the recent advent of the CRISPR-Cas9 system has recently boosted its development in insects

$\underset{\text { mosquito }}{\text { gene drive }} \mathrm{X}$ wild-type
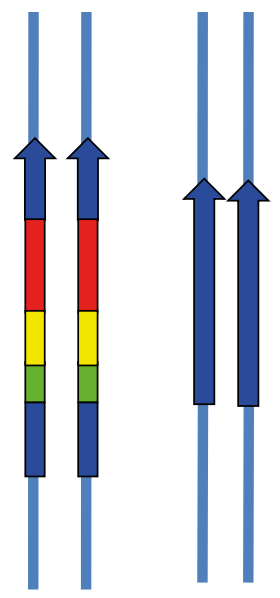

in germ cells of
F1 progeny

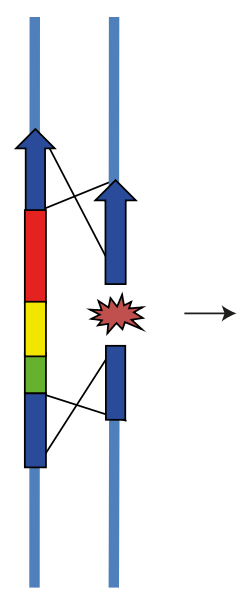

germ cells become

homozygous

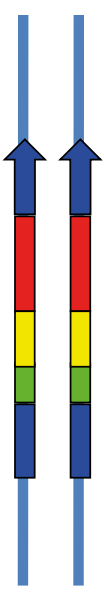

target locus

Cas9

gRNA coding gene(s)

cargo

Figure 2. Principle of a CRISPR-Cas 9 based gene drive in mosquitoes. A compound transgene composed of Cas 9 and guide RNA (gRNA) coding genes, and in some designs of a cargo and/or selection marker genes, is inserted on a mosquito chromosome exactly inside the target site recognized by the gRNA. In embryos formed when such a transgenic mosquito fertilizes a wild mosquito, the wild chromosome gets cut by Cas 9 at the gRNA recognition site. This DNA break is usually repaired by homologous recombination with the intact chromosome. As a consequence, the transgene is copied onto the repaired chromosome, which becomes transgenic. Should this mechanism be $100 \%$ efficient, inheritance of the transgene would be $100 \%$ instead of $50 \%$. 
(Gantz et al. 2015; Hammond et al. 2016). Two vector control approaches based on gene drive are envisioned: gene drives aiming at population suppression or even elimination, and gene drives aiming at population modification to decrease vectorial capacity (see below).

\section{Case studies}

\section{Recent improvements in the use of SIT against tsetse flies}

One of the recent examples of the use of the SIT against vectors is a tsetse fly eradication project that has been implemented in the Niayes area of Senegal, based on an integrated pest management strategy (Vreysen et al. in press). In 2005, this country initiated a project entitled 'Projet de lutte contre les glossines dans les Niayes' (tsetse control program in the Niayes) with the aim of creating a zone free of Glossina palpalis gambiensis (Vanderplank) in that area. The project received technical and financial support from the IAEA, the FAO, the Centre de Coopération Internationale en Recherche Agronomique pour le Développement (CIRAD), and the US Department of State through the Peaceful Uses Initiative (PUI), and was implemented in the context of the Pan African Tsetse and Trypanosomiasis Eradication Campaign (PATTEC). The project was implemented following a phased conditional approach (PCA), that entails programme implementation in distinct phases in which support to the next phase is conditional upon completion of all (or at least the majority) activities in the previous phase. In the case of the tsetse project in Senegal, the PCA consisted of 4 phases: (1) training and commitment of all stakeholders; (2) baseline data collection and feasibility studies; (3) pre-operational activities; and (4) operational activities.

Activities of phase 2 focused on the collection of entomological (Bouyer et al. 2010), veterinary (Seck et al. 2010), socio-economic (Bouyer et al. 2014) and environmental baseline data, and a population genetics study that proved that the target population was isolated (Solano et al. 2010). These data enabled the tsetse infested area to be delimited, the impact of animal trypanosomosis on the farmers' welfare to be quantified, and the formulation of an area-wide integrated pest management strategy to eradicate the isolated tsetse populations from the Niayes that included a sterile insect (SIT) component.

This project benefited from a strong applied research component and each stage involved methodological and technological developments. During the pre-operational phase, a series of activities were carried out that were needed to implement the operational phase.

The operational phase included a reduction of tsetse densities using insecticide traps and insecticide treatment of cattle followed by the aerial release of sterile males. Habitats suitable to the tsetse fly were identified by remote sensing based on a population distribution model (Dicko et al. 2014). Using this model, a low trap density of only 1-3.4 per $\mathrm{km}^{2}$ allowed reducing tsetse populations by more than $95 \%$, thanks to the targeting of the most suitable habitats.

The SIT component included the development of transport methods for long distance shipments of sterile male pupae. These sterile males were actually produced and irradiated in Burkina Faso at CIRDES (Centre International de Recherche-Développement sur l'Elevage en Zone Subhumide), and in Slovakia, by the Slovak Academy of Sciences. They were transported to Senegal by express delivery under regulated conditions. A specific transport method was developed to guarantee their survival (in particular, a temperature of $10^{\circ} \mathrm{C}$ ) (Pagabeleguem et al. 2015) and a quality control method originally developed in fruit flies SIT programmes was transferred to tsetse to ascertain that highly competitive sterile males would be released (Seck et al. 2015). They were 
then reared for six days in an insectarium at ISRA, Senegal (Institut Sénégalais de Recherches Agricoles). Sterile males were released by an automatic machine loaded onto a gyrocopter (Figure 3). This machine, developed by a Mexican company, is piloted by a geographic information system (GIS) that can adjust the density of sterile males to be released in order to achieve the objective (Mubarqui et al. 2014). Installed on an Android tablet, this GIS enables the pilot to concentrate on following release lines, with the machine automatically beginning the release upon entering the target zone, and ceasing upon exit. Release densities of sterile males were thus adjusted depending on the availability of suitable habitat predicted by the distribution model (10 sterile males per $\mathrm{km}^{2}$ if the habitat is unsuitable; 100 if it is suitable). The competitiveness of sterile males was very good (Fried ${ }^{3}$ index ranging from 0.3 to 0.5) (Bouyer et al. 2012; Fried 1971).

The innovative elements of this project are currently being transferred to other tsetse fly eradication projects in Africa, like the tsetse eradication campaign in the Deme valley of Ethiopia, within the framework of PATTEC. This successful experience can be used as an example for improving or starting vector control in other parts of the world.

\section{RIDL against mosquitoes}

RIDL has been developed (Phuc et al. 2007) and tested by the British-based company Oxitec against Ae. aegypti in Cayman Islands, Panama and Brazil within small pilot trials (10 to $16 \mathrm{ha}$ ) (Carvalho et al. 2015; Harris et al. 2011; Lacroix et al. 2012). It is currently extended to larger areas in Brazil, encompassing $12 \mathrm{~km}^{2}$ with nearly 60,000 residents which will allow evaluating its potential

\footnotetext{
${ }^{3}$ Fried index measures the propensity of a wild female to mate with a sterile male if it is given the choice between this sterile male and a wild male. It is calculated using the ratio of sterile to wild males and the induced sterility in the target population. For example, the female will mate twice more often with a wild than a sterile male if the Fried index is 0.5 .
}

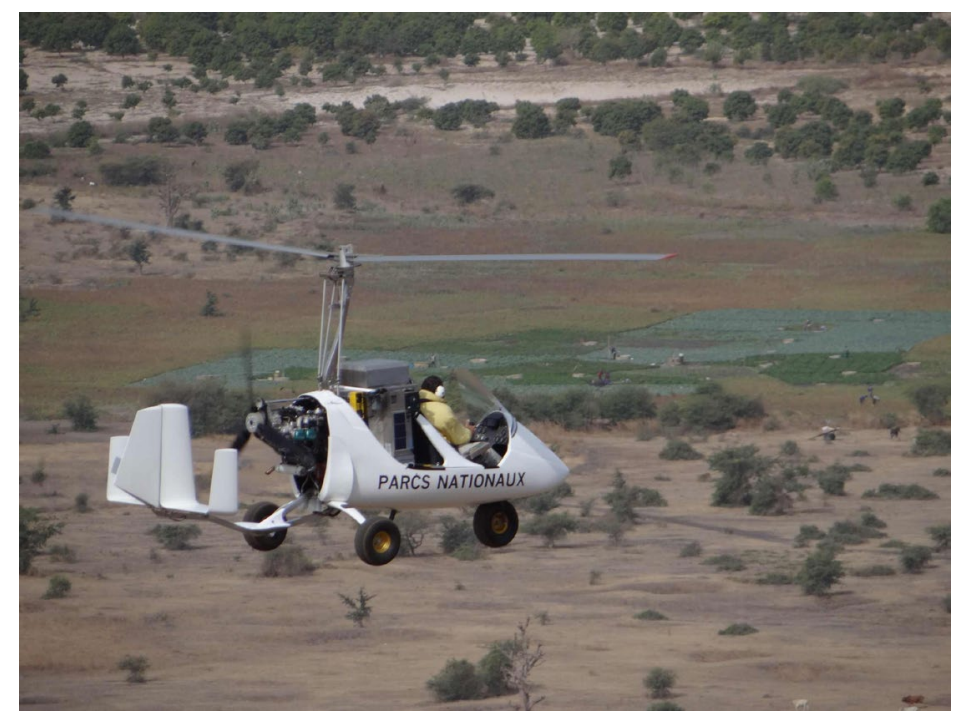

Figure 3. A gyrocopter releasing sterile tsetse male Glossina palpalis gambiensis in Senegal thanks to an automatic release machine (photo by Jérémy Bouyer). 
impact on virus transmission (Servick 2016). In the system tested (OX513A strain), the progeny of transgenic males are killed by the transgene (Alphey 2014). In pilot trials, the target larvae populations were suppressed by 80 to $96 \%$ but an overall low competitiveness of the transgenic males was observed in the field, ranging from 3 to $6 \%$ (Carvalho et al. 2015). This can be partly blamed on the current legislation inappropriately considering transgenic strains as medicines, making it necessary to use the originally registered strain everywhere without adjusting its genetic background (Mexican for OX513A in this case) (Haut Conseil des Biotechnologies 2017), which is better adapted to laboratory conditions than to the environmental conditions in the target areas.

Finally, the cost of this technique is still high, estimated at $\$ 5$ per person per year in Brazil (Servick 2016) while the total treated area remains minuscule compared to the extent of Ae. aegypti distribution in this country.

\section{Use of transinfected Wolbachia to transform mosquito populations}

A successful trial was conducted in 2011 in two natural Ae. aegypti populations in Australia, using a strain of Ae. aegypti transinfected with the wMel Wolbachia strain from Drosophila melanogaster (Hoffmann et al. 2011). The Wolbachia strain, released using infected males at a density allowing to reach the invasion threshold of $30 \%$ (which is quite high due to a loss of fitness in the infected adults), successfully invaded the two wild populations, reaching near-fixation in a few months, in the release areas only, following releases (Hoffmann et al. 2011). Pathogen interference from this Wolbachia strain blocks the transmission of various pathogens including dengue virus, thanks to a mechanism that is still not fully understood at the time of writing.

A follow-up study demonstrated that the strain was still fixed in the Australian target populations in 2013 and 2014 (Hoffmann et al. 2014). The strain also did not diffuse outside of treated areas, which is probably due to the low dispersal capacities of Ae. aegypti combined with the high invasion threshold necessary to fix wMel Wolbachia because of its intrinsic fitness cost. Moreover, wMel mosquitoes collected from these localities in January 2012 and challenged with three dengue virus serotypes still showed little vector competence in comparison to the wild type (Frentiu et al. 2014).

These open releases were approved by the Australian Pesticides and Veterinary Medicines Authority (Hoffmann et al. 2011). However, these field releases stimulated debates in the scientific community about the potential risks associated with this technique (Dobson et al. 2016; Loreto and Wallau 2016b; O'Neill 2016). One of the controversial points was that this particular symbiotic association would have a very low probability to appear without human intervention, even if other Wolbachia strains were previously detected in Ae. aegypti (Coon et al. 2016; Klasson et al. 2009; Woolfit et al. 2009). Still, the man-stimulated spread of such a symbiotic association in a natural population is currently not raising as much public concern as transgenes. Nevertheless, predicting how transmitted viruses will react to this new selection pressure is difficult, all the more so as the mechanism blocking their transmission is still unknown. It cannot be excluded that virus strains evolve to evade the Wolbachia-mediated mosquito resistance, perhaps with increased pathogenicity for vectors or even hosts.

Finally, this technique is also costly at the time of writing, although the Eliminate Dengue program in Brazil plans to bring its cost down to $\$ 1$ per person or less (Servick 2016). 


\section{Gene drive to control mosquitoes}

The elimination approach was tested by designing gene drive cassettes homing into three different female fertility genes (Hammond et al. 2016). In preliminary cage experiments, the frequency of one of the constructs increased from an initial 50 to $75 \%$ within 4 generations, consistent with a model of invasion - and suppression- of a wild mosquito population in case of release of this construct. However, the efficacy of these gene drives is currently limited by the unexpected sterility of heterozygous females. The loss-of-function mutations in these fertility genes should be recessive, but due to somatic activity of the drive - in addition to the desired activity in the germ line - females that are initially heterozygous become somatically homozygous and sterile, hampering a female contribution to the spread of the transgene. In addition, gene drive spread in nature is likely to be limited by natural genetic diversity and by the appearance of drive-resistant mutations due to the drive mechanism itself (Champer et al. 2017; Unckless et al. 2017). Work is in progress to solve these roadblocks and generate more efficient elimination drives. However, it is important to note that decisions regarding field implementation of elimination drives will have to consider the possible undesired ecological consequences of the elimination of an entire species (Hochkirch et al. 2017).

In contrast to these elimination drives, an example of a population modifying gene drive has been provided for An. stephensi, in which antipathogen effector genes targeting Plasmodium falciparum and transcribed in female only thanks to blood meal-regulated expression were successfully transmitted at a very high rate ( 99.5\%) (Gantz et al. 2015). These results illustrate the possibility to transform wild populations of vectors in order to interrupt pathogen transmission.

In Ae. aegypti, it was suggested that CRISPR-Cas9 might be used to target the mosquito male determining factor ( $M$ factor) to drive maleness to control wild mosquito populations (Adelman and $T u$ 2016). Ectopic expression of $M$ in genetic females would result in female masculinization or death, whereas males may carry the masculinizing transgene on to their progeny. This would lead to a fast spread of the transgene in the population, ideally each new transgenic generation being constituted of transgenic males only. The $M$ factor has only been recently discovered in Ae. aegypti (Hall et al. 2015) and even more recently in An. gambiae (Krzywinska et al. 2016).

Although very attractive - regarded by some as a silver bullet - gene drive strategies offer the possibility to alter entire wild populations and therefore ecosystems, and must include robust safeguards and methods of control (Esvelt et al. 2014). Moreover, two primary risks related to undesired spread have been identified. First, rare fertile hybridisation events may allow the drive to affect closely related species (in the Anopheles complex for example). It should be possible to mitigate this risk by using precision drives (subspecies-specific sequences) to target sequences unique to the targeted vector. Second, where this method would be employed against invasive species such as Aedes mosquitoes, the construct might spread from the targeted invasive populations back into the native distribution range and lead to species eradication. This risk poses ethical concerns about the desirability of eradicating entire species. It will thus be critical that all decisions involving the use of suppression drives involve extensive deliberations including but not limited to ecologists and citizens of potentially affected communities (Esvelt et al. 2014). In addition, given that these transgenes have the potential to disseminate through borders, their use will demand international debates and regulations.

A committee of experts appointed by the US National Academies of Sciences, Engineering, and Medicine has recently estimated that current evaluation and regulatory procedures are 
not adequate to address the risks and requirements of this technology (National Academies of Sciences, Engineering and Medicine 2016). Therefore, a wide consultation involving regulatory bodies, scientists, medical authorities, vector control agents and the public is needed to establish the risk/benefit evaluation and regulatory procedures that are necessary to move this research from the lab to field implementation (James et al. 2018).

\section{Involving public research and industries}

Collaboration between public research and the private sector is needed for the large-scale use of genetic methods that require mass-rearing and area-wide releases of insects. Gene drive is a notable exception, since it theoretically requires the release of only few individuals to initiate target population invasion by the transgene.

In the case of SIT, the large-scale use of sterile insects implies mobilising extensive research and development programmes, such as the ones leading to the eradication of the screwworm in North America, Central America and Libya, and of the Mediterranean fruit fly, of agricultural concern, in Mexico (Enkerlin et al. 2015). However, public research cannot implement these programmes alone, but can contribute to them by conducting the operational research essential to their optimisation, success and regulation.

Pesticide industries are faced with increasingly strict regulations limiting the number of approved molecules and with the shorter lifespan of these molecules because of the spread of genetic resistance in insects. It is therefore in their interest to invest in the development of new non-toxic control methods, and to deploy these within an integrated pest control framework, taking into account lessons learned from past experience. Public research can make significant contributions to both processes.

Another area of collaboration is the assessment of risks associated with the use of transgenic insects which requires a case-by-case analysis of risks, which depend on the genetic mechanisms used, and in particular on their potential for dissemination. In Europe, this analysis can be based on the European Food Safety Authority recommendations (EFSA 2013).

Although genetic control has potential that must be exploited, no technique should be automatically ruled out, and it is essential to analyse the advantages, disadvantages and risks associated with each one. This implies gaining further knowledge of the ecology of target vector populations, but also of the socio-ecosystems concerned by vector control. It will then be possible to achieve an optimal and integrative combination of several different methods, with the help of modelling studies.

\section{Perspectives}

Beyond the emerging genetic methods described above, other integrated strategies have been proposed to amplify the impact of genetic control. For example, implementing SIT requires sufficient quantities of sterile males to overwhelm wild males in the target population, which is generally very costly or even impossible when suppression techniques are not fully efficient, like in the case of Aedes mosquito species. To overcome these obstacles, a variant of SIT, named 'boosted SIT', has been proposed (Bouyer and Lefrançois 2014). It aims to reduce by 90 to $99 \%$ the quantity of sterile males needed to control or eradicate a target population. In this variant strategy, sterile males are considered as a specific means of contaminating females with a control 
agent. This agent may be an active substance, bacteria, a fungus or a virus, or even recombinant versions of these pathogens. An example of proposed association to sterile males are densoviruses (Bouyer et al. 2016), that are very specific to the target species or closely related species (Carlson et al. 2006). In the case of the AeDNV virus isolated from Ae. albopictus for example, only other Aedes or Culex species are sensitive to the virus, while all other insects or crustaceans are not. The double specificity of action warranted by specific contamination of the target species and larval habitats through the sterile males and the restricted host range of the virus might allow proposing efficient and safe vector control methods in the near future.

\section{Conclusion}

Genetic control appears as a major alternative to the use of large spectrum insecticides in the global war opposing humans to pest and vector arthropod species. No technology represents a solution on its own, but the SIT, the ancestor of all the techniques presented in this chapter, represents a biological alternative with validated successes in the past and that appears as the safest alternative at the time of writing. Many opportunities have emerged recently, stimulated by the spread of vector-borne diseases, particularly mosquito-borne arboviruses like dengue, chikungunya or Zika. But these opportunities are also accompanied by ecological and ethical challenges that will necessitate unbiased assessments of potential benefits and risks at an international level before they can be used at an operational scale.

\section{Acknowledgements}

The authors would like to thank K. Bourtzis (Insect Pest Control Sub-programme, Joint FAO/IAEA Division of Nuclear Techniques in Food and Agriculture, Vienna, Austria) for the revision of the draft chapter.

The work of J. Bouyer is funded by the REVOLINC (revolutionizing insect control) ERC Consolidator Grant. This project has received funding from the European Research Council (ERC) under the European Union's Horizon 2020 research and innovation programme (grant agreement No 682387).

\section{References}

Adelman ZN and Tu Z (2016) Control of mosquito-borne infectious diseases: sex and gene drive. Trends Parasitol 32: 219-229.

Akiner MM, Demirci B, Babuadze G, Robert V and Schaffner F (2016) Spread of the invasive mosquitoes Aedes aegypti and Aedes albopictus in the Black Sea region increases risk of chikungunya, dengue, and Zika outbreaks in Europe. PLoS Negl Trop Dis 10: e0004664.

Aksoy S, Weiss B and Attardo G (2008) Paratransgenesis applied for control of tsetse transmitted sleeping sickness. In: Aksoy S (ed.) Transgenesis and the management of vector-borne disease. Springer, New York, NY, USA, pp. 35-48.

Alphey L (2014) Genetic control of mosquitoes. Ann Rev Entomol 59: 205.

Atyame CM, Pasteur N, Dumas E, Tortosa P, Tantely ML, Pocquet N, Licciardi S, Bheecarry A, Zumbo B, Weill M and Duron $\mathrm{O}$ (2011) Cytoplasmic incompatibility as a means of controlling Culex pipiens quinquefasciatus mosquito in the islands of the south-western Indian Ocean. PLoS Negl Trop Dis 5: e1440.

Barclay HJ, Matlock R, Gilchrist S, Suckling DM, Reyes J, Enkerlin WR and Vreysen MJB (2011) A conceptual model for assessing the minimum size area for an area-wide integrated pest management program. International Journal of Agronomy, Article ID: 40932.

Bellini R, Balestrino F, Medici A, Gentile G, Veronesi R and Carrieri M (2013) Mating competitiveness of Aedes albopictus radio-sterilized males in large enclosures exposed to natural conditions. J Med Entomol 50: 94-102. 
Benedict MQ, Levine RS, Hawley WA and Lounibos LP (2007) Spread of the tiger: global risk of invasion by the mosquito Aedes albopictus. Vector-Borne Zoonotic Dis 7: 76-85.

Bourtzis K, Lees RS, Hendrichs J and Vreysen MJB (2016) More than one rabbit out of the hat: radiation, transgenic and symbiont-based approaches for sustainable management of mosquito and tsetse fly populations. Acta Trop 157: 115-130.

Bouyer F, Seck MT, Dicko A, Sall B, Lo M, Vreysen M, Chia E, Bouyer J and Wane A (2014) Ex-ante cost-benefit analysis of tsetse eradication in the Niayes area of Senegal. PLoS Negl Trop Dis 8: e3112.

Bouyer J (2015) Integrated management of insect vectors of human and animal diseases. Developing genetic control. CIRAD Perspectives 34, 4 pp. Available at: https://tinyurl.com/y88phugf.

Bouyer J, Chandre F, Gilles J and Baldet T (2016) Alternative vector control methods to manage the Zika virus outbreak: more haste, less speed. Lancet Glob Health 4: e364.

Bouyer J, Grimaud Y, Pannequin M, Esnault $O$ and Desquesnes M (2011) Importance épidémiologique et contrôle des stomoxes à la Réunion. Bull Epidémiol 43: 53-58.

Bouyer J and Lefrançois T (2014) Boosting the sterile insect technique to control mosquitoes. Trends Parasitol 30: $271-273$.

Bouyer J, Seck MT, Pagabeleguem S, Sall B, Lo M, Vreysen MJB, Balenghien T and Lancelot R (2012) Study of the competitiveness of allochtonous sterile males during the tsetse eradication campaign in Senegal. $18^{\text {th }}$ Conférence E-sove 2012, EID, CIRAD, IRD, Montpellier, France.

Bouyer J, Seck MT, Sall B, Guerrini L and Vreysen MJB (2010) Stratified entomological sampling in preparation of an area-wide integrated pest management programme: the example of Glossina palpalis gambiensis in the Niayes of Senegal. J Med Entomol 47: 543-552.

Burt A (2003) Site-specific selfish genes as tools for the control and genetic engineering of natural populations. Proc Royal Soc London B: Bio Sci 270: 921-928.

Carlson J, Suchman E and Buchatsky L (2006) Densoviruses for control and genetic manipulation of mosquitoes. Adv Virus Res 68: 361-392.

Carvalho DO, McKemey AR, Garziera L, Lacroix R, Donnelly CA, Alphey L, Malavasi A and Capurro ML (2015) Suppression of a field population of Aedes aegypti in Brazil by sustained release of transgenic male mosquitoes. PLoS Negl Trop Dis 9: e0003864.

Champer J, Reeves R, Oh SY, Liu C, Liu J, Clark AG and Messer PW (2017) Novel CRISPR/Cas9 gene drive constructs in Drosophila reveal insights into mechanisms of resistance allele formation and drive efficiency in genetically diverse populations. bioRxiv: 112011.

Concha C, Palavesam A, Guerrero FD, Sagel A, Li F, Osborne JA, Hernandez Y, Pardo T, Quintero G and Vasquez M (2016) A transgenic male-only strain of the New World screwworm for an improved control program using the sterile insect technique. BMC Biol 14: 72.

Coon KL, Brown MR and Strand MR (2016) Mosquitoes host communities of bacteria that are essential for development but vary greatly between local habitats. Mol Ecol 25(22): 5806-5826.

Dicko AH, Lancelot R, Seck MT, Guerrini L, Sall B, Lo M, Vreysen MJB, Lefrançois T, Williams F, Peck SL and Bouyer J (2014) Using species distribution models to optimize vector control: the tsetse eradication campaign in Senegal. Proc Nat Acad Sci 111: 10149-10154.

Dobson SL, Bordenstein SR and Rose RI (2016) Wolbachia mosquito control: regulated. Science 352: 526-527.

Durel L, Estrada-Peña A, Franc M, Mehlhorn H and Bouyer J (2015) Integrated fly management in European ruminant operations from the perspective of Directive 2009/128/EC on sustainable use of pesticides. Parasitol Res 114: 379-389.

European Commission (2009) Directive 2009/128/EC of the European Parliament and of the Council of 21 October 2009 establishing a framework for Community action to achieve the sustainable use of pesticides. OJ L 309: 1-26.

European Food Safety Authority (EFSA) (2013) Panel on Genetically Modified Organisms (GMO) Guidance on the environmental risk assessment of genetically modified animals. EFSA J 11: 3200. 
Enkerlin W, Gutiérrez-Ruelas JM, Cortes AV, Roldan EC, Midgarden D, Lira E, López JLZ, Hendrichs J, Liedo P and Arriaga FJT (2015) Area freedom in Mexico from mediterranean fruit fly (Diptera: Tephritidae): a review of over 30 years of a successful containment program using an integrated area-wide SIT approach. Florida Entomol 98: 665-681.

Esvelt KM, Smidler AL, Catteruccia F and Church GM (2014) Concerning RNA-guided gene drives for the alteration of wild populations. Elife 3: e03401.

Food and Agriculture Organization of the United Nations (FAO) (2015) Glossaire des termes phytosanitaires NIMP 5. FAO, Rome, Italy.

Frentiu FD, Zakir T, Walker T, Popovici J, Pyke AT, Van den Hurk A, McGraw EA and O'Neill SL (2014) Limited dengue virus replication in field-collected Aedes aegypti mosquitoes infected with Wolbachia. PLoS Negl Trop Dis 8: e2688.

Fried M (1971) Determination of sterile-insect competitiveness. J Econ Entomol 64: 869-872.

Galizi R, Doyle LA, Menichelli M, Bernardini F, Deredec A, Burt A, Stoddard BL, Windbichler N and Crisanti A (2014) A synthetic sex ratio distortion system for the control of the human malaria mosquito. Nature Com 5:3977.

Galizi R, Hammond A, Kyrou K, Taxiarchi C, Bernardini F, O'Loughlin SM, Papathanos P-A, Nolan T, Windbichler N and Crisanti A (2016) A CRISPR-Cas9 sex-ratio distortion system for genetic control. Sci Rep 6: 31139.

Gantz VM, Jasinskiene N, Tatarenkova O, Fazekas A, Macias VM, Bier E and James AA (2015) Highly efficient Cas9mediated gene drive for population modification of the malaria vector mosquito Anopheles stephensi. Proc Natl Acad Sci 112: E6736-E6743.

Hall AB, Basu S, Jiang X, Qi Y, Timoshevskiy VA, Biedler JK, Sharakhova MV, Elahi R, Anderson MAE, Chen X-G, Sharakhov IV, Adelman ZN and Tu Z (2015) A male-determining factor in the mosquito Aedes aegypti. Science 348: 1268-1270.

Hammond A, Galizi R, Kyrou K, Simoni A, Siniscalchi C, Katsanos D, Gribble M, Baker D, Marois E, Russell S, Burt A, Windbichler N, Crisanti A and Nolan T (2016) A CRISPR-Cas9 gene drive system targeting female reproduction in the malaria mosquito vector Anopheles gambiae. Nature Biotech 34: 78-83.

Harris AF, Nimmo DD, McKemey AR, Kelly N, Scaife S, Donnelly CA, Beech C, Petrie WD and Alphey L (2011) Field performance of engineered male mosquitoes. Nature Biotech 29: 1034-1037.

Haut Conseil des Biotechnologies (2017) Avis du Comité Scientifique en réponse à la saisine du 12 octobre 2015 concernant l'utilisation de moustiques génétiquement modifiés dans le cadre de la lutte antivectorielle. Haut Conseil des Biotechnologies, Paris, France.

Hochkirch A, Beninde J, Fischer M, Krahner A, Lindemann C, Matenaar D, Rohde K, Wagner N, Wesch C and Wirtz S (2017) License to kill? - disease eradication programs may not be in line with the convention on biological diversity. Conservation Lett 11: e12370.

Hoffmann AA, Iturbe-Ormaetxe I, Callahan AG, Phillips BL, Billington K, Axford JK, Montgomery BL, Turley AP and O'Neill SL (2014) Stability of the wMel Wolbachia infection following invasion into Aedes aegypti populations. PLoS Negl Trop Dis 8: e3115.

Hoffmann AA, Montgomery BL, Popovici J, Iturbe-Ormaetxe I, Johnson PH, Muzzi F, Greenfield M, Durkan M, Leong YS, Dong Y, H. Cook, Axford J, Callahan AG, Kenny N, Omodei C, McGraw EA, Ryan PA, Ritchie SA, Turelli M and O'Neill SL (2011) Successful establishment of Wolbachia in Aedes populations to suppress dengue transmission. Nature 476: 454-457.

James S, Collins FH, Welkhoff PA, Emerson C, Godfray HCJ, Gottlieb M, Greenwood B, Lindsay SW, Mbogo CM, Okumu FO, Quemada H, Savadogo M, Singh JA, Tountas KH and Touré YT (2018) Pathway to deployment of gene drive mosquitoes as a potential biocontrol tool for elimination of malaria in sub-Saharan Africa: recommendations of a Scientific Working Group. Am J Trop Med Hyg 98 (Suppl 6): 1-49.

Klasson L, Kambris Z, Cook PE, Walker T and Sinkins SP (2009) Horizontal gene transfer between Wolbachia and the mosquito Aedes aegypti. BMC Genomics 10: 1.

Krzywinska E, Dennison NJ, Lycett GJ and Krzywinski J (2016) A maleness gene in the malaria mosquito Anopheles gambiae. Science 353: 67-69.

Kumar A, Wang S, Ou R, Samrakandi M, Beerntsen BT and Sayre RT (2013) Development of an RNAi based microalgal larvicide to control mosquitoes. Malaria World J 4: 1-7.

Lacroix R, McKemey AR, Raduan N, Wee LK, Ming WH, Ney TG, Siti Rahidah AA, Salman S, Subramaniam S and Nordin O (2012) Open field release of genetically engineered sterile male Aedes aegypti in Malaysia. PLoS ONE 7: e42771. 
Loreto ELS and Wallau GL (2016a) Risks of Wolbachia mosquito control. Science 351: 1273.

Loreto ELS and Wallau GL (2016b) Risks of Wolbachia mosquito control. Science 351: 1273-1273.

Mancini MV, Spaccapelo R, Damiani C, Accoti A, Tallarita M, Petraglia E, Rossi P, Cappelli A, Capone A and Peruzzi G (2016) Paratransgenesis to control malaria vectors: a semi-field pilot study. Parasite Vectors 9: 1.

McBeath J (2015) An industry perspective on the development of new insecticide - based tools for vector control. International Society for Neglected Tropical Diseases - ISNTD Bites, London, UK.

McGraw EA and O'Neill SL (2013) Beyond insecticides: new thinking on an ancient problem. Nature Reviews Microbiology 11: 181-193.

Mubarqui RL, Perez RC, Angulo Kladt R, Zavala Lopez JL, Parker A, Seck MT, Sall B and Bouyer J (2014) The smart aerial release machine, a universal system for applying the sterile insect technique. PLoS ONE 9: e103077.

National Academies of Sciences, Engineering and Medicine (2016) Gene drives on the horizon: advancing science, navigating uncertainty, and aligning research with public values. National Academies Press, Washington, DC, USA.

O'Connor L, Plichart C, Sang AC, Brelsfoard CL, Bossin HC and Dobson SL (2012) Open release of male mosquitoes infected with a Wolbachia biopesticide: field performance and infection containment. PLoS Negl Trop Dis 6: e1797.

O'Neill SL (2016) Wolbachia mosquito control: tested. Science 352: 526-526.

O'Brochta DA, Atkinson PW and Lehane MJ (2000) Transformation of Stomoxys calcitrans with a Hermes gene vector. Insect Mol Bio 9: 531-538.

Pagabeleguem S, Seck MT, Sall B, Vreysen MJB, Gimonneau G, Fall AG, Bassene M, Sidibé I, Rayaisse JB, Belem A and Bouyer J (2015) Long distance transport of irradiated male Glossina palpalis gambiensis pupae and its impact on sterile male yield. Parasite Vectors 8: 259 .

Patterson RS, LaBrecque GC, Williams DF and Weidhaas DE (1981) Control of the stable fly, Stomoxys Calcitrans (Diptera: Muscidae), on St. Croix, US Virgin Islands, using integrated pest management measures: III. Field techniques and population control. J Med Entomol 18: 203-210.

Phuc HK, Andreasen MH, Burton RS, Vass C, Epton MJ, Pape G, Fu G, Condon KC, Scaife S, Donnelly CA, Coleman PG, White-Cooper $\mathrm{H}$ and Alphey $\mathrm{L}$ (2007) Late-acting dominant lethal genetic systems and mosquito control. BMC Biol 5: 1.

Ranson H, N'Guessan R, Lines J, Moiroux N, Nkuni Z and Corbel V (2011) Pyrethroid resistance in African anopheline mosquitoes: what are the implications for malaria control? Trends Parasitol 27: 91-98.

Rezza G, Nicoletti L, Angelini R, Romi R, Finarelli AC, Panning M, Cordioli P, Fortuna C, Boros S and Magurano F (2007) Infection with chikungunya virus in Italy: an outbreak in a temperate region. Lancet 370: 1840-1846.

Seck MT, Bouyer J, Sall B, Bengaly Z and Vreysen MJB (2010) The prevalence of African animal trypanosomoses and tsetse presence in western Senegal. Parasite 17: 257-265.

Seck MT, Pagabeleguem S, Bassene MD, Fall AG, Diouf TAR, Sall B, Vreysen MJB, Rayaissé J-B, Takac P, Sidibé I, Parker AG, Mutika GN, Bouyer J and Gimonneau G (2015) Quality of sterile male tsetse after long distance transport as chilled, irradiated pupae. PLoS Negl Trop Dis 9: e0004229.

Servick K (2016) Brazil will release billions of lab-grown mosquitoes to combat infectious disease. Will it work? Science, Washington, DC, USA. Available at: https://tinyurl.com/h383qek.

Solano P, Kaba D, Ravel S, Dyer N, Sall B, Vreysen MJB, Seck MT, Darbyshir H, Gardes L, Donnelly MJ, De Meeûs T and Bouyer J (2010) Tsetse population genetics as a tool to choose between suppression and elimination: the case of the Niayes area in Senegal. PLoS Trop Negl Dis 4: e692.

Sow A, Sidibé I, Bengaly Z, Bancé Z, Sawadogo GJ, Solano P, Vreysen MJB, Lancelot R and Bouyer J (2012) Irradiated male Glossina palpalis gambiensis (Diptera: Glossinidae) from a 40-years old colony are still competitive in a riparian forest in Burkina Faso. PLoS ONE 7: e37124.

Suckling DM, Stringer LD, Stephens AE, Woods B, Williams DG, Baker G and El-Sayed AM (2014) From integrated pest management to integrated pest eradication: technologies and future needs. Pest Manag Sci 70: 179-189.

Unckless RL, Clark AG and Messer PW (2017) Evolution of resistance against CRISPR/Cas9 gene drive. Genetics 205: 827-841.

Vargas-Terán M, Hursey BS and Cunningham EP (1994) Eradication of the screwworm from Libya using the sterile insect technique. Parasitol Today 10: 119-122. 
Vreysen MJB, Saleh KM, Ali MY, Abdulla AM, Zhu Z-R, Juma KG, Dyck VA, Msangi AR, Mkonyi PA and Feldmann HU (2000) Glossina austeni (Diptera: Glossinidae) Eradicated on the island of Unguja, Zanzibar, using the sterile insect technique. J Econ Entomol 93: 123-135.

Vreysen MJB, Saleh KM, Lancelot R and Bouyer J (2011) Factory tsetse flies must behave like wild flies: a prerequisite for the sterile insect technique. PLoS Trop Negl Dis 5(2): e907.

Vreysen MJB, Seck MT, Sall B, Mbaye AG, Bassene M, Fall AG, Lo M and Bouyer J (in press) Eradication of Glossina palpalis gambiensis from the Niayes area of Senegal: a review of operational research in support of a phased conditional approach. In: Hendrichs J, Pereira R and Vreysen MJB (eds.) Area-wide integrated pest management: development and field application. CRC Press, Boca Raton, FL, USA.

Walshe DP, Lehane SM, Lehane MJ and Haines LR (2009) Prolonged gene knockdown in the tsetse fly Glossina by feeding double stranded RNA. Insect Mol Biol 18: 11-19.

Whyard S, Erdelyan CN, Partridge AL, Singh AD, Beebe NW and Capina R (2015) Silencing the buzz: a new approach to population suppression of mosquitoes by feeding larvae double-stranded RNAs. Parasit Vectors 8: 96.

Windbichler N, Menichelli M, Papathanos PA, Thyme SB, Li H, Ulge UY, Hovde BT, Baker D, Monnat RJ and Burt A (2011) A synthetic homing endonuclease-based gene drive system in the human malaria mosquito. Nature 473:212-215.

Woolfit M, Iturbe-Ormaetxe I, McGraw EA and O'Neill SL (2009) An ancient horizontal gene transfer between mosquito and the endosymbiotic bacterium Wolbachia pipientis. Mol Biol Evol 26: 367-374.

Wyss JH (2006) Screwworm eradication in the Americas. Ann NY Acad Sci 916: 186-193.

Zhang D, Zheng X, Xi Z, Bourtzis K and Gilles JRL (2015) Combining the sterile insect technique with the incompatible insect technique: I-impact of Wolbachia infection on the fitness of triple-and double-infected strains of Aedes albopictus. PLoS ONE 10: e0121126. 


\title{
15. Biosecurity: methods to reduce contact risks between vectors and livestock
}

\author{
Bastiaan G. Meerburg ${ }^{1,2^{*}}$ and Bruce Schoelitsz ${ }^{3}$ \\ ${ }^{1}$ Wageningen Livestock Research, Wageningen University \& Research, P.O. Box 338,6700 AHWageningen, \\ the Netherlands; ${ }^{2}$ Dutch Pest \& Wildlife Expertise Centre (KAD), P.O. Box 350, 6700 AJ Wageningen, \\ the Netherlands; ${ }^{3}$ HAS University of Applied Sciences, P.O. Box 90108, 5200 MA's-Hertogenbosch, the \\ Netherlands; bastiaan.meerburg@wur.nl
}

\begin{abstract}
In order to prevent direct contact between livestock and pest animals and thus decrease the risk of pathogen transmission, the implementation of preventive or sometimes even curative measures is required. The concept of biosecurity refers to implementation of such measures, but it is difficult to quantify the results as the situation between farms may vary substantially. In this chapter we investigate the position of biosecurity and the evolution of this concept, especially in relation to pest management. We stress the need for such a strategy not only because of the potential transmission of (zoonotic) pathogens to livestock, which can have significant consequences for livestock health and the food chain, but also because of structural damage to buildings and crops. As there are large differences in both farm conditions and between vectors, implementation of a pest management strategy can come with serious difficulties. Thus, we present a generic framework that helps to develop a more tailor-made approach for a pest management strategy on farms, which will hopefully contribute to more effective interventions.
\end{abstract}

Keywords: biosecurity, framework, livestock, pests, pest management

\section{Introduction}

Originally, the concept of biosecurity mainly focused on the introduction of new livestock animals to a farm, consequently leading to introduction of new pathogens within the herd (Casal et al. 2007; Moore 1992). To reduce such risks, it was necessary to reduce the number of source farms from which livestock is purchased or to keep new animals under quarantine. Moreover, biosecurity also dealt with the risks of visitors or even employees: people that work on a farm should not visit other farms, and wear overalls and boots for on-farm use exclusively. A track record of the visits and the visitors is also necessary to trace possible emerging disease threats.

However, two important aspects of biosecurity that have historically received less attention are: (1) the geographical location of the farm (probably as it is a non-modifiable parameter as soon as the farm is built); and (2) the risk due to vectors. Although some studies recommend the installation of bird-proof nets in windows and the need of proper disinfection, disinfestation and rodent-control programmes, one of the main problems is how to implement these measures on-farm in practice as each farm is different.

In this chapter we will focus on this point, the risks of vectors, and discuss methods to reduce their influence on the farm. Because of the differences between farms, we will present a generic framework that will help to develop a tailor-made pest management approach for each farm. 
It should be noted that for this chapter, we define vectors as all animals that potentially transmit pathogens to livestock, including arthropods, mammals and birds, even if the pathogen does not depend on the vector species to complete its life cycle. Furthermore, we classify pests as all unwanted animals at a specific time and location, including vectors.

\section{Risks of vector presence: introduction of pathogens to livestock farms}

Although much remains to be discovered, we know that vectors can have a substantial influence on the introduction of pathogens to farms and should therefore be carefully monitored. Phylogenetic analysis of an highly pathogenic avian influenza $A(H 5 N 8)$ virus outbreak in the Netherlands in 2014, provided evidence of four separate introductions and one between-poultry farm transmission (Bouwstra et al. 2015). Evolution of the virus seemed to follow a parallel track in Japan and Europe, which supports the hypothesis that H5N8 virus was exchanged between migratory wild waterfowl at their breeding grounds in Siberia and from there was carried by migrating waterfowl to Europe. In the Netherlands, the virus was then introduced to indoor poultry farms, for example via persons with contaminated clothing/boots/materials/feed, or by vermin including flies (Bouwstra et al. 2015). Moreover, these authors suggest to house poultry that normally has outdoor access, indoors during such epidemics in order to prevent exposure to possibly infected wild waterfowl and their excrement (Koch and Elbers 2006).

In a study on the epidemic of high pathogenicity avian influenza (HPAI) that occurred in the Netherlands in 2003, a census survey of 173 infected and 401 uninfected commercial poultry farms was carried out to identify factors associated with the introduction of the HPAl virus into poultry farms (Thomas et al. 2005). These authors found an increased risk of HPAl virus introduction in layer finisher type poultry if biosecurity was inappropriate: Odds Ratio $=2.05$ ( $95 \%$ confidence interval, $\mathrm{Cl}=1.29-3.27)$. An explanation for this increased risk was the high number of contacts between these farms, especially via cardboard egg trays used for removal of eggs during the epidemic. Moreover, this study identified size and housing type as possible confounders in this study. Although literature on Avian Influenza does not report accordingly, from observational studies on transmission of infectious diseases in intensively reared stock, a tendency arises for larger farms to become infected more often with infectious disease agents than small farms (Akey 2003; Dewey et al. 2003; Kaneene et al. 2002; Refregier-Petton et al. 2001) and thus farm size being a risk factor for the presence of these agents.

In China, sparrows (Passer domesticus) around poultry farms in the southern part of the country were shown to carry virulent Newcastle disease virus strains (Zhu et al. 2010). These strains were of a similar genotype as those circulating in poultry. Although the epidemiological link between sparrows and poultry is still difficult to illustrate (Zhu et al. 2010), it shows the potential of birds to transmit a hazardous pathogen.

During a study in Denmark (Hald et al. 2004) it was investigated how flies contribute to the transmission of Campylobacter jejuni to chickens during summer (April-October). It was found that hundreds of flies per day passed through the ventilation system into the broiler house. Of these, $8.2 \%$ of flies caught outside a broiler house were infected. The authors conclude that flies may cause severe problems in poultry houses (Hald et al. 2004). Beside Campylobacter, flies are also capable to carry a number of other bacteria, protozoan parasites and viruses (Baldacchino et al. 2018; Meerburg et al. 2007; Sparagano et al. 2018). In general, transmission of pathogens by adult flies occurs by: (1) mechanical dislodgement from their exoskeleton (e.g. by hair and bristles on 
their legs or by cushion-like structures, pulvilli that are used for adherence to vertical surfaces); (2) faecal deposition; and (3) the regurgitation of incompletely digested food (Meerburg et al. 2007).

A study from the Netherlands shows the importance of good pest management practices and what it may contribute to the health of the farm animals and also to consumer of pig meat (Kijlstra et al. 2008). Toxoplasma gondii is a parasite that can be transmitted via rodents and shrews to pigs and via those pigs to the consumer and may result in huge health hazards. In a study on three organic pig farms with a confirmed rodent problem, a 4-month rodent control campaign and a 7-month longitudinal analysis of $T$. gondii seroprevalence in slaughter pigs was organised. Initial T. gondii seroprevalence in the slaughter pigs ranged between 8 and $17 \%$ and dropped on the three farms during the rodent control campaign to $0-10 \%$, respectively. After four months of rodent control, T. gondii infection was absent from pigs from two of the three farms investigated and appeared again in one of those two farms after the rodent control campaign had stopped. This study emphasizes the role of rodents and shrews in the transmission of T. gondii to pigs and the importance of rodent control towards production of T. gondii-free pig meat (Kijlstra et al. 2008).

In the UK, wild brown rats (Rattus norvegicus) from 11 rural UK farmsteads were found to carry 13 zoonotic and 10 non-zoonotic parasitic species (e.g. Cryptosporidium, Listeria, Yersinia, Coxiella and Hantavirus). This study (Webster and Macdonald 1995) demonstrates that wild brown rats, serving as vectors of pathogenic organisms, can represent a serious risk to the health of domestic animals and also humans.

Litter beetles are the adult stage of the lesser mealworm Alphitobius diaperinus (Panzer). These beetles are known transmitters of a number of poultry pathogens including bacteria (e.g. Salmonella, Escherichia coli), fungi (Aspergillus spp.), and viruses such as infectious bursal disease virus (birnavirus), fowl poxvirus and Newcastle disease virus (Barin et al. 2010; Goodwin and Waltman 1996). In addition, excessive beetle populations can adversely affect feed conversion ratios and chick body weight gains and can cause damage to poultry house insulation (Goodwin and Waltman 1996). A problem with litter beetles is that cleaning the poultry house during the different rounds of poultry is hardly sufficient as this vector may hide itself in the insulation and may appear again if a clean flock is present. Thus, infections may re-emerge.

Culicoides spp. (Diptera: Ceratopogonidae) are world players in the epidemiology of many important arboviral diseases (Mellor et al. 2000), which they can transmit to sheep and cattle. Of these, bluetongue virus (BTV) is among the most devastating. Some suggest that the spread of BTV has been driven by recent changes in European climate that have allowed increased virus persistence during winter, the northward expansion of Culicoides imicola Kieffer, the main BTV vector in southern Europe, and, beyond this vector's range, transmission by indigenous European Culicoides species - thereby expanding the risk of transmission over larger geographical regions (Purse et al. 2005). However, the outbreak of bluetongue virus in northern Europe in 2006-2008 has led to an urgent need to identify control measures for the Culicoides biting midges that transmit the virus (Ansari et al. 2011). Although their control is difficult because of the biology of the species, recently it has been shown that entomopathogenic fungi are potential biocontrol agents against adult specimens (Weeks et al. 2018), through the application of 'dry' conidia on surfaces (e.g. manure, leaf litter, livestock) where the midges rest. Subsequent conidial transmission between males and females may cause an increased level of fungi-induced mortality in midges thus reducing the incidence of disease (Ansari et al. 2011). 
In swine farms in North Carolina and other southeastern states of the USA, German cockroaches (Blattella germanica Linnaeus) are a common pest (Zurek and Schal 2004). With laboratory assays their vector competence for one of the most important porcine bacterial pathogens, verotoxigenic E. coli F18, was evaluated. In addition, the populations of faecal coliforms from the faeces of piglets and cockroaches collected from a swine nursery were assessed (Zurek and Schal 2004). It was found that the population of faecal coliforms in cockroach faeces was high $\left(4.4 \times 10^{5} \mathrm{cfu} / \mathrm{g}\right)$ and comparable to that of piglet faeces $\left(1.9 \times 10^{6} \mathrm{cfu} / \mathrm{g}\right)$. This study demonstrates that cockroaches may serve as important mechanical vectors of pathogenic $E$. coli in the swine industry of the USA (Zurek and Schal 2004).

Another vector that is of serious importance in the livestock industry is the poultry red mite Dermanyssus gallinae (De Geer). This threat to the laying-hen industry affects the production adversely and threatens hen health and welfare throughout the globe, both directly and through its role as a disease vector (George et al. 2015). The potential medical significance of $D$. gallinae is exacerbated by the fact that these mites can carry and transmit a number of zoonotic diseases of both bacterial and viral origin (George et al. 2015).

In sheep farms, abortions cause great important losses. Although the etiology of such abortions can be diverse, most of the abortifacient agents are zoonotic pathogens. One of these is Anaplasma phagocytophila that parasitizes granulocytes and monocytes in domestic ruminants in several countries in Europe causing a syndrome called tick-borne fever. The main vector of A. phagocytophila in Europe is the tick Ixodes ricinus (Linnaeus), which may benefit from warm temperatures and rainfall for its development and survival. In a study from Spain, this pathogen was found in association to abortions in around $25 \%$ of the investigated flocks (Garcia-Perez et al. 2003).

Contact between domestic pig populations and wild boar may also result in cross infections. Indications for such transfer are supported by findings on e.g. Mycobacterium bovis (the causative agent of bovine tuberculosis) in Spain (Parra et al. 2003), classical swine fever virus (Brugh Jr. et al. 1964), and hepatitis E virus in Japan (Meng et al. 2009).

West Nile virus is endemic in the Mediterranean Basin and in eastern Europe. The European strain can be locally detected in wild birds and may cause sporadic local cases in horses and people (Gortázar et al. 2007; Hubálek and Halouzka 1999).

In a study where remote surveillance was used to monitor red fox (Vulpes vulpes) activity in buildings on cattle farms in southwest England over a period of two years, frequent visits were observed through the year (Tolhurst et al. 2011). During such visits foxes excreted and scentmarked on stored feed, which carries potential risks of pathogen transmission to cattle. Fox visits were positively correlated with maximum temperature in the preceding 24 hours, and were most frequent to buildings where cattle were absent. The frequency of fox visits varied widely amongst farms, potentially reflecting variation in local farm management practices. On some farms, visits were frequent and involved excretion behaviour with the potential to result in contamination of the environment with infectious pathogens (Tolhurst et al. 2011).

It is concluded that a large number of wild vectors can be present in farming systems and their presence can lead to veterinary or even zoonotic problems and eventually, economic losses. We present a table of frequently encountered wild vectors that are known to impose a threat for livestock (Table 1). However, it should be noted that the risks of some vector species (e.g. common 
Table 1. Frequently encountered wild vectors associated with different types of livestock.

\begin{tabular}{ll}
\hline Livestock type & Problematic frequent wild vectors \\
Pigs & $\begin{array}{l}\text { rodents (Rattus rattus, Rattus norvegicus, Mus musculus), flies (Musca, Ophyra), wild boars, } \\
\text { shrews, cockroaches } \\
\text { rodents (R. rattus, R. norvegicus, Mus musculus), poultry red mite (Dermanyssus gallinae), flies } \\
\text { (Musca domestica, Stomoxys calcitrans), litter beetles (Alphitobius diaperinus), swallow bugs }\end{array}$ \\
& $\begin{array}{l}\text { (Oeciacus vicarius), waterfowl, marine and other birds } \\
\text { badgers, cervids (deer), rodents (M. musculus, Rattus spp.), flies (M. domestica, Hippoboscidae), } \\
\text { pigeons, ticks (Ixodes ricinus), foxes }\end{array}$ \\
Sheep \& goats & $\begin{array}{l}\text { mosquitoes (genus Culex), midges (genus Culicoides), ticks (I. ricinus) } \\
\text { birds, mosquitoes (e.g. genus Culex), ticks (I. ricinus) }\end{array}$ \\
\hline Horses & Pind
\end{tabular}

moles or insectivores that may function as intermediate hosts of pathogens) are sometimes poorly investigated (Krijger et al. 2014). Also, the capability of a significant vector species such as the brown rat (R. norvegicus) to spread OIE-listed diseases (such as foot and mouth disease virus or classical swine fever; see www.oie.int) needs to be further elucidated. Moreover, it should be remembered that beside wild vectors also domestic vectors such as cats and dogs may pose a serious threat to the well-being of the livestock on farms (and sometimes even later in the food chain).

\section{Additional risks of vector presence: structural damage to buildings or crops}

Beside transmission of hazardous pathogens, vector presence may also cause structural damage to buildings and crops, which is another reason to be careful with biosecurity. In the Netherlands, it is assumed that a large number of farm fires can be attributed to commensal rodents. Rats and mice start gnawing on the electric wiring and then insulation material catches fire because of short-circuiting. But not only mammals that can cause structural damage, as the lesser mealworm is known to cause destruction in insulation materials in poultry houses such as polystyrene (Despins et al. 1987; Vaughan et al. 1984).

Moreover, the presence of pests may also cause substantial pre- and postharvest losses on farms. In developing countries, rodents for example are known to cause huge pre- and post-harvest losses (Meerburg et al. 2009). Also in developed countries problems may occur. An Italian study (Santini 1986) presents an overview of damage caused in agriculture and forestry by several rodent species (Table 2 ).

In the winter of 2014/2015, there was a very significant outbreak of Microtus arvalis in the northern parts of the Netherlands, where grasslands of dairy farmers were damaged to a large extent. Particularly high densities of $M$. arvalis were observed in peat, clay-on-peat and clay, with much visible damage and bare eaten grasslands (Figure 1). In total, it was estimated that this outbreak caused a total direct damage of 15 million euros (Wymenga et al. 2016). As such high rodent densities do not only cause direct and indirect damage, but may also lead to additional health risks for livestock and the rural population, such outbreaks should be closely monitored. 
Table 2. An overview of damage caused in agriculture and forestry by several rodent species (adapted from Santini 1986).

\begin{tabular}{|c|c|c|c|}
\hline Species & Agriculture & Forestry & Damage \\
\hline \multirow[t]{2}{*}{ Rattus norvegicus } & & tree-seedlings in nurseries & sporadic and localized \\
\hline & $\begin{array}{l}\text { sunflower seedlings and ears } \\
\text { maize seedlings and ears }\end{array}$ & & $\begin{array}{l}\text { relevant and diffused } \\
\text { relevant and diffused }\end{array}$ \\
\hline Rattus rattus & & ornamental shrubs (bark removal) & frequent but localized \\
\hline Mus musculus & carnation in greenhouses & & frequent but localized \\
\hline Apodemus sylvaticus & $\begin{array}{l}\text { seedlings of Papilionaceae, } \\
\text { Cucurbitaceae }\end{array}$ & tree seedlings & relevant and diffused \\
\hline Microtus arvalis & $\begin{array}{l}\text { apple orchards (root bark } \\
\text { removal) }\end{array}$ & & very serious \\
\hline Microtus savii & $\begin{array}{l}\text { citrus orchards (root bark } \\
\text { removal) }\end{array}$ & & very serious \\
\hline & artichoke & pine trees (root bark removal) & relevant and diffused \\
\hline Glis glis & & pine trees, poplars (bark removal) & frequent but localized \\
\hline Sciurus vulgaris & & pine trees, poplars (bark removal) & frequent but localized \\
\hline
\end{tabular}

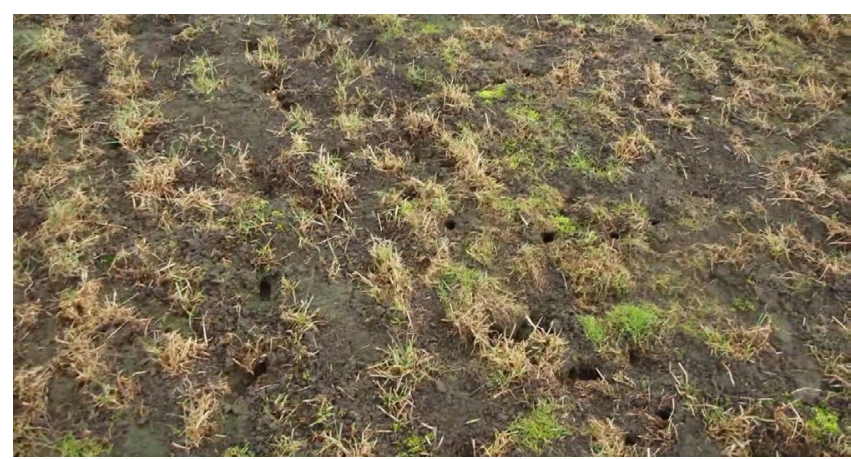

Figure 1. Damage caused to grasslands by the common vole (Microtus arvalis) in Friesland in 2014/2015 (photo by Wageningen University).

\section{Influencing vector presence: possible biosecurity measures and implications for integrated pest management}

Vectors that are present on farms can manifest themselves in many forms. One of the main difficulties is that advice to prevent vectors is most of the time quite generic, while the particular situation on each farm may cause different patterns of vector presence and therefore lead to different levels of risk. Vectors can be attracted to spilled feedstuffs, the availability of water and the presence of shelter, but there are also a number of other factors that may influence their levels. A number of such characteristics are described in Table 3. 
Table 3. Characteristics that may influence presence of vectors.

\begin{tabular}{ll}
\hline Factor & Example \\
Livestock species raised on the farm & e.g. pigs/poultry/cows \\
Farm size & hobby farm, family farm or factory farm type \\
Husbandry system & organic, free-range or conventional \\
neographic location of the farm & neater sanitation facility or waste disposal site \\
& near a nature conservation area or a lake \\
& near a deforestation area \\
& near a town or city \\
near other farms & presence of shrubs near or on farm buildings \\
presence of storage of materials near farm buildings & hygienic situation in general \\
Terrain characteristics on the farm & functioning of sewer system \\
& placement of garbage containers \\
& manure storage \\
pipes through walls are properly sealed & quality of doors \\
possibility for vectors to enter via chimneys, ventilation openings, joints, \\
the drainage system, etc. \\
quilding characteristics of farm \\
buildings
\end{tabular}

Two examples that shows the importance of a tailor-made approach to vector presence on farms are discussed.

If one has to deal with a cattle farm or organic livestock farms, it is generally difficult to prevent introduction of vectors, such as Norway rats ( $R$. norvegicus), because of the open stables. In a situation as this, hygienic measures, to prevent the presence of spilled food, are extremely important. Furthermore, storage of hay and other material in the stable may provide shelter and harbourage and should be avoided. Because of the opportunity of introduction that rats are given by the open structure of the stables, increased monitoring is necessary. If rat presence is detected in the vicinity of the stables, snap traps could be installed to trap them before they are able to establish a colony within the stable. A good measure that could also be taken by farmers is the installation of owl boxes, which will help to reduce the rodent population on the farms. Moreover, this step is often also positively evaluated by the general public (but beware of the use of bio-accumulative rodenticides, as this may lead to secondary poisoning of the owls!). Use of cats on intensive (pigs and poultry) farms is not a good preventive measure, as this can lead to transmission of the hazardous parasite Toxoplasma gondii. However, on cattle farms, transmission 
risk is much lower as this type of livestock is less prone to the parasite. Nevertheless, one should remember that not all cats are good rodent catchers.

The common house fly (Musca domestica L.) is a common pest in poultry stables (Sparagano et al. 2018). Different chemical control methods may be used at a single location, such as adulticide spraying, larval growth inhibitors and lures with active ingredients, to control the population. This is often done with little, or a short term effect, because of the fast development of resistance within a population. Strategies based on these methods only focus on population reduction and do not take into account the prevention of introduction (which may be difficult to achieve completely) and reduction of suitability of the environment. A good preventive as well as curative measure can be to remove manure once a week, as the development of larvae of the common house fly to pupae takes approximately one week. Thus, by doing so, not only the suitable oviposition sites and feed for larvae are reduced (preventive), but eggs, larvae and pupae, which make up for a large part of the population are removed as well (curative). By disrupting their life-cycle in this way, few adults emerge and the total population decreases extensively.

As presented in Table 3, there are many factors that may influence the presence of vectors on farm. A generic approach should be based on the performance of a risk analysis and the development of a pest management strategy. One of the key issues in the development of such a strategy is the knowledge of the biology of the vector species.

In our opinion, a pest management strategy should focus on the following aspects:

- $\quad$ prevent introduction of the vector(s);

- reduce suitability of the farm environment for vector species;

- reduce number of vectors present.

A pest management strategy should aim for the development of a tailor-made strategy that incorporates different tactics that take into account the three aspects mentioned above and is based on specific farm characteristics (Table 3). A tailor-made pest management strategy may be challenging to develop, because of the huge variety in circumstances. The lack of a sound pest management strategy is likely to result in the implementation of a single or series of pest control tactics, quick fix solutions and the use of a vast amount of pesticides when vectors are encountered (reduce number of vectors present). By developing a generic framework (Figure 2), however, the development of a sustainable tailor-made strategy is supported.

It should be noted that this framework for the development of pest management strategies is not restricted to arthropod management, but can be applied for the management of vertebrate vectors as well.

To detect the presence of vectors, regular inspections and monitoring are essential. The frequency and methods of inspection and monitoring is dependent on the potential species present and the risks they present for the infection of livestock. It is, therefore, crucial to identify potential species and the risks they pose if introduction would occur, even if vectors are not found to be present. Both potential vector species and their risk of infection are affected by geography, climate and type of farm or livestock (Table 3). If potential vectors cause a risk, preventive tactics must be implemented to reduce the suitability of the environment and prevent introduction. These tactics include proper hygiene and exclusion measures. Knowledge of the biology of the vector species is essential to successfully implement these measures. 


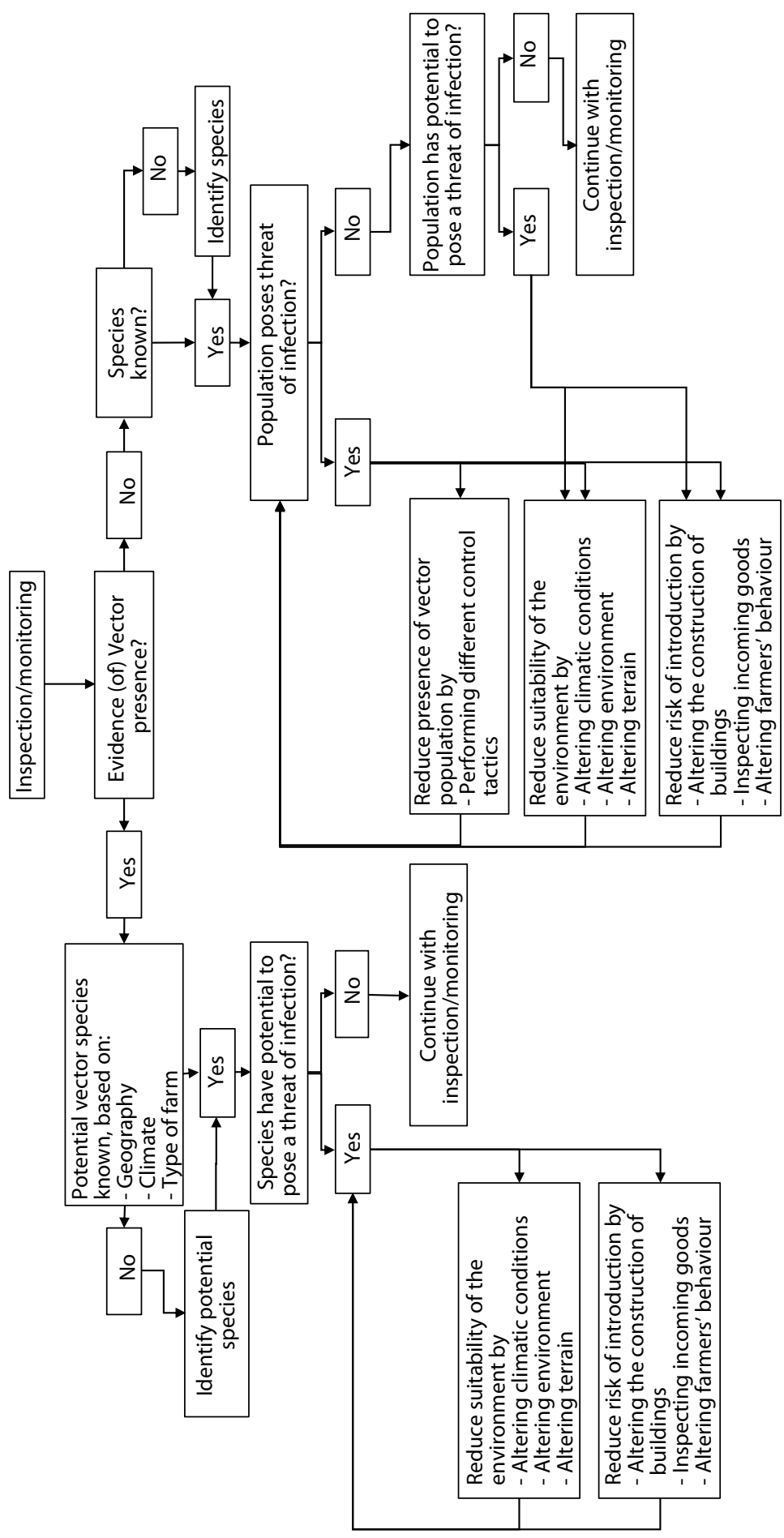

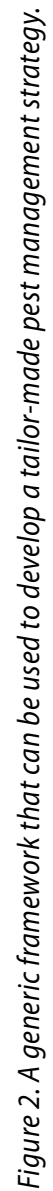


If vectors are present, the species need to be identified to asses: (1) the risk for livestock; and (2) the ecological requirements of the species and whether these requirements are met. In case of early detection, as a result of a thorough inspection and/or appropriate monitoring, of a small vector population that does not pose a risk, it should be assessed whether the population has a potential to affect livestock in the future. If so, the same steps are taken as described above: preventive measures to make the environment for this species less suitable, and prevent its introduction.

Because the prevention of introduction and reduction of the suitability of the environment have an indirect or slow effect on population size, it may be necessary to perform curative measures to quickly reduce population size and protect livestock. This is only done if the present population poses a risk of infection, and is always performed in combination with preventive measures. Curative measures can consist of chemical control, biological control such as the use of entomopathogenic fungi, heat treatment or trapping/lure and kill. It should be noted that the use of chemical control measures are only performed if their use is justified in terms of economic and environmental damage.

Finally, the effectiveness of the measures taken has to be evaluated, in order to adjust or finetune the pest management strategy if necessary. If it is not possible to prevent introduction of the vector or to alter the suitability of the environment, the consequences of interventions and alternative solutions need to be carefully considered.

The generic framework we developed (Figure 2) can be used for every farm and for each vector species. The pest management strategy that is developed based on this framework is as variable, however, as farms and vector species. Hopefully, application of this framework leads to the insight for its users that quick fixes such as the use of vast amounts of pesticides are not the best solution and that in order to have the best results more focus should be pointed to the biology of the vector species and the farm characteristics.

\section{References}

Akey BL (2003) Low-pathogenicity H7N2 avian influenza outbreak in Virginia during 2002. Avian Dis 47(s3): 1099-1103. Ansari MA, Pope EC, Carpenter S, Scholte E-J and Butt TM (2011) Entomopathogenic fungus as a biological control for an important vector of livestock disease: the Culicoides biting midge. PLoS ONE 6: e16108.

Baldacchino F, Desquesnes M, Duvallet G, LysykT and Mihok S (2018) Veterinary importance and integrated management of Brachycera flies in dairy farms. In: Garros C, Bouyer J, Takken W and Smallegange RC (eds.) Pests and vector-borne diseases in the livestock industry. Ecology and Control of Vector-borne diseases. Vol. 5. Wageningen Academic Publishers, Wageningen, the Netherlands, pp. 55-90.

Barin A, Arabkhazaeli F, Rahbari S and Madani SA (2010) The housefly, Musca domestica, as a possible mechanical vector of newcastle disease virus in the laboratory and field. Med Vet Entomol 24: 88-90.

Bouwstra R, Koch G, Heutink R, Harders F, Van der Spek A, Elbers A and Bossers A (2015) Phylogenetic analysis of highly pathogenic avian influenza a (h5n8) virus outbreak strains provides evidence for four separate introductions and one between-poultry farm transmission in the Netherlands, November 2014. Euro Surveill 20: 21174.

Brugh M Jr., Foster J and Hayes F (1964) Studies on the comparative susceptibility of wild European and domestic swine to hog cholera. Am J Vet Res 25: 1124-1127.

Casal J, De Manuel A, Mateu E and Martín M (2007) Biosecurity measures on swine farms in Spain: perceptions by farmers and their relationship to current on-farm measures. Prev Vet Med 82: 138-150.

Despins JL, Turner EC and Ruszler PL (1987) Construction profiles of high rise caged layer houses in association with insulation damage caused by the lesser mealworm, Alphitobius diaperinus (panzer) in Virginia. Poult Sci 66: 243-250. 
Dewey C, Carman S, Pasma T, Josephson G and McEwen B (2003) Relationship between group A porcine rotavirus and management practices in swine herds in Ontario. Can Vet J 44: 649-653.

Garcia-Perez AL, Barandika J, Oporto B, Povedano I and Juste RA (2003) Anaplasma phagocytophila as an abortifacient agent in sheep farms from northern Spain. Ann NY Acad Sci 990: 429-432.

George DR, Finn RD, Graham KM, Mul MF, Maurer V, Moro CV and Sparagano OA (2015) Should the poultry red mite Dermanyssus gallinae be of wider concern for veterinary and medical science? Parasite Vectors 8: 178.

Goodwin MA and Waltman WD (1996) Transmission of Eimeria, viruses, and bacteria to chicks: darkling beetles (Alphitobius diaperinus) as vectors of pathogens. J Appl Poult Res 5: 51-55.

Gortázar C, Ferroglio E, Höfle U, Frölich K and Vicente J (2007) Diseases shared between wildlife and livestock: a European perspective. Eur J Wildlife Res 53: 241.

Hald B, Skovgård H, Bang DD, Pedersen K, Dybdahl J, Jespersen JB and Madsen M (2004) Flies and Campylobacter infection of broiler flocks. Emerg Infect Dis 10: 1490-1492.

Hubálek Z and Halouzka J (1999) West Nile fever - a reemerging mosquito-borne viral disease in Europe. Emerg Infect Dis 5: 643-650.

Kaneene JB, Bruning-Fann CS, Granger LM, Miller R and Porter-Spalding BA (2002) Environmental and farm management factors associated with tuberculosis on cattle farms in northeastern Michigan. J Am Vet Med Assoc 221: 837-842.

Kijlstra A, Meerburg B, Cornelissen J, De Craeye S, Vereijken P and Jongert E (2008) The role of rodents and shrews in the transmission of Toxoplasma gondii to pigs. Vet Parasitol 156: 183-190.

Koch $\mathrm{G}$ and Elbers A (2006) Outdoor ranging of poultry: a major risk factor for the introduction and development of high-pathogenicity avian influenza. NJAS-Wag J Life Sci 54: 179-194.

Krijger IM, Cornelissen JB, Wisselink HJ and Meerburg BG (2014) Prevalence of Toxoplasma gondii in common moles (Talpa europaea). Acta Vet Scand 56: 48.

Meerburg BG, Singleton GR and Leirs H (2009) The year of the rat ends - time to fight hunger! Pest Manag Sci 65: 351-352.

Meerburg BG, Vermeer HM and Kijlstra A (2007) Controlling risks of pathogen transmission by flies on organic pig farms: a review. Outlook Agri 36: 193-197.

Mellor PS, Boorman J and Baylis M (2000) Culicoides biting midges: their role as arbovirus vectors. Ann Rev Entomol 45: 307-340.

Meng XJ, Lindsay DS and Sriranganathan N (2009) Wild boars as sources for infectious diseases in livestock and humans. Philos Trans R Soc Lond B Biol Sci 364: 2697-2707.

Moore C (1992) Biosecurity and minimal disease herds. Vet Clinic N Am: Food Anim Pract 8: 461-474.

Parra A, Fernández-Llario P, Tato A, Larrasa J, Garćıa A, Alonso JM, Hermoso de Mendoza M and Hermoso de Mendoza $J$ (2003) Epidemiology of Mycobacterium bovis infections of pigs and wild boars using a molecular approach. Vet Microbiol 97: 123-133.

Purse BV, Mellor PS, Rogers DJ, Samuel AR, Mertens PPC and Baylis M (2005) Climate change and the recent emergence of bluetongue in Europe 3: 171-181.

Refrégier-Petton J, Rose N, Denis M and Salvat G (2001) Risk factors for Campylobacter spp. contamination in French broiler-chicken flocks at the end of the rearing period. Prev Vet Med 50(1-2): 89-100.

Santini LA (1986) Agriculture and forestry rodent problems and control in Italy. In: Proceedings of the $12^{\text {th }}$ Vertebrate Pest Conference, University of California, Davis, CA, USA, pp. 54. Available at: http://tinyurl.com/ybg4qb7o.

Sparagano O, Di Domenico D, Venturelli C, Papadopoulos E, Smallegange RC and Giangaspero A (2018) Arthropod pests in the poultry industry. In: Garros C, Bouyer J, Takken W and Smallegange RC (eds.) Pests and vector-borne diseases in the livestock industry. Ecology and Control of Vector-borne diseases. Vol. 5. Wageningen Academic Publishers, Wageningen, the Netherlands, pp. 17-53.

Thomas ME, Bouma A, Ekker HM, Fonken AJM, Stegeman JA and Nielen M (2005) Risk factors for the introduction of high pathogenicity avian influenza virus into poultry farms during the epidemic in the Netherlands in 2003. Prev Vet Med 69: 1-11.

Tolhurst BA, Ward Al and Delahay RJ (2011) A study of fox (vulpes vulpes) visits to farm buildings in Southwest England and the implications for disease management. Eur J Wildlife Res 57: 1227-1230. 
Vaughan JA, Turner EC and Ruszler PL (1984) Infestation and damage of poultry house insulation by the lesser mealworm, Alphitobius diaperinus (panzer). Poult Sci 63: 1094-1100.

Webster JP and Macdonald DW (1995) Parasites of wild brown rats (Rattus norvegicus) on UK farms. Parasitol 111:247-255.

Weeks ENI, Machtinger ET, Leemon D and Geden CJ (2018) Biological control of livestock pests: entomopathogens. In: Garros C, Bouyer J, Takken W and Smallegange RC (eds.) Pests and vector-borne diseases in the livestock industry. Ecology and Control of Vector-borne diseases. Vol. 5. Wageningen Academic Publishers, Wageningen, the Netherlands, pp. 337-387.

Wymenga E, Latour J, Beemster N, Bos D, Bosma N, Haverkamp J, Hendriks R, Roerink GJ, Kasper GJ, Roelsma J, Scholten $\mathrm{S}$, Wiersma P and Van der Zee E (2016) Terugkerende muizenplagen in Nederland: inventarisatie, sturende factoren en beheersing, Altenburg \& Wymenga, Feanwâlden, the Netherlands.

Zhu W, Dong J, Xie Z, Liu Q and Khan MI (2010) Phylogenetic and pathogenic analysis of Newcastle disease virus isolated from house sparrow (Passer domesticus) living around poultry farm in southern China. Virus Genes 40: 231-235.

Zurek L and Schal C (2004) Evaluation of the German cockroach (Blattella germanica) as a vector for verotoxigenic Escherichia coli f18 in confined swine production. Vet Microbiol 101: 263-267. 


\title{
16. The Fly Simulator: a simulation model of stable flies and their control
}

\author{
Gösta Nachman ${ }^{1 *}$ and Henrik Skovgård ${ }^{2 *}$ \\ ${ }^{1}$ Department of Biology, Section of Ecology and Evolution, University of Copenhagen, Universitetsparken \\ 15, 2100 Copenhagen $\varnothing$, Denmark; ${ }^{2}$ Department of Agroecology, Section of Pathology and \\ Entomology, University of Aarhus, Forsøgsvej 1, 4200 Slagelse, Denmark; gnachman@bio.ku.dk; \\ henrik.skovgaard@agro.au.dk
}

\begin{abstract}
The blood-sucking stable fly (Stomoxys calcitrans) is a serious pest associated with cattle production. The species is therefore subject to intensive control efforts, primarily by means of insecticides. As chemical control may have some long-term negative side-effects, biological control applying natural enemies has for several decades attracted interest. In Denmark, experiments with massreleases of the pteromalid parasitoid Spalangia cameroni have been conducted on a number of small farms with good results. Parasitoids are now marketed, but used primarily by organic farmers since the method seems economically unattractive for conventional farmers. However, as the use of insecticides is likely to be restricted in the future, this may change the balance in favour of biological control. In order to understand how control of stable flies can be conducted most efficiently, an interactive and user-friendly simulation model, called The Fly Simulator, has been developed. The model applies experimental data on temperature-dependent development, survival and oviposition rates of stable flies and S. cameroni to predict how populations of stable flies will develop when subjected to ambient temperatures, both in absence of parasitoids or after releases of parasitoids. The model shows that flies can be suppressed if parasitoids are released in large numbers at regular intervals, i.e. by inundative releases. This makes biological control relatively expensive compared with chemical control. However, the model also points to frequent removal of manure as an effective and low-cost way of managing flies. It is therefore recommended to apply a combination of high hygienic standard and inundative releases of parasitoids to maintain stable flies at an acceptable low level.
\end{abstract}

Keywords: biological control, chemical control, IPM, population dynamics, Stomoxys calcitrans, Spalangia cameroni, temperature-dependence

\section{Introduction}

Among ancient Egypt's ten plagues described in the Bible, flies are mentioned as the fourth and since the biblical times, the troubles with flies have probably not diminished. One of the fly species that Noah should have walloped before it embarked on his ark is the stable fly Stomoxys calcitrans (L.) (Diptera: Muscidae) as this species today is a major problem associated with livestock production. While the eggs, larvae and pupae develop in animal manure and other decomposing organic substrate, the adult flies move around in search for warm-blooded animals, including humans, to feed on. The insects use their sharp mouthparts to cut through the victim's skin to get access to its blood since such meals are necessary for the survival of both genders as well as for egg-laying. Cattle being exposed to the painful bites from sometimes myriads of bloodthirsty flies will be severely disturbed during their feeding activities, which may lead to anaemic conditions of the affected animals and reduce production of meat or milk. Thus, Taylor et al. (2012) estimated the annual losses caused by stable flies to be more than one billion US dollars in USA alone. 
Furthermore, stable flies may also serve as mechanical vectors for various diseases, such as trypanosomid parasites, African horse sickness, African swine fever, West Nile and Rift Valley viruses (Baldacchino et al. 2013).

In order to reduce fly nuisance, the farmers have several options: use insecticides, prevent adult flies from getting access to suitable manure, take advantage of the flies' natural enemies or use a combination of all three control strategies as part of an integrated pest management strategy. However, as the control options are likely to interfere with each other, the outcome of mixing them can be difficult to anticipate. Besides, some options are more expensive than others, which mean that the choice of control measures should take the costs and benefits of the various methods into consideration. Finally, although chemical control can be both cheap and effective, there are some constraints on their use to avoid adverse effects, such as environmental pollution, pesticide residues in farm products or development of resistance in the fly population (Cilek and Greene 1994; Durel et al. 2015; Pitzer et al. 2010). Therefore, to obtain a better understanding of how the different control strategies interact with each other and to optimize their use, the Danish Ministry of the Environment offered financial support to a project aiming at developing a simulation model of stable flies inhabiting cattle facilities. The primary goal of the project was to investigate whether biological control by means of the pupal parasitoid Spalangia cameroni Perkins (Hymenoptera: Pteromalidae) could be used as a realistic alternative to chemical control. More specifically, the model should serve as a strategic tool to decide how often and in which numbers parasitoids should be released in a stable in order to achieve acceptable control of stable flies.

The present model, called The Fly Simulator, is not the first to simulate pest control of flies in animal production systems (Weidhaas et al. 1977; Wilhoit et al. 1991a,b). However, in contrast to previous DOS-based models, The Fly Simulator is a Windows-based interactive simulation model, allowing the user to interfere with the system during a simulation. The models also differ with respect to fly species to be controlled by natural enemies since The Fly Simulator focuses on stable flies, whereas the other models simulate house flies. The model by Wilhoit et al. (1991a) (called PARMOD) permits incorporation of two parasitoid species of which one can be S. cameroni, while The Fly Simulator uses $S$. cameroni as the only biological control agent. In the more comprehensive and detailed management model by Wilhoit et al. (1991b), a whole complex of natural enemies, including both parasitoids and predators, is considered. It should also be noted that the various modelling approaches differ with respect to mathematical relationships and data used to parameterize these relationships.

\section{The stable system and the species inhabiting it}

A Danish cattle facility is typically inhabited by several fly species of which house flies Musca domestica L. and stable flies (Diptera: Muscidae) are the most common. Their natural enemies are represented by predators (e.g. the predaceous mite, Macrocheles muscaedomesticae (Scopoli) (Mesostigmata: Macrochelidae), beetles within the genus Carcinops (Coleoptera: Histeridae), Alphitobius diaperinus (Panzer) (Coleoptera: Tenebrionidae) and predacious larvae of the black dump fly, Hydrotaea (Ophyra) aenescens (Wiedemann) (Diptera: Muscidae)), parasitoids (e.g. Muscidifurax raptor (Girault \& Sanders), Nasonia vitripennis (Walker) and S. cameroni (Hymenoptera: Pteromalidae)) and various microorganisms (e.g. Beauveria bassiana L. (Hypocreales: Clavicipitaceae) and Entomophthora muscae (Cohn), (Entomophthorales: Entomophthoraceae)). 


\section{Biology and ecology of stable flies}

The stable fly undergoes complete metamorphosis, i.e. its life cycle includes the following morphological stages: egg, larva, pupa and adult. The egg is white, elliptical and about $1 \mathrm{~mm}$ long. It hatches within 1-2 days depending on the temperature. Because the larvae are saproand coprophagous, favourable habitats for oviposition are silage, bedding mixed with urine and faeces, rotting hay, fermenting feed or other decomposing and fermenting plant materials (Axtell 1986; Thomsen 1938). The maggot-like larva passes through three instar stages before pupation. At optimum temperature, larval development normally takes 6-8 days, while lower and upper temperature thresholds for larval development are around 10 and $30^{\circ} \mathrm{C}$, respectively (Gilles et al. 2005; Thomsen 1938). Prior to pupation, the larva begins to move out of the organic media in search for dryer and cooler $\left(15-20^{\circ} \mathrm{C}\right)$ areas. During pupation the larval integument (the larval skin) contracts to form a cylindrical puparium which is about 3-5 mm long and with a colour ranging from light to dark brown. One to two days after the formation of the puparium, a pupa will start developing inside and full development requires 4-21 days depending on temperature (Gilles et al. 2005). The adult female fly (Figure 1) copulates shortly after eclosion and after her first blood meal she starts egglaying, typically 4-8 days after copulation. After each blood meal, the female will be able to produce an egg clutch, typically containing 25-50 eggs. A female usually deposits 4- 6 clutches of eggs during her lifetime resulting in 100-300 eggs (Friesen and Johnson 2012). Oviposition rate peaks while a female is still young (Skovgård and Nachman 2017). In the laboratory, females are reported to survive 20-30 days (Lysyk 1998), but under field conditions life expectancy ranges between 4 and 11 days (Berry et al. 1981; Scholl 1986; Skovgård and Nachman 2012). The sex ratio (males:females) is close to 1:1 (Lysyk 1998; Salem et al. 2012).

The complete life cycle of the stable fly takes about 13-18 days during summer in areas where the mean air temperature is $24-30{ }^{\circ} \mathrm{C}$, but may take $20-25$ days in northern Europe where summer temperatures are lower (Axtell 1986; Gilles et al. 2005; Kunz et al. 1977; Thomsen 1938). The species does not enter diapause during winter time, but development is very slow and hinges mainly on the overwintering larvae (Berry et al. 1978; Thomsen 1938). Thus, eggs hatching in late autumn will reach the pupal stage in early spring, though it should be noticed that larval development is likely to be faster than predicted from the ambient air temperatures due to the heat produced by decaying and fermenting organic matter. As seen from Figure 2, the abundance of stable flies is closely correlated

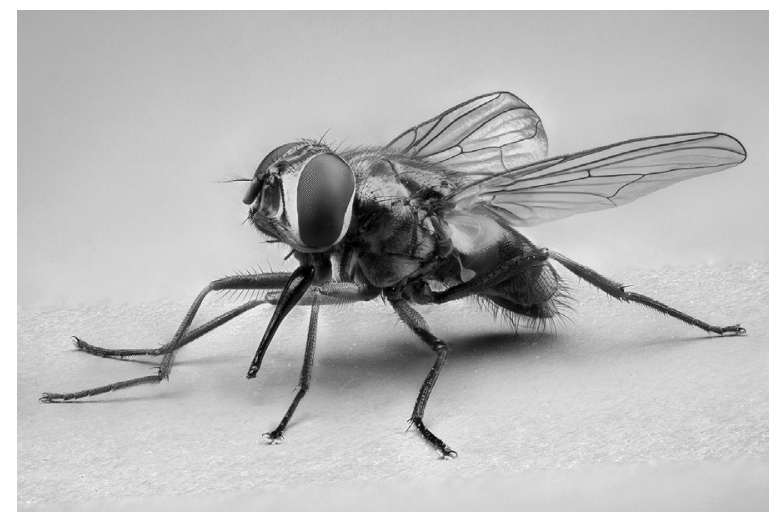

Figure 1. Adult female stable fly (photo by Hans Smid, www.bugsinthepicture.com). 


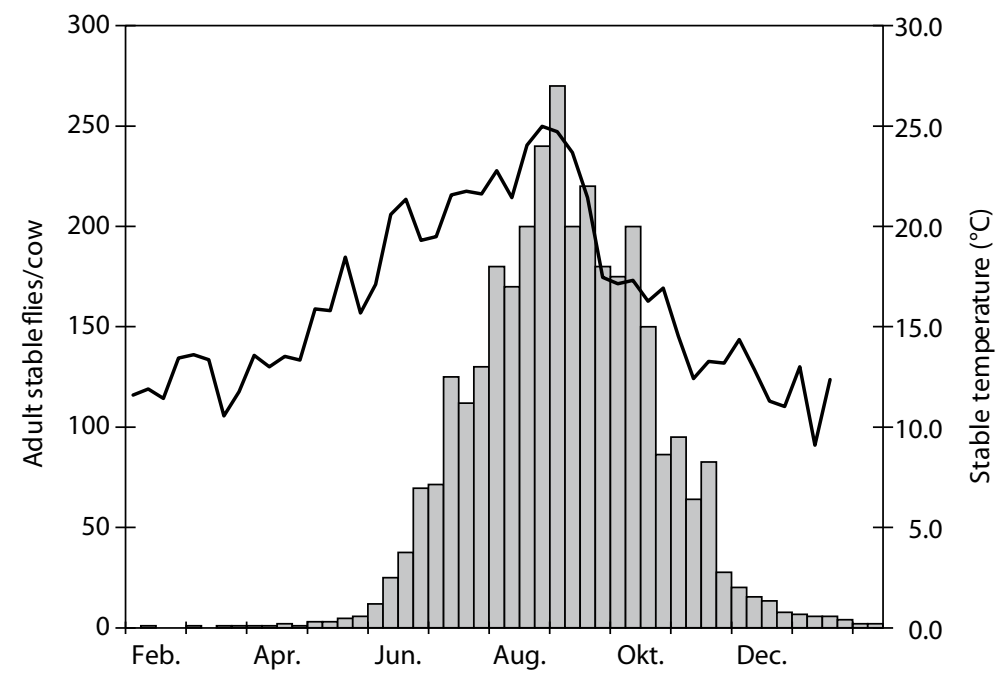

Figure 2. Average weekly temperatures measured inside a Danish dairy cattle stable hosting 60-70 adult cows (line) and the abundance of adult stable flies based on a visual estimate of the average number of flies occurring on and around a cow (bars).

with ambient temperatures. Under laboratory conditions, the maximum intrinsic rate of increase $\left(r_{m}\right)$ has been estimated to be around 0.22 day $^{-1}$ (Lysyk 1998), but under natural conditions, the per capita growth rate is likely to be considerably lower. Thus, Skovgård and Nachman (2012) estimated the maximum realized growth rate in a Danish stable to be 0.070 day $^{-1}$.

\section{Biology and ecology of Spalangia cameroni}

Adult females of S. cameroni parasitize pupae of various fly species (Figure 3). Development from egg to adult takes place inside the fly puparium and lasts about 30 days at $25^{\circ} \mathrm{C}$ (Skovgård and Nachman 2016a). The sex ratio tends to be female-biased as about $65 \%$ of the eclosed parasitoids are females (Skovgård and Nachman 2016a). Survival and oviposition rates of the adults depend on temperature. For instance, at $25{ }^{\circ} \mathrm{C}$ females live on average 25 days after eclosion and can produce about 100 eggs during their entire lifetime (Skovgård and Nachman 2016a). They usually lay one egg per fly pupa, but not all attacks lead to a viable offspring as the adult parasitoids can supplement their energy reserves by feeding on host pupae. Thus, host feeding may account for up to $20 \%$ of all host kills (Skovgård and Nachman 2015a). The attack rate per female depends on both the ambient temperature and on the density of suitable hosts (Figure 4). When offered fly pupae ad libitum, the intrinsic rate of natural increase $\left(r_{m}\right)$ was found to peak at $33.5^{\circ} \mathrm{C}(0.182$ day $^{-1}$ ) and to be around 0.1 day $^{-1}$ at $25^{\circ} \mathrm{C}$ (Skovgård and Nachman 2016a).

In contrast to many species of pupal parasitoids, which confine their activity to the upper $0-3 \mathrm{~cm}$ of the manure layer, S. cameroni is capable of moving as deep as 10-15 cm (Geden 2002; King 1997; Skovgård 2006; Skovgård and Nachman 2015a). The species is thermophile with a preference for temperatures above $25^{\circ} \mathrm{C}$ (Geden 1997). Though S. cameroni occurs naturally in Danish stables, it 


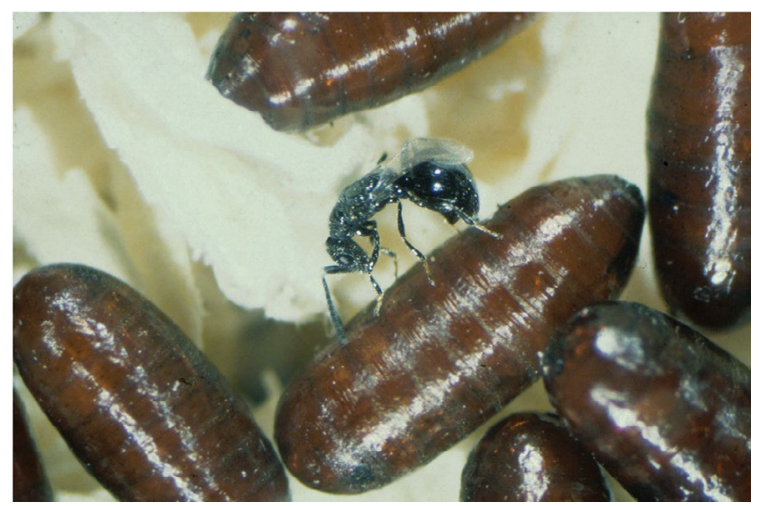

Figure 3. An adult female Spalangia cameroni using its ovipositor to penetrate the puparium of a house fly to lay an egg on the outside of the pupa (photo by Henrik Skovgård).

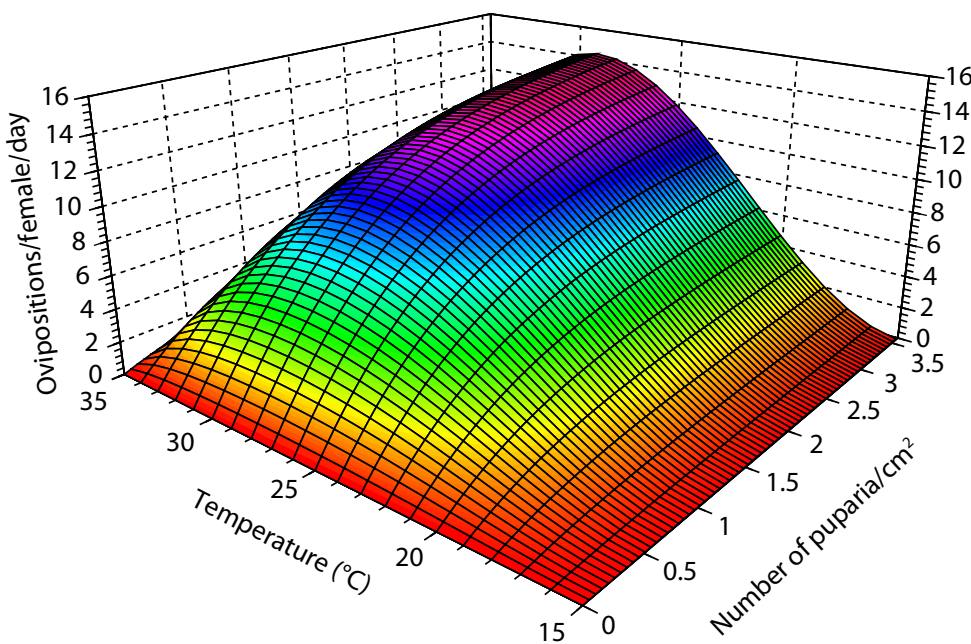

Figure 4. The rate of successful parasitism (attacks leading to an oviposition) of female Spalangia cameroni when exposed to different temperatures and densities of stable fly pupae.

suffers from almost 100\% winter mortality (Floate and Skovgård 2004). Seasonal activity begins in April, which coincides with the increase in outdoor temperatures and the growing populations of house and stable flies (Skovgård and Jespersen 1999, 2000). From May and onwards, parasitism increases gradually and attains a maximum in September-October (Skovgård 2004). Because of the high winter mortality, the density of parasitoids in spring is far too low to cope with the exploding fly populations. To remedy this problem, one strategy is to apply inoculation where the parasitoids are reintroduced once or twice in sufficient numbers with the advent of higher temperatures 
in spring. Another strategy is inundation where the parasitoids are released abundantly several times throughout the fly season - more like a bio-pesticide. In the case of S. cameroni it seems that inundation is the only release strategy that works in practice, though with variable success (Andress and Campbell 1994; Birkemoe et al. 2009; Skovgård 2004; Skovgård and Nachman 2004).

The properties that make S. cameroni a promising candidate for biological control of stable flies in Denmark are: (1) it is a native species; (2) it is an effective natural enemy of flies under laboratory conditions; (3) in absence of stable flies, it can feed and reproduce on other fly species; (4) it is adapted to the high temperatures prevailing inside stables during summer time (usually $20-30{ }^{\circ} \mathrm{C}$ ); (5) it is willing to move into the manure to find hosts; and (6) it can be mass-produced on pupae of house fly or black dump fly H. aenescens (Skovgård 2004).

\section{Biological control of stable flies by means of Spalangia cameroni}

In Denmark, S. cameroni has been used successfully as biological control agent against flies in cattle facilities on organic farms, which often suffer from severe fly problems (Skovgård 2004). While adult cows during summer time usually spend most of the time grazing outside the stable and only come to the stable to be milked, young calves are raised indoors in calf boxes where they stay on a mixture of straw and dung (in the following called manure). As this bedding often is allowed to accumulate, it serves as an excellent substrate for development of flies.

To assess the efficacy of S. cameroni under conditions prevailing in Danish stables, large numbers of S. cameroni were released on one dairy and two swine farms (Skovgård 2004). The parasitoids were introduced as house fly puparia that had been parasitized by $S$. cameroni females under laboratory conditions. Introductions took place every second week from April through October and about 200 adult parasitoids, emerging from the puparia, were applied per $\mathrm{m}^{2}$ treated area. Since the farms differed with respect to number of animals, management, etc., the individual farms were used as their own controls since sampling of flies took place over two successive seasons, where parasitoids were only released during the second year. As seen from Figure 5, showing the numbers of adult stable flies and house flies on one of the selected farms, the difference between the two years with respect to fly abundances was very clear, and the same applies to the percentage of the fly pupae that were parasitized. The results of the above experiments were used as the background for the simulation model described below.

\section{The Fly Simulator}

The Fly Simulator is a deterministic simulation model consisting of four modules: (1) a module describing the environmental conditions in the stable (e.g. temperatures in the air and in the manure, amount of manure, larvicide concentration in the manure); (2) a module modelling the population dynamics of stable flies without parasitoids; (3) a module modelling the population dynamics of S. cameroni and its interaction with stable flies; and (4) a management module where the user can try to control stable flies by (a) treating the manure with a larvicide; (b) release parasitoids; (c) remove manure; or (d) apply a combination of all three methods.

It has to be emphasized that the management module is tentative and is included mainly for educational purposes, i.e. to give the user a feeling of the system's complexity and the problems associated with controlling nuisance flies. By making the model interactive, the user can interfere with the system during a simulation and decide what to do in order to obtain a desired level of fly control. At the end of a simulation, the user's performance as farmer is evaluated in terms 

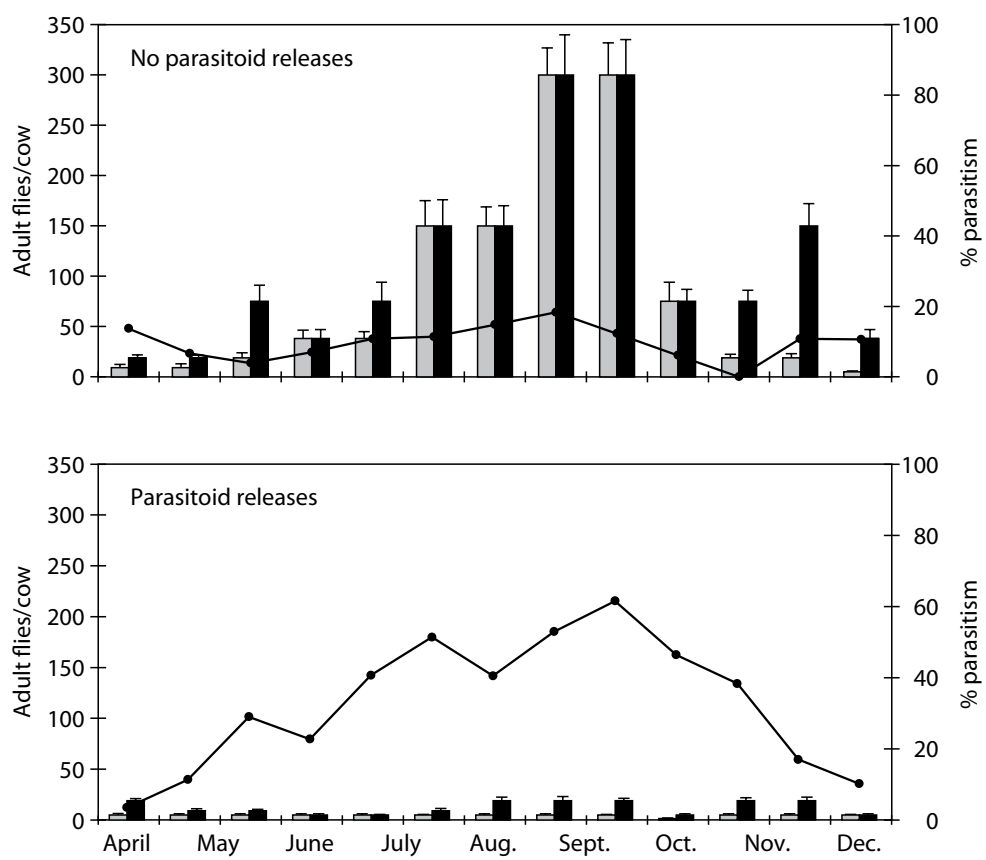

Figure 5. Average number of adult house flies (grey bars) and stable flies (black bars) per cow (see Figure 2) in a Danish cattle stable hosting 60-70 milking cows the year before (upper panel) and the year when releases of Spalangia cameroni took place (lower panel). Parasitoids were introduced as house fly puparia that had been parasitized by S. cameroni in the laboratory. The puparia were placed into 200-300 $\mathrm{m}^{2}$ of accumulated manure where prior inspection had revealed development of house flies and stable flies. Typically around 200 emerged adult parasitoids were applied per $m^{2}$ manure every second week from April through October (after Skovgård 2004).

of the achieved reductions in fly abundance compared with the amount of money invested in fly control. Thus, the model is designed as a game where the player can to try to improve his/ her performance based on a combination of ecological knowledge, intuition, trial-and-error and previous experience with the model. As all costs (in Danish currency DKK) associated with the various control options are preliminary, the user can modify the default values to make them more realistic. The same also applies to most of the other parameters determining the system's dynamics. Thereby, The Fly Simulator can serve as a tool box for obtaining a better understanding of the factors that make fly control more cost efficient.

In the following we will give an overview of the model. Readers with interest in more mathematical details are referred to a research report by Skovgård and Nachman (2016b), which also describes how the model's parameters were estimated from empirical data. However, it has to be emphasized that some parameter values given in this report differ from those given here (Table 1-3) because more data have become available since the report was published. Besides, data used to parameterize temperature-dependent sub-models of $S$. cameroni are available as separate scientific papers: The functional response in Skovgård and Nachman (2015a), mutual interference 
Table 1. Estimated parameter values used to model temperature- and age-dependent functions. Where males and females have been tested separately, values apply to the females $\left(\Delta \mathrm{Q}, \mathrm{p}_{s^{\prime}} \mathrm{p}_{h}\right.$ and $\mathrm{F}$ have unit day ${ }^{-1} ; \varphi(\mathrm{y}), \pi(\mathrm{x})$ and $\mathrm{v}(\mathrm{x})$ are dimensionless; $\mathrm{x}$ and $\mathrm{y}$ denote the biological age of Stomoxys calcitrans ( $\mathrm{S}$. cal.) and Spalangia cameroni (S. cam.), respectively; values in squared brackets are by definition set to 1).

\begin{tabular}{|c|c|c|c|c|c|c|c|c|c|c|}
\hline Equations & $\begin{array}{l}\text { Species and } \\
\text { stage }\end{array}$ & $y_{\max }$ & $T_{\min }$ & $T_{\max }$ & $a$ or $q$ & $b$ or $r$ & $Q_{\min }$ & $Q_{\max }$ & c & $d$ \\
\hline $\operatorname{Aging}(\Delta Q)$ & S. cal. eggs ${ }^{1}$ & [1] & 2.597 & 44.14 & 2.267 & 0.034 & - & - & - & - \\
\hline \multirow[t]{5}{*}{ (Equation 8) } & S. cal. larvae ${ }^{1}$ & [1] & 2.514 & 39.82 & 2.993 & 0.436 & - & - & - & - \\
\hline & S. cal. pupae ${ }^{1}$ & [1] & 0.703 & 36.48 & 3.081 & 0.464 & - & - & - & - \\
\hline & S. cal. adults ${ }^{1}$ & [1] & 8.523 & 48.73 & 3.641 & 3.343 & - & - & - & - \\
\hline & S. cam. imm. ${ }^{2}$ & [1] & 9.417 & 35 & 1.589 & 0.049 & - & - & - & - \\
\hline & S. cam. adults ${ }^{2}$ & {$[1]$} & 0 & 45 & 2.241 & 0.0002 & - & - & - & - \\
\hline Survival $\left(p_{s}\right)$ & S. cal. eggs ${ }^{1}$ & 0.999 & 2.597 & 44.14 & 0.006 & 0.085 & - & - & - & - \\
\hline \multirow[t]{5}{*}{ (Equation 9 and 11) } & S. cal. larvae ${ }^{1}$ & 0.998 & 2.514 & 39.82 & 0.062 & 0.056 & - & - & - & - \\
\hline & S. cal. pupae ${ }^{1}$ & 0.991 & 0.703 & 36.48 & 0.141 & 0.099 & - & - & - & - \\
\hline & S. cal. adults ${ }^{1}$ & 1.0 & 8.523 & 48.73 & 0.328 & 0.713 & - & 32.82 & 2.452 & - \\
\hline & S. cam. imm. ${ }^{2}$ & 0.996 & 9.417 & 35 & 0.003 & 0.006 & - & - & - & - \\
\hline & S. cam. adults ${ }^{2}$ & 1.0 & 0 & 45 & 0.059 & 0.041 & - & 12.26 & 3.271 & - \\
\hline Hatching $\left(p_{h}\right)$ & S. cal. eggs ${ }^{1}$ & [1] & - & - & - & - & - & 0.492 & 14.88 & - \\
\hline \multirow[t]{3}{*}{ (Equation 10) } & S. cal. larvae ${ }^{1}$ & [1] & - & - & - & - & - & 5.685 & 9.582 & - \\
\hline & S. cal. pupae ${ }^{1}$ & [1] & - & - & - & - & - & 4.794 & 15.34 & - \\
\hline & S. cam. imm. ${ }^{2}$ & [1] & - & - & - & - & - & 19.64 & 7.226 & - \\
\hline Fecundity $(F)$ & S. cal. fem. ${ }^{1}$ & 35.86 & 8.523 & 48.73 & 1.504 & 0 & 3.373 & 32.82 & 3.226 & 8.098 \\
\hline (Equation 12) & S. cam. fem. ${ }^{2}$ & 20.24 & 8.069 & 36.56 & 4.342 & 1.692 & 0 & 12.26 & 0.545 & 4.299 \\
\hline $\begin{array}{l}\text { Success rate }(\varphi(y)) \\
\text { (Equation 18) }\end{array}$ & S. cam. fem. ${ }^{2}$ & 0.900 & 15.00 & 35.47 & 0.294 & 0.174 & 0 & 12.26 & 0.004 & 0.700 \\
\hline $\begin{array}{l}\text { Preference }(\pi(x)) \\
\text { (Equation 19) }\end{array}$ & S. cam. fem. ${ }^{2}$ & [1] & - & - & - & - & 0 & 4.682 & 13.06 & 5.698 \\
\hline $\begin{array}{l}\text { Host quality }(v(x)) \\
\text { (Equation 19) }\end{array}$ & S. cam. fem. ${ }^{2}$ & [1] & - & - & - & - & 0 & 4.682 & 0.434 & 0.144 \\
\hline
\end{tabular}

${ }^{1}$ Skovgård and Nachman (2017).

2 Skovgård and Nachman (2016a).

in Skovgård and Nachman (2015b), and life-history parameters (i.e. stage- and age-dependent survival, development and fecundity rates) in Skovgård and Nachman (2016a). Finally, parameter values related to the life-history of stable flies are taken from Skovgård and Nachman (2017). All biological parameters used in the model are based on laboratory experiments except the parameter expressing intraspecific competition among stable fly larvae ( $\psi$ in Equation 13), which was estimated by calibrating the model to the observed dynamics. 
Table 2. Miscellaneous parameters.

\begin{tabular}{|c|c|c|c|c|}
\hline Equation & Description & Symbol & Value & Dimension \\
\hline \multirow[t]{2}{*}{4} & Female proportion (Stomoxys calcitrans) ${ }^{1}$ & $p_{f}$ & 0.5 & None \\
\hline & Female proportion (Spalangia cameroni) ${ }^{2}$ & $p_{f}$ & 0.707 & None \\
\hline 13 & S. calcitrans larval competition ${ }^{5}$ & $\psi$ & 28.2 & $\mathrm{~cm}^{3} /$ larva \\
\hline \multirow[t]{2}{*}{15} & S. cameroni female attack rate ${ }^{3}$ & $a$ & 1.120 & $\mathrm{~cm}^{2} /$ female \\
\hline & & $\beta$ & 1.0 & None \\
\hline \multirow[t]{2}{*}{16} & Effect of mutual interference among S. cameroni & $r$ & 2.620 & $\mathrm{~cm}^{2} /$ female \\
\hline & females on attack rate ${ }^{4}$ & $q$ & 1.666 & None \\
\hline \multirow[t]{2}{*}{16} & Effect of mutual interference among S. cameroni & $r$ & 0.203 & $\mathrm{~cm}^{2} /$ female \\
\hline & females on success ratio 4 & $q$ & 0.028 & None \\
\hline \multirow[t]{2}{*}{22} & Heat production due to fermentation ${ }^{5}$ & K & 0.09 & ${ }^{\circ} \mathrm{C} /$ day \\
\hline & Heat conduction in manure ${ }^{5}$ & $c$ & 0.47 & day $^{-1}$ \\
\hline
\end{tabular}

${ }^{1}$ Skovgård and Nachman (2017).

${ }^{2}$ Skovgård and Nachman (2016a).

${ }^{3}$ Skovgård and Nachman (2015a).

${ }^{4}$ Skovgård and Nachman (2015b).

${ }^{5}$ Estimated by calibration to stable data.

Table 3. Estimated costs of the intervention options (7 DKK is approximately equal to 1 US dollar).

\begin{tabular}{llll}
\hline Costs & Parasitoids & Insecticide & Removal of manure \\
Fixed & $100 \mathrm{DKK}^{\text {release }}{ }^{1}$ & $400 \mathrm{DKK} /$ treatment $^{1}$ & $500 \mathrm{DKK}^{1}$ cleansing $^{1}$ \\
Variable & $0.014 \mathrm{DKK} /$ parasitoid $^{2}$ & $243 \mathrm{DKK} / \mathrm{kg}^{3}$ & $0.20 \mathrm{DKK} / \mathrm{kg} \mathrm{manure}^{1}$ \\
\hline
\end{tabular}

1 Guestimate.

2 Price found on an American web-page.

${ }^{3}$ The larvicide Neporex containing $2 \%$ cyromazin; price found on a Danish web-page.

\section{How to get access to The Fly Simulator?}

The Fly Simulator is part of a comprehensive simulation program called Fitom, which together with a manual to The Fly Simulator can be freely downloaded from https://tinyurl.com/yblul7ho.

\section{Modelling dynamics of insect populations}

When an individual stable fly or parasitoid undergoes a transition from one stage to the next it is said to 'hatch'. An individual may either survive during a time interval $(\Delta t)$ with probability $p_{s}$ or die with probability $1-p_{s}$. A surviving individual may either remain in stage $s$ (with probability $p_{s}\left(1-p_{h}\right)$ ) or hatch to the stage $s+1$ (with probability $p_{s} p_{h^{\prime}}$ where $p_{h}$ is the hatching probability). Both $p_{s}$ and $p_{h}$ may vary over time and, for a given species, may also depend on gender ( $g=1$ for males and $g=2$ for females), stage ( $s=1$ for eggs, $s=2$ for larvae, $s=3$ for pupae and $s=4$ for adults) and age $(x)$, 
where $x$ is the number of days an individual has spent in the stage. However, since development of the egg, larval and pupal stage of $S$. cameroni takes place inside the fly puparium, these three stages are lumped into one stage (called the immature stage), so for this species s only takes two values (i.e. $s=1$ for immatures and $s=2$ for adults).

For a given species, the number of individuals of gender $g$ in stage $s$ belonging to age class $x$ at time $t$ is denoted $N(g, s, x)$. At time $t+1$, the surviving individuals have age $x+1$ and the size of this age class is found as:

$$
N(g, s, x+1)=p_{s}\left(1-p_{h}\right) N(g, s, x)-H(g, s, x)+H(g, s-1, x)
$$

where $H(g, s, x)$ denotes the number of individuals hatching from $x^{\text {th }}$ age class during the time interval and $H(g, s-1, x)$ the number of individuals entering stage $s$ from the previous stage. If we assume that individuals neither emigrate from nor immigrate into the population (i.e. the population is closed), $H(g, s, x)$ can be modelled as:

$$
H(g, s, x)=p_{s} p_{h} N(g, s, x)
$$

As all new individuals entering stage $s$ have age $x=0, H(g, s-1, x)$ will be 0 for $x>0$ and otherwise found as:

$$
H(g, s-1,0)=\sum_{x} p_{s} p_{h} N(g, s-1, x)
$$

where $x$ is taken over all age classes for which $N(g, s-1, x)$ is $>0$. Besides, $H(g, s, x)$ is 0 for the adult stage.

For $s=1, H(g, s-1,0)$ corresponds to the total number of new eggs of gender $g$ produced by all adult females during a time interval. This is the same as $N(g, 1,0)$ at time $t+1$ obtained from:

$$
N(g, 1,0)=\sum_{x=0}^{\infty} p_{g} F(x) N(2, S, x)
$$

where $F(x)$ is the age-dependent fecundity rate (number of eggs produced per day per female), $p_{g}$ the proportion of eggs belonging to gender $g$ and $N(2, S, x)$ the number of adult females in age class $x$ at time $t$. $S$ denotes the last (adult) stage of the life-cycle, i.e. $S$ is 4 for the flies and 2 for the parasitoids.

\section{The concept of limiting factors}

Survival probability $\left(p_{s}\right)$, hatch rate $\left(p_{h}\right)$, and fecundity rate $(F)$ are specific for a given gender $(g)$, stage $(s)$ and age-class within a stage $(x)$ of the species in focus and depend on the ambient environment as well as on the conditions to which individuals have been exposed earlier in life. We assume that each of these vital rates has a maximum for an optimum combination of the factors that simultaneously affect the respective rates. To illustrate this, let $y$ denote the value of a given rate and let $y_{\max }$ be the maximum value $y$ takes if all $n$ factors affecting $y$ simultaneously were optimal. In accordance with the multiplicative Mitscherlich model (Harmsen 2000; Johns and Vimpany 1999; Nijland et al. 2008) y can be modelled as a product of limiting factors:

$$
y=y_{\max } f_{1}(\cdot) f_{2}(\cdot) \cdots f_{n}(\cdot)=y_{\max } \prod_{j=1}^{n} f_{j}(\cdot)
$$


where $f_{j}(\cdot)$ is a function expressing the effect of the $j$ th factor on $y \cdot f_{j}(\cdot)$ can take values between 1 and 0 . Thus, if $f_{j}(\cdot)=1$ for all $n$ factors, the maximum value of $y$ is attained, whereas if one or more values of $f_{j}(\cdot)$ are $<1, y$ will be smaller than $y_{\max }$. Finally, $y$ will be 0 if at least one of the limiting functions is 0 .

\section{The concept of biological age}

The factors affecting $y$ are typically external, such as the ambient temperature, humidity, amount and quality of food, the influence of conspecifics, competitors or natural enemies. However, $f(\cdot)$ in Equation 5 can also be used to represent internal factors, such as an individual's age. In arthropods, aging is typically associated with the ambient temperature, which tends to speed up the aging process. So instead of using an individual's chronological age $(x)$, i.e. the number of days since the individual entered the stage, its biological age can be used. The biological age of an individual with chronological age $x$ is denoted $Q(x)$. As The Fly Simulator updates chronological age with a time step of one day, while development and survival of individuals are influenced by their biological age, it is necessary to link $x$ to $Q(x)$ so as to allow for a daily update of the latter. Thus, $Q(x)$ represents the cumulated effect of temperature experienced by an individual until age $x$ and is calculated as:

$$
Q(x)=\sum_{i=0}^{x-1} \Delta Q_{i}
$$

where $\Delta Q_{i}$ denotes the increase in biological age while the individual belonged to age class $i$ $(i=0,1,2, . ., x-1) . Q(x)$ will increase with time until the maximum biological age, denoted $Q_{\text {max }}$ is reached. As $Q_{\max }$ denotes the maximum biological age an individual can attain before it either completes the life stage or dies, $Q(x) / Q_{\max }$ will take values between 0 and 1 , which corresponds to physiological age used by e.g. Van Straalen (1983) and Régnière et al. (2012).

Due to variation among individuals with respect to developmental rate, not all individuals entering a given stage at the same time will leave the stage again in synchrony (Wagner et al. 1984). The distribution of developmental times is modelled by assuming that the proportion of individuals still in the stage at time $t$, but completes development during $\Delta t$ and enters the next stage will increase as $Q(x)$ approaches $Q_{\max }$ (see below). This implies that the mean developmental time will be shorter than the maximum (see e.g. Son and Lewis 2005; Wagner et al. 1984).

\section{The SANDY model}

The function $f(\cdot)$ can be modelled by means of the SANDY model (Nachman and Gotoh 2015) ${ }^{4}$. It is given as:

$$
f(V)=\mathrm{S}(V)=C\left(\frac{V-V_{\min }}{V_{\text {max }}-V_{\text {min }}}\right)^{a}\left(\frac{V_{\text {max }}-V}{V_{\text {max }}-V_{\text {min }}}\right)^{b}
$$

where $S(V)$ is short-hand notation for the SANDY function relating the actual value of a factor $(V)$ to its limiting effect $f(V) . V_{\min }$ and $V_{\max }$ define lower and upper thresholds for the factor $V$. $C$ is a scaling factor ensuring that $S(V)$ is 1 when $V$ is optimal. As $C$ is determined by the shape

\footnotetext{
${ }^{4}$ Mathematically, the SANDY model is similar to the equation used by Pleydell and Chretien (2010) to model habitat suitability. It seems likely that the equation has been developed independently several times and been used for different purposes. Basically, the SANDY model can be regarded as a special case of the Beta distribution (https://en.wikipedia. org/wiki/Beta_distribution).
} 
parameters ( $a$ and $b$ ) and given as $C=\left(\frac{a}{a+b}\right)^{-a}\left(\frac{b}{a+b}\right)^{-b}$, Equation 7 can also be written as:

$\mathrm{S}(V)=\left(\frac{(a+b)\left(V-V_{\min }\right)}{a\left(V_{\max }-V_{\min }\right)}\right)^{a}\left(\frac{(a+b)\left(V_{\max }-V\right)}{b\left(V_{\max }-V_{\min }\right)}\right)^{b}$

The maximum value of $\mathrm{S}(V)$ is achieved when $V=V_{o p t^{\prime}}$ where $V_{o p t}=\frac{a V_{\max }+b V_{\min }}{a+b}$. The curve described by Equation 7 is very flexible, depending on the values of the two shape parameters (Nachman and Gotoh 2015). Three special cases are relevant for describing biological phenomena: (1) when both $a$ and $b$ are $>0$, the curve has a maximum (i.e. $S(V)=1)$ for $V_{\text {min }}<V<V_{\text {max }}$, while $S(V)=0$ when $V \leq V_{\min }$ or $V \geq V_{\max } ;(2)$ for $a>0$ and $b=0, S(V)=0$ when $V \leq V_{\min }$ and increases monotonically when $V_{\min }<V<V_{\max }$ to become 1 when $V \geq V_{\max }$; and (3) for $a=0$ and $b=1, S(V)$ $=1$ for $V \leq V_{\min }$ and decreases monotonically when $V_{\min }<V<V_{\max }$ to become 0 when $V \geq V_{\max }$.

\section{Modelling biological age}

The increase in biological age during a time interval (e.g. one day) is described by means of Equation 7a as:

$$
\Delta Q_{i}=\mathrm{S}(T) \Delta t=\left(\frac{(q+r)\left(T-T_{\min }\right)}{q\left(T_{\max }-T_{\min }\right)}\right)^{q}\left(\frac{(q+r)\left(T_{\max }-T\right)}{r\left(T_{\max }-T_{\min }\right)}\right)^{r} \Delta t
$$

where the two shape parameters $q$ and $r$ are used to characterize the species- and stage-specific aging process as a function depending on temperature $(T) . T_{\min }$ and $T_{\max }$ are the lower and upper temperature thresholds for survival, implying that aging stops when an individual dies, i.e. $\Delta Q=$ 0 when $T \leq T_{\min }$ or $T \geq T_{\max }$. For each time step $\Delta t$, Equation 8 is used in Equation 6 to update the biological age of the individuals in each age class $(x)$ within each developmental stage. Aging proceeds fastest when $T=T_{\text {opt }}$ where $\Delta Q$ is seen to be equal to $\Delta t$, i.e. the increase in biological and chronological age is the same, when the temperature is optimal. For all other temperatures, $\Delta Q$ will be less than $\Delta t$, so typically biological age $(Q)$ will lag behind chronological age $(x)$. At low temperatures, aging may proceed very slowly, but individuals will rarely become very old (in chronological time) as mortality tends to increase steeply as $T$ approaches the lethal threshold.

\section{Modelling survival probabilities of the immature stages}

Survival probabilities of eggs, larvae and pupae are assumed to be independent of their age but to depend on the ambient temperature $(T)$. Thus, the probability that an individual survives from day $t$ to day $t+1$ is modelled as:

$$
p_{s}=s_{\max } \mathrm{S}(T)=s_{\max }\left(\frac{(a+b)\left(T-T_{\min }\right)}{a\left(T_{\max }-T_{\min }\right)}\right)^{a}\left(\frac{(a+b)\left(T_{\max }-T\right)}{b\left(T_{\max }-T_{\min }\right)}\right)^{b}
$$

where the parameters may depend on species, gender and stage. $s_{\max }$ is the maximum survival probability $\left(0 \leq s_{\max } \leq 1\right)$ achieved when $T=T_{\text {opt }}$. 
Note that Equation 9 assumes that all factors other than temperature are optimal for survival. Survival of fly larvae is reduced when intraspecific competition among the larvae is taken into consideration, while survival of fly pupae is reduced by parasitism.

\section{Modelling hatch probabilities of the immature stages}

The probability that an individual hatches (i.e. transforms from one stage to the next) during $\Delta t$ is assumed to be 0 when $Q(x)$ is 0 and to increase monotonically with biological age. Thus, by replacing $a$ and $b$ in Equation $7 a$ with $c$ and $d$, we get by setting $d=0$ and $Q_{\min }=0$ :

$p_{h}=h_{\max } \mathrm{S}(Q)=h_{\max }\left(\frac{Q(x)}{Q_{\max }}\right)^{c}$

where $h_{\max }$ is the maximum hatching probability. The shape parameter $c$ determines how steeply hatch probability increases when $Q(x)$ approaches $Q_{\max }$. As it is assumed that $p_{h}=1$ when $Q(x)=$ $Q_{\text {max }} h_{\text {max }}$ is per definition equal to 1 .

\section{Modelling survival probabilities of adults}

In contrast to the immature stages, adults do not hatch to the next stage. However, conceptually age-dependent mortality can be interpreted as if an individual hatches into death. Thus, its risk of dying during a time step is likely to increase when its biological age $Q(x)$ approaches the maximum age $Q_{\max }$ and to become 1 when $Q(x)=Q_{\max }$. We therefore model the per capita death rate due to aging by an expression similar to Equation 10. Thus, the probability of not dying is given as $1-$ $\left(Q(x) / Q_{\max }\right)^{c}$. Besides, the ambient temperature may also have an effect on survival probability, especially at extreme temperatures. Thus, survival probability of adults is modelled as a product of the ambient temperature $(T)$ and biological age $(Q(x))$. In combination this yields:

$$
p_{s}=s_{\max } \mathrm{S}(T)(1-\mathrm{S}(Q))=s_{\max }\left(\frac{(a+b)\left(T-T_{\min }\right)}{a\left(T_{\max }-T_{\min }\right)}\right)^{a}\left(\frac{(a+b)\left(T_{\max }-T\right)}{b\left(T_{\max }-T_{\min }\right)}\right)^{b}\left(1-\left(\frac{Q(x)}{Q_{\max }}\right)^{c}\right)
$$

\section{Modelling oviposition rates of adult females}

Age-dependent oviposition rates of females are also modelled as a function of the ambient temperature $(T)$ and their biological age $(Q(x))$. Typically fecundity increases initially, which is attributed to maturation of eggs, and then declines as aging proceeds. Thus, temperature- and age-dependent fecundity rates, assuming that all other factors are optimal, are modelled as:

$$
\begin{aligned}
& F=F_{\max } \mathrm{S}(T) \mathrm{S}(Q)= \\
& F_{\max }\left(\frac{(a+b)\left(T-T_{\min }\right)}{a\left(T_{\max }-T_{\min }\right)}\right)^{a}\left(\frac{(a+b)\left(T_{\max }-T\right)}{b\left(T_{\max }-T_{\min }\right)}\right)^{b}\left(\frac{(c+d)\left(Q(x)-Q_{\min }\right)}{c\left(Q_{\max }-Q_{\min }\right)}\right)^{c}\left(\frac{(c+d)\left(Q_{\max }-Q(x)\right)}{d\left(Q_{\max }-Q_{\min }\right)}\right)^{d}
\end{aligned}
$$

where $Q_{\min }$ defines the preoviposition period, i.e. the biological age at which oviposition commences. 


\section{Modelling sex ratio}

If $p_{f}$ denotes the proportion of females then $p_{f} F$ of the produced eggs will be female. It is assumed that $p_{f}$ does not change with time and age so that females constitute a fixed proportion of all individuals in each stage and age class.

\section{Modelling intraspecific competition among fly larvae}

High densities of fly larvae in the manure are likely to reduce larval survival due to intraspecific competition (Nicholson 1954). Thus, the effect of competition on survival is included in the model as a limiting factor given as:

$$
f\left(H_{2}\right)=\frac{1}{1+\psi\left(H_{2} / M\right)}
$$

where $\mathrm{H}_{2}$ is the number of all larvae and $M$ is the volume of the manure occupied by the stable flies, while $\psi$ is a constant expressing the strength of competition among larvae. For simplicity and due to lack of sufficient experimental data, it is assumed that $\psi$ is the same for both genders and for all instar stages, though it seems likely that older larvae have a stronger impact on young larvae than vice versa. $f\left(H_{2}\right) \approx 1$ when larval density is low, and approaches 0 when $H_{2} / M \rightarrow \infty$. Total daily survival probability of fly larvae is modelled as the product of Equation 9 and Equation 13.

\section{Modelling the interactions between parasitoids and hosts}

Adult females of $S$. cameroni cause mortality on stable flies by attacking their pupae. An attack may result in a successful oviposition, yielding a viable parasitoid offspring developing inside the puparium. However, not all attacks are successful as the pupa may die without yielding a parasitoid, either because the offspring dies before being fully developed or because the adult parasitoid feeds on the host. Parasitoid attacks not resulting in a viable offspring are characterized as parasitoid induced mortality (PIM). Therefore, the realized fecundity of the parasitoids can be modelled as $F=\varphi E$ where $E$ is the number of attacks per time unit and $\varphi$ is the proportion of attacks resulting in a successful oviposition, while $1-\varphi$ is the proportion dying due to PIM (Skovgård and Nachman 2015a).

Equation 12 describes the fecundity of an adult female parasitoid if the availability of suitable fly pupae is unlimited, all attacks are successful (i.e. $\varphi=1$ ) and no other parasitoids interfere with the female. To incorporate host density, host feeding and mutual interference, we model the attack rate $E$ as:

$$
E=E_{\max } f_{1}(T) f_{2}(Q) f_{3}\left(H_{3}\right) f_{4}\left(P_{2}\right)
$$

where $E_{\text {max }}$ is the maximum rate of encounters with hosts, $f_{1}(T)$ the effect of temperature, $f_{2}(Q)$ the effect of parasitoid age, $f_{3}\left(H_{3}\right)$ the effect of host density and $f_{4}\left(P_{2}\right)$ the effect of parasitoid density on the attack rate. $H_{3}$ denotes the number of host pupae and $P_{2}$ the number of adult female parasitoids.

As in Equation 10, $f_{1}(T)$ and $f_{2}(Q)$ are modelled by means of the SANDY model, i.e. $f_{1}(T)=S(T)$ and $f_{2}(Q)=S(Q)$. The effect of host density is modelled by means of the Weibull (1951) distribution given as: 


$$
f_{3}\left(H_{3}\right)=1-e^{-\alpha\left(H_{3} / A\right)^{\beta}}
$$

where $A$ is the surface area of the manure, while $\alpha$ and $\beta$ are positive constants, which are assumed to be independent of parasitoid age and temperature. Equation 15 is based on the assumption that fly larvae pupate in the upper layer of the manure, which coincides with the depth at which female S. cameroni can move (Skovgård and Nachman 2015a).

Finally, the effect of parasitoid density (mutual interference) is modelled as:

$$
f_{4}\left(P_{2}\right)=\frac{1}{1+r\left(\left(P_{2}-1\right) / A\right)^{q}}
$$

where $r$ and $q$ are positive parameters (Skovgård and Nachman 2015b).

The probability that a host is attacked during the time it spends in the pupal stage depends on the total number of attacks carried out by the $P_{2}$ female parasitoids, the number of host pupae and the time the pupae have been exposed to the parasitoids. In the following, we will use $x$ and $y$ to denote the chronological age of host and parasitoid individuals, respectively. Thus, the maximum number of attacks carried out during a time interval is found as:

$R=\sum_{y=0}^{\infty} E(y) P(2,2, y)$

where $E(y)$ is the attack rate of a female parasitoid of age $y$ (Equation 14) and $P(2,2, y)$ is the number of adult female parasitoids of age $y$.

As not all attacks lead to a successful attack, we calculate the maximum number of ovipositions as:

$$
R^{\prime}=\sum_{y=0}^{\infty} \varphi(y) E(y) P(2,2, y)
$$

where $\varphi(y)$ depends on the ambient temperature, the biological age of the parasitoids and the effect of mutual interference, and can be modelled as $\varphi(y)=\varphi_{\max } \mathrm{S}(T) \mathrm{S}(Q) f_{4}\left(P_{2}\right) \cdot f_{4}\left(P_{2}\right)$ is modelled by Equation 16, but with parameter values that are specific to the effect of mutual interference on the success ratio $(\varphi(y))$ (see Skovgård and Nachman 2015a). Equation 17 accounts for the number of killed hosts per time unit, while Equation 18 predicts the number of attacks leading to a viable parasitoid offspring.

Host pupae of age $x\left(H_{3}(x)\right)$ are assumed to be encountered in proportion to the age group's share of the total number of healthy pupae, i.e. $H_{3}(x) / H_{3}$, but when a host has been encountered, its probability of being attacked depends on its age. Thus, a pupa of age $x$ has probability $v(x)$ of being attacked upon an encounter with a parasitoid. However, when parasitoids are offered pupae of different ages, they seem to have higher preferences for attacking hosts of intermediate ages and to avoid attacking young and old pupae (Larsen 2006). Thus, the risk that a given pupa is attacked not only depends on its own age, but also on the age distribution of all the other pupae. Consequently, the total rate of attacks on pupae of age $x$ is modelled as:

$$
Z(x)=v(x) R \frac{\pi(x) H_{3}(x)}{\sum_{x=}^{\infty} \pi(x) H_{3}(x)}
$$

where $\pi(x)$ denotes the preference function, which peaks at the preferred age class. 
As the mean number of attacks per pupa during $\Delta t$ is $Z(x) \Delta t / H_{3}(x)$, the proportion of host pupae of age $x$ avoiding being attacked, assuming random encounters between hosts and parasitoids, can be found as the 0 -term of the Poisson distribution, i.e. as $e^{-Z(x) \Delta t / H_{3}(x)}$. Consequently, the probability that a pupa of age $x$ will be attacked during $\Delta t$ is found as:

$$
z(x)=1-e^{-Z(x) \Delta t / H_{3}(x)}
$$

so that the combined survival probability of a host pupa of age $x$ is found by combining Equation 9 and 19, i.e. $p_{s}(x)=s_{\max } \mathrm{S}(T)(1-z(x))$.

To find the proportion of successful attacks against pupae during $\Delta t, R$ in Equation 19 is replaced with $R^{\prime}$ (Equation 18) and $Z(x)$ in Equation 19 with $Z^{\prime}(x)=\varphi(x) Z(x)$. Using $Z^{\prime}(x)$ in Equation 20, yields $z^{\prime}(x)$, which is the probability that a healthy pupa of age $x$ is parasitized during $\Delta t$. Consequently, the total number of eggs belonging to gender $g$ added to the parasitoid population during $\Delta t$ is found as:

$$
P(g, 1,0)=p_{g} \sum_{x=0}^{\infty} H_{3}(x) z^{\prime}(x)
$$

The three age-dependent functions $v(x), \pi(x)$ and $\varphi(x)$ are assumed to be independent of the ambient temperature as experiments to reveal the functions were only conducted at $25^{\circ} \mathrm{C}$ (Larsen 2006). The functions are modelled by means of SANDY, using biological age of host pupae instead of chronological age, i.e. $v(x)=v_{\max } \mathrm{S}(Q), \pi(x)=\pi_{\max } \mathrm{S}(Q)$ and $\varphi(x)=\varphi_{\max } \mathrm{S}(Q)$.

\section{Modelling temperature variation in manure}

Manure temperature in a given depth is assumed to depend on fermentation rate, the ambient temperature and the ability of manure to conduct heat. We consider manure as consisting of $L$ vertical layers each of width $\Delta L=1 \mathrm{~cm}$. The change in temperature in layer $i$ (corresponding to depth $L_{i}=i \Delta L$ ) during $\Delta t$ is modelled as:

$\frac{\Delta T_{i}}{\Delta t}=K_{i}-c\left(T_{i}-T_{i-1}\right)-c\left(T_{i}-T_{i+1}\right)$

where $T_{i}$ denotes the current temperature of layer $i . K_{i}$ is the heat produced per time unit due to fermentation processes, and $c$ is a constant expressing heat conductivity of manure. The temperature at the surface (i.e. $\left.T_{0}\right)$, corresponds to the current air temperature $\left(T_{\text {air }}\right)$ and $T_{L+1}$ to the floor temperature $\left(T_{\text {floor }}\right)$. Until more information becomes available, $K_{i}$ is regarded as a constant (i.e. $K_{i}=K$ for all $i$ ), though it is likely to depend on the temperature and humidity in layer $i$.

\section{Modelling vertical distributions of insects inside a stable}

Though stable fly larvae can actively move around in the manure to find suitable sites, movements are constrained by many factors of which temperature, texture, water content and anoxia are likely to be the most important. Typically, manure becomes more compressed, wetter and with less oxygen at increasing depths, so even though the temperature may be favourable at greater depths, immature flies are more likely to be found in the upper layers of the manure. For simplicity we assume that eggs are deposited in the upper $3 \mathrm{~cm}$ of the manure and exposed to the average temperature in this layer, while larvae can occupy manure down to $8 \mathrm{~cm}$, which implies that they typically develop at higher temperatures than eggs. When larvae are going to pupate, they tend to move closer to the surface, so we assume that fly pupae, as well as the parasitoids, are exposed 
to the average temperature of the upper $3 \mathrm{~cm}$. Adult stable flies are typically found sitting where the temperature is higher than in the air, especially during cold seasons, for instance by spending time on the cattle, the ceiling above cattle, sunny spots on the walls, etc. We therefore assume that adult female flies are always capable of finding places where the temperature is optimal $\left(T_{\text {opt }}\right)$ for maximizing their fitness defined as the product of survival probability $\left(p_{s}\right)$ and fecundity rate $(F)$. Yet, during the winter period adult stable flies are normally reduced to very low numbers in Danish stables (Skovgård 2004). Likewise, the extreme cold winters in Canada and the northern parts of USA are responsible for the decline of adult flies observed in these areas (Beresford and Sutcliffe 2009). Males of both species are assumed to be exposed to the same temperature as the females.

\section{Modelling management of stable flies}

The management module of The Fly Simulator gives the user three options for controlling stable flies: (1) release parasitoids; (2) treatment of the manure with larvicide; and (3) removal of manure. The user can either apply a preplanned strategy, which is used independently of the actual abundance of flies, or use the model tactically by only interfering with the system when the abundance of flies exceeds a certain threshold or threatens to become a problem. In this context, it should be noted that the user of The Fly Simulator has perfect knowledge of the number of flies inhabiting the simulated cattle facility, whereas in the real world such exact information is unavailable. In practice, the farmers usually describe fly problems in terms like 'nothing to talk about', 'irritating' or 'intolerable', which can be difficult to translate into numbers. More exact information can be obtained by sampling, either by using indices (e.g. the average number of flies counted on an animal or a selected part of an animal (e.g. Kristiansen and Skovmand 1985) or the number caught on sticky cards and strings (Williams 1973) or by using a mark-recapture method (e.g. Nachman and Skovgård 2012; Skovgård and Nachman 2012). However, as a rule of thumb the more precise the information needed for making correct decisions, the more time and money have to be invested into sampling. As The Fly Simulator does not incorporate the costs of information, the model underestimates the total costs associated with ad hoc control measures based on assessment of fly abundance.

To assess the efficiency of a given control strategy, The Fly Simulator applies the concept of FlyDays, which is calculated as the sum of daily counts of adult flies over a given period. Thus, the model can first be run without any control measures to calculate maximum FlyDays. When the model is subsequently run, the success of a given control effort can then be expressed by the control index $(\mathrm{Cl})$ calculated as the percentage reduction in FlyDays compared with no control. Furthermore, if we know (or guess) the price of the various control options (Table 3), we can calculate the gain of controlling flies as the difference in FlyDays without and with control and relate the gain $(G)$ to the costs (C), e.g. in Danish crowns (DKK). Thus, the exercise is to use The Fly Simulator as a tool for optimizing control of stable flies defined as the control strategy that keeps the abundance of flies below a given threshold level (e.g. 10,000 adult flies) and at the same time maximizes $G / C$. The optimal strategy can be further constrained if the user wants to minimize or completely avoid the use of insecticides.

\section{Simulating management of fly populations}

\section{Stable fly dynamics without control measures}

Data from a Danish organic dairy farm was used to test the ability of the model to simulate the observed population dynamics of stable flies from March 1, 2003, through December 20, 2004 
(Skovgård and Nachman 2012). The farm had about 70 milking cows and 25-50 calves. The calves were raised in a separate building connected to the cow stable through two open doorways. It is the calf facility which is in focus of the modelling efforts, because stable flies were especially abundant here. The area of the calf building was approximately $300 \mathrm{~m}^{2}$ of which about $144 \mathrm{~m}^{2}$ were taken up by two separate confinements, each $3 \times 24 \mathrm{~m}$, at each side of the building and separated by a $3.5 \mathrm{~m}$ broad gangway. Depending on current needs, the confinements could be divided into smaller boxes of variable size ( $\min .3 \times 3 \mathrm{~m})$. The calves were free-roaming within the boxes where they were provided with straw-beddings. The manure thus consisted of a mixture of straw, decomposing feed, faeces and urine. Removal of manure took place at irregular intervals and not necessarily at the same date in both confinements. As manure depth was not measured experimentally, it was assumed that the area covered with manure in each confinement was 50 $\mathrm{m}^{2}$ with the same depth $(20 \mathrm{~cm})$ everywhere.

Air temperature inside the stable was measured during the experimental period yielding daily average temperatures from March 1, 2003 through January 1, 2005. Stable temperatures were calibrated to outdoor temperatures measured at the same dates by a nearby weather station. This enabled estimation of the air temperature inside the stable on a given day from the corresponding outdoor temperature during the period where stable temperatures had not been measured directly, i.e. from January 1 through 28 February, 2003. By using daily average temperatures, the model ignores diurnal variation, e.g. on an hourly basis. This is justified by the fact that the daily amplitude between minimum and maximum temperatures inside stable buildings is rather small compared with outdoor conditions.

Because the initial stage distribution of stable flies on January 1,2003, is unknown, it was assumed that it was the same as the simulated distribution on January 1, 2005. An iterative procedure was used to gradually narrow the difference between the initial and final stage distributions. Starting with an arbitrary initial distribution and by successively using the final distribution as input for the next simulation, the procedure could be repeated until the two distributions become the same, usually after three or four cycles. This happens when the initial population consists of 627,098 stable flies of which 189,219 are eggs (30.2\%), 320,461 larvae (51.1\%), 107,800 pupae (17.2\%) and 9,628 adults (1.5\%) (Figure 6).

A stable age distribution was applied to initialize the model with respect to the age distribution of individuals within each life stage. It was assumed that parasitoids were absent unless they had been intentionally introduced.

The initial temperature profile in the manure was estimated by using an iterative calibration procedure similar to the way the initial stage distribution was assessed so that the profile on January 1, 2003, was the same as on the same date in 2005 (Figure 7).

Figure 8 demonstrates that The Fly Simulator describes sampling data of stable flies reasonably well. Thus, $66.4 \%$ of the total variation in the observed numbers of adult stable flies can be explained by the model. Agreement between observed and predicted numbers of adult flies is best during the first year, while the model predicts higher abundances during the following spring than observed. Peak density during the second summer is predicted to occur around September 1, which agrees with data, but at a markedly lower level than actually observed. The model predicts, in accordance with data, very high densities of stable flies (80,000-120,000 adults) during summertime, which is clearly unacceptable. Consequently, FlyDays reached $26,754,850$ over the two years period. In the following, Figure 8 will be referred to as the base-line (or worst-case) scenario against which the various control measures can be compared. 


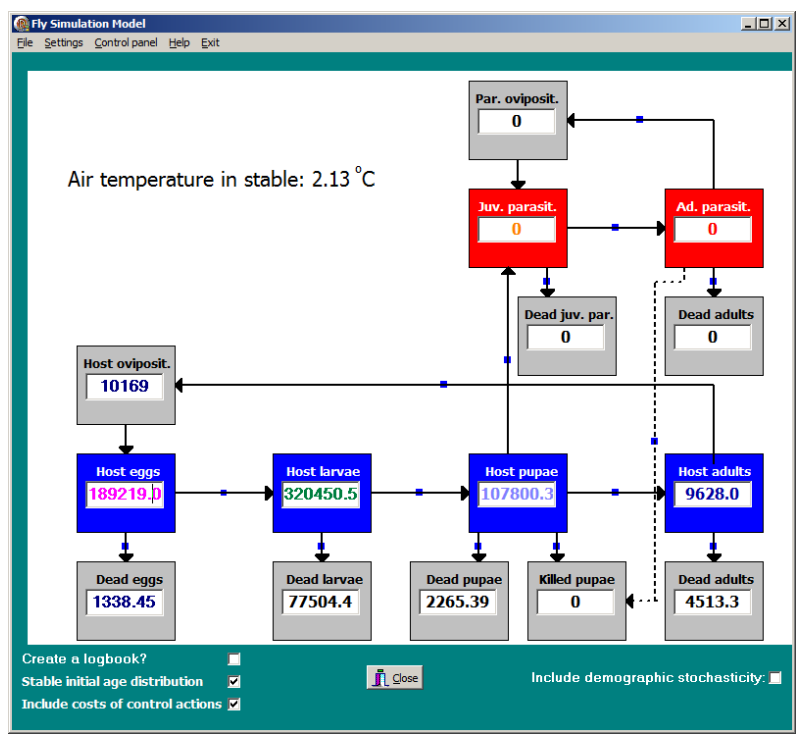

Figure 6. System diagram describing the interactions between stable flies (dark boxes) and parasitoids (dark grey boxes). The initial numbers of individuals in each developmental stage can be entered into the boxes. The grey boxes are the expected numbers of individuals added or lost from the populations per day. These rates are determined by the current temperature and the model's parameters, which can be changed by clicking the small blue squares on the arrows. The simulation was started on January 1 when the air temperature inside the stable was $2.13^{\circ} \mathrm{C}$.

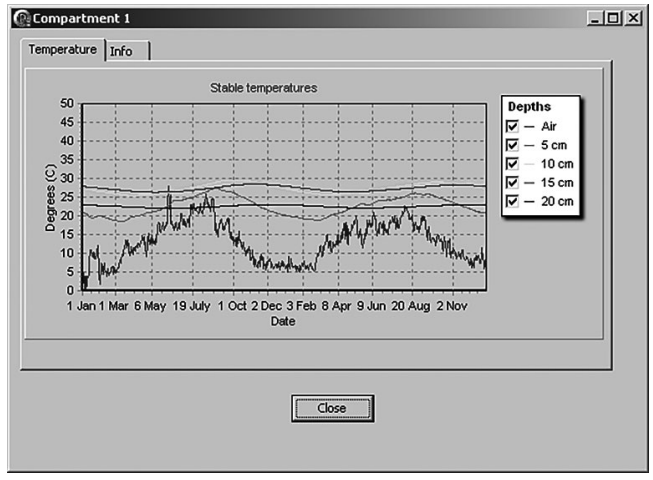

Figure 7. Stable temperatures used for the simulation initiated in Figure 6. The air temperature (jiggered curve) was measured daily while the manure temperatures (smoothed curves) at different depths were found by means of Equation 22. The seasonal variation in manure temperatures declines with depth. 


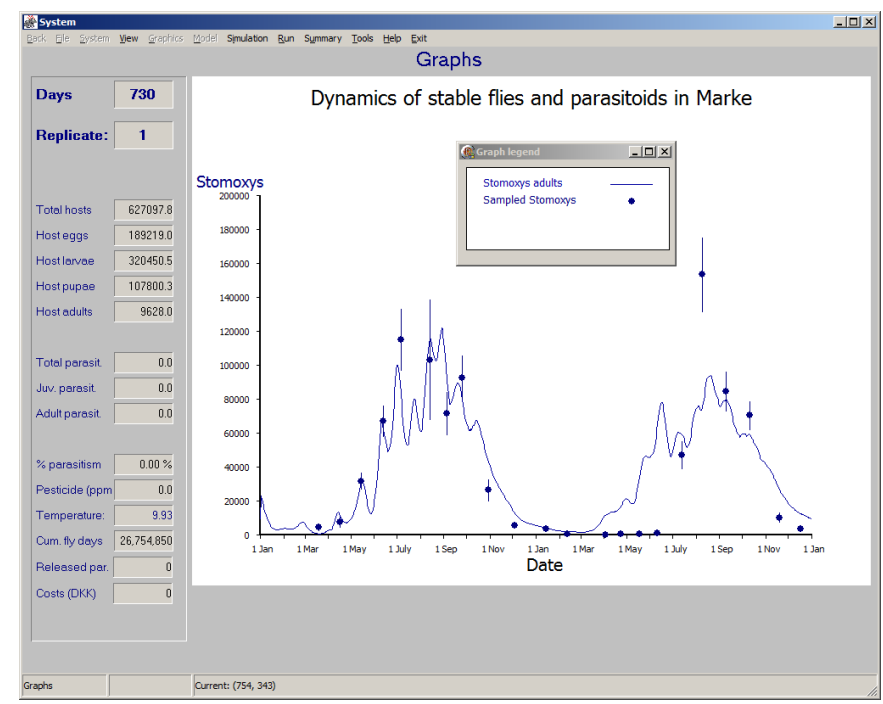

Figure 8. The observed (dots) and model predicted (line) dynamics of stable flies from January 1, 2003, through January 1,2005, using the parameter values given in Table 1 and 2. The model was initiated with the values shown in Figure 6 and with temperature variations shown in Figure 7. The observed numbers of stable flies were obtained from mark-recapture data which also yielded 95\% confidence intervals for the true value (horizontal lines).

\section{Biological control of stable flies by means of parasitoids}

Since none or very few $S$. cameroni can survive the low temperatures prevailing in a Danish stable during winter time, biological control of stable flies by means of $S$. cameroni should be based on either inoculation or inundative releases when flies start to become a nuisance in early spring. As practical experience, as well as model simulations, has shown that inoculative releases with small numbers of parasitoids are insufficient to prevent serious outbreaks of flies, inundation seems to be the only way of using this parasitoid species in practice. The Fly Simulator offers four different options for introducing parasitoids into the stable: (1) when a simulation is stopped by the user; (2) at fixed time intervals from when a simulation is stopped and until the simulation finishes; (3) at fixed time intervals during a chosen period (e.g. every 14 days from spring to autumn); and (4) every time the abundance of stable flies exceeds a pre-defined threshold.

Figure 9 shows an example of an inundative release strategy where 20,000 S. cameroni (introduced as parasitized fly puparia) are released on $100 \mathrm{~m}^{2}$ of straw bedding every second week from April 15 through October 15. The model assumes that an adult parasitoid ecloses from each puparium within 24 hours after introduction.

The chosen strategy requires releases of 560,000 parasitoids at an estimated cost of DKK 21,280 (appr. 3,000 US dollars). Compared with the base-line scenario, the reduction in FlyDays is $18,678,744$, corresponding to a $\mathrm{Cl}$ of $69.8 \%$. Thus, for each released parasitoid the reduction is 33.4 FlyDays or 877.8 FlyDays/DKK. As seen from Figure 10, the predicted reduction in FlyDays $(C l)$ increases asymptotically towards $100 \%$ with the number of released parasitoids, whereas the 


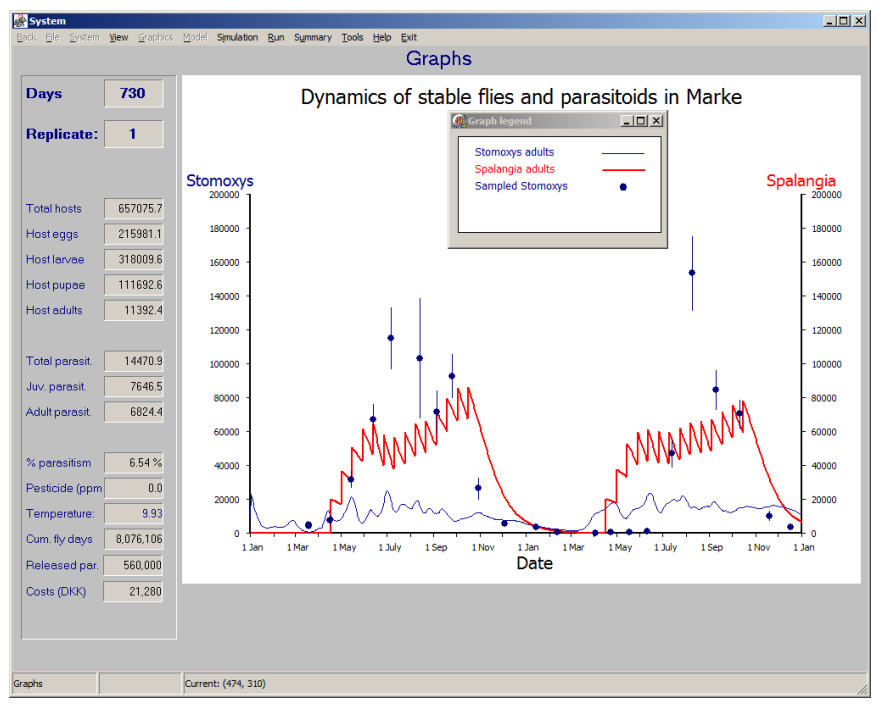

Figure 9. A simulation showing the predicted outcome of a pre-planned control strategy based on releasing 20,000 parasitoids every second week from April 15 through October 15 in both years. The effect of parasitoids can be measured as the decline in FlyDays compared with the base-line value shown in Figure 8.

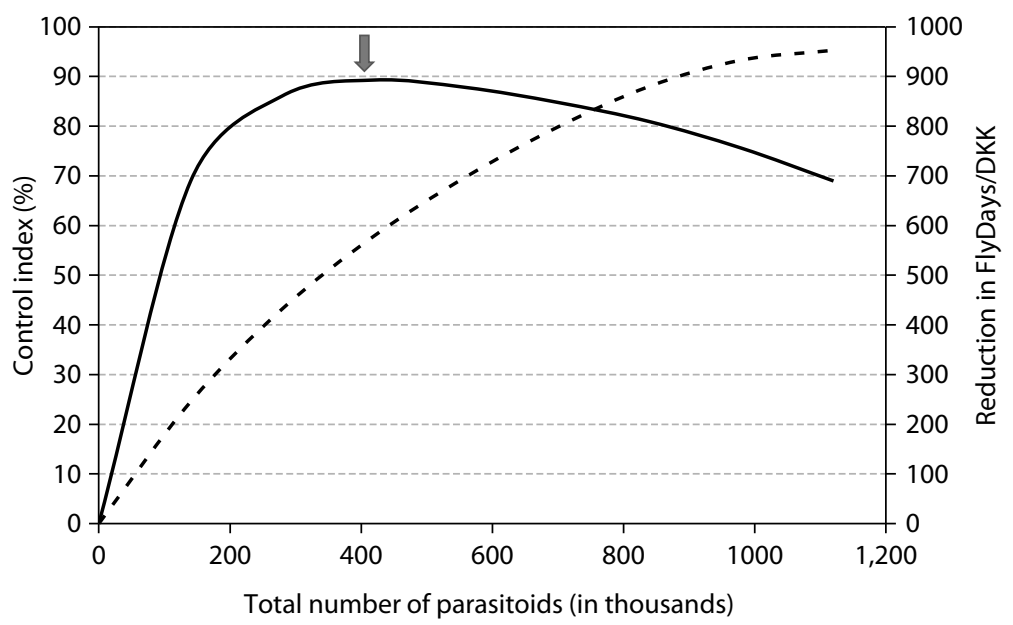

Figure 10. The expected reduction in FlyDays expressed by the control index (CI) (broken line) and the reduction in FlyDays per invested DKK (G/C) (full line). Both variables are shown against the total number of released parasitoids. Releases are assumed to take place every second week from April 15 through October 15 as in Figure 9. The arrow shows the optimal number of released parasitoids. 
reduction compared with the costs $(G / C)$ peaks at around 400,000 parasitoids $(15,000$ parasitoids per release), which corresponds to a predicted $\mathrm{Cl}$ of $57.9 \%$ with a stipulated cost of DKK 17,360 . If the farmer wants to raise $\mathrm{Cl}$ further (e.g. to $90 \%$ ), he would need to increase the number of parasitoids to a level where it is no longer economically affordable, in particular if he has other control options to choose among.

\section{Chemical control of stable flies}

The Fly Simulator provides an option to apply insecticides against stable flies. To model chemical control, we used data for the larvicide Neporex containing $2 \%$ cyromazin, which is a growth inhibitor affecting moulting of larvae from one instar stage to the next and from the third and last instar stage to the pupal stage (Vazirianzadeh et al. 2007). The average concentration of larvicide in the manure depends on its volume $\left(\mathrm{m}^{3}\right)$, the amount of larvicide used at each treatment, and the rate at which larvicide disappears either by degradation or when the manure is removed. Mortality rate of fly larvae increases according to a dose-lethality curve based on $\mathrm{LC}_{50}$ and $\mathrm{LC}_{90}$ values given by Vazirianzadeh et al. (2007). Insecticide can be applied in the same four ways as used for introducing parasitoids, giving the user options to decide when, how often, and in which dose larvicides should be used.

In contrast to biological control, which requires considerable planning and logistics, pesticides can be stored at site and applied when needed. The following mimics how insecticides are used ad hoc (or tactically) when the abundance of stable flies exceeds a given threshold. It is assumed that the chosen threshold corresponds to the density at which stable flies becomes intolerable. As the threshold varies considerably among individual farmers, the consequences of being more or less sensitive to the presence of flies are analysed. Thus, the more sensitive a farmer is, the lower should the threshold be. Figure 11 shows a simulation, where the treatment threshold was set to 10,000 adult flies. The amount of Neporex used per treatment was set to $2.5 \mathrm{~kg}$ (equivalent to $50 \mathrm{~g}$ cyromazin), which corresponds to the recommended dose of $25 \mathrm{~g}$ Neporex per $\mathrm{m}^{2}$. Since the insecticide only affects larvae when they moult from one larval stage to another, the abundance of adult flies is not reduced immediately. The minimum time interval between two successive treatments was therefore set to be 14 days. Finally, the estimated cost per treatment is seen to be DKK 1007.5.

The simulation shows that it was necessary to apply larvicide 16 times over two years, which corresponds to $40 \mathrm{~kg}$ insecticide at a total cost of DKK 16,120, resulting in 5,039,488 FlyDays ( $\mathrm{Cl}=$ $81.2 \%)$. This indicates that use of larvicides gives much more control for the money than parasitoids do, but it has to be noted that the model 'knows' exactly how many flies that are in the stable and therefore applies insecticide exactly at the right time. If sampling costs and wrong decisions based on imperfect knowledge of the current population size are taken into consideration, it will make pest management strategies based on sampling less profitable compared with pre-planned strategies.

Figure 12 shows the predicted outcome of simulations, where the larvicide was applied as in Figure 11, but with increasing dose per treatment. As increasing doses may lead to fewer treatments, the relationship between dose and the total amount of larvicide applied is not straightforward and explains why the curves in Figure 12 are not smooth. $\mathrm{Cl}$ increases steeply at low doses up to around $10-15 \mathrm{~kg}$ Neporex (e.g. 22 treatments of $0.5 \mathrm{~kg}$ ) and then levels off at higher doses. This also corresponds to the treatment that is most cost efficient. Thus, the model predicts that a dose of $0.5 \mathrm{~kg} /$ treatment will result in $70.1 \%$ reduction in FlyDays, while the recommended dose of $2.5 \mathrm{~kg}$ will increase $\mathrm{Cl}$ to $81.2 \%$, but to an extra cost of 4,629 DKK. However, it has to be emphasized that though Figure 12 indicates that the recommended dose leads to a marginal gain in $\mathrm{Cl}$, the predicted results depend on the assumptions incorporated in the analyses, including the 
16. The Fly Simulator: a simulation model of stable flies and their control

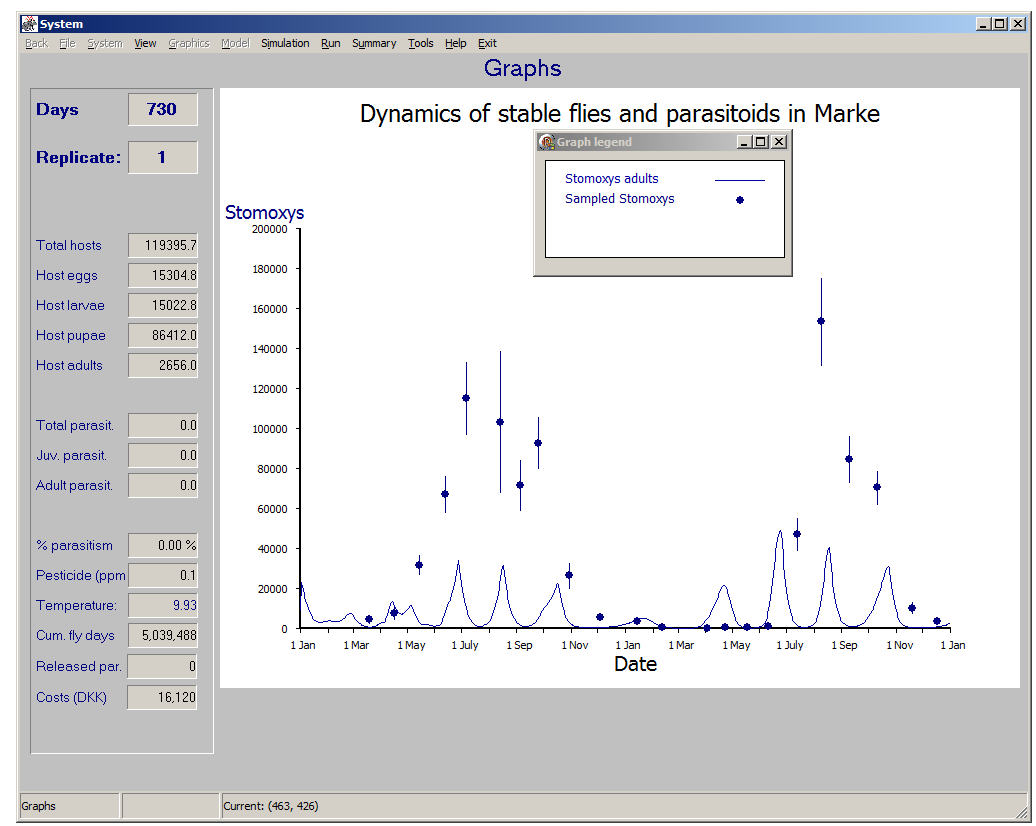

Figure 11. A simulation showing an example of chemical control by means of a larvicide using a dose of $2.5 \mathrm{~kg}$ Neporex (50 g cyromazin) each time the number of stable flies in the stable exceeds 10,000 , but with a minimum interval between treatments of 14 days.

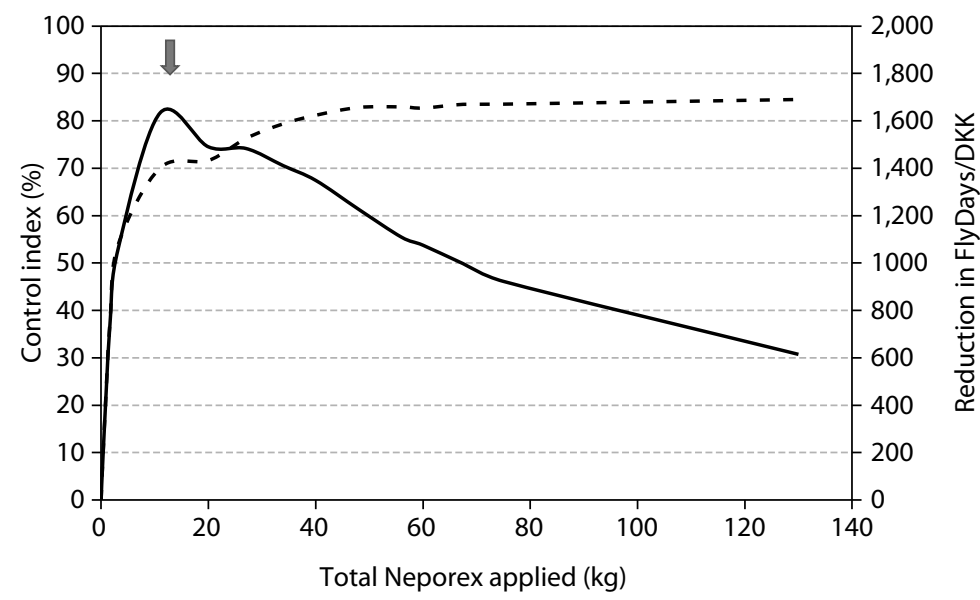

Figure 12. The expected reduction in FlyDays expressed by the control index $(\mathrm{CI})$ (broken line) and the reduction in FlyDays per invested DKK (G/C) (full line). Both variables are shown against the total amount of larvicide applied in a control strategy where treatment takes place each time the number of stable flies exceeds 10,000, but with a minimum interval of 14 days between two successive treatments as in Figure 11. The arrow shows the optimal amount of larvicide to be applied. 
presumed decay rate of Neporex in a stable system (10\% disappearance rate per day) as well as the chosen treatment threshold of 10,000 adult flies. Finally, the farmer may not aim at optimizing control costs, but to keep fly populations as low as possible, which may be affordable as long as insecticides are fairly cheap to purchase. However, even at very high doses it seems as $\mathrm{Cl}$ will not exceed $85 \%$.

Though the current version of The Fly Simulator is limited to a larvicide, it makes good sense to include an adulticide in future versions. It is because a sensitivity analysis of lifetable data indicates that factors affecting the mortality of adult stable flies will have a greater impact on population growth than factors influencing mortality of any other developmental stage (Skovgård and Nachman 2017).

\section{Removal of manure}

In the previous simulations, the costs of removing manure from the stable were not included. Besides it was assumed that the depth of manure is constantly $20 \mathrm{~cm}$. However, removal of manure can be modelled explicitly by The Fly Simulator together with the estimated costs. To include cleaning, it is necessary to specify how much manure the cattle produce per day. Without cleaning, manure will gradually accumulate under the animals. Removal can take place when the user stops the simulation. Alternatively, the user may set an option where cleaning occurs automatically each time the depth of manure exceeds a certain threshold.

In the following example, it is assumed that the initial manure depth is $20 \mathrm{~cm}(10,000 \mathrm{~kg} /$ confinement). The amount of manure produced per day is set to $35 \mathrm{~kg}$ in confinement 1 and to $30 \mathrm{~kg}$ in confinement 2 . If manure is not removed, but allowed to accumulate, its depth after two years is predicted to reach $71 \mathrm{~cm}$ in confinement 1 and $64 \mathrm{~cm}$ in confinement 2 . Under these circumstances, total FlyDays are predicted to reach $38,613,970$, which in the following will serve as the maximum value to which different removal strategies can be compared.

To demonstrate the effect of manure removal, a simulation was run where manure is removed each time its depth exceeds $40 \mathrm{~cm}$ (Figure 13). The standard of cleansing is set to $10 \mathrm{~cm}$, which is the average depth of manure (the left-over) after cleaning has taken place. Though we have no experimental evidence of how the actual turn-over of manure in the stable was, the hygienic standard was definitely low and probably not very different from the practice used in the simulation shown in Figure 13. Thus, the model explains $79.4 \%$ of the observed variation in fly abundance, which is an improvement compared with Figure 8 . However, the model is still unable to catch the extreme peak during the second summer and all attempts to explain this apparent discrepancy will be purely speculative. The predicted value of FlyDays is $26,589,759$ and the total cost of cleaning the stable is estimated to be DKK 10,529. Thus, even with the moderate cleaning effort applied in Figure 13, the reduction in FlyDays $(C I)$ is $31.1 \%$ compared with no cleaning.

Figure 14 shows that $\mathrm{Cl}$ decreases the higher the chosen treatment threshold is, which is the same as increasing the intervals between two successive cleanings. However, this will also decrease the costs so that the ideal threshold lies around $30 \mathrm{~cm}$, which corresponds to a $\mathrm{Cl}$ around $60 \%$. If a higher level of $\mathrm{Cl}$ is required, it may pay to improve the cleaning procedure by reducing the left-over. For instance, a left-over of only $1 \mathrm{~cm}$ and a cleaning threshold of $25 \mathrm{~cm}$ will increase $\mathrm{Cl}$ to $91.1 \%$ and on top of this reduce the stipulated costs by $2,440 \mathrm{DKK}$. If sufficient cleaning is not possible of practical reasons, it may be necessary to supplement manure removals with either biological or chemical control. 


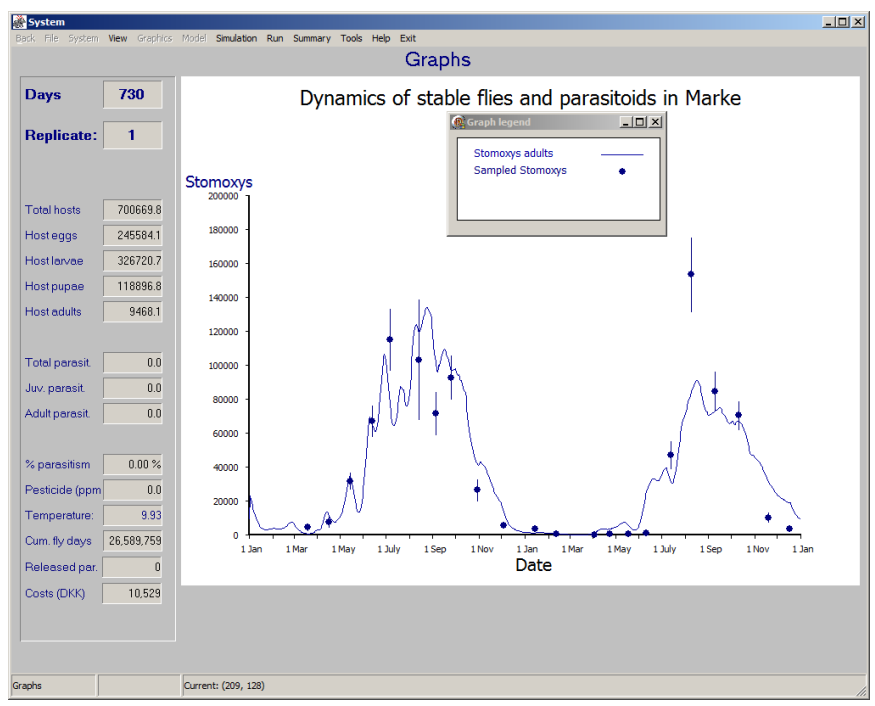

Figure 13. An example on stable fly dynamics if manure removal is modelled explicitly. It is assumed that the animals produce 35 and $30 \mathrm{~kg}$ manure/day in confinement 1 and 2, respectively. The manure is removed each time its depth exceeds $40 \mathrm{~cm}$ in a confinement. After removal the average depth is $10 \mathrm{~cm}$.

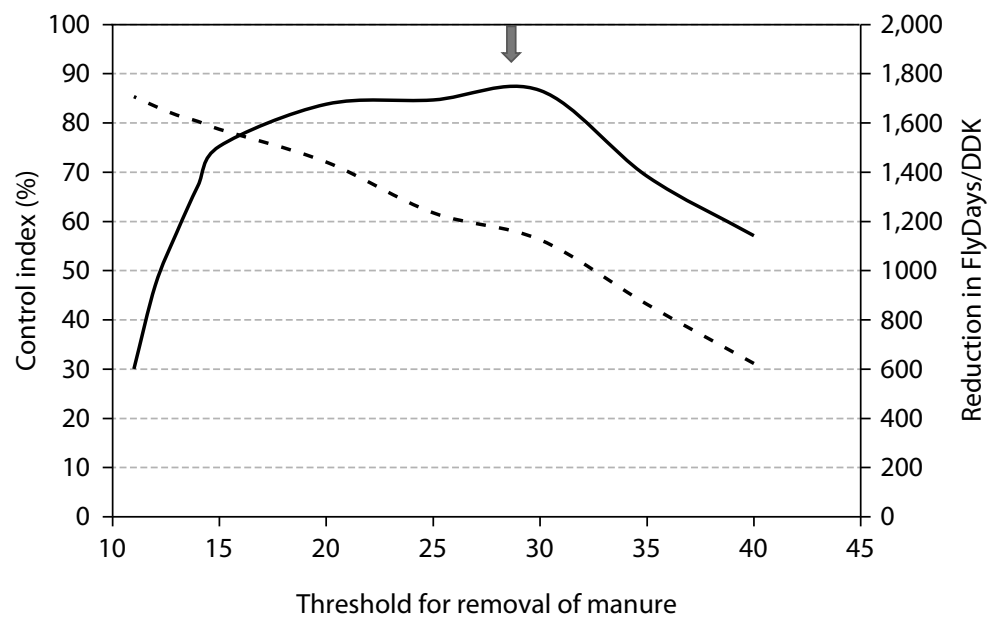

Figure 14. The expected reduction in FlyDays expressed by the control index $(\mathrm{CI})$ (broken line) and the reduction in FlyDays per invested DKK (G/C) (full line). Both variables are shown against the threshold depth when cleaning takes place. The curves are not smooth because the number of cleanings is a discrete variable that depends on the chosen threshold. The arrow shows the optimal threshold for cleaning. 


\section{Combining removal of manure with other control methods}

As shown above, cleaning the stable is by itself a way of controlling stable flies, which means that the total costs associated with fly control should include cleaning costs. The Fly Simulator allows for combining cleaning with biological and/or chemical control. To demonstrate this, it is assumed that an organic farmer uses a cleaning scheme similar to the one in Figure 13, but combines it with regular releases of 20,000 parasitoids every second week from April 15 through October 15.

As seen from Figure 15, this results in 7,866,627 FlyDays $(\mathrm{Cl}=79.6 \%)$ at a total cost of DKK 31,809. Compared with Figure 13, the reduction in FlyDays is 18,723,132 at an extra cost of DKK 21,280. Thus, the marginal gain by using biological control is calculated as 879.8 FlyDays/DKK. On the other hand, a non-organic farmer may decide to supplement cleaning with application of $2.5 \mathrm{~kg}$ Neporex each time the number of stable flies in the stable exceeds 10,000 (as in Figure 11). This control strategy results in 5,342,804 FlyDays $(C l=86.2 \%)$ at an extra cost of DKK 16,120 , which gives a marginal gain of 1,318 FlyDays/DKK, demonstrating that chemical control is economically more attractive than biological control. However, if the farmer instead of controlling flies actively decides to remove manure more frequently, for instance each time its depth exceeds $25 \mathrm{~cm}$ and at the same time makes the cleaning process more effective so the left-over is only $1 \mathrm{~cm}$ on average, this removal strategy alone will reduce FlyDays to $3,446,983(\mathrm{Cl}=91.1 \%)$ at a total cost of DKK 11,641 , corresponding to a marginal gain of 20,812 FlyDays/DKK.

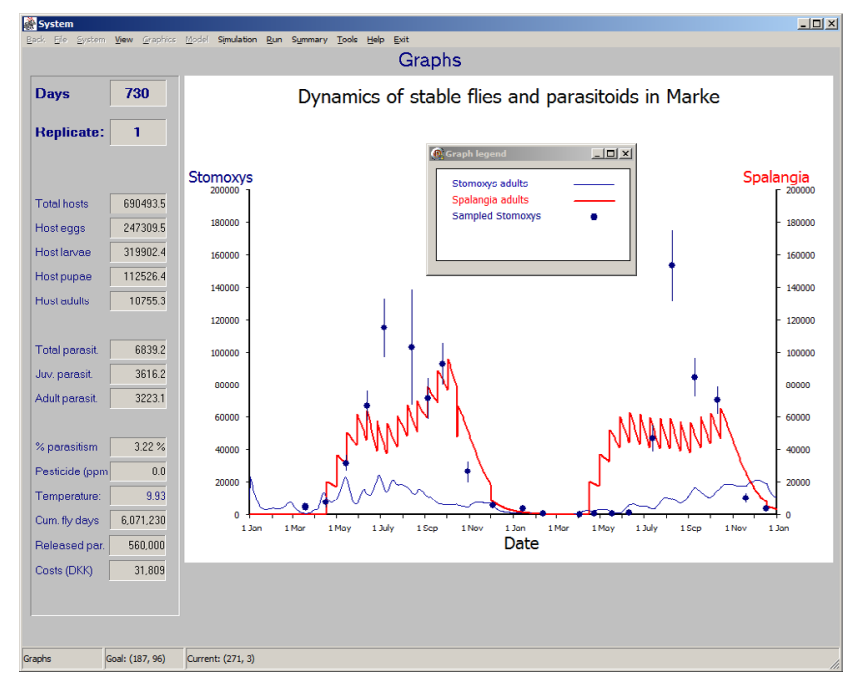

Figure 15. A simulation showing how pre-planned releases of parasitoids as in Figure 9 can be combined with removal of manure as in Figure 13. 


\section{Conclusions}

The simulations indicate that maintaining a high hygienic standard in a stable is by far the most effective way of avoiding serious fly problems and may render the use of other control measures superfluous. However, if the farmer for one reason or another is unable to remove manure as frequently as needed, he has the option of falling back on chemical control. For the organic farmer, the toolbox is more limited as parasitoids are unsuitable for responding to emergency situations. Although non-synthetic biocides exist (e.g. SPY med 1\% Spinosad), their efficacy seems low compared with the synthetic insecticides. Consequently, we recommend that organic farmers supplement fly control with a pre-planned program for releasing parasitoids, though the number per release may be lower than 200 per $\mathrm{m}^{2}$ and/or the interval between releases longer than 14 days, depending on how tolerant the farmer is to the presence of stable flies. Admittedly, the conclusions are based on tentative assessments of the costs associated with the various control options, but even if these costs are changed markedly, simulations show that the above results and conclusions are rather robust. However, if more farmers switch to organic production it is expected that natural enemies will become cheaper, while the costs of applying insecticides are likely to increase, partly because of legal constraints and partly because of declining efficiency due to resistance. In combination, this will make biological control of stable flies more economically competitive.

\section{Future developments and perspectives}

A model, like the one presented in this chapter, will always be an imperfect - some may even say a parodic - representation of the real world. However, in spite of their limitations, models may be useful tools for obtaining better insight into complex processes that are too difficult to understand intuitively and/or too expensive to study experimentally. The advantage of a model is that its assumptions and premises can be stated explicitly and communicated to others, which can open up for discussions regarding the model's realism, and how to increase it, based on current knowledge and new experiments. We hope that our presentation of The Fly Simulator will stimulate interest in its application for improving management strategies of stable flies or other nuisance flies.

The current version of The Fly Simulator has a number of limitations, which have to be overcome in order to solve specific fly problems. Consequently, its predictions cannot simply be extrapolated to other farms or areas around the world, which may differ with respect to size, number and composition of farm animals, farming practice, and climatic conditions. Although some of the processes included in the model are likely to be site independent, it is required to incorporate information on local temperature variations, since the simulations show that this external variable is paramount for predicting fly abundance. If conditions differ markedly from those prevailing in a Danish stable, it may be necessary to replace or supplement S. cameroni with one or more parasitoids from the complex of species associated with flies (Machtinger and Geden 2018). Furthermore, it is assumed that humidity is not a limiting factor for survival, development or reproduction of neither species. Though this is a reasonable assumption inside farm buildings, other studies have shown that precipitation in combination with temperature are important drivers for development of fly populations attacking free ranging cattle in North America (Taylor et al. 2007). Finally, the next version of The Fly Simulator should include an option to apply adulticides as a supplement to larvicides. 
Seen in a wider perspective, the next step will be to develop The Fly Simulator from being a strategic research tool to become an operational tactical model that can be part of a computeraided support system available to farmers and advisors for on-farm management of fly problems. The most critical input to such a management system is information about the current population sizes of flies and their natural enemies. Reasonable precise estimates of total population size of adult flies can be obtained by a mark-recapture procedure (Nachman and Skovgård 2012), but as this is very time-consuming and therefore also very costly, there is a need of testing whether some simple fly indices (e.g. flies per animal (Kristiansen and Skovmand 1985)) are acceptable proxies for the real population size. Research is also needed to investigate the relationship between sampling effort (e.g. sample size or time allocated for monitoring) and the precision of the estimated population size based on abundance indices. This will allow for incorporating sampling error into the simulations which, in combination with reliable weather forecasts, can produce short-term predictions (i.e. with a time horizon of e.g. 2-4 weeks) of best and worst case scenarios with or without interventions. The model may also provide recommendations as to the time until the next survey of fly abundance has to be made in order to adjust prognoses to reality since the discrepancy between the observed and predicted abundance of flies is likely to increase with time. Although there is still a long way to go before such a tactical model can be operational, a first step has been taken by The Fly Simulator.

\section{Acknowledgements}

The authors thank The Danish Ministry of the Environment for supporting our research (Grant J. No. 667-00136).

\section{References}

Andress ER and Campbell JB (1994) Inundative releases of pteromalid parasitoids (Hymenoptera: Pteromalidae) for the control of stable flies, Stomoxys calcitrans (L.) (Diptera: Muscidae) at confined cattle installations in west central Nebraska. J Econ Entomol 87: 714-722.

Axtell RC (1986) Fly control in confined livestock and poultry production. Technical Monograph, CIBA-GEIGY Corporation, Dover Township, NJ, USA, p 59.

Baldacchino F, Muenworn V, Desquesnes M, Desoli F, Charoenviriyaphap and Duvallet G (2013) Transmission of phatogens by Stomoxys flies (Diptera: Muscidae): a review. Parasite 20: 26.

Beresford DV and Sutcliffe (2009) Local infestation or long-distance migration? The seasonal recolonization of dairy farms by Stomoxys calcitrans (Diptera: Muscidae) in South Central Ontario, Canada. J Econ Entomol 102: 788-798.

Berry IL, Foerster KW and Campbell JB (1978) Overwintering behaviour of stable flies in manure mounds. Environ Entomol 7: 67-72.

Berry IL, Scholl PJ and Shugart JJ (1981) A mark and recapture procedure for estimating population sizes of adult stable flies. Environ Entomol 10: 88-93.

Birkemoe T, Soleng A and Aak A (2009) Biological control of Musca domestica and Stomoxys calcitrans by mass releases of the parasitoid Spalangia cameroni on two Norwegian pig farms. BioControl 54: 425-436.

Cilek JE and Greene GL (1994) Stable flies (Diptera, Muscidae) insecticide resistance in Kansas cattle feedlots. J Econ Entomol 87: 275-279.

Durel L, Estrada-Pena A, Franc M, Mehlhorn H and Bouyer J (2015) Integrated fly management in European ruminant operations from the perspective of directive 2009/128/EC on sustainable use of pesticides. Parasitol Res 114: 379-389.

Floate KD and Skovgård H (2004) Winter survival of nuisance fly parasitoids (Hymenoptera: Pteromalidae) in Canada and Denmark. Bull Entomol Res 94: 331-340. 
Friesen KM and Johnson GD (2012) Reproductive potential of stable flies (Diptera: Muscidae) fed cattle, chicken, or horse blood. J Med Entomol 49: 461-466.

Geden CJ (1997) Development models for the filth fly parasitoids Spalangia gemina, S. cameroni, and Muscidifurax raptor (Hymenoptera: Pteromelidae) under constant and variable temperatures. Bio Cont 9: 185-192.

Geden CJ (2002) Effect of habitat depth on host location by five species of parasitoids (Hymenoptera: Pteromalidae, Chalcidiae) of house flies (Diptera: Muscidae) in three types of substrates. Environ Entomol 31: 411-417.

Gilles J, David J-F and Duvallet G (2005) Temperature effects on development and survival of two stable flies, Stomoxys calcitrans and Stomoxys niger niger (Diptera: Muscidae), in La Réunion Island. J Med Entomol 42: 260-265.

Harmsen K (2000) A modified Mitscherlich equation for rainfed crop production in semi-arid areas. Theory Neth J Agric Sci 48: 237-250.

Johns GG and Vimpany JA (1999) Interaction of pH amendment and potassium fertilizer on soil chemistry and banana plant growth. Austral J Agric Res 50: 199-210.

King BH (1997) Effects of age and burial of house fly (Diptera: Muscidae) pupae on parasitism by Spalangia cameroni and Muscidifurax raptor (Hymenoptera: Pteromalidae). Environ Entomol 26: 410-415.

Kristiansen K and Skovmand O (1985) A method for the study of population size and survival rate of house flies. Entomol Exp Appl 38: 145-150.

Kunz SE, Berry IL and Foerster KW (1977) The development of the immature forms of Stomoxys calcitrans. Ann Entomol Soc Amer 70: 169-172.

Larsen D (2006) A stage structured simulation model based on the interaction between the parasitoid Spalangia cameroni and its host Stomoxys calcitrans, a nuisance pest in animal stables. MSc thesis, University of Copenhagen, Copenhagen, Denmark.

Lysyk TJ (1998) Relationships between temperature and life-history parameters of Stomoxys calcitrans (Diptera: Muscidae). J Med Entomol 35: 107-119.

Machtinger ET and Geden CJ (2018) Biological control with parasitoids. In: Garros C, Bouyer J, Takken W and Smallegange RC (eds.) Pests and vector-borne diseases in the livestock industry. Ecology and Control of Vector-borne diseases, Volume 5. Wageningen Academic Publishers, Wageningen, the Netherlands, pp. 299-335.

Nachman G and Skovgård H (2012) A simulation study of how simple mark-recapture methods can be combined with destructive subsampling to facilitate surveys of flying insects. Envir Entomol 41: 141-151.

Nachman G and Gotoh T (2015) Modeling the effects of constant and variable temperatures on the vital rate of an age-, stage, and sex-structured population by means of the SANDY approach. Environ Entomol 44: 821-834.

Nicholson AJ (1954) An outline of the dynamics of animal populations. Austral J Zool 2: 9-65.

Nijland GO, Schouls J and Goudriaan J (2008) Integration the production functions of Liebig, Michaelis-Menten, Mitscherlich and Liebscher into one system dynamics model. Neth J Agric Sci 55: 200-224.

Pitzer JB, Kaufman PE and TenBroeck SH (2010) Assessing permethrin resistance in the stable fly (Diptera: Muscidae) in Florida using laboratory selections and field evaluations. J Econ Entomol 103: 2258-2263.

Pleydell DRJ and Chrétien S (2010) Mixtures of GAMs for habitat suitability with overdispersed presence/absence data. Comput Stat Data An 34: 1405-1418.

Régnière J, Powell J, Bentz B and Nealis V (2012) Effects of temperature on development, survival and reproduction of insects: experimental design, data analysis and modeling. J Insect Phys 58: 634-647.

Salem A, Franc M, Jacquiet P, Bouhsira E and Liénard E (2012) Feeding and breeding aspects of Stomoxys calcitrans (Diptera: Muscidae) under laboratory conditions. Parasite 19: 309-317.

Scholl PJ (1986). Field population studies of Stomoxys calcitrans (L.) in eastern Nebraska. Southwest. Entomol 11: 155-160.

Skovgård H and Jespersen JB (1999) Activity and relative abundance of hymenopterous parasitoids that attack puparia of Musca domestica and Stomoxys calcitrans (Diptera: Muscidae) on confined pig and cattle farms in Denmark. Bull Ent Res 89: 263-269.

Skovgård H and Jespersen JB (2000) Seasonal and spatial activity of hymenopterous pupal parasitoids (Pteromalidae and Ichneumonidae) of the house fly (Diptera: Muscidae) on Danish pig and cattle farms. Environ Entomol 29: 630-637. 
Skovgård H (2004) Sustained releases of the pupal parasitoid Spalangia cameroni (Hymenoptera: Pteromalidae) for control of house flies, Musca domestica and stable flies Stomoxys calcitrans (Diptera: Muscidae) on dairy farms in Denmark. Biol Contr 30: 288-297.

Skovgård H and Nachman G (2004) Biological control of house flies Musca domestica and stable flies Stomoxys calcitrans (Diptera: Muscidae) by means of inundative releases of Spalangia cameroni (Hymenoptera: Pteromalidae). Bull Entomol Res 94: 555-567.

Skovgård H (2006) Search efficiency of Spalangia cameroni and Muscidifurax raptor on Musca domestica pupae in dairy farms in Denmark. BioControl 51: 49-64.

Skovgård H and Nachman G (2012) Population dynamics of stable flies Stomoxys calcitrans (Diptera: Muscidae) on an organic dairy farm in Denmark based on mark-recapture with destructive sub-sampling. Environ Entomol 41:20-29.

Skovgård H and Nachman G (2015a) Temperature-dependent functional response of Spalangia cameroni (Perkins) (Hymenoptera: Pteromalidae), a parasitoid of Stomoxys calcitrans (L.) (Diptera: Muscidae). Environ Entomol 44: 90-99.

Skovgård H and Nachman G (2015b) Effect of mutual interference on the ability of Spalangia cameroni (Hymenoptera: Pteromalidae) to attack and parasitize pupae of Stomoxys calcitrans (Diptera: Muscidae). Environ Entomol 44: 1076-1084.

Skovgård H and Nachman G (2016a) Temperature- and age-dependent survival, developmental and oviposition rates of the pupal parasitoid Spalangia cameroni. Environ Entomol 45: 1062-1075.

Skovgård H and Nachman G (2016b) Modelling biological control of stable flies by means of parasitoids. Report published by The Danish Ministry of the Environment. Available at: https://tinyurl.com/yblul7ho.

Skovgård H and Nachman G (2017) Modeling the temperature- and age-dependent survival, development, and oviposition rates of stable flies (Stomoxys calcitrans) (Diptera: Muscidae). Environ Entomol 46: 1130-1142.

Son $Y$ and Lewis EE (2005) Modelling temperature-dependent development and survival of Otiorhynchus sulcatus (Coleoptera: Curculionidae). Agric Forest Entomol 7: 201-209.

Taylor DB, Berkebile DR and Scholl PJ (2007) Stable flies population dynamics in eastern Nebraska in relation to climatic variables. J Med Entomol 44: 765-771.

Taylor DB, Moon RD and Mark DR (2012) Economic impact of stable flies (Diptera: Muscidae) on dairy and beef cattle production. J Med Entomol 49: 198-209.

Thomsen T (1938) Stuefluen (Musca domestica) og stikfluen (Stomoxys calcitrans). Undersøgelser over biologi og bekæmpelse samt en oversigt over andre til husdyr eller boliger knyttede fluearter. Den Kongelige Veterinære Landbohøjskole, Landsøkonomiske Forsøgslaboratorium, AG Bangs forlag, Copenhagen, Denmark.

Van Straalen NM (1983) Physiological time and time-invariance. J Theor Biol 104: 349-357.

Vazirianzadeh B, Jervis MA and Kidd NAC (2007) The effects of oral application of cyromazine and triflumuron on housefly larvae. Iranian J Arthropod-Borne Dis 1: 7-13.

Wagner TL, Wu H-I, Sharpe PJH and Coulson RN (1984) Modeling distributions of insect development time: a literature review and application of the Weibull function. Ann Entomol Soc Amer 77: 475-487.

Weibull W (1951) A statistical distribution of wide applicability. J Appl Mech 18: 293-297.

Weidhaas DE, Haile DG, Morgan PB and LaBrecque GC (1977) A model to simulate control of house flies with a pupal parasite, Spalangia endius. Environ Entomol 6: 489-500.

Wilhoit LR, Stinner RE, Axtell RC, Bacheler JE and Mann JA (1991a) PARMOD: a simulation model for the population dynamics of Muscidifurax spp. and Spalangia ssp. (Hymenoptera: Pteromelidae), parasites of house fly pupae (Diptera: Muscidae). Environ Entomol 20: 1418-1426.

Wilhoit LR, Stinner RE and Axtell RC (1991b) Computer simulation model of house fly management in confined-animal production systems. Technical bulletin 296. North Carolina Agricultural Research Service, Raleigh, NC, USA.

Williams DF (1973) Sticky traps for sampling populations of Stomoxys calcitrans. J Econ Entomol 66: 1279-1280. 


\section{Impact of vector control}




\title{
17. Case study: costs of Culicoides-borne arboviral diseases
}

\author{
Helen Roberts ${ }^{1 *}$, Emily Nixon ${ }^{2,3}$ and Clemens Matt ${ }^{4}$ \\ ${ }^{1}$ Exotics and Risk team, Animal and Plant Health Agency, Defra, 17 Smith Square, London, SW1P 3JR, \\ United Kingdom; ${ }^{2}$ APHA Exotics and Risk Team, Advice Services, Animal and Plant Health Agency, \\ Defra, 17 Smith Square, London SW1P 3JR, United Kingdom; ${ }^{3}$ Veterinary Parasitology and Ecology \\ Group, University of Bristol, Bristol Life Sciences Building, 24 Tyndall Avenue, Bristol, BS8 1TQ, United \\ Kingdom; ${ }^{4}$ Exotic Disease policy team, Defra, 17 Smith Square, London, SW1P 3JR, United Kingdom; \\ helen.roberts@apha.gsi.gov.uk
}

\begin{abstract}
The costs of control for veterinary vector borne diseases can fall to government, industry or a combination of both. Cost effectiveness and cost benefit analyses frameworks can help governments to take decisions on intervention but uncertainty can lead to ambiguity. This chapter concentrates on the type of issues which arise with viral diseases such as bluetongue, Schmallenberg and African horse sickness; comparing the different approaches with regards to the best prevention and control measures available and where the economic costs make government intervention beneficial for society. All three diseases are transmitted by Culicoides biting midge species, are challenging to control because of their large population sizes and diversity of breeding sites. The diseases are caused by viruses which can reassort and in some cases appear in multiple serotypes with limited cross-protection following infection. The diseases which these viruses cause are variable in terms of species affected, age of animal affected and clinical signs observed. What is common for all three is that they can rapidly spread in a naïve population and that as notifiable exotic diseases, they may have a high impact on the ability of an affected country to trade in susceptible animals and their products, requiring government to take action.
\end{abstract}

Keywords: arbovirus, bluetongue, economics, Schmallenberg, disease control

\section{Introduction}

This chapter will deal with notifiable or emerging Culicoides-borne diseases of livestock and how decision makers can use economic and disease modelling to develop policy. Disease contingency planning for government relies on several pre-defined steps: preparedness; surveillance (before, during and after an outbreak) and control and; exit strategies. Each of these depends on the resources available and the risk appetite of the government. Economic analysis, when applied to animal health, is not simply a matter of applying monetary units to a situation; it is about decision making in the light of limited resources and competing alternatives. The considerations need to go wider than a static assessment of the cost-effectiveness of different control options and capture differing incentives offered by the control programme.

Economic models conceptually consider the impact of disease, resources and effectiveness of differing control options and their differing distributional impacts (e.g. Brooks 2010; Velthuis et al. 2010). There are multiple ways a disease may impact on a livestock production system: by depleting the livestock population, by reducing productivity (e.g. reduced feed conversion), tangible outputs such as reduced milk production, spoiled or damaged skins and hides, by increasing the costs for the producer (through vaccination costs or veterinary services) or through restricting market access. But there are also less tangible outputs such as animal welfare, consumer confidence, 
access to the countryside which are all important issues even if the financial costs cannot be weighed up easily (FAO 2016; Oxford Analytica 2012).

We will consider three arboviral animal diseases which can be transmitted by Culicoides vector species: bluetongue virus (BTV) (a notifiable disease but where vaccination is available), African horse sickness (AHS) (a notifiable disease, but lacking an EU approved vaccine) and Schmallenberg virus (SBV) (a newly emerging disease in 2010/11, not made notifiable and where a vaccine is available). Within these arboviruses the most interesting comparison between SBV and BTV because for AHS the risk of introduction is exceedingly low (Carpenter et al. 2017; DEFRA 2008) and because we are discussing risk mitigation such as vaccination, which is not available for AHS, not just standard control measures, such as movement restrictions and culling. Mitigation must fundamentally pay its way in terms of expected, avoided disease costs.

When a disease is given notifiable status (a useful guidance is given in the OIE Terrestrial Animal Health Code for how to determine this: http://tinyurl.com/y7jjl5u2), outbreaks must be reported to the European Commission and trade partners and the OIE (World Animal Health Organisation). The status of notifiable means that clinical suspicion of disease and any positive test results must be reported to the competent authorities resulting in area- or premises-level movement restrictions being put in place under EU rules. Trade restrictions on live animals and products of animal origin may be put in place and there is a set time under EU rules for return to disease freedom which will depend on the epidemiology of the disease. For vector borne diseases, it is often two vector transmission seasons (usually two years).

In any disease outbreak or epizootic, the stages of action taken by government are similar no matter what the disease may be. In the case of Culicoides-transmitted arboviruses, there are considerations around the requirements in EU disease control regulations and what is epidemiologically feasible.

\section{Economic analysis}

There are essentially two differing approaches to an economic analysis:

- A cost-benefit analysis (CBA) assesses whether or not benefits of the policy outweigh its costs.

- A cost-effectiveness analysis (CEA) to assess which option is the cheapest to meet a certain objective.

The cost of a disease is the sum of the losses and expenditures and this is balanced against the returns (or profits) to give a benefit cost ratio (BCR). For an individual farmer, their $B C R$ will depend on many factors associated with market prices of land, services and the actual livestock product. Therefore this can only be assessed at the farm level. However, for government, the BCR will take into account the average costs for livestock production systems, the value to society with taxes and subsidies included. There is frequently a non-linear relationship between cost and losses and understanding this relationship and the avoidable cost of disease are what help government make decisions. The comparison of different control options against a baseline of 'do nothing' may be used. For example, the cost of vaccination versus a culling strategy, movement controls or herd isolation and the impact these have on disease spread and the costs of such measures. Conceptually, government may also use CEA where monetizing a health benefit is difficult, for example the costs of preventing an animal welfare issue. CEA compares the costs and outcomes of different control measures, where this is the ratio between the benefit gained (fewer abortions, longer life, increased production) against the cost of the control measure. In public health terms 
this is expressed as a quality adjusted life year but there is no equivalent measure for animal health.

For the purpose of this chapter, we will be focussing on the costs to government of disease control and its role in decision making by comparing three different diseases.

Other important factors to bear in mind are:

- Uncertainty (for example of disease incursion and spread) needs to be explicitly considered - this matters in two ways: (1) expected costs/benefits are quite different than in a situation where there is a deterministic setup and full certainty; and (2) the size of the variance matters more for risk-averse people.

- The market impact of disease (reduction in supply and demand) which is often modelled using multi-market models and includes switching from export to domestic markets.

- Incentive effects - the traditional approach is that government takes care of disease control and mitigation. Placing responsibility on producers increases the need to highlight longer term incentives and expectations.

\section{The diseases}

It is important to document the differences between the three diseases which will be considered in this chapter (Table 1.), as these differences go part way to explain the very different approaches to disease control, particularly when comparing bluetongue and Schmallenberg in Europe.

Where BTV and AHS are notifiable diseases, there are EU rules around disease control measures and OIE recommendations for trade. SBV presented a quite different proposition to government. As an emerging disease, initial concerns were around how it was transmitted, what the impact would be on adult animals in the long term and whether there was any human health risk (EC 2012). Once some of these questions were answered, decisions were made in consultation with stakeholders and producers to not make the disease notifiable. This would reduce the impact the disease would have on trade as well as the requirement for government intervention. Instead, at a government level, the onus was placed on improving the evidence base through a series of joint research programmes, joint development of diagnostic tests and preventing where possible, trade bans (Afonso et al. 2014).

Three stages of a country's disease status exist: (1) a disease-free status; (2) the high risk period (HRP); and (3) post HRP (Saatkamp et al. 2014) and prevention and control measures will therefore change depending on this cycle. During the disease-free status, preparation is important understanding the likely incursion of disease, what control options are available, what the likely scale of outbreak will be, whether a vaccine is available or if investment in developing a vaccine is necessary, and what diagnostic tools are available. In the HRP, the focus is on prevention using tools such as preventive vaccination, which may be government or industry led. The requirement for surveillance to detect the first case, for early detection or to prove disease freedom will drive the different surveillance components and may be applicable before, during or post the HRP. The cost effectiveness of the different surveillance strategies depend on rate of spread, the clinical signs (for a passive surveillance system) and the diagnostic tests available (virus identification or antibody detection). In the early stages of the Schmallenberg epizootic, no serology test was available and diagnosis relied on a PCR test but as animals were viraemic for only a few days, such tests were not suitable. Instead a case definition for clinical signs (arthrogyposis and developmental signs in new born lambs or calves) was used to given an indication of the prevalence of infection (Afonso 


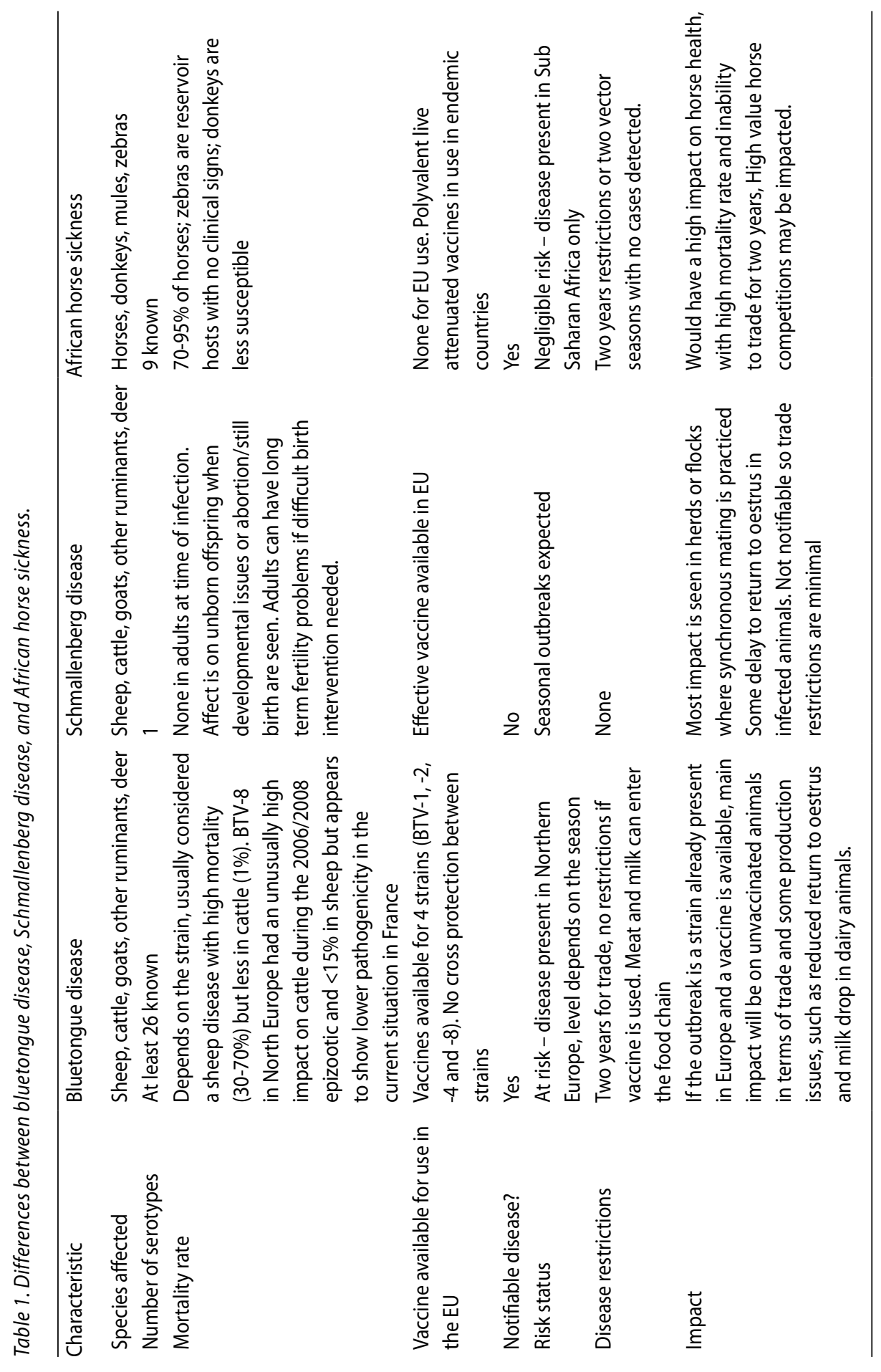


et al. 2014). For BTV-8, passive surveillance or syndromic surveillance was considered the most cost-effective measure for detecting early incursion (Welby et al. 2016). Post HRP and the focus is on control measures which may be required by legislation and a return to either disease-free status or to accepting a disease may become endemic with associated trade measures required.

\section{Disease preparedness}

Key to decision making for disease control is preparedness in understanding the risk of disease incursion, the impact it may have on your livestock populations and what are the control requirements and options. For this, understanding livestock demographics and disease epidemiology are paramount.

Predicting disease outbreaks for vector borne and transboundary diseases can be difficult and frequently risk assessors deal with high levels of uncertainty or unknowns. Horizon scanning for notifiable and emerging disease is a relatively straightforward daily task. The OIE has an open data source of notifiable disease outbreaks (see www.oie.org) and the EU Animal Disease Notification System is available to registered users in the competent authorities. These data can be used to inform a risk of incursion model, based on the OIE guidelines for import risk assessment (Roberts et al. 2011), to provide a semi-quantitative output for the comparison of likelihood of disease incursion depending on the pathway. For a vector borne disease, the most important pathways are usually proximity to a region with circulating disease for wind borne transport of vectors, or importing a live animal incubating disease. Imports of products such as blood products or germplasm can also be important pathways; but generally meat or other products of animal origin and fomites are not important routes of transmission in a vector borne disease where a biological, rather than mechanical vector is involved. However, it is important to mention to source of BTV and SBV incursion into Europe is still unknown.

As the risk increases, there may be a requirement for disease surveillance. For example, the Bluetongue Directive, EU/1266/2007 (EC 2007) requires Member States to undertake surveillance if they are adjacent to a restriction zone. The level of surveillance is not stipulated and it should be noted that adopting a system to detect $<5 \%$ prevalence with $95 \%$ confidence will not necessarily lead to detection of the first case. When infection causes few clinical signs, a passive surveillance system may also not detect circulating disease. Targeted surveillance is likely to be the most effective, and this may include post import checks, testing at slaughter houses or bulk milk testing in at-risk areas, testing animals associated with certain risk factors, or syndromic surveillance. For a vector borne disease, where the vector transmission is affected by environmental conditions, meteorological and environmental modelling is useful to indicate the trigger points in increased risk. For example, with BTV-8 and the midge vector Culicoides obsoletus in Northwest Europe, the average daily temperature of more than $12^{\circ} \mathrm{C}$ is necessary to reach a basic reproductive rate of greater than unity $\left(R_{0}>1\right)$ leading to disease spread (Gubbins et al. 2008). When the Schmallenberg epizootic started in NW Europe, having a regional approach to sharing information and gathering data meant affected Member States were able to more cost-effectively produce joint risk and impact assessments (Afonso et al. 2014; Roberts et al. 2014). When the risk of disease incursion is either very low, or very high, there would be little cost benefit in gold-plating year-round surveillance, albeit for opposite reasons, but when disease incursion is as likely to occur as not (medium risk or 33-66\% likelihood) then the decision about what to do becomes less clear (DEFRA 2016). 
A good understanding of the population demographics will require investment in systems for collating data, such as animal movements, veterinary medicine records, breed, age and identity of the animals. Some of these data are a legal requirement, for example the individual identification of cattle and horses, flock or herd identifiers for sheep and pigs, animal passports for trade purposes. These systems are often better placed with industry bodies than with government, but access to the data within is vital during disease outbreaks and this may require a cost-sharing exercise between government and industry for collecting the data. Investment in outbreak exercises for testing these systems as well as testing disease contingency plans are a requirement in EU Legislation in Council Directive 2003/85/EC Article 73, for testing foot and mouth disease plans (EC 2003), Directive 2001/89/EC for classical swine fever (EC 2001) and Directive 2005/94/EC for avian influenza (EC 2006) but there are options for testing plans for other diseases as well. In the UK, exercises have been held for AHS but not for BTV or SBV (although the reference laboratory carry out regular simulation exercises), the reasons being that these two diseases have been or are present in the UK. This initial investment in both time and resource improves preparedness for outbreak responses.

Although AHS is considered a low priority in terms of risk of incursion, the example of BTV-8 arriving and persisting in Europe in the last ten years has raised concerns that AHS may mount similar incursions (Carpenter et al. 2017).

\section{Disease costs}

As mentioned earlier, the impact of a disease incursion can have an economic or financial cost to both the farmer and government. The complexities associated with the cost of a disease outbreak are shown in Figure 1. At each step, additional investment by the farmer can reduce the level of losses through disease control, however as a counterbalance, when the cost of control is excessive and the loss due to disease is low, then the farmer may not increase his profits through additional investment.

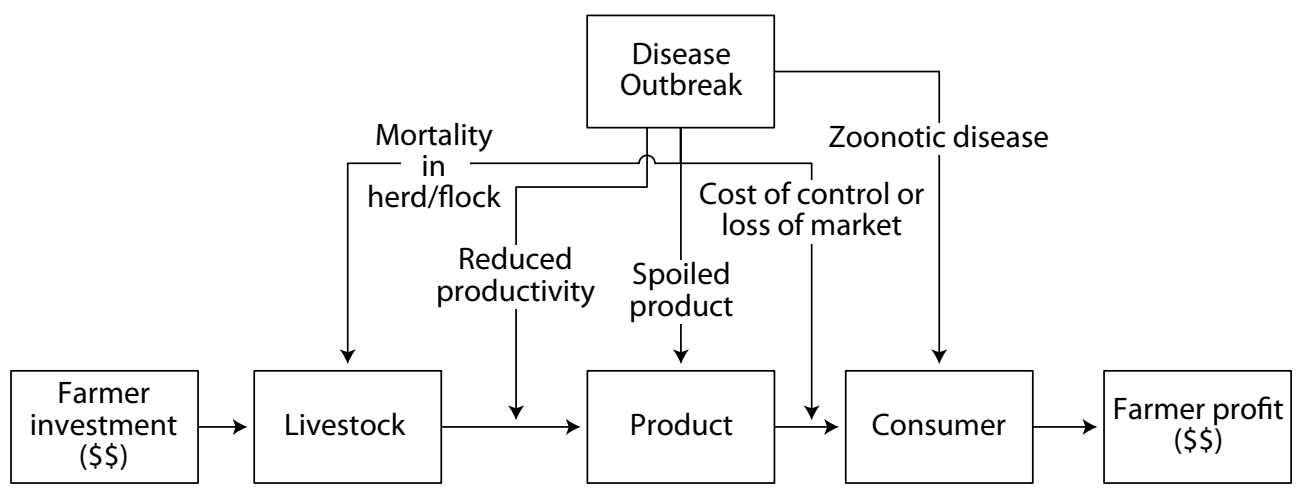

Figure 1. Schematic for the flow of economic costs associated with an animal disease (adapted from FAO 2016). 


\section{Outbreak control}

Veterinary measures taken for disease control can be divided into: those aimed at eradicating disease or looking at how far it has spread, such as culling, tracing and surveillance; those for preventing further spread, such as movement controls, vaccination or preventive culling or; those aimed at regaining disease freedom or resuming trade. Modelling of disease spread and effects of control measures is frequently used to help inform these decisions (Sumner et al. 2013).

The economic impacts of disease control measures can also be classified by those which have an immediate impact on the individual livestock keeper, such as loss of animals or products which have been destroyed, inability to trade in vaccinated animals, closing markets and auctions. Alternatively, control measures may be less measurable but still very important for decision makers for their impact on such factors as welfare losses, loss of consumer confidence, reputational impacts or public health concerns. Finally, controls may exert a positive externality in that the benefit is for a third party rather than the individual, by preventing further disease spread. Encouraging positive externalities is a role for government in terms of economic policies and therefore can also be used when considering disease control measures. Negative externalities may arise through the loss of export markets as products can be redirected to the domestic market, resulting in loss of foreign exchange, reduced prices for the producer but weighted against cheaper process for the domestic consumer.

Using Figure 1 as above, if the case of bluetongue virus is considered, the CBA model shows that control costs may not always be a sensible option for the livestock keeper. An initial investment in raising livestock, balanced with low losses due to disease of live animal or products mean that investing in further control costs will reduce the profit margin for the producer. Government incentives or subsidies may make these control measures more favourable. Farmers ideally need a fully integrated way to make decisions around vaccination according to all the different factors that might be involved. As an industry issue this approach requires them to make informed decisions themselves. Measures which could be used to help this process could be consideration of whether vaccinated animals entering the food chain are acceptable to the consumer and if animals moving directly to slaughter are required to be vaccinated; for BTV and SBV, as there are no vaccination requirements for such moves, it would only be a cost benefit for the farmer to vaccinate the breeding stock and not the fattening stock. However, this may impact on disease control measures for the wider society.

Vector control may be both a preventive measure against incursion of disease, for example, as required for livestock transport when consigning animals, and as a control measure to prevent further spread within a herd in certain circumstances, using a combination of baited traps and insecticide but it is highly unlikely to work in the vector season over wide distances as over $2 / 3$ of the midge population would be required to visit a trap with a constant level of insecticide capable of killing midges with an efficiency of at least 0.75 and it may simply not be possible to cover such a wide area (White et al. 2017). However, for a vector such as the Culicoides biting midge species found in the Northern hemisphere, population sizes can reach very high density, biting rates are very high, the larval development sites are varied and ubiquitous and the midges themselves may be dispersed over several kilometres each day (White et al. 2017).

Movement controls of animals in certain disease situations is a realistic and necessary disease control option to limit virus spread. Where diseases are transmitted by direct contact and therefore spread through the movement of infected animals, movement controls are effective. However, as 
soon as other transmission pathways are involved, such as aerosol or vector borne transmission, movement controls are less effective. For highly contagious diseases, such as foot and mouth disease, movement controls are put in place for all susceptible animals on a regional basis, with the size of the region depending on epidemiological expert advice, and with a minimum of $3 \mathrm{~km}$ protection and $10 \mathrm{~m}$ surveillance zones. For BTV, the size of the disease control zones are much larger - a $20 \mathrm{~km}$ control zone, a $100 \mathrm{~km}$ protection zone and an additional $50 \mathrm{~km}$ surveillance zone, because of the involvement of midges. The same size zones will be used for AHS. These zones may not control the vector itself and instead impact on the business as usual activities for the sector. The movement controls in France during the 2006/2007 BTV-8 epizootic meant the average farmer sold $21 \%$ fewer animals (Tago et al. 2014). If movement controls alone are used in disease models for vector borne disease, they do have a limited impact which is greater if the restriction is targeted to the infected farms but the environmental factors affecting vector transmission should also be taken into account (Ensoy et al. 2013; Turner et al. 2012). Movement from one zone to another can only happen under licence. For SBV, these zones were not used, because it was recognised that disease had already spread: detection occurred only several weeks after disease incursion, because the diagnosis was made on malformed offspring, rather than on infected adults at the time of infection.

Vaccination can be used in one of two ways. To vaccinate animals and prevent a disease incursion, such as currently recommended for areas at risk of BTV incursion in the UK, or as a disease control measure, where animals surrounding a disease outbreak may be vaccinated to act as a 'firebreak'. There are currently four vaccines approved for use in the EU against BTV- $1,-2,-4$ and -8 , whereby trade can continue is the animal is vaccinated an appropriate time before movement. Other vaccines are available for other strains of BTV but as these are live attenuated vaccines, and as there is no DIVA (differentiating infected from vaccinated animals) vaccine available, the animals may not be consigned for trade and the region must remain under trade restrictions. This is the situation in Italy where such vaccines for other strains have been used in the past (see EU website for details of such restriction zones, http://tinyurl.com/yc $8 z 2 f w g)$. Therefore where a vaccine is used in a preventive manner, there is a direct BCR to the farmer, if the risk of disease incursion is high. This risk can itself be difficult to predict and therefore the advice to vaccinate should be to consider the cost of vaccinating a whole flock or herd of animals and the likely number of animals which may become infected and would develop clinical signs and economic loss. For example, the benefit of vaccinating a beef cattle herd of 500 animals is likely to be less than the benefit of vaccinating a dairy herd, because milk drop, the most common clinical sign of BTV in cattle, is only a financial loss for dairy farmers. These considerations must be made clear to farmers to allow them to take decisions about the need to vaccinate.

Vaccine costs can be high, particularly for new vaccines or if several vaccines are needed to protect against multiple strains of disease. In 2007, when BTV-8 first arrived in the UK, the government helped farmers by providing a vaccine bank which farmers could access. This involved estimating the number of animals which may be at risk from mathematical modelling of the possible disease spread and knowledge of the livestock demographics. However, it did not take into account farmers' perception of risk, their own economic margins and veterinary costs involved in vaccination. This meant that take-up rates were high in regions where disease had been identified, but low in other areas of the country and vaccine stocks were under-utilised. Modelling work on sheep scab and farmer behaviour has looked at when it is more economically viable for the farmer to pay for preventive treatment in comparison to a neighbour's treatment choices, and what incentives (either subsidies or penalties) could be used by government (Nixon et al. 2017). The conclusion 
was that most farmers are more likely to implement a reactive or therapeutic control measure than a prophylactic one, in the absence of incentives.

\section{Exit strategy}

EU and OIE rules for regaining disease free status requires no disease outbreaks to be confirmed for two vector seasons. The impact on trade is reduced if vaccination is allowed and provided animal health certificates allow the use of approved vaccines. Government has a role in negotiating the certificates with the third country authorities only, while for EU trade the certificates and trade rules are harmonised. As the decision to trade depends on the producer, then any costs of preexport testing or vaccination lie with them and should not rest with the tax payer.

For BTV, the EU and national governments harmonised the trade requirements such that vaccination is allowed and pre-export testing provides additional guarantees that animals are safe to be traded. Meat and milk products do not come under any trade restrictions as they are not considered risk commodities. Approved vaccines for the most commonly detected virus strains were produced by several companies and their use for trade was included in health certificates.

For SBV, EU and national governments worked together to ensure that trade restrictions were not put in place on countries reporting disease. Part of that assurance for trade partners was about providing data on the disease impact and spread through the European Food Safety Authority (Afonso et al. 2012).

For AHS, there have been no outbreaks in Europe since 1987-1990, when the last cases of AHS in Spain and Portugal occurred (as a result of imported infected zebras from Namibia). For other countries in Africa, where disease occurs, there is no trade in Equidae to the EU unless the animals move to another country first, which is free of AHS, for a period of residency (known as triangulation). In South Africa, where the export of horses is considered important enough for government to take action, regionalisation was adopted in 1997. A small area in Cape Town is the AHS free area and no Equidae can enter unless they have been vaccinated annually against AHS. Regular surveillance is carried out and outside this area there is a protection zone and a surveillance zone, where again all Equidae must be vaccinated annually and movements of equines to the AHS controlled area are subjected to strict movement control and require certification and permits. However, this is currently suspended as outbreaks have in the last two years been detected in the protection zone, through the illegal movement of unvaccinated horses.

SBV was an emerging disease in 2010/2011 and governments across Europe had to take decisions about how much intervention was possible, necessary and should be provided by government as opposed to the producer. If a disease is notifiable the livestock keeper must immediately notify the veterinary authorities if there is suspicion of disease observed in livestock. This can have a serious impact on the individual producer as the farm will usually be placed under movement restrictions until disease can be ruled out, if necessary through laboratory tests. This is primarily to prevent undetected spread during the high risk period. But with SBV, it was recognised that clinical signs in adult animals were so mild and transient it was unlikely a farmer would suspect disease; suspicion only occurred when birth defects occurred in new born or aborted animals several weeks later. Secondly, as infection had occurred earlier in the year, restrictions on animals at that point were unnecessary. Finally, diagnostic tests were in their infancy and at that point there was no serology test therefore historical infection could not be ruled out. A vaccine was produced in a relatively short time so livestock producers could make the decision whether they 
were at risk and if vaccination made economic sense on the type of farm they were running. As SBV is not notifiable, there is no requirement for stamping out or declaring disease freedom. We expect SBV will become a seasonal (although not necessarily annual) issue in Northern Europe, but the economic losses for farmers were not long term and could be avoided through careful timing of breeding animals by staggering mating times (http://tinyurl.com/yb9pah9m). Government continues to provide testing facilities and information to farmers about SBV, but not to provide financial aid when disease is reported.

When a disease become endemic and a decision is taken to not control or eradicate the disease, the economic costs may shift away from immediate losses in a naïve population such as mortality and morbidity towards longer term fertility problems leading to changes in herd structure and adoption of different breeding strategies or technology (Rushton and Lyons 2015). It is difficult to assess how BTV endemicity would impact on the livestock in Northern Europe. The recent reemergence of disease in France suggests the BTV-8 virus has little clinical impact on the cattle affected, as so few animals have been reported with clinical disease: just 3,330 positive cases, 7 deaths out of $\sim 360,000$ susceptible cattle and just 39 positive sheep (OIE 2017), but the requirement for vaccination to move and trade animals means the government has procured enough vaccine for around 8.5 million cattle and 4.5 million sheep, while the costs of sentinel surveillance in animals and vectors across the whole mainland region are unknown but together with vaccination costs will probably outweigh any cost resulting from the direct impact of disease (Ministère de I'Agriculture et d'Alimentation 2017; Pinior et al. 2015). It remains to be seen whether this same cost benefit would be observed if disease spread into other regions with a large proportion of naïve animals, which highlights the complexities around determining government action.

\section{Conclusion}

There are many models which are used to measure economic costs of disease control measures whether for cost benefit analysis or cost effectiveness analysis. The area where data are sparse and therefore where decisions are difficult to make, is around understanding the impact of disease on production, changing markets and farmer behaviour as well as externalities, either positive or negative. Cost sharing where disease in one region can impact on another region, may be interesting to consider with respect to transboundary diseases such as the vector borne diseases. Cost effectiveness or CBA offers a consistent framework for analysis; however, there is often a lot of uncertainty which can lead to ambiguity; risk appetite of the decision maker matters.

Comparing the different control measures for two vector borne diseases of ruminants in Europe is an interesting contrast. One (SBV) is a disease where vaccination is possible and has been relatively effective in controlling disease but the risk remains through seasonal virus circulation the (rare) serious impacts of which can be managed by the farmer. The other (BTV) is a disease with variable impacts in livestock, multiple strains with no cross-protection between strains where either vaccine-induced or natural immunity is present. AHS on the other hand would have a heavy impact on a relatively small sector of society (albeit one with high economic profile - horse racing and competition horses) but where there are limited opportunities for control and a very small risk of disease occurring in the EU.

The difficulties of determining government action in the face of an emerging disease, with unknown financial consequences are substantial and may result in long delays in decision making. We may believe we have good evidence to help us predict the impact of known diseases such as BTV and AHS, nevertheless government and experts can underestimate unseen indirect 
economic impacts. Proportionality is key and therefore this remains an area where economists and veterinarians should continue to work together and understand one another's disciplines to improve this important area of animal health economics.

\section{References}

Afonso A, Cortinas Abrahantes J, Conraths F, Veldhuis A, Elbers A, Roberts H, Van der Stede Y, Méroc E, Gache K and Richardson J (2014) The Schmallenberg Virus epidemic in Europe - 2011-2013. Prev Vet Med 116: 391-403.

British Horse Society (2015) Guide to African horse sickness. Available at: https://tinyurl.com/jp7r6kr.

Brooks $L$ (2010) A model of the economic impact of African horse sickness to the equine breeding industry in South Africa. Available at: https://tinyurl.com/y8cz597c.

Carpenter S, Mellor PS, Fall AG, Garros C and Venter GJ (2017) African horse sickness virus: history, transmission, and current status. Ann Rev Entomol 62: 343-358.

Ensoy C, Aerts M, Welby S, Van de Stede Y and Faes C (2013) A dynamic spatio-temporal model to investigate the effect of cattle movements on the spread of bluetongue BTV-8 in Belgium. PLOS ONE 8: e78591.

European Commission (EC) (2001) Council Directive 2001/89/EC of 23 October 2001 on Community measures for the control of classical swine fever. OJ L 316: 5-35.

European Commission (EC) (2003) Council Directive 2003/85/EC of 29 September 2003 on Community measures for the control of foot-and-mouth disease repealing Directive 85/511/EEC and Decisions 89/531/EEC and 91/665/EEC and amending Directive 92/46/EEC. OJ L 306: 1-87.

European Commission (EC) (2006) Council Directive 2005/94/EC of 20 December 2005 on Community measures for the control of avian influenza and repealing Directive 92/40/EEC. OJ L 10: 16-65.

European Commission (EC) (2007) Commission Regulation (EC) No 1266/2007 of 26 October 2007 on implementing rules for Council Directive 2000/75/EC as regards the control, monitoring, surveillance and restrictions on movements of certain animals of susceptible species in relation to bluetongue. OJ L 283: 37-52.

European Commission (EC) (2012) Commission Implementing Decision 2012/349/EU concerning a financial contribution by the Union to Belgium, Germany, Spain, France, Italy, the Netherlands and the United Kingdom for studies on Schmallenberg virus. OJ L 173: 12-22.

Food and Agriculture Organization of the United Nations (FAO) (2016) Economic analysis of animal diseases. FAO animal production guidelines No 18. FAO, Rome, Italy.

Gubbins S, Carpenter S, Baylis M, Wood JL and Mellor PS (2008) Assessing the risk of bluetongue to UK livestock: uncertainty and sensitivity analysis of a temperature-dependent model for the basic reproduction number. JR Soc Interface 5: 363-371.

Ministère de l'Agriculture et d'Alimentation (2017) Questions - réponses sur les cas de fièvre catarrhale ovine détectés en 2015. Available at: https://tinyurl.com/ycz32kxg.

Nixon EJ, Vineer HR and Wall R (2017) Treatment strategies for sheep scab: an economic model of farmer behaviour. Prev Vet Med 137: 43-51.

Oxford Analytica (2012) The costs of animal disease. A report produced for the International Federation for Animal Health. https://tinyurl.com/y8z6eov2.

Pinior B, Brugger K, Kofer J, Schwermer S, Loitsch A and Robel F (2015) Economic comparison of the monitoring programmes for bluetongue vectors in Austria and Switzerland. Veterinary Record 176: 464.

Roberts H, Carbon M, Hartley M and Sabirovic M (2011) Assessing the risk of disease introduction in imports. Vet Rec 168: 447-448.

Roberts HC, Elbers ARW, Conraths FJ, Holsteg M, Hoereth-Boentgen D, Gethmann J and Van Schaik G (2014) Response to an emerging vector-borne disease: surveillance and preparedness for Schmallenberg Virus. Prev Vet Med 116: 341-349.

Rushton J and Lyon N (2015) Economic impact of Bluetongue: a review of the impacts on production. Vet Ital 51:401-406.

Saatkamp HW, Mourits MCM and Howe KS (2014) A framework for categorization of the economic impacts of outbreaks of highly contagious livestock diseases. Transbound Emerg Dis 63: 422-434. 
Sumner T, Burgin L, Gloster J and Gubbins S (2013) Comparison of pre-emptive and reactive strategies to control an incursion of Bluetongue virus serotype 1 to Great Britain by vaccination. Epidemiol Infect 141: 102-114.

Tago D, Hammitt JK, Thomas A and Raboisson D (2014) Cost assessment of the movement restriction policy in France during the 2006 bluetongue virus episode (BTV-8). Prev Vet Med 117: 577-589.

Turner J, Bowers RG and Baylis M (2012) Modelling Bluetongue virus transmission between farms using animal and vector movements. Sci Rep 2: 319.

UK Department for Environment, Food and Rural Affairs (DEFRA) (2008) African horse sickness: potential risk factors and the likelihood for the introduction of the disease to the United Kingdom. Available at: https://tinyurl.com/y7ar2tj9.

UK Department for Environment, Food and Rural Affairs (DEFRA) (2016) Risk assessment for Bluetongue Virus (BTV-8): risk assessment of entry into the United Kingdom. Available at: https://tinyurl.com/yawdof54.

Velthuis AGJ, Saatkamp HW, Mourits MCM., De Koeijer AA and Elbers ARW (2010) Financial consequences of the Dutch bluetongue serotype 8 epidemics of 2006 and 2007. Prev Vet Med 93: 294-304.

Welby S, Van Schaik G, Veldhuis A, Brouwer-Middelesch H, Peroz C, Santman-Berends IM, Fourichon C, Wever P and Van der Stede $Y$ (2016) Effectiveness and cost efficiency of different surveillance components for proving freedom and early detection of disease: Bluetongue serotype 8 in cattle as case study for Belgium, France and the Netherlands. Transbound Emerg Dis 64: 1771-1781.

White S, Saners CJ, Shortall CR and Purse BV (2017) Mechanistic model for predicting the seasonal abundance of Culicoides biting midges and the impacts of insecticide control. Parasit Vectors 10: 162.

World Animal Health Organisation (OIE) (2017) Bluetongue in France. Follow-up report No 78. Available at: https:// tinyurl.com/yat39ddq. 


\title{
18. Controlling tsetse - what does it cost?
}

\begin{abstract}
Alexandra P.M. Shaw
Division of Infection and Pathway Medicine, School of Biomedical Sciences, Edinburgh Medical School, College of Medicine and Veterinary Medicine, The University of Edinburgh, 1 George Square, Edinburgh EH8 9JZ, United Kingdom; A P Consultants, 22 Walworth Enterprise Centre, Duke Close, Andover, SP10 5AP, United Kingdom; alex@apconsultants.co.uk
\end{abstract}

\begin{abstract}
The costs of currently used methods to control tsetse are reviewed. Three methods: stationary baits (insecticide-treated traps, targets with or without odour baits, screens and fences), mobile baits (insecticide-treated cattle using spraying, pour-ons, footbaths or dips) and aerial spraying (sequential aerosol technique applied from fixed wing aircraft) were studied, using current published information and historical information from 1990 onwards. For comparability all data were converted to 2016 United States dollars (USD). Cost calculation methodologies are analysed and best practice approaches for full cost calculation and data collection are suggested. Despite the approximations and assumptions involved in converting historic costs to 2016 price levels, the accumulated evidence since 1990 shows remarkably consistent results. Traps, currently principally used for tsetse monitoring, cost USD 7-10 each, tiny targets, for use against riverine tsetse flies, USD 1.5-4.0 depending on size and including fixings and supports, whereas the larger traditional targets cost USD 15-20. Adding the cost of delivery, deploying the larger targets at 4 per $\mathrm{km}^{2}$ costs USD 200-300 per $\mathrm{km}^{2}$ whereas the tiny targets cost under USD 100 per $\mathrm{km}^{2}$ per year. For insecticide-treated cattle using dips or footbaths, costs ranged from about USD 0.15-0.35 per bovine per treatment, including insecticide and an allowance for dip/footbath depreciation and paid manpower. Pour-ons cost about USD 0.50 per treatment for the insecticide and an applicator, where delivery is included the cost roughly doubles. Spraying cattle monthly, using the restricted application protocol, costs USD 7 per year per bovine including delivery. Aerial spraying, for 5 cycles, costs USD 500-600 per km² when applied on a large scale. These costs are briefly discussed in relation to likely benefits. In most circumstances targets or insecticide-treated cattle provide sufficiently high returns for traditional livestock keepers largely to fund control themselves. Encouraging uptake, through better communication and some financial and entomological support, remains the main challenge.
\end{abstract}

Keywords: aerial spraying, cost, fences, insecticide-treated cattle, targets, traps, tsetse control, screens

\section{Introduction}

The study of the economics of trypanosomosis and tsetse control began in the 1950s with cost analyses of tsetse control schemes (Shaw 2009). Thus, Wilson (1953) reported on the costs of bush clearing and DDT spraying of fringing vegetation against Glossina fuscipes fuscipes Newstead. Davies $(1964,1971)$ reported on the use of ground spraying with DDT to clear tsetse along the watercourses of north-eastern Nigeria's Lake Chad basin. Jahnke (1974) produced the first published account of the benefits and costs of tsetse control, looking at ground spraying and the, at the time, cheaper alternative of game elimination in Uganda. The use of residual insecticides, bush clearance and even more so game elimination, as methods of tsetse control have long been abandoned in favour of more environmentally benign approaches. The methods in current use can be grouped into four categories: 
1. Stationary baits - targets, traps, screens and fences made of insecticide-impregnated blue or black cloth and netting and placed in tsetse habitats where they attract tsetse visually (with the help of odour baits for morsitans group flies, and animal odour in the case of fences around livestock pens) and the flies pick up a lethal dose of insecticide. The acronym ITT for insecticide-treated targets/traps will be used here for all stationary baits.

2. Mobile baits - insecticide-treated cattle (ITC) in the form of pour-ons, by spraying, dipping or footbaths - which may apply insecticide to the whole of the animal or part (using the restricted application protocol; RAP).

3. Aerial spraying - which is undertaken by fixed wing aircraft using the sequential aerosol technique (SAT).

4. Sterile insect technique (SIT) - in which sterile male tsetse are released from aircraft to outcompete wild male tsetse in a population which has been reduced using other techniques.

Comprehensive summaries of these methods and how they are applied can be found in various book chapters (e.g. Allsopp and Hursey 2004; Feldmann 2004; Gimonneau et al. 2018; Vale and Torr 2004; Van den Bossche and De Deken 2004) as well as published journal articles. Those relating to newer techniques are cited as relevant in the text. The remit of the present chapter is to focus on the options for ongoing tsetse control activities which are applied continuously for a period of time and which aim to reduce transmission and thus the incidence of trypanosomosis in people and animals, and thus does not include SIT.

The challenge for today's decision-makers in an Africa where veterinary services have been to some extent privatised and many of the government tsetse control departments absorbed into other organisational units, is to find ways of delivering or facilitating tsetse control to livestock keepers and those dealing with human African trypanosomosis (HAT). In order to do this effectively, coherent and reliable estimates of tsetse control costs are an essential prerequisite.

\section{Cost calculation methods}

Clearly there are major differences in the methods of calculating costs as well as variations from country to country Jordan (1986)

The quote above from the classic text by Jordan (1986) expressed his frustration at the difficulties in comparing costs from different sources and places. This continues to pose problems. This section seeks to analyse some of these and to illustrate what the options are for producing more standardised and comprehensive costings.

There are two main reasons for the different approaches used. The first is essentially administrative. As Tibayrenc and Gruvel (1977) noted: 'the cost of treatment per unit of surface area is most often calculated using only the elements that feature directly in the budget line (insecticide, staff payments, vehicle running) at the time of the intervention. The more remote cost components (vehicle purchases, construction of premises or lodging, etc.) are not taken into account when a tsetse control activity falls under a permanent government service and not a temporary body set up for a few years'. The second is that much of the published work presents the results of scientific research which aimed to produce cheaper devices (such as traps or targets) or to find ways of reducing the amount of insecticide used, etc. and thus focuses on the cost of the devices, insecticide, etc. rather than on the cost of delivery, project preparation and overall project administration. 


\section{Terminology}

The classic economic terminology distinguishes between fixed and variable costs. Variable costs increase with the volume of production and are zero if nothing is produced, whereas fixed costs, often described as overheads, remain constant unless there is a major increase in production and can still be incurred if nothing is produced. Some costs are highly variable (e.g. targets, their odour baits and cloth) others less so (number of days in the field and associated travel costs and staff allowances) and others relatively fixed (headquarter supervision and administration costs). Other terms are also often found in the literature. The terms marginal and variable are often used interchangeably. However, the term marginal cost in economics has a very specific meaning, referring to the extra cost of producing an extra unit of output, as compared to the average cost (total fixed and variable costs divided by the total amount produced). The concept of marginal costs is an important one when costs are compared either to monetary benefits or to a measure of effectiveness (e.g. people protected, $\mathrm{km}^{2}$ treated, cattle sprayed). In such cases the 'incremental' cost (overall extra costs in relation to the extra units of impact due to a change, such as an improved intervention) is usually calculated. The word 'incremental' has a specific meaning in economics, however, again in everyday language the 'incremental' costs of a project are often deemed to be those additional budget lines which are added when it is implemented. Another commonly used distinction is between 'operational' or 'running' costs and total costs. These also tend to refer to specific budget lines and exclude capital items.

The categorisation of costs is further complicated by a tendency to use the terms 'direct' and 'indirect' indiscriminately. To date no real consensus exists in either the animal health or the entomological field. This is in contrast to the human health sector where there are generally accepted cost categories (Meltzer 2001). In tsetse control, usually 'direct' costs are considered to be the cost of the core items associated with each control technique: traps, targets, drugs, insecticide and flying time, sometimes they refer to all field costs, sometimes to a project's budget line. Indirect has been used refer to administrative costs, entomological surveys and to additional research activities. However, often 'indirect' can become a euphemism for cost categories that are difficult to quantify.

Figure 1 lists the main costs components of tsetse control activities, in approximate order of 'variableness' or 'directness'. In fact, there is a continuum, and it is difficult to draw a clear dividing line. Thus, pending a consensus on terminology, in an international and multi-organisational context some of these terms are probably best avoided as they mean different things to different constituencies.

\section{A full cost economic model for tsetse control}

In Table 1 a practical model for categorisation and calculation of costs is proposed. This is based on three distinctions:

1. the difference between a 'financial 'or 'budgeting' approach, which looks at the costs accruing to particular stakeholders (government, livestock keepers and local communities, researchers, donors) and an 'economic' approach which aims to combine all the costs to derive the total 'societal' cost of the activity;

2. the difference between capital items, which are durable i.e. can be used for more than a year and recurrent cost items which have to be replaced annually or more often;

3. the difference between specialised items, which are only used for tsetse control and other items, which may be shared with other activities. 


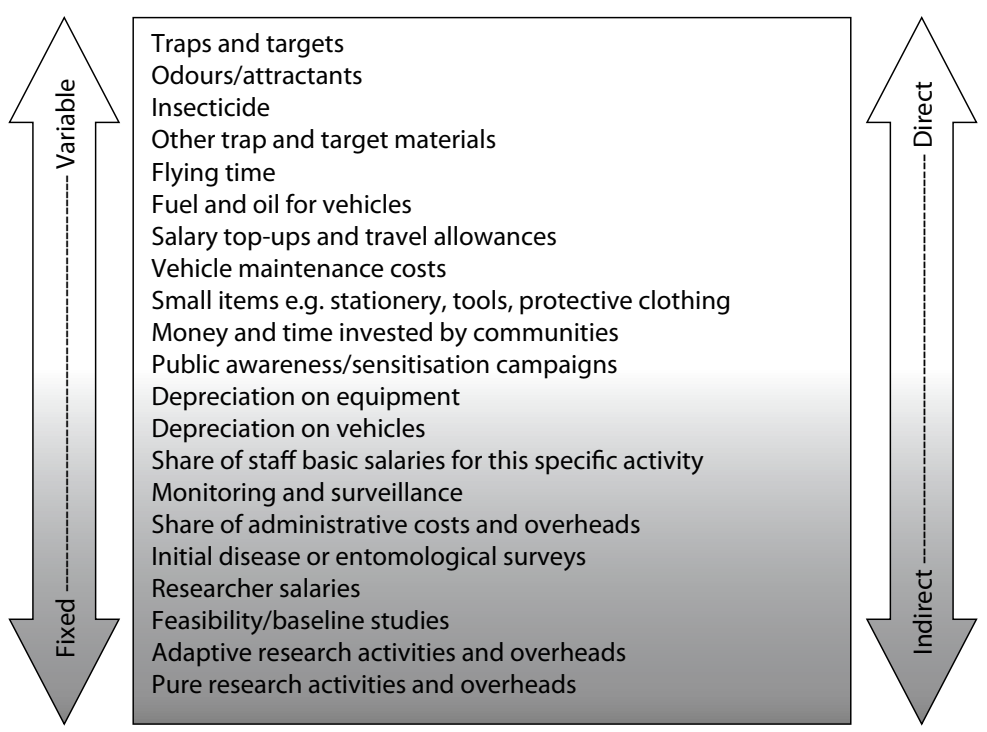

Figure 1. Tsetse control cost components: a continuum from the most variable/direct to the most fixed/indirect.

\section{Data collection}

Collection of cost data in the form of project expenditure is a requirement for most activities. This necessarily focuses on budget lines - what the individual stakeholder has had to spend. However, to calculate the full economic costs of tsetse control, and thus to provide a valid statistic for project planning and economic decision-making, data collection has to go beyond this. As the scientific component of such activities usually already involves very detailed data collection, for example on fly catches, this should not add appreciably to the project workload. The key thing for success is to collect information for every outing and intervention the time it is undertaken. The main components are as follows:

- Identification of vehicles used, kilometres travelled and associated vehicle running costs whether or not paid for directly by the activity.

- Listing all individuals contributing time, whether preparing for field work or during field work, to include not just full time staff, their allowances and salaries, but also temporary workers and, importantly members of local communities who may be paid, or whose time inputs should be noted and ultimately assigned a notional value equivalent to local labour cost.

- Recording all durable equipment used, whether directly bought by the project, or shared with other activities, as well as its current replacement cost and an estimation of how many years of useful life are expected, so that depreciation can be estimated.

- Recording all consumable items used, whether directly bought by the project, or shared with other activities. 


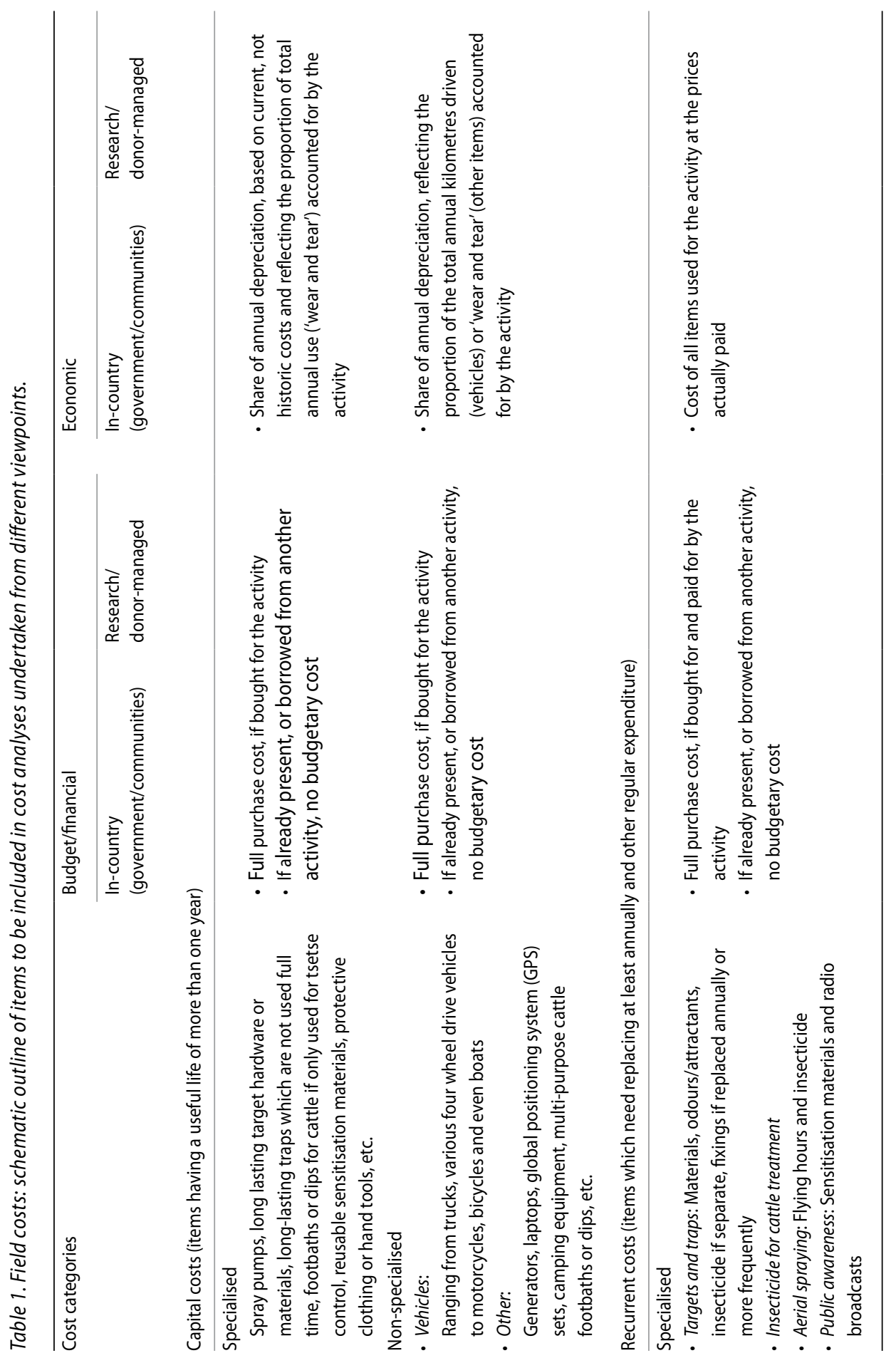




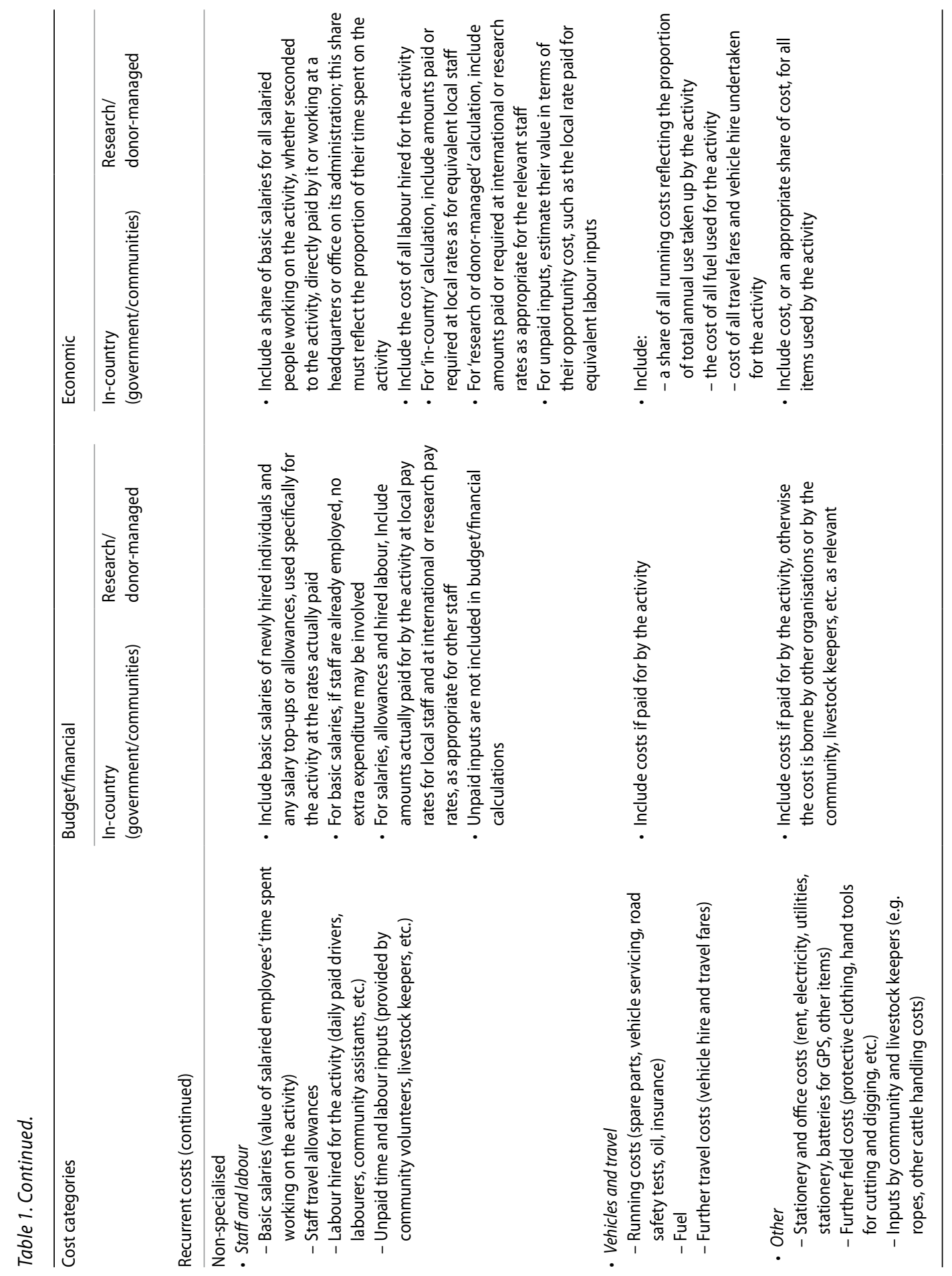




\section{Review of published cost data}

\section{Methodology}

As stated in the introduction, interest in the economics of trypanosomosis and tsetse control began with the collection and reporting of cost data. Having outlined the options for presenting and calculating costs, and the type of data that needs to be collected to underpin cost calculations, the next section goes on to look at the data that has accumulated. As well as the historical studies mentioned (Davies 1964, 1971; Jahnke 1974; Tibayrenc and Gruvel, 1977; Wilson 1953) two later studies provide a detailed analysis of different methods of tsetse control, based on actual field data. The first covered ground spraying and helicopter aerial spraying in Nigeria (Putt et al. 1980) and the second trapping in Côte d'Ivoire, aerial spraying in Nigeria and Cameroon, while focussing on the SIT in Burkina Faso (Brandl 1988).

The tables below (Tables 2-8) go on to analyse the more recent main published sources of such information, taking the year 1990 as an approximate cut-off date. Three sources provide detailed information which covers multiple schemes in some detail. First and foremost, Barrett (1997) undertook what remains the most detailed and comprehensive field-based study of different techniques for controlling or eliminating tsetse, covering ground-spraying, aerial spraying with fixed wing aircraft, targets and a range of options using ITC. This study analysed not only average costs, but also a range of project sizes, different intensities of control and different terrains. Secondly, the chapter by Allsopp and Hursey (2004) provides a collection of published costs for ground spraying, aerial spraying, targets and dipping cattle, from Botswana, Kenya, Nigeria, Uganda, Zambia and Zimbabwe. Lastly, both a template for full economic cost calculation and updated costs for a range of interventions were produced by Shaw et al. (2007, 2013a, 2015b) based on Ugandan price levels. In addition to these multiple technique cost studies, a selection of key papers were consulted to add to the range of schemes analysed for each technique.

In each case, the historic costs given at the time of publication were converted to United States dollars (USD) at the rate applicable at the time the costs were collected and calculated (usually a year or more earlier than the publication date). The figures were then converted to 2016 prices using historic USA inflation rates (http://tinyurl.com/ydbt6gh7). This method provides consistent results, with a low USA inflation rate being set off against generally falling exchange rates against the USD. Importantly, it also maintains the relationships between cost items which applied at the time the data was collected. The disadvantages are that the prices of individual cost components may have changed at different rates (for example local labour remaining at a very low price while imported items have become relatively more expensive) and that some historical official exchange rates overstated the value of their currencies (e.g. it was thought that the CFA franc was somewhat overvalued prior to devaluation in 1994).

The costs are thus those calculated in the studies cited, so they are at local, in-country, market prices at the time the study was undertaken. These have always been the prices cited for tsetse control. The alternative of converting all costs to their 'purchase power parity' levels by using so-called 'international prices' has not been considered here, partly because for the historic costs the data to support this would be very difficult to extract, but primarily because within the community of researchers, implementers and decision-makers in the field of tsetse and trypanosomosis control, national market prices have always been used. These are transparent, easily interpreted and cost components can be recalibrated for different prices, ecological situations or administrative structures. 
Where costs are expressed per $\mathrm{km}^{2}$, unless stated otherwise, this refers to what is usually described as the 'area protected' or for some schemes to control animal African trypanosomosis (AAT), the 'grazing area' made safe. This is in contrast to the area 'treated', which especially for palpalis group (riverine) tsetse, usually consists of the fringing vegetation along water courses. This area is difficult to measure and citing the density of traps or targets in a treated area can give a misleading impression of overall trap requirements. For example, in Côte d'Ivoire, when controlling sleeping sickness in the forest zone, the trap density was 25 per $\mathrm{km}^{2}$ protected, as against 130 per $\mathrm{km}^{2}$ treated (Laveissière et al. 1994).

\section{Devices and doses}

As discussed above, researchers have often focussed on the 'core' cost of devices or approaches that they have developed or improved. Tables 2, 3, and 4 give a selection of field-based figures for these core (direct/variable/incremental/marginal costs) that everyone agrees have to be costed and included. These core costs also represent the cutting edge of research - the basic unit for measuring efficacy and effectiveness.

\section{Traps, targets, screens and fences}

Looking at Table 2, it is noticeable that costs for traps have not changed that much over time. At 2016 prices, traps used without odours or insecticide mostly fall into the USD 7-10 price range. In the cited studies these were used either to control palpalis group flies, or more often, just for entomological surveys and monitoring. The Ngu trap, however, was developed for use in an area with a morsitans and a fusca group fly (Glossina pallidipes Austen and Glossina longipennis Corti). Including added odour but using no insecticide, it would cost over USD 15 at current prices, while being significantly cheaper than the targets in use at the time.

For targets the price range is greater. The classic large targets developed in Zimbabwe and recently deployed in Ghana come out at USD 17 in Ghana, and USD 32.50 in Zimbabwe. The latter includes depreciation of the metal supporting frames and is possibly a figure slightly exaggerated by the exchange rate conversion and inflation calculations. At about USD 7, the much smaller screens used to control HAT in Côte d'Ivoire, which could be hung up as well as stood in the ground, were cheaper.

Since then, one major change is, of course, the availability of insecticide-impregnated netting and cloth, which has markedly reduced costs because it is not necessary to carry out re-impregnation visits to maintain the devices. The second is the development of a new generation of 'tiny targets.' For areas where only palpalis group flies are present, the very small targets currently being deployed in Uganda cost only USD 1.50 in-country including wooden supports and ties, the slightly larger ones being used in Guinea at around USD 3.5-4.00 if freight and fixings are included. When used against subspecies of Glossina fuscipes they do not require odours, however, odours have sometimes been added when deployed against Glossina palpalis gambiensis Vanderplank. Lastly, the use of insecticide-impregnated fencing to protect livestock, for example pigs or zerograzed cattle, provides another option. Definitive costs are not yet available, although an incountry price of USD 1.50 per $\mathrm{m}^{2}$ of insecticide-impregnated netting is likely. The amount of netting per unit could bring the cost up to USD 50, however it should be kept in mind that such units would impact on tsetse populations in the vicinity and thus act like a large target - and require deployment at similar densities to achieve a similar effect. 


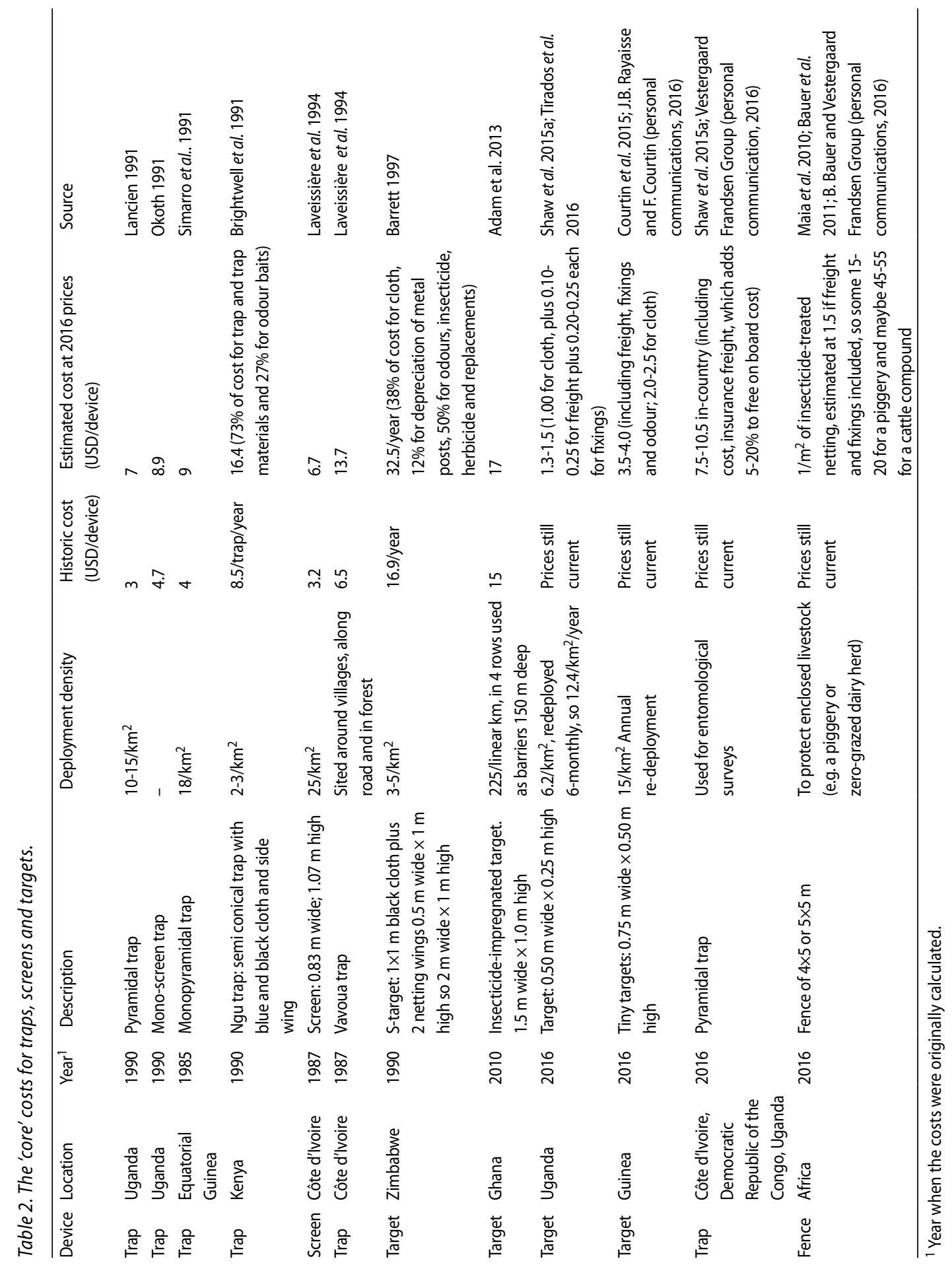




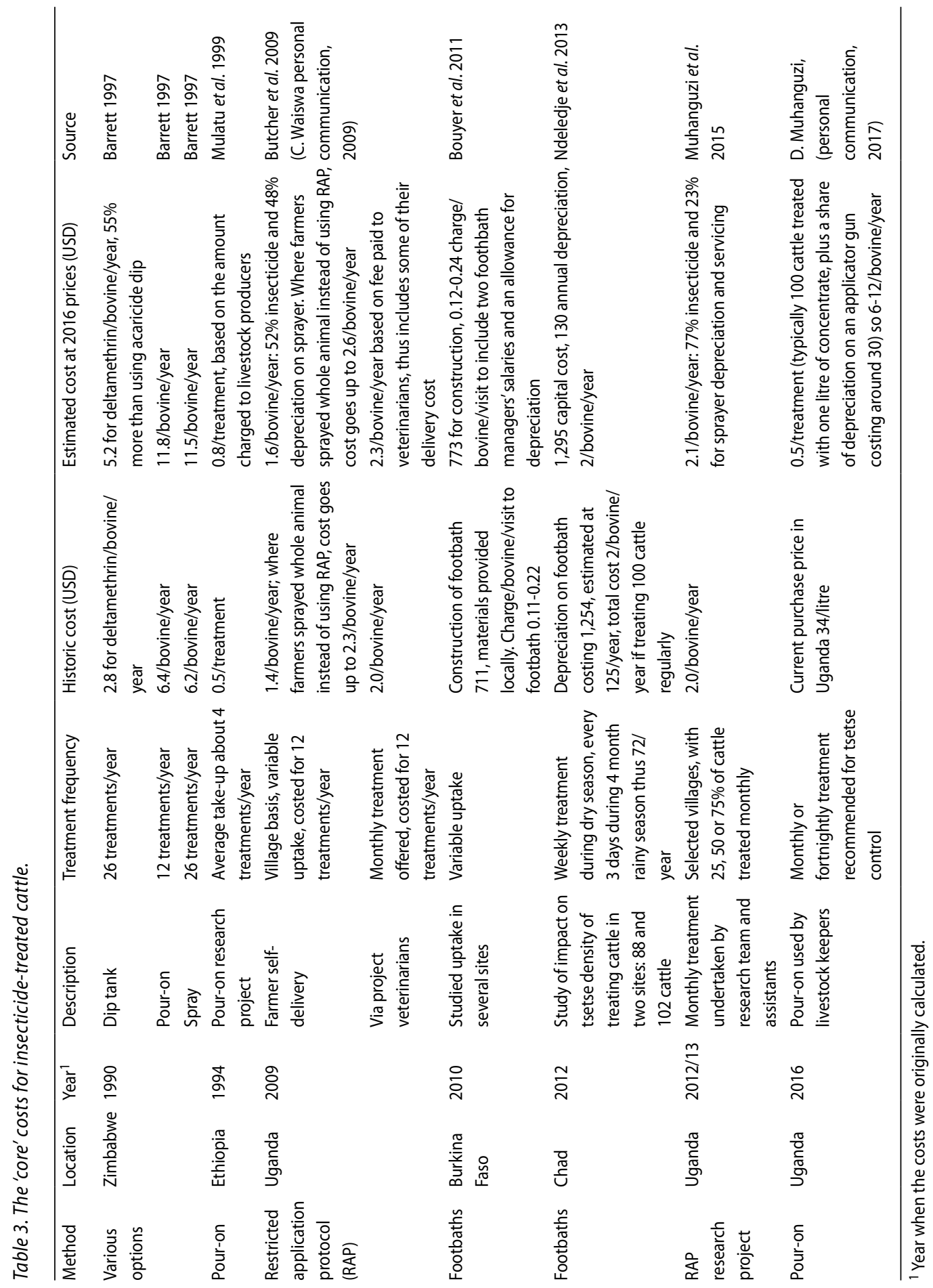




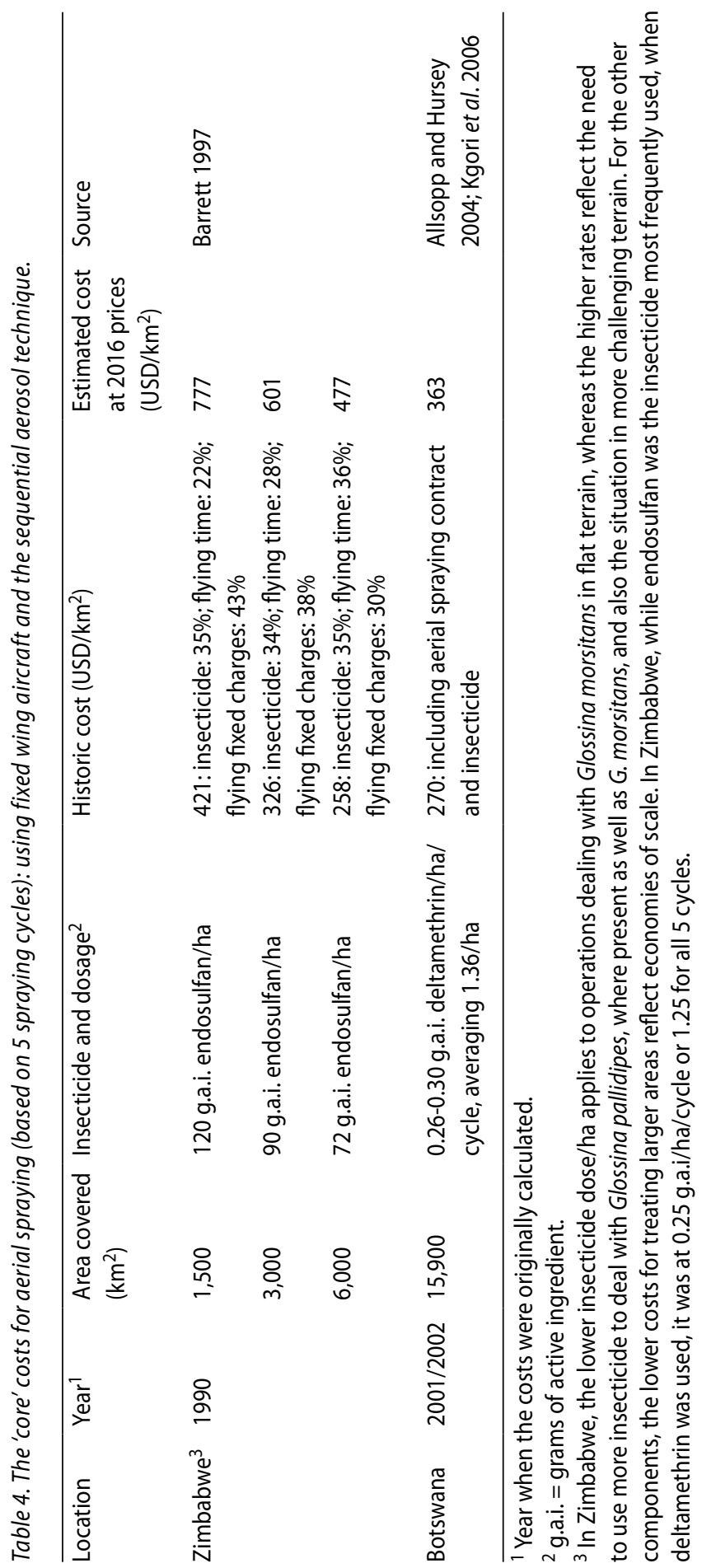




\section{Insecticide-treated cattle}

Turning to ITC, Table 3 gives costs for the core items (insecticide, spray pump and dip tank) for various methods for applying the insecticide. Again, the costs are surprisingly consistent over time. Pour-on costs for 12 applications a year ranged from USD 6-12.The cost of spraying with insecticide appears relatively more costly in Zimbabwe; it would work out at USD 5.3 for 12 applications although undertaken at a higher frequency in practice. In Uganda, two studies were undertaken analysing the cost of using the RAP which requires as little as $10 \%$ of the insecticide used for full body spraying (Torr et al. 2007). Butcher (2009) looked at the costs of RAP to farmers either delivered by private veterinarians for a fixed fee, costing USD 2.3 per year for 12 applications, or by the farmers themselves purchasing insecticide and a sprayer, costing USD 1.6 per year, with the difference largely accounted for by the fact that the veterinarian's fee included some of their delivery cost. As part of large research project, Muhanguzi et al. (2015) investigated the impact of RAP applied to different proportions of village cattle populations. Over the whole study, the cost of insecticide and sprayer servicing and depreciation came out at just over USD 2 per bovine per year for 12 applications.

The cost of dipping with deltamethrin to control both tsetse and ticks was investigated in Zimbabwe, which in the 1980s and early 1990s had a functioning network of dip tanks which were visited by cattle owners every two weeks to dip their herd to control tick-borne diseases. The cost for 26 applications would be USD 5.2, an additional cost of USD 3.4 over the cost of the acaricides normally used.

Another option for applying insecticide is via footbaths. In Burkina Faso livestock keepers were charged USD 0.12-0.24 per visit, which covered the recorded insecticide consumption, estimated footbath depreciation and the footbath managers' wages and thus is close to being a full cost. In Chad the estimated cost for the insecticide and footbath depreciation only was appreciably lower, amounting to USD 2 per bovine per year if treating a herd of 100 head, but for a far higher treatment frequency, 72 applications a year, designed to stop ticks attaching themselves to cattle, particularly during the rainy season. Thus, on the basis of core costs alone, dipping or footbaths appear cheapest, followed by the RAP spraying.

\section{Aerial spraying}

For aerial spraying, using fixed wing aircraft and the SAT an analysis looking at the impact of scale was conducted in Zimbabwe (Table 4). This showed the price increasing from USD 477 for a large area of $6,000 \mathrm{~km}^{2}$ to USD 777 for an area of $1,500 \mathrm{~km}^{2}$. The difference reflected the impact of different doses of insecticide and the spreading of the fixed (as against flying hour) charges levied by the aerial spraying contractor. The higher dose of insecticide also reflected the requirements for dealing with more than one tsetse species in more difficult terrain (see footnote to Table 3 ). For the core items of insecticide and aerial spraying, the estimated cost of the operation in Botswana's Okavango delta came to USD 363 per km², a lower cost which may reflect scale as well as a tendency for the long term conversion from historic costs to current costs for Zimbabwe to err on the high side, as was the case for targets.

\section{Delivery}

The picture becomes more complex once delivery, which here is taken to mean all the additional costs required to get the core items/technology in place, is taken into account. Relatively few 
published costings take into account all the components required for a full cost estimation from an economic point of view (as defined in Table 1). Again the key studies published from 1990 onwards, which have cited more than just the costs of core items, are reviewed in Tables 5, 6 and 7. These mostly focus on field costs. Recently there have been a number of studies which have covered the full costs for tsetse control (Muhanguzi et al. 2015; Shaw et al. 2013a, 2015a, 2015b). Of these Muhanguzi et al. (2015) and Shaw et al. (2015a) are based on implemented work and careful field data collection and they represent recent and low cost options for ITC and ITT, so they are analysed in more depth in Table 8.

\section{Traps, targets and screens}

Looking at the costs presented in Table 5, these fall into several categories. The costs for traps in Uganda, and full size targets in Zimbabwe, Botswana and Uganda mostly fall in the range of USD 200-300 per km². As presented, these costs include most field level delivery costs, but not all administrative overheads, as detailed in the comments column in each case. In each case the core cost (trap/targets, insecticide and odours) accounts for $30-50 \%$ of the total field cost. The similarity between the Zimbabwe and Uganda costings in particular reflects the fact that the latter were based in part on estimates of the African Development Bank, International Atomic Energy Agency and Pan-African Tsetse and Trypanosomosis Eradication Campaign (ADB/IAEA/ PATTEC 2004), which also used information from Barrett (1997).

The Côte d'Ivoire programme (Shaw et al. 1994) was conducted on a very large scale, and costs were kept down by not servicing traps during the rainy season, simply uplifting and replacing them once a year. Trap density was very low at 0.3 per $\mathrm{km}^{2}$ compared to $4-10 \mathrm{~km}^{2}$ for the other interventions, which was reflected in achieving good control over Glossina tachinoides Westwood, but less consistently so for Glossina palpalis gambiensis Vanderplank (Rowlands et al. 1996). This and the fact that this is a fully comprehensive calculation, with all administrative and overseas costs included, explains the low share (17\%) of core costs in the total whereas the low overall cost of USD 45 per $\mathrm{km}^{2}$ reflects the low deployment density and single annual visit for replacement. The slightly high cost for Zimbabwe, as mentioned before, may reflect the fact that the exchange rate conversion rate and inflation adjustments tended to exaggerate the amount calculated at 2016 prices. It also reflects a relatively intensive operation, with at least one maintenance visit a year. The areas were often relatively isolated, with denser vegetation, requiring access provision, maintaining access and construction of camps, which accounted for $14 \%$ of total costs.

Turning to the Côte d'Ivoire screens, despite the much higher density, the cost is similar to the other schemes, as $92 \%$ of the costs are core costs, with some overheads not being included. The source, Laveissière et al. (1994) also includes a very informative chapter on costs, with a detailed breakdown of all the components taken into account. Lastly, the cost of the tiny target scheme in Uganda worked out at just under USD 80 per $\mathrm{km}^{2}$, excluding administrative support and USD 88 per $\mathrm{km}^{2}$ if this is included.

\section{Insecticide-treated cattle}

The field level costs for various types of ITC are given in Table 6. To provide some comparability with the per $\mathrm{km}^{2}$ figures, these are calibrated for 10 cattle being treated a year at the frequency used in the scheme described. The cost per treatment is provided in brackets. The first set of results, the estimates for Zimbabwe, follow on from the core costs itemised in Table 3. The cheapest option is dipping, where $58 \%$ of costs are core costs, and the cost of dip and water 


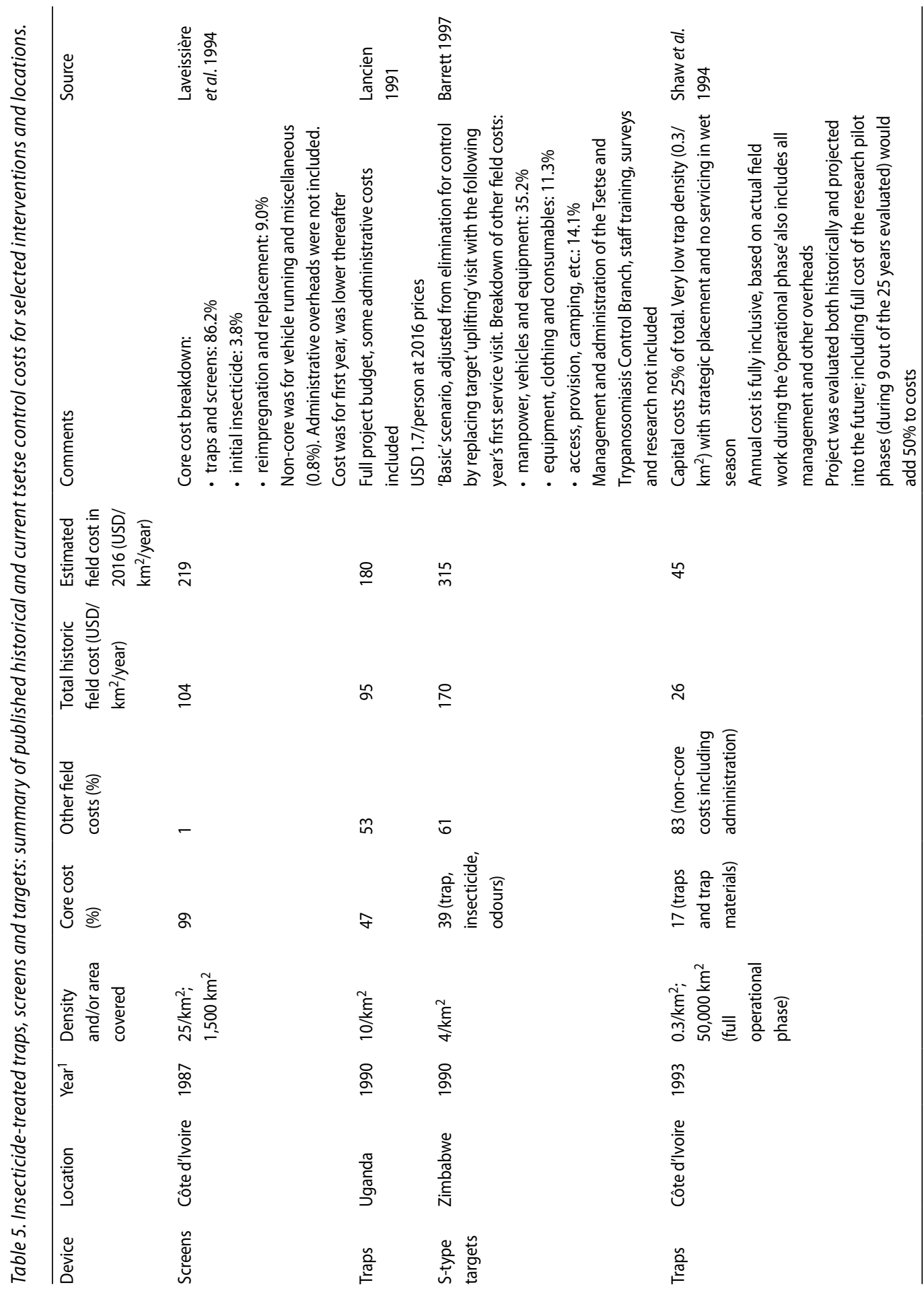




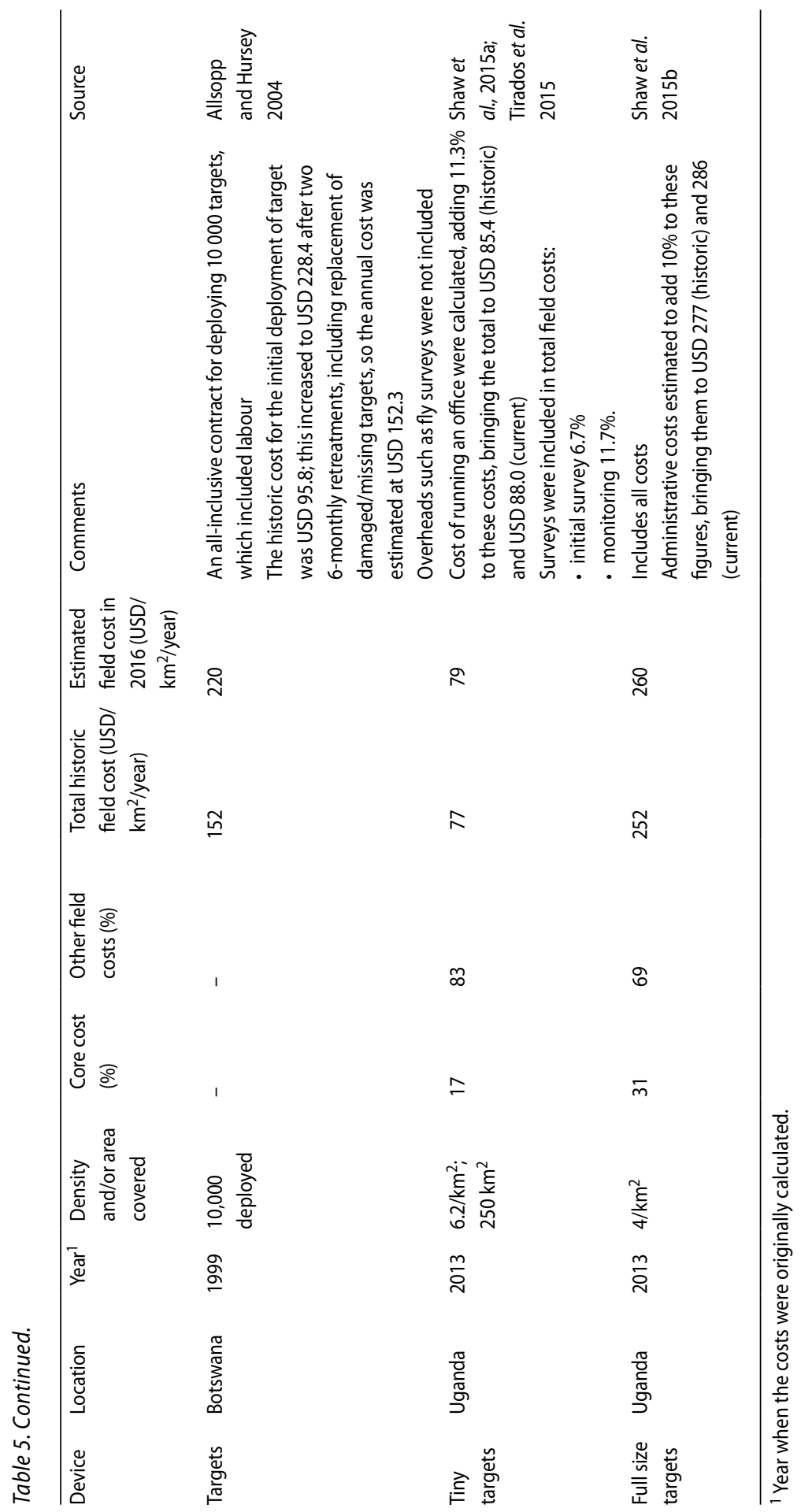




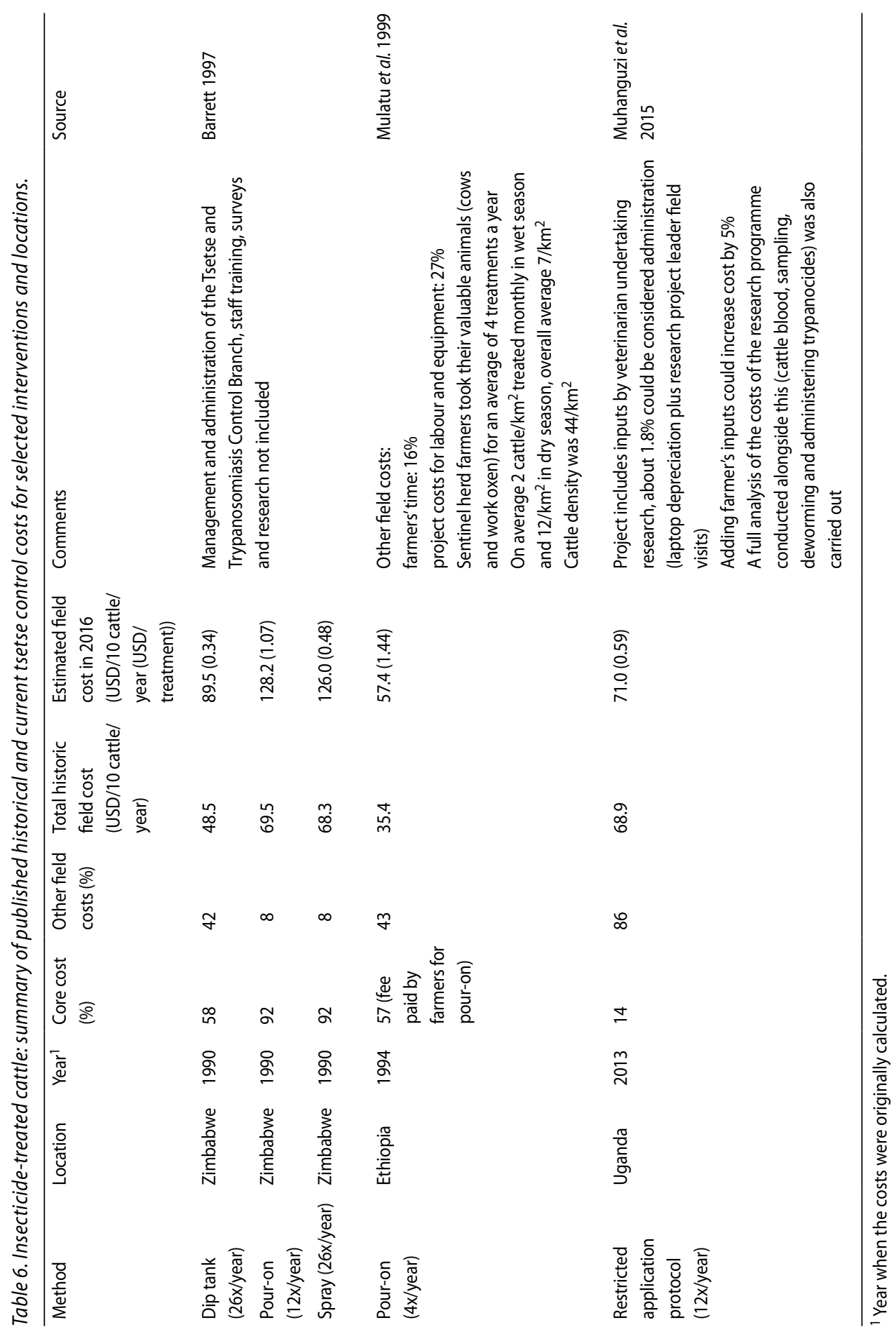




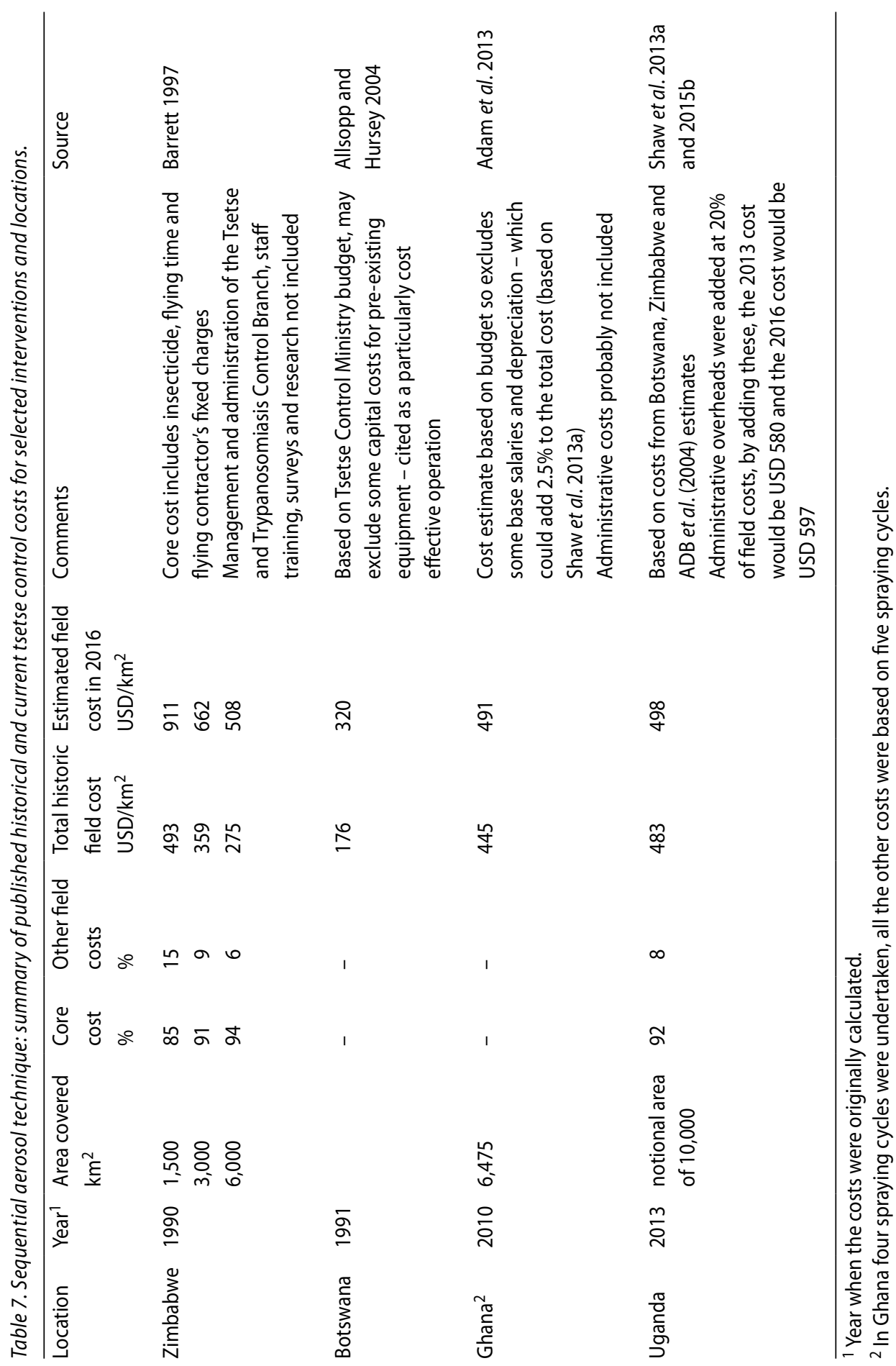


Table 8. Full costings for tiny targets (ITT) and insecticide-treated cattle (ITC) in Uganda in 2012/2013 (2013 USD) (adapted from Muhanguzi et al. 2015 and Shaw et al. 2015a).

\begin{tabular}{|c|c|c|c|c|}
\hline \multirow[b]{2}{*}{ Cost categories } & \multicolumn{2}{|l|}{ ITC } & \multicolumn{2}{|l|}{ ITT } \\
\hline & $\begin{array}{l}\text { USD/10 } \\
\text { cattle/12 } \\
\text { treatments }\end{array}$ & $\%$ breakdown & $\begin{array}{l}\text { USD } / \mathrm{km}^{2} / \\
\text { year }\end{array}$ & $\%$ breakdown \\
\hline \multicolumn{5}{|l|}{ Depreciation on capital items } \\
\hline \multicolumn{5}{|l|}{ - Specialised } \\
\hline Spray pump & 2.7 & 3.8 & - & - \\
\hline \multicolumn{5}{|l|}{ - Non-specialised } \\
\hline Vehicle & 3.6 & 5.3 & 9.8 & 11.5 \\
\hline Laptops & 0.1 & 0.2 & 0.8 & 0.9 \\
\hline GPS sets & 0.2 & 0.2 & 2.6 & 3.0 \\
\hline Office equipment & - & - & 0.6 & 0.7 \\
\hline Sub-total capital costs & 6.6 & 9.5 & 13.8 & 16.1 \\
\hline \multicolumn{5}{|l|}{ Recurrent costs } \\
\hline \multicolumn{5}{|l|}{ - Specialised } \\
\hline Servicing of spray pump & 2.0 & 3.0 & - & - \\
\hline Vectocid & 15.4 & 22.4 & - & - \\
\hline Targets and target materials & - & - & 13.1 & 15.3 \\
\hline Traps for monitoring & - & - & 0.3 & 0.4 \\
\hline Extension materials & - & - & 0.8 & 1.0 \\
\hline Sub-total specialised recurrent costs & 17.4 & 25.4 & 14.2 & 16.7 \\
\hline \multicolumn{5}{|l|}{ - Non-specialised } \\
\hline \multicolumn{5}{|l|}{ Staff } \\
\hline Basic salaries & 20.5 & 29.8 & 9.3 & 10.9 \\
\hline Staff travel allowances & 7.5 & 10.8 & 0.5 & 0.6 \\
\hline $\begin{array}{l}\text { Daily paid drivers, labourers, community } \\
\text { assistants, etc. }\end{array}$ & - & - & 20.6 & 24.2 \\
\hline \multicolumn{5}{|l|}{ Vehicles and travel } \\
\hline Vehicle fuel and running costs & 14.5 & 21.0 & 13.2 & 15.5 \\
\hline Other travel costs & - & - & 6.5 & 7.6 \\
\hline \multicolumn{5}{|l|}{ Other } \\
\hline Stationery and office running & - & - & 5.0 & 5.8 \\
\hline GPS and other batteries & & & 1.0 & 1.1 \\
\hline Protective clothing for staff, machetes, etc. & 1.1 & 1.6 & 1.0 & 1.1 \\
\hline $\begin{array}{l}\text { Village head payment for communication } \\
\text { (ITC) airtime, refreshments, T-shirts (ITT) }\end{array}$ & 1.3 & 1.9 & 0.3 & 0.4 \\
\hline Sub-total non-specialised recurrent costs & 44.9 & 65.1 & 57.4 & 67.2 \\
\hline Overall total & 68.9 & 100.0 & 85.4 & 100.0 \\
\hline
\end{tabular}


pump depreciation, water carrier and dip attendants wages, allowances and protective clothing and a dip inspection are added. For pour-on (the most expensive option per animal treated) and spraying, just over $90 \%$ of costs are core costs. Turning to Ethiopia, the breakdown of costs is $57 \%$ for the cost for the pour-on (which was recovered from the farmers using a fixed fee) and $43 \%$ for the other delivery costs, which in this case were very complete and even included the opportunity cost of the livestock keepers' time for walking their animals back and forth to the place where they were treated, the latter accounting for $16 \%$ of the total. However, the research component and administrative overheads were not included. Although the overall treatment rate was relatively low, the impacts both on cattle productivity in the monitored sentinel herd and on overall tsetse density were significant (Mulatu et al. 1999). The calculation for RAP in Uganda is fully comprehensive in including all delivery costs, with core costs at $29 \%$ of the total.

\section{Aerial spraying}

Turning to SAT, Table 7 summarises the information from five situations. The Zimbabwe costs follow on from the estimates given in Table 3 and again highlight the issue of scale, with the addition of other field costs (physico-chemical monitoring, tsetse survey teams, ground marker teams, access provision, camp and airstrip construction and maintenance) being more than four times as much per $\mathrm{km}^{2}$ for an area of 1,500 than for an area of $6,000 \mathrm{~km}^{2}$, thus further enhancing the effect of scale demonstrated in Table 3. An additional factor commented on in Barrett (1997) is the impact of dealing with areas with multiple tsetse species, which could increase costs by about $25 \%$. One estimate of the cost per $\mathrm{km}^{2}$ from Botswana put total costs at USD 320 per $\mathrm{km}^{2}$, cited by Allsopp and Hursey (2004) as a particularly cost-effective operation. Ghana provides a recent example of actual field costs, coming to USD 491 per $\mathrm{km}^{2}$, excluding depreciation and some base salaries and for only four spray cycles. This compares well with the figure of USD 498 estimated for a large scale operation in Uganda. However, these figures do not include administrative overheads. Thus it seems that an appropriate guideline figure for a large scale operation is USD 500-600 per km².

\section{Overhead costs}

The various costings analysed, Tables 5, 6 and 7 focus on field costs, with indications given in the comments table as to what other overheads might be and to what extent these have been quantified. Such overheads broadly consist of the following:

- The administrative costs associated with running a tsetse control programme by the organisation which manages it - an entomological service, an independent research or other project. In some cases these costs have been estimated. For the recent examples of ITT using tiny targets in Uganda, office costs account for $10.2 \%$ of total costs (Shaw et al. 2015a).

- The costs of preliminary fly surveys and other studies. These costs depend very much on the nature of the project. For the ITT project in Uganda, the essential preliminary surveys and tsetse population monitoring during target deployment respectively accounted for 6.0 and $10.6 \%$ of total costs. In Shaw et al. (2013a), an additional 5, 10 and 20\% were added to field costs for ITC, ITT and SAT. However, where the objective is localised tsetse elimination, more extensive studies are usually undertaken and the costs of these can be very high, adding USD 100-350 per km² (Shaw et al. 2013a, 2015b) in turn partly based on the projected elimination costs in the report of the ADB/IAEA/PATTEC (2004). In Senegal, Bouyer et al. (2014) calculated the cost of preliminary studies for SIT at USD 2,500 per $\mathrm{km}^{2}$, although it is likely that this included a research component.

- Sensitisation of local populations. Many schemes include this cost, however in some cases it is seen as an overhead and not quantified alongside other field costs. Also, it is a cost which 
may be incurred annually, or such activities may be undertaken less frequently, so that the cost can be spread over several years.

- As the information on costs has been mainly sourced from scientific publications it is inevitable that research is an important component. It is not always easy to separate this from the costs of implementing tsetse control. For example, the project implemented in Côte d'Ivoire (Shaw et al. 1994) was preceded by a nine year research phase and the work reported on in Mulatu et al. (1999) had an important research component. In other cases, tsetse control is undertaken alongside research activities, for example for ITC in Uganda in 2012/13, where cattle bleeding to sample for trypanosomosis and tick-borne diseases was an integral part of the whole project and the research component accounted for $55 \%$ of total field costs, although this was excluded from the control costs described in Table 8 (Muhanguzi et al. 2015).

\section{Case study: tiny targets and insecticide-treated cattle in Uganda}

Lastly, to illustrate a fully comprehensive cost calculation, Table 8 goes on to provide a detailed breakdown of the costs of two recent schemes, showcasing low cost technologies for ITC and ITT, coincidentally both undertaken in Uganda in 2012/13. Both involved rigorous cost recording with an emphasis on including the costs of items that are sometimes overlooked, such as a share of salaries of people already employed or of depreciation for vehicles already owned and the cost of supervision and office overheads. Here the original price levels are used. It is likely that there has been only limited price change - to be fully comparable with the figures and methodology used in Tables 3-7, an annual inflation rate of $3 \%$ to convert to 2016 prices would be applicable.

Looking at the core costs, using the RAP minimises the amount of insecticide required, and the tiny targets used are the cheapest target available, so that the core costs are low in each case, 29.2 and $17 \%$ of total costs for ITC and ITT respectively. Vehicle and travel costs in each case came to around a third of costs: $26.3 \%$ for ITC and $34.6 \%$ for ITT. Staff and labour costs were also similar proportions, $40.6 \%$ for ITC and $35.7 \%$ for ITT. The delivery cost for tiny targets is much smaller than has historically been the case for ITT, because the small size of the targets meant that they could be transported in rucksacks on motorcycles or even bicycles, if the distances to the field were short. The project area was close to the town of Arua and densely populated (estimated at 500 people per $\mathrm{km}^{2}$ ) so that access was comparatively easy, which helped to keep costs down. For ITT, the proportion of costs for each of the component project activities was also calculated. These were initial fly surveys: $6.0 \%$, sensitisation of local populations: $18.6 \%$, target deployment: $34.5 \%$, tsetse monitoring: $10.6 \%$, target maintenance: $20.1 \%$ and office overheads: $10.2 \%$.

\section{Discussion}

\section{Costs and benefits}

Ultimately these control costs need to be assessed alongside likely benefits.

For AAT, a number of studies have attempted to do this in relation to cattle. To cite results just from the studies included here, Mulatu et al. (1999) estimated benefit-cost ratios for the pour-on intervention of 8.1 to cattle owners and 4.3 for the project as a whole, while Shaw et al. (1994) estimated a baseline benefit-cost ratio of 3.2. A full discussion of earlier studies can be found in Shaw (2004). Estimating the potential benefits from controlling trypanosomosis in cattle is complex, as disease losses per bovine need to be very carefully estimated for individual production systems and looked at alongside other disease problems, notably tick-borne diseases, 
where there may be some overlap. Disease challenge and livestock keepers' ability to mitigate this varies greatly. With these caveats in mind, the analysis by Shaw et al. (2014), indicated that losses per bovine could be in the range of USD 10-25 per year at 2016 prices, with the higher levels applying where a significant proportion of cattle are used for draught power or are crossbred dairy cows. Recent field work (Okello 2016; Okello et al. 2015) reinforces this. Such loss levels imply that with the annual cost per insecticide-treated bovine currently ranging from USD 3-12, and half or less of animals needing treatment, benefit-cost ratios could range from 1.5 to 15 . Thus, from the cattle keepers' perspective, tsetse control using ITC offers good returns, especially as only a proportion of animals need to be treated, particularly at higher cattle population densities. For low-cost target operations the benefits to cattle production also easily outweigh the costs, especially at higher cattle densities. Aerial spraying on the other hand, as a short term and more expensive intervention which needs to be undertaken on a large scale, does cover costs in many situations and has been regarded as a useful intervention for control where it is important to stop transmission quickly - as during an outbreak of sleeping sickness. A full discussion is beyond the scope of this chapter, however, Shaw et al. (2015b, 2017) compare these benefits and costs, presenting the results as maps.

For HAT, Sutherland et al. (2015) review the economic studies undertaken and Sutherland et al. (2016) calculate how tsetse control could contribute to the overall goal of the elimination of the gambiense form of HAT. The tiny target programme assessed in Shaw et al. (2015a) primarily aimed at tsetse control in an endemic gambiense HAT focus in northwestern Uganda, while the study undertaken by Muhanguzi et al. $(2014,2015)$ was part of a wider programme to control rhodesiense HAT in its southeastern Uganda focus.

\section{Alternative strategies}

Although this chapter is about tsetse control, it is appropriate briefly to set it into the context of two alternatives.

\section{Trypanocides}

Trypanocides, whether curative (diminazene aceturate) or prophylactic (isometamidium chloride, which confers about 3 months protection) are widely used by livestock keepers in Africa. Recent estimates of the overall volume used are lacking, however historically it was thought that about 0.8 doses were given per year per bovine living in tsetse-infested areas (Geerts and Holmes 1998). These would mainly be given to draught males, adult females or cattle herds on transhumance. Field studies showed 1.5 doses per bovine per year in areas with high draught oxen populations (Doran 2000; Van den Bossche et al. 2000).

The price of trypanocides varies by country, drug and presentation, and between urban and rural areas. Estimated trypanocide costs in Uganda in 2016 for a bovine of $200 \mathrm{~kg}$ were about USD 1.4 for isometamidium and 1.0 for diminazene and delivery adds some USD 0.7-1.0 to that, so that a total for a single dose would be around USD 2.3 for isometamidium and 1.9 for diminazene (Muhanguzi et al. 2015; Shaw et al. 2015b). Comparisons with the costs of ITC and ITT are made in Shaw et al. (2013a, 2105b, 2017). Some use of trypanocides alongside vector control will usually be necessary, particularly when vector control work first starts, or when animals move in and out of a tsetse controlled area. At low cattle population densities, where ITC is not feasible and ITT is unlikely to be cost-effective, more reliance on trypanocides is necessary despite the risks of resistance (Geerts and Holmes, 1998). 
In human health, finding and treating patients is an essential step independent of, or alongside, vector control for both the gambiense and the rhodesiense form of HAT (see costs in Sutherland et al. 2015, 2016 and the effect of RAP on infection rates in the cattle reservoir of rhodesiense HAT in Muhanguzi et al. 2014).

\section{Elimination scenarios}

The focus in this chapter has been firmly on tsetse control, viewed as an ongoing, continuous activity rather than on the creation of tsetse-free zones (localised elimination, although historically and traditionally referred to as eradication in the tsetse community). The only additional tsetse control technique not costed here is the SIT as, unlike the techniques costed here, which play a role in both control and elimination, SIT is only considered if elimination is the ultimate objective. The cost is very sensitive to scale and, for a single tsetse species, is likely to add between USD 1,800 to 3,800 per $\mathrm{km}^{2}$ over and above the costs of the initial reduction of the tsetse population using one of the methods costed in this chapter (Shaw et al. 2015b, 2017). In addition, it would be necessary to factor in the costs of preliminary research, other overheads and barriers to prevent reinvasion, composed of high density ITT or ITC. Taken together, these would increase baseline costs by between 50 and $75 \%$. These figures tie in with the high overall costs reported from recent elimination projects, amounting to USD 7,400 per $\mathrm{km}^{2}$ at 2016 prices in Tanzania (Msangi et al. 2000) and to USD 8,500 in Senegal (Bouyer et al. 2014). For other techniques, if used in an elimination context, overheads would add about 300 per $\mathrm{km}^{2}$ and the need for reinvasion barriers, conservatively costed for 5 years on a quarter of the circumference of the area, would add between 100 and 900 per $\mathrm{km}^{2}$ depending on terrain and the fly species involved, with the higher costs reflecting the use of targets (Shaw et al. 2015a, 2017). The idea of a once-and-for-all cost to achieve permanent elimination is very attractive. However, to be cheaper than ongoing tsetse control activities, reinvasion must be sustainably prevented (Brandl 1988; Shaw et al. 2015b) or the relevant tsetse population must be identified as isolated, which is the case only in a limited proportion of tsetse-infested areas (Bouyer et al. 2015). A comparison of costs and outcomes and discussion of the issues involved can be found in Brandl (1988), Bouyer et al. (2013) and Shaw et al. (2013a, 2013b, 2015b, 2017).

\section{Determinants of uptake}

Farmers and communities have not controlled tsetse, because with few exceptions, they were not given the opportunity Dransfield and Brightwell (2004)

The published costs summarised in Tables 2-8 are all linked either to government or donorfunded schemes, usually with a strong research component. The cost analyses indicate that costs have fallen and both ITC and ITT are now cheaper and mostly easier to implement than when these techniques were first introduced. However, it seems that uptake has lagged behind. It was hoped that the reduction in funding of veterinary services and their partial privatisation from the late 1980s onwards would provide a more flexible and sustainable delivery of animal health to livestock keepers (Leonard 2000). This restructuring also saw a parallel reduction in the size and funding of many of the continent's government tsetse control units. However, privatisation has been implemented very patchily, with success in some areas while in many of the more isolated rural areas where tsetse and trypanosomosis are abundant, livestock keepers have little access to any form of veterinary care or drugs for their animals. This, together with funding limitations, led to great hope being put in the implementation of community-based tsetse control schemes. 
The late 1990s and early 2000s saw many studies of such schemes both in the field of HAT control (e.g. Gouteux and Sinda 1990; Laveissière et al. 1994) and AAT control (reviewed in Barrett and Okali 1998; Dransfield and Brightwell 2004). Alongside these were a number of studies which examined farmers' willingness-to-pay for tsetse control using traps/targets, providing either labour or cash (Echessah et al. 1997; Kamuanga et al. 2001; Swallow and Woudyalew 1994). These emphasised the public/private nature of ITC, where the tick-control component confers a private benefit and tsetse control can be described as a 'local public good' (Swallow and Woudyalew 1994). Kamuanga et al. (2001) found that it was unlikely that communities would fully fund such operations. However, this applied to the larger, higher cost targets in use at the time.

So, has the situation changed since the turn of the century as analysed by Dransfield and Brightwell (2004)? More recent evidence gives room for optimism. In Arua district of Uganda, an action research programme found that local women were happy to supply labour to monitor and maintain tiny targets for HAT control, and that even when quantifying the opportunity cost of their time, the cost for target deployment and maintenance was almost identical to that for a project led by entomologists (Kovacic 2015; Kovacic et al. 2013; Shaw et al. 2015a). Uptake is difficult to predict and can be highly variable, as found for footbaths in Burkina Faso by Bouyer et al. (2011) where the potential for tick control was often a driver for adoption and the type of waiting or holding area for cattle near the footbath also a crucial factor. Past experience does indicate that in some situations a lower than expected uptake is nevertheless sufficient to accomplish project objectives, as in Ethiopia where cattle keepers focussed on their valuable animals, cows and draught males, and applied pour-on to these about four times a year in an area with relatively high cattle population densities (Mulatu et al. 1999). Livestock keepers in many areas practice tick control using acaricides, and one option is to ensure that the locally available products are pyrethroids which will control both tsetse and ticks (Bardosh et al. 2013). Lastly, sometimes it is found that tsetse control is being undertaken autonomously, apparently without external promotion from government agencies or non-government agencies. Thus in Serengeti District, Tanzania, preliminary results from a questionnaire survey carried out amongst agropastoralist households in 2016 indicated widespread use of alphacypermethrin insecticide, via sprays or dips, in order to control tick and tsetse-borne disease as well as abundant use of prophylactic trypanocides (F. Allan, personal communication, 2016).

\section{Conclusion}

This chapter analyses a selection of published costs for different methods of tsetse control from 1990 onwards, focusing on field-based studies and converting them to 2016 prices. The data is divided into 'core' costs (such as targets, traps, insecticide and flying time), which are those most often reported by researchers and the delivery costs and overheads which complete the cost picture.

The costs of the core items for all three groups of tsetse control techniques have remained relatively similar over time and across countries, with differences generally reflecting technical changes. Smaller targets for use against riverine (palpalis) group tsetse species without odour baits and the availability of insecticide-impregnated cloth and netting for targets and traps have significantly reduced the cost of ITT and the restricted application of insecticide that of ITC. For SAT, core costs have not changed.

Delivery costs have also been affected by these technical improvements for ITT, with simpler or fewer maintenance visits as insecticide re-impregnation was not required and tiny targets can be 
transported in rucksacks using motorbikes or even bicycles. For ITC although using less insecticide does reduce costs, the cost of delivery does not necessarily reduce proportionately. For SAT, scale is again a major determinant of overall cost. Where livestock keepers or communities are the main implementers, the opportunity cost of people's time has only been quantified in a few studies.

The component about which the least amount of information is available is overheads: administration, office back-up and preparation, such as preliminary fly surveys. This is because this component is necessarily linked to the organisational structure involved. These costs are likely to vary more, and the lack of information reflects the fact that the overheads are often absorbed in the organisation's ongoing costs, rather than appearing as specific budget lines for specific tsetse control activities. Overall, the two comprehensive field studies cited on ITC and ITT do indicate that the $5-10 \%$ range suggested (Shaw et al. 2013a) for these methods is probably correct. It may be that the suggested $20 \%$ mark-up for aerial spraying is an overestimate.

As regards future research agendas, from the cost point of view the main topics would be the following. A better consensus on how many ITC per $\mathrm{km}^{2}$ are required to control tsetse and reduce disease incidence in both people and animals is urgently needed. This would need to monitor both tsetse and trypanosomosis and factor in the scale of such operations, in terms of the area covered. Field studies on the delivery side, especially on the more novel approaches are also needed. Two important topics are the use of low-cost footbaths installed near cattle pens for ITC and fencing of livestock compounds such as zero-grazed cattle or pigs. Other new or modified delivery approaches are being tested, for example targets could be mounted on boats in mangrove swamps (Rayaisse et al. 2015). More information is also needed on the non-cash costs to livestock keepers or other community members, which are a major determinant of uptake levels. It is vital that new field trials and research initiatives involve rigorous monitoring of costs. The cost calculation template proposed above has been tested and applied in a number of field situations and proven viable. The cost recording required is not particularly onerous, it just needs to be consistent and timely.

In terms of orders of magnitude, for ITT, the cost of conventional large targets with odours for use against all tsetse species is likely to remain at USD 200-300 per $\mathrm{km}^{2}$, that of tiny targets at under USD 100, as evidenced by the recent full cost calculation. For ITC, different dosages, different application frequencies per bovine and very different numbers of bovines to be treated per $\mathrm{km}^{2}$, make for a more complex picture. ITC, using the RAP with a spray at 12 doses per year, would cost USD 0.6 per dose or 7 per treated bovine including full delivery costs and all overheads, thus USD 70 per $\mathrm{km}^{2}$ if 10 bovines were treated. For other forms of ITC, estimates including most delivery costs would be: for footbaths and dips USD 0.15-0.35 per dose and for pour-ons USD 1.0-1.5 per dose. The cost of aerial spraying using SAT is about USD 500-600 for larger areas, for example over $5000 \mathrm{~km}^{2}$, with the cost increasing substantially for smaller areas.

The often repeated statement that 'control is unsustainable' is refuted by these cost calculations. The lower cost ITC and ITT options could be applied for several decades before matching the cost of elimination using SIT or even SAT - even without allowing for the cost of barriers, the likelihood of reinvasion and the time value of money, whereby economics discounts future monetary sums in relation to current ones. Furthermore, control activities are not necessarily designed to be fully applied, without interruption, for many years. Instead it is likely that they would be deployed where and when there are particular needs, such as maintaining draught oxen or dairy cows in good health or dealing with HAT. The control activities might be applied at different levels of intensity, for different lengths of time, in combination with other tsetse control methods and 
alongside some use of trypanocides. For example, some livestock farmers might use insecticideimpregnated fencing, while the community as a whole was using targets.

However, despite the availability of off-the-shelf ready-to-use technologies and some instances such as the Tanzanian example described above, uptake still tends to be limited to situations where research is being undertaken or there is a clear short term goal with high level government or donor support. This has been the case in recent years where the control of both forms of HAT and the possible elimination of the gambiense form has been a major objective. Elsewhere, a focus on so-called area-wide integrated pest management has encouraged the use of various control technologies, but at low intensity or for a limited period as a precursor to the creation of tsetsefree zones, usually using more capital-intensive high technology interventions such as the sterile insect technique or aerial spraying, which have often failed to materialise or be sustained.

Thus the ultimate challenge remains now, as at the turn of the century, of making the highly affordable tsetse control technologies available to communities where they are needed. This needs to include the provision of some appropriate government co-funding, of support and information to relevant non-governmental organisations and communities, as well as a basic level of suitable entomological oversight so that the technologies are applied in a way that effectively controls tsetse so as to protect both people and their livestock.

\section{Acknowledgements}

As well as providing an opportunity to update estimates for tsetse control made in the past, writing this chapter has given the author an opportunity to compile and compare costs for different techniques from several projects that she has worked on over the last decade. For this, heartfelt thanks are due to all colleagues in this work, and in particular to Giuliano Cecchi, Fabrice Courtin, Johan Esterhuizen, Vanja Kovacic, Mike Lehane, Clement Mangwiro, Raffaele Mattioli, Ian Maudlin, Dennis Muhanguzi, Walter Okello, Jean-Baptiste Rayaisse, Tim Robinson, Iñaki Tirados, Steve Torr, Charles Waiswa, Sue Welburn and William Wint. Particular thanks go to Fiona Allan for generously sharing her experience from her research work in Tanzania. The editorial inputs and suggestions made by Jérémy Bouyer, Giuliano Cecchi and Steve Torr were very helpful in improving the text and were gratefully received.

\section{References}

Adam Y, Cecchi G, Kgori PM, Marcotty T, Mahama Cl, Abavana M, Anderson B, Paone, M, Mattioli R and Bouyer J (2013) The sequential aerosol technique: a major component in an integrated strategy of intervention against Riverine Tsetse in Ghana. PLoS Negl Trop Dis 7: e2135.

African Development Bank, International Atomic Energy Agency and Pan-African Tsetse and Trypanosomosis Eradication Campaign (ADB/IAEA/PATTEC) (2004) Integrated area-wide programme for trypanosomiasis-free zones in Uganda. ADB, IAEA and PATTEC, Kampala, Uganda.

Allsopp R and Hursey BH (2004) Insecticidal control of tsetse. In: Maudlin I, Holmes PH and Miles MA (eds.) The trypanosomiases. CABI Publishing, Wallingford, UK, pp. 491-507.

Bardosh K, Waiswa C and Welburn SC (2013) Conflict of interest: use of pyrethroids and amidines against tsetse and ticks in zoonotic sleeping sickness endemic areas of Uganda. Parasit Vectors 6: 204.

Barrett J (1997) Economic issues in trypanosomiasis control. Natural Resources Institute, Chatham, UK.

Barrett K and Okali C (1998) Partnerships for tsetse control - community participation and other options. World Anim Rev 90: 38-46. 
Bauer B, Holzgrefe B, Mahama Cl, Baumann MPO, Mehlitz D and Clausen P-H (2011) Managing tsetse transmitted trypanosomosis by insecticide treated nets - an affordable and sustainable method for resource poor pig farmers in Ghana. PLoS Negl Trop Dis 5: e1343.

Bouyer F, Hamadou S, Adakal H, Lancelot R, Stachurski F, Belem AMG and Bouyer J (2011) Restricted application of insecticides: a promising tsetse control technique, but what do the farmers think of it? PLoS Negl Trop Dis 5: e1276.

Bouyer F, Seck MT, Dicko AH, Sall B, Lo M, Vreysen MJB, Chia E, Bouyer J and Wane A (2014) Ex-ante benefit-cost analysis of the elimination of a Glossina palpalis gambiensis population in the Niayes of Senegal. PLoS Negl Trop Dis 5: e3112.

Bouyer J, Dicko AH, Cecchi G, Ravel S, Guerrini L, Solano P, Vreysen MB, De Meûs T and Lancelot R (2015) Mapping landscape friction to locate isolated tsetse populations that are candidates for elimination. Proc Natl Acad Sci USA 112(47): 14575-14580.

Bouyer J, Seck MT and Sall B (2013) Misleading guidance for decision making on tsetse eradication: response to Shaw et al. (2013). Prev Vet Med 112: 443-446.

Brandl FE (1988) Cost of different methods to control riverine tsetse in West Africa. Trop Anim Health Prod 20: 67-77.

Brightwell R, Dransfield RD and Kyorku C (1991) Development of a low-cost tsetse trap and odour baits for Glossina pallidipes and G. Iongipennis in Kenya. Med Vet Entomol 5: 153-164.

Butcher C (2009) Synthesis report - from research into use: monitoring and evaluation of a public-private partnership. Stamp out sleeping sickness case study. With contributions from JF Morton and A Shaw. DFID Research into Use, Edinburgh, UK.

Courtin F, Camara M, Rayaisse J-B, Kagbadouno M, Dama E, Camara O, Traoré IS, Rouamba J, Peylhard M, Somda MB, Leno M, Lehane MJ, Torr SJ, Solano P, Jamonneau V, Bucheton B (2015) Reducing human-tsetse contact significantly enhances the efficacy of sleeping sickness active screening campaigns: a promising result in the context of elimination. PLoS Negl Trop Dis 9: e0003727.

Davies $\mathrm{H}$ (1964) The eradication of tsetse in the chad river systems of north-eastern Nigeria. J Appl Ecol 1: 387-403.

Davies $\mathrm{H}$ (1971) Further eradication of tsetse in the chad and gongola river system of northern Nigeria. J Appl Ecol 8: 568-578.

Doran M (2000) Socio-economics of trypanosomosis. Implications for control strategies within the common fly-felt of Malawi, Mozambique, Zambia and Zimbabwe. Bovine trypanosomosis in Southern Africa. Vol. 3. Regional Tsetse and Trypanosomosis Control Programme for Southern Africa. Harare, Zimbabwe, $156 \mathrm{pp}$.

Dransfield RD and Brightwell R (2004) Community participation in tsetse control: the principles, potential and practice. In: Maudlin I, Holmes H and Miles MA (eds.) The trypanosomiases. CABI Publishing, Wallingford, UK, pp. 533-546.

Echessah PN, Swallow BM, Kamara DW and Curry JJ (1997) Willingness to contribute labour and money to tsetse control: application of contingent valuation in Busia district, Kenya. World Dev 25: 239-253.

Feldmann U (2004) The sterile insect technique as a component of area-wide integrated pest management of tsetse. In: Maudlin I, Holmes PH and Miles MA (eds.) The trypanosomiases. CABI Publishing, Wallingford, UK, pp. 565-582.

Geerts S and Holmes PH (1998) Drug management and parasite resistance in bovine trypanosomiasis in Africa. Programme against African Trypanosomosis (PAAT) Technical and Scientific Series No. 1. FAO, Rome, Italy. Available at: https://tinyurl.com/ybr8o9g8.

Gimonneau G, Rayaisse JB and Bouyer J (2018) Integrated control of trypanosomosis. In: Garros C, Bouyer J, Takken W and Smallegange RC (eds.) Pests and vector-borne diseases in the livestock industry. Ecology and Control of Vectorborne diseases. Vol. 5. Wageningen Academic Publishers, Wageningen, the Netherlands, pp. 147-174.

Gouteux JP and Sinda D (1990) Community participation in the control of tsetse flies. Large scale trials using the pyramid trap in the Congo. Trop Med Parasitol 41: 49-55.

Jahnke HE (1974) The economics of controlling tsetse flies and cattle trypanosomiasis examined for the case of Uganda. Forschungsberichete der Africa-studienstelle 48. Institut für Wirtschaftsforschung, Weltforum Verlag, Munich, Germany.

Jordan AM (1986) Trypanosomiasis control and African rural development. Longman, New York, NY, USA.

Kamuanga M, Swallow BM, Sigué $H$ and Bauer B (2001) Evaluating contingent and actual contributions to a local public good: tsetse control in the Yale Agro-pastoral Zone, Burkina Faso. Ecol Econ 39: 115-130. 
Kgori PM, Modo S and Torr SJ (2006) The use of aerial spraying to eliminate tsetse from the Okavango Delta of Botswana. Acta Trop 99: 184-199.

Kovacic V (2015) Women-led tsetse control - a pilot study in northwest Uganda. PhD thesis, University of Liverpool, Liverpool, UK.

Kovacic V, Tirados, I, Esterhuizen J, Mangwiro CTN, Torr SJ, Lehane MJ and Smith H (2013) Community acceptance of tsetse control baits: a qualitative study in Arua District, North West Uganda. PLoS Negl Trop Dis 7: e2579.

Lancien J (1991) Lutte contre la maladie du sommeil dans le sud-est Ouganda par piégage des glossines. Ann Soc Belg Méd Trop 71, Suppl. 1: 35-47.

Laveissière C, Grébaut O, Lemasson J-J, Meda AH, Couret D, Doua F, Brou N and Cattand P (1994) Les communautés rurales et la lutte contre la maladie du sommeil en forêt de Côte d'Ivoire. WHO, Geneva, Switzerland. Available at: https://tinyurl.com/yb5ajhre.

Leonard DK (2000) The new institutional economics and the restructuring of animal health services in Africa. In: Leonard DK (ed.) Africa's changing markets for health and veterinary services - new institutional issues. Macmillan Press Limited, Basingstoke, UK, pp. 1-39.

Maia M, Clausen, P-H, Mehlitz, D, Garms, R and Bauer B (2010) Protection of confined cattle against biting and nuisance flies (Muscidae: Diptera) with insecticide-treated nets in the Ghanaian forest zone at Kumasi. Parasitol Res 106: 1307-1313.

Meltzer MI (2001) Introduction to health economics for physicians. Lancet 358: 993-998.

Msangi AR, Saleh KM, Kiwia N, Malele II, Mussa WA, Mramba F, Juma KG, Dyck VA, Vreysen MJB, Parker AG, Feldmann U, Zhu ZR and Pan H (2000) Success in Zanzibar: eradication of tsetse. In: Tan K-H (ed.) Area-wide control of fruit flies and other insect pests. Penerbit Universiti Sains, Penang, Malaysia, pp. 57-66.

Muhanguzi D, Okello WO, Kabasa JD, Waiswa C, Welburn SC and Shaw APM (2015) Cost analysis of options for management of African animal trypanosomiasis using interventions targeted at cattle in Tororo District; southeastern Uganda. Parasit Vectors 8: e387.

Muhanguzi D, Picozzi K, Hattendorf J, Thrusfield M, Welburn SC, Kabasa JD and Waiswa C (2014) Improvements on restricted insecticide applicaton protocol for control of human and animal African trypanosomiasis in Eastern Uganda. PLoS Negl Trop Dis 8: e3284.

Mulatu W, Swallow B, Rowlands GJ, Leak SGA, d'leteren GDM and Nagda SM (1999) Economic benefits to farmers of six years of application of an insecticidal 'pour-on' to control tsetse in Ghibe, southwest Ethiopia. Proceedings of the $24^{\text {th }}$ Biennial Conference of the International Scientific Council for Trypanosomiasis Research and Control (ISCTRC). Scientific, Technical and Research Commission of the Organisation of African Unity (OAU/STRC), Nairobi, Kenya, pp. 578-586.

Ndeledje N, Bouyer J, Stachurski F, Grimaud P, Belem AMG, Mbaïndingatoloum FM, Bengali Z, Alfaroukh IO, Cecchi G and Lancelot, R (2013) Treating cattle to protect people? Impact of footbath insecticide treatment on tsetse density in Chad. PLoS ONE 8: e67580.

Okello WO (2016) An economic analysis of zoonotic disease control in Uganda and the Lao People's Democratic Republic. PhD thesis, University of Edinburgh, Edinburgh, UK.

Okello WO, Muhanguzi D, MacLeod ET, Welburn SC, Waiswa C and Shaw AP (2015) Contribution of draft cattle to rural livelihoods in a district of southeastern Uganda endemic for bovine parasitic diseases: an economic evaluation. Parasit Vectors 8: e571.

Okoth JO (1991) Description of a mono-screen trap for Glossina fuscipes fuscipes Newsted in Uganda. Ann Tropl Med Parasitol 85: 309-314.

Putt SNH, Shaw APM, Matthewman RW, Bourn DM, Underwood M, James AD, Hallam MJ and Ellis PR (1980) The social and economic implications of trypanosomiasis control - a study of its impact on livestock production and rural development in northern Nigeria. Study no. 25, Veterinary Epidemiology and Economics Research Unit (VEERU). Reading University, Reading, UK.

Rayaisse J-B, Salou E, Courtin F, Yoni W, Barry I, Dofini F, Kagbadouno M, Camara M, Torr SJ and Solano P (2015) Baitedboats: an innovative way to control riverine tsetse, vectors of sleeping sickness in West Africa. Parasit Vectors 8: 236. 
Rowlands GJ, Coulibaly L, Hecker PA, d'leteren GD, Leak SG and Authié E (1996) Effect of tsetse control on trypanosome prevalence in livestock: problems of experimental design and statistical interpretation - a case study in northern Côte d'Ivoire. Vet Parasitol 63: 199-214.

Shaw A (2004) Economics of African trypanosomiasis. In: Maudlin I, Holmes PH and Miles MA (eds.) The trypanosomiases. CABI Publishing, Wallingford, UK, pp. 369-402.

Shaw A, Torr S, Waiswa C and Robinson T (2007) Comparative costings of alternatives for dealing with tsetse: estimates for Uganda. FAO/PPLPI Working Paper, no. 40. FAO, Rome, Italy. Available at: http://www.fao.org/3/a-bp194e.pdf.

Shaw A, Wint W, Cecchi G, Torr S, Waiswa C, Alemu, T, Eregae M, Abdi A, Muchina M, Mugasi M, Mattioli R and Robinson T (2017) Intervening against bovine trypanosomosis in eastern Africa: mapping the costs and benefits. PAAT Technical and Scientific Series No. 11. FAO, Rome, Italy. Available at: http://www.fao.org/3/a-i7342e.pdf.

Shaw APM (2009) Assessing the economics of animal trypanosomosis in Africa - history and current perspectives. Onderstepoort J Vet Res 76: 27-32.

Shaw APM, Cecchi G, Wint GRW, Mattioli RC and Robinson TP (2014) Mapping the economic benefits to livestock keepers from intervening against bovine trypanosomosis in Eastern Africa. Prev Vet Med 113: 197-210.

Shaw APM, Tirados I, Mangwiro CN, Esterhuizen J, Lehane MJ, Torr SJ and Kovacic V (2015a) Costs of using 'tiny targets' to control Glossina fuscipes fuscipes, a vector of Gambiense sleeping sickness in Arua District of Uganda. PLoS Negl Trop Dis 9: e3624.

Shaw APM, Torr SJ, Waiswa C, Cecchi G, Wint GRW, Mattioli RC and Robinson TP (2013a) Estimating the costs of tsetse control options: an example for Uganda. Prev Vet Med 110: 290-303.

Shaw APM, Torr SJ, Waiswa C, Cecchi G, Wint GRW, Mattioli RC and Robinson TP (2013b) Reply to the letter to the editor by Bouyer et al. (2013). Prev Vet Med 112: 447-449.

Shaw APM, Wint GRW, Cecchi G, Torr SJ, Mattioli RC and Robinson TP (2015b) Mapping the benefit-cost ratios of interventions against bovine trypanosomosis in eastern Africa. Prev Vet Med 122: 406-416.

Shaw APM, Zessin K-H and Muenstermann S (1994) Modelling the economics of tsetse control using mono-pyramidal traps in Cote d'Ivoire. Kenya Veterinarian 18: 244-246.

Simarro PP, Sima FO, Mateo MJ and Roche J (1991) La lutte contre la trypanosomiase humaine africaine dans le foyer de Luba en Guinée équatoriale: bilan de trois méthodes. Bull World Health Organ 69: 451-457.

Sutherland CS, Stone CM, Steinmann P, Tanner M and Tediosi F (2016) Seeing beyond 2020: an economic evaluation of contemporary and emerging strategies for elimination of Trypanosoma brucei gambiense. Lancet Glob Health 5: e69-e79.

Sutherland CS, Yukich J, Goeree R and Tediosi F (2015) A literature review of economic evaluations for a neglected tropical disease: human African trypanosomiasis ('sleeping sickness'). PLoS Negl Trop Dis 9: e0003397.

Swallow BM and Mulatu W (1994) Evaluating willingness to contribute to a local public good: application of contingent valuation to tsetse control in Ethiopia. Ecol Econ 11: 153-161.

Tibayrenc R and Gruvel J (1977) La campagne de lutte contre les glossines dans le bassin du lac Tchad. II. Contrôle de l'assainnissment glossinaire. Critique technique et financière de l'ensemble de la campagne. Conclusions générales. Rev d'Elev Med Vet Pays Trop 30: 31-39.

Tirados I, Esterhuizen J, Kovacic V, Mangwiro TNC, Vale GA, Hastings I, Solano P, Lehane MJ and Torr SJ (2015) Tsetse control and Gambian sleeping sickness; implications for control strategy. PLoS Negl Trop Dis 9: e0003822.

Torr SJ, Maudlin I and Vale GA (2007) Less is more: restricted application of insecticide to cattle to improve the cost and efficacy of tsetse control. Med Vet Entomol 21: 53-64.

Vale G and Torr S (2004) Development of bait technology to control tsetse. In: Maudlin I, Holmes PH and Miles MA (eds.) The trypanosomiases. CABI Publishing, Wallingford, UK, pp. 509-523.

Van den Bossche P and De Deken R (2004) The application of bait technology to control tsetse. In: Maudlin I, Holmes PH and Miles MA (eds.) The trypanosomiases. CABI Publishing, Wallingford, UK, pp. 525-532.

Van den Bossche P, Doran M and Connor RJ (2000) An analysis of trypanocidal drug use in the Eastern Province of Zambia. Acta Trop 75: 247-258.

Wilson SG (1953) The control of Glosssina palpalis fuscipes (Newstead) in Kenya colony. Bull Entomol Res 44: 711-728. 


\title{
19. Acceptability of vector control actions or co-production of innovations?
}

\author{
Fanny Bouyer ${ }^{1,2,3^{*}}$ and Eduardo Chia ${ }^{4}$ \\ 'Unité Mixte de Recherche ASTRE 'Animal, Santé, Territoires, Risques et Ecosystèmes', Campus \\ international de Baillarguet, Centre de Coopération Internationale en Recherche Agronomique pour le \\ Développement (CIRAD), 34398 Montpellier, France; ${ }^{2}$ ASTRE, Univ Montpellier, CIRAD, INRA, Montpellier, \\ France; ${ }^{3}$ 3Finnovation S.A.S., 9 rue des Coteaux, 68550 Saint-Amarin, France; ${ }^{4}$ INRA UMR Innovation \\ 0951, 2 place Vialat, Bat 27, 34060 Montpellier, France; fanny.bouyer@3finnovation.com
}

\begin{abstract}
Concerning the insect pest management, two different views of the world face each other: those of the partisans of the social acceptability and those of the co-conception of innovations. There is no definition of the concept of acceptability, which can appear either a grey area or polysemous, with managerial origin and inputs from political philosophy, sociology, social psychology and political sciences. In a first part, thanks to an analytic frame based on the simplified actor-network theory (ANT), we will analyse how the question of acceptability was addressed in 3 situations of vector control. The studied vectors include screwworms, tsetse, and stomoxes, and implemented control packages include innovation transfer, partial co-production and innovation by users. Thus, we will see that we need to use an accurate theory of innovation to understand innovation processes to support them. At last, we will discuss how necessary it is to go beyond acceptability to appropriation and co-production when symbolic acceptation of methods is not sufficient.
\end{abstract}

Keywords: actor-network theory, appropriation, footbath, participation, screw-worm, stomoxes, tick, tsetse

\section{Introduction}

In insect pest control, the partisans of the social acceptability face the partisans of the coconception of innovations. In a context where participation is ever-present, the social acceptability of insect pest control strategies became one of the main concerns of politics and researchers. The purpose is the acceptation of the technical measures mostly implemented by the research and in particular the direct and indirect effects of insect control. However, instead of only consulting the population about an exogenous product (the opinion), we can choose to associate the people to the process of co-conception of acceptability in order to reduce the risk of failure.

Relations between insects and humans are paradoxical. On one side, insects and humans conducted battles for centuries, without ever winning the war, according to Lindquist (Lindquist, 2000). On the other side, they achieved alliances in order to live together (Akrich et al. 2006; Dupé 2015). Whereas the vectors ways of adaptation are variant and powerful, comparable to high technology, humans have had for a long time only a restricted access to high and adaptive technology, new strategies being mainly limited to new ways of using insecticides within the global framework of a defensive strategy (Lindquist 2000) while, despite many successes, genetic control is still a purpose of socio-technical controversies.

In the case of livestock farming, vector control represents an individual concern, on the one hand, for the livestock keepers to avoid the harmful biting of insects and the associated diseases. On 
the other hand, it is also a collective concern for the governments and institutions in charge of controlling zoonoses, diseases submitted to an obligatory declaration and those whose sanitarian and economic importance justifies the intervention of the public institutions or the implementation of collective actions by associations.

Two main types of vector control strategies are distinguished by entomologists: on the one hand the conventional control, of defensive nature and issued by individual initiatives and, on the other hand, the area-wide insect pest management (AW-IPM), of offensive nature, which aims to the suppression or eradication of an insect species targeted at the scale of the population and which implementation is necessarily collective and coordinated.

According to the French Ministry of Research, the control of conditions of appropriation and thus of uses of technologies represents a major issue in the competitiveness race (Terrade et al. 2009). This view accurately corresponds to the innovation regime of the technoscientific promises into which the elaboration of public policies generally fit (Joly et al. 2013). Fontenille advocates for an integrated vector control approach that must be targeted, environment-friendly and accepted by the population (Fontenille 2008). The Centre National d'Expertise sur les Vecteurs underlines the importance of taking into account the acceptability of new technologies since the first steps of research to avoid blockages like for the GMOs in Europe. But there is no definition of the concept of acceptability, which can appear either a grey area or polysemous (Dupé 2015).

\section{The concept of acceptability}

The risk management cannot be seen only according to the economic dimension. In relation to its managerial origins, there is a huge suspicion (of manipulation) around this concept that became popular by the managers of big projects of land settlement in the 90 's. So it is necessary to disconnect this concept from the managerial field to get it appropriated by the social sciences (Mayaux 2015). Moreover, the sociological analyses are necessary as the economical point of view appeared to be ineffective to explain many cases of non-adoption of innovation or social protest (Bouyer et al. 2011a; Mayaux 2015): a positive economical, technical or material impact is not sufficient to obtain acceptation of innovation.

Starr declares that the political driver is perhaps the most important determinant of the feasibility of risk management (Starr 1985), what parasitologists called 'contingencies' (Klassen 2000). He advocates for a comparison by quantitative assessment of risk probabilities and consequences (of several strategies) that would be used to give better information and to increase the public's confidence in new technologies.

Political decision makers or technicians of control implementation mobilize very often the political dimension of acceptability as a concern. The non-acceptation of new vector control strategies is a danger with several aspects: a political danger and a technical one. But who is concerned by the acceptability? The concept of public does not refer to a homogeneous group of people, so several groups of actors must be distinguished.

The question of acceptability stands for different actors. In the case of the pharmaceutical industries, the goal is to increase their sales and drive their research-development activities. In a commercial view, the research of social acceptability stands as a conquering and manipulative logic, and the sociology of uses appeared by consequence as a mercantile avatar (Roy 2013). In the case of vectors control, livestock farmers have to take decisions of collective actions in the 
framework of participative democracy and the public institutions to build public policies. For governments, the various approaches can be categorized within a large scale of participation, from sensitization to collaboration. Some strategies are entirely implemented by professionals of vector control without an active participation of beneficiary populations (or the groups concerned, as the livestock farmers), which adoption of the strategy relies on the principle of acceptation, with an ethical or political dimension. An unpopular action can get the initiative illegitimate and then the actors as illegitimate representative. However, some insect management strategies are implemented by the actors impacted by the vectors (general population, livestock farmers, etc.) or need combined actions as very well illustrated by the control campaigns against the human arboviruses or the control of stomoxes. The question of acceptability stands in a more complex way when the impacted actors must implement new practices, new tools and strategies. Their effective appropriation can be very constraining in the case of area-wide management.

Acceptability appears as a fantastic and sprawling 'kraken' for the trail-blazers (Roy 2013): fantastic in the relation to the difficulties of anticipation, sprawling for the difficulties of control. Many very ambitious and mythic projects (such as the Great Wall of China or the pyramids) have been driven acceptable. Thus this issue could be seen as an ideological one in relation to its practicalsocial function more than the theoretical or knowledge function (Roy 2013). Acceptability has, however, many different dimensions according to the sociology of innovation, political science and psychology and the social resistance to innovation should be seen as an exciting element that obliges innovators to take all these dimensions into account.

\section{General inputs of political philosophy and sociology}

The political philosophy, following a long tradition from Platon to John Rawls, interrogates the legitimacy of governing. Why do people accept the rules and laws of the society, per example in the case of AW-IPM, thus submitting themselves to the Leviathan (state, sovereign or tyrant), who monopolizes the speaking, the will and thus practices violence or coercion (Callon et al. 2014)? According to the political sciences, the question of acceptability fluctuates between the physical constraint (coercion, domination, slavery) and the moral constraint (awareness, advertisement, propaganda, manipulation, and disinformation). The moral constraint is not indeed less violent than the physical violence, but violence cannot sum up a process that requires an active enrolment to get a stabilized situation. The people must not be reduced to weak and ignorant crowds as rulers can always be knocked down (Callon et al. 2014). On the other hand, the liberal tradition heads toward overestimating the free will of individuals and underestimating the relations of power and leads to the conclusion that rejection of innovation is those of the progress that cannot be free or rational (Roy 2013). However, since Aristote and the Greek philosophy, the weak of the will and the fact that people can act against their interest (the 'akrasia' phenomenon) is taken into account.

In an uncertain world, in front of many crises linked to the management of sciences and technics (such as the bovine spongiform encephalitis, GMOs, Zika, etc.), the limits of the delegated democracy to manage the technoscientific challenges are questioned (Callon et al. 2014). Callon, Lascoumes and Barthes arise the question of the fairness of the decisions and the 'fair effect process' (the mechanism by which the people get convinced of the fairness of a decision rises to social psychology), which depends on the degree of control that the stakeholders have on the decision-making process. In the delegated democracy, the aim is to take into account opinions of the different groups, according to each point of view, and thus to get closer to impartiality. The decisions will be perceived as fair according to the degree of trust previously established. In that matter, the interest of formalized procedures is central. Moreover, when the concerned groups 
validate a decision as fair, the fair effect process spread to the others groups. Only the procedural justice can get the technical democracy possible, with fairness and effectiveness. The dialogical procedures of the hybrids forums are equitable procedures following principles such as the early involvement of profanes, the representation of the diversity/minorities, the clarity of the rules, the equality of information (Callon et al. 2014). These procedural innovations are the opportunity to create new conditions of science and decision making to face new challenges.

\section{Inputs of social psychology}

The changes of opinions, of alliances between actors, can be so fast and so extreme that it leads to the question of how opinions are built. What are the limits and mechanisms of the norm of acceptability? How to take into account the social determinants in the analysis of acceptability of new vector control technologies? The social acceptability is a very used concept, but despite not often expressed and rarely defined. Models of acceptability have been created to predict the uses, and the importance of distinguishing the analyses which aim to predict from those which aim to explain is underlined because the role of the social context is less prominent in the last ones (Terrade et al. 2009). Many competing models exist for predicting user acceptance of technologies. They are derived from the 'Theory of Reasoned Action' and from the 'Theory of Planned Behaviour', which are two classical models for predicting new behaviours. Behavioural intention and intention are mostly predicted by attitude, perceived behavioural control and subjective norms, in particular, the injunctive ones.

The importance of the individual acceptance described by utility and usability in the models is overestimated. The social psychology research considers that this is necessary to take into account 4 levels of analyses in order to predict the use of technology (Terrade et al. 2009): (1) the intraindividual level (intra-individual mechanisms which help to organize individual experiences); (2) the inter-individual level (the dynamic of inter-individual relationships for a particular situation); (3) the positional level (previous differences of social positions and the social organization); and (4) the ideological level (the role of ideological beliefs and culture). A multi-level analysis highlights how social norms and social context are essential to understand and predict user acceptability of technologies.

\section{Inputs of political sciences}

Social norms correspond to a 'should be': norms have meaning only if they include a part of a contingency, something that might not be (Roy 2013). Norms indicate which behaviours must be realized and they differ necessarily from the 'natural laws' which come under necessity. According to the concept of biological normality (capacity to stay in good health and to overcome crises) (Canguilhem 1943) and the synthetic theory of evolution, living things are considered to be an innovative process, the scientific, social or ethic norms must be able to evolve.

Otherwise, it has been observed that the paradigm of modern science and related new knowledge reshape the view of the society and thus politics (Roy 2013). Concerning the conception of environmental norms that should concern many countries or even be applied worldwide, Roy indicates the challenge to reach a consensus about the merits and so the degree of universality (Roy 2013). Vector control AW-IPM meets the same challenge when the scale increases.

The political science considers that acceptation corresponds to a normative compromise that can be a hybridization between old and new norms, a layering or a slow substitution contrasting with 
a quick change which leads to a normative confrontation (Mayaux 2015) and thus social protest. When decision-makers who are not the users decide an innovation, the change of norms can follow two different strategies: generating legitimacy or acceptability. The choice of one of these two strategies depends on the influence of previous predominant actors.

The work of legitimacy consists of convincing people of the compatibility of old with new ones or of the need to change the perceptions and the associated norms. Legitimacy relies on a general adhesion to some normative principles that are mobilized by rulers in their political speeches (Mayaux 2015). Thus a flourishing democracy is strongly associated to a popular legitimacy given to the rulers and their decisions. The legitimacy of norms is thus an ethical and political issue that is linked to the civilization project chosen (Roy 2013). According to the classical sociology of Max Weber (Weber 1978), legitimacy gives to citizens some normative reasons that justify their subordination, or their adhesion to the decisions of the rulers.

The work of acceptability describes a process of change to get acceptation of a situation (or a strategy) more than an adhesion to some principles. The work of justification is weak. Acceptation can be defined by tolerance, the absence of public and general mobilization. In fact, the concept covers a broad range of behaviours and intensity from the acknowledgment of full legitimacy, tolerance, disinterest, apathy, resignation, and fear of coercion. Thus the Britannic sociologist Colin Crouch links social acceptability to post-democracy: the public debate is focused on a limited choice of issues selected by professional teams of communication (Crouch 2004). Citizens are thus considered as a passive crowd submitted to react to stimuli sent by professionals (Mayaux 2015). A centralized political control is not the condition, but it corresponds to a non-democratic pluralism, to governance beyond the state (Mayaux 2015). The speakers are selected according to the conformity of their knowledge and perceptions, so that containment of debate or avoidance is observed. It leads to a democracy of low intensity in which the discreet civil disobedience (the hidden transcripts of Scott) allow reproduction of this kind of post-democracy (Mayaux 2015).

\section{Our approach}

For veterinarians, the use of a theoretical approach from the sociology of innovation has been recommended (Bouyer 2015) to study innovation in relation to the sanitarian context: the actornetwork theory (ANT) (Akrich et al. 2006). The ANT is a theory that allows a better understanding of the innovation process but it is not a method. It was mobilized in many different fields, such as medical controversies (Callon et al. 2014), management of natural resources (Denayer and Collard 2014) or even HR management (Dervaux et al. 2011). The ANT authors have shown the need for not separating the macro and the micro-actors in the analysis, in the continuity of Hobbes about the Leviathan. Adopting the principle of free association allows raising, with the same analysis frame, to the rationales of the actors, that could be economic, social, technical, political, etc. (Akrich et al. 2006). It allows understanding and analysing the swirling process of innovation without any a priori. The process is seen as a succession of enrolments and alliances between human and nonhuman actors shaping a network with different evolving functions and relations of power (Akrich et al. 2006; Callon 2001). The focus is thus done on the many negotiations that are successive translations of the identities and wills of the groups of actors by their spokespersons that allow action through alliances.

In the first part, we will see how the question of acceptability stands in different situations of vector management in relation with the implemented control packages. The common analytic frame will be based on simplified ANT (Vitry and Chia 2016): 
- starting objectives given to the control strategy according to the different points of view;

- the description of the enrolment package (their main evolution phases);

- the role given to the different stakeholder's groups;

- the main controversies or scientific questions which illustrate the redefinition of the human and nonhuman actors (the control package included).

In the second part, we will discuss the need of methods and approaches that allow a better understanding of the socio-technical network and could assist the swirling process of innovation. At last, we will discuss the importance of going beyond the question of acceptability to come up to those of appropriation. The co-conception of vector management strategies together with the beneficiaries is an alternative way to build consensus.

\section{Different situations of vector management strategies}

\section{The control of Cochliomyia hominivorax (new world screw worm) AW-IPM}

The biggest technical and economic success of a pest (flies with carnivore larvae) eradication concerning animal health (and secondarily human health) is the eradication of Cochliomyia hominivorax (Coquerel) from the North American continent up to Panama. The larval stages grow on the living flesh of warm-blooded animals, including human. A screwworm infestation can kill an adult animal in 7-10 days if not treated, so it is an important animal health constraint, a human health concern and an important economic constraint (Wyss 2000).

This program began in 1957 in the USA and ended in 2000 in Panama (Wyss 2000), where preventive releases of sterile males are still in course to prevent re-invasion. In 1996, the annual direct benefits (for producers) were assessed to be US\$ 796 million for the USA, 292 for Mexico and 77,9 for Central America (Wyss 2000). Moreover, the positive indirect effects are multiplied by 3,5: they thus correspond to US\$2,8 billion for the USA, 1 billion for Mexico and 272,6 million for Central America. The benefits to perpetuity are especially huge as a low discount rate of $3 \%$ (less than the actual cost of money) is customarily used for public-good program, with double impact on both animal and human health!

The concept of AW-IPM and the eradication by sterile insect technique (SIT) have both been created and redefined during this process. As the success is complete as well from an entomological point of view than from an economic one, we will see how the enrolment package has been build step by step and how the definition of the two concepts (AW-IPM and SIT) as well as the definition of the identity of the different actors have been shaped following a swirling process. The feasibility and acceptability were determined very late.

\section{The building of a network at the national scale}

The south-western part of the USA has been infected since a long time before the East of the Mississippi River and livestock farmers of South-west identified screw-worms as a severe pest for animals before 1933. But, it is after the introduction of infected animals and the quick spread of the pest in the east of Mississippi (Georgia, Florida, etc.) that the livestock industry requested help in controlling this severe pest. As a matter of fact, the observation of the losses by mortality, decreases in production and increases of veterinary costs by the livestock owners was tremendous in the situation of before/after introduction in the Southeast and has become the beginning of the enrolment of researchers by the livestock industry. 
In 1933, the work of the researchers began by giving a better definition of the parasite to control. The species was defined as different from the secondary blowfly, Cochliomyia macellaria (Fabricius). In 1934, the United States Department of Agriculture (USDA), the Agricultural Research Service (ARS), opened a research station in Georgia and assigned two researchers (E.W. Laake and E.F. Knipling) to this station. In 1935, another researcher (R.C. Bushland) was hired on the same topic in another centre located in Texas. In 1936, screw-worm colonization was studied and artificial media and technique developed. In 1937 the (new) concept of autocidal theory of screw-worm population suppression was discussed by Knipling and Bushland. The research work was suspended during the Second World War between 1937 and 1947. In 1947, the interest of a new control strategy was raised again. In 1950, Muller described the way of sterilizing insects by $\mathrm{X}$-ray in Drosophila. The following year, the sterilizing effect of X-ray applied to pupal stages was demonstrated for the two sexes of adult screw-worm by Bushland and Hopkins. These hypotheses were field tested two times in a small island off the Southeastern coast of Florida. The results indicated the efficiency of sterilization (by measuring the sterile egg masses). The effective suppression of the population was reached but without eradication. So a second field trial was initiated in a bigger island (Curaçao, West Indies) located farther from the continent (65 versus $3 \mathrm{~km}$ ) to test the role of the re-infestation and the feasibility of eradication by SIT. The protocol was adapted by increasing the density of sterile male released. In 1954, after several months of release, scientists gave the status of screw-worm-free to the island. This technical success (with the findings of Linquist in 1955) played a significant role in the enrolment package who sealed all the actors to get engaged in a strategy based on the eradication by SIT. The negotiation led to a cost-shared programme: one-quarter was paid by the livestock industry, one-quarter by the state, and one-half by the federal government (Klassen 2000). The climate variations of the year 1957 brought its stone to the building of the enrolment: livestock owners of the southeast experienced the worst losses from screw-worms since pest introduction during spring and summer 1957. During the same summer, a pilot field trial of control by SIT was conducted in Florida, which led to a reduction of $70 \%$ of clinical cases in the centre of the area $\left(5,180 \mathrm{~m}^{2}\right)$. Politics decided to begin an eradication campaign at the end of the year in the South-eastern USA. By luck, the winter 1957-1958 was one of the coldest and wettest winter in the history of Florida and contributed to the success of the early stage of the campaign. A new sterile fly facility was built to increase the number of sterile males released to take advantage of these favourable conditions. The last two cases detected (except imported cases) occurred in February and June 1959, less than two years after the beginning of the campaign; the dispersal of sterile males continued until November 1959 , and the status of screw-worm-free area was given at this time. Since this step, cases of infected animals were distinguished into two groups: imported or autochthonous. This distinction helped to adapt the control measures and the enrolment through the status of states.

\section{Growth of the network as solution to maintain its stability}

The success of the campaign in the Southeast have been observed with much interest by the livestock breeders associations and industry in Southwestern USA, who asked the Southwest Animal Health Research Foundation (SWAHRF) to start a similar campaign. The steps were: to get technical information, to get trained, to obtain support from the authorities (for the foundation), to struggle to get the authorization and to raise producer-contributed funds. The authorization, obtained in February 1962, was a necessary condition to begin the expanding of the existing facility in Kerrville, Texas by the Animal Disease Eradication Division of the USDA and to build a new one in Mission, Texas, by the SWAHRF in June 1962. The alliance with sterile insects appeared through the increased capacity of 200 million sterile males per week obtained before the beginning of the campaign. The campaign began in Texas and New Mexico and then was expanded to Arizona 
and California after getting the screw-worm-free status by the two first states. Meanwhile, during the first months of 1966, no screw worms case was observed, the cattle industry and the state governments persuaded the USA secretary to declare the entire USA free of screw-worms (Klassen 2000). At this time, the new world screw-worm became an exotic pest: in consequence, the federal government became solely responsible for any further control in case of reinfestation and would endorse any costs. It was the end of the cost-shared programme. However, the observation of screw-worm cases continued at a low level, even at the end of the year 1966. Up to this date, the pest population to fight had been the population of each state. After getting the status of screw-worm free, the new goal was to prevent reintroduction and the population targeted has then become the flies migrating from Mexico as well as the larva infesting the cattle coming from Mexico too. So weekly dispersals of sterile male along the border were used to maintain a sterile fly biological barrier and inspection of animals entering with cattle dipping into insecticide were organized. Discussions with the Mexican government began in 1966 to build an agreement and organize the control of screw-worm in Mexico in order to implement a real eradication strategy.

Up to this step, the protocol (the 'alliance' between all the actors to control the wild flies in the USA) seemed to be quite efficient but the 'betrayal' of the flies obliged to rethink the protocol entirely: in 1968, the number of cases increased up to 9,877, and in 1972 a significant outbreak reached the 'pre-eradication' levels. Some imported cases continued to occur up to 1982 . The raising question was more how to redefine the screw-worm population to control and the location of the barrier than to change the control methods.

For the American livestock industry and the American scientists, the problem to solve became how to change the location of the barrier (and the definition of the screw worm population to control would be deduced). As the border between the USA and Mexico is long $(2,400 \mathrm{~km})$, the reintroductions have shown the lack of effectiveness of this barrier. To find a smaller barrier would ease the feasibility and decrease the costs. The representatives of livestock producers were interested in controlling the pest in Mexico so that the enrolment of all actors was easier. The Isthmus of Tehuantepec in Mexico was identified to build the barrier of $190 \mathrm{~km}$ instead of the previous $2,400 \mathrm{~km}$. An agreement was signed on the 28 August 1972 to create the Mexico-United States Commission for the Eradication of Screw-worms (Commission). The primary task of the Commission was to build a new facility and to organize the eradication southwards down to the Isthmus of Tehuantepec in South Mexico. The campaign began in 1972 using the sterile flies produced in Texas. The Mexican sterile flies rearing facility was inaugurated four years after the 28 August 1976 in Chiapas by the Secretaries of Agriculture from the USA and Mexico. Although the need to maintain the barrier at the Isthmus was 150 million flies per week, the rearing facility had a bigger capacity in comparison with the facility of Mission, Texas, which closed in 1982.

In the early 1970s, a huge controversy about the eradication feasibility arose in the American scientist's community in relation to the large-scale experiment mounted by the USDA on the feasibility of the eradication of the boll weevil of the USA (without SIT) (Klassen, 2000). The impact on the concurrence between states for the cotton production was an issue. The USDA was convinced regarding the screw-worm experience that an iterative fashion could lead to eradication if suppressive measures were iteratively applied to surviving populations. Concerning the boll weevil the experiment was asked by the cotton industry who sealed an alliance with the USDA but neither the experiment's Technical Guidance Committee, a committee of the Entomological Society of America nor a committee appointed by the National Academy of Sciences, recommended implementation of an eradication programme. The eradication feasibility 
by SIT for the screw-worm was still the purpose of debates with however some scientists more involved (in comparison with the boll weevil).

Following this controversy, in 1977, a controversy shared in many American universities was voiced by Dr L.D. Newsom (Louisiana State University) who criticized the gap between the free status and the true prevalence and thus the way this status was given 'by fiat but not in fact'. He stated that the research work had been insufficient, that the eradication was not reached in the USA as the programme was extended to other areas without any guaranty of the feasibility. He took the role of spokesperson for scientists who felt a concern for the credibility of the American community of scientists threatened by the authoritative pronouncement of the USDA. In 1982, the real eradication was reached in the USA as no more imported cases were observed.

\section{Shift of the network to maintain his stability}

Then the eradication of screw-worm in North Mexico to the Isthmus of Tehuantepec was achieved in 1984, but a social tension was increasing in the South: the livestock farmers reproached the government to neglect them, in comparison with the farmers north to the barrier. Further feasibility studies were conducted and led to the conclusion that Panama (from the Panama Canal to the border with Colombia) was a better site for a permanent barrier. A new enrolment was built by scientists with the following arguments: the entire country of Mexico will be free of screw worm and the livestock breeders of the South will support the initiative, the number of sterile males needed to be dropped from 150 million to 40 million (the financial support needed would decrease), the efforts of inspection would decrease as the cattle movements used to be much less in this area. The conditions were favourable for negotiation with countries of Central America who all showed signs of interest.

In 1985, a technical proposition was elaborated to plan and coordinate national campaigns of eradication in Guatemala and Belize in a first step, in El Salvador and Honduras in a second, then Nicaragua, Costa Rica and Panama (Table 1). First, the eradication campaign was enlarged to the entire country of Mexico, which was not screw-worm free when the campaign began in Guatemala and Belize.

The prominent elements of the global implementation are a very good coordination and a quick implementation based on field results. Agreements were signed between the ministries of agriculture of each nation (in 1896 for Guatemala, in 1988 for Belize, in 1991 for El Salvador, in 1991 for Honduras, in 1991 for Nicaragua, in 1993 for Costa Rica and 1994 for Panama) and the Commission or the USDA (Table 1). The campaigns began quickly after signature and before the end of the campaign of the previous country (in the order from north to south) (Table 1). The peak of aerial releases was generally maximal (with a maximum of sterile flies between 24 and 120 million) the first year and then decreased. The releases began in the north before being over the entire country except for Honduras and Panama where the releases began in the southwestern and western parts respectively for epidemiologic reasons. The surveillance system revealed the maximum number of cases the first year of campaign (except for Honduras where the surveillance system was slower to implement), due to greater surveillance efforts and then a regular decrease. The results shared by the scientists (with a distinction of autochthonous and imported cases) were an important intermediary object to adapt the technical measures and to keep the alliance between all the stakeholders. Moreover, the livestock farmers did generally observed less clinical cases after the beginning of the campaigns. The last cases did rapidly been noted after the beginning of the releases (five years for Guatemala, two for Belize, three for El Salvador, four for 


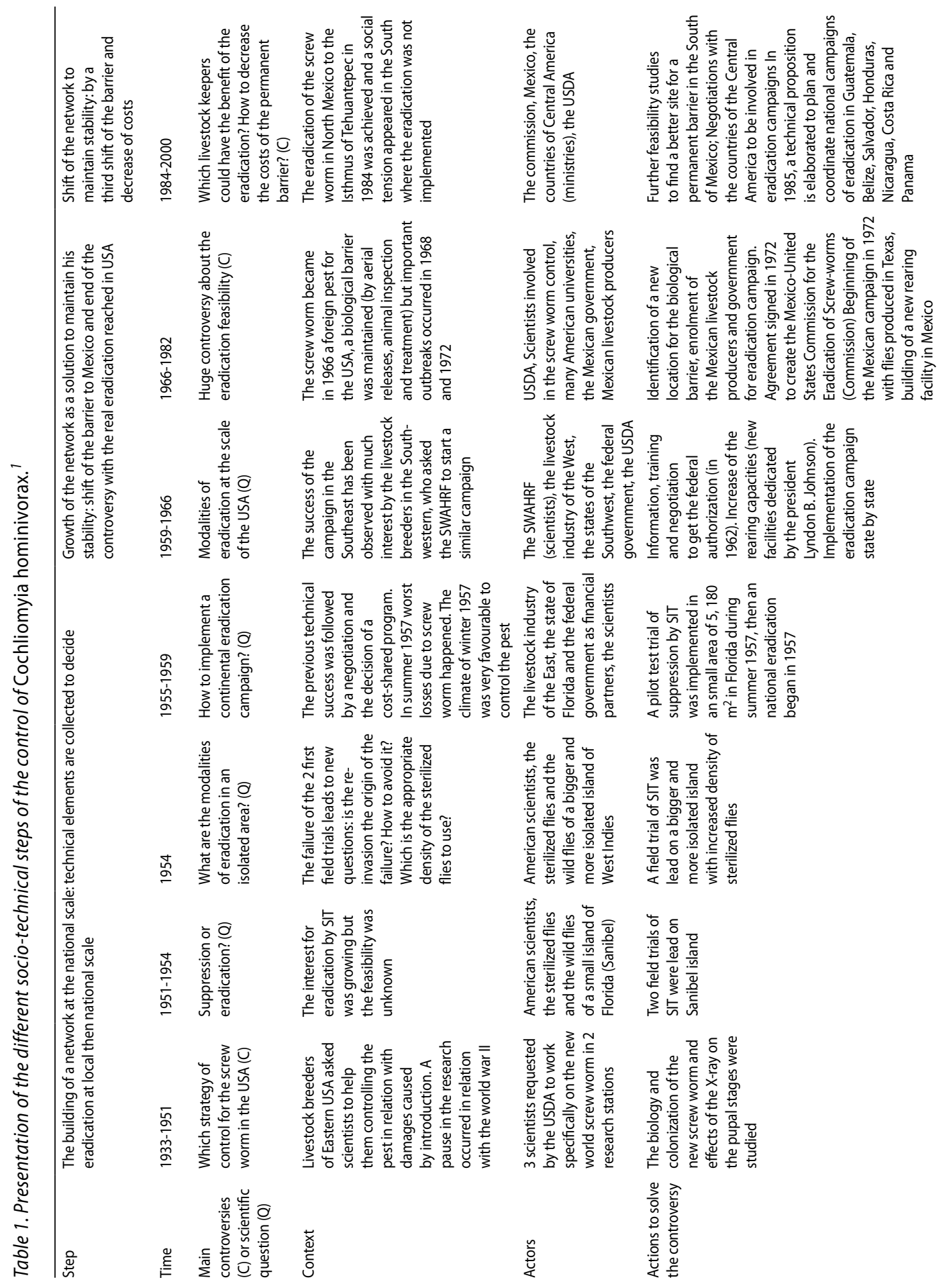




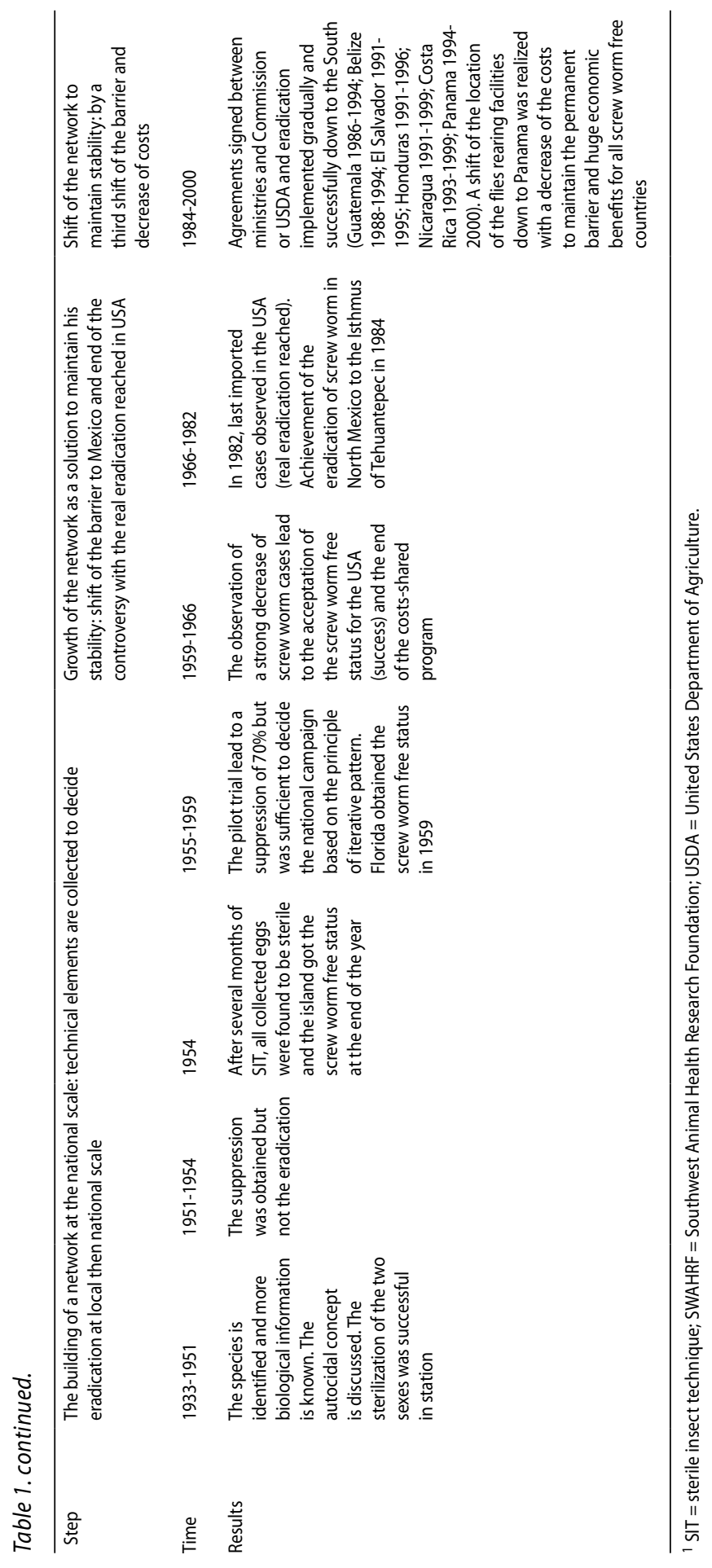


Honduras, five for Nicaragua, three for Costa Rica and Panama) and the screw-worm free status given quickly.

Honduras was declared screw-worm free only in August 1996 (20 months after the last case) and a small contingent was still in activity for prevention and surveillance activities. Ever more precaution was taken in Nicaragua: the free status was given much later than the others countries at the end of 1999 (2.5 years after the last case). Surveillance activities and prevention of reinfestation continued at a much lower level up to 2002.

The agreement between the USDA and the Ministry of Agriculture and Livestock Development of Panama was different of the others as it concerned the establishment of a permanent biological barrier too. The building of a sterile fly facility was planned. The regional screw-worm programme office was relocated from Mexico to Panama in July 1994. Field surveillance activities began one year later. Releases of sterile males began in the western part of the country in July 1998, using over-flights out of Nicaragua. Then a new dispersal centre was implemented near Panama City, and releases covered $50 \%$ of the country in October 1998, using this new centre. Negotiations began to build a new sterile fly production plant east of Panama City to close the one of Mexico. The commission argued that the costs of operating and maintaining the plant were increasing and the costs of transport were too high. A concern for Mexico scientists was the danger of fertile flies escaping from the plant located in the middle of the screw-worm free area. The eradication activities were stopped in 2001 and since 2002 only activities of containment have been going on (Table 1).

\section{Failure of the enrolment of countries of Caribbean region and South America: solidification of the socio-technical network in Panama}

Since 1995, the Caribbean region is seen by scientists and International Atomic Energy Agency (IAEA) as 'the next most logical area to eradicate this pest. Of all the Caribbean Islands, only Cuba, Hispaniola (Haiti and Dominican Republic), Jamaica, and Trinidad-Tobago are infested with screwworms. Their proximity, with the exception of Trinidad-Tobago, to the USA, Mexico and Central America makes them a possible source of re-infestation for the screw-worm-free countries' (Wyss, 2000). Parasitological studies showed that Cuba was the largest infested Island (from 1995 to 2003). An agreement was signed between the government of Cuba and the FAO to plan a suppression pilot trial on the Isla de la Juventud followed by an island-wide effort with SIT (Garcia et al. 2007). An economic study was lead to estimate the costs of eradication and the benefits (Grant et al. 2000). The Instituto de Medicina Veterinaria implemented the trial and IAEA provided technical assistance through a technical cooperation project. On the one hand, the technical results used to indicate that a properly implemented insecticidal wound treatment programme can lead to an effective suppression (as seen in the North) but bad results in the South, where the density of livestock was low, were observed. Following this result coupled with political difficulties, the SIT campaign was not initiated as planned.

In Jamaica, an eradication campaign was undertaken in 1996 and cooperation was established between Jamaican Veterinary Services Division, IAEA, Food and Agriculture Organization of the United Nations (FAO) and USDA in 1997, but little progress were obtained until 2005. Several difficulties were met in particular concerning the communication and organization. This program was mainly funded by the USA at the beginning and the involvement of the local actors (in particular the livestock owners represented by the Jamaica Agricultural Society) and their coordination was insufficient. The public outreach campaigns were funded inadequately and 
by consequence implemented only sporadically. The message delivered to the livestock farmers used to be inadequate at the beginning of the project: at first, the farmers did not perceive the importance of the preventive wound treatment (and so their active participation) and they used to think that the SIT was sufficient for eradication. In relation to the lack of full-time staff and the low involvement of the livestock farmers, the field data collection was inadequate and not systematic and the passive surveillance and active monitoring (based mainly on the 'reported screw-worm cases) ineffective. The backup systems and contingency plans were inadequate: only one dispersal centre was available, and material was lacking, all these problems used to be disruptive for the campaign.

Others feasibility studies had been lead: a genetic one in Uruguay (Torres et al. 2007) as well as economic studies in order to involve other countries into eradication approach (Vo 2000). These studies were supported by IAEA or FAO (Grant et al. 2000).

\section{Conclusions}

The American livestock industry (powerful representatives of industrial farmers) used to have an active role to initiate the eradication campaign, by making the enrolment of some scientists and politicians. The alliance was strong through the costs-shared program and allowed to pass over the long period of doubt of the scientific community about the eradication feasibility. Then, the USDA and the Commission took the relay by negotiating with the others countries the shift of the barrier. The technical and financial support to the countries down to Panama was important for maintaining the stability of the new sanitarian situation: in a first time, the strategy relied on the growth of the socio-technical network (shift of the barrier to Mexico and end of the controversy concerning the eradication feasibility thank to the real eradication reached in the USA) then it relied on the shift of the control area in Panama. This strategy fitted to the view of agricultural development of the political leaders (and society) and the sustainable economic and sanitarian impact on national scales was sufficient to contain the question of acceptability to negotiations of the financial modalities of short time campaigns (except for Panama). The failures in the Caribbean region had shown that a strong involvement of the livestock farmers and politics that must give accurate human and material means is essential and highlight the way they are enrolled.

Note: After an accidental introduction of $C$. hominivorax in Libya, a successful eradication was lead in Libya with a military discipline between 1988 and 1992 but is not detailed here. It represents an example of excellent monitoring components based on the American experience (Vreysen et al. 2007).

\section{The control of tsetse and ticks by acaricide-insecticide footbath}

This control method against tsetse and ticks has been designed on the basis of new knowledge of vector ecology. The study of the innovation process revealed how the question of the acceptability of the method (by the livestock farmers and the cattle) has been led by the researchers in terms of adoption, even though the process revealed an appropriation which could be explained by the ANT and which showed the importance of innovation by the users (humans and non-humans). 
A new concept based on a set of observations and trials about the behavioural ecology of Amblyomma variegatum

Initial observations about the behaviour of Amblyomma variegatum Fabricius were made during the 1994 rainy season at the Centre de Recherches Zootechniques et Vétérinaires de Wakwa, Cameroon (Stachurski 2000a). A. variegatum is a species of ticks of animal health and economical importance for cattle and small ruminants in Africa. The first observations done on a small sample of cows grazing daily on an enclosed pasture and kept nightly on another pasture led an acarologist of the French Agricultural Research Centre for International Development (CIRAD) to the hypothesis of temporary attachment of ticks to the interdigital areas during the grazing time. The idea of a targeted tick control, using a footbath, emerged at this moment but the hypothesis regarding the invasion process of cattle by the tick needed to be confirmed with animals managed according to the traditional practices, i.e. grazing during the day and lying down only once back in the night pen. Complementary experiments were carried out in 1997 and 1998 at Banankélédaga Station, Burkina Faso and confirmed the hypothesis. Then, the paper 'Invasion of West African cattle by the tick $A$. variegatum' based on trials implemented in both Cameroon and Burkina Faso was published.

In 1997, at the same time as the study concerning tick behaviour, the first trial of targeted treatment, based on spraying of acaricide on the limb ends thanks to a portative spray (as it was cost-effective), was implemented (Stachurski 2000b). The treatment based on local spraying of flumethrin in the morning appeared not to be efficient, in relation with a low impregnation during the spraying, an early rinsing in the river leading to a short persistency. The additional problem of cross infestation between treated and untreated cattle kept in the same night pen was discovered after this trial when night movements of ticks from one animal to another were observed.

A new method of targeted insecticide treatment was thus tested in August 2000: a first footbath was built near the night pen of two traditional cattle farmers, located in the peri-urban area of Bobo-Dioulasso, the protocol was defined and implemented for assessing the ability of the treatment to limit ticks infestation to predilection sites. Two herds were treated every two and then three days, when back from the pasture, by passage in the vat of the footbath filled on average by $20 \mathrm{~cm}$ depth of a flumethrin formulation. A first economic comparison of different control methods (spraying, pour-on, dipping tank, footbath, targeted spraying) showed that control by footbath was the less expensive (by head and by season) (Stachurski 2000b) with the targeted spraying but this strategy appeared not so easy to implement and with a less important impact on the tick infestation rate. Academic debate concerned the ratio effect/cost, the initial investment and depreciation expenses.

A second footbath with modified dimensions (without pre-footbath cleaning vat) was built in another settlement and used since 2001. The acarologist considered the building of two footbaths as a gift to livestock farmers who used to work with him for other trials. Some farmers asked these livestock farmers the right to treat their drought bulls and used it at a different frequency (one time a week). The evolution of infestation was monitored thanks to the data recorded in 2001 by the use of the second footbath. The crossed analysis of results obtained in 2001 and 2005 with the first footbath added to the results of the second obtained in 2001 (Stachurski and Lancelot 2006) confirmed the ability of the treatment (with the defined frequency) to limit ticks infestation to predilection sites. An important work 'behind the scenes' of the trial was done by the acarologist with the livestock farmers to design a method of maintenance, management documents, a way of training the cattle to go through the footbath and a way of building the 
waiting pen. A second economic assessment showed that the treatment was even less expensive with the second footbath than with the first footbath (Stachurski and Lancelot 2006; Stachurski et al. 2005). At this step, the controversy raised by the acarologist was the constraint represented by the distance that each herd must walk from the footbath to the night pen and the initial investment (for building the footbath). The idea of collective investment and use by an association (or settlement) appeared as a solution to researchers (Table 2).

At this time, the method based on footbath appeared as an efficient, cost-effective and easy to use strategy for sedentary or semi-transhumant herds. Moreover, interest in controlling tsetse and mosquitoes was cited as an opportunity to assess. In the same research centre (CIRDES - Centre International de Recherche-Developement sur I'Elevage en zone Subhumide), this hypothesis was discussed between the acarologist and an entomologist (both of CIRAD) who used to observe the tropism of tsetse for distal parts of human and animals.

\section{From tick to tsetse outdoor experiments: a redefinition of the concept}

The tsetse entomologist of the CIRAD decided to study the tropism of tsetse (Glossina palpalis gambiensis Vanderplank and Glossina tachinoides Westwood) for distal parts of cattle legs in a first step (October 2002) and, in a second step, the effectiveness of the tsetse control based on local insecticide treatment. So indoor experiments to compare the effect of insecticide administration with a footbath versus a manual sprayer were implemented from April to June 2003 (Bouyer et al. 2007). The interest of the scientists for insecticide-treated cattle for controlling mosquitoes and mosquitoes-borne diseases were growing (Habtewold et al. 2004).

The director of the ARIOPE (Projet d'Appui au Renforcement Institutionnel des Organisations Professionnelles d'Eleveurs modernes) project of the French Embassy began to discuss with the entomologist of CIRAD to find technical solutions to the trypanosomias constraint. His interest for acaricide-insecticide footbaths led to introduce debates into livestock farmers' associations of Bobo-Dioulasso facilitated by the assistant of the project (about animal health constraints and solutions, as well as a technical presentation of footbaths).

\section{Change of scale from a socio-technical network supported by research to one supported by both research and development projects}

Constructions of footbaths supported by the ARIOPE project began in 2004: for the traditional livestock farmers, a collective use of the footbaths was proposed by the project, which supported $80 \%$ of the building costs ( $20 \%$ was supported by the association, and help for the building was asked); only two associations of livestock farmers asked for the construction the first year. A workshop was then organised by the CIRDES about the topic 'control of the tick A. variegatum' to which researchers, representatives of groups of livestock keepers (of associations of BoboDioulasso and Ouagadougou) and development agents were invited in May 2004 (Stachurski et al. 2005) (Table 2).

In 2005, footbath effect on the apparent tsetse density (field conditions) was studied near a water point in a livestock farmer's settlement located $15 \mathrm{~km}$ north of Bobo Dioulasso, from May to June, i.e. at the beginning of the rainy season. As the settlement was chosen on scientific purpose, the livestock farmers of the settlement were 'enrolled' by the regular presence of the scientific team of the CIRDES (for the monitoring of its efficiency). The natural tsetse-fly population present around this water point was considered as isolated from other tsetse-fly populations and cohorts of reared 


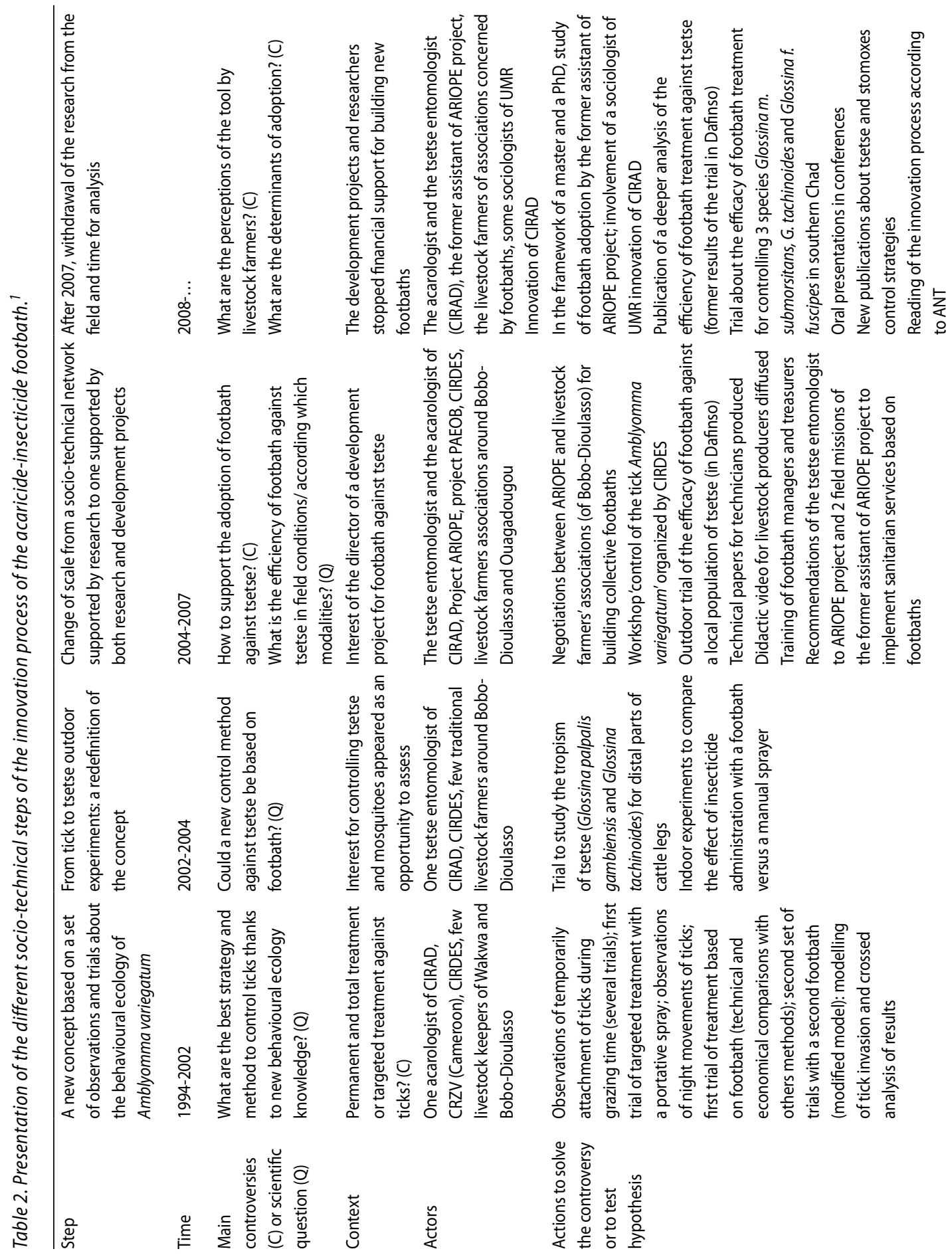




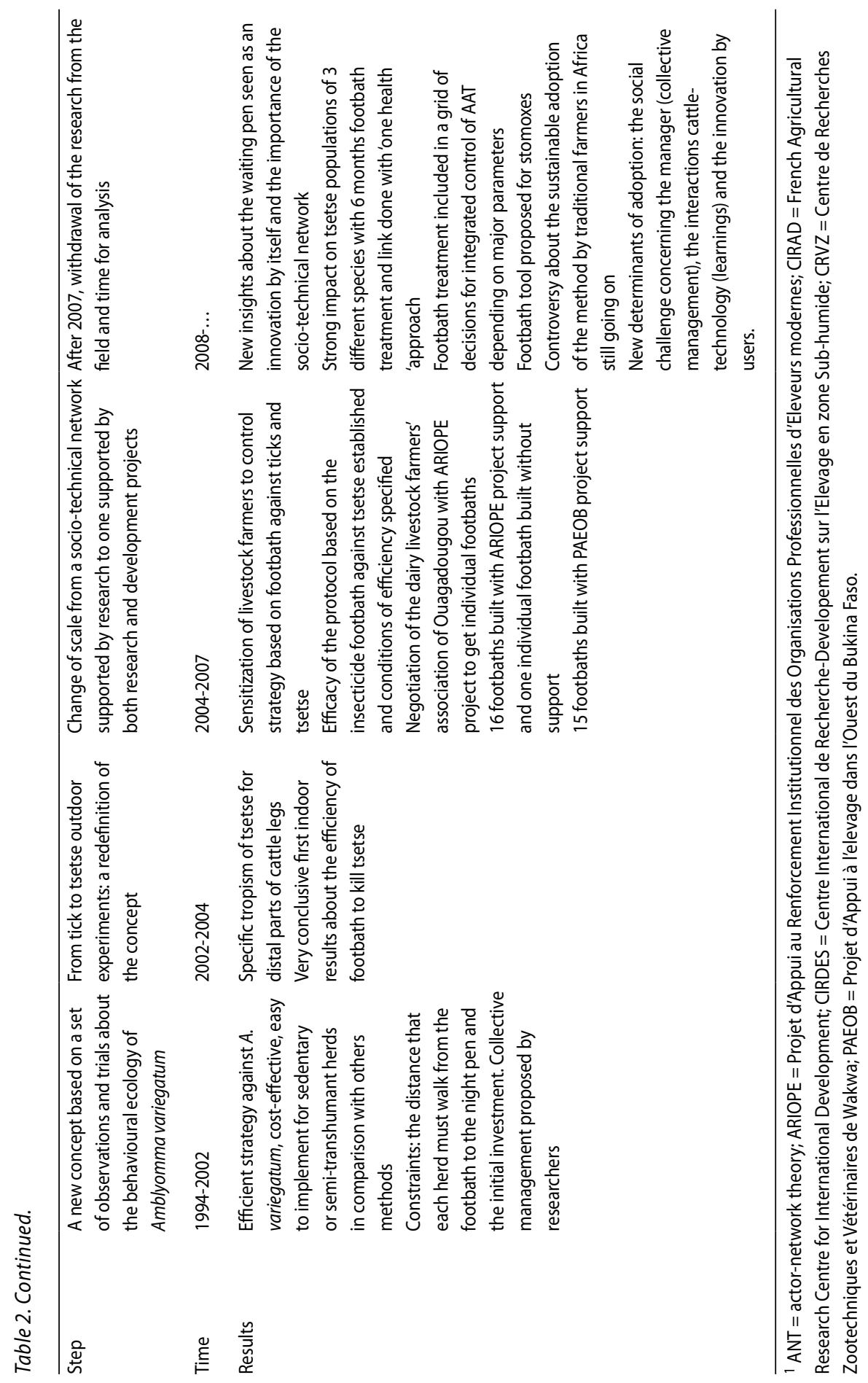


sterile male flies were released at this point: the apparent density of wild flies was modelled, and daily mortality was assessed thanks to the sterile males. The efficacy of the protocol based on the insecticide footbath was established and conditions of efficiency specified (Bouyer et al. 2007).

In 2004-2005, technical papers concerning acaricide-insecticide footbath treatment and insecticide treatment were produced (the targeted public was 'decision-makers, researchers and development actors') in the framework of a European project devoted to the transfer of innovation (Bouyer et al. 2005; Stachurski 2005). A didactic video explaining the construction and the use of the footbath was produced by CIRDES in French and Dioulà and diffused in the livestock farmers associations of Bobo-Dioulasso and Ouagadougou in 2005. The main concerns of the acarologist were the ability of livestock farmers to train the herds to go through the footbath, the training of the managers to do the maintenance and the respect of the protocol. As the livestock farmers appreciated the quick knock down effect of treatment based on amitraz, the acarologist wanted to test the hypothesis about the weight of the perception of the footbath effect on ticks according to the livestock keepers (that allows the invasion of some ticks, and thus keeps an efficient immunity against cowdriosis, babesiosis and anaplasmosis) as a determinant of adoption. At first, the associations seen as potential beneficiaries of the ARIOPE project were those of Bobo-Dioulasso but the association of intensive dairy farmers of Ouagadougou showed interest for this control method against ticks and stomoxes (as the trypanosomian risk is absent around Ouagadougou) and individual constructions were allowed (and supported by the project) under the condition of financial participation. The main concern of the project was the technical training of one manager for each footbath and the implementation of a sustainable collective management (in particular for the financial aspects). Some improvements of the tools of management and the method were done thanks to the interactions between researchers, associations and development agents during the implementation of 'the sanitarian services based on acaricide-insecticide footbaths'. The association of dairy livestock farmers of Ouagadougou decided to train their technician to the maintenance of footbaths and to get available a specific service of footbath maintenance to their members. In 2005, 12 footbaths were built in settlements of Bobo-Dioulasso and Ouagadougou. In 2006, only two supplementary footbaths supported by the project ARIOPE were built, and an intensive livestock farmer of Ouagadougou built one footbath without support of the project. This event was seen as a sign of success.

In January 2007, following the recommendations of the expertise of the entomologist, the project PAEOB (Projet d'Appui à l'elevage dans l'Ouest du Bukina Faso) that targeted the development of livestock farming in the Western South of Burkina Faso at a larger scale than the peri-urban area asked two field missions to the former assistant of the project ARIOPE in order to support the implementation of collective services based on the acaricide-insecticide footbath (Bouyer 2007, 2009). 15 footbaths were built in 2007. Meanwhile the conciliation and signature of contracts between livestock farmers and the project were a main concern for the assistant, many learnings were realized in relation with the implementation of the footbaths The betrayal of the technical obligations, the absence of use of wire fence for the waiting pen, and a new initiative of the farmers (building the waiting pen with wood like a night pen) gave new insights about the interactions between humans, cattle and technologies.

\section{After 2007, withdrawal of the research from the field and time for analysis}

In 2007, the former assistant of the project ARIOPE took the decision of studying the adoption of the acaricide-insecticide footbaths in Burkina Faso in the framework of a Master 'Integrated management of natural resources' at the University of Bobo-Dioulasso. All the potential 
beneficiaries of the 22 footbaths (built before the rainy season of 2007) around Bobo-Dioulasso and Ouagadougou were questioned in 2008. The first analysis was based on variables describing the livestock farming system, the implementation and perception of the method and the knowledge of the epidemiological system. These multivariate analyses allowed discriminating three clusters of cattle farmers that were then compared using indicators of adoption. As the identification of the group of intensive dairy farmers of Ouagadougou as good innovators was obvious, the novelty was the discrimination of the more traditional farmers into two clusters, one of which showed a good adoption rate, whereas the second failed to adopt the method. The economic benefit and the farmers' knowledge of the epidemiological system appeared to have a low impact on the early adoption process whereas some practices of intensive farming, as well as social factors appeared critical. The high variations in risk perceptions and benefits were put in relation with the geographical position, to the quality of technical support provided to the farmers and led the researchers to the approach by the socio-technical networks (Bouyer et al. 2011b).

In 2009, a deeper analysis of the results of the trial conducted in Dafinso was published. It gave a better assessment of the effect of the restricted application of insecticides on cattle to control trypanosomosis and allowed to discuss further the conditions of efficiency (Bouyer et al. 2009b). The same year, the efficacy of restricted application of insecticides on cattle by footbath for controlling three species Glossina morsitans submorsitans Newstead, G. tachinoides and Glossina fuscipes fuscipes Newstead was tested in southern Chad (Ndeledje et al. 2013). It was demonstrated that the impact on tsetse populations was strong with a reduction of $80 \%$ of the local population by the end of the 6-month footbath treatment. So the interest of the method for the 'One Health' approach was discussed as it could be a very important innovation, as a new use of footbath for its impact on human health (Ndeledje et al. 2013).

In December 2009, an oral presentation of the adoption of the footbath was done in a conference about innovation organized by researchers of the UMR Innovation of CIRAD and CIRDES (Bouyer et al. 2009a). The debate opened interesting insights about the waiting pen seen as an innovation by itself and the importance of the communication network. Contacts were taken with sociologists of the UMR innovation of CIRAD.

The control method based on footbaths was taken into account for general recommendations and included in a grid of decisions for integrated control of animal African trypanosomiasis (AAT) depending on major parameters (type of eco-epidemiological cycles, breeds of cattle) in a sustainable way (Bouyer et al. 2010, 2013). But the controversy about the sustainable adoption of the method by traditional farmers in Africa is still going on (few information concerning the use of footbath by livestock farmers is available since 2008). The footbath tool was proposed for others vectors (stomoxes) in other contexts (La Réunion Island) (Bouyer et al. 2011c).

In the framework of the thesis 'Trypanosomian risk and innovation: the case of livestock farmers in West Africa', a reading of the innovation process according to ANT (Akrich et al. 2006; Callon 2001) was suggested to be adapted for a better understanding of the innovation process by the thesis co-director of the UMR Innovation (CIRAD) (Bouyer 2015). The analysis showed that a cobuilding approach had been implemented, but it was incomplete and the first 'spokespersons' of the livestock farmers appeared not to be representative of the diversity of the traditional farmers of the peri-urban associations of Bobo-Dioulasso. As a matter of fact, the livestock farmers who used to work with the acarologist were in a particular situation as the management of their footbath was done at the scale of the family (with an organization similar to their usual way of daily working) and they got a special training and support in relation with their regular relationships 
with the CIRDES. In the group of traditional agro-pastoralists who did not adopt the footbath, which was revealed by multifactorial analyses, emerged a social challenge around the position of the footbath manager. A comprehensive approach allowed to get the action logic that lead to reject the footbath management recommendations and thus to reject the tool. The technical and financial management and maintenance by a young and literate manager would change the usual social relationships at the local scale ('we do not pay our children') and was unacceptable for the bigger settlements, well before any technical or financial difficulties. Moreover, difficulties of going through the footbath in relation with cattle breeds illustrate well the fact that non humans actors have their own relationships with technology, some trainings and that these ones are able to validate or reject innovation as well as humans do. At last, the non-respect of recommendations for building the waiting pen (done with wood like the traditional night pens and without wire fence as recommended) showed the importance of innovation by users and the whirling process (no use of the recommended pen and use of a new model).

\section{Conclusions}

A reading according to the ANT allows a better understanding of the process, in particular the 'betrayals' (livestock farmers not using footbaths or not wishing to get one): the co-building process appeared insufficient, closer to an intermediary process between co-building and technology transfer. The social challenge and thus its social acceptability emerged at a late step. The first spokespersons of livestock farmers did not represent the diversity of situations. At last, the importance of the quality of the waiting pen (an innovation by itself), modified or not by the livestock farmers in an effective way, showed the need to allow innovation by users for a better acceptability of humans or non-humans.

\section{The control of stomoxes}

The control of stomoxes in cattle farming on La Réunion Island illustrates a collaboration between a Groupe de Défense Sanitaire (GDS) and the research to design an operational vector control program. The control tools are numerous, and some of them are still on the probe step. Currently, several control tools are mobilized by the livestock farmers in routine at the scale of the farm and the GDS proposed to their members the furniture of new services like the delivery of biological control. We observe the elaboration of an integrated pest and area-wide management in which the (difficult) appropriation of the control by the livestock farmers and their spokespersons became the major challenge.

The scientific interest for stomoxes at La Réunion Island is not recent: 'Nowhere else perhaps, the insects have such an influence on animal health', according to N. Barré (Barré 1981; Squarzoni 2001). Already, in the 80 's, with the intensification of cattle production, several veterinarians and entomologists (N. Barré and P.C. Morel for La Réunion Island; Ramsamy, Moorgessenpillay for Mauritius) used to study stomoxes populations in relation to their animal health and economic importance, to the stress induced on cattle, to the blood spoliation and to the transmission of anaplasmoses that are responsible for mortalities and production drops. Moreover, a heterogeneous repartition of Stomoxys niger niger Macquart and Stomoxys calcitrans L. was observed on La Réunion Island, unlike in Mauritius, which implies a different strategy of control. stomoxes are supposed to be the important mechanical vectors of anaplasmosis (Anaplasma marginale) on La Réunion Island in relation with their huge densities (Bouyer and Stachurski 2009). Scientific interest for ticks grew up at the same time in relation to their role of cyclical transmission of hemoparasites (Rhipicephalus (ex Boophilus) microplus (Canestrini) ticks are cyclical vectors 
of babesioses (Babesia bovis and Babesia bigemina) and anaplamoses and A. variegatum ticks of cowdriosis). We distinguished three socio-technical dynamics to manage the relations between humans, cattle and stomoxes since the first control program.

First implementation of the chemical and biological control strategies by veterinarians: the $1^{\text {st }}$ POSEIDOM program (1994-1998)

In 1994, following the loss of more than 1 million euros linked to a lumpy skin disease outbreak (1992) and a very high infection rate of cattle by hemoparasitoses (with an unstable epidemiological situation for anaplasmosis and babesiosis), the services supporting livestock breeding were reorganized. The POSEIDOM (Program of specific options to remoteness and insularity in the overseas departments) used to offer the means to do it. The veterinary service of POSEIDOM, the quartering service and the RESIR (network of epidemiological monitoring of La Réunion Island) became the three main components of the GRDSBR (Regional Group of animal health control of La Réunion Island). The vet POSEIDOM 'program of eradication of anaplasmosis and babesiosis on La Réunion Island' was implemented thanks to the will of the veterinary services and the GRDSBR to better control ticks and stomoxes to avoid the vector-borne diseases. It was a five-year program (1994-1998) that was based on integrated management. The GRDSBR thus became the contracting authority and project manager of the vet POSEIDOM since 1994, with the DSV (Direction of Veterinary Services), the CIRAD (French Agricultural Research Centre for International Development), the IDE (Institute of livestock breeding) and the LVD (Departmental Veterinary Laboratory) as partners and with a multiple European, national and regional funding. A prefectural decree concerning the global and mandatory control was published. The long-term objectives were to maintain cattle immunity against hemoparasitoses, to control the vector by a general biological control strategy and to eradicate hemoparasitoses (Squarzoni 2001).

In addition to the pour-on adulticide insecticide (deltaméthrine, Butox ${ }^{\circledR}$; MSD Santé animale (Intervet), Beaucouze, France), a larvicide insecticide was proposed too in order to treat stomoxes breeding sites (Néporex ${ }^{\circledast}$, Cyromazine; Novartis Santé Animale, Rueil-Malmaison, France). The seasonal treatment of cattle by pour-on became mandatory and checked by the DSV. Livestock farmers used to get the pour-on for free from the private veterinarian of their choice (an initial subsidy of $100 \%)$. A tapering rate of subsidy was planned to decrease from 100 to $25 \%$ during the 5 -year program, to give the farmers the 'sense of responsibility' (Squarzoni 2001).

In 1995, an expert report of CIRAD showed that the number of stomoxes was mainly influenced by climatic conditions (particularly the temperature) and by the kind of livestock rearing and associated practices (management of manure) (Squarzoni 2001).

The work of GRDSBR was focused on the implementation of mass rearing of stomoxes and parasitoids (6 technicians were dedicated): rearing of stomoxes began in March 1995 after bibliographic research, then that of Tachinaephagus stomoxicida Subba Rao. Twenty-four farms were selected for the 'suivi en ferme' (monitoring of the releases in farms). The initial objective of discrete augmentative (inondative) releases of $T$. stomoxicida, a specific parasitoid of stomoxes used in Mauritius, was discussed by the technical team because this species was very sensitive to environmental conditions and had a limited activity duration: its production used to be low and its survival in the various conditions of the Island was doubtful. A complex of three parasitoids was found in some sites and raised the interest for two other indigenous parasitoids, Spalangia nigroaenea Curtis and Trichopria sp. The rearing of the parasitological complex thus appeared as a better option than the rearing of a single sensitive species. The first releases of Tachinaephagus 
were implemented in December 1995 and the rearing of Spalangia nigroaenea Curtis and Trichopria the following month.

In September 1996, a first pilot area including 30 farms was defined on technical criteria. In October 1996, releases of parasitoids concerned the three species and became more intensive up to the end of the program. The biological control appeared more promising and durable than chemical control, seen as 'curative'. The control at the scale of the Island in an area-wide perspective (Hendrichs et al. 2007) was thus essential.

In April 1998, a second pilot area was opened, and in July 1998, a monitoring began (with sentinel larvae). In October, releases became more frequent. The production of pupae parasitized by Spalangia gradually increased up to become the predominant parasitoid at the end of the program in relation with its ease of rearing. During the same year, an entomopathogen fungus was found and brought to the laboratory for culture and identification (it was identified as Entomophthora muscae (Cohn), considered by N. Barré in 1981 as the main regulator of stomoxes populations on La Réunion Island). Unfortunately, it was not possible to use this species as a biocontrol agent as the results of the contamination between adults were negative. At the end of the program, the rate of use of the pour-on dropped to $20 \%$ (the evolution of the covering rate followed that of the subsidy rate). The chief of the POSEIDOM project pointed out that the withdrawal mostly concerned the farmers with few cattle and with several jobs. The objective of getting a responsible and well-reasoned treatment was not reached, and it did not go in the direction of eradication. An increase of milk and meat production was however observed during the program, and these performances were attributed to a body of positive factors (climate, animal health and technical) (Squarzoni 2001). As the two last years of the program used to be drier, the hypothesis of a natural decrease of stomoxes that could have decreased the motivation of the livestock keepers to treat was set.

At the end of this first program, the main conclusion was that the integrated management could not lead to the eradication of vectors. No significant effect was found on vectors populations and the epidemiological appraisal of hemoparasitoses. However, scientists of the program kept hope on biological control. The control of ticks seemed to be more favoured than that of stomoxes. The steering of the program by the technical team of GRDSBR was possible thanks to the POSEIDOM funding and was realized in a top-down way, and livestock farmers used to be in a position of passive acceptability.

Diversification of enrolment systems of scientists and livestock farmers: production of new knowledge, exploration of new methods: $2^{\text {nd }}$ program of control POSEIDOM (1999-2007)

In 2000, the subsidy of Butox ${ }^{\circledast}$ was stopped because of abuses. Stomoxes control was mainly based on Vavoua traps, glue traps and Néporex ${ }^{\oplus}$. However, the enzootic situation of anaplasmosis and babesiosis appeared to be still unstable (Bouyer and Stachurski 2009; Squarzoni 2001). New scientific questions were raised concerning the atypical clinical charts of anaplasmosis: were they linked to a very pathogenic stem, to under-dosed treatments or incorrect diagnosis?

The livestock breeding hub of CIRAD decided to work on stomoxes ecology: a thesis 'Ecology and biology of stomoxes populations at La Réunion Island' began. Since 2002, individual contracts of commitment were signed between the GRDSBR and livestock farmers, with an incentive goal. Technicians showed control measures to apply and gave a batch of traps and Néporex ${ }^{\circledR}$ for free during the first visit. The subsidy concerned routinely the Néporex ${ }^{\circledR}$ and traps according to the 
size of the herd (for the traps) and the results of the assessment visits. Technicians assessed the implementation of recommendations with a notation grid twice a year. The biological control was a free service proposed to adherents and funded by the Conseil Général. Satisfaction inquiries conducted by the GRDSBR between 2002 and 2005 showed a high level of satisfaction of livestock farmers concerning the technical support in the frame of commitment contracts. It was confirmed by the increase in number of adherents having signed a commitment contract from 483 in 20022003 to 1265 in 2005-2006 (Hue and Ceniceros 2007). Vavoua and glue traps were appreciated for their visual results and Néporex ${ }^{\circledR}$ for its ease of use. The intensity of problems linked to ticks and stomoxes was semi-quantitatively assessed. Only 10 to $19 \%$ of livestock farmers used to have any problems of stomoxes. The serological enquiries (prophylaxis) of 2002 and 2003 showed very high levels of enzootic bovine leucosis in the dairy cattle and called for further complementary studies.

The technicians began to give advises of environmental management against ticks and stomoxes since 2003 (thanks to their biological knowledge and experience). It concerned the good practices of manure management, the destruction of breeding sites and resting places for stomoxes as well as other specific practices for ticks.

In 2004, one entomologist of CIRAD did an expert mission to support the GRDSBR and the PhD work on stomoxes ecology. The first results of the thesis work concerned gene flows of stomoxes throughout the island. The student tried hard to solve the controversy of the importance of temperature versus livestock breeding factors as a driver of stomoxes abundance. It brought out that the livestock breeding characteristics had more impact than climate to explain the distribution and abundance of the species. New samples of the lethal fungus for stomoxes were realized and sent to $3 \mathrm{P}$ laboratory (hub of vegetal protection) for culture, confirmation of identification and assessment of culture conditions.

In 2005, a new control method was proposed: the spray of geranium hydrolat, produced locally (Bouyer et al. 2011c). The spraying system was set up by the GRDSBR and cattle were treated automatically before milking (repellent effect).

In 2006, a study carried out in the framework of a veterinary thesis concerned the factors of livestock farming and environmental factors influencing the abundance of stomoxes at the scale of the farm. The veterinarian implementing this study became the chief of the POSEIDOM project in 2006. 60 livestock farmers were under contract for the biological control and the technicians implemented the releases once a month. Since 2002, three pairs of technicians-advisors visited the farms to train farmers to integrated vector management and to assess its efficiency. In fact, data have not been analysed and the technicians felt not comfortable with this approach that put them in a difficult double position of advisor and assessor. The livestock keepers all received traps, and the refunding of external insecticide was easily granted. Thanks to funding of mechanical control methods, the Vavoua and glue traps were freely provided. The assessment of control practices was stopped in 2007 as it was not seen as reliable and useful.

A training of veterinarian thesis in the GRDSBR concerned the efficiency assessment of essential oils: whereas their larvicide effect was demonstrated (Bastien 2008), it was not used in the field (too expensive).

Some knowledge about stomoxes ecology has been collected, but a more active enrolment of farmers and stomoxes was needed to increase the efficiency of the control strategy. Not only was more accurate knowledge needed but the global strategy had to be revised. 
Third step: revision of the integrated management and the program in the context of change of funding modalities and spokespersons (2008-2016)

In 2008, the GRDSBR expressed the will to change their control strategy against hemoparasitoses in the frame of an European contract EAFRD (European Agricultural Fund for Rural Development) 2008-2013. Contacts were taken with GDS France. The program veterinary POSEIDOM became the CMTV (control of vector-borne diseases) program. Three teams were organized for the implementation of the program: the team of the laboratory of biological control and development of durable control methods (four agents), the team 'integrated management' (two agents) and the team 'technical support and training of livestock farmers' (two agents). The new actions planned by the CMTV were: the design of a new contract of objectives, the implementation of a communication campaign, a strategy of customized support to the control (ATP) and the maintenance of experimental activities in order to propose new control methods. With the end of the generous POSEI funding, more justifications of results were asked.

A retrospective inquiry implemented by the GRDSBR supported by CIRAD confirmed the regular increase of the cattle mortality since 2000. In this difficult animal health context, several audits of the cattle production highlighted hemoparasitoses as one of the main causes of cattle mortality at La Réunion Island (from 18\% of mortalities in 2000 to $12 \%$ in 2006 according to data of RESIR presented by the LVD). However a joint expert work of IDE, CIRAD and AFSSA underlined the probable involvement of intensive livestock farming practices that were not adapted to the epidemiologic context to explain the huge prevalence of hemoparasitoses (Brunschwig et al. 2009). Although this driver had already been suggested by N. Barré, no specific protocols to decrease the incidence were designed to modify the risky practices like the distribution of not immunized heifers (Bouyer and Stachurski 2009).

The efficacy of biological control was very much questioned by the Department of La Réunion. A laboratory engineer was hired in 2008 for the assessment of the biological control based on all past studies (SEF from 1999...) and new monitoring efforts of parasitological prevalence.

In 2009, the analysis of the study implemented in 2006 showed some risk factors for S. niger (manure management, proximity of sugar cane crops, etc.), abundant in the farms located 'in the bottoms' (valleys), but none for S. calcitrans (in relation with its endophily and difficulties to assess the densities), frequent in farms located 'in the tops' (Pannequin 2009). The role of Entomophthora as a regulator for $S$. niger was highlighted.

An entomologist and an acarologist of CIRAD were mandated for an expert mission about vectors ecology in February 2009. It revealed that the technical and financial means of the first POSEIDOM program did not correspond to those needed to an eradication campaign. The strategies of integrated management were not sufficiently specific to each livestock farm and were applied in a too variable way. Thus some examples of success at the scale of the farm did exist, but the failures were preponderant. The integrated management was described as a 'good collaboration with the livestock farmers', in relation to the important number of commitment contracts $(1,400)$ signed in 2009. The main prospect was to extend integrated management practices because of the limits of the chemical control. Thus a redefinition of the integrated management was expressed that underlined the need to better understand the vector ecology specific to the island and to be able to do ecological, economical and sociological predictions (Bouyer and Stachurski 2009). 
As the return to a stable enzootic situation of hemoparasitoses did not seem to be probable, some technical solutions were proposed (trivalent vaccination, eradication of $B$. microplus, use of footbaths, eradication of stomoxes by SIT) that should be decided in the long term research framework on integrated management and following a feasibility study in order to choose a control strategy (Bouyer and Stachurski 2009). Some scientific protocols were proposed: the study of stomoxes dispersal by release-recapture protocols, the study of the cycle of Batkoa apiculata (to be able to use it for biological control), the assessment of the efficiency of insecticide treated mosquito nets and the assessment of biological control.

In 2009, trials were numerous. The assessment of deltamethrin treated mosquito nets against stomoxes and at the same time the assessment of traps as indicators of stomoxes densities were achieved (Pannequin 2009).

The assessment of the biological control showed that the control until 2000 was not cost-effective (and that the assessment of the efficiency of the control was limited by methodological failures) and the strategy chosen since 2000 of producing the single species Spalangia endius Walker, naturally abundant and not much specific, was criticized. By consequence, the upgrading to standards of the insectarium to mass rear the three parasitoids species was recommended (Bouyer and Stachurski 2009).

Since April 2009, some strikes of the GRDSBR employees have slowed down control activities: the management practices of the direction and the lack of representation of the 'real livestock farmers' posed a problem. The laboratory was totally renovated and integrated to the GRDSBR following the will of the chief of the project to improve collaboration between the different services.

As the number of adherents to the GRDSBR increased from $10 \%$ of livestock farmers in 2000 up to $50 \%$ in 2010 (thanks to the proposed services, in particular, the sales of material), a change of governance of the GRDSBR happened in 2010 (Table 3): a new direction board and a new president were elected, and also a new chief of project was hired. The GRDSBR became a GDS, with a more similar organization to other GDS.

The GDS turned toward a collective control strategy at big scale including all potentially infected farms (of cattle, horses, pigs). The communication campaign continued with the farm visits conducted by six technicians (with didactic material and facilities) in the adherents' farms as well as in the non-adherents' ones.

The customized control strategy was established by the signature of an individual commitment contract (last year) and should be based on accurate epidemiological data (not realized because of a failure of data stream). A technical support to livestock farmers experiencing difficulties was proposed as well as the option of getting involved in epidemiological surveys of the RESIR.

In 2010, the information that only the half of the farms of the GDS used to get the technical visits lead to a radical change of strategy: visits were replaced by the opening of collection points of material (Vavoua and glue traps), that was based on a voluntary move of the livestock farmer to get equipped. The livestock farmers received the information of availability by SMS or phone calls, and the agents did not go into the farms anymore. As the financial participation of the livestock farmers to get the control tools used to be low previously, these campaigns appeared to be huge failures: only 5 to $10 \%$ of them got equipped. The price of the traps, still low, had increased in relation with the decrease of the subsidy. The livestock farmers gradually preferred the glue trap 


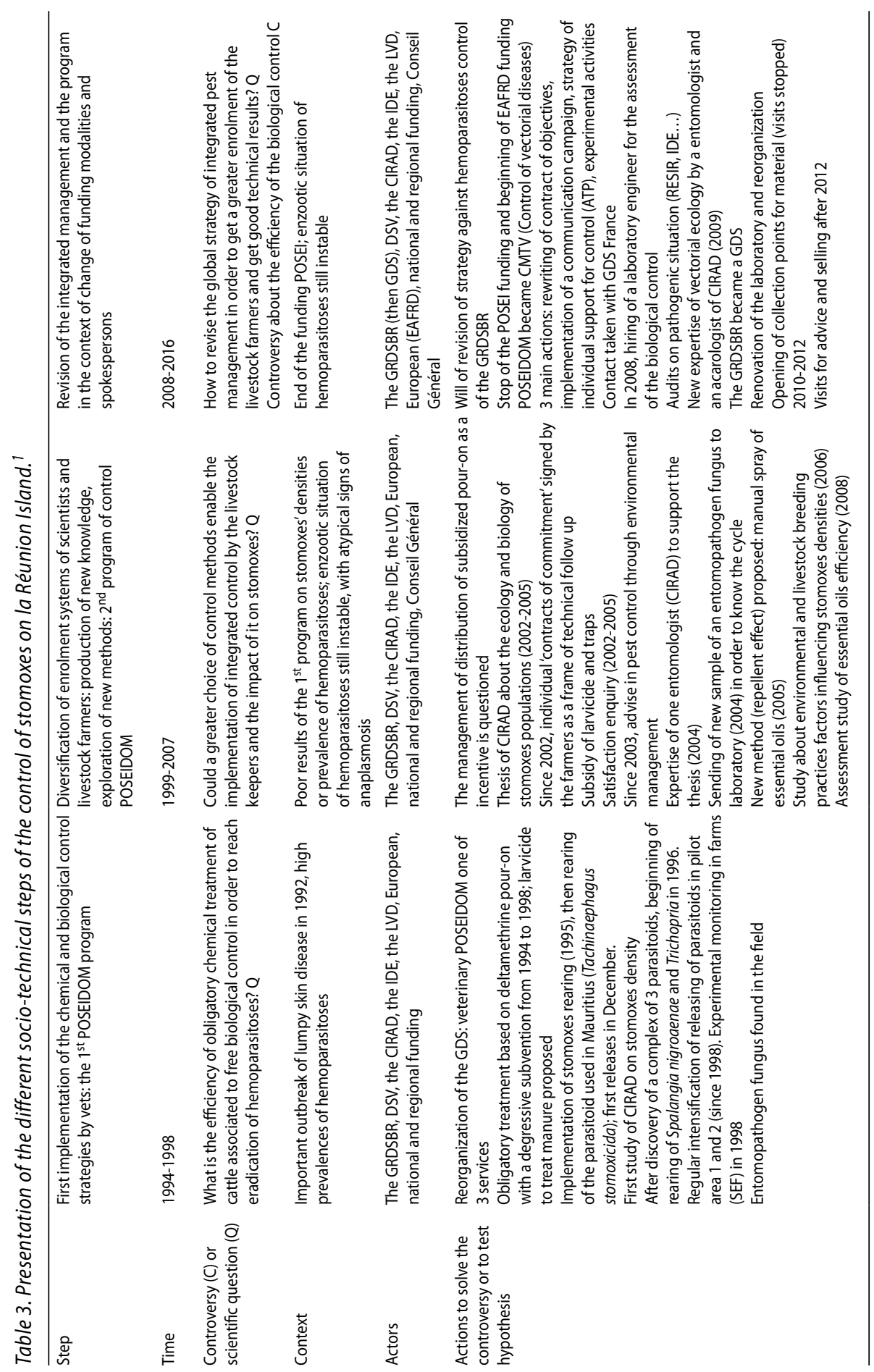




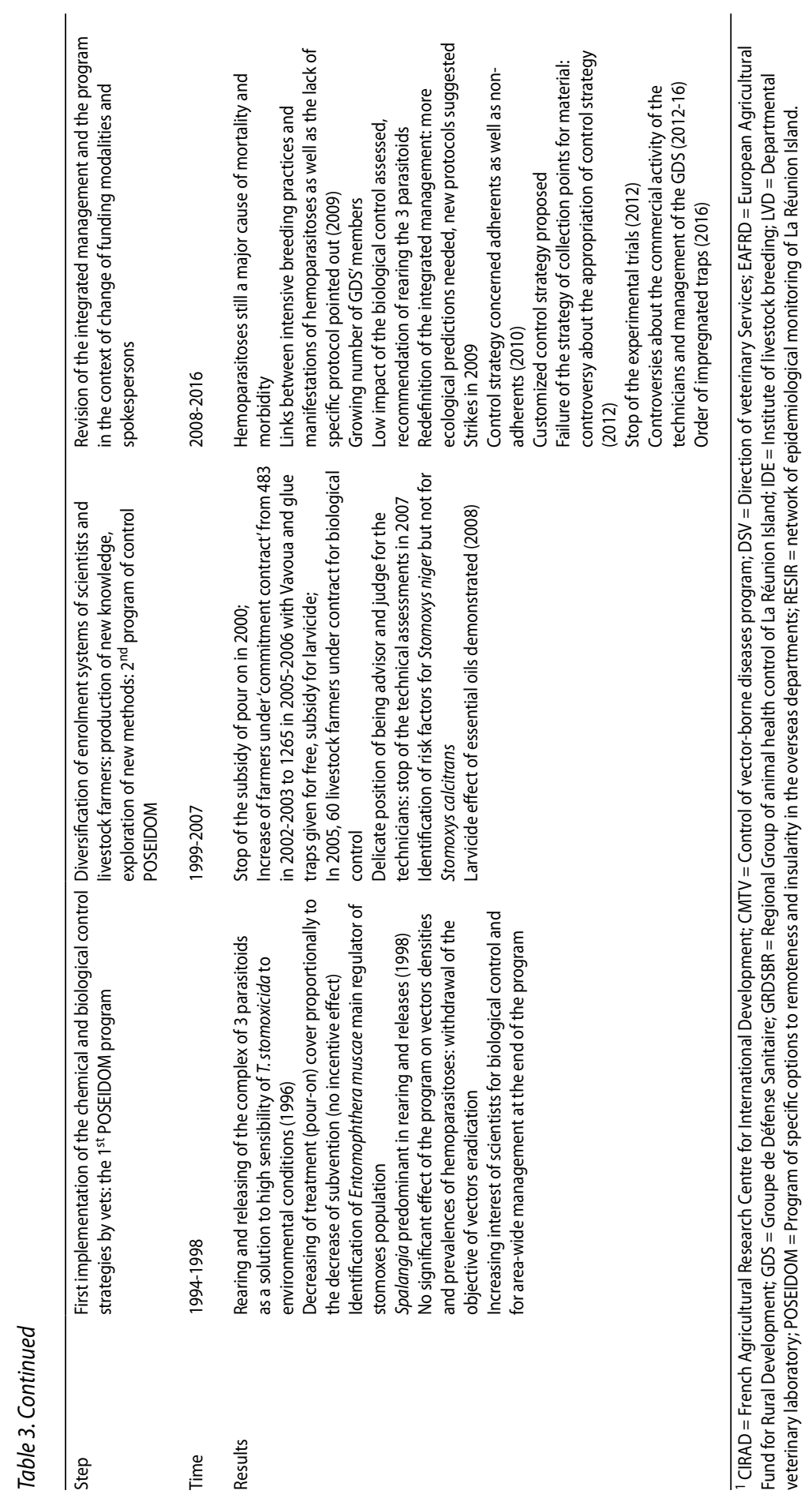


to the Vavoua trap because stomoxes catches were more visible. This failure was analysed by the veterinarians of the GDS as a failure of communication between the technical team and the farmers. The controversy about the low appropriation of the vector control fed some 'political stories' between the pro and the anti-GDS.

Since 2012, the collection points of material were stopped and the visits of technical support by technicians started again. The goal was to equip systematically the farms with traps at the beginning of the hot season. Six technicians from whom two had an important experience of advising and sales composed the team. However, the agents of the GDS assumed two different functions at the same time contrary to the GDS France that had two different teams of advisor technicians and sales assistants. During their visits, they sold different products (of cattle farming) that had a negative effect on the confidence concerning the advice, which seemed to be too much correlated to commercial issues.

However, although veterinarians pointed out the problem, some difficulties were met to negotiate a solution as the direction board of the GDS gave importance to the commercial dimension, in the context of reduction of public funding. At the same time, controversies about management practices (of the GDS) created some tensions.

While the insectarium allowed producing pupae parasitized by Spalangia, in 2012, the new managers (direction), with few experience of management of the GDS, decided to stop the trials and to develop further the commercial activities. The control campaigns continued with the no experimental tools and veterinarians had difficulties to engage new activities. In 2016, the chief of the CMTV decided to order 1000 Vavoua traps impregnated with insecticide that will be received in late 2016 (for the next hot season).

The Néporex ${ }^{\circledR}$ will be replaced by the corresponding generic to increase the distribution and to reach more farms. The glue trap will still be distributed to satisfy livestock farmers, although its efficiency against stomoxes was seen to be low.

After 20 years of control, the impact of vector control appeared to be low concerning the incidence of hemoparasitoses or the durable adoption of control practices. According to the technicians, the active will of stomoxes control by farmers appeared to be more justified by attacks on humans during the milking than by problems on cattle. At this step, the technicians stated that the preferences of livestock farmers for the tools seemed to be more in relation to the importance of the subsidy than in relation to their efficiency, as illustrated by the example of Butox ${ }^{\circledR}$ pour-on, livestock farmers preferring the less expensive spray formulations (but more difficult to use and leading to more mistakes of under-dosing). The action logics remained confused and without relation with identified groups. The need of understanding reasons and drivers of failures by 'a socio approach' appeared in the technical team, with a current debate about the means to implement it.

At the beginning of the POSEIDOM program, the control strategy was more focused on ticks than stomoxes but the intensification of the stomoxes control failed. After a step while the veterinarians expected to eradicate stomoxes at the scale of the farm, they change their view for the control of hemoparasitoses, with a strategy including livestock farming practices as well as entomological aspects ('experience feeds the advice'), with the particular importance of controlling stress levels. 
In 2016, the aim was to completely revise the way to work in order to end the contentious situation between the direction board and the employees of the GDS who felt difficulties to reach the technical objectives. The technical team stated that there was a lack of communication concerning the different objectives and action logics of livestock farmers. Tensions still occurred about the GDS management, and the director was dismissed. The livestock farmers' spokespersons showed low interest for the stomoxes control and no appropriation occurred.

\section{Conclusions}

The control strategy against stomoxes was gradually implemented through middle term projects. A top-down approach of proposal of technical options to livestock farmers was selected to test different methods. During the first ambitious program of vectors eradication, the GDS organized a mandatory chemical control with subsidy as well as the free biological control in an experimental way. The subsidy strategy was an enrolment system that appeared not to have any sustainable effect. Then, during the second step, the enrolment of scientists was more important but on a short-term mode. The enrolment of the livestock farmers was tried by the diversification of the control tools and the system of 'individual commitment contracts'. New methods were proposed and in particular practices of environmental management. At the $3^{\text {rd }}$ step, following a change of funding and spokespersons, the revision of the program with new definitions of the integrated management strategy was implemented with the help of audits and expert missions. Eradication of vectors was shifted to control of hemoparasitoses. Several strategies of active enrolment of the livestock farmers succeeded each other (customized control support, collection points of material, visits of advice and sales) to reduce the huge variability of implementation of control strategies which acceptability was expressed through the (low) use of proposed methods but was not sufficiently negotiated by the different spokespersons. The difficulties were the non-stabilized identity of technicians as advisors, the lack of involvement of scientists on the long term, and the failing of translation of the diversity of livestock farmers and their action logics letting by consequence the farmers in a passive role concerning vector control.

\section{Discussion: the question of acceptability and innovation and the ways of studying it}

\section{Understanding to support innovation}

ANT (Akrich et al. 2006) is an efficient theory for non-sociologists that allows to exit from the paradigm of the linear innovation process and to understand the whirling and irreversible innovation process, being the result of a mish-mash of various decisions, negotiations, successive socio-technical controversies or scientific questions during which the identities of human or non-human actors are redefined (the translation). The steps of raising interest and enrolment are decisive, which highlights the way how innovations are implemented without focusing on the primary characteristics of the technical object (its acceptability) but in taking into account the dynamic interaction between the innovation and the socio-technical network. The innovation is seen as the result of the activation of the socio-technical network and it is necessary to approach actors of the nature, the science and the society, and their interactions symmetrically.

The three main principles of the ANT (widespread agnosticism, widespread symmetry, and free association) allow understanding the innovation process being based on a technical object (like the acaricide insecticide footbath) or when following innovation trajectories within a given challenge (the stomoxes control). With these principles, this is possible to catch new phenomenon 
and to understand the process in front and behind the scenes without neglecting any actors (from the society, the science or the nature) or any action (creative or destructive).

However, this theory does not give any turnkey method. The participation represents an answer to the need of enabling attitudes and methods to design and implement development programs, in particular in poor countries. It was defined as: 'A process through which stakeholders influence and share control over development initiatives and the decisions and resources that affect them' (World Bank 1996). The participation is thus the process that helps the citizens to influence decision taking that concerns them and to take control over it from the design of a public policy up to technical choices. The goal is thus double: social and political (d'Aquino 2007). The participative approaches allow enabling the knowledge of complex situations, in an uncertain world (Callon et al. 2014), as the study of action in the considered system is needed. Four main kinds of participation (d'Aquino 2007) can be defined according to the way that concerned stakeholders are involved: the participative sensitization, the participative diagnostic, the combined management and the participative democracy. As the designing of the four approaches is very different, it is necessary to choose the approach since the beginning. Hybridization between biological and social sciences or multidisciplinary approach open many options in relation to the kind of participation considered.

Participative epidemiology (PE) has the vocation to study and support innovation in the field of animal health. The three main current uses of PE are the development of animal health services, epidemiosurveillance and veterinary research (Jost et al. 2007). The specific tool to support innovation is the problem tree (Calba et al. 2014; Veselý 2008) that examines the reasons and consequences of identified difficulties. This tool as well impact matrixes (Bellet et al. 2012) and all the participative tools used in focus groups are used to elicit the perceptions and consider the stakeholders without any predefined innovation theory. The participative methods have been criticized (Bouyer 2015; Lavigne Delville et al. 2000) since they can recreate the biases that they want to fight. The aim of the MARP (Méthode Accélérée de Recherche et de Planification) or 'participatory rural appraisal', the most common method of $\mathrm{PE}$, is to co-produce knowledge and to allow the shared learning between farmers and technicians by an open discussion using visual tools to drive and plan action. The initial multidisciplinary approach of the MARP has been lost and is mostly focused on the animal health management implemented by the veterinarians (Catley et al. 2012). However, the criticism concerns its 'ideological populism' (Lavigne Delville et al. 2000): the idealization of 'the population', with an insufficient differentiation of social groups and an under-estimation of divisions, challenges and conflicts. Three illusions are reported: the community illusion (seen as consensual), the communication illusion (an information of good quality is linked to the choice of the tools) and an empiricist illusion (the speech of actors is sufficient and no special theory is needed). Without further inputs of sociology of innovation and a deeper method to prepare, drive debate and to analyse the discourses (Bouyer 2015), it is difficult to fight these 3 illusions. Moreover, the innovation model stays the linear one in an implicit way. The study cases illustrated well the need to exit from a determinist view of the innovation that should be acceptable or not and the need to adopt a constructivist approach (Bouyer 2015).

An inductive approach followed by abduction allowed renewing the approach of biologists in areas and contexts where the data are lacking. According to Lavigne, the inductive approach is possible only in a comprehensive approach (Lavigne Delville et al. 2000). The case study is a particular accurate strategy when object and context are highly linked.

The GERDAL (Group of Experimentation and Research, Development and Localized Actions) designed a method for the co-active research of solutions between development agents 
and farmers with a research position based on a comprehensive sociology (Weber 1978): the comprehensive socio-technical analysis and support. Others methods exist like the hybrid forums (Callon et al. 2014) or the scenarios workshops (Bicaïs 2007).

\section{Acceptability, appropriation and co-production}

The problem of the relations between researchers and populations and the need of taking nonscientists opinions into account are often seen as a nightmare by the researchers (Dupé 2015). The major model of the 'transfer of technology' was predominant until the 70's and is still very frequent for the scientists, veterinarians and engineers. But in the 70's the observation that many efficient technical solutions, supported by development projects, were not adopted lead to the revision of this model and the emergence of the concept of participation (Bouyer 2015; Catley et al. 2012). The acaricide insecticide footbath is an example of efficient and economical technical solutions with a low adoption. It is necessary to end with the technico-economical myth in the community of biologists and to see the innovation process as non-linear and multidimensional.

The acceptability reaches the idea of probability of adoption of vector control and corresponds, like the adoption, to a binary choice between acceptation and reject. To go over this binary view based on the split conception-use model, De Vaujany (Vitry and Chia 2016) introduces the concept of appropriation to understand the process in a constructivist way by the transformation of actors and control network.

What are the lessons learned from the assessment of the social acceptability about the use of TIS against mosquitoes (Dupé 2015) and the control of stomoxes on La Réunion Island? The universalizing view of the societies and the natures of the authorities and the naturalist view of the new control strategies were confronted to several interpretative systems owning to this overseas department at this period (post-colonial) (Dupé 2015). The enrolment of the population is more symbolic than effective for a SIT project. Concerning the stomoxes control on La Réunion Island, the biological control needed only a symbolic participation too but the enrolment of the livestock farmers had to be really effective for the chemical and the environmental control techniques. The scientific works allowed better defining the stomoxes populations but the diversity of the livestock farmers and their action logics stayed in a black box. It appeared that the strategies should be adapted to the epidemiological characteristics at the scale of the farm (in relation to the different behaviour of the two species, in particular) around 2009, and later, the need of taking into account the action logics of the farmers, in their diversity, was highlighted to enable the communication and to adapt the recommendations too. It would have been relevant that the livestock farmers change from objects of policy to actors, like in the sanitarian practices against mosquitoes on La Réunion Island. The example of the control of stomoxes shows how difficult it was to shift from an innovation transfer approach to those of appropriation, as the representatives of the livestock farmers used to stay passive towards the control strategy. The technical challenge disappeared behind the issue of governing (of the professional group of livestock farmers) and the complexity of various and non-identified socio-technical dynamics. Thus, after 20 years of control programs, the challenge would be to identify the groups of peers (members of the same socioprofessional group sharing objectives and action logics) and their own innovation processes. As a matter of fact, each group of peers has priority challenges to solve so that a global access to their action logics is mandatory. A comprehensive socio-technical analysis is particularly adapted to this kind of challenges, to understand the different socio-technical dynamics and to manage a process of appropriation or co-building of the control strategy (Darré 1999, 2006). It allows giving an active role to users of control methods and the different groups concerned. 
Many control cases at a large scale like the new screwworm control had been successful although the approach was not a participative one. The cattle industry was particularly active to initiate the control process and even to drive it by asking the scientists for an eradication strategy, but powerful institutions monopolized the following negotiations and small farmers (or the general population) were not involved. The reasoning was mainly based on general economic issues at a large scale, without any assessment of the impact for small producers. The acceptability of the approach was thus based on the legitimacy of the institutions and a preliminary view of the society on the technical progress and the development of agriculture. The political stakeholders took some shortcuts, as their project appeared to match with the view of the majority. That explains why the linear innovation process 'top down' can be effective in some situations and will keep credibility. However, the possibility to be challenged by minority groups or when majority norms change must not be underestimated. Communication could control the institutional risk (Dupé 2015).

The way that the social acceptability appears depends mainly on the view of the management of the animal health risk: it could be a collective management following a process of dialogic democracy or a technocratic management that drives the political action. The two approaches of management bring different and complementary innovation capacities. The emerging regime of collective experimentation becomes more and more popular and supported by politics as it brings new capacities in comparison with the centralized regime of technoscientific promises that dominated the scene for a long time (Joly et al. 2013). However, this last regime keeps its benefits for scientists and it is not relevant to suppress it when the goal is to maximise the innovation capacities. There is no perfect dichotomy between these two innovation regimes and the challenge is to better coordinate the different innovation systems (Joly et al. 2013).

To promote innovation in the field of vector control, the question of acceptability must go further, up to appropriation and co-production. The co-production offers the maximum guarantee of acceptability, of consensus of interests and sustainability. However, the implementation is not easy and fast, and needs the help of social sciences, which explains why many scientists still rely on the innovation transfer strategy. How to support innovation?

\section{Acknowledgements}

We thank Jérémy Bouyer, Olivier Esnault, Thomas Hue, Marion Pannequin and Frédéric Stachurski for the interviews and corrections.

\section{References}

Akrich M, Callon M and Latour B (2006) Sociologie de la traduction. Textes fondateurs. Collection sciences sociales. Presses des Mines, Paris, France.

Barré N (1981) Les stomoxes ou mouches boeuf à La Réunion. Pouvoir pathogène, écologie, moyen de lutte. MaisonsAlfort, France.

Bastien F (2008) Effet larvicide des huiles essentielles sur Stomoxys calcitrans à La Réunion. Ecole Nationale Vétérinaire de Toulouse, Toulouse, France.

Bellet C, Vergne T, Grosbois V, Holl D, Roger F and Goutard FL (2012) Evaluating the efficiency of participatory epidemiology to estimate the incidence and impacts of foot-and-mouth disease among livestock owners in Cambodia. Acta Tropica 123: 31-38.

Bicaïs M (2007) Imaginaire de la fonctionnalité, de l'acceptabilité sociale à l'émergence du projet technicien. Université Pierre Mendès France, Grenoble, France. 
Bouyer F (2007) Elaboration avec les organisations d'éleveurs de cahiers des charges en vue de la mise en place de services de prophylaxie sanitaires basés sur le pédiluve acaricide/insecticide et identification de sites d'implantation des pédiluves. PAEOB/CIRAD, Bobo-Dioulasso, Burkina Faso.

Bouyer F (2009) Appui technique et organisationnel aux organisations professionnelles d'éleveurs pour la mise en place et le suivi de services de prophylaxie sanitaire basés sur le pédiluve acaricide/insecticide (seconde phase). PAEOB/CIRAD, Thiès, Senegal.

Bouyer F (2015) Risque trypanosomien et innovation: le cas des éleveurs d'Afrique de l'Ouest (Trypanosomosis risk and innovation: the case of livestock farmers in West Africa). Université Montpellier II, Montpellier, France.

Bouyer F, Belem AMG, Seyni H, Adakal H, Lancelot R and Bouyer J (2009a) Adoption d'une nouvelle méthode de lutte sanitaire en milieu paysan: le pédiluve acaricide-insecticide au Burkina Faso. In: Andrieu N (ed.) Evaluation des impacts des innovations dans les systèmes de production et les territoires agropastoraux d'Afrique de l'Ouest: quelles méthodes, quels indicateurs? December 1-4, 2009. CIRAD, CIRDES, Ouagadougou, Burkina Faso, $19 \mathrm{pp}$.

Bouyer F, Belem A, Seyni H, Adakal H, Lancelot R, Stachurski F and Bouyer J (2011a) Adoption d'une nouvelle méthode de lutte sanitaire en milieu paysan: le pédiluve acaricide/insecticide au Burkina Faso. Rev Elev Méd Vét Pays Trop 64(1-4): 21-32.

Bouyer F, Hamadou S, Adakal H, Lancelot R, Stachurski F, Belem AMG and Bouyer J (2011b) Restricted application of insecticides: a promising tsetse control technique, but what do the farmers think of it? PLoS Negl Trop Dis 5(8): e1276.

Bouyer J, Bouyer F, Donadeu M, Rowan T and Napier G (2013) Community- and farmer-based management of animal African trypanosomosis in cattle. Trends Parasitol 29(11): 519-522.

Bouyer J, Grimaud Y, Pannequin M, Esnault $O$ and Desquesnes M (2011c) Importance épidémiologique et contrôle des stomoxes à la Réunion. Bull Épidémiol, Santé Animale Alimen 43: 53-58.

Bouyer J, Kaboré I, Stachurski F and Desquesnes M (2005) Epicutaneous treatment of cattle. Santé animale en Afrique de l'Ouest. Recommandations Techniques. Vol. 8. CIRDES/CIRAD, Bobo-Dioulasso, Burkina Faso.

Bouyer J, Solano P, Cuisance D, Itard J, Frézil J-L and Authié E (2010) Trypanosomosis: control methods. In: Bouyer J, Solano P, Cuisance D, Itard J, Frézil J-L, Authié E and Uilenberg G (eds.) Infectious and parasitic diseases of livestock. Éditions Lavoisier (Tec \& Doc), Paris, France, pp. 1936-1943.

Bouyer J and Stachurski F (2009) Mission d'appui pour les orientations du programme CMTV (Contrôle des Maladies à Transmission Vectorielle). UMR Cirad/INRA CMAEE, Dakar, Sénégal, 108 pp.

Bouyer J, Stachurski F, Gouro A and Lancelot R (2009b) Control of bovine trypanosomosis by restricted application of insecticides to cattle using footbaths. Vet Parasitol 161(3-4): 187-193.

Bouyer J, Stachurski F, Kaboré I, Bauer B and Lancelot R (2007) Tsetse control in cattle from pyrethroid footbaths. Prev Vet Med 78: 223-238.

Brunschwig P, Lancelot R and Zanella G (2009) Expertise sur la mortalité des bovins laitiers à la Réunion. Available at: https://tinyurl.com/y6v8put2.

Calba C, Ponsich A, Nam S, Collineau L, Min S, Thonnat J and Goutard FL (2014) Development of a participatory tool for the evaluation of village animal health workers in Cambodia. Acta Tropica 134: 17-28.

Callon M (2001) Actor network theory. International encyclopedia of the social \& behavorial sciences. In: Smelser $\mathrm{NJ}$ and Baltes PB (eds.) International Encyclopedia of the Social \& Behavioral Sciences. Elsevier, Amsterdam, the Netherlands, pp. 62-66.

Callon M, Barthe $Y$ and Lascoumes P (2014) Agir dans un monde incertain. Essai sur la démocratie technique. Seuil, Paris, France.

Canguilhem G (1943) Le normal et la pathologique (Quadrige). PUF, Paris, France.

Catley A, Alders RG and Wood JLN (2012) Participatory epidemiology: approaches, methods, experiences. Vet J 191: 151-160.

Crouch C (2004) Post-democracy (1 ${ }^{\text {st }}$ Ed.). Polity Press, London, UK.

Darré J-P (1999) La production de connaissance pour l'action. Editions de la Maison des sciences de l'homme, Institut National de la Recherche Agronomique, Paris, France. 
Darré J-P (2006) La recherche coactive de solutions entre agents de développement et agriculteurs. GRET, Etudes et travaux, Paris, France.

d'Aquino P (2007). Empowerment et participation: comment mieux cadrer les effets possibles des démarches participatives? Proposition d'un cadre d'analyse à partir d'une synthèse bibliographique. CIRAD, Montpellier, France.

Denayer D and Collard D (2014) Ce que gérer la faune implique: une approche par la théorie de l'acteur-réseau. Le cas de la conservation de l'ours brun dans les Pyrénées Françaises. Ann Mines- Gérer Compr 118(4): 78-88.

Dervaux A, Pichault F and Renier N (2011) L'apport de la théorie de l'acteur-réseau à la professionnalisation de la GRH en milieu hospitalier. J Gestion D'écon Méd 29(1): 62-73.

Dupé S (2015) Séparer les moustiques des humains à La Réunion. Co-production d'un nouvel ordre socio-naturel en contexte post-colonial. Université de La Réunion - Muséum National d'Histoire Naturelle, Réunion, France.

Fontenille D (2008) Écosystèmes, entomologie et lutte anti-vectorielle. Ann Mines-Respons Environ 3: 55-60.

Garcia R, Mendez L, Serrano E, Gil Morales T and Vreysen MJB (2007) Insecticidal wound treatment of livestock on Isla de la Juventud, Cuba: an efficient suppression method of new world screwworm Cochliomyia hominivorax prior to the release of sterile insects. In: Vreysen MJB, Robinson AS and Hendricks J (eds.) Area-wide control of insect pests. From research to field implementation. Springer, Vienna, Austria, pp. 393-403.

Grant GH, Wendell Snow J and Vargas-Terán M (2000) The New World Screw-worm as a pest in the Caribbean and plans for its eradication from Jamaica and the other infested Caribbean islands. In: Tan K-H (ed.) Area-wide control of fruit flies and other insect pests. Penerbit Universiti Sains Malaysia, Penang, Malaysia, pp. 87-94.

Habtewold T, Prior A, Torr SJ and Gibson G (2004) Could insecticide-treated cattle reduce Afrotropical malaria transmission? Effects of deltamethrin-treated Zebu on Anopheles arabiensis behaviour and survival in Ethiopia. Med Vet Entomol 18(4): 408-417.

Hendrichs J, Kenmore P, Robinson AS and Vreysen MJB (2007) Area-wide integrated pest management (AW-IPM): principles, practice and prospects. In: Vreysen M, Hendrichs J and Robinson AS (eds.) Area-wide control of insect pests, from research to field implementation. Springer, Dordrecht, the Netherlands, pp. 3-34.

Hue T and Ceniceros R (2007) Gestion de la lutte contre les hémoparasitoses et leurs vecteurs - lle de la Réunion - Etat des connaissances 2007. Réunion, France, 106 pp.

Joly P-B, Rip A and Callon M (2013) Réinventer l'innovation? Available at: https://tinyurl.com/ydccuejv.

Jost CC, Mariner JC, Roeder PL, Sawitri E and Macgregor-Skinner GJ (2007) Participatory epidemiology in disease surveillance and research. Rev Sci Tech Off Int Epiz 26: 537-547.

Klassen W (2000) Area-wide approaches to insect pest management: history and lessons. In: Tan K-H (ed.) Area-wide control of fruit flies and other insect pests. Penerbit Universiti Sains Malaysia, Pulau Pinang, Malaysia, pp. 21-38.

Lavigne Delville P, Sellamna N and Mathieu M (eds) (2000) Les enquêtes participatives en débat: ambitions, pratiques, enjeux. Karthala, Paris, Montpellier, France.

Lindquist DA (2000) Pest management strategies: area-wide and conventional. In: Tan K-H (ed.) Area-wide control of fruit flies and other insect pests. Penerbit Universiti Sains Malaysia, Penang, Malaysia, pp. 13-19.

Mayaux P-L (2015) La production de l'acceptabilité sociale. Privatisation des services d'eau et normes sociales d'accès en Amérique latine. Rev Française Sci Politiq 2(65): 237-259.

Ndeledje N, Bouyer J, Stachurski F, Grimaud P, Belem AMG, Mbaïndingatoloum FM, Bengaly Z, Alfaroukh IO, Cecchi G and Lancelot R (2013) Treating cattle to protect people? Impact of footbath insecticide treatment on tsetse density in Chad. PLoS ONE 8: e67580.

Pannequin M (2009) Étude des pratiques d'élevage et des facteurs environnementaux influençant l'abondance en stomoxes dans les élevages bovins laitiers. Ecole Nationale Vétérinaire de Toulouse, Toulouse, France.

Roy L (2013) Jalons pour une recherche sur l'acceptabilité sociale de l'innovation éco-conçue: aspects éthiques et épistémologiques de la norme environnementale. Marché Org 1(17): 83-99.

Squarzoni C (2001) Lutte biologique contre les stomoxes des bovins à La Réunion: l'exemple du 'Poseidom vétérinaire.' Ecole Nationale Vétérinaire de Toulouse, Toulouse, France.

Stachurski F (2000a) Invasion of West African Cattle by the tick Amblyomma variegatum. Med Vet Entomol 14: 391-399. 
Stachurski F (2000b) Modalités de la rencontre entre la stase adulte de la tique Amblyomma variegatum (Acari, Ixodida) et les bovins: applications potentielles à la lutte contre ce parasite. Université Montpellier II, Montpellier, France.

Stachurski F (2005) The acaricide footbath, Santé animale en Afrique de l'Ouest. Recommandations techniques. Vol. 1. CIRDES/CIRAD, Bobo-Dioulasso, Burkina Faso.

Stachurski F, Bouyer J and Bouyer F (2005) La lutte contre les ectoparasites des bovins par pédiluve: une méthode innovante utilisée en zone péri-urbaine sub-humide du Burkina Faso. Rev d'Elev Méd Vét Pays Trop 58: 221-228.

Stachurski F and Lancelot $\mathrm{R}$ (2006) Foot-bath acaricide treatment to control cattle infestation by the tick Amblyomma variegatum. Med Vet Entomol 20: 402-412.

Starr C (1985) Risk management, assessment and acceptability. Risk Anal 5(2): 97-102.

Terrade F, Pasquier H, Reerinck-Boulanger J, Guingouain G and Somat A (2009) L'acceptabilité sociale: la prise en compte des déterminants sociaux dans l'analyse de l'acceptabilité des systèmes technologiques. Trav Hum 72: 383-395.

Torres TT, Lyra ML, Fresia P and Azeredo-Espin AML (2007) Assessing genetic variation in New World Screwworm Cochliomyia hominivorax populations from Uruguay. In: Vreysen MJB, Robinson AS and Hendrichs J (eds.) Areawide control of insect pests. From research to field implementation. Springer, Vienna, Austria, pp. 183-191.

Veselý A (2008). Problem tree: a problem structuring heuristic. Central Europ J Public Policy 2: 60-81.

Vitry C and Chia E (2016) Contextualisation d'un instrument et apprentissages pour l'action collective. Manag \& Avenir 1(83): 121-141.

Vo TT (2000) Economic impact of eradicating the New World Screw-worm (Cochliomyia hominivorax). In: Tan KH (ed.) Area-wide control of fruit flies and other insect pests. Penerbit Universiti Sains Malaysia, Penang, Malaysia, pp. 113-116.

Vreysen MJB, Gerardo-Abaya J and Cayol JP (2007) Lessons from area-wide integrated pest management (AW-IPM) programmes with an SIT component: an FAO/IAEA perspective. In:Vreysen MJB, Robinson AS and Hendrichs J (eds.) Area-wide control of insect pests. From research to field implementation. Springer, Vienna, Austria, pp. 723-744.

Weber M (1978) Economy and society: an outline of interpretive sociology. University of California Press, Berkeley and Los Angeles, CA, USA.

World Bank (1996) The World Bank participation sourcebook. The World Bank, Washington, DC, USA. Available at: http:// tinyurl.com/yb28207t.

Wyss JH (2000) Screw-worm eradication in the Americas - overview. In: Tan KH (ed.) Area-wide control of fruit flies and other insect pests. Penerbit Universiti Sains Malaysia, Pulau Pinang, Malaysia, pp. 79-94. 
Conclusion 


\title{
20. Control of vector-borne diseases in the livestock industry: new opportunities and challenges
}

\begin{abstract}
Claire Garros ${ }^{1,2 *}$, Jérémy Bouyer ${ }^{2,3,4}$, Willem Takken ${ }^{5}$ and Renate C. Smallegange ${ }^{5}$
${ }^{1}$ CIRAD, UMR ASTRE, 97491 Sainte-Clotilde, La Réunion, France; ${ }^{2}$ ASTRE, University of Montpellier, CIRAD, INRA, Montpellier, France; ${ }^{3}$ Unité Mixte de Recherche ASTRE 'Animal, Santé, Territoires, Risques et Ecosystèmes', Campus international de Baillarguet, Centre de Coopération Internationale en Recherche Agronomique pour le Développement (CIRAD), 34398 Montpellier, France; ${ }^{4}$ Insect Pest Control Laboratory, Joint FAO/IAEA Programme of Nuclear Techniques in Food and Agriculture, 1400 Vienna, Austria; ${ }^{5}$ Laboratory of Entomology, Wageningen University \& Research, P.O. Box 16, 6700 AA Wageningen, the Netherlands; gnachman@bio.ku.dk; claire.garros@cirad.fr
\end{abstract}

\begin{abstract}
A large number of state-of-the-art issues of livestock pests and diseases, and their control, has been reviewed in the current volume of the Ecology and Control of Vector-borne diseases book series. The combined reviews demonstrate a clear difference in livestock pests between northern and southern regions, where the former are more subjected to pest control and the southern areas are more confronted with infectious vector-borne diseases. It appears that arthropod pests and associated vector-borne diseases in livestock have received less attention than required, leading to a lack of novel and innovative strategies for their prevention and control. Yet, the rapid expansion of the livestock industry as a result of population growth and global change call for solutions that are essential for economic and sustainable livestock production systems. The One Health concept, by now well known in human health, still needs to be integrated into the livestock industry. This becomes more urgent given the recent emergence of novel arthropod-borne diseases and the invasion of arthropod pests in new territories. It is concluded that a multidisciplinary approach to tackle the problem of arthropod pests and associated diseases in livestock is required, which should include novel genetic and microbiological tools and strategies in order to prevent the near-unity reliance on chemical pesticides and vaccines.
\end{abstract}

Keywords: integrated control, One Health, livestock pest and disease, innovation, future strategies

\section{Introduction}

This book presents with 18 state-of-the-art chapters the diversity of pests and vector-borne diseases that affect livestock and animal production. It also underlines the differences in vector control strategies and methods available between territories and according to epidemiological situations. A large section is also dedicated to the impact of vector control and cost-benefits evaluation of surveillance and vector control measures (Roberts et al. 2018; Shaw 2018). Today, disease control for animal health is essentially founded on insecticide-based vector control and vaccination when available. The regulatory requirements relating to vector control in the livestock industry is limited and there is no overall animal-health oriented vector policy with a statutory framework.

The usual dichotomy with northern countries free of disease while southern countries fighting against vector-borne diseases, is continuously challenged by new invasions. Vector species colonize new territories, pathogens cross borders, and climate and environment cause changes in disease epidemiology. Several, mostly unexpected, outbreaks such as: West Nile virus in the 
USA, African swine fever in eastern Europe, bluetongue (Miranda 2018) and Schmallenberg viruses justify renewed attention to vector-borne diseases of veterinary and public health.

The recent illegal use of the insecticide fipronil in several European countries (see Sparagano et al. 2018; http://tinyurl.com/y7gm8lhk) shows the challenges faced in the poultry industry caused by only one of the arthropod pests, the poultry red mite (Dermanyssus gallinae De Geer), demonstrating the urgency for effective and sustainable control methods to be implemented in this sector (Sigognault Flochlay et al. 2017; Sparagano et al. 2018). Both in poultry, sheep, and in dairy farms flies pose a direct and indirect threat to animal and human health, even though several control methods are available (Baldacchino et al. 2018; Colwell and Wall 2018; Sparagano et al. 2018). An expected growth and expansion of fly populations urges us to look for efficient fly control programmes (Sallam et al. 2017). In (sub)tropical areas producers of beef, dairy and leather encounter financial losses due to high levels of resistance to acaricides in the cattle tick (Rhipicephalus microplus (Canestrini)). One avenue to develop new control tools is to take advantage of the semiochemicals emitted by the host and the differential attractiveness between cattle (Brugman et al. 2018; Kinney Ferreira de Miranda Santos et al. 2018; Smallegange et al. 2011). Another one is the development of genetic control, in particular the sterile insect technique, which has been used to eradicate screwworms from the entire northern and central America (Bouyer and Chia 2018) and more recently against tsetse flies (Bouyer and Marois 2018). New methods like gene drive or population replacement using symbiotic bacteria are under development against mosquitoes (Bouyer and Marois 2018) but still necessitate huge research efforts to be used in a sustainable way. These new tools should be integrated with current vector control methods within an area-wide integrated pest management (IPM) perspective, which has been the paradigm for more than 50 years in the case of agricultural pests (Vreysen et al. 2007). Such control tools require a centralized approach with the involvement of public authorities. Most of the time, however, vector control will still rely on beneficiary farmers and the use of insecticides which must still be done within an IPM perspective, as nicely illustrated in the control of African animal trypanosomes (Gimonneau et al. 2018). Models will then be instrumental to optimize the integration of biological control tools to existing techniques. The Fly Simulator (Nachman and Skovgård 2018) shows promising results: a biological control agent combined with sanitary measures can be effective in reducing an arthropod pest.

Although this book aims to be as comprehensive as possible, some topics are not discussed, for shortage of available experts who would contribute to this volume. This was the case with the arthropod control in the pork industry and horse breeding. In particular, African swine fever is a devastating infectious disease of pigs, usually deadly, and to be reported as a OIE listed disease (http://www.oie.int/animal-health-in-the-world/oie-listed-diseases-2018). It does not affect humans nor does it affect other animal species other than pigs and wild boars. It can be transmitted either via direct animal contact or via dissemination of contaminated food (e.g. sausages or uncooked meat) or via tick transmission. No vaccine exists to combat this virus which limits its control. In infected areas, control is done through slaughtering of all pigs and destruction of cadavers and litter, cleaning and disinfection, designation of infected zones, and control of pig movement, as well as epidemiological investigation (tracing of sources and possible spread of infection). The reason for these actions is its transmissible nature that has the potential for very serious and rapid spread, irrespective of national borders, having serious socio-economic and public health consequences, and is of major importance in the international trade of animals and animal products. 
On another perspective, although it has not a high economic impact, sweet itch (insect bite hypersensitivity), caused following the bite of Culicoides spp., is a major concern for horse breeders and owners as very limited preventive and curative treatments are available. Advances in immunological research for the development of a rapid diagnostic tool and eventually a vaccine based on salivary proteins of Culicoides are promising (Jonsdottir et al. 2015), but these tools are not yet available, leaving the horse industry to methods such as poor-on insecticides and sitespecific insecticidal treatment of midge breeding sites.

\section{One Health}

Despite these emergences and diseases touching or threatening the livestock production, there are new opportunities ahead. One main change is the use of the One Health approach for disease surveillance and control. One Health is an integrated approach and the term used to tackle diseases (particularly zoonoses) considering all components that might lead to, or increase the disease threat. These include environmental and ecological, wildlife components as well as domestic animal and human factors. It also requires the interactions between the various experts and scientists, policy makers and funding bodies. Indeed, building relationships across disciplines and actors require time and a major paradigm change. Because vector-borne diseases are difficult to control due to their complex epidemiology, the management, control and prevention of most economically important vector-borne diseases could benefit from an One Health approach. Coordinated interdisciplinary approach could help answering questions on the wildlife reservoirs, vector competence of autochthonous species/exotic virus strains, laboratory capacity diagnosis, optimization of current control measures (vaccination, vector control, animal movements) and social and economic impacts of the outbreaks or control strategy.

Even though a promising concept, and several initiatives have been started in the past decade, implementation lacks behind and the barriers causing this should be determined and removed (Sikkema and Koopmans 2016). One aspect might be that further discussions are necessary to define the exact meaning of One Health (Kingsley and Taylor 2017).

\section{Opportunities and challenges}

An opportunity for the future of vector control in the livestock industry is integrated control. If integrated vector control is recommended or imposed by health organizations, the assessment of operational impacts is mandatory both at the implementation and use of vector control tools and return on investment/cost-benefits for disease control. Effectiveness of control measures depends highly on the understanding that people (from farmers, local communities to policy markers) under the threat of vector-borne diseases have of the importance of the issues, and on the acceptability of strategies adopted. Cost effectiveness of surveillance and control methods should be part of the integrated control strategies as they are relevant to societal concerns and public health budgets.

New challenges also need to be tackled. Risk assessment and impact of intervention methods on non-targeted organisms, human populations and environment need to be investigated to minimize exposure and side effects. Insecticides are often considered a quick, easy, and inexpensive solution for controlling pests and vector species but it has to come with insecticide resistance monitoring. 
Recently, the absence of vaccines, effective vector control strategies, the limited number of novel insecticide molecules and the environmental considerations have resulted in the need for novel arthropod control strategies. Today, novel control strategies are under development or already in use such as biocontrol agents (Bti and Wolbachia strains, fungi), sterile insect techniques, insecticide-treated durable wall lining, sugar-baited traps, anti-tick vaccines, and push-pull strategies, among others (e.g. Machtinger and Geden 2018; Pages and Cohnstaedt 2018; Pérez de León et al. 2018; Pfeffer et al. 2018; Weeks et al. 2018). Ironically, most of the time these techniques target zoonotic diseases with a clear objective to protect human populations and only in second instance livestock. Even though promising new strategies, they will be only applied if the society and government allow it.

\section{Conclusions/future directions}

From the available evidence (this volume) it seems that progress on solving livestock pests and the prevention of livestock vector-borne diseases has not received the attention that the sector requires: the vastly increasing livestock production for the consumer market (poultry, swine, beef, etc.) and the intensification of production systems places the industry at greater risk of livestock pests. Hence, sustainable solutions, that are acceptable for society and environment, are still required. Against vectors of human diseases, numerous new methods of genetic control have been proposed recently, but still need to be tested at the operational scale to meet the challenges related to the reduction of the spectrum of authorized insecticides and the spread of insecticide resistance. This is even more the case for the control of vectors of animal diseases, which clearly remains at the backyard.

A key element for success is proper identification of disease agents and vector species, either already present or posing a potential threat. Once this has been established, measures should be taken, depending on the source and scale (globally, nationwide, locally). A multidisciplinary approach, taking into consideration all aspects involved, i.e. the One Health concept, is recommended to warrant effect. Durel et al. (2014) consider prevention, suppression, and monitoring of pest species the only realistic options within the implementation of IPM strategies in livestock facilities. Ample examples of methods that are or could be implemented are described in this book that still require more research. Local circumstances differ enormously, which requires tailor-made approaches (Meerburg and Schoelitsz 2018). Moreover different options, either targeting a single or several species, may have unexpected interactions that are sometimes additive, synergistic or antagonistic. Much lessons can be taken from the control of agricultural pests to foster innovation in vector control (Suckling et al. 2012, 2014).

\section{References}

Baldacchino F, Desquesnes M, Duvallet G, LysykT and Mihok S (2018) Veterinary importance and integrated management of Brachycera flies in dairy farms. In: Garros C, Bouyer J, Takken W and Smallegange RC (eds.) Pests and vector-borne diseases in the livestock industry. Ecology and Control of Vector-borne diseases. Vol. 5. Wageningen Academic Publishers, Wageningen, the Netherlands, pp. 55-90.

Bouyer F and Chia E (2018) Acceptability of vector control actions or co-production of innovations? In: Garros C, Bouyer J, Takken W and Smallegange RC (eds.) Pests and vector-borne diseases in the livestock industry. Ecology and Control of Vector-borne diseases. Vol. 5. Wageningen Academic Publishers, Wageningen, the Netherlands, pp. 537-571.

Bouyer J and Marois E (2018) Genetic control of vectors. In: Garros C, Bouyer J, Takken W and Smallegange RC (eds.) Pests and vector-borne diseases in the livestock industry. Ecology and Control of Vector-borne diseases. Vol. 5. Wageningen Academic Publishers, Wageningen, the Netherlands, pp. 435-451. 
Brugman VA, Smallegange RC and Logan JG (2018) Semiochemical tools for a new generation of livestock pest control. In: Garros C, Bouyer J, Takken W and Smallegange RC (eds.) Pests and vector-borne diseases in the livestock industry. Ecology and Control of Vector-borne diseases. Vol. 5. Wageningen Academic Publishers, Wageningen, the Netherlands, pp. 389-434.

Colwell DD and Wall R (2018) Sheep myiasis: a one health perspective. In: Garros C, Bouyer J, Takken W and Smallegange RC (eds.) Pests and vector-borne diseases in the livestock industry. Ecology and Control of Vector-borne diseases. Vol. 5. Wageningen Academic Publishers, Wageningen, the Netherlands, pp. 135-144.

Durel L, Estrada-Peña A, Franc M, Mehlhorn H and Bouyer J (2015). Integrated fly management in European ruminant operations from the perspective of directive 2009/128/EC on sustainable use of pesticides. Parasitol Res 114: 379-389.

Gimonneau G, Rayaisse JB and Bouyer J (2018) Integrated control of trypanosomosis. In: Garros C, Bouyer J, Takken W and Smallegange RC (eds.) Pests and vector-borne diseases in the livestock industry. Ecology and Control of Vectorborne diseases. Vol. 5. Wageningen Academic Publishers, Wageningen, the Netherlands, pp. 147-174.

Jonsdottir S, Hamza E, Janda J, Rhyner C, Meinke A, Marti E, Svansson V and Torsteinsdottir S (2015) Developing a preventive immunization approach against insect bite hypersensitivity using recombinant allergens: a pilot study. Vet Immunol Immunopathol 166: 8-21.

Kingsley $P$ and Taylor $E$ (2017) One Health: competing perspectives in an emerging field. Parasitology 144: 7-14.

Kinney Ferreira de Miranda Santos I, Rocha Garcia G, Silva Oliveira P, Veríssimo CJ, Morita Katiki L, Rodrigues L, Juan Szabó MP and Maritz-Olivier C (2018) Acaricides: current status and sustainable alternatives for controlling the cattle tick, Rhipicephalus microplus, based on its ecology. In: Garros C, Bouyer J, Takken W and Smallegange RC (eds.) Pests and vector-borne diseases in the livestock industry. Ecology and Control of Vector-borne diseases. Vol. 5. Wageningen Academic Publishers, Wageningen, the Netherlands, pp. 91-134.

Machtinger ET and Geden CJ (2018) Biological control with parasitoids. In: Garros C, Bouyer J, Takken W and Smallegange RC (eds.) Pests and vector-borne diseases in the livestock industry. Ecology and Control of Vector-borne diseases. Vol. 5. Wageningen Academic Publishers, Wageningen, the Netherlands, pp. 299-335.

Meerburg BG and Schoelitsz B (2018) Biosecurity: methods to reduce contact risks between vectors and livestock. In: Garros C, Bouyer J, Takken W and Smallegange RC (eds.) Pests and vector-borne diseases in the livestock industry. Ecology and Control of Vector-borne diseases. Vol. 5. Wageningen Academic Publishers, Wageningen, the Netherlands, pp. 453-464.

Miranda MA (2018) Case studies of vector-borne diseases in livestock: bluetongue virus. In: Garros C, Bouyer J, Takken W and Smallegange RC (eds.) Pests and vector-borne diseases in the livestock industry. Ecology and Control of Vector-borne diseases. Vol. 5. Wageningen Academic Publishers, Wageningen, the Netherlands, pp. 221-271.

Nachman G and Skovgård H (2018) The Fly Simulator: a simulation model of stable flies and their control. In: Garros C, Bouyer J, Takken W and Smallegange RC (eds.) Pests and vector-borne diseases in the livestock industry. Ecology and Control of Vector-borne diseases. Vol. 5. Wageningen Academic Publishers, Wageningen, the Netherlands, pp. 465-494.

Pagès N and Cohnstaedt LW (2018) Mosquito-borne diseases in the livestock industry. In: Garros C, Bouyer J, Takken W and Smallegange RC (eds.) Pests and vector-borne diseases in the livestock industry. Ecology and Control of Vectorborne diseases. Vol. 5. Wageningen Academic Publishers, Wageningen, the Netherlands, pp. 195-219.

Pérez de León AA, Mahan S, Messenger M, Ellis D, Varner K, Schwartz A, Baca D, Andreotti R, Rodríguez Valle M, Rosario Cruz R, Domínguez García DI, Pagan MC, Canabal CO, Urdaz J, Collazo Mattei F, Soltero F, Guerrero F and Miller RJ (2018) Public-private partnership enabled use of anti-tick vaccine for integrated cattle fever tick eradication in the USA. In: Garros C, Bouyer J, Takken W and Smallegange RC (eds.) Pests and vector-borne diseases in the livestock industry. Ecology and Control of Vector-borne diseases. Vol. 5. Wageningen Academic Publishers, Wageningen, the Netherlands, pp. 275-298.

Pfeffer M, Król N and Obiegala A (2018) Prevention and control of tick-borne Anaplasmosis, Cowdriosis and Babesiosis in the cattle industry. In: Garros C, Bouyer J, Takken W and Smallegange RC (eds.) Pests and vector-borne diseases in the livestock industry. Ecology and Control of Vector-borne diseases. Vol. 5. Wageningen Academic Publishers, Wageningen, the Netherlands, pp. 175-194. 
Roberts H, Nixon E and Matt C (2018) Case study: costs of Culicoides-borne arboviral diseases. In: Garros C, Bouyer J, Takken W and Smallegange RC (eds.) Pests and vector-borne diseases in the livestock industry. Ecology and Control of Vector-borne diseases. Vol. 5. Wageningen Academic Publishers, Wageningen, the Netherlands, pp. 497-508.

Sallam MF, Pereir RM and Koehler PG (2017) Fly populations and problems in a changing climate. In: Dhang P (ed.) Climate change impacts on urban pests. CAB International, Wallingford, UK.

Shaw APM (2018) Controlling tsetse - what does it cost? In: Garros C, Bouyer J, Takken W and Smallegange RC (eds.) Pests and vector-borne diseases in the livestock industry. Ecology and Control of Vector-borne diseases. Vol. 5. Wageningen Academic Publishers, Wageningen, the Netherlands, pp. 509-536.

Sigognault Flochlay A, Thomas E and Sparagano $O$ (2017) Poultry red mite (Dermanyssus gallinae) infestation: a broad impact parasitological disease that still remains a significant challenge for the egg-laying industry in Europe. Parasite Vectors 10: 357.

Sikkema R and Koopmans M (2016). One Health training and research activities in Western Europe. Infect Ecol Epidemiol 6: 33703.

Smallegange RC, Verhulst NO and Takken W (2011) Sweaty skin: an invitation to bite? Trends Parasitol 27: 143-148.

Sparagano O, Di Domenico D, Venturelli C, Papadopoulos E, Smallegange RC and Giangaspero A (2018) Arthropod pests in the poultry industry. In: Garros C, Bouyer J, Takken W and Smallegange RC (eds.) Pests and vector-borne diseases in the livestock industry. Ecology and Control of Vector-borne diseases. Vol. 5. Wageningen Academic Publishers, Wageningen, the Netherlands, pp. 17-53.

Suckling DM, Tobin PC, McCullough DG and Herms DA (2012) Combining tactics to exploit Allee effects for eradication of alien insect populations. J Econ Entomol 105: 1-13.

Suckling DM, Stringer LD, Stephens AE, Woods B, Williams DG, Baker G and El-Sayed, AM (2014) From integrated pest management to integrated pest eradication: technologies and future needs. Pest Management Sci 70: 179-189.

Vreysen M, Robinson AS and Hendrichs J (2007) Area-wide control of insect pests, from research to field implementation. Springer, Dordrecht, the Netherlands.

Weeks ENI, Machtinger ET, Leemon D and Geden CJ (2018) Biological control of livestock pests: entomopathogens. In: Garros C, Bouyer J, Takken W and Smallegange RC (eds.) Pests and vector-borne diseases in the livestock industry. Ecology and Control of Vector-borne diseases. Vol. 5. Wageningen Academic Publishers, Wageningen, the Netherlands, pp. 337-387. 


\section{About the editors}

Claire Garros (1979) is a medical and veterinary entomologist at the French agricultural research and international cooperation organization working for the sustainable development of tropical and Mediterranean regions (Cirad), Montpellier, France. After studying biology and parasitology at the university, she obtained her PhD in entomology from the University of Montpellier in 2005 with a work on the systematic and taxonomy of anopheline species in Southeast Asia. Then, she joined the University of California, Irvine, USA, as a post-doc followed by a second post-doc at the University of Louvain-La-Neuve, Belgium in 2007. When she started at Cirad, Montpellier, in 2009 while the bluetongue and Schmallenberg viruses were touching Europe, her focus shifted towards studying the taxonomy and ecology of Culicoides biting midges. She also coordinated for 7 years the national surveillance network for Culicoides populations. In addition to national activities, she takes part in international networks and research projects related to vector-borne diseases in Europe and in Africa. Since 2016, she is localized in La Réunion Island, French territory in the Indian ocean, where she developed research on mosquitoes and Culicoides for the whole south-west Indian ocean region.

Jérémy Bouyer (1977) is a medical entomologist leading the Human Disease Vectors group of the Insect Pest Control Laboratory, Joint FAO/IAEA Division of Nuclear Techniques in Food and Agriculture (NAFA), Vienna, Austria. After studying veterinary medicine at the École Nationale Vétérinaire de Maisons Alfort, France he obtained his $\mathrm{PhD}$ in entomology from Montpellier University, France, in 2006. Subsequently, he worked seven years in Burkina Faso, seven years in Senegal and two years in Ethiopia on tsetse flies, vectors of African trypanosomoses. With extensive experience in the ecology and control of vectors and diseases, he returned to Europe to work at the IAEA in 2017. He is also coordinating the ERC REVOLINC (revolutionizing insect control) project for UMR Cirad-INRA ASTRE.
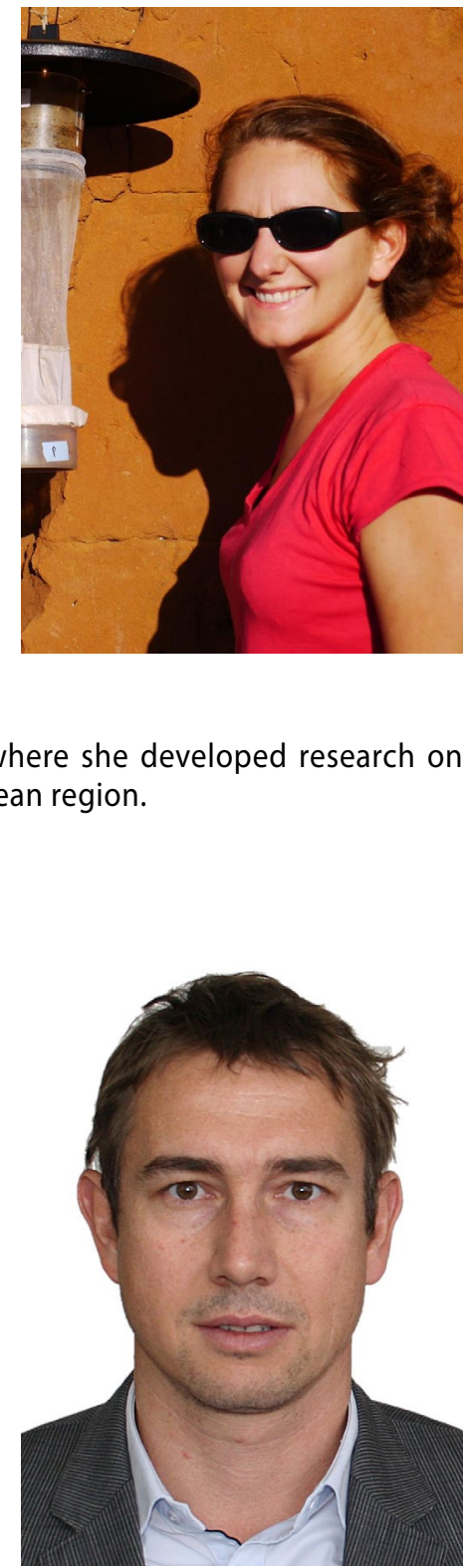
Willem Takken (1951) is professor in Medical and Veterinary Entomology at Wageningen University \& Research, the Netherlands. He studied in Wageningen and obtained his PhD degree in 1980 based on research on the biology and feeding behaviour of tsetse flies. He worked in several African countries on the control of animal trypanosomiasis. Upon his return to Europe, he was appointed as lecturer at the Laboratory of Entomology in Wageningen, where he introduced medical and veterinary entomology to the Wageningen academic society. His work involved mosquitohost interactions, in particular the host-seeking behaviour of malaria mosquitoes. He later expanded this work to include field research in Tanzania, Kenya, and other tropical countries. Today, his work involves the ecology of mosquitoes, biological control of mosquitoes and the impact of environmental change on malaria vectors. In Europe, Willem studies the ecology of Lyme disease vectors and vectors of other, emerging infectious diseases. He emphasises collaboration with other institutions, and has an extensive network of national and international collaborators. He serves on several editorial boards and advisory committees.

Renate Smallegange (1969) is a medical and veterinary entomologist. After studying biology at Wageningen University, the Netherlands, she obtained her PhD in 2003 from Groningen University, the Netherlands, where she researched the response of the house fly, Musca domestica, to visual and olfactory stimuli. Subsequently, she worked for 10 years at the Laboratory of Entomology of Wageningen University \& Research. During this period, she took part in studies identifying human volatiles as attractants for malaria mosquitoes in order to disrupt malaria transmission. In recent years she was initiator and consultant of a BBSRC project and a ZonMw project (both collaborations between the London School of Hygiene and Tropical Medicine and Wageningen University \& Research) examining the olfactory mechanisms underlying behavioural manipulation of mosquitoes by malaria parasites. During a short visit in 2011 at the London School of Hygiene and Tropical Medicine a M. domestica colony was established to run olfactometer experiments with this important livestock pest. Currently, she is commissioning editor at Wageningen Academic Publishers.
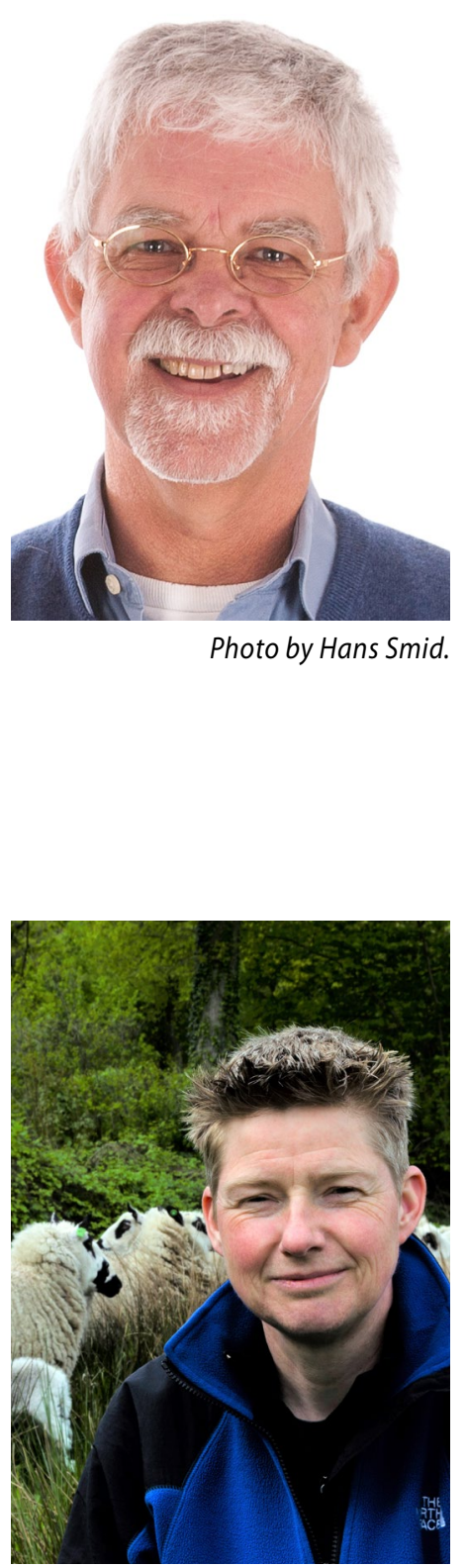

Photo by Marcel de Haas (with courtesy of Karin Terpstra). 


\section{Contributors}

\section{Renato Andreotti}

Embrapa Gado de Corte, Av. Rádio Maia, No. 830, Zona Rural, CEP 79106-550 Campo Grande, MS, Brazil

\section{Dan Baca}

United States Department of Agriculture-Animal and Plant Health Inspection Service, Veterinary Services, San Jacinto Blvd., Austin, TX 78701, USA

\section{Frédéric Baldacchino}

Research and Innovation Centre, Fondazione Edmund Mach, Via Mach 1, 38010 San Michele all'Adige, Italy; ${ }^{2}$ Direction départementale de la protection des populations Nord, 93-95 boulevard Carnot, 59046 Lille cedex, France

\section{Fanny Bouyer}

CIRAD, UMR ASTRE, 34398 Montpellier, France; INRA, UMR 1304, ASTRE, 34398 Montpellier, France; 3Finnovation S.A.S., 9 rue des Coteaux, 68550 Saint-Amarin, France

\section{Jérémy Bouyer}

ASTRE, University of Montpellier, CIRAD, INRA, Montpellier, France; Unité Mixte de Recherche ASTRE 'Animal, Santé, Territoires, Risques et Ecosystèmes', Campus international de Baillarguet, Centre de Coopération Internationale en Recherche Agronomique pour le Développement (CIRAD), 34398 Montpellier, France; Insect Pest Control Laboratory, Joint FAO/IAEA Programme of Nuclear Techniques in Food and Agriculture, 1400 Vienna, Austria

\section{Victor A. Brugman}

Evolution Biotechnologies, Colworth Science Park, Sharnbrook, Bedford, MK44 1LQ, United Kingdom

\section{Eduardo Chia}

INRA UMR Innovation 0951, 2 place Vialat, Bat 27, 34060 Montpellier, France

\section{Lee W. Cohnstaedt}

United States Department of Agriculture, Agricultural Research Service, Arthropod-borne Animal Diseases Research Unit, 1515 College Ave, Manhattan, KS 66502, USA

\section{Francisco Collazo Mattei}

United States Department of Agriculture-Animal and Plant Health Inspection Service, Veterinary Services, 8100 NW 15 $5^{\text {th }}$ Place Gainesville, FL 32606, USA

\section{Douglas D. Colwell}

Agriculture and Agri-Food Canada, Lethbridge Research and Development Centre, $54031^{\text {st }}$ Ave. S., Lethbridge, $A B, T 1 J$ 4B1, Canada

\section{Myrna Comas Pagan}

Department of Agriculture of Puerto Rico, P.O. Box 10163, San Juan 00908-1163, Puerto Rico 


\section{Marc Desquesnes}

Cirad, Avenue Agropolis, 34398 Montpellier Cedex 5, France; Kasetsart University, 50 Ngam Wong Wan Rd, Ladyaow Chatuchak, Bangkok 10900, Thailand

\section{Davide Di Domenico}

Mellivora Pest Management and Consulting, Via F. Coppi n²0, 40033 Casalecchio di Reno (BO), Italy

\section{Gérard Duvallet}

Centre d'Écologie fonctionnelle et évolutive, UMR5175, Université Paul-Valéry, Route de Mende, 34199 Montpellier Cedex 5, France

\section{Dee Ellis}

Institute for Infectious Animal Diseases, Texas A\&M University System, 1500 Research Parkway, Suite B270, College Station, TX 77843-2129, USA

\section{Claire Garros}

Unité Mixte de Recherche ASTRE 'Animal, Santé, Territoires, Risques et Ecosystèmes', Campus international de Baillarguet, Centre de Coopération Internationale en Recherche Agronomique pour le Développement (CIRAD), 34398 Montpellier, France; CIRAD, UMR ASTRE, 97491 SainteClotilde, La Réunion, France; ASTRE, University of Montpellier, CIRAD, INRA, Montpellier, France

\section{Gustavo R. Garcia}

Department of Biochemistry and Immunology, Ribeirão Preto School of Medicine, University of São Paulo, Avenida Bandeirantes 3900, Monte Alegre, Ribeirão Preto, SP 14049-900, Brazil

\section{Christopher J. Geden}

United States Department of Agriculture, Agricultural Research Service, Center for Medical, Agricultural and Veterinary Entomology, 1600 SW 23 ${ }^{\text {rd }}$ Drive, Gainesville, FL 32608, USA

\section{Annunziata Giangaspero}

Department of Science of Agriculture, Food and Environment, University of Foggia, Via Napoli 25, 71121 Foggia, Italy

\section{Geoffrey Gimonneau}

CIRAD, UMR INTERTRYP, Département BIOS TA A-17/G Campus International de Baillarguet 34398 Montpellier Cedex 5, France; Centre International de Recherche-développement sur l'Élevage en Zone Subhumide, BP 454, Bobo-Dioulasso, Burkina Faso

\section{Felix Guerrero}

United States Department of Agriculture-Agricultural Research Service, Knipling-Bushland United States Livestock Insects Research Laboratory, and Veterinary Pest Genomics Center, 2700 Fredericksburg Rd., Kerrville, TX 78028, USA

\section{Delia Inés Domínguez García}

Laboratorio de Investigación en Biotecnología, Salud y Ambiente de la Unidad Académica de Ciencias Naturales de la Universidad Autonoma de Guerrero. Campus el Shalako, Petaquillas, Guerrero, C.P. 39105, Mexico 


\section{Luciana M. Katiki}

São Paulo State Animal Science Institute, Rua Heitor Penteado, 56, Nova Odessa, SP 13460-000, Brazil

\section{Nina Król}

Institute of Animal Hygiene and Veterinary Public Health, Veterinary Faculty, University of Leipzig, An den Tierkliniken 1, 04103 Leipzig, Germany

\section{Diana Leemon}

Department of Agriculture and Fisheries (Queensland), Agri-Science Queensland, Ecosciences Precinct, GPO Box 267, Brisbane, Queensland, 4001, Australia

\section{James G. Logan}

London School of Hygiene and Tropical Medicine, Keppel Street, London, WC1E 7HT, United Kingdom

\section{Timothy Lysyk}

Research Centre, Agriculture and Agri-Food Canada, $54031^{\text {st }}$ Avenue South Lethbridge, AB, T1 J 4B1, Canada

\section{Erika T. Machtinger}

Penn State University, Department of Entomology, 501 ASI Building, University Park, State College, PA 16082, USA

\section{Suman Mahan}

Zoetis, Veterinary Medicine Research and Development, 333 Portage Street, KZO-300-329.4SW, Kalamazoo, MI 49007, USA

\section{Christine Maritz-Olivier}

Faculty of Agricultural and Natural Sciences, University of Pretoria, Department of Genetics, Private bag X20, Hatfield 0028 Pretoria, South Africa

\section{Eric Marois}

INSERM U963, CNRS UPR9022, Université de Strasbourg, Institut de Biologie Moléculaire et Cellulaire, Strasbourg, France

\section{Clemens Matt}

Exotic Disease policy team, Defra, 17 Smith Square, London, SW1P 3JR, United Kingdom

\section{Bastiaan G. Meerburg}

Wageningen Livestock Research, Wageningen University \& Research, P.O. Box 338, $6700 \mathrm{AH}$ Wageningen, the Netherlands; Dutch Pest \& Wildlife Expertise Centre (KAD), P.O. Box 350, 6700 AJ Wageningen, the Netherlands

\section{Matthew Messenger}

United States Department of Agriculture-Animal and Plant Health Inspection Service, Plant Protection and Quarantine, 4700 River Road, Unit 140, Riverdale, MD 20737, USA 


\section{Steve Mihok}

388 Church Street, Russell, ON, K4R 1A8, Canada

\section{Robert J. Miller}

United States Department of Agriculture-Agricultural Research Service, Cattle Fever Tick Research Laboratory, 22675 North Moorefield Rd. MAB 6419, Edinburg, TX 78541, USA

\section{Miguel Á. Miranda}

Laboratory of Zoology, University of the Balearic Islands, Carretera de Valldemossa Km 7.5, 7122 Palma de Mallorca, Spain

\section{Isabel K.F. de Miranda Santos}

Department of Biochemistry and Immunology, Ribeirão Preto School of Medicine, University of São Paulo, Avenida Bandeirantes 3900, Monte Alegre, Ribeirão Preto, SP 14049-900, Brazil

\section{Gösta Nachman}

Department of Biology, Section of Ecology and Evolution, University of Copenhagen, Universitetsparken 15, 2100 Copenhagen $\emptyset$, Denmark

\section{Emily Nixon}

APHA Exotics and Risk Team, Advice Services, Animal and Plant Health Agency, Defra, 17 Smith Square, London SW1P 3JR, United Kingdom; Veterinary Parasitology and Ecology Group, University of Bristol, Bristol Life Sciences Building, 24 Tyndall Avenue, Bristol, BS8 1TQ, United Kingdom

\section{Anna Obiegala \\ Institute of Animal Hygiene and Veterinary Public Health, Veterinary Faculty, University of Leipzig, An den Tierkliniken 1, 04103 Leipzig, Germany}

\section{Carmen Oliver Canabal}

Department of Agriculture of Puerto Rico, P.O. Box 10163, San Juan 00908-1163, Puerto Rico

\section{Nonito Pagès}

CIRAD/INRA UMR ASTRE (Animal, Santé, Territoires, Risques et Ecosystèmes), CRVC - Centre for Research and surveillance on Vector-borne diseases in the Caribbean, Domaine Duclos-Prise d'Eau, 97170-Petit Bourg, Guadeloupe, France

\section{Elias Papadopoulos}

Laboratory of Parasitology and Parasitic Diseases, School of Veterinary Medicine, Faculty of Health Sciences, Aristotle University of Thessaloniki, Thessaloniki, Greece

\section{Adalberto A. Pérez de León}

United States Department of Agriculture-Agricultural Research Service, Knipling-Bushland United States Livestock Insects Research Laboratory, and Veterinary Pest Genomics Center, 2700 Fredericksburg Rd., Kerrville, TX 78028, USA

\section{Martin Pfeffer}

Institute of Animal Hygiene and Veterinary Public Health, Veterinary Faculty, University of Leipzig, An den Tierkliniken 1, 04103 Leipzig, Germany 


\title{
Jean-Baptiste Rayaisse
}

Centre International de Recherche-développement sur l'Élevage en Zone Subhumide, BP 454, Bobo-Dioulasso, Burkina Faso

\section{Helen Roberts}

Exotics and Risk team, Animal and Plant Health Agency, Defra, 17 Smith Square, London, SW1P 3JR, United Kingdom

\section{Leandro Rodrigues}

São Paulo State Animal Science Institute, Rua Heitor Penteado, 56, Nova Odessa, SP 13460-000, Brazil

\section{Manuel Rodríguez Valle}

The University of Queensland, Queensland Alliance for Agriculture and Food Innovation, 306 Carmody Rd., Bldg. 80, Level 3; St. Lucia, Qld 4072, Australia

\section{Rodrigo Rosario Cruz}

Laboratorio de Investigación en Biotecnología, Salud y Ambiente de la Unidad Académica de Ciencias Naturales de la Universidad Autonoma de Guerrero. Campus el Shalako, Petaquillas, Guerrero, C.P. 39105, Mexico

\section{Bruce Schoelitsz}

HAS University of Applied Sciences, P.O. Box 90108, 5200 MA's-Hertogenbosch, the Netherlands

\section{Andy Schwartz}

Texas Animal Health Commission, 2105 Kramer Lane, Austin TX 78758-4013, USA

\begin{abstract}
Alexandra P.M. Shaw
Division of Infection and Pathway Medicine, Edinburgh Medical School, Biomedical Sciences, College of Medicine and Veterinary Medicine, The University of Edinburgh, Chancellor's Building, 49 Little France Crescent, Edinburgh, EH16 4SB, United Kingdom; A P Consultants, 22 Walworth Enterprise Centre, Duke Close, Andover, SP10 5AP, United Kingdom
\end{abstract}

\section{Priscila Silva Oliveira}

Department of Biochemistry and Immunology, Ribeirão Preto School of Medicine, University of São Paulo, Avenida Bandeirantes 3900, Monte Alegre, Ribeirão Preto, SP 14049-900, Brazil

\section{Henrik Skovgård}

Department of Agroecology, Section of Pathology and Entomology, University of Aarhus, Forsøgsvej 1, 4200 Slagelse, Denmark

\section{Renate C. Smallegange}

Laboratory of Entomology, Wageningen University \& Research, P.O. Box 16, 6700 AA Wageningen, the Netherlands

\section{Fred Soltero}

United States Department of Agriculture-Animal and Plant Health Inspection Service, Veterinary Services, 654 Munoz Rivera Ave. Plaza Bldg. Suite 700 San Juan 00918, Puerto Rico 


\section{Olivier Sparagano}

Centre for Agriculture and Water Resilience (CAWR), Coventry University, Coventry, CV1 5FB, United Kingdom

\section{Matias P.J. Szabó}

School of Veterinary Medicine, Federal University of Uberlândia, Av. Pará, 1720, Campus UmuaramaBloco 2T, 38400-902 Uberlândia, MG, Brazil

\section{Willem Takken}

Laboratory of Entomology, Wageningen University \& Research, P.O. Box 16, 6700 AA Wageningen, the Netherlands

\section{Jose Urdaz}

United States Department of Agriculture-Animal and Plant Health Inspection Service, Veterinary Services, 2150 Centre Ave. Bldg. B, MS-3E13, Ft. Collins, CO 80526, USA

\section{Kevin Varner}

United States Department of Agriculture-Animal and Plant Health Inspection Service, Veterinary Services, San Jacinto Blvd., Austin, TX 78701, USA

\section{Claudio Venturelli}

AUSL of Romagna, U.O. Igiene e Sanità Pubblica - Cesena, Via M. Moretti, 99, 47521 Cesena (FC), Italy

\section{Cecília J. Veríssimo}

São Paulo State Animal Science Institute, Rua Heitor Penteado, 56, Nova Odessa, SP 13460-000, Brazil

\section{Richard Wall}

University of Bristol, Life Sciences Building, 24 Tyndall Avenue, Bristol BS8 1TQI, United Kingdom

\section{Emma N.I. Weeks}

University of Florida, Entomology and Nematology Dept., 970 Natural Area Drive, Gainesville, FL 32653, USA 


\title{
Reviewers
}

\author{
Diego Ayala \\ MIVEGEC, IRD, CNRS, University of Montpellier, 911 Av. Agropolis, BP 1864501, 34394 Montpellier, \\ France
}

\section{Frédéric Baldacchino}

Direction départementale de la protection des populations Nord, 93-95 boulevard Carnot, 59046 Lille cedex, France

\section{Tariq M. Butt}

Swansea University, College of Science, Department of Biosciences, Singleton Park, Swansea SA2 8PP, United Kingdom

\section{Simon Carpenter}

The Pirbright Institute, Ash Road, Pirbright, Woking, GU24 ONF, United Kingdom

\section{Giuliano Cecchi}

FAO Sub-regional Office for Eastern Africa, CMC Road, Next to ILRI, P.O. Box 5536, Addis Ababa, Ethiopia

\section{Lee W. Cohnstaedt}

United States Department of Agriculture, Agricultural Research Service, Arthropod-borne Animal Diseases Research Unit, 1515 College Ave, Manhattan, KS 66502, USA

\author{
Marcel Dicke \\ Laboratory of Entomology, Wageningen University \& Research, P.O. Box 16, 6700 AA Wageningen, \\ the Netherlands
}

\section{Gérard Duvallet}

Université Paul-Valéry Montpellier, UMR 5175 CEFE (Centre d'Ecologie fonctionnelle et évolutive), Route de Mende, 34199 Montpellier Cedex 5, France

\section{Christopher J. Geden}

United States Department of Agriculture, Agricultural Research Service, Center for Medical, Agricultural and Veterinary Entomology, 1600 SW 23 ${ }^{\text {rd }}$ Drive, Gainesville, FL 32608, USA

\section{David George}

Stockbridge Technology Centre, Cawood, Selby YO8 3TZ, United Kingdom

\section{Flavie L. Goutard}

Centre de Coopération Internationale en Recherche Agronomique Pour le Développement (CIRAD), Département ES, UPR AGIRs, TA C22/E, Campus International de Baillarguet, 34398 Montpellier Cedex 5, France

\section{Lara Harrup}

The Pirbright Institute, Ash Road, Pirbright, Woking, GU24 ONF, United Kingdom 


\section{Andrei D. Mihalca}

University of Agricultural Sciences and Veterinary Medicine Cluj-Napoca, Calea Mănăştur 3-5, 400372, Cluj-Napoca, Romania

\section{Bradley A. Mullens}

Department of Entomology, University of California, Riverside, CA 92506, USA

\section{Henrik Skovgård}

Department of Agroecology, Section of Pathology and Entomology, University of Aarhus, Forsøgsvej 1, 4200 Slagelse, Denmark

\section{Frédéric Stachurski}

UMR Cirad-Inra ASTRE, Campus international de Baillarguet, 34398 Montpellier cedex 05, France

\section{Willem Takken}

Laboratory of Entomology, Wageningen University \& Research, P.O. Box 16, 6700 AA Wageningen, the Netherlands

\section{Sip E. van Wieren}

Resource Ecology Group, Wageningen University \& Research, P.O. Box 47, 6700 AA Wageningen, the Netherlands 


\section{Keyword index}

A

AAT - See African animal trypanosomosis

Acadrex $^{\circledast} 241$

Acari 41

acaricide $12,45,91,94,97,100,118,175,179,181$,

$184,186,187,275,276,278,283,284,287,413$,

$414,531,549,552,554,565,567$

- residues in beef and dairy 93

- resistance 43, 93, 98, 100, 102, 276, 288

acaropathogenic fungi 278

6-acetoxy-5-hexadecanolide 405

acetylcholinesterase 44,96

Acinetobacter spp. 67

Actinomyces pyogenes - See Trueperella pyogenes

adenotrophic viviparity 65,401

adulticides 238

Aedes 197, 199, 201, 204, 229, 345, 347, 402, 404, $406,415,437,439,447$

- aegypti 212, 213, 345, 347, 348, 396, 398, 400, $404,408,415,439,443,444,445$

- albopictus 210, 212, 213, 404, 405, 406, 435, 439, 447

- campestris 210

- caspius 406

- dorsalis 210

- mcintoshi 201,404

- nigromaculis 345

- notoscriptus 207

- ochraceus 404

- polynesiensis 404,439

- procax 207

- sierrensis 345

- vexans 201, 202, 210

- vigilax 207

Aereobacter 28

aerial

- sampling 231

- spraying 509,510

Aeromonas spp. 67

Africa 57, 58, 59, 63, 65, 66, 70, 72, 102, 104, 114, $147,148,175,180,182,184,185,199,201,202$, $206,207,212,318,340,354,363,401,406,435$, $436,443,505,510,529,550,555$

African

- animal trypanosomosis (AAT) 55, 58, 59, 70, $147,157,160,161$
- horse sickness (AHS) 221, 222, 339, 407, 466, 497, 498, 500

- swine fever 12

African swine fever 466, 576

aggregation 99

- pheromone 369

Al - See avian influenza

air entrainment 391,412

Akabane virus 222, 339

aldrin 244, 245

allelochemicals 390

allergies 37

Allium

- cepa 103

- sativum 101, 103

allomones 390, 392

alpha-cypermethrin 93, 241, 531

Alphavirus 208

Alphitobius diaperinus 17, 31, 341, 360, 361, 369, $455,457,466$

- control 34

- habitat 32

- hot spots 35

Alsynite traps 79

Altosid 245

Amblyomma 111, 340

- americanum 111, 176, 368

- hebraeum 180,414

- maculatum 414

- variegatum 151, 164, 176, 180, 357, 367, 414, 550,557

Ambystoma tigrinum 415

America 57, 64, 102

American

- cockroach - See Periplaneta americana

- Samoa 318

aminidines 93

amitraz 94, 167

- resistance 95, 98, 100

ammonia 80

Ammotragus lervia 222

amphibians 395

Anacardium occidentale 405

Anadenanthera macrocarpa 101

anaemia 22, 162

anaphylactic shock 409

anaplamoses 557 


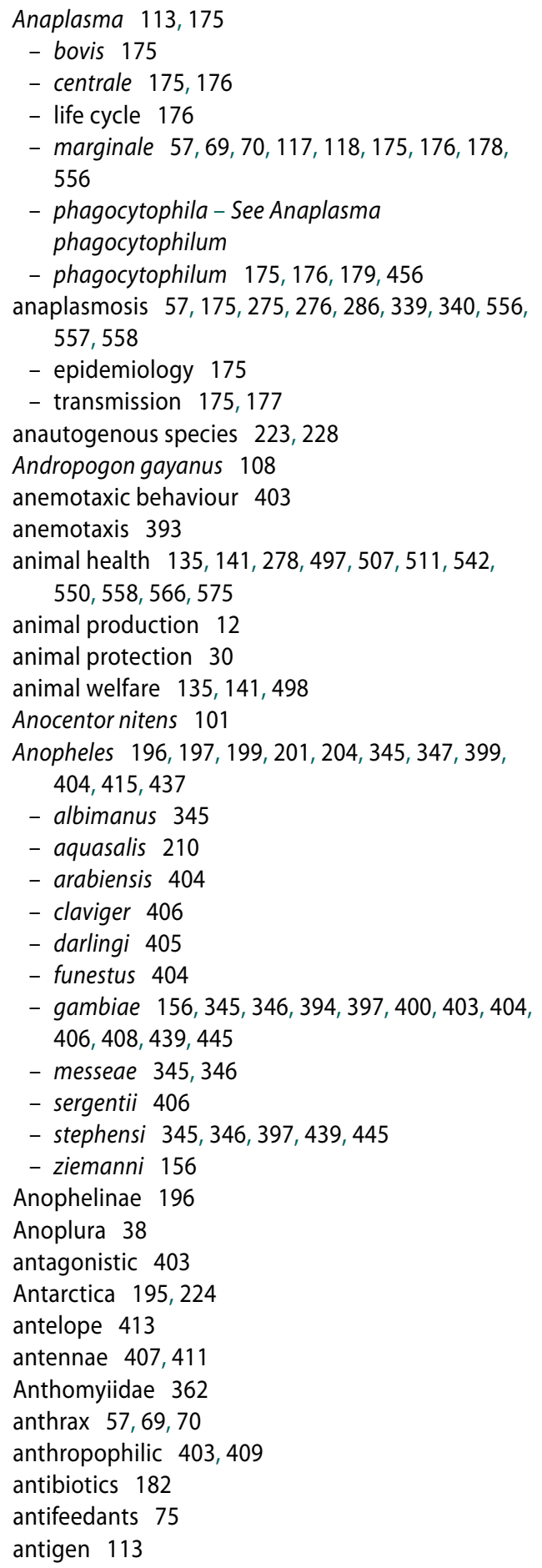

antigenic variation 179

anti-tick vaccine 275,578

Apodemus sylvaticus 458

Arabian Peninsula 200

Araucaria angustifolia 101, 103

arboviral disease 221,455, 497

arbovirus 199, 213, 223, 250, 251, 405, 435, 447

- surveillance 394

- transmission 234

Argasidae 340, 355

Argentina 93, 322

Argus persicus 341

Arkofly ${ }^{\oplus} 241$

Armigeres 204

arrhenotokous development 302

arthrogyposis 499

arthropod 401

- behaviour 111

- -borne diseases 11, 13, 389, 398, 575

- pests 17

- resistance 370

Artiodacyla 200

Asaia 439

Ascaris 28

Asia 24, 58, 59, 63, 64, 66, 102, 105, 114, 175, 176 $184,203,205,206,212,318,340,435$

Aspergillus 33, 455

- ochraceus 355

associative learning 415

Athericidae 55, 61

ATSB - See attractive toxic sugar bait

attractants 154

attraction-aggregation-attachment pheromones 413

attractive toxic sugar bait (ATSB) $398,406,409$

Atylotus 411

- agrestis 71

- fuscipes 71

- loewianus 411

- quadrifarius 411

augmentative biological control 319

Australia $58,59,61,64,69,72,76,93,96,97,102$, $114,137,138,139,175,184,203,205,206,227$ $228,231,232,235,236,238,248,249,278,306$, $318,352,405,406,444$

autodissemination 348,371

avermectins 395

avian influenza (Al) 24, 26, 42, 454

Azadirachta indica 101, 236 
azadirachtin 348

B

Babesia 113, 179, 183

- bigemina 104, 175, 183, 184, 186, 187, 276, 557

- bovis 104, 175, 183, 184, 186, 187, 276, 557

- divergens 183

- jakimovi 185

- occultans 185

babesioses 278,557

babesiosis $12,175,183,275,276,277,278,286$, $340,557,558$

- epidemiology 184

- transmission 184

Bacillus 33

- anthracis 57,69,70

- thuringiensis (Bti) 30, 75, 76, 338, 370, 409, 578

- thuringiensis thompsoni 75

- thuringiensis var. israelensis 249, 405

bacteria $11,12,22,28,33,37,67,69,111,392,402$, 439,454

Bacteroides 68

Barmah Forest virus 394

Bartonella bacilliformis 408

basic reproductive rate 501

Batkoa apiculata 561

Beauveria 110, 362

- bassiana 30, 34, 46, 75, 248, 324, 337, 341, 343, $344,345,346,347,348,349,350,351,352,353$, $354,355,356,357,358,359,360,361,370,466$

bed bugs - See Cimex lectularius

behaviour-modifying chemicals 389

Belgium 227, 231

Belize 545

benefit cost ratio 498

Y-benzene hexachloride 244

Besnoitia besnoiti 55, 70, 71, 411

BG-lure $404,406,410$

BG-Sentinel trap 229, 244, 246, 404, 406

bifenthrin 352

binary mixtures 79

biocide $37,234,491$

biocontrol 305,455

- agents 578

biological age $475,476,479$

biological control $13,17,26,34,45,74,109,248$, $253,299,337,436,462,466,470,484,490,557$, $559,560,561,565,567,576$

- augmentative 319
- housefly 26

- non-target effects 111

biopesticide $12,348,470$

biorational control 13

biorational Insecticides 245

biosecurity $31,278,453$

bioterrorism 212

birnavirus 455

Bison bison 176

biting flies 19, 22

black dump fly - See Ophyra; See Hydrotaea (Ophyra) aenescens

black fly 390, 396, 397, 399, 400, 401, 409

black fruit fly - See Drosophila repleta

black garbage fly - See Ophyra

black soldier fly - See Hermetia illucens

blastospores 343,346

Blatta orientalis 35,36

Blattella germanica $35,36,456$

Blattodea 35

blowfly $27,135,352,398$

bluetongue $12,212,497,499,500,503,576$

bluetongue virus (BTV) 221, 339, 407, 455, 498

- transmission 221, 223, 228, 241

- vaccines 234

Bm86 113

- -based vaccine 279, 281

Bombyx mori 110

Boophilus 340

boric acid 398

Borrelia 112

- burgdorferi 57, 117

Bos

- indicus 105, 114, 182

- taurus 105, 114, 182

Botswana 515, 519, 520, 521, 523, 525, 527

bovine

- anaplasmosis 70

- besnoitiosis 70,80

- spongiform encephalitis 539

- thelaziosis 72

Brachycera 55

Braconidae 363

Brazil 17, 42, 92, 93, 101, 106, 209, 228, 276, 287, $318,356,357,405,406,443,444$

breeding for resistance 105,114

bridge vectors 208

brown chicken louse - See Goniodes dissimilis

Brucella spp. 27, 57, 69 
brucellosis 57

Brugia 199

Bti - See Bacillus thuringiensis

BTV - See bluetongue virus

Bubalus bubalis 176

Bubulcus ibis 76, 180

buffalo 252

- fly - See Haematobia

- worm 31

Bulgaria 137, 138, 227

Bunyaviridae 199, 402

Buphagus

- africanus 110

- erythrorhynchus 110

Burkina Faso 77, 164, 410, 515, 531, 554

bush fly - See Musca vetustissima

Butox $^{\circledast}$ 240, 241, 243, 558, 564

\section{C}

Caesalpinia gaumeri 103

Calliphora 18, 27

- augur 138

- stygia 138

- vicina 30,138

- vomitoria 138

Calliphoridae $18,21,135,339,362$

camels 200, 340, 414

Cameroon 515

Campylobacter 22, 23, 24, 26, 29, 67

- jejuni 67,454

campylobacteriosis 23

Canada 206, 305, 318, 322, 366, 481

cannibalism 33

Caprapyrenaica 222

Capreolus capreolus 222

Carapaguianensis 101

carbamates 96

carbaryl 41,43

carbon dioxide $42,79,229,251,347,394,401,402$, $403,404,405,406,407,408,410,411,412,413$

carboxylesterase gene 96,99

Carcinops 466

card agglutination test (CAT) 178

Cardinium 250

Cardiofilaria 199

Caribbean 180, 287, 548, 549

carrot fly - See Psila rosae

carvacrol 102, 103

CAT - See card agglutination test catnip - See Nepeta cataria

cattle $11,160,175,200,203,222,226,227,240$, $242,276,278,283,284,285,286,299,306,307$, $309,317,321,322,323,338,340,345,353,354$ $390,395,402,407,409,410,442,455,456,459$, $465,466,481,488,502,515,528,549,550,556$, $557,560,564,576$

- fever tick eradication 275

- fly - See Musca crassirostris

- tick - See Rhipicephalus (Boophilus) microplus

cavitary myiasis 136

Cayman Islands 443

CDC light trap

- miniature 229, 247

CDC light traps $404,408,409$

cDNA library 251

cedrol 399

Central America 114, 139, 184, 207, 446, 542

Cephenemyia 393

- apicata 399

- jellisoni 399

Ceratopogonidae 221, 248, 249, 339, 390, 394, 398, 407,455

Ceratopogoninae 224

Cervus elaphus 222

Chalcidoidea 300

chemical control $17,30,34,236,436,460,462,465$, $466,486,488$

Chenopodium ambrosioides 101

chicken 227

- body louse - See Menacanthus stramineus

- lice 38

- red mite - See Dermanyssus gallinae

chikungunya $394,435,437,447$

Chile 318

China $17,64,136,203,205,318,454,539$

Chironomidae 248, 249

Chlamydia spp. 67

chlordane 244, 245

chlorpyrifos 244, 246

chronological age 476,479

Chrysanthemum cinerariaefolium 95

Chrysomya

- albiceps 138

- bezziana 135, 139

Cimex lectularius $341,343,360$

cineole 102

Cinnamomum verum 103

citronella 101,237 
classical swine fever 456,457

climate change 213,435

clorfenvinfos 370

clorpyrifos 238

Clostridium 28

- perfringens 24

cluster fly - See Pollenia rudis

Cochliomyia 437

- hominivorax 135, 139, 542, 546

- macellaria 543

cockroach 17, 35

- life cycle 35, 36

- prevention 38

- reproduction 35

coevolution 114

colleterial glands 36

Colombia 209, 210, 236, 545

community bath 151

Comoros 199

control $11,12,18,24,30,35,401,498,499,503,542$

- biological 13,26,34, 45, 319

- biorational 13

- chemical 17,30,34

- mechanical 13,31

- non-chemical 30

copaiba 101

coprophagous 467

Coquillettidia 201

- perturbans 210

Corymba citriodora 102

Corynebacterium glutamicum 112

Costa Rica 70, 318, 545, 548

cost-effectiveness 154

Côte d'Ivoire $515,516,521,522,528$

coumaphos 41,96

coupled GC-mass spectrometry 391

Cowdria ruminantium 180

cowdriosis $175,180,557$

- epidemiology 180

- transmission 180

Coxiella 112,455

- burnetii 57,69

coxsackievirus 69

Crescentiacujete 103

CRISPR-Cas9 441, 445

crop protection 29

crossbreeding 116

cross-protection 183

cross resistance 97 cryptosporidiosis 68

Cryptosporidium 455

- parvum 68

Cryptotylus unicolor 71

Cuba 548

Cuclotogaster heterographus 38

Culex 196, 199, 201, 204, 210, 213, 345, 347, 402, $404,406,447$

- annulirostris 204,205, 207

- antennatus 207

- erraticus 210

- fuscocephala 204

- gelidus 204, 207

- modestus 207

- nigripalpus 207

- pipiens 196, 201, 202, 204, 207, 208, 212,397, 400, 405, 406

- pipiens pipiens 345,346

- pseudovishnui 204, 207

- quinquefasciatus 212, 345, 346, 400, 405, 439

- restuans 207,415

- sitiens 204

- tarsalis 201, 204, 207, 208, 210, 345, 400

- tritaeniorhynchus 203, 204, 207

- univitattus 207

- vishnui 204, 207

Culicidae 195, 339, 390, 394, 398, 402

Culicinae 196

Culicinomyces

- clavisporus 248, 344, 345

Culicoides 221, 222, 223, 234, 237, 238, 239, 241, $242,252,339,344,395,396,397,407,455,497$, 503,577

- actoni 235

- arakawae 228

- arboricola 249

- (Avaritia) actoni 225

- (Avaritia) brevitarsis 225

- (Avaritia) chiopterus 225

- (Avaritia) dewulfi 225

- (Avaritia) fulvus 225

- (Avaritia) imicola 225

- (Avaritia) obsoletus 225

- (Avaritia) scoticus 225

- (Avaritia) wadai 225

- barbosai 249, 252

- bolitinos 226, 235

- boydi 249

- brevipalpis 225 
- brevitarsis 228, 235, 238, 242, 248, 250, 344, 345

- brunnicans 230

- bundyensis 231

- cacticola 249

- chiopterus 227, 228, 230, 235

- circumscriptus 226, 245, 250

- clastrieri 249

- crepuscularis 224

- cubitalis 249

- (Culicoides) lupicaris 225

- (Culicoides) newsteadi 225

- (Culicoides) pulicaris 225

- dewulfi 226, 227, 228, 235, 243

- edeni 249

- fulvus 235

- furens 226, 235, 244, 246, 252, 408

- guttipennis 249

- heliophilus 227

- histrio 231

- (Hoffmania) insignis 225

- hollensis 227

- imicola 223, 226, 227, 228, 229, 233, 234, 235, $236,237,239,244,245,246,250,251,252,396$, 407,455

- immaculatus 236

- impunctatus 226, 236, 244, 249, 407, 408

- insignis 228

- lahontan 249

- melleus 244

- mississippiensis 244,246,249, 252

- molestus 248

- (Monoculicoides) sonorensis 225

- nanus 249

- nubeculosus 236, 237, 239, 241, 247, 248, 251, $344,407,408$

- obsoletus 224, 225, 227, 228, 229, 230, 233, 235, $239,240,242,243,252,501$

- occidentalis 249

- odibilis 249

- ornatus 236

- oxystoma 250

- pachymerus 236

- paraflavescens 250

- parroti 230, 243

- peregrinus 245, 248

- pulicaris 235, 240,242, 243, 250, 408

- punctatus 228, 250

- pusillus 228

- (Remnia) schultzei 225
- schultzei 250

- scoticus 224, 227, 228, 233, 235

- sonorensis 224, 226, 228, 229, 235, 236, 237, $242,249,250,251,253$

- subimmaculatus 249

- varipennis 238, 242, 245

- wadai 235, 242, 250

Culiseta 196, 406

- melanura 210

cumin 101

Curculionidae 370

cutaneous

- myiasis 21

- streptothricosis 68

cyclical vectors 556

cyclodienes 93

Cymbopogon

- citratus 412

- nardus 101

cypermethrin 76, 241, 243, 246, 247

Cyprus 232

cyromazine 76

cytochrome P450 oxidase 98

cytoplasmic incompatibility $436,438,439$

\section{D}

daily activity patterns 61

dairy cattle $179,287,321,323,339,504$

dairy farms $55,363,576$

Dama dama 222

DDT 244, 245, 509

deer $226,252,285,500$

- bot fly 393

DEET (N,N-diethyl-3-methylbenzamide) 77, 236, $253,395,396,415$

Deinocerites 197

Delia spp. 362

deltamethrin $76,77,93,237,239,240,243,244$, $246,357,360,370,402,405,413,519,520,561$

dengue $223,348,435,437,444,447$

- transmission 405,439

Denmark 63, 230, 232, 233, 306, 318, 349, 454, 470

densoviruses 447

Dermacentor 176,340

- albipictus 176

- andersoni 70, 108,112

- occidentalis 176

- reticulatus 176

- variabilis $111,176,414$ 


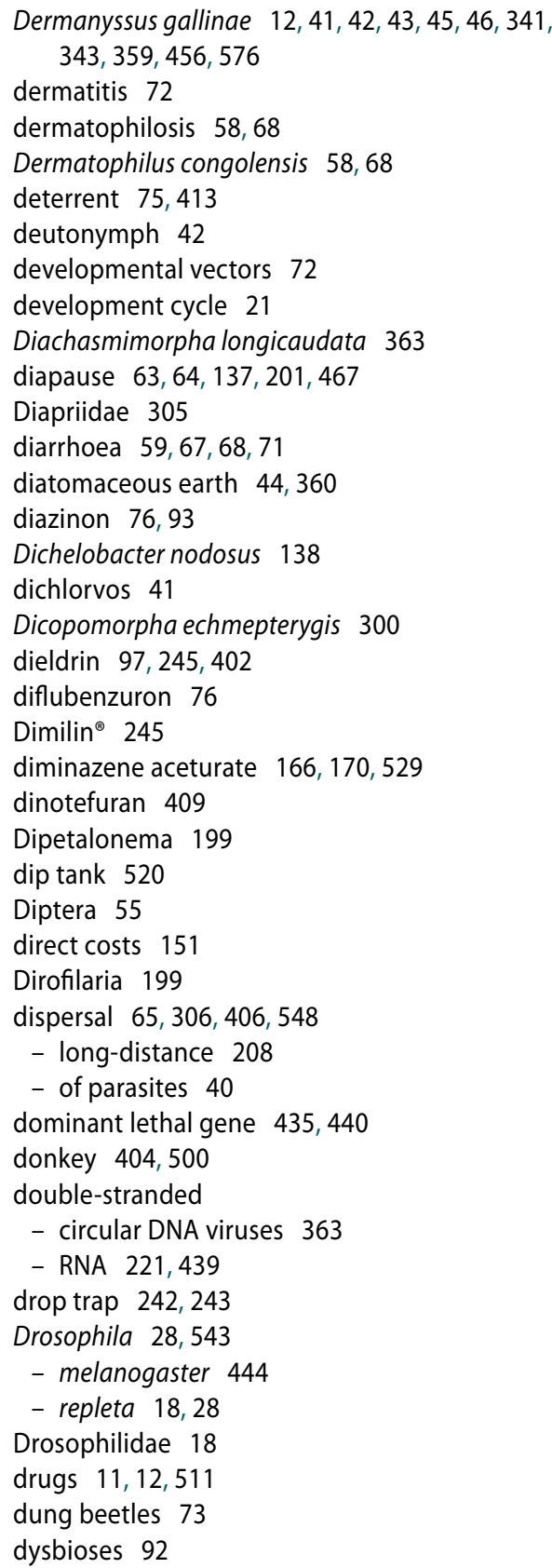

\section{E}

early warning system 30

ear-tags, insecticide-impregnated 243

eastern equine encephalitis virus (EEEV) 209, 339 economic

- benefit 555

- costs $138,506,512$

- impact 73

- loss 55, 56, 93, 175, 179, 413, 456, 506

- threshold 66, 277, 287, 370

ecosystems 445

ecosystem service 60,73

ecotourism 161

ectoparasite $11,38,40,41,46,100,105,109,114$, $242,337,340,341,343,353,359,360,389$

ectoparasitic 42

ectoparasitoid 300, 305

Ecuador 93, 98

EEEV - See eastern equine encephalitis virus

effector genes 445

Egypt 199, 200, 202, 359

Ehrlichia

- equi 176

- phagocytophilum 176

- ruminantium 180,181, 182, 183

EIP - See extrinsic incubation period

ELISA - See enzyme-linked immunosorbent assay

El Niño Southern Occilation (ENSO) 201

El Salvador 545

enantiomers 252

encephalitis 207, 339

- transmission 211

Encyrtidae 299, 300

Endolimax nana 68

endoparasite 92,99

endoparasitoid 300

endosulfan 519

endosymbiont 439

endosymbiotic bacteria 250

England 64

engorgement 106, 108

ENSO - See El Niño Southern Occilation

Entamoeba spp. 25, 68

Enterobacteriaceae 23

Enterobacter spp. 67,68

Enterococcus spp. 67

enteropathogens 67

enterovirus 27, 28, 69

entomopathogen $92,106,117,337,558$

entomopathogenic

- bacteria 249, 370

- fungi (EPF) 12, 44, 55, 60, 74, 110, 248, 337, 338, $341,347,348,350,353,356,357,358,455,462$ 
- nematodes (EPN) 253, 337, 365, 366, 369

- viruses 249

Entomophthora 560

- muscae 337, 362, 466, 558

- schizophorae 362

enzootic 184, 558, 559, 561

- circulation 182

- cycles 403

- stability 117

enzyme-linked immunosorbent assay (ELISA) 178, 186

EPF - See entomopathogenic fungi

epidemics 211

epidemiology $55,72,501,566$

epimastigotes 72

epizootic 195, 201, 202, 209, 362, 365, 498, 499, 501,504

- haemorrhagic disease 221, 222, 339

- vectors 213

EPN - See entomopathogenic nematodes

eprinomectin 93

equine $299,317,323,343,351$

equine encephalitis 195, 205, 206, 208

- cycle 211

- epidemiology 211

- vaccine 211

Eretmapodite 201

Erysipelothrix rhusiopathiae 69

erythro-6-acetoxy-5-hexadecanolide 400

Escherichia coli 22, 23, 24, 25, 26, 27, 28, 33, 55, 58, $67,68,455,456$

essential oil $44,60,75,101,102,103,278,396$

Ethiopia 443, 524, 527, 531

etiology 456

EU 201, 202

eucalyptus 101,237

Eucalyptus maculata var citriodora 236

eugenol 102, 103

Euphorbia prostrata 100

Europe 24, 31, 41, 42, 58, 59, 63, 64, 66, 77, 80, 136, $137,138,139,175,176,184,200,205,206,207$, $212,213,221,222,225,226,228,229,234,235$, $238,239,243,244,318,340,352,411,435,436$, $446,454,456,499,500,501,502,505,506,576$

European Union 17

exophilic 62,235

- behaviour 246

extrinsic incubation period (EIP) 213, 223

\section{$\mathbf{F}$}

face fly - See Musca autumnalis

facultative myiasis 28

false stable fly - See Muscina stabulans

Fannia 309, 362

- canicularis 18, 24, 25, 309, 339, 349, 351

- femoralis 351

Fanniidae 18, 362

Farinocystis tribolii 34

farming systems $12,159,161$

Faviviridae 199

feeding behaviour 22

- Stomoxys calcitrans 29

fenitrothion 238

fenthion 238, 244, 246

fenvalerate 76,242

filth fly 30

Finegoldia magna 112

fipronil 12, 43, 76, 93, 94, 97, 352, 576

fish 395

fixed costs 511

Flavivirus 203, 205

flea 12

fluazuron 93

fluff louse - See Goniocotes gallinae

flumethrin 550

Flypor $^{\oplus} 241$

fly strike $138,139,352$

Foleyella 199

foot and mouth disease 502,504

- virus 27

footbaths 164

formamidine 93

formamidine resistance 95

fowl pox virus 29, 339, 455

fowl ticks - See Argus persicus

France $71,137,227,230,232,239,241,500,504$, 506,564

French Polynesia 439

functional response 471

fungal

- infection resistance 350

- pathogens 30

- proteases 110

fungi 33,34

Fusobacterium necrophorum 68,138

G

GABA-gated chloride channel 97 
Galindomyia 197

Galleria mellonella 31, 369

gas chromatography (GC) 391

- -electroantennography 408

gastroenteritis 33

gastrointestinal (Gl) pathogens 24

Gavia immer 410

GC - See gas chromatography

gene drive $437,441,445,446,576$

gene silencing 439

genetic

- control 250,435, 537, 576, 578

- diversity 288

- heterogeneity 177

- modifications 437

genetically modified organisms 436

genotype $99,100,116$

German cockroach - See Blattella germanica

Germany 177, 229, 232, 240, 243, 318

Ghana 156, 525

GI - See gastrointestinal

Giardia spp. 25, 28, 59, 68

Glis glis 458

Glossina 13, 55, 59, 65, 71, 74, 77, 340, 354, 363, $390,397,399,400,401,415,436,439,442,509$, 549,576

- brevipalpis 170

- fusca 65

- fuscipes 354

- fuscipes fuscipes 65, 154, 156, 168, 355, 509, 555

- life cycle 72

- longipalpis 65

- longipennis 355,516

- morsitans centralis 400,402

- morsitans morsitans 77, 400,402

- morsitans submorsitans 65,555

- pallidipes $77,170,354,355,402,516$

- palpalis gambiensis $154,155,164,168,402,442$, 516, 521, 551

- palpalis palpalis 402

- swynertoni 170

- tachinoides 65, 154, 155, 164, 402, 521, 551, 555

Glossinidae 55, 65, 340, 390, 398

glutathione S-transferase 96,98

goat $11,137,181,182,202,226,340$

Goniocotes gallinae 38

Goniodes

- dissimilis 38

- gigas 38 gonotrophic

- cycle 228

- status 228

GPCR - See G-protein coupled receptor

G-protein 102

- coupled receptor (GPCR) 94

Great Britain 63

Greece 137, 227

Guadeloupe 182

Guatemala 545

Guinea 156,168

H

habitat modification 234

Habronema 339

Haemaphysalis 176, 340

- concinna 176

- punctata 176

Haematobia 64, 69, 395

- irritans $12,58,59,62,64,67,69,72,73,74,75$, $76,77,79,321,353,354,390,395,397,398,401$, 408,412

- irritans exigua $64,66,67,76,340$

- irritans irritans $64,66,340$

Haematobosca 64, 65, 69

- latifrons 64

- squalida 64,71

- stimulans 64

haematophagous insects 29, 63, 64, 66, 92, 221

Haematopota 61

- pluvialis 71

haemoglobinemia 185

haemoparasites 117

haemorrhagic bovine filariasis 72

Haemosporidians 222

Haller's organ 390, 413

Halophytophthora 249

- batemanensis 249

Hammondia spp. 68

Hantavirus 455

haplodiploid 319

haplotype 100, 115

Havardia albicans 103

Hawaiian islands 224

head louse - See Cuclotogaster heterographus

health risks for livestock 457

heartwater 180

hedgehogs 111 


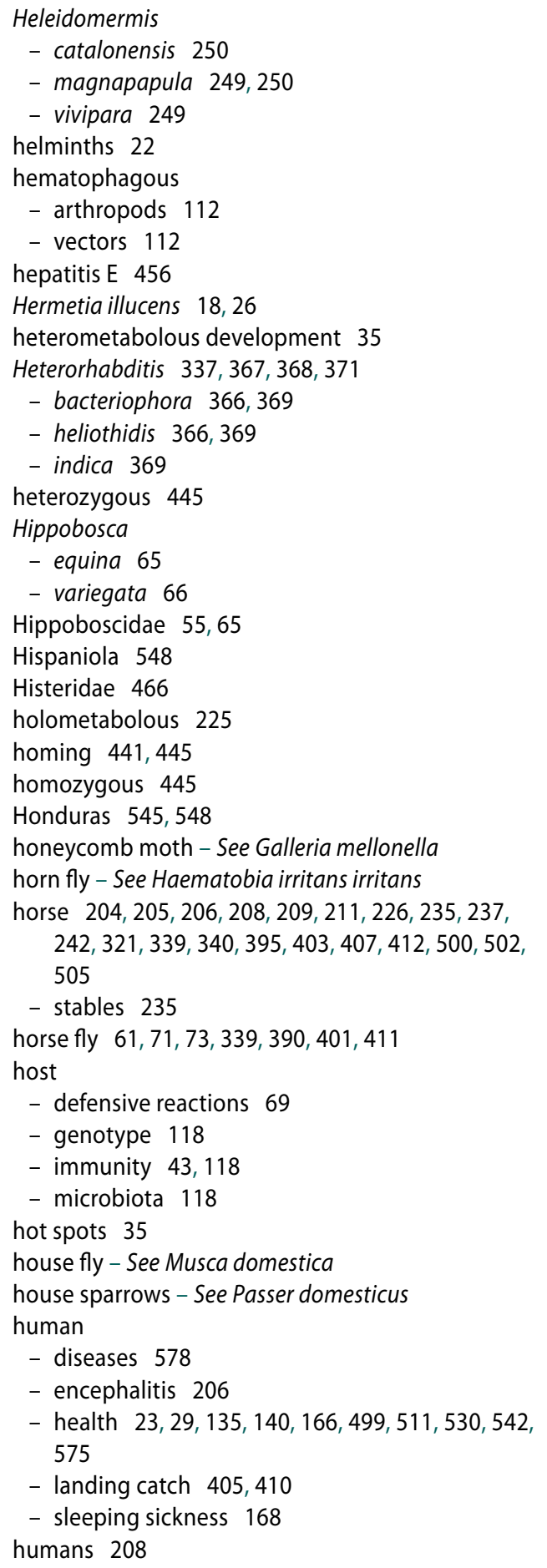

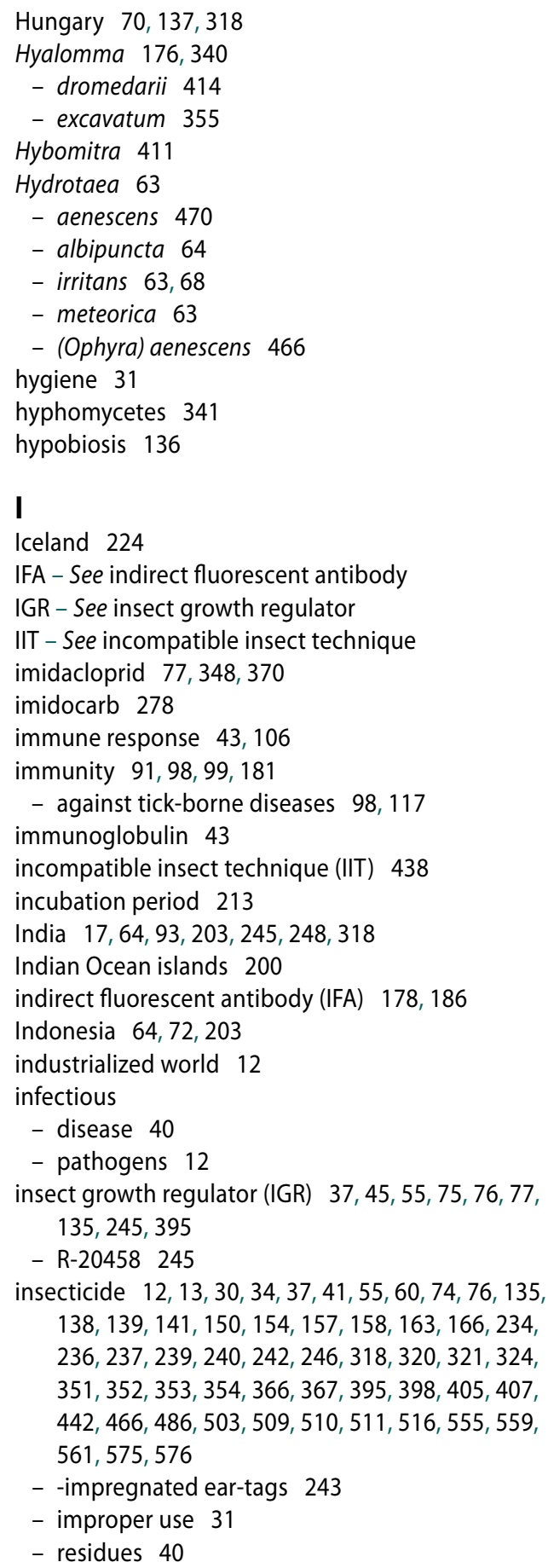


- resistance $12,31,77,157,247,338,349,370$, $408,412,415,435,577,578$

- toxicity 435

- -treated cattle (ITC) 148, 526, 528

- -treated fences 156, 163

- -treated nets (ITN) 77, 245, 516

- -treated targets (ITT) 148, 152, 155

insect pest management 537

integrated control 171,402,577

integrated pest management (IPM) $13,17,29,43$, $45,46,55,60,73,108,147,148,276,299,321$, $324,338,348,352,360,370,372,404,442,446$, $466,533,558,560,561,576,578$

integrated tick management 277

intensively reared stock 454

interspecific

- communication 390

- competition 308

- variation 396

intestinal parasites 37

intraspecific

- communication 390

- competition $472,477,478$

- variation 396,412

intrinsic

- fitness 444

- rate of increase 468

inundation 470

inundative releases 465,484

IPM - See integrated pest management (IPM)

Ireland 232

iridescent virus

- transmission 249

Iridoviridae 249

Isaria

- farinosa 345, 346, 353

- fumosorosa 344, 353, 354

isobenzam 414

isoeugenol 102

isometamidium chloride $166,170,529$

Isospora spp. 68

Israel 137, 206, 207, 227, 232, 233, 318

Italy 232,406

ITC - See insecticide-treated cattle

ITN - See insecticide-treated nets

ITT - See insecticide-treated targets

ivermectin 76, 93, 94, 238, 276

Ixodes 340,355

- hexagonus 111
- neitzi 413

- pacificus 176

- persulcatus 176

- ricinus 111, 176, 456

- scapularis 92, 176, 358, 368, 413

- spinipalpis 176

Ixodidae 340, 355

Ixodoidea 390, 413

J

Jamaica 548

Japan 58,454

Japanese encephalitis virus (JEV) 195, 203, 212

- cycle 204

- epidemiology 204

- genotypes 203

- transmission 203

- vaccines 205

JEV - See Japanese encephalitis virus (JEV)

K

kairomone 79, 251, 252, 390, 391, 392, 393, 394, $398,401,402,403,405,408,410,413$

Kenya 76, 170, 199, 318, 404, 405, 515

keratoconjunctivitis 58,68

Klebsiella spp. 67

knock-down effect 283

koinobionts 300, 305

Korea 203, 228, 318

Kunjin (KUNV) 203, 206

KUNV - See Kunjin

L

La Crosse virus 223

lactones 93

Lagenidium giganteum 248

Lama glama 222

lambda-cyhalothrin 239, 240

landscape management 135

La Réunion 157, 437, 439, 555, 556, 558, 560, 567

large chicken louse - See Goniodes gigas

larval development 20

Larvicides 244

Latin America 113, 209

LD50-LD90 239, 240, 241, 486

Lecanicillium

- longisporum 248,344, 345

- psalliotae 355,358 


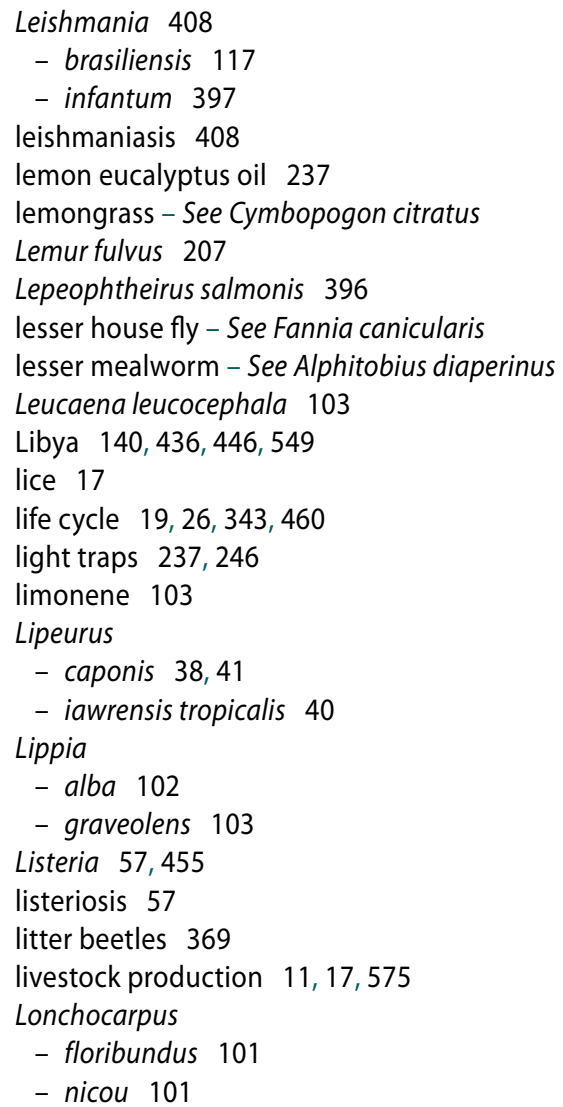

lower income countries 12

Lucilia 18, 27, 137, 339, 398

- caesar 138

- cuprina 135, 137, 340,352, 353

- illustris 138

- sericata 135, 136, 137, 340, 352

lumpy skin disease $55,57,71,80,557$

lure-and-kill approach $30,371,393,397,398,400$, $405,409,414$

Lutzomyia 408

- longipalpis 390, 395, 399, 400, 401, 408, 409

lyme disease 57

\section{M}

Macrocheles muscaedomesticae 76, 466

Macrochelidae 76, 466

macrocyclic lactone acaricides $93,94,97,135,141$

Madagascar 199, 201, 206

malaria $92,348,404,406,435,437$

malathion $41,93,96,238,244,245,246$
Malaya 198

Malaysia $64,203,318$

Mallophaga 38

- bird louse 39

mammillitis 71

Manilkara zapota 100

Mansonia 196, 201, 204, 404

manual spraying 150

manure $20,26,488$

- poultry 28,30

mark-recapture 481,492

mass-trapping 406, 408

mastitis 69,92

material safety data sheet (MSDS) 34

mathematical modelling 212

Mauritania 199, 200

Mauritius 318, 556

maxillary palps $38,390,407,411$

Mayotte 199

m-cresol 79

mechanical

- control 13,31,78, 234

- vector 25,69,411,466,501, 556

Mediterranean 136

Melinis minutiflora 108

Menacanthus stramineus $38,39,40,341$

meningitis 207

meningoencephalitis 204

Menopon gallinae 38,40

Mentha piperita 75

Mermithidae 249

Merodon equestris 363

metamorphosis 136, 196, 467

Metarhizium 110, 362

- acridium 345

- anisopliae 30,75, 110, 248, 337, 341, 342, 344, $345,346,347,348,349,350,351,352,353,354$ $355,356,357,358,359,360,361,370$

- brunneum 344,358

- flavoviridae 355, 358

- pingshaense 344

- robertsii 344, 346

metathorax 198

meteorological conditions 33

methomyl 367

methoprene $37,76,245$

6-methyl-5-hepten-2-one 408,412

metofluthrin 77 
Mexico 93, 96, 108, 114, 206, 209, 276, 277, 287, $436,446,542,544,545,548,549$

microbes 275

microbiota $91,106,111,112$

microclimate 33,368

microencapsulation 396

microenvironment 107,108

microhabitat 317,319

microorganisms 195, 340, 399, 466

microsatellites 113

Microsporidia 249

Microtus

- arvalis 457,458

- savii 458

Middle East 57, 58, 63, 199, 202, 205, 206

midges $224,227,228,234,240,243,244,246,248$, $253,339,344,390,396,397,401,455,501,503$, 577

migration 33

mites 17

model simulations 484

monitoring 31

Moraxella bovis $55,58,68$

Morellia 63

- hortorum 63,64

- simplex 63

Morganella morganii 67

Morocco 137, 200, 406, 409

mosquito 201, 234, 238, 244, 248, 251, 339, 343, $345,370,371,390,396,398,399,400,401,402$, $409,415,435,436,440,441,443,561,567,576$

- -borne diseases 195

- control 403

Mosquito Magnet trap 252, 405

mouthparts 56,69

MSDS - See material safety data sheet

multivoltine 62

Murray Valley 203

Musca 18, 22, 395

- autumnalis 55, 58, 59,62,63,66, 68, 69, 72, 73, $76,79,321,411$

- crassirostris 63

- inferior 63

- planiceps 63

- vetustissima $58,63,67,73,76$

Musca domestica $12,18,23,24,25,30,31,55,57$, $58,59,62,67,69,70,73,74,75,76,79,299,306$, $309,323,337,339,349,350,351,362,363,366$, $370,371,395,399,460,466,470$
- biological control 26

- overwintering 24

- parasitoids 317

Muscidae 18, 24, 55, 62, 299, 339, 362, 390, 398, 412,465

Muscidifurax 299, 305, 306, 307, 308, 309, 310, 319

- raptor 74, 300, 301, 303, 307, 308, 309, 317, 318, $319,321,322,323,466$

- raptorellus 300, 301, 304, 306, 318, 321, 322, 323

- uniraptor 302, 322

- zaraptor 300, 301, 303, 306, 308, 318, 321, 323

Muscina stabulans 18,27

- life cycle 27

muscoid flies 22

Mus musculus 458

Mycobacterium bovis 456

mycoinsecticides 362

mycopesticides 344

myiasis $11,21,55,135,339$

- cavitary 136

- cutaneous 21

- facultative 28

- wound 21

\section{N}

nagana $70,71,77,160,340,401$

naled 238

napier grass 108

Nasonia vitripennis $300,301,305,307,308,318$, $319,322,323,324,466$

neem $101,236,348,370$

Nematocera 55, 201

nematode $11,30,34,72,222,249,250,337,366$, $367,368,370$

neonicotinoids 77,322

Nepal 203

Nepeta cataria 75,412

- catnip oil 75, 395

New Caledonia 106, 278

Newcastle disease $24,25,454,455$

New Zealand 58, 137, 138, 139, 224, 318

Nicaragua 545, 548

Nigeria 515

non-biting-flies 22

non-chemical control 30

non-target effects 111

North Africa 58,64 
North America $41,59,61,63,96,138,139,176,232$, $235,305,318,403,446,491$

northern fowl mite - See Ornithonyssus sylviarum

Norway 318,323

Nosema 319

- disease 320

Notonecta 400

novaluron 76,93

\section{0}

obligate parasite 135

Obsoletus group 225, 228, 231

Oceania 114, 318, 340

Ochlerotatus 201

- canadensis 210

- melanimon 210

- sollicitans 210

- taeniorhynchus 201,210,406

Ocimum 104

1-octen-3-ol 111, 252, 347, 394, 407

octenol 79

octopamine receptor 94

Odocoileus hemmionus 176

odour baited traps 30

Oestridae 135

Oestrus ovis 135, 136

olfactometer 79

olfactory

- cues 307, 389, 394, 407, 411

- receptor neuron (ORN) 390, 391

- receptors 111, 390

- stimuli 410

- system 389

omnivorous 36

Onchocerca

- ochengi 410

- volvulus 409,410

onchocerciasis 409

Onderstepoort light trap 229, 237, 244, 246

One Health 135, 203, 278, 279, 555, 575, 577

Onthophagus 73

ootheca 36

Ophyra 22

- aenescens 18,25, 26, 362

- capensis 25

- leucostoma 25

Oreotragus oreotragus 413

organochlorides 97

organophosphates $93,94,135,141,238$
- resistance 96,97

organophosphorus insecticides 93

oriental cockroach - See Blatta orientalis

ORN - See olfactory receptor neuron

Ornithonyssus sylviarum 41, 42, 341, 359

ornithophilic

- mosquitoes 208, 211

- species 227,410

Oropouche virus 222

Orthobunyaviridae 224

Oswaldofilaria 199

outbreak 500

- control 503

oviparous 62

Ovis

- aries musimon 222

- canadensis 222

ovoviviparous 36

oxytetracycline 182

$\mathbf{P}$

Pachycrepoideus vindemmiae 317,318

Paecilomyces fumosoroseus 248, 346, 355

Pakistan 203

palindromic repeats 441

Panama 211, 443, 542, 545, 548, 549

Panicum 108

Papua New Guinea 64, 203, 205

Parafilaria bovicola 55,72

parameres 198

parasitaemia 162

parasites $18,31,33,39,66,68,70,72,186,250,275$, $276,282,287,338,341,347,365,366,402$

- dispersal 40

- life cycle 105

parasitism 250, 300, 302, 307, 317, 371, 477

parasitoid 30, 55, 60, 74, 248, 249, 299, 372, 465, $466,468,478,484,486,557,561$

- house fly 317

- wasp 74

paratransgenesis $112,436,439$

Passer domesticus 397, 454

Pasteurella multocida 69

pasture 108,110

- rotation 108

pathogen $12,22,24,26,27,31,33,34,37,55,60$, $68,91,107,175,179,180,184,250,299,320$, 339,389

- of ticks 109 
- surveillance 393,409

- transmission 29,62, 66, 176, 445, 453

- transmission routes 56

pathogenic

- agents 36

- microorganisms 22

- microorganisms, transmission 29

- viruses 408

pathogenicity 454

$\mathrm{PCR}$ - See polymerase chain reaction

p-cresol 79

pediculosis 38,238

Pennisetum purpureum 108

Peptoniphilus indolicus 68

Peribunyaviridae 222

Periplaneta americana 35,36

peritrophic membrane 249

permethrin $43,76,95,237,240,242,243,247$

pesticide $11,29,30,34,45,73,203,370,371,372$, 446,575

- resistance 337

pest(s) 12, 343, 390, 393, 394, 395

- control 13,23,34,372,397

- management 389,460,462

phenol 79

phenotype 105, 116

phenyl acetaldehyde 398

phenylpyrazole 76,77

pheromone $37,79,251,369,390,400,407,409$, $413,414,415$

- z-9-tricosene 367

Philippines 203, 318

Philonthus 76

Phlebotominae $390,398,408$

phlebotomine sand flies 396

Phlebotomus 395, 408

- argentipes 409

- papatasi 409

Phlebovirus 199

Phormia 18, 27

- regina 138

Photorhabdus 365, 371

- luminescens 371

phoxim 43

Phthiraptera 38

phylogenetics 113

phytochemicals 398

phytophagous 36

Phytophthora gonapodyides 249
Pichia pastoris 281

pig 11, 12, 203, 321, 323, 340, 455, 456, 502, 576

Pilocarpus microphyllus 101, 103

pinkeye 58,68

piperonyl

- alcohol 102

- butoxide 77, 95, 98, 237, 242

Piper tuberculatum 100

pirimiphos methyl 240

piroplasmosis - See babesiosis

piroplasms 183

plant oils 139

Plasmodium 199, 348

- chabaudii 397

- falciparum 397,445

- gallinaceum 199

- relictum 397

Plistophora sp. 249

p-menthane-3,8-diol 236, 396

poliovirus 69

Pollenia rudis 362

polyandry 65

polymerase chain reaction (PCR) 178, 181, 186, 196 population

- management 139

- modification 442

- suppression 442

Portugal 227, 505

poultry $11,12,17,299,309,317,321,322,324,337$, $338,340,341,343,351,352,359,360,366,369$, $372,395,454,457,460$

- diseases 18

- facilities 30,34

- farms 22, 23, 28, 32

- houses 23, 26, 31, 33, 35, 36, 37, 42

- manure 28,30

- mites 41

- mites, life cycle 42

- pests 45

- production 18,21,24

- red mite - See Dermanyssus gallinae

pour-on products $77,149,150,157,163,169,237$, $238,240,241,242,253,509,510,520,524,527$, $550,557,558,564$

pour-on treatments 171,221

predators $18,44,46,60,62,76,107,248$

- of ticks 109

prevention $11,12,18,29,31,37,46,95,140,179$

proboscis $18,19,22$ 
production systems 528

prophylaxis 559

propoxur 246, 414

Proteus spp. 27, 28,67

protonymph 42

Protophormia terraenovae 138

protozoa 11, 22, 28, 34, 68, 70, 222, 339, 340

protozoan parasites 454

Providencia stuartii 67

Pseudomonas spp. 67

Psila rosae 362

Psorophora 197

- columbiae 210

- confinnis 210

Pteromalidae $74,299,300$

public health $37,213,339,498,576$

public-private partnerships $275,276,279$

Puerto Rico 107, 279, 281, 286, 318

pupation 20

push-pull strategy 397,413

pyrethroid $34,37,41,76,93,94,95,135,141,149$, $150,152,156,159,164,165,166,168,170,236$, $238,239,435$

- resistance 96,98,99

pyriproxifen 37,76

Pythium prolatum 249

Q

Q fever 57

quality control 319

quarantine 277

Quassia amara 101

\section{$\mathbf{R}$}

Rana rinibunda 207

Rattus

- norvegicus 455, 457, 458, 459

- rattus 458

recombinant antigen 275

red fox - See Vulpes vulpes

refractoriness 364

refractory 364

- ticks 367

remote sensing 442

Reoviridae 221, 222

repellent $77,91,106,157,186,234,236,247,253$, $308,359,390,391,393,394,396,407,409,410$, 412,559

reservoirs 341 resistance $45,91,116,187,360,444,466$

- acaricide 276, 288

- arthropod 370

- insecticide 370

- to fungal infection 350

- to insecticides 247, 349

- to pesticides 337

resistant parasites 99

Rhagionidae 55,61

Rhipicephalus 175,340

- annulatus 185, 275, 276, 282, 285, 355, 367, 368

- appendiculatus 91, 176, 357

- australis 91, 106, 118, 278, 279, 282

- decoloratus 91, 104, 185

- evertsi 185

- geigyi 185

- sanguineus $101,176,355,356,357,358,414$

- simus 176

Rhipicephalus (Boophilus) microplus 70, 91, 176, 185, $275,276,281,282,283,285,356,357,367,368$, $370,556,561,576$

- genome 92

- life cycle 107

- natural enemies 110

- resistance 115

rickettsiae 22, 340

Rickettsia ruminantium 180

RIDL 440, 443

Rift Valley fever virus (RVFV) 12, 57, 71, 195, 199, $212,339,402,404,466$

- epidemiology 201

- life cycle 201

- transmission 201

- vaccines 202

risk analysis 460

RNA interference 251, 439

RNA-sequencing 251

Romania 137, 206, 208

Rosmarinus officinalis 103

Ross River virus 394

rotavirus 24,69

rotenone 101

RT-qPCR 228

Russia 208, 232

Ruta graveolens 103

RVFV - See Rift Valley fever virus

\section{S}

salivary 
- gland hypertrophy virus 337,363

- proteins 113

Salmonella 22, 24, 26, 27, 28, 33, 45, 58, 67, 68, 455

- dublin 67

- typhimurium 33,67

Salmo salar 396

sandfly - See Lutzomyia longipalpis

Sanger DNA sequencing 196

sanitation 32,73

Sapindus saponaria 101, 103

saprophagous 62,467

Sarcocystis spp. 68

sarcocystosis 59

Sarcophagidae 135

Saudi Arabia 200, 202

SBV - See Schmallenberg virus

scabies 238

scanning electron microscope 356

Scarabaeidae 73

Scathophaga stercoraria 362

Scathophagidae 362

Schmallenberg virus (SBV) 222, 224, 339, 407, 497, $498,499,500,501,576$

Sciurus

- carolinensis 207

- niger 207

- vulgaris 458

Scotland 226, 236, 244, 252

screening $235,395,407$

screens $154,155,159,245,509,510,521,522$

screwworm 13, 135, 139, 446, 568

sea lice - See Lepeophtheirus salmonis

semiochemicals $45,109,111,231,251,253,308$, 389,576

Senegal $182,199,228,527,530$

sensilla coeloconica 251

sensory receptors 24

septicaemia 365

sequence 441

Serratia marcescens 112,370

Setaria 199

sexual dimorphism 28, 36

shaft louse - See Menopon gallinae

shearing 115

sheep $11,135,200,202,210,221,222,226,230$, $237,240,241,243,252,338,340,352,404,455$, $456,500,502,506$

- blowfly 339

- head fly - See Hydrotaea irritans
Shigella spp. $25,28,67,68$

Siberia 185

Silene 398

Simarouba versicolor 101, 103

simulation model 466,470

Simuliidae 248, 390, 394, 398, 409, 410

Simulium

- arcticum 410

- damnosum 400,410

- euryadminiculum 410

- ochraceum 410

SIT - See sterile insect technique

sleeping sickness 166,516

slow release 44

snipe fly $61,62,74$

social challenge 556

socio-economic

- data 442

- development 11

- impacts 13

socio-ecosystems 446

Sodalis 438,439

Solanum verbascifolium 110

solid phase microextraction 391

South Africa 93, 99, 137, 151, 180, 182, 199, 202, $212,230,235,237,318,352,505$

South America 58, 71, 114, 160, 184, 207, 209, 211 , $212,213,232,318$

South East Asia 63,64

Spain 70, 137, 202, 223, 227, 230, 233, 239, 456, 505

Spalangia $74,299,305,309,310,319,564$

- cameroni 30, 74, 300, 301, 304, 306, 307, 308, $309,317,318,319,321,322,323,324,465,466$, $468,471,478,484,491$

- drosophilae 317

- endius 74, 300, 301, 305, 308, 309, 317, 318, $321,322,323,324,561$

- nigra 300,317, 318

- nigroaenea 300, 307, 309, 317, 318, 322, 323, 557,558

Spaniopsis 61

spinosad 43, 74, 322, 370, 398, 491

spirochaetes 340

Splangia 309

splenomegaly 178

Sri Lanka 203

stable fly - See Stomoxys calcitrans

staphylococci 27,28 
Staphylococcus 22, 23

- aureus 55, 58,67,68,69, 112

- chromogenes 112

- sciuri 112

Steinernema 337, 366, 367, 368, 371

- arenarium 369

- carpocapsae 366, 368, 369, 370

- feltia 34

- feltiae $366,367,369$

- glaseri 366, 368

Stephanofilaria 72

- stilesi 55, 72, 340

sterile insect technique (SIT) $139,140,148,402$, $435,436,437,442,446,510,527,530,542,545$, $549,561,567,576,578$

sticky traps 30,37

Stomoxys 19, 22, 64, 69, 73, 156, 157, 176, 395

- calcitrans 13, 18, 22, 28, 29, 30, 58, 59, 62, 64, $66,70,71,73,74,75,76,79,299,305,309,323$, $339,349,350,390,395,397,399,400,401,412$, $413,437,465,470,556,560$

- niger 64,71,556, 560

- sitiens 64

stored food products 31

Stratiomyidae 18

streptococci 24

Streptococcus 33

- dysgalactiae 68,112

Stylosanthes 108

suction traps 252

summer mastitis 58

- pathogens 68

Suna trap 404

Suragina 61

surra $58,70,160$

surveillance $225,229,230,231,277,278,389,390$, $401,403,404,405,415,456,497,499,501,503$, $505,545,575,577$

sweat flies 62,64

Sweden 227

sweet itch 222, 339, 407, 577

swine 317

Switzerland 235

Sylvilagus floridanus 207

symbiont $44,302,365,435,436,437,439$

symbiotic association $367,436,444,576$

Symphoromyia 61

synanthropic

- pests 339
- species 25

synergism 79, 351, 359, 371, 394, 401, 403, 407, 411

synomones 390

synthetic pyrethroids 77,93

Syrphidae 363

T

Tabanidae $55,61,390,398,411$

tabanids $57,69,70,72,77,79$

Tabanocella denticornis 70

Tabanus 176, 411

- bovinus 70

- fuscicostatus 69,71

- importunus 71

- nebulosus 71

- pallipennis 69

- rubidus 70

Tachinaephagus

- stomoxicida 557

- zealandicus $300,301,306,310,318,320$

Tagetes minuta 103

Taiwan 203

Tamias striatus 207

Tanzania 200, 530, 531, 533

target $55,60,78,154,157,159,163,171,509,510$, $511,521,522,528,529,531,532$

tarsomeres 67

temephos 244,409

Tenebrionidae 466

terrestrial species 35

tetracycline 440

tetramethrin 247

Thailand 67, 203, 318, 405, 439

The Fly Simulator 465

Theileria

- annulata 183

- parva 183

theileriosis 340

Thelazia 55

- rhodesii 72

- skrjabini 72

thelytokous development 302

the Netherlands $43,138,243,454,455,457$

thermophile 468

thymol 102

tick $41,70,157,201,355,390,401,413,520,549$, 557,564

- aggregation 118

- behaviour 109 
- control 181,358,367,531, 550

- genotype 100

- survival 107, 108

tick-borne disease $92,111,117,159,175,276,340$, $413,520,528$

- immunity 98

tick-resistance breeds 186

time savings 151

Togaviridae 199

TOT - See transmission, transovarial

toxaphene 414

toxic baits 79

toxicity 77,158

- of insecticides 435

toxic sugar baits 79

toxins 60

Toxoplasma 28

- gondii 68,455, 459

Toxorhinchites 198

Toxorhynchitinae 196

transboundary diseases 501,506

transfluthrin 77

transgene $436,441,444,445$

transgenic insects $435,437,440,441,444,446$

transhumance 164

transmission $22,55,347,362,404,413,444,454$, $456,501,576$

- biological 58,59,71

- cyclical 22,71

- iatrogenic 179

- maternal 320

- mechanical 57,59,69,176

- risk 198

- threshold 148

- transovarial (TOT) 176, 180, 185, 201, 223

- transstadial 180

transposable elements 437

traps $30,55,60,75,78,148,152,153,155,226,229$, $246,252,324,353,371,393,396,397,400,401$, $402,403,404,405,406,410,411,412,442,503$, $509,510,511,516,521,522,531,558,559,561$, 564

- BG Sentinel 229, 244

- drop 230

- emergence 233

- light 229, 237

- miniature CDC light 229

- Onderstepoort 229, 237

- sticky 231
- suction $\sim 231$

- truck 231

Trichogrammatidae 319

Trichomalopsis sarcophagae $300,301,305,318,323$

Trichomonas spp. 68

Trichopria 305, 310, 557, 558

- nigra 305

- stomoxydis 305

Trichuris 28

Trinidad-Tobago 548

triphenyl phosphate 95

Tropidurus torquatus 110

Trueperella pyogenes 68

trypanocides $163,166,168,529,531,533$

trypanosensitive cattle 163

Trypanosoma 55, 59, 71, 340, 401

- brucei 70,72, 168, 340

- brucei brucei 59,160

- brucei rhodesiense 166,167

- brucei sensulato 164

- congolense 59, 70, 71, 72, 148, 160, 164, 166, 168,340

- evansi $58,70,80,160$

- theileri 72

- vivax $58,70,71,72,148,160,164,166,168,340$

Trypanosomatidae 222

trypanosome $55,71,162,163,168,170,397$

- resistance 171,439

- transmission 164

trypanosomosis $12,147,340,401,442,509,510$, $515,516,528,532,555$

- cyclical 160

- endemic 160, 163

- interface 160, 163

- mechanical 160,164

- sylvatic 160

trypanotolerance $161,162,163$

trypomastigotes 72

tsetse fly - See Glossina

- control 148, 156, 157, 159, 163

- density 157

- eradication 147

Turkey 202, 232

U

Uganda 156, 160, 166, 205, 318, 509, 515, 521, 522, $524,525,526,527,528,531$

UK $139,227,230,231,232,237,367,455,502$

ultraviolet light 30 


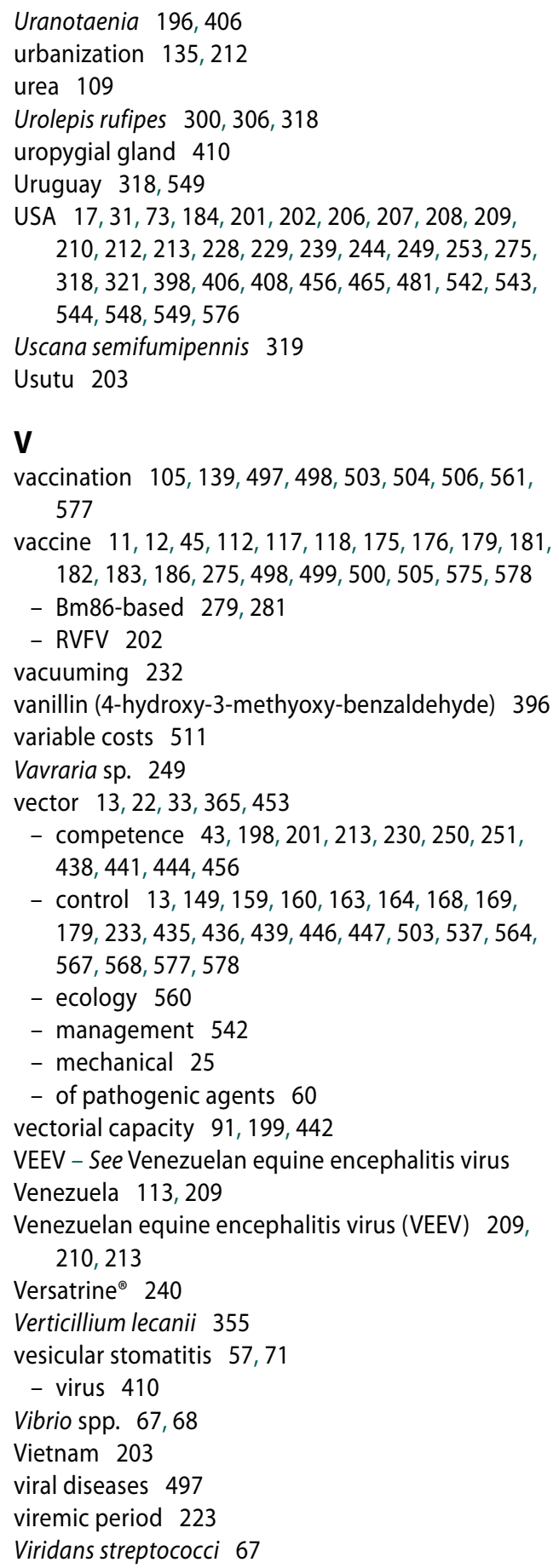

virus $11,12,22,33,37,55,69,71,198,199,337$, $340,402,454$

- avian influenza 26

- foot-and-mouth disease 27

- H5N1 24

- HPAl-H5N1 24

- lumpy skin disease 55

- transmission 444

visceral leishmaniasis 397

visual

- cues 411

- targets 30

volatile(s) 111

- compounds 395

voltage-gated sodium channel $95,96,98,99$

voracious 36

Vulpes vulpes 456

\section{W}

warble fly 55

waterbuck 390

water buffalo 408

WEEV - See Western equine encephalitis virus

West Africa 160

West Bengal 203

Western equine encephalitis virus (WEEV) 209

West Nile virus (WNV) 195, 203, 205, 212, 223, 339, $398,403,456,466,575$

- epidemiology 208

- transmission cycle 207, 208

- vaccines 208

WHO cone test 237,247

whole-farm systems analysis 141

wind-borne dispersal 212

wing louse - See Lipeurus caponis

wMel Wolbachia 444

WNV - See West Nile virus

Wohlfahrtia magnifica 135, 136

wohlfahrtiosis 137

Wolbachia 112, 250, 302, 435, 438, 439, 444, 578

worms 28

wound myiasis 21

Wuchereria 199

$\mathbf{X}$

Xenorhabdus 365

- poinarii 371 


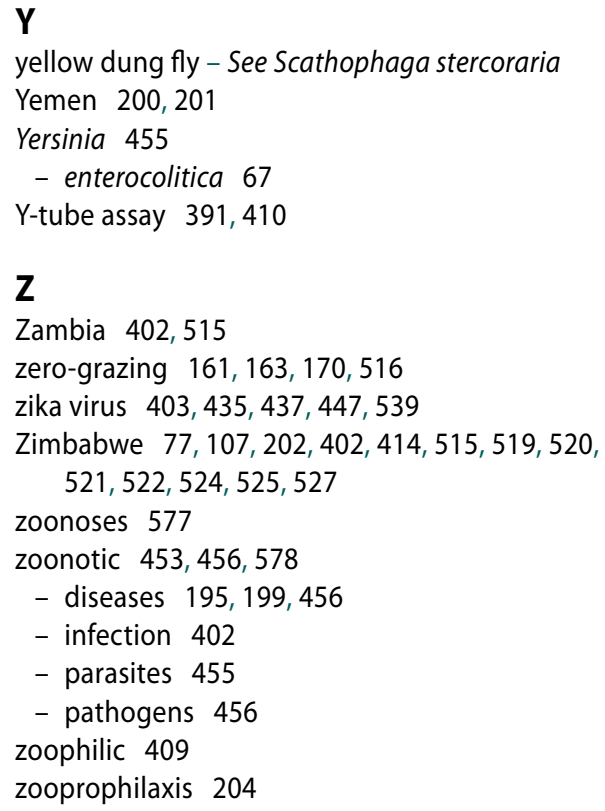


